\title{
Potter
}

2nd. EDITION

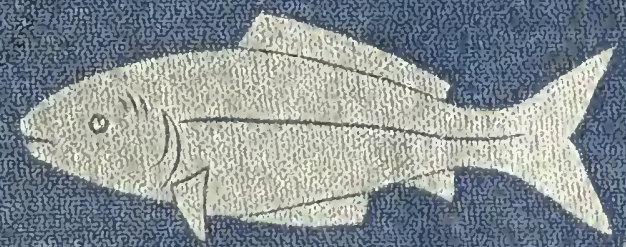

(1) 가 


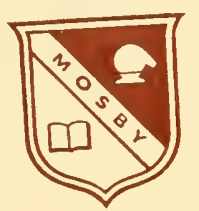

INSTRUCTOR'S COPY

Compliments

of

THE C. V. MOSBY COMPANY

ST. LOUIS, MO. and SAN FRANCISCO, CAL.

SENT AT THE REQUEST

OF

MR. FRANK A. VOLK

Your opinion of this book will be appreciated when your review of it bas been completed. 
几

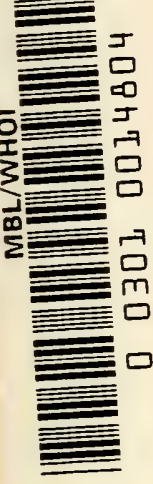





\section{TEXTBOOK $\mathrm{OF}$ \\ ZOOLOGY}

BY

\section{GEORGE EDWIN POTTER, Ph.D.}

Professor of Zoology, Agricultural and Mechanical College of Texas, Formerly Professor of Zoology, Baylor University

SECOND EDITION

With 445 Text Illustrations

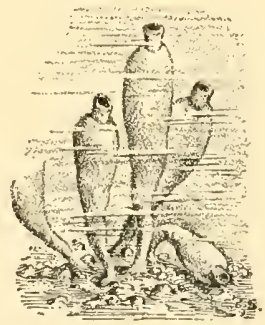

ST. LOUIS

THE C. V. MOSBY COMPANY 
Copyright, 1938, 1947, By The C. V. Mosby Company (All rights reserved)

Printed in U. S. A.

Press of

The C. V. Mosby Company

St. Louis 


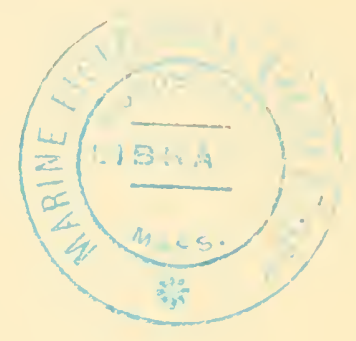

DEDICATED TO

\section{PROFESSOR FRANK A. STROMSTEN}

A friend and an inspiration

to the student 



\section{PREFACE TO SECOND EDITION}

The present edition represents a revision of certain parts of several ehapters, such as those dealing with Annelida, Genetics, Eugenies, Internal Regulation and Endoerines, Physiology, and Phylogenetic Relations of Animals. A bricf section on Mammalian Development has been added. Numerous minor corrections or improvements have been made throughout. Several illustrations have been added and others improved.

In addition to the acknowledgements ineluded in the preface to the first edition, the author wishes to acknowledge the help of Dr. Kelshaw Bonahm of the Fish and Game Department, Agricultural and Iechanieal College of Texas, on the ehapter dealing with Pisees. At this point recognition is made of assistance given by Mr. Gordon Gunter in revising the list of animals of the Texas Gulf Coast in the chapter on Marine Zoology. The author is also indebted to Dr. Fred L. Kohlruss, Biology Department, University of Portland (Oregon), for numerous useful suggestions. Many valued suggestions have been reeeived from individuals in a number of other institutions where the first edition has been in use. The author's indebtedness and appreciation is also expressed to $\mathrm{Mr}$. Phil T. Williams who has furnished a number of the new illustrations. Finally, appreciation is expressed to Agricultural and Mechanical College of Texas for cooperation in numerous ways to assist in making this revision.

George E. Potter.

College Station, Texas 


\section{PREFACE TO FTRST EDTTION}

The important problems of life are common to all animals (including man) as well as to plants. It should be the purpose of a texthook in general zoology to present the animal kingdom in a logical and natural way and at the same time carry the interpretation of the facts in terms of the principles involved. It is exceedingly difficult to strike the ideal balance between the necessity of presenting sufficient, factual, "type" material in order that the student will have the requisite knowledge of classification, structure, function, development, and organography to appreciate the discussion of principles, and the opposite temptation to go into endless discussions of theories and rules, the comprehension of which is unquestionably beyond the capacity of the student who has not become grounded in fundamentals of animal make-up. Of course it is usually hoped that the laboratory division of the course will supply this needed foundation. It seems reasonable that the ultimate aim of the teacher of introductory zoology should be to bring the student to a fundamental and well-grounded understanding of the principles involved in all of the living processes. It is extremely difficult to skim this information from the top of the entire body of zoological knowledge, as one can skim eream from a crock of milk, and hand-feed it to the waiting student mind. Apparently there must be a certain amount of personal acquisition of the principles of the subject through attaining a clear-cut knowledge of the complete biology of a series of representative animals. Each of these representatives, since it is a living organism, demonstrates certain of these principles. In order to bring this out there must be a lather close coordination between the studies of the laboratory and the presentation of principles by the textbook.

Based on a recognition of the above-mentioned situation and also on the realization that the majority of students taking elementary zoology plan to go no further in the field, the author has attempted to strike a workable combination of the two schools of teaching and still cover the fundamental knowledge of the subject. There has been a definite effort to lead the student to think of biology as related to humankind and to himself. It is hoped that the book will overlap the laboratory studies just far enough to lift the student out of the laboratory into his own correct interpretation of the facts discovered 
there. It is of course assumed that the teacher will naturally elaborate upon particular phases of the topies taken up in the course. The anticipation of this and limitations of space have reduced the volume of detailed information included.

Many animals from west of the Mississippi River are featured in this book. There has been no attempt to limit the scope of the work to this region, but since many southwestern and western forms are available and serve as very good illustrative material, they have been utilized. It is hoped this will make the book more useful and meaningful to students in these regions, as well as more teachable.

The introduction of chapters on Animal Anomalies, Animal Regeneration, Biological Effects of Radiation, Marine Zoology, and Wildlife Conservation is a slight departure from the usual textbook outline, but each of these seems to the author to have enough of special value and eurrent interest to warrant presentation. The chapters on Regulatory Glands, Animal Distribution, The Animal and Its Environment, Animal Parasitism, Comparative Embryology, Animal Behavior, and Paleontology are also presented with the feeling that they are of exceptional general interest to all students, as well as being thoroughly zoological.

The arrangement of the chapters on animal groups has been somewhat in the order of complexity and systematic relationships. The chapters are written in such a way, however, that this order may be modified in any manner to suit the teacher. The chapters dealing with typical Protozoa, Hydra, Planaria, Annelida, Arthropoda, and Amphibia are somewhat amplified and include more detail because they are so often chosen as typical groups for study. Throughout the book the genus and species names have been italicized, and many names of structures and functions have also been italicized the first time they occur.

The author is indebted and extremely grateful for the cooperation of several teachers and specialists who have contributed manuseript for chapters in their fields. For this service acknowledgment is made to: J. Teague Self, University of Oklahoma, Annelida; Elmer P. Cheatum, Southern Methodist University, Mollusca, and assisted with Marine Zoology; Vasco M. Tanner, Brigham Young University, Arthropoda; Mary Fickling, Public Schools, Waco, Texas, collaboration on Elasmobranchii; Rose Newman, Baylor University, collaboration on Pisces; Ottys Sanders, Southwestern Biological Supply Co., 
Amphibia, and assisted with Marine Zoology; Leo T. Murray, Baylor University, assisted by James E. Blaylock, Ranger Junior College, Reptilia; IIelen Joe Talley, University of Oklahoma, collaboration on Regulatory Glands; T. C. Byerly, United States Bureau of Animal Industries, Animal Regeneration; Titus C. Evans, University of Iowa, Medical College, Biological Effects of Radiation; Willis Hewatt, Texas Christian University, Animal Distribution, and assisted with Marine Zoology; A. O. Weese, University of Oklalioma, The Animal and Its Environment; Sewell II. Hopkins, Texas Agricultural and Mechanical College, Animal Parasitism; J. G. Burr, Texas Game, Fish, and Oyster Commission, marine data; Walter P. Taylor, Texas Cooperative Wildlife Service and United States Bureau of Biological Survey, Wildlife Conservation; A. Richards, University of Oklahoma, Comparative Embryology; Frank G. Brooks, Cornell College, Genetics and Eugenics; Iva Cox Gardner, Baylor University, Animal Behavior; W. M. Winton, Texas Christian University, Paleontology.

To Mr. Ivan Summers goes immeasurable eredit for the excellent art work lie has put into this edition. Dr. Titus Evans, of the University of Iowa, Medical College, has also been of great service with his excellent talent in creating illustrations. Mrs. Ruth M. Sanders, Miss Joanne Moore, and Mr. Edward O'Malley have each assisted by contributing certain illustrations. The drawings used in Chapter XLII on Genetics and Eugenics were made by Miss Betty R. Smith of Cornell College. The author is grateful to all of these individuals for their valuable services.

The author wishes to acknowledge also the friendly and helpful advice which has been offered by Professors D. B. Casteel, T. S. Painter, and E. J. Lund of the University of Texas, and Professor Asa Chandler of Rice Institute. Finally, appreciation is expressed to Baylor University for the cooperation which has made the writing of this book possible.

George E. Potter.

Waco, Texas. 


\section{CONTENTS}

CHAPTER I

INTRODUCTION

The Biological Point of View, 17; Science and the Scientific Method, 15; Zoology, a Biological Science, 19; The Subdivisions of Zoology, 19; Classification of the Animal Kingdom, 25; Vital Relations of Animals and Plants, 27 ; Attributes of Life, 30 ; Balance in Nature, 31 ; Zoology as Related to Man, 33; Agriculture and Zoology, 34; Fisheries and the Application of Zoology, 34 .

\section{CHAPTER II}

History OF ZOOLOGY

\section{CHAPTER III}

Protoplasi and the Cell ${ }_{-} \quad \ldots$

Liring Matter, or Protoplasm, 49; The Cell Principle, 49; General Characteristics of Protoplasm and the Material of the Cell, 53; Fundamental Properties or Activities of Protoplasm, 54; Physical Nature of Protoplasm, 55; Chemical Nature of Protoplasm, 56; Structure of a Typical Animal Cell, 5S; Cell Division, 61.

\section{CHAPTER IV}

Phylum Protozoa in General _ $\ldots$ Characteristies, 65; Classification, 65; Colonial Protozoa, 75 ; Tropisms and Animal Reaction, 77 ; Economic Relations of Protozoa, 77 .

\section{CHAPTER Y}

Euglexa OF ClaAs MAStigophora

Habitat and Characteristies, S1; Structure, 81; Food and Assimilation, 31; Respiration and Excretion, 83; Reproduction and I.ife Cycle, 83; Behavior, 84; Locomotion and Flagellar Morement, 84.

\section{CHAPTER VI}

AMOEBA OF ClaAs SARCODINA

Characteristics and Habitat, 85; Structure, 86; Metabolism, 86; Reproduction and Life Cycle, 89; Behavior, 91; Amoeboid Movement and Locomotion, 91.

\section{CHAPTER VII}

Paranecium uf Class Infusoria

Characteristics and Habitat, 93; Structure, 93; Metabolism, 95; Reproduction and Ifife History, 96; Behavior, 100; Locomotion, 102. 
Metazoan Organization

General Characteristics, 103; Cellular Differentiation, 104; Cellular Organization, 105; Development of Sexual Reproduction, 111; Metazoan and Ontogeny, 113.

\section{CHAPTER IX}

Phylum PORIFera $-\overline{-}--\overline{-}-\overline{-}-\overline{-}-\overline{-}-\overline{-}-\overline{-}-$
Sponges, 119; Classification, 120; Fresh-Water Sponges, 121; The Simple Sponge, 122; Habitat and Behavior, 122; External Anatomy, 123; Internal Anatomy, 124; Metabolism, 127; Reproduction and Life History, 127; Economic Relations, 129; Phylogenetic Advances of Sponges When Compared With Protozoa, 129.

\section{CHAPTER $\mathrm{X}$}

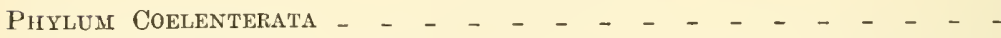

Classification of the Phylum, 131; Hydra, 144; Habitat and Behavior, 144; External Anatomy, 146; Internal Anatomy, 148; Metabolism, 151; The Nervous System and Nervous Conduction, 153; Reproduction and Life Cycle, 153; Regeneration, 156; Economic Relations of the Phylum, 156; Phylogenetic Advances of Coelenterates, 156.

\section{CHAPTER XI}

\section{Phylum Ctenophora $-\overline{-}-\overline{-}-\overline{-}$
Habitat and Behavior, 157 ; Anatomy, 157.}

\section{CHAPTER XII}

Phylum Platyhelminthes

Classification, 160; Planaria, 163; Habitat and Behavior, 163; External Anatomy, 165; Internal Anatomy, 165; Metabolism, 170; Reproduction and Life History, 170; Regeneration, 173; Economic Relations of the Phylum, 174; Phylogenetic Advances of Platyhelminthes, 174 .

\section{CHAPTER XIII}

Phylum Nemathelminthes - $-\overline{-}-\overline{-}-\overline{-}-\overline{-}-\overline{1}-\overline{-}$
Classification, 175 ; Ascaris, A Representative Roundworm, tat and Behavior, 179; External Anatomy, 179; Internal Anatomy, 181; Reproduction and the Life Cycle, 183; Relations to Man, 183.

\section{CHAPTER XIV}

Molluscoida, Trochelminthes, and Chattognatha -

Molluscoida, 184; Bugula, 184; Trochelminthes, 188.

\section{CHAPTER XV}

Phylum Annelida (By J. Teague Self) - $\quad$ - - - $\quad$ - $\quad$ - -

Earthworm, 199; Internal Anatomy, 201; Reproductive Organs, 202;

Digestive System, 203; Circulatory System, 205; Respiratory System, 
206; Excretory System, 206; The Nervous System, 208; Reproduction, 209; Regeneration, 212; Importance of Annelids to Man and Other Animals, 215; Phylogenetic Adrances of Annelida, 216.

\section{CHAPTER XVI}

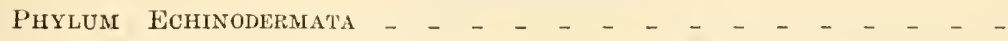

Classification, 217; Starfish of Class Asteroidea, 226; Habitat and Behavior, 226; Exterual Anatomy, 226; Internal Anatomy, 227; Reproduction and Life Cycle, 234; Regeneration and Autotomy, 234; Economic Relations, 235.

\section{CHAPTER XVII}

Phylum Mollusca (By Elmer P. Cheatum)

General Characters, 236; The Snail, 237; Habitat and Belavior, 237; External Anatomy, 239; Internal Morphology, 243; Respiration, 244; Circulation, 244; Nervous System, 245; Excretory, 245; Reproduction and Life Cycle, 245; Fresh-Water Clams, 248; Habitat and Behavior, 248; External Features, 249; Internal Anatomy, 250; Digestion, 250; Respiration, 251; Circulation, 252; Nervous System and Sense Organs, 252; Excretion, 253; Reproduction and Life Cycle, 253; Economic Relations of the Phylum, 255; Classification, 256.

\section{CHAPTER XVIII}

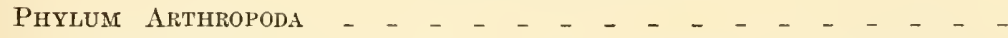

Classification, 263; Crayfish of Class Crustacea, 266; Habitat and Behavior, 267; External Structure, 268; Internal Structure, 271; Metabolism, 278; Reproduction, 278; Regeneration and Autotomy, 281; Economic Relations, 281; Characterization of Other Crustacea, 282; Recapitulation Theory, 284; Phylogenetic Advances of Arthropoda, 286.

\section{CHAPTER XIX}

Phylum Arthropoda (Cont'd) (By Vasco M. Tanner) - _ _ - - 287

Onychophora and Myriapoda, 287; Onychophora, 287; Myriapoda, 288.

\section{CHAPTER XX}

Phylum Arthropoda (Cont'd) (By Vaseo M. Tanner') - _ _ _ Arachnida, 292; Spiders, 292; Classification of the Arachnida, 295.

\section{CHAPTER XXI}

Phylum Arthropoda (Cont'd) (By Vaseo M. Tanner) - - - - - 300

Class Insecta, 300; Insect Characteristics, 301; Head, 301; Thorax, 305; Abdomen, 307 ; Body Wall, 30s; Metamorphosis, 308; Classification, 309; Hemimetabolous Insects With Incomplete Metamorphosis, 320; Holometabolous Insects With Complete Metamorphosis, 321; Other Orders, 333; Social Life Among the Insects, 334; Guests, 339; Economic Relations, 340; Useful Inseets, 341. 


\section{CHAP'TER XXII}

Representative Insects (By Vasco M. Tanner) _ _ _ _ _ $~{ }_{-} 343$

The Loenst, 343; The June Bug, 354; The Honey Bee, 357.

\section{CHAPTER XXIII}

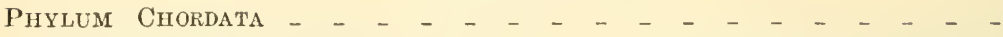

Characteristics, 360; Classification, 361; Phylogenetic Advances of Chordata, 362; Protochordata (Lower Chordates), 362; Subphylum Hemichordata, 362; Subplyylum Urochorda, Molgula, 365; Subphylum Cephalochorda, Amphioxus, 368.

\section{CHAPTER XXIV}

The Vertebrate Animal: Subphylum Verterrata _ $\ldots$ _ $\ldots$ Classification, 410 .

\section{CHAPTER XXV}

Cyclostomata

Classification, 412; Economic Relations of the Class, 413; The Lamprey, 413; Habitat, 413; Habits and Behavior, 415; External Structure, 415; Internal Structure, 415.

\section{CHAPTER XXVI}

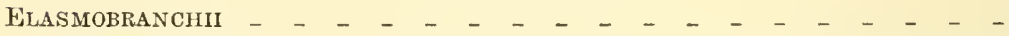

Classification, 422; Economic Relations of the Class, 425; The Spiny Dogfish, 426; External Features, 426; Muscular System, 427; Skeletal System, 427; Digestive System, 430; Cireulatory System, 431; Respiratory System, 435; Nervous System, 435; Urinogenital System, 437; The Bonnethead Shark, Reniceps (Sphyrna) Tiburo Compared to Squalus, 439.

\section{CHAPTER XXVII}

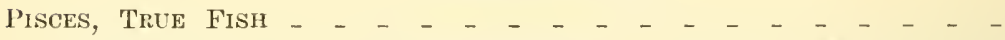
Classification, 445; Economic Relations of the Class, 455; Typical Bony Fish-Yellow Bullhead and Some Comparisons With Yellow Perch, 457; External Features, 457; Digestive System and Digestion, 458; Circulatory System and Circulation, 459; Respiratory System, 463; Excretory Organs, 464; Skeletal System, 464; Muscular System and Locomotion, 467; Nervous System, 469; Reproduction and the Life History, 470.

\section{CHAPTER XXVIII}

Class Amphibia (By Ottys Sanders) 472

Classification, 482; A List of Families of the Amphibia in the United States, 484; Order Caudata (Urodela) (Tailed Amphibians), 484; Order Salientia (Anura) (Tailless Amplibians), 485; Economic Importance, 486; Necturus Maculosus, the Mud Puppy, 487; Food and Digestive System, 489; Cireulatory Systen, 490; Respiratory System and Breathing, 491; Urinogenital System, 492; Skeletal System, 
494; Museular System, 496; The Nervous System and Sense Organs, 497; The Bullfrog, 497; Habitat, 497; External Structure, 497; Digestive System and Digestion, 499; Cireulatory System, 502; Respiratory Organs and Respiration, 514; Excretory System and Excretion, 515; Skeletal System, 517; Muscular System, 523; Nervous System, 525; The Sense Organs, 528; Reproductive Organs, 531; Embryology, 532; The Toad, 538; Habitat, 538; External Features, 539; Internal Structure, 541; Respiratory and Digestive Organs, 541; Urinogenital Organs, 541; Blood Vascular System, 542; Skeleton and Muscles, 543; Nerrous System and Sense Organs, 543; Embryology, 544 .

\section{CHAPTER XXIX}

Reptilia (By Leo T. Murray and James E. Blaylock) - _ _ - Fossil Reptiles, 546; Classification of Living Reptiles, 547; ClassReptilia, 547; Order Testudinata (Chelonia), 548; Order Squamata, 551; Order Rhincocephalia, 560; Order Crocodilia, 560; The Horned Lizard, 561; Habits and Behavior, 561; External Structure, 562; Digestive System, 563; Respiratory System, 566; The Circulatory System, 566; The Urinogenital System, 571; The Nervous System, 573; The Skeletal System, 574; Muscular System, 576; The Turtle, 577; Habits and Behavior, 577; External Structure, 578; Digestive System, 578; Respiratory System, 579; Circulatory System, 580; Urinogenital System, 581; The Nervous System, 581; The Skeleton, 581; The Muscular System, 581.

\section{CHAPTER XXX}

Classification, 584; Economic Relations, 596; Domestic Chicken, 598; Habits and Behavior, 598; External Strueture, 599; Digestive System, 601; Respiratory System, 603; Cireulatory System, 604; Exeretory System, 606; Nervous System, 607; Skeletal System, 609; Muscular System, 612 ; Reproduction and Life History, 613.

\section{CHAPTER XXXI}

Mammalia $-\overline{-}-\overline{-}-\overline{-}-\overline{-}-\overline{-}-\overline{-}$
Classification, 616; Economic Relations, 637; The Cat, A Representative Mammal, 639; External Structure, 639; Skeleton, 641; Muscular System, 644; The Digestive System, 646; Circulatory System 648; Respiratory Systen,, 649; Nervous System, 650; Excretory System, 650; Reproduction and Life History, 652.

\section{CHAPTER XXXII}

\section{Animal ANomalies}

Harelip and Cleft Palate, 656; Diaphragmatic Hernia (Open Diaphragm), 657; Polydactylism (Extra Digits), 659; Conjoined Twins, 659; Hermaphroditism, 663; Cardiae Anomalies, 664; Abnormalities of Brain and Sense Organs, 664 . 
The Endocrine Glands and Their Functions -

The Thyroid Gland, 667; The Parathyroid Glands, 670; The Suprarenal Bodies, 671; The Pituitary Gland, 672; The Thymus Gland, 675; The Gonads and Sex Hormones, 675; The Pancreas, 677.

\section{CHAPTER XXXIV}

Regeneration (By T. C. Byerly) -

Introduction, 681; Regenerative Capacity, 681; Protozoa, 681; Porifera, 682; Coelenterata, 682; Platyhelminthes, 683; Annelida, 684; Mollusca, 686; Arthropoda, 686; Echinodermata, 686; Chordata, 657; Amplibia, 688; Reptilia, 690; Aves, 690; Mammalia, 690; Basis for Regeneration, 692; Adaptability and Regeneration, 695; Summary, 696.

\section{CHAPTER XXXV}

Biological Effects of Radiations (By Titus C. Evans) - - - The Structure of the Atom, 697; Biological Effects of Sunlight, 700; Infrared Radiation, 700; High Frequency Oscillations, 701; Effects of Ultraviolet Radiation, 701; Roentgen Radiation, 702; The Fundamental Action of Roentgen Radiation, 707; Biological Action of Radium, 708; Effects of Other Radiations, 709; Summary, 710.

\section{CHAPTER XXXVI}

Animal Distribution (By Willis Hewatt) - _ $-{ }_{-}+\ldots$ Life Regions and Zones of the Earth, 711 ; Migration of Animals, 716; Means of Dispersal and Barriers, 717; Effects of Man Upon Distribution, 718.

\section{CHAPTER XXXVII}

The Animal and Its Environment (By A. O. Weese) - _ The Principal Biotic Formations, 724; Adaptation, 727; Succession, 727 ; Animal Populations, 730 ; Seasonal Changes, 733 ; Summary, 733.

\section{CHAPTER XXXVIII}

Animal Parasitism (By Sewell H. Hopkins) -

Social Relations of Aninals, 735; Origin of Parasitism, 736; Degrees of Parasitism, 736; The Successful Parasite, 737; Means of Infection and Transmission, 740; Parasitism and Host Specificity, 741; Parasites and the Groups in the Animal Kingdom, 742; Some Representative Parasites, 747 . 
CHAPTER XL

PAGE

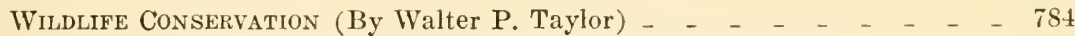

The Abundance of Wild Animals, 784; The Natural Range of Wild

Animals, 789; The Coming of Civilization and a Declaration of Inde-

fensibles, 792 ; The Problem of Restoration, 794.

\section{CHAPTER XLI}

Comparative Embryologi (By A. Richards) _ _ _ _ _ $\ldots$

\section{CHAPTER XLII}

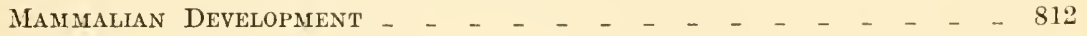

Organs and Systems, 817.

\section{CHAPTER XLIII}

Genetics And Eugenics (By Frank G. Brooks) _ _ _ _ 821

The History of a Great Discovery, 8:1; Mendel's Law, 821; Derivatives of Mendel's Law, 823; The Physical Basis, 824; Plotting Crosses, 825; Complications of Mendelian Inheritance, 827; Inheritance of Sex, 831; Linkage, 832; Sex Linkage, 832; Crossing Over, 834; Mutations, 836; Human Heredity, 836; Matings Among Defectives, 839; The Differential Birth Rate, 839; Family Size in Eugenic Groups, 841; Family Size in Dysgenic Groups, 842; What Can Be Done? 844; Some Eugenic Measures, 844.

\section{CHAPTER XLIV}

Animal Behavior (By Ina Cox Gardner) _ _ _ _ _ _ _ _

Introduction, 846; Tropistic Behavior, 849; Reflex Behavior, 850; Chain Reflex Behavior, 851; Habitual Behavior, 852.

\section{CHAPTER XLV}

Paleontologi (By W. M. Winton) - $-{ }_{-}-{ }_{-}-{ }_{-}-$

\section{CHAPTER XLVI}

Phylogenetic Relations of Animal groups and the Theory of Evo-

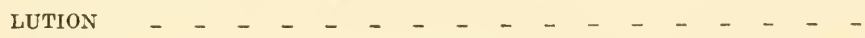
Colony Formation in Certain Protozoa, 864; Development of the Gastrula, 865; Trochophore Larva, S65; Peripatus and the Wormlike Ancestry of Arthropoda, 865; Eehinoderms and Their Larval Relations, 866; Ancestry of the Vertebrates, 866; Basis for the Theory of Evolution, 870; Darwin and Studies of Evolution, 885. 



\title{
TEXTBOOK OF ZOOLOGY
}

\author{
CHAP'TER I \\ INTRODUCTION
}

In whichever direction we turn or wherever we go, whether in the air, on land, or in the sea, we are surrounded by living creatures. Their very presence presents problems and fills us with curiosity. We ask questions. From whence do they come? What is the source of their energy? Why are there so many different kinds? What is our relation to other living things? What is life? Such questions and endless numbers of similar ones kindle the interest of every thinking person. The constant endeavor on the part of man to answer these questions and solve the problems of the origin and nature of life has given us the field of study known as biology.

Biology is a word derived from two Greek words, bios, life, and logos, discourse, and is the name universally applied to the study of living organisms and life processes. Since living things fall largely into two general categories, plants and animals, such a study deals with the forms and phenomena exhibited by both.

\section{The Biological Point of View}

Nature is ever inviting investigation; her forces are in constant operation about us, but she hides the truth. The biologist looks upon himself as a seeker after truth, as one striving to get a glimpse into the mysteries of life. As he succeeds in obtaining these glimpses, he soon realizes the existence of certain fundamental features common to the structure and function of all living forms. He soon recognizes the oneness of all life, and himself as a part of one great organic system, each unit of which has some relation to the whole. A biological concept may rest upon observations, which may be changed from day to day by the discovery of new facts, but the biologist, like the chemist or physicist, is justified in holding to a theory or hypothesis as long as it provides a true working basis for further investigation. 


\section{Science and the Scientific Method}

"Trained and organized common sense" was the definition of science given by Thomas H. Huxley, an eminent English biologist who lived from 1825 to 1895 . That was his way of saying that scientific knowledge is simply an extension and organization of the knowledge based upon common observation and experiment concerning the facts of nature. Facts are indispensable building stones of science. Facts must be gleaned from careful observations and experiments which have been rigidly checked and will yield identical results with frequent repetition and by numerous observers. Science lays its foundation on accurate observations and depends on the ability of the senses to reveal the truth. Established facts represent truth, and the scientist respects truth while to him tradition or mere opinion counts for little as such.

There is nothing mysterious in the scientific method, although the steps are often tedious and much involved. The method is simplicity itself; to observe, to identify by comparison, to experiment, to coordinate, to deduce, to conclude. The scientist is not a magician who can draw his conclusions from thin air as Thurston seemed to produce almost anything imaginable. Complete and accurate observations of the objects or phenomena under investigation followed by honest interpretation, are the aims of the scientist. Our powers of observation have been increased by the development of the microscope and many other instruments. Experiments are devised to bring to light the features not readily revealed by direct observation.

After the facts are thus established, the qualities of the thing observed are compared and the essential or fundamental ones are separated from the nonessential. This requires accurate logic and keen judgment.

These fundamental facts are then classified with respect to previously established facts, and, on the basis of the relationships of qualities, deduction may be made of the principles involved.

It is by this systematic method of investigation that science has been established. In idea, as it first develops from preliminary observation and experiment, is known as a hypothesis. When the conclusions have been further verified by repeated examination, observation, and experimentation, the hypothesis becomes a theory. 
There is always a considerable volume of evidence which supports the theory and gives all indication that it is a true statement. Finally, the theory advances to a principle or law after it has been so thoroughly and critically tried as to be generally accepted and assumed as a truth. This process requires the accumulation of the combined results of numerous investigators over a long period of time. To many people truth is absolute, not relative, and a conclusion once drawn is fixed and may not be withdrawn for any reason. In science conclusions are always subject to modification or even abandonment as investigation continues. Scientific hypotheses are frequently shown to be untenable, theories are occasionally found fallacious and discarded, but up to the present time our scientific principles have remained valid. However, at any time sufficient evidence is produced to show the absolute fallacy of a so-called principle, the scientist will put aside sentiment and prejudice and accept the results of repeated investigation. Science is, therefore, a changing, increasing body of knowledge which is ever becoming more thoroughly established.

\section{Zoology, a Biological Science}

The name, zoology, which is derived from the Greek words zoos, animal, and logos, discourse on, refers to the study or science of animals. The natural sciences, as distinguished from the social sciences, are conveniently divided into two groups: the physical sciences, such as ehemistry, physics, and astronomy, which deal with nonliving bodies; and the biological sciences, such as botany and zoology, which are concerned with living organisms. Zoology and botany together constitute the science of biology. The expression animal biology is often used as a synonym for zoology. A person who specializes in the study of zoology is known as a zoologist. There was at one time an erroneous popular impression that zoologists were simply "bug-hunters." This conception of the field has been greatly expanded until now it is considered one of the valuable and serious fields of science.

\section{The Subdivisions of Zoology}

Although zoology is only one of the divisions of the general field of biological science, it is such a broad field in itself that it is necessary to subdivide it into several divisions for convenience in study. It has been a relatively short time since all of the known biology, geology, and related subjects were studied under the head of natu- 
ral history. But now the subject matter of zoology alone has grown to such magnitude that it has become necessary to divide it into numerous special fields. These subdivisions may be summarized as follows:

1. Morphology is the study of the form and structure of the bodies of animals. Its organization as a special field of study oc-

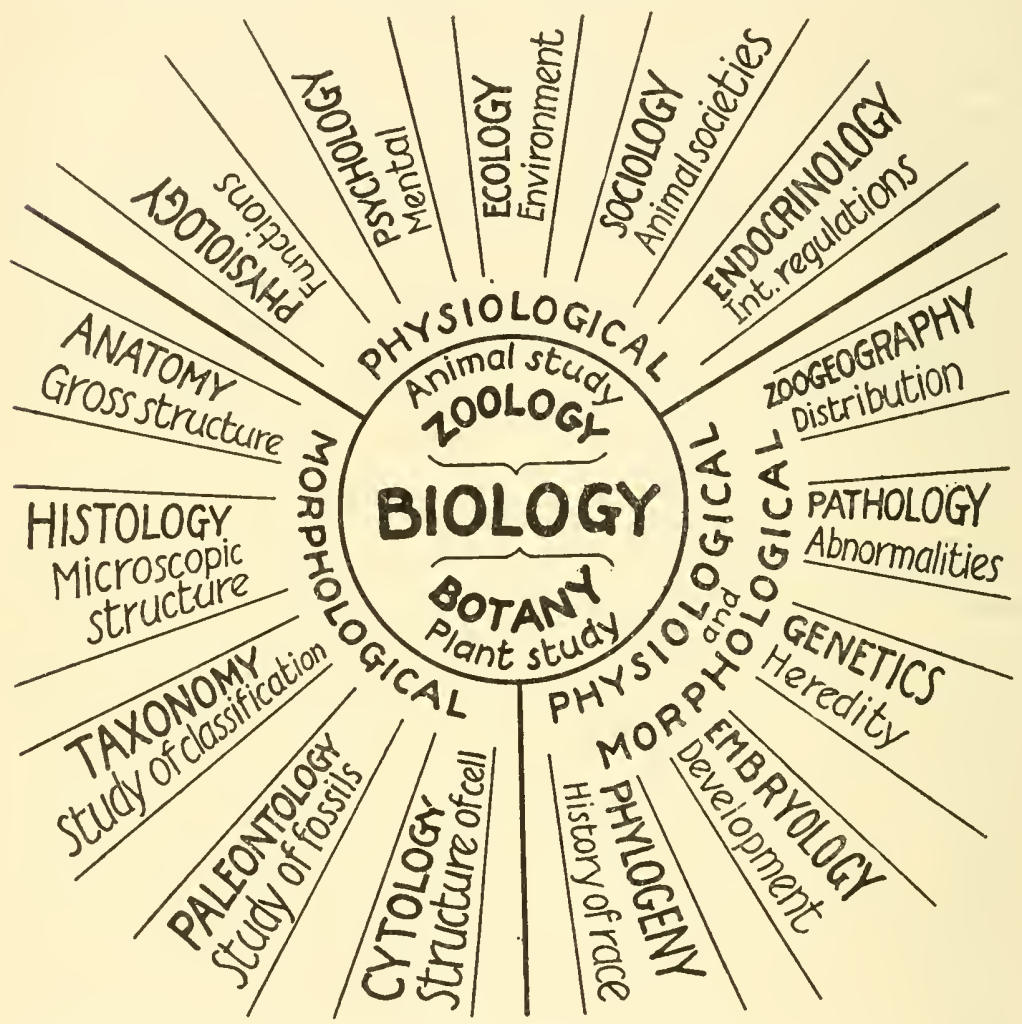

Fig. 1.-Divisions of study in the fleld of biology. (Modified from several authors.)

curred at about the beginning of the nineteenth century. It is now further divided into several branches.

A. Gross anatomy, which literally means cutting up, includes all that may be studied of form and structure of bodies by dissecting them. Human anatomy, which is one of the fundamental subjects of study in the preparation of the medical student, is usually separated from comparative anatomy. The latter study comprises a compara- 
tive study of the form and structure of the other animals and these in turn are compared, finally, with the human anatomy. The dissection, observation, and study of the parts, form, and relationship of parts of the digestive system of the eat would be a good example of anatomical study. Galen, A.D. 131-201; Vesalius, 1514-1564; Cuvier, 1769-1832.

B. Histology or Microscopic Anatomy, is a study of the microscopie structure of the various parts of the animal body. The histologist studies the relationship and arrangement of the cells as they cooperate to comprise the substance of the organism.

C. Cytology is the study of the minute structure of the cells which, we will learn, are the units of structure of all living matter. Cytology, as usually studied, includes not only the morpliology of the cell but a great deal of the physiology in addition. This field of study has yielded many fundamental concepts of the factors involved in the living process.

2. Taxonomy is the subdivision which deals with the elassification or orderly arrangement of organisms according to their natural relationships. This field is often spoken of as systematic zoology. The number of described species of animals as given by different authorities ranges from 840,000 to well over a million. One well-known writer says there are probably no less than 2,000,000 species of living animals. Besides these, there are large numbers of extinct forms. It can readily be seen that a system for putting these large numbers of different kinds of animals into a known order is one of the first prerequisites for dealing with them. On a much smaller scale, the department store is systematized for some of the same reasons. One can see that it would be next to impossible to do business if a company were to provide a large floor space, go out and buy the thousands of different kinds of articles that are handled by a department store, and just throw all of them on its floors at random. Few customers would returu a second time if they had to wait hours while the clerk hunted among ladies' shoes, children's toys, and men's underwear for the toothbrush the customer desired. Instead of this, the store is divided into general departments, and the goods are completely classified within these departments. To get the toothbrush, the customer can be directed to the proper department and counter, where kind, color, size, and 
price are all orderly arranged. In this way the large unwieldy number of different kinds of articles become simply managed.

The relationships of animals are discovered from similarity of structure, from facts of distribution, from embryological similarities, and many other comparisons. A group in which the members are very closely related is likely to be comparatively small. These groups are ranked together according to evident relationships. Zoologists recognize a number of large divisions of the animal kingdom based on certain general characteristics. Each of these divisions is known as a phylum and is divided into classes, each class is divided into orders, each order into families, each family into genera, and each genus into species. Taking the classification of man as an example we have:

Phylum :Chordata

Subphylum : Vertebrata

Class :Mammalia

Order:Primates

Family:Hominidae

Genus :Homo

Species :sapiens

The scientific name of man is written, Homo sapiens Linnaeus. Such a name is composed of the genus name and species name, and followed by the name of the person who wrote the first authoritative description of the particular species. This always gives a double name to a kind of animal, and for that reason it is the binomial system of nomenclature. This system was originated by Linnaeus. The names are in Latin instead of common vernacular because Latin is a constant and almost universal language. The common names would be almost certain to vary with each different language, but the Latinized form Homo sapiens Linn. is the same in Russian as it is in English.

3. Physiology is the study of the functions of the various parts of the organism as well as its living process as a whole. It involves a consideration of metabolism, growth, reproduction, sensitivity, and adaptation. In this field is included the study of many special functions, such as digestion, circulation, respiration, excretion, glandular secretion, nervous activity, muscular contraction, and 
others. Many of the processes which occur in the developing embryo are also included here. Much of the present study referred to as eytology is physiological. Physiology, like morphology, is an old branch of zoology; physiology, however, remained in a crude state long after morphology was fairly well developed. Physiology depends upon an understanding of physies and chemistry on one hand, and anatomy on the other. This field of study could not develop until the sciences of physics and chemistry came forward during the nineteenth century.

4. Pathology is the study of the abnormal structures and abnormal functioning of life processes. It is really the science of disease in all of its manifestations. There is a fundamental similarity in diseases in the different groups of animals, and a study of pathology is likely to involve certain forms from all groups of animals. This field has advanced rapidly during the last seventy years.

5. Embryology is a study of the origin and development of the individual. It usually involves the changes occurring in the organism from the time of fertilization by the union of two cells, one derived from each parent, through the numerous cell divisions, growth, organization, and differentiation leading to the adult condition. This process includes both morphological and physiological changes. The beginning of this study dates back to the work of $\mathrm{K}$. E. von Baer before the middle of the nineteenth century. In recent years the field of experimental embryology has developed rapidly. The development of the individual may be referred to as ontogeny.

6. Genetics is the division which deals with the study of variations, resemblances, and their inheritance from one generation to the next or from parent to offspring. The characteristic features of an animal or plant may be transmitted to the offspring somewhat independently of one another, bringing about a variety of combinations in the progeny. Fairly definite laws governing this inheritance of qualities have been established by the geneticists. Some of the factors which control this distribution of characteristics are morphological in their nature, others are physiological.

7. Phylogeny is a study of the origin and relationships of the different groups and races of organisms. It is based on the results of studies of morphology, embryology, genetics, zoogeography, and paleontology. 
8. Ecology is a study of the relation of the organism to its environment. Many adjustments in structure and function have been made by animals to bring them into harmony with the conditions of the environment. Such conditions as the relation of the organism to the medium in which it lives to temperature, to light, to food, to competition, to enemies, to mating, and many other factors, all become a part of an ecological study. This study usually draws somewhat upon a knowledge of all branches of biology. This branch of the field has become prominent in comparatively recent years.

9. Zoogeography or geographical distribution of animals is concerned with the extent of the regions over which species are distributed and the association of species in individual regions. In some respects this field is closely related to ecology. It is concerned with the regions in which species exist and with the factors affecting their distribution. The regional distribution of an animal group is limited in part by the extent and relations of favorable environmental conditions, but no species occupies all of the regions where environment would permit. The point of origin of the group may be cut off from other favorable regions by unsurmountable obstacles. Conditions which prevent dispersal of animals from one area to another are known as barriers. Oceans, mountains, forests, deserts and land are all barriers to different types of animals. Even a slight difference in the salinity or acidity of the water becomes a barrier to many aquatic animals. The failure of a species to occupy a suitable region usually means that it has been umable to reach that region, perhaps because of the topography of the region, its geological history, or the remoteness of the place of origin of the species. The English sparrow, which originated in Europe, was not found in America until after it was introduced by man, and in relatively few year's it became a dominant bird.

10. Paleozoology is a study of the animals of the past as they are presented by their fossil remains. Parts of many of the ancient animals are embedded and preserved in the sedimentary rocks. The relative age of the fossils is determined from the depth of the rock strata in which they are found. Many of the probable lines of descent of animals have been discovered by studies of the fossils. Much concerning the facts and the fate of extinct species has been learned through this field of study. Paleozoology is ordi- 
narily studied under the head of geology, and the geologist uses it in determining the relative ages of the rock strata which compose the crust of the earth.

\section{Classification of the Animal Kingdom}

Very few people realize how many different kinds of animals there are and how greatly they vary in size, structure, and habits of life. The estimated number of kinds is all the way from $1,000,000$ to $10,000,000$. To date, approximately 840,000 species have been

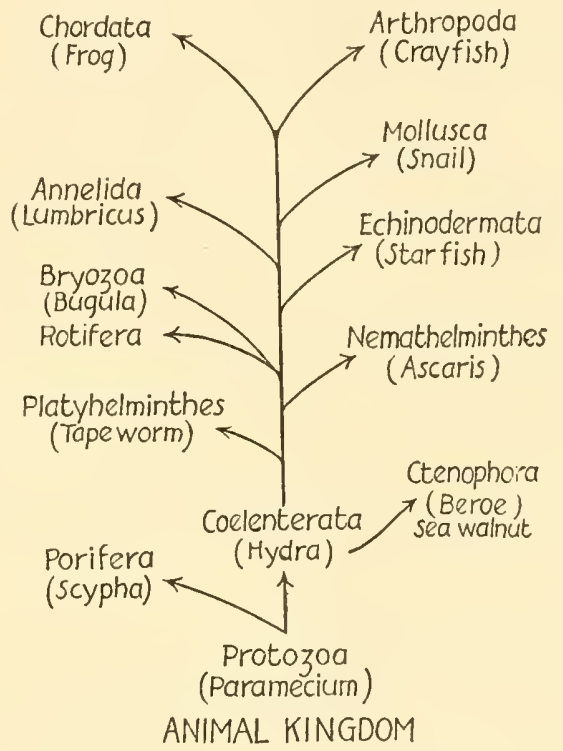

Fig. 2.-Phylum relations in the animal kingdom.

named and described. In order that different forms of animals may be known and definitely recognized, a scheme of grouping related kinds has been devised.

The entire kingdom is divided into two subkingdoms: Protozoa, or all single-celled animals, and Metazoa, the many-celled animals. The secondary groups are phyla, and they in turn are divided into classes. The principal groups subordinate to the class are order, family, genus, and species. The principal phyla are listed and described briefly:

Phylum Protozoa.-Individuals consist either of a single cell or of aggregates of cells, by each of which are performed all the essential 
functions of life. They are mostly microscopic in size and largely aquatic in habit. Some live in the ocean, some in fresh water, others in soil water, and still others as parasites in man and other animals. About 15,000 are known.

Phylum Porifera (Sponges).-Aquatic metazoans which live attached. Most of them are marine. The body is supported by fibrous, calcareous, or siliceous spicules, and the body wall is perforated by many pores. There are approximately 3,000 known species.

Phylum Coelenterata (Jellyfish).-All are aquatic and most of them are marine. They possess radial symmetry, a single gastrovascular cavity, and tentacles provided with stinging bodies, nematocysts. The described species number at least 4,500.

Phylum Ctenophora (Sea Waluuts or Comb Jellies).--Free swimming, delicate, marine animals that possess biradial symmetry. They are triploblastic and hermaphroditic. Less than one hundred species are known, and twenty-one of these are American.

Phylum Platyhelminthes (Flatworms).-These are flat, unsegmented, bilaterally symmetrical, triploblastic worms. "Flame cells" are characteristic excretory structures. These animals may be free-living or parasitic. Tapeworms, liver flukes, and the freeliving, aquatic Planaria are commonly known. Approximately 6,500 species have been described.

Phylum Nemathelminthes (Threadworms or Roundworms).-Unsegmented, bilaterally symmetrical, elongated worms which possess both a mouth and an anus. Some are free-living, others are parasitic. The hookworm, ascaris, and the "loorsehair worm" are common representatives. About 3,500 species are known.

Phylum Echinodermata.-Marine animals which have a spiny skin and the body wall usually supported with calcareous plates. They are radially symmetrical and have tube feet as organs of locomotion. The common representatives are starfishes, sea urchins, sea cucumbers, and sea lilies. There are about 4,500 known living species.

Phylum Annelida (Jointed worms).-This group is characterized by segmented body, well-developed body cavity, and nephridia as tubular excretory structures. They live in marine waters, fresh water, and in the soil. The earthworm and leech are well-known examples of the phylum. There are at least 4,500 known species. 
Phylum Arthropoda.--The representatives of this group may be aquatic or terrestrial. Their bodies are segmented, and they have segmented appendages. The group includes crayfishes, lobsters, crabs, centipedes, scorpions, and all insects, such as bugs, beetles, butterflies, flies, etc. This is by far the largest single phylum. Some authors believe as many as 675,000 species belong to it.

Phylum Mollusca.--Unsegmented animals that are usually enclosed in a calcareous shell. The single muscular "foot" is a characteristic structure. Common forms include clams, snails, slugs, and octopuses. About 78,000 species have been recognized.

Phylum Chordata.--Segmentally constructed animals with bilateral symmetry and an endoskeletal axis or notochord at some stage. Many of our best known animals belong here; the phylum includes lampreys, sharks, bony fish, frogs, salamanders, alligators, snakes, turtles, rats, birds, horses, sheep, cows, monkeys, and men. Approximately 40,000 species have been described in the group.

In addition to the above generally recognized phyla, there are several other more or less independent smaller but distinct groups. Most of these groups have certain of the wormlike characteristics. Many authors have dignified each of these as a phylum. They are: Nemertinea-nearly unsegmented, contractile, wormlike forms; Trochelminthes-unsegmented and frequently similar to certain larval stages of annelids and molluses, rotifers being typical; Bryozoa-colonial, marine, or fresh-water forms, of which there are about 1,750 known species; Brachiopoda-marine animals enclosed in a bivalve shell, the majority of which are fossil; Phoronideasessile marine worms living in chitinous tubes in shallow water; Chaetognatha-marine, transparent, carnivorous worms of which Sagitta is an example; Sipunculoidea-unsegmented, elongated marine worms, living either free, in tubes, or in snail shells. A number of these are sometimes described under the phylum name Molluscoida.

\section{Vital Relations of Animals and Plants}

There are certain single-celled organisms that are claimed as animals by zoologists and as plants by botanists. As a matter of fact, it is not easy to draw an absolutely clear-cut line of distinction. Of course, it is easy to recognize the extremes. Anyone 


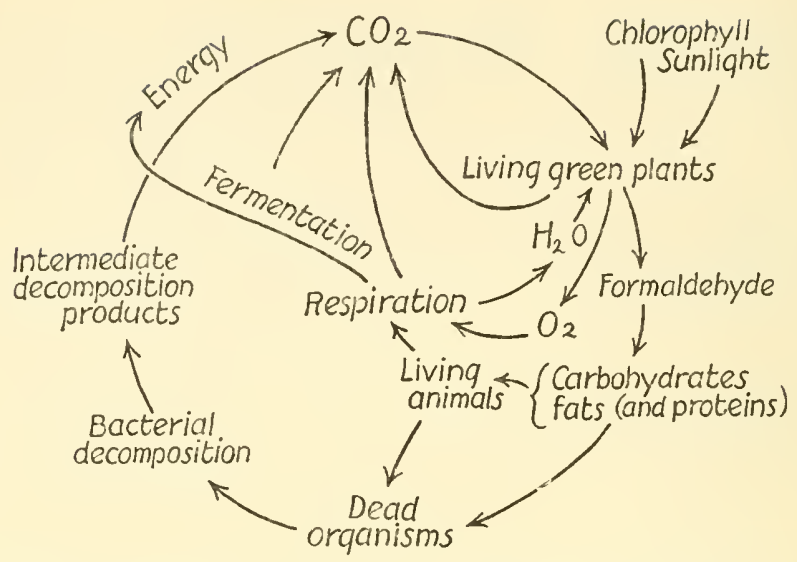

Fig. 3.- The carbon cycle and the fundamental living process.

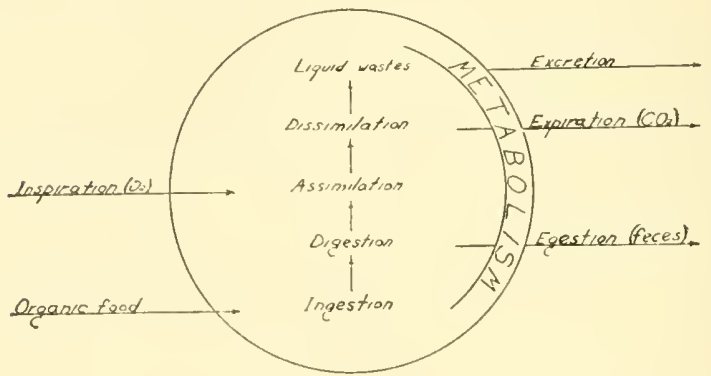

a Animal

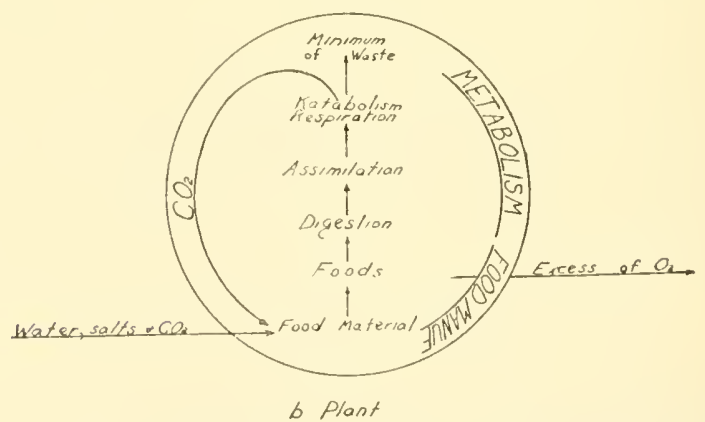

Fig. 4--The metabolic processes of plants and animals as well as the food Fig. 4.- The metabolic processes of plants and (Redrawn by permission from Wolcott. Animal Biology, published by McGraw-Hill Book Company, Inc.) 
holding a sunflower in one hand and a frog in the other has no difficulty in determining which is animal and which is plant. 'The distinctly typical animal forms depend on green plants for their

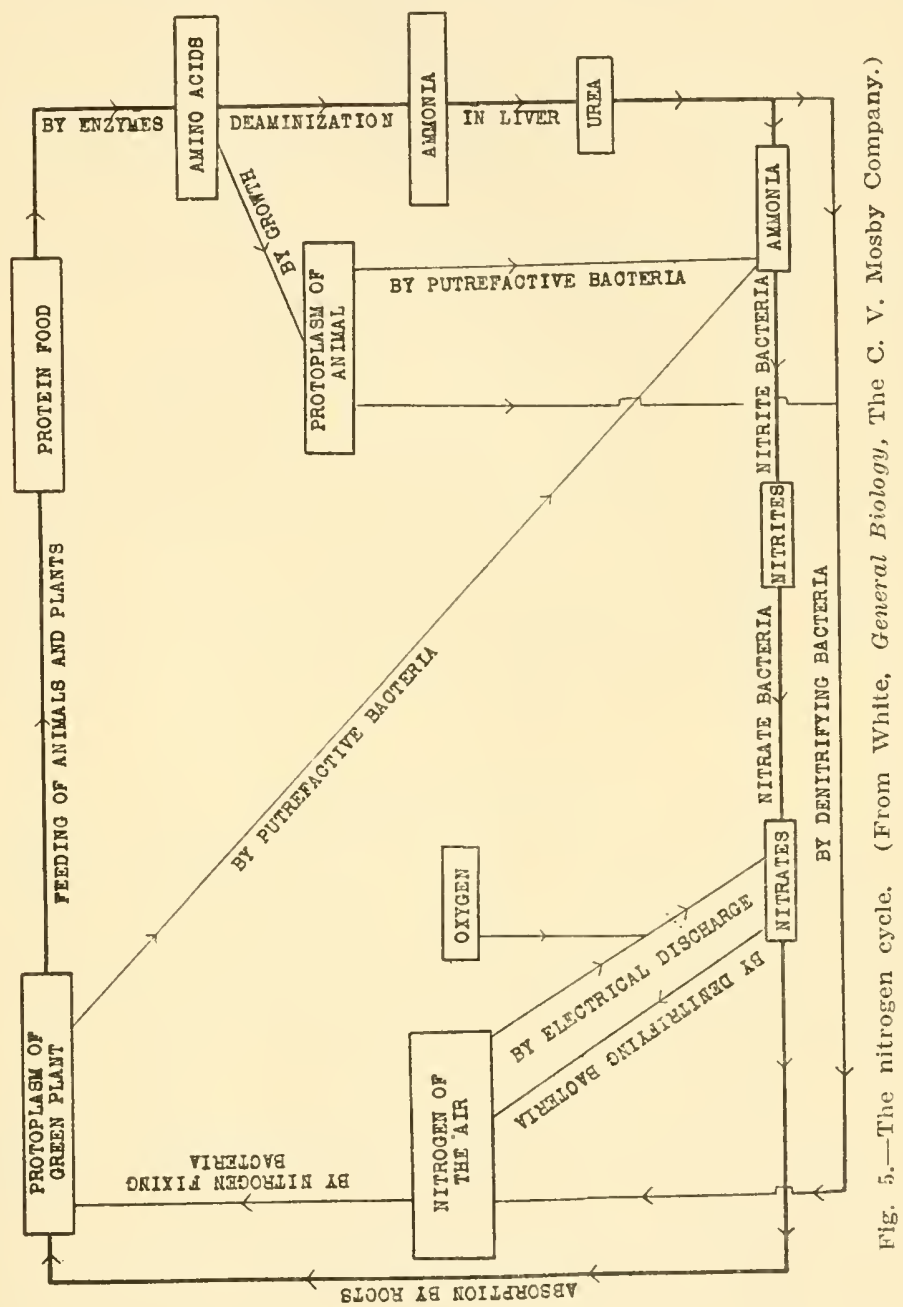

existence. All organic animal food is derived either directly or indirectly from the process of photosynthesis carried on by green plants to manufacture starch which is stored in plant tissue. Green plants by effect of their chlorophyll (green pigment) in sunlight 
cause carbon dioxide, a gas of the air, and water to unite in the formation of a simple carbohydrate. This is the basic food material of plants as well as of animals. During daylight hours while photosynthesis is in progress, oxygen is discharged into the air as a product of the process. This oxygen adds to the atmospheric supply and is used by animals in respiration. The carbon dioxide discharged by respiration of plants and animals is made use of by plants in this synthesis of material. The excretory products of animals contain nitrogen which is easily transformed into a soluble form and absorbed by plants to be combined with the simple carbohydrate, already described, to produce protein.

In general, plants, by utilizing the radiant energy of the sun, and chlorophyll, as a catalyst in causing the combination of water and carbon dioxide, form the potential material for the animals, because they extract simple substances from the earth and unite them into complex foodstuffs, such as the starches and proteins. Plants are devoured by animals, and some animals are in turn devoured by others. The complex substances are then broken down with liberation of energy, and the by-products excreted are again incorporated in the earth to be available to other plants.

\section{Attributes of Life}

Most of us think we know what life is, but if asked to define it, we find ourselves confronted by an almost hopeless task. The question, What is Life?, is the greatest riddle in the biological world.

The term life is an abstraction with no objective reality except as it is a phenomenon related to the activities of living units. The following statement has been given and is probably as nearly a definition as can be found: Life is a continuous series of reactions in a complexly organized substance, by means of which the organization tends to adjust itself to a constantly varying environment. Numerous attributes of living material may be given. Living material has the ability to carry on active chemical reactions without losing its body form. It is responsive to changes in the environmental conditions; therefore, it is said to be adaptive. Living material is able to sustain and reproduce itself under favorable conditions. The size of living organisms varies within definite limits. Much more will be said of living material in the following chapter. 


\section{Balance in Nature}

The influence exerted by one animal or one group of animals on another can hardly be estimated until one of them leaves the picture. In an established animal community which might be said to be balanced, all groups are held in bounds by their enemies. Balanced animal communities can be found the world over, and we are only beginning to get a notion of the extensive ramifications of the forces concerned in maintaining that balance. Quite clearly most animals live in a state of repression because relatively few of them become pests and overrun the country. About eighty-five years ago someone who had admired the remarkable spirit of the English sparrow in its native European home thought this hardy little bird would be a cheerful addition on this side of the Atlantic. Consequently, a few pairs were landed in Brooklyn. In the short years that have elapsed, this sparrow has proved so hardy and free of enemies here that it is now our dominant bird. Every city in the United States, as well as many in Canada and Mexico, has a large permanent population. Its nesting and perching habits in the heart of great cities are a source of great amnoyance and expense to building owners. Also, they consume enormous quantities of the farmer's' grain.

The story of the rabbit in Australia is likewise an interesting example of the effect of balance or lack of it. Not many year's ago Australia did not have a rabbit within its boundaries. It was hoped and intended by English immigrants there, that a few imported pairs of rabbits would increase sufficiently so that the old English sport of riding to the hounds might be developed in Australia. To the surprise and dismay of these people the rabbits flourished until now they are jeopardizing the enterprises of man. Nany men are kept employed full time doing nothing but hunting rabbits.

Again, we have an example of the effect of the natural agents of repression. The Japanese beetle which was recently introduced in the United States by accident has ravaged the vegetation in several eastern states and threatens other areas. When our investigators went to Japan to study the enemies of the beetle in an effort to find a means of control, they had to search for weeks to find a seriously infested area. So impressed are some biologists becoming with the potential danger of interfering with the natural balance, that even when some irritating pest is under discussion, 


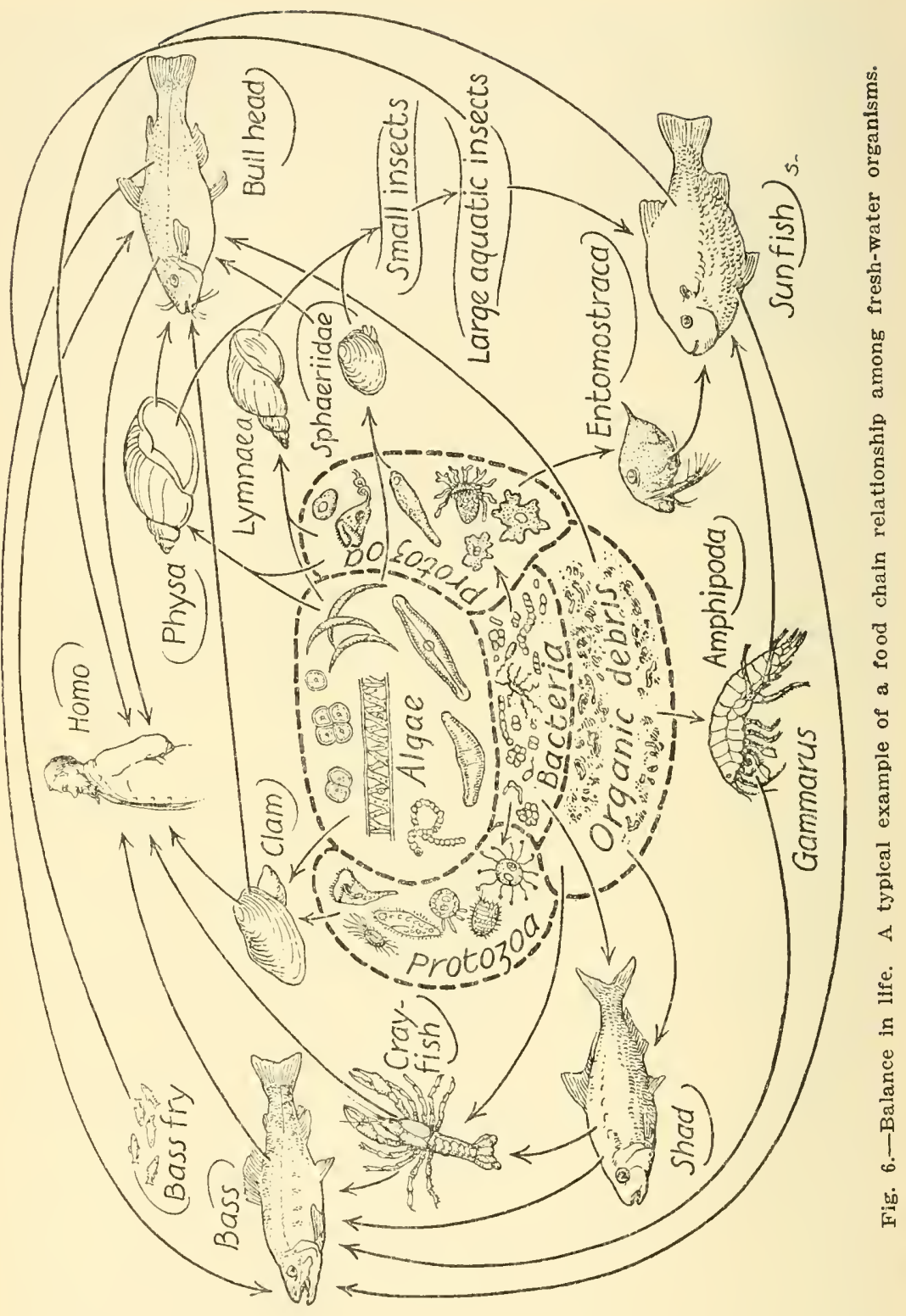


whose extermination is easily possible, they will advise against it until all phases of the animal's existence are thoroughly investigated. To wipe out this form might remove the check on others that are still more obnoxions. Because of the danger of interfering with the normal balance or equilibrium in nature, our government and many others have placed a restriction on importation of plants or animals. One must have permission to bring either into this country.

\section{Zoology as Related to Man}

The values of the study of zoology may be placed in two classes: cultural and practical. There is hardly a field of endeavor in the realm of human activities which is not greatly influenced by zoology and biology generally. The study of philosophy, the formulation of our conception of religion, the comprehension of social welfare problems, and many other similar intellectual and social accomplishments are greatly facilitated by a knowledge and recognition of biological principles. From the purely practical or economic side, of course, agriculture, medicine, and their related sciences have profited enormously. In fact, these fields are in themselves applied biology. Most of the great cliscoveries as to the nature and control of disease, the manner of inheritance of human characteristics, and the knowledge of fundamental physiological processes occurring in our own bodies have been attained by studies on other animals. What is found to be true in a dog, frog, rabbit, rat, monkey, or guinea pig, usually has its application to man. The lives of these laboratory animals have made untold and inestimable contribution to the welfare and comfort of man. The loss of their lives is constantly saving millions of human lives. One of the most obvious uses of other animals is as a source of food supply. All of the phyla and classes of larger animals furnish at least a few species that find places on our menu cards, particularly mammals, birds, turtles, frogs, fish, crabs, lobsters, clams, oysters, and even snails.

Many animals are important because of their destructive tendencics in regard to articles valued by man, or to the health and life of man. Most of the predaceous animals today are not a menace to man directly, but they do destroy many domesticated as well as useful wild animals. It is likely that the parasites which live on and in the bodies of men, and on domesticated plants and animals have been much more costly than the depredations of the more conspicuous predators. 


\section{Agriculture and Zoology}

It may frequently bring a smile to the lips of an onlooker to see a full-grown and perhaps intelligent zoologist enthusiastically attempting to learn what, when, and how much a little boll weevil eats or when, where, and how it lays its eggs; and yet, the discovery of such information may influence the activities of our entire cotton industry. A recent instance of the economic importance of zoological knowledge is found in the saving of the entire citrus industry in Florida from the Mediterranean fruit fly. Injurious insects alone cause an annual loss in the United States of more than one and one-half billion dollars' worth of products if they could be sold at the price the remaining portion brings. With proper knowledge of animal life and application of this knowledge it is likely that at least half of this loss could be prevented. Losses almost as important are caused each year by the parasitism of our domestic animals by bacteria, protozoans, worms, and insects. The knowledge and application of parasitology, which is a field of zoology, would avoid this loss.

Agriculture has benefited greatly from the application of the principles of heredity to plant and animal breeding. Much fundamental knowledge has come from the extensive studies on the genetics and breeding of the common fruit fly, Drosophila. It is easily kept in the laboratory and mated. It produces a new generation about once every nine days. More improvement of strains of animals and plants too, can be made in one man's lifetime than was previously possible through ages. The United States Department of Agrienlture and the United States Department of Interior have taken the lead in much of this type of zoology.

\section{Fisheries and the Application of Zoology}

A very practical and profitable application of zoology has been made in the fishing industry. The annual salmon catch alone on the Pacifie coast has been known to be worth $\$ 25,000,000$. The fishing industry cultures, collects, and markets not only fish of many kinds but also oysters, clams, lobsters, erabs, shrimp, and even sponges. The United States Fish and Life Service docs an extensive and remarkable work in the study, propagation, and care of this natural zoological resource. Even with this work and that of all the State Fisheries Departments, the natural fish life does not flourish as it might, had 
our public more appreciation of conditions necessary for a fish to live. A fish needs suitable water conditions including proper gas content, salt balance, nesting places, vegetation, and freedom from chemical or oil pollution.

The strictly intellectual and cultural endowments which zoology has given man are no less valuable than the tangible gifts. To understand something of the orderly conduct of Nature and to see that her operations are in accord with definite principles, gives one insiglit to the solution of many of the problems of life. Many of the superstitious dreads of unseen monsters have been eliminated by the knowledge of the fundamental principles of life processes. In recent times, it is probably true that nothing has influenced the thinking of the world more than the ideas, principles, and knowledge growing out of biological study. 


\section{CHAPTER II}

\section{HISTORY OF ZOOLOGY}

This brief chapter is organized to afford a slight preview of the works and lives of a selected few of the historic pioneers of zoology. This is not all attempt to give a complete history of the subject. The works of numerous pioneers in special fields are being considered throughout the text rather than in a given chapter.

There were individual persons interested in and studying natural history long before there was any organized field of study recognized under the name of natural history or the more limited divisions of it, including zoology. Some of the translations from the early Egyptians and later from the Greeks indicate that there had been some concern for the problems of life as well as medicine a number of centuries before Christ. Some of the early Greek scholars believed that the ocean supported all of the original life. Hippocrates, a Greek living from 460 to 370 B.C., was the first to think of medicine on a scientific basis. Aristotle (384-322 B.c.) was an outstanding Greek philosopher and scholar. To him goes the credit for establishing the scientific method of study which is based on gathering facts from direct observations and drawing conclusions from a study of these facts. His observations on the structure and development of embryo sharks, chicks, and many other animals, as well as his introduction of animal classification, are contributions which caused him to be called a biologist. He had the assist. ance of the armies of Alexander the Great in collecting materials. Alexander had been one of Aristotle's pupils and had become interested in the development of scientific endeavor. He made a grant of 800 talents $(\$ 200,000$ or more) for use by Aristotle in his investigations. Thus even in those times endowments were being set up for the support of research. The other Greeks who followed Aristotle added very little of importance.

Early Roman Scholars.-From shortly before the time of Christ and extending for about sixteen centuries was a period of "dark ages" in scholarly endeavor. However, a few contributions of note were made. Pliny (A.D. 23-79), a Roman general, compiled a 37volume work in which much of the scientific knowledge of the time 
and traditional superstitions are woven together. His work was limited to compilations, and because of the indiscriminate mixing of fact and fancy it is not scientifically valuable. It does reflect the tendency of the time in that scientific observation had given way to speculation.

Galen (A.D. 131-201), coming in the midst of the "dark ages" as he does, should be particularly credited for the contributions he made. He was of Greek ancestry but moved to Rome early and became a successful physician. His anatomical studies were made principally from direct observations on elephants, Barbary apes, and

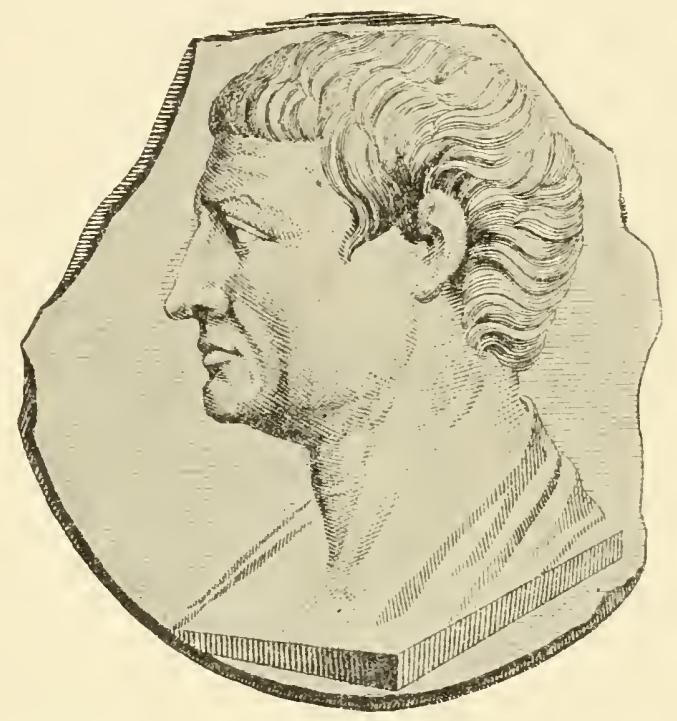

Fig. 7.-Aristotle (384-322 B.C.), father of naturalists. From a bas-relief found in the collection of Fulvius Ursinus. (Visconti, Iconographic grecque.) (From Locy, Growth of Biology, published by Henry Holt and Company. Inc.)

swine. During his time it was strictly against the law to make dissections of the human body so he was not allowed this privilege. Unfortunately, Galen did not take advantage of the work of certain of his predecessors who had been privileged to study liuman bodies. His conviction in the matter of direct observation as a basis of study handicapped him in this respect. His textbook on anatomy became the authority for the next eleven or twelve centuries.

Andreas Vesalius (1514-1564).-The return of interest in zoology eame about through the medical schools. Vesalius was an active 


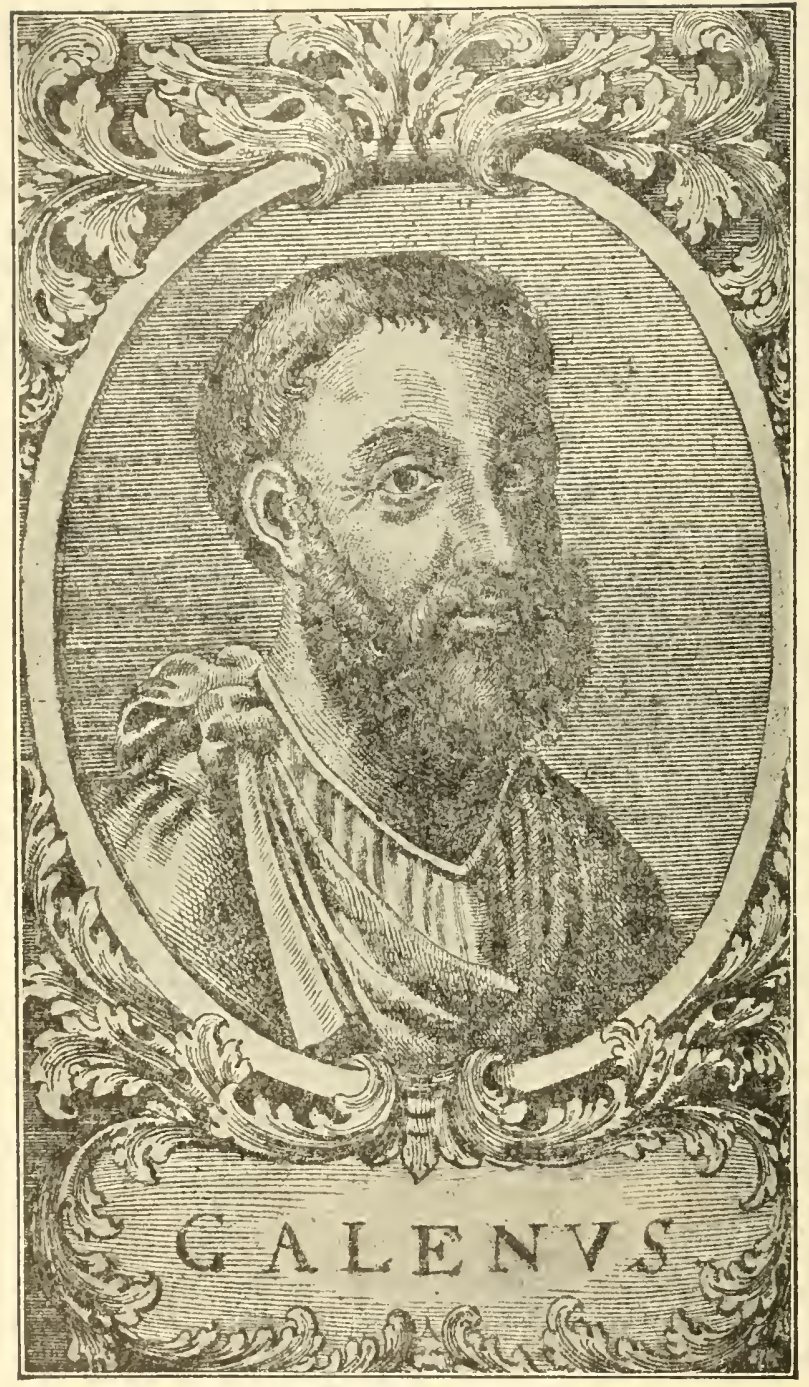

Fig. 8. Galen (A.D. 131-200), anatomist. Acta Medicorum Berolinensium, Vol. 5. 1719. (From Locy, Growth of Biology, published by Henry Holt and Company, Inc.) 
young student and was not satisfied to accept the authority of Galen's textbook. Therefore, after beginning his medical education at Brussels, he transferred to Padua where human dissection was

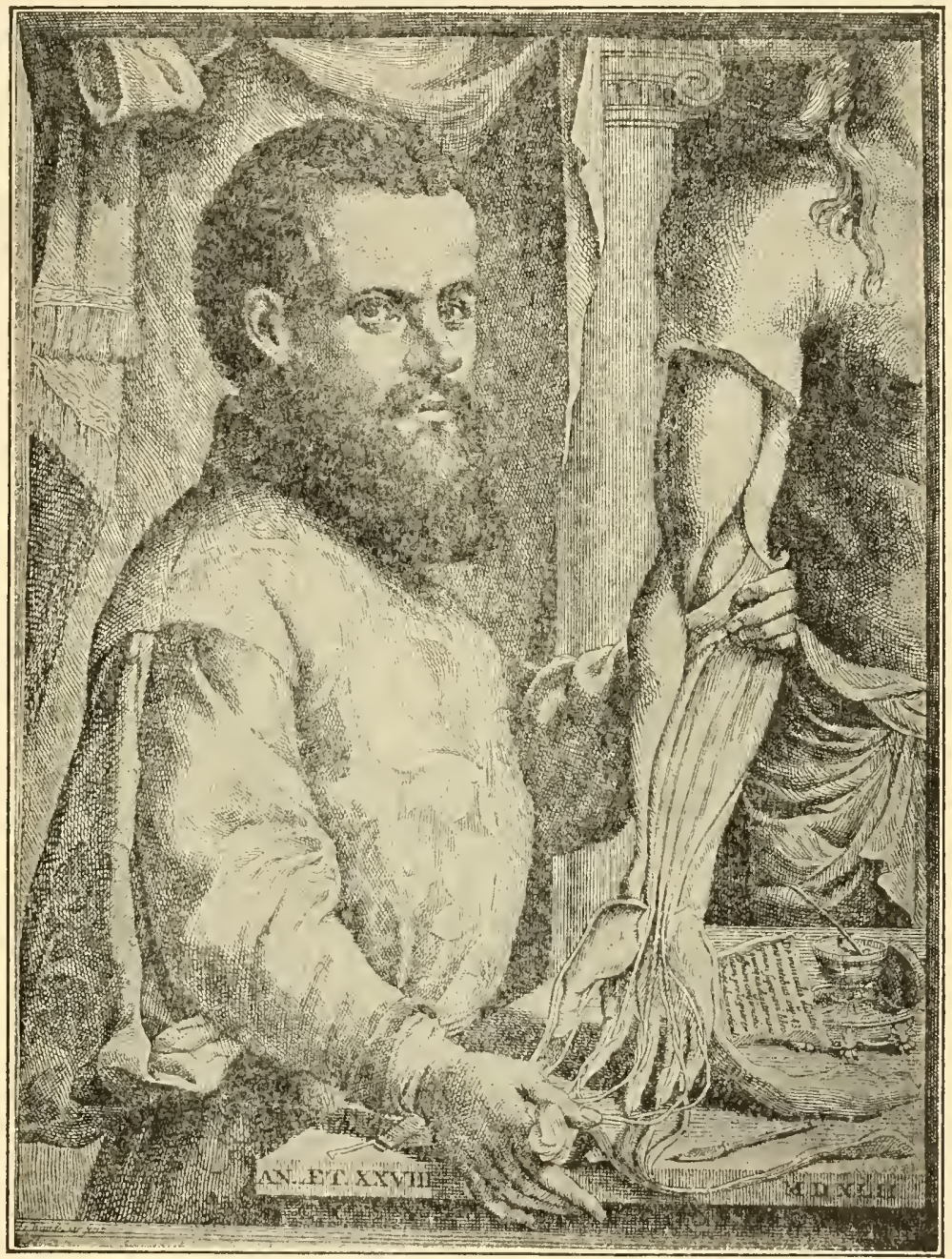

Fig. 9.-Vesalius (1514-1564), anatomist. Frontispiece, facsimile edition of 1728. Northwestern University Libraly. (From Locy, Growth of Biology, published by Henry Holt and Company, Inc.)

then allowed. He later became professor of surgery there. He was the first, since the time of Aristotle and Galen, to prove that direct 
observation is the only true eriterion of knowledge. Vesalius is thought of as the "father of modern anatomy," and his teaching is really responsible for the rapid development of biology and medicine following his time.

William Harvey (1578-1657).-Following closely upon the epochmaking work of Vesalius and inspired by several of his pertinent observations on the anatomy of the circulatory system, William Harvey, an Englishman, began experiments on the movement of blood in the vessels. Galen, Vesalius, and three or four others had

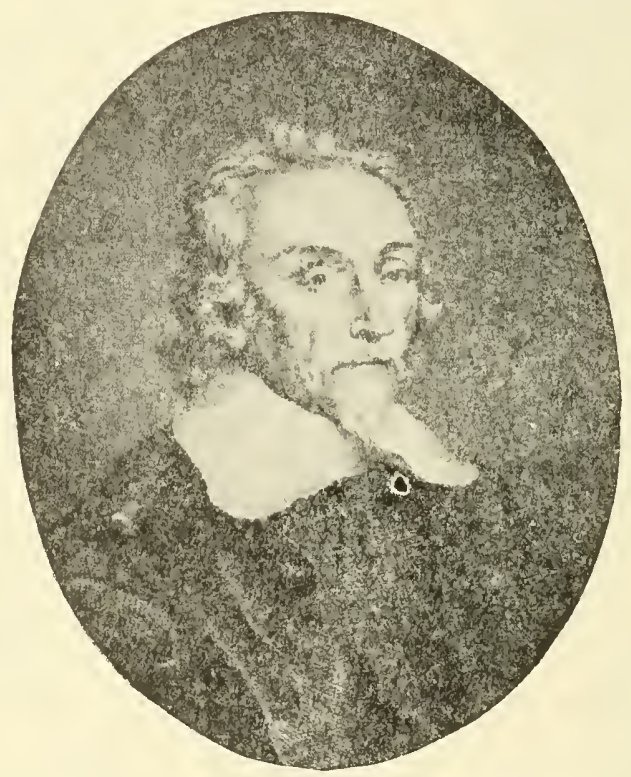

Fig. 10.-William Harvey (1578-1657), father of physiology. (From Garrison, History of Medicine, published by W. B. Saunders Company.)

suspeeted a cireuit of the blood from the heart to the lungs and return, but Harvey was the first to demonstrate circulation, and the first to arrive at an idea of a complete circulation of all of the blood through a closed system of vessels. This new idea was presented in 1628. He also did notable work in embryology.

Marcello Malpighi (1628-1694) was a famous Spanish anatomist, histologist, and embryologist. His observation of blood corpuseles in capillaries, studies on glands, and his work on the structure and metamorphosis of the silkworm take rank with outstanding con- 
tributions to zoological knowledge. Numerous organs of the human body are named for this renowned scientist of his time. Like other early microscopists, he had to build his own microscope.

Antonj van Leeuwenhoek (1632-1723) lived almost contemporaneously with Malpighi and like him made many contributions to the

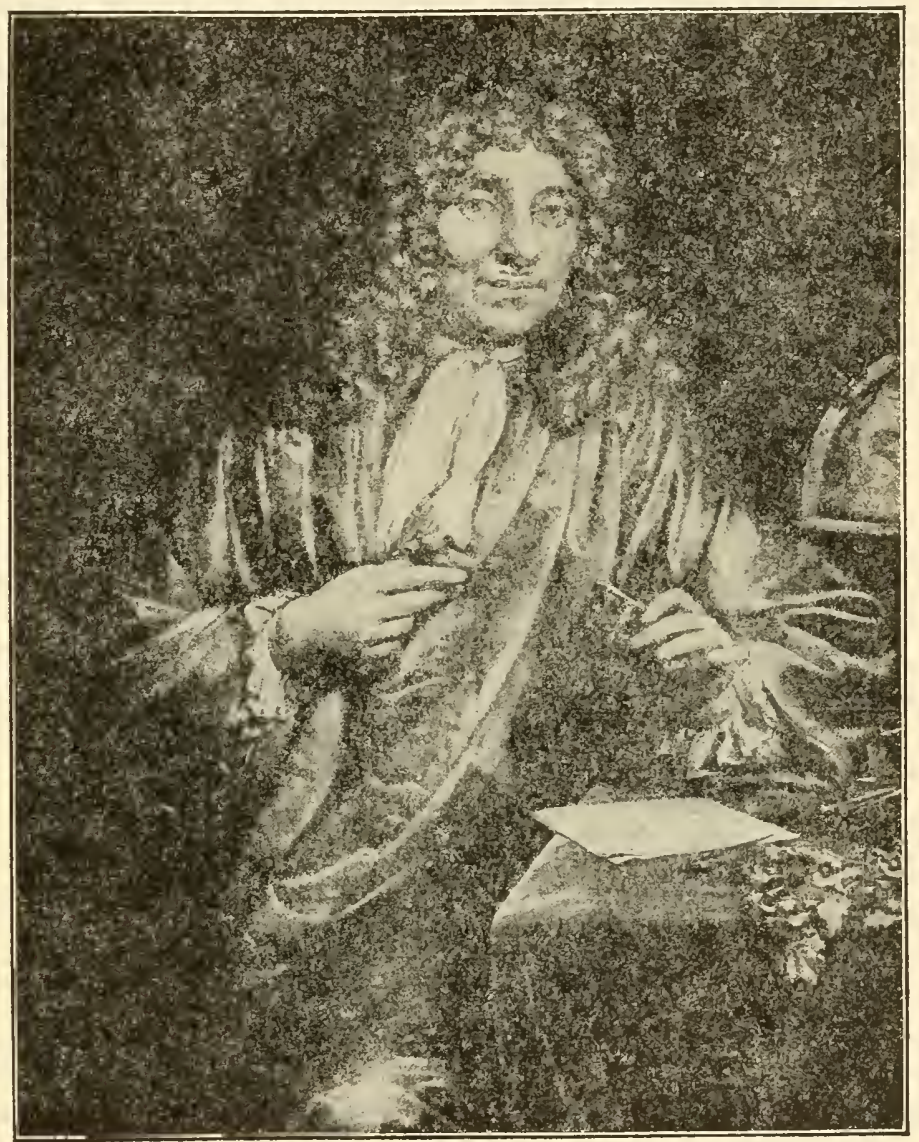

Fig. 11.-Leeuwenhoek (1632-1723), pioneer micromotist. (From a painting by Veekolje, 1685 . Reprinted by permission from Locy, Growth of Biology, published by Henry Holt and Company, Inc.)

development of the microscope. He is said to have possessed a total of 419 lenses, most of which he had ground. Further study on capillary blood circulation, first descriptions of spermatozoa, extended observations on bacteria and microscopic animals, and his 
raluable contributions to the development of the microscope are the enviable accomplishments of this man.

Carolus Linnaeus (1707-1778) was a rery eminent Swedish biologist, who, like many early students of this subject, was educated as a physician. He followed somewhat in the footsteps of Ray (1628-1705), who had paved the way by fixing a definite conception

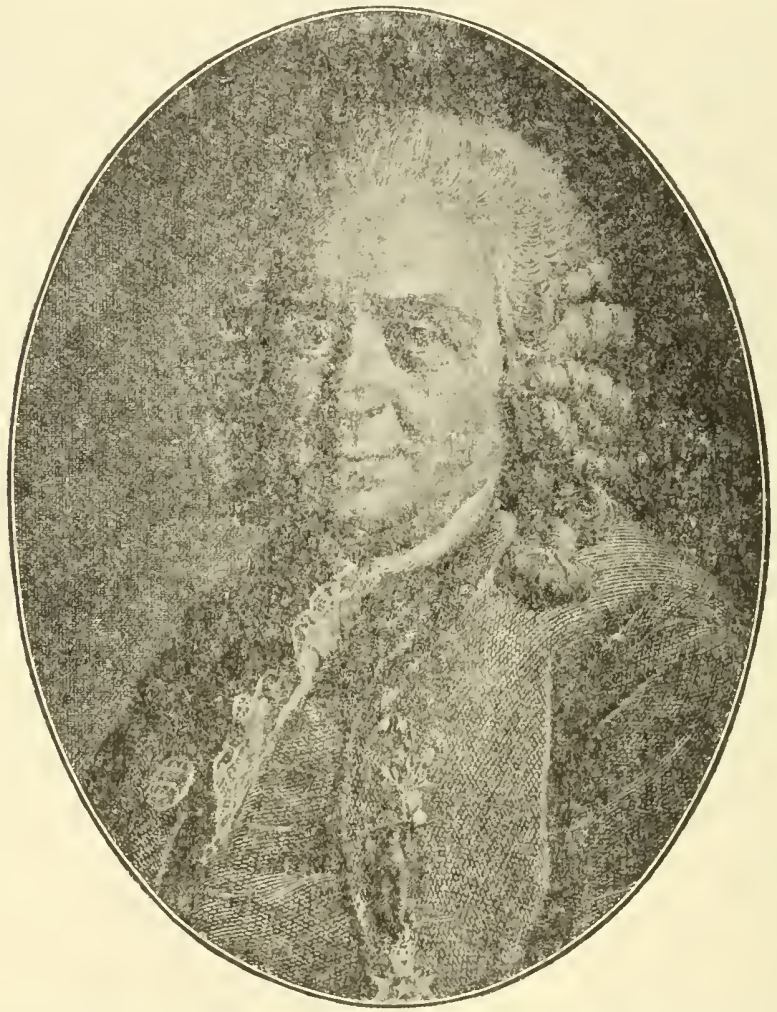

Fig. 12.-Linnaeus (1707-1778), an outstanding Swedish biologist of his time. (Reprinted by permission from Locy, Growth of Biology, published by Henry Holt and Company, Inc.)

of a species and introduced the use of anatomical features in distinguishing the larger groups. Linnaeus believed in a rigidly fixed species and had divided the animals into six classes, 32 sub-classes, and numerous genera and species. In spite of his idea of the invariability of species his classification system was so simple, clear, and flexible that it has persisted to the present time. His was the 
first natural system of classification, and it is known as the Binomial System of Nomenclature. Each individual not only fits into larger general groups by this system, but it is specifically known by the genus and species names used together, hence the two names. Linnaeus is said to have classified and listed 4,378 species of plants and animals.

Almost immediately following Limnaeus came the Frenchman, Lamarck (1744-1829), who among other important things is credited with being first to realize that there are different lines of descent and that no living species is absolutely fixed. Much later, in 1866, Ernst Haeckel organized the modification of this system as used in modern times.

Georges Cuvier (1769-1832) is eredited with establishing the field of comparative anatomy. He was of French ancestry and largely self-educated by his studies at the seashore. A number of anatomieal structures bear his name.

Karl Ernst von Baer (1792-1876), a Russian biologist, is one who really established embryology as a field of study. His notable papel' on the development of the chick was published in 1832. He established the "germ layer theory," thus explaining the unfolding and differentiation of the various organs of the developing animal. The recapitulation theory, which is explained elsewhere, came as a result of his work and thought.

Johannes Müller (1801-1858), a German scientist, is referred to as the founder of comparative physiology and the first to apply the facts of physics and chemistry to living protoplasm. His work was a great impetus to modern physiology.

Matthias Schleiden (1804-1881) and Theodor Schwann (1810-1882) are the two Germans who in 1838-1839 arrived at one of the most important generalizations of biology, the cell theory (principle). This is to be discussed further in the following chapter.

Louis Agassiz (1807-1873) is commonly regarded as the father of American zoology and a renowned student of comparative anatomy. His great inspiration has permeated through his students to nearly every institution in the land. He was a recognized paleontologist as well as zoologist. He is responsible for one of our first and oldest Marine Biological Laboratories. 
Charles Darwin (1809-1882), an Englishman, made extensive studies on the problem of the manner and means by which new species of organisms arise. He very effectively developed the thesis that they originate by a process of natural selection. This was based on the idea that no two individuals are exactly alike, that new variations are constantly appearing, and finally that those individuals or groups best suited to their environment would be the ones to persist and produce progeny. His conception of the factors and

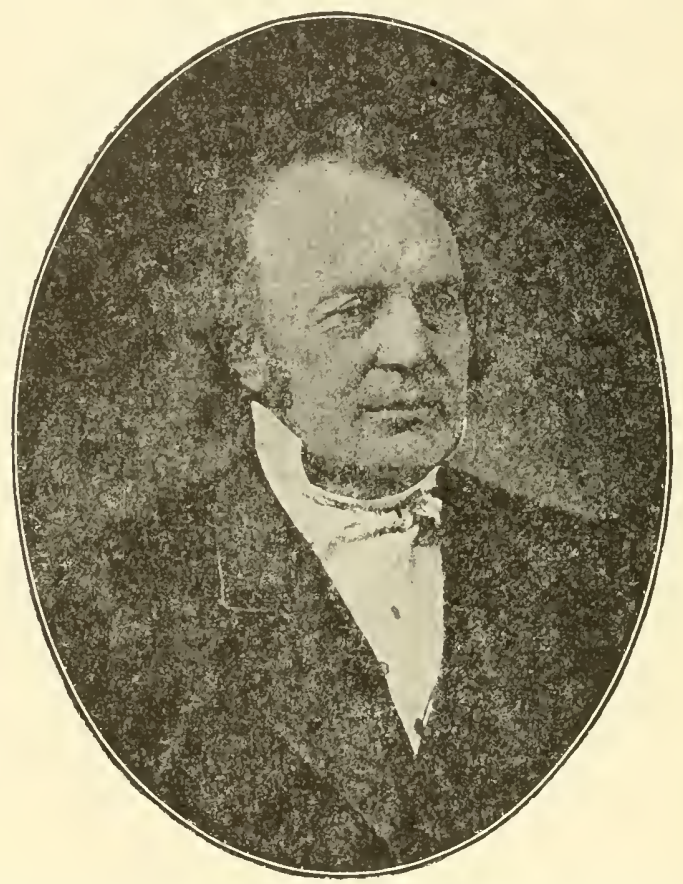

Fig. 13.-Agassiz (1807-1873), great American pioneer zoologist. (From Locy, Biology and Its Makers, published by Henry Holt and Company, Inc.)

limitations determining the development of new species, pictures a constant struggle for existence among organisms, with those whose natural variations happen to fit them best to the changing features of the environment persisting as dominant species and others being crowded out. Those least fitted to the environment would naturally become extinct.

Darwin did not claim originality in his idea. Lamarck, Buffon, and Erasmus Darwin, grandfather of Charles, had presented similar 
ideas before him. It was the vast accumulation of facts covering a period of twenty years which commanded the attention of scientists as well as the public generally. In 1858 he read a joint paper with Alfred Russel Wallace, a contemporary who had reached the same conclusion, on the theory of natural selection. That same year Darwin published his book Origin of Species which is a classic in its field and familiar to all scholars.

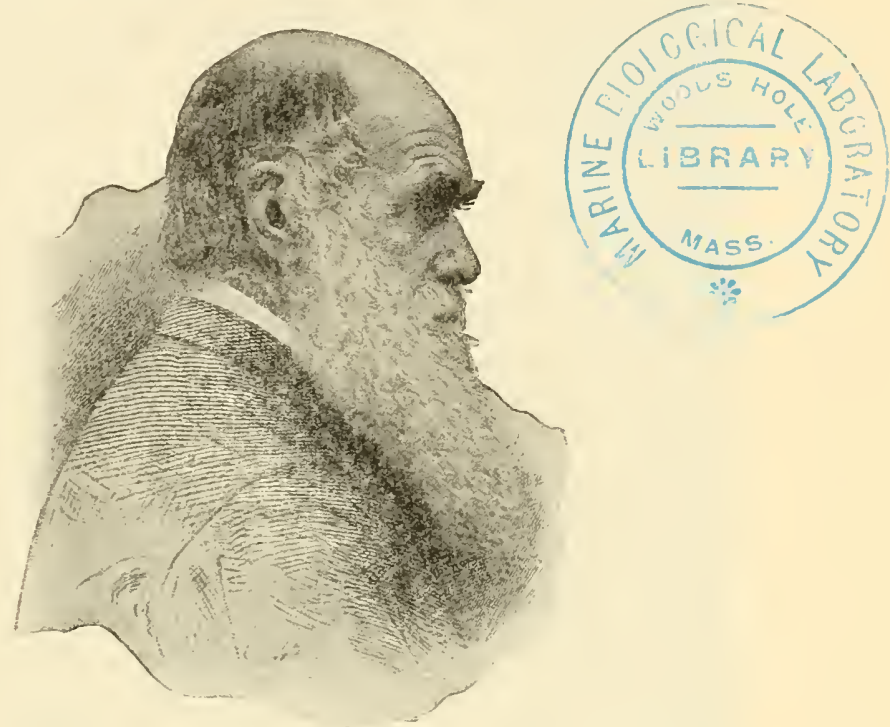

Fig. 14.-Charles Darwin (1809-1882), the author of Origin of Species. (From Garrison, History of Medicinc, published by W. B. Saunders Company.)

Gregor Mendel (1822-1884) was an Austrian monk who carried on experiments with the breeding of garden peas in the cloister garden. From his work there, he derived the original laws of heredity. His results were first published in an obscure Swiss paper in 1866 and were not really discovered and appreciated until 1900. He was the founder of genetics. He crossed different kinds of peas and found that the offspring in the first generation all resembled one parent. When these offspring were interbred he found that three-fourths of their progeny resembled one grandparent, and the remainder resembled the other. From these facts he referred to characteristics of the former group as dominant and those of the latter as recessive. The facts which he established are now known as Mendel's Laws of Heredity. 

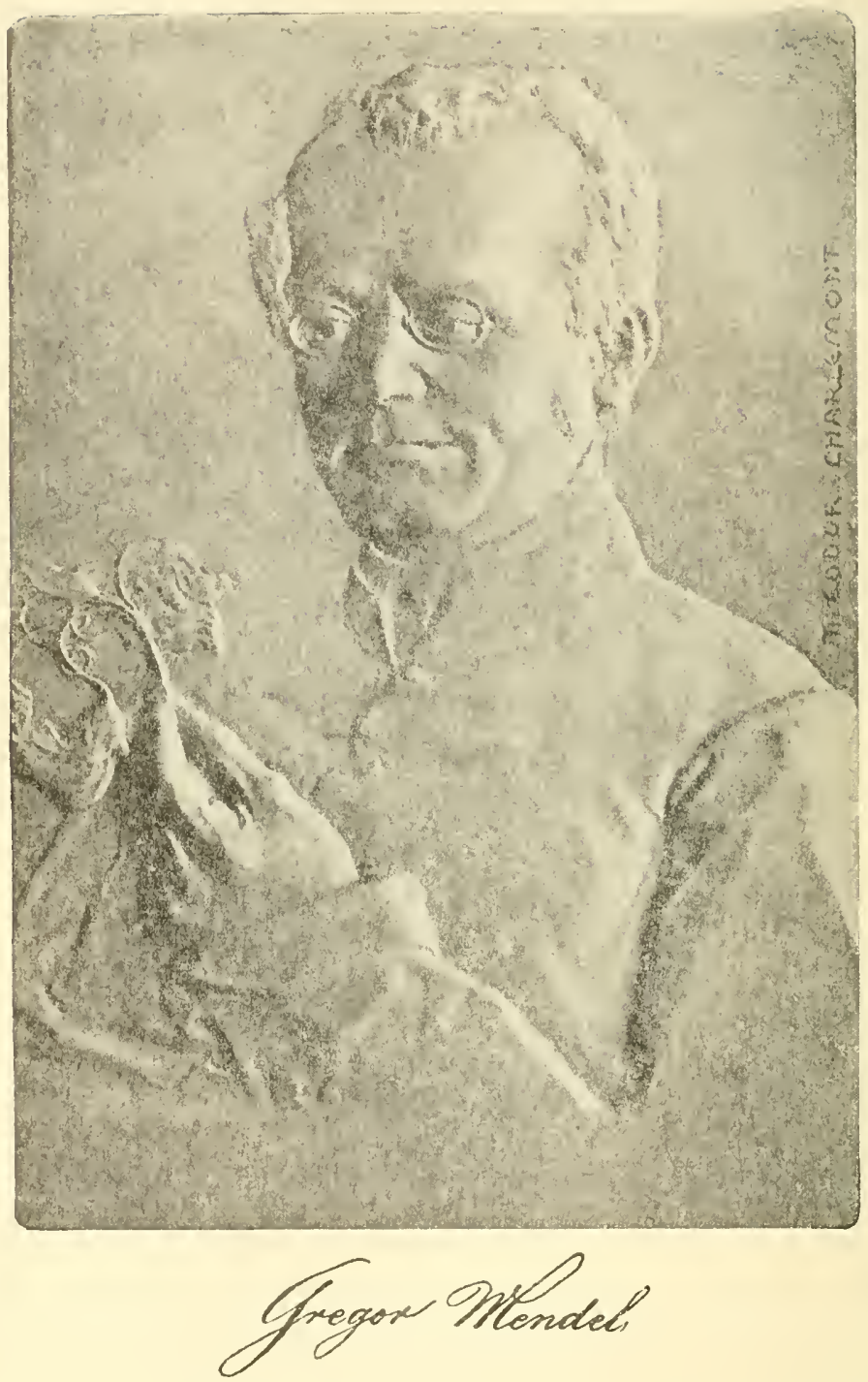

Fig. 15.-Gregor Mendel (1822-1884), the Austrian monk who pioneered in studies of heredity. Plaque by Theodor Charlemont based on "Fuschia picture" made between 1861 and 1864. The signature is his, taken from an autobiography. (Courtesy of the Journal of Heredity.) 
Louis Pasteur (1820-1895) was a French scientist who had been trained in chemistry but became one of the outstanding pioneers in applied biology and medicine. In 1861 he put an end to the controversy regarding spontaneous generation of living organisms and established the idea that all life in present times comes from preexisting life. He showed that living organisms cause fermentation and demonstrated that these organisms and others could be killed by heating them to a certain temperature. He showed that materials thus heated and then sealed would not ferment until after they were exposed to the organisms in the air. The pasteurization

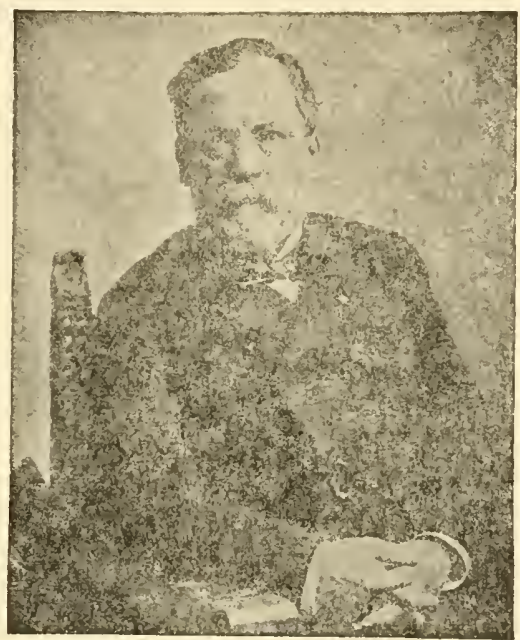

Fig. 16.-Louis Pasteur (1822-1895), one of the benefactors of mankind. (Froin Garrison, History of Medicine, published by W. B. Saunders Company.)

process grew out of these experiments. He rescued the silk industry of southern Europe by discovering the organism which killed the insects, and he also discovered an immunization process and treatment for hydrophobia.

Thomas Henry Huxley (1825-1895) was one of the most popular English scientists of his day. He was one of the principal champions of Darwin's ideas and theories. Comparative anatomy and paleontology were greatly advanced through his influence.

August Weismann (1834-1914) was a German zoologist who started out as a physician after having been trained in that field. He was 
an outstanding scholar in the fields of heredity and embryology. $\mathrm{He}$ is best known for his theory, that there is continuity of germ plasm from generation to generation.

Hugo DeVries (1848-1935), a Dutch botanist, brought out the mutation theory, which is important to all modern biological conceptions. His idea was that species have not arisen through gradual selection requiring thousands of years for each but by jumps through sudden, though small, transformations. He is widely known for his experimental studies in plant breeding and genetics, particularly with evening primrose.

E. D. Cope (1840-1897) was one of the greatest comparative anatomists of America. He dealt not only with living forms but with fossil materials as well.

The work of all those mentioned and hundreds of others has given us the background for our present knowledge and grasp of zoology and medicine. History is being made so rapidly in these fields during the current years that it is difficult even to catalogue the important contributions. It is an extremely active field, particularly in the realm of the experimental endeavors. The printed program for the annual meeting of the American Zoological Society, which is made up largely of titles and abstracts of new papers to be presented, is a small book in itself.

The works and lives of such prominent pioneer zoologists of the Southwest as Jacob Boll, Gustaf W. Belfrage, Lincecum, Vliet, Walker, Webb, and others have been described in the recent book by Dr. S. W. Geiser of Southern Methodist University, entitled Naturalists of the Fronticr. This book is extremely interesting to read. 


\section{CHAPTER III}

\section{PROTOPLASM AND THE CELL.}

\section{Living Matter, or Protoplasin}

Little is known concerning the origin of living matter, or protoplasm, as it is called, but more and more is being learned about its nature, characteristics, structure, and activities. Living matter is always active in some degree, and this activity attracted the attention of scholars at a rather early date, but serious study of the material was not begun until approximately one hundred years ago. A Frenchmas by the name of Dujardin, in 1835, realized that some of the simple microscopic animals he was studying were composed of a soft, gummy substance and called it sarcode, which means "flesh." He was able to test its solubility and its behavior with alcohol and acids sufficiently to satisfy himself that it differed from ordinary gelatin or albumin, with which it might be confused. In 1840, Purkinje, a Bohemian biologist, gave living matter the name protoplasm, which comes from the Greek protos, first, and plasma, anything formed or molded. In 1846 von Mohl, a German botanist, saw in plants a granular, viscous substance similar to that already seen in animals, and called it protoplasm. He was instrumental in bringing this name into common use. During these years it had gradually dawned on biologists that this matter is found in all living things.

\section{The Cell Principle}

Cells had been seen and even superficially described during the latter part of the seventeenth century and numerous times during the eighteenth century, but their significance was not realized. Hooke, an Englishman, in 1665 in observing cork with the microscope he had made, saw the spaces in it and called them cells because they reminded him of prison cells. This name later came to be applied to the real cells. It was an unfortunate term, for cells do not have a hollow structure but are typically semisolid bodies. Leeuwenhoek saw spermatozoa and bacteria and included them with single-celled animals as "little beasties"; Malpighi had described 
the nature and appearance microscopically of several organs of the body; Grew had made rather extensive microscopic studies of plants, and in 1831 Robert Brown had discovered the nucleus of the cell, but not until the work of Schleiden in 1838 and Schwann in 1839 was the coll theory formally enunciated. The former a botanist and

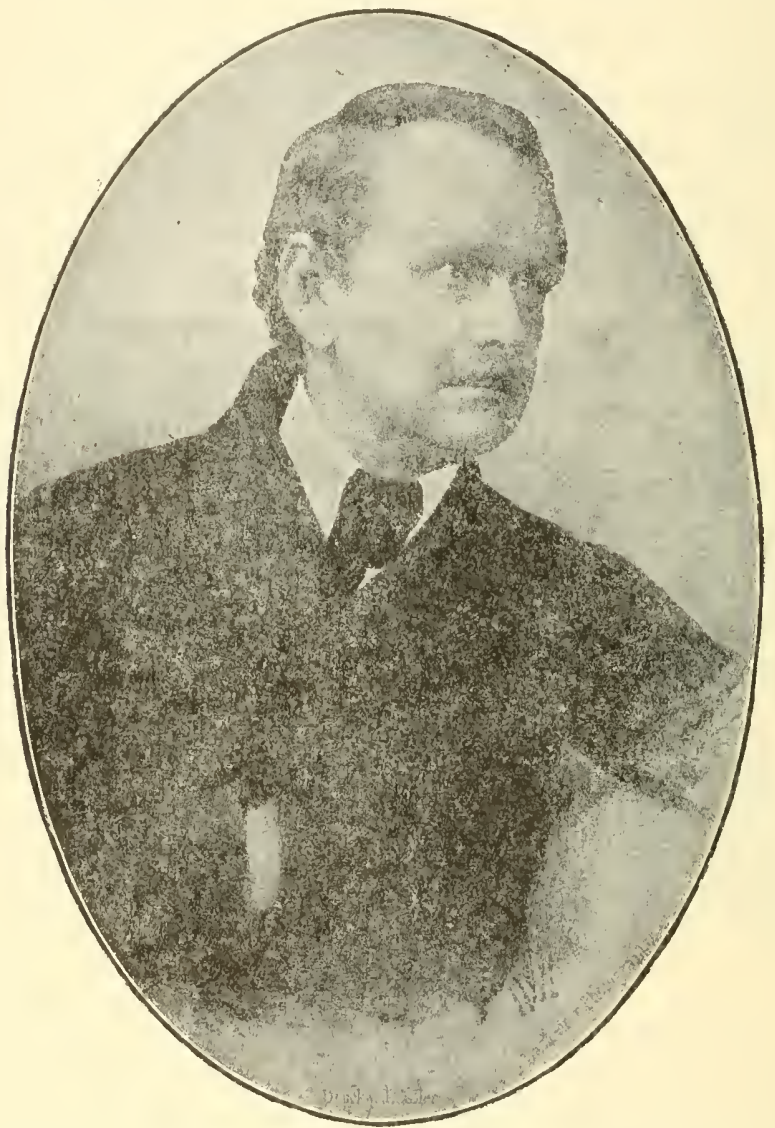

Fig. 17.-Mathias J. Schleiden (1804-1881), noted German botanist who helped establish the cell theory. (From Locy, Growth of Biology, published by Henry Iolt and Company, Inc.)

the latter a zoologist, each working independently, came to the same conclusion and in 1839 collaborated their ideas. This theory, as they gave it, was in substance, All living things (plants and animals) are composed of cells. 
It is no discredit to this theory or these men that they and many other biologists of the time had erroneous ideas concerning the essential features of the cell. Although Brown had recently discovered the nucleus, the cell wall was thought to be the essential part, though now we know it is not a universal structure of all cells since practically no animal cells have a cell wall. The notions of the origin of cells and the functional significance were almost wholly fantastic, yet the cell theory proved to be such a unifying generalization and inspiring stimulus to investigation that it became the turning point in the development of biological study.

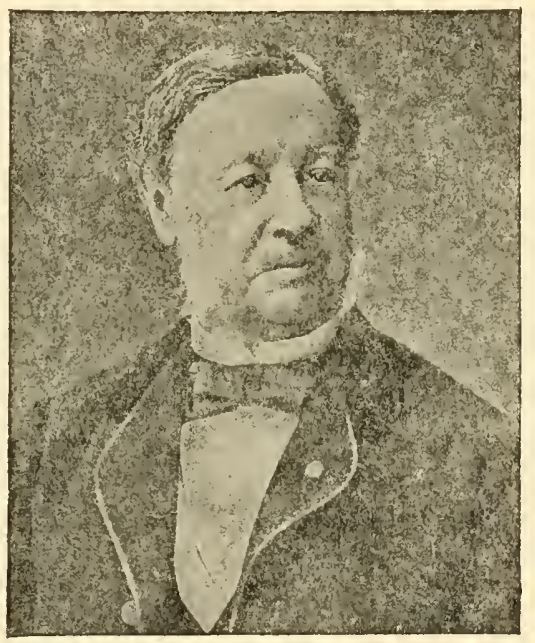

Fig. 18. - Theodor Schwann (1810-1882), the German zoologist who, in 1838 and 1839, collaborated with Schleiden in formulating the cell theory. (From Garrison, History of MIedicine, published by W. B. Saunders Company.)

The bare statement that living beings are composed of cells soon became inadequate as studies of cells progressed. It was soon found that some tissues are made up not only of cellular structures but included also certain noncellular materials produced by the cells. The matrix, so abundant between the cells of cartilage, was soon found to be noncellular and to be produced by the cartilage cells which became cmbedded in it. This matrix is not strictly living matter since it is inactive and passive as far as life processes are concerned. Connective tissue fiber's fall in the same category. Since living bodies are composed of such an abundance of this noncellular 
material produced by the cells, the cell principle soon came to be stated thus: all living things are composed of cells and cell products. With the years, the conceptions of the nature of the nucleus, the cell membranes, and the composition of protoplasm itself have all added their contributions to the present understanding of the meaning and application of the cell principle. The cell is now regarded as a physiological unit as well as a structural one, and as almost a corollary to the original statement of the principle, namely, that the activities of the organism equal the sum of the activities of its cells.

With the embracing of the functional activity of the cell as a part of the principle underlying living processes comes also the inclusion of heredity and development. Cell division, growth, tissue formation, migration of cells, formation of cell products, chromosome relationships and modifications have come to be recognized as being brought about in or by the cells. Through the rather rigid and constant set of developmental changes for which the cells are responsible, there is developed a new individual which usually resembles its parents quite closely.

The influence of the eell theory on biological thinking and progress as well as its effect on fundamental thinking generally, can hardly be over-estimated. The conception of this idea was one of the great landmarlss in development of biological and scientific thinking. It was the first great generalization in biology. It is comparable in the field of biology to Newton's law of gravitation in the field of physics. Up until this time there had been no single fundamental idea applied to living material that was recognized as being universally true. This conception focused the thinking of all biologists in the same direction and therefore it had a great unifying influence. Deliberation and meditation on this fundamental idea seemed to prepare biologists for other great generalizations which followed quite rapidly. Many new problems arose with this new knowledge of plants and animals. Comparative morphology was extensively investigated, and pliysiology now has become physiology of cells as a result of this impetus. An understanding of the permeability of cellular membranes, the transformation of energy by chemical reaction within cells, the roles of electrolytes in living substance, and the principles of heredity are some of the results of this new conception of life embraced in the cell theory. 
General Characteristics of Protoplasm and the Material of the Cell

To begin with, it may be said that this substance has a variable degree of fluidity under different conditions. The range of this variation may be from semisolid to semiliquid. It is viscid and gelatinous in consistency. It is more or less granular, nearly colorless, and more or less translueent; however, it is never perfectly transparent. The transluceney eauses a mass of it to have a lustrous gray appearance. As a constituent of protoplasm there is always a considerable pereentage of water, which conditions the degree of viseosity.

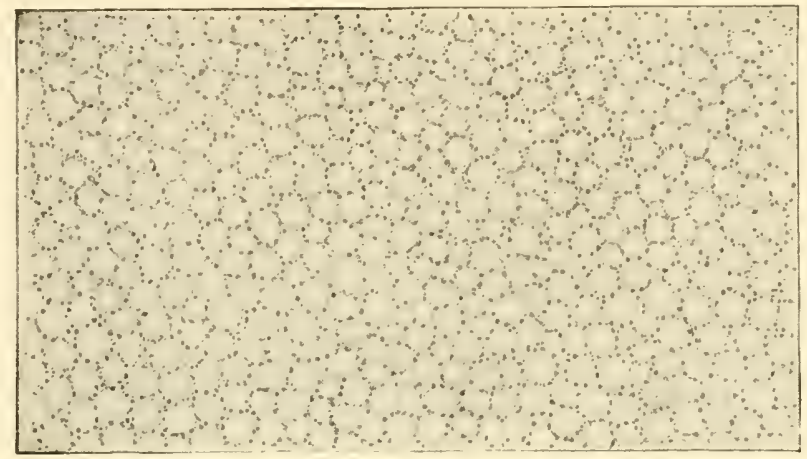

Fig. 19. - Structure of living protoplasm as seen in the starish. (From Wilson, The Cell, published by The Nacmillan Company.)

Protoplasm is in a colloidal state of the emulsoid type. A colloid is a substance of gelatinous nature, permeable by crystalloid solutions, and diffusing not at all or very slowly through animal or vegetable membranes. In the emulsoid, or eolloidal emulsion, the substances are distributed through the more watery or dispersion medium. A colloid is identified by the presence of particles whieh are groups of molecules dispersed through a more fluid or watery phase. These particles, of course, are larger than molecules, but they are too small to be seen with the ordinary microscope. It is possible for water and substances in solution to enter protoplasm from without, and this is reversible. With loss of water from the dispersion medium the dispersed particles of the colloid become eongested by loss of general fluidity. This eondition is known as the gel state. When there is increased water in the dispersion medium and the partieles move with greater ease in the more fluid medium, the colloidal state tends to be- 
come sol. This transfer of water may be due to chemical changes in the dispersed particles or in the dispersion medium of the colloid. The ability of protoplasm, because of its colloidal nature, to change from sol to gel state and back to sol repeatedly is the basis of many of the rital activities, such as utilization of food, disposal of waste, and movement.

\section{Fundamental Properties or Activities of Protoplasm}

In addition to the general characteristics, there may be mentioned and described briefly a number of important activities common to all protoplasm. These properties are:

1. Irritability, which refers to the capacity present in all protoplasm for responding to clianges in environmental conditions, or external stimuli.

2. Conductivity refers to the fact that the impulses produced by stimuli or irritations at one point in protoplasm are conducted to other parts of not only a single cell but also to adjoining cells.

3. Contractility, which is the power of contraction and relaxation that is common to the substance of every cell.

4. Mctabolism, the process of continual exchange of food and fuel materials being built into the protoplasm, while, at the same time, materials there are being oxidized to liberate kinetic energy, such as heat and movement, and produce waste by-products.

5. Growth is recognized as any increase in volume. When the rate of the building side of metabolism exceeds the oxidation rate in the protoplasm, there is storage of materials in the mass of the protoplasm and hence growth. All protoplasm has this capacity.

6. Reproduction is the capacity for producing new individuals of the same kind. All living organisms are capable of this by some means. Simple cell division is the most primitive process of reproduction among animals.

Consciousness, which refers to the awareness of one's own existence, is frequently given as a property of protoplasm. It is certain that some protoplasm possesses consciousness, but evidence of this quality is rather intangible. Spontaneity is also considered a property of protoplasm by some. To be certain that the activity and source of all reaction comes from within is likewise rather difficult of definite proof, so this is simply mentioned here as another property which is often listed. 


\section{Physical Nature of Protoplasm}

Protoplasm is a semifluid material which is heavier than water and somewhat more refractive to light. Its physical constitution is similar to glue or gelatin, rather than to crystalloids, such as sugar or ordinary table salt (sodium chloride). Instead of being in the form of a true solution like salt in water, it consists of suspensions of relatively large molecular aggregations varying roughly betweell 0.0001 and 0.000001 of a millimeter in diameter. These particles keep up an expression of energy in that they move against each other as though they were dancing in a limited space. This activity ean be seen only with a special optical arrangement known as the ultramicroscope and the phenomenon is known as Brownian movement (characteristic of colloidal substances). Protoplasm diffuses slowly or not at all through animal membranes. It changes from a fluid or sol state to a more solid or gel state and may return in the other direction. Ordinarily the viscosity of the continuous phase or supporting liquid is only three or four times that of water, while with the dispersed particles included it is only eight or ten times that of water. The viscosity of the nuclear fluid is only twice that of water. Since glycerin has a viscosity about a thousand times as great as water, it will be realized that most protoplasm is quite fluid in its active state. Changes in viscosity accompany and are essential to the activity and functioning of it.

Protoplasm is not a single compound; it is a colloidal system of a number of chemical compounds existing together. Colloidal systems are known as disperse systems of the emulsoid type. The more watery or continuous part of the system is known as the dispersion medium, while the particles or molecular aggregations constitute the dispersed phase. An important consequence of the colloidal systems in protoplasm is the enormous surface of particles exposed to the continuous phase. If a sphere of material has a radius of one centimeter its total surface will be 12.6 square centimeters. Now, if the same volume of material is in colloidal particles of the average size given above, the total surface of these will be approximately 7,000 square meters. This increase in surface is one of the significant effects of colloidal organization of substances, because many important reactions occur at these surfaces. By the presence of salt ions in the continuous phase and these becoming adsorbed 
upon the surfaces of the colloidal particles, they acquire an electric charge. Protoplasm exhibits these several phenomena because of its colloidal nature.

\section{Chemical Nature of Protoplasm}

Up to the present time, protoplasm has eluded complete and exact chemical analysis. Nevertheless the compounds of living matter are composed of several elements, many of them the most ordinary and abundant in the world. The list of elements necessary to make human protoplasm could be gathered in almost any locality on the face of the earth. As a rule the elements found in protoplasm are oxygen, carbon, hydrogen, nitrogen, sulphur, phosphorus, calcium, sodium, chlorine, magnesium, iron, potassium, iodine, and frequently others like silicon, aluminum, copper, manganese, bromine, and fluorine. The most abundant of these are found named in the first part of the list. A few of them are usually given as constituting approximately the following percentages of protoplasm: oxygen 65 pel' cent, carbon 18 per cent, hydrogen 10 per cent, nitrogen 3 per cent, calcium 2 per cent, phosphorus 1 per cent, and all others making up the remaining 1 per cent. These elements are found combined to form compounds. The organic compounds include carbohydrates, fats, proteins, and also enzymes. The inorganic compounds consist of several inorganic salts and water.

The carbohydrates, which include starches and sugars, are compounds of carbon, oxygen, and hydrogen. The proportion of the hydrogen to oxygen in the molecule is the same as found in water, two to one. The principal carbohydrate found in protoplasm is the monosaccharid, or simple sugar, glucose, whose formula is $\mathrm{C}_{6} \mathrm{H}_{12} \mathrm{O}_{6}$. This is actually built into some parts of the cell, but its chief function is to furnish the most available source of energy by its ready oxidation. When a molecule of glucose is burned, the potential energy is released as kinetic or mechanical energy, and there are formed six molecules of water $\left(\mathrm{H}_{2} \mathrm{O}\right)$ and six molecules of carbon dioxide $\left(\mathrm{CO}_{2}\right)$. Glucose is converted to a starchlike substance, glycogen, for storage in the various animal tissues. This substance must be reconverted to glucose before it is available for production of energy.

Fats, like carbohydrates, are composed of carbon, hydrogen, and oxygen but in more complex molecular arrangement. There is much more carbon and hydrogen with less oxygen, which allows the fats 
to combine with more oxygen in oxidation and therefore release more energy. Fat is extremely well adapted as a form of material for storage, since weight by weight it contains more potential energy than any of the organic group. Such common substances as lard, butter, tallow, whale blubber, and cottonseed oil are good examples. Fats serve a double function in protoplasm: constitution of a part of the structure of the cell and, secondly, the storage of food.

Proteins constitute the bulk of the foundation or framework of the cellular structure and are the most abundant organic constituents. They are composed of carbon, hydrogen, oxygen, and nitrogen, with the frequent addition of traces of sulphur, phosphorus, magnesium, and iron. All of the proteins have large molecules, each being composed of thousands of atoms; as an illustration, take hemoglobin of the red blood corpuscles with its formula $\mathrm{C}_{712} \mathrm{H}_{1130} \mathrm{~N}_{214} \mathrm{O}_{245} \mathrm{FeS}_{2}$. Proteins have a slow rate of diffusion, high resistance to electric eurrent, and usually coagulate upon heating or upon addition of acids, alcohol, or salts to form a clot. Egg albumen, gelatin, and lean meat are common examples of proteins. They are split into numerous amino acids which serve as the building stones of the stable portions of protoplasm.

Enzymes are substances whose exact chemical nature is not yet known, but whose importance to protoplasm is probably unequaled. Chemically and physically they seem to be more like proteins than anything else. These substances are found not only in the cells, but they are also secreted by cells into the digestive tract and into the blood stream, where they act as organic catalysts. The general function of the catalyzer or catalytic agent is that of facilitating and speeding up certain chemical exchanges without the agent itself entering into the reaction. The well-known example of catalysis is the effect of a small amount of platinum in increasing the rate at which hydrogen and oxygen combine to form water. A particular enzyme is usually specific for one kind of reaction, but not for the species of animal in which it will function. Enzymes taken from one species will usually facilitate the same kind of specific reaction in other species. The digestive enzymes may be thought of as an example. Of these, pepsin will bring about the same general reaction, whether it is in the stomach of a frog or of a man, under favorable conditions. Since many enzymes influence only one specific type of chemical reaction and sinee there are numerous 
types of reactions going forward in active protoplasm, it is seen that there must be numerous enzymes present in the cells of every organism.

Water constitutes 60 to 90 per cent of protoplasm and maintains many substances in solution. Water is not only a very efficient solvent; but it is important to protoplasm because of its comparatively high surface tension, because its presence gives the protoplasm a consistency compatible with the range of variation necessary for metabolism, and because of its ligh specific heat. This latter point is important in maintaining protection against sudden and extreme temperature changes in the living organism. Young cells contain more water than old ones, young organisms likewise contain more than old ones. The relative amounts of water in relation to other materials of the protoplasm vary in different cells and in different species.

The inorganic salts are present in considerable numbers but in relatively small amount. They are electrolytes, and therefore split up in aqueous solution into ions, which are able to combine with all the other substances in protoplasm. The chlorides, phosphates, iodides, carbonates, and sulphates of sodium, potassium, calcium, magnesium, and iron are important salts of living cells. The relative proportion of these salts is kept at a fairly constant level, and slight changes in this balance have regulatory effects on metabolism.

From the chenical standpoint, living protoplasm is considered the most complex of all systems of compounds. Even the proteins, as a part of protoplasm, are more complex than any other substances. In a sense, protoplasm is quite unstable in that it changes its composition in response to every change in the environment, and when active it is not the same for any two consecutive moments. The exceeding variability of protoplasm chemically, makes possible all of the necessary adjustments of living matter to its environment. On account of the extreme complexity of protoplasm it is not surprising that the chemistry of all of its activities is not yet completely understood.

\section{Structure of a Typical Animal Cell}

The quantity of protoplasm comprising a single cell varies within wide limits; therefore cells vary greatly in size. The majority of cells, but not all of them, require considerable magnification to be seen. Cer- 
tain of the single-celled blood parasites are about as small as any cells known. They are barely seen with our highest magnifications. At the other extreme of size we may refer to another parasitic single-celled animal, Porospora gigantea, which lives in the intestine of the lobster, and may reach from one-half to two-thirds of an inch in length. Egg cells, including the yolk, may exceed this size. Some of the nerve cells, though of less mass, may be several feet in length. Minscle cells are relatively long also.

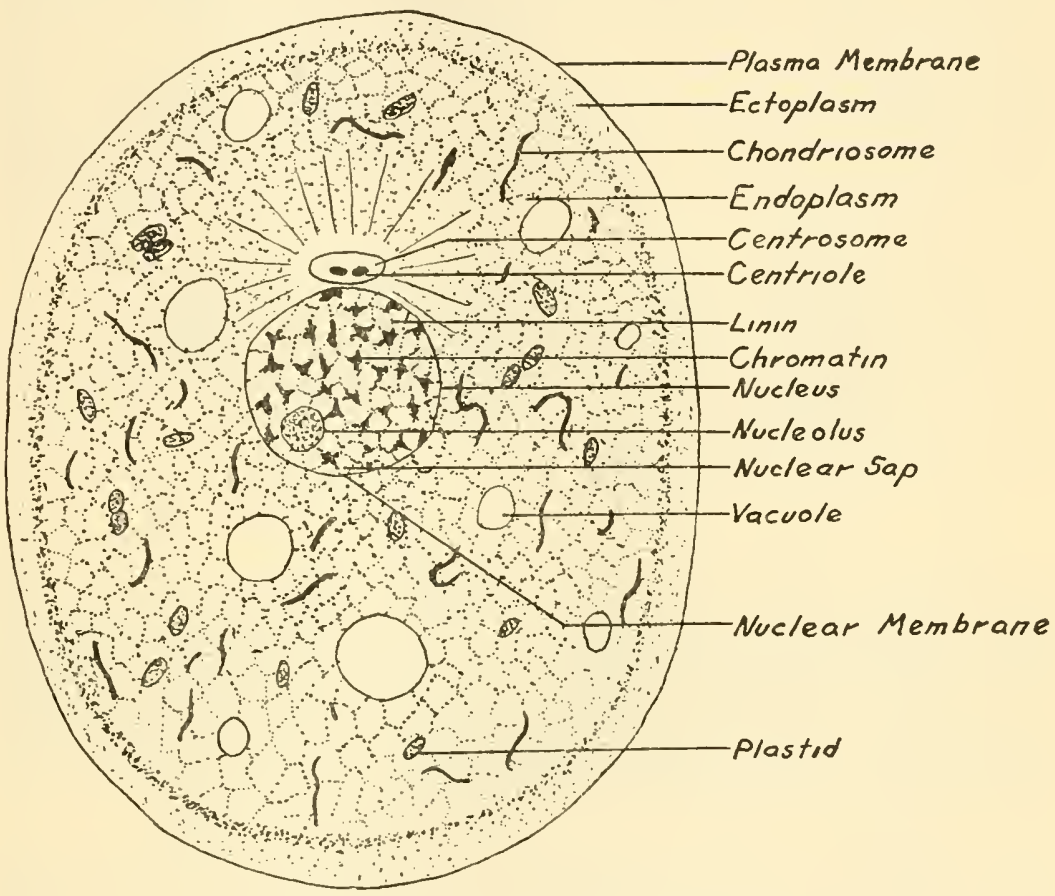

Fig. 20.-Diagram showing a typical animal cell.

The shape of the typical cell is spherical; but due to the effects of mechanical pressure, specialized functions, and unequal growth almost all cells are far from this shape. They vary greatly in shape and include platelike, cubical, columnar, polygonal, and spindleshaped forms. The particular form of any cell is not a haphazard matter but strictly controlled by morphological and functional necessities. 
A cell consists of a mass of jellylike cytoplasm surrounding a nucleus. The outer surface of the cytoplasm is modified, the protoplasm having more density here to form the plasma membrane, or cell membrane, which is the outer covering of the animal cell. This membrane is living and semipermeable. In some types of cells two separate membranes may be distinguished. In plant cells the plasma membrane is covered by a cellulose cell wall.

The cytoplasm usually includes the larger part of the substance of the cell. It may be subdivided into the more nearly clear, structureless fluid, hyaloplasm, and the interspersed fibrillar substance known as spongioplasm. Within the eytoplasm, lying near the nucleus, in most animal cells is the centrosome. Its substance is known as kinoplasm and is made up of two parts, the larger centrosphere, enclosing a (two if divided) centriole. Vacuoles are often present as small

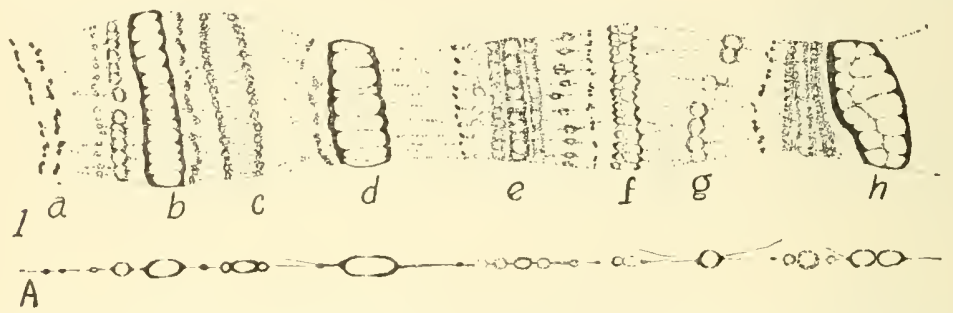

Fig. 21.-A camera lucida drawing, showing the details which appear on the upper surface of a fully developed salivary gland chromosome (large vesicle type) from simulium fly larvae. The longitudinal threadlike bands are called chromonemata, and these consist of a linear series of granules, the chromomeres, which have a specific arrangement of grouping. $1 \mathrm{~A}$ is a semidiagrammatic representation of the types of chromomeres and the ways in which they are connected. At $a$ in the main figure there are two rows of dotlike chromomeres which tend to associate in pairs. The band labeled $b$ is composed of 15 or 16 vesiculated chromomeres closely pressed together. $c$ to $h$ are other groupings of chromomeres along the chromonemata of the chromosome. (From Painter and Griffen: Chromosomes of Simulium, Genetics 22: 616, 1937.)

cavities filled with water, gases, or oils. Scattered through the cytoplasm also are numerous rod-shaped bodies known as mitochondria. Threadlike Golgi elements or apparatus may be observed in the cytoplasm, particularly near the nuclens. Secretions produced in the cell may be stored as granules in the cytoplasm, also certain inclusions may be seen here.

The nucleus, which is usually round and centrally located, is surrounded by the eytoplasm and separated from it by the nuclear membrane. This membrane, like the plasma membrane, consists of a part of the protoplasm whose density is somewhat greater than the adjacent portions. The protoplasm which constitutes the nucleus is usually known as karyoplasm. The more nearly fluid, transparent 
portion of this is karyolymph, or nuclear sap, while the meshwork of fine fibers extending through it is called linin net. Supported on this net is a dark-staining granular or fibrillar substance known as chromatin, which is thought to be the center of functional activities of the nucleus. The threads of chromatin are called chromonemata. During division of the cell this granular material becomes arranged into definite bodies, the chromosomes. It is generally thought that in these bodies are located the units of material (genes) which function in the transmission of hereditary characteristies from one generation to the next. There are usually one or two knots of more dense chromatin in the nucleus which are called karyosomes. Then besides these, most nuclei have another body composed of material thought to be temporary storage products of nuclcar metabolism, the nuclcolus, or plasmosome. Mitochondria, similar to those of the cytoplasm, are also found in the nucleus. The cell is often spoken of as the unit of structure and function in living material. Both nucleus and cytoplasm are necessary for its normal activities. It is not entirely possible to define the part each plays in the metabolism of the whole. Since the development of the microdissector by Dr. Chambers, it is possible to dissect the nucleus of a cell. Cells that are deprived of their nuclei are unable to carry on assimilation, although catabolism goes on until the cytoplasm is depleted.

\section{Cell Division}

The cell is limited in its size, as is the complete organism. This limit of size is fixed primarily by the physiological necessities which are transmitted through the surface of the cell. There is a definite relation between volume and surface in any mass of material, and this may be expressed in a ratio. With variation of the size of the mass, the volume varies according to the cube of the diameter while the surface area varies according to the square of the diameter. When the limit of growth is reached the cell divides, and this restores the proportion of the surface area to volume that will again permit growth. Remak, in 1855, was the first to describe cell division. His idea was that the nucleolus split first, then the entire nucleus, and finally the cytoplasm divided, placing each portion with its share of the nucleus. This direct method of division was called amitosis. Its actual ocenrrence is quite rare. The usual method of cell division is far more complex and less direct. There are several preliminary changes or phases which must occur before the actual cleavage of the cell into two new ones. This is mitotic 
cell division, more briefly mitosis, or indirect cell division. This method of division was first described by Fleming in 1878, though Schneider in 1873 described much of the complicated process.

Although the process of mitosis is a continuous series of changes, for convenience in study, these changes will be set out as six phases.
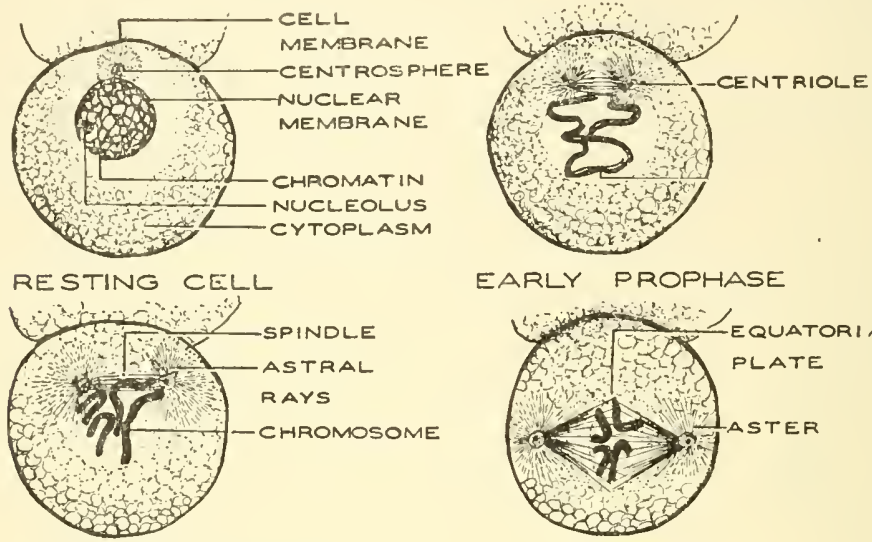

EARLY PROPHASE

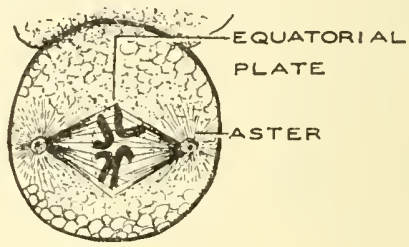

LATE PROPHASE

METAPHASE

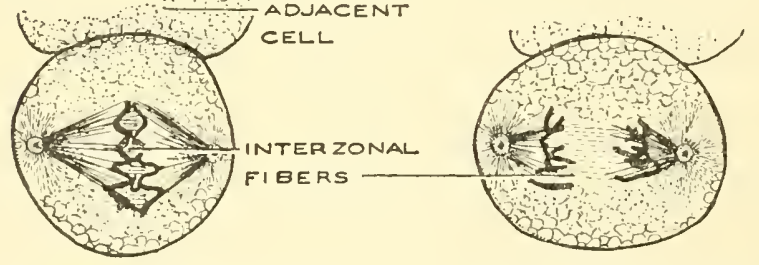

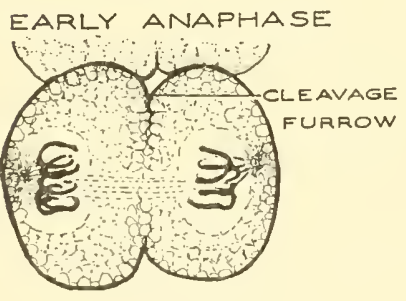

TELOPHASE
LATE ANAPHASE

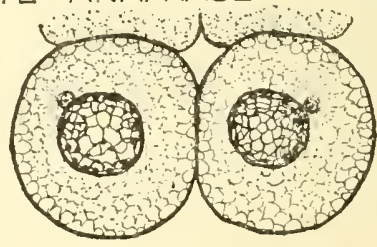

DAUGHTER CELLS

Fig. 22,-The stages in typical nuitosis (indirect cell division) as shown in fertilized Ascaris eggs. They follow each other in order: resting cell, early prophase, late prophase, metaphase, early anaphase, late anaphase, telophase, an. daughter cells. (Drawn by Titus Evans.)

Following the resting cell condition come the first changes, and the early prophase condition is seen. In this stage the centriole has divided, and the two pieces have moved considerably apart. The 
surrounding protoplasm has produeed some rays radiating from each centriole. These two bodies are now known as asters because of their starlike appearance. The two asters taken together are called the amphiaster. The mucleolus disappear's and the chromatin which apparently up until this time has been somewhat dispersed through the nuelear substance in the form of granules, beeomes organized into long, possibly tangled, chromatin fibers or threads. (Some hold that the chromatin retains its linear arrangement from one eell generation to the next.) Each consists of a double linear series of chromatin bodies like two chains bound elosely together to form the thread. A single one of these two series in the thread is known as a chromonema (pl., chromonemata). The ehromatin bodies comprising the chromonemata are often called chromomeres (Fig. 21).

In the middle prophase the centrioles have migrated still farther from each other and the splindle fibers between the centrioles as well as the astral rays around them have become well established. According to modern explanation, each of the ehromatin threads now shortens and eondenses to beeome a chromosome. There is a constant number of these in the eells of each speeies. During the above ehanges, the nuelear membrane begins to degenerate. In the late prophase, the eentrioles have reached the polar positions on opposite sides of the nucleus. The spindle extends between the two asters and the chromosomes become arranged on the spindle fibers in an orderly fashion midway between the centrioles to form the equatorial plate. It has been reported that, in certain cells at least, the prophase stage requires about eight minutes. The nuclear membrane now has almost completely disappeared.

The metaphase follows with no interruption. The chromosomes, still arranged in the equatorial plate, now each split longitudinally, placing one chromonema in each part. The characteristic feature of the metaphase is this splitting. Following this stage each 'of the new chromosomes, resulting from this splitting or division, migrates along the spindle fibers toward its respective eentriole, or pole. This period is the anaphase. These "half-ehromosomes" each soon come to have two chromonemata, and they earry the chromatin material of the new eells which result from the ensuing division. The explanation of the movement of these chromosomes from the equatorial plate out to the poles is not entirely fortheoming, although there is general belief that the spindle fibers, with whieh they are assoeiated, are involved in the phenomenon. 
As the chromosomes approach the poles of the spindle they crowd very close to each other. At this time a constriction of the cytoplasm begins in the plane of the equatorial plate. This is the beginning of the telophase stage. The cytoplasm perfects its constriction and divides into two parts. A nuclear membrane forms to enclose each chromosome group, and immediately the chromosomes begin to separate from the group, although certain ones still clump together. The chromosomes progressively lose their identity and their staining qualities. The nucleus resumes its granular appearance of the resting cell. One or more nucleoli soon become evident. The formation of the new nuclear membrane excludes the centrosome, so it takes its normal position just outside the nuclear membrane in the cytoplasm. At about this time the centriole divides into two. These two new cells resulting from the division are spoken of as daughter cells. These cells have each received the same quantity and quality of chromatin material.

Following the organization of these daughter cells, which are in the resting stage as far as division is concerned, growth is rapid until they reach their typical limit of size. For most average cells under optimum conditions, it is stated that this requires less than two hours. Then after a further period of from one to twelve hour's, another mitotic division may take place. The universality of this process in all types of organisms, both plant and animal, and the regularity of the occurrence of the phases of the process suggest that it is of vital significance. The great precision with which the chromatin is divided between the two cells seems to indicate that this is a most significant step. Chromatin is recognized as the material which makes possible the inheritance of qualities from cell to cell and, in case of sex cells, from generation to generation. The purpose of the splitting of the chromosomes in the metaphase stage seems to be to provide each daughter cell with identical hereditary qualities. This equal division of chromatin, both qualitatively and quantitatively, has given rise to the thought expressed in the phrase, "continuity of protoplasm," and that present chromatin comes from pre-existing chromatin. In 1855 Virehow, a German pathologist, declared the doctrine that all cells must be derived from previously existing cells, in his statement, "omnis cellula e cellula." This supposes that in the first living material created were inherent all of the possibilities which have been realized in all living things that have existed since. 


\section{CHAPTER IV \\ PHYLUM PROTOZOA IN GENERAL}

The animals included in this group are usually said to be the first to have existed on earth and, therefore, they are considered the oldest. Being single-celled, they are usually referred to as the simplest known animals, although many of them are perliaps more complicated than numerous many-celled or metazoan forms because of the extensive modifications of the one cell. Protozoa are universally placed first when animal groups are placed in the order of complexity, beginning with the simplest. It has been supposed and with reasons to support the supposition, that modern Protozoa have descended, without changing their single-celled condition, from primitive organisms that were also the ancestors of Metazoa.

\section{Characteristics}

The great majority of Protozoa are microscopic creatures. Most of them live in water while a few live in the body fluids of other animals. Certain types are found living rather abundantly in the soil water. They are found in almost all conceivable shapes. Some have irregular, changing shapes; others are nearly spherical, oval, spindle-shaped, cylindrical, and vase-shaped. Most Protozoa exist singly as an independent cell, but some are organized into groups called colonies. A few arc encased in hard coverings or shells which are made up of a secretion from the cell alone, or of a combination of such a secretion with a foreign material like sand. With the exception of one class the Protozoa have charactaristic locomotor organs.

\section{Classification}

This group is often spoken of as a sublingdom as well as the first phylum of the animal kingdom. In spite of the exceedingly large number of species and microscopic size, the phylum has been quite systematically classified and is divided into classes, orders, families, genera, and species. The phylum is usually divided into four classes, each characterized by a distinctive locomotor structure or by the total lack of such features, as in one of the classes. 
1. Class Mastigophora (măs tĭ gŏf'ó rả) which meaus whip bearers, includes forms that possess one or more whiplike extensions of the eytoplasm, or flagella. The number of flagella is limited, and they serve the animal as its means of locomotion. In some species they serve the organism in feeding. The flagellum is a contractile structure. There are some species in which exist both flagellate and amoeboid stages. This seems to show a rather close relation of this class to the next. This class also has a close relationship with plants in that many of its representatives possess ehlorophyll.

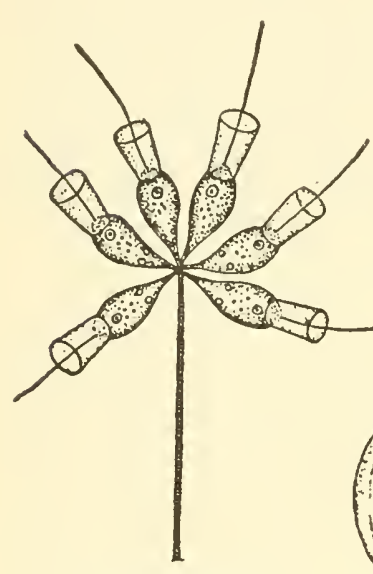

Codonosiga

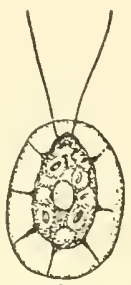

Sphâerella

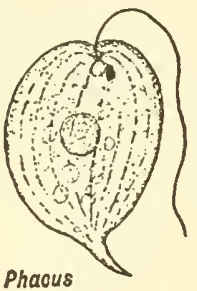

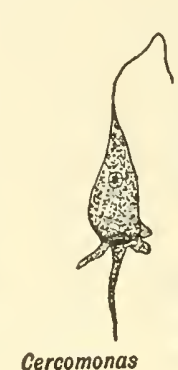
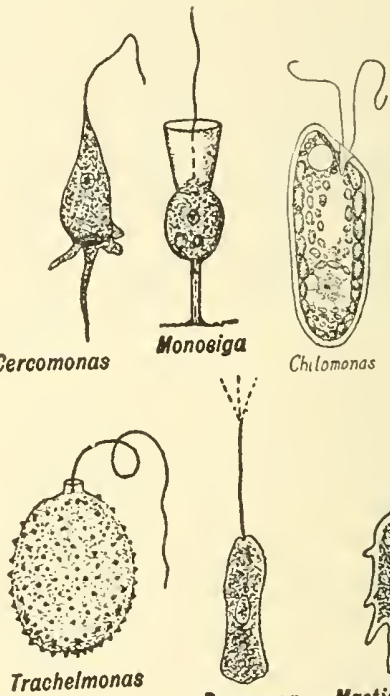

Chilomonas

Fig. 23.-Group of representative Mastigophora. (Reprinted by permission from Curtis and Guthrie, Textbook of General Zoology, published by John Wiley and Sons, Inc.) (Figure of Chilomonas modified.)

These forms are frequently classified as plants by botanists. The class Mastigophora is divided into two groups: (a) the animal-like forms which may be holozoic, saprophytic, or entozoic, and (b) those more plantlike forms which may be holophytic, saprophytic, or entozoic. Holozoic refers to forms which ingest and digest food material. Saprophytic refers to the habit of absorbing nonliving organic matter in solution directly through the surface of the body. Entozoic is a name applied to forms which live within the bodies of other animals, as in the intestine or the blood stream. 
A large number of Mastigophora live in quiet streams, ponds, lakes, and in the ocean. Euglena is a very commonly studied fresh-water form. Noctiluca is an interesting marine form which is pelagic (lives at the surface) in its habits and appears as a thick, creamy scum. This soupy mass of organisms may cover an area of hundreds of square rods. When stimulated, these animals are luminescent and at night frequently give up an attractive greenish or bluish white light; Uroglena is a fresh-water form which is often found in water supply basins and causes a pungent, oily odor and unpleasant taste in the water. Giardia (Fig. 386), Trichomonas, Chilomastix, Retortamonas and Enteronomas are all genera with representatives occurring in the digestive tract of man.

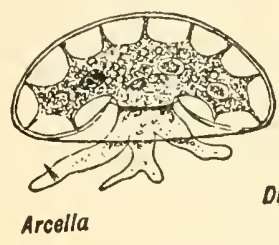

Arcella
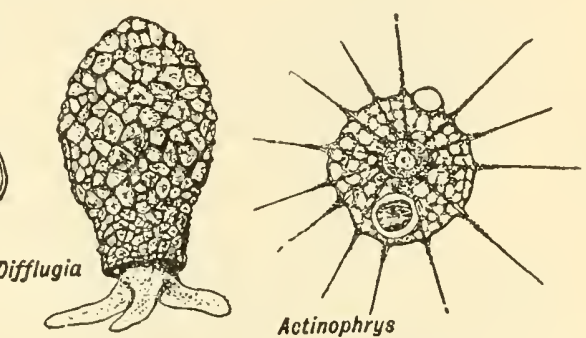

Fig. 24.-Group of typical Sarcodina. (From Curtis and Guthrie, Textbook of General Zoology, published by John Wiley and Sons, Inc.)

2. Class Sarcodina (sär kố dī'nả, fleshy) or Rhizopoda (rī zŏp'ó dả, root foot).-A distinctive feature of nearly all species of this class is the capacity to form protoplasmic processes called pseudopodia (false feet) which are temporary structures and can be withdrawn. The animal is able to accomplish locomotion by extending the protoplasm into these pseudopodia. The representatives of this class include many freeliving forms as well as numerous parasitic ones. A number of the representatives of class Sarcodina secrete an external shell of lime, silicon, chitin, cellulose, or some bind in sand or other solid substances with one of the secretions. The class is commonly divided into five orders. (a) Amoebina are irregularly-shaped forms with lobelike pseudopodia. Some of the species are naked, and others are covered by a shell. Amoeba proteus is the free-living naked form which is commonly studied. Endamoeba histolytica is the most common parasitic form. Arcella, which secretes its shell, and Diffugia, which constructs its 
shell of sand cemented together by a secretion, are two of the most commonly observed shell-bearing forms. (b) Foraminifera is an order of shelled forms whose pseudopodia are very slender and reticular. The psendopodia are extended through small pores in the shell. Only a very few of this group live in fresh water. The vast

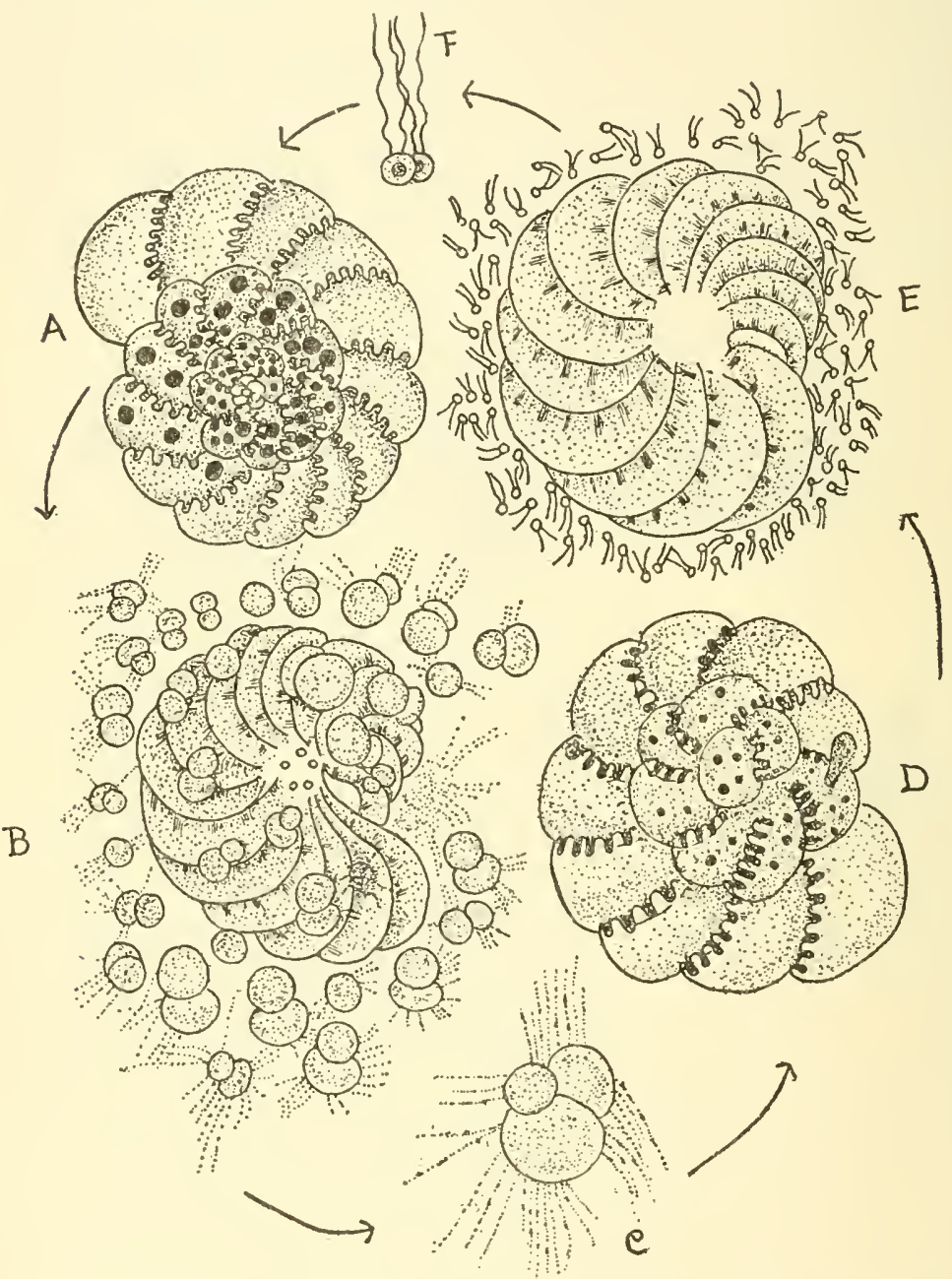

Fig. 25 - micropheric individual; $B$, amoeboid cells escaping from it: $C$, young macrospheric individual developing from amoeboid cell ; $D$, macrospheric individual; $F$, flagellated gametes escaping from it; $F$, union of gametes.

Haswell, Zoology, published by The Macmillan Co.) 
majority are marine, and Globigerina is a typical example. The disintegrating calcareous shells of this organism constitute a great mass of material on the bottom of the ocean which is known as globigerina ooze and from which chalk is formed. (c) Mycetozoa, are characterized as being able to produce enormous plasmodia containing hundreds of nuclei and contractile vacuoles, as well as having ability to

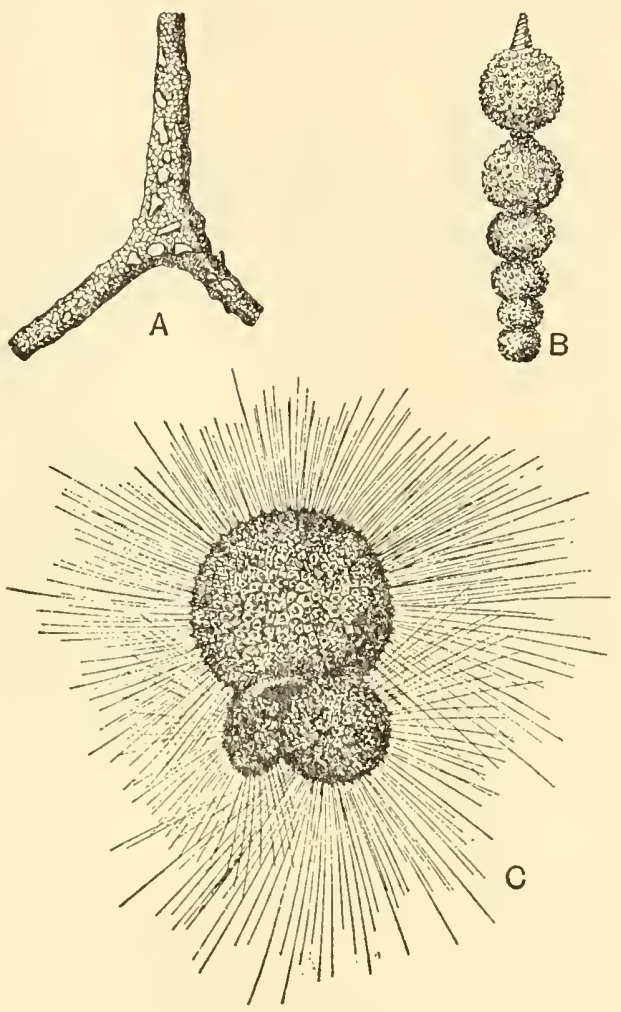

Fig. 26.-Shells of different Foraminifera. A, Rhabdamina abyssorum ( $\times 4.5)$; $B$, Nodosaria hispida $(\times 18) ; C$, Globigerina bulloides $(\times 55)$. (From Borradalle and Potts, The Invertebrata, published by The Macmillan Company, after varlous authors.)

reproduce by spore formation. They live quite commonly in masses of decaying vegetable material upon which they feed. (d) Heliozoa is a group with thin, radially arranged, threadlike, unbranched pseudopodia. Actinophrys sol is a common one found in fresh-water streams and ponds. (e) Radiolaria is a marine group with fine, ray- 
like pseudopodia and a shell composed largely of silica. The pseudopodia extend through the relatively large apertures in the shell.

3. Class Infusoria ( $\mathrm{n}$ fú sō'rì à, crowded).-This group includes those single-celled animals covered with small hairlike, cytoplasmic processes known as cilia. They occur in both fresh and marine waters as free-swimming organisms. There are a few parasitic forms, notably Balantidium coli. Paramecium, Stentor, and Vorticella are
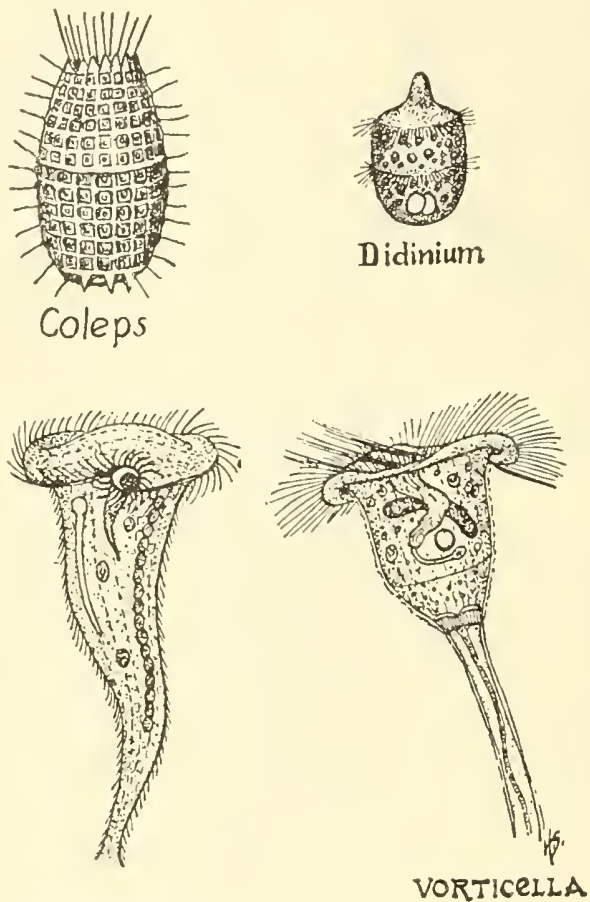

STENTOR

VORTICELLA

Fig. 27.-Group of typical Infusoria. (Courtesy of General Biological Supply

the commonly studied infusorians. The class is now divided into two subelasses, Ciliata and Suctoria. The first, Ciliata, is composed of four orders. (a) Holotrichida, possess cilia of equal length over the body, or they are restricted to particular regions in specialized forms; a cell-mouth is present in most forms. Paramecium is our most common genus living in fresh water. Didinium, Frontonia, Chilodon, and Coleps are other common forms. Opalina is a well-kwown para- 
sitic genus which inhabits the large intestine of the frog. (b) Heterotrichida possess a well-developed undulating membrane in the cytopharynx. The body cilia are small or partially absent, but the cilia of the oral region are well developed. In some forms this oral region possesses membranelles. Stentor, Halteria, and Bursaria are common fresh-water genera while Balantidium (Fig. 389) is a parasite in the intestine of man and some other mammals. (c) IIypotrichide possess eirri or structures formed by fusion of eilia; these are found prineipally on the rentral side. The cell is flattened dorsoventrally and

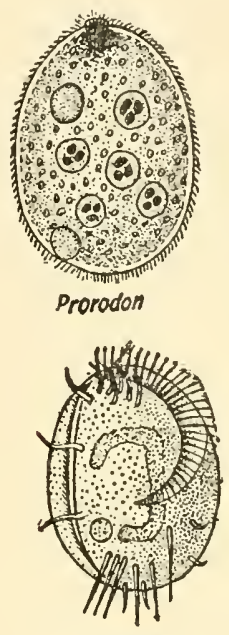

Euplotes
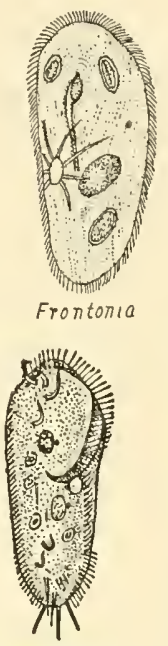

Stylonyohio

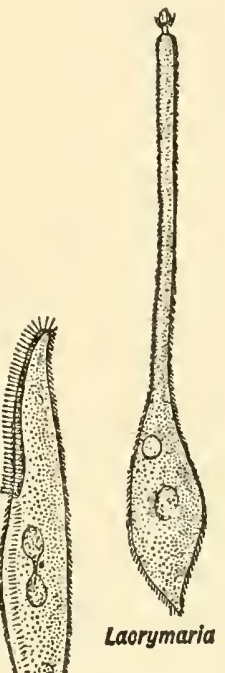

Lionotus

Fig. 28.-Representatives from class Infusoria. (Reprinted by permission from Curtis and Guthrie, Textbook of General Zoology, John Wiley and Sons, Inc.) (Figure of Frontonia modified.)

most of the genera use creeping as their means of locomotion. Stylonychia, Oxytricha and Euplotes are common fresh-water genera. Kerona is a parasitic form and is often found creeping over the external surface of fresh-water Hydra. (d) Pcritrichida is an order composed of sedentary ciliates with a whorl of oral cilia continued into a depression in which are located the oral spot and aperture of the contractile vacuole. At the base of this depression is loeated the mouth. There are no body cilia in certain phases of the life history. These forms are typically attached by stalks. Torticella is probably 
the most common living genus. Epistylis and Carchesium are wellknown colonial gencra. Vorticella and Carehesium have contractile stalks while Epistylis is attached by noncontractile branching stalks.

The second subclass, Suctoria or Tentaculifera, as it is sometimes called, includes animals that are not ciliated, except during a freeswimming stage which may occur following division or encystment. These are attached forms with protoplasmic projections which are used in the capture of food. Most of them are marine, but Podophrya is an example of a fresh-water genus.

4. Class Sporozoa (spō rớ zō'á, seed animal).--These protozoans in their early stages are often amoeboid, but in the completed life history locomotor structures are wanting. During the life cycle there is a spore stage. The animals of this class are entirely parasitic

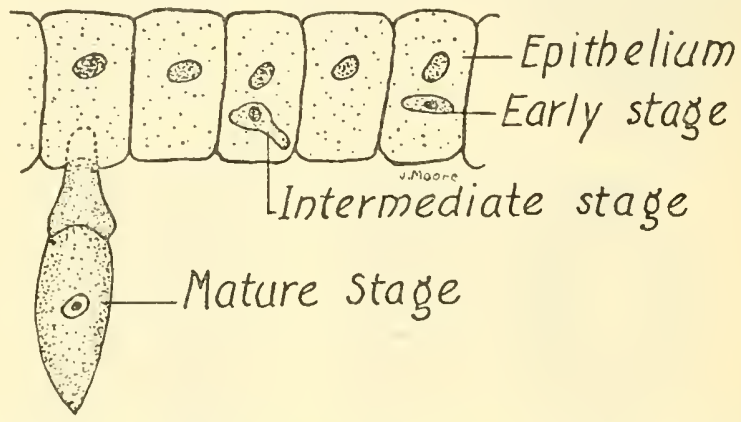

Fig. 29.-Gregarina attached to an epithelial cell of host's intestine. Other stages of its development are shown withln adjacent cells.

and they are usually transmitted to other animals in the spore stage. They often pass from one host in its feces and enter another in contaminated food or drink; or they are drawn from one host by a blood-sucking animal and transmitted to the blood of another. All Sporozoa reproduce by sporulation in which asexual, multiple fission is followed by gamete formation, and the gametes fuse to form a zygote. The spores are produced by the parent animal dividing into fragments while it is encysted. These little cysts, which are secreted by the protoplasm of the animal, are protective and enable them to withstand adverse conditions. The cyst is dissolved upon entrance to a lost and liberates the organisms.

This class of Protozoa is among the most widely distributed of the animal parasites, and their life cycles are often quite compli- 
cated. There are three subclasses of the class, and each of these is divided into some orders. The first subclass is Tclosporidia in which the spores produced have neither a polar capsule nor polar filament. In this group are three orders. (a) Gregarinida (Fig. 29), commonly called gregarines, inhabit the cells (cystozoic) of earthworms, cockroaches, other insects, and occasionally vertebrates in their carly stages, but later they may become free in the cavities of the host. They may attain considerable size. (b) Coccidia are minute monoeysted forms which are permanent intracellular parasites of molluses, arthropods, and rertebrates, including man. The life history involves a period of asexual reproduction (schizogony) and a period of so-called sexual reproduction which ends in spore formation (sporogony). (e) Haemosporidia. The representatives of this order live chiefly in the red blood corpuscles of vertebrates. Again the life cycle involves both schizogony and sporogony. The former oceurs in the blood of the vertebrate and the latter takes place in such hosts as insects, leeches, and ticks. The malaria parasite and the causal agent of Texas fever in cattle are the most important forms.

Cnidosporidia is the name of a second subclass, the spores of which contain from one to four polar capsules cach with a coiled polar filament. There are two orders: (a) Myxosporidia are found chiefly as fish parasites, but occur occasionally in reptiles and amphibia. The gall-bladder, uriniferous tubes, and minary bladder are usual seats of infection for the free forms, while the gills and muscles of the fishes are choice tissues for the cysts. Myxidium and myxobolus are characteristic genera. (b) Microsporidia have in each spore a single polar capsule. This group is parasitic chiefly in arthropods, and occasionally in other invertebrates, fish and amphibia.

The third subclass Acnidosporidia includes forms which produce simple spores. Again there are two orders: (a) Sarcosporidia. As the name infers these oceur in muscles of several mammals. The encysted forms attain a length of several millimeters, and ultimately each becomes a mass of sickle-shaped spores. The complete life cycle is not known, but the saclike, encysted form in muscles of mammals is known as Miescher's tube or sac. (b) Haplosporidia are single cells, each with a single nucleus, and they have a relatively simple strueture. This order parasitizes fishes and certain insects, notably the cockroach. 
Plasmodium, the malaria parasite, is one of the Haemosporidia, and its life cycle will be given to illustrate the intricate life history of certain of these forms. Life cycles in which there are primary and intermediate hosts are quite common among parasites. This example will illustrate also the relationship of insects to diseaseproducing organisms. There are three species of human malariacausing organisms: (a) Plasmodium vivax, which causes tertian fever, is characterized by an attack each forty-eight hours. (b) Plasmodium malariac, which causes quartan fever, is characterized by an attack every seventy-two hours. (c) Plasmodium falciparum, causing estivo-autumnal or subtertian fever, the attacks of which recur each day, or there may be a somewhat constant fever. The parasite (see Fig. 393) may live in the blood of man by a series of asexual generations which may continue throughout the life of the person. The parasite, while in the spore stage, invades the red corpuscles, where it reproduces by a sort of multiple division called sporulation, in which there are numerous nuclear divisions before the mass of cytoplasm divides. The new individuals (merozoites) are freed by destruction of the corpuscle and almost immediately enter new corpuscles where repetition of events occurs. Some of these merozoites become sexual cells (gametocytes). Part of the gametocytes develop into macrogametes, spoken of as female, and others become microgametes which develop from the male gametocyte. If a female Anopheles mosquito bites and sucks blood from this person, the mosquito becomes infected with gametocytes of Plasmodium. A union of the flagellate mierogametes with the egglike macrogametes takes place in the stomach of the mosquito. The union is commonly called fertilization, and a fused cell or zygote thus formed soon becomes a motile, wormlike form, known as an oökinete. This oökinete enters the wall of the mosquito's stomach where it encysts in the form of a ball with a shell, and is now called an oöcyst which grows at the expense of the adjacent tissue. This cyst protrudes like a little wart on the outside of the wall of the stomach. Inside of the oöcyst the nucleus divides repeatedly, forming sporoulasts. These enlarge and coalesce, while slender, spindle-shaped sporozoites develop within, each with a chromatin dot as a nuclens. The capsule of this oöcyst is crowded full of these sporozoites which may number 10,000 or more, and there may be 500 capsules in one mosquito. Depending somewhat on the temperature, it requires twelve days or more for 
this development to go on in the mosquito. These little parasitic sporozoites make their way to the salivary gland of the mosquito where they may remain for weeks. When this mosquito "bites" a man, some of the saliva with sporozoites flows into the wound, and the process of asexual multiplication begins over again in the red corpuscles of this person as a new host.

\section{Colonial Protozoa}

There are some species of Protozoa in which the individual cells exist in groups called colonies. This formation frequently results from incomplete separation of the cells following division. In some
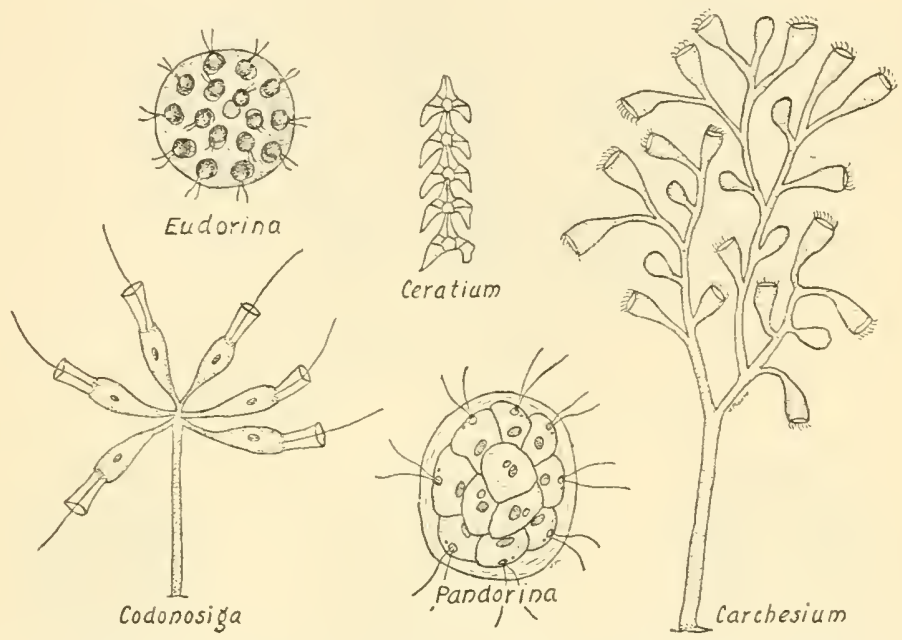

Fig. 30.-Different types of colonial Protozoa. Eudorina, a simple colony: Pandorina, within gelatinous envelope; Ceratium, a linear colony; Carchesium, stalked infusorian colony; Codonosiga, a stalked flagellate colony. (Drawn by Joanne Moore.)

of these forms only two cells adhere, but in others the cells may remain attached after many divisions, with the result that thousands of cells are built into the group. In some species there is a jellylike, spherical envelope inside of which the colony of cells remains. In certain species the cells are stalked, and the new cells remain attached to the stalk, giving a branching colony. Pandorina and Eudorina are typical examples of the former, while Epistylis and Carchesium are typical examples of the latter. These types of colonies are known as spheroid and arboroid or dendritic respectively. Colonies like that of Ceratium with individuals ar- 
ranged in a line form a linear one, and colonies of irregular arrangement are spoken of as gregaloid. The difference between these colonial Protozoa and simple Metazoa is a difference in the relationship of single cells to the group as a whole and not a simple difference
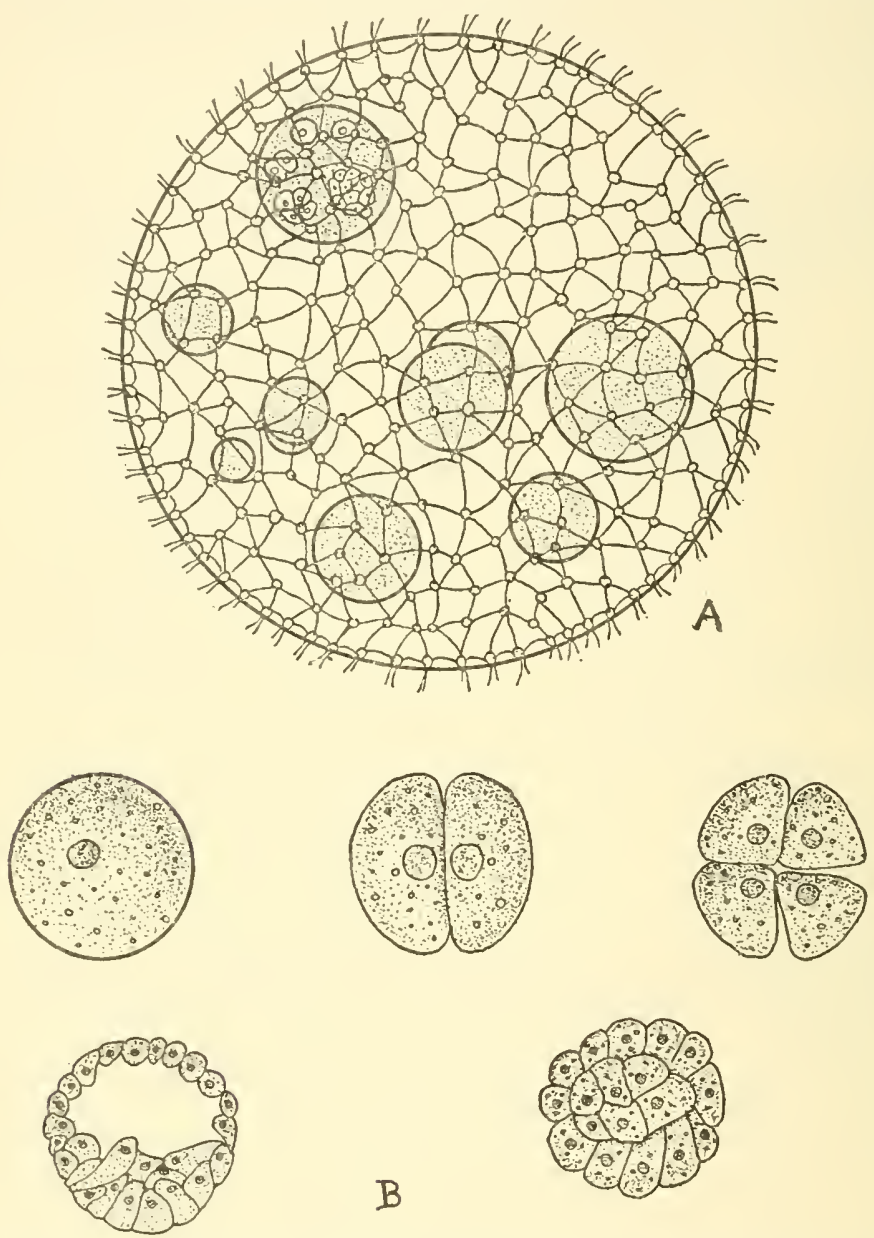

Fig. 31.-Volvox. A, Mature colony containing several daughter colonies. $B$, Fornation of daughter colony by development of a parthenogonidium. (From White, General Biology, The C. V. Mosby Company.)

in numbers of cells. In the colony each cell is an independent or almost independent individual so far as the functions of living are concerned. In the structure of Metazoa the cells are specialized and 
distributed, so that certain groups carry out a definite portion of the entire metabolism. They are classified into general body (somatic) cells and reproductive (germ) cells. Certain of the spheroid protozoan colonies, such as Volvox, have a rather striking resemblance to the blastula stage in the early development of metazoans. Both are spherical organizations of cells.

\section{Tropisms and Animal Reaction}

Organisms, whether plant or animal, of all degrees of complexity respond to various kinds of stimuli. The important stimuli which call out immediate or direct response by the animal are light, bodily contact, chemical change, temperature, gravity, mechanical currents, and electric emrrents. The response to a stimulus may be either positive or negative. Tropism, which means turning, refers to the reaction of an organism to a stimulus. Taxis may also be used here if the response involves the movement of the organism as a whole. Tropisms are named with respect to the stimulating agent, and the common ones usually recognized are:

a. Phototropism, response to light

b. Thigmotropism, response to contact

c. Chemotropism, response to chemical changes

d. Thermotropism, response to temperature

e. Geotropism, response to gravity

f. Rheotropism, response to mechanical currents

g. Electrotropism or galvanotropism, response to electric currents

If the animal is attracted to the source of the stimulation and turns toward it, the response is said to be positive. If the organism is repelled by the stimulus, the response is negative. It has not been thoronghly determined why an animal responds to a specific stimulus in a certain way. The minimum strength of stimulus which is necessary to get a response is known as the threshold. The simpler animals under a given set of conditions respond to these stimuli in a certain way not because of power of choice, but because they cannot behave in any other way. The Protozoa are controlled in their behavior largely by tropisms.

\section{Economic Relations of Protozoa}

Man has not yet found a way or need to eat Protozoa directly as food material, although he does draw on it indirectly by a food 
chain including water fleas, larger crustaceans, and fish. Too, the protozoans are not classed as predators on man as would be the lion, but many of them are parasites. Many diseases of man and animals are caused by Protozoa. Most of the diseases of this origin are more prevalent in the tropical and subtropical regions of the earth. Such diseases may attain sufficient importance to render large portions of continents uninhabitable by man; for example, much of northern South America and Central America was, at one time, ruled by yellow ferer and malaria, and the same applies for sleeping sickness in Africa. There are other Protozoa that render water unfit for drinking or help fertilize the soil.

Amoebic Dysentery.--Ulcers on the inside of the walls of the intestine of man are caused by this disease. There results from this, severe diarrhea and dysentery. From the intestine the infection, if allowed to continue, will be carried to the liver where serious abscesses are formed. The infection is usually obtained directly through drinking water or eating food which has been contaminated with the encysted organisms from fecal matter. About 10 per cent of our population are said to be carriers of these organisms. The causal agent is one of the Amoebae, Endamoeba histolytica (see Fig. 391), and it can be rather successfully eliminated from human beings by use of such drugs as emetine, carbarsone, and chiniofon, administered by a physician. Some other Amoebae have been found in human beings, but, so far as known, they are not pathogenic. Endamoeba coli, Endolimax nana, and Endamoeba gingivalis are such examples.

Foraminifera which is an order in class Sarcodina has some economic importance because of the limestone which is formed by the concentration of the material of the dead tests or shells. A genus by the name of Globigerina is one of the best known members of the group. It is about the size of a pinhead, and as it dies, it sinks to the bottom of the ocean where the mass forms the globigerina ooze which hardens into solid chalk.

Radiolaria is another order in the same class. Each of its representatives has a complicated skeleton of silica. From their skeletal remains comes an ooze on the sea floor sometimes hundreds of feet deep. From this is formed quartz or flint.

African Sleeping Sickness.-This malady is the most important disease of man caused by flagellate Protozoa. Technically the disease 
is called trypanosomiasis for the genus name of the animal that causes it, Trypanosoma gambiense or Trypanosoma rhodesiense. These organisms are transmitted by the tsetse fly, Glossina palpalis (Fig. 388), and the disease is limited to that area in Africa where this fly is found. The organisms (Fig. 387) live free in the blood and collect in the lymph glands, spleen, liver, and other organs. In final phases it collects and attacks the brain. The infection will bring about loss of appetite, severe emaciation, extended coma, which ends in death usually within three or four months, or it may be extended into years. Such animals as antelope, cattle, and some wild game are susceptible to the disease and may serve as carriers. This complicates the control of it. The disease has been considered absolutely fatal, but recently a drug, arsphenamine, an arsenic compound, has been tried with partial success.

Chagas' Disease.-A closely related flagellate, Trypanosoma cruzi, causes this disease in Central and South America. It is transmitted through the bite of Triatoma, one of the true bugs which is closely related to our common blood-sucking form, the "kissing bug." Chagas' disease affects dogs, monkeys, guinea pigs, armadillos, as well as man. The symptoms are continued fever; swollen lymph glands, liver, and spleen; anemia; and disturbance of the nervous system.

Malaria.-The life histor'y of Plasmodium, the sporozoan which causes this disease, has already been discussed under the general topic of Sporozoa. The disease is one of the oldest and most widely distributed among men. It was the first disease proved to be directly caused by a protozoan parasite. As early as 1718 a worker by the name of Lancisi ventured the statement that mosquitoes or gnats might transmit malaria; however, it was not until about the opening of the present century that this relationship was understood. In 1881, Dr. Laveran found a curious parasite in the blood of malaria patients. Several years later Laveran and Manson independently suggested that the organism might be transmitted by some bloodsucking insect. After several years more of investigation, Major Ronald Ross, an Englishman, was able to prove that the female Anopheles mosquito is responsible for the transmission of malaria.

If houses are sereened to keep out mosquitoes at all times, or if all malaria patients or carriers are thoroughly screened in, or if all mosquitoes and mosquito breeding places are destroyed, the chain 
of necessary relations for production of the disease is broken. Mosquitoes are destroyed by draining swamps which serve as breeding places, by placing mosquito fish (top minnows) in the pools to eat the larvae, or by covering the water with a film of oil which keeps out air and smothers the larvae as well as discourages females from laying eggs in such water. Another means by which the chain may be broken is to cure the carriers by killing all of the Plasmodia in their blood by use of quinine, properly administered under a physician's direction. Quinine is a specific drug for this disease.

Texas Fever.-The small sporozoan, Babesia bigemina, canses this disease in cattle by destroying red blood corpuscles. The red corpuscle count of the host may be reduced from an average of $7,000,000$ per cu. ml. to less than $1,000,000$ per cu. ml. The disease is transmitted from cow to cow by the cattle tick and its young.

Nagana, similar to African sleeping sickness in man, dourine, a sexual disease of the horse, and surra, are all diseases of domesticated animals and are cansed by trypanosomes. In some parts of the world they have considerable economic importance.

There are many other diseases that are rather similar to the above which may be caused by Protozoa, although the organisms have not been specifically isolated. Such diseases as Rocky Mountain spotted fever, transmitted by the Rocky Mountain spotted fever tick and fatal to man; dengue or breakbone fever, a very umpleasant and uncomfortable disease, transmitted by the yellow fever mosquito Aedes (Stegomyia); as well as perliaps rabies, scarlet fever, typhus fever, smallpox, and trachoma should be considered with this possibility.

The cost of the above-mentioned and other Protozoa to man throughout the world in money, loss of time, and suffering is almost inestimable. A good protozoologist is one of our most valuable economic assets. 


\section{CHAPTER V}

\section{EUGLENA OF CLASS MASTIGOPHORA}

\section{Habitat and Characteristics}

The most common species are Euglena viridis and Euglena gracilis which are found abundantly in fresh water. This genus is also quite well represented among marine animals; many Euglenae possess chloroplastids which give them the possibility of photosynthesis. They are usually found living in the surface waters of ponds, sluggish ereeks, and lakes. Euglenae are sometimes classified as plants by botanists because of the presence of ehlorophyll. It is a form which illustrates certain plant characteristics and animal characteristics in the same organism.

\section{Structure}

The microscopic, single-celled body has about the shape and proportions of a cigar with a blunt anterior and a sharp posterior end. At the anterior end, attached near the mouth, it bears a very slender, almost transparent, whiplike filament, the flagellum. This is an extension of the cytoplasm. The superficial layer of the cell or ectosarc (ectoplasm) is covered by an extremely thin portion, the cuticle. Most of the euglenoid forms have spiral markings (striations) on the surface of the body. The mouth of the cell is near the anterior end, and extending inward from it is the gullet or cytopharynx. Beside the eytopharynx is the reservoir or large vesicle. Just anterior to this is the stigma, which is red in many individuals of $E$. viridis. Bodies of collected protein material may be seen in connection with most of the chloroplasts which are distributed through the cytoplasm. These bodies are called pyrenoid bodies. Within the inner portion of the cell or endosare (endoplasm) is located the nucleus. It is usually obliterated from view by the abundant chloroplasts. Small contractile vacuoles empty from the endoplasm into the reservoir.

\section{Food and Assimilation}

The food problem among Euglenae as a group is interesting from the biological standpoint. It seems that some Euglenae are able to ingest other small organisms through the mouth and cytopharynx 
to be digested in a vacuole within the endoplasm; this has been called holozoic nutrition as typical of animals. E. viridis probably does not possess this possibility. Others, like E. gracilis, are able to assimilate dissolved nutriment by absorption through the general cell surface (saprophytic nutrition). In fact, this species has been maintained for more than two years in a nutrient solution in darkness.

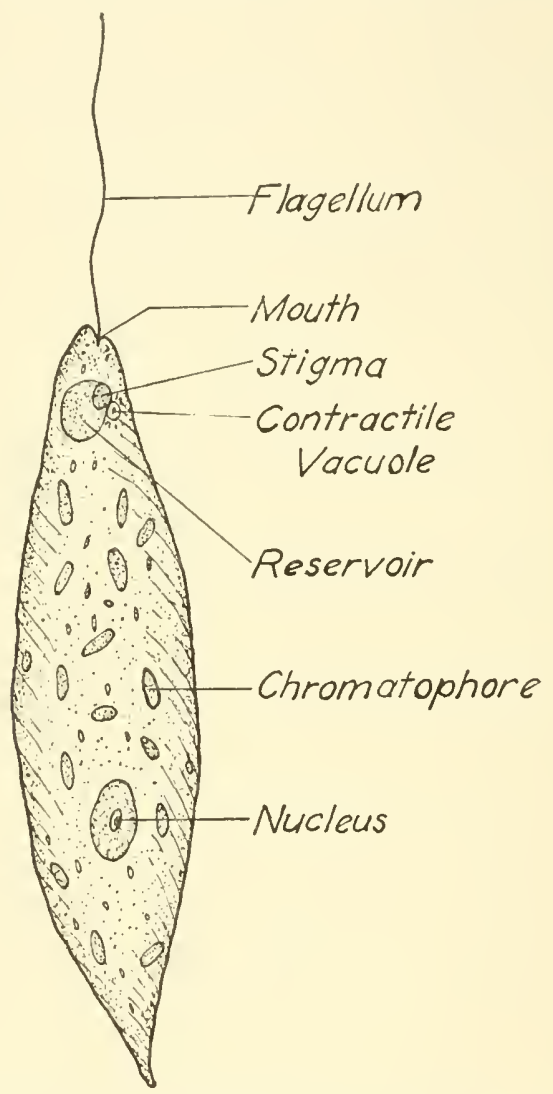

Fig. 32.-Euglena viridis Ehrenberg. A chlorophyll-bearing flagellate.

Those forms like $E$. viridis that are abundantly endowed with chlorophyll obtain their food largely by photosynthesis as does the green plant. This process utilizes water, carbon dioxide, dissolved mineral salts, and with the aid of light and ehlorophyll builds up organic food substances. The final stage of the carbohydrates formed by this 
process is paramylum, a granular substance much like starch. Grains of this substance may be observed throughout the endoplasm of these Euglenae when living in favorable conditions. It is not likely that all three of these fundamental types of nutrition are found in any one species of Euglena, but all are represented in closely related species of these flagellates.

\section{Respiration and Excretion}

Respiration is carried through the general surface of the cell membrane. There may be some utilization of the carbon dioxide produced in the metabolic activity by the process of photosynthesis in forms where it exists. Likewise, some of the excess oxygen produced by photosynthesis may be used in metabolism. Water and waste products collect in the several small contractile vacuoles which empty into the reservoir, a permanent vesicle communicating with the exterior.

\section{Reproduction and Life Cycle}

Binary longitudinal fission is the common means of reproduction. This division occurs only in the motile state (or active phase) in some species, in a quiet but not encysted condition in other species, and in a few others, fission occurs only while encysted (encysted phase). E. viridis may divide by longitudinal binary fission in either the motile or encysted condition. According to some authors the original flagellum is retained by one-half, while a new flagellum is developed by the other, but there is also some rather authentic work which shows that the old flagellum entirely disappears during division, and a new one is developed in each daughter cell. During adverse conditions, such as drought or increased chemical concentration, Euglena becomes encysted. In this condition it becomes spherical in shape, nonmotile, and secretes a thick gelatinous envelope about itself. During the encysted phase, division takes place. There may be a single division or there may be several. Upon the return of normal, favorable conditions these cells emerge from the cyst and assume the active phase. Some observers have reported as many as thirty-two young flagellated individuals coming from a single cyst. On rare occasions two individual Euglenae come together side by side and fuse permanently into a single cell. This is somewhat similar to the zygote formation in sexual reproduction. 


\section{Behavior}

Euglena usually lives near the surface of the water if the light there is not too intense, and when in the active phase swims about. This animal displays positive phototropism and is easily stimulated by changes in intensity of light. If the light is too intense, there will be a negative response. A medium light is optimum for it. There is naturally an attraction to light in those forms which utilize it in the manufacture of food by photosynthesis. Direct, intense sunlight, however, is injurious to them. When Euglena swims through the water, its anterior end with the flagellum goes foremost and is first to reach any injurious or distasteful environment. When it encounters such a condition in the medium, it stops and turns sharply in another direction and attempts to move out of danger. This is known as the avoiding reaction. In these and other reactions this cell exhibits the irritability that is characteristic of all protoplasm.

\section{Locomotion and Flagellar Movement}

Contractions and expansions take place in Euglenae when they are not actively moving about. These movements resemble waves of contraction (peristaltic contraction) passing over the cell. Some of the larger species move about in a crawling fashion by taking advantage of this movement. This activity is known as euglenoid movement. The chief method of locomotion is swimming by means of the whiplike movements of the flagellum through the water. A spiral path is followed due to the continuous turning of the body. The flagellum is made up of an elastic onter sheath which encloses an axial filament composed of one or more contractile fibrils. 


\section{CHAPTER VI}

\section{AMOEBA OF CLASS SARCODINA}

It is likely that no microscopic organism has attracted so much attention and popular interest as Amoeba. Amoeba is recognized by the public generally as a simple and low form of life. Even the writers of fiction speak of the range of the span of complexity of animal life as extending "from Amoeba to Man." The pedigree of Amoeba is probably as long as that of any of the animals we know and involves hundreds of times as many generations as many of the common animals; yet Amoeba remains in a relatively primitive and simple state. Little or nothing is known about the real ancestry of Amoeba. There are many kinds or species of Amoeba, some simpler and some more complex than Amoeba proteus. Chaos diffuens is a very desirable species for study. Recently Chaos chaos Schaeffer has been rediscovered. It is enormous in size and can be seen with the unaided eye.

\section{Characteristics and Habitat}

The many kinds of Amoeba live in fresh water, marine water, soil, or as parasites in the fluids of the visceral organs of higher types of animals. Amoeba proteus may be collected in a variety of places where conditions of water, temperature, and organic food are favorable, such as debris from watering troughs, bottoms of ponds, spring pools, drain ditches, abandoned tanning pits, in streams where the watcr runs over rocky ledges, and wherever there is abundant aquatic vegetation. It is often found on the surface of submerged lily pads. A mass of pond weed may be brought into the laboratory in some of the pond water and allowed to stand in the container a few days. If amoebae are present, they will likely be in the brown scum which forms, or in the sediment at the bottom.

The general appearance of this animal is that of a slate-colored, lustrous, irregular mass of gelatinlike substance with slowly-moving, fine particles within. When it is active, the outline is constantly changing. 


\section{Structure}

Amoeba proteus is one of the largest of the fresh-water forms. Its average diameter is about $1 / 100$ inch $(0.25 \mathrm{~mm}$.), while its extreme diameter is $1 / 50$ inch or barely visible as little specks to the unaided human eye. The animal owes its irregular shape to the fact that protrusions of its own substance are formed at its surface. These are known as pseudopodia, and they are constantly changing in shape in the active animal by the flowing of the protoplasm.

Under favorable conditions the protoplasm can be differentiated into two portions. The firmer, somewhat tougher outer portion, the ectosarc (ectoplasm), is nearly homogeneous and includes the plasma membrane (or plasmalemma) : the more fluid inner portion, endosarc (endoplasm), is much more gramular and contains the cytosome, cell

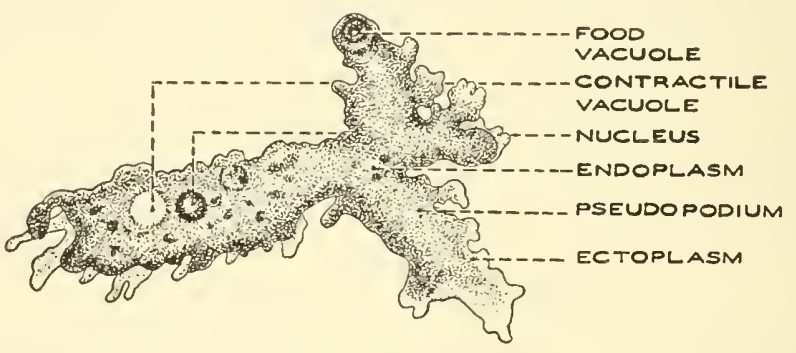

Fig. 33.-Drawing to show the appearance and structure of a living Amoeba proteus.

inclusions as well as the nucleus. The larger bodies in the cytosome are food vacuoles, single, shiny, contractile vacuoles containing watery fluid and varying in size; water vacuoles; various granules; mitochondria; fat globules; and erystals. Some authors distinguish two types of protoplasm in the endosare; the inner more fluid, plasmasol in which the streaming movements take place and, surrounding this a more viscous, passive portion, the plasmagel. The nucleus usually appears somewhat dense and granular, and is located in the portion away from the end which is advancing in a moving specimen.

\section{Metabolism}

This refers to the constant building up (anabolism) of living protoplasm and its concurrent oxidation (catabolism). It includes all activities necessary for maintenance of itself and its race. These 
phenomena are the same as those found in the highest forms of life but reduced to very simple terms. Here we may study the entire metabolic cycle in progress within the confines of a single cell. Its phases are as follows:

Food.-Its prey consists chiefly of smaller Protozoa, small singlecelled plants, such as diatoms and desmids, and portions of filamentous algae. Bacteria may be used to some extent and rotifers (small Metazoa) are sometimes devoured.

Ingestion.-Amoeba has no definite mouth but the food is taken into the body by engulfing it at any point that comes in contact with it. A pseudopodium is formed at this point, and the end of it flows around the food particle until the particle is entirely enclosed. A droplet of water is included with the food to form what is called a food vacuole. These vacuoles move about in the endoplasm.

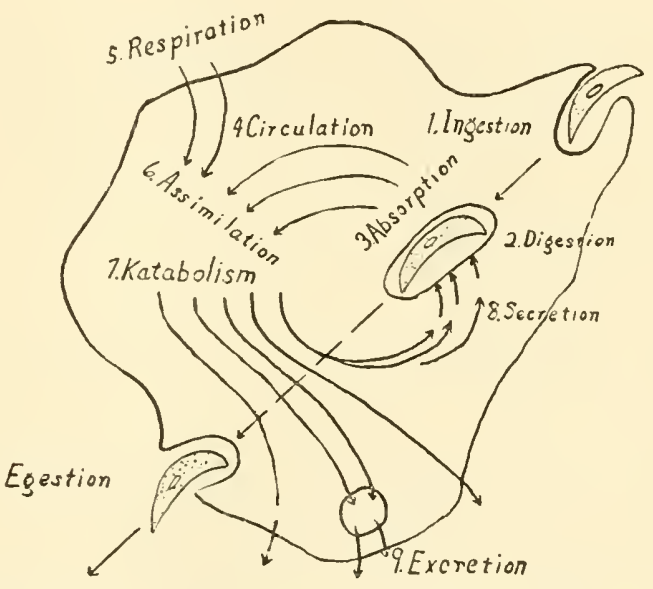

Fig. 34.-Diagram to show the phases of the metabolic process as it occurs in amoeba. (Redrawn by permission from Wolcott, Animal Biology, published by MeGraw-Hill Book Company.)

Digestion.-The food gradually disintegrates and much of it goes into solution in the fluid of the vacuole. The function of digestion is to convert complex materials into a soluble, absorbable form. It is assumed that the surrounding cytoplasm secretes enzymes into the food vacuoles of Amoeba to perform this function, since enzymes serve this purpose in larger animals where exact study can be made on the process. A circulatory system is not necessary since the 
vacuoles with the food in the process of digestion circulate so widely in the endoplasin that all parts of the cell may receive nourishment by direct absorption.

Egestion.-Indigestible material or debris that has been ingested with the food is carried to the surface of the cell and cast out or egested by simply being left behind as the animal moves away.

Assimilation.-This is the process of transforming the digested food material into protoplasm. In Amoeba the digested food material is absorbed directly from the food vacuoles by the surrounding cytoplasm. Since the vacuoles move rather generally through the endosare, most of the protoplasm of the cell is in rather close contact with the dissolved food.

Respiration.-This is a process whereby the gas, carbon dioxide $\left(\mathrm{CO}_{2}\right)$, leaving the protoplasm, is exchanged for oxygen $\left(\mathrm{O}_{2}\right)$ entering it. Such a process is essential to all living protoplasm. In Amoeba this exchange is carried on primarily through the general body surface. The water in which the animal lives must contain dissolved oxygen in order that this diffusion may go on. Amoebae, however, are able to and do live in rather foul water where the oxygen content is rather low and the carbon dioxide high because of the decaying vegetation present. Amoebae may live several hours in water from which the oxygen is removed before asphyxiation occurs. The contractile vacuole likely assists in discharging $\mathrm{CO}_{2}$.

Catabolism or Dissimilation.-The chemical union of the oxygen with the organic substance of the protoplasm liberates kinetic energy and heat. This is known as oxidation and is a burning process which goes on within the protoplasm. Water, some mineral matter, urea, and carbon dioxide are residual products of this process.

Excretion.- - These by-products of metabolism in the form of waste liquids must be disposed of. They cannot be allowed to accumulate beyond certain limits in the living organism if life is to continue. Urea and uric acid, which are protein by-products, excess water, and salts, are discharged from the body of Amoeba by way of the contractile vacuole along with some carbon dioxide. The contractile vacuole is formed by the union of small droplets of liquid under the plasma membrane. It fills out with liquid which is forced out through the membrane as the vacuole disappears. Its location apparently is not fixed in the cell but is often near the nucleus. The contractile vacuole is absent in some forms, and in such cases, ex- 
cretion occur's only by diffusion through the cell surface. There is likely some excretion by this means in all Amoebae.

Growth.-If there is increase in the volume of a body, this is spoken of as growth. In all living organisms growth is accomplished by addition to the protoplasm. If food is plentiful, more material is added to the protoplasm than is used up in the oxidation which produces active cnergy. In other words, growth occurs when the rate of anabolism exceeds the rate of catabolism in the organism.

\section{Reproduction and Life Cycle}

The life history of the many-celled animals to be studied later includes a series of changes from egg, through embryo state, to adult. In Amoeba the cycle is likely only partly known, because it
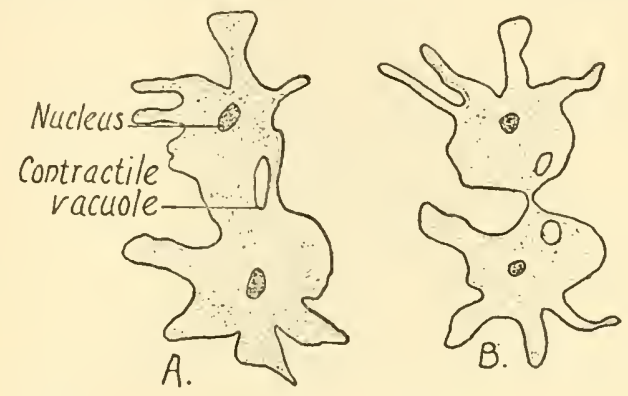

Fig. 35.-Diagram to show flssion in amoeba. $A$, Beginning of the process; $B$, fission nearing compietion. (Drawn by Joanne Moore.)

is difficult to maintain cultures in perfectly normal conditions for sufficiently long periods to get this complete story. Ordinarily, the animal grows when conditions are favorable until it attains a certain size; when this limit of size has been reached growth ceases. Why does the cell cease to grow? Why should it not attain the size of a man? Or why should a tree not continue to grow until it reaches the sky, or a man take on the proportions of an elephant? We have not been able to put our fingers on any one factor that completely controls growth. We do know of certain relationships that influence it. It will be recalled that all materials used by a cell must pass through the cell membrane, and likewise all waste substances must be discharged in a similar manner. Mathematics states that the volume of a cell increases according to the cube of 
its diameter; while its surface increases only according to the square of its diameter. In other words, the amount of material in a growing cell increases approximately twice as fast as the plane surface needed to surround it. It is logical, then, to assume that a point may be reached when the surface area will not be sufficient for the passage of necessary materials into and out of a cell. There is, however, considerable variation in the size of cells; hence it seems there must be other factors besides volume and surface relation in operation. Modified surface and difference in the rate of metabolism certainly would be factors affecting the size of the organism. When Amoeba reaches the limit of size, a division occurs. Binary fission, by which two new individuals are produced, has been definitely established, and some other methods of reproduction have been presented. Calkins, an authority on Protozoa, states

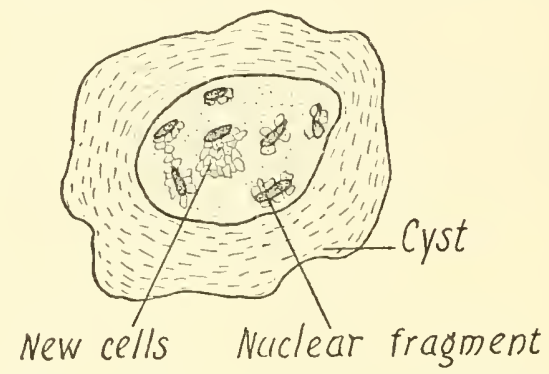

Fig. 36.-Diagram to show amoeba encysted and undergoing the process of sporulation. (Drawn by Joanne Moore.)

that Amocba starts out as a tiny pseudopodiospore which has only one pseudopodium. It then passes through a growth period and increases in complexity until it reaches the full-grown condition. It then divides by binary fission into two danghters. When each daughter has grown to nearly twice its original size, fission is repeated. Environmental conditions and the variety of Amoebae determine the number of times this phase is repeated. Occasionally the fission seems to be an amitotic one. At the close of the fission phase, there is a period of encystment and subsequent sporulation. During the eneystment the protoplasm undergoes several divisions to produce the several pseudopodiospores which later break from the cyst as infant Amoebae. It is felt that the complete details of the life cycle of many common Sarcodina are not yet available. 


\section{Behavior}

All of the activities of an animal which come in response to internal or external stimuli make up the "behavior." The activities of the animal under discussion include the formation of pseudopodia, ingestion of food, locomotion, and others. Amoeba proteus exhibits either positive or negative reactions to various stimuli. An environmental change to which an animal reacts is known as a stimulus, while the reaction of the animal is called the response. The movements made by an animal in response to stimuli are called tropisms. Amoeba exhibits all of the tropisms discussed in Chapter IV. To physical contact, it responds positively if the impact is gentle; otherwise the response is negative. It responds negatively to strong light and finds its optimum in a moderately reduced light. When some part of the body surface of this animal comes quietly into contact with food, there is a characteristic response. This part of the protoplasm stops flowing while other parts continue, thus forming a pocket around the particle of food. The edges of the pocket fold in, meet, and join so as to enclose the object. This attraction to food is likely a positive chemotropism. Amoeba reacts negatively to concentrated salt, cane sugar, acetic acid, and many other chemicals which have been tried. Amoebac have an optimum temperature range between $15^{\circ}$ and $25^{\circ} \mathrm{C}$. Temperatures approaching the freezing point inactivate the animal, while temperatures above $30^{\circ}$ C. $\left(86^{\circ} \mathrm{F}\right.$.) also retard their activities and may soon become fatal. A weak electric current has an effect on the physical condition of the protoplasm on the side nearest the cathode. The tendency is toward the sol state here, hence the animal turns toward the cathode. According to Jennings, who has done extensive research on behavior of Protozoa, these activities are "comparable to the habits, reflexes, and automatic activities of higher animals." He also feels that Amoeba probably experiences pain, pleasure, hunger, desire, and the other simple sensations.

\section{Amoeboid Movement and Locomotion}

The flowing or streaming of the protoplasm and extending the cell in some direction by the formation of pseudopodia is usually called amoeboid movement. It is so named from the perfect exemplification of such activity by Amoeba. Locomotion is accomplished by the pseudopodia, and the process of their formation in most Amoebae. 
Successive pseudopodia are formed in the moving Amoeba proteus as it goes in a given direction. The pseudopodia are temporary locomotor structures. Most zoologists explain this movement as being due to the contraction of the more viscous ectoplasm, particularly in the "posterior" region. This brings about a forward movement in the more fluid endoplasm (plasmasol) which causes an outflow at points where the ectoplasm is thinnest, or where surface tension is lessened. As this plasmasol approaches the advancing tip of the pseudopodium, it turns to the sides and changes to more solid endoplasm (plasmagel). This process contiuues, pushing the advancing tip farther and farther forward. At the opposite side, the plasmagel continues to become plasmasol to provide for fluent material. At the side of the animal away from the advancing pseudopodium, the cell membrane (plasmalemma) moves upward and over the upper side of the body; it continues to move forward to the tip of the

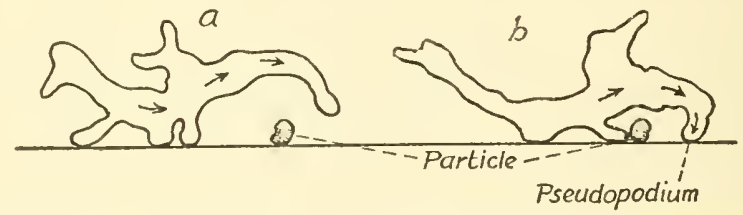

Fig. 37.-Successive positions in the movements of an amoeba viewed from the side. Notice the formation of new pseudopodia and the engulfing of the particle on the surface. (Modified from photographs by Dellinger, 1906, Journal of Experimental Zoology.)

pseudopodium where it dips down and is laid on the substratum over which the animal is moving and becomes a part of the stationary portion. If the specimen has several pseudopodia, one or more may be developing while others are receding. In the latter, the flow of plasmasol is back through the centers of the pseudopodia toward the main mass. Temperature and other environmental factors affect the rate of locomotion.

Dillinger mounted some of the animals on the edge of a slide in a groove formed by the projecting edges of two cover glasses and observed their movement from side view by tilting the microscope to a horizontal position. He describes their movement as a sort of walking on the progressively forming pseudopodia. The new pseudopodia are formed at the advancing margin of the cell. 


\section{CHAPTER VII}

\section{PARAMECIUM OF CLASS INFUSORIA}

This animal has been the subject of much study and the victim of considerable experimentation. Paramecium caudatum is probably the species most commonly studied. It is easily available and is large in size, ranging between 0.2 and $0.3 \mathrm{~mm}$. in length.

\section{Characteristics and Habitat}

Paramecium is an active, cigar-shaped animal, just about large enough to appear as small white specks in the water. It has a definite axis and permanent anterior and posterior ends, but it is asymmetrical in shape. Paramecia are easily cultured by collecting some submerged pond weeds and allowing them to stand in a jar of the pond water for several days. Or some natural creek or pond water may be placed in a jar with some old dry grass and allowed to stand about ten days. These animals occur abundantly in any water which contains considerable decaying organic matter. They thrive in all streams, creeks, or ponds polluted by sewage. They tend to congregate at the surface and particularly in contact with floating objects, where they frequently form a white scum. This animal is a great favorite in zoology laboratories.

\section{Structure}

Paramecium is sometimes described as being slipper-shaped. The anterior portion, which is blunt but generally narrower, represents the heel part; while the posterior portion, which is generally broader but pointed, represents the sole portion.

At one side is a depression, the oral groove, which passes diagonally from the anterior end to about the middle of the body. It is broad and shallow anteriorly but it becomes narrow and deeper as it ends in a mouth, which leads to the gullet. The groove usually extends obliquely from right to left in $P$. caudatum as the animal is viewed from the oral side. Occasionally cultures are found in which the majority of the individuals show the groove extending from left to 
right from this view. The body is covered with fine hairlike cilia which are of even length except in the oral groove and at the posterior extremity, where they are noticeably longer. The cilia within the gullet are fused togetler into a sheet, forming the undulating nembrane.

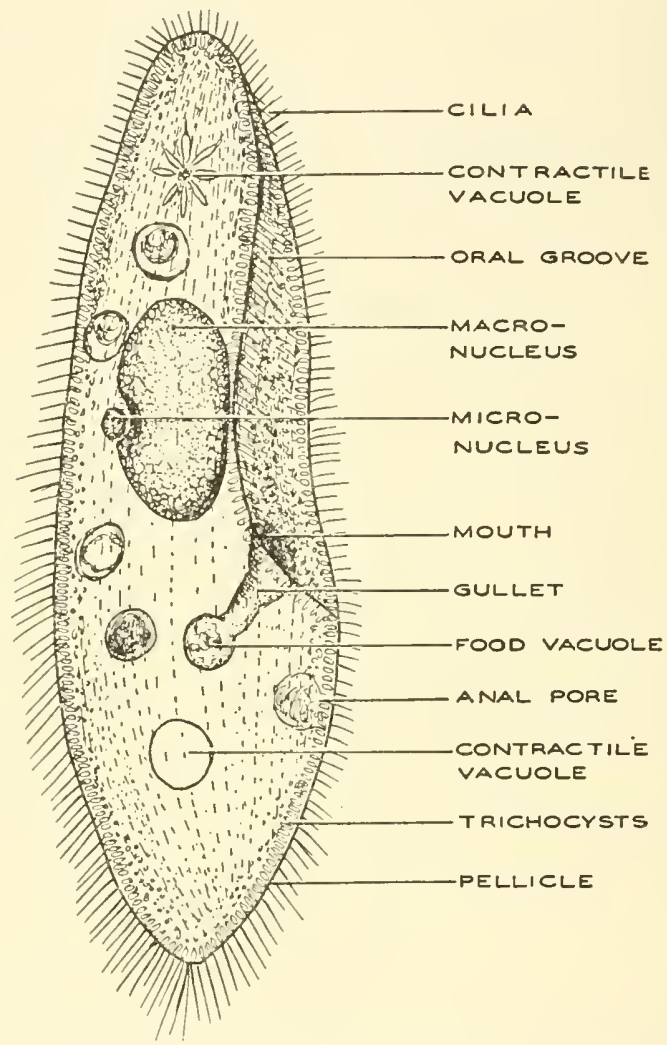

Fig. 38.-Diagram showing the structure of paramecium, much enlarged. (Drawn by T. C. Evans.)

The cell is divided into the outer, tough, nongranular ectosarc which is composed of ectoplasm. The outer surface of it is a thin, elastic cuticle or pellicle which is marked in hexagonal areas by the distribution of the cilia. The cilia are direct outgrowths of the ectosarc. There are a great many spindle-shaped cavities located in the ectosare with their long axes perpendicular to the surface. These structures, trichocysts, are filled with a semifluid substance and each opens to the outside through the pellicle. The endosarc, composed of 
endoplasm, is within. It contains food vacuoles, two contractile vacuoles, macronucleus, and other granular masses. The numerous food vacuoles are formed, one at a time, at the inner end of the gullet by a mass of food material coming in with a droplet of water, a process similar to that described in Amoeba. The vacuoles circulate through the endoplasm in a rather definite course. This activity is called cyclosis. The contractile vacuoles are located near each end of the animal. Each vacuole has several radiating canals entering it. These vacuoles expand and contract alternately. The macronucleus is located slightly posterior to the center and somewhat beside the mouth. It is relatively large and rather bean-shaped. The micronucleus is located in the curved surface of the macronucleus and is much smaller. $P$. aurelia, another species, ordinarily has two micronuclei instead of one.

\section{Metabolism}

The same general activities as described in Amoeba and others occur, differing only in certain details. These same vital functions must take place in all living things (organisms).

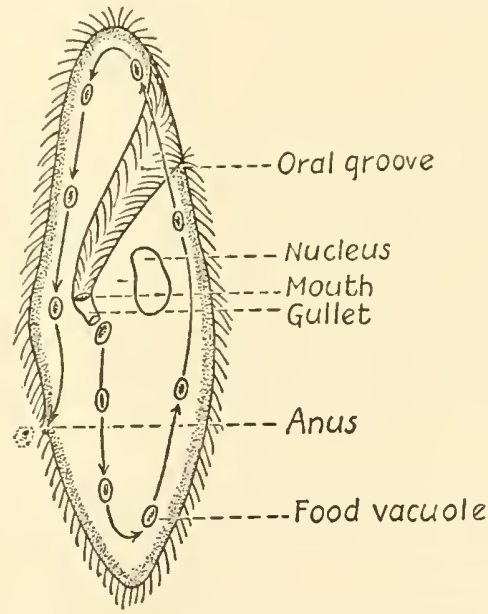

Fig. 39.-Cyclosis in paramecium, showing the course of the food vacuoles through the endoplasm while digestion is in progress.

Food.-Smaller protozoans, bacteria, and particles of debris constitute the principal items on the menu for Paramecia.

Ingestion.-This animal liunts its food, and when it locates a region where food is abundant, it settles down and becomes relatively 
quiet. The food is swept through the oral groove by the beating action of the cilia, and carried back through the mouth into the gullet. Finally it passes by means of the action of the undulating membrane into the endoplasm in the form of one food vacuole after another. These food racuoles move in a definite course through the endoplasm. Since this course is in the form of a cycle, the circulation is known as cyclosis.

Digestion, Assimilation, Respiration, and Catabolism or Dissimilation all occur in a manner very similar to that described for Amoeba. Egestion occurs at a definite anus.

Excretion of the waste products of metabolism in solution is by means of the alternate filling and expelling of fluid by the two contractile vacuoles, or it may occur to some extent by diffusion through the entire cell membrane.

Growth occurs as it does in Amoeba and in all other organisms. Under favorable conditions the storage of nutrient materials, like starch and fats, occurs in the cytosome. Nutrition in this animal is holozoic, and its living process is essentially like that of all higher forms of animal life.

\section{Reproduction and Life History}

The actual reproduction is by transverse binary fission which in itself is asexual. The cell divides transversely into individuals, and this is repeated for long series of generations, one after another. During this division process in $P$. caudatum, both the macronucleus and the micronucleus divide, the old gullet divides into two, and two new contractile vacuoles are formed by division of the old ones. The micronucleus divides by mitosis, but the division of the macronucleus is not distinctly so. The time required for the completion of a division ranges between thirty minutes and two hours, depending on environmental conditions. Division is repeated at least once each twenty-four hours and under especially favorable conditions, twice a day. It has been estimated that if all survived and reproduced at a normal rate, the descendants of one individual over a month's time would number $265,000,000$ individual paramecia.

$P$. caudatum is a conjugating form of paramecinm, while $P$. aurelia and others seem not to conjugate. Conjugation is a temporary union of two individuals with exchange of nuclear material. Calkins carried some cultures of $P$. caudatum through a long series of generations and observed that coujugation occurs at intervals of approxi- 
mately every two hundred generations. When two paramecia are ready to conjugate, they come in contact, with their oral surfaces together, and adhere in this position. A protoplasmic bridge is formed between the two individuals. This union resembles a sexual act and has recently been described as such. The conjugants are usually small, rather unhealthy appearing individuals. Shortly after the adherence of the conjugants the nuclei of each undergo changes. The micronucleus enlarges and divides, forming two micronuclei, while the macronucleus undergoes disintegration and final disappearance. Each of these two new micronuclei again divides to form four, three of which disintegrate, but the fourth divides again, forming one large and one small micronucleus. Sometimes the smaller of these nuclei is spoken of as the "male" nucleus and the larger, as the "female." In each animal the smaller nucleus moves across the protoplasmic connection to the other animal and fuses with the larger nucleus there. Each individual now has a fusion nucleus. The two conjugants now separate, and very shortly the fusion nucleus of each divides by mitotic division; each of these divides, forming four nuclei in each animal, and these four divide to form eight. The descriptions of the subsequent events vary somewhat. At least it is known that four of the eight nuclei enlarge and become macronuclei; three of the others degenerate, and one remains as a micronucleus. This micronucleus divides, and almost immediately the entire animal divides by binary fission with two macronuclei and one micronucleus going to each cell. These daughter cells then divide to produce a total of four Paramecia which have the typical number of one micronucleus and one macronucleus of the active phase. Following this comes the long series of generations formed, one after the other, by transverse binary fission.

The whole series of changes involved in conjugation has been compared to maturation of germ cells and fertilization in sexually reproducing metazoans. The degeneration of the three micronuclei is compared with reduction division in maturation, and the fusion of the small "male" micronucleus with the lar'ger "female" micronucleus of the other conjugant is compared to fertilization.

A phenomenon, known as endomixis, has been found occurring in $P$. aurelia by Woodruff. It occurs in a single individual. This species has two micronuclei and one macronucleus. At regular intervals of about every forty or fifty generations, the macronucleus 


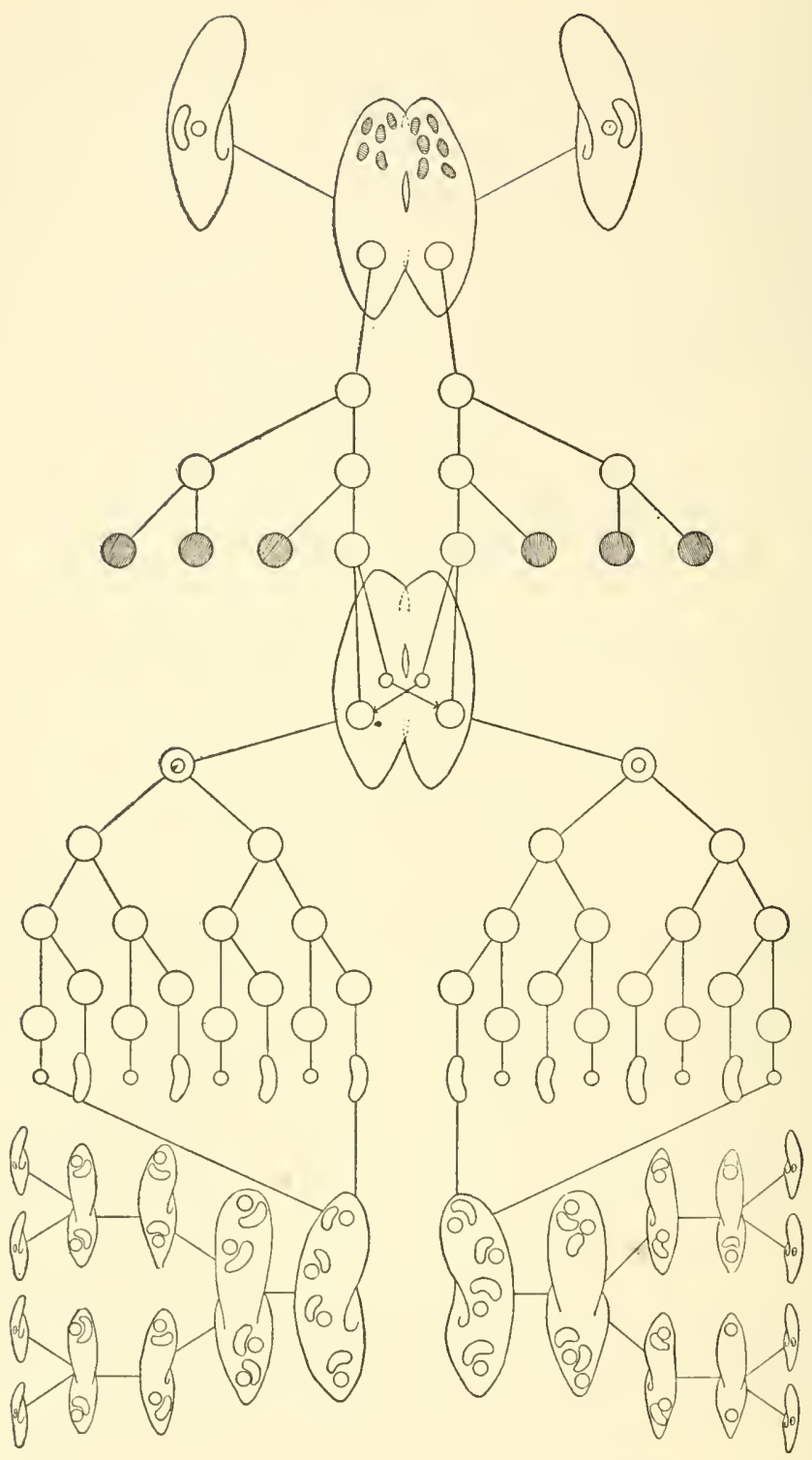

Fig. 40.-Conjugation and subsequent divisions in paramecium, showing activities of the micronucleus. Circles are micronuclei and crescents are macronuclei. The shaded ones have been resorbed. The divisions for micronuclei actually occur within the cells instead of outside, as figured for convenience. (From White, General Biology.) 
disintegrates, and the micronuclei undergo two divisions which produce a total of eight. Six of these disappear, and then the cell divides; one of the remaining micronuclei goes to each. This nucleus then undergoes two divisions. Two of these four become macronuclei, and two remain as micronuclei. The micronuclei then divide again as the entire cell divides to form daughters, each with two micronuclei and one macronucleus, the typical condition for this species. Endomixis may oceur in P. caudatum also. Endomixis seems to have about the same effect as conjugation.

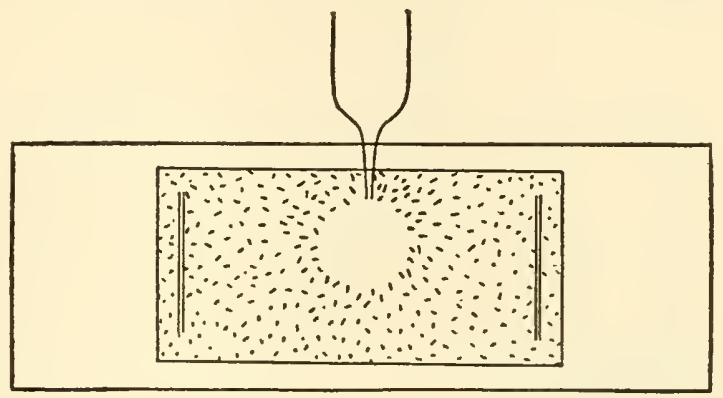

A.

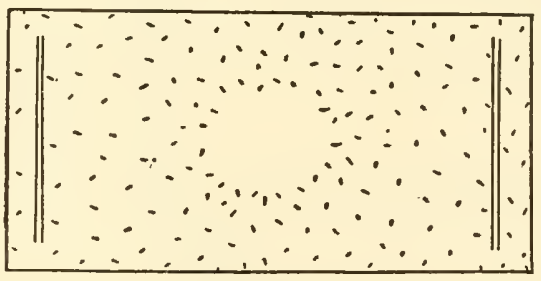

$B$.

Fig. 41.-Reaction of paramecia to a drop of 0.5 per cent $\mathrm{NaCl}$. A, Introduction of the drop beneath the cover glass: $B$, four minutes later. (From Jennings, $B e$ havior of the Lower Organisms, published by The Columbia University Press.)

There is still difference of opinion as to the exact function of conjugation and endomixis, but the chief result of the processes seems to be the reorganization of the nuclear substance. This may allow for variations in the fundamental constitution of the race. According to some authors these processes rejuvenate or renew the vitality of the individuals. In recent years, not only sexual reproduction but also distinct sexes have been described for Paramecium.*

* Sonneborn, Science News Letter, Aug. 21, 1937. 


\section{Behavior}

This animal is an active swimmer and necessarily shows ready response to environmental factors. Its behavior consists of its spiral course in locomotion, avoiding reactions, responses to food material, contact and other minor reactions. Its reactions to stimuli are somewhat similar to those described for Amoeba; however, it seems not to be affected by ordinary light. It reacts either positively or negatively to contact, change of chemical constitution, change in temperature, to gravity, and to electric current. The response to contact is positive, negative to ultraviolet light, negative
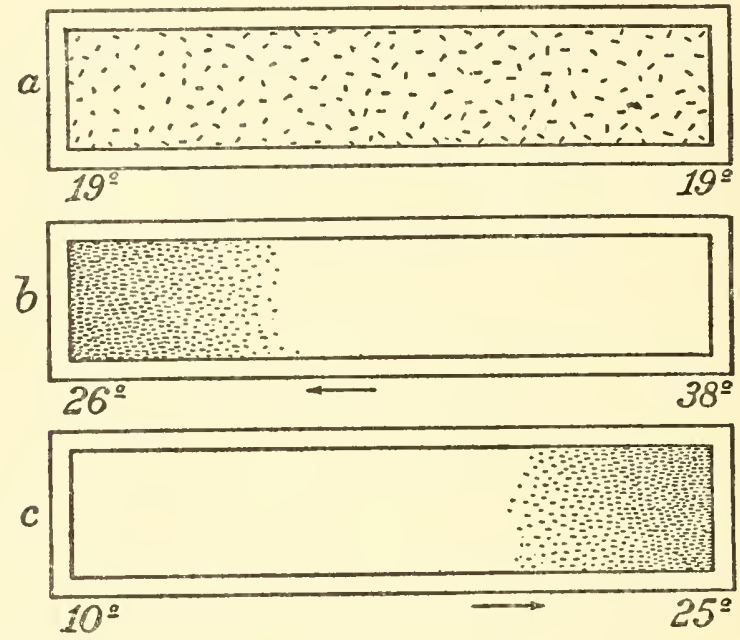

Fig. 42.-Reactions of paramecia to temperature. $a$, Paramecia are in a trough with temperature at $19^{\circ} \mathrm{C}$. uniformly through the water. The animals are generally scattered. In $b$ the temperature is held at $26^{\circ} \mathrm{C}$. at the left end and $38^{\circ} \mathrm{C}$. at the other. The animals are collected in the end of lower temperature. In $c$, the temperature is $25^{\circ} \mathrm{C}$. at one end and $10^{\circ} \mathrm{C}$. at the other, and the Paramecia are congregated in the region of higher temperature. (From Jennings, Behavior of the Lower Organisms, published by The Columbla University Press.)

to sodium chloride, positive to weak acetic acid, and positive to the negative pole of a weak, galvanic electric current. The optimum temperature for Paramecium ranges between $24^{\circ}$ and $28^{\circ}$ C. $\left(71^{\circ}\right.$ F.). Gravity causes the anterior end to point upward, and when placed in moving water, the animals will swim upstream. If Paramecium comes in contact with a solid object when it is moving, it will back away, swing on its posterior end to a slightly different 
direction and try again. This may be repeated, and is known as the "avoiding reaction." Such a reaction really involves simply one or more negative responses. These animals are constantly sampling the water and avoiding the conditions which are least favorable. This may be repeated in all directions. The same type of persistence is practiced in attempting to surmount a solid barrier.

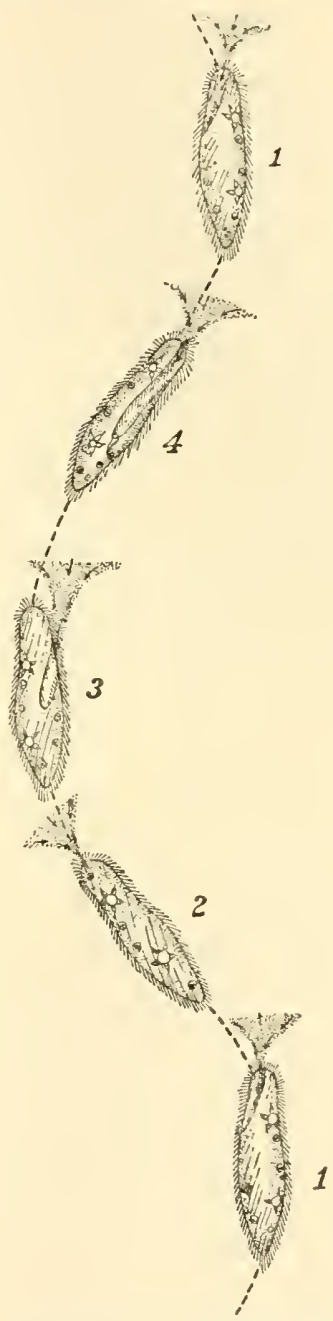

Fig. 43.-Diagram of the course and movement of paramecium through the water. Notice the spiral path. (From Jennings, Behavior of the Lower Organisms, published by The Columbia University Press.) 
Such successive attempts to gain the result desired constitute what is known as the "trial and error" mode of behavior.

In an effort to defend itself when severely irritated, Paramecium will discharge the contents of the trichocysts, which harden on contact with the water and form a mass of fine threads. These threads will entangle many of the aquatic enemies of these animals.

\section{Locomotion}

The beating action of the cilia against the water serves as the principal means of locomotion. The stroke of the cilia is rather oblique and this coupled with the increased length of the cilia along the oral groove causes the body to turn on its long axis while swimming. The total effect of these activities causes the course followed through the water to be that of a spiral. Paramecium may reverse the direction of the stroke of the cilia and thus move backward just as a car can be thrown in reverse.

The eilia are contractile outgrowths of the ectosare. Each has an elastic sheath and a fibrillar core. Contraction of the protoplasmic substance on one side, bends the cilium in that direction. The reverse stroke is much more passive. The movement of one tier of cilia seems to stimulate the adjacent ones to bring about coordinated, rhythmic ciliary activity and movement. 


\section{CHAPTER VIII}

\section{METAZOAN ORGANIZATION}

All animals whose bodies consist of few or many cells functioning as a unit are called metazoans. In most respects the vital activities of Metazoa are similar to those of Protozoa. Since Metazoa are more or less like compound Protozoa with some degree of intercellular differentiation, it is thought by many authorities that they arose through organization of single-celled organisms. In some forms of compound or colonial Protozoa, only two cells adhere together after cell division, but in others the cells may remain attached after many divisions. The size of different colonies may range from two to two thousand similar cells. In the most complicated protozoan colonies there may be several different types of cells. The representatives of class Mastigophora are the most likely ancestral forerunners of Metazoa. The colonial forms, such as Gonium, Pandorina, Eudorina, Pleodorina, and Volvox, are rather plantlike in characteristics, but a series of this type shows the possibility of the relative complexity of different colonial forms. There are several genera of animals which are intermediate between Protozoa and Metazoa, but for the most part the two groups are fairly distinet.

\section{General Characteristics}

This group includes all of the strictly many-celled animals. The cells are definitely organized and classified morphologically as well as physiologically. There is a well-regulated division of labor. Among the single-celled animals each cell, like primitive man, is largely independent of its fellows, doing for itself all that is necessary to carry on living processes. In the many-celled animal, as in a highly developed society of men, certain individual cells become more proficient in doing certain kinds of work, and as a result, a special group is able to care for a particular function necessary to the life of the entire organism. In return, other special groups care for other functions. In this way each exchanges the products of its labor for the products of the labors of the other groups. In human society this becomes more and more complicated as civiliza- 
tion advances; so it is with development of complexity in metazoans. Another characteristic of Metazoa is the presence of a definite center of control localized in a particular group of cells which becomes the nervous system in higher forms.

\section{Cellular Differentiation}

In Protozoa there is seen fair development of intracellular differentiation, making it possible for one part of a cell to perform a particular function, and for other parts to perform other functions. The complexity of Metazoa is not the result of great complexity of the individual cells, but it is due to the special differences between them. The presence of a variety of cells within one body is spoken of as intercellular differentiation. The modification of metabolic
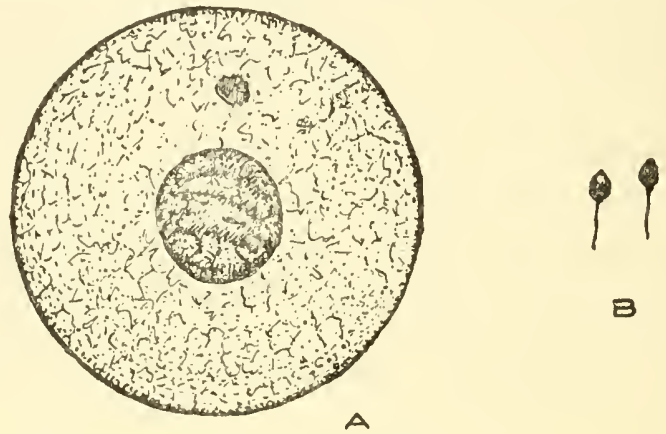

$B$

Fig. 44.-Typical germ cells. $A$, ovum of the female; $B$, spermatozoa of the male.

activity is the basic factor in the development of all differentiation. Certain groups of cells become specialists in a particular phase of the metabolic activity. Some become protective surface cells, others secrete special enzymes, still others specialize in excretion, and so on.

The entire metazoan body is usually divided into germ cells, which are specialized for reproduction, and somatic cells or body cells, which compose the remainder of the body and are grouped in layers. The germ cells are set aside early in the life of the individual for reproductive purposes. They develop in the reproductive glands or gonads of the two sexes. The protoplasm of these cells is known as germ plasm. The female germ cells are eggs or ova, and those of the male are spermatozoa. When the germ cells reach maturity, they become separated from the body and may give rise to a new generation. 
About forty years ago Weismann presented the idea of the continuity of heredity from generation to generation by way of the germ plasm. The germ plasm, according to this idea, gives rise not only to the protoplasm of the germ cells of the new individual but to the somatic cells as well. In Protozoa the entire material of the individual is passed on to the two offspring and, for this reason, this protoplasm is spoken of as being immortal. Potentially, germ plasm is likewise immortal.

The protoplasm of the somatic cells is known as somatoplasm. This is rebuilt with each generation, and when the individual dies, all of the somatoplasm perishes. In final analysis, the somatoplasm serves as a means of conveyance for the germ plasm through the current generation.

\section{Cellular Organization}

The simpler Metazoa are composed of only two kinds of somatic cells. These cells are grouped according to kind in two layers. With advanced differentiation, a rather wide variety of cells has been produced.

A tissue is an organization of similar cells into a group or layer for the performance of a specific function. A certain amount of intercellular substance is characteristic of most tissues and enhances their usefulness. The entire living mass of the metazoan animal body may be classified under five fundamental (four by some authors) kinds of tissues, and when it is so distributed, there is nothing left. These classes of tissues are: epithelial, protective or covering; sustentative, connective or supporting; muscular, contractile; nervous, irritable or conductive; vascular, circulatory.

Epithelial Tissue.-A sheet of cells that covers external or internal surfaces of the body is known as an epithelium. The epidermis or outer layer of the skin and the layer of column-shaped cells lining the inside of the intestine are good examples. According to function, this type of tissue can be classified as protective epithelium, glandular epithelium, and sensory epithelium. The epithelium which covers external surface of an organism usually develops various protective structures in the different groups of animals : the hard, horny chitin of insects; scales of fish; horny plates and scales of reptiles; feathers of birds; hair and nails of mammals. The glands of the body are developed from epithelium. Secretions 
from these various glands lubricate the surfaces, contain enyzmes for digestion of food, supply regulatory substances directly to the blood, serve as poison to other animals, and some are repellent to enemies.

Sustentative Tissue.-This type comprises all tissues whose function is to bind together or support the various parts of the body. Connective tissue is, in most cases, composed of slender cells with an abundance of intercellular material. This tissue is almost universally present in the various organs throughout the body. Tendons, the tough cords that comnect muscles to bones, of which the "hamstring" is a good example, and much of the dermis of the skin are composed of commective tissue. Bone and cartilage, which make up the framework of the body and support the other tissues, are called supporting tissues. In crayfishes and grasshoppers the supporting tissue is chitin instead of bone or cartilage. Cartilage is composed of scattered cells interspersed with abundant, homogeneous, granular, semisolid matrix or intercellular substances. Bone is somewhat similar, except that the matrix has been replaced by a heavy deposit of calcium phosphate and calcium carbonate, two solid salts. The scattered cells are present as bone cells.

Muscular or Contractile Tissue.-This is distinctive because of its ability to contract and in that way produce movements. Cells adapted to this function are more or less elongated and fiberlike. There are three types of muscular tissue: smooth, involuntary, and nonstriated, as found in the wall of the intestine; striated, voluntary, skeletal, as found in the muscle of the arm; and striated, involuntary, cardiac, as found in the wall of the heart. Skeletal, voluntary muscle is made up of large multinucleate (many nuclei) fibers, each composed of many fibrils (myofibrils) along which are evenly distributed dense and light areas, which give the general appearance of stripes across the cell, because the dense areas on the adjacent fibrils come at the same level. The smooth involuntary muscle is composed of individual, spindle-shaped (fusiform) cells, the cytoplasm of which is largely myofibrils but without striations and therefore smooth. There is a single oval nucleus, centrally located. The outer membrane of a muscle cell is the sarcolemma. The cardiac involuntary muscle is said to be made up of individual cells, highly modified in arrangement. The definition of cells in 
this tissue is rather difficult, but the fibers are faintly segmented by thin intercalary disks which define areas each with a single nucleus. The cells branch laterally to join each other quite frequently, producing a condition of netlike branching known as anastomosis.

Nervous Tissue.-This is specialized to receive stimuli and transmit impulses which have been set up by some stimulating agent in some part of the body. The structural features consist of nerve cell bodies and their processes. Two kinds of processes are recognizable: (a) the axone, usually a single unbranched fiber except for infrequent collateral branches; and (b) dendrites, frequently mucb

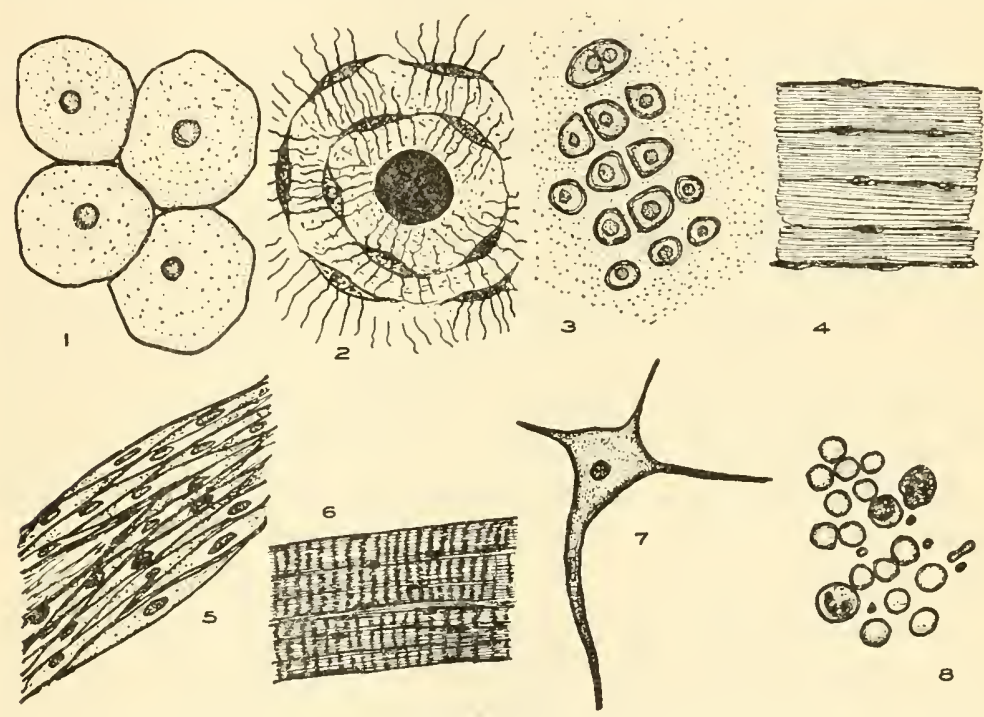

Fig. 45.-Typical cells and tissues from vertebrate animals. 1, Squamous epithelial cells; 2, section through a portion of bone showing Haversian canal (in center), bone cells, lacunae, canaliculi, and matrix; 3 , section of hyaline cartilage showing cartilage cells in lacunae, and matrix between lacunae; 4, sec. tion of tendon composed of white fibrous connective tissue; 5, longitudinal view of smooth (involuntary) muscle cells; 6, striated (voluntary) muscle; 7 , motor nerve cell, showing process; 8, human red blood (nonnucleated) corpuscles and human white (nucleated) corpuscles. (Drawn by Titus Evans.)

branched and arborlike. An axone may be several feet long, e.g., one extending from the spinal cord to the hand or foot. Dendrites may be lacking. The impulses are conducted toward the cell body over the dendrites and away over the axone. A nerve cell body together with its processes is called a neuron. The neurons approach each other and pass impulses from one to the other at the synapses, where 
the brushlike ending of the axone of one comes into close proximity with a dendrite of another. In this way an impulse can be transmitted from one part of the body to other parts. The chief function of the nervous tissue is to relate the organism to its environment.

Vascular Tissue.- This is fluid tissue consisting of cells known as corpuscles in a fluid medium called plasma. The cells are the red corpuscles (erythrocytes) and white corpuscles (leucocytes), while the plasma or fluid is the intercellular substance. Blood and lymph are the two common vascular tissues. Lymph has no red corpuscles. In the blood of mammals the red corpuscles are without nuclei; while in fish, frogs, turtles, and birds these cells are nucleated. The chief function of this tissue is the transportation of digested food and oxygen to the cells of the body and the removal of waste byproducts of metabolism from them.

An organ is an arrangement of two or more tissues as a part of the body which performs some specific function or functions. Some organs are made up of all of the different types of tissues just described. For example the stomach is an organ with an internal cavity. It is covered and lined with epithelium; the wall contains two strong layers of muscular tissue; blood vessels carrying blood, and lymph spaces bearing lymph, branch through the wall; nervous tissue reaches all parts of the organ to receive stimuli and distribute impulses; and connective tissue serves to bind all the others in proper relation.

A system is an aggregation of organs properly associated and related to perform some general function of life. There are ten different systems usually recognized:

a. The Integumentary System is composed of the skin and its outgrowths, such as hair, nails, scales, horns, hoofs, and similar structures. Its principal purposes are protection, primarily, with some degree of excretion and respiration, some absorption, and regulation of body temperature.

b. The Skeletal System composes the supporting framework of the body. The bony and cartilaginous tissues make up the material of this system. The vertebral column, skull, ribs, sternum, and bones of the limbs are the general parts of the vertebrate skeleton, and they serve for the support of the body as a whole and for the protection of the internal, vital organs. 
c. The Muscular System consists of muscles, the voluntary, striated group moves skeletal parts and accomplishes locomotion; the nonstriated, involuntary group is concerned with the movements of the internal organs (viscera), and the cardiac muscle produces the heart action.

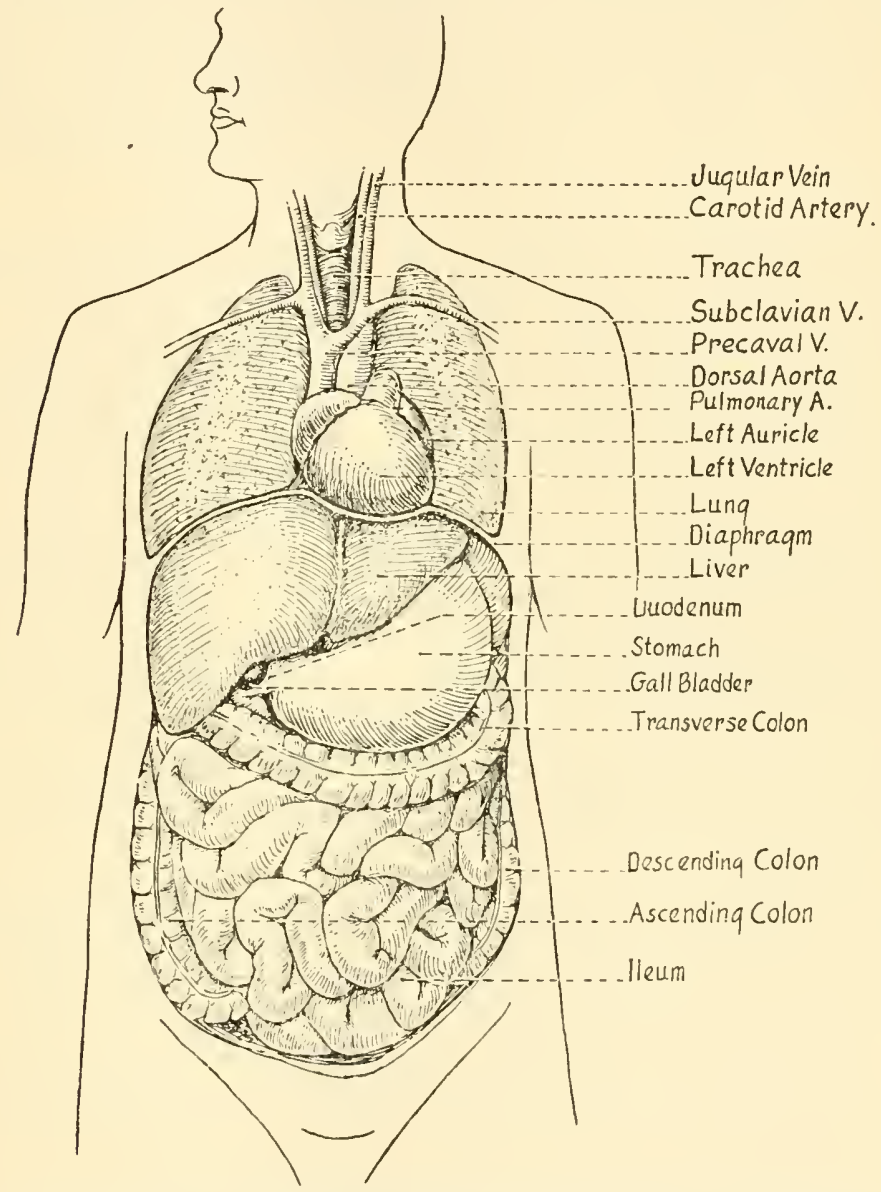

Fig. 46.- Ventral view of human manikin showing parts of the principal systems. (Drawn by Edward O'Malley.)

d. The Digestive System of the higher animals includes the mouth, pharynx, esophagus, stomach, small intestine, large intestine, and accessory glands. The general form of the system is that of a tube, and it is frequently called the alimentary canal. The functions of in- 
gestion, digestion, egestion, absorption, secretion, and very little exeretion are performed by this system. In general, it puts the food in solution so that it may be absorbed by the blood.

e. The Respiratory System consists of structures capable of delivering oxygen to the body and eliminating carbon dioxide. In some forms the general surface of the body serves the purpose, but in all higher forms there are special structures for this function. Tracheae are found in insects, gills of various modifications in many aquatic Metazoa, and lungs in the terrestrial vertebrate forms; accessory to the lungs are the nasal passages, pharynx, larynx, trachea, and bronchi.

f. The Circulatory or Vascular System is a very extensive one consisting of the heart, arteries, veins, capillaries, lymph spaces, lymph nodes, and lymphoid glands. The general functions are: (1) to distribute blood carrying food, oxygen, and hormones from glands of internal secretion to the tissues; (2) collect and transport to the point of exit carbon dioxide, liquid wastes, bacteria, and other foreign matter.

g. The Excretory or Urinary System is made up of tubular structures and accessory parts, such as flame cells, nephridia, Malpighian tubules, green glands, and kidneys. In the mammals, the ureters, urinary bladder, and urethra are accessory to the kidneys. The kidneys withdraw liquid waste products of metabolism from the blood and deliver them to the outside of the body. The nitrogenous substances, urea and uric acid dissolved in water, are the principal products discharged.

h. The Endocrine System includes a number of different glands located in various pal'ts of the body. These glands discharge chemical substances, known as hormones, directly into the blood. The hormones cooperate to regulate the metabolic activity of the entire body. The thyroid gland of the neck region, adrenals located near the kidneys, and the islands of Langerhans of the pancreas are typical examples of these organs. They go under the names of ductless glands and organs of internal secretion also.

i. The Nervous System is an organization of the nerve cell bodies and their processes in such a way as to receive stimuli, carry sensations, correlate them, and coordinate the activities of the parts of the body. By the function of the sensory portion of the system, the animal becomes aware of the enviromment and relates itself to it. In vertebrates the principal parts of the system include the brain, spinal cord, peripheral nerves, autonomic nerves, sense organs, and ganglia. 
j. The Reproductive System is an organization of glands, duets, and accessory structures which function in the reproduction of the species. More discussion of this system is found below.

The body might be thought of as being eonstrueted by relating eells to eells to form tissues, tissues to tissues to form organs, organs to organs to form systems, and systems to systems to form the metazoan organism. These will all be studied in more detail in conneetion with the study of speeific animals.

\section{Development of Sexual Reproduction}

Reproduction makes great advances among the metazoans. The simple fundamental process of reproduction by eell division or binary fission has been studied already. This is not possible for most metazoan animals, but, in general, this type of animal begins life as a single cell resulting from the fusion of two sex eells, one produced by each parent. In some of the colonial Protozoa and also in Sporozoa, as well as possibly in Paramecium, there seems to be the beginning of sexual reproduction. The individuals in a colony by peculiarities in cell division become differentiated into two types: (a) the ordinary, nutritive individuals, whose means of reproduetion is fission and (b) reproductive individuals or gametes of two forms: the large, egglike, inaetive macrogametes and the smaller, motile microgametes. In reproduetion these two types of cells unite to form a single zygote, from which a new eolony arises by repeated divisions. In a number of the Sporozoa, both sexual and asexual generations oceur. The zygotes, which are formed in the sexual phase or generation, produce a number of spores which develop sporozoites (already studied under Plasmodium). These become nutritive trophozoites and are eapable of production of another generation of gametes. Conjugation of paramecium is also looked upon as a forerunner of sexual reproduction.

In simple Metazoa there are likewise two forms of reproduction: asexual (without sex), including budding and fission, and sexual, which involves the union of two germ (sex) cells, one male and one female. In simple forms like sponges and jellyfish the germ cells arise from general formative interstitial cells between the two primitive germ layers to form temporary gonads. When the germ cells are mature, they break through the wall to the outside of the body. Again, among the simpler metazoans a single individual produces both male and female germ cells. Such an organism is said to be 
hermaphroditic or monoecious. Most of the types of animals in the phylogenetic scale, up to and including the worms, are normally hermaphroditic.

Infrequent examples of hermaphrodites occur either normally or occasionally abnormally here and there among the higher groups of metazoans, even in man.

In higher forms the usual method of reproduction involves germ cells produced by two individuals. Each cell is either male or female, the gonads of the other sex having degenerated in that individual. The sexes are separate under such conditions and are said to be dioecious.

There are some forms, particularly insects, in which it is possible for the unfertilized egg cell to develop without union with another germ cell. This is known as parthenogenesis. The case of the ordinary aphids or plant lice, known to every gardener, is a good example. In the spring an egg which was fertilized and laid the previous fall hatches to produce an individual known as a stem-mother. This individual feeds on the sap of the particular plant on which she lives and grows to maturity. Instead of mating (there are no males in her generation) she produces a series of eggrs (macrogametes) which continue to develop without union with a sperm (male germ cell). Another generation of female aphids arises from these eggs which in turn reproduce in a similar manner. A series of female generations appears in succession during the summer. No males are produced until the last generation of the season, and this time there are both males and females. These mate, the females lay fertilized eggs which pass through the winter and hatch as the first generation next spring. These individuals are the stem-mother's for the new season. Somc authors speak of this process as "virgin birth." The honey bee queen ean control her offspring to some degree. If her eggs are not fertilized, the offspring are all males (drones). If the eggs are fertilized, as most of them are, only females are produced, these becoming queens if fed abundantly on proper food or workers if fed otherwise. In regard to this state of affairs Lane puts it this way, "So it comes about, that though a drone bee may become the father of thousands of daughters, he never has a son, nor did he himself have a father."

The eggs of a number of animals, such as frogs, molluses, worms, sea urchins, and others have been artificially stimulated to continue 
development by application of chemical, electrical, or mechanical agents. This goes under the name of artificial parthenogenesis.

Metagenesis is a phenomenon occurring in the life history of a number of scattered species of Metazoa, including the coelenterate, Obelia; two or three marine worms; and Salpa, the tunicate (a chordate animal). This process is an alternation of production of sexual individuals in one generation and asexual in the next. The offspring in each case differs from its parents. This is spoken of as alternation of generation. In Obelia, a coelenterate related to Hydra (to be studied shortly), there is a plantlike, asexual, colonial form, which gives rise to sexual, free-swimming medusae (Fig. 59). The medusae produce eggs and sperms which unite in the water and develop into asexual colonies. Metagenesis really involves two methods of reproduction in successive generations of the same species. The significance is somewhat uncertain, but possibly it insures better and more complete distribution of individuals than could be secured by only the budding colony. Many of the sexually reproducing plants have a similar alternation of sexual and asexual generations.

\section{Metazoan and Ontogeny}

Ontogeny refers to the development and life history of the individual organism, produced sexually from the union of germ cells or gametes. This process is quite generally similar wherever it occurs, differing only in detail. Embryological development is an expression referring to the processes which occur during the earlier portion of the life of the individual.

The male and female germ cells or gametes are produced in their respective gonads as previously described. They are in a very immature state when they are first differentiated, and are called primordial germ cells.

The maturation (gametogenesis) or development of the germ cells occurs while they are still within the gonads, except for the latter part of the process in ova which reaches completion after the cells leave the ovary. It consists of a series of mitotic cell divisions which is modified at one point to bring about a fusion and subsequent reduction in the number of chromosomes in the eells. In brief maturation is the preparation of germ cells for fertilization which may follow. The devclopment of the male germ cell is known as spermatogenesis, and the development of the female germ cell is oögenesis. 
Oögenesis begins with the primordial germ cell within the ovary. These cells are typically spherical or oval with a prominent nucleus, having the normal number of chromosomes for the somatic cells of the species. This number of cliromosomes is known as the diploid number. For purposes of illustration the process will be deseribed for a form whose diploid number of chromosomes is eight. The primordial cell divides by mitosis to form two oögonia. Each of these divides similarly. As is typical of mitotic division, each chromosome divides with the division of the cell. This series of divisions constitutes the multiplication period of the maturation process. In some

Oögenesis Spermatogenesis

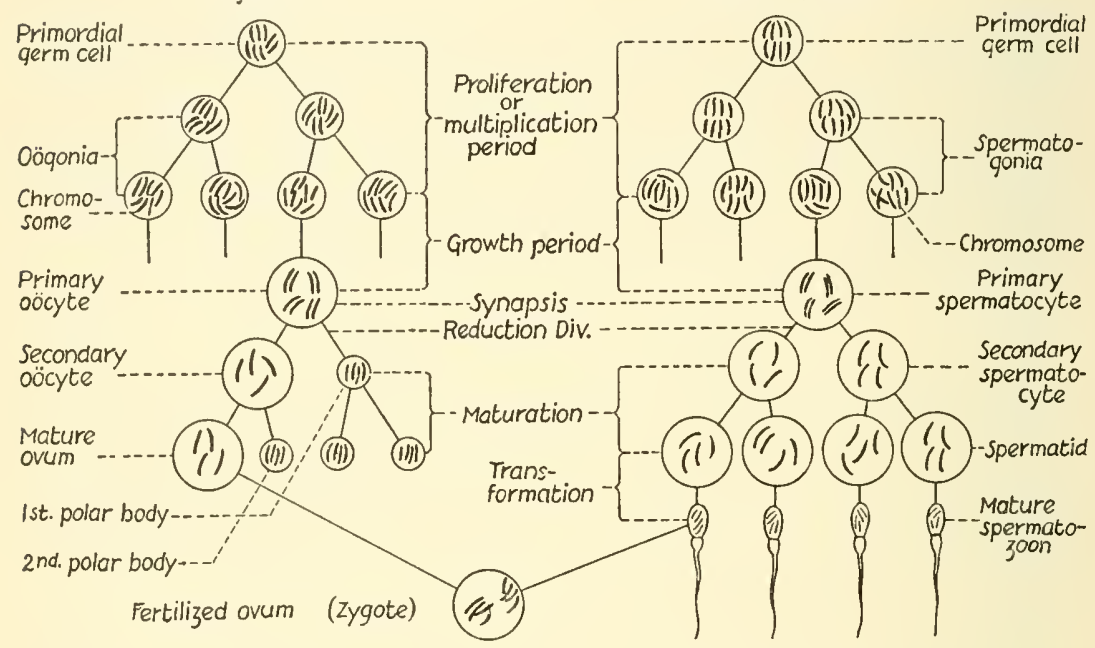

Fig. 47.--Maturation of the germ cells. Oögenesis includes the maturation divisions of the female germ cells or ova, and spermatogenesis is a similar process of division in the development of mature male germ cells or spermatozoa.

instances each of these cells divides once more. Next, each of these oögonia passes through a growth period without division. During this time the chromosomes in each mite in pairs and fuse together. This fusion is spoken of as synapsis of chromosomes. At the close of this growth each of these cells is called a primary oöcyte. Each of these oöcytes divides by meiosis, the fused chromosomes dividing as though they were single ones in normal division. This division, therefore, results in cells with half the somatic (diploid) number of chromosomes and is spoken of as the reduction division. The cytoplasm does not divide equally; nearly all of it goes to one of the 
cells in each case. This large cell is called the secondary oöcyte and the small one is the first polar body. Each of these cells has four chromosomes. Following this the secondary oöcyte divides to form the mature ovum and another polar body. Occasionally the first polar body divides, but none of them have any further significance after carrying away half of the chromosomes. They now degenerate, and their protoplasm is reabsorbed by the surrounding tissue. The series of divisions and changes following the primary oöcyte stage constitute the maturation period of the process. The ovum containing the haploid number of chromosomes is now prepared to unite with a mature spermatozoon in fertilization.

Spermatogenesis is completed within the tubules of the testis, and, like oögenesis, is a series of mitotic cell divisions. The primordial germ cells divide by mitosis to form spermatogonia, and this process continues just as it does in oögenesis, until the division of the primary spermatocytes which have developed during the growth period. When the primary spermatocytes divide, the division is an equal one and all of the resulting cells are typical secondary spermatocytes with the haploid number of chromosomes. These cells divide to form spermatids. Each spermatid then undergoes a change of shape or transformation to form the mature spermatozoa, each with its half number or, in this case, four chromosomes. The change from spermatid to spermatozoa does not involve a cell division but simply rearrangement. The spermatozoon is a slender, motile cell composed of head, middle piece, and tail. It is now able to swim in fluid and prepared to unite with a mature ovum.

The maturation process is very significant for at least two important reasons. First, during the fusion and subsequent divisions of the cells, there is given opportmity for variation of the genetic composition. Secondly, the number of chromosomes is reduced to half in each mature germ cell, thereby making it possible for the germ cells to unite without doubling the typical number of chromosomes in each new generation. Each species has a definite and constant number of chromosomes.

Fertitization involves the union of a mature ovum and mature spermatozoon to produce a fertilized ovum or zygote. The spermatozoon swims to the egg and enters it by penetrating the outer membrane which is called the vitelline membrane. For most animals, as soon as one sperm enters an egg, the chemical nature of the vitelline 
membrane changes and prevents entrance of others. The head of the sperm carries the nucleus and soon takes the form of a rounded male pronucleus inside the cytoplasm of the egg. The egg nucleus is known as the female pronucleus. The male and female pronuclei finally fuse to form the fusion nucleus, and the fertilization is complete. The significance of fertilization is largely centered around two important functions. First, it is the impetus for the development of an embryo from the egg under most normal circumstances; however, parthenogenesis replaces this function in some cases. Secondly, it brings about

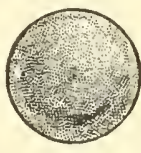

I

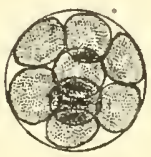

4

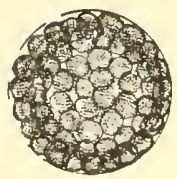

7

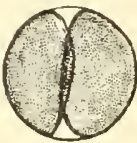

2

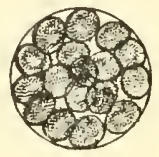

5

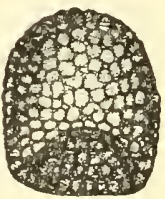

8

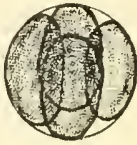

3

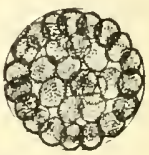

6

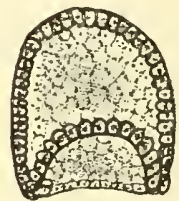

9

Fig. 48.-Diagrams showing cleavage in the young embryo of Asterias. 1, Fertilized egg (zygote); 2, two-celled embryo following first cleavage divislon; $;$, the four-cell stage; 4, the eight-cell stage; 5 , the sixteen-cell stage; 6 , morula stage (solid) ; 7 , blastula stage (hollow) ; 8, early gastrula stage (infolding of cell layer at one side); 9 , later stage of gastrulation. The infolded layer is the endoderm. (Drawn by T. C. Evans.)

the means for inheritance of characteristics from two different lines of ancestry. This union also restores the diploid number of chromosomes.

Cleavage is a series of mitotic cell divisions beginning in the zygote immediately following its formation. These divisions occur in rapid order with but very little intervening growth, and the resulting cells adhere to each other in a body. In eggs where the yolk material is scant and evenly distributed, the ensuing cleavage 
divisions extend completely through the zygote, forming nearly equal cells. If the yolk is concentrated in one end of the egg, the divisions of the developing embryo are unequal. During the early divisions all of the cells of the body divide at so nearly the same rate that it appears as if the zygote were being cut with a knife or cleaver into smaller parts. This process provides for the rapid increase in the number of eells and growth of the embryo which is necessary before any special parts can be formed. Cleavage will be described more fully in a later chapter under the discussion of the development of the frog.

As divisions proceed, a blastula is formed by the development of a cavity (blastocoele) within the spherical mass of cells, the wall of which is now a single layer. The formation of the blastula, which usually comes at the sixty-four cell stage or later, marks the end of cleavage. The blastula stage of an animal like a starfish or a frog resembles somewhat a hollow rubber ball whose wall is made up of a large number of pieces cemented together.

As cell divisions continue in the blastula, a gastrula is finally formed. The blastula does not simply increase in circumference, but there comes a time when the wall on one side pushes in (invaginates), finally meeting the wall of cells from the other side. This gradually crowds out the cavity and forms a wall of two layers of cells. The outer layer is known as the ectoderm (outer skin) and represents the portion of the wall of the blastula which has not folded in. The inner layer, or that resulting from the infolding of the wall of the blastula, is called endoderm (inner skin). As division of cells in this wall proceeds and the infolding continues, the two margins of the infolded part come nearer and nearer each other. This gradually encloses an outside space which is lined by the endoderm and represents the primitive digestive tract or archenteron. This is the beginning of the two primitive germ layers, ectoderm and endoderm. In sponges and coelenterates development stops here.

In higher forms, immediately following gastrulation, a third germ layer, the mesoderm (middle skin), is organized from cells usually contributed by one or the other or both of the other gel'm layers. In some cases it arises as two saclike outgrowths from the endoderm, one on each side in the gastrula. These pouches push into the remains of the blastocoele. In other cases separate cells are shed from ectoderm or endoderm or both, or from an undifferentiated 
portion to organize as a distinct layer between the other two. The position of the mesoderm is external to the endoderm and internal to the ectoderm. It nearly encircles the endoderm. Sooner or later a space forms within the mesoderm, causing the outer limb of it to join the ectoderm and the inner to join the endoderm. This cavity is the coelom or future body cavity. From each of the germ layers, particular parts of the body are derived.

The fate of the germ layers is determined as cell division and development continue. The division proceeds at different rates in different regions and at different times resulting in various infoldings, outpushings, and extensions which finally bring about the formation of all parts of the mature individual. The ectoderm gives rise to the external surface cells or epidermis of the skin and to the nervous system; the mesoderm furnishes the muscles, skeleton, circulatory system, blood, excretory, and reproductive systems besides nearly all comnective tissue; and the endoderm produces the internal linings of the digestive tract, respiratory tract, and such outgrowths as the liver and pancreas. 


\section{CHAPTER IX \\ PHYLUM PORIFERA}

\section{SPONGES}

The name of this phylum, Porifera (pōruffẽrá), means "porebearers," and this, these animals certainly are. This group is thought to be sort of an aberrant type with peculiar relations, but the group is often considered the simplest and lowest type of Metazoa, notwithstanding the presence of a simple mesoderm which is lacking in Coelenterata. For a long time sponges were thought to be plants, and it was not until 1857, only a little over ninety years ago, that they were fully acknowledged as animals.

They are sessile in habit, being fastened to piers, pilings, shells, rocks, etc., for life. There is entire lack of locomotion. Most sponges, bath sponges included, live in the sea. There are only a few small fresh-water forms. They have tissues but are without organs. The body is in the form of a hollow sac with many canals piercing the walls and making connection between the internal cavity and the outside. The pores of these canals are essentially mouths. There is only one general exit from the cavity. All sponges have some type of skeletal structure; some possess hard, calcareous, or siliceous spicules, and others have a flexible fiberlike material as a skeleton.

The organization of the sponges is a loose one, and the interdependence of part upon part is not great. An animal with hundreds of mouths cannot be very highly organized. Some authorities show a rather close comparison between sponges and colonial Protozoa. The sponges possess collar cells or choanocytes which are similar to the cells of the colonial mastigophoran, Proterospongia.

There are workers who hold that sponges may have arisen from a common ancestor with the choanoflagellate type of colonial Protozoa. For a time sponges themselves were considered colonial Protozoa. The sponges do not have a distinct enteron or digestive cavity, but digestion is entirely intracellular (within cells). The germ layers are not well-established; the layer which seems to begin like endoderm develops into the external layer. The so-called ecto- 
derm comes to line the internal cavities and its function is circulating the water. The middle layer is very poorly differentiated, being hardly more than a matrix, and is hardly recognizable as the mesoderm of the typical triploblastic animal. If the type can be classified according to germ layers, it might be considered a modified diploblastic (two germ layers) form. Because of these peculiarities, some author's have called sponges Mesozoa or Parazoa.

\section{Classification}

Class Calcispongiae.--Single, shallow-water, marine forms, characterized by calcareous spicules. There are two orders.

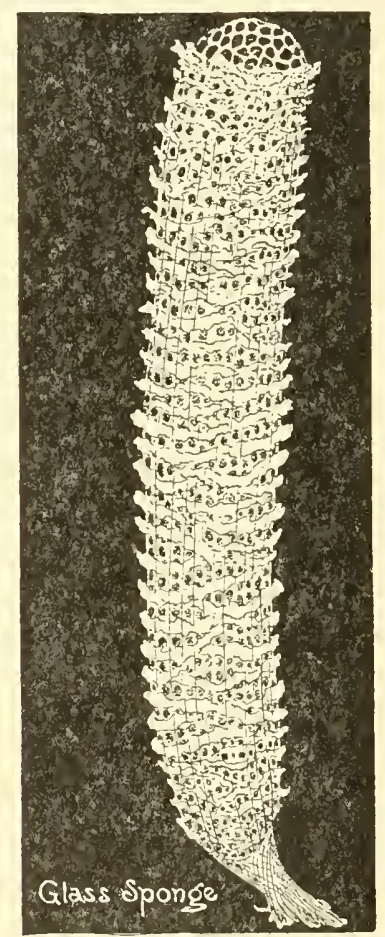

Fig. 49.-Glass sponge or Venus's flower basket, Euplectella sp., is probably the most beautiful of the sponges. (Courtesy of General Blological Supply House.)

Order Homocoela.-Simplest type, possessing a very thin body wall with pores as perforations in individual cells. The internal cavity is lined with choanocytes. Leucosolenia. 
Order Heterocoela.-Moderately complex wall. Choanocytes in radial canals. Scypha (Grantia).

Class Hyalospongiae.-Sponges which possess siliceous spicules with three axes and six rays or a multiple of six. Spicules are white and like spun glass. Often called glass sponges because of this skeleton. Venus's flower basket.

Class Demospongiae.-Forms which have either nontriaxial siliceous spicules or spongin or no skeleton. They have complicated canal systems and are often quite large and brightly colored. A few fresh-water forms are known.

Order Tetraxonida.-These are ordinarily attached to the bottom in deep water. Thenea.

Order Monaxonida.-Includes shallow-water, marine forms and one family of fresh-water sponges (Spongillidae). There are less than two dozen fresh-water sponges known in this country. Spongilla, Haliclona, or finger sponge, and Cliona, or boring sponge.

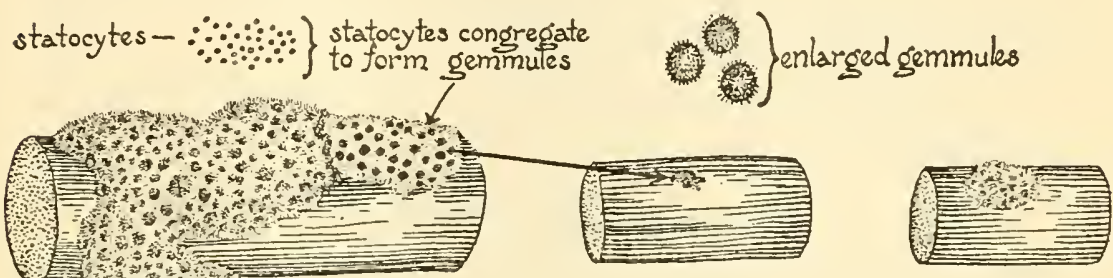

Fig. 50.-Spongilla, showing reproduction. (Courtesy General Biological Supply House.)

\section{Fresh-IVAter Sponges}

In the southwestern part of the United States, at least in central Texas, there are four species of fresh-water sponges: Spongilla fragilis, Trochospongilla horrida, Asteromeyenia plumosa, and Ephydatia crateriformis. Of the four, Spongilla fragilis seems to be the most abundant in this area. Most of the colonies of this species are irregular in shape, averaging approximately $1 / 3$ of an inch in diameter; but there are some as large as 6 inches by $2 \frac{1}{2}$ inches. Usually they are not over $1 / 4$ of an inch in height. Most of the colonies are irregular in shape, but some are cushion-shaped and a few are branched. Most of the large colonies of sponges in this region are dark grey or chocolate brown in color and are found 
on logs either floating in the water or submerged. In some parts of the country there is the idea that sponges require clear water, but in the region referred to they (particularly Trochospongilla horrida and Ephydatia crateriformis) grow abundantly in muddy ponds and in muddy streams whose turbidity equals 110 parts of solid matter per million. In this region again the growth of the sponge and apparently gemmule formation is a perennial process. The maximum production of gemmules seems to be in the late autumn and throughout the winter, even following periods of low temperature in the spring. These gemmules are ordinarily deposited in a pavementlike layer on the object to which the sponge is attached, sometimes covering several square inches. The species are usually identified by means of microscopic differences in the gemmule spicules as seen when crushed.

Order Ceratosa.-A group of important sponges of which man uses at least a dozen different ones. The representatives of this order have skeletons of spongin and are found in subtropical and tropical marine waters. Euspongia, the bath sponge.

Order Myxospongida.-These sponges are entirely devoid of skeleton. Haliscara.

\section{THE SIMPLE SPONGE}

Scypha coronata* (Ellis and Solander) has been mistakenly called "grantia," the European form, by most textbooks for years. This is a commonly studied representative of the phylum. It is available and is also comparatively simple in structure. It is not as simple, however, as Leucosolenia.

\section{Habitat and Behavior}

This type lives attached to rocks in relatively shallow marine water. The animal is attached by the basal or proximal end; the opposite end is free or distal. A colony may be formed by budding. Water is drawn in through the pores or ostia on the sides of the body, then by way of the canals into the internal eavity. This water is forced up through the eavity and out at the osculum or exit opening at the top. The osculum and ostia may be closed and there

* A Case of Incorrect Identification American genus is Scypha. M. W. de Laubenfels, Pasadena, Calif., Science Vol. 85, No. 2199, Feb. 10, 1937, p. 199. 
may be contractions of the entire body. These movements are accomplished by individual contractile cells. These reactions may involve the entire body, or they may be local. Laymen and many zoologists think of sponges as sluggish, inactive forms, because they are sessile. On the contrary, these animals work day and night to keep a continuous current of water to supply their vital needs. It is reported that an average sponge will pump approximately forty-five gallons of water through his body in forty-eight hours.

Activities and coordination in Scypha and sponges generally are quite limited by lack of a nervous system. Individual cells respond directly to stimuli, and impulses are conducted simply from cell to cell in a primitive fashion. This results in very slow transmission of impulses and is called neuroid transmission.

\section{External Anatomy}

The average length of Scypha is about three-fourths of an inch. It is rather goblet-shaped with the excurrent opening, oseulum, at the top. A row of picketlike spicules or spines encircles the oseulum,

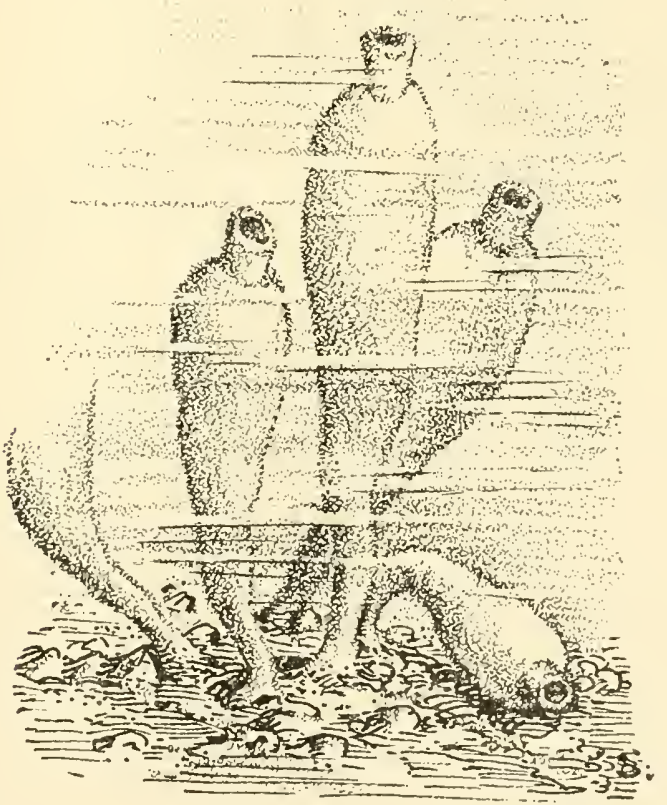

Fig. 51.-Scypha coronata (Grantia), showing habit of life. 
and other less conspicuous spicules are distributed over the body. The ostia are the incurrent pores through which water is taken into the body. They are quite evenly distributed over the wall. A dermal epithelium covers the outer surface of the animal.

\section{Internal Anatomy}

Internally there is a large central or gastral cavity which is simply a water cavity and is not comparable to a stomach or enteron. In
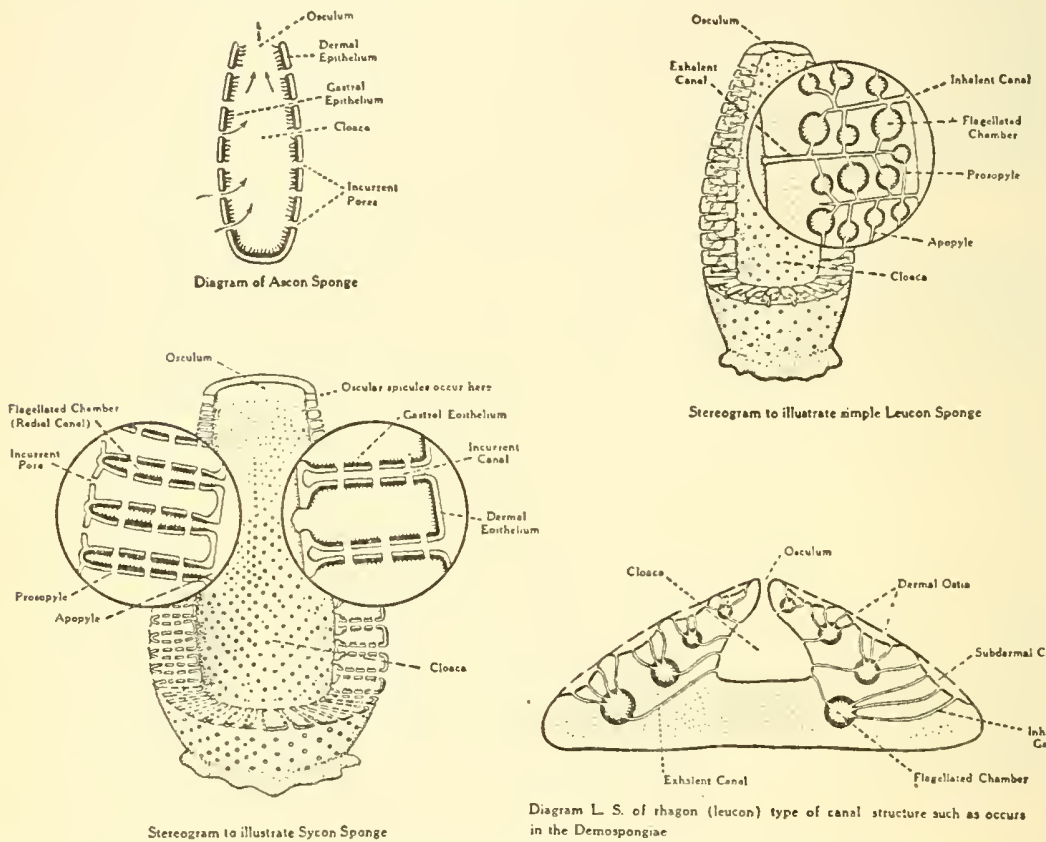

Fig. 52.-Structure of different types of sponges, shown diagrammatically. (Courtesy Pacific Biological Laboratories.)

more complex sponges there may be several or even many such cavities, each one opening distally by an osculum. Communicating with and radiating from this cavity is a set of radial canals. They join the cavity through small pores called apopyles and extend nearly to the outer surface of the wall where they end blindly. Lying between these and extending inward from the ostia are the incurrent canals. They connect with the radial canals by rather numerous apertures called prosopyles. This canal system not only serves to 
carry the water, but it substantially increases the surface area of the animal. This seems to be a definite provision to allow increase in volume by keeping the ratio of surface to volume.

In sponges generally, there are three types of canal systems, identified as the ascon, sycon, and rhagon types of which the first is the simplest, the second intermediate, and the third, the most complex. The canal system of Scypha is of the sycon type.

The character of the skeleton is a diagnostic feature in the classification of sponges. Some have a skeleton of calcareous spicules, others of siliceous spicules, others of the fibrous spongin, and still others have no skeleton. Spongin of the ordinary bath sponge, which is simply the skeleton of one of these animals, resembles silk chemically. It is formed by some special cells called spongioblasts. The

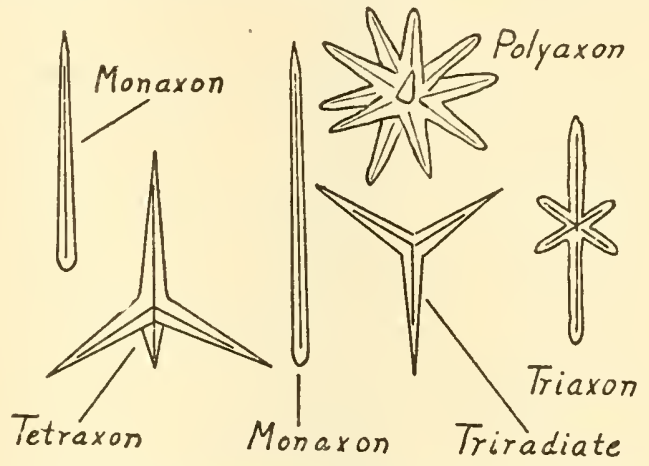

Fig. 53.-Types of calcareous skeletal spicules found in different sponges. (Drawn by Joanne Moore.)

spicules are of several types with a number of modifications of each. The monaxon type consists of simple straight spines; the triradiate type consists of those that have three rays joining each other in one plane; the tetraxon type has four rays radiating from a common point in four different planes; the triaxon type possesses six rays lying in three axes; and the polyaxon type has numerous rays. The cells which produce spicules are known as scleroblasts.

The histology of Scypha presents a peculiar arrangement of a number of different types of cells. The outer, dermal layer is composed of simple, flat, epithelial cells, contractile cells (myocytes), gland cells which secrete the substance for anchorage, as well as the scleroblasts. A great many of the cells in this layer do not have distinct boundaries, making it a syncytium. In Order Ceratosa, the 
spongioblasts are located in this layer. The incurrent canals are lined with flat pavement cells. At each prosopyle there is a single large dermal cell, a porocyte, which surrounds the aperture. In the middle layer are found the reproductive cells and some amoeboid wandering cells. The radial canals and the internal wall of the central eavity have similar histological structure since the former are outpouchings of the latter. The cells here are primarily special shaped choanocytes, peculiar to sponges, interspersed with scat-

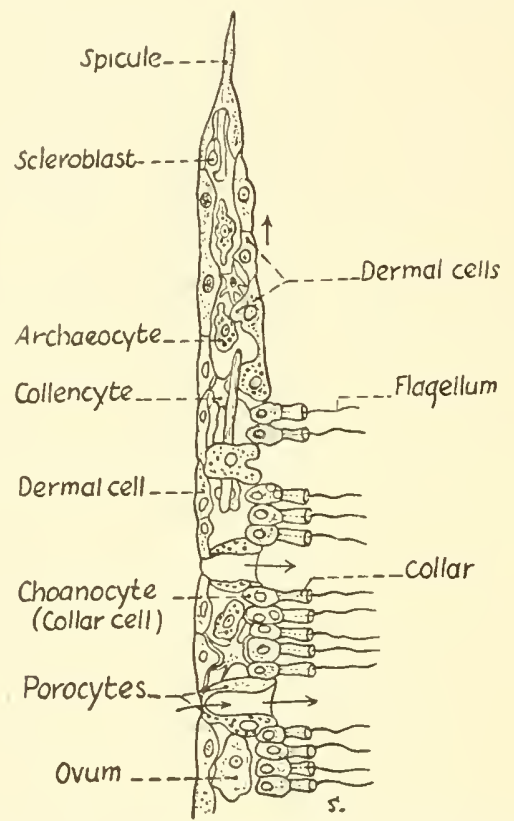

Fig. 54.- Histology of wall of a simple sponge in longitudinal section. (Redrawn and modified from Lankester, Treatise on Zoology, published by The Macmillan Company, after Minchin.)

tered, flat, epithelial cells. Each choanocyte has at its free margin a funnel or collar opening to the central cavity and a flagellum or whip extending from the fumel. The flagella agitate the water and drive the suspended food particles into the fumnellike mouths of the collar cell where a food vacuole is formed in the cell in amoeboid fashion. Of course the spicules appear in a histological section. The entire arrangement is quite similar to a large colony of semiindependent cells which do not function as integral parts of a tissue as do the cells of higher animals. It has been found that indi- 
vidual cells can be separated from each other by squeezing some types of sponges through the meshes of a silk clotli. From these living cells, if kept in favorable conditions, a new sponge will reorganize.

\section{Metabolism}

A sponge obtains food from the water which is continually passing by way of ostia, through the canals and central cavity, and out the osculum. Microörganisms and other particles of organic matter are drawn in with the water. The current is produced by the flagella of the choanoeytes and contractility of the walls. It is controlled by the contractility of the cells surronnding the ostia. As the current sweeps the potential food particles into the collar cells they are seized and ingested by pseudopodia, according to some authors. At any rate the food particles are taken into the cytoplasm of certain of the cells. Digestion is intracellular (within cells) in the food vacuoles and the process is much the same as has been described in Protozoa. The digested material is assimilated by diffusion from cell to cell. This may be augmented by the amoeboid wandering cells. Respiration is carried on by diffusion through the general surfaces, and the exchange of gases $\mathrm{O}_{2}$ and $\mathrm{CO}_{2}$, is made with the surrounding water. Catabolism, or the union of oxygen with the fuel substance of the cell to liberate energy, goes on in the cells in some degree as long as they are alive. Excretion is largely by general diffusion through the surfaces, perhaps assisted by the wandering cells. Egestion is probably accom. plished much as it is in Amoeba.

\section{Reproduction and Life History}

Scypha is able to reproduce both asexually and sexually. The former may be by budding or by the formation of gemmules. Budding involves the branching of new individuals from the external surface of an old one. These new individuals finally become free from the parent. Sometimes a colony is formed by the buds remaining attached to the parent. Gemmule formation or internal budding is another type of reproduction, found particularly in fresh-water sponges. Groups of cells become separated from the surrounding deep tissue by limiting membranes, which become infiltrated with siliceous materials. They are usually formed during adverse conditions and can withstand desiccation and other severe 
circumstances. In fresh-water forms these gemmules are formed in the middle layer of cells; the parent individual then dies; and the following spring new individuals emerge from the gemmules.

Sexual Reproduction occurs here for the first time in our discussions. Sponges are usually hermaphroditic, but the germ cells
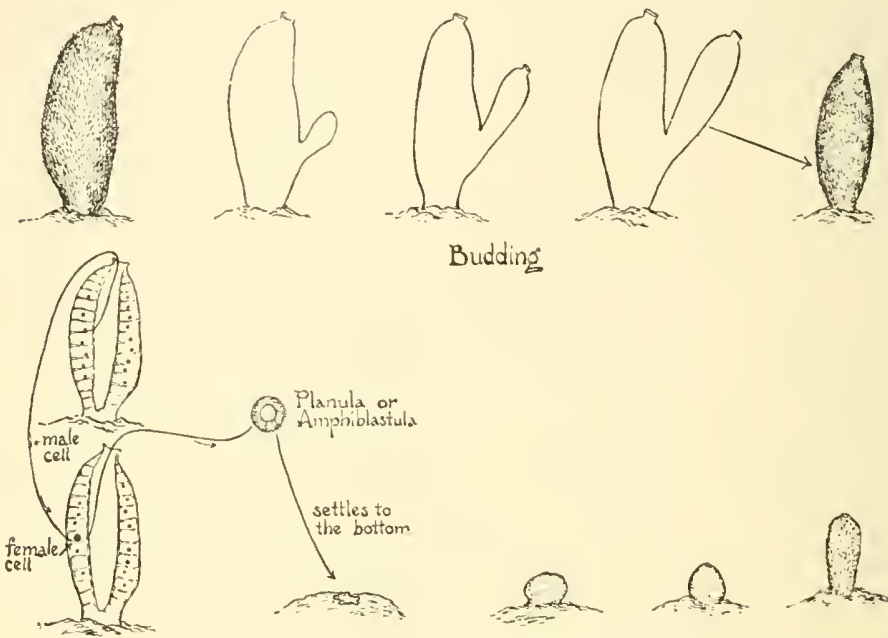

Budding
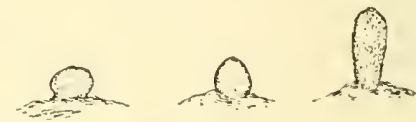

Sexual Reproduction

Turroa

Fig. 55.-Methods of reproduction in Scypha. (Courtesy General Biological Supply House.)

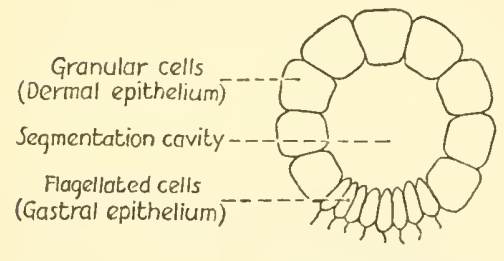

Typical free-swimming Amphiblastula
Osculum breaks thru here

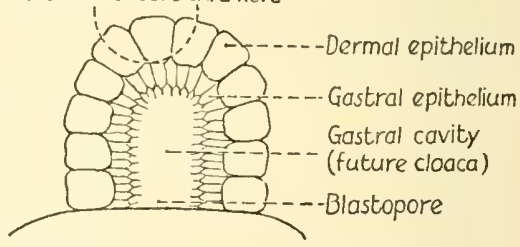

Typical Amphiblastula at time of attachment

Fig. 56.-Diagrammatic sections of Scypha larvae.

of the male usually mature before those of the female. The reproductive cells are produced in the jellylike middle layer. Fertilization takes place here and cleavage division progresses. At about the blastula stage the embryos are liberated through the wall of the body as free-swimming, ciliated larvae. These later settle down, become attached, and are modified to form adult, sessile sponges. 


\section{Economic Relations}

Many sponges are beneficial to man, and there are a few which are detrimental. Oyster's and some other Mollusca are injured or destroyed by certain sponges which attach themselves to the molluse's body or by other's which bore through its shell and thus kill it.

Of positive importance, lesser items include the large flint deposits from siliceous spicules of some species and those used for ornaments. The chief importance lies in the use of the spongin skeletons of certain groups for bath and surgical sponges. With rapid industrial development sponges have also become useful as a fabric material. The demand has brought about the establishment of sponge farms where they are raised from fragments about an inch square or slips, like plants. Selected sponges of good quality are cut up, and the pieces fastened to hooks or wire on a weighted frame. The "seeded" frame is sunk in the ocean and left for a year or two for the sponges to grow. If everything has gone as expected, the slips will then be marketable.

Sponges are usually harvested by diving or hooking between May and October. Dredges which are sometimes used for this purpose are not very satisfactory, because they drag down and kill so many young sponges. Sponges die quickly when taken. They are allowed to rot for a day or two, then beaten and squeezed under water, washed, dried, and sometimes bleached. Next they are trimmed, sorted, and placed on the market. The value of the crop each year is at least $\$ 5,000,000$. Most of the commercial sponges come from the Mediterranean Sea, Red Sea, coasts of Florida, the Bahamas, the West Indies, and Central America.

\section{Phylogenetic Advances of Sponges When Compared With Protozoa}

This group appears to be somewhat advanced over the Choanoflagellata of the Genus Proterospongia in Class Mastigophora. In sponges the cells are partially organized into layers and are differentiated for separate functions. Sexual reproduction is developed here in a simple way. The group has advanced so little that little else can be said. 


\section{CHAPTER $\mathrm{X}$ \\ PHYLUM COELENTERATA}

The phylum name, Coelenterata (sēl ěn tẽr $\bar{a}^{\prime}$ tá), means “hollow intestine," and all of the representatives bear this out by possessing a single large cavity in the body. There is a single opening to this cavity, and it functions as both mouth and anus. There are two general types of coelenterates; the polyp form and the jellyfish form. They are all modified gastrulas, have radial symmetry, and possess tentacles with "sting bodies" or nematocysts. Most of the species are marine, but there are a few fresh-water forms. The body wall is composed of two layers of cells, and for that reason they are said to be diploblastic. These two layers are the outer ectoderm and inner endoderm. Most of the representatives do not develop skeletal structures, but coral polyps produce hard, calcareous cases around themselves. In several species there is the typical alternation of generations of attached and free-living forms. Most coelenterates are attached or very sedentary for at least a part of the life span.

The radial symmetry is correlated with an attached habit of life. A good many of the attached forms look much like plants and were so described for a long time.

The digestive process is principally extracellular, being accomplished by enzymes which are secreted by special cells of the endoderm into the internal or gastrovascular cavity. A limited amount of the digestion, however, takes place within the endoderm cells after particles of partially digested food have been engulfed by these cells. This is called intracellular digestion. Excretion and respiration are carried on by the general surfaces of the body. Asexual reproduction is accomplished by budding and fission. Sexual reproduction, involving production of ova and spermatozoa and their union in fertilization, occurs here too.

The group is considered among the simplest of metazoans and shows, in a simple way, typical features of this great division of the animal kingdom. Hydra will be studied in detail, because it is 
readily available, easily collected and handled, and is representative of multicellular animals of simple formation. The study of Hydra as a simple metazoan will go far in giving insight into the much more complex make-up of the body and life of man.

\section{Classification of the Phylum}

The phylum is divided into three classes, each with three or four orders.

Class Hydrozoa.-These are typical polyp forms, many of which produce medusae forms by budding. The group includes marine, colonial polyps, or hydroids, floating colonial hydroids, such as Portuguese man-of-war, one special group of corals, some smaller jellyfishes, and the fresh-water polyps.

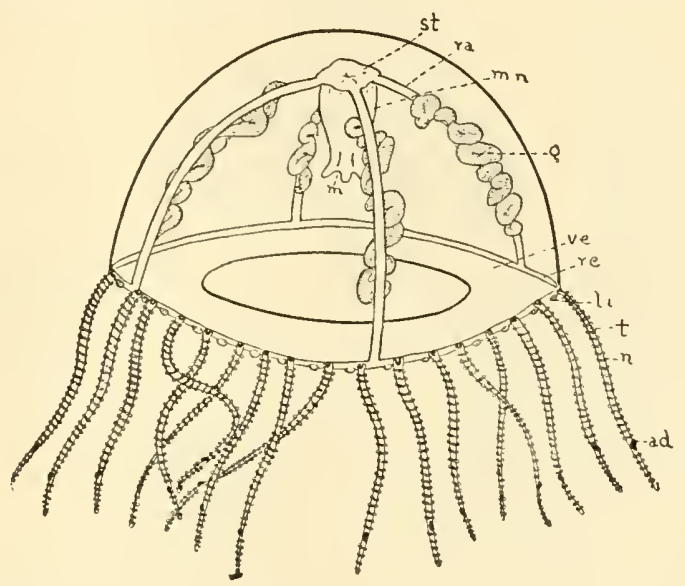

Fig. 57.-Structure of Gonionemus. ad, Adhesive pad; $g$, gonads; $l i$, lithocyst ; $m$, mouth ; $m n$, manubrium : $n$, nematocyst; $r a$, radial canal; $r c$, ring canal ; st, stomach; $t$, tentacle; ve, velum. (From White, General Biology.)

Order Leptolina-a group which has a sedentary or sessile polyp stage. Such examples as Hydra, Obelia, Gonionemus, Campanularia, Tubularia, and Craspedacusta are well-known forms. The first one is a fresh-water polyp form and is the best known of the group. The last one listed is a fresh-water form with a small polyp stage lacking tentacles but with a disclike medusa possessing many tentacles. Hydra, of this order, will be discussed as a general representative of the phylum, but since Gonionemus and Obelia are common marine forms, a brief description of them may be included here. 
Gonionemus is a small jellyfish form, measuring about a centimeter across, and is found in the pelagic waters, along our eastern shores. Its shape reminds one somewhat of an umbrella with a fancy fringe but with practically no handle and made of clear cellophane. The exumbrella is the convex upper, or aboral side while the subumbrella is the concave, lower, oral side. A short stalklike part, the manubrium hangs down from the center of the subumbrella. At its distal end is the mouth, bordered by four oral lobes. The mouth is the aperture leading into the internal or gastrovascular cavity which has four radial branches or canals. These radial eanals join a circumferential or marginal or ring canal. A circular ledge or fold of tissue which extends inward from the margin of the subumbrella and partially encloses this saucer-shaped cavity, is called the velum (craspedon). From a few to more than eighty almost solid tentacles hang down from the margin of the subumbrella. The cell structure of this animal is made up of an outer ectoderm and an inner endoderm, with a large amount of jellylike mesoglea between these two germ layers. Wavy, leaflike folds hanging in the subumbrella and radiating from manubrium to margin are the gonads. A planula-like hydroid form develops from the egg. The animal is able to swim about by drawing water into the partially enclosed eavity of the subumbrella and expelling it through the aperture formed by the velum with enongh force to move the animal in the opposite direction. The pressure is developed by contraction of the body.

Obelia is a marine, colonial type resembling a branched plant in appearance. The individuals are attached to each other in the colony, and it is fastened to a rock or other substratum by a rootlike hydrorkiza. They are distributed in the Atlantic Ocean and Gulf of Mexico out to forty fathoms in depth. The colony begins as a single individual which buds, but they do not separate from the preceding or parent generation. This may continue for several generations. From the hydrorhiza there is an upright stem, the hydrocaulus. This stemlike part gives off lateral branches, hydranths; at the end of each is a mouth and tentacles. These are feeding polyps. Also as branches of the stem, there are the blastostyles which are modified, nonfeeding polyps capable of producing medusae. The medusa is the third type of individual connected with an Obelia colony. The perisarc, which is composed of chitin, covers the colony. In some parts this is ringed, and it expands at the base of the 

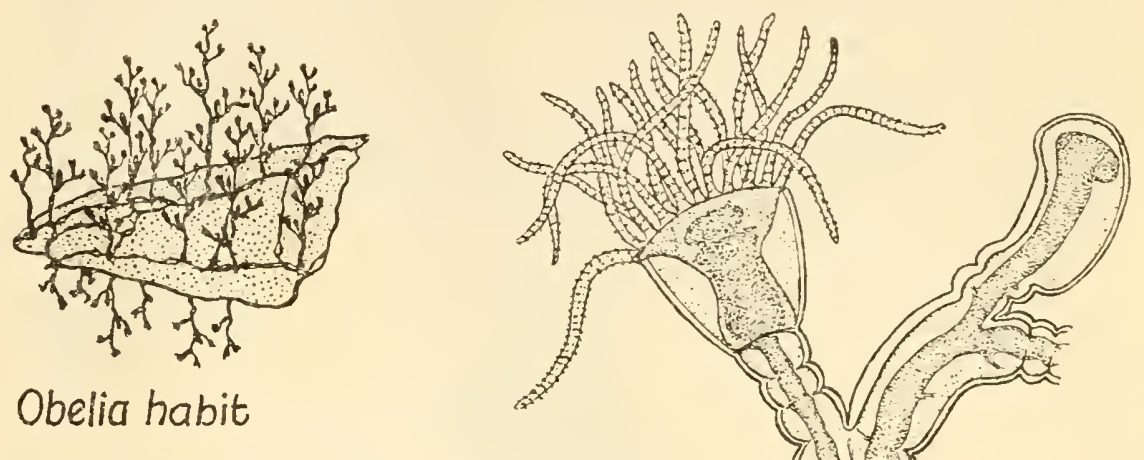

Obelia habit

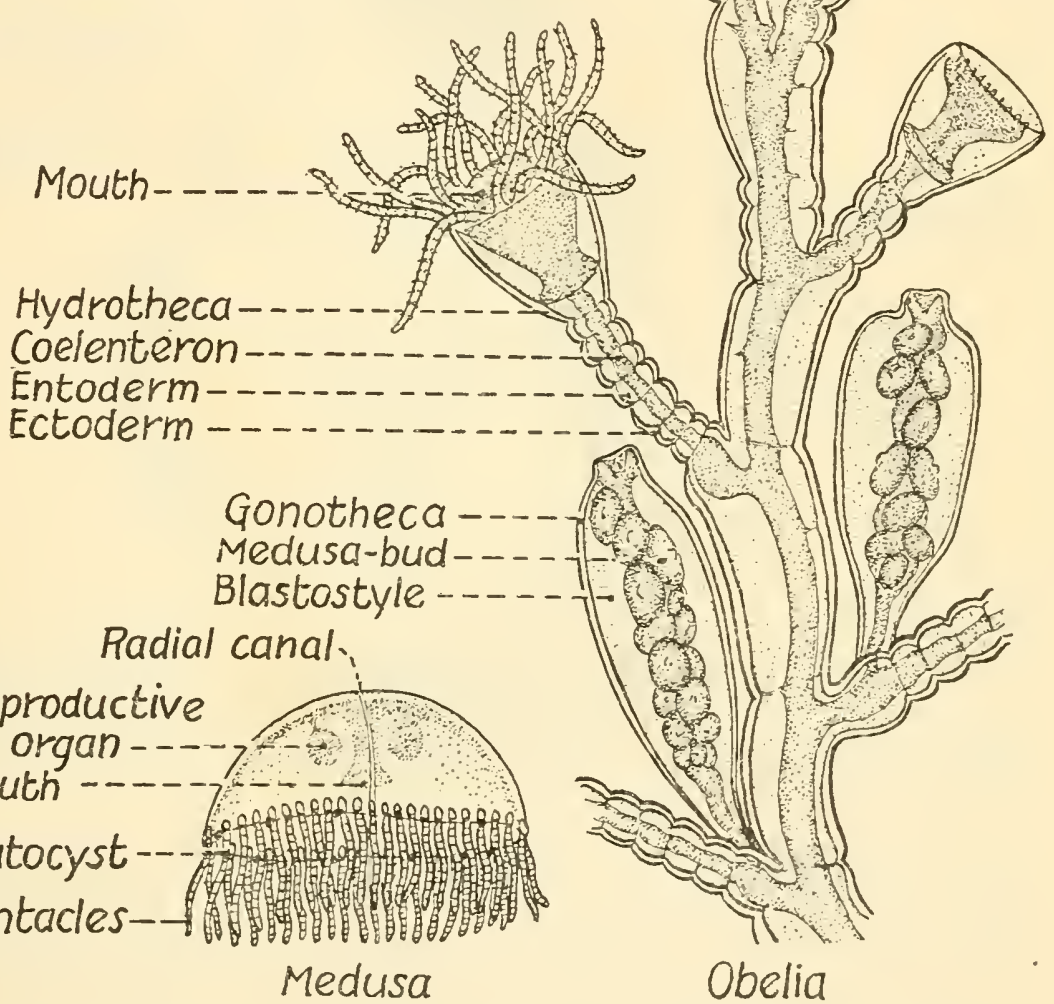

Fig. 58.-Obelia, hydrozoan colonial coelenterate, showing asexual generation, sexual generation (medusa), structure, and habit of life. (Courtesy of General Biological Supply House.) 
hydranth to form a bowllike ease or hydrotheca which supports it. Another modification is the taller, more enclosed case, gonotheca, which nearly encloses the blastostyle. The blastostyle with this covering is often called the gonangium. Fibrous processes connect the perisare to the soft, inner parts (coenosarc). The cavity of the hydranth is continuous with that of the hydrocaulus, and is, therefore, a part of the gastrovascular cavity.

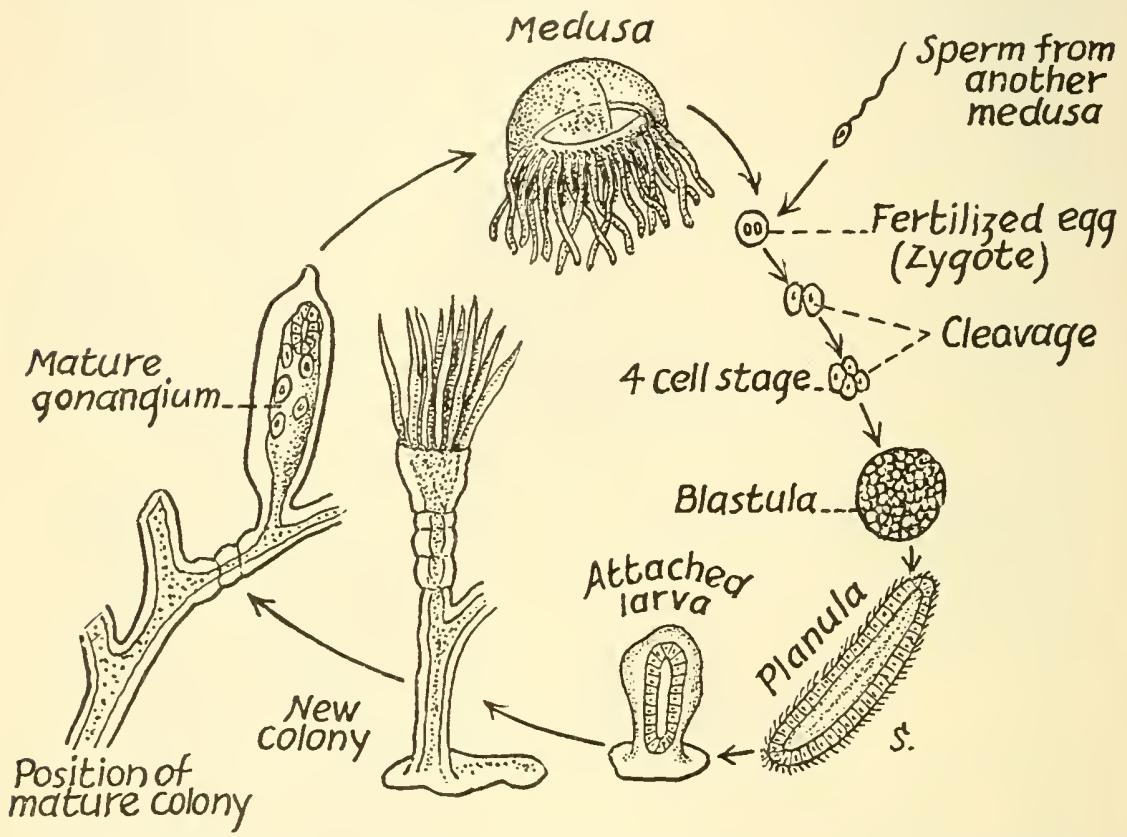

Flg. 59.-Life cycle of Obelia, illustrating polymorphism and metagenesis, Adult hydroid colony with mature gonangium gives rise to sexual medusa which is produced in the gonangium and set free in the water. Germ cells produced by the medusae complete the cycle. Blastula and planula are free-swimming. (Redrawn and modified from Wolcott, Animal Biology, published by McGraw-Hill Book Company, Inc.)

The coenosare is made up of an outer layer of cells, the ectoderm, just beneath the perisare, and an inner endoderm layer lining the cavity. The mouth of the hydranth is located in a domelike hypostome at the free end. There are between twenty and thirty solid tentacles attached around the basal margin of the hypostome. The hydranth captures and ingests small aquatic organisms as food by the aid of stinging bodies (nematocysts) produced in certain ectoderm cells of the distal portions of the tentacles. The digestion of this food is accomplished 
in the internal cavity. With the exception of reproductive processes, a single hydranth of Obelia will be found similar to an entire hydra, to be studied soon.

The reproductive cycle is both sexual and asexual, alternating between the sexually produced polyp or hydroid generation and the asexually produced sexual generation, the medusa or jellyfish form. The medusae arise as buds from the special individuals, blastostyles, escape through the distal pores, and develop to sexual maturity as free-swimming individuals. The sexes of these are separate; some produce eggs, and others, spcrmatozoa, which are discharged into the water at maturity and unite to form zygotes.

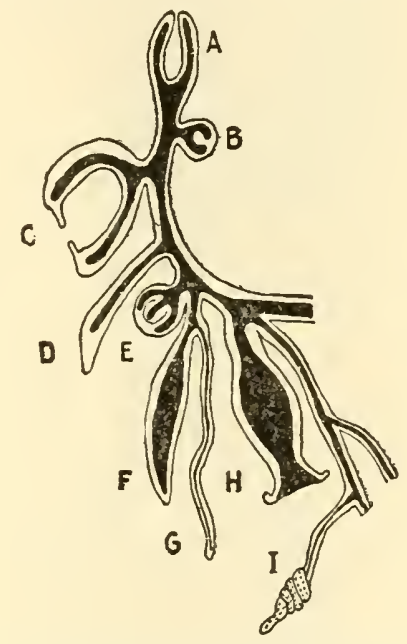

Fig. 60.-Diagram of a siphonophore colony (Physophorida). A. Pneumatophore; $B, C$, swimming bells; $D$, protective zooid; $E$, sporosac; $F$. $G$, dactylozooids: $H$, feeding polyps (gastrozooids); I, nettling cells. (From 'Van Cileave, Invertebrate Zoology, published by McGraw-Hill Book Company, after Claus.)

The zygote develops into the free-swimming, ciliated planula stage which soon attaches and develops into a polyp from which a new colony arises. After producing a generation of medusae, this colony disintegrates, and after producing germ cells, the medusae die. This process, involving alternation of generation, is described as metagenesis in Chapter VIII.

Obelia presents a very good example of metagenesis as represented in animals. The medusae of this sort are spoken of as hydromedusae to distinguish them from the seyphomedusae or jellyfishes of Class Scyphozoa. 
Order Trachylina.-This order includes two suborders of hydromedusae which come from the egg directly with no polyp stage. Campanella and Liriope are generic examples.

Order Hydrocorallina.-This group resembles the corals by producing strong calcareous skeletons. They have extensive, branched hydrorhiza and powerful nematocysts (stinging bodies). Rudimentary medusalike bodies develop on the coenosarcal canals. Millepora, the staghom or stinging coral, as it is called, is a good example.

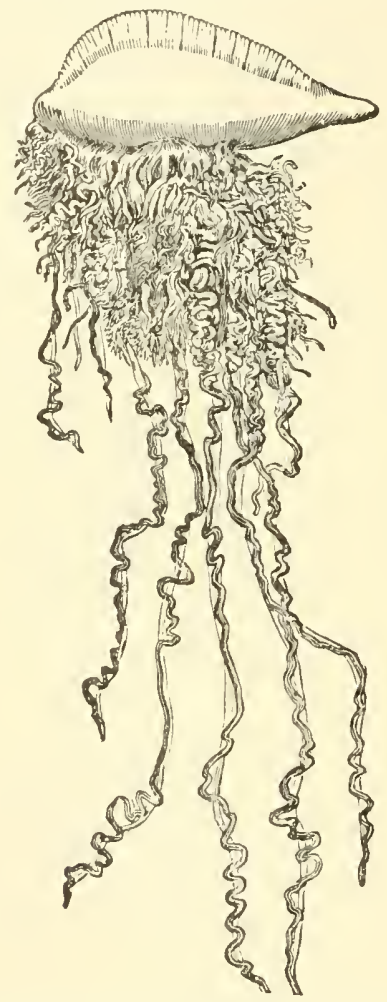

Fig. 61.-Physalia, the Portuguese man-of-war a floating colonial coelenterate. (From Hegner, College Zoology, published by The Macmillan Company.)

Order Siphonophora.-This is a pelagic order of colonial coelenterates with extreme polymorphism. A common tube of the coenosare unites the five kinds of individuals of the colony, and this eavity is continuous from one individual to another. The blind end of the coenosarcal tube is an air-filled, bladderlike float (pneumatophore) 
with a superior crest. The polyps lhang down into the water beneath this float. The types of polyps include: gastrozooids (nutritive or feeding), dactylozooids with nests of nematocysts and having long tentacles (tactile and protective), gonozooids which are male, reproductive zooids, and other's which produce ova-bearing medusae. Swimming bells (nectocalyees) often oceur just below the pneumatophore. Most of the individuals are specialized to such a degree that they care for only limited functions. This specialization and diversity of forms is such that the entire colony appears as a single individual. Physalia, the Portuguese man-of-war, is a typical example. Its sting is quite poisonous; bathers coming in contact with the trailing tentacles, which bear batteries of nematocysts, suffer severe pain.

Class Scyphozoa.-The coelenterates belonging here are large jellyfishes having an alternation of generation in which the medusa form is dominant. The seyphomedusa has an eight-notehed margin, lacks the vclum (therefore acraspedote), and has gonads connected with the endoderm. The polyps have four longitudinal endodermal folds, called taeniolae, which form gastral tentacles or filaments in the medusa. These jellyfish have a complex system of branched radial canals and abundant marginal tentacles as well as oral tentacles. Several of the representatives of this class are thought by some zoologists to exist generation after generation only as medusae, but it may be that tle polyp form has not been discovered yet, if it exists. There are records of individuals of this group twelve feet in diameter, and possessing tentacles one hundred feet in length.

Order Stauromedusae.-Conical or vase-shaped medusae which usually lack marginal sense bodies (tentaculocysts). The tentacles are distributed perradially and interradially. Lucernaria and Haliclystus are usually cited as examples.

Order Peromedusae.-These are cup-shaped, free-swimming forms with four interradial tentaculocysts. The tentacles are adradial and perradial. They occur in the open sea. Pericolpa and Periphylla.

Order Cubomedusac.-Forms which have rather cubical shape, four perradial tentaculocysts, interradial tentacles, and are chiefly tropical. Charybdea is an example.

Order Discomedusae.-Scyphozoa whose medusae are dominant, saucer-shaped, and almost transparent. Some of them are more than seven feet in diameter. There are usually eight or more tentaculocysts perradially and interradially distributed on the margin 
of the bell. Tentacles are usually present also on the margin of the bell. This is the most numerous and extensively distributed group of Scyphozoa. Aurcllia and Stomolophus are common examples.

Aurellia* is the typical example, and, like most jellyfishes, is composed largely of water. When they are dried, only a thin film remains. This is a common one and ranges from New England to the Gulf of Mexico. It may reach a foot in diameter.

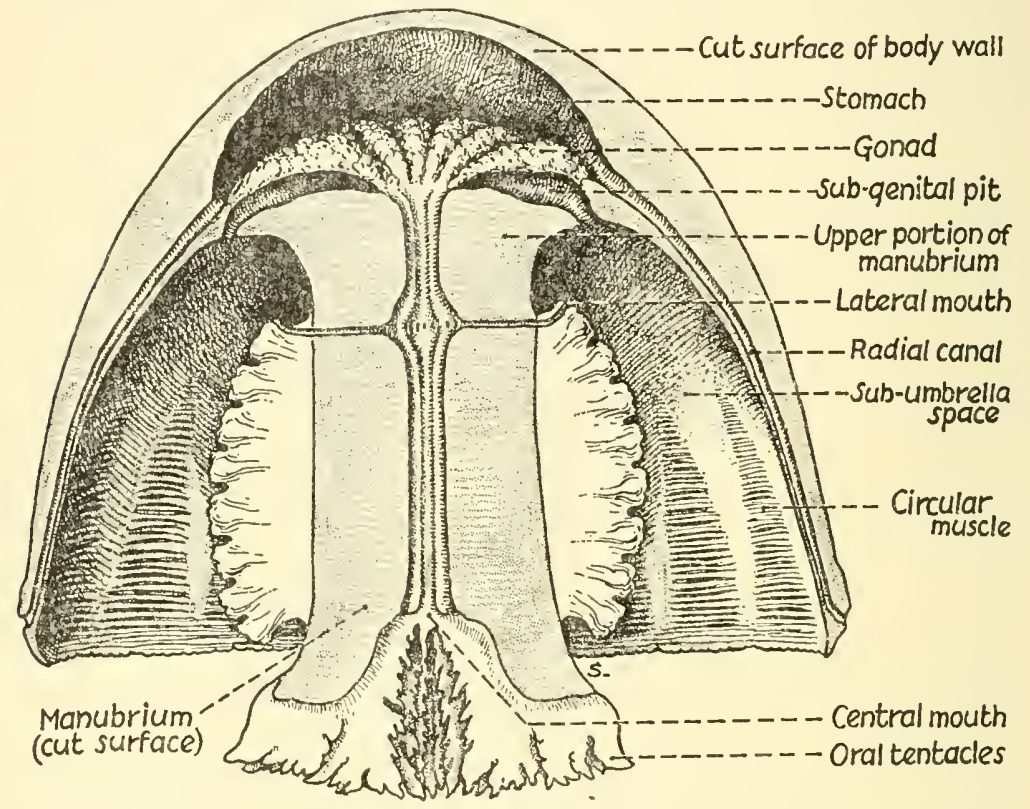

Fig. 62.-Cabbage-head jellyfish, Stomolophus meleagris, a very common form in the Gulf of Mexico. Bisected to show internal structure.

The animal has no velum as do the hydromedusae, but there is a square mouth on the subumbrellar side with wing-shaped, liplike oral lobes or arms. A subgenital pit lies in each quadrant of this side of the animal. The mouth leads through a short passageway into the angular gastrovascular cavity which in turn has four lateral gastric pouches containing the fringelike gonads. There is also a row of small gastric filaments here carrying nematocysts. A large number of branching radial canals extend from the gastrovascular cavity out to the margin of the bell, there joining a circumferential

\footnotetext{
- This spelling is according to Mayer's monograph. The generic name first proposed by Peronas Le Sueur was so spelled.
} 


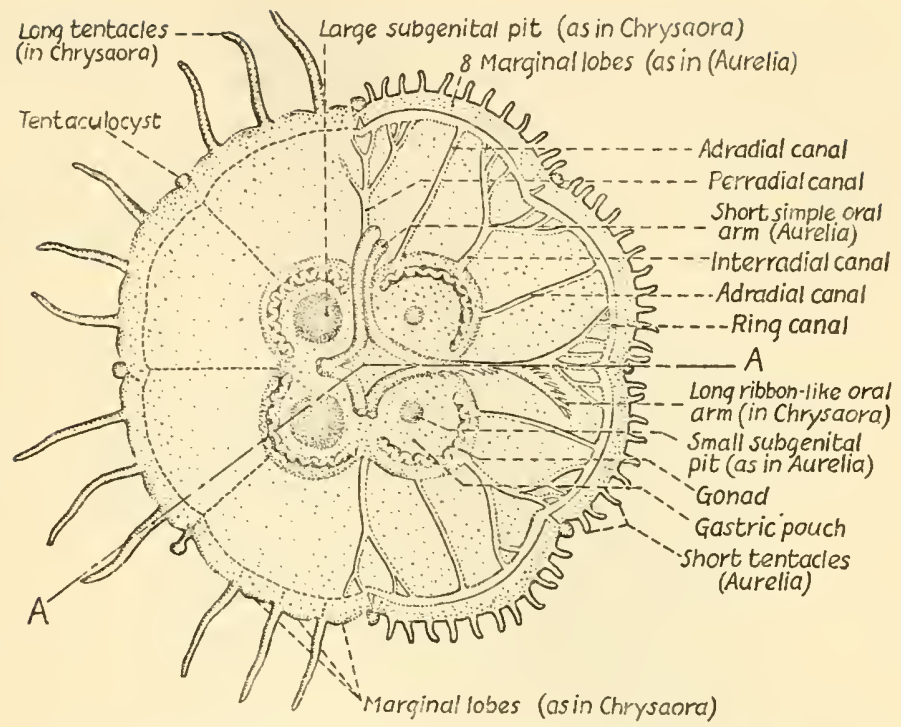

Fig. 63.-Aurellia and comparative structure of jellyfishes. (Modifled from figure in Pacific Biological Laboratories' catalogue.)

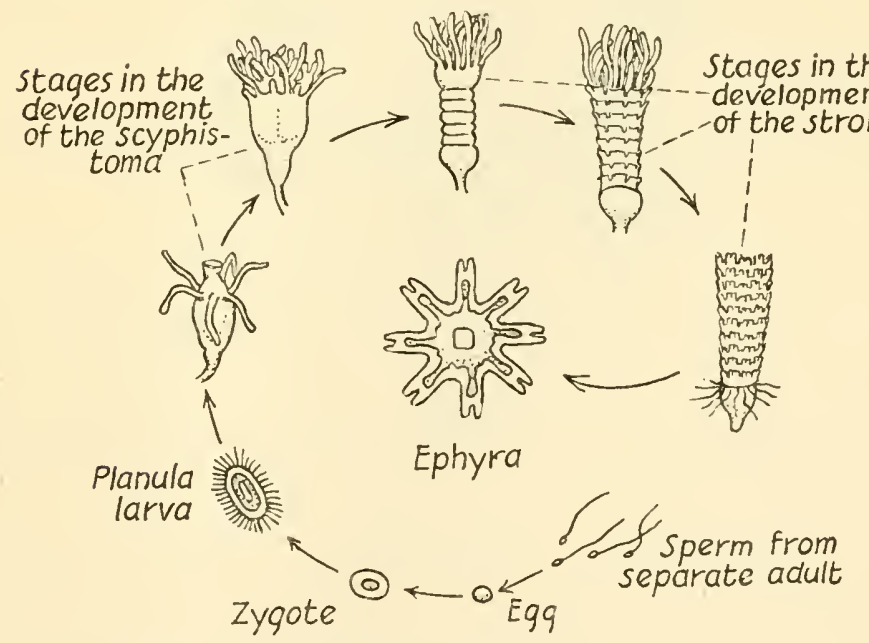

Fig. 64.-Life cycle of Aurellia aurita, showing stages from germ cells to the ephyra stage which precedes the adult condition. 
canal. The eight tentaculocysts are symmetrically located at eight points on the margin, each between marginal lappets. The tentaculocysts are sense organs of equilibrium. The pigment spot over each is likely sensitive to light. Near it is the olfactory pit.

Reproduction involves both sexual and asexual generations. Germ cells are produced by the pinkish gonads in the gastric pouches, and they pass out through the mouth with the water. Fertilization takes place, and the egg develops into a free-swimming planula which after attachment becomes a tubelike polyp that reproduces by budding most of the season. Then the polyps form medusae by strobilization, i.e., constrictions are formed around the body making it resemble a stack of saucers; the upper one periodically frees itself and swims away. The polyp with all of these constrictions is known as a strobila, and the new medusa is called an ephyra.

Class Anthozoa.-All animals in this class conform to polyp organization and may be colonial or solitary. They have an ectodermal esophagus and longitudinal partitions called septa (mesenteries) incompletely dividing the gastrovascular cavity. Muscular tissue bands are found in the septa. The mesogloea is quite abundant and contains a good many cells that resemble primitive connective tissue cells. Many of these animals produce a calcareous external skeleton called coral. Both sexual and asexual reproduction are common.

Subclass Zoantharia.-This group has numerous paired septa, typically occurring in multiples of six, and plain tubular tentacles. It includes sea anemones and corals.

Order Actinaria.-These anemones are usually solitary polyps; they have many complete septa and numerous tentacles but no skeleton. Sagartia, Cerianthus, and Metridium are common examples.

Metridium usually lives attached to rocks or to solid bodies in the water near shore, even in tide pools. They average about three or four inches in height and two or two and a half inches in diameter. The free end of the jar-shaped body is covered with tentacles which are provided with nematocysts. The entire body can be expanded and contracted, and it canl change its location by "scooching" on its basal disc (attacherd end). The mouth is located in the center of the crown, and food is forced into it and on through the gullet (stomodeum) by action of cilia on the tentacles and part of the lining of the mouth. At each side of the gullet is usually a 
ciliated groove, the siphonoglyphe, through which water is constantly carried into the gastrovascular cavity for respiratory purposes. The gastrovaseular cavity is divided into radially arranged compartments by the primary septa or mesenteries which extend from the wall of the gullet to the inside of the body wall. The primary septa in the axis of and extending parallel with the siphonoglyphes are called directives. At the basal end these cavities are continuous

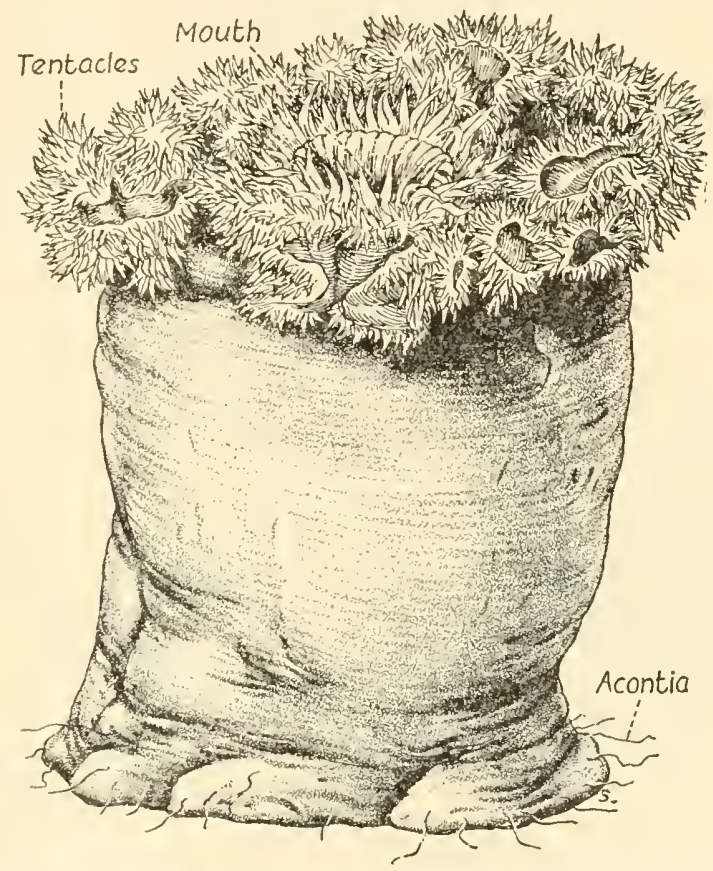

Fig. 65.-Sea anemone, Metridium marginatum, showing external features.

with the main central cavity. Between the primary septa are secondaries which do not quite reach the wall of the gullet, hence their medial ends ar'e free in the cavity. Between these and the primaries are some tertiary septa which are still shorter and also attached to the inner surface of the body wall. A quarternary set is represented by mere ridges on the inner surface of the wall and is interspersed among the others. There is a band of muscle running vertically on the face of each septum next to the muscle on the adjacent septa of the same rank. Below the gullet the mesentery has secretory filaments which in turn bear long, threadlike acontia. 
These protrude through pores (cinclides) in the body wall to the outside, and they are supplied with nematocysts and secretory cells. They serve as defensive as well as offensive structures.

Asexual reproduction by budding from the margin of the basal dise is practiced by this animal. Occasional longitudinal fission may occur. The gonads develop on the edges of the lower part of the septa to provide for sexual reproduction. The sexes are distinct.

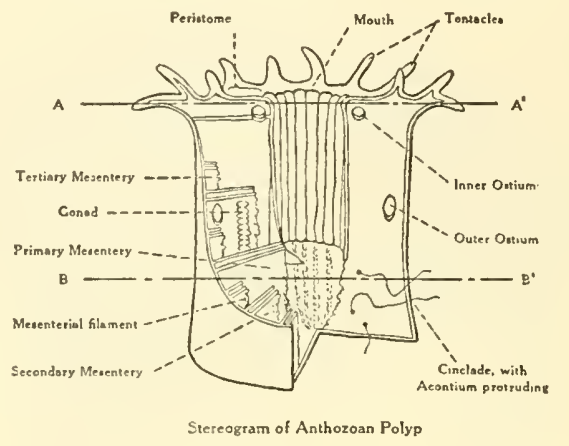

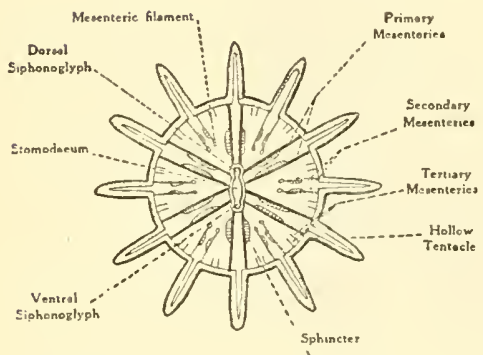

Diagrammatic T. S. of Anthozoan Polyp at level A-A

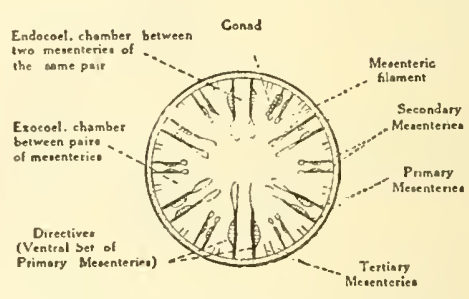

Diagrammatic T S. of Anthozoan Polyp at level B-B'

Fig. 66.-Diagrams to show the structure of the anthozoan, Metridium. (Courtesy of Pacific Biological Laboratories.)

Mature ova and spermia are discharged into the water of the eavity and escape through the mouth to unite in fertilization outside. The development includes cleavage and planula stages, before the new individual attaches and changes form.

Order Madreporaria.-The representatives of this order secrete an external limestone skeleton; most of them are colonial. The individuals of colonies communicate with each other by coenosarcal connections. Otherwise they are similar to anemones. Astrangia, Madrepora, and Oculina are examples. 
Astrangia is the common coral polyp, and it is quite similar to a small sea anemone to which calcium carbonate has been added by secretion from the ectoderm cells as well as having budded to form a colony of numerous individuals. Coral polyps vary in size from one-sixteenth of an inch to several inches in diameter. In time continually growing colonies of these animals can produce enormous stony barriers (reefs) in the sea. One such reef is over 1,100 miles in length and from ten to twenty-five fathoms deep. Many corals are of beautiful colors.

Order Antipathidea.-An order composed of branching colonies whose individuals are joined by a branched tubular axis which is covered by an epidermal layer. Cirripathes and Antipathes are typical examples.
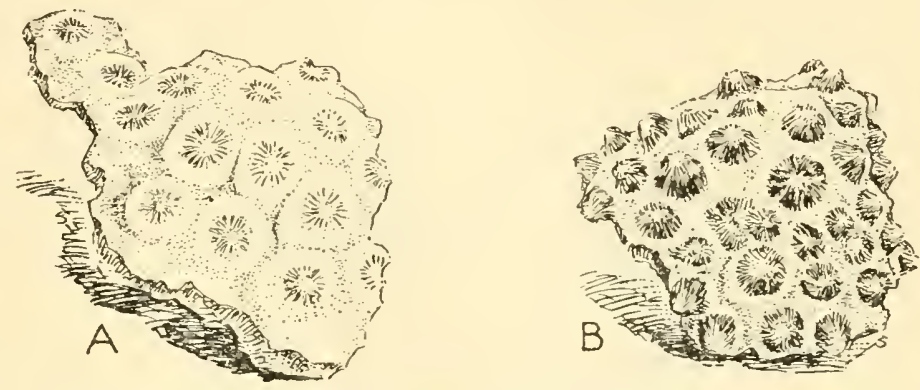

Fig. 67.-Common coral, Astrangia danae. A, Stone produced by the animals when cleaned; $B$, polyps in natural habitat.

Subclass Alcyonaria.-The features of this division include eight hollow, feathered tentacles, eight mesenteries, and one siphonoglyphe. Colonial and polymorphic forms are not uncommon.

Order Alcyonacea.-A colonial group which has calcareous spicules but lacks an axial rod. Body walls of individuals fuse together as one. Alcyonium is the type example. Organ pipe coral belongs in this order.

Order Gorgonacea.-This is another colonial coral which is sessile and has a calcareous axial rod. The colonies are bilaterally symmetrical. The common sea fan, Gorgonia, as well as the precious Corallium rubrum are well known examples.

Order Pennatulacea.-Another colonial form whose body is modified so that one portion is submerged in the substratum. The colony 
takes a bilateral form, and the individuals are born on a dise or axial stem which is supported by a liard skeleton. There may be dimorphism of zooids within the colony. Renilla and Pennatula, sea pens and sea feathers, are typical examples.

\section{HYDRA}

\section{Habitat and Behavior}

Hydra (Chlorohydra) viridissima is likely the most common hydra of the Southwest. It is the small green hydra which is very uctive and has short tentacles. This species has the green color because of the presence of a unicellular alga, Chlorella vulgaris, in the endoderm cells. The plant uses some of the by-products of metabolism of the hydra, and the hydra benefits by receiving oxygen from the photosynthesis of the alga. This kind of a relationship is called symbiosis.

Most of the hydras are found in cool fresh water, attached to the surface of plant leaves, smooth sticks, debris, or even the surface film of the water. The brown hydras, such as $H$. americana, $H$. carnea, and Pelmatohydra oligactis, are sluggish and have longer tentacles than the green ones.*

Hydra is a sedentary kind of animal and may remain stationary for a considerable period of time if living conditions are uniformly good. When the environmental conditions are changing, and the animal is in need of food, it becomes quite active, moving about from place to place. It keeps the tentacles extended, ready to grasp any food which may come into its reach. Nematocysts or sting bodies are discharged when the tentacle comes in contact with potential food, and if it chances to be a small animal, it will likely be paralyzed by the toxin which is injected by the nematocysts. The prey is then carried to the mouth and tucked into it by the tentacles. Frequently hydra is able to stretch its body over articles of food which are actually larger than the hydra usually is in normal condition. Hydra will eat only when it is hungry and will not react to food at other times. It is more sensible than many people in this respect. On the other hand, it has been authentically reported that a hungry hydra will perform the characteristic feed-

* Recent taxonomic information concerning Hydras of the United States may be found in the papers of Libbie H. Hyman, published in the Transactions of the American Microscopical Society, Vols. 48, 49, and 50 . 
ing movements when only beef extract is in solution in the water. Thus it responds to a chemical stimulus alone, but it will not respond to a mechanical stimulus only.

These animals show response to a number of environmental conditions. Any sudden change is likely to bring about a negative response. If the stimulus is of a general nature and of considerable
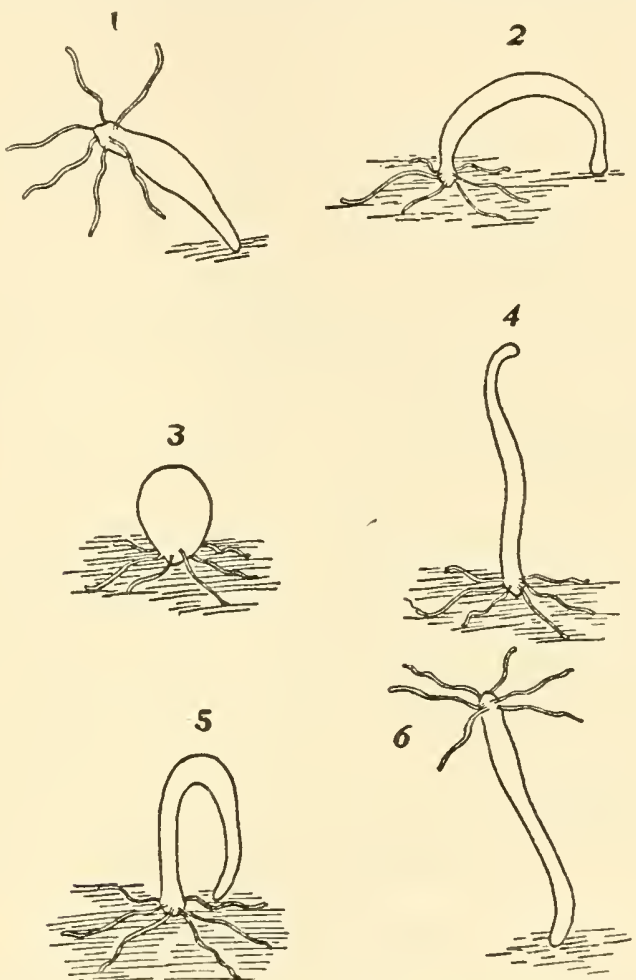

Fig. 68.-Locomotion in hydra. Successive positions taken when progressing by somersaults. (From Jennings, Behavior of the Lower Organisms, published by The Columbia University Press.)

intensity, the animal will contract all of the tentacles and the body also. If the stimulus is restricted to one locality and is not too strong, the animal will contract in the affected area, by the withdrawal of one tentacle. The movements of the animal are performed by contraction and relaxation of the contractile fibers connected with certain of the cells. The activities come in response to internal as well as external stimuli. 
The common tropisms, which have been described previously, are present in hydras. They respond to light and will find an optimum intensity which varies with the different species. Green hydras react positively to sunlight and withstand moderate temperature; hence they are adapted to the Southwest. They likewise possess an optimum for temperature and prefer relatively cool water. They seem not to become particularly uncomfortable until the temperature gets up to $31^{\circ} \mathrm{C}$; then they attempt to find lower temperature. As the temperature is lowered on them, they simply become less and less active and finally cease to move as the freezing point is approached. As pointed out previonsly, both chemotropisms and thigmotropism are concerned in food-taking. Contact stimuli are of considerable significance in a sedentary animal like this. It remains attached in contact with some solid body most of the time. Sudden mechanical stimulation like stirring the water or jarring the attachment of the animal will cause it to contract vigorously.

Locomotion is accomplished in at least four ways. Gliding from one point to another by partially releasing the basal disc and slipping it to a new location is common. Or the animal may bend over and cling to the substratum by the tentacles, release the basal disc, then draw the body toward this point, where the basal dise is reattached. This process is consecutively repeated and is called "looping." Occasionally the animal bends over, holds by the tentacles, then turns a "handspring" or "somersault" to attach the basal dise on the substratum beyond this point. The fourth means by which locomotion is effected is by dropping to the bottom, then secreting a bubble of gas at the basal disc and floating back to the top on that.

\section{External Anatomy}

Hydra is a macroscopic animal, but it is relativcly small. Its body is quite contractile, being able to extend from a contracted length of two or three millimeters to a length of eighteen or twenty millimeters. The column or body is a tubular, cylindrical trunk which ordinarily stands in a vertical position. In some forms the distal (free, oral, or anterior) end of the column is much stouter than the proximal (attached, aboral, or posterior) end, but in $H$. viridissima there is only a slight tapering toward the basal end. Attached around the free end of the column is a circlet of from four to seven fingerlike tentacles, which extends free in the water. Tentacles may 
stretch out to be slender threads five to seven centimeters in length. They are very useful either singly or as a group in capturing and delivering food to the mouth. The mouth is located in the center of the distal end of the column and is surrounded by the tentacles. This conical elevation between the bases of the tentacles in which the mouth is located is called the hypostome. The mouth when closed and viewed from the top looks something like an asterisk.

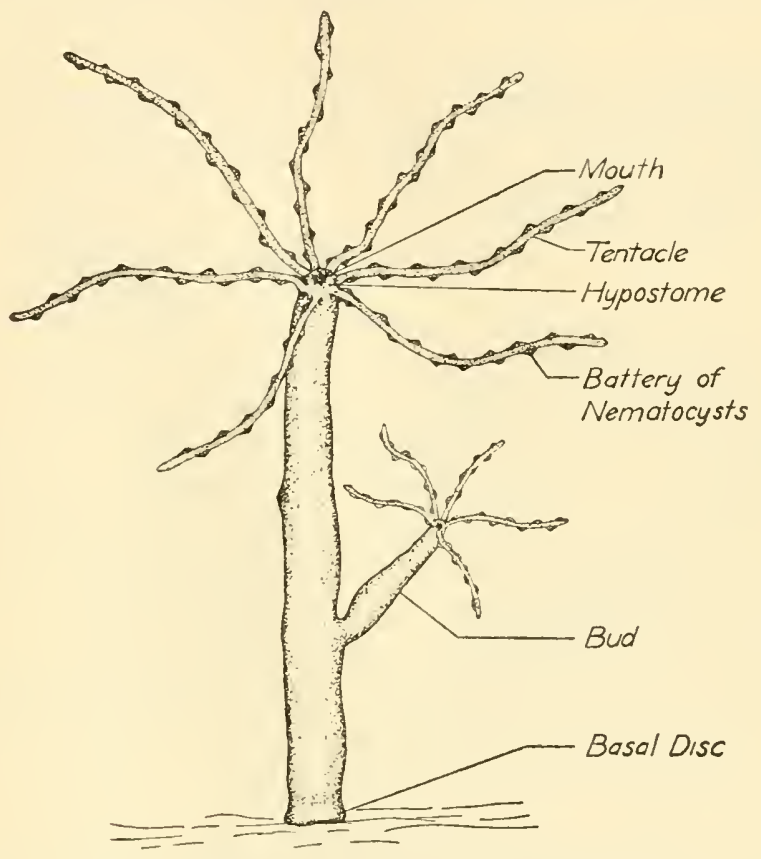

Fig. 69.-Hydra showing external features.

From the side it appears simply as an indentation or notch in the conical end of the hypostome. The proximal or attached end terminates in a basal disc or foot, which secretes an adhesive substance which helps the animal in attaching to objects. From one to several buds are often found on the sides of the trunk, and these occasionally bear buds before the first is separated from the original parent. Buds are lateral outgrowths of the column and are found when the animal has favorable living conditions. Budding usually occurs at about the middle of the body in $H$. viridissima. Occasionally there may be observed rounded projections on the side of the column which 
are seasonal reproductive organs. Both ovaries (female gonads) and testes (male gonads) may be formed on a single individual, but they are usually seen on separate individuals. If these projections are conical and located nearer the tentacles, they are testes or spermaries; if they are more nearly knoblike and are located nearer the base, they are ovaries. This animal possesses radial symmetry, but it is arranged with an axis of polarity from basal dise to hypostome, which is essentially equivalent to what is called the ventrodorsal axis of more advanced forms. All of the metazoans have a primary axis. Sedentary and sessile animals very commonly have radial symmetry, while the motile or free-living organisms tend toward bilateral symmetry.

\section{Internal Anatomy}

Another feature of the organization of this animal is the diploblastic structure which consists of two layers of cells or the germ layers surrounding an internal space, the gastrovascular cavity or enteron. These are studied on stained sections. The outer one is the ectoderm, which is thinner and is composed of four types of cells. The most numerous ones are typically cuboidal in shape and serve both as contractile units and as the general external surface of the body; they are appropriately called epitheliomuscular cells. Each of these cells consists of a polyhedral outer or epithelial portion and a basal portion which is drawn into one or two long, slender, fibrils which extend in a direction parallel to the length of the animal. These cells contract to shorten the length of the animal. Interspersed occasionally among these cells are the larger cnidoblasts in which develop the nematocysts, stinging cells or nettle cells. These are distributed over all the body except the basal disc, but they are much more numerous near the distal part of the column and on the tentacles. The nematocysts are usually contained in little raised tubercles in the ectoderm. Each tuberele contains a large barbed one and several of a smaller variety. Four different kinds have been described. Since the large barbed type is the most conspicuous, it will be described here. In the cnidoblast the nematocyst appears as a sac of fluid within which is inverted a barbed stalk with a coiled thread attached. Projecting out of the superficial surface of the cnidoblast is a triggerlike process called the cnidocil, which when chemically stimulated causes the cnidoblast to discharge the nemato- 
cyst. Chemicals, such as weak iodine, acetic acid, or methyl green, when added to the water, will bring this about. Contact will not. In this reaction the stalk and thread are everted, probably by development of pressure. This type of nematocyst produces a hypnotoxin which anesthetizes the animals into which it is discharged. In another form the sac is small, the stalk is barbless, and the thread is elastic; it becomes coiled around the object against which it is discharged, and thus impedes locomotion of the victim.

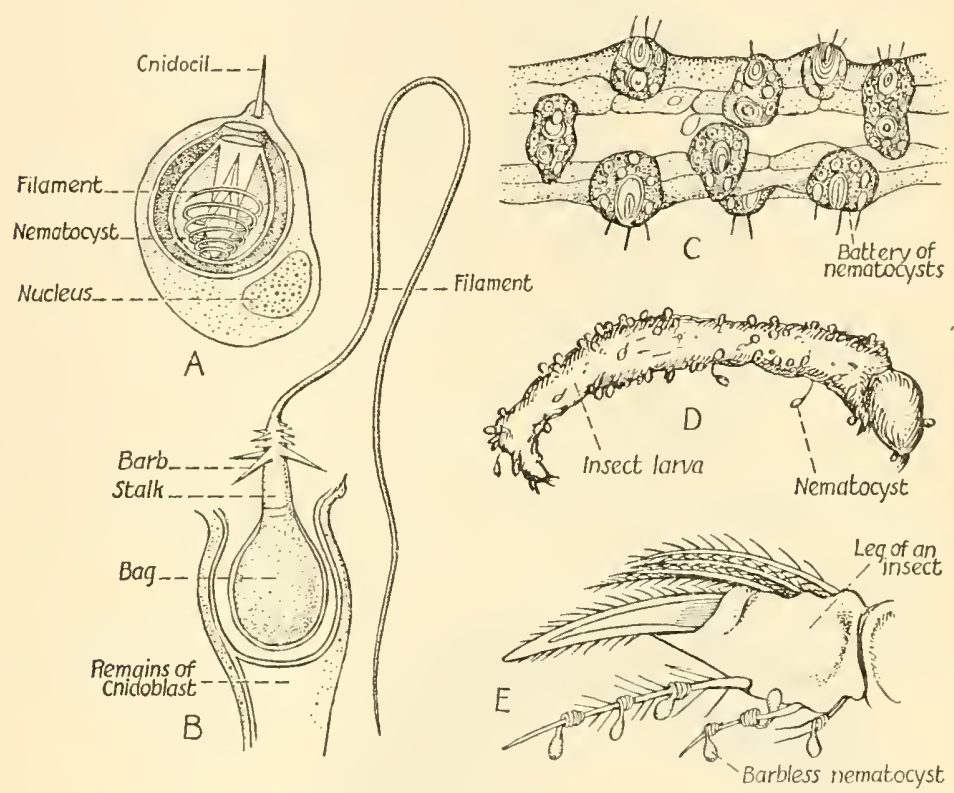

Fig. 70.-Nematocysts and their function. A, Cnidoblast containing an undischarged nematocyst, after Schneider: $B$, nematocyst everted and extended but still held in the cnidoblast, after Schneider; $C$, portion of tentacle, after Jennings : $D$ insect larva attacked by hydra, after Jennings: $E$, leg of small aquatic insect with barbless nematocysts on its spines, after Toppe. (Redrawn and modified from Wolcott, Animal Biology, McGraw-Hill Book Company, Inc.)

The enidoblasts are produced by a third type of cell, the interstitial cell, which is small and rounded. These are formative cells about the size of the muclei of the epitheliomuscular cells and quite densely granular in nature. They crowd in between the other cells, especially near their bases. As a nematocyst is discharged, the entire cnidoblast is replaced by an interstitial cell migrating into position. A damaged or spent cell of the body may be replaced from the interstitial cell. Besides these three types, there are the scattered, ir- 


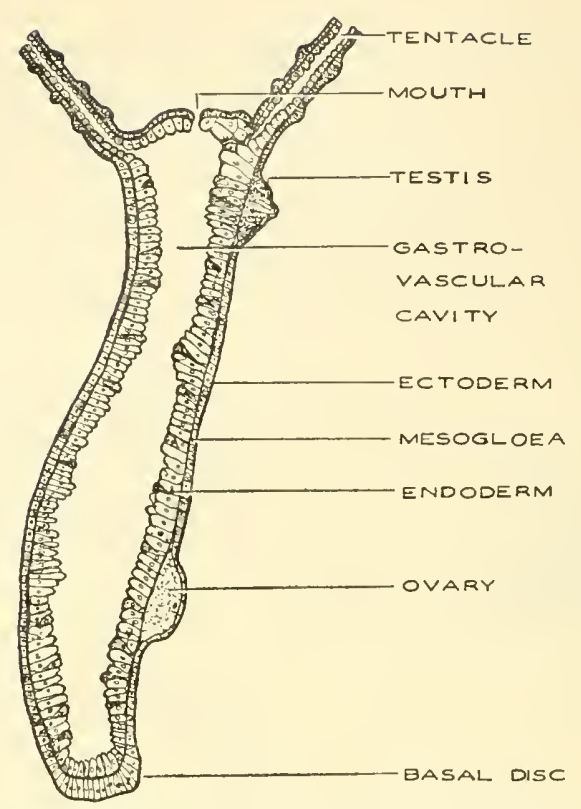

Fig. 71.-Diagrammatic longitudinal section of hydra, showing mature gonads and typical cell layers. (Drawn by Titus C. Evans.)

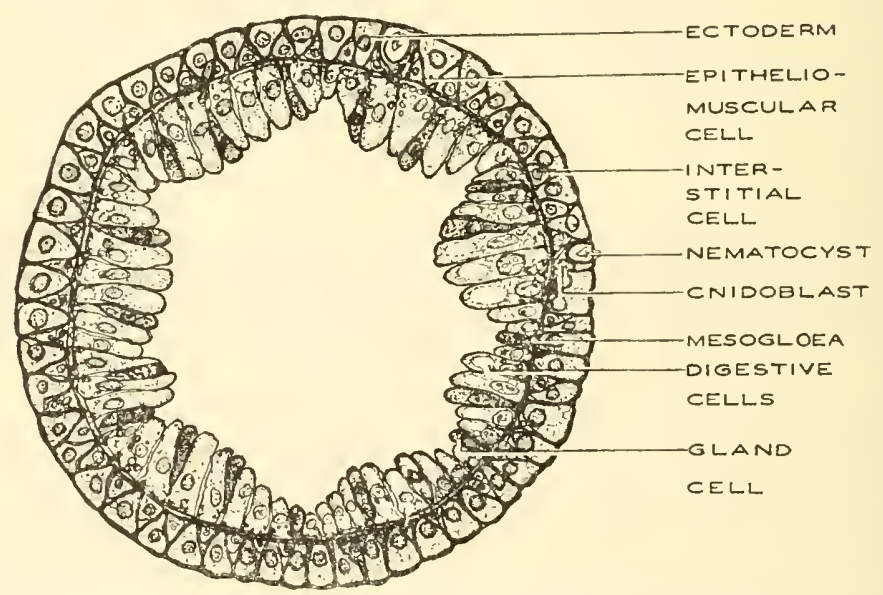

Fig. 72.-Cross-section through the column of hydra. The central space is the gastrovascular cavity or enteron. (Drawn by Titus C. Evans.) 
regular, slender, neuroepithelial cells which are joined into a net by intercellular processes. These cells fit between the others and are either sensory or motor in function, thus receiving external stimuli and also causing contraction of the contractile cells at proper times.

Beneath the ectoderm and embedding the bases of the cells is a very thin layer of noncellular substance called mesogloea. It is produced by the cell layers and serves as attachment for them, particularly for the fibrils of the epitheliomuscular cells. In some of the other coelenterates, this layer is exceedingly thick and heavy.

The inner, thicker cell layer of the wall is the endoderm which lines the lumen of the gastrovascular cavity. The most conspicuous cells here are the nutritive-muscular cells which are long, vacuolated structures attached to the mesogloea by fibrils which extend in it parallel to the circumference of the animal. By contraction these cells increase the length of the animal by reducing its circumference. These cells often possess flagella at the free margin and at times engulf particles of partially digested food like an amoeba. It is seen then, that they serve both as muscles and as digestive cells. Glandular cells are also present in this layer. Being slender, they wedge themselves between the nutritive-muscular cells and secrete what is probably a digestive fluid into the gastrovascular cavity. Neuroepithelial and interstitial cells are also interspersed amoug the other cells of this layer. The general morphology of the adult animal is very similar to the gastrula stage of the developing embryo of more complex metazoans.

\section{Metabolism}

The food of hydra consists of small insect larvae, minute worms, small bits of organic matter in the water, water fleas, and other small crustacea. Ingestion of the food has been described already. Upon entering the mouth the morsel of food is moved some distance down in the cavity by successive wavelike contractions of the column progressing from distal to proximal. Such serial contractions are usually called peristaltic contractions. Here in the upper half of the enteron digestion takes place. The wall possesses many more of the gland cells in the endoderm, and the food material disintegrates into smaller particles here in this region. The digestion which occurs here is spoken of as intercellular digestion and is brought about by enzymes produced by the secreting cells of the endoderm. The dis- 
solution of the food by the enzymes is augmented by the churning effect of the contractions of the body. The flagella present on the nutritive-muscular cells create currents of water which also hurry the process. The dissolved material is presumably absorbed by the cells of the endoderm, and by diffusion the nutrient solution reaches the ectoderm cells just outside. Small particles of the partially digested substance are engulfed by the free ends of many of the nutritive-muscular cells by virtue of their amoeboid activity. These particles are taken in food vacuoles, and the digestion is completed there just as it is in an amoeba or paramecium. This illustrates something of the primitive organization of hydra as a metazoan.

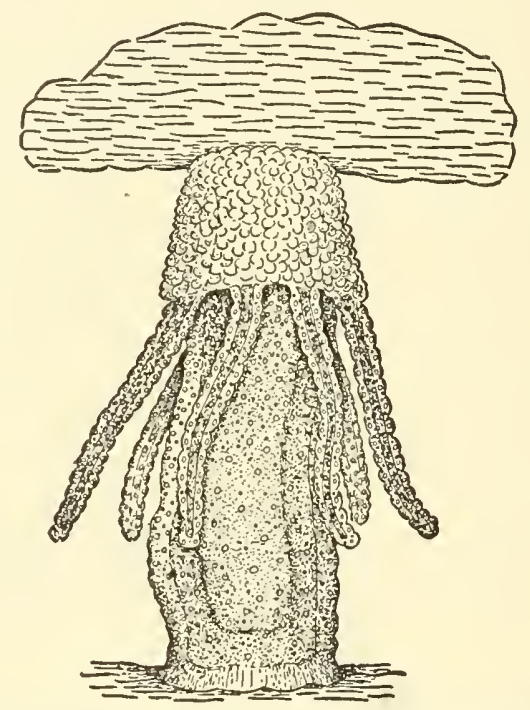

Fig. 73.- Hydra with body turned inside out in attempting to ingest a piece of meat. (From Curtis and Guthrie, Textbook of General Zoology, published by John Wiley and Sons, Inc.)

As will be remembered, this process of converting the digested food into an integral part of the protoplasm is known as assimilation. The food is distributed to all parts of the enteron, which extends into the tentacles and buds, by the action of the flagella and by bodily contractions. There is no separate system of transportation or circulation of nutriment. This dissolved material reaches the remote parts of the protoplasm by diffusion through the membranes and protoplasm generally. The gastrovascular cavity has the dual function of digestion and circulation. 
Many of the animals used as food have hard skeletal parts that will not digest. These indigestible portions are ejected from the cavity through the mouth by reverse peristalsis, and the process is known as egestion. Respiration furnishes the necessary exchange of oxygen and earbon dioxide by diffusion through the plasma membranes. The dissolved oxygen in the water in which the animal lives is the source of this element.

Catabolism or dissimilation takes place in the protoplasm and involves the union of oxygen with the substance of the protoplasm to transform potential energy there to kinetic energy and heat. Accompanying this oxidation there are prodnced some waste byproducts in solution including urea, uric acid, and water which must be expelled from the body. In hydra this excretion is accomplished by diffusion through the general surface of the body. There is some indication that there may be accumulation of waste products in endoderm cells as cytoplasmic granules. which finallv escape through the gastrovascular cavity and mouth. It will be noticed that these phases of metabolism are, in general, very similar to the comparable processes in Protozoa and the same similarity will be noticed when they are compared later with the higher forms of animals, because the protoplasmic requirements are the same in all animals.

\section{The Nervous System and Nervous Conduction}

The neuroepithelial cells are distributed among the other cells of the germ layers. There is a greater abundance of them on the hypostome, basal dise, and tentacles than along the length of the column. The greatest concentration of these eells is in the hypostome around the month, which makes this region in a sense comparable to a primitive brain. These cells all over the body are in contact with each other by means of their processes forming what is called a nerve net. When one sensory cell is stimulated, all of the sensory cells seem to be stimulated in some degree. A sufficiently strong stimulus affecting any sensitive point will stimulate the entire body. This is a definite organized type of nervous system but not a very efficient one.

\section{Reproduction and Life Cycle}

Reproduction is both asexual and sexual. Asexual reproduction is accomplished very efficiently and quite rapidly. This process is essentially reproduction by somatic cell division. Nutrient mate- 
rial accumulates at some point near the middle of the column. The bud first appears as a slight superficial bulge. The cell division at this point is very rapid, involving considerable activity in interstitial cells. This enlargement rapidly increases in size to form a stalk. An extension of the enteron extends into the bud, which is essentially an outgrowth of the body wall. Tentacles appear as outpushings of ectoderm and endoderm, and in the terminal position a mouth is developed. After the bud has attained some size, a constriction occurs between it and the parent. This closes the enteron between bud and parent, and the bud finally separates to become a free individual.
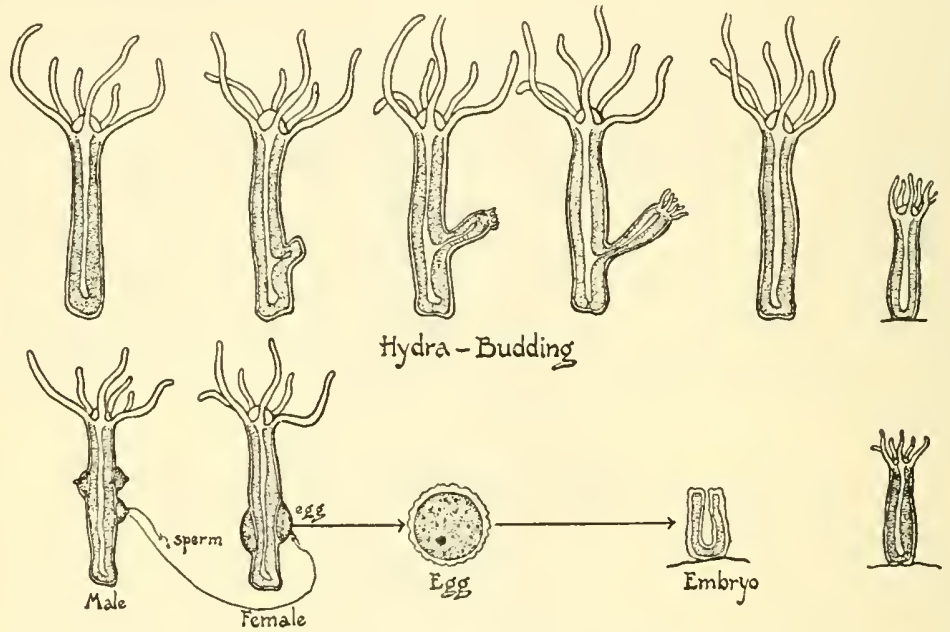

Hydra- Sexual Reproduction

Fig. 74.-Methods of reproduction in hydra. (Courtesy General Biological Supply

Sexual Reproduction.-During the summer and fall particularly, hydra reproduces sexually. This involves the production, maturation, and union of germ cells. Testes may appear first and ovaries later on the same individual or both gonads may be present at the same time in which case self-fertilization is possible. As a rule, these animals are hermaphroditic or monoecious as suggested before, but it has been reported that individuals of separate sex (dioecious) have been found. The germ cells or gametes develop from interstitial cells which accumulate at a certain place between the ectoderm and endoderm, where they multiply by division to form oögonia in 
the female gonad and spermatogonia in the male. All phases of maturation (gametogenesis) may be observed in the testis and ovary. The testis produces large numbers of motile spermatozoa, which when mature emerge periodically from an opening in the tip of the testis and are discharged free in the water. In the ovary a single egg develops at the expense of the other oögonia, which are engulfed bodily and used for food. This one cell grows rapidly, and when

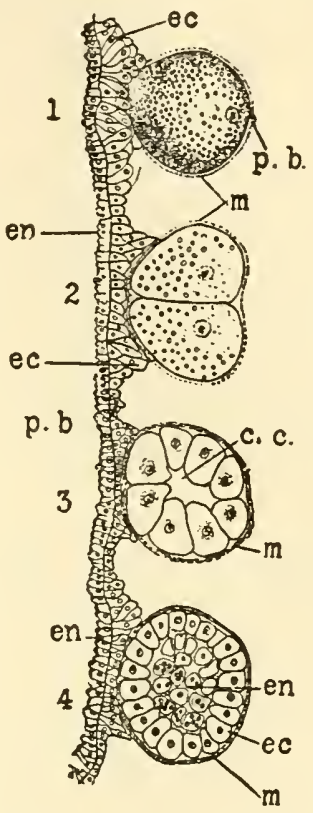

Fig. 75.-Development of hydra. 1, Fertilized ovum; 2, two-cell stage; 3 , blastula stage; 4 gastrula, showing ectoderm $(e c)$ and endoderm $(e n) ; c c$, cleavage cavity (blastocoele); $m$, cyst; $p . b$. , polar bodies. (After Tanreuther, Biological Bulletin, Vol. 14.)

mature it fills the ovary. Fertilization is accomplished by the entrance of spermatozoa through a rupture in the overlying ectoderm and cross-fertilization usually prevails. A single sperm unites with the mature ovum, and this zygote undergoes the total and equal divisions of cleavage here in place. The process continues until a hollow blastula of many cells is formed. Then follows the formation of the gastrula by a shedding of. cells into the cavity (blastocoele) from the inside of the original layer of cells. These new cells on the inside become organized as an endoderm layer, while the original 
outer layer is now known as ectoderm. Further changes involve the secretion of the thin mesogloea which seals the two layers together. In the meanwhile a shell is produced abont the outer surface of the embryo, and this encysted body falls from the parent to the bottom. If conditions are favorable for development, it increases in length within the cyst; when it has attained some size it breaks out, after which tentacles and a mouth appear at one end, while the enteron develops within the endoderm. This individual steadily grows and soon attains adult condition. When the zygote is formed in the fall, the embryo does not emerge from the cyst until spring.

\section{Regeneration}

As is the case in many invertebrate and a few vertebrate animals, Hydra is able to replace mutilated parts or an entire animal from a portion of one. Complete animals may be formed from very small pices ( $1 / 6 \mathrm{~mm}$. in diameter) of a hyclra. This process is known as regeneration, and while it is not normally a method of reproduction or multiplication, it is of great advantage to the animal. This phenomenon was first discovered in animals from studies on Hydra in 1744 by Trembly.

\section{Economic Relations of the Phylum}

The entire group is not worth much in dollars and cents to man directly. A number of different ones are used as food by some of the useful fish. The corals are of importance both positively and negatively. Many of them are valuable as ornaments, while the large coral reefs are very costly to navigation of marine waters. Many corals are quarried for builching stone, and in some instances they protect the shore from being washed by the waves.

\section{Phylogenetic Advances of Coelenterates}

(1) Definite organization of diploblastic condition; (2) welldefined gastrovascular cavity with one opening, the mouth; (3) presence of tentacles with (4) nematocysts or sting-bodies; (5) continuance of sexulal reproduction; (6) distinct radial symmetry and, (7) a nerve net. 


\section{CHAPTER XI}

\section{PHYLUM CTENOPHORA}

This is a group of exclusively marine animals, most of which are pelagic (float near the surface). There is a limited number that lives and moves about on the bottom. Ctenophora (tē nŏf'ó rácomb-bearing), because of their similarity to coelenterates, are often classified as a class in this phylum. There are only twenty-one American species representing this phylum, and they are commonly called sea walnuts or comb jellies. Most of them swim by means of eight rows of fused cilia, called swimming plates or combs. These animals are quite clear and transparent, with a faint tint of pink, purple, or blue. They are often phosphorescent. There are two classes in the phylum: (1) Tentaculata, with a pair of tentacles present either in the larva stage or throughout life. Mnemiopsis leidyi is a luminescent, transparent form; Pleurobrachia bachei has long tentacles on a relatively short, oral-shaped body; and Cestus veneris, Venus's girdle, niay be four feet long and only two inches in width, bandlike, transparent, with an iridescence showing violet, blue, and green colors. (2) $N u d a$, with no tentacles at any stage; Beroë ovata, about 10 to $12 \mathrm{~cm}$. in length, conical in shape, and rather common, is an example.

\section{Habitat and Behavior}

These are primarily surface-living forms with rather wide distribution but most abundant in tropical seas. They move about very slowly through the water with the oral end forward and the two long tentacles trailing if tentacles are present. The tentacles have adhesive or glue cells (colloblasts) which produce a secretion, and with these they capture any small organisms making contact with them.

\section{Anatomy}

The size of different individuals of this group ranges from five millimeters to four feet in length, and the shape may be spherical, pear-shaped, ribbonlike or eylindrical. The symmetry is said to be biradial since there are eight rows of radially arranged paddles or plates which are equally distributed on each side of the median 
line. These paddles are the locomotor organs. When seen from the side, the paddles resemble a comb. The mouth is in the oral end of the body and leads into the stomachlike stomodeum which is connected with a series of canals running through the body. This stomodeum is lined with ectoderm and leads to an infundibulum or gastrovascular cavity proper which joins the stomodeum at right

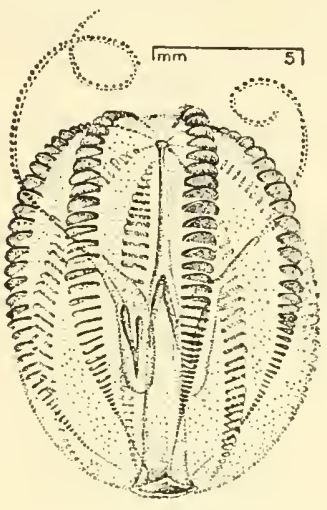

Pleurobrachia bachei

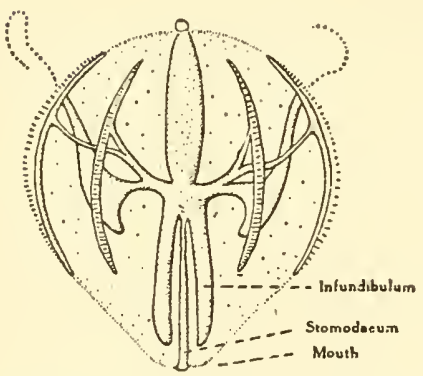

Perspective Drawing along inf undibulas plane

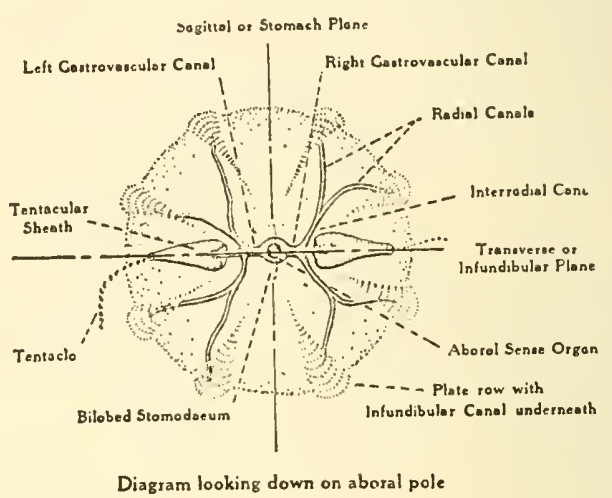

Diagram looking down on aboral pole

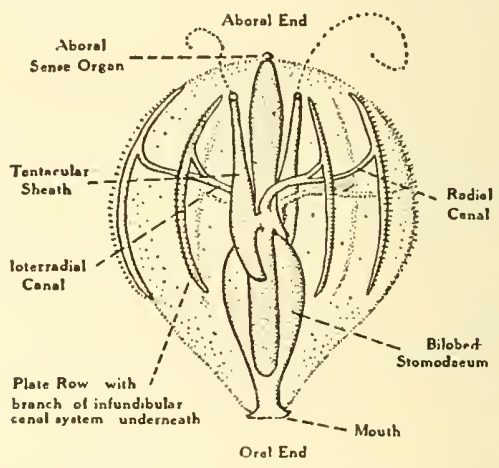

Perspective Drawing along sagittal or stomodaeum plane

Fig. 76.-Pacific comb jelly, showing external features and structure. (Courtesy of Pacific Blological Laboratories.)

angles. This cavity is lined with endoderm; undigested food is egested through the mouth. The six canals mentioned above are called excretory canals. There are two blind canals extending from the infundibulum beside and parallel to the stomodeum; these are called paragastric canals. The tentacular canals lead to the meridional canals lying beneath the ciliated plates. There are two blind 
tentacular pouches connected with the outside; one lies on each side of the infundibulum. The solid prehensile tentacles emerge from these sacs.

Around the aboral surface of the body is a collection of sense structures or statocysts, which serve as organs of equilibrium by stimulating the cilia of the bands on the side against which the internal calcareous ball (statolith) rolls as the level of the body changes. These animals are monoecious (hermaphroditic); the ova are formed in ovaries along one side of each meridional canal and spermatozoa

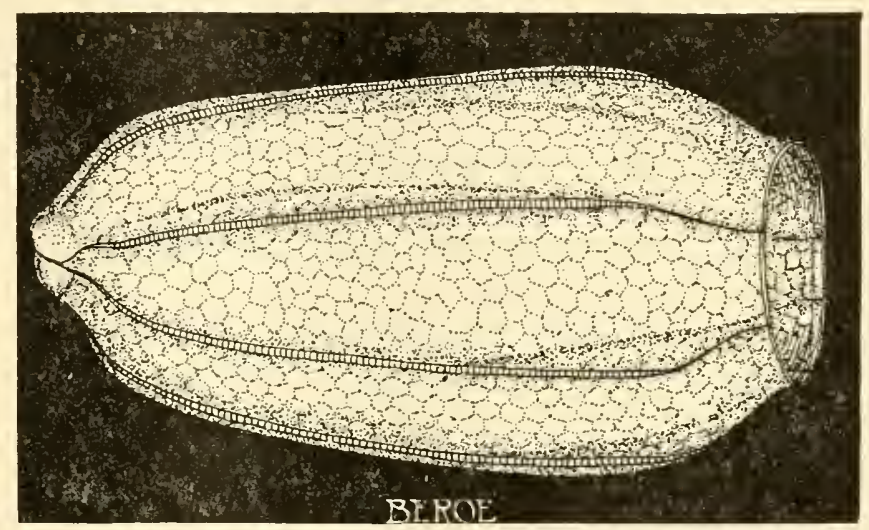

Fig. 77.- Sea walnut or comb jelly, Beroë ovata, from Atlantic Ocean and Gulf of Mexico. (Courtesy of General Blological Supply House.)

along the other. The mature germ cells rupture into the infundibulum and pass through the stomodeum to the exterior. The fertilized ovum develops and finally metamorphoses to the adult stage. There is no alternation of generation. The animals are triploblastic instead of diploblastic as are Hydras, because, instead of a simple mesogloea, there are, in addition, differentiated muscle fibers lying between the ectoderm and endoderm. This is a morphological advance when compared to the coelenterates. These animals have no nematocysts and therefore do not irritate bathers as do jellyfish, but they do serve as food for a large number of valuable fish. Otherwise they have no economic importance. 


\section{CHAPTER XII}

\section{PHYLUM PLATYHELMINTHES}

The representatives of Phylum Platyhelminthes (plăt $\check{i}$ hĕl minn' thēz, broad worm) are usually called flatworms and in many ways show considerable advance over the coelenterates. Some of the species are parasitic, and the remainder of them are free-living. The common fresh-water planaria is an example of the free-living type; while the parasitic flatworms are known as fukes or trematodes and tapeworms or cestodes. All of these worms are bilaterally symmetrical and triploblastic. The nervous system in the free-living forms is of the "ladder-type," and centralization is developed. They possess a fairly well-differentiated mesoderm, and along with it have developed some systems of organs. The alimentary cavity functions as a gastrovascular cavity and has only one opening to the exterior. The excretory system is composed of a pair of longitudinal tubules, branch tubules, and "flame cells." The gonads are within the body and are connected with the exterior by accessory organs. There are definite muscle cells, and excretory and reproductive systems composed of the new mesoderm layer.

The representatives of the two parasitic classes have, for the most part, quite complex life histories and special adaptations. They are very important economically because of their injury to man and the domesticated animals.

\section{Classification}

There are four recognized classes in the group.

Class Turbellaria (tûr bě lā'rĭ à--little stirring). - This class consists of a gromp of soft-bodied, elongate and usually free-living forms. The surface layer or epidermis is ciliated in patches, and there is a plentiful supply of secreting cells in this layer. The digestive tube is single, three-branched, or many-branched. The mouth is located ventrally. There are both land and water forms. Four orders are known: Acoela, Rhabdocoelida, Tricladida, and Polycladida. Planaria and Stenostomum are examples. 
Class Trematoda (trè mả tō'dả-having pores).-These animals, commonly called flukes, have no epidermis but a thick nonciliated cuticle. The body is either leaf-shaped or elongate and has from one to many ventral suckers. This entire class is parasitic, and the immature stages frequently make use of snails and crabs as hosts for a phase of their life history. This group is divided into only two subclasses: Monogenea and Digenea with orders Gasterostomata and Prosostomata. Paragonimus, Clonorchis (Fig. 397), Fasciola (Figs. 398 and 399) are genera representing the class.

Class Cestoda (sěs tō'dá-girdle form). - This group is also characterized by a heavy cuticular cover, and a long, ribbonlike body divided into sections called proglottides.

These tapeworms each have a knoblike "head" or scolex on the anterior proglottid. This structure is supplied with suckers for attachment and sometimes has hooks. There is no alimentary tract, and the group is parasitic. A developmental stage of the life history is the bladder worm or cysticercus which lives embedded in the muscular tissue of several different animals. The class includes five orders: Pseudophyllidea, Cyclophyllidea, Tetraphyllidea, Trypanorhyncha, and Heterophyllidea. Taenia (Figs. 400, 401, and 402), Diphyllobothrium, IIymenolepis are examples.

Class Nemertina.-It seems difficult to know where to classify this group since some systematists give it the rank of phylum while others give it lower ranking. The Nemertinea (něm ẽr tǐn'ēảunerring) as individuals, are unsegmented "band worms." Nost of them are free living and marine. A long proboscis, the newly developed blood vascular system, the alimentary canal, two apertures, and cilia over the body are all characteristic of this type. There is present a mesoderm, nervous system, and excretory system, but there seems to be no coelom. The animals feed on the bodies of other animals and on certain types of general organic matter. They usually live in burrows in sand or mud or beneath solid objects. The larger ones reach a length of ninety feet. The animals are frequently brightly colored. There are numerous mucus-secreting glands in the skin which may produce a tubelike dwelling for the worm. The two muscular layers of the body are so effective that an extended worm of fifteen feet may contract to less than two feet in length. Locomotion is accomplished by the cilia and the contractility of the body. The proboscis is a very characteristic organ which is 
in the form of a hollow tube turned back through the body inside of a cavity called the proboscis sheath. By contracting the saclike sheath, the proboseis may be everted and extended from the anterior part of the body.

The sexes are ordinarily separate and each individual possesses gonads which are located laterally and between the intestinal

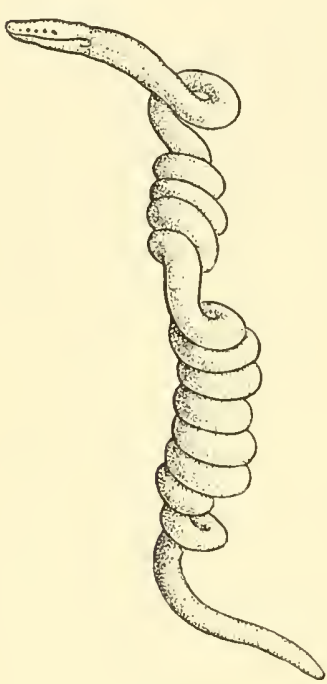

Fig. 78 .

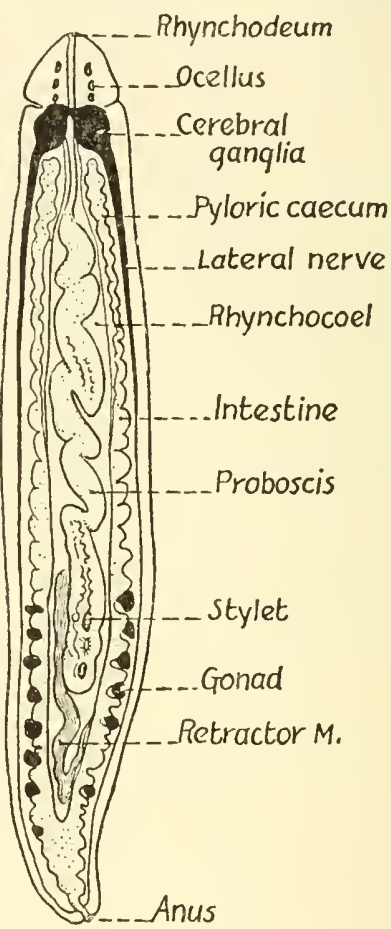

Fig. 79.

Fig. 78.-A nemertine worm, Lineus socialis, with the body coiled. Natural length about $15 \mathrm{~cm}$. (Redrawn and modified from Hegner, College Zoology, published by The Macmillan Company, after Coe.)

Fig. 79.-Structure of the nemertine worm, Prostoma rubrum, as it appears when flattened. (Redrawn and modified from Hegner, College Zoology, published by The Macmillan Company, after Coe.)

pouches. Both egg's and sperm are discharged from respective individuals through a dorsal pore and fertilization occurs in the surrounding water. Following cleavage there is a helmet-shaped larva called pilidium. Cilia develop on the lappets at the lower margins of the body and on a patch at the opposite pole or apical plate. This plate is the principal nerve center of the animal in this stage. The 
adult appears after metamorphosis. In some forms there is a creeping larva known as Desor's larva. The vascular system is composed of longitudinal vessels comnected by transverse loops. The vascular fluid is usually colorless. The excretory system includes the usual longitudinal tubules and flame cells characteristic of the phylum. Either one pore or several communicate with the exterior. The central nervous system consists of two ganglia and three longitudinal cords passing through the body. A pair of grooves with cilia along each side of the cephalic portion are sensory and are called cerebral organs. Other tactile organs and eyes are usually developed. Prostoma, Cerebratulus, Tetrastemma are representatives.

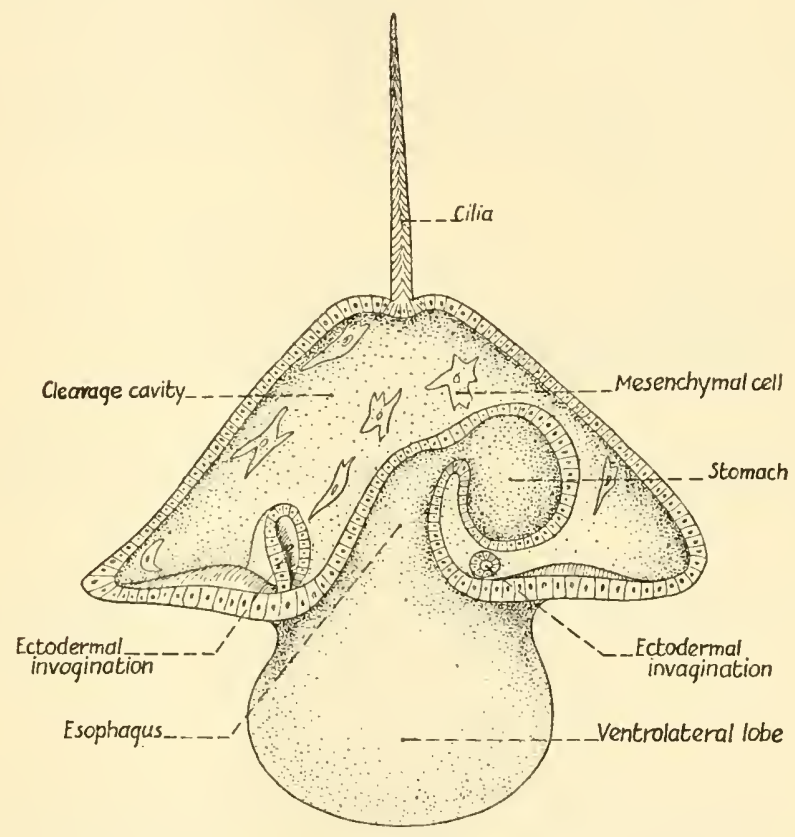

Fig. 80.- Structure of pilidium larva of the nemertine worm in partial section. (Redrawn and modified from Wolcott, Animal Biology, published by McGraw-Hili Book Company, Inc.)

\section{PLANARIA}

\section{Habitat and Behavior}

This free-living, fresh-water, flatworm thrives beneath the rocks, logs, leaves, algae, or debris at the bottom of shallow spring-fed brooks and pools. They must have pure, clear, cool water. These 
animals are rather gregarious and when at rest will group together beneath objects where the light is not intense. They respond negatively to bright light. They usually feed upon minute plants and animals, dead animal bodies, and living forms, such as small arthropods and molluses. Planaria partially encompasses the food with the body, while the pharynx is protruded to eat it. If tiny scraps of meat are placed in a dish with hungry planarians, they will form a wad of living protoplasm about it. The mouth is located at the middle of the ventral side of the body, and the pharynx is everted throngh it as a proboscis which is used to draw food within. It is interesting to watch these animals passing the proboscis about over the surface of fresh meat, apparently sucking up the nourishing fluids from the meat. If very minute quantities of meat juice are liberated in the water at specific points, the planarians are attracted to those points.

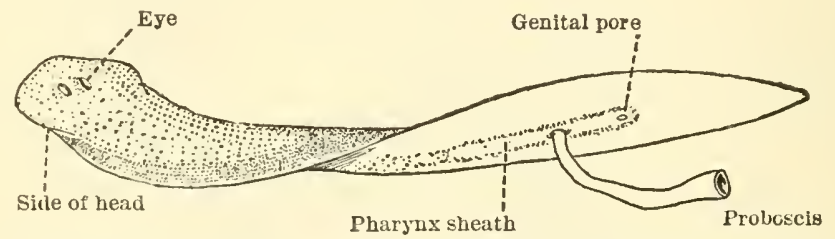

Fig. 81.-Entire planaria with pharynx extended in position for feeding. (From Hegner, College Zoology, published by The Macmillan Company, after Shipley and McBride.)

The locomotion is accomplished in an easy gliding fashion by the action of the beating cilia and muscular contractions of the body. The ability to move along in this way is enhanced by the secretion of slippery mucus which essentially lays a smooth track for the moving animal. It glides over a surface, even the under side of the surface film of water, and adjusts itself easily to any irregularities because of the soft, flexible nature of the body. The ciliary action and muscular contractions are both rhythmic and progress in waves from anterior to posterior.

The behavior of this animal is of a reflex or automatic type. The receiving or receptor sensory cell transfers the impulse produced by a stimulus to a ganglion cell or adjustor in the central nervous system which in turn transmits an impulse to an efferent cell carrying it to a muscle or gland. The planarians respond to several tropisms. They possess negative phototropism and thermotropism (as regards high 
temperatures). They react positively to contact (thigmotropism) and water currents (rheotropism). The responses to chemicals are positive in case of food juices and the like; while they are negative to alkalies, acids, strong salts, alcohol, etc. The common species are Planaria maculata, $P$. agilis, and $P$. dorotocephala.

\section{External Anatomy}

The body is elongated, flat, broadly wedge-shaped at the anterior and tapering to a point at the posterior end. It is triploblastic since the ectoderm, endoderm, and mesoderm are all differentiated and present in a clear-cut fashion for the first time in our studies so far. The symmetry is distinctly bilateral. In Planaria maculata there is considerable pigment in the skin; while in Dendrocoelum lacteum there is much less. On the dorsal side of the anterior region are two pigment bodies called eyespots which are sensitive to light. At each side of the "head" region is a pointed, sensitive, extension of the epidermis in the form of a lappet or "ear," called an auricle. These are sensitive to touch and chemical stimulations but not to sound. The mouth is located in the midventral portion of the body. The pharynx may be protruded through the mouth in the form of a long, trunklike proboscis which is used in feeding. Posterior to the mouth is a small, constricted, scarlike aperture, the genital pore. Externally the epidermal cells are soft and the general surface is nearly covered with patches of cilia which are cytoplasmic extensions of these cells. These cilia along with muscular contractions accomplish locomotion. The average length of fully developed active $P$. maculata is about three-fourths of an inch.

\section{Internal Anatomy}

The ectoderm covers the outer surfaces of the body and composes the nervous system; the endoderm lines the intestine and its branches; while the mesoderm constitutes the muscular, excretory, and reproductive systems. The undifferentiated mesoderm lying outside the intestine is composed of a meshwork of large cells and is called mesenchyme or parenchyma. Many of the structures of the animal, which have been observed in none of the forms previously studied, have come into existence with the development of mesoderm.

The digestive system is composed of a mouth in the midventral position; a prehensile pharynx held in the pharyngeal chamber or 


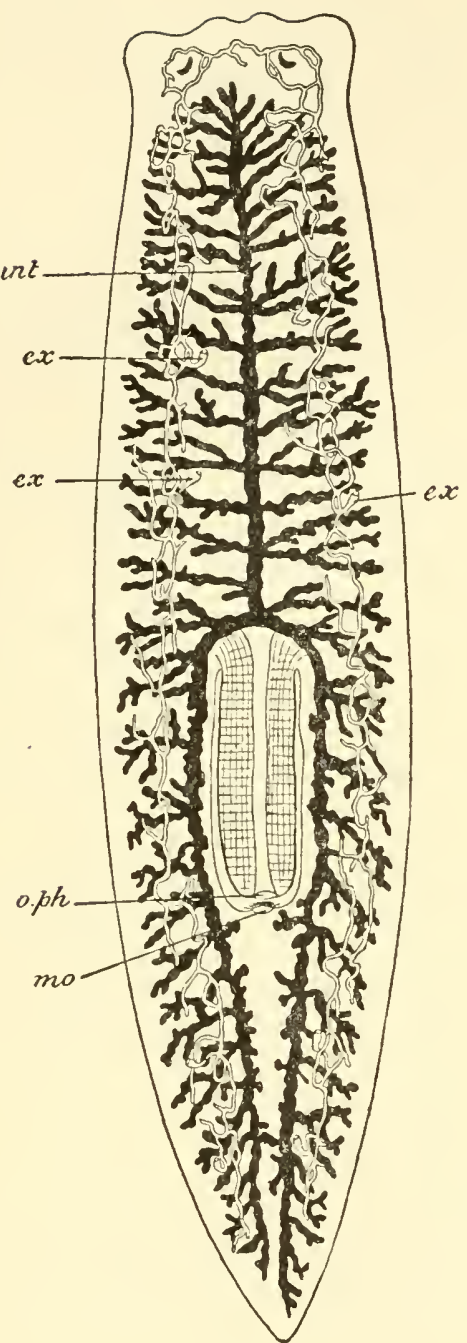

A.

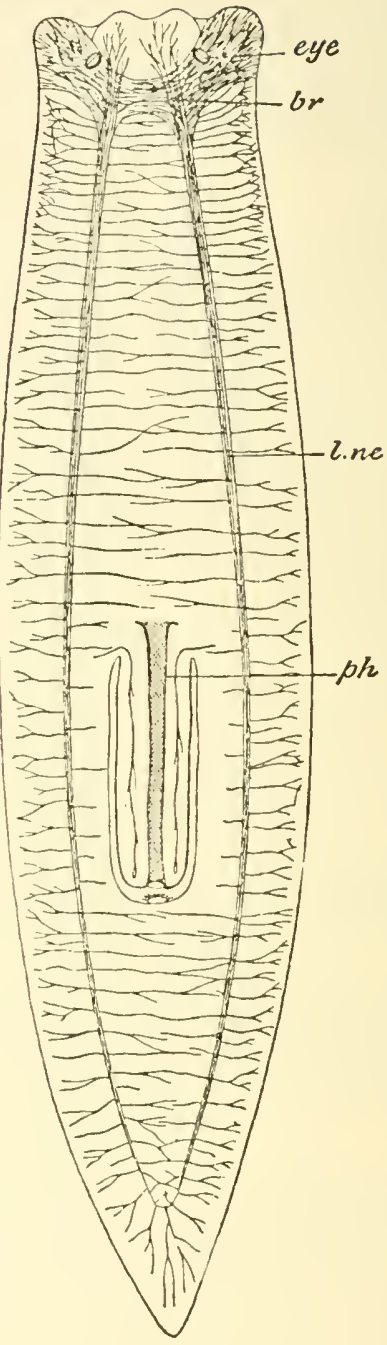

B.

Fig. 82--Structure of planaria. $A$, Digestive and excretory systems; $\boldsymbol{B}$, nervous system; br. cephalic ganglia (brain); $e x$, excretory pore; int, intestine: no mouth: oph, opening of pharynx; $p h$, pharynx. From Parker and Haswell, Zoology, published by The Iacmillan Company.) 
buccal cavity which it nearly fills; a three-branched enteron or intestine, which branches immediately from the anterior end of the pharynx into an anterior trunk; and two lateral trunks that turn posteriorly, one along each side of the pharynx, and extend nearly to the posterior end. The pharynx is in the form of a cylindrical fold projecting through the full length of the pharyngeal chamber. It is attached only at its proximal or anterior end and is perfectly free otherwise. When it is extended or protruded through the mouth opening which it fills, it forms a proboscis whose length may be as great as, or greater than, that of the entire body. The trunks of the enteron have many lateral, blind extensions or pockets called diverticula which greatly increase the surface exposure of the organ and project among most of the other tissues of the body. The whole arrangement represents a complicated gastrovascular cavity whose wall is endodermal.

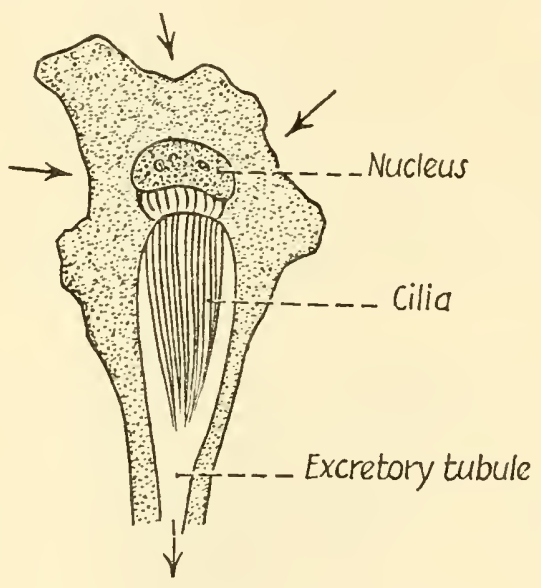

Fig. 83.-Flame cell of planaria.

The excretory system is new to our study and is composed of a set of tubules which relate themselves to all parts of the body. There are two principal, longitudinal, coiled tubules, one along each side of the body, which receive many small branches and open by minute pores located just posterior to the eyespots, and by scveral other pores along the length. Each of the numerous smaller branch-tubules has at its blind end a flame cell which is hollow and contains a mass of long cilia that are continually beating in a direction toward the tubule, the movements appearing something like a flickering flame. 
The cellular walls of the tubules as well as the flame cells arise in the mesoderm. Under strict definition, some authors object to calling this arrangement a system.

Another mesoderm organization is the muscular system. It is composed of an outer circular layer just under the epidermis; an outer longitudinal layer just medial to the circular layer; oblique bundles of fibers; and at the medial margin of the mesoderm is another irregular, internal, longitudinal layer just medial to a circular layer. By the alternate activity of these layers the animal is capable of great extension and contraction.

Another advanced development is the "ladderlike" nervous system which consists of two contiguous lobes of nerve cells just ventral to the eyespots, two ventrolateral longitudinal nerve cords, transverse commissures, branch nerves, and sensory end areas of the epidermis. The double ganglion at the anterior is the central portion of the system. It is known as the cephalic ganglion and gives branches to sensory areas of the head, auricles, etc., besides joining the longitudinal nerve cords. The transverse commissures connect the two longitudinal cords at from 15 to 20 points like the rungs of a ladder. At each point where a transverse commissure meets a longitudinal cord, is a small ganglion composed of a few nerve cell bodies. The branch nerves extend to the surrounding tissue from these points.

The reproductive system is fairly well developed in most species except $P$. dorotocephala which rarely develops sexual organs. Its reproduction is entirely by asexual fission. The sexual reproduction of other planarians is hermaphroditic, which is rather characteristic of sedentary animals. The male organs consist of numerous globular testes located in the parencliyma through most of the length of the body. Vasa efferentia are slender, thin-walled ducts leading from the testes to two larger, longitudinal ducts, the vasa deferentia. These in turn lead posteriorly, enlarge to become seminal vesicles, and converge to form the penis or cirrus, the copulatory organ. This opens into the common cavity called the genital atrium or genital cloaca, which opens externally at the genital pore. Some authors describe glands which pour a seminal fluid into the system. The female organs in the same animal consist of two ovaries located well toward the anterior, a tubular oviduct leading posteriorly from each to join the genital atrium at a common point near its posterior end 
by way of the vagina. There are numerous yolk glands joining each oviduct along its length; a glandular structure of questionable func-

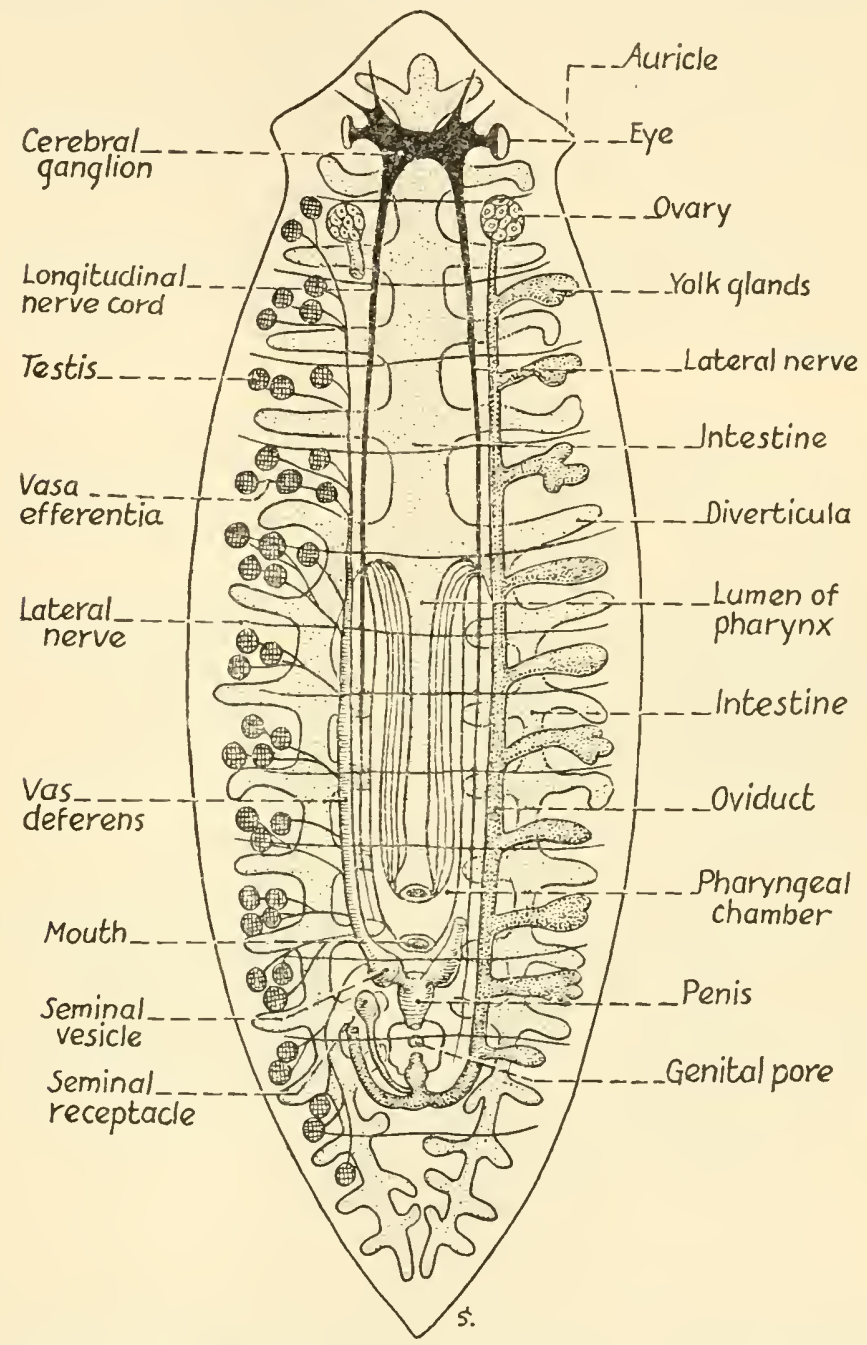

Fig. 84.-Reproductive system of the planarian worm. Male organs shown on one side only.

tion, in the form of a blind tube with an inflated end, is connected with the genital atrium. It has been suggested that the fertilized eggs accumulate and are retained here for a time. The system is 
notably quite elaborate, and it is found generally that the flatworms have a highly specialized reproductive system.

The planarian worms and the representatives of this phylum possess no skeletal system, no respiratory system (breathe through the skin); no coelom or body cavity; and no circulatory system; this function, however, is performed by the branched enteron. It is significant that the reproductive system upon which the continuance of the race depends is highly specialized, this succeeded by the digestive system responsible for nourishment of the individual, and this followed by the nervous system which relates the organism to its surroundings.

\section{Metabolism}

The food is principally animal tissue with some plant matter, and ingestion takes place through the proboscis. The food may be partially digested by a fluid produced in the pharynx. The principal process of digestion occurs in the cavity of the enteron. Here the process is similar to that of Porifera and Coelenterata, being both intercellular and intracellular; that is, part of the food in the intestinal cavity is digested by secretions from cells in their walls, while other food particles are engulfed by pseudopodia extended from cells lining the cavity and are digested in food vacuoles inside the cells. Absorption and assimilation take place through the plasma membranes of adjacent cells. Since the diverticula of this system penetrate all parts of the body, and the diffusion of materials supplies all other cells, no circulatory system is necessary to transport nutriment. There is no anus, so all indigestible material is egested by way of the mouth. Respiration is accomplished through the general surface epithelium, and oxygen is distributed by diffusion through the protoplasm and fluid-filled spaces of the parenchyma. Catabolism or dissimilation takes place in the cells by union of the oxygen with the organic components of the protoplasm. Excretion or elimination of nitrogenous waste liquids is cared for by the flame cells and system of tubules. The flame cells absorb these wastes from the surrounding tissues and force the fluid into the tubules by the action of the cilia.

\section{Reproduction and Life History}

Sexually the individuals are hermaphroditic. The spermatozoa or male germ cells mature in the testes, then pass through the vasa efferentia and vasa deferentia, to the seminal vesicles where they 
are stored in advance of copulation. Here they become organized into pockets known as spermatophores. The ova mature in the ovaries, pass down the oviducts where yolk cells or nurse cells are added by the yolk glands, through the vagina to the genital atrium, and probably from lere to the uterus or seminal receptacle where

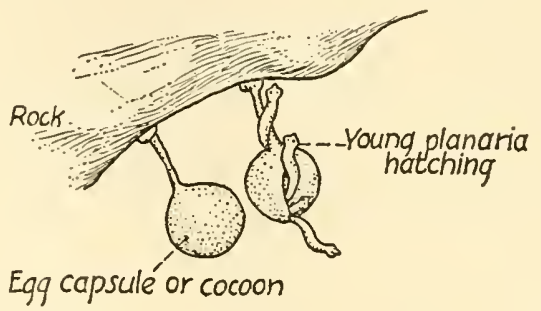

Fig. 85.-Planarian cocoons and the young hatching.

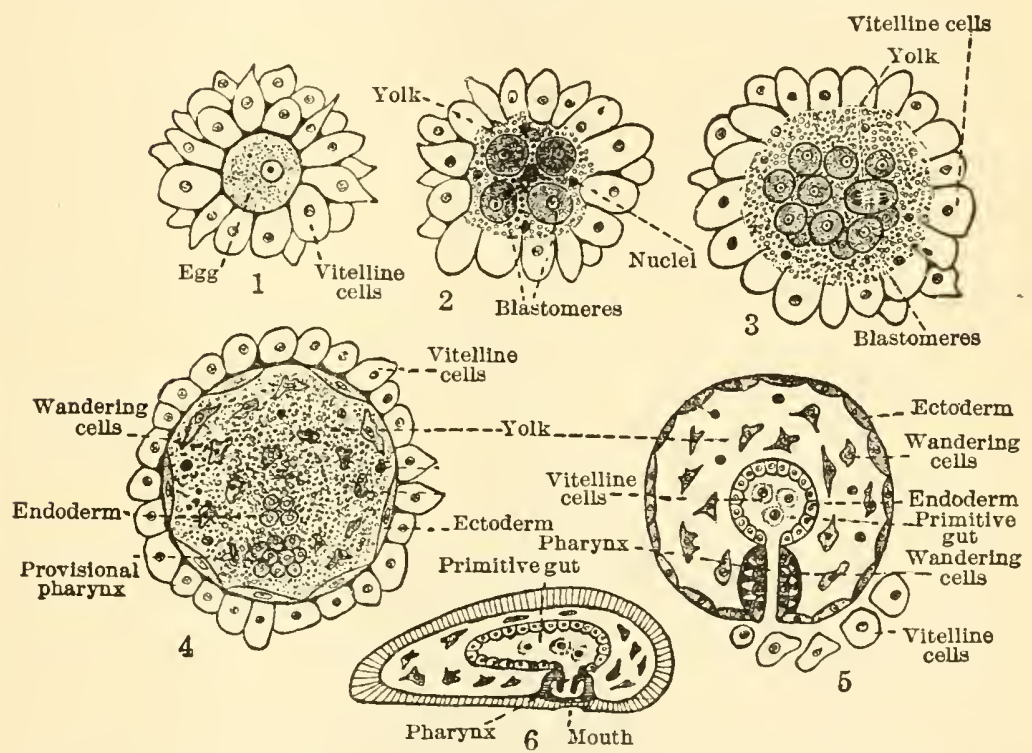

Fig. 86.-Development of Planaria lactea. 1, Egg surrounded by yolk; 2, four blastomeres from segmented egg; 3 , later stage; 4, still later, after blastomeres have differentlated into ectoderm, endoderm, a provisional pharynx, and wandering cells: 5, cellular differentiation more advanced; 6 , embryo becomes flattened and ovoid. (From Hegner, College Zoology, published by The Macmillan Company, after Lankester after Hallez.)

they are thought to be stored. Cross-fertilization is practiced by these animals. Planarians have been observed to copulate with an apparent exchange of spermatozoa in the form of spermatophores. In copula- 
tion the cirrus or penis is protruded from the genital pore to enter the genital pore and extend into the uterus of the other copulant. In this way spermatozoa may be transferred from each animal to the other. Spermatozoa have been found along the oviduct as far as the anterior portion, so fertilization likely occurs somewhere along this tube. At breeding time zygotes are found in the atrium, and each is surrounded by a large number of yolk cells (nurse cells). Each yolk cell contributes its store of nourishment to the egg cell to which it is attached. From one to several zygotes, surrounded by many thousands of yolk cells, become enclosed in a capsule-like shell

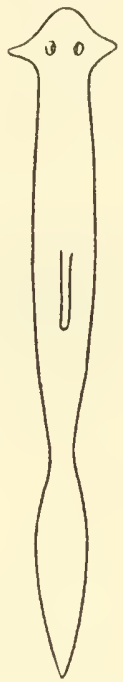

Fig. 87.-Fission as it occurs in Planaria dorotocephala.

secreted by the genital atrium and known as a cocoon. These are expelled from the atrium and each is attached by a stalk to the under sides of submerged stones or vegetation in the water. In the cocoon the embryo passes through cleavage divisions, blastula stage, gastrulation and even later stages before the cocoon ruptures and the small wormlike planarians escape into the water.

Asexual reproduction by transverse fission occurs quite frequently when the mature animals become slowed down. The individual constricts and then divides into anterior and posterior portions each of which forms the missing parts by rapid eell division. The axial 
orientation of the tissue is retained; i.e., an anterior portion develops in the position of the original anterior portion, and a posterior portion at the original posterior position. This process is not fundamentally different from budding in Hydra or strobilization in the Scyphomedusae.

The retention of the axial orientation during fission has been explained by Dr. Child of Chicago University. The animal possesses a well-defined axial organization in which the "head" portion as usual has the highest metabolic activity of the body. Beginning at the anterior there is a gradient of decreasing metabolic activity until a level just posterior to the mouth is reached, and here a sudden increase occurs. Posterior to this the decreasing gradient again follows to the posterior tip of the body. The level where the metabolic rate suddenly rises represents the point of fission or the anterior end of the second individual. This seems to indicate a kind of zoöid organization in the animal. In larger, older individuals there may be other such points of increased metabolism posterior to this first one. Such zoöids are the result of successive functional isolations of the basal structure accompanying growth in length. This graduation of the rate of metabolism along the principal axis of an axiate animal has been called an axial gradient by Dr. Child. When the animal is young, it is relatively short and the entire body, but particularly the "head," carries on a high rate of metabolism. The head at this time holds a dominance over the length of the organism. As the animal grows older, it becomes longer, and the entire metabolic rate decreases. This means that the head loses its dominance over the entire lengtl. A new center of dominance and increased metabolism is established just posterior to the point where this "head" dominance fades out.

\section{Regeneration}

This group shows remarkable power's of replacing lost or mutilated parts of the body. It can be cut into several pieces, and each piece will replace the missing parts about as the process is carried out in fission. A piece from the middle of the animal will regenerate a head portion at the anterior margin and a tail portion at the posterior margin. A more complete discussion of this phenomenon will be found in a later chapter on Animal Regeneration. 


\section{Economic Relations of the Phylum}

The planarians and other free-living flatworms are of practically no economic importance, but the phylum includes a large number of forms, principally Trematodes and Cestodes, which are parasitic in higher vertebrate animals, including man. Such groups as the intestinal flukes, liver flukes, lung flukes, blood flukes, pork tapeworm, beef tapeworm, margined tapeworm of dog, gid worm, hydatid worm, common tapeworm of dog, chicken tapeworm, dwarf tapeworm, sheep tapeworm, tapeworm of horse, and fish tapeworm are all serious parasites. They cost many thousands of dollars and much debility each year. A more complete discussion of this topic will be found in the chapter on Animal Parasitism.

\section{Phylogenetic Advances of Platyhelminthes}

(1) Anteroposterior principal axis, (2) bilateral symmetry, (3) a distinct third germ layer, the mesoderm, (4) an excretory system of flame cells, (5) central nervous system extending with the axis of the body, (6) specialized gastrovascular cavity, and (7) permanent sexual reproductive organs. 


\section{CHAPTER XIII}

\section{PHYLUM NEMATHELMINTHES}

This group is known as the unsegmented roundworms or threadworms. Some of the Nemathelminthes (nĕm á thĕl minn'thēz, threadworms) are free-living in soil, fresh water, and salt water; some are found living in plant tissues; and others live in animal tissues as parasites. The majority of them are microscopic, but a few are macroscopic in size. These worms are long, slender animals whose bodies are more or less cylindrical but tapering toward each end. The range of length is from $1 / 4 \mathrm{~mm}$. to four feet. They differ from the flatworm not only in shape, but also in that the intestine has two openings, there is a dorsal as well as a ventral nerve cord, they are mostly dioecious, and there is a total absence of cilia. They also lack respiratory and circulatory systems, true coelom, and definite locomotor organs. The group is very widely distributed and is deserving of considerable attention. Some of the better known forms are Ascaris (pigworm or eelworm), "horsehair snake," hookworm, pinworm, Trichinella, Filaria, Guinea worm, whipworm, and eye worm. The former will be discussed in some detail in this chapter, and several others will be considered in the chapter on Animal Parasitism.

\section{Classification}

Three classes are usually recognized, although some authors prefer to use only two. The three classes are Nematoda, Gordiacea, and Acanthocephala.

Class Nematoda (něm à tō'dä-threadworm) is a group occupying almost every possible habitat capable of supporting life. There are many free-living, fresh water, marine, and soil-inhabiting species, and large numbers of parasitic forms living at the expense of other animals and plants. This is a very important class parasitically. In size they range from $1 / 10 \mathrm{~mm}$. to more than a meter in length. Locomotor organs are found in a few forms, no segmentation is present, and there is no true coelom. Chemical sense organs called amphids are nearly universal, while eyes and tactile organs are common in the free-living forms. The skeletonlike cuticle, common to all, is shed periodically like the molting of arthroporls. The nervous 
system is composed of a circumpharyngeal ring from which cords extend posteriorly. It is a sensory-neuro-muscular system. The structure of the free-living forms is generally more complex than that of the parasitic forms. They are adapted to a wide variety of habitats and can withstand many rigors of natural adversity, such as freezing, high temperatures, droughts, and other unfavorable conditions. Large numbers of free-living forms have not been named and described. Representatives of this class have an intestine but no proboscis.

Order Ascaroidea.-It includes both parasitic and free-living forms. Ascaris (Fig. 90), the common intestinal worm, is the most abundant. Enterobius vermicularis, the human pinworm; Strongyloides stercoralis, another parasite of man; Ascaridia lineata, the chicken worm, and Toxocara canis of dogs are other familiar examples. Ascaris lumbricoides will be discussed later as a typical example of Nemathelminthes.

Order Strongyloidea.-This is an entirely parasitic group. The males have caudal bursae with rays. The club-shaped esophagus is without a posterior bulb. The hookworms of man, the Strongylus roundworms of horses, and Syngamus trachea which causes gapes in birds by obstructing the windpipe are all common representatives.

Order Filaroidea.-This is a completely parasitic order, modified for living in such tissues as lymph, blood, connective tissue, and muscle of chordate animals, and transmitted by certain insects. Two distinctive characteristics are: (1) lack of bulb on esophagus; (2) lateral paired lips or entire absence of lips. Guinea worm, eye worm, and Filaria are the common human parasites. Some species cause elephantiasis (Fig. 390) through occlusion of blood and lymph vessels. This disease lesults in enormous swelling of the affected parts. These organisms are transmitted by mosquitoes. Several Filaroidea are parasites of horses and dogs.

Order Dioctophymoidea.-This is another parasitic group living in the kidneys, body cavity, and alimentary canal of mammals and birds. The genus Dioctophyme includes the largest roundworms, some reaching more than three fect in length.

Order Trichinelloidea.-This parasitic group has a peculiar cuticle lining the esophagus, outside of which is a single layer of epithelial cells. The common trichina (Fig. 396) and the whipworm are wellknown examples. 
Class Gordiacea (gôr dĭ ā'shēả, a kuot).- Superficially these animals resemble the nematodes, but the fundamental structure is quite different, and therefore, it is likely proper to give them the rank of a class. They are free-living as adults but as larvae are parasitic on May flies and other insects. They leave this host and take up abode in a terrestrial form like that of grasshoppers or beetles. After complete development the adult "hair snakes" escape into

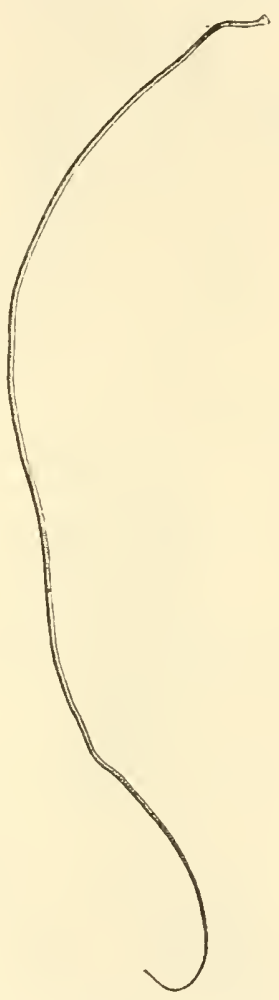

Fig. 88.-Hair snake, Gordius, an aquatic roundworm.

the water of some stream, puddle, or watering trough. These fcmales again lay eggs in the water in long strings. In the adult worm the intestine is a straight tube, often without a mouth, but opening at the posterior end by an anus. Some have no intestine at all. The outer surface of the body is covered by a cuticle. The body is cylindrical and witlout lateral lines, excretory organs, or circulatory system. There are four longitudinal spaces or sinuses 
in the parenchyma; in the adult female the two lateral spaces are lined with peritoneum. The nervous system is composed of a midventral cord with a nerve ring at the anterior end. There are rudimentary eyespots and scattered sensory cells over the body.

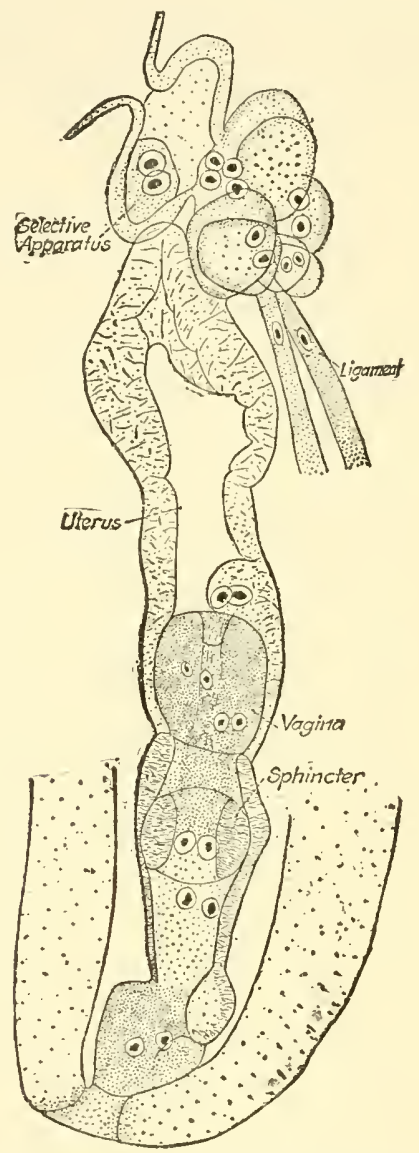

Fig. 89.-Structure of Acanthoccphala. Internal structure of the genital organs of a young female nematode, Neoechinorhynchus emydis. (From Van Cleave, Invertebrate Zoology, first edition, second impression, published by McGraw-Hill Book Company, Inc.)

Sexually the group is dioecious with the gonads opening into the posterior end of the digestive tract. Fertilization takes place within the body of the female. Gordius aquaticus and Paragordius varius are the common examples of the group. 
Class Acanthocephala (ả kăn thố sěf'à lá, thorn head) includes a group, known as "spiny-headed worms," which is absolutely parasitic in its habits. The adults are from a few millimeters to fifty millimeters in length and have an elongated, flattened body when found in the intestine of a vertebrate but become distended to a cylindrical shape when removed to some solution outside the body. The protrusible proboscis is a peculiar and characteristic structure located at the anterior end of the body. It bears numerous recurved hooks or spines, and in many species it is capable of receding into a proboscis receptacle or sheath. There is no digestive tract in this parasite, and its food is absorbed through the surface of the body even though it is covered with a cuticle. A single ganglionic mass constitutes the central nervous system.

The male reproductive organs are the testes and a set of cement glands joining the cirrus which is held in the copulatory bursa at the posterior portion of the body. The bursa is capable of eversion. The sexes are separate, but the female has no permanent gonads. Egg masses develop early and completely rupture to produce a considerable number of embryos in the body cavity. Finally the embryos are discharged by way of the uterus through the genital pore which is located posteriorly and is the only external aperture of the body. Not only man, but mice, rats, pigs, fish, turtles, and in fact all classes of vertebrates serve as hosts for these animals.

\section{ASCARIS, A REPRESENTATIVE ROUNDWORM}

\section{Habitat and Behavior}

The animal which is frequently studied as a representative of this phylum is Ascaris lumbricoides which frequents the digestive tract of men and hogs. It is entirely dependent on its host for furnishing suitable food and environment. The only time this organism is at the mercy of the elements of nature is during the egg stage when it may remain potent for months or even years if it falls in an environment unsuitable for development.

\section{External Anatomy}

This is one of the largest nematodes, females commonly reaching a length of from eight to fourteen inches and males averaging six 
to twelve inches. Males are always more slender and have a curled tail instead of the blunt tail of the female. The mouth is guarded by three lips, two in lateroventral positions and one dorsal. These lips have small papillae on their surfaces, two on the dorsal and

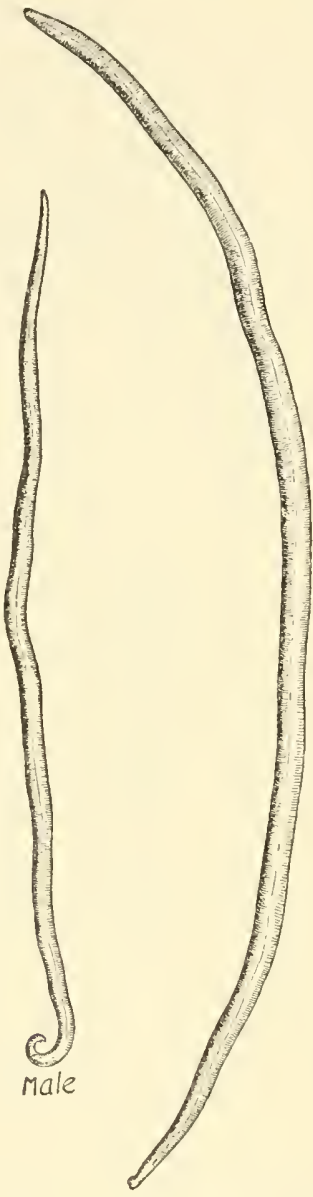

Fig. 90.-Male and female Ascaris or eel-worm.

one on each of the ventral. The shape of the body is generally cylindrical with tapering ends. The smooth surface is marked by four longitudinal lines, two lateral, one dorsal, and one ventral. The genital pore in the female is located on the ventral midline approximately one-third of the length of the body from the anterior 
extremity. The anus is located near the posterior tip of the body, and in the male the reproductive aperture and two penial setae or spicules are located just within this opening.

\section{Internal Anatomy}

The body wall is composed of the thin, outer, smooth cuticle, the epidermis, whose cells rum together, and a thick layer of longitudinal muscle fibers, whose medial margins are rather baggy. There

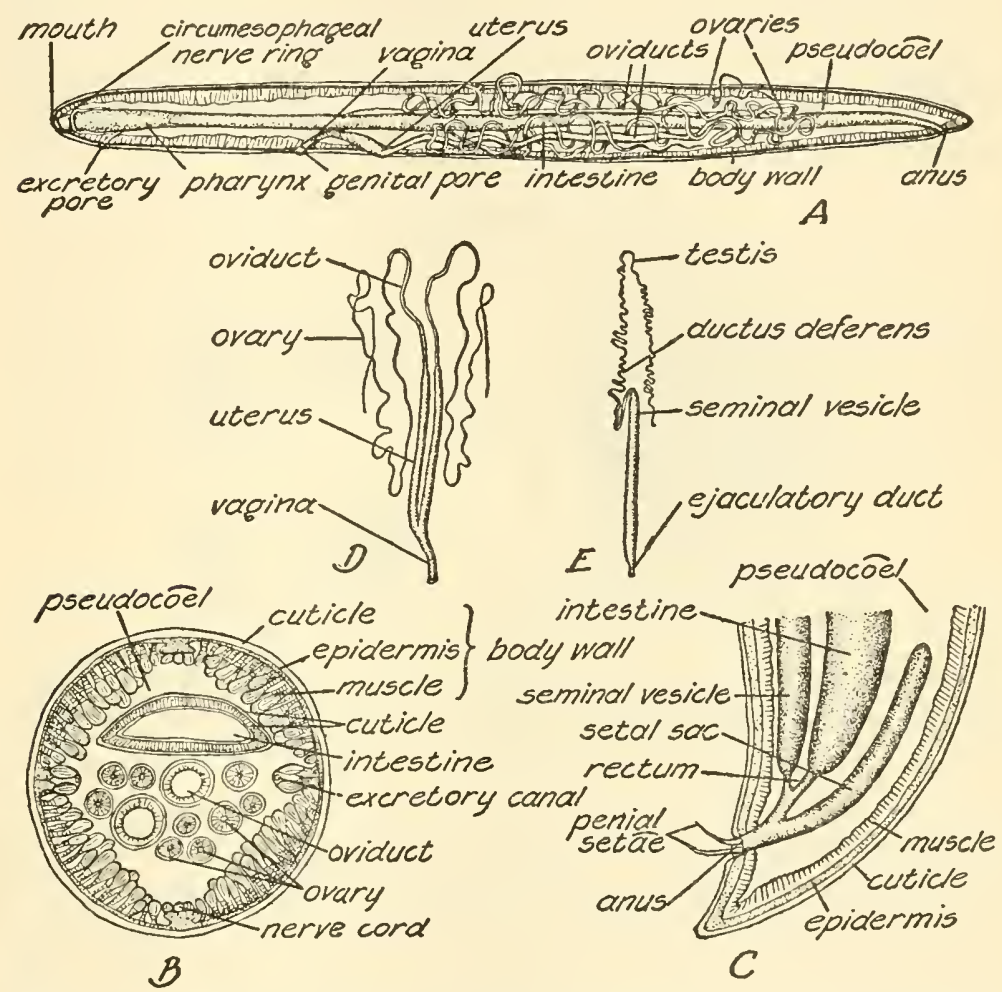

Fig. 91.-Internal anatomy of Ascaris lumbricoides. A, Diagram of lateral view of dissection of female; $B$, cross-section of the midregion of the body of female: $C$, longitudinal section of posterior portion of male; $D$, reproductive system of female: $E$, reproductive system of male. (From Curtis and Guthrie, Textbook of General Zoology, published by John Wiley and Sons, Inc., modified from Leuckart.)

are thickenings of the epidermis in the positions of the longitudinal lines. The excretory tubes follow the lateral lines. The body cavity of this animal is a primitive or false coelom which is lined externally by the mesoderm of the body wall and internally by the endoderm 
of the intestinal wall. Ordinarily the coelom, when fully developed, is lined both laterally and medially with mesodermic peritoneum. This is the simplest type of animal in which the body cavity or coelom is found. In higher forms the outer coat of the intestine is mesodermic. The alimentary canal is quite straight and simple and lies in the dor'sal part of the body cavity. There is no need for great specialization of the digestive system since the food is taken from the digested material in the intestine of the host. A contractile pharynx, which acts as a pump, draws fluid into the long epithelial intestine from which it is absorbed by the other tissues. The narrowed posterior portion is the rectum and leads to the anus

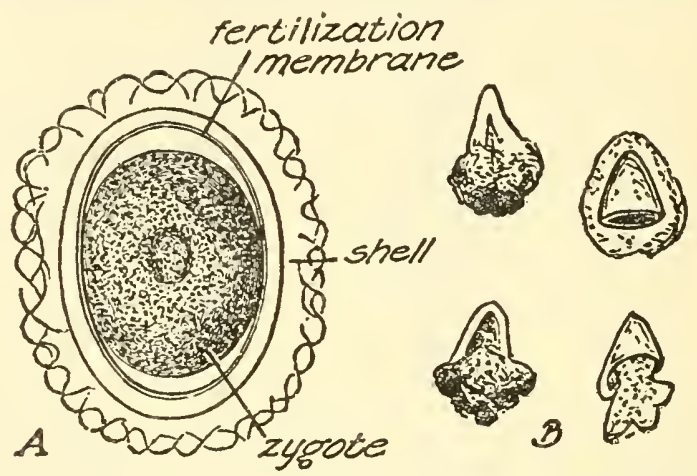

Fig. 92.-Fertilized ovum, $A$, and amoeboid spermatozoa, $B$, of Ascaris lumbricoides. (From Curtis and Guthrie, Textbook of General Zoology, published by John Wiley \& Sons, Inc., after Leuckart.)

at the posterior portion of the body. The two laterally located, longitudinal ducts open externally by a single pore near the anterior end of the body. There is a nerve ring around the pharynx which gives off a large dorsal longitudinal nerve and a large ventral longitudinal nerve. There are usually four other smaller longitudinal nerves and some connectives. In the males the testis is a threadlike structure which is much coiled in the cavity. This tube enlarges posteriorly to become the vas deferens which in turn enlarges still more before reaching the aperture to become the ejaculatory duct. In the female the threadlike ovaries join the coiled oviducts which lead forward and join the two uteri. These tubes join in the vagina, which is a short tube leading to the genital pore. 


\section{Reproduction and the Life Cycle}

The animals copulate, and at this time the spermatozoa are introduced into the vagina of the female to fertilize the mature ova in the oviducts. A mature female may contain as many as 27,000,000 eggs. These eggs pass from the host with the feces. Some workers have reported that each female worm in an infected host may produce a crop of eggs in excess of two thousand per gram of feces. Based on this figure, the daily production is computed to be something like 200,000 eggs. These eggs are so resistant that they can be successfully cultured in 1 to 2 per cent formalin, and they may be stored successfully for four years in a refrigerator. The life history is completed only in case the eggs are swallowed by a susceptible host. They hatch in the small intestine of the host and then go on a ten-day journey by way of the blood stream to the liver, thence to the heart, and thence to the lungs. By burrowing out from here, these larvae make their way to the throat, esophagus, and back to the stomach and intestine. After reaching the intestine, the larval worms, 2 to $3 \mathrm{~mm}$. long, grow to maturity in two to two and one-half months. They likely live a little less than a year in the host.

\section{Relations to Man}

Heavy infestation in man may cause severe hemorrhages and set up pneumonia that is often fatal. Anemia is often the result of such infection; in certain cases the organisms may even tangle in masses and block the intestine until surgical operation is necessary to remove them. The toxic substances from these parasites may bring on coma, convulsions, delirium, nervousness, and other similar symptoms. Drugs like chenopocium, santonin, and hexylresorcinol have been used successfully under physicians' directions as a cure. Effective sanitary disposal of fecal material is the most successful preventive. 


\section{CHAPTER XIV}

\section{MOLLUSCOIDA, TROCHELMINTHES, AND CHAETOGNATHA}

These groups are rather conveniently considered in the same chapter, beeause they are more or less isolated, small groups of the unsegmented worm type.

\section{MOLIUSCOIDA}

This is the name of a group composed of two classes, as they are treated here. It is usually considered a phylum name, but many authors prefer to give each of the classes phylum rank. The justification of the latter plan may be questionable.

Class Bryozoa (brí ó zō'ámoss animals) includes a group of colonial animals often called Polyzoa, which are similar to colonial hydroids in their manner of growth and forms. It is true that their structure distinguishes them very readily. Nearly all bryozoans are marine, although there are a few fresh water forms. In external appearance they resemble certain of the corals and hydroids. It was a long time after their existence was known that they were separated from that group. The subclass Ectoprocta includes forms in which the mouth is surrounded by tentacles and the anus is not enclosed in this area. Bugula is an example of a treelike type of this subclass. Another type is one that grows as an incrusting organism. The second subclass Endoprocta is characterized by the circlet of oral tentacles which also encloses the anus.

\section{Bugula}

Bugula is a common marine genus, the individuals of which are associated in a treelike colony that lives attached to some object in the water. These individuals are ealled zooids of which the soft parts are known as polypide. They are within the primitive coelomic cavity, the wall of which is the zooccium. The presence of retractor muscles make it possible for each zooid to be withdrawn into the vaselike part of the chitinous skeleton. There are some smaller individuals whose shape is similar to that of a bird's head and whose bodies are smaller than the other zooids. These are called avicularia, and they are found on the surface of the colony. Their 
movable jaws seem to serve as grappling hooks which operate to keep the colony free from other small organisms and debris which may be present in the habitat. Vibracularia (vibracula), which con-

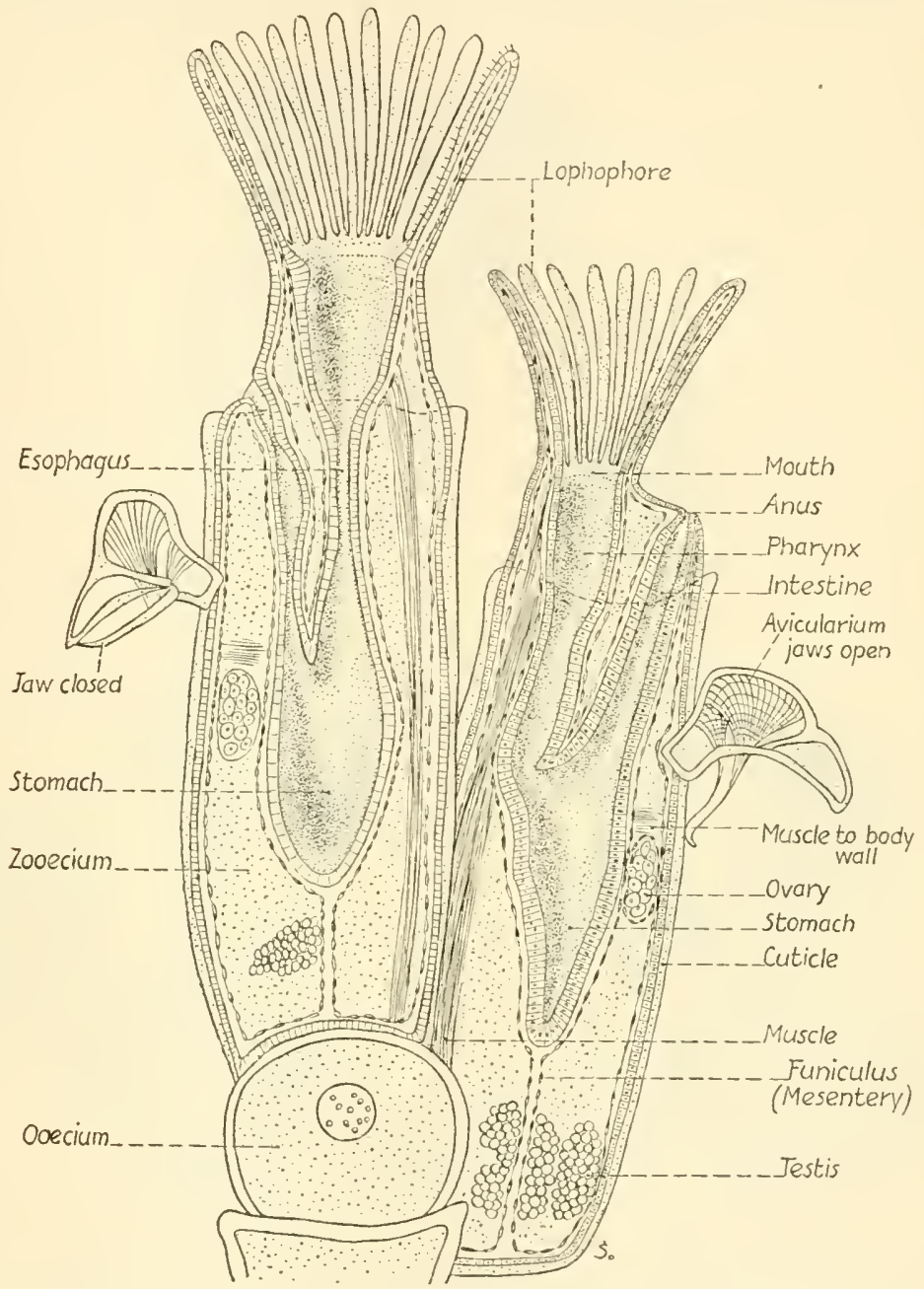

Fig. 93.-Bugula, a marine bryozoan, showing the structure and habit of life of two zooids from a colony.

stitute another modified type of zooid, are filamentous, whiplike appendages. They are thought to be variations of the avicularian modification. 
The mouth of the larger, regular zooid is located at the free end and is surrounded by a tuft of ciliated tentacles. This arrangement is known as the lophophore and has the shape of a horseshoe when expanded. Within, the digestive tube is U-shaped and termi-
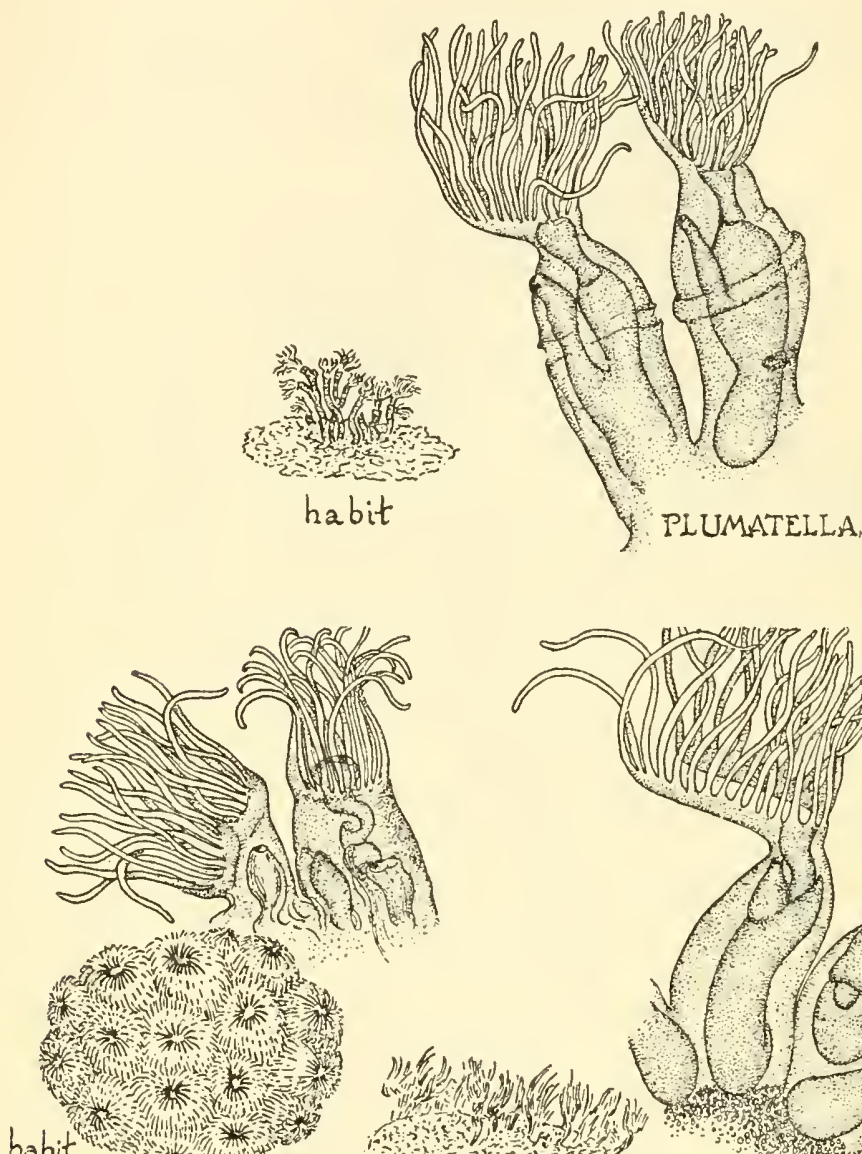

PECTINATELLA.

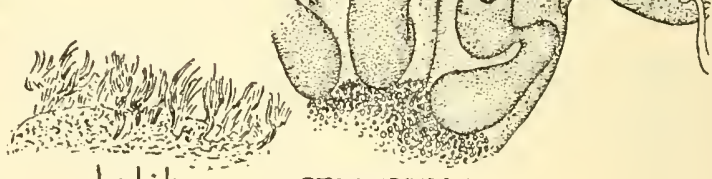

CRISTATELLA

Fig. 94.-Three forms of fresh-water Bryozoa showing the habit of life for each. (Courtesy of General Biological Supply House.)

nates at the anus, which is located just outside the lophophore. The digestive tract is held in place by strands of mesenchymatous tissue extending from the wall of the coelom. Special strands of this tis. sue are termed the funiculus. The body is triploblastic and there- 
fore composed of ectoderm, endoderm, and mesoderm. The nervous system is centered in a ganglion or mass of nerve cells located in the region of the mouth and from it, nerves extend to the tentacles.

Reproduction is accomplished either by budding or sexually. Ovaries and testes make their appearance either in the funiculus or in the lining of the coelom and fertilization occurs in the body cavity. The early development goes on in a modified region of the zooecium, called the broad-pouch or ooecium. When the embryo escapes, it is a free-swimming, ciliated larva which is similar to the trochophore larva found as a developmental stage of certain Annelida and Mollusca. Its form resembles certain adult Rotifera. This larva becomes attached and transforms into a parent individual, the zooid of which will form a new colony by budding.

The branching Plumatella, which is supported by a secretion of calcium carbonate, and the slimy Pectinatella, whose skeleton is in the form of a gelatinous mass, are the two forms most frequently found in fresh water. These fresh water types may be developed from winter eggs, enclosed in shells, or new individuals may be produced as internal buds. These buds are called statoblasts. They are produced in autumn and may either float on the water or sink to the bottom. They witlistand the rigors of winter and are stimulated by it. So far as is known this group has little if any economic value.

Class Brachiopoda (brăk $\breve{1}$ ŏp'ó dả-arm and foot) is a group of marine forms, the individuals of which possess bivalve shells. For this reason they are sometimes confused with the clamlike molluses. The brachiopods, however, have dorsoventral valves, while the molluscan valves are lateral. The shell is secreted by a mantle which lines the valves. The tip of the beaklike valves is penetrated by a foramen which serves as an opening for the peduncle. This fleshy organ makes permanent attachment to some object in the water. Internally, the lophophore is a conspicuous and characteristic structure of this type of animal. This organ is composed of two coiled appendages which bear numerous ciliated tentacles. The cilia produce water currents in the longitudinal groove and carry food particles to the mouth.

The digestive tract is U-shaped and is composed of the mouth, lophophore, gullet, stomach, and ventrally directed intestine. This 
tract ends blindly in many brachiopods. The entire tube is lined internally with eiliated epithelium. A segmented, true coelom is present, but the septa are a little bit difficult to distinguish. Extensions of the coelom enter the arms and mantle of this type of animal. About two pairs of nephridia are comnected with the coelom and serve in excretion. The coelomic cavities produce the gonads also. The sexes are distinctly separate and mature germ cells are discharged into the coelom, thence to nephridia and outside. Fertilization takes place in the water, and a free-swimming, ciliated larva hatches from the egg.

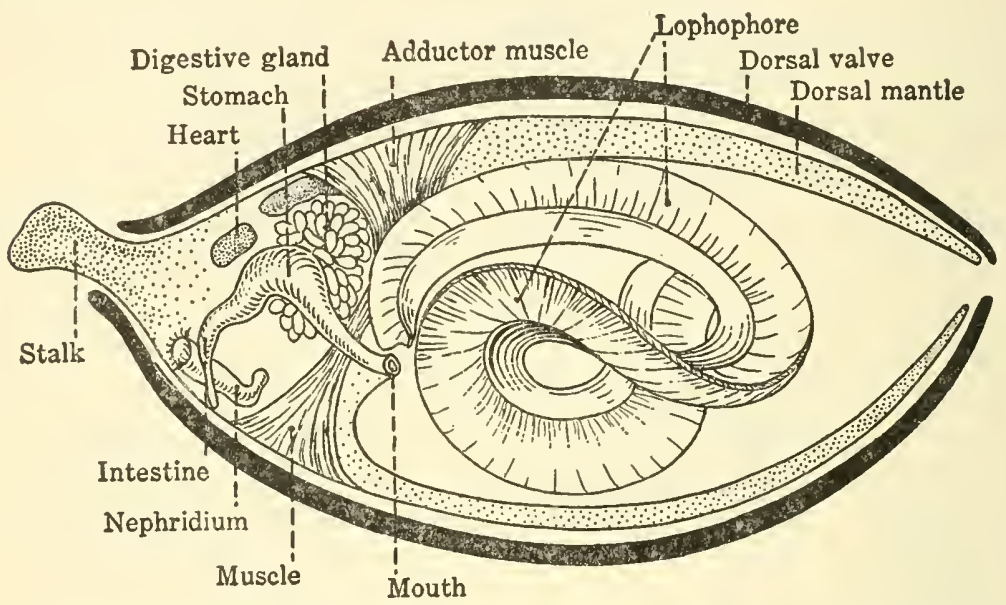

Fig. 95.-Diagram of a sagittal section of a brachiopod to show internal organs. (From Hegner, College Zoology, published by The Macmillan Company.)

Magellania flavescens and $M$. lenticularis are commonly studied forms. They are entirely marine and represent an old line of animals. There are relatively few modern forms in existence. The group is of little economic significance.

\section{TROCHELMINTHES}

The rotifers (Rotifera) are common examples of this group known as the Trochelminthes (trŏk ĕl m̌nn'thēz-wheel wor'm). In early times they were called "whecl animalcules." There is very little difference between the trochophore larva of this group and the adult animal. Gastrotricha constitutes another small division of this group but will not be discussed in detail here. 
Rotifers are plentiful in fresh water, and a few of them inhabit the sea. They are microscopic in size, and they are often associated with Protozoa. They are very resistant to adverse conditions produced by drought and may be distributed in dry form.

The body of a rotifer is bilaterally symmetrical and can be divided into head, trunk, and foot. It is covered externally by a cuticle. The so-called head is rather largely a trochal disc composed of various modifications of two bands of cilia over the anterior end and around the mouth. These cilia are in active motion, often ereating two sets of water currents so as to resemble two rotating wheels. They are responsible for obtaining food and for locomotion. The mouth is located in an anteroventral position. The trunk tapers toward the posterior and contains numerous organs. At the posterior end is the tail or foot which is forked or toelike in many species. Here, too, in many forms, are located some cement or adhesive glands which assist the animal in adhering to most surfaces. The foot as a whole serves in locomotion, pushing the animal along.

The internal organs include several systems which lie in the rather extensive body cavity or false coelom. The digestive system begins anteriorly at the mouth which receives other small organisms as food. It is a cavity leading to the pharynx. Inside the pharynx is a mill-like organ or mastax, composed of chitinous jaws, which masticates the particles of food. The movements of these jaws may be observed in certain rotifers when alive. A short tubular esophagus leads to the pouchlike stomach, and extending posteriorly is the smaller cylindrical intestine which leads by way of the cloaca to the anus. Nearly the entire internal surface of the alimentary canal is lined with cilia which aid the movement of the food material through it. The stomach and intestine are lined internally with endoderm.

The excretory system is well developed and consists of a number of flame cells, similar to those of flatworms; and two winding nephridial ducts which lead posteriorly to a contractile bladder. This bladder is pouchlike and empties into the cloaca. (The name cloaca is applied to any cavity which serves as the posterior portion of the alimentary canal and also receives products of the urinogenital system. It opens externally by way of the anus.) The flame cells are distributed in the body wall from the anterior, posteriorly. Some authors believe that the bladder functions also to assist in 
respiration by collecting the excess water and carbon dioxide. The oxygen is received into the body with water which diffuses through the body wall. A large ganglion, located in a dorsoanterior position and several nerves extending to sense organs and muscles con-
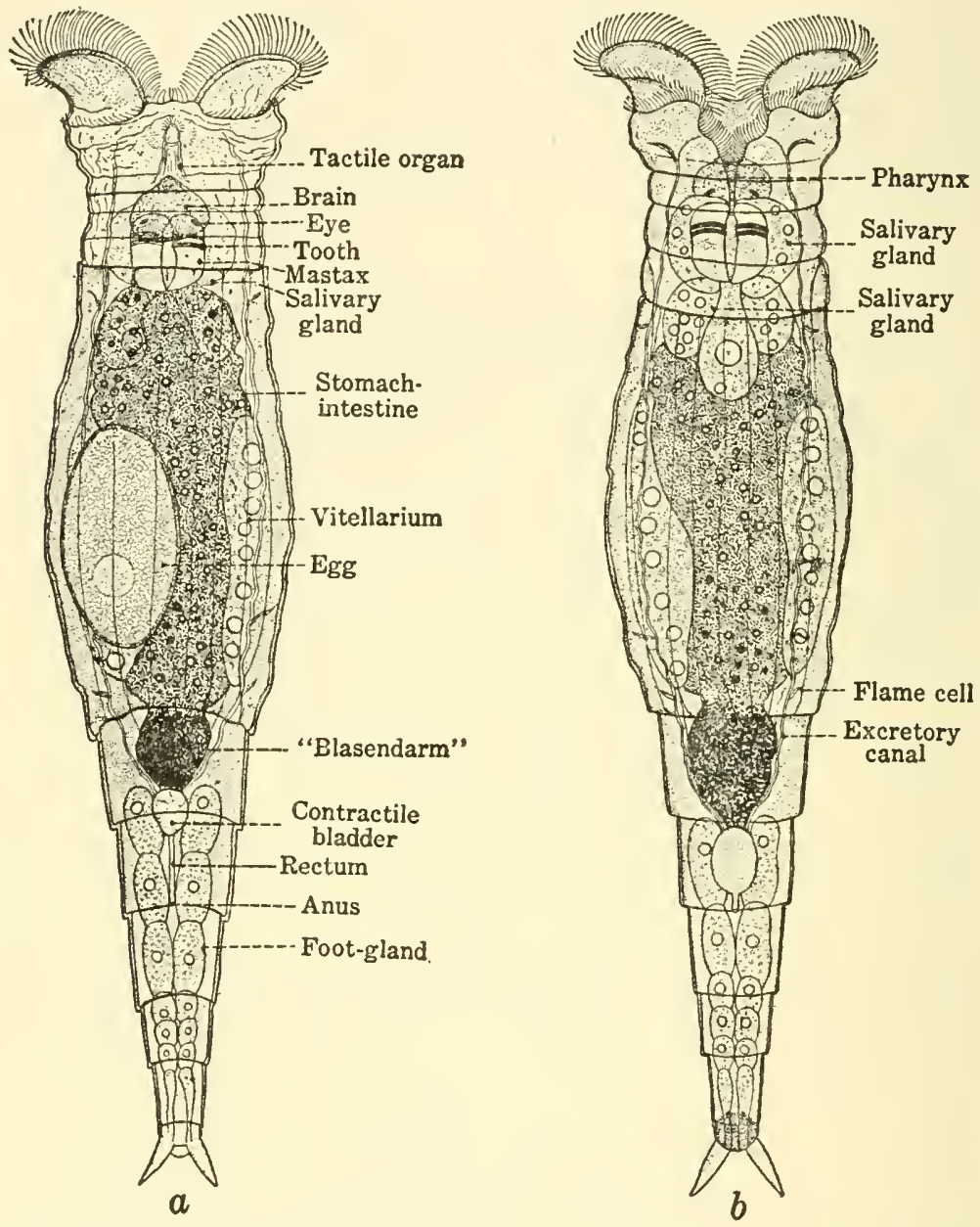

Fig. 96.-A common fresh-water rotifer, Philodina roseola, showing internal structure. $a$, dorsal view; $b$, ventral view. (From Hegner, College Zoology, published by The Macmillan Company, after Hickernell.)

stitute the nervous system. The body wall is composed of an outer cuticle over a thin layer of ectoderm. Under this layer is the mesodermal tissue which includes mesenchymatous cells and muscle fibers. 
This group of animals is bisexual, and dimorphism (striking differences in form of the two sexes) is present. The males are usually much smaller and may even live as a parasite on the female. The males lack a well-developed digestive system and are therefore very short lived. In the female of most species there is one ovary which produces the eggs. Connected with this gonad is a yolk gland or vitellarium. In a few forms there are two ovaries with no distinct yolk gland. Rotifers may be oviparous (lay eggs), ovoviviparous, or even a few are viviparous. The eggs produced during the summer are thin-shelled, of two sizes, and develop parthenogenetically.

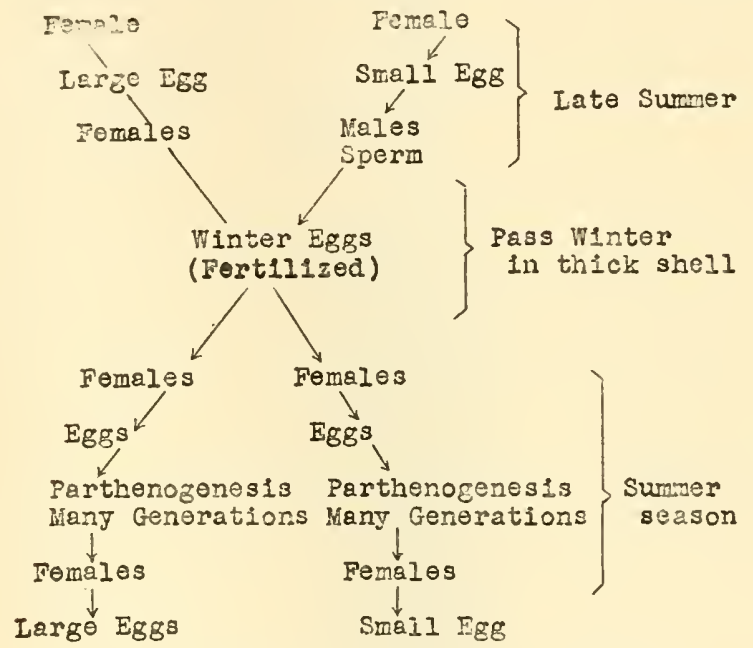

Fig. 97.-I,ife cycle of the rotifer, Hydatina.

The smaller type produce males. The eggs produced during the winter are thick-shelled, produce females only, and require fertilization. The eggs when mature, or the young if born alive, are carried by the tubular oviduct to the cloaca and are discharged to the exterior through the anus. The less highly developed males possess a single testis in which spermatozoa are produced. In some there is a peculiar type of copulation during which the special copulatory organ composed of a protrusible cirrus seems to perforate the body wall of the female. At this time the eggs of the female are fertilized. In oviparous forms, the fertilized eggs are usually carried in the body for a time and then discharged by way 
of the oviduct. They then lie dormant and inactive in the water for a period before hatching. There is considerable similarity between the adult rotifer and the trochophore larva of some annelids,

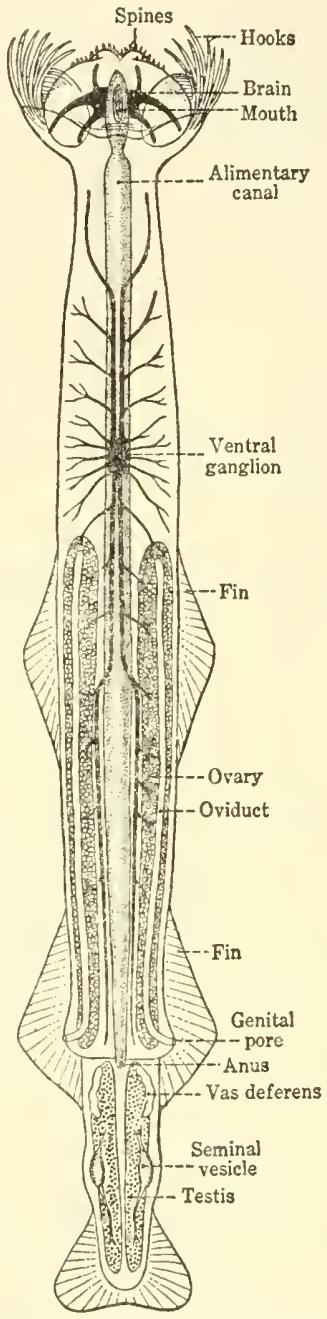

Fig. 98.-Sagitta hexaptera, an arrowworm, drawn to show internal organs. (From Hegner, College Zoology, published by The Macmillan Company.)

mollusks, Nemertinea and others. This resemblance has prompted the theory that the above groups are rather closely related to the rotifers. 
Class Chaetognatha (kè tŏg'nả thả-horse's mane, jaw).-These small marine worms are often called arrowworms, and they are well adapted to living at the surface of the ocean. Horizontal fins support the animal at the surface and also make it possible for it to move about rapidly. The prehensile mouth with its bristles have given the animal the name of "bristle jaws" in addition to other names. The body is divided into three divisions: head, trunk, and tail. These are separated by septa and the coelomic cavity is separated into right and left cavities by a longitudinal mesentery.

Internally is a tubelike intestine which extends from the mouth at the anterior, to the anus located near the base of the caudal fin or tail. The nervous system consists largely of a supraesophageal ganglion or brain, ventral ganglion, branch nerves, two eyespots, and other sensory organs. These animals are lacking in circulatory or excretory structure.

Each individual is eapable of producing both ova and spermatozoa, that is, the hermaphroditic condition prevails. The ovaries are located in the posterior portion of the body cavity and the mature ova are carried to the exterior by an oviduct on each side. The testes are located in the eavity of the tail portion. The spermatozoa are discharged into this cavity and delivered to the exterior by a pair of slender vasa deferentia or sperm ducts, which enlarge to become seminal vesicles near the aperture. The fertilized ova become small adults without a typical ciliated larval stage. Sagitta is the best known genus of the group. 


\section{CHAPTER XV \\ PHYLUM ANNELIDA}

\section{(By J. Teague Self, University of Oklahoma)}

The Phylum Annelida (ă nĕl'í dả, form of a little ring) comprises an extremely large group of worms characterized by (1) the presence of a coelom surrounded by two layers of muscle, (2) metameres or segments, (3) a ventrally located segmental nervous system, (4) segmented, nonjointed, chitinous appendages in most cases, (5) an excretory system composed of nephriclia, and (6) a nonchitinous cuticle covering the body. These worms are found in almost every type of free-living habitat where moisture is present. There are many forms which live in the ocean, either swimming freely, burrowing in the sand, or living in especially prepared tubes. Freshwater streams and ponds are inhabited by numerous forms of annelids, and moist soil is usually alive with terrestrial earthworms. From this it is evident that the phylum as a group has become adapted to many varied living conditions and comprises one of the large groups of the animal kingdom from the standpoint of numbers. In the process of adaptation the annelids have become diversified in their anatomical features until only a very few characters, such as those mentioned in the beginning of this chapter, are common to the entire phylum. Even then, these distinguishing features are sometimes modified until only an expert can recognize them.

The Phylum Annelida may be divided into four classes:

Class I. Chaetopoda

Order 1. Polychaeta

Order 2. Oligochaeta

Class II. Archiannelida

Class III. Hirudinea

Class IV. Gephyrea

Order 1. Echiuroidea

Order 2. Sipunculoidea

Class Chaetopoda (kè tŏp'ó dả, hair and foot).--This class includes the most commonly known forms of the phylum. There are marine, 
fresh-water, and terrestrial forms; and they all possess setae (chaetae), or bristlelike appendages on the body segments. The setae are chitinous and are embedded in pits of the integument. They bear muscle attachments which make them movable and therefore useful in locomotion. The coelom, which surrounds the straight digestive tract, is divided between the segments by partitions known as septae.

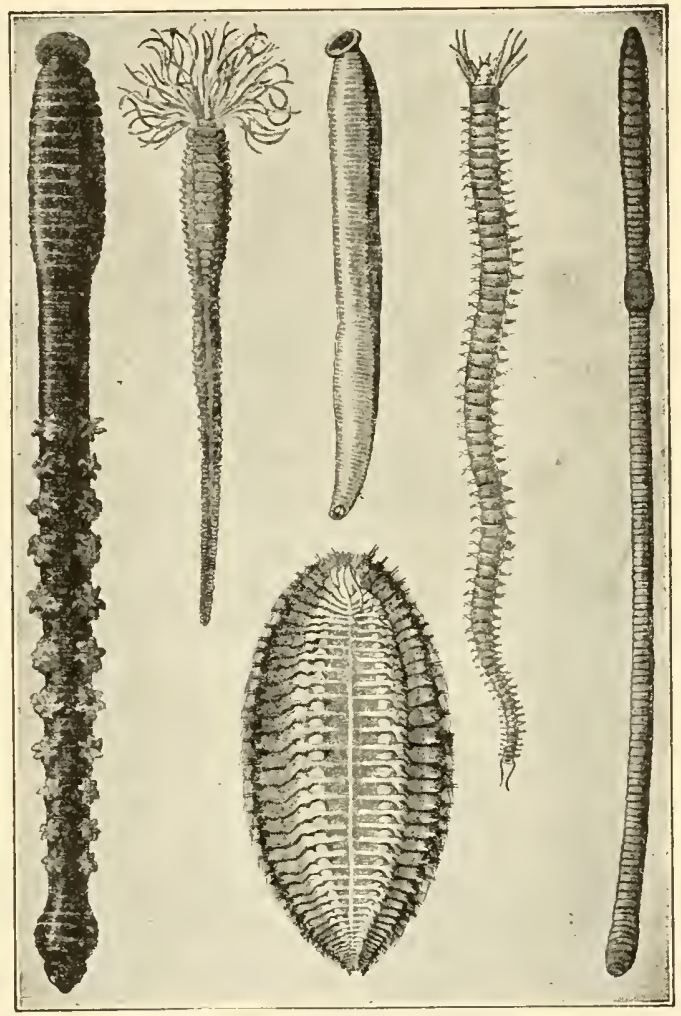

Fig. 99.-Representative annelids. From left to right, Arenicola cristata, lug worm; Amphitrite ornata, marine annelid with branching gills; Hirudo medicinalis, large medicinal leech (upper center) ; Aphrodita ornata, sea mouse (lower center): Nereis virens, sand worm or clam worm; Lumbricus terrestris, earthworm or angleworm. (Courtesy of Denoyer-Geppert Company.)

Typically, each coelomic space possesses a pair of nephridial tubules which communicate with the coelom at one end by means of a ciliated, funnellike opening, the nephrostome. The other end opens to the outside by means of a nephridiopore. The nephridia remove nitrogenous waste materials from the coelomic cavities and from the blood. 
The inner body wall of each segment is made up of an inner longitudinal layer and an outer circular layer of muscle. Segmental nerves which are derived from segmental nerve ganglia innervate the metameres and coordinate the movements of the body. The segmental ganglia communicate with each other through connections extending from one segment to the other. At the anterior end is the brain, which is composed of a suprapharyngeal and a subpharyngeal ganglion joined together by a pair of commissures. The brain, however, has little to do with the coordination of different parts of intersegmental and intrasegmental reflexes, so that the stimulation in one segment automatically stimulates the adjoining ones. Reactions which require immediate coordination of the whole body are controlled by three giant nerve fibers which run through the entire length of the nerve chain. The primary function of the suprapharyngeal and subpharyngeal ganglia is to relay sensory impulses.

The principal vessels of the circulatory system are a dorsal one, through which the blood moves forward, and a ventral one through which the blood moves posteriorly. These are connected in the anterior region of the body by a varying number of paired, segmental hearts or comnectives. The dorsal vessel exhibits wavelike contractile movements (peristaltic contractions) which force the blood anteriorly. The latter passes through the hearts, which also pulsate, then backward through the ventral vessel to the skin, intestine, and other organs. Hemoglobin is suspended in the blood plasma of some Chaetopoda; in others, a green pigment known as chlorocruorin is found; in still others no known blood pigment occurs. The principal vessels and hearts have valves on their inner surfaces which prevent the blood from flowing in the wrong direction.

The class Chaetopoda may be divided into two orders; namely, (1) the Polychaeta and (2) the Oligochaeta.

Order Polychacta.-The polychaetes (many bristles) are typically marine Cliaetopoda. One of the most widely known forms of this group is Nereis virens or the clamworm, which may be studied as a representative form. It possesses many setae (chaetae) located in fleshy parapodia. In this case the parapodia with their setae constitute the segmental appendages. The parapodium is divided into a dorsal notopodium and a ventral neuropodium, and each surrounds a large seta, or aciculum, which serves as a point of attachment for 
the parapodial muscles. A dorsal and a ventral cirrus are usually present. The notopodium and the neuropodium each have a large group of setae. The parapodia are used principally as locomotor and respiratory organs.

The head of Nereis seems to have resulted from the fusion and specialization of the anterior segments. It is composed of a prostomium, which bears a pair of tentacles, a pair of palps, and two pairs of eyes. The peristomium constitutes the first segment and bears four pairs of cirri or tentacles. The pharynx is equipped with muscles by which it can be everted, and a pair of chitinous jaws which protrude

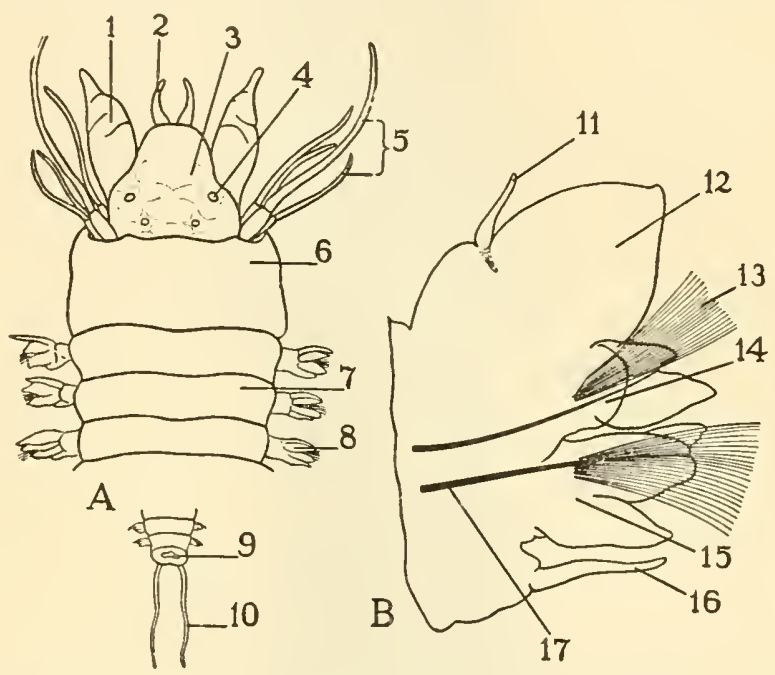

Fig. 100.-External anatomy of Nereis virens and parapodium. A, anterior end and posterior end; $B$, parapodium (enlarged); 1 , palp; 2 , terminal tentacle; 3 , prostomium; 4, eye ; 5 , lateral tentacles; 6 , peristomium; 7 , segment; 8 , parapodium; 9, anus; 10, anal cirrus; 11, dorsal cirrus; 12, gill plate; 13, setae (chaetae): 14, notopodium; 15, neuropodium; 16, ventral cirrus; 17, aciculum. (Courtesy of General Biological Supply House.)

when the pharynx is extended. The jaws serve in capturing small organisms and crushing anything which is to be swallowed. The succeeding segments are all alike except the posterior one which bears a pair of ventral cirri extending posteriorly.

The digestive tract consists of an essentially straight tube. The mouth opens directly into the muscular protrusible pharynx, which may be everted by use of protractor muscles to form a sort of prohoscis. The pharynx leads into the relatively narrow esophagus 
which extends through about six segments and which has a digestive gland opening into it from both sides. The remainder of the digestive tract is a straight intestine which continues to the last segment, where it opens to the outside.

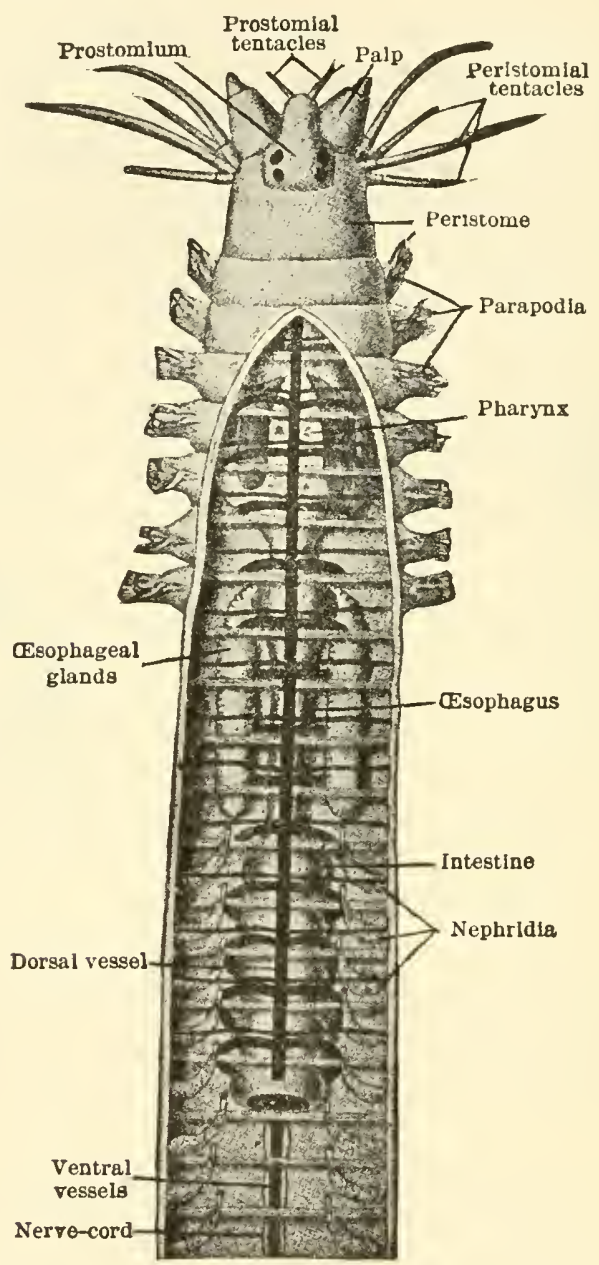

Fig. 101.-Internal anatomy of Nereis virens. (From Hegner, College Zoology, published by The Macmillan Company, after Parker and Haswell.)

The circulatory system is composed principally of a dorsal and a ventral blood vessel joined in each segment by a pair of connecting vessels. The blood is forced anteriorly through the dorsal vessel and 
passes posteriorly through the ventral one. Its movement is effected by wavelike contractions in the walls of the dorsal vessel. It reaches the parapodia and digestive tract through lateral branches of the ventral vessel and is then returned to the dorsal one by parietal branches.

Each segment of the body except the peristomium has two nephridia opening directly from the coelom to the outside. The nephridium consists of a ciliated funnel, nephrostome, and a coiled tubule which ends in its external opening, the nephridiopore. The nephridia serve to convey the excretory and reproductive products to the outside. The sexes are separate and there are gonads in all the segments except those in the anterior end of the body. The sex cells arise from the walls of the coelom and when ripe pass to the outside, fertilization taking place in the water. The fertilized egg develops into a trochophore larva, which metamorphoses into the adult animal.

In the central nervous system there are two suprapharyngeal ganglia dorsal to the pharynx. These are connected by means of commissures to the subpharyngeal ganglion ventral to the pharynx. A nerve chain, composed of segmental ganglia joined by intersegmental connections, extends posteriorly on the ventral side of the body to the anal segment. Lateral nerves from the ventral nerve chain innervate the various organs of the worm. Two eyes receive nervous connections from the brain and the animal is apparently able to detect moving objects.

Order Oligochaeta.-The best known example of the order Oligochaeta is Lumbricus terrestris, the common earthworm, which is used almost universally as a laboratory specimen. Lumbricus is not as common in the Southwest as are other large forms of earthworms, (Dipocardia) but is used here as an example because it is so well known and because its features represent so well those common to the entire order.

\section{EARTHWORMI}

The body of Lumbricus terrestris varies from six to fourteen inches in length and gives the appearance of a number of rings joined in a linear arrangement. The rings are the body segments, or metameres, and vary in number up to 175 . In the adult the number of segments from the anterior end to the posterior end of the clitellum 
remains constant, while the number posterior to this varies. This is because growth is accomplished by the addition of segments posterior to the clitellum.

The prostomium is a sort of knoblike lobe at the anterior end, projecting out over the mouth. It is not considered a true metamere. The first segment is incomplete due to the opening of the
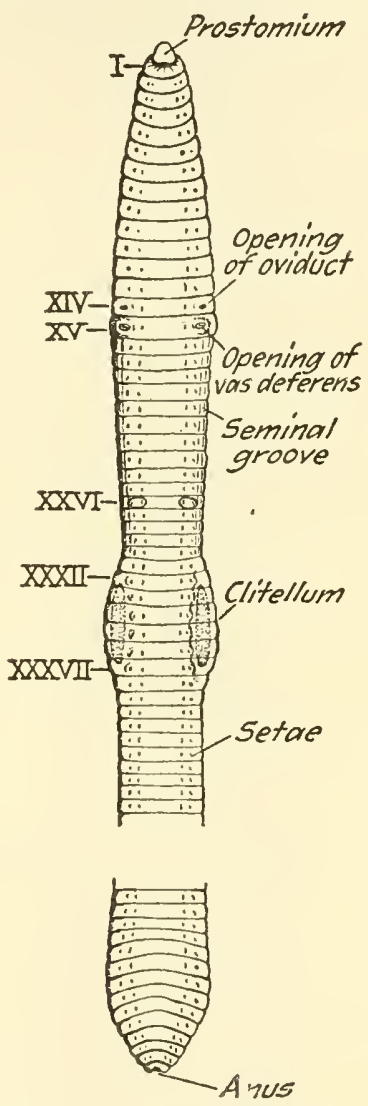

Fig. 102.-External anatomy of earthworm, ventral view, segments in roman numerals. (From Wolcott, Animal Biology, published by McGraw-Hill Book Company, Inc.)

mouth through its ventral side. In studying the earthworm it is customary to number the segments with Roman numerals beginning at the anterior end. This simplifies the study because external as well as internal structures are definitely related to specific seg- 
ments. The openings of the oviducts through which the eggs pass to the outside are seen as minute pores, one on each side of segment XIV. The pores of the seminal receptacles occur in pairs, one pair in the groove between segments $\mathrm{IX}$ and $\mathrm{X}$, and one between $\mathrm{X}$ and XI. The openings of the vasa deferentia (sperm ducts), which convey sperms to the outside, are located, one on each side, in the anterior part of segment XV. In sexually mature worms, segments XXX, XXXI, or XXXII to segment XXXVII are swollen to form the clitellum, a sort of saddle-shaped structure, the function of which is to secrete the cocoon in which eggs are deposited during reproduction.

Each segment except the first and last bears four pairs of chitinous setae. They are fine, stiff bristles which may be located by passing the hand lightly over the worm. They are moved by protractor and retractor muscles and serve to help the worm move through the soil. A pair of nephridiopores (the external openings of nephridia) is situated on the posterior ventral side of each segment except the first two or three.

The body of the earthworm is covered by a thin, transparent cuticle which is secreted by the epidermal cells just beneath it. It contains numerous minute pores through which secretions of the unicellular glands beneath are poured and through which gaseous exchanges between the blood and moist soil can take place. It serves also as a protection against physical and chemical injury to the animal's body.

\section{Internal Anatomy}

The body of the earthworm, if cut open along the mid-dorsal line, gives the general appearance of a tube within a tube, the digestive tube being the inner one and the body wall the outer one. The space between them is the coelom. The constricted regions dividing the segments on the outside correspond to the positions of the septae which divide the coelom into separate segmental compartments. These coelomic divisions communicate with each other by means of pores in the septae so that the clear fluid which fills the coelom can circulate freely. The septae are absent between segments I and II and incomplete between segments III and IV, and XVII and XVIII. The walls of the coelom are lined by a thin layer of cells known as peritoneum (mesothelium). 


\section{Reproductive Organs}

The earthworm is hermaphroditic, the organs of both sexes being present in every animal. The seminal receptacles, oviducts, and ovaries are female organs, and the testes and seminal vesicles are male organs. The seminal vesicles are three pairs of light-colored bodies located in segments IX, XI, and XII. In sexually mature individuals they may extend back through the septae as far as the fifteenth segment. If their contents are examined with a microscope, they

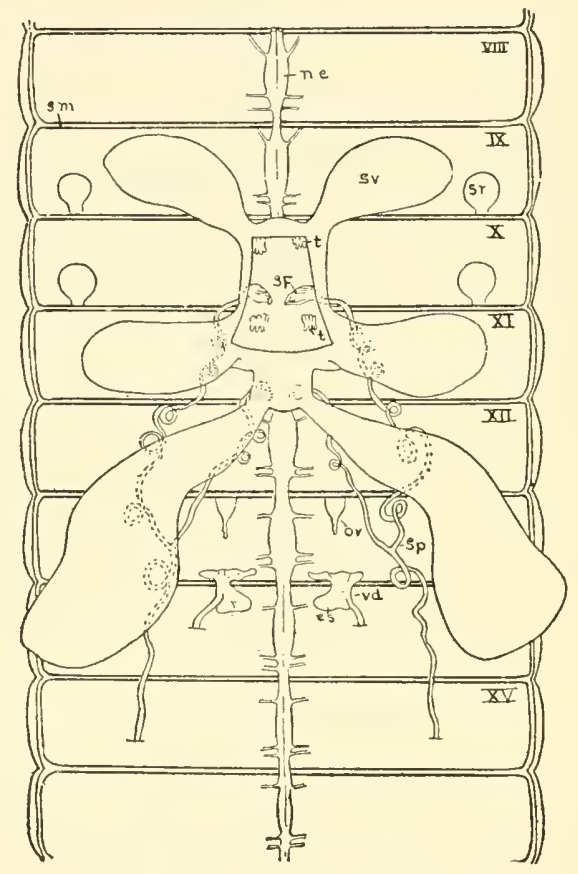

Fig, 103.-Diagram showing reproductive system and nervous system in segments VIII to XV of an earthworm. The seminal vesicles have been cut away in somites $\mathrm{X}$ and $\mathrm{XI}$ to disclose the testes and sperm funnels. es, egg sac; nc, nerve cord: $o v$, ovary; $s f$ seminal funnel: $s m$, septum between two somites; $s p$, sperm duct (vas deferens) opening in the fifteenth somite; $s r$, seminal receptacle; $s v$, seminal vesicle; $t$, testis; vd, oviduct. (From White, General Biology, published by The C. V. Mosby Company.)

are seen to contain the various stages of developing spermatozoa coming from the sperm mother cells. The testes are the two pairs of very minute bodies projecting into the seminal vesicles in segments $\mathrm{X}$ and $\mathrm{XI}$ and cannot be seen without first removing the dorsal part of the seminal vesicles. The union of the vasa effer- 
entia coming from the vesicles on each side forms a single pair of vasa deferentia in segment XII. The seminal receptacles are pairs of small white bodies located in segments IX and $\mathrm{X}$. The ovaries are two minute bodies located one on each side of segment XIII.

\section{Digestive System}

The mouth cavity extends through segments I to III and leads into the bulbous, muscular pharynx which extends through segment V. The pharynx plays the part of a sucker in securing food for

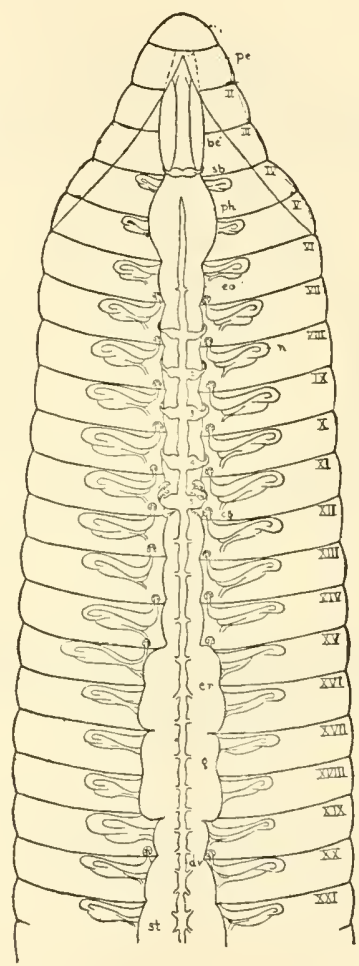

Fig. 104.-Diagram of dorsal dissection of an earthworm in reglon of segments I to XXI. $b c$, buccal cavity; $c g$, calciferous glands; $c r$, crop: $d v$, dorsal blood vessel; eo, esophagus; $g$, gizzard ; $n$, nephridium ; $s b$, subpharyngeal ganglion; $s t$, stomach-intestine; pe, peristomium ; $I I-X X I$, somites; $p h$, pharynx; pr, prostomium. (From White, General Biology.)

the animal. The esophagus is a straight narrow tube extending from the pharynx through the fourteentl segment. In segments X to XII three pairs of yellow lateral pouches open into it. These are the 
calciferous glands, the secretions of which help to neutralize the acid organic matter taken as fooc. The esophagus opens into the crop, a larger, thin-walled structure, which extends through segment XVI. This is followed by the muscular gizzard in segments XVII and XVIII. A thin-walled intestine extends to the anus, which opens to the outside throngh the last segment.

The intestine is not a simple tube but has a large fold, the typhlosole, protruding into its lumen from the dorsal side giving it more absorptive surface for the assimilation of food. The coelomic side of

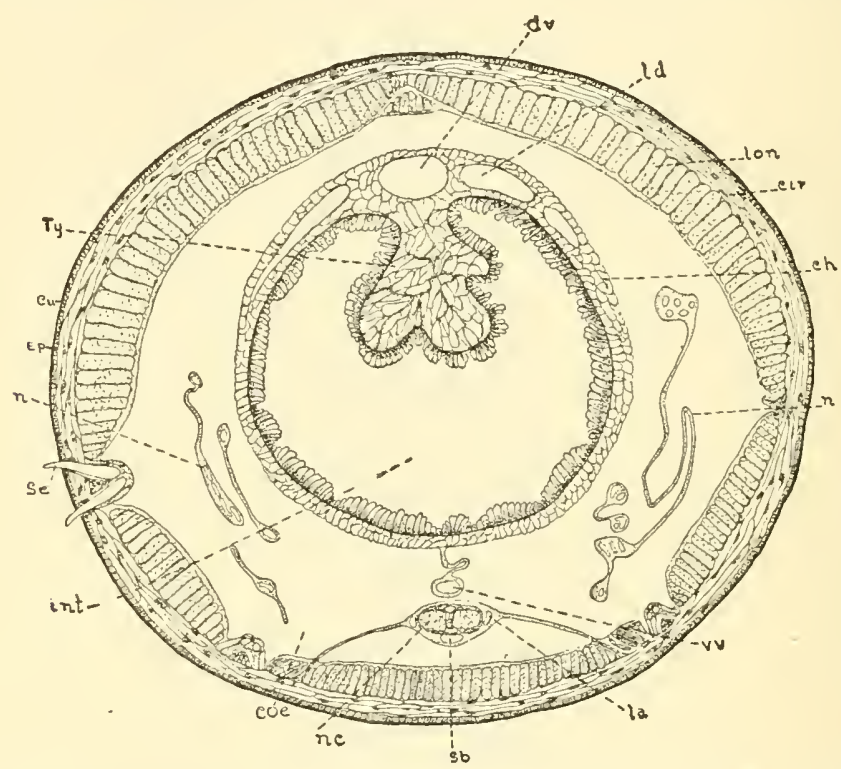

Fig. 105.-Cross section of the earthworm through a posterior segment. ch, chloragogue cells; cir, circular muscle fibers; coe, coelom; cu, cuticle; $d v$, dorsal blood vessel; eq, epidermis: int, intestine; la, lateral neural vessel; $l d$, lateral branch of dorsal vessel: lon, longitudinal muscle fibers; $n$, nephridium; $n c$, nerve cord; $s b$, subneural blood vessel; se seta, $t y$, typhlosole; vv, ventral blood vessel. (From White, General Biology.)

the intestine is covered with a layer of brown cells, known as chioragogen cells, whose function is doubtful. They are generally believed, however, to play a part in the excretion of nitrogenous wastes.

The food of the earthworm consists of almost any kind of organic matter which may pass through its digestive tract. The animals remain in the soil during the daytime and work their way through 
it by passing it continually through the digestive tract. At night they come to the surface of the ground, usually remaining partly within or very close to the burrow, and feed on dead organic matter, such as leaves. Food is drawn into the mouth by suction produced by the muscular pharynx. In the pharynx it receives the secretions from the pharyngeal glands and is then passed on through the esophagus, where it receives the secretions from the calciferous glands. It is then passed into the crop and is stored there long enough for the secretions of the calciferous glands to neutralize the organic acids which may be present in the food. It is then passed into the gizzard, where it is ground by contractions of the muscular walls of that organ. This process is aided by sand grains which are swallowed along with the food. From the gizzard the food is passed into the intestine where digestion is completed and the absorption of digested materials is accomplished.

\section{Circulatory System}

The blood of the earthworm consists of a clear liquid plasma in which there are numerous colorless cells. The red color of the blood, as seen in a living specimen, is due to a pigment known as hemoglobin suspended in the plasma and not in the corpuscles as is the case in many animals. A complicated system of blood vessels makes up the circulatory path of the blood. The principal ones are: (1) the dorsal blood vessel, (2) the paired hearts (usually five) in segments VII to XI, (3) the ventral blood vessel, (4) the subneural trunk, (5) the parietal vessels, (6) the typhlosolar vessel, and (7) the intestinointegumentary vessels. The dorsal vessel conveys the blood anteriorly and forces it along by wavelike contractions. The paired hearts receive the blood from the dorsal vessel and by pulsating movements force it into the ventral vessel which distributes it to the body wall, the nephridia, and the intestine. In the intestine food is taken up; in the skin gaseous exchanges are made with the water in the moist soil; and in the nephridia the nitrogenous wastes are removed. The lateral neural vessels receive freshly oxygenated blood from the skin; hence the nervous system receives the most highly oxygenated blood. From the lateral vessels it passes into the subneural, where it flows posteriorly, and then returns to the dorsal vessel by way of the parietal connectives. The blood flows from the intestine through the typhlosole into the dorsal vessel by dorso-intestinal vessels. An- 
terior to the hearts the dorsal vessel carries the blood posteriorly and the ventral vessel carries it anteriorly. The circulatory system is equipped with numerous valves which keep the blood from flowing in the wrong direction.

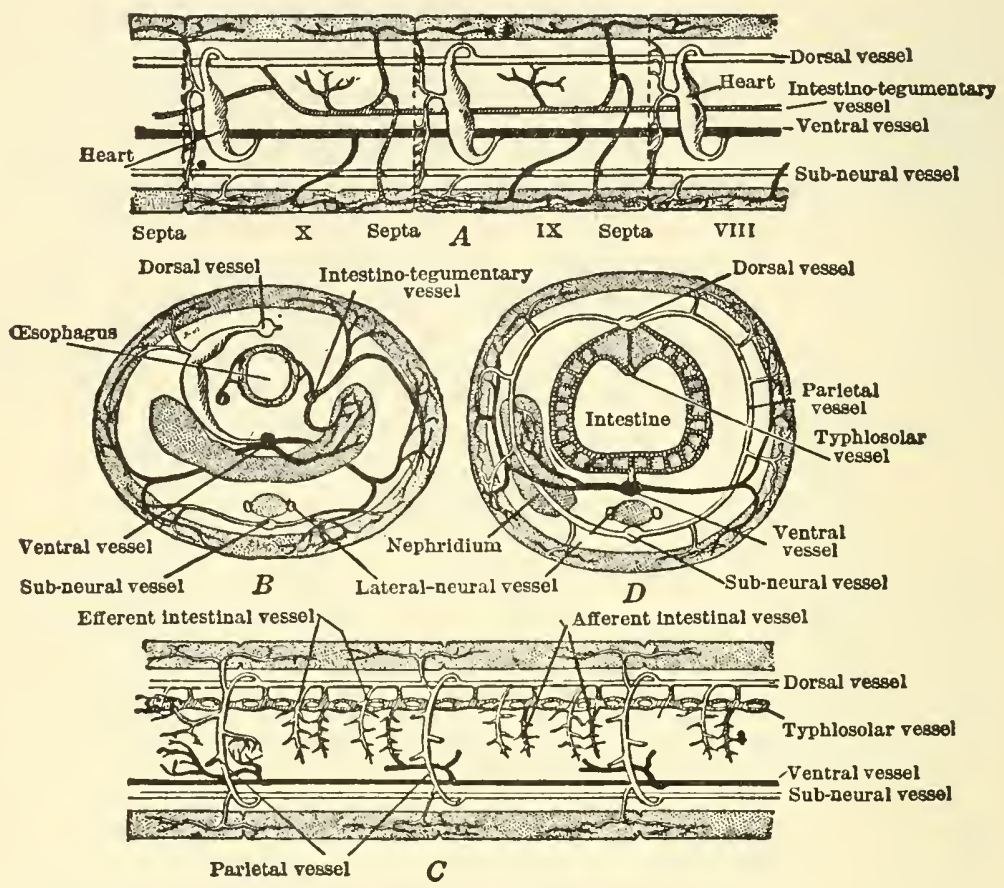

Fig. 106.-Circulatory system of the earthworm. $A$, longitudinal view of vessels in somites VIII, IX, and $\mathrm{X} ; B$, transverse section of same region; $C$, longitudinal view of the intestinal region; $D$, transverse section of the same region. (From Hegner, College Zoology, published by The Macmillan Company, after Bourne, after Benham.)

\section{Respiratory System}

Respiration in the earthworm is carried on through the skin which is well supplied with blood. Since the animal always lives in a moist environment, this type of respiration is possible.

\section{Excretory System}

The function of excretion is cared for principally by the paired nephridia, which are found in each segment except the first two and the last one. A single nephridium consists of a ciliated funnel (the nephrostome), a thin coiled tube, and a nephridiopore. The cilia of the nephrostome create a current which takes the fluid containing 
nitrogenous wastes from the coelom into the tubule where it can pass to the outside through the nephridiopore. Also the wastes in the blood are excreted by way of the nephridial tubules. The nephrostome is located in the posterior part of the segment and leads into the tubule of the segment just posterior to it. The nephridium coils two or three times before reaching the nephridiopore.

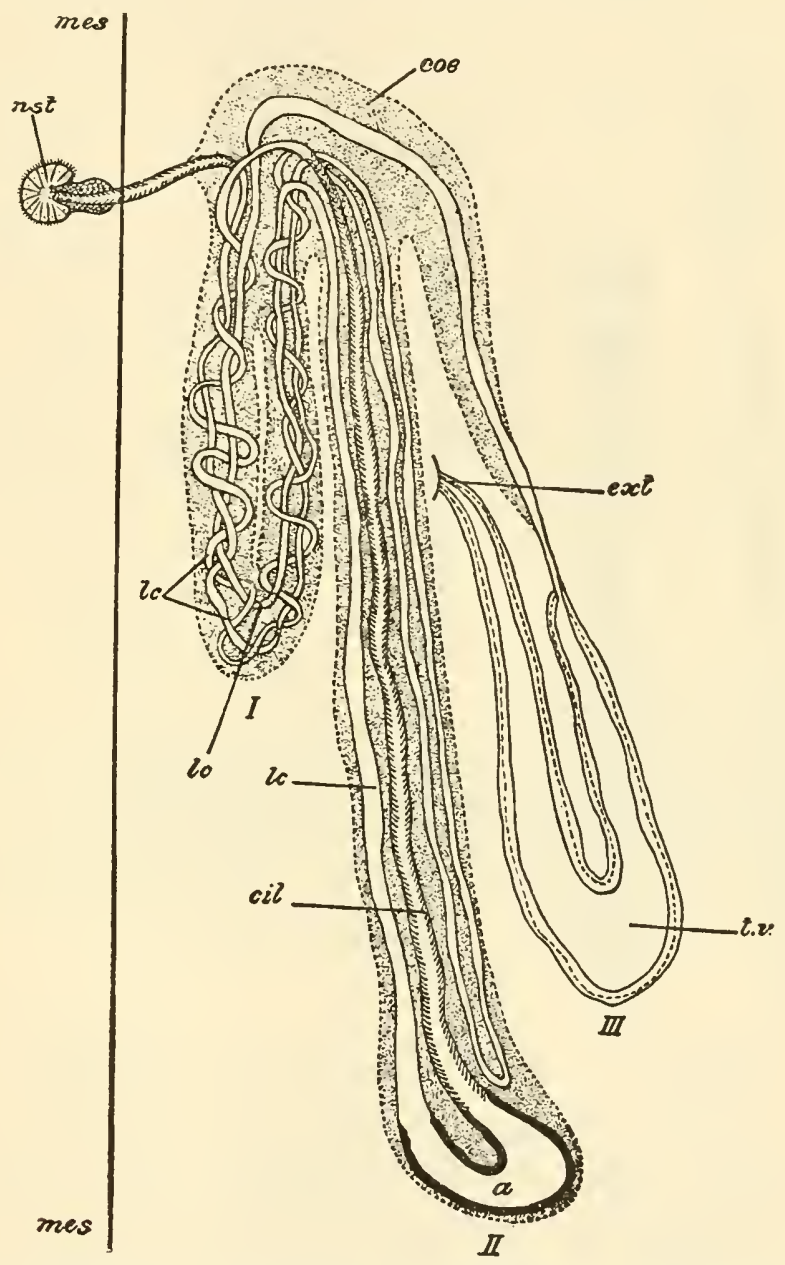

Fig. 107.-Structure of a nephridium from earthworm. $a$, ampulla between the ciliated and nonciliated portions of the intracellular canal ; cil, ciliated canal; coe, part derived from coelomic epithelium; ext, external opening (nephridiopore) : $l c$, nonclliated canal; mes, septum between somites; nst, nephrostome (internal opening); tu, intercellular canal. (From Parker and Haswell: Textbook of Zoology, published by The Macmillan Company, after Meisenheinmer, after Mazairski.) 


\section{The Nervous System.}

The "brain" of the earthworm consists of the suprapharyngeal ganglion, two circumpharyngeal connectives, and the subpharyngeal ganglion. The ventral nerve cord extends posteriorly the length of the body with a ganglion and three pairs of nerves in each segment.

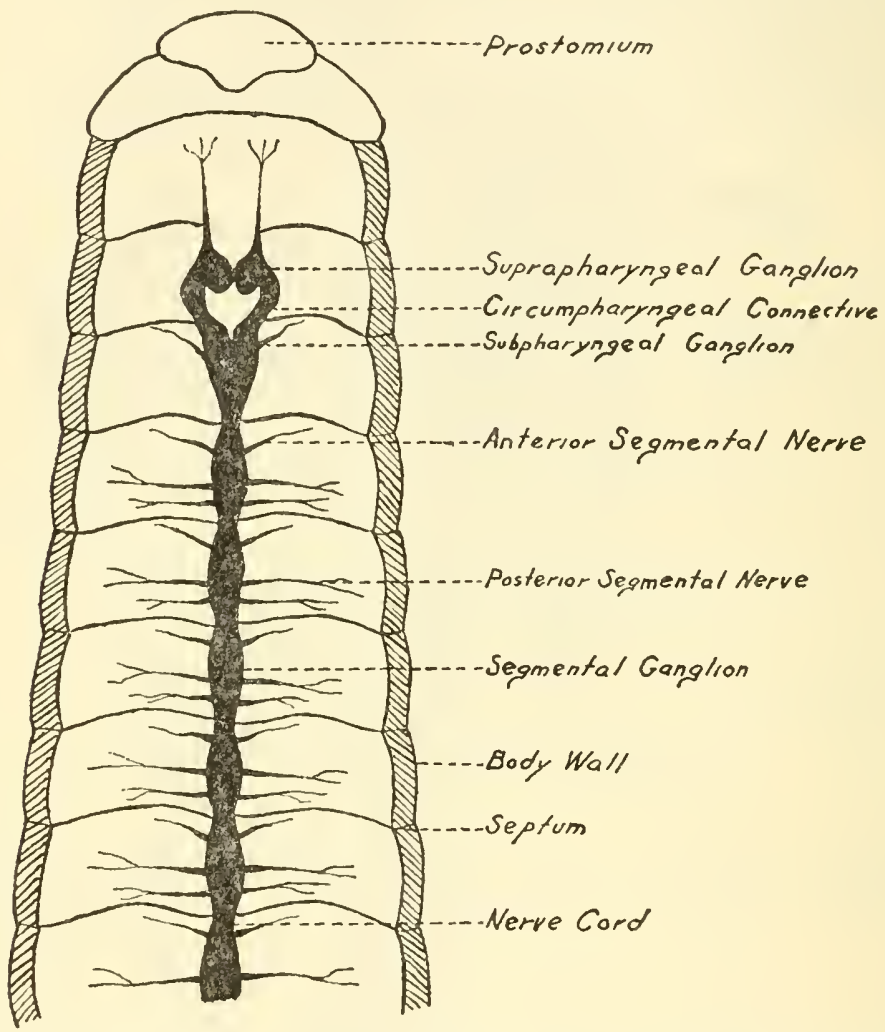

Fig. 108.-Anterior portion of the nervous system of an earthworm from dorsal view.

Each ganglion is really the fusion of two, a deviation from the condition found in many annelids and artluropods where there are two ganglia in each segment and the nerre cord is double. The suprapharyngeal ganglion lies dorsal to the pharynx in the third segment and the subpharyngeal ganglion lies ventral to the pharynx in the fourtl segment. Nerves from these two ganglia innervate the first three segments and the prostomium. 
Stimuli are received by sensory cells and are passed into the ventral nerve ganglia by the afferent nerves. The stimulus is modified in the ventral ganglia and sent to the responding organs by efferent neurons. Nerve impulses then have the nature of a simple reflex except that the ventral ganglia are connected by association neurons which conduct stimuli from one to the other. Because of this arrangement a stimulus applied to any part of the body will cause responses to occur in a wavelike manner in both directions from the point of stimulation. Located in the dorsal part of the nerve cord are three giant fibers which serve as the sole means of conducting an impulse directly from one end of the body to the other. By this means the worm can contract its entire body at one time.

\section{Reproduction}

As has already been described, the earthworm is hermaphroditic. Self-fertilization does not occur, however, each egg being fertilized by a sperm from another individual. In reproduction two animals come together with their anterior ends pointing in opposite directions and the ventral surfaces of their bodies in close contact from
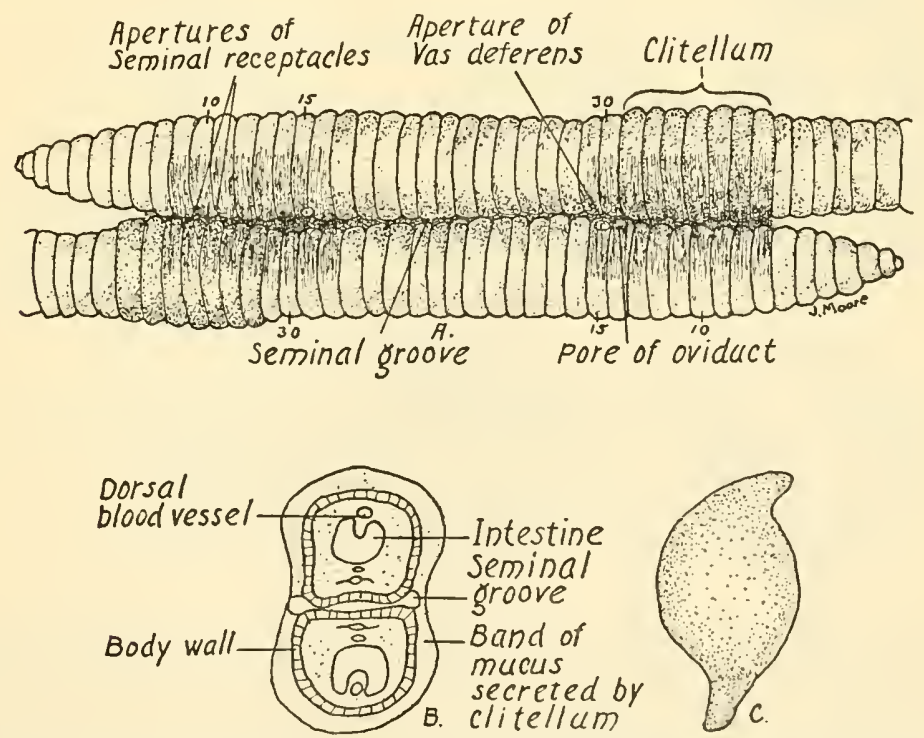

Fig. 109.-Reproduction in earthworm showing copulation and the cocoon. $A$, two worms enclosed in bands of mucus; $B$, transverse section showing the seminal grooves; $C$, cocoon. 
the anterior end to the clitellum. With their bodies in close contact a closed passage is formed between the genital openings of the two individuals. Sperms pass from the testes out through the seminal vesicles and vasa deferentia to the closed passage and move through it
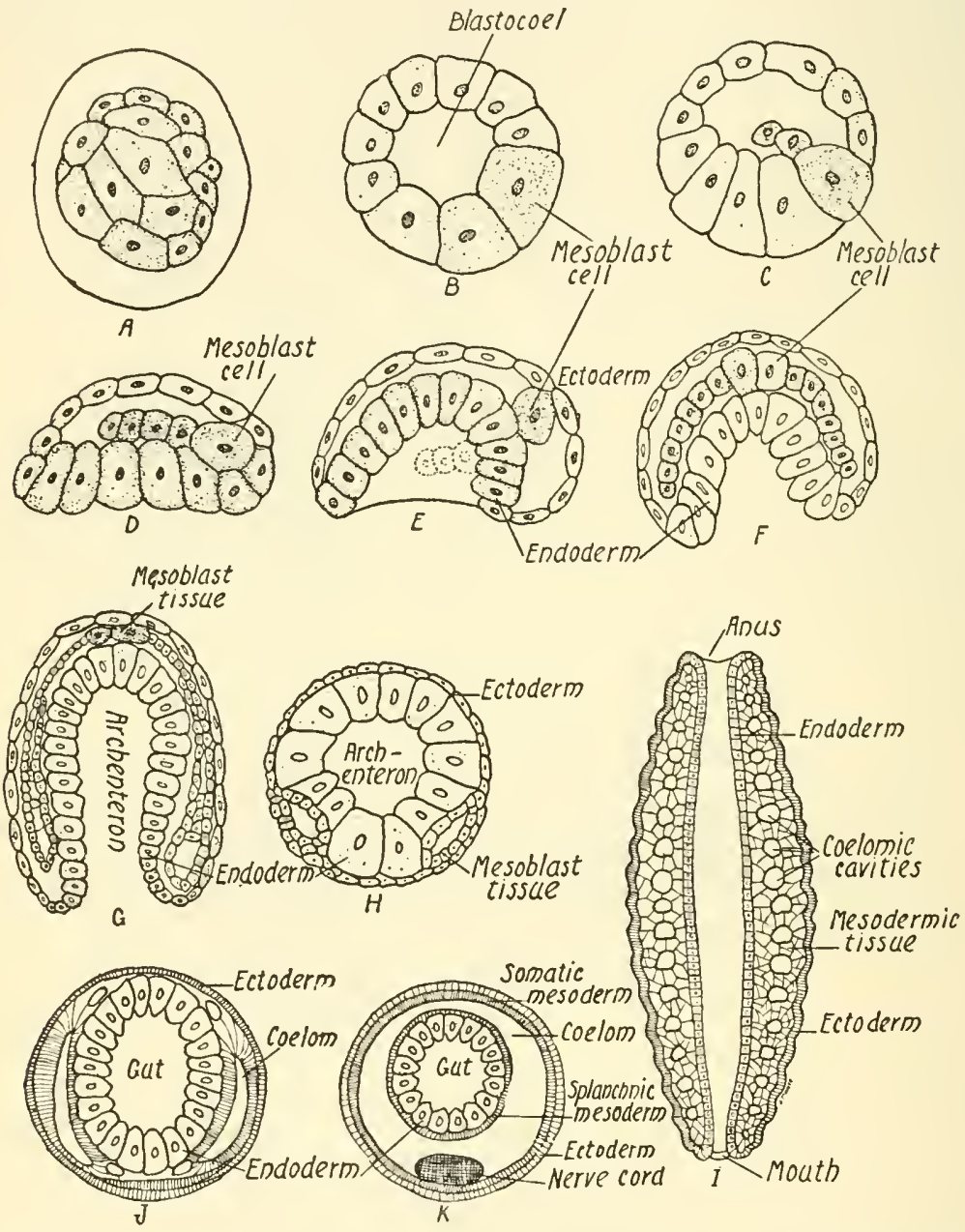

Fig. 110.-Development of the earthworm. A, blastula, surrounded by a membrane: $B$, section of a blastula showing blastocoele and one of the mesoblast cells (primary cell) of mesoderm layer; $C$ and $D$, showing stages in the beginning of gastrulation: $E$, side view of gastrula showing invagination; $F$, section of gastrula along a line to show polar cells, mesoderm layers on each side of them and the archenteron; $G$, later stage showing cavities in the mesoderm; $H$, gastrula in cross section; $I$, longitudinal section of a young worm after formation of the mouth and anus: $J$, same as $i$ but in cross section; $K$, cross section of later stage. (After Wilson, Embryology of the Earthworm, Journal of Morphology, 1889.) 
to the seminal receptacles of the mate, where they are stored. In the meantime, the clitellum of each individual secretes a band which binds them together at these two points. After each has received sperms from the other, they separate by working themselves through the bands secreted by the clitella. This leaves each animal with a band which is gradually worked off toward the anterior end. As the band passes over the openings of the oviducts eggs are released into it, and as it passes the openings of the seminal receptacles sperms which came from the reproductive mate are released.

Both ends of the band close, forming a cocoon in which fertilization and development take place.
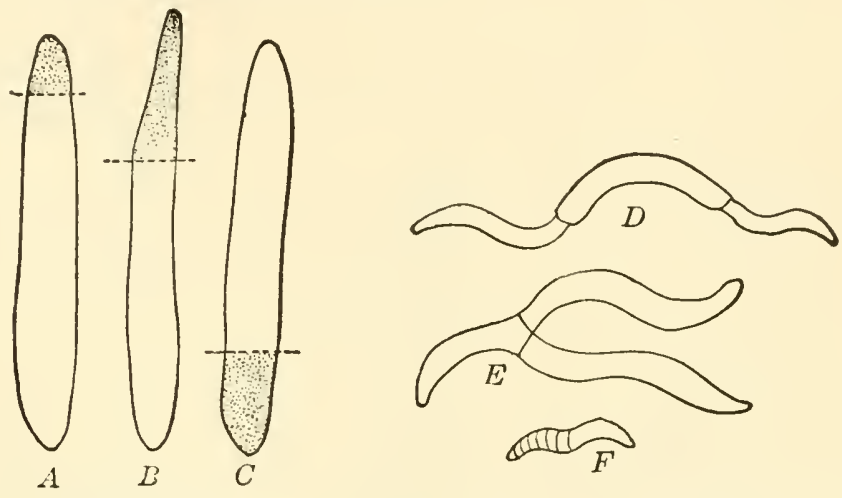

Fig. 111.-Regeneration and grafting in earthworm. $A$, anterior five segments regenerated from posterior part of a worm; $B$, tail regenerated from the posterior portion of a worm; $C$, tail regenerated from an anterior piece of a worm; $D$, union of three pieces to make a long worm: $E$, grafting of two pieces to form a double bodied worm; $F$, anterior and posterior pieces grafted together to form a new worm. The stippled areas in $\mathrm{A}, \mathrm{B}$, and $\mathrm{C}$ represent regenerated tissue. (From Hegner, College Zoology, published by The Macmillan Company, after Morgan.)

Cleavage in earthworms is of the unequal holoblastic type. Soon after the segmentation cavity is formed, a certain cell, known as the mesoblast cell, is set off, and the cells resulting from its divisions move into the cleavage cavity, and will form the mesoderm. As the mesoblast cells move into the cleavage cavity, gastrulation occurs by invagination to form the endoderm and ectoderm. The gastrula elongates and the archenteron opens at both ends to form the mouth and anus. The mesodermal cells which fill the space, between the ectoderm and endoderm develop segmental cavities which are the coeloms of the metameres. At this stage the animal constitutes a tube within a tube and from it the organs of the adult develop. 


\section{Regeneration}

Earthworms have becn used extensively in regeneration experiments because they possess the ability to regenerate lost parts. It has been demonstrated that when the anterior end is cut off, in front of the eighteenth segment, the segments from one to five will be regenerated. If the cut is made posterior to segment eighteen a new anterior end will not regenerate on the tail half, but instead another tail will develop from the cut surface. This produces an animal with two tails and no head, and death from starvation results. When any part of the tail region is cut off, the lost parts readily regenerate. Numerous grafting experiments have also been performed on earthworms. Almost any part of an individual grafted to the cut surface (if properly located) of another will fuse to it and grow. In this way numerous unusual forms of earthworms have been produced.

Class Archiannelida (är kĭ ă nĕl’ú dả, first Annelida).-This class includes numerous small marine forms which resemble Chaetopoda in a number of ways. It is now believed that they have been derived from that group by changes usually involving the reduction or loss of certain structures. They are very small and lack both setae and parapodia. Internally they are very similar to the earthworm. The best known example of this group is Polygordius, which has a long cylindrical segmented body with a pair of tentacles on the prostomium. Two ciliated pits are present as a retention of juvenile characters. The trochophore larva is common to the entire group.

Class Hirudinea (h̆̌r t̆ dìn'è ả, leech). -These animals are commonly known as the leeches. They are usually flattened dorsoventrally, possess both an anterior sucker and a posterior sucker, have characteristically thirty-two segments and possess no external appendages. The anterior sucker is formed from the prostomium and first two segments, and the posterior one comes from the last seven. Each segment shows externally a variable number of annuli or rings, making the animal appear to possess more segments than are really present. Lecches are commonly parasitic and live by sucking blood from other animals.

In a typical leech, of which Hirudo medicinalis is a good example, there is a muscular pharynx, a short esophagus, midgut or crop, 
intestine, and ectodermal rectum. Blood which is sucked from another animal receives a ferment from the salivary glands of the pharynx, which prevents it from coagulating. It is then stored in the diverticulae of the crop. The animal is capable of ingesting three times its own weight in blood, and, since several months may elapse before it is all digested, frequent feedings are not necessary. The

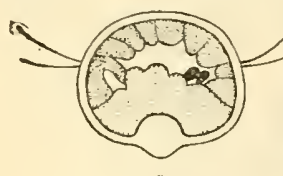

A
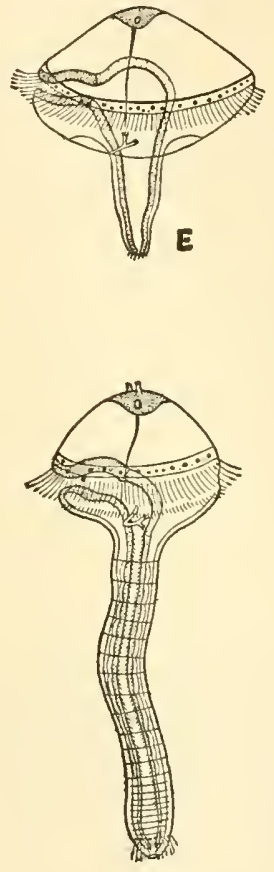

$\boldsymbol{F}$

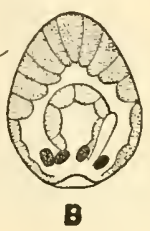

B

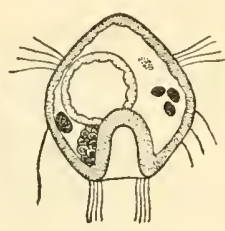

C

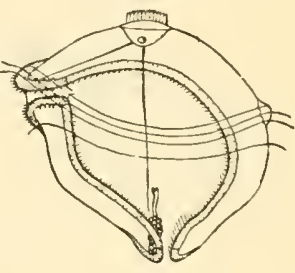

D

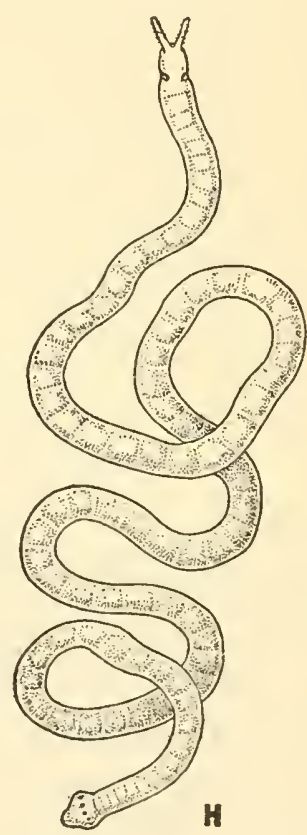

Fig. 112.-Development stages of Polygordius, an archiannelid. A, blastula: $B$, gastrula; $C$, early trochophore larva; $D$, optical section of trochophore larva showing apical plate with eyespot, head kidney; and preoral band of cilia; $E$, trochophore larva with posterior growth region; $F$, segmenting larva: $G$, advanced larval condition in which the trochophore larva is seen with head, mouth, eyespots, and tentacles: $H$, adult worm, showing faint external segmentation. (From Newman, Textbook of General Zoology, published by The Macmillan Company, after Whitman.) 
coelom is very much reduced due to the excessive development of the mesodermal tissue. Each animal is hermaphroditic. Sperms are placed on the skin of another leech, and they apparently work through it into the ovaries, where fertilization occurs. Development takes place in a cocoon produced by the clitellum. Two nephridia are present. The nervous system is typical of the annelids.

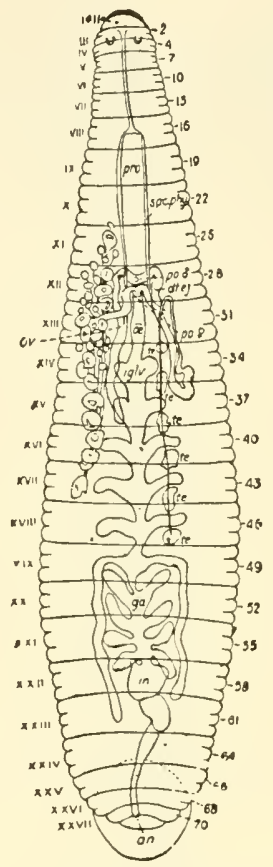

Fig. 113.-Glossiphonia fusca, a leech, showing annulation, segmentation, and internal organs. I to XXVII, somites; $2-70$, annuli ; $a n$, anus; $d t$. ej., ductus ejactulatorius; $g a$ and in, intestine; iglv, crop or stomach; oe, esophagus; ov, ovary; po o, male aperture; po ơ, female aperture; pro, proboscis : te, testes. (From Ward and Whipple, Fresh-water Biology, published by John Wiley \& Sons, Inc, as modified from Castle, Bulletin of Museum of Comparative Zoology.)

Class Gephyrea (jè fī rè'á, bridge). - This class is a group of marine annelids which are nonsegmented, have no appendages, and possess a trochophore larva. They are usually comparatively large and live in shells, erevices, and such other places as will afford protection. In this class, the representatives of the order Echiuroidea have a well-developed prostomium, used in capturing prey and in locomotion. In Bonellia, the female is the normal individual, while the 
male has no proboscis, is eiliated, and lives in the segmental organ of the female. Representatives of the order Sipunculoidea have no prostomium in the adult.

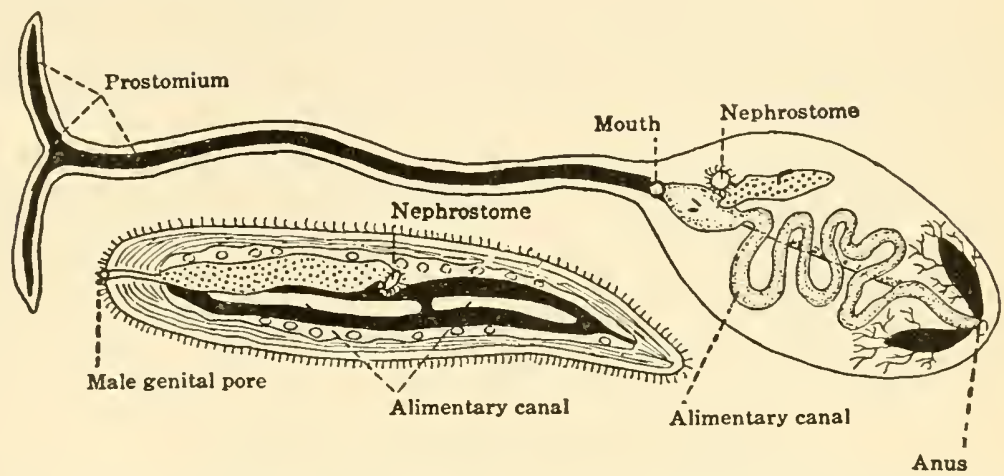

Fig. 114.-Bonellia viridis. Female (above) has bifurcated proboscis: male (below) is ciliated over the surface and much smaller. (From Hegner, College Zoology, published by The Macmillan Company.)

\section{Importance of Annelids to Man and Other Animals}

Even though no casual observer would consider that annelids have any important relationship to other living organisms, they have been found to be of great importance in a number of ways. Darwin concluded from some forty years of observation that the earthworms in an acre of ground could bring to the surface in one year as many as eighteen tons of feces, known as castings. This indicates without doubt that these animals are of great value, because in stirring the soil they cover up objects, causing them to decay. Their continuous burrowing through the soil also makes it porous, a necessary condition for plant growth.

Earthworms have also less desirable qualities. They serve as secondary hosts for parasites of several animals. Most of the parasites having the earthworm as a secondary host live as adults in birds, pigs, and other animals which use the worms as food.

They have ereated a serious problem in some of the irrigation districts of the Southwest by burrowing through levees until they are too porous to hold water. Before irrigation was started they did not appear to be at all numerous, but with the presence of water they have become very abundant. 
The medicinal leech was once used in the bleeding of individuals as a treatment for various ailments. Various forms of leeches live as parasites on turtles and other forms of animal life in the water. They are not at all averse to attacking human beings when the opportunity presents itself; however, they cause no great injuries and are important only as pests and as secondary hosts for some parasites, thus spreading certain diseases.

Marine annelids are important only as food for larger forms. In many regions the burrowing forms along the tide levels literally form a good grazing ground for fishes. The fish simply swim along, biting off that part of the worms protruding from the mud or sand. Instead of dying the injured worms regenerate one or more new heads and go about their business.

\section{Phylogenetic Advances of Annelida}

(1) Segmentation, (2) coelom, (3) alimentary canal with defined parts, (4) closed circulatory system, (5) excretory system of nephridia, (6) muscular system, (7) concentrated mid-ventral nerve cord connected to a dorsal pair of suprapharyngeal ganglia, (8) respiratory system (gills on parapodia of Nereis), and (9) improved sense organs (eyes, palps, and tentacles of Nereis). 


\section{CHAPTER XVI}

\section{PHYLUM ECHINODERMATA}

The Echinodermata (è kī nố dûr'má tá-hedgehog skin) constitute a rather backward phylum of animals which are thought to have undergone a certain amount of retrogression in structural features. That is, they seem to have a lower level of organization than that possessed by some of their ancestors. The modern echinoderms, as the group is commonly called, possess several distinctive characteristics. Some of these characteristics are as follows: skin covered with spines; lack of segmentation; triploblastic radial symmetry, subduing a primitive bilateral symmetry; water vascular or ambulacral system and tube feet; circumoral nerve ring and radial nerves; a calcareous skeleton composed of plates; pedicellariae; and a coelom. The external opening into the water vascular system is called the madreporite. It is located on the dorsal or aboral side, at an interradius betreen arms in such a position that a line drawn through it and on through the radius of the opposite ray, will divide the body into two similar halves. There will be a half ray and two complete rays in each half of a five-rayed animal divided in this way.

\section{Classification}

In earlier classifications as in the case of Cuvier, this entire group was included along with coelenterates in a group called Radiata. The basis for this was the apparent similarity of radial symmetry. It was later discovered that the coelenterates have a typical primitive radial symmetry while the Echinodermata have only a secondary radial symmetry which is derived from a bilateral condition. This is indicated quite definitely by the fact that the larvae of echinoderms have a typical bilateral symmetry. The change which occurs seems to be an adaptation to a sedentary habit. This phylum is usually divided into five classes of modern forms including such common animals as starfishes, brittle stars, sea urchins, sea cucumbers, and sea lilies.

Class Asteroidea.--The general features of the body include a central disc usually with five arms or rays radiating from it. There are some species which do not adhere to this pentamerous condition 
and have up to forty rays. The rays are not usually sharply constricted from the central disc. An ambulacral groove is present in each ray. This groove is formed by a particular arrangement of skeletal plates. Slieletal plates also support the aboral side of the central disc as well as the region around the mouth. Surrounding the spines on the skin are distributed some pincherlike calcareous

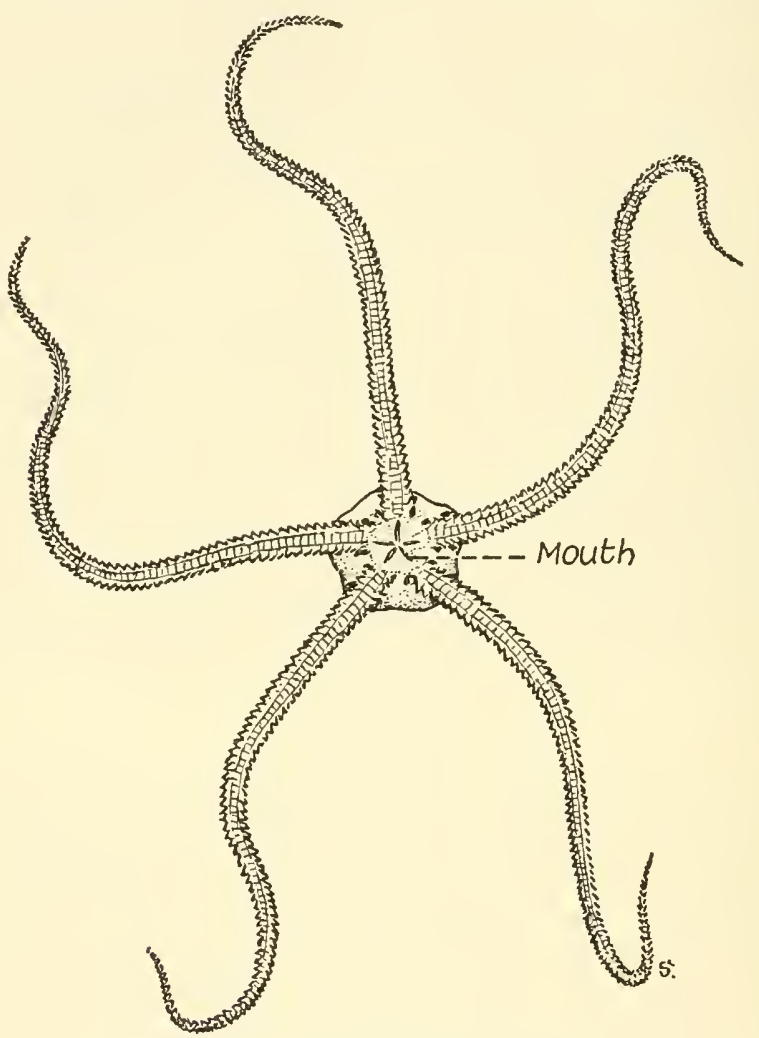

Fig. 115.-A brittle star, oral view.

organs called pedicellariae. These vary considerably in different species. The anus and madreporic plate are located aborally. The mouth is located on the oral or ventral side of the central disc, and the radiating parts of the vascular, ambulacral, and nervous systems lie orally along the ambulacral groove of each arm. Asterias, Astropecten, Pisaster, Solaster, Oreaster and Echinaster are representative genera. 
Class Ophiuroidea.-Brittle stars and serpent stars. There is a small central dise with five long, slender rays which are elearly marked off from the dise. The rays are lacking in ambulacral grooves. The tube feet do not serve in locomotor functions but are tactile only. Both the madreporite and mouth are located ventrally. On the oral side of the central dise, five interradial sets of skeletal plates project toward the mouth in the formation of jaws which are operated by muscles in chewing food. The anus is not present. The viscera are confined to the central disc. The brittle stars and serpent stars are quite active and live in the shore waters. At low tide they may be found under rocks and debris, but they move about and feed during high tide. The ability of autotomy

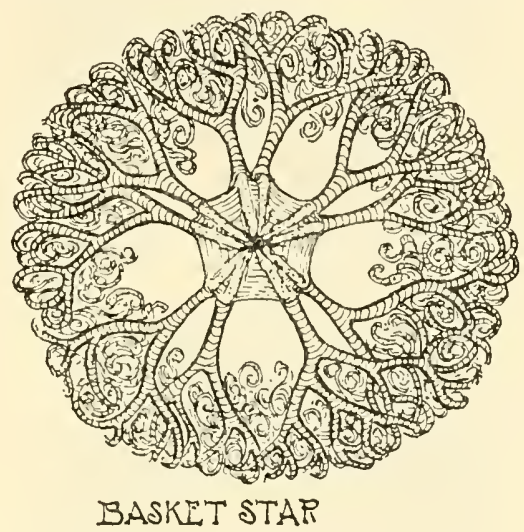

Fig. 116.-Oral view of a basket star belonging to class Ophiuroidea. (By courtesy of General Biological Supply House.)

(self-mutilation) is so well developed here that arms will become detached by merely grasping them. This makes it difficult to collect entire animals alive. Ophioderma, Ophiura, Ophiothrix and Gorgonocephalus are common Atlantic and Gulf Coast genera.

Class Echinoidea.--Sea urchins and sand dollars are representatives of this group the members of which have lost the rays but still retain the pentamerous (five division) condition of the body. The sea urchins are globular or hemispherical, while the sand dollars are disc-shaped. The skeleton or test is composed of five rows of closely fitting plates which are usually arranged into five pairs of inter-ambulacral rows. The position and condition of these rows 
of plates can be compared to a starfish with its arms turned up over its body until the tips all touch each other. The surface of the skeleton bears processes which support movable spines. Tube feet may be thrust out through perforations in the plates of the ambulacral rows. These rows correspond in position to the ambulacral grooves of the starfish. The plates of the inter-ambulacral rows

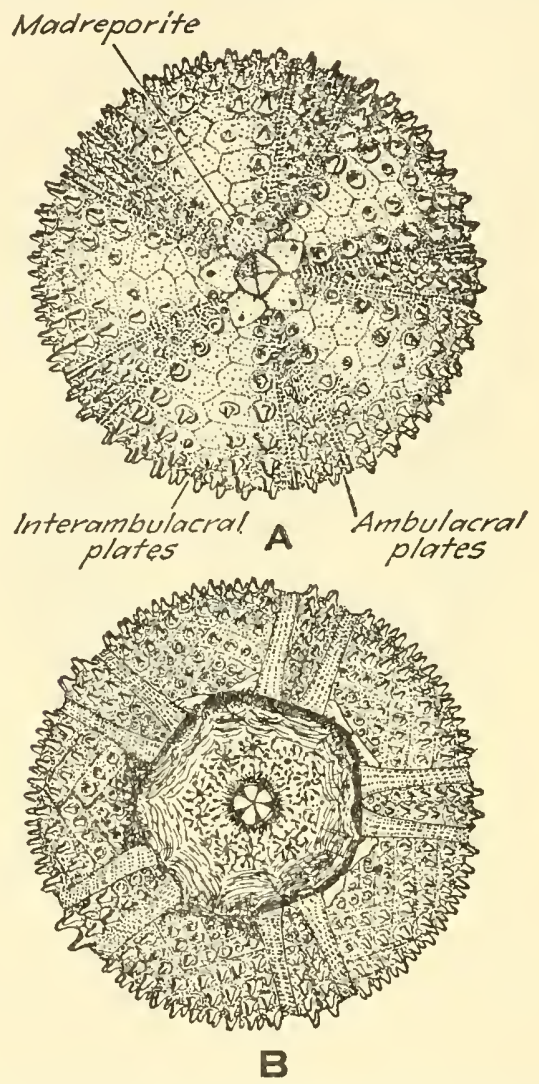

Fig. 117.-Dried test of the sea urchin, Arbacia. A, shows arrangement of the plates on the aboral side; $B$, oral view showing mouth and perioral area. (From Wolcott, Animal Biology, published by McGraw-Hill Book Company, Inc.)

are not perforated. The mouth of this type of animal is located ventrally (orally), and it is guarded by five projecting skeletal processes called teeth. These converge over the aperture and are set in a skeletal case which is composed of many hard ossicles and contains the muscles for moving the teeth. The teeth are used in 
removing algae from rocks for food. This arrangement constitutes Aristotle's lantern, and the esophagus leads internally from its aboral part. Aborally, each ambulacral area ends in a single ocular plate at the periproct. A series of genital plates interspace the ocular plates of the periproct, and one of these genital plates has become the madreporite. The anus is a small pore located near the center of the periproct.

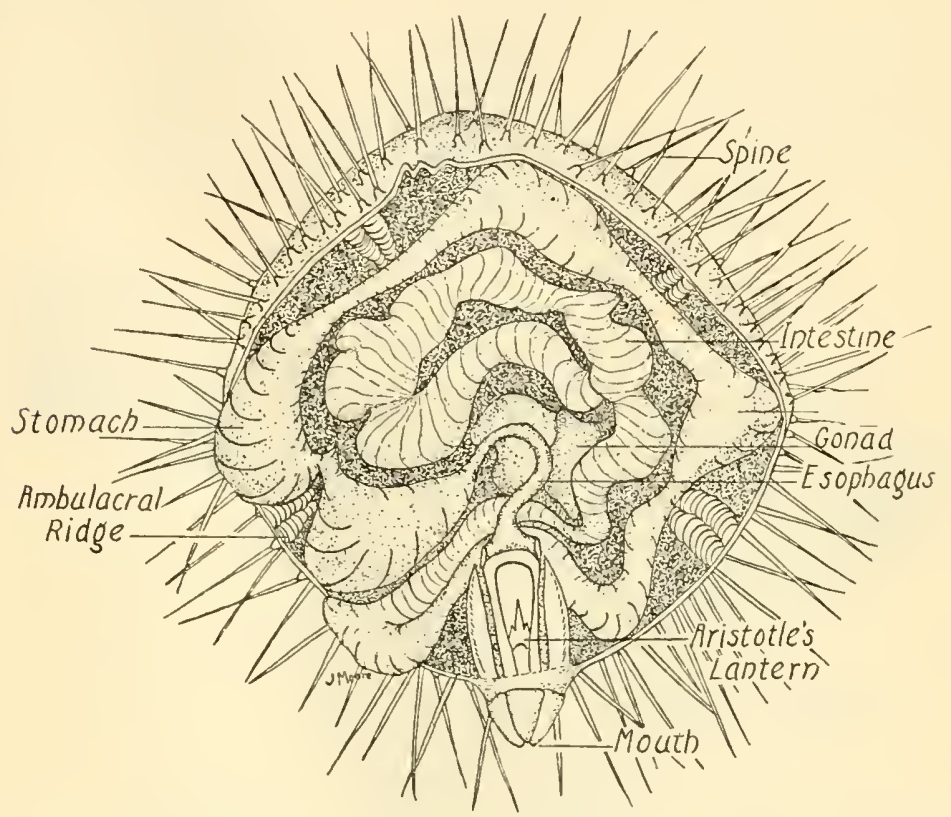

Fig. 118. - A portion of the test of the sea urchin has been removed to show the internal organs.

Upon first view, a dissected sea urchin seems to be almost entirely gonads, internally. In each pentagonal section there is a mass of the gonadal structure which is held in place by a band of tissue known as the genital rachis extending from the aboral wall. Small genital ducts lead from each gonad through a pore in its adjacent genital plate. The general arrangement internally includes a water vascular system composed of a stone canal leading from the madreporite to a circumoral or circumesophageal canal which encircles the mouth. A radial vessel extends from the circumoral canal through the length of each ambulacral arch. In each ambulacral plate are found two pores for a tube foot which is a lateral branch of the 
circumoral canal. Each foot is comnected with a bulblike ampulla. These tube feet along' with some mobile spines constitute the locomotor system. Five interradial pouches or branchiae or "Polian vesicles" are in conmunication with the circumoral canal. The esophagus leads from the aboral part of Aristotle's lantern into the flat, dilated stomach. The stomach extends almost around the internal wall of the body. From it the intestine leads in an opposite direction and in sea urchins finally opens externally by the median aboral anus. In the sand dollar the intestine passes along the posterior ambulacral plate to the anal opening which is near the margin of the dise-shaped body. A branch from the esophagus runs parallel to the stomach and finally joins it. This tube is heavily

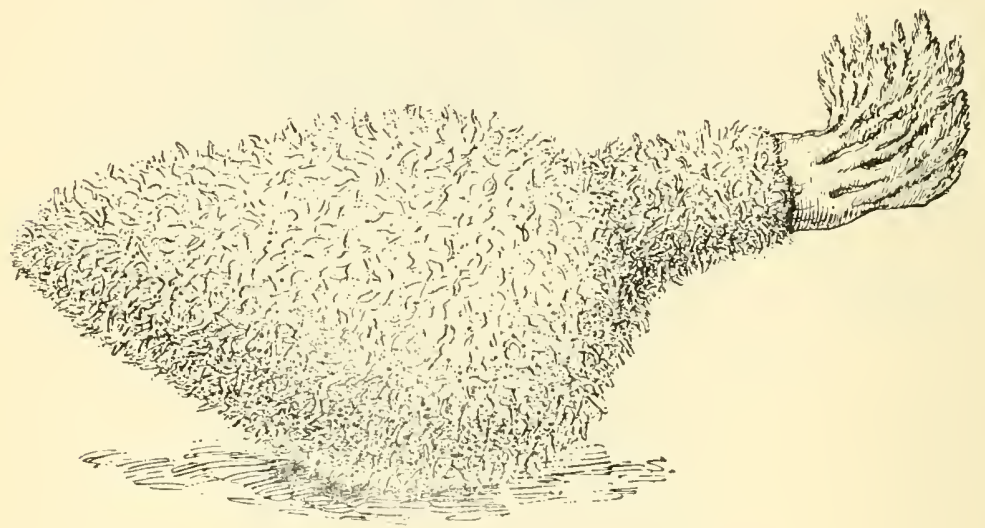

Fig. 119.- Thyone, the common sea cucumber.

ciliated internally and is known as the siphon. Its function is conjectured to be either respiratory or a means of washing refuse from the intestine. The principal organs of respiration are the interradial pouches and the tube feet. The nervous system is composed of a circumoral ring with radial cords extending into the ambulaeral areas. Strongylocentrotus, Arbacia, Tripneustes, Clypeaster, and Echinarachnius (sand dollar) are representative genera of the group.

Class Holothurioidea.-The echinoderms of this class have only an incomplete skeleton, the body is elongated, the mouth surrounded by tentacles is located at one end and the anus is at the other. These animals are called sea cucumbers because of their shape and 
color. There is some remnant of the pentamerous condition in that there are five double rows of tube feet extending lengthwise on five sides of the body of some forms, others have less or none. The expanded body of a holothurian is soft like a bladder partly filled with liquid and the body wall is very muscular. The madreporite is located internally. The rows of tube feet serve as structures of

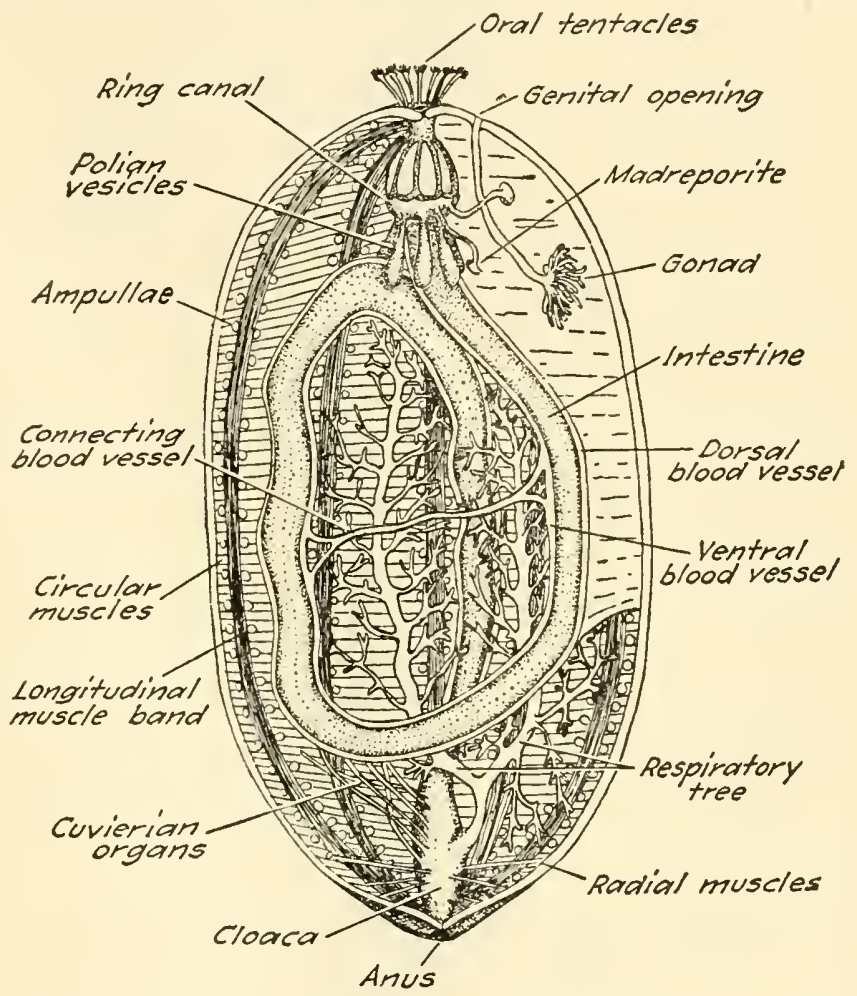

Fig. 120.-Diagram showing the internal anatomy of a sea cucumber. (From Wolcott, Animal Biology, published by McGraw-Hill Book Company.)

locomotion and for clinging. Some of them, next to the mouth, assist the tentacles in procuring food. Within the cloaca are the openings of two long tubular respiratory trees which receive water to assist in respiration. The tube feet, tentacles, cloaca, and other organs serve in respiration. These respiratory trees function also as excretory organs. The madreporite draws water from the inside of the body cavity. 
The digestive system of most sea cucumbers consists of a short esophagus which is supported by a skelctal structure at the point where it enters the body cavity. This structure serves as attachment for the tentacles and retractor muscles. Following the esophagus is a short but rather inflated stomach which leads to the long, coiled intestine. This tube is partially supported by a mesentery which is attached to the midventral line of the body wall. The intestine is thickened in its posterior portion to become the muscular cloaca which contains the openings of the two respiratory trees. In the coelom are fine longitudinal muscles that lie in the ambulacral areas. The gonad and genital duct are in ambulacral areas. They are found free at one side of the esophagus and stomach. This duct opens exteriorly by a pore beside the mouth. The food of the sea cucumbers is largely the organic material derived from mud which is ingested. This class of animals possesses a striking power of autotomy and subsequent regeneration. When they are irritated or disturbed, the muscles of the body cavity contract and produce internal pressure sufficient to cause either the body wall to split near the anus where the viscera are ejected or the viscera are forced out the mouth. Other animals, attempting to attack the sea cucumber, are rendered helpless by becoming entangled in the visceral mass. The sea cucumbers eqan then regenerate the lost viscera in a short time. This power to eviscerate itself is a unique characteristic of the group. Representative genera of this class include; Thyone, Holothuria, Cucumaria, Leptosynapta, Aphelodactyla and Caudina.

Class Crinoidea.-Miost of the sea lilies live attached by long stalks, but a few are free. At the free end of the stalk are located the five, many-branched arms which make up the calyx. The branches of the anus are called pinnules. In most forms some lateral projections, called cirri, are distributed at regular intervals along the stalk. The mouth is located in the uppermost center of the calyx and is surrounded by the anus. The anus is also to be found on the oral side of the calyx within the enclosure made by the arms. On the oral surfaces of the arms are ciliated ambulacral grooves which serve to transport food to the mouth. Modified tube feet are present, but they serve more as tentacles than as feet. They lack ampullae 
and are tactile and slightly respiratory in function. Gonads are borne on the arms. The skeleton is well developed in all crinoids and for that reason many of the ancient forms are preserved as fos-

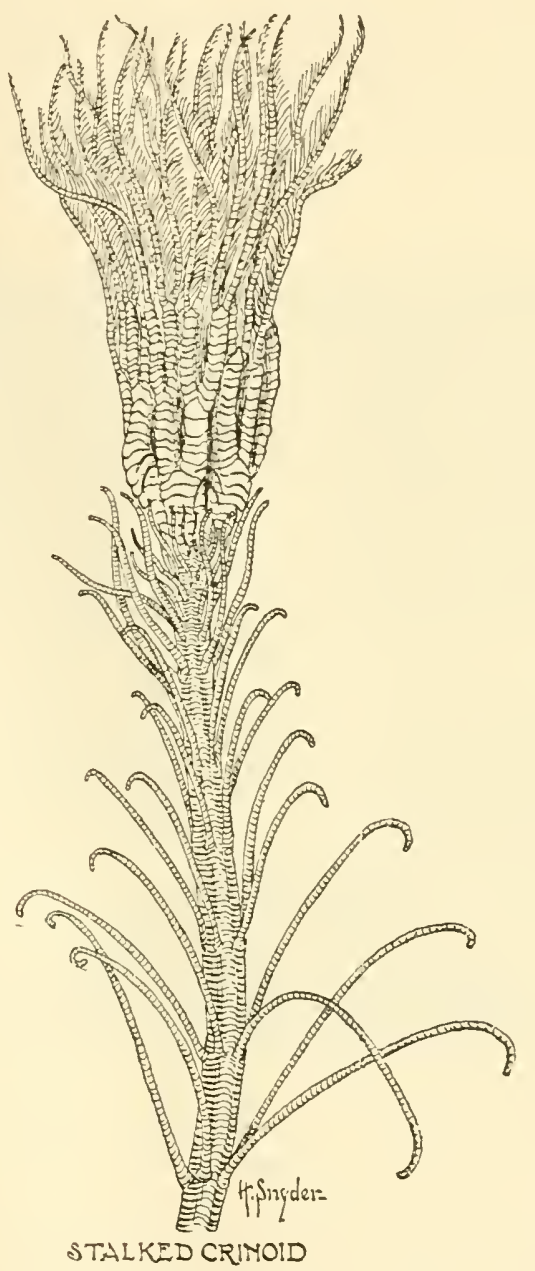

Fig. 121.-Distal portion of a stalked crinoid. (Courtesy of General Biological sils in widely distributed limestone layers of the earth's surface. The nervous and circulatory structures parallel the ambulacral groores and encircle the mouth. Neocomatella, Pentacrinus, Rhizocrinus, Metacrinus and Antedon are representative genera. 


\section{STARFISH OF CLASS ASTEROIDEA}

\section{Habitat and Behavior}

The starfish lives along the shores and in the shore waters (to a depth of over 125 feet) of our stony coasts of the Atlantic and Pacific, with scattered ones occurring in the Gulf of Mexico. A few scattered individuals may be found on muddy or sandy shores, but they are quite scarce. They are often found clinging to pilings, old boats, and other objects in the water. By action of the tube feet they are able to cling very tenaciously to almost any solid object. At low tide they may be found under the rocks, out of the sun, where they are protected from the heat and drying. Due to a food relationship they are usually found in the same area with marine clams, oysters, and rock barnacles. During the day they are rather inactive, but at night they are much more active and respond to such stimuli as light, temperature, contact, and chemicals. It has been demonstrated experimentally that starfishes may form habits. They ordinarily live and move about with the oral side next to the substratum, and if turned over, will right themselves in the same way time after time. If the arms which are habitually used for this are incapacitated, they will acquire the habit of using another combination of rays in this act.

\section{External Anatomy}

The body is composed of a central disc and some (usually five) radiating arms or rays. The mouth is located in the center of the under or oral surface while the upper or aboral surface is covered with spines of various lengths. On the arms these spines are arranged somewhat in rows. Between the spines the exposed skin is extended into projections known as papula or dermal branchiae. There are some small pincherlike structures, called pedicellariae, arranged around the bases of the spines, which serve to keep the surface of the exposed papulae clear of debris and foreign material. The pedicellariae are composed of two jaws or blades and a basal plate with which the jaws articulate. There are large and small pedicellariae. In an eccentric position on the aboral side of the central disc is found the calcareous, sievelike madreporite. The portion of the central dise and two rays adjacent to the madreporite 
constitute the bivinm. The other three arms and their adjacent portions of the central disc compose the trivium. On the oral side surrounding the mouth is a perioral membrane or peristome. An ambulacral groove, containing rows of tube feet, radiates from this along the oral side of each arm. A reddish pigment spot in the end of each arm is called an eye. The spines are longer and stronger around the mouth and along the margins of the ambulacral grooves than elsewhere.

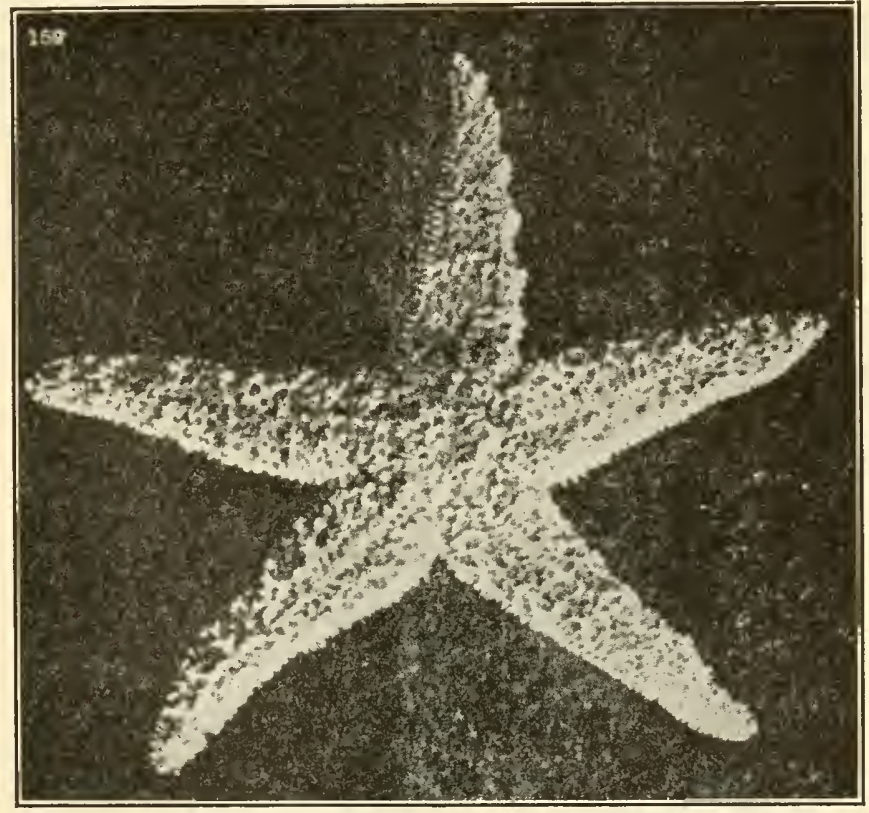

Fig. 122.-The ochre starfish, Pisaster ochraceus, an abundant form along the Pacific coast $\left(X^{1 / 4}\right)$. (Johnson and Snook, Seashore Animals of the Pacific Coast., published by The Macmillan Company.)

\section{Internal Anatomy}

The body wall is relatively strong and hard without being perfectly rigid. This condition is due to the presence of the calcareous skeletal plates throughout, which are bound together by connective tissue and muscular fibers. These plates are often called ossicles. They lie in a flat position in the aboral portions of the body wall. The skeleton of the ambulacral grooves consists of four rows of articulated, oblong ossicles in each arm. These ossicles are ar- 
ranged with the flat sides together, like cards in a filing ease. The two middle rows of ossicles are called ambulacral plates. Ambulacral pores, through which the tube feet project, are located between these plates. The outer rows of plates, forming the margin of the groove,

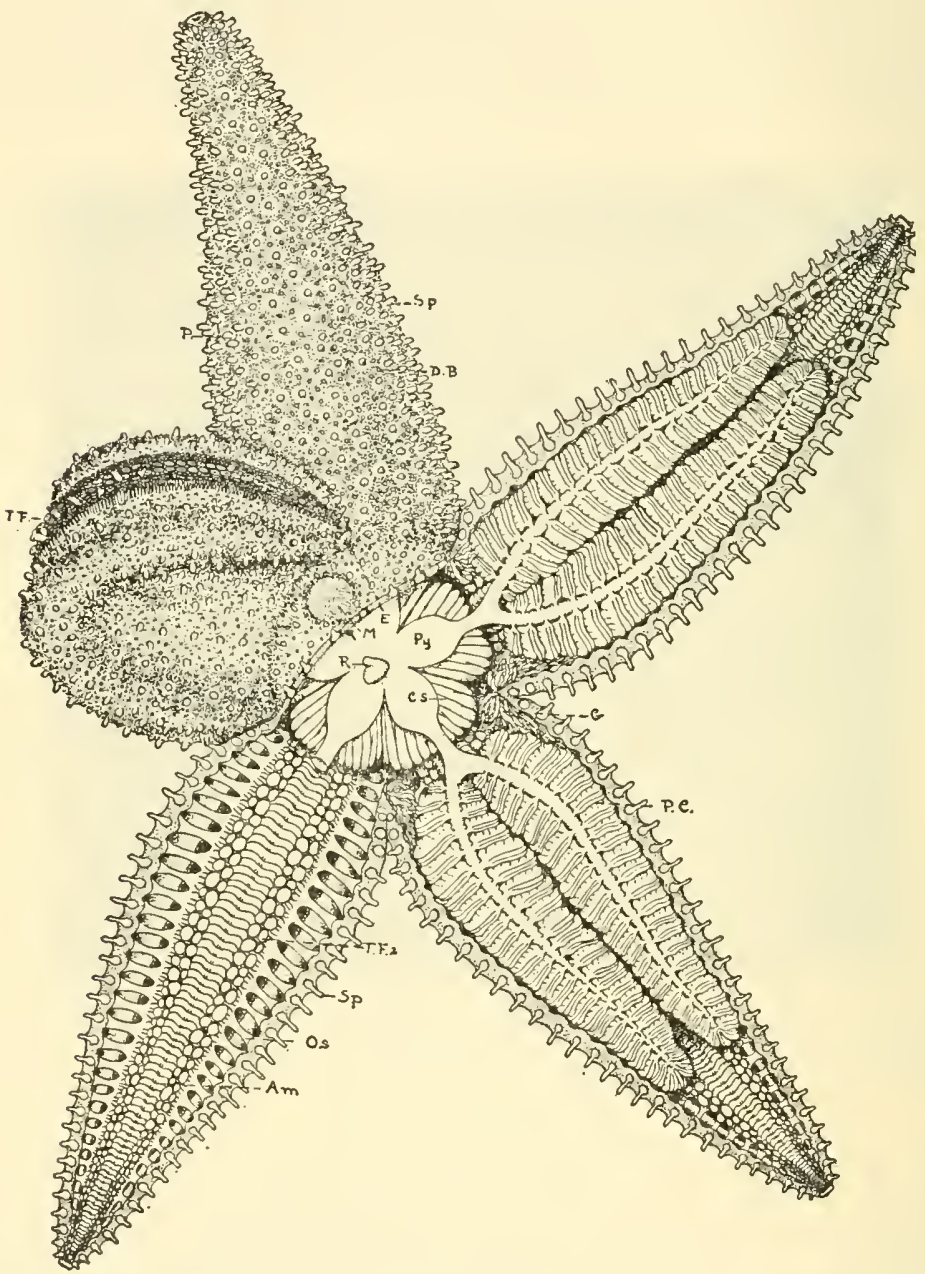

Fig. 123.-Dissection of the starfish, Asterias. The aboral wall has been removed from the trivium and a portion of the central disc. One ray of the bivium has been turned to expose the oral surface and tube feet. The organs have been removed from one ray of the trivium to expose the skeleton. Am., ambulacral groove: C.S., cardiac stomach; $D . B$. dermal branchiae; $E$, eyespot; $G$, gonads ; $M$, madreporite; Os., ossicle; $P$, pelicellariae; $P$.C., ploric caeca; $P y$., pyloric sac; $R$, rectal gland; T.F., tube feet: T.F.2, arrangement of tube feet in skeletal ray. (From White, General Biology, published by The C. V. Mosby Company.) 
are shorter and are known as adambulacral plates. Five flat oral ossicles surround the mouth.

Within the body wall and extending into the arms is a large coelom which is lined by a peritoneum and filled with coelomic fluid. In this cavity are located the organs of most of the systems. The digestive system is a modified tube extending vertically from the mouth on the oral side to the minute arms at the aboral surface. From the mouth a short esophagus leads to the double-pouched stomach. The larger cardiac portion (or pouch) receives the esophagus and is separated aborally from the pyloric portion by a marked constriction. A large pair of branched glandular structures, known

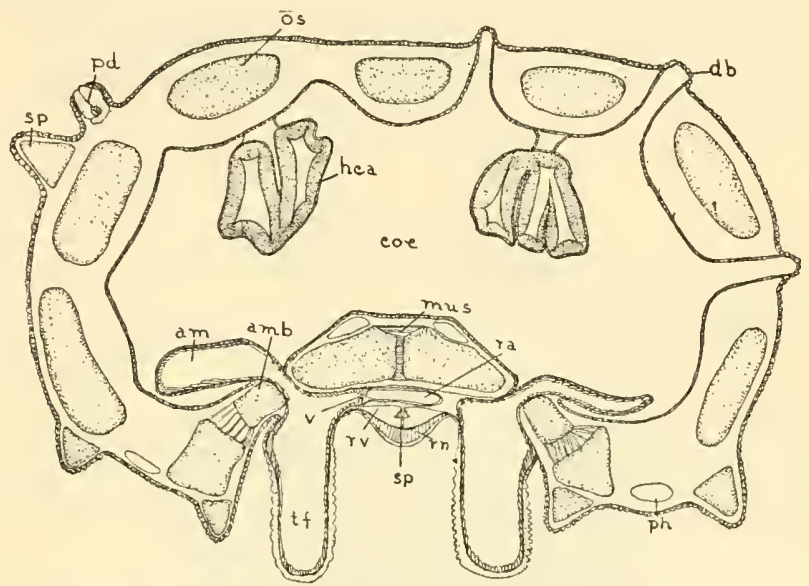

Fig. 124.-Diagram of a cross section through a ray of a starfish. am, ampulla: $a m b$, ambulacral ossicle: coe, perivisceral coelom; $d b$, dermal branchia; $h c a$, hepatic caeca; mus, muscle; os, ossicle; $p d$, pedicellaria; $p h$, perihemal space; $r a$, radial canal: $r n$, radial nerve; $r v$, radial blood vessel; $s p$, spine; $S p$, septum in radial blood vessel $(r v)$; $t f$, tube feet; $v$, valve between tube foot and radial canal. (From White, Generai Biology.)

as hepatic or pyloric caeca, is located in each arm, and each pair joins the pyloric pouch by a duct which seems to be a continuation of this pouch. These glands and possibly the pyloric pouch produce digestive enzymes in solution. The fluid secreted by the wall of the cardiac portion probably does not contain enzymes. A short rectum or intestine leads aborally from the pyloric pouch to the porelike anus at the exterior surface of the central disc. Two brown, branched pouches arise from the rectum. These are known as rectal caeca or glands and probably have excretory function. In feeding, the starfish catches its bivalve prey in the five arms and humps 
over it. The tube feet are attached to the shells, and, by cooperative activity, an enormous pull is exerted on the valves of the shell. After the shell is open, the stomach of the starfish is everted through its mouth and is spread over the tissues of the prey which are digested in situ. An abundance of digestive fluid secreted over the food causes the mollusk to be digested in its own shell and this material is then taken into the stomach of the starfish. It is reported that between four and five dozen clams may be eaten by a single starfish in a week. It has also been shown that a starfish may survive after months of fasting. After feeding, the stomach is withdrawn into the body eavity by five pairs of retractor muscles, one pair extending from the pyloric portion to the ambulacral skeleton of each arm. The branched, treelike gonads fill the remaining space in each arm and the external pores from them are located in the crevice between adjacent arms.

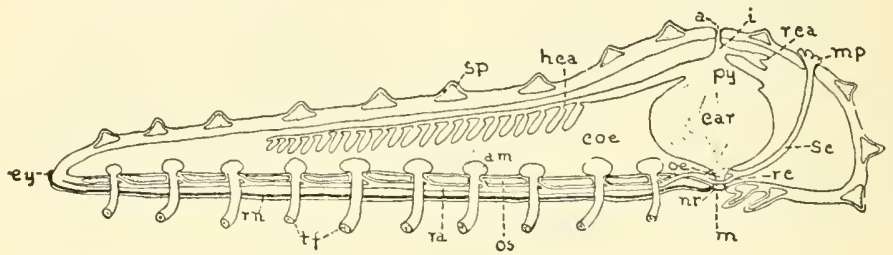

Fig. 125.-Longitudinal section through the central disc and one ray of a starfish. $a$, anus : am, ampulla; car, cardiac stomach; coe, perivisceral coelom; $e y$, eyespot; hca, hepatic caeca $; i$, intestine $; m$, mouth $m p$. madreporic plate; $n r$. nerve ring; oe, esophagus; os, ambulacral ossicle; $p y$, pyloric sac; ra, radial canal; rc, ring canal; rca. rectal caeca; sc, stone canal; sp, spine; $t f$, tube feet. (From White, General Biology.)

The water-vascular system is composed of the madreporite, stone canal, circumoral or ring canal, radial canals, Tiedemann's bodies, lateral canals, ampullae, and tube feet. Water is taken in through the sievelike madreporite on the aboral side of the central disc and is conducted by the S-shaped, calcareous stone canal (hydrophoric canal) to the ring canal, which encircles the mouth. The movement of the water through the madreporite and stone canal is accomplished by the action of eilia, which line them. On the medial surface of the ring canal are nine small Tiedemann's (racemose) bodies, the stone canal joining the ring eanal where the tenth might be expected. These bodies produce amoeboid cells. The five radial eanals extend distally, one in the roof of the ambulacral groove of each ray. Numerous paired lateral canals arise along the length of each radial canal. Each ends shortly by connecting with its ampulla and tube 
foot. The ampulla is bulblike and is located above the roof of the ambulacral groove in the coelom. It is connected through its ambulacral pore with the contractile tube foot which hangs down into the ambulacral groove. The distal or free end of the foot has a slightly inverted, suckerlike shape. The proximal pair of

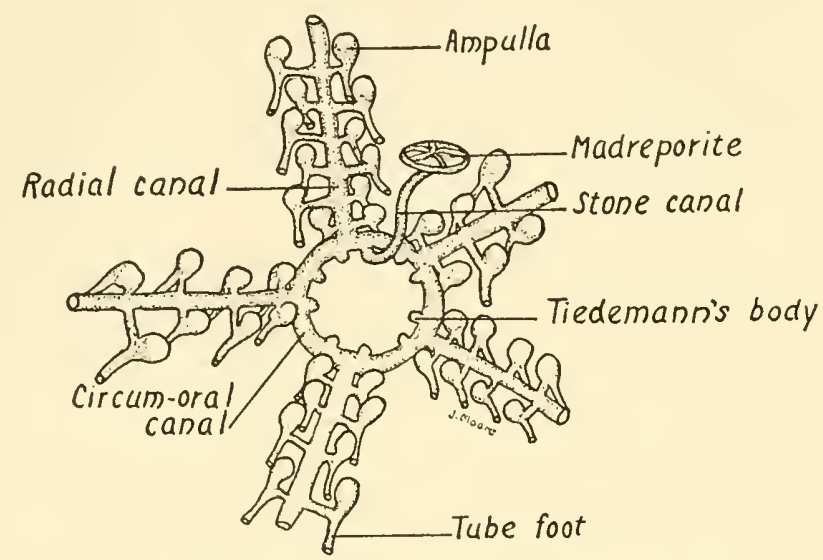

Fig. 126.-Diagram of water-vascular system of the starfish.

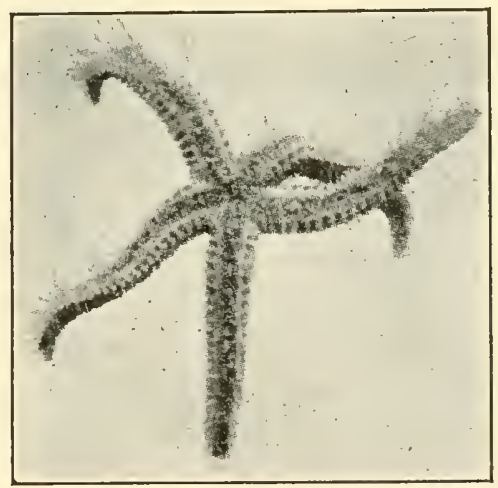

Fig. 127.-Starfish "walking" on glass. Notice the extended tube feet. (Courtesy ampullae in each arm of some starfish lack the tube feet and are sometimes erroneously called Polian vesicles. Alternate tube feet are farther from the radial canal than the others on each side. The ampullae and tube feet function effectively in locomotion, the ampullae contracting to force water into their respective tube feet 

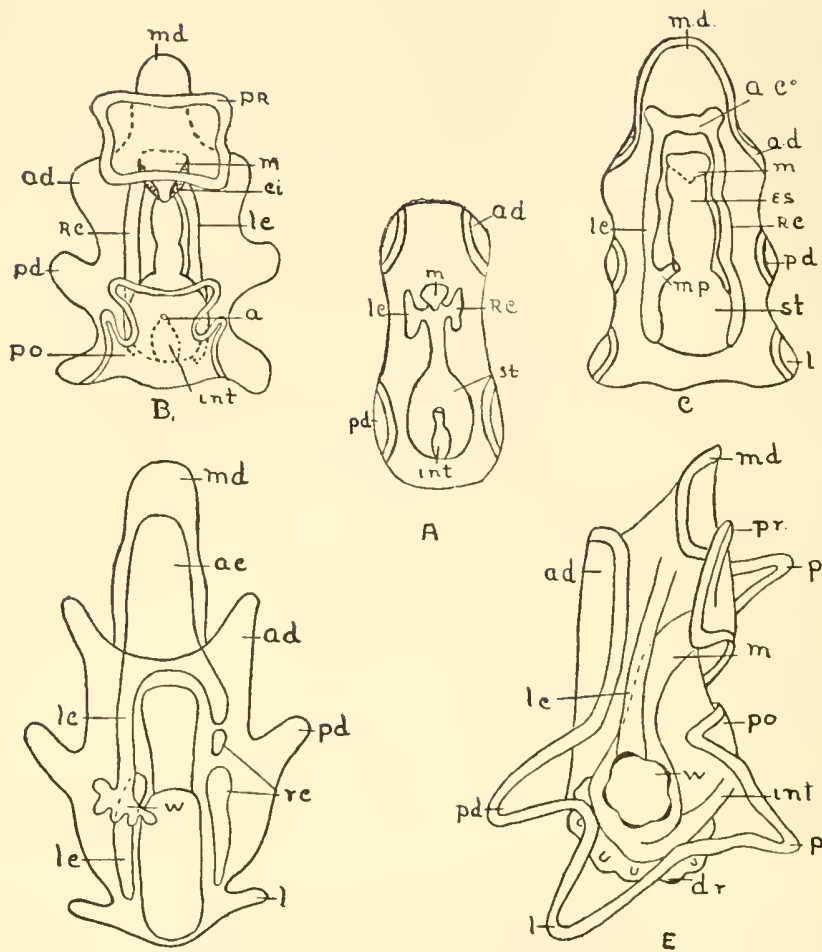

A

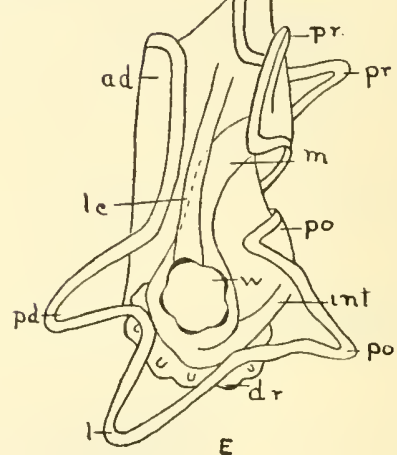

D.
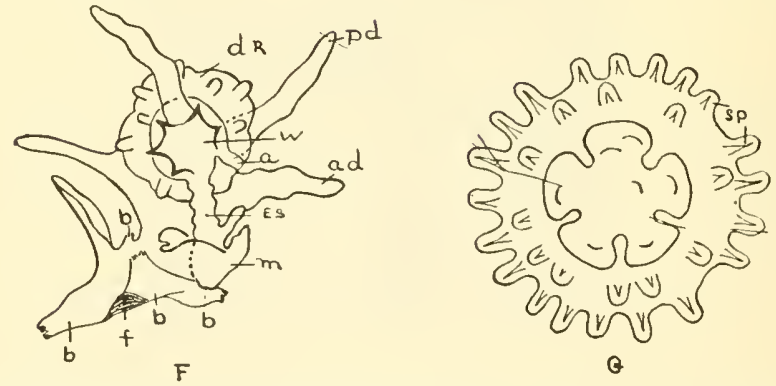

Fig. 128.-Development and metamorphosis of the starfish. A, Dorsal vlew of early ciliated larva showing ciliated bands, and left and right coelomic pouches. $B$, Ventral view of bipinnaria larva showing the extension of the left and right coelomic pouches. $C$, Dorsal view of the same larva showing the left madreporic pore and water tube, and the fusion of the left and right coelomic pouches to form an anterior coelom. $D$. Dorsal view of an older larva showing the budding of the five water tubes from the left coelom. $E$, Left side view of a still older larva showing the water vascular system developing from the water tubes, and the rays of the adult starfish developing on the dorsal side $F$. Brachiolaria larva in process of metamorphosis. The larva has settled on the preoral region which is greatly shortened. $G$, Aboral view of a young starfish showing the developing spines. 
to extend them. The walls of both ampullae and tube feet are muscular. In large starfish the tube feet may be extended an inch or two. The sucker ends of these tube feet work like a vacuum cup and will adhere effectively to surfaces over which the animal is drawing itself. When the pressure is released by the ampulla, the tube foot contracts and draws the animal forward. When water is again forced into the tube, it releases its grip and is again extended. By alternation of the activity of tube feet in different parts of the body the animal is able to move itself from one place to another. The entire water vascular system is a modified part of the coelom.

A thin-walled system of vessels running parallel to the water vascular is the circulatory system. It is enclosed in a perihemal space. In addition to this the coelomic fluid, which occupies the coelom and bathes all of the organs, serves as a circulatory medium in that it absorbs the digested food and distributes it. This fluid bears amoebocytes which are cells capable of picking up particles of waste material and carrying them to the dermal branchiae, where they pass through the membrane to the exterior. These dermal branchiae are pouches of the coelomic wall which extend outward between the skeletal plates and have the additional function of respiration. When these pouches are completely extended, they nearly cover the exterior surface of the animal, and thus expose an enormous area to the water for respiration.

Excretion is carried out in part by the amoebocytes which have been produced by the Tiedemann's bodies and have migrated to the coclomic cavity. The rectal caeca serve in respiration to some extent also. There is a certain amount of diffusion of dissolved wastes through the dermal branchiae and the walls of the tube feet.

The nervous system is radially arranged about the oral ring which encircles the mouth just orally to the ring canal. From the oral ring, a radial nerve extends the length of each arm and ends in the pigmented eyespot. These nerves lie in the roof of the ambulacral grooves. The aboral surface is supplied by a less conspicuous aboral

$a$, anus; $a c$, anterior coelom; $a d$, anterodorsal arm; $b$, brachiolar arms; $c i$, adoral ciliated band; $d r$, dorsal surface developing rays; $e s$, esophagus; $f$, point of fixation; int, intestine; $l$, lateral arm; $l c$, left coelomic pouch; $m$, mouth; md, median dorsal arm; $m p$, madreporic pore and water tube; pad, posterodorsal arm; po, postoral ciliated band; $p r$, preoral ciliated band; $r c$, right coelomic pouch: $s p$, spines: st, stomach; $w$, five water tubes of the water vascular system. (Modified from Wilson and McBride. By permission, The Macmillan Co.) 
nerve which extends from an anal nerve ring. Branches of these nerves extend to the numerous nerve cells distributed in the epidermis above the nerve cords. The pigment eyespots at the tips of the arms are photosensitive and sensitive to touch. The pedicellaria and tube feet are also sensitive to touch. There is little centralization except in the oral ring and radial cords, still there is sufficient centralization for the necessary coordination exercised by the animal.

\section{Reproduction and Life Cycle}

The starfish is dioecious; i. e., the sexes are separate. The reproductive systems of the two are similar and each consists of five paired gonads lying in the cavity of the rays beside the pyloric caeca. They open to the exterior by pores in the angles between arms. Mature eggs produced in ovaries of females and mature spermatozoa discharged from testes of males are freed in the ocean water where they unite in fertilization. Total, equal cleavage is the type of division which follows fertilization, and this finally gives rise to the many-celled, free-swimming, ciliated blastula. The wall of this infolds to form a gastrula. Following this the rounded body becomes somewhat elongated and lobed. Ciliated bands develop over its surface and it is known as bipinnaria. This larval stage has bilateral symmetry, and the larva swims about near the surface for weeks by the aid of its ciliated bands. A later modification of the bipinnaria in which there are several extended symmetrical processes, is known as the brachiolarian stage (Fig. 128). Following this condition is a metamorphosis during which many processes are formed, and the radial symmetry superimposes the bilateral. The presence of the bilateral symmetry in these larval stages seems to indicate that the ancestors of echinoderms were likely animals with this type of symmetry.

\section{Regeneration and Autotomy}

Regeneration is the name applied to the power some animals have to replace mutilated or lost parts. The starfish has this phenomenon quite well developed with regard to its alms. Any or all of the arms of the starfish may be lost and the missing parts regenerated. (Fig. 367.) An arm with a small portion of the central dise will regenerate the missing parts under favorable conditions. A mutilated arm or one caught in the grip of some enemy may be cast off 
by breaking loose at the constricted point where it joins the central disc. This ability of self-mutilation is known as autotomy. Following autotomy there is regeneration of a new part.

\section{Economic Relations}

Compared with many other animals the echinoderms are relatively unimportant economically. The sea cucumbers of several different species are used as food by the Chinese and other oriental people. The larger animals, some of them two feet long, are eviscerated, boiled, soaked in fresh water, dried or smoked and sold under the name of beche-de-mer or trepang. This dried product is semileathery and gelatinous. It is quite expensive and is usually served as a very palatable soup. The chief fisheries are found along the shores of China, the East Indies, Australia, and the Philippines; some, however, are taken in California, Hawaii, and the West Indies.

Sea urchins of several kinds furnish a sort of caviar known as "sea eggs." The egg masses are taken from the sexually mature females and are eaten either raw or cooked. Each specimen contains a considerable quantity of roe at the season just before spawning. Production of "sea eggs" has become quite an industry in the Orient, Italy, and the West Indies. The Barbados are particularly noted for their production of this commodity.

Perhaps the starfish is the most important of the group, but its relationship is almost entirely of negative importance. It is one of the worst enemies of clams, oysters, and snails. The starfish grows in enormous numbers around the oyster beds of the Atlantic, attacks the oysters, and feeds on them, leaving only the empty shells. A single starfish may eat as many as two dozen oysters in a day. Oyster hunters formerly attempted to protect the oysters and clams by dragging "tangles" made of frayed rope over the beds, catching large numbers of starfish, breaking them in two, and dumping the scraps back into the water. The fallacy of this was realized when their power of regeneration was learned, so at present they are usually dropped into boiling water or thrown on the bank to dry. Salted or smoked starfish roe (eggs) are considered a delicious food by many people.

The brittle stars and crinoids have little value except as geological indices and biological specimens. Their skeletal parts contribute to the formation of limestone. 


\section{CHAPTER XVII \\ PHYLUM MOLLUSCA}

(By Elmer P. Cheatum, Southern Methodist University)

\section{GENERAL CHARACTERS}

The phylum Mollusca includes such familiar animals as the snails, clams, oysters, and cuttlefish. Even though they appear different externally, all are soft-bodied, unsegmented, usually bilaterally symmetrical, and most of them produce a shell composed principally of calcium carbonate. A muscular foot is present which may be modified for different functions. In the snail it is used for creeping; in the clam for plowing through the substrate, and, in the nautilus or squid for seizing and holding prey. Covering at least a portion of the body is a mantle or dermal fold, the outer surface of which secretes the shell in most species. Between the mantle and main body is a mantle cavity which is usually either provided with gills or modified into a primitive pulmonary sac for use in respiration. Jaws are present in the snails, slugs and cephalopods. Within the mouth cavity of many species is the radula, which is an organ composed of fine chitinous teeth arranged in rows and used in rasping food.

Approximately 78,000 species of mollusks have been described, hence they constitute one of the largest groups of animal life. With very few exceptions they are sluggish animals and occupy a diversity of habitats, occurring abundantly on land, in fresh water, and in the sea. Although most of the species live in moist surroundings, a few inhabit arid regions. Some species, such as the cuttlefish, are strictly carnivorous; many of the snails are herbivorous, and others feed as scavengers. The oyster and other species that are attached during adulthood feed on the floating organisms in the sea.

From the standpoint of their ancestry, the veliger larva of various marine forms bears close resemblances to the trochophore larva of the annelids. Whether or not they are direct descendants of the 
annelids is a matter for conjecture since some morphologists regard this similarity in larval forms as an example of adaptive parallelism in a similar type of environment. Certainly morphological evidence shows a close relationship.

\section{THE SNAIL}

(Detailed description based on Helix)

\section{Habitat and Behavior}

Snails occupy a variety of habitats. They occur abundantly in fresh water, salt water, brackish water, and thermal springs; they live in the arid sections of the country and occur abundantly in the tropics where certain arboreal forms are found. Some species belonging to the genera Caecilianella and Helix live underground, feeding
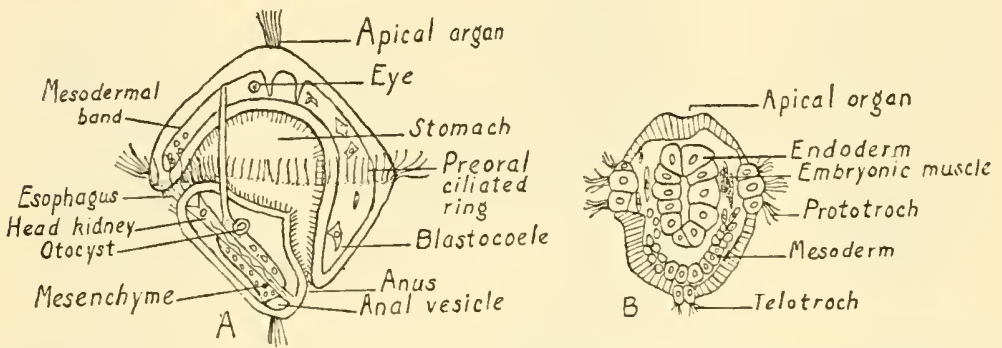

Fig. 129.-A, Trochophore larva of Eupomatus (a polychaete annelid), side view. (After Shearer.) B, Veliger larva of Patella (a marine snail) frontal section. (After Patten. (Drawn by Joanne Moore.)

on roots of plants; many other species live deeply embedded in moist humus. Certain species, such as Helix hortensis and Helix aspersa, excavate holes in rocks and live in them. Although most snails are not tolerant to extremes of cold, Vitrina glacialis lives in the Alps above the timberline where the rocks are covered with snow most of the year; even some of our fresh-water snails in this country, such as Lymnaea palustris, Physa gyrina and Helisoma trivolvis, when frozen gradually, can live at least several weeks in solid cakes of ice.

Land snails are most active either during a light rain or immediately following. In heavily shaded woodlands where surface moisture prevails, snails are active during the day as well as at night. The same species of snail that exhibits both diurnal and nocturnal activity in the woodland may show only nocturnal activity in an open, 
exposed habitat. Movements of most land snails appear to be coincident with moisture rather than darkncss. Preceding prolonged periods of cold, land suails may move to protected places, such as beneath dead logs, dense mats of humus, crannies in or under
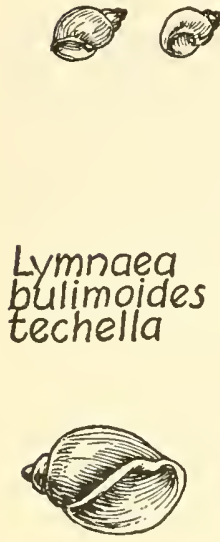

\section{Physa humerosa}
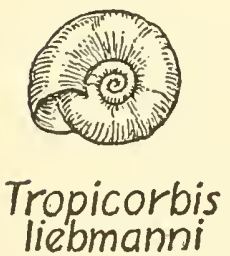
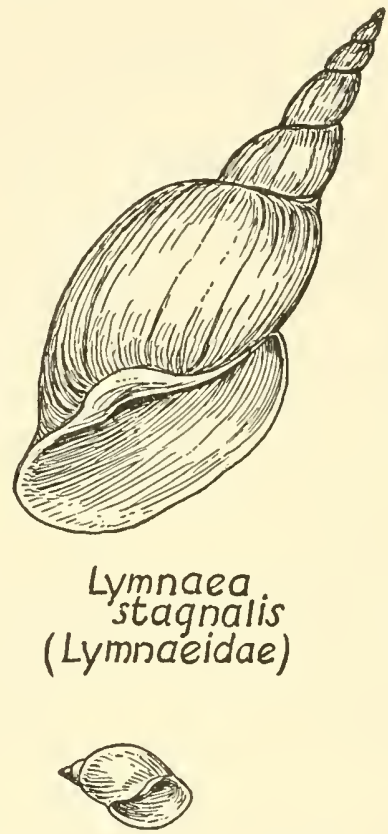

Physa anatina (Physidae)

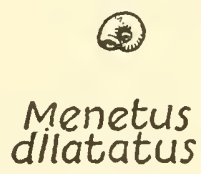

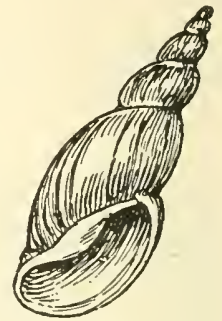

\section{Lymnaea} palustris

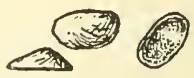

Ferrissia excentrica (Ancylidae)

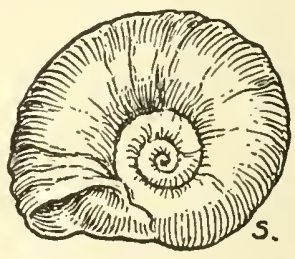

Helisoma trivolvis lentum (Planorbidae)

Fig. 130.-Some common fresh water pulmonate snails.

rocks, and there begin their period of hibernation. During this condition of torpidity the body of the snail may be well protected by one or several thin parchmentlike membranes called epiphragmas which are stretched across the shell aperture. When warm weather arrives, the membranes are broken and the snail resumes its activities. 
Water snails are active all four seasons, provided open water is available. Naturally their movements are slowed down in the winter due to cold, but when the pond or stream is frozen over, the movements of Lymnaea, Physa, or Helisoma may be observed through the ice. During periods of dry weather when ponds and creeks dry up, snails embed themselves in moss and mud, and in this manner are able sometimes to survive long periods of drouth. During this condition epiphragms may be formed in certain species (Lymnaca palustris), the same as in land snails; these structures probably function in retarding water loss.

At least a few species of land snails possess a homing instinct. Helix aspersa, H. pomatia, and Polygyra roemeri have all been observed to occupy as "home" a definite place and go out from this "home" on nocturnal foraging trips, then return by sunrise the next morning.

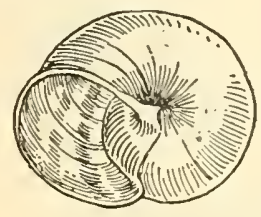

Humboldtiana chisosensis
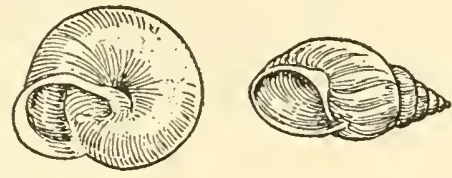

\section{Bulimulus}

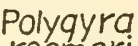
roemeri dealbatus liquabilis

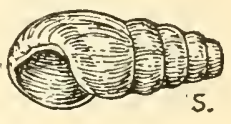

Rumina decollata

Fig. 131.-Common terrestrial snails.

The life span seems to vary considerably in snails; some of the aquatic genera, such as Lymnaea and Helisoma may live two to four years whereas some species of Helix may live to be six or eight years old.

Parasitism and commensalism are both exemplified by certain species of snails. A commensalistic relationship exists between the rare mollusk Lepton squamosum and the crustacean Gebia stellata. The former feeds on secretions produced by the latter. A few species of sponges, echinoderms, annelids, and mollusks are parasitized by various species of mollusks.

\section{External Anatomy}

Shell.-The shell of the snail may be in the form of a low, broad, or flattened spiral (Humboldtiana chisosensis and Polygyra roemeri), or a long, tapering spire (Lymnaea stagnalis); on the other hand, 
some shells are shaped like house roofs (Patella that lives in the sea or Ferrissia, a fresh-water form). The worm shell (Vermetus spiratus) is so loosely coiled that it superficially resembles a worm. Some shells, such as those belonging to the genus Murex, may have long peculiarly eurved spines extending out from the main shell body that give to the shell a grotesque appearance. In the sea and land slugs the shell is either rudimentary, internal, or absent.

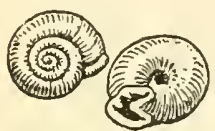

Polygyra texasiana
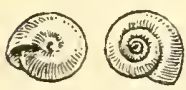

Zonitoides arboreus

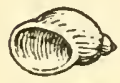

succinea avara

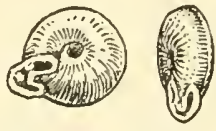

Polygyra dorfecilliana

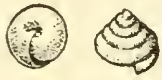

Euconulas chersinus trochulus
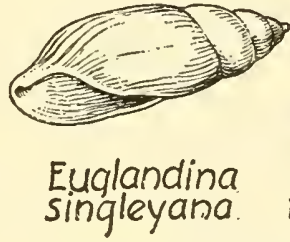

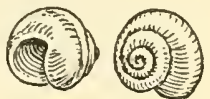

Helicina orbiculata tropica

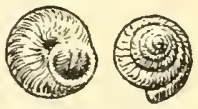

Strobilops labyrinthica texasiana

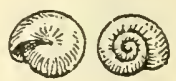

Retinella indentata paucilirata

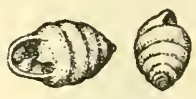

\section{Gastrocopta} armifera

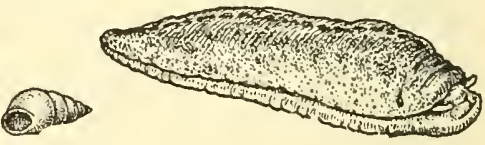

Philomycus carolinensis

Fig. 132.-Common terrestrial snails.

If the shell is held with the aperture toward the observer and the aperture is on the left, the shell is said to be sinistral; if on the right, the shell is dextral. Most species are normally dextral, but occasionally a reversal occurs which has been found to be inherited.

The shell which is largely composed of carbonate of lime is secreted by the mantle and usually consists of three layers. Embedded within the latter may be pigments that give the occasional brilliant colors to certain species. The thickness of the layers is dependent on the richness of lime salts in the environment; thus, snails living in an acid bog have thin transparent shells, whereas the same species inhabiting an area rich in lime salts have thicker, 
perhaps opaque shells. Certain species, such as Polygyra roemeri, $P$. albolabris and $P$. texasiana are capable of repairing broken shells if the damage is not too severe.

Body.-The body of the snail consists of a head, neck, foot, and visceral hump. The head of a land snail (Helix) has one pair of

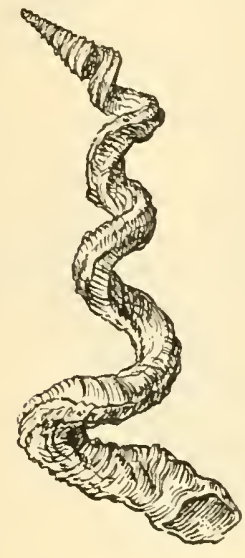

vermetus spiratus
(Worm shell)

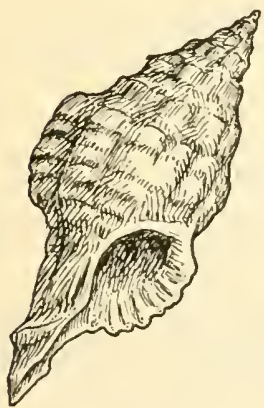

Urosalpinx
(Oyster drill)

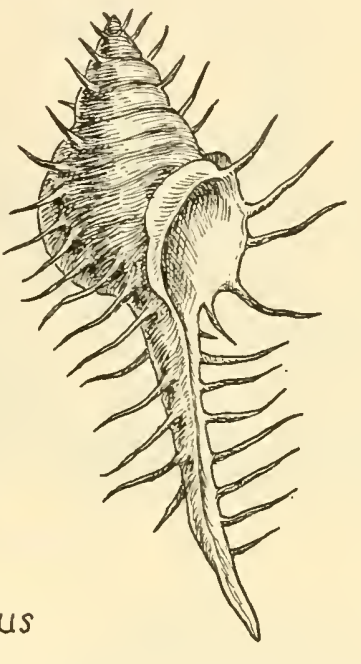

Murex tenuispina (Venus's comb)

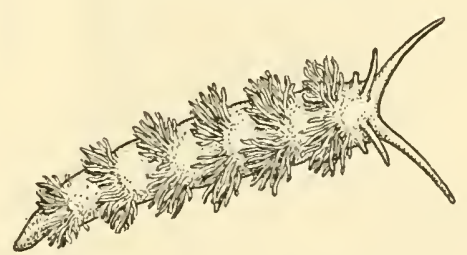

Aeolis
(sea slug)

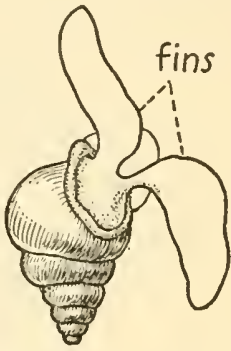

Limacina australis

(Pteropod)

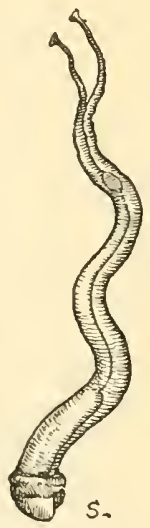

Teredo navalis. (Ship worm)

Fig. 133.-Marine mollusks.

true tentacles which are probably sensitive to contact and smcll, and a pair of stalked "eyes" which can possibly detect different light intensities, but are not sight organs. Our common genera of water snails (Lymnaea, Physa, Helisoma) have their eyes situated at the 
base of the tentacles. Just in front of and below the tentacles is a mouth. Located on the side of the head is the genital pore. The broad muscular foot is covered with a mucus-secreting integument. Just ventral to the mouth is the opening of the pedal gland which deposits a highway of mucus over which the snail usually glides;
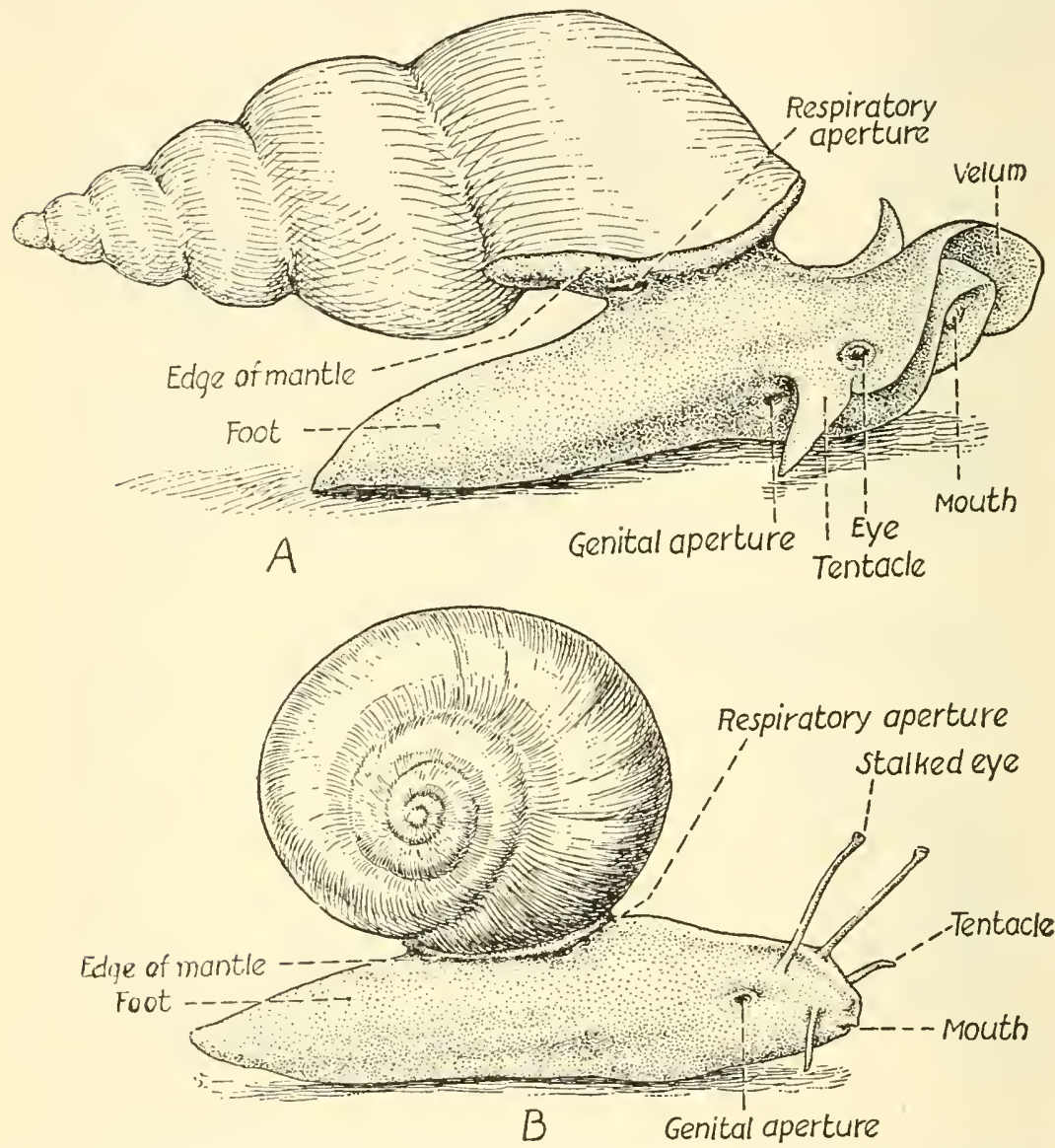

Fig. 134.-Fresh-water and land snails with bodies expanded. $A$, fresh-water snail, Lymnaec; $B$, land snail, Humboldtiana.

the gliding movements are scarcely perceptible. In some marine snails the surface of the foot is covered with cilia, the latter facilitating movement. The visceral hump, which encloses the digestive, circulatory, respiratory, excretory, and reproductive systems, is protected by the shell which is lined with the mantle. A thick 
collar is produced where the mantle joins the foot, and just beneath this mantle-collar is the respiratory aperture; back of the latter is the anal opening.

\section{Internal Morphology}

Digestion.- -Just within the mouth of a snail is a rounded organ known as the buccal mass. The latter is composed of a ribbon of

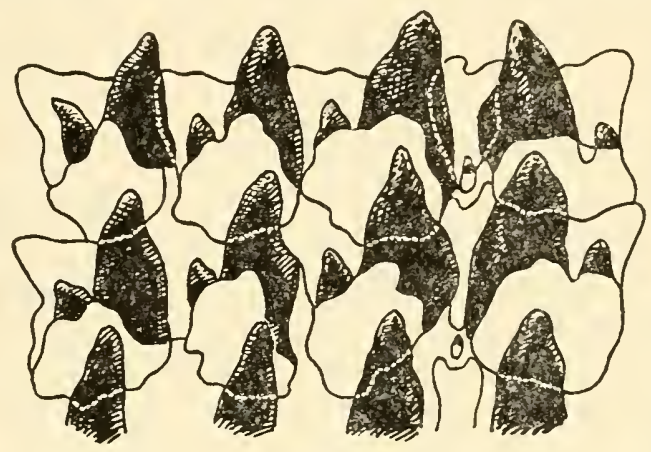

Fig. 135.-Arrangement of teeth in the radula of a snail.

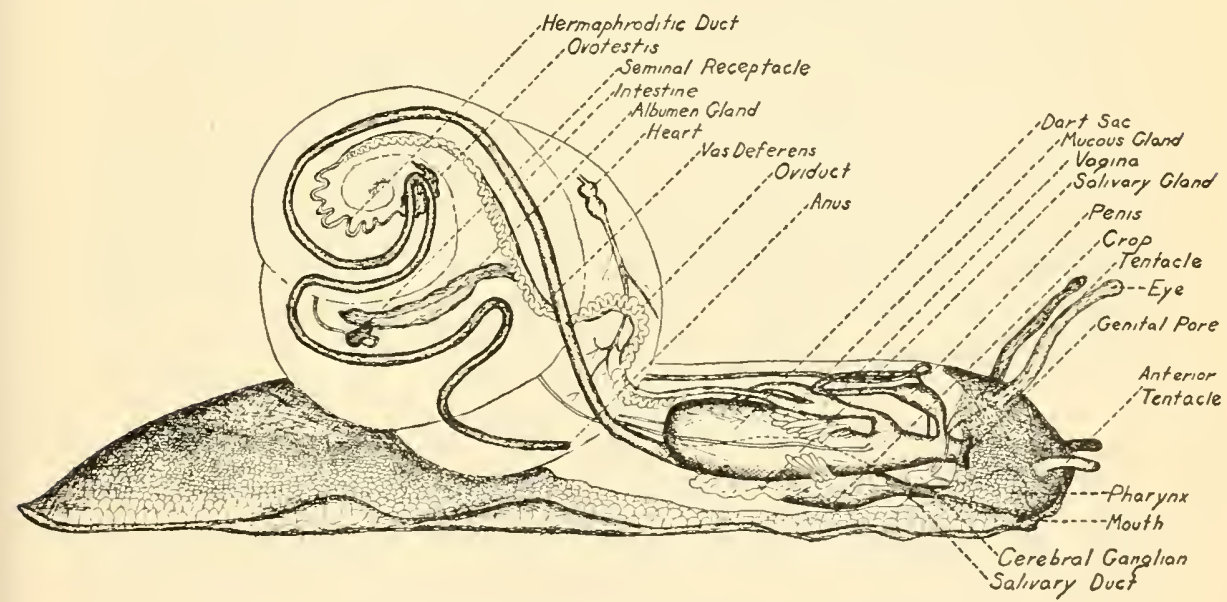

Fig. 136.-Internal anatomy of Helix. Shell removed.

minute recurved teeth, the radula, supported and moved by connective tissues and muscles. On the roof of the mouth is a horny jaw which pulls food into the mouth cavity. It is then rasped by the radula into fine particles and mixed with saliva which flows into the buccal cavity from salivary glands that lie on each side of 
the crop. The masticated food is then passed into the esophagus which widens, forming the crop. Here the food may be mixed with a brown liquid produced by the digestive gland which occupies most of the visceral hump. Enzymes produced by this gland convert starches into glucose, and, in the case of Helix, the ferment is powerful enough to dissolve the cellulose of plant cells, thus releasing the protoplasm so that it may be utilized. From the crop, food enters the stomach and is passed on into the intestine where absorption takes place. Feces are discharged to the outside through the anus.

\section{Respiration}

Land and most fresh-water pulmonate snails breathe by a fold of the richly vascularized mantle which has been modified into a primitive lung, whereas the branchiate snails breathe by true gills.

In all probability pulmonate snails that inhabit the deep water of lakes use the pulmonary sac as a gill and breathe like the branchiates. When the water is cold, it is not necessary for aquatic pulmonate snails to make periodic trips to the surface in order to renew their air supply, but when the water becomes sufficiently warm, cutaneous respiration alone is inadequate and the snail must come to the surface to get additional oxygen. The pulmonary sac of aquatic pulmonates not only serves in the capacity of a gill or lung but also may serve as a hydrostatic organ, thus enabling snails to ascend to the surface by flotation. Such movements are probably made possible through contraction of the mantle walls, thus decreasing or increasing the rolume of air. Most of the marine species are gill breathers, and some, such as the sea slugs, have external feather-like gills.

\section{Circulation}

The blood of the snail consists of a plasma which is usually colorless, but in Helisoma, hemoglobin is dissolved in the plasma, thus giving it a red color, and in Lymnaea and some species of Helix the blood has a bluish tinge due to the presence of a copper-containing pigment, hemocyanin. In the plasma, float the colorless corpuscles. The blood serves as a transporting medium whereby digested food, excretions, secretions, and gasses may be carried from one part of the body to another. The heart, which consists of an auricle and a ventricle, lies in the pericardial cavity. Blood is pumped from the 
ventricle through a common aorta which divides into two branches, one of which supplies the head and foot, and the other carries blood to the visceral hump. The terminal branches of these arteries communicate with a hemocoele or series of sinuses. Veins carry the blood from the hemocoele to the mantle walls where it is purified and then passed through the pulmonary vein to the single auricle and on into the ventricle.

\section{Nervous System}

Encircling the esophagus is a ring of nerve tissue which includes three pairs of ganglionic swellings: the cerebral ganglia, situated above the esophagus, supply nerves to the anterior regions of the body; the pleural, pedal, and visceral ganglia lie below the esophagus and are connected to the cerebral ganglia by commissures. From them, nerves extend out to the visceral hump and basal parts of the body. The arrangement of ganglia and their connectives is of taxonomic importance.

\section{Excretory}

The kidney is a yellow gland situated near the heart. Its ureter, a thin-walled tube, parallels the rectum and opens near the anus.

\section{Reproduction and Life Cycle}

Most fresh-water and terrestrial pulmonate snails, as well as the sea slugs, are hermaphroditic. The majority of the marine shelled gastropods and our fresh-water branchiates, such as Pleurocera, Goniobasis, and Amnicola are unisexual. The reproductive system of a unisexual snail is relatively simple but is exceedingly complex in the hermaphroditic species.

In bisexual (hermaphroditic) snails cross-fertilization ordinarily occurs. The ova, as well as spermatozoa, are produced by the ovotestis. Some snails are protogynous, since the ovotestis functions first as an ovary and later as a testis; others are protandrous, since male gametes are first formed, followed by the production of ova. Spermatozoa pass from the hermaphroditic duct into the sperm duct, and enter the vas deferens which terminates in a muscular penis. By means of the latter organ sperm are transferred into the seminal receptacle of another snail. Ova are passed from the ovotestis into the hermaphroditic duct, and from there into the oviduct 
which terminates in a thick-walled muscular vagina. During this journey the ova are fertilized by sperm from the seminal receptacle and coated with albumin from the albumin glands. Both the penis and vagina have a common genital opening to the exterior.

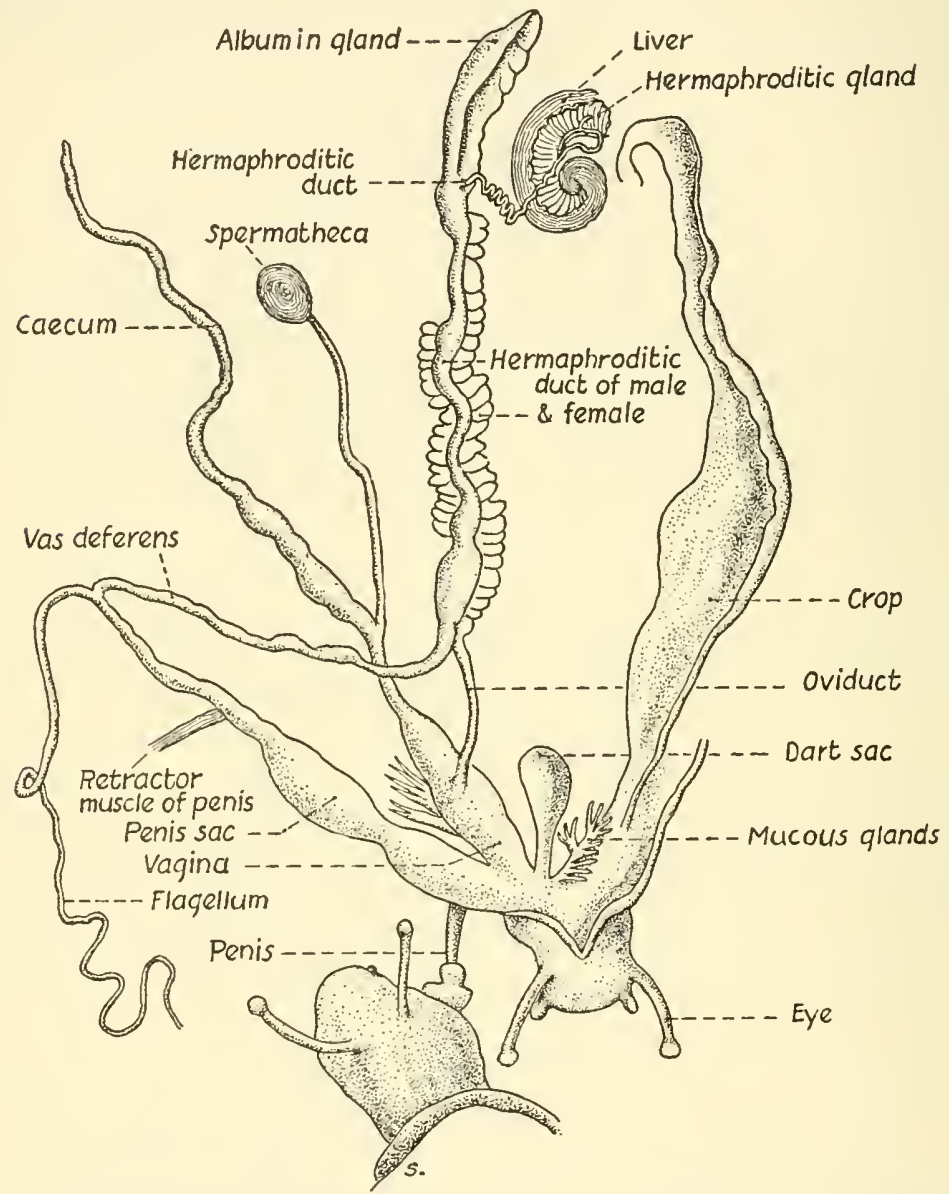

Fig. 137.-Genitalia of Helix aspersa; act of union. (Modified, after Cooke, Cambridge Natural History. By permission of The Macmillan Company.)

Most of the fresh-water snails deposit eggs in clear gelatinous masses on submerged objects, such as twigs and rocks. The land snails usually deposit their eggs singly or in clusters in well-protected places, such as in rotten wood or beds of humus. The eggs 


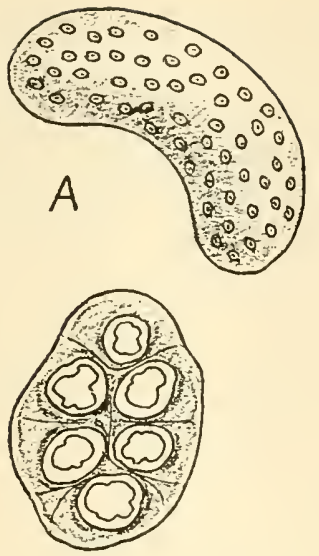

D

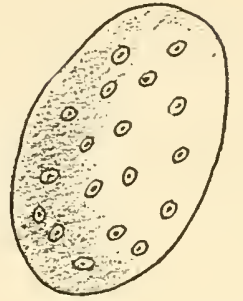

B

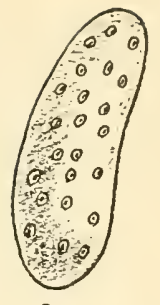

C

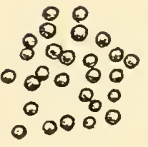

$E$

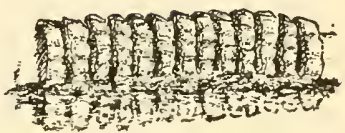

F

Fig. 138.-Egg masses of common snails. A, Lymnaea (fresh-water; gelatinous mass); $B$, Heliosoma (fresh-water; gelatinous mass); $C$, Physa (fresh-water ; gelatinous mass) ; $\boldsymbol{D}$, Pleurocera (fresh-water branchiate; tough gelatinous mass) $E$, Polygyra texasiana (terrestrial; eggs in cluster); $\boldsymbol{F}$, egg capsules of Busycon.

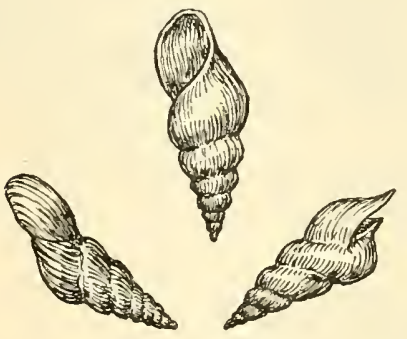

Goniobasis comalensis (Pleuroceridae)

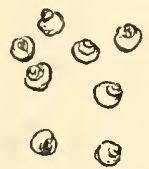

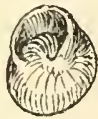

Amnicola comalensis
6

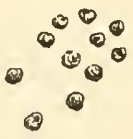

Cochliopa texana

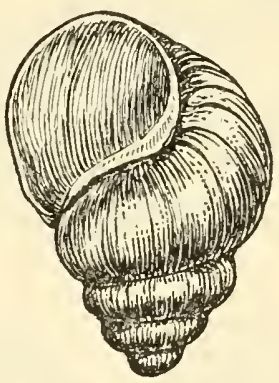

Campeloma decisum (Viviparidae)

(Amnicolidae)

Fig. 139.-Some common fresh-water branchlate snalls. 
are covered with thin shells which prevent undue water loss. In some marine snails, such as Busycon, eggs are deposited in discshaped capsules which are spaced equally apart and held together by a tough band. Some snails, such as the fresh-water Campeloma, have a brood pouch in which eggs are deposited and the young are born alive. The latter is ovoviviparous reproduction in contrast to oviparous reproduction, as described above.

\section{FRESH-WATER CLAMS}

(Detailed description based on Lampsilis)

\section{Habitat and Behavior}

Mussels or clams are usually found partly buried in the mud, sand or gravel of ponds, lakes, or streams. By means of the muscular foot which is protruded from between the two valves at the

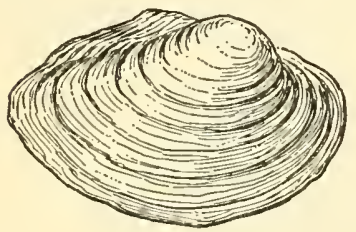

Anodonta stewartiana

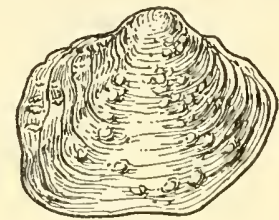

Quadrula forsheyi

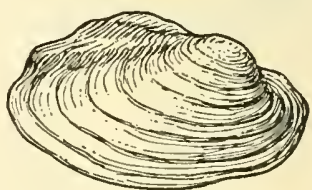

Proptera purpurata

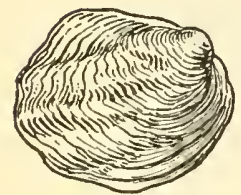

Amblema costata
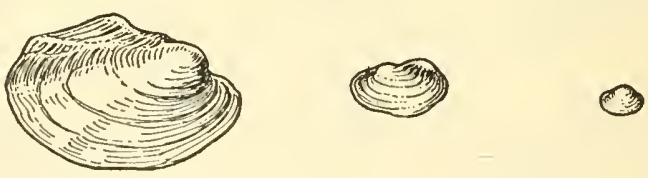

\section{Leptodea fragilis.}

\section{carunculina texasensis}

\section{Musculium ferrissi}

Fig. 140.-Some common fresh-water bivalves.

anterior end of the shell they plow their way slowly through the stream or pond bed, feeding on the microscopic organisms in the water. At the posterior end of the shell are two openings: the ventral siphon which pulls in food and water, and the dorsal siphon through which wastes and deoxygenated water are eliminated. 
Movement is varied among the pelecypods. Scallops may move rapidly by suddenly contracting the valves, thus ejecting a jet of water. Oysters are motile in their larval stages but in the adult stage are attached to rocks and other objects. Many marine mussels are attached to objects on the bottom or along the shore. Attachment is made possible by the dissolution of a part of the under valve and adherence of a portion of the body thus exposed.

The life span of clams may be relatively long. It has been estimated that Anodonta, one of our common genera of fresh-water clams, attains its maximum growth in twelve to fourteen years.

\section{External Features}

Shell.-Unlike the snail whose shell is of one piece, the clam shell is composed of two parts called valves (hence, bivalves) which are attached together at the dorsal surface by a hingelike ligament.

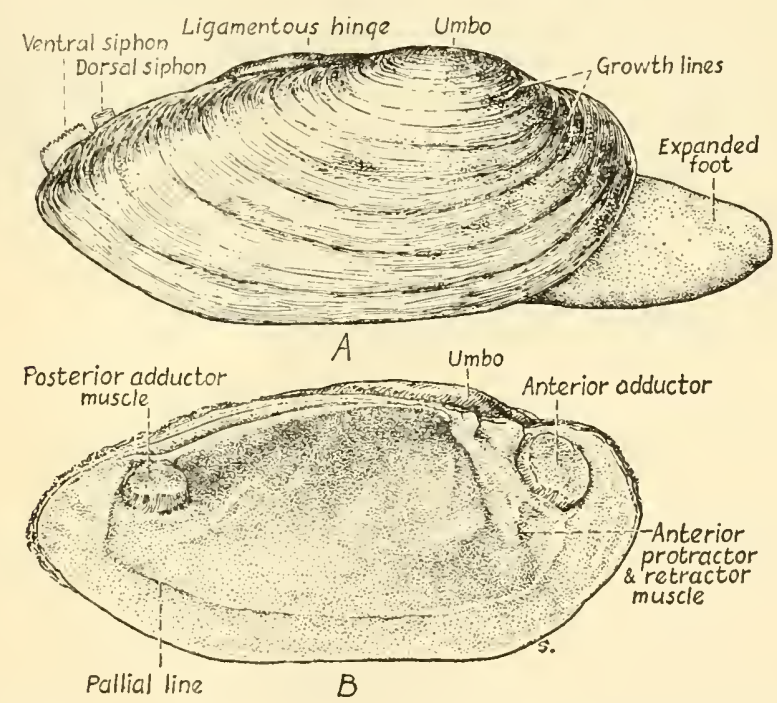

Fig. 141.-External (A) and internal (B) shell features of Lampsitis anodontoides.

The oldest part of the shell is the umbo which is usually a rounded protuberance near the top of the valves and is frequently eroded due to carbonic acid in the water. Extending out from the umbo on each valve in a concentric manner are the growth lines of the shell, evidenced as slight, medium, or heavy ridges. 
The shell is covered by a horny, pigmented periostracum. Underlying this is the prismatic layer composed of carbonate of lime. The inner mother-of-pearl or nacreous layer consists of many thin, usually smooth plates, that in reflected light produce an iridescence in many species.

\section{Internal Anatomy}

The valves are held together by two powerful transverse muscles, the anterior and posterior adductors. Upon cutting these muscles the shells gape open, exposing the underlying organs. The valves are lined with a mantle which secretes the shell. On the inner sur-

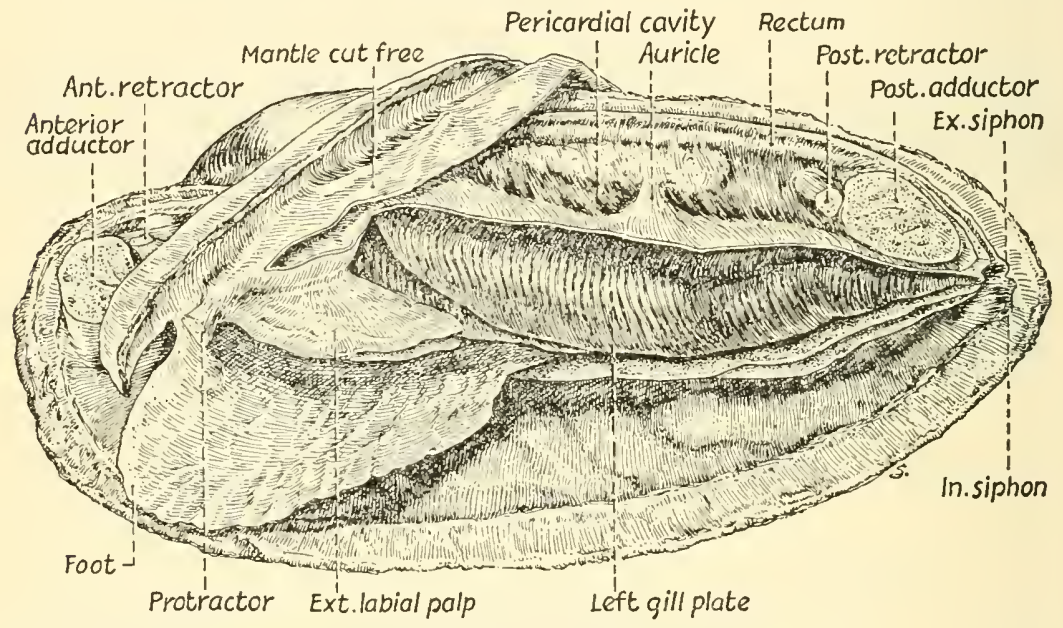

Fig. 142.-Lampsilis anodontoides with the left mantle partially removed and turned back to expose the underlying organs.

face of each shell may be seen the curved pallial line which extends between the two adductor muscles and indicates the partial attachment of the mantle. Teetl which strengthen the closure of the shell may be present where the two valves come together. Between the two walls of the mantle is the mantle cavity which contains the leaflike gills, the foot, and visceral mass.

\section{Digestion}

During the activity of the clam a constant current of water is maintained in the mantle cavity. Food material is circulated forward to the mouth which lies between ciliated labial palps. Upon 
entering the mouth, food is passed through a short esophagus into the saclike stomach. Here it comes in contact with a digestive ferment produced by the digestive gland which is discharged into each sice of the stomach through ducts. The crystalline style, a diverticulum of the intestine, and found only in mollusks, produces an enzyme mixed with the stomach content which undoubtedly facilitates the digestion of carbohydrates. The food, having been mostly digested and partly absorbed in the stomach, is passed on

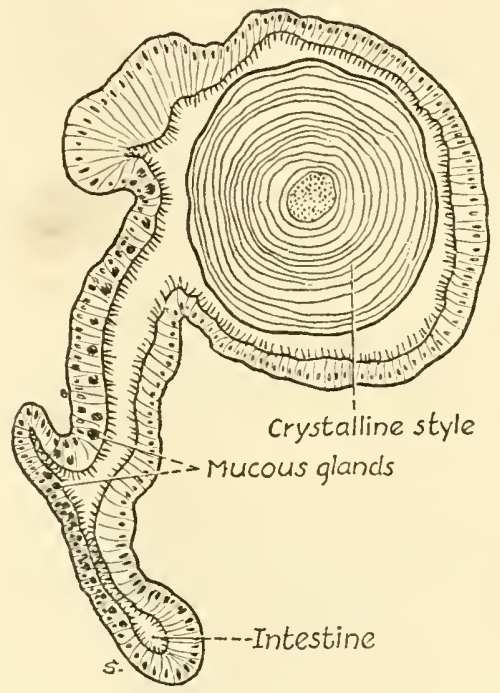

Fig. 143.-Cross section through the style sac and intestine of Lampsitis anodontoides. (Modified after Nelson.)

into the intestine which makes one or more loops in the foot, passes through the pericardium and terminates in the anus near the dorsal siphon.

\section{Respiration}

Respiration is carried on through two pairs of vascularized gills which hang down into the mantle cavity on each side of the foot. Oxygenated water drawn in through the ventral siphon is passed through a rather complicated series of water tubes in the gills. Oxygen is absorbed by the capillaries and carbon dioxide passed into the water where it is discharged to the outside through the dorsal siphon. 


\section{Circulation}

The heart which is composed of a ventricle and two auricles lies in the pericardium. The ventricle, a muscular organ, surrounds the rectum and drives blood forward through the anterior aorta and backward through the posterior aorta. Both aortae give off arteries which ramify all parts of the body. Most of the returning blood is carried to the kidneys by means of the vena caval vein. Within the latter, nitrogenous wastes are removed, and the blood then flows to the gills through afferent branchial veins; after purification in the gills it is returned to the auricles by way of the efferent branchial veins. The blood is colorless and contains several types of white corpuscles.

\section{Nervous System and Sense Organs}

Situated on each side of the esophagus is a cerebropleural ganglion, the two ganglia being connected by means of a cerebral com-

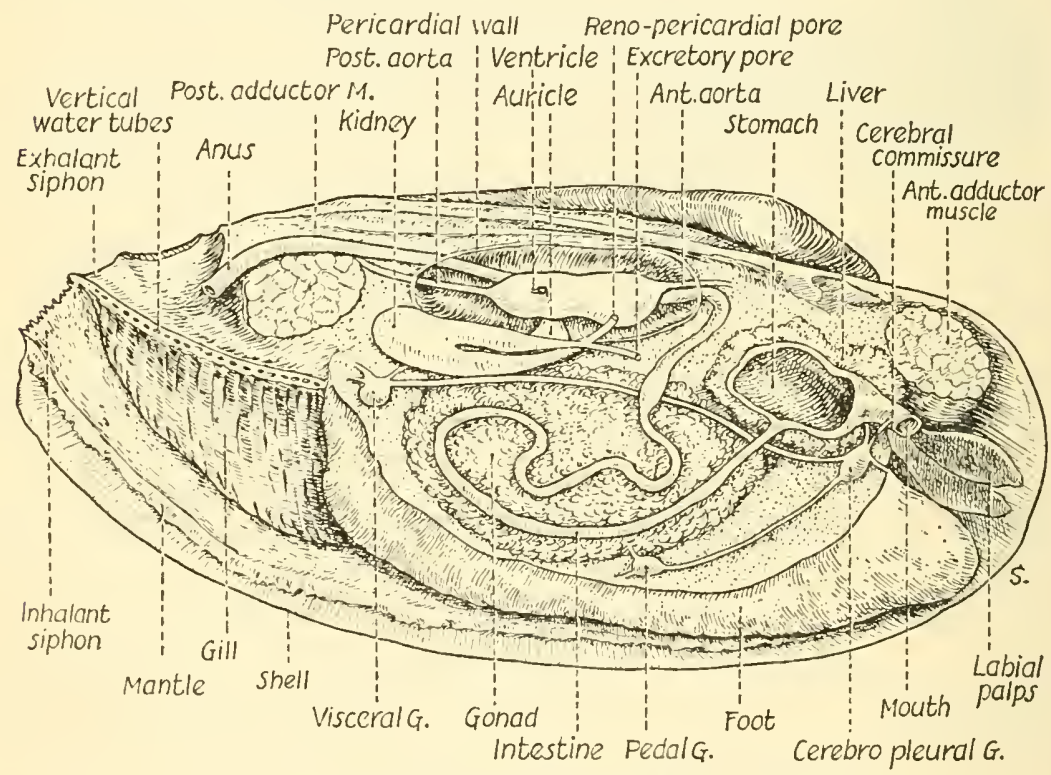

Fig. 144.-Internal organs of Lampsilis anodontoides.

missure which passes above the esophagus. Each ganglion gives off two nerve cords, one of which passes ventrally and posteriorly to the pedal ganglion situated at the junction of the visceral mass 
with the foot. The other nerve cord extends backward, terminating in a visceral ganglion which is usually located just ventral to the posterior adductor muscle. The visceral as well as the pedal ganglia are united.

The sensory organs of the clam are primitive. Covering each visceral ganglion is a patch of sensory epithelium called the osphradium, the function of which may be to test the purity of the water brought in through the respiratory system. A short distance back of each pedal ganglion is a statocyst which functions in equilibrium. It is composed of a small calcareous concretion, the statolith, which is surrounded by sensitive cells. In addition to the sensory organs named, there are many sensory cells distributed along the mantle edges and elsewhere which probably react to light and touch.

\section{Excretion}

Paired kidneys lie on each side of the body just below the pericardium. Each consists of a glandular portion which excretes waste, and a thin-walled bladder that is connected with an excretory pore through which wastes are discharged to the outside.

\section{Reproduction and Life Cycle}

The small bivalves belonging to the family Sphaeriidae (Sphaerium) are hermaphrodites, but in the larger ones the sexes are usually separate. The paired gonads are situated in the foot; the testis is usually whitish in color and the ovary reddish. A short duct leads from the gonad and opens just in front of the excretory pore. Sperm are passed to the outside through the dorsal siphon and enter the female clam through the ventral siphon. The ova, having been discharged through the genital apertures, become lodged in various parts of the gills, depending upon the species. Within the gills the eggs are fertilized. Thus, the gills serve as brood pouches or marsupia and may become greatly distended due to the tremendous number (as many as three million) of developing embryos.

The small bivalve larva, which ranges in size from about 0.05 to 0.5 millimeter in diameter, is called a glochidium and has a single adductor muscle for closing the valves which may or may not be hooked. Extending out from the center of the larva is a long secretory thread, the byssus. In most clams the glochidia are discharged to the outside through the dorsal siphon. They fall to the floor of 
the river, pond, or lake, and lie with their jaws agape, or snap their jaws on any object. If the soft filament of a fish's gill or a fin of the fish comes in contact with the glochidium, it will close

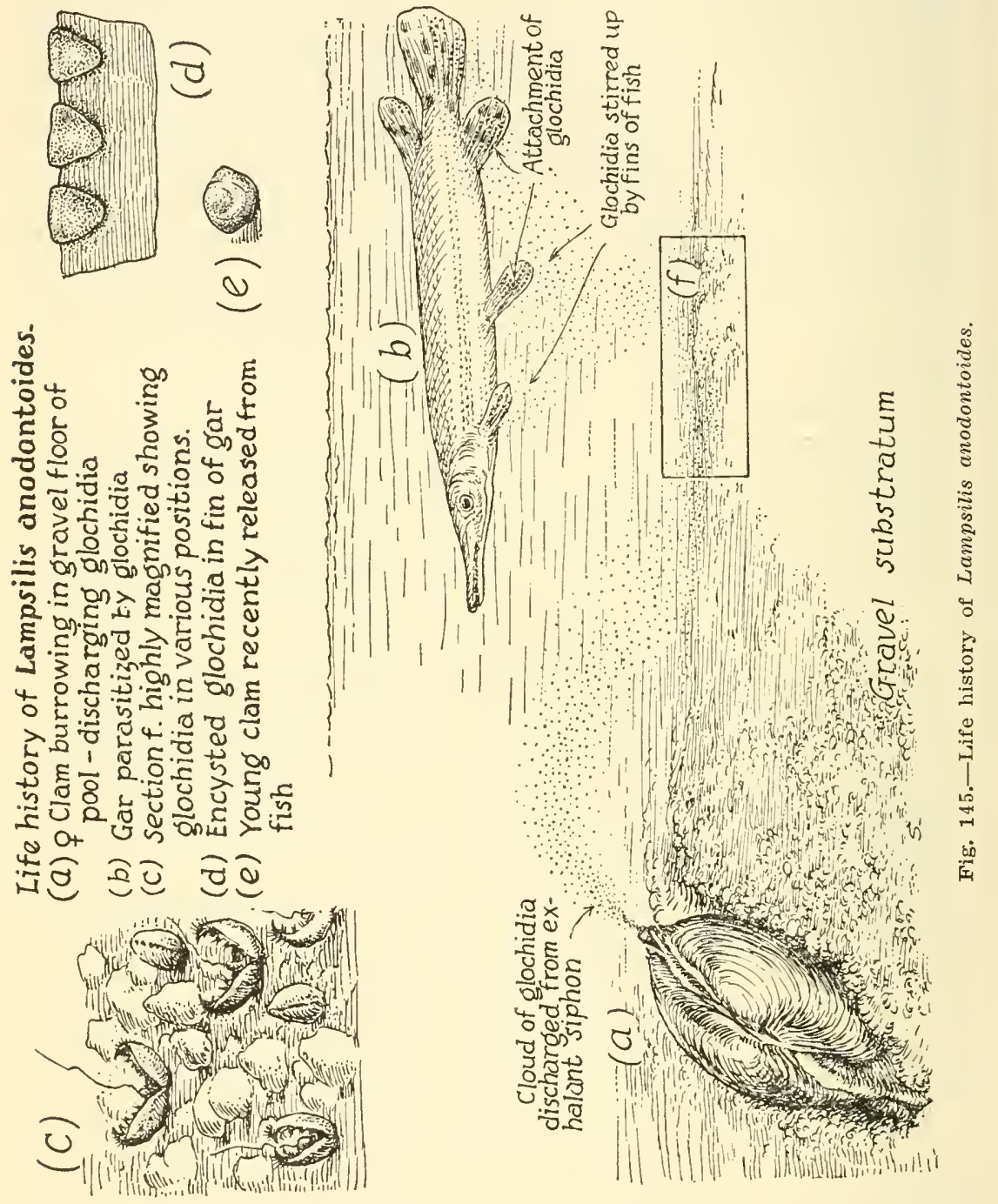

down upon it and remain attached if the fish is the suitable host for the particular species of clam. The tissues injured due to the attachment of glochidia produce by proliferation new cells which 
group up around and eventually cover the parasites. Thus a cyst is produced about the glochidium and within this structure the larval clam undergoes metamorphosis. It shortly breaks loose from its host, drops to the stream or pond bed, and leads an independent life. The rapid dissemination of mussels in a river system can be accounted for by the movements of their fish-hosts.

\section{Economic Relations of the Phylum}

Mollusks have been used as food by man from the beginning of civilization. Oysters, clams, scallops, suails, and the arms of cuttlefish are found in the menus of peoples all over the world. It has been estimated that the oyster industry along the Atlantic Seaboard approximates 40,000,000 dollars annually. Along the Texas coast alone, Federal statistics show that 51,719 barrels of oysters were sold in 1932. Buttons are made from the shells of the large heavy river clams and along the Ohio, Missouri, and Mississippi rivers the button industry amounted to 5,000,000 dollars in 1931 .

Within some of the clams are found pearls which are formed by some irritating particle, such as a parasite or sand grain that becomes lodged between the mantle and the shell. Iridescent protective layers of mother-of-pearl are deposited around the foreign particle, thus producing the pearl. The Japanese have been successful in artificially stimulating pearl production by planting small objects, such as pieces of mother-of-pearl, between the mantle and shell of pearl-oysters.

Pulverized clam shells are also being used as a calcium supplement to chicken feed. Shells have also been used as a medium of exchange. The wampum of the eastern coast of North America consisted of strings of cylindrical beads made from brightly colored clam shells. Shells have always been and still are used for ornamentation. Crushed shells are used in road construction.

Some mollusks are injurious to human interests. Among these might be mentioned the marine snail, Urosalpinx cinerea, which drills into and feeds on oysters and other pelecypods; the common shipworm, Teredo navalis, attacks the wood of ships and pilings, making extensive excavations. Certain species of snails serve as the intermediate host of parasitic flatworms or flukes. The liver fluke 
(Fasciola hepatica) whose intermediate host is the small fresh-water snail, Lymnaea bulimoides, causes the disease, liver rot in livestock, particularly in the sheep of the Southwest.

Since shells are easily fossilized they serve as excellent guides to the geologists in determining the type of rock formation and relative age of the strata.

\section{CLASSIFICATION}

Classification of this phylum is based on the nature of the foot, and respiratory organs; shape and structure of the shell; arrangement and structure of the nervous and reproductive systems.

Class I. Amphineura

Includes the Chitons, which are found abundantly on rocks between tide marks along the Atlantic and Pacific Coasts. This class appears to be the most primitive in the phylum, and its members have departed least from the ancestral condition. Bilaterally symmetrical body; tentaculess head, eyes absent; shell, if present, consists of eight overlapping plates. Most species have a flattened foot but other species are slender and wormlike Ischnochiton conspicuus.

Class II. Pelecypoda

Includes the bivalve mollusks, such as the oysters, clams, scallops, and cockles. More than ten thousand species have been described, of which approximately four-fifths live in the ocean. Division of the class into orders is based on gill characters.

Order 1. Protobranchiata

Marine species; gills consist of short, flattened leaflets; distribution along the Atlantic and Pacific Coasts.

Order 2. Filibranchiata

Marine species; gills composed of long filaments which hang down into the mantle cavity. The edible scallops and the sea mussel, Mytilus, exemplify this order.

Order 3. Eulamellibranchiata

Fresh-water and marine species; with two platelike gills which hang down into the mantle cavity on each side of the foot.

Family 1. Unionidae

Fresh-water clams or mussels; shell large or relatively large; valves equal and umbo anterior to center.

Family 2. Sphaeriidae

Fresh-water species. Shell small; umbo median or posterior to middle of shell. 
Order 4. Pseudolamellibranchiata

Marine species; gills plaited into rertical folds; shell frequently inequivalve. The oyster (Ostrea) and Pecten illustrate this order.

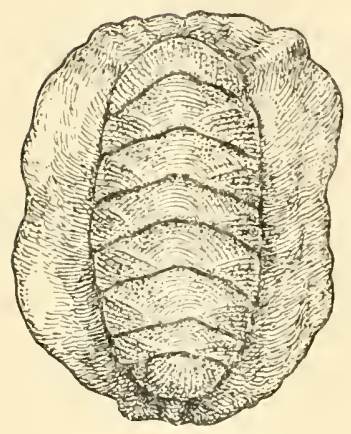

Ischnochiton

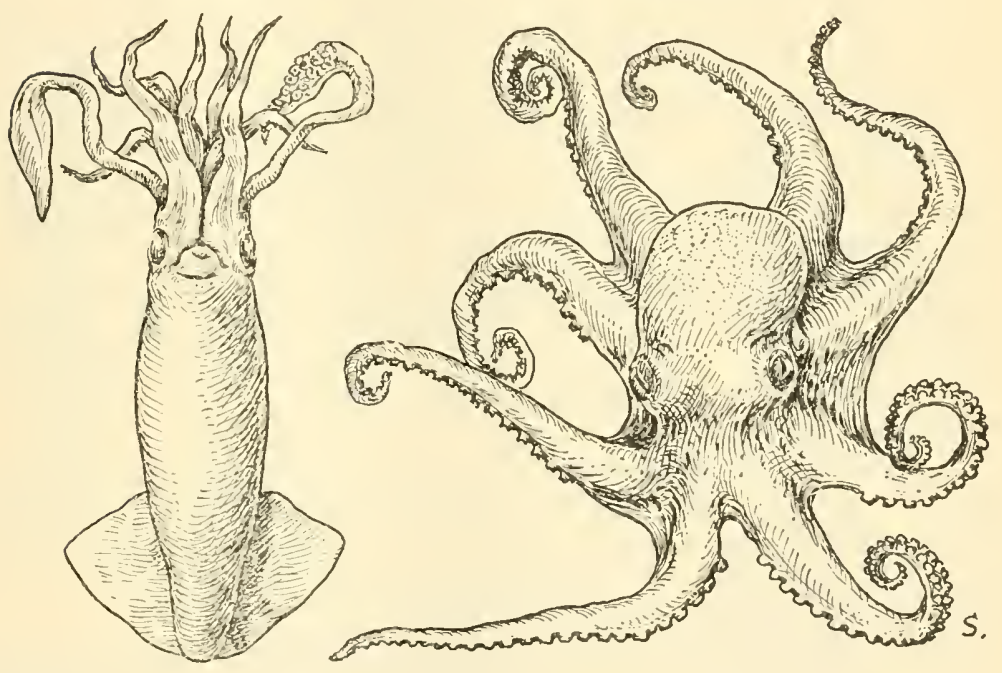

Loligo brevipennis Polypus bimaculatus (Octopus)

Fig. 146.- Representatives of three classes of mollusks. Class Amphineura, Ischnochiton; Class Scaphopoda, Dentalium; Class Cephalopoda, Loligo brevipennis (squid) and Polypus bimaculatus (octopus).

Class III. Gastropoda

Includes the snails and slugs. Approximately fifty-five thousand species hare been discovered and described. Shell, if present, univalve. 
Order 1. Prosobranchiata

Mostly marine, but fresh-water and land forms are represented. As the name implies, the gills are situated in the mantle cavity anterior to the heart. This order embraces such animals as the limpets, abalones, and periwinkles, all of which live in the sea; also a few fresh-water genera, such as Goniobasis, Campeloma and Pleurococera; Helicina orbiculata, a terrestrial southern species which is frequently arboreal in habit, comes under this order.

Order 2. Opisthobranchiata

Strictly marine. Gills, when present, are situated posterior to the heart; shell, if present, small. Includes the sea slugs. In the sea butterflies (pteropods), the foot may be modified into two fins which are used in swimming. Some of the heavier types have broad cephalic dises, adapted for burrowing in the sand. Many are found in coral beds and in seaweeds, their vivid colors harmonizing with the background.

Order 3. Pulmonata

Mostly terrestrial and fresh-water snails. Gills are absent, the mantle cavity serres as a pulmonary sac; shell usually present, sometimes rudimentary or absent.

Suborder 1. Basommatophora

Fresh-water species; eyes located at base of tentacles; external shell present. Includes the families Lymnaeidae, Physidae, Planorbidae and Ancylidae.

Suborder 2. Stylommatophora

Terrestrial snails and slugs; stalked retractile eyes, and one pair of retractile tentacles; shell in form of elevated or depressed spire, rudimentary and concealed, or absent.

Class IV. Scaphopoda

Marine. Mantle edges grown together along ventral side forming tube, with a shell of same shape and open at both ends. Commonly known as tooth shells. Approximately 300 known living species. (Dentalium.)

Class V. Cephalopoda

Marine. The most highly organized of the mollusks. A definitely formed head is present which bears a pair of eyes that superficially resemble the eyes of vertebrates. The foot is modified into arms or tentacles. They are carnivorous animals and many of them are used as food by man. (Nautilus, Loligo, Polypus.)

Order 1. Tetrabranchiata

The chambered nautiius (Nautilus) is a representative of this order. The animal inhabits the last chamber of a flattened spiral calcareous shell. As the name Tetrabranchiata implies there are four gills; also four primitive kidneys and 


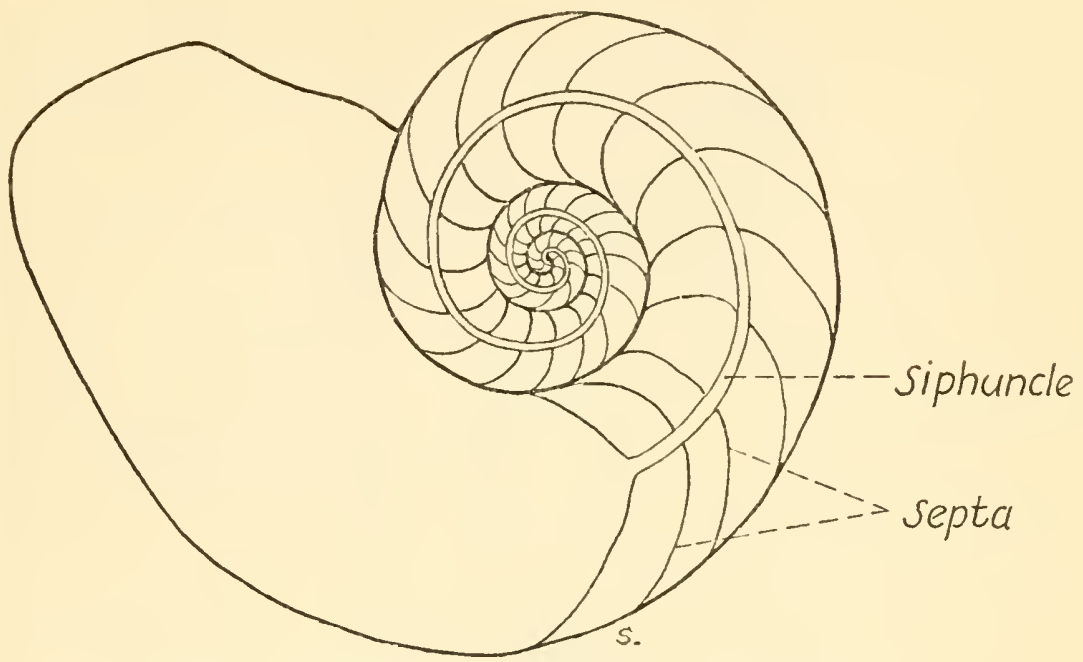

Fig. 147.- Sectional view of internal structure of Nautilus.

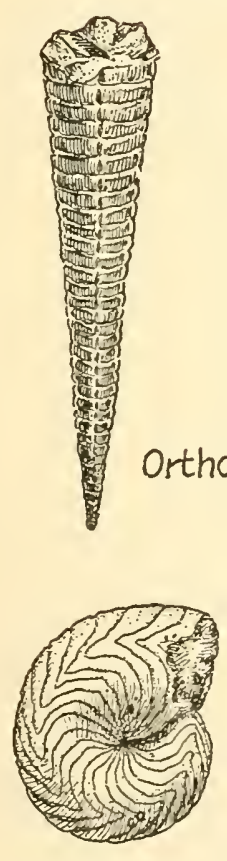

Goniatite

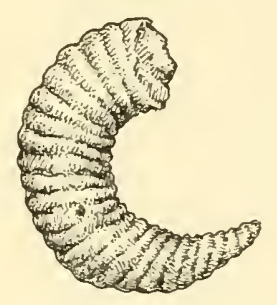

Cyrtoceracone

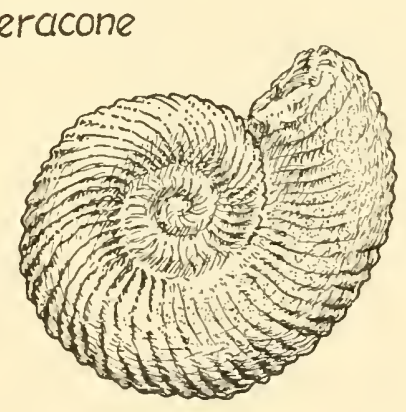

ceratite

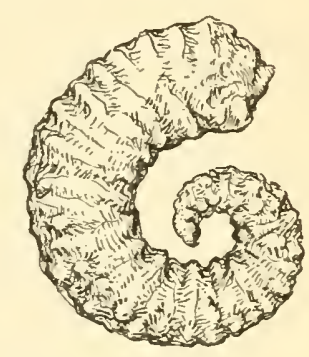

Gyroceracone

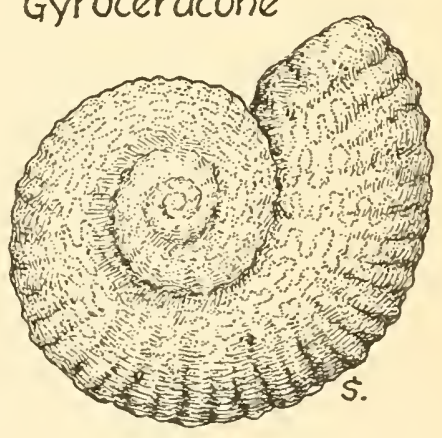

Ammonite

Fig. 148.-Evolution of the cephalopods. 
four auricles; ink sac absent. This suborder reached its peak of development in the Silurian and Devonian periods and is one of the most elear-cut examples of evolutionary development in the invertebrates.

During the Ordovician period the cephalopods constituted one of the chief groups of marine animals. Even though at that time cephalopods with coiled shells existed, the predominant ones were the orthocones (those with straight conical shells). This latter group in all probability gave rise to the entire series of coiled shells, culminating in Nautilus. In all nautiloids a series of partitions, termed septa, extend the full length of the shell. The point of union with the septa and sides of the shell hay appear as a straight, curved, angulate or highly complex line. This line is called the suture and in fossil shells whose outer shell coating is lost, it stands out rather conspicuously. The suture line is used as a taxonomic character for the group.

\section{Order 2. Dibranchiata}

Octopods and squids are representative types. Shell internal or absent; two gills and two primitive kidneys; ink sac present; mouth surrounded by 8 to 10 tentacles which are furnished with suckers. This order includes the largest of all mollusks, the giant squid (Architenthis princeps) which may attain a total length, including arms, of over fifty feet. The squids and octopods are noted for their ability to change color by the rapid contraction or expansion of chromatophores in their skin. Their unvements are rapid and are produced by expelling water from the mantle cavity througl the museular siphon with such force that the animal is jerked backward. In the squids, fins along the sides of the body facilitate locomotion.

Loligo brevipennis is the small squid found along the Gulf coast. When taken out of the water it is usually a mottled red or tan. The visceral mass and mantle cavity are enclosed by a thick muscular mantle. Beneath the skin along the back is a primitive endoskeleton in the form of a feather-shaped shell. The squid is predatory, feeding on almost any animal it can capture. Within the pharynx are two large jaws moved by powerful muscles. The pharynx connects with an esophagus which in turn terminates in a muscular stomacl. Digestive juices from the liver and pancreas are emptied into the stomach, and after the food is partially digested, it is passed into a thin-walled cecum where digestion is completed and absorption takes place. Wastes are discharged through the anus which opens near the base of the siphonal fold. The blood 


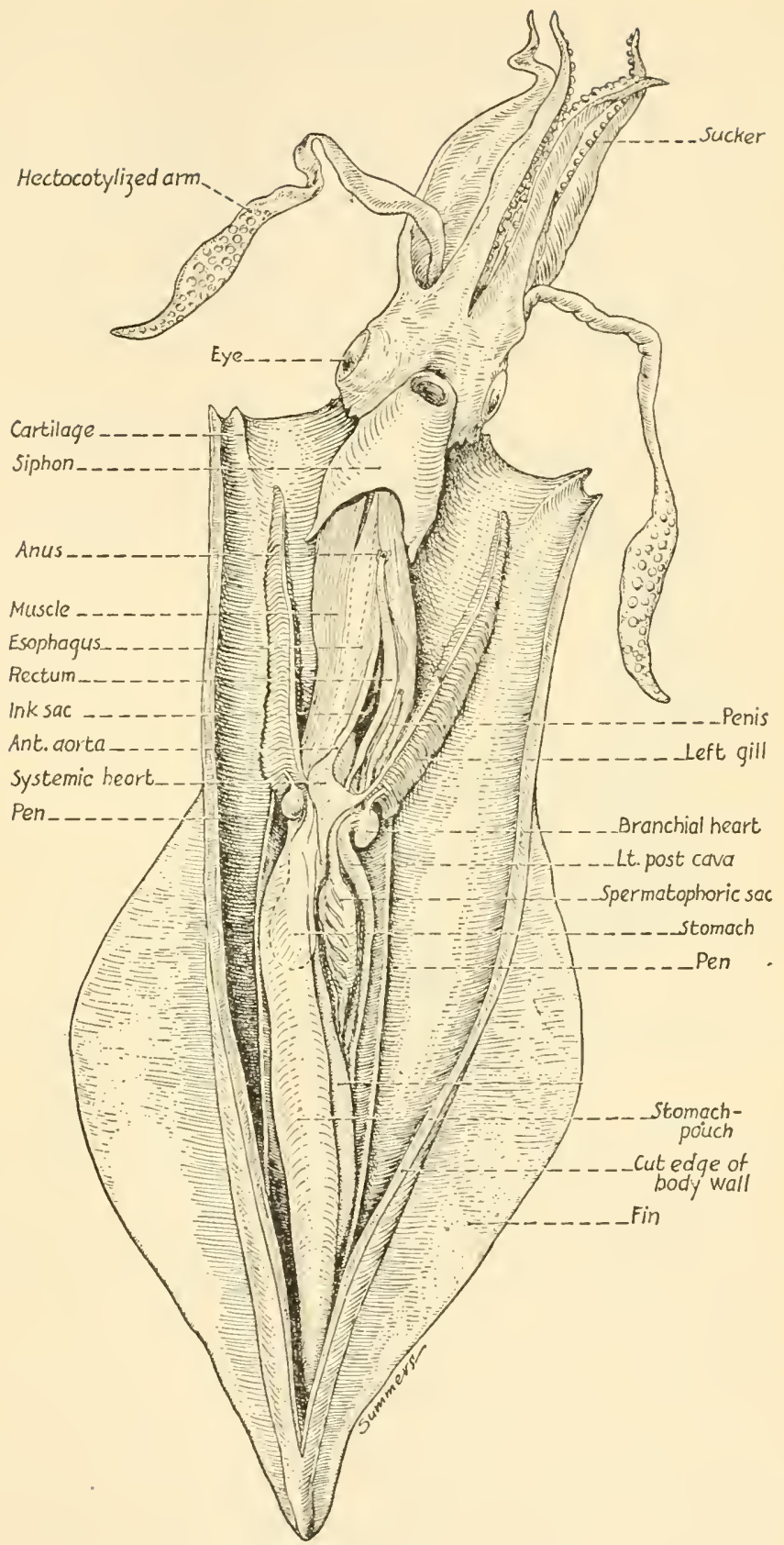

Fig. 149.--Dissection of squid to show internal anatomy. 
system, which is closed, is composed of arteries, veins, and two branchial hearts. Blood is oxygenated in two feathery gills which project into the mantle cavity. The two light-colored triangular kidneys are situated anterior to the branchial hearts and discharge their contents through small papillae, one located on each side of the intestine. In squids the sexes are separate. The male reproductive system is composed of a testis, vas deferens, spermatophoric sac, and penis; the female system consists of an ovary, oviduct, oviducal gland, and nidamental gland.

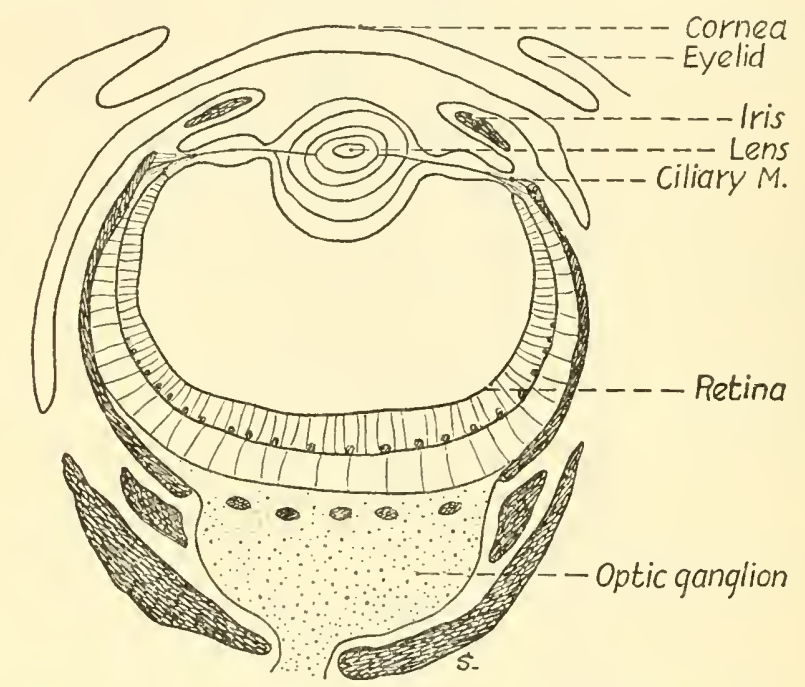

Fig. 150.-Longitudinal section through eye of squid. (Redrawn and modified after Borradaile and Potts by permission of The Macmillan Co.)

The nervous system of cephalopods shows a high degree of specialization when compared with the nervous system of other mollusks. The "brain" is composed of a close association of ganglia around the esophagus and is protected by a capsule of tough tissue resembling cartilage. Nerves radiate out from the central nerve mass to the various parts of the body; some of the nerves terminate in large ganglia, such as the stellate ganglia in the mantle. The eyes of the squid are supported by pieces of "cartilage" and are relatively complicated. Statocysts, which are similar but more complicated than those described for the clam, are situated near the brain mass. Ciliated pits which are supposed to be olfactory in function open in the form of a slit just back of each eye. 


\section{CHAPTER XVIII}

\section{PHYLUM ARTHROPODA}

Arthropoda (är thrǒp'ó dả, joint foot) is the name of the largest known group of animals. As the name implies, all representatives of the phylum have paired, jointed appendages and a definite tendency toward specialization of them. Their bodies are triploblastic, segmented, bilateral, and covered by a chitinous exoskeleton. The coelom is modified by a marked reduction as a result of specialized vascular spaces. The segmentation or metamerism of the body is expressed in a high degree in this phylum and there is a definite relation of appendages to segments. The segments have undergone greater specialization and greater regional differentiation than was the case in annelids. In forms where there is little or no differentiation of segments, the condition is referred to as homonomous, while a highly differentiated condition of segments as found in most arthropods is spoken of as heteronomous. This group has fairly distinct head, thorax, and abdomen. The appendages on various segments are typically homologous with each other. Some are modified as sense organs, others as mouth parts, others for walking, swimming, and reproduction.

The skeleton is entirely exoskeletal, composed of chitin, and fits exactly the shape and contour of the body. Since it is fairly unyielding to growth, it becomes necessary for the arthropod to shed the skeleton periodically during its growing periods. This molting or ecdysis, as it is called, is quite characteristic of many of the divisions of this phylum.

The circulatory system is of the open type, since there are large sinuses or spaces surrounding most of the organs instead of a continuous circuit of blood vessels. The nervous system is of a modified ladder type with a ventrally located cord. The digestive system shows specialization in that it is divided into distinct regions as an adaptation to special types of food which require mastication.

\section{Classification}

This phylum is divided into two sections and at least five classes; some authors recognize as many as eight. The sections are determined according to the means of respiration. 
Section I. Branchiata (brăn kĭ ă'tà, gill) gill-breathing, aquatic forms for the most part.

Class I. Crustacea, crayfish, crab, pill bug, barnacle, water flea, etc.

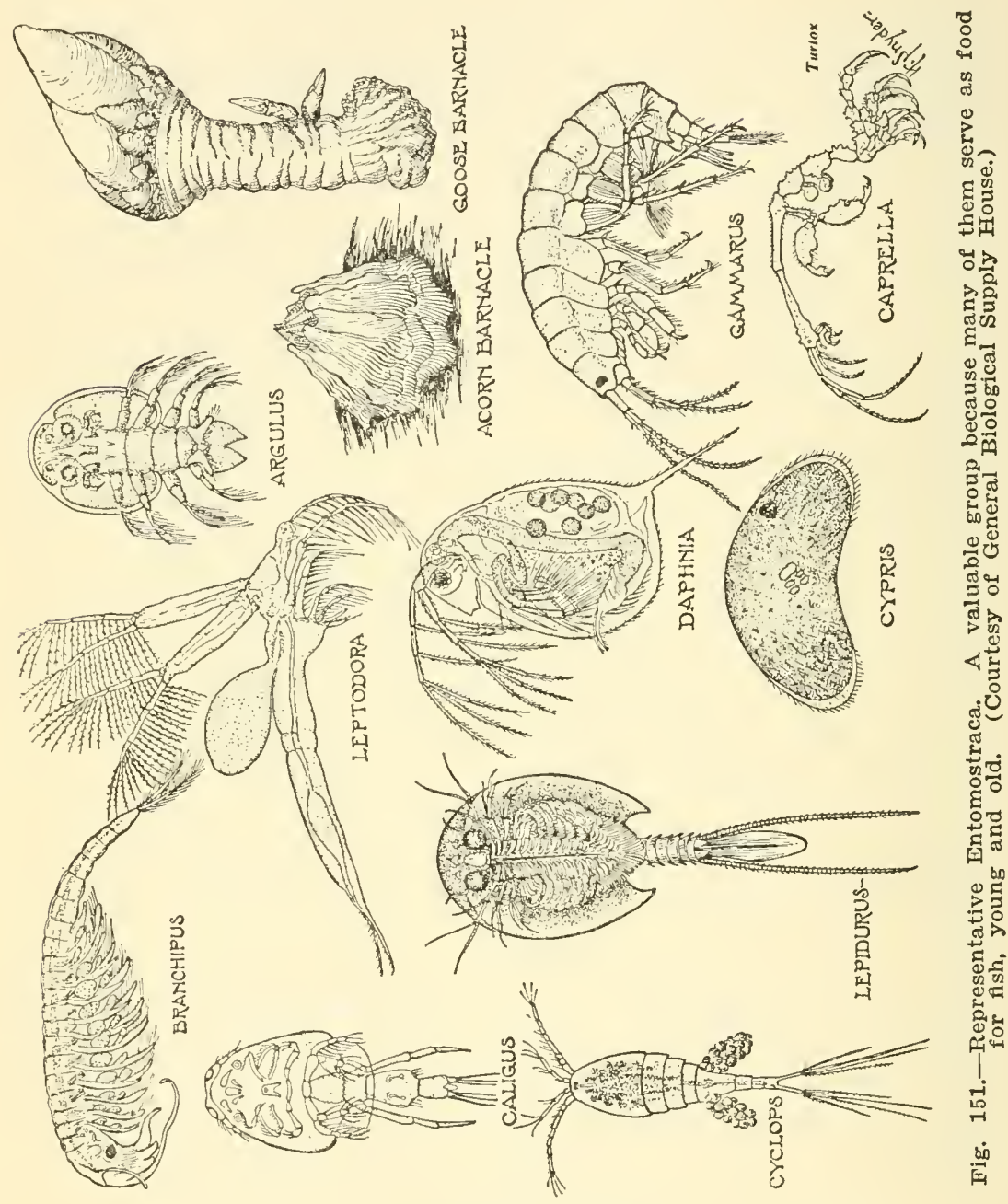

Subclass Entomostraca, fairy shrimps, water fleas, and barnacles. Order Branchiopoda, fairy shrimp (Branchipus), water flea (Daphnia).

Order Ostracoda, Cypris. 
Order Copepoda, cyclops, fish louse (Argulus).

Order Cirripedia, goose barnacle (Lepas), rock barnacle (Balanus), Sacculina (Fig. 404).

(Some authors prefer to rank Branchiopoda, Ostracoda, Copepoda, and Cirripedia as subclasses, thereby dispensing with Entomostraca.)

Subclass Malacostraca, pill bugs, sow bugs, sand fleas, lobsters, crayfish, and crabs.

Order Isopoda, pill bugs and sow bugs.

Order Amphipoda, sand fleas and beach fleas.

Order Decapoda, crabs, crayfish, lobsters, and shrimps.

Section II. Tracheata (trà kiè à'tá, rough) both terrestrial and aquatic arthropods which breathe by tracheae, book lungs or book gills. This section is divided into three divisions depending on the primitiveness of the characteristics.

Division A. Prototracheata. The primitive form with some arthropod characteristics and certain annelid features, such as nephridia.

Class II. Onychophora, Peripatus, the wormlike arthropod.

Division B. Antennata. More highly specialized forms with one pair of antennae.

Class III. Myriapoda, centipedes and millepedes (thousand legs) having one or two pairs of appendages on each segment.

Order Chilopoda, centipedes.

Order Diplopoda, millepedes.

Class IV. Insecta, beetles, bees, locusts, etc., all with three pairs of thoracic appendages and most of them with wings.

Order Thysanura, silver moth.

Order Collembola, springtails.

Order Ephemerida, mayflies.

Order Odonata, dragonflies and damsel flies.

Order Plecoptera, stone flies.

Order Embiidina, embicls. (Texas, Califomia, Florida.)

Order Orthoptera, erickets, grasshoppers, roaches.

Order Isoptera, termites or "white ants."

Order Dermaptera, earwigs.

Order Coleoptera, weevils and beetles.

Order Strepsiptera, stylopids (parasites in insects).

Order Thysanoptera, thrips. 
Order Corrodentia, book lice.

Order Mallophaga, bird lice.

Order Anoplura, body lice ("cooties"), crab louse.

Order Hemiptera, true bugs, as squash bug.

Order Homoptera, plant lice, seale insects, eicadas.

Order Neuroptera, aphis lions, ant lions.

Order Trichoptera, caddis flies.

Order Lepidoptera, butterflies and moths.

Order Mecoptera, scorpion flies.

Order Diptera, true flies, mosquitoes.

Order Siphonaptera, fleas.

Order Hymenoptera, wasps, ants, bees.

Division C. Arachnoidea (ăr ălk noi'dè á, spiderlike). A group without antennae but with tracheae, book lungs or book gills, and four pairs of thoracic appendages.

Class V. Arachnida, spider, mite, scorpion, king erab, etc.

Order Scorpionida, scorpions.

Order Pedipalpi, vinegarroon and tarantula.

Ordex Pseudoscorpionida, book scorpion.

Order Phalangida, daddy longlegs or harvestmen.

Order Palpigradi, one Texas species.

Order Araneida, spiders.

Order Acarina, ticks and mites.

Order Xiphosura, king erab or horseshoe crab.

This summary of the classification of the phylum has been placed early in the chapter in order that the student may realize the magnitude of its size and the great variety of animals included. The number of species described under the phylum is approximately one-half million, and there are large numbers still undescribed and unnamed.

\section{CRAYFISH OF CLASS CRUSTACEA}

Since this animal represents a relatively simple type of arthropod and is so generally well known, it serves ideally as a representative species for a more detailed study. The genera Cambarus and Potamobius or Astacus are commonly found in the streams of North America. The former is distributed east of the Rocky Mountains and the latter on the Pacific slope. 


\section{Habitat and Behavior}

For the most part crayfishes (crawfishes, crawdads, fresh-water lobsters) are inhabitants of fresh-water streams and ponds where there is sufficient calcium carbonate in solution for purposes of skeleton formation. These animals may be found moving about on the bottom, or they may be in hiding under some stone or log, or they may be in the mouth of a burrow beneath the water's edge. Some species carry air tumels vertically from the original horizontal burrow to the surface of the earth and deposit mud around the opening of a tumnel. They are much more active at night than during the day. It is possible for them to walk about on the bottom of the stream or pond, moving the body in almost any direction. Their

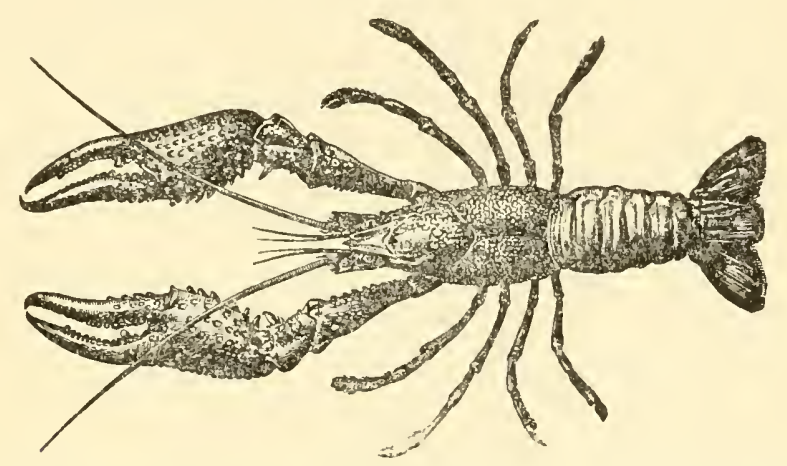

Fig. 152.-Cambarus clarkii, the swamp crayfish, a very common species in the swamps of the Southern States. (Courtesy of Southern Biological Supply Co.)

swimming habits are rather peculiar in that they dart backward through the water, as a result of the strong downward stroke of the tail. One stroke of the tail will carly the animal a yard and this is commonly sufficient to avoid the enemy. The daytime is usually spent in hiding under objects or in the mouth of the burrow. Crayfishes may at times desert their aquatic habitat and go foraging out over swampy land. In some localities certain species build their burrows down to the subterranean water table right out in the fields and become important pests. Sight, touch, and chemoreception are important senses in this animal.

The crayfish captures other animals, such as tadpoles, small fish, and aquatic insects, by waiting in hiding and suddenly seizing 
them. The crayfish is quite well protected, due to its protective color which matches the background, its chitinous skeletal covering, and its pinchers. In spite of this, they are captured by water snakes, alligators, turtles, fish (such as bass and gars), frogs, salamanders, herons, and raccoons in particular. Many have been exterminated by the drainage of swamps, and by their use as food for man.

\section{External Structure}

The chitin-covered body is divided into cephalothorax, abdomen, and appendages. The cephalothorax is a compound division of the body including the thirteen most anterior segments and is divisible into head and thorax. The boundary between these is marked by the oblique cervical groove on each side of the region. The shelllike covering whose lateral edges are free, is known as the carapace. The portion anterior to the cervical groove is the head or cephalic portion, while the portion posterior to the grooves is the thorax. The anterior end of the cephalothorax is drawn out to almost a point, and this portion is called the rostrum. The mouth is located on the ventral side of the head portion and not at the tip of the rostrum where most people look for it. The lateral portions of the carapace are known as branchial arcas or branchiostegites, and they cover the gills. Their ventral edges are free. On the ventral side of the thorax between the twelfth and thirteenth segments (about the level of the fourth walking leg) of the femalc is a cuplike pouch called the annulus or seminal receptacle. It serves in reproduction for the receipt and storage of spermatozoa.

The portion posterior to the thorax, which is frequently called "tail" by fishermen, is really the abdomen, and the tail proper is at the posterior end of this. The abdomen is divided into six typical segments and the terminal telson, which has no appendages but is often called the seventh abdominal segment. The anus is found on the ventral side of this part. The skeletal part of the abdominal segment consists of: the dorsally arched tergum; a thin, overhanging lateral plate, the pleuron; and the slender ventral sternum in the form of a narrow bar extending from side to side. A thin arthropodial membrane extends between successive sterna and allows for movement of the segments upon one another. 

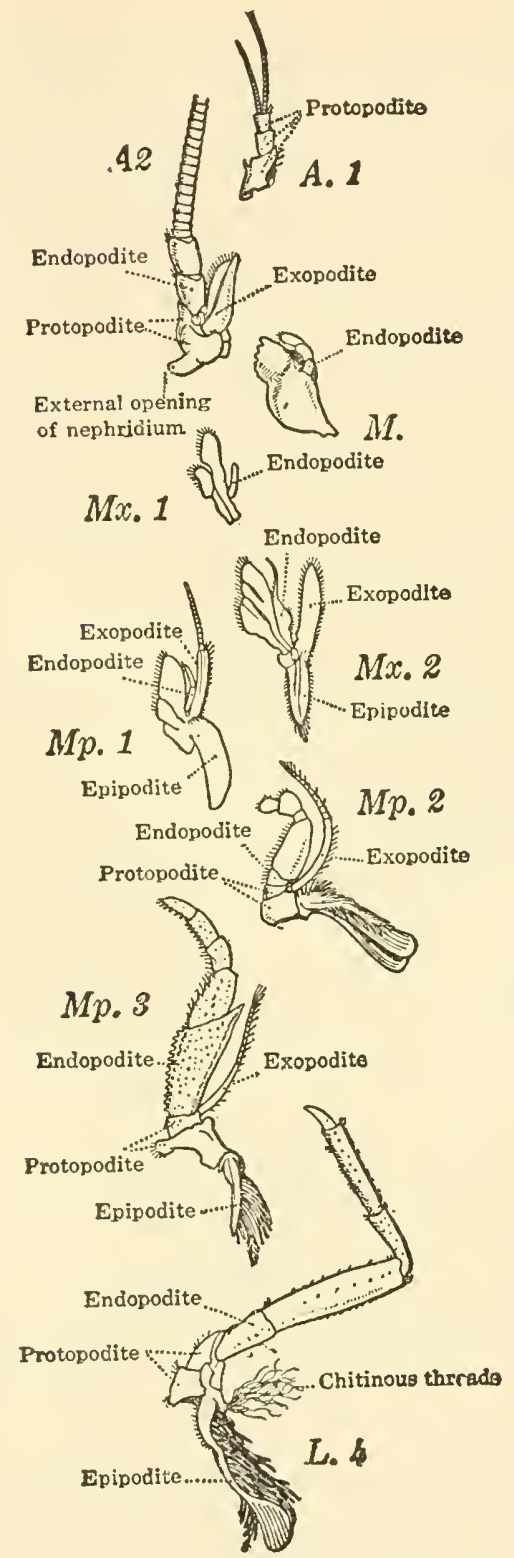

Fig. 153.-Examples of cephalic and thoracic appendages of the crayfish, ventral view. $A .1$, Antennule; $A .2$, antenna; $L$. 4 , fourth walking leg; $M$, mandible $M p$. 1. first maxilliped; 'MID. 2, second maxilliped: $M P$. 8, third maxilliped : $M x$. 1, frst maxilla; $M x$. 2, second maxilla. (From Newman, Outlines of General Zoology, published by 'The Macmillan Company, after Kerr.) 
The appendages are paired, with one pair attached to each typical segment. There are nineteen such pairs. They are all developed on the same plan from the typical biramous (two branched) appendage. The five anterior pairs of abdominal appendages are quite typical of the primitive form except for the modification of the first two in comnection with reproduction. This group is known as swimmerets or pleopods and all have the fundamental parts consisting of a basal protopodite composed of coxopodite, joining the body and the basipodite; the exopodite or lateral branch and the endopodite or medial branch each have many joints. The first two are much reduced in the female, but in the male the protopodite and endopodite are fused and extended to serve as an organ for transfer of spermatozoa. The posterior pair of swimmerets, attached to the sixth abdominal segment, are broadened into fanlike structures for swimming. They are known as uropods and have oval, platelike exopodite and endopodite. The posterior five thoracic appendages are the walking legs or perciopods. These are uniramous due to the complete reduction of the exopodite. Each is composed of the two joints of the protopodite and five of the endopodite. Joining the coxopodite (first segment of protopodite) is a sheetlike structure which supports a gill and some chitinous threads. The three anterior walking legs possess pinchers or chela which are formed by the terminal segment being set on the side of the second segment. The walking legs are used in locomotion, offense, and defense. The three anterior segments of the thorax bear three pairs of biramous maxillipeds. The parts are quite typical in most respects. Each has an epipodite joining the basipodite and all except the first bear gills. These appendages are used in getting food to the mouth.

To the segments of the head are attached five pairs of appendages. Just posterior to the month and immediately in front of the first maxilliped are two pairs of maxillae, the second of which overlies the first. They are both leaflike and modified. The epipodite and exopodite of the second are fused to form a bladelike bailer or scaphognathite which fits over the gills and by its movement helps circulate the water for respiration. Its endopodite is slender, but the protopodite is broad and foliate. The first maxilla is reduced to a leaflike protopodite and small endopodite. The jawlike mandible at each side of the mouth is composed of hard protopodite 
with teeth and a fingerlike endopodite, which is tucked under the anterior edge of the former. This appendage is used for chewing. In front of these are the antcnnae which are biramous and are sometimes called "feelers." They consist of the protopodite of two parts, a long many-jointed, filamentous endopodite and a relatively short, fan-shaped exopodite. Anterior to these are the antennules which are biramous and feelerlike. The exopodite and endopodite are similar in these.

The principle of homology is excellently illustrated by the appendages of the crayfish. In general, homologous structures are those which have similar structure and similar origin but may have similar or different functions. By way of contrast, analogous struetures are those which, when compared, show different structure and origin but similar function. During early development each of the appendages of the crayfish is similar to all others. Some become modified with development. Other illustrations of homologous struetures are the human arm and the bird's wing. In organisms like crayfish where the appendages of successive segments are homologous to each other, the condition is spoken of as serial homology. Homologous structures are found in many animal groups and are used in establishing relationships. It has been suggested that the parapodia of Nereis represent possible forerumners of erustacean legs. They are both typically biramous and both take about the same position on the body, as well as having a similar segmental distribution. There is also considerable similarity in their structure.

\section{Internal Structure}

Beneath the shell-like, chitinous exoskeleton there is a very representative set of systems. As in most higher animals the segmentation is retained in the muscular system, nervous system, and to a degree in the circulatory system. Earlier in the chapter it was pointed out that the coelom is modified as a provision for increased blood sinuses which have occupied mueh of the space.

Respiratory System.-Under the branchial areas of the carapace may be found the paired, feathery gills held in the gill cavity or branchial chamber. There are three types of gills present here: pleurobranchiae, attached to the sides of the thorax; podobranchiae, arising from the epipodites of the thoraeic appendages; and arthro- 
branchiae, which arise from the coxopodites of the thoracie appendages. Several of the segments have lost the pleurobranchiae. The scaphognathite moves in such a way over the external surface of the gills as to move the water in an anterior direction. The water is brought under the free edge of the branchiostegite or branchial area of the carapace and moved forward to be discharged by an anterior aperture. An almost constant stream of water is pumped over the gills to facilitate the exchange of oxygen and carbon dioxide between the blood in the capillaries of the gills and the surrounding water. The aerated blood is then carried to all of the tissues of the body.

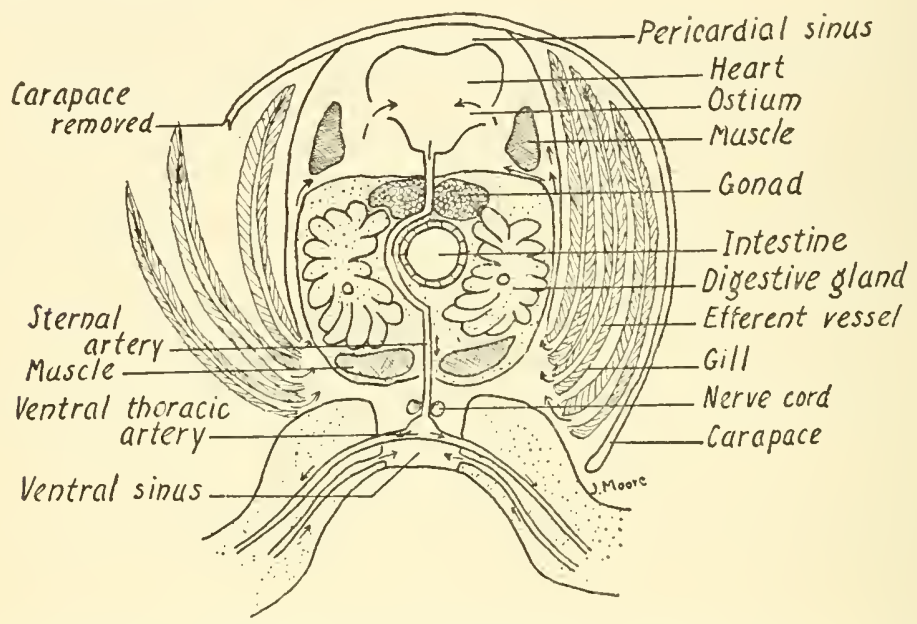

Fig. 154.-Diagram of cross section through the posterior thoracic region of a crayflsh. Arrows indicate flow of blood.

The digestive system is in the form of a modified canal and is composed of mouth, esophagus, stomach, and intestine. The mouth opens between the mandibles on the ventral side of the third segment. From this the short, tubular esophagus leads dorsally and joins the ventral side of the stomach almost directly above the mouth. This larger anterior portion of the stomach is the cardiac chamber. Within its wall are a number of hard chitinous bars, known as ossicles, which bear teeth capable of mastication of food when moved over each other by the muscular activity of the wall. This grinding apparatus is known as the gastric mill. Between the cardiac chamber and the posterior or pyloric chamber is an arrange- 
ment of bristles which serve as a strainer that allows only properly masticated food to pass through. The pyloric chamber is considerably smaller and curves downward to continue posteriorly as the tubular intestine which extends almost directly posteriorly through the center of the abdomen to the anus in the last segment. Large digestive glands (hepato-pancreas) lead into the pyloric chamber through hepatic ducts. The secretion of these glands contains digestive enzymes.

The vascular system consists of a heart, the pumping organ; the arteries, definite vessels; the sinuses, a series of blood spaces; and the blood which circulates. It consists of the fluid plasma containing

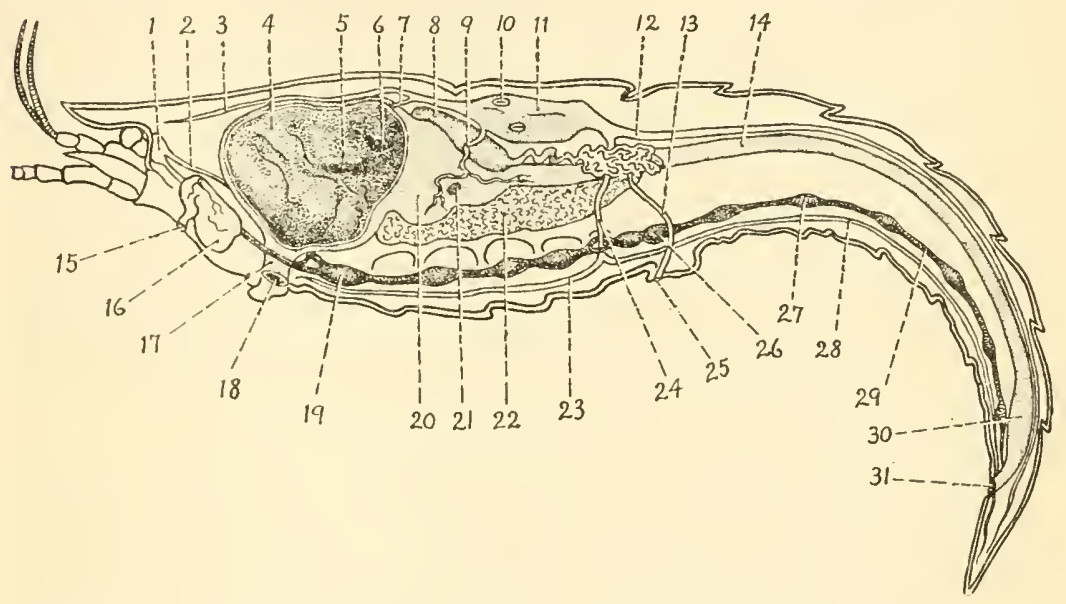

Fig. 155.-Lateral view of a dissection of the crayfish to show many of the internal organs. 1, supraesophageal ganglion; 2 , circumesophageal connective ; 3 , ophthalmic artery ; 4 , stomach, cardiac portion; 5 , lateral teeth; 6 , median teeth: 7 , antennary artery ; 8 , testis ; 9 , hepatic artery: 10 , ostium; 11 , heart; 12 , dorsal abdominal ganglion; 28 , ventral abdominal artery; 29 , nerve cord; 30 , rectum ; gland; 17, esophagus; 18 , mouth; 19 , subesophageal ganglion; 20 , stomach, pyloric portion; 21 , opening of hepatic duct; 22 , digestive gland; 23 , ventral thoracic artery ; 24 , sternal artery; 25 , opening of vas deferens; 26 , thoracic ganglion ; 27 , abdominal artery: 13, vas deferens; 14 , intestine; 15, renal opening; 16, green 31 , anus. (Modified from Turtox Key Card of Crayfish. Courtesy General Biological Supply House.)

white corpuscles but without red ones. The hemocyanin which absorbs oxygen is dissolved in the plasma. Fresh blood is almost clear and colorless, but it takes a blue color after standing in the air for a short time. The heart is somewhat flattened and angular in outline, and has a muscular wall which is perforated with three pairs of slitlike ostia. When the muscular wall of the heart is relaxed, the slits open, 
and blood is drawn in from the surrounding pericardial sinus in which the aerated blood accumulates. When the heart contracts, blood is foreed into the anterior region of the body through the single anterior median artery, paired antennary, and paired hepatic arteries all of which arise from the anterior end of the heart. The large dorsal abdominal artery extends from the posterior tip of the heart posteriorly throngh the abdomen just dorsal to the intestine. It supplies the intestine and muscles of the body wall. The sternal artery is a large branch arising from the dorsal abdominal artery just after it leaves the heart. It passes ventrally through the nerve cord and divides into a posterior, ventral abdominal artery and an anterior, ventral thoracic artery. These branehes carry blood to the ventral portions of the body. Besides the pericardial sinus already mentioned, there are others retmrning the blood to this one. The sternal sinus is the main one, and it is located beneath the thorax. From it several branches lead into the gills. This provides for a course through the gills. From them blood is collected by branchio-cardiac canals and delivered to the perieardial sinus. A perivisceral sinus surrounds most of the alimentary canal and collects the venous blood from it. This kind of system is called the open type because of the large irregular spaces or sinuses instead of an evenly constructed set of veins which make a complete circuit of the course.

The excretory system eonsists principally of a pair of large bodies located in the ventrolateral portion of the head. These are richly supplied with blood and draw the nitrogenous wastes and excess water from the blood to deliver them externally through cxcretory pores located in the eoxopodites of the antennae.

The nervous system is of the same structural plan as that of the earthworm, which is a modified "ladder type." The two longitudinal cords have come together in the ventral line and run the entire leugth of the body to form a ventral nerve cord with ganglia. This arrangement constitutes the central nerrous system. The ganglia of the anterior three segments are fused into the "brain" or supraesophagcal ganglion which is located anterior to the esophagus and is joined to the cord by two circumesophageal commissures or connectives, one passing on each side of the esophagus. From this dorsal ganglionic mass, norves pass to the eyes, antemnae, and antemnules. The most anterior portion of the ventral cord receives these com- 
missures. This portion, which consists of the fused ganglia from segments three to seven, is known as the subesophageal ganglia. Nerves go from it to the mouth parts, first and second maxillipeds, green glands, esophagus, and muscles of the thorax. Each segment posterior to the subesophageal ganglia possesses a segmental ganglion with branches to its respective appendages and muscles. The sense organs include antennae, antemules, sensory hairs, statocysts, and

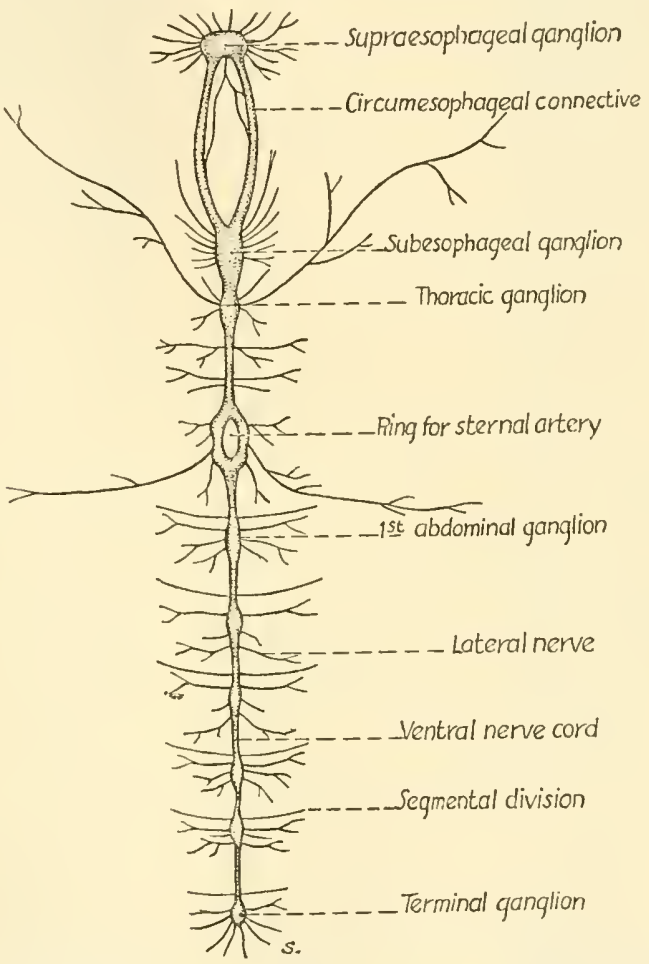

Fig. 156.-Dorsal view of nervous system of crayfish. Notice merging of anterior thoracic ganglia with subesophageal ganglion.

eyes. The antennae are tactile organs (sensitive to touch), the endopodite of which is a relatively long jointed filament. The exopodite is much shorter and fan-shaped. The basipodite and coxopodite are closely fused to the ventral side of the cephalic region. An excretory pore opens to the exterior through the coxopodite of each antenna. The hairlike processes along the edge of the carapace, on the legs, and other parts of the body are also sensitive to touch. The anten- 
nules are tactile and each has two slender filamentous processes, the exopodite and endopodite. In addition to these slender jointed processes each antemule has a saclike statocyst in its coxopodite. This structure is an infolding from the outside and is lined with exoskeleton and sensory hairs. Inside of each are small particles of solid material, such as grains of sand, which are called statoliths. As the animal changes its position the statoliths move about inside of the statocyst and stimulate the sensory hairs. From these stimulations the erayfish is able to determine its orientation in space, i.e., it knows whether it is in normal walking position, on its back, or standing on its head. These organs serve for equilibrium. When the crayfish molts, the statocysts are temporarily lost and new ones form as the new skeleton develops. If there are no solid objects in the water in which a crayfish lives during molting, there will be no statoliths in the statocysts and the animal has an impaired sense of equilibrium. Experimenters have placed only iron filings in the water at such a time and the animals present have used them for statoliths. By bringing a magnet near the crayfish in this condition the statoliths are moved and the animal goes through numerous peculiar contortions in attempting to respond to these stimulations of orientation. Besides the above functions the antennules provide the chemical senses of smell and taste.

The eyes, which are of the compound type, are mounted on movable stalks, one on each side of the head region. They are described as compound because each one is composed of a large number of individual sight units, each of which is essentially an eye. Each of these units is called an ommatidium, and the crayfish has about 2,500 in its eyes. A single one is rather spike-shaped, tapering from the broader superficial end to the rather pointed internal extremity. A single ommatidium has an outer cornea which is transparent and supported by some corneagen cells on the vitrella. Beneath this is the rather long crystalline cone beneath which is the rhabdom, another lenslike structure. Surrounding the latter are sensory cells making up the retinula. The wall of the ommatidium possesses pigment cells along the sides of the crystalline cone and in the retinula. The distribution of the pigment varies with the intensity of the light. The stronger the light the more these cells are expanded and the more direct must be the ray of light to reach the retinula, because 
the possibility of reflection within the ommatidium is reduced. In dim light the pigment is concentrated partly toward the outer and partly toward the basal portion of the ommatidia which allows more refraction of rays by the crystalline cones and a combination of images in several adjacent units. In brighter light only the ray from

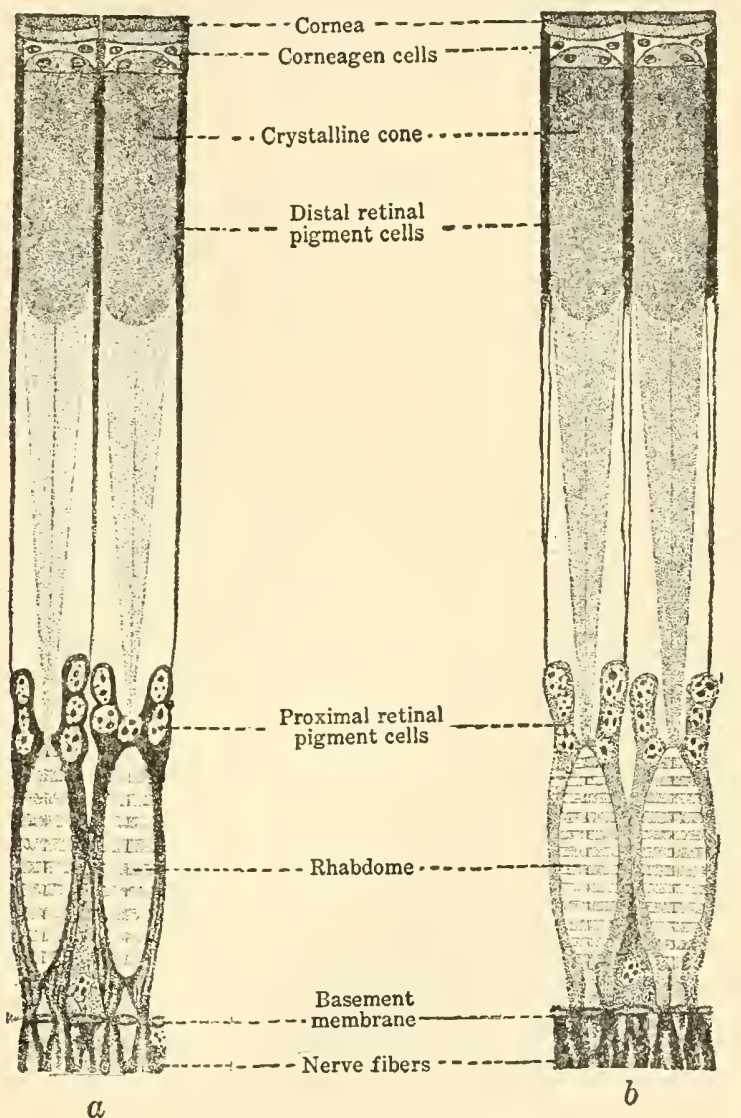

Fig. 157.-Longitudinal section of ommatidia from eye of crayfish. $a$, position of piginent when light is present; $b$, position of pigment when in the dark. Notice in the latter the distal pigment is in the outward position and the proximal pigment is concentrated inwardly. (From Hegner, College Zoology, published by The Macmillan Company, after Bernhards.)

directly in front of the cornea will reach the retinula and stimulate the nerve cells there. These cells are commected internally with the optic nerve. The type of vision produced in the compound eye 
is "mosaic" in that there is registered only a single image by the eye. Each ommatidium which is in focus on the object registers an image of that part. As the object moves, new ommatidia are stimulated and movement is indicated by the rate of stimulation of successive ommaticlia. The farther the object is from the eye, the fewer ommatidia will be stimulated. The crayfish eye is often termed a modified appendage because an antennalike structure will regenerate in case an eye is mutilated.

\section{Metabolism}

The crayfish ingests principally flesh from bodies of fish, snails, tadpoles, insects, and other animals, some caught alive and others found dead. The maxillae and maxillipeds hold the morsels while they are crushed by the mandibles. Mastication continues in the cardiac chamber of the stomach and chemical digestion begins in the pyloric portion. The digestive juices possess enzymes which convert the food into soluble form, and as it passes along the intestine, it is absorbed by the blood and distributed to the tissues over the body. This conversion of food material into protoplasm is assimilation. The external phase of respiration has put oxygen in the blood, and it is distributed throughout the protoplasm of the cells. The energy stored in the food material is released or converted to kinetic form by mion with the oxygen (oxidation) in the protoplasm. From this union there is excess heat produced. Mechanical and chemical activity is the result of the harnessing of this energy. As a by-product of this catabolism, excretory materials, such as excess water, urea, uric acid, and other substances are formed in solution and are collected by the blood. The green glands relieve the blood of these and deliver them to the exterior. Of course growth results when excess food materials are built into the cells at times when the rate of anabolism exceeds that of catabolism.

\section{Reproduction}

These animals are dioecious (sexes separate) and the mating takes place either in the spring or fall or perhaps both. The spring hatch become well developed before winter. The eggs produced in the fall may not be laid before spring. 


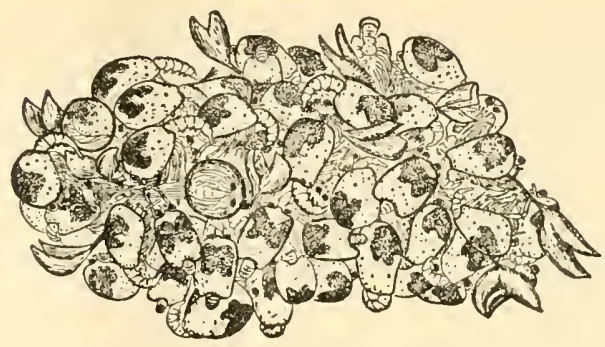

A.

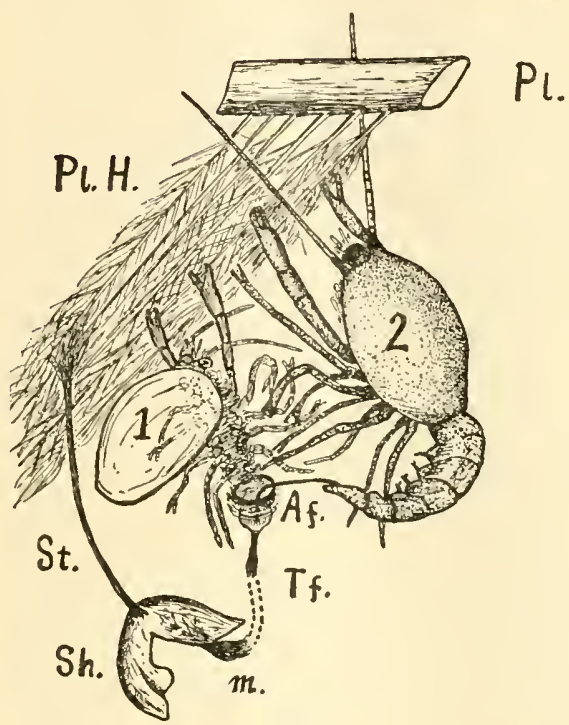

B.

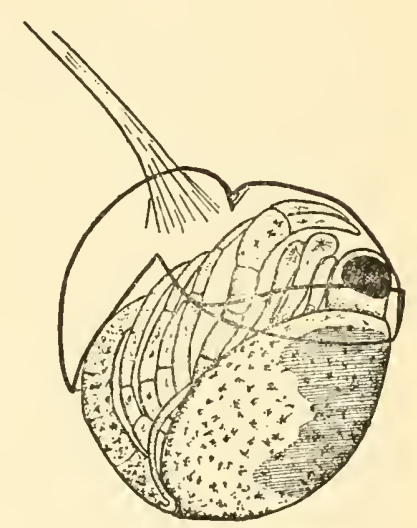

C.

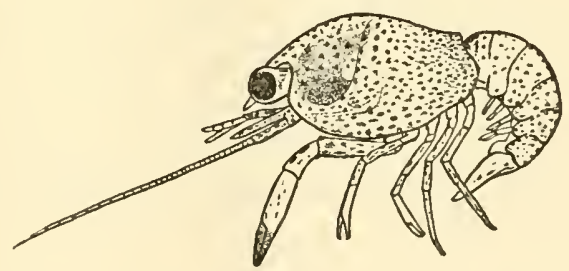

$D$.

Fig. 158.-Development of the crayflsh. A, Young crayfish clinging to swimmerets of mother. $B$, Second larval stage (2) attached by its chelipeds to hairs $(P l . H$.$) on a swimmeret (P l$.$) of the parent. The molted shell of the first larval$ stage $(1)$ is clinging by chelipeds. A portion of the egg-membrane ( $m$ ) and shell (Sh.) are still attached to the swimmeret by a stalk (St.). When the first larva hatches it remains attached to the shell by a flament (Af.). By means of these flaments the young remain fastened to the mother during development. $C$, First larva hatching through shell. D, The second larva. (Reprinted by permission after Andrews, 1916, Smithsonian Contributions, Vol. 35.) 
In the case of Cambarus clarkii the adults retire to holes or burrows at the water's edge during the summer. It is here that the eggs are laid and carried by the female until after hatching; then the young cling to her swimmerets. In late summer or fall, soon after the young hatch, the adults become very migratory at night, particularly in rainy weather. In this way they help to distribute the young to new water holes.

The female reproductive organs are composed of a bi-lobed ovary located beside the pyloric chamber of the stomach and beneath the pericardial sinus. During development the eggrs appear in the ovary. Two oviducts lead, one from each side of the ovary, to a genital pore in the coxopodite of the third walking leg (pereiopod) of each respective side. The ova develop in follicles in the ovary. The maturation divisions (oögenesis) take place here and, when mature, the eggs break into the central cavity of the ovary, from which at the time of laying, they pass out through the oviducts. The male reproductive organs are composed of the bi-lobed testis located dorsal to the pyloric stomach and ventral to the heart. Spermatogenesis takes place here and mature spermatozoa are shed. The tubular vasa deferentia extend posteriorly and ventrally to open externally on the coxopodite of each fifth walking leg. During copulation (mating) the sperm cells are transferred by the two pairs of anterior swimmerets (pleopods) of the male from the apertures of the vasa deferentia to the annulus (seminal receptacle) on the ventral side of the thorax of the female. Later, when the mature eggs are laid, they are likely fertilized as they pass posteriorly in the groove between the legs on the two sides of the body. The fertilized eggs are fastened to the swimmerets by a secretion and appear much as small bunches of shot-sized grapes hanging there. The later development continues here, and they are aerated by movements of the swimmerets through the water.

Cleavage divisions follow over the surface of the egg and the em bryo develops on one side of the mass. The body form with segments and limb buds appears, and hatching occurs in from five weeks to two months. The larvae grasp the swimmerets with their chela and remain with the mother for about a month. Two or three days after hatching they pass through the first molt or ccdysis; that is, they 
shed the outer cuticle. 'This is repeated seven or eight times during the first season to allow for growth. The average life span of the crayfish that reaches maturity is about four years.

\section{Regeneration and Autotomy}

This power is limited to the appendages and eyes in this animal, but it is quite well developed in these parts. The possibilities and rate of regeneration are greater in younger animals. Mutilated or lost legs or mouth parts are readily restored.

The genus Cambarus has the ability to allow a walking leg to break off at a certain line or joint if it is caught or injured. A new leg will develop from this stump. This phenomenon is called autotomy. There are special muscles to help in this and a membranous valve stops the passage of blood through the leg, thus preventing excessive bleeding. Bleeding will stop more quickly if the break occurs at such a point than it would otherwise. Autotomy often makes it possible for the animal to sacrifice a leg to save its life.

\section{Economic Relations}

Crayfish and the entire class Crustacea are of considerable importance to man. The crayfish, lobster, crab, shrimp, and others are used directly as food to the extent that it is an industry valued at several million dollars annually in the United States. The numerous smaller genera, like Daphnia, Cyclops, Cypris, Gammarus, Asellus, and Eubranchipus, comprise a large part of the food of many of our food fish either directly or indirectly. The more minute ones also feed many clams and oysters and finally end in human consumption. The shrimp and crab fisheries are the most important of the crustacea on the Texas coast of the Gulf of Mexico. In the Mississippi valley and on the Pacific Coast the crayfish is used extensively as a food. It becomes a serious pest in the cotton and corn fields of Louisiana, East Texas, Mississippi, and Alabama. They fill the swampy land with their burrows where they come up to the surface and eat the young plants. Frequently their burrows do serious damage to irrigation ditches and earthen dams. Crayfish also capture numerous small fish which are either immature food fish or potential food of such fish. 


\section{Characterization of Other Crustacea}

Besides erayfish the order Decapoda includes lobster, shrimp, and crab. They all have ten walking legs for which they are named. The crayfish and lobster are very similar except in size. The shrimps and prawns are marine and resemble the crayfish except that they do not have the great pinchers (chela) and the abdomen is bent sharply downward. The crabs are quite different in shape in that the cephalothorax is broader than it is long, the abdomen is poorly developed, and folded sharply beneath the thorax. Crabs of different kinds vary in diameter from a few millimeters to several inches. There are four species of swimming crabs in the Gulf of Mexico, of which the blue or edible crab (Callinectes sapidus, Fig. 408) is the most important and best known. The lady crab and calico crab are also interesting species. When the blue crab is captured at molting time it is called the soft-shelled crab. At other times it is the hard-shelled crab. They may be caught in baited nets or on pieces of meat on a line with which they are brought to the surface and lifted out in a dip-net. The hermit crab (genus Pagurus, Fig. 408) is smaller and lives in empty gastropod shells by backing into the shell and carrying it around. Due to the cramping and inactivity the abdomen has become soft and partly degenerate. The fiddler erab (genus $U c a$, Fig. 408) is another very abundant form found on the coast of the Gulf of Mexico and elsewhere. These are small semiterrestrial crabs which burrow in tumnels, and may thus honeycomb large areas of salt marshes. They can run quite rapidly, often moving sidewise, and they are peculiar in that one pincher of the male, usually the right, is much enlarged. This gives the appearance of a fiddle and the other, reduced pincher resembles the bow. The large pincher is used in a nuptial dance, and oceasionally a large number of these little crabs will be seen raising and lowering these enormous pinchers in concert.

Asellus communis is a common fresh-water form found in streams and pools. A salt-water genus, Idotea, is found in the ocean. The pill bug (Armadillidum) and the sow bug (Oniscus asellus or Porcellio sp.) are terrestrial, living in damp places under logs, stones, or heavy vegetation, and in cellars or greenhouses. Their legs are arranged in two groups, which point in opposite directions. Respiration is carried on by gills on the ventral side of the body and for this 
reason they must live in moist places. They are a garden pest in that they eat leaves of delicate plants. There are a number of acquatic forms which are parasitic on fish and others, such as the gribble (Limnoria), which tunnel in submerged wood.

The amphipods are sand and beach dwellers which may be found burrowing or jumping around on the seashore or walking on the bottom in fresh water. Gammarus is the best known fresh-water form. The legs of representatives of this order are divided into two groups, with the legs of each group pointing toward each other. These are of particular value as fresh-water fish food.

Entomostraca as a group is composed of many smaller crustaceans occurring in great numbers in both marine and fresh waters. The fairy shrimps (Eubranchipus) are delicate, transparent and feathery appearing. They are about three-fourths of an inch in length. They

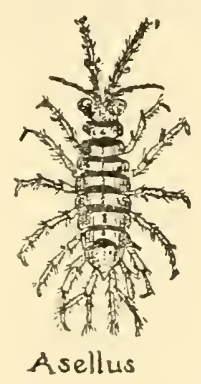

Fig. 159.-Asellus, a common fresh-water crustacean. (Courtesy of General Biological Supply House.)

swim with the ventral side up and their long, leaf-like appendages hang from the body; these appendages serve also as respiratory organs. They live in cool streams during the spring and fall. The summer is passed in the egg, which can withstand complete dryness. Many of them are parthenogenetic, hence, males are rare. The common marine form is Artemia, often called brine shrimp.

The water fleas including Daphnia of order Branchiopoda, Cyclops and Diaptomus of order Copepoda and other small Crustacea constitute an important common group. Daphnia is one that is enclosed in a delicate bivalve shell. The second pair of antennae are very large and are used in swimming. The shell is beautifully marked and terminates in a caudal spine. They are only about one-tenth of an inch in length. Cyclops is another common freshwater form with the antennae shorter than the cephalothorax whose 
body length is also about one-tenth inch. It has a single median eye, and the females frequently are seen with a pair of egg sacs attached at the base of the abdomen. Diaptomus, another Copepod, is a common form of about the same form and size as Cyclops, except that the antennae are nearly as long as the body. Argulus is a genus of Copepods which is parasitic on fish, and the individuals are called fish lice or carp lice. They are flat creatures and are found running around over the scales of their hosts. Some of the other forms are parasitic on the gills and fins of fish and their bodies become greatly modified.

The ostracods are small, swimming, bivalve forms that are sometimes called swimming clams. This group has beautifully marked valves; in fact, these animals are the most beautiful found in the plankton.

Adult barnacles of order Cirripedia bear so little resemblance to other crustacea that they are usually overlooked as such by the layman. They are completely encased in a thick shell of several sections and have the general appearance of an oyster or clam. They are sessile in habit as adults, though free-swimming in the larval stage. Their entire life is spent in marine waters. There are several characteristic barnacles, rock barnacles on rocks, whale barnacles from ships and pilings, and gooseneck barnacles of the stalked type. After attachment, the legs become modified into featherlike bristles which are used in gathering food. Sacculina (Fig. 404) is a genus related to true barnacles which has gone parasitic on crabs and has lost all resemblance to animal form. It settles on the body of a crab, makes its way to the interior and there becomes a branched mass of tissue which penetrates by roots to all parts of the body of the crab. After a time a baglike portion forms and projects externally on the ventral side of the abdomen of the crab.

\section{Recapitulation Theory}

A statement of this idea, which was developed by von Baer, Haeckel, and others, and is so well illustrated by the comparison of the phylogenic and embryonic stages of certain crustacea, may well come at this point. This theory maintains that certain developmental stages or structures of the individual are related to ancestral conditions. That is, the individual in its development tends to repeat in an abbreviated fashion the history of the development 
of the race. Briefly stated ontogeny recapitulates phylogeny. There is still some doubt as to the validity of this generalization in direct application.

A classical example which is frequently cited is that of the development of the shrimp, Penaeus, which hatches out as a nauplius larva,

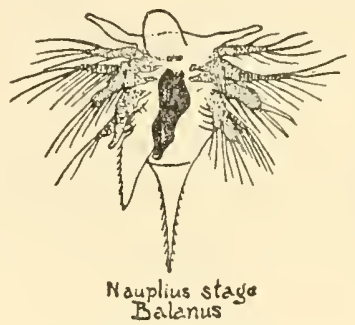

Fig. 160.-Nauplius stage of the barnacle, Balanus. (Courtesy of General Biological Supply House.)

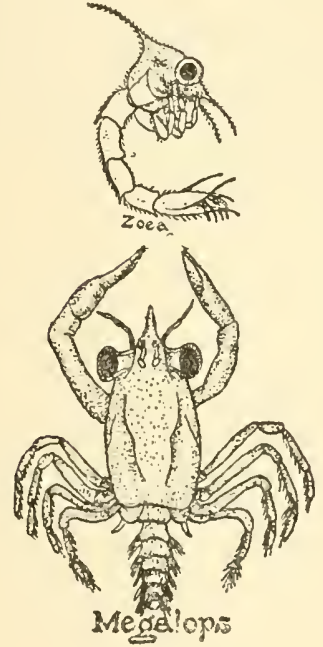

Fig. 161.

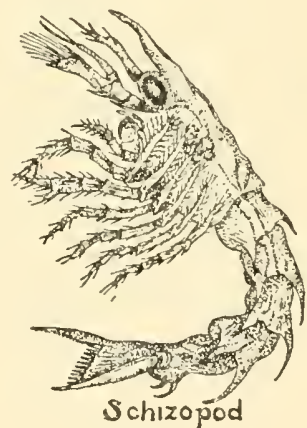

Fig. 162

Fig. 161.-Zoea and Megalops stages of developing Crustacea. Crabs include these stages in their development. (Courtesy of General Biological Supply House.) Fig. 162.-Schizopod or mysis stage through which the shrimp and lobster pass. (Courtesy of General Biological Supply House.)

having a single median cye and only three pairs of appendages. Following the molt, this nauplius changes to become the Prozoea stage, possessing six pairs of appendages. T'he next molt brings on segmentation and some change in form. This stage is called the Zoea 
and resembles very closely the adult Cyclops of modern Copepoda. The Zoea transforms during further molts and growth to a stage with thirteen segments and a distinct cephalothorax which resembles the adult Mysis and therefore is called the Mysis stage. Gammarus is also in about this category of phylogenetical development. Following the next molt the mysis stage becomes a juvenile shrimp with nineteen segments. The life history of the barnacles and Sacculina has illustrated quite foreibly the possibility of such a relationship. There are extinct forms also whose adult condition was that of one of these developmental stages. This idea generally has served as a great stimulus to the study of embryology and the theory of evolution as well as serving to establish natural relationship of animal groups.

\section{Phylogenetic Advances of Arthropoda}

(1) Greater specialization of segments, (2) paired, jointed appendages, (3) chitinous exoskeleton, (4) gill and tracheal respiration, (5) dioecious reproduction, (6) development of eyes and other sense organs, (7) green glands and malpighian tubules (insects) as excretory organs, (8) organization of social life. 


\section{CHAPTER XIX}

\section{PHYLUM ARTHROPODA (CONT'D)}

\section{ONYCHOPHORA AND MYRIAPODA}

(By Vasco M. 'Tanner, Brighadi Young University)

\section{Onychophora}

An interesting group of arthropods, now considered as the class Onychophora, is restricted to the more tropical and semitropical regions of the earth south of the Tropic of Cancer. These primitive nocturnal forms, according to Austin $H$. Clark, are found in areas that vary in annual temperature from $50^{\circ}$ to $80^{\circ} \mathrm{F}$.; in fact, most of the species are confined to habitats in which the temperature does not vary beyond the limits $60^{\circ}$ to $70^{\circ} \mathrm{F}$.

The onychophores are characterized as soft-bodied, wormlike, terrestrial forms with internally segmented bodies. The body may be divided into a head and abdomen. On the head is one pair of annulate antemnae and a pair of jaws. The body bears many pairs of legs which are not distinctly jointed, but are provided with transverse pads and apical claws. Respiration is by means of tracheae which communicate with spiracles that are, in some species, arranged in rows on the body. The exeretory system consists of nephridia arranged in pairs in the body segments and opening to the outside by a pore at the base of the fourth and fifth legs. The genital organs discharge at the posterior end of the body. The nervous system is ventral, consisting of separate longitudinal nerve cords connecting a number of ganglia. A pair of eyes is located at the base of each antenna. The body wall consists of an unsegmented dermomuscular covering.

In commenung on the ancestry of the Onychophora, Prof. J. W. Folsom has the following to say: "Onychophora, as represented by Peripatus, are often spoken of as bridging the gulf that separates the Insecta, Chilopoda and Diplopoda from the Annelida. Peripatus indeed resembles the chaetopod Annelids in its segmentally arranged nephridia, dermomuscular tube, coxal glands and soft integument, and resembles the three other classes in its tracheae, dorsal vessel with paired ostia, lacunar circulation, mouth parts, and salivary glands. These resemblances are by no means close, however, and Peripatus 
does not form a direct link between the other tracheate arthropods and the annelid stock, but is best regarded as an offshoot from the base of the arthropodan stem."

Very little is known about the habits of the onychophores, except that they live under stones and the bark of trees, feeding upon small inseets and spiders which they capture in a slime produced and forcefully discharged from glands which open on the oral papillae. Many of the species are viviparous, a single female producing as many as thirty living young in a season.

About seventy-three species and fifteen genera are known from the two families Peripatopsidae and Peripatidae. A number of species in the family Peripatidae are found in tropical America; Macroperipatus perrieri (Bouvier) is found at Vera Cruz, Mexico; while the species of the family Peripatopsidae are confined to New Guinea, Australia, Tasmania, New Zealand, Cape Colony, and Chile.

The classes Diplopoda and Chilopoda are eonsidered by some anthors as orders of the class Myriapoda. The more recent students of these groups, however, have adopted the plan of classification followed here.

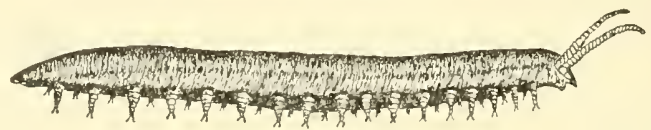

Fig. 163.-Peripatus capensis. Natural size. (After Moseley from Folsom's Entomology. Redrawn by Nelson A. Snow.)

\section{Myriapoda}

The Diplopoda are terrestrial arthropods commonly called millepedes. The body is composed of three regions: the head, thorax or trunk, and the abdomen. The head bears a pair of short antennae, occlli, and mouth parts consisting of a pair of mandibles and a pair of maxillae. Just back of the head is a segment with a well-developed tergite, the collum, considered by some students of this group as a segment which has played an important role in the formation of the head in some of the other groups of arthropods. A single pair of legs seem, however, to belong to this first segment, as does a single pair of legs to the three following segments. These four segments are said to constitute the thorax. Ducts from the reproductive organs open at the base of the second pair of legs on the third body segment.

The abdomen consists of an indefinite number of segments, each consisting of a tergum and two sterna. Each sternum bears two pairs 
of legs and two pairs of spiracles. The spiracles are elosed by a valve and communicate with tracheal pockets and unbranched tracheae. Embryological evidence supports the belief that the abdominal segments have resulted from the fusion of two segments.

The legs are jointed very much as in the insects. The tarsus consists of the three segments.

The heart is a dorsal structure with side valves and an anterior tube to the head similar to the arrangement in the insects. The digestive tract consists of a mouth, esophagus, stomach, and intestines. The exeretory wastes of the body are removed by two or four pairs of Malpighian tubules which discharge their excretions into the intestines.

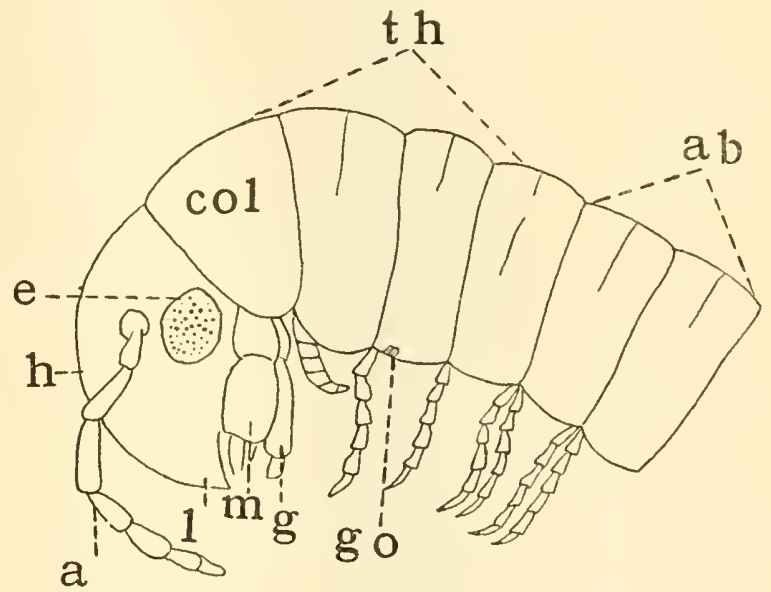

Fig. 164. Julus terrestris. A common millepede. Side view of anterior end. $a$, antenna; $a b$, abdomen; col, collum (first thoracic segment) : $e$, a group of ocelli; $g$, gnathochilarium; go, genital opening; $h$, head; $l$, labium; $m$, mandible ; th, thorax. (After Borradaile and Potts, Invertebrata. Redrawn by Nelson A. Snow.)

The millepedes feed upon vegetable matter, decaying as well as living plant substanee. They are slow-moving, wormlike ereatures, living in dark, moist places. When disturbed they usually roll themselves up into a little coil. The eggs are laid in damp earth, and when the young hateh they are very small, consisting of only a few segments and three pairs of legs.

The diplopods are found in most parts of the world. In the United States there are six important families and about 120 speeies. The family Julidae is widely distributed. The speeies Julus hortensis Wood; J.virgatus; J.hesperus Chamberlin; and Spirobolus margin- 
atus are fairly common in many parts of the United States. In the family Polydesmidae, Polydesmus serratus Say is a common species in the United States west to the Mississippi River.

Members of the class Chilopoda differ from the Diplopoda by their dorsoventrally flattened bodies, consisting of fewer segments which bear but one pair of legs, and by their long antennae. The Chilopoda, as a rule, move faster than millepedes.

The Chilopoda are carnivorous, preying upon adult as well as immature insects, also upon spiders and Mollusea. The mouth parts consist of a pair of mandibles and two pairs of maxillae. A pair of

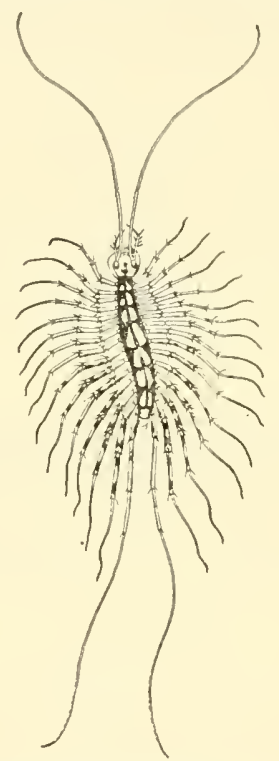

Fig. 165.-Scutigera forceps. The long-legged house centipede. (From a paper by C. L. Marlatt, Farmer's Bulletin, No. 627, U. S. Dept. of Agriculture, 1914.)

poison claws are located on the first body segment, the last two segments are legless, and each of the other body somites bear a pair of seven-jointed walking legs.

The circulatory system is well developed; it extends the full length of the body and gives off in each segment lateral vessels or arteries.

The nervous system consists of connected paired ganglia in each leg-bearing somite and a subesophageal and supra-esophageal ganglia which supply nerves to the eyes, mouth parts. antennae, and other parts of the head. 
The digestive system is fairly simple, consisting of the esophagus, stomach, and intestines. A pair of Malpighian tubules empty into the anterior part of the intestines. The tracheae are branched, being connected to a pair of spiracles on each body segment.

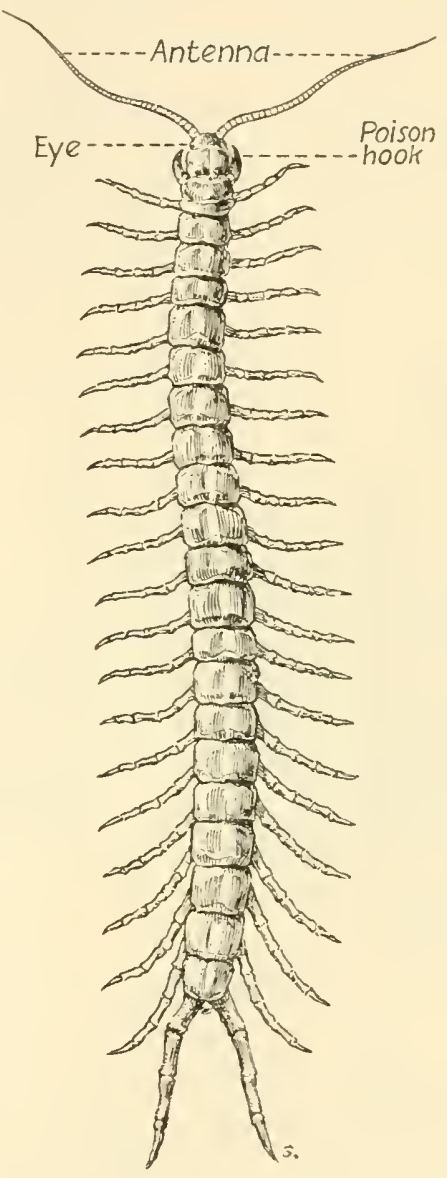

Fig. 166.-Scolopendra. The large centipede of the Southwest.

Dr. R. V. Chamberlin, one of the leading students of the millepedes and centipedes, reports the following species of centipedes for the Western United States: Scolopendra morsitins Linn.; S. heros Girard and Arthrorhabdinus pygmaeus Pocock of the family Scolopendridae. The species $S$. heros is a large dark greenish colored species found in Kansas, Arizona, Southern California, and Texas. 


\section{CHAPTER XX}

\section{PHYLUM ARTHROPODA (CONT'D)}

\section{ARACHNIDA}

\section{(By Vasco M. Tanner, Brigham Young University)}

Probably the most heterogeneous class of arthropods is the class Arachnida, which is now a pigeon-hole for the spiders, scorpions, mites, ticks, pseudoscorpions, harvestmen, whip scorpions, bear animalcules, king crabs, and several other less common orders, such as the Pentastomoids and Pycnogonids.

In attempting to give the characteristics of this class we will list only the obvious characters and then remind the reader that the members of this group have more likenesses to the Arachnida than to any other assemblage of animals, hence, no doubt, the reason for having this class intact.

The spiders and their relatives are characterized by having two main divisions of the body, the cephalothorax and the abdomen; no antennae or wings; mouth parts consisting of but two pairs of appendages, the chelicerae and pedipalpi; four pairs of walking legs, each consisting of seven joints; lung books, which are remarkable respiratory organs; simple eyes, that are definite in number and arrangement in the various families; abdominal appendages which have been modified into spinning organs; and no metamorphosis, the development being direct.

\section{Spiders}

The spider's body consists of two divisions: the cephalothorax and abdomen. The first division of the body, the cephalothorax, results from the fusion of the head and thorax. Six pairs of appendages are to be found upon this part of the body. The first pair, the chelicerae, are two-jointed, consisting of a basal part or mandible and a terminal claw. The mandible is stout and covered on the inner surface with small teeth and setae. The poison glands in the mandibles discharge their poison through the movable sharp-pointed claws. The poison serves as an aid to kill insects and other animals used by the spider for food. The pedipalpi, consisting of six joints, are located in front of the legs. They are used in handling the food, 
as feelers, and in the males the terminal segments are used as copulatory organs. These palpal organs are useful in the classification of many of the families of spiders. The basal segments, or maxillae, are used in chewing the food. The four following pairs of appendages are the legs. Each leg is made up of seven segments; namely, the coxa, trochanter, femur, patella, tibia, metatarsus, and tarsus.

The eyes are located on the front of the head and usually are eight in number; some species have fewer, but never more than this number.

The cephalothorax and abdomen are connected by a narrow waist. On the under side of the abdomen, just back of the last pair of legs,

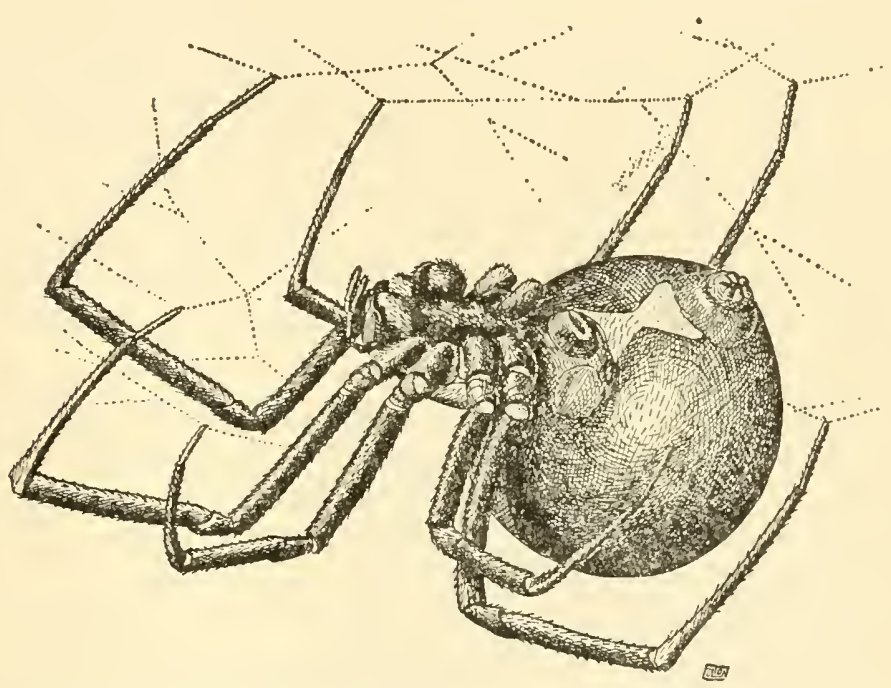

Fig. 167.-Ventral view of adult female, black widow spider, Latrodectus mactans, hanging from web. The hour glass on the ventral part of the abdomen is clearly shown. (From Knowlton, by permission of the Utah Agricultural Experiment Station.)

are the front pair of breathing openings or slits which communicate with the lung books. Near these is the opening of the reproductive organs, which in the females is protected by a plate called the epigynum. Near the end of the abdomen are three pairs of spinnerets. In front of these is an opening to the tracheae and just posterior to them is the anus.

The digestive system of the spiders is well adapted for its fluid food. The mouth is located just behind the chelicerae. Through its small opening the liquid portion of the prey is sucked up by means of muscles which are attached to the dorsal wall of the cephalothorax 
and the anterior portion of the stomach, which is called the sucking stomach. The posterior portion of the stomach gives off five eaeea which are supplied with blood vessels from the anterior aorta. The intestine passes through the small waist eomnection into the abdomen and becomes enlarged in two portions, one for the reception of the hepatie duets from the liver and the other at the posterior end for the formation of a stereoral sac or pocket.

The exeretory wastes are removed by the Malpighian tubules, which discharge into the posterior portion of the intestines, and by vestigial coxal glands.

The vascular system consists of a museular tube or heart, arteries, and veins, located above the intestine. The heart receives its blood from the body by means of three pairs of ostia. It is then foreed anteriorly through the aorta into the eephalothorax and also posteriorly into the abdomen.

The nervous system is eomposed of a large ganglion in the eephalothorax which is eonneeted with a dorsal brain by a nerve ring around the esophagus. Nerves pass from the ganglion to the limbs and the abdomen.

The respiratory functions are earried on by the lung books or saes, which eontain a number of thin plates through which the blood passes on its way to the heart. In the posterior part of the abdomen is a pair of branching air tubes or tracheae. This system of breathing is found only in the arachids.

The reproductive organs consist of the ovaries in the female and the testes in the male. The female has two sets of openings, one to reeeive the sperms into the saes, from the tips of the pedipalps, and the other is the exterior opening of the oviducts. The male and female openings are near the lung books. The eggs are fertilized within the body of the female and then laid in silken bags or eocoons. In many species these egg bags are earried on the abdomen of the female. The rate of growth of the eggs varies aecording to the conditions. For example, eggs laid in the antumn develop slowly all winter, while those laid in the summer develop within a few days. The hatehing takes but a day or two. The young spider is pale and soft bodied, but in a few days it moits for the first time and then begins to look like an adult spider. As the spider grows, it molts a number of times, the development being direet. If the young spiders do not get out of the cocoon soon enough, so that they have their freedom, they 
begin to eat one another. Many spiders live only one year, developing from over-wintering eggs in the spring, growing to maturity during the summer, laying eggs, and then dying in the fall. Some spiders live two or three years, while other species live many years.

\section{Classification of the Arachnida}

For purposes of this chapter we shall confine our discussion to but five of the common orders of the class Arachuida: (1) Araneida, the true spiders; (2) Acarina, the mites and ticks; (3) Scorpionida, the scorpions; (4) Phalangida, the daddy longlegs; and (5) Xiphosura, the king crabs.

According to a recent study, A Natural Classification of Spiders, by Dr. A. Petrunkevitch, the order Araneida is made up of sixty-two families. Species of many of these families are rarely encountered, and some of them are not found in America. The following eight families, however, are common in the United states: 1. Lycosidae, the wolf spiders. This family contains many of the largest native species. They are found mainly upon the ground running around in search of food. The females carry the cocoons attached to their abdomens. The eyes are arranged in three rows of four, two, and two. The species, Lycosa gulosa, L. kochii, L. frondicola, and Allocosa parva are common species in the western states.

2. Theridiidae, the comb-footed spiders. The spiders of this family are found on low growing regetation, fences, buildings, and at times under boards and rocks. They build rather loose webs from which they hang back downward. Members of this family have a toothed comb on the tarsi of the fourth pair of legs, three claws, and eight eyes. The black widow, Latrodectus mactans, reported as a poisonous species, is a member of this family. The hourglass on the ventral part of the abdomen is scarlet in color in the live spider.

3. Thomisidae, crab spiders. These crablike spiders do not construct webs, but lie in wait in the flowers for insects that visit them. They are often highly colored for protection. They possess eight eyes in two rows; the two anterior pairs of legs are longer than the other legs; and the body is rather flat. Misumena vatia and Xysticus nervosus are common species.

4. Drassidae. The drassids live mainly on the ground, under stones, or in silk tubes on shrubs and grass. The spinnerets are generally long enough to extend a little behind the abdomen. The eyes are eight 
in number and arranged in two rows, but in this family there are only two tarsal claws. They differ from the wolf spiders in that the body is much longer than wide and flattened slightly on the back. This is one of the large families of spiders. Drassus neglectus is a representative species.

5. Attidae, jumping spiders. Members of this family attract attention by their jumping and bright coloration. They live on fences, buildings, plants, and on the ground. They do not spin webs for the capture of prey but only for their protection and cocoons. The eyes of this group are most distinctive. They are arranged in three rows, oceupying an area on the cephalothorax known as the ocular quadrangle. The eyes on the front row are the largest. The males and females differ considerably in size and structure. This family is world-wide in distribution. It is represented by three hundred and fifty species in America north of Mexico. Some of the common species of our fauna are: Phidippus workmanii; P. formosus; Icius similis; Euophrys monadnock; and Salticus senicus.

6. Argiopidae, the orb-web spiders. The spiders of this family build rather large typical webs for the purpose of capturing their prey. After building the web they lie in wait for some insect which may serve for food to become entangled in it. This is a large family, the members of which have eight eyes, and three claws on the tarsus. They are confined to suitable places on vegetation, buildings, fences, and holes, where they construct their webs and then remain near by to watch them. Some of the common species of this family are Tetragnatha laboriosa; T. extensa; Metargiope trifasciata; Neoscona benjamina; and Aranea gemma.

Most of the species of Tetragnatha are found in moist places; they build their webs over running water. Their bodies are round and long. Metargiope trifasciata is the common garden spider, found in the fall of the year in potato, tomato, and beet fields. It has a silvery white color and is about an inch in length when full grown.

7. Aviculariidae, the tarantulas. Representatives of this family are confined to the south and southwestern United States. They are large, hairy, black spiders with eight eyes and chelicerae projecting forward. The trap-door spiders live in tunnels dug in the ground and provided with a hinged door which closes the entrance to the tunnel so perfectly that it is almost impossible to locate the tunnel. The tarantulas, which are large nocturnal species, live under roeks, 
in holes, and under débris in the daytime. They feed upon beetles and other ground-living insects. Eurypetma steindachneri and $E$. hentzi are common tarantulas.

S. Pholcidae, the pholeids, are spiders with long legs and six or eight eyes. They build loose webs in dark places, such as cellars and protected corncrs of buildings. There are six genera in the United States. Pholcus phalangioides is a large species. The abdomen is elongated in form, and the legs are one and one-half to two inches long. In this species the ehelicerae are used to carry the eggs. Three species are found in the genus Physocyclus: two of them are Physocyclus glabosus which is found in Florida and P. tanneri, in Utah.

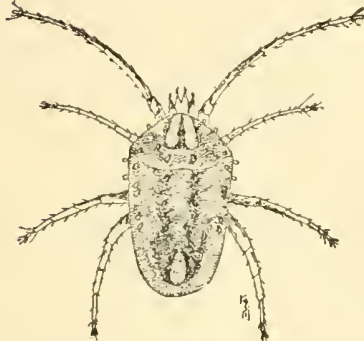

Fig. 168.

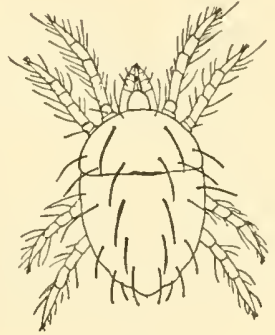

Fig. 169.

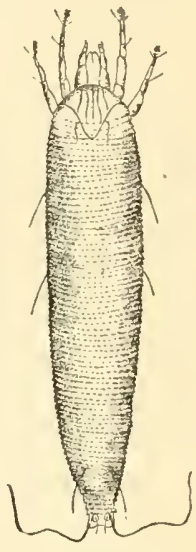

Fig. 170 .

Fig. 168.-The brown mite, Bryobia praetiosa. (Greatly enlarged.) (After Sorenson, by permission of the Utah Agricultural Experiment Station.)

Fig. 169.-An adult female, red spider, Tetranychus telarius. (After Inowlton, by permission of the Utah Agricultural Experiment Station.)

Fig. 170.-An adult blister mite, Eriophyes pyri. (After Sorenson, by permission of the Utah Agricultural Experiment Station.)

For a diseussion of the other spider families found in this country, the student should consult the most valuable treatise on this subject, The Spider Book, by Professor John H. Comstock.

Order Acarina.-Mites and ticks are mostly small creatures with the eephalothorax and abdomen fused solidly together. They are, in the main, ectoparasites or endoparasites; however, some of the aquatic species are able to shift for themselves, living upon varions small water animals. All mites lay eggs which hatch into six-legged nymphs. After some growth and moulting they develop into adults with eight legs. 
A group of mites belonging to the family Tetranychidae, known as brown mites and red spiders, are widely distributed, feeding upon practically all kinds of eultivated plants. The elover or brown mite, Bryobia practiosa, is eosmopolitan in distribution. It feeds upon fruit and shade trees, garden plants, and eommon annual and perennial plants. The common red spider, Tetranychus telarius, which is common in this country, attacks field plants, fruit trees, forest trees, shade trees, and shrubs. It is reported that it attacks over 250 species of plants. Another common mite pest is the leaf blister mite, Eriophyes pyri, which belongs to the family Eriophyidae. This species attacks the leaves of the pear and apple. The damage to the leaves results in the development of brownish blotches and partial defoliation.

The ticks are all fairly large parasitic species. Those belonging to the family Argasidae attack only warm-blooded animals. After taking a meal of blood, they leave the animal and go into hiding. At times they are a serious pest to poultry. Ticks of the family Ixodidae attach themselves to their host, suck blood, and grow to many times their original size. 'Texas eattle fever and Rocky Mountain spotted fever are dreaded diseases that are carried by ticks.

The order Scorpionida consists of large-sized arachnids with pedipalpi which resemble the chelipeds or pinchers of the crayfish; also with a flattened body and elongated abdomen terminating in a specialized stinging organ. They are nocturnal, hiding during the day under rocks and burying themselves in the sand. They feed upon insects and spiders.

They are viviparons; the mother takes eare of the young, protecting them by carrying them around on her back and by helping them to eateh their prey. They breathe by means of lung books and have a direct development like the spiders. A peculiar comblike strueture, called the peetine is found in the ventral part of the seeond abdominal segment. Four families are represented in the United States, and they are found only in the southern and western states. Hadrurus hirsutus, and Vejovis mexicanus of the family Vejovidae are common species.

Another interesting little group of arachnids is the order Solpugida, found in the same territory as the scorpions and represented by twelve species contained in three genera. Eight of the species belong in the genus Eremobates. 
The Phalangida, commonly ealled harvestmen and daddy longlegs, can be distinguished from other arachnids by their body which is composed of a broadly fused eephalothorax and abdomen, the abdomen consisting of nine segments; long legs; and the presence of only two eyes on the cephalothorax. The reproductive organs, an ovipositor in the female and a penial organ in the male, are loeated on the obscure division between the cephalothorax and abdomen. The respiratory organs consist of tracheae which open through abdominal spiracles. The harvestmen do not have silk glands and therefore do not construct cocoons for the eggs, which they lay under stones and under the bark of trees. There are about seventy species in the United States, representing six families. Species of the family Phalangididae are the most common and widely distributed.

The order Xiphosura, king crab, is represented by only one living primitive genus and five species. Limulus polyphemus is the American species found along the Atlantic Coast from Maine southward. Because of its shape and resemblance to the crabs, it has been called the horseshoe crab. The body consists of two regions; the cephalothorax and the abdomen. There are six pairs of appendages on the cephalothorax. The basal parts of the appendages situated around the mouth are used for crushing the food, which consists mainly of worms. On the abdomen are six pairs of appendages, the last five pairs bearing book-gill structures used in respiration. The males are a little smaller than the females, but similar in appearance. The eggs are deposited in the summer in shallow water in small sandy depressions where they are then fertilized by the male. 


\section{CHAPTER XXI}

\section{PHYLUM ARTHROPODA (CONT'D)}

\section{CLASS INSECTA}

(By Vasco M. Tanner, Brigham Young University)

Insects are the most abundant creatures on the earth today. There is said to be over 650,000 living species, many of which have never been seen by the great majority of mankind. This, no doubt, is because insects exist in every type of habitat known. They are found in sea water along the shore; in fresh water that ranges in temperature from $50^{\circ} \mathrm{C}$. to ice cold; in the soil ; in dry desert conditions; on the vegetation of plain and swamp; from the tundra of the north to the tropical pampas; in trees; on and in animals, as well as man, many of which are carriers of disease. They ravage our crops and damage our stored foods. In short, we may say that insects are omnipresent. One noted entomologist has said that this is an age of insects, and to this we may add that every man's farm is "no man's land" and that the contending forces are insects and man.

This great class Insecta has been upon the earth from the Pemnslyramian times, of the late Paleozoic era, to the present. This means that for probably one hundred million years these arthropods have been adjusting to a changing environmental complex, and the success with which they have met the challenge is quite evident today. Various explanations have been advanced to account for the great adaptability of insects in filling practically every niche in nature. The Russian biologist, S. S. Chetverikov, argues that the chitinous exoskeleton has been of great value in the evolution of this group, in that it has permitted them to develop strong appendages, unlimited external features, and a small size which has opened up an entirely new place in the world of living animals. Dr. C. H. Kennedy, however, has pointed out that there are advantages as well as disadvantages to the possession of an exoskeleton. He says: 
"The exoskeleton has made possible very definite advances in the evolution of insects, but at the same time has limited their evolution in fully as many other ways.'"

Aside from the chitinous exoskeleton, other distinctive characteristics, such as power of flight, which is possessed by no other invertebrate animal; a tracheal system, which keeps the hemolymph or blood from becoming impure; and finally their great variability and power to reproduce, have made the insects, no doubt, the successful creatures they are today. This leads us to wonder how successful man will be in his evolution during the next fifty million years. Will he be able to meet the demands of a changing environment as the insects have?

\section{INSECT CHARACTERISTICS}

Insects are Arthropoda in which the body is divided into three regions, the head, thorax, and abdomen. The head, which consists of six segments, bear's a single pair of antennae, the eyes, and the mouth parts; the thorax consists of three segments and is the region which bears three pairs of legs and two pairs of wings, when they are present in the nymphs and adults; the abdomen bears a variable number of segments in the rarious groups of insects, also the genital apertures which are situated near the anus at the posterior end of the body.

\section{Head}

The head consists of a number of immovable plates or sclerites forming the head capsule, to which are attached the paired appendages. In many insects the sutures separating the sclerites are visible, and these plates and depressions have been given definite names. The paired appendages furnish much evidence that the head has resulted from the fusion of several segments. The eyes, antennae, mandibles, maxillae, and labium are considered as developing on distinct somites. Evidence concerning the fusion of the six anterior segments of the body in the formation of the head comes through an embryological study of the insect.

There are two kinds of eyes: ocelli, or simple eyes, and compound eyes. The simple eye is a small area consisting of a single cornea. Simple eyes are generally found in varying numbers along with the compound eyes in adult insects; they are, however, usually lack- 
ing in beetles. The compound eye consists of many facets, which are the hexagonal-shaperl corneal ends of structures called ommatidia. The facets are convex, and insects are short-sighted.

It has been shown by experimentation that many insects are able to deteet eolors. Some sindents of the insects maintain that the

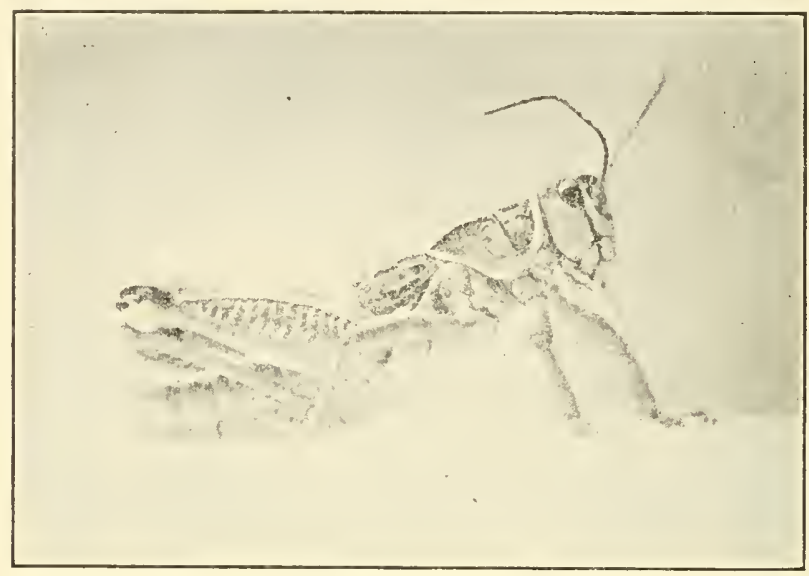

A.

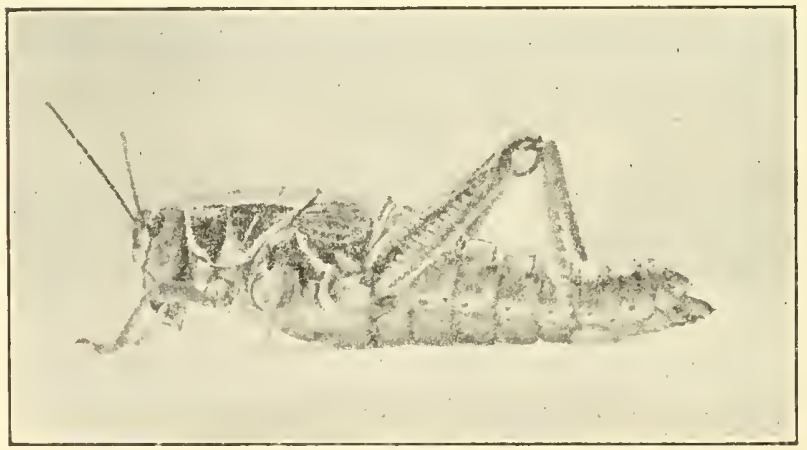

$B$.

Fig. 171.-Western lubber grasshopper, Brachypephus magmus. $A$, male, and $B$, female. The form found on the plains. (Photographed by Leo T. Murray.)

compound eyes function mainly in detecting movements, while ocelli are used to deteet light intensities.

There is but one pair of antenuae or feelers, and they vary in size, shape, and position on the head among the various groups of 
insects. The feelers function in many ways; in some insects they are tactile; in others they are respiratory or olfactory, or auditory, or may be used to hold the female during copulation. Antennae are useful structures in the classification of insects.

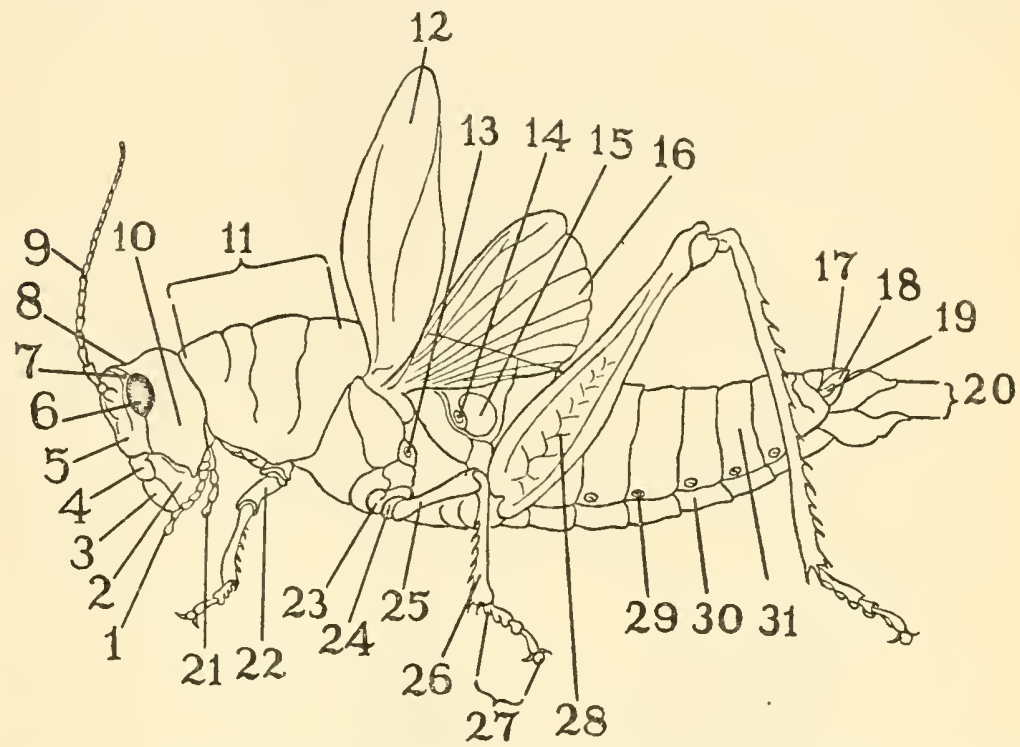

Fig. 172.-The external features of a grasshopper. 1, maxillary palp; 2, mandible: 3 , labrum ; 4, clypeus ; 5 , frons ; 6 , compound eye ; $\%$, ocellus ; 8 , vertex ; 9 , antenna: 10 . gena : 11 , pronotum; 12 , wing, mesothoracic; 13 , spiracle, thoracic: 14, spiracle. first abdominal sezment; 15 , auditory apparatus; 16 , wing, metathoracic: $1 \%$, supra-anal nlate; 18 , podical plate; 19 , cercus: 20 , ovipositor: 21 , labial nalp; 22 , femur, prothoracic leg; 23 , coxa, mesothoracic les; 24, trochanter: 25 , femur; 26 , tibia; 27 , tarsus; 28 , femur, metathoracic leg; 29 , spiracle; 30 , sternum; 31, tergum. (After Turtox key card, courtesy General Biological Supply House.)

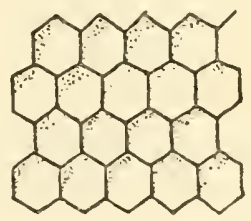

Fig. 173.-Detail of ommatidia, magnified. (From White, General Biology, published by The C. V. Mosby Company.)

The mouth parts are chitinous structures and are represented in the insects by two distinct types: mandibulate or biting, and suctorial or sucking mouths. Some of the insect orders which possess mandibulate mouth parts are Coleoptera, Odonata, Neuroptera, 
Nallopliaga, Dermaptera, Isoptera, and Orthoptera. The Lepidoptera, Diptera, Heteroptera, Homoptera, and Siphonaptera are suctorial orders. A knowledge of the mouth parts is very useful if insects are to be effectively controlled and classified. No other group of organs within the insect body vary in form as do the
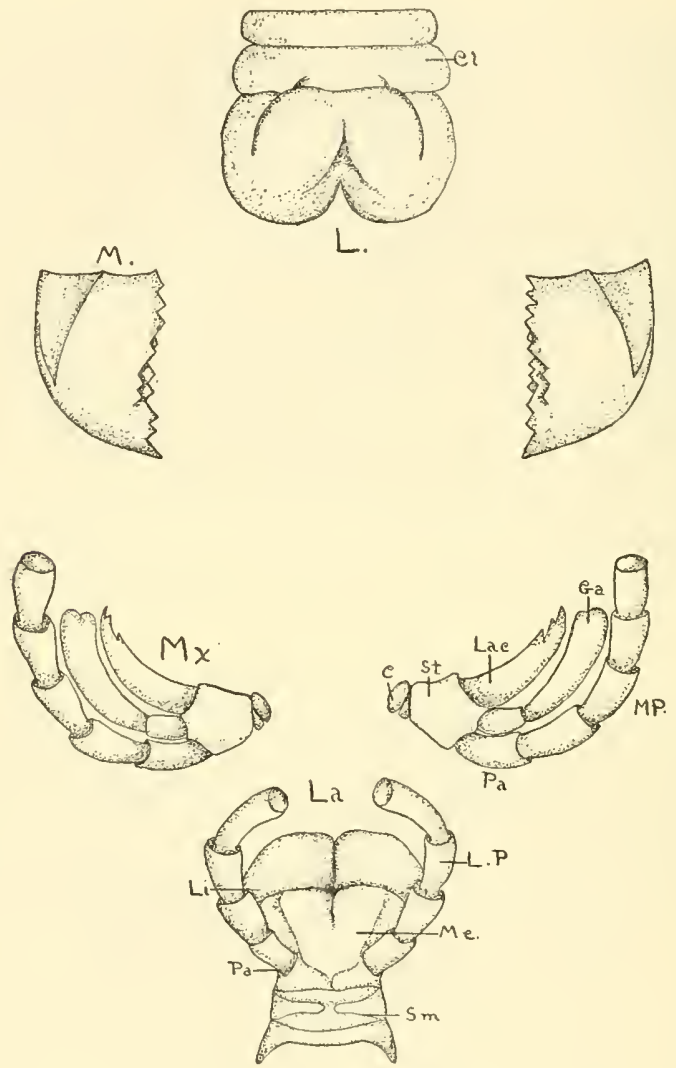

Fig. 174.-Mouth parts of Rhomaelia microptera. $C$, cardo; $C l .$, clypeus: Ga., galea: $L$, labrum; La, labium (second maxilla) ; Lac., lacinia; Li., ligula; L.P., labial palp: M. mandible; Me., mentum; $M P$., maxillary palp; $M x$., first maxilla; Pa., palpifer; Śm., submentum; St., stipes. (From White, General Eiology.)

mouth parts. The mandibulate mouth parts are the type from which the suctorial type has been evolved. In the mandibulate mouth parts there is a labrum or upper lip which is attached on its upper border to the clypeus and extends down over the mandibles. The mandibles or jaws are true appendages which move in a trans- 
verse plane. They are hard, thick plates of chitin with toothed edges adapted for cutting or crushing food. In some beetles the mandibles become greatly enlarged and apparently worthless. The maxillae or second pair of jaws lie under the mandibles and move in a similar plane. The maxillae, which consist of sclerites, are much more complex. The cardo is the piece which hinges the maxillae to the head; attached to the distal portion of the cardo is the stipes, which bears three sclerites-the lacinia, galea, and palpus. The lacinia is provided with teeth or spines which aid in holding and chewing the food. The palpus is composed of four or five segments and is sensory in function.

The labium or lower lip is formed by the fusion of what is believed to have been a second pair of maxillae. The labium is hinged to the head by the mentum from which extends one or two pairs of lobes, the ligula. Projecting from the mentum on each side is a palpus which consists of one to four segments; it functions as a sensory organ, probably detecting senses similar to our own senses of taste and smell.

The hypopharynx or tongue arises from the labium into the cavity of the mouth, and bears the opening of the salivary duct.

In the typical sucking insect the mouth parts consist of two pairs of sicklelike or styletlike structures which are modified mandibles and maxillae, as found in the mandibulate orders discussed above. The labium forms a long sheathlike structure in which the styletlike mandibles and maxillae lie. The upper lip or labrum covers orer the proximal portion of the beak. Food is taken in a liquid form by being sucked up through the labial sheath. In the mosquito the hypopharynx is long and slender like the mandibles and maxillae; the salivary duct extends throughout the entire length of the food channel. The saliva causes an irritation, and if the mosquito is infected with malarial organisms they are introduced into the blood stream of man.

\section{Thorax}

The thorax is composed of three segments, which are called the prothorax, mesothorax, and metathorax. A pair of jointed legs is attached to each scgment and most adult insects bear a pair of wings on the mesothorax and metathorax. The dorsal or back surface of 
the segments of the thorax is called the tergum, the ventral or under surface, the sternum, and each side, the pleurum. The legs are made up of five main segments: the coxa, trochanter, femur, tibia, and tarsus. The coxa forms the joint by means of which the leg is attached to the body. The trochanter is small, while the femur, or thigh, and tibia are large, forming the greater part of the leg. The tarsus or foot is composed of five smaller segments and a pair of claws. In insects the tarsal segments may differ in size, length,

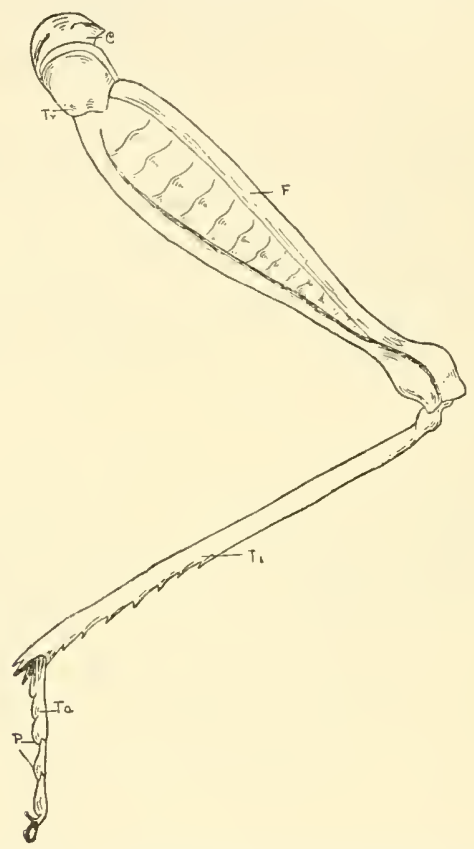

Fig. 175.-Jumping leg or third thoracic appendage of Rhomaelia microptera. $C$, coxa ; $F$, femur; $P$, pulvilli ; $T a$, tarsus; $T i$, tibia; $T r$, trochanter. (From White, General Biology.)

shape, and number, and are useful in classification. The legs are g'reatly modified for obtaining food, ruming, walking about, swimming, and jumping. They are also modified for the production or reception of sound, for the collection of food, such as pollen, and for copulation. In some species they also exhibit secondary sexual characters.

The wings are thin folds of the skin, shaped and strengthened with veins in various ways. The presence of wings is one of the most 
characteristic features of the insects. Because of their great variation, wings are of much value in classification. The wing is composed of a network of thickened lines called veins and thin areas between the veins called cells. The number, arrangement, and character of the reins and cells are an aid in grouping insects into families, genera, and species. The majority of insects possess two pairs of wings; there are some, however, that have but one pair and some groups are wingless.
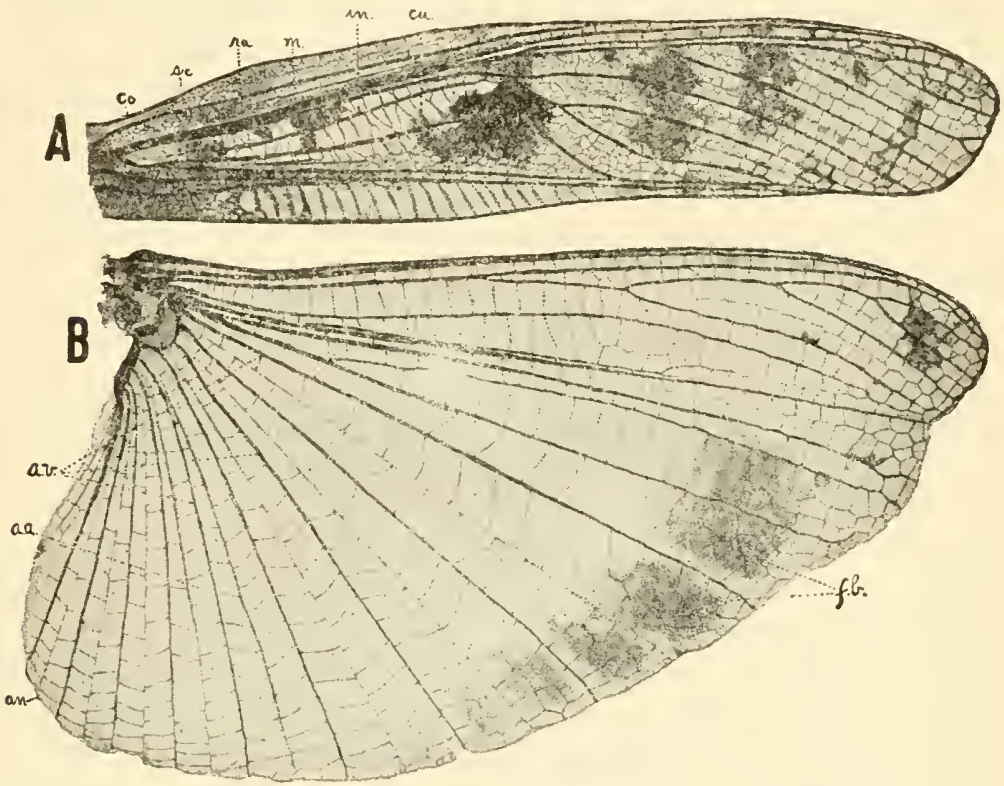

Fig. 176.--Right wings of a grasshopper. $A$, the fore wing; $B$, the hind wing. (From Henderson, permission of Utah Agricultural Experiment Station.)

\section{Abdomen}

The segments of the abclomen are usually simple, but the number varies greatly in different insects. There are only ten present in many insects, yet in the embryo of the insect there are eleven. The jointed appendages have been almost entirely lost in adult insects. On the eighth and ninth segments of the female and the ninth of the male are paired structures forming the genitalia which are the external organs of reproduction. Within the abdomen are found the respiratory, digestive, and genital systems. 


\section{Body Wall}

Another distinctive feature found in the arthropods is the chitinous body wall, which provides the only rigid support for the body. The exoskeleton consists of three layers known as the cuticula or outer layer which is impreguated, more or less, with calcareous matter, the hypodermis or intermediate layer, and the basoment membrane. The hypodermis has its origin in the ectoderm and is the active growing layer of the body wall. Chitin is a substance found in many parts of the insect body, but it especially serves to give firmness to the cuticula. Chitin is not destroyed by caustic potash. It is a most interesting organic substance, resembling horn in some physical ways.

All the tubercles, spines, setae, and scales of the body wall are formed by the cuticula. These structures are of importance in the identification of insects.

\section{Metamorphosis}

Metamorphosis includes the alterations which an insect undergoes after hatching from the egg, and which alters, extensively, the general form and life of the individual. All the changes which are undergone by a butterfly in passing from egg to adult-each change from egg to larva, from larva to pupa, and from pupa to adult-constitute metamorphosis.

There are four types of development or metamorphosis: first, anetabolous or development without metamorphosis; second, paurometabolous or gradual metamorphosis; third, hemimetabolous or incomplete, and fourth, holometabolous or conplete metamorphosis. The ametabolous insects are the Thysanura, Collembola, Mallophaga, and Pediculidae, which after hatching, grow through a number of instars, remaining practically the same form as the adult insect during all the development. This is development without metamorphosis. In the following orders: Orthoptera, Hemiptera, Homoptera, Isoptera, Thysanoptera, and Dermaptera, there is a type of development in which the nymphs gradually increase in size and the rudimentary wings and genital appendages become adult structures. This is known as paurometabolous development.

In the Odonata, Ephemerida, and Plecoptera, the newly hatehed naiads pass through an incomplete metamorphosis. All of the 
hemimetabola naiads live an aquatic life which necessitates changes and physiological adjustments not required in the adult aerial existence. In these orders there are greater changes during development than are found in gradual metamorphosis.

The Holometabola, in which the larva hatched from the egg bears no resemblance to the adult, goes through a complete metamorphosis. The holometabolous insects include the following orders: Trichoptera, Neuroptera, Coleoptera, Lepidoptera, Siphonaptera, Diptera, and Hymenoptera. The larva is varionsly called the maggot, grub, or caterpillar. It eats almost constantly since this is the growth period in an insect's life history. After molting several times it comes to rest and prepares for the pupal stage. The pupa gradually takes on the adult form and after a few days or even months, the adult or imago emerges.

The remarkable adaptation of the immature stages of insects to their food supply has undoubtedly had much to do with their great success as a group. Their food habits, minute size, use of flight in locomotion, and rate of multiplication, along with otler distinctive characteristics mentioned above, have made possible the development of this dominant group.

\section{CLASSIFICATION}

Because of the important role insects play in the life of man it is worth while to be able to recognize some of the common orders. The character's most used for the separation of the order's of insects are the structures of the wings and mouth parts, and the type of development, or metamorphosis. The number of orders recognized in this class varies considerably, depending on the authority followed. It has been divided into the subclasses: Apterygota, the two wingless orders Thysanura and Collembola, and Pterygota, which includes all the other order's of insects. Since practically all the orders fall into the subclass Pterygota, the arrangement followed here is that of discussing them according to their development.

Subclass Apterygota.-Ametabola are insects without metamorphosis.

Order Thysanura.-The members of this order have retracted mouth parts, elongated rather flattened bodies, long antennae, and abdominal appendages. They are soft-bodied small insects, com- 
mon in warm climates. They feed largely upon dead plant tissue, and are found in the soil and beneath stones, dry leaves, and loose bark. One of the commonest species of this order" is the "fish moth" or "silverfish," which attacks book bindings, the paste of wall paper, and starched clothes. It is about half an inch long and has three bristles extending from the tip of the abdomen.

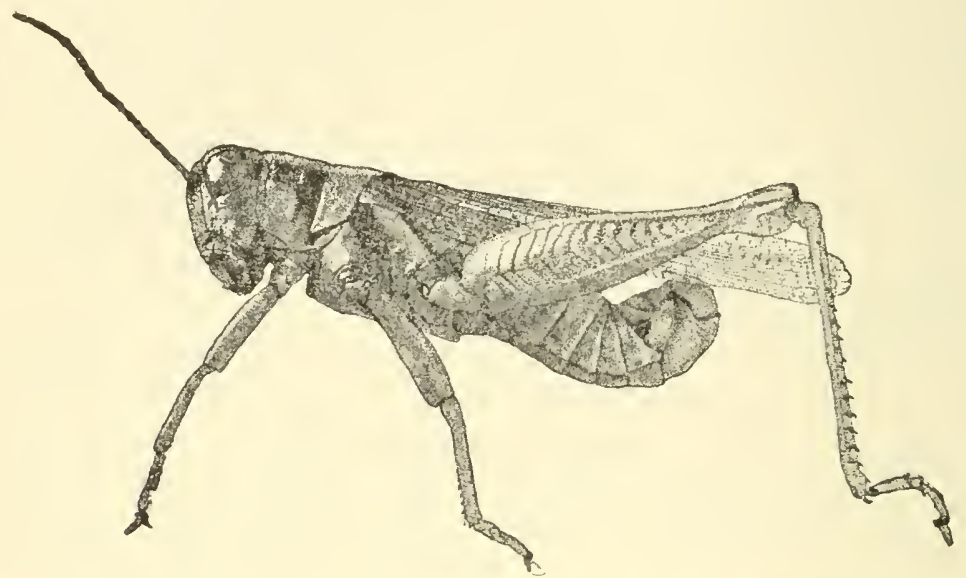

A.

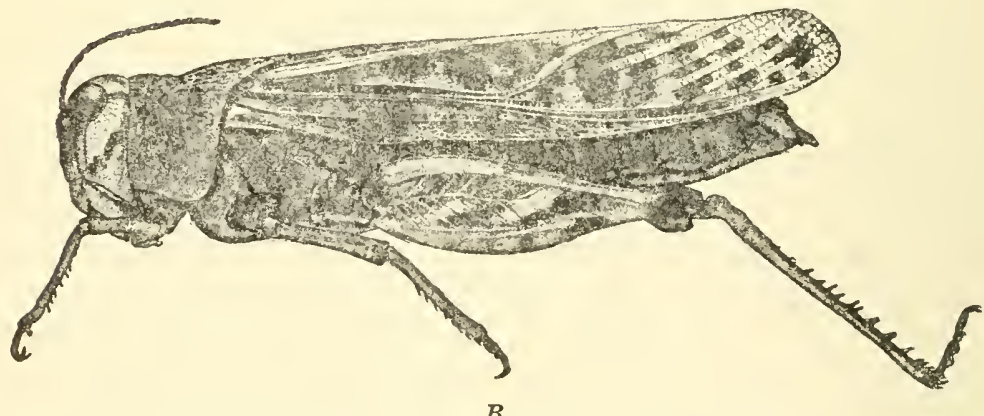

Fig. 177.-Grasshoppers common to western United States. A, Red-legged locust, Melanoplus femur-rubrum DeGeer, male; $B$, Haldeman's locust, Hippiscus corallipes Hald.; $C$, Shoshone grasshopper, Schistocerca shoshone Thomas, female; $D$, two striped Mermiria, Mermiria bivittata Serville: $E$, western meadow grasshopper, Conocephalus vicinus Morse, female. (From Henderson, Itah Agricultural Experiment Station.)

The following are some of the families and species found in the western United States: Family Lepismidae. Lepisma saccharina Linn. The silverfish moth; Machilidae, Machilis orbitalis Packard; 
Campodeidae, Campodea folsomi Silvestri ; and Eutrychocampa wilsoni Silvestri; and Japygidae, Japyx hubbardi Cook and Evalljapyx sonoranus Silvestri.

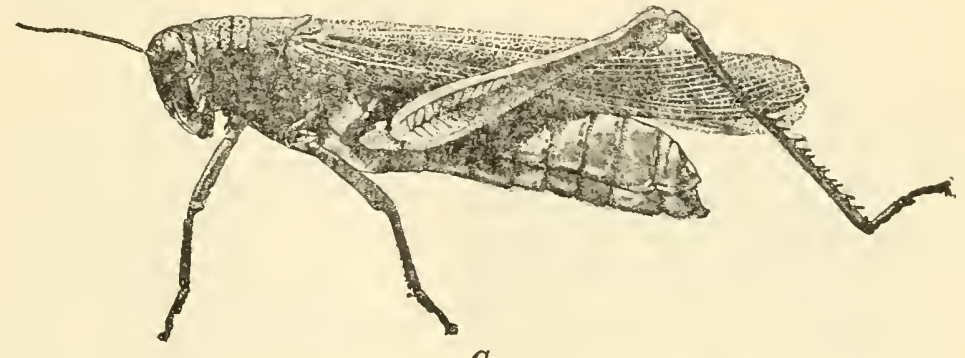

o.

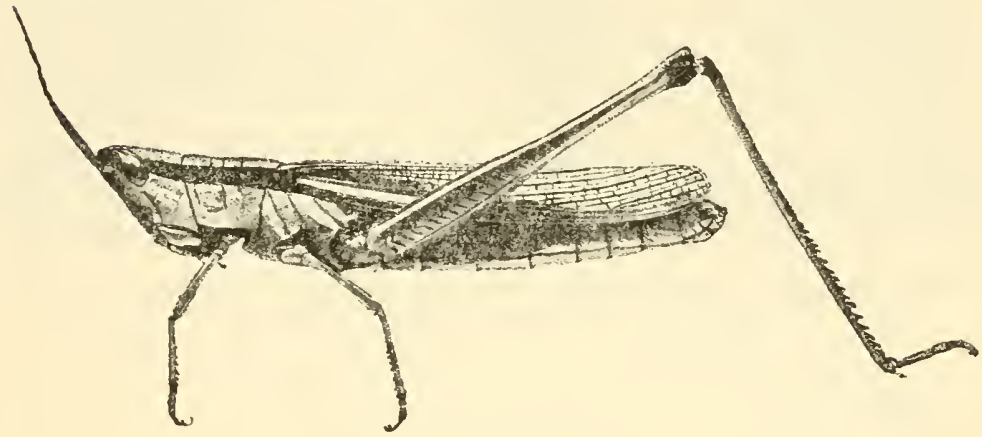

$D$.

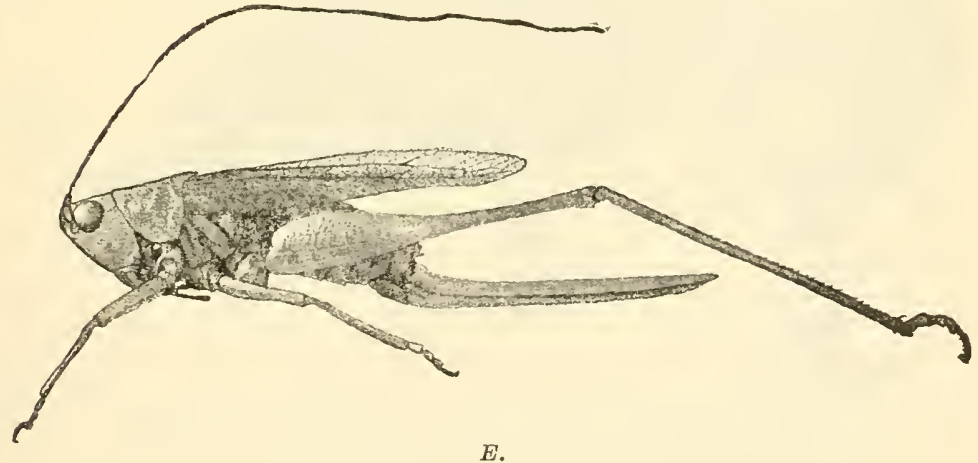

Fig. 177.-(Continued.)

Order Collembola.-Small insects with retracted mandibulate mouth parts; simple eyes; antennae with four segments in most genera; abdomen with six segments, which often carries three ap- 
pendages modified for jumping. The body is often cylindrical. About one thousand species have been described, some of the common species follow: Family Poduridae. Podura aquatica L.; Achorutes maturus Fols.; Family Entomobryidae Folsomides decemoculatus

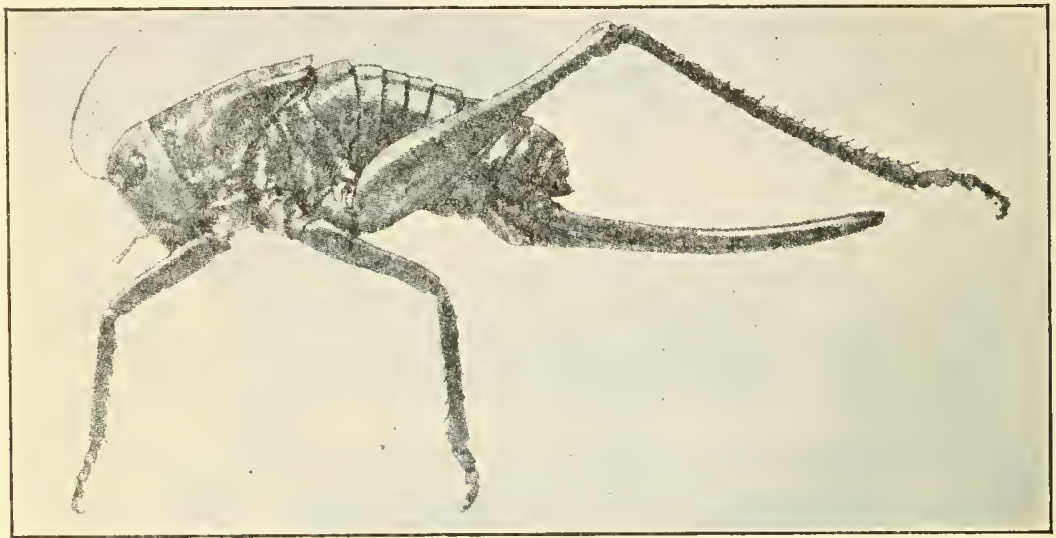

Fig. 178.- Western or Mormon cricket, Anabrus simplex Haldeman, female. (From Henderson, permission of Utah Agricultural Experiment Station.)

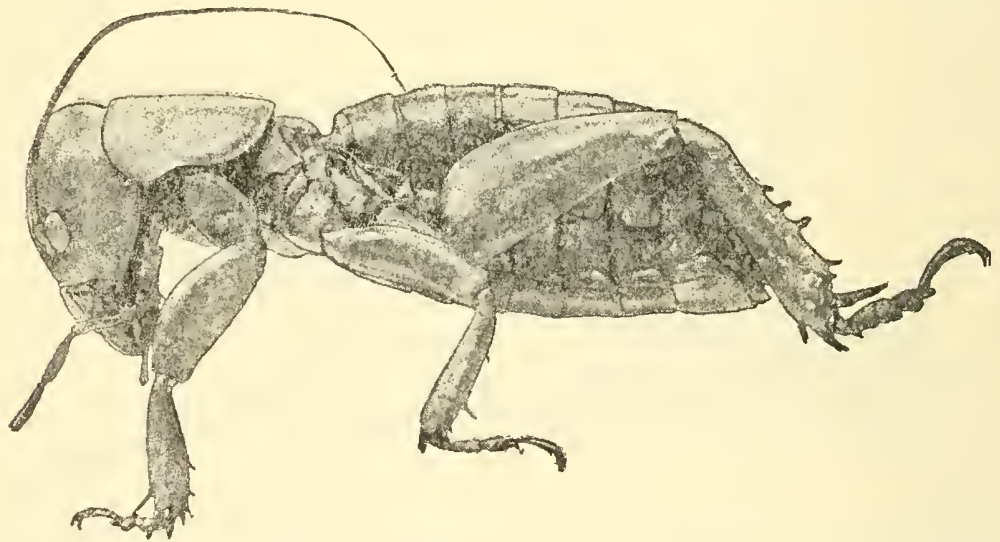

Fig. 179.- Sand cricket, Stenopelmatus fasciatus Thomas. (From Henderson, permission of Utah Agricultural Experiment Station.)

Mills; Isotoma clongata Mac G.; I. titusi Fols; and Tomocerus vulgaris Tull.; Family Sminthuridae, Sminthurus niger (Lubbock); S. eisenii Schott; and Papirius maculosus Schott; and Family Neelidae, Megatothorax incestoides Mills. All the springtales listed above are found 
in many of the states of western America. Mills reports that the Collembola are of some economic importance, damaging, in the main, tender plant tissue.

Subclass Pterygota.-Paurometabola are insects with gradual metamorplosis. The following are some of the more important paurometabolous orders.

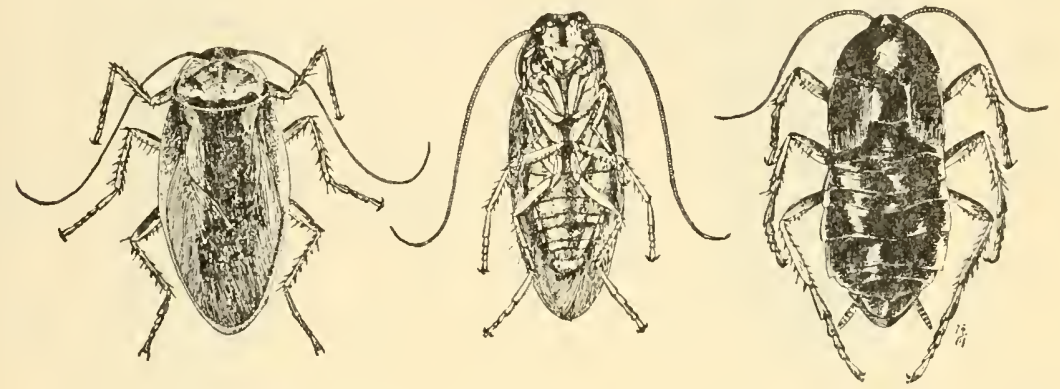

Fig. 180.-American, German (ventral view), and Oriental Cockroaches (left to right). (From Knowiton, permission of Utal Agricultural Experiment Station.)

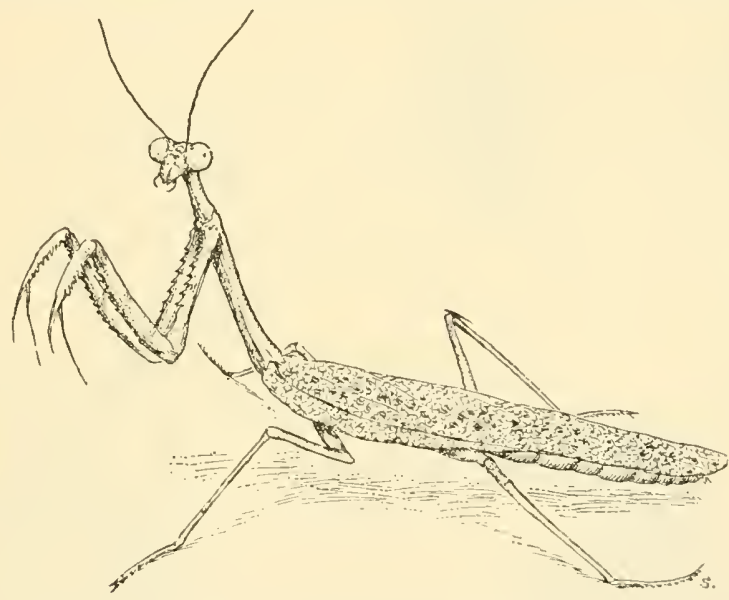

Fig. 181.-Praying mantis, Stagmomantis sp. It is named for its pose.

Order Orthoptera.-This order contains the grasshoppers, katydids, crickets, cockroaches, mantids, and walking sticks. Members of this order usually possess two pairs of wings; some species, however, have their wings greatly modified or reduced, and some do not possess wings at all. The mouth parts are of the biting type. The legs are highly developed for use in getting and holding food and 

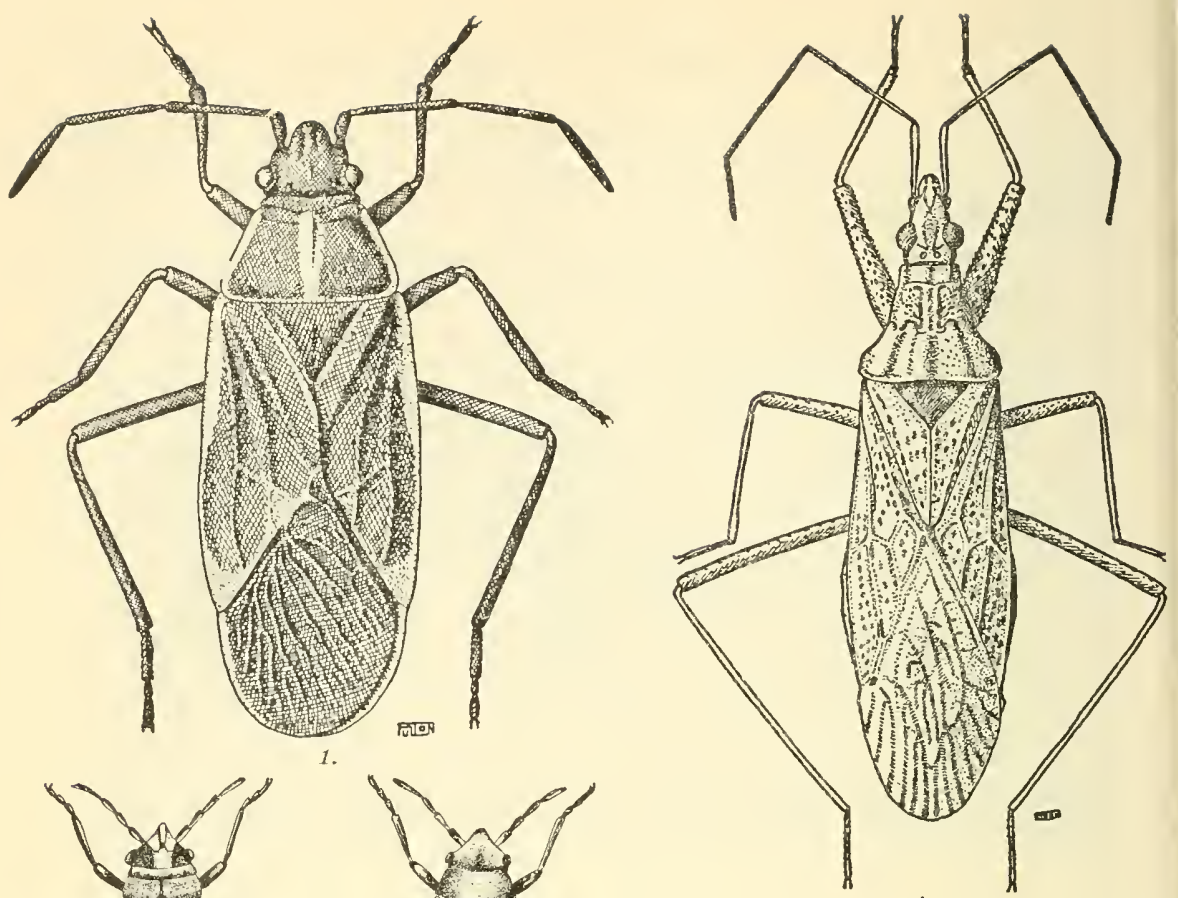
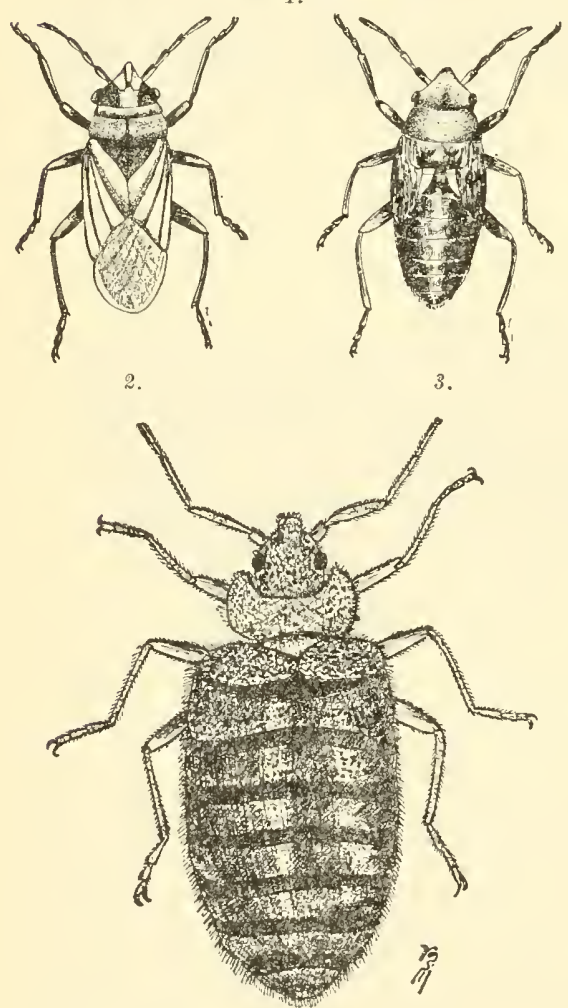

5.
4.

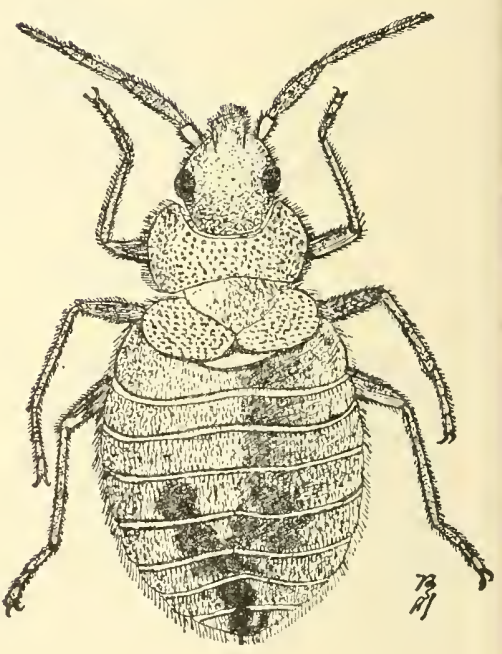

6.

Fig. 182.-(Legend on opposite page.) 
for jumping. The abdomen is usually provided with jointed cerci, and an ovipositor is generally present. About seventeen thousand species have been described.

Some of the common families and species found in many of the western states are, first, the Locustidae, or grasshoppers; the species of this family are widely distributed and are of economic importance, doing great damage to crops and forage plants. The family is divided into three subfamilies, Locustinae, Tryxalinae, and Oedipodinae. The red-legged locust, Melanoplus femur-rubrum (Fig. 177) (DeG.); Haldeman's locust, Hippiscus corallipes Hald; the Shoshone grasshopper, Schistocerca shoshone (Thomas); the twostriped Mermiria, Mermiria bivittata. (Scrville); and the western meadow grasshopper, Conocephalus vicinus Morse, are of considerable cconomic importance and are widely distributed thronghout the western states.

The family Tettigonidae consists of the katydids, cave crickets, camel crickets, and sand crickets. The MIormon cricket, Anabrus simplex Hald, is one of the most destructire insects found in this order. It has attracted much attention since the Mormon pioneer days of $18+8$ when it overran the fields of the pioneers and would have destroyed all the grain crops, had the sea gulls not deroured them in great quantities and so reduced their numbers that the growing grain was saved. A sea gull monument commemorating this event has been erected on the temple grounds in Salt Lake City (Fig. 178).

The katydids. Scudderia furcata Brumner and Hicrocentrum retinerve (Burmeister) are willely distributed in the western states. Hubbell in his classical study of cave crickets and camel erickets reports more than eighty species. The following species Ceuthophitus utahensis Thomas; C. agassizi Scudder; C. conicaudus Hubbell; and C. nodulosus Brunner are fairly common.

The sand cricket, or "child of the earth," Stenopelmatus fasciatus (Thomas), is also a rery interesting member of this family.

The family Blattidae is represented by such common species as the American cockroach, Periplaneta americana (L.); the German roach, Blatella germanica (L.); the oriental cockroach, Blatta orientalis L.; and Orenivaga erratica (Rehn), a native species of the western United States (Fig. 180).

Fig. 182.- Some common Hemiptera. 1, adult box-elder bug, Leptocoris trivittatus Say: 2, adult false chinch bug; 3, nymph or immature false chinch bug; 4, tatus Say; 2 , adult false chinch bug; 3 , nympis ferus L: 5. male bedbug; 6 , female bedbug, Cimex lectularius $L$. (From Inowlton, permission Utah Agricultural Experiment Station.) 

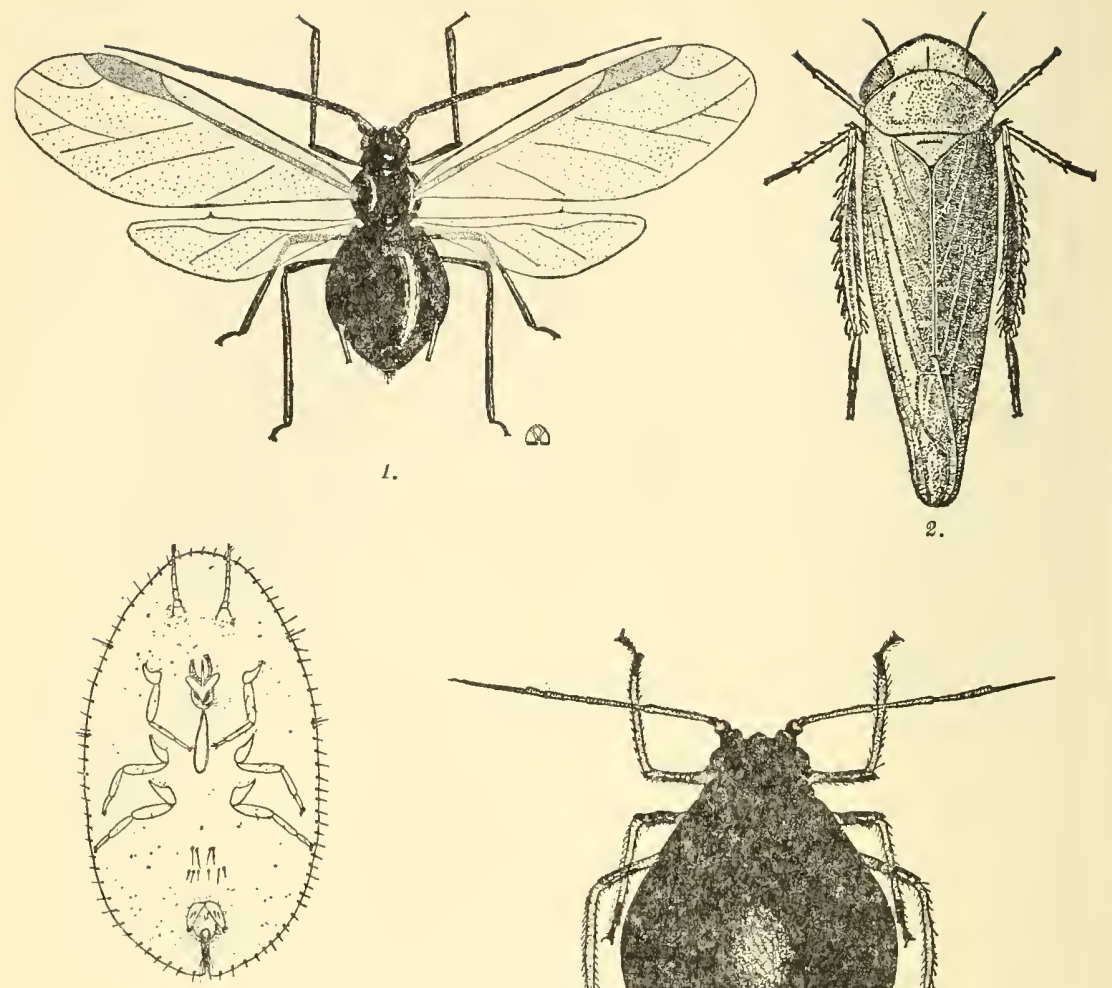

2.

s.

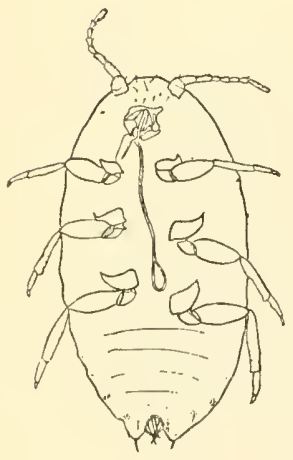

4.

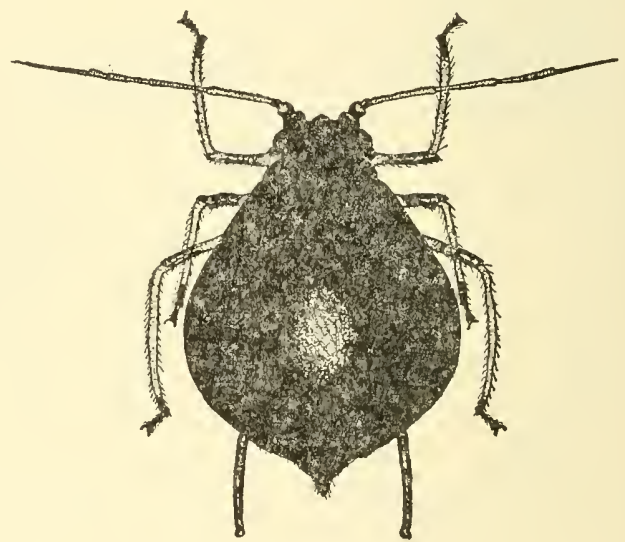

5 .

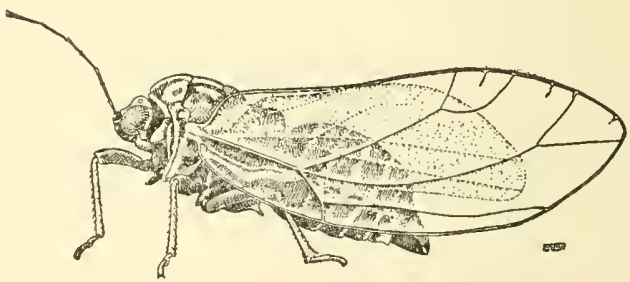

6.

Fig. 183.-Some common Homoptera. 1, Spring migrant of rosy apple aphid ; 2, adult beet leafhopper: 3 , cottony maple scale, Pulvinaria vitis L., ventral view of body; 4, Balker's mealybug, Pseudococous maritimus Ehrh, ventral view of body; 5, wingless female black cherry aphid; 6, lateral view of adult female potato psyllid; 7 , San Jose scale, Aspidiotus perniciosus Comst., pygidium; 8, purple scale, Lepidosaphes becki Newman, pygidium; 9, pine scale, Chionaspis pinifoliae Fitch, 

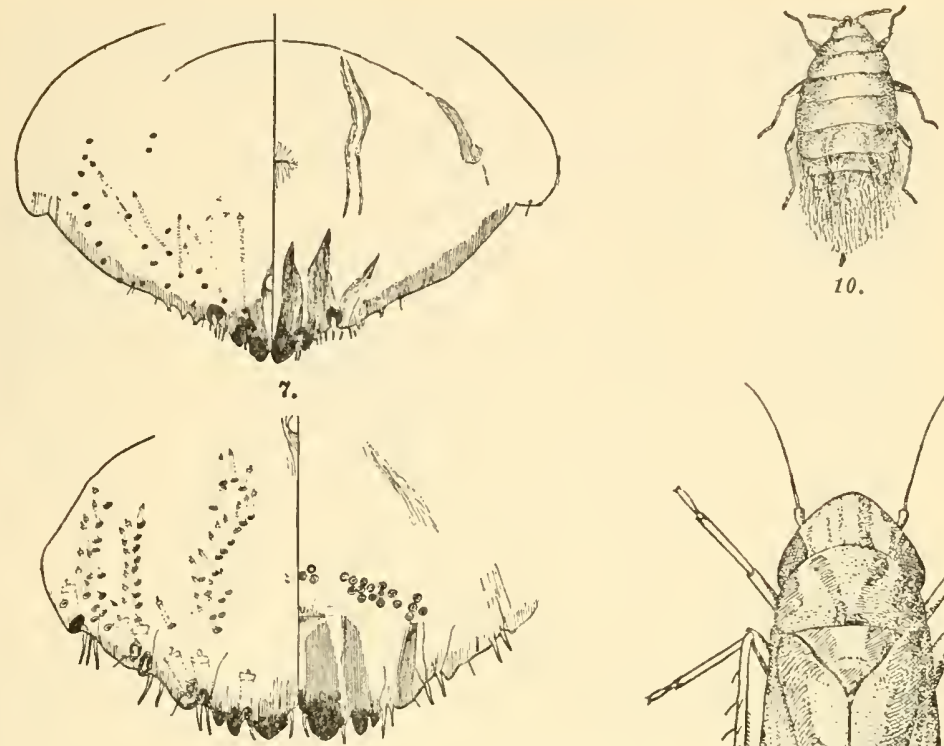

8.
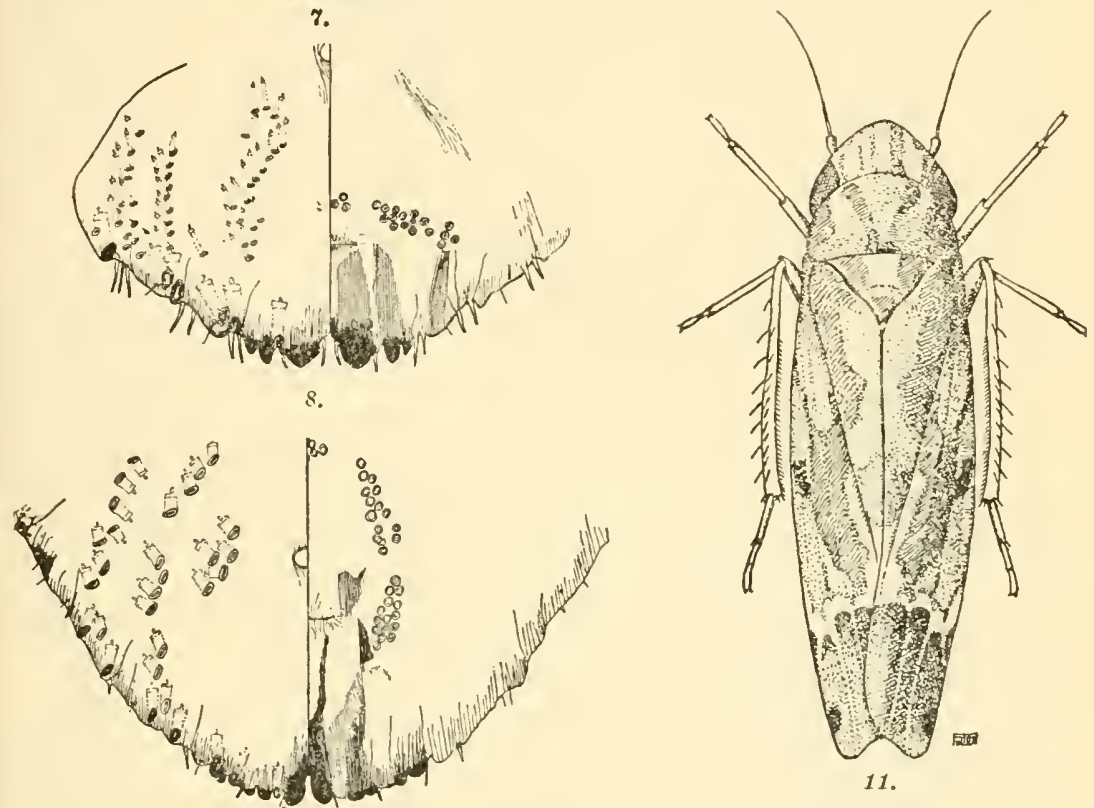

9.

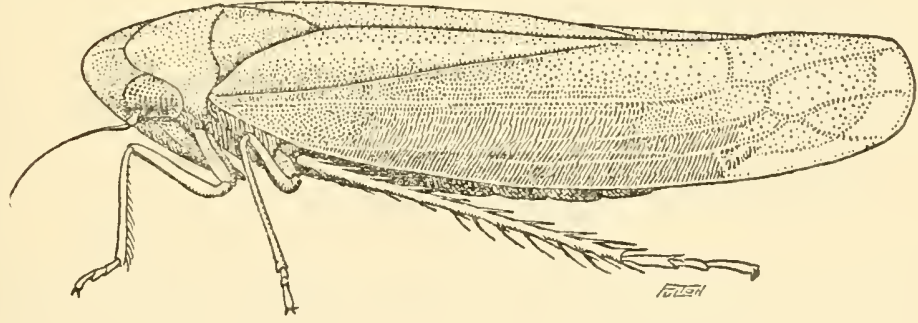

12.

pygidium; 10, beet aphid, adult wingless female; 11 , adult of one of the varieties of grape leafhopper common in Utah, Erythroneura ziczac Walsh; 12, adult male of Empoasca filamenta De L. (Nos. 1, 2, 5, 6, 10, 11, 12 from Knowiton, permission Utah Agricultural Experiment Station. Nos. 3, 4, 7, 8, 9 from Jorgensen, courtesy Utah Academy of Sciences, Arts and Letters.) 
A common species of Phasmidae is Parabacillus coloradus (Scudder) and of the Mantidae is Litanscutric obscura Scudder (Fig. 181).

Order Hemiptera.-This order includes the true bugs, insects with piercing and sucking mouths; the winged species with the front wings leathery and hard near the base and membranous over the outer half. Over twenty thousand species of bugs have been described. They are widely distributed, and many are of considerable economic importance. The following represent some of the common families and species: Family Pentatomidae, stink bugs, Chlorochroa sayi Stal, and the harlequin bug, Murgantia histrionica (Hahn) are common species. The family Coreidae is represented by the squash bug, Anasa tristis (DeG.) ; and the family Corizidae by the box-elder bug, Leptocoris trivittatus (Say). The false chinch bug, Nysius ericae (Schilling), is a common species of the family Lygaeidae. The lace bug, Corythucha distincta Osborn and Drake is a handsome Tingitidae; the common damsel bug Nabis ferus (I.) is typical of the family Nabidae. The members of the family Niridae are numerous and widely distributed. The tarnished plant bug, Lygus pratensis (L.) is one of the commonest mirids in the United States. The water striders, Gerridae; the back swimmers, Notonectidae; and the giant water bugs, Belostomatidae are familiar to all who are acquainted with the life of streams and ponds. The bedbugs belong to the family Cimicidae, and Cimex lectularius L. is an example of a bloodsucking species which is world-wide in distribution (Fig. 182).

Order Homoptera.-Many of the most serious insect pests belong to this order, also some species that are beneficial to man. Insects with membranous wings and sucking mouths, such as the cicadas, aphids, leaf hopper's, and scale insects, constitute this order. The plant lice or aphids, belonging to the family Aphididae, are probably one of a half dozen species of insects known by all. The rosy apple aphid, Aphis roseus (Baker) is one of the most common and destructive apple aphids in the West. The beet root aphid, Pemphigus betae Doane, and the black cherry aphid, Myzus cerasi (Fab.), are destructive species. The potato psyllid, Paratriozoa cockerclli (Sulc), is one of the very destructive Chermidae. The family Coccidae is a small obscure group of insects, yet they are very destructive and hard to control. Baker's mealy bug, Pseudococcus maritimus Ehrh.; the cottony maple scale, Pulvinaria vitis L.; the San José scale, Aspidiotus 
perniciosus Comst.; the purple scale, Lepidosaphes becki (Newman); and the pine scale, Chionaspis pinifoliae Fitch are important scale insect pests. The Cicadellidae or leaf hoppers are represented by the following insect enemies : the sugar-beet leaf hopper, Eutettix tenellus (Baker); Delong's leaf hopper, Empoasca filomenta DeL.; and the grape leaf hopper, Erythroneura comes (Say). Insects of this order are all plant feeders, and they are very numerous; over sixteen thousand species have been described (Fig. 183).

Oider Isoptera.-Nore than five hundred species of termites, often wrongly called white ants, have been named. Termites are white, soft-bodied, mandibulate insects. They feed principally upon wood, and in the tropies they are one of the most destructive insects known. Termites are social in habits, forming large colonies which are used for years and contain as many as five hundred thousand to a million individuals. The Nevada termite Termopsis nevadensis (Hagen); and the western termite, lieticulitermes hesperus Banks, are common and destructive. More will be said of these insects under the discussion of social insects, later in this chapter.

Order Thysanoptera.-Thrips are very small insects, not more than two to three millimeters in length. They are mostly plant feeders, sucking the juices from the plants. The banded thrip, Aeolothrips fasciatus (L.) and the onion thrip, Thrips tabaci Lindeman are common insect pests. About five hundred species of thrips are known.

Order Dermaptera.-The earwigs are small terrestrial, mandibulate insects with a pair of forcepslike appendages at the tip of the abdomen. The winged species have a short leathery anterior pair of wings which resemble the elytra of some beetles. The small earwig, Labia minor (L.) ; and the toothed earwig, Spongovostox apicedentatus (Caudell) are species commonly found in the western United States.

\section{Hemimetabolous Insects With Incomplete Metamorphosis}

Order Odonata.-The dragonflies and damsel flies are insects with large compound eyes, mandibulate mouth parts, four membranous wings that are finely veined, and a long slender abdomen. The naiads are aquatic and possess a labium which has been highly modified. It can be greatly extended for the catching and holding of 
prey. The adults are swift flying, brightly colored, predaceous insects. Their food consists of mosquitoes, gnats, and many other kinds of flying insects. Mruch has been written on the dragonflies of the United States. About twenty-eight hundred species have been described. The order is divided into the suborders, Zygoptera (damsel flies), and Anisoptera (dragonflies). There are two families of damsel flies, the Agrionidae and Caenagrionidae; also two families of dragonflies, Aeschinidae and Libellulidae. The beautiful ruby spot, Hetaerina americana Fabr; and the stalked-winged, Lestes uncatus Kirby are damsel flies that are widely distributed. The dragonflies Libellula pulchella Drury and Sympetrum rubicundulum are common west of the Mississippi River in the United States (Fig. 184).

Order Ephemerida.-The Mayflies are aquatic insects, with mandibulate naiads, but since the adult stage lasts but a day, the mouth parts are vestigial. The adults have well-developed wings and two or three long abdominal cerci. The life cycle occupies from one to three years. The food of the naiads consists of small aquatic plants and organic matter which is obtained from the rocks and mud on the bottom of streams and along the shores of lakes where they live. They serve as food for larger insects and fishes. The prickleback, Ephemerella grandis Eaton; and the western bunchgill, Siphlurus occidentalis Eaton are common species.

Order Plecoptera (Stone flies).--The stone flies are found near streams, flying low over the water. They have mandibulate mouth parts, four wings that are not so thickly netted with veins as are the Odonata, but with longer antennae than the Odonata. They are found on stones along lakes and streams where they pass their naiad stage. They require running water that is well aerated. Their food consists largely of aquatic insects, such as May flies. They are sometimes used as bait for trout. There are four families: the little curler, Pteronarcella badia Hagen; and Perla modesta Banks are representative species.

\section{Holonetabolous Insects With Complete Metaniorphosis}

The following are some of the important orders that fall within this group.

Order Trichoptera (Caddis flies).-This order includes about eighteen hundred species of "case flies" or "rock rollers," as they 
are sometimes called. The adults are less than an inch long, with well-developed wings, but with vestigial mouth parts since they probably take no food. The larvae inhabit the bottoms of lakes, ponds, rivers, and creeks, and as a means of protecting their soft bodies they build cases or tubes of small rocks, shells, bits of wood, and plants. The larvae feed upon plant tissue and small animals which they capture in little nets that are placed near the entrance to their case. Pupation takes place in the water. The adults lay their eggs in the water on sticks or stones. About eighteen families are recognized. The species Hydropsyche partita Banks and $H$. scalaris Hagen of the net-making family Hydropsychidae, and Platyphylax designata (Walker) of the family Limnophilidae are common in the western states.

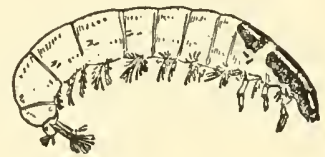

Fig. 185.-Larva of net-making caddis worm, Hydropsyche. (From Needham and Christensen, permission of Utah Agricultural Experiment Station.)

Order Neuroptera (Nerve Winged Insects).-This order contains the doodlebugs, lacewings, snake flies, dobson flies and mantispids. It is probably the most heterogeneous order of insects; all the species, however, have biting mouths and two pairs of net-veined membranous wings. The larvae are both terrestrial and aquatic, and feed mainly upon other insects. There are thirteen families, but probably the families Raphidiidae, snake flies; Chrysopidae, lacewing flies; and the Myrmeleonidae, doodlebugs or ant lions contain insects most generally encountered.

The lacewing or golden eyes, Chrysopa californica Coquillett, is a beneficial and widespread species. It feeds in the larval stage upon aphids, thrips, scale insects, and psyllids.

Order Coleoptera (Beetles).-The beetles are world-wide in their distribution and contain the largest number of species of any order in the animal kingdom. They are adapted for an almost unlimited variety of conditions, living on plants and animals, on land, and in the water. They have biting mouth parts, and the first pair of wings, the elytra, are leathery or hard. They feed on all possible kinds of 


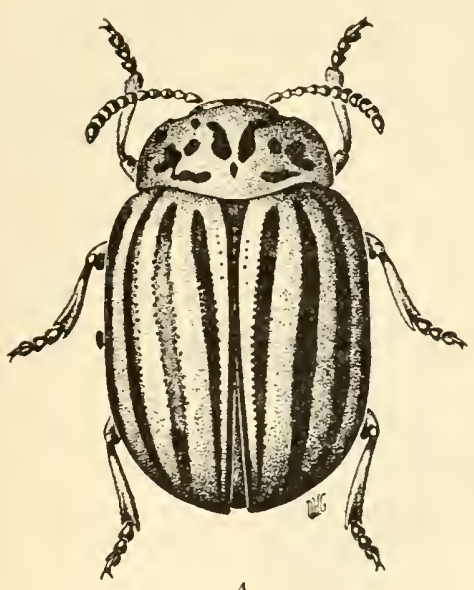

A.

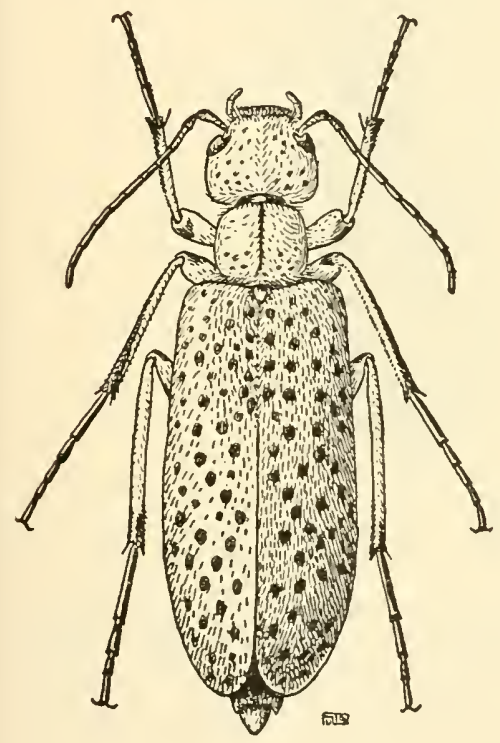

C.

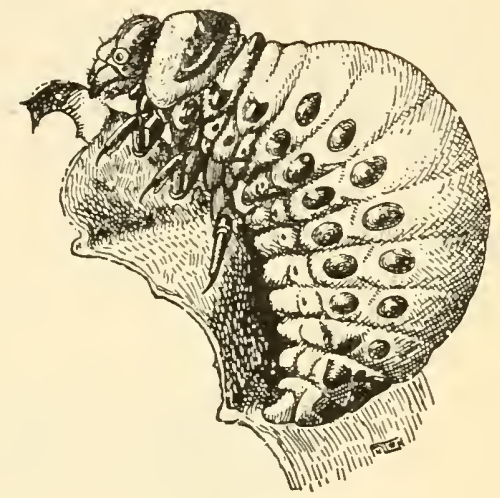

$B$.

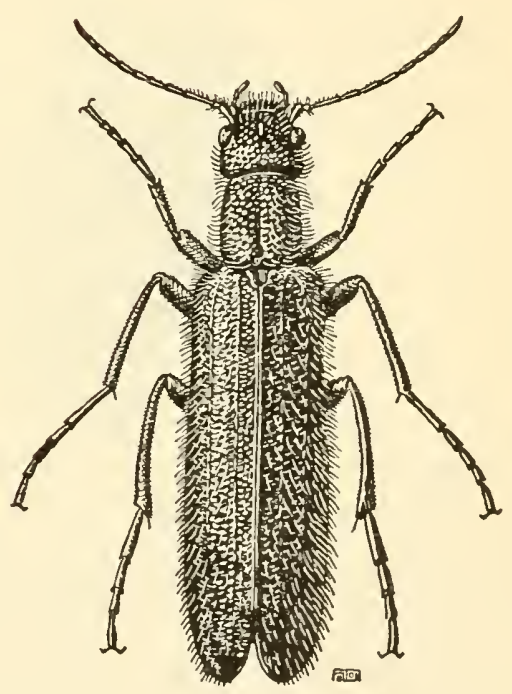

D.

Fig. 186.-Three species of Coleoptera, A, adult Colorado potato beetle, Leptinotarsa decimlineata Say; $B$, larva or slug of Colorado potato beetle; $C$, spotted blister beetle, Epicauta maculata Say; $D$, common blister beetle, Epicauta puncticollis Mann. (From Knowlton and Sorenson, permission Utah Agricultural Experiment Station.) 
food. Many species do an enormous amount of damage, while in contrast, some of the most beneficial insects are beetles. In the United States, north of Mexico there are one hundred and nine families and twenty-four thousand species recognized. Over 200,000 species from all parts of the world have been described.

Some of the families which contain the most destructive species are the leaf beetles, Chrysomelidae; the long-horned wood-boring beetles, Cerambycidae; the click beetles, Elateridae; the June beetles, Scarabaeidae; the metallic wood-boring beetles, Buprestidae;
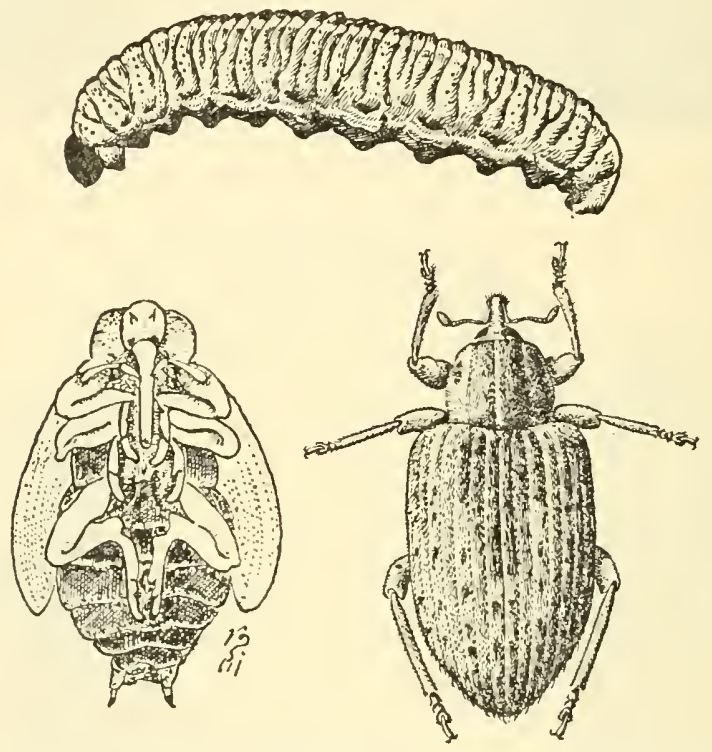

Fig. 187.-Alfalfa weevil, Phytonomus porticus. Above, Iarva; lower left, pupa; lower right adult. (From Knowlton and Sorensen, permission Utah Agricultural Experiment Station.)

and the weevils, Curculionidae. The following families are, in the main, very beneficial: the tiger beetles, Cicindelidae; ground beetles, Carabidae; ladybird beetles, Coccinellidae; and the carrion beetles, Silphidae. The cotton boll weevil, Anthonomus grandis, and the alfalfa weevil, Phytonomus posticus, have done millions of dollars' worth of damage. Other groups of weevils of which the following are typical do considerable damage: the billbugs, Calendra mormon Chitt.; Rhynchites bicolor var. cockerelli Pierce; and Apion proclive Lec. (Fig. 188). 
Order Lepidoptera (The Butterflies and Moths).--In the Lepidoptera the larvae have biting mouth parts, while the adults have a highly specialized suctorial structure. The antennae are of various shapes and sizes. The two pairs of wings are covered with scales, which are highly colored in many species.

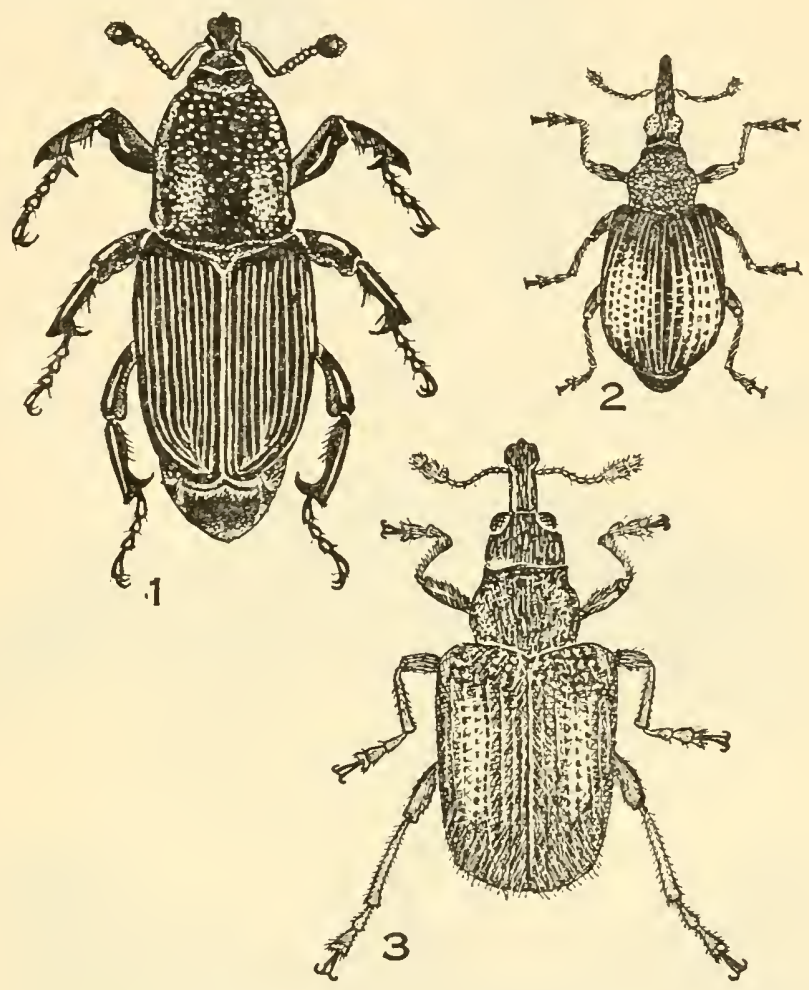

Fig. 188.-Common weevils. 1, the bill-bug, Calendra mormon Chitt.; 2, Apion proclive Lec.; 3 , the rose weevil, Rhynchites bicolor var. cockerlli Pierce. (Drawings by Tanner.)

This is the second largest order of insects. Approximately ninetyfive thousand species are recognized, of which about eight thousand are found in the United States. The order is divided into the suborders Rhopalocera, butterflies, and Heterocera, the moths.

The larvae or caterpillars are among our most destructive insect pests. They attack the foliage and fruit of the forest, orchard, field, and garden; also, stored food and animal products. 

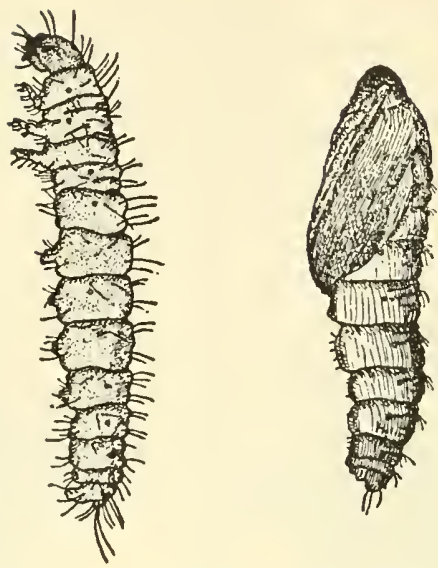

Fig. 189.-At left, larva of Capitophorus potentillae (Walker) ; right, strawberry leafroller, Ancylis comptana var. fragariae (TV, and R.) (From Knowlton and Smith, courtesy of Utah Academy of Sciences, Arts and Letters.)
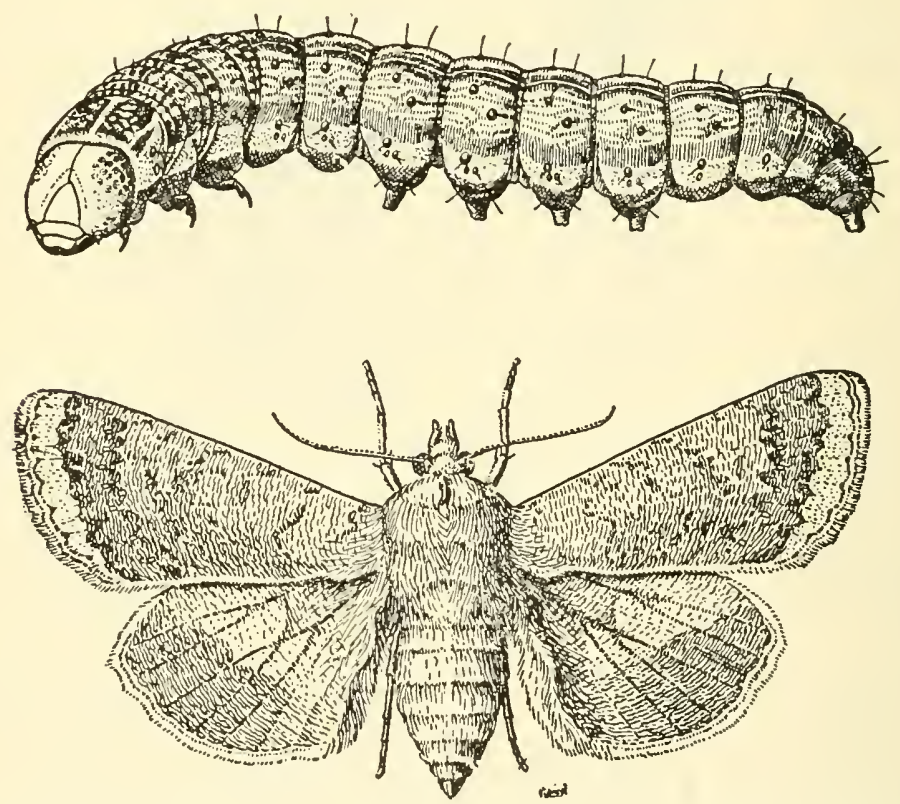

Flg. 190.-Representative of order Lepidoptera. Above, tomato fruitworm (or corn-ear worm); below, adult tomato fruitworm moth, Heliothis obsoleta. (From Sorensen and Knowlton, permission Utah Agricultural Experiment Station.) 
The following are some examples of common species: the monarch butterfly, Danaüs menippe (Hubner), is widely distributed through the United States, parts of Canada, and south into the tropics. This species is typical of the family Danaïdae which is one of the nine families of butterflies in this country.
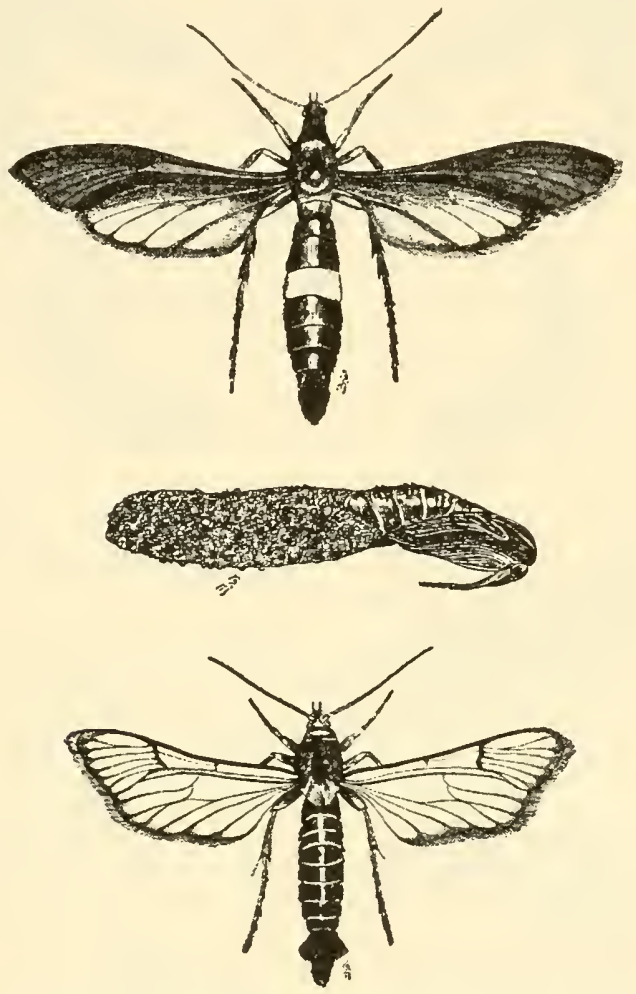

Fig. 191.-Insects of the order Lepidoeptera. Above, adult female moth of peach tree borer. Aegeria exitiosa; center, cocoon and empty pupal case; below, adult male moth of peach borer. (From Sorensen and Knowlton, permission Utah Agricultural Experiment Station.)

Some of the most destructive species of this order are among the moths. The Noctuidae (millers) is a large family of injurious species. The corn-ear worm or cotton bollworm, Heliothis obsoleta (Fabr.), feeds upon many plants, a few of which are tomatoes, corn, the green bolls of cotton, squash, strawberries, cabbage, and at times alfalfa (Fig. 190). The gooseberry fruitworm, Zophodia grossulariae Riley, is a pest belonging to the snout moths or Pyralididae. The clear- 
wing moths, Aegeriidae, a rather distinctive family, are represented by the peach-tree worm, Aegeria exitiosa Say, a serious enemy of the peach in most parts of the United States (Fig. 191). The strawberry
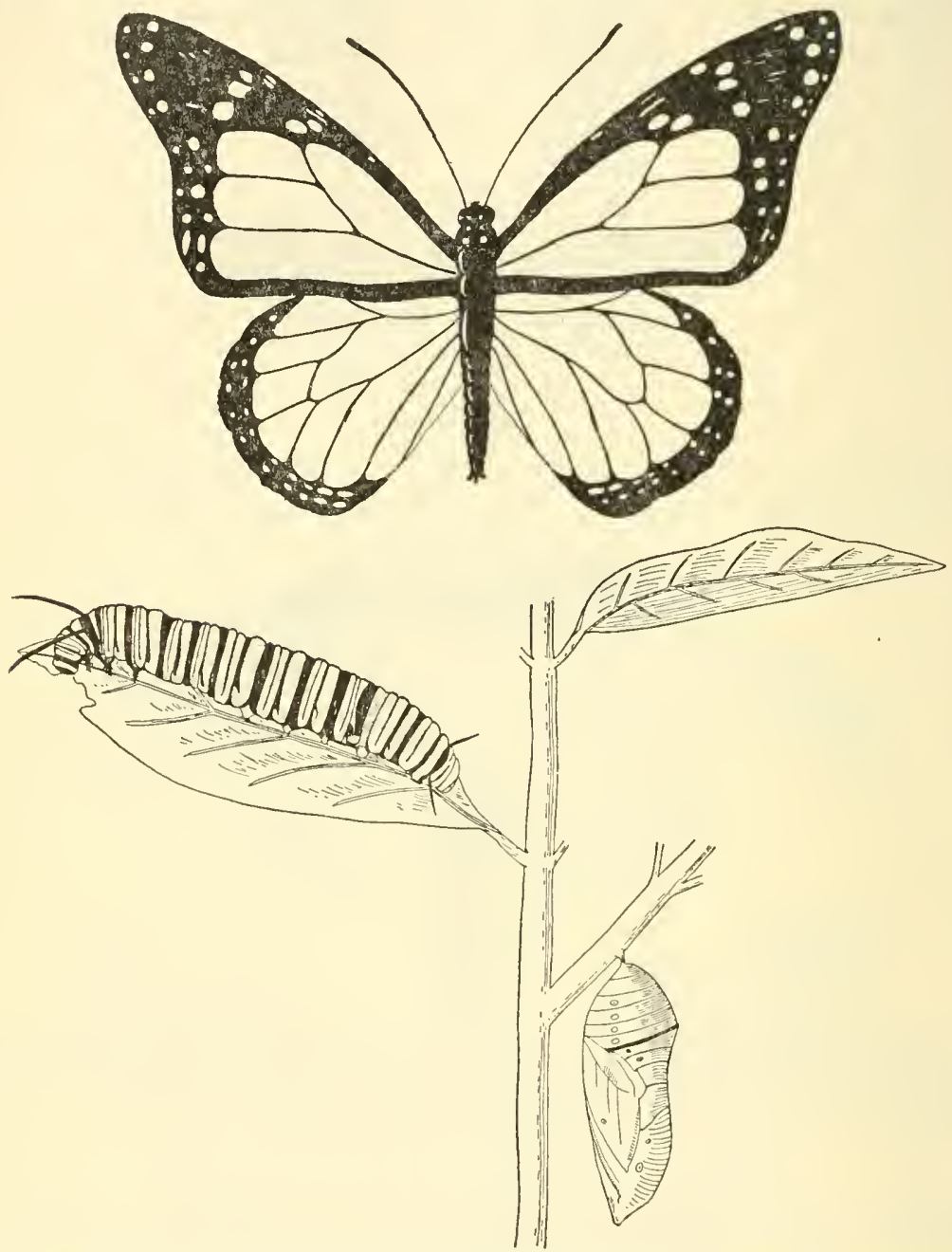

Fig. 192.-Life history of monarch butterfly. (From White, General Biology, published by The C. V. Mosby Company.)

leaf roller, family Eucosmidae, is an imported species from Europe; it feeds on both wild and cultivated strawberries, blackberries, and raspberries and is found in many parts of the United States. 
Order Siphonaptera (Fleas).-Fleas have strong jumping legs, piercing and sucking mouth parts, laterally flattened bodies, but no wings. They are world-wide in distribution; about four hundred species have been described. All of the species in the adult stage are external parasites on warm-blooded vertebrates. They are pests on cats and dogs and known to be carriers of bubonic plague.

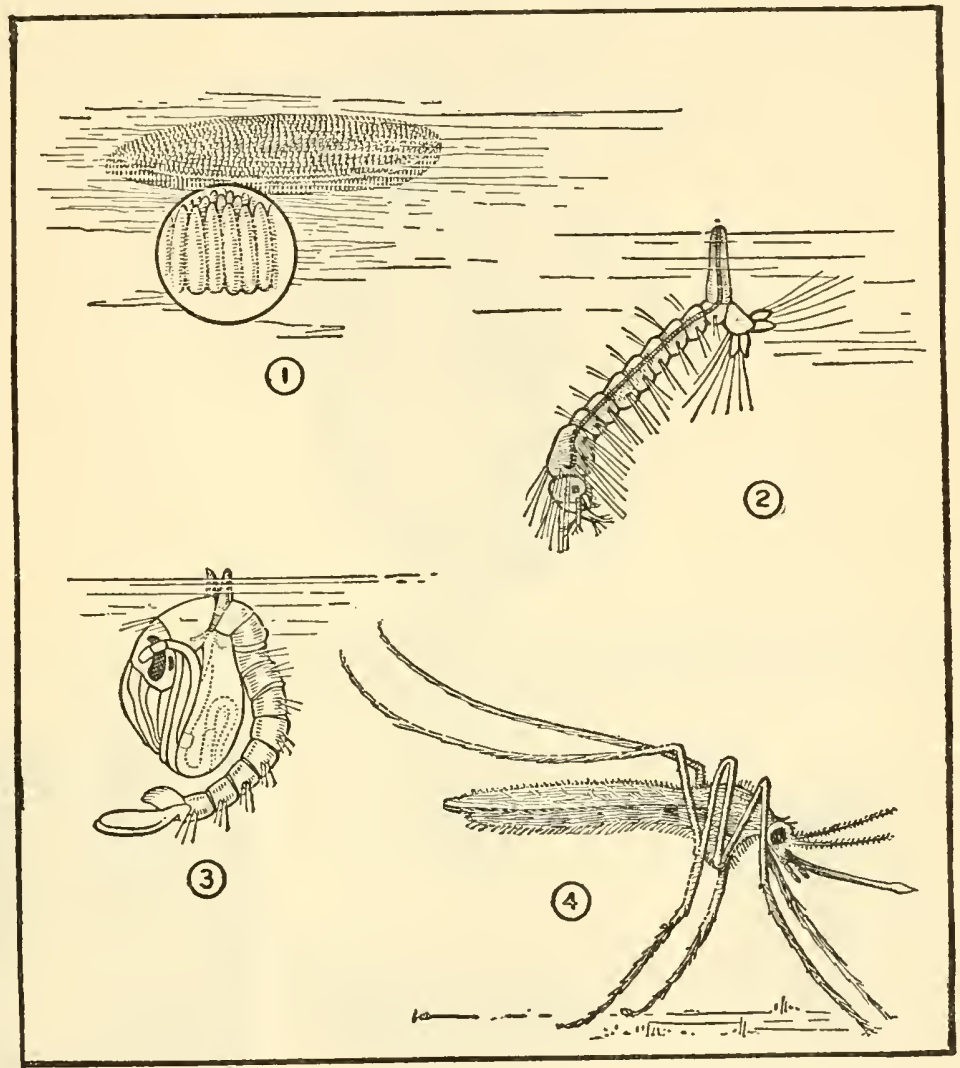

Fig. 193.-Life history of the mosquito. 1, mosquito eggs floating in the water (slightly magnified); 2 mosquito larva or wiggler; 3 , mosquito pupa or tumbler; 4, adult (From Turner, Personal and Community Health, published by The C. V. Mosby Company, after Turner and Collins.)

Order Diptera (Flies and Mosquitoes).-The Diptera may be characterized as insects with mouth parts specialized for sucking, in some species for piercing; and with only two wings, the halters or second pair being vestigial structures. 
Many of the most useful insects are found in this order. The robber flies, Asilidae; the syrphids, Syrphidae; the bee flies, Bombyliidae; and the tachinids, Tachinidae, contain many species that are valuable to mankind. On the other hand, the mosquitoes, Culicidae;

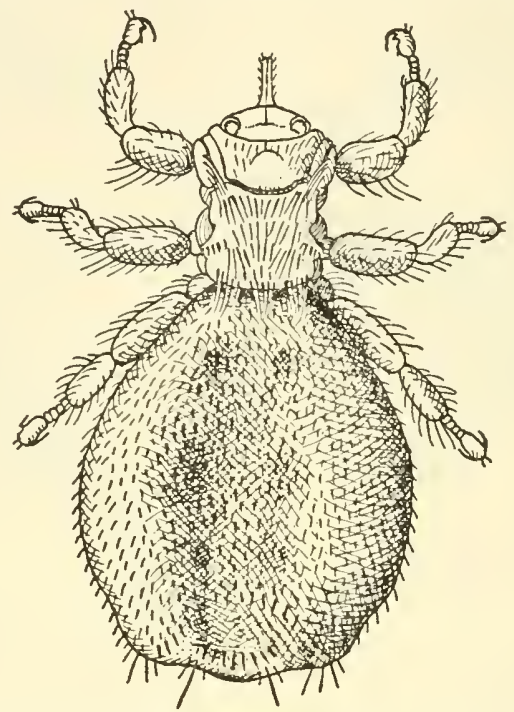

Fig. 194.-Adult female sheep tick, Melophagus ovinus Linn. (From Knowlton, Rowe, and Madsen, by permission of the Utah Agricultural Experiment Station.)

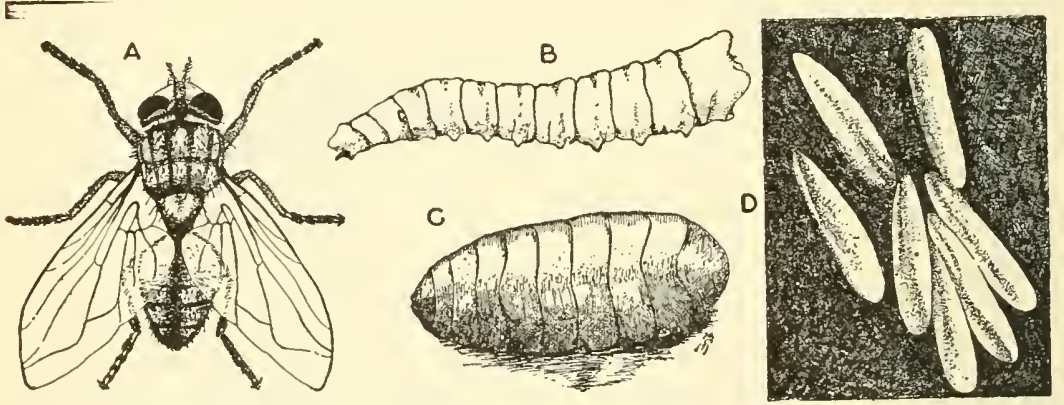

Fig. 195.-Life history of the housefly, Musca domestica I. A. A, adult; $B$, mature larva; $C$, pupa inside puparium; $D$, eggs. (From Knowlton, Rowe, and Madsen, by permission of the Utah Agricultural Experiment Station.)

the fruit flies, 'Trypetidae; the houseflies, Muscidae; the botflies, Oestridae; and the sheep tick, Hippoboscidae, damage food and spread disease and suffering. The larvae of some families are called maggots. Some larvae are parasitic, others predacious, or scaven- 
gers. There are over fifty thousand species of Diptera, ten thousand of which are known to occur in the United States. The suborder Pupipara is a most interesting group, containing the bloodsucking ectoparasites which live upon bats, birds, and mammals. The sheep tick is a fairly common species.

Order Hymenoptera (Bees, Wasps, and Ants).-The Hymenoptera are so named because of their membranous wings; the word hymen means membrane. In the winged species there are two pairs of wings, the second pair being smaller than the first pair. The mouth
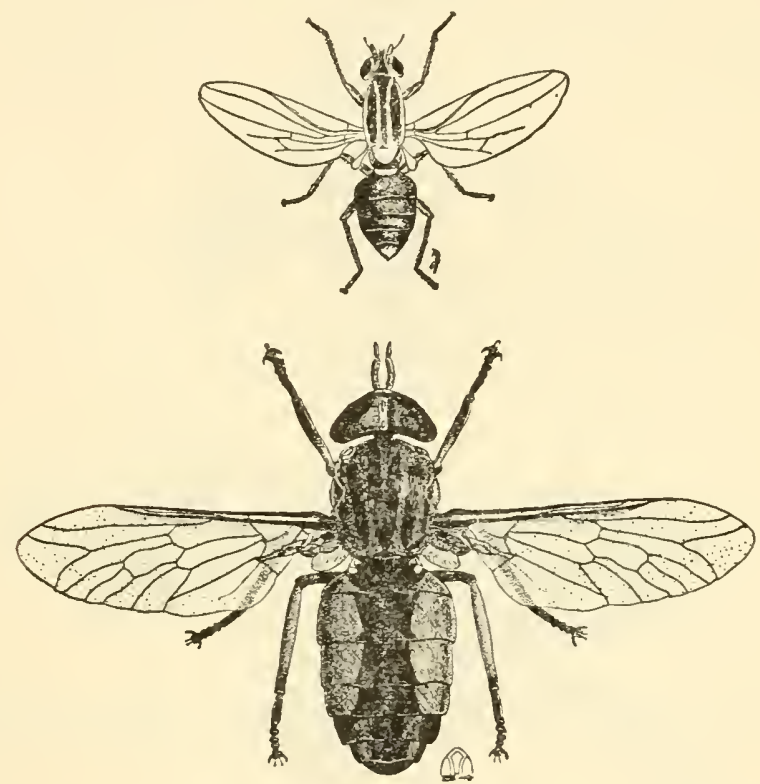

Fig. 196.-Flies. Above, Chloropisca glabra Meig. Its maggots feed upon beet root aphids. Below, adult western green-headed horsefiy, Tabanus phaenops O. S. (From Knowlton, Rowe, and Madsen, by permission of the Utah Agricultural Experiment Station.)

parts are both biting and sucking, and the females are provided with ovipositors that have become greatly modified. In the ichneumon flies, the ovipositor is composed of long slender bristlelike structures, which are used for drilling through the bark of trees and depositing their eggs upon insect larvae under the bark. The ants, mutillids, and bees use their ovipositors for stinging as well as for depositing eggs. The pigeon horntails bore into trees, causing considerable damage. 
Many of the Hymenoptera live as parasites and are of great value in biological control work. The braconids, ichneumon flies, and chalcid flies are examples of this group of parasites. A number of the Hymenoptera are not beneficial, since they feed upon the leaves of
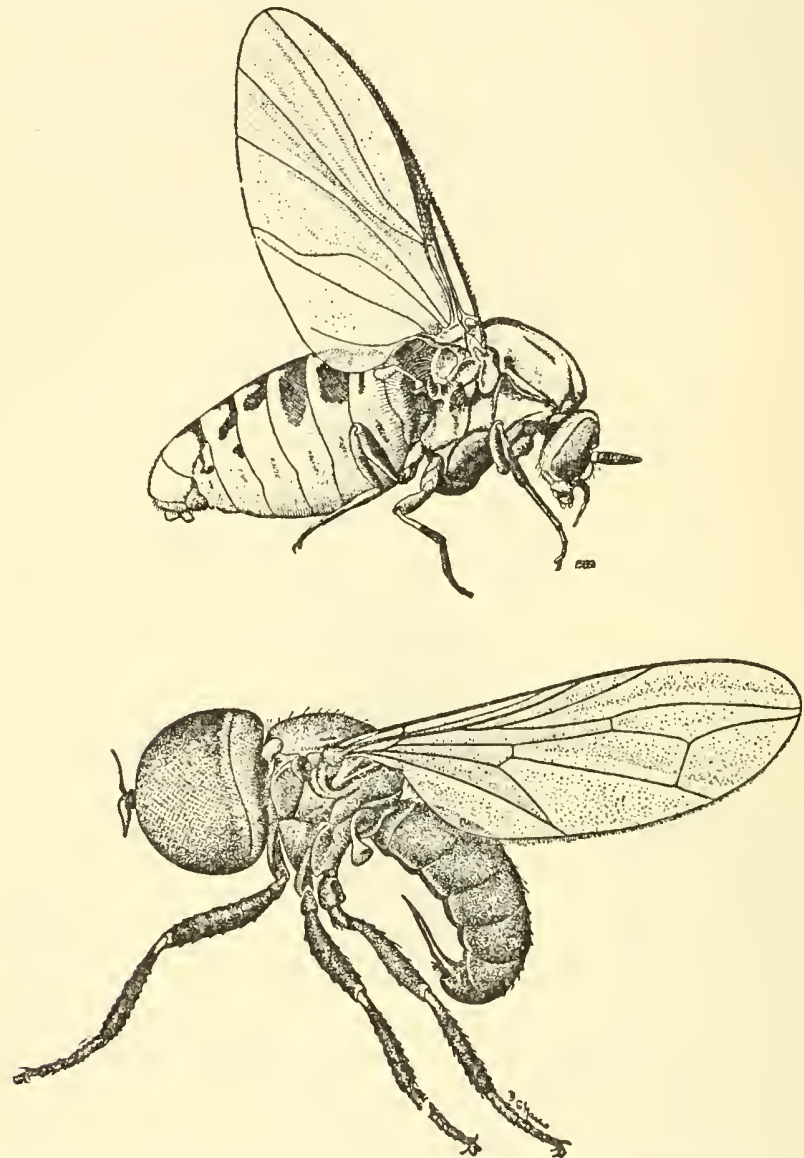

Fig. 197.-Above, adult female Simulium vittatum Zett. (From Knowlton, Rowe, and Madsen, by permission of the Utah Agricultural Experiment Station.) Below, female big-headed fly, Pipunculus subvirescens Loew. (From Knowlton. courtesy of Utah Academy of Sciences, Arts, and Letters.)

our garden, orchard, and forest vegetation. There are many species that are gall makers, attacking a wide variety of plants. Many species are highly developed as far as social organization is concerned, thousands of individuals living in a single colony. The 
ants, honey bees, and social wasps are examples. The Hymenoptera found in this country are divided into three suborders, twentyeight families and about twelve thousand species. The honey bees and silkworms are the only really domesticated insects.

\section{Other Orders}

Other orders than the ones discussed above are included in the notable treatises on entomology. These are in the main, however, rare and little known insects. Professor Comstock in his An Introduction to Entomology, recognizes twenty-five orders: the Zoraptera, insects resembling termites in many respects, and consisting of but

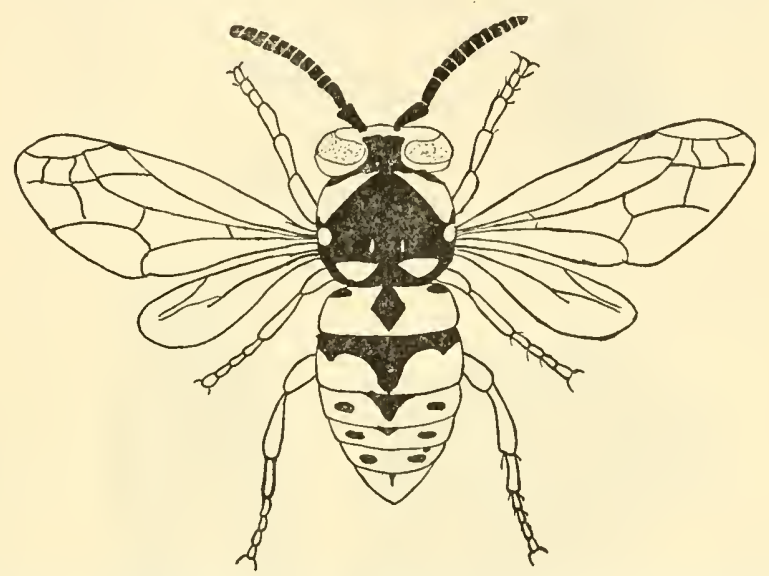

Fig. 198. - The common wasp, or yellow-jacket, Vespula pennsylvanica Saussure. (From Sorensen and Knowlton, by permission of the Utah Agricultural Experiment Station.)

six known species in a single genus Zorotypus; the Corrodentia, psocids and book lice; the Mallophaga, wingless ectoparasites of birds; the Embiidina, a small group of about seventy species found in the warmer parts of the world, living under stones and in the detritus of the soil; the Anoplura, the true lice, an order consisting of sixty-five species of blood-sucking parasites found on the mammals; the Strepsiptera, a group of small twisted-winged insects that live as parasites within the body of other insects; and the Mecoptera, a group of about forty American species, commonly called scorpion flies, in addition to the eighteen orders discussed above. Brues and Melander in their Classification of Insects recognize thirty-four or- 
ders; while Imms, the noted English entomologist, has included twenty-three orders in his A General Textbook of Entomology.

In this elementary consideration of insect classification we have tried to include information and illustrations which will be of value in interesting the student in the thousands of insects of our environment.

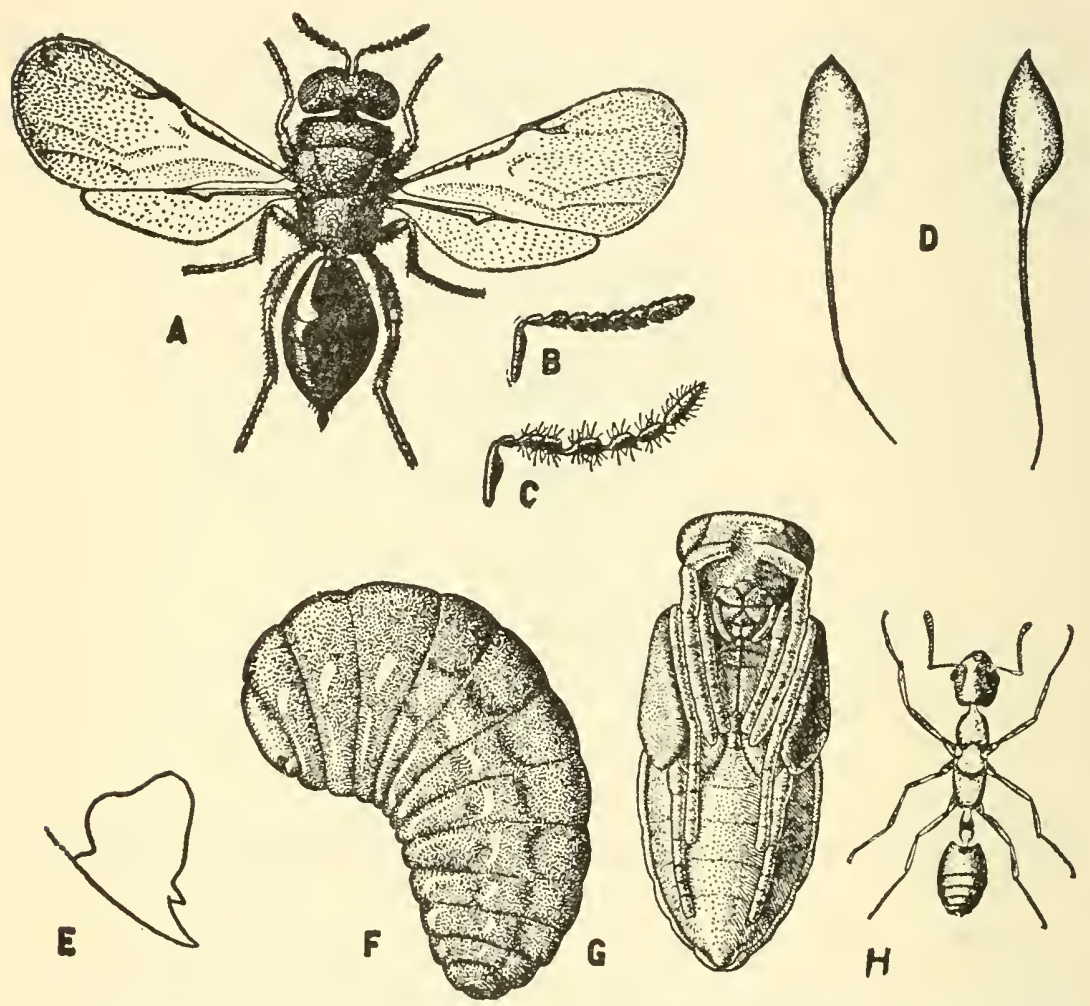

Fig. 199.-Hymenoptera. Alfalfa-seed chalcis-fly, Brucophagus funebris How. $A$, female; $B$, female antenna ; $C$, male antenna; $D$, eggs (greatly enlarged); $E$, anterior view of right mandible; $F$, larva; $G$, pupa, (enlarged); $H$, worker of the black ant. (From Sorensen and Knowlton, permission Utah Agricultural Experiment Station.)

\section{SOCIAL LIFE AMONG THE INSECTS}

The great majority of insects live an individual existence, without any cooperation or filial relationship existing between parents and offspring. The processes that have ever been operative have emphasized the importance of the individual in the scheme of prog- 
ress. Despite this, Wheeler, the great authority on insect societies, pointed out that at least twenty-four different times communism or societies have appeared in the class Arthropoda. He reports that social life occurs in six families of Coleoptera, fifteen families of Hymenoptera, and in the Dermaptera, Embiidina, and Isoptera. Let us look at some of the ways social life has manifested itself.

In the beetle family, Scarabaeidae, we find a number of species in which there is a cooperation between the male and female for the perpetuation of their offspring. A common species, Canthon simplex var. corvinus Harold, which the writer has ofttimes observed, rolls up small spheres of fresh cow manure, and then excavates beneath the roll, letting it gradually down into a hole in the ground. The male helps to dig and cover over the sphere of manure upon which the female has deposited an egg. The French naturalist and entomologist, J. H. Fabre, reported many interesting observations relating to the preparation of manure pellets for the deposition of eggs by several different scarabaeids.

Another beetle, Passalus cornutus, in the family Passalidae, lives in rotten logs. The developing larvae feed upon wood that has been prepared by the adult beetles. The colony is kept together by audible noises made by the mature beetles.

The ambrosia beetles of the families Scolytidae and Platypodidae form colonies by making their burrows into the wood of both living and dead trees. Each species of beetle grows a species of fungus which is fed to the developing larvae by the adult beetles.

The beetles are probably the least social of all the orders listed. No castes have been developed, and the males take but little part in colony life.

In the Hymenoptera are found varying stages of social life. In the solitary wasps, the female digs a burrow in the ground which is provisioned and then an egg is sealed in the cell. No other attention is given to the developing young and the new generation never knows the old.

The following excerpt from a study of the nesting habits of Odynerus dorsalis Fabr. made by Mr. Edwin Vest gives a good picture of the activities of this solitary wasp.

"Odynerus dorsalis is a solitary wasp in that each female builds a separate nest, yet there are often several nesting individuals in the same vicinity forming a kind of community. The labor of dig- 
ging the hole for the nest and gathering the provisions is apparently done entirely by the female. At no time was the male seen to engage in any part of this work. After the nesting is begun the females spend the night in the burrows with the head uppermost, while the males roost upon nearby herbs or shrubs.

"Their attempts at copulation are very amusing as well as interesting. Beginning about one or two o'clock in the afternoon the males become very active. They fly rapidly back and forth over the community usually from six to eight inches above the ground. They often alight on a female as she is working about the nest or returning to the nest with food and knock her to the ground. One female was resting on the ground when a male flew down and alighted on her back as if attempting to copulate; another male attacked with such vigor that the female flew away with still another male in pursuit.

"The ground where the nests are made is hard, dry, and composed principally of clay. In order to penetrate it the female fills a thin pouchlike sac, located within the second segment of the abdomen, with water and uses this to moisten the ground. With her mandibles she digs the dirt out in small pellets, varying in size from $2 \mathrm{~mm}$. in diameter to $6.8 \mathrm{~mm}$. These pellets are carried a short distance away from the hole. This work is continued until the hole is as deep as desired, the depth varying from 48 to $110 \mathrm{~mm}$. There are usually one or two, rarely three, cells constructed in the tunnel for the deposition of eggs. The bottom of the hole is enlarged slightly into a cell and is made very smooth on the inside. The cell might be lined with a secretion from the body which forms a cementlike protection to the larva during the winter. The average size of the cells is 23 by $14 \mathrm{~mm}$. In general they are ovoid-elliptical in shape.

"Each cell is provisioned with from five to twelve Pieridae larvae. The wasp carries these larvae by grasping them with her mandibles just back of the head and supporting them somewhat with her two front legs. Desiring to learn how Odynerus handled the larvae before putting them in the nest, the writer attempted to induce several wasps to pick up worms that were dropped on the ground about the nests. Favorable results were obtained in two cases. When the wasp found the worm she applied her mandibles to various places on the body but spent most of her time biting just back of the head as if 
trying to cut it off. This is probably a part at least of the process of paralyzing the victim. These paralyzed Pieridae larvae have been kept in the laboratory in bottles for two weeks in warm weather before there began to be any change in their appearance. After that time they began to decompose rapidly.

"After the cell is provisioned with the Pieridae larvae the female attaches the egg to the upper part of the cell by a short hairlike process $1.8 \mathrm{~mm}$. in length with the point of attachment to the cell wall concave and about $2 \mathrm{~mm}$. in diameter. Only one egg is deposited in each cell. The cell is then sealed over by wetting the soil at the surface and then carrying it down to be moulded into an apparently air-and water-tight compartment. In order to observe this process, the writer used a small pocket mirror to reflect the light down into the hole. This did not seem to interfere with the activity of the wasp.

"Most of the nests observed in this study consisted of two cells, with single-celled nests ranking second and three-celled nests third in frequency. The writer was not successful in hatching out all the individuals of any three-celled nest dug from the ground but those containing one or two cells were often hatched successfully. Of those individuals successfully reared in the laboratory it was found that in the case of the one-celled nests the individual invariably developed into a female, while with the two-celled nests the larva in the lower cell always developed into a female and the upper individual into a male. No successful observations were made on the three-celled nests. The facts of the case would seem to indicate that the male develops more rapidly than the female, since the egg in the lower cell is laid before that in the upper cell. It was noted that the wasp in the lower cell did not emerge until three days after the top cell had been vacated. The above condition applies primarily to two-celled nests, although it might be equally true of the three-celled types.

"It is evident from this study that the eggs laid in July and August hatch and remain in a late larval instar throughout the winter. On August 2 a number of larvae were collected and placed in glass vials. During the warm weather they were kept moistened by placing a few drops of water on blotting paper covering the cells. About the middle of September they were placed in a north room of the writer's home where they were left throughout the 
winter. Some of the larvae spun their cocoons in the vials while others had already done this before being removed from the ground. The room in which they were kept was cold, the temperature sometimes going slightly below the freezing point of water. About the last of May the specimens were removed to the Brigham Young University where they were kept on the writer's desk. The adults emerged fully developed about the middle of July. One female was kept in a breeding cage and fed on a syrup of cane sugar and distilled water.

"It is thought that under natural conditions the insects emerge somewhat earlier in the summer than was indicated by the artificially reared specimens since they have been observed to be very active even during the early part of May. It seems evident that these early wasps build their nests in the spring and that their young emerge during the same season. Only the individuals nesting in the late summer spend the winter in the larval stage."

The social wasps, belonging in the genera Vespula, Polistes, and Polybia, of the family Vespidae, start new colonies each spring from overwintering queens. After the nests are built and the eggs begin hatching, the queen feeds the larvae until they are completely developed. These workers then come to the aid of the exhausted founder of the colony by taking over the enlarging of the nest and the feeding of the larvae and the queen. The queen's only duty now is to lay eggs. It will be noted that the Vespidae attend their young by gathering food and feeding them; also that in turn the adults may feed upon the saliva of the larvae. Wheeler believes that the exchange of food in many of the social insects, which he chooses to call "trophallaxis," has been the source of the social habit.

In the family Bremidae, the bumblebees also start a colony in the spring by overwintering queens seeking out an unoccupied mouse hole or some other suitable hole in the ground. The queen gathers pollen and nectar with which she fills a few cells. She then deposits an egg in each cell and waits for them to hatch and develop into workers. The workers assist in building and feeding the colony. When the winter comes on, the queen, workers, and males die, leaving only the females, which developed late in the summer and which hibernate, to carry on the life cycle. All this is very similar to the life habits of the social wasps. 
In the honey bees, ants, and termites, social life is carried to its highest state of perfection. In these groups the colony is probably perpetuated for hundreds of years. Some ant and termite queens live from ten to fifteen years, building up large colonies consisting of fifty to eighty thousand individuals. Other queens take up the job of continuing the colony.

A well-developed caste system, also polymorphism, is found in these social insects. In a swarm of bees there are three kinds of individuals, males, females, and workers. The workers are females that are undeveloped sexually. Ants and termites have many different forms of individuals in each species. In a termite colony there are many castes. The principal kinds are perfect males and females, or the royal stock, the fecund pair of the colony; a less fully developed sexual caste, with rudimentary wings; a worker caste, of fairly small, sterile, wingless individuals; a soldier caste, morphologically distinct from other individuals because of their large heads and strong jaws; and finally a caste known as nasuti, which are small individuals with the head produced into a kind of snout. Both males and females are found in the various castes of termites. There is also an interesting symbolic relationship existing between numerous intestinal protozoa and the termites. The wood eaten by the termites is made soluble by the infusoria found in their digestive tracts.

Ants are world-wide in their distribution; they are also very numerous as individuals and species, since about four thousand species are known today. Wheeler believed that ants are the most highly developed as well as the dominant group of social insects. The Formicidae have a highly developed caste system and usually the workers and at times the males and queens are polymorphic.

\section{Guests}

There are many species of insects that live in the nests of the social insects; these guests are called myrmecophiles when found with ants, and termitophiles when with the termites. Wheeler reports that fully two thousand species of myrmecophiles and one thousand termitophiles have been described. Many of the guests have become so dependent upon living with ants or termites that they are never found outside of the colonies. Aphids and mealy 
bugs are kept as guests for the droplets of honeydew which they excrete when stroked by the antennae of the symbiont. Dr. S. A. Forbes has reported most intercstingly upon the activities of the cornroot aphid, Aphis maidi-radicis Forbes and the brown ant, Lasius niger var. americanus Emery. The little ants gather the aphid eggs in October and take care of them during the winter. In the spring before the corn commences to grow, the aphids, after hatching, are placed upon the roots of smartweed and some of the grasses. As soon as the corn has started to grow the agamic female aphids are transferred onto the roots. Here many generations are produced parthenogenetically. Then in later September or October wingless males and females are produced. After mating, eggs are laid, which are gathered and stored for the winter by the ants. The ants are repaid for the care they bestow on the aphids by receiving a honeydew given off by the aphids, which they greedily feed upon.

Many of the insect guests are beetles, Histeridae, Staphylinidae, Pselaphidae, and Scarabaeidae. The two histerids, Hetaerius tristriatus Horn and $H$. zelus Fall are fairly common in ant nests in the states west of the Rocky Mountains. Several species of Xenodusa, members of the family Staphylinidae, are found in ant hills in the United States and Mexico. A number of species of Batrisodes and Reichenbachia, pselaphids, and Cremastocheilus angularis LeC. and $C$. Knochi LeC., scarabaeids, are found in the colonies of several of the mound ants. Some Diptera are also guests in ant colonies.

\section{ECONOMIC RELATIONS}

Insects attack all kinds of growing crops and plants. The destruction of plants and their products valuable to man amounts to over a billion dollars annually. This great loss goes on because of the unabated and persistent struggle of the insects to maintain their "place in the sun." Plants are not only eaten and damaged by insects, but many plant diseases are spread by them.

Animals and man suffer greatly from the attacks of insects. Many species live as endoparasites or ectoparasites on animals and man, and in so doing also spread disease. Some of the most dreaded diseases known to man are carried by insects. Because of this there has recently developed a new branch of entomology known as "medical entomology." Some of the most notable progress during 
the past thirty or forty years has been made in the field of medical entomology. Diseases such as malaria, yellow fever, typhus fever, African sleeping sickness, bubonic plague, Rocky Mountain spotted fever, tularemia, and elephantiasis are now known to be insect borne. Much remains to be done in this new entomological field.

After man has produced his crops and harvested them for use, he finds many insects ready to take their toll from these concentrated products. The "board bill" of the insect pests of stored foods annually amounts to about four times the cost of all higher institutions of learning in this country. Insects belonging to the orders Coleoptera and Lepidoptera are the main offenders. The pea weevil, bean weevil, granary weevil, and confused flour beetle feed upon and damage practically all kinds of grains and seeds and their products. Much damage is also done to the same products by such species as the Mediterranean flour moth and the Indian meal moth. Practically all pests of stored foods are world-wide in their distribution, which makes it difficult to ship food products long distances or store them for future use without running the hazard of insect damage.

Many insects have taken up their abode with man, living upon his upholstered furniture, clothing, furs, and rugs. Great losses are suffered annually by the producers of clothing, as well as in the homes, due to clothes moths. Termites also attack the wooden parts of dwellings, even furniture and books. The tobacco beetles and drugstore beetles live upon tobacco products, home furniture, and many drugs.

\section{Useful Insects}

Fortunately not all insects are our enemies. Many species are allies of man in the struggle against the injurious insects, as well as in many other ways.

Everyone knows that honey is produced by the honey bee and silk by the silk moth, but there are many people who do not know that certain insects produce shellac, the pigment cochineal, tannic acid, formic acid, cantharidin or "Spanish fly," inks and dyes, and beeswax. In India a small scale insect, Tachardia lacca Kerr, lives on trees and produces a secretion that forms a layer over the branches. This substance, shellac, is removed by the natives in 
various ways, millions of pounds being sold throughout the world. Shellac is used for making varnishes and polishes, as an electrical insulating material, in airplane construction, and many other ways.

Insects serve as food for many fishes, amphibians, reptiles, birds, and mammals, including man. It is important that insects be recognized as playing a major role in this connection. Without the insects the food habits of many of the vertebrates would be entirely changed.

Finally, many plants depend upon insects to assist in pollenizing the blossoms. Only as the insect helps in transferring the pollen from plant to plant or from the stamens to the pistil of the same plant is it possible for some fruits, seeds, vegetables, and ornamental plants to develop. 


\section{CHAPTER XXII}

\section{REPRESENTATIVE INSECTS \\ (By Vasco M. Tanner, Brighani Young University)}

\section{THE LOCUST}

The locust or grasshopper is one of the most common insects, being known to practically all people, because very few boys and girls grow up without having some experience with a grasshopper. They are widely distributed throughout the world, living on grass and low-growing plants of the fields and open country. In the United States many destructive species are found. As early as 1743 Mr. Smith reported the damaging activities of Melanoplus atlanis in the New England states, and from 1855 to 1877 many outbreaks of grasshoppers were reported in the western United States. Even today the national government is expending large sums annually to keep down the activities of the many destructive species.

The grasshopper is a typical insect, and along with the beetles and bees, to be discussed later in this chapter, may serve to illustrate the general structure of the class Insecta.

The insect body is divided into a series of rings, or segments, and the segments are made up of hardened plates. These plates are known as sclerites, and the depression between the plates is called a suture. The hardness of the plates is due to the deposition of a horny substance called chitin. In many places two or more of these rings have grown together, or are fused. Again, in certain regions of the body, parts of the segments may be lost. Regardless of the amount of variation in this respect, we find that the segments are always grouped into three regions, known as the head, thorax, and abdomen.

The head is made up of a number of segments, which are fused together, forming a boxlike structure known as the epicranium. This boxlike piece which surrounds the eyes and forms the basis of attachment for the movable parts of the head extends down the front of the head, between the eyes, to the transverse suture, and down the sides of the liead to the base of the mouth parts. The sides of the epicranium below the compound eyes are known as the genae, or cheeks, while the front of the head between the eyes is called the frons. 


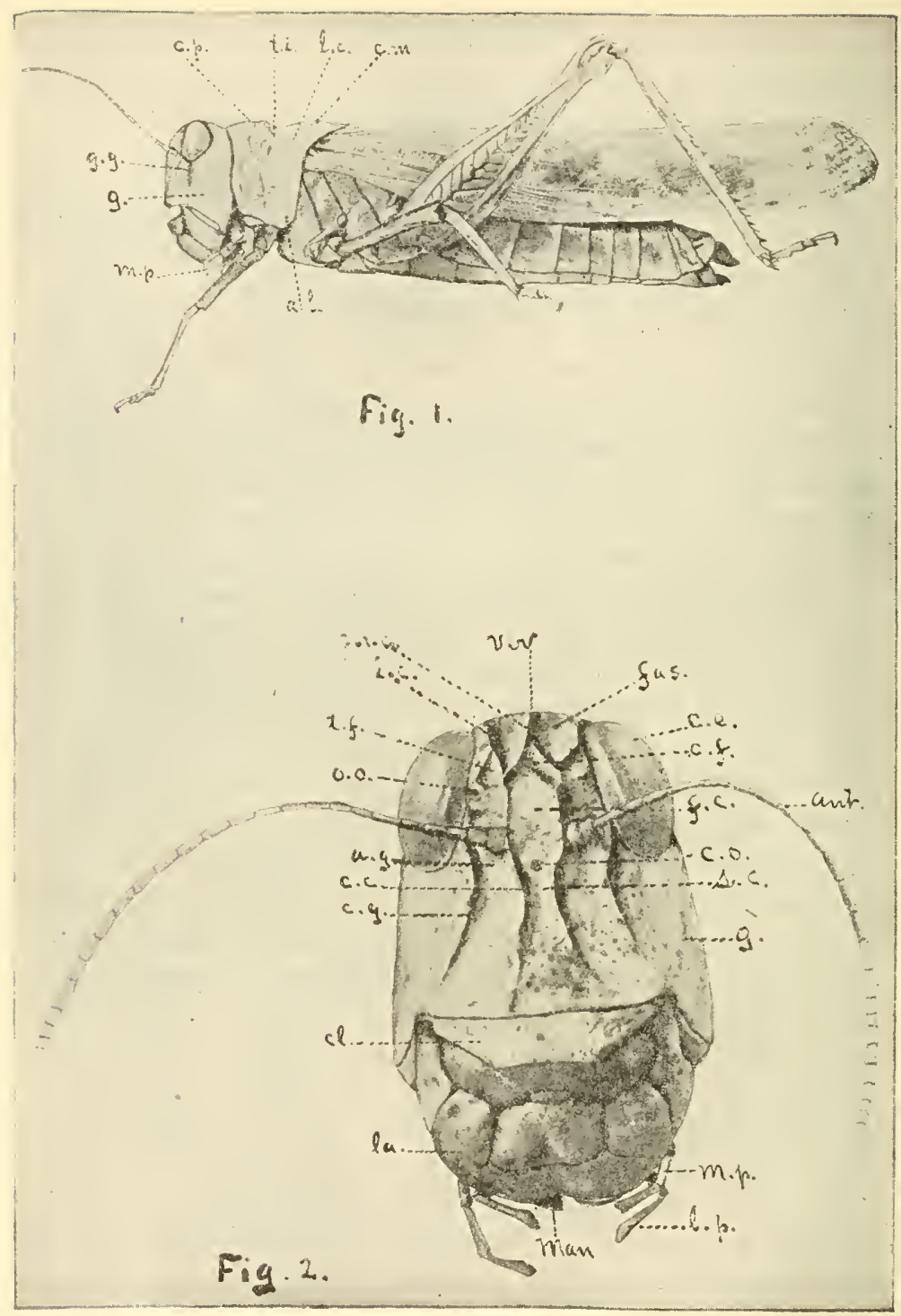

Fig. 200.-1, The external structure of the grasshopper, Dissosteira spurcata. $a l$., Hind angle of lateral lobe; c.m., crest of the metazone; c.p., crest of the prozone; g., gena; g.g., genal groove; l.c., lateral carina of the metazone; m.p., maxillary palpus; $t . i .$, transverse incision. '2, Front view of the head of the grasshopper, Dissosteira spurcata, a.g., Antennal groove; ant., antenna; c.c., lateral carina; c.e., compound eye; c.f., central foveola; c.g., carina of the antennal groove : cl., clypeus; c.o., central ocellus; fas., fastigium of the vertex; f.c., frontal costa: g., gena; la., labrum; l.c., lateral carina of the fastigium; l.p., labial palpus ; man., mandible; m.c., median carina of the fastigium; m.p., maxillary palpus : o.c., ocellus; s.c., sulcation of the frontal costa; $t . f$., tempora, temporal foveola : ver., vertex. (From Henderson, by permission of the Utah Agricultural Exper1ment Station.) 
The grasshopper has both compound and simple eyes. The compound eyes are situated upon the upper portion of the sides of the head, and are large, oval areas with smooth, highly polished surfaces. If the eye is examined with a dissecting microscope, the surface will be seen to be made up of a number of hexagonal areas, which are known as facets. The simple eyes or ocelli consist of three small, almost transparent, oval areas. One of the ocelli is situated on the front of the head, just below the margin of the impression which contains the bases of the antennae, and in contact with the upper portion of the compound eye.

The antennae or feelers are two threadlike processes situated median to the compound eyes. Each consists of about twenty-six segments. On the front of the head there is a short rectangular piece, called the clypeus, which is attached by its upper edge to the epicranium, and on the lower edge to the labrum.

The mouth parts consist of a number of separate parts attached to the ventral region of the epicranium. The first noticeable part is the labrum, or upper lip, a flaplike piece attached to the lower edge of the clypeus. The free edge is deeply notclied on the median line. Just beneath the labrum are the mandibles, or first pair of jaws. Each mandible consists of a single piece which is notched on the inner grinding surface to form a number of ridges or teeth. A second pair of jaws, the maxillae, may be exposed by the removal of the mandibles. Each maxilla is composed of a number of parts, consisting of the cardo or proximal hinge part of the structure; the stipes, the lacinia, a sclerite which bears some teeth on its terminal end; the outer lobe or galea; and the maxillary palpus. The caudal part of the mouth parts is the lower lip or labium, which is composed of the submentum which acts as a hinge on the epicranium above; a mentum; labial palpi, and two large outer flaps, the ligulae (Fig. 200).

The prothorax is the segment to which the head is attached. It may be divided into two regions, the dorsal part known as the pronotum and the ventral portion known as the sternum. The pronotum is a saddle or bonnetlike piece extending over the dorsal and lateral regions of the prothorax. It is made up of a fusion of four plates, which are indicated by the transverse sutures. The sternum or ventral side of the pronotum is also made up of separate plates, or sclerites. The anterior sclerite bears a spine on the median line. 

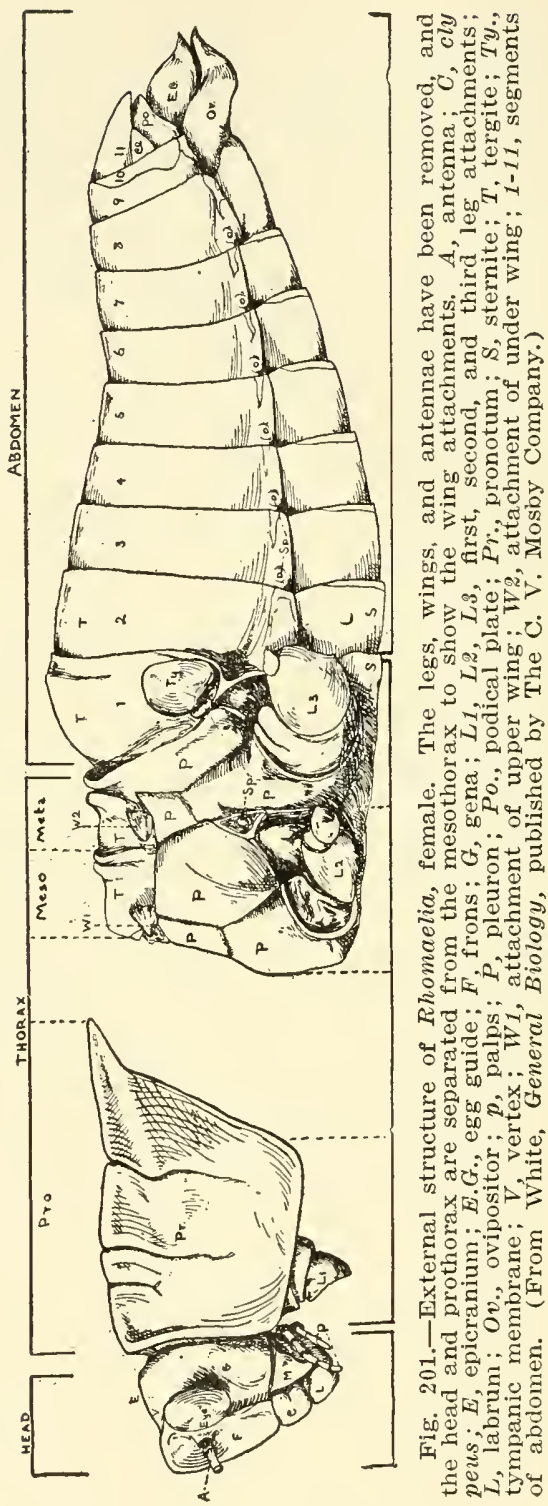
The next two segments, the mesothorax and metathorax, are made up of sclerites that are intimately associated, and their structure will be discussed together. The mesothorax is joined to the prothorax by a membrane which permits of more or less movement. Posteriorly the metathorax is joined immovably with the first abdominal segment. The mesothorax and metathorax form a strong, boxlike structure for the support of the wing and leg muscles. Like the prothorax these segments are made up of separate plates, held together by a tough, connecting membrane. These plates may, however, be divided into three groups: the tergum, or dorsal region; the sternum, or ventral region; and the pleuron, or lateral region. On the dorsal and ventral regions of the body the sutures separating the mesothorax from the metathorax are not very distinct. On the sides of the body, however, there is a very distinct line, or suture, running from the posterior border of the attachment of the second pair of legs toward the dorsal part of the body. This suture divides the mesothorax from the metathorax. The pleura of each of the posterior thoracic segments are again divided by transverse sutures, so that each pleuron consists of two selerites.

A pair of legs arises from the lateral and ventral portions of each of the segments of the thorax. Each leg is composed of five parts. The coxa is the first segment and is attached to the thorax by a tough elastic membrane. The next segment, the trochanter, is a very short piece which is hard to distinguish except in the first pair of legs. The femur is the third and largest segment of the leg, and in the case of the metathoracic leg contains the muscles used in jumping. The fourth segment, the tibia, is slender, but about the same length as the femur. The last division of the leg is the tarsus which is made up of three segments, each movable with the other. The segments bear a series of pads, which terminate on the last one in a large suckerlike dise known as the pulvillus.

There are two pairs of wings. The first pair or wing covers, also called tegmina, is attached to the dorsal region of the mesothorax. They are leathery in texture and do not fold fanlike over the abdomen. They are strengthened by many veins and eross veins. The second pair of wings is attached to the metathorax. They are membranous, with many veins to strengthen them, and fold fanlike over the abdomen when not in use. The metathoracic wings are used in flight. 
The last main division of the insect body is the abdomen. It is composed of eleven segments. The seven anterior segments are similar in both the male and female. In the male the first abdominal segment is made up of a curved dorsal shield, the tergum, which terminates just above the attachment of the third pair of legs. This piece partially surrounds the tympanic membrane, or ear, which is a large, crescent-shaped area covered with a semitransparent membrane. The ventral part of the first segment, the sternum, is not attached to the tergum, owing to the large size of the attachment of the legs. The pleura are entirely absent. The second to the eighth segments are all quite similar, consisting of a dorsal tergum, which extends laterally to near the ventral part of the body, where it joins the sternum. The pleura, or side pieces, noted in connection with the thorax, have been inseparably fused to the tergum. In the ninth and tenth segments the terga are partially fused together, the union of the two being indicated by the presence of a transverse suture. The sterna of these two segments are entirely fused and much modified, forming a broad, platelike piece. The eleventh segment is represented only by the tergum, which forms the terminal, dorsal, shield-shaped piece (Fig. 201).

The cerci constitute a pair of plates attached to the lateral posterior border of the tenth segment, and extending back, past the end of the eleventh tergum. The podical plates lie directly beneath the cerci and ventral to the eleventh tergum. 'The anus opens between these plates, and the genital chamber lies directly below them. Attached to the ninth sternum is the subgenital plate which forms the most posterior ventral plate of the body.

In the female the eighth segment rescmbles the other segments, except that the sternum is nearly twice as long, and known as the subgenital plate. The ninth, tenth, and eleventh segments are essentially like those of the male, the terga of segments nine and ten being partially fused, and tergum eleven forming the terminal, dorsal shield. The plates called cerci and podical plates are similar to those in the male, except that the podical plates are much more prominent.

The ovipositor consists of three pairs of movable plates. The dorsal pair lies just ventral to the eleventh tergum and each plate is long, lance-shaped, and with a hard, pointed tip. The ventral pair arises just dorsal to the eighth sternum and resembles the dorsal 
pair. When these four pieces are brought together, their points are in contact, forming a sharp organ by means of which the female bores the holes in the ground in which to deposit her eggs. The third set of plates are known as the egg guides. These are much smaller and are located median to the plates of the true ovipositor.

There are ten pairs of spiracles, or openings in the respiratory system on the body of the grasshopper. Two pairs of these liplike structures are situated on each side of the thorax on the anterior margin of the pleural plates. The mesothoracic spiracle is concealed by the posterior edge of the pronotum. The metathoracic spiracle is located just dorsal to the mesothoracic leg, near the suture separating the two segments. There is another spiracle just dorsal to the attachment of the metathoracic leg, but this belongs to the first abdominal segment. From the second to the eighth abdominal segments there is one pair of spiracles located on the anterior margin of each segment near the union of the sternum and tergum. The spiracles are one of the most useful sets of structures for determining the segmentation of an adult insect body. This is because there are never more than eight pairs of abdominal spiracles present in any fully developed insect. Air passes through the spiracles into the tracheae and is carried to the tissues of the body. This unique system of breathing enables the insect to keep the body tissues well aerated and the carbon dioxide eliminated from the body.

The circulatory system consists of a single dorsal tube, or heart, which extends along the length of the median dorsal part of the body. In the abdomen of the fully developed insect this vessel is divided into a number of chambers with side valves, which allows the blood to enter but not to escapc, except through the vessel toward the head. Due to the pulsating of this portion of the tube, which has been called the heart, the blood is forced to the anterior part of the body where it flows out into the body cavity and slowly returns to the abdominal region. In this process the tissues are supplied with nourishment from the food materials carried in the blood. It will be noted that the circulatory system has practically nothing to do with the carrying of oxygen to the tissues.

The digestive system of the grasshopper consists of a practically straight tube extending from the mouth to the anus through the 
central portion of the body. The food after being ground up by the mouth parts passes into the mouth or pharynx where it is mixed with the salivary mucin and the action of the enzyme, invertase, begins. From the mouth the food is conveyed through the esopha-

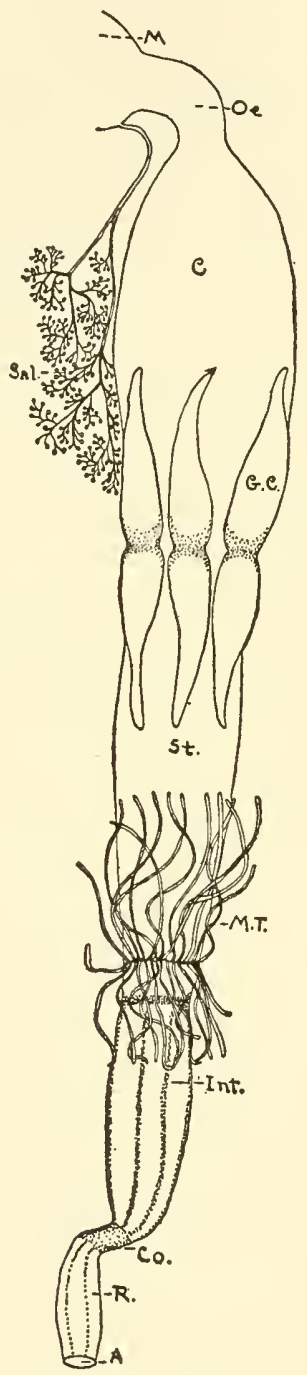

Fig. 202.-Digestive system of Rhomaelia microptera. $A$, anus; $C$, crop; Co., colon; G.C., gastric caeca ; Int., intestine; $M$, mouth ; $M . T$. Malpighian tubules; Oe., esophagus; $R$, rectum; Sal., salivary glands. (From White, General Biology. The C. V. Mosby Co.) 
gus to the crop and gizzard which are dilatations of the tract filling a great portion of the thorax. The gizzard is muscular and lined with chitinous ridges which strain the coarse particles of food and prevent their entering the next division of the system, the stomach.

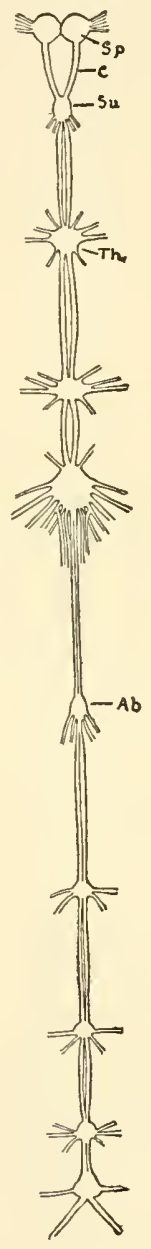

Fig. 203.-Nervous system of Rhomaelia microptera. Ab., first abdominal ganglion: $C$, circumesophageal commissure; Sp., supraesophageal ganglion; $S u$., subesophageal ganglion. (From White, General Biology. The C. V. Mosby Co.)

The food is acted upon in the stomach by the secretions of the gastric caeca, which are glandular bodies opening into the anterior end of the stomach. They secrete a weak acid which helps in the 
emulsification of fats and the conversion of albuminoids into peptones. Much of the food is absorbed into the hemolymph from the stomach. Between the stomach and the intestines is a pyloric valve which permits the contents of the system to pass in only one direction. In the intestine, which is divided into the ileum, colon, and rectum, absorption of food continues, especially in the ileum. Just back of the stomach many threadlike tubes enter the intestine. These tubes are the excretory organs, known as Malpighian tubules, and perform a similar function to the kidneys of higher animals. The rectum has thick muscular walls with six-surface rectal glands. The feces are expelled from the rectum to the outside of the body through the anus.

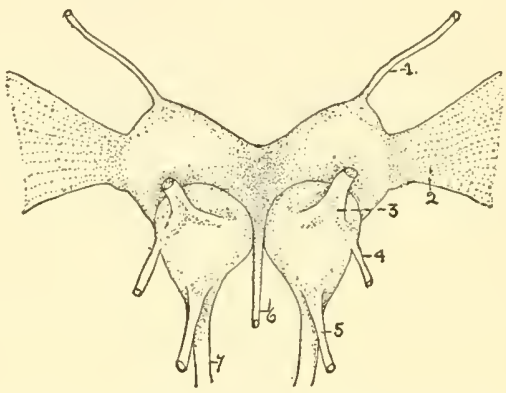

Fig. 204.-Anterior aspect of brain (supraesophageal ganglia) of Rhomaelia microptera. (Magnified.) 1, nerve to paired ocellus: 2, nerve to eye, showing fibers to ommatidia; $s$, nerve to antenna; 4 and 5 , nerves to mouth parts; 6 , nerve to unpaired ocellus; $\%$, circumesophageal commissure. (From White, General Biology. The C. V. Mosby Co.)

The nervous system consists of a series of ganglia or nerve cells connected by a double set of commissures or connecting nerve fibers lying along the ventral body wall. Five ganglia are located in the abdomen. Since there are at least eleven segments in the abdomen of the adult grasshopper, it is apparent that the ganglia of some of the segments have fused together. In the larvae of insects there is usually a ganglion to each segment. Three large, well-developed ganglia are found in the thorax; the anterior one is connected with the subesophageal ganglia which in turn are connected with the brain or supraesophageal ganglia by nerve fibers which pass on each side of the esophagus. Nerves pass from the brain to the eyes, antennae, and palpi of the head. The subesophageal ganglia supply the mouth parts with nerves. The legs and wings are coordinated in their movements by the thoracic ganglia. In the vertebrates the 
nervous system is dorsal to the digestive tract, and the foreshadowing of this evolutionary change is initiated in the insects by the development in the cephalic region (Figs. 203 and 204).

The grasshopper is dioecious; the abdominal structures separating the two sexes are distinctive. The external genital structures have been discussed above. The male organs consist of testes located dorsal to the intestines. The sperms are borne in ducts which communicate with the penis, which consists of chitinous styles used in copulation with the female. In the female there are two ovaries,

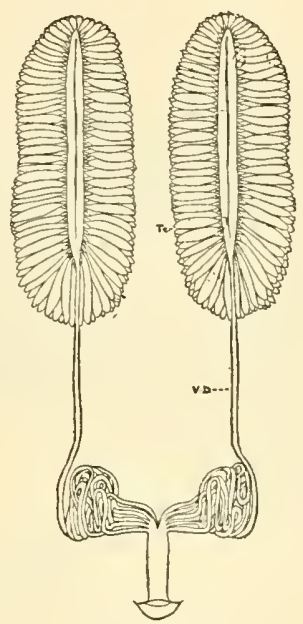

Fig. 205.

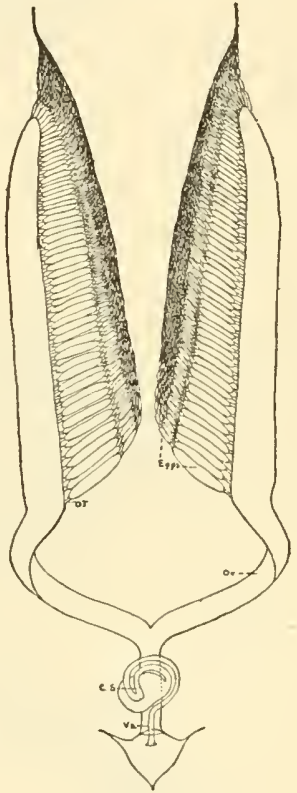

Fig. 206.

Fig. 205.-Male reproductive organs of Rhomaelia microptera. Te., testes; V.D., vas deferens. (From White, General Biology.)

Fig. 206.-Female reproductive organs of Rhomaelia microptera. C.S., copulatory sac; O.T. ovarian tube with eggs; Ov., oviduct; Va., vagina. (From White, General Biology. The C. V. Mosby Co.)

which when mature fill the major portion of the abdomen. The oviducts convey the eggs to the vagina, a duct made by the union of the two oviducts, which discharges the eggs through the opening at the base of the egg guide to the outside of the body. The eggs are fertilized by the sperms from the spermatheca, which is dorsal to the vagina and which is comnected by means of a sperm duct. The female is able to dig a hole in the ground with the ovipositor 
and deposit the eggs to the depth of an inch or more. The eggs are covered with a frothy substance which protects them from moisture and, to some extent, from the frost. The eggs are laid in the fall and hatch in the spring of the year. The development of the grasshopper is by gradual metamorphosis.

\section{THE JUNE BUG}

The June bugs or May beetles are members of the family Scarabaeidae, a very large and important family of beetles. More than one hundred and twenty-five species of these beetles have been reported as occurring in the United States and Canada, the majority of them being considered as pests. The larvae or white grubs live underground, destroying the roots of grain, cereal, truck, and garden crops, as well as great tracts of pasture and grasslands. The adults live upon the leaves of many kinds of trees and shrubs, often completely defoliating the trees. Because of the general distribution of these beetles, they have been selected as a type to illustrate the characteristics of Coleoptera, the largest order of arthropods.

An examination of a specimen of the genus Phyllophaga reveals that there are three body regions: the head, thorax, and abdomen. The rather small, retracted head bears antennae of nine or ten joints and a club composed of three elongate leaflike joints. The antennae are located just beneath the lateral edge of the prominent clypeus. The compound eyes are on the sides of the head near the prothorax. There are no ocelli. The mouth parts are of the biting type, similar to those of the grasshopper.

The thorax consists of three segments. The metathorax is fused with the first abdominal segment and with the mesothorax, leaving the prothorax free and movable. Attached to the dorsal portion of the mesothorax are the fore wings that are modified into horny sheaths, or elytra, which cover and protect the back of the thorax and abdomen. The hind wings are membranous and folded under the elytra. The legs are well developed, the prothoracic ones being adapted for digging in the ground. The thorax is provided with yellow setae.

The abdomen, which is broadly fused with the metathorax, consists of eight external segments. When the elytra are removed, the spiracles may be seen in the lateral margins of the dorsal surface of the abdomen. The genital organs of both sexes are simple. 


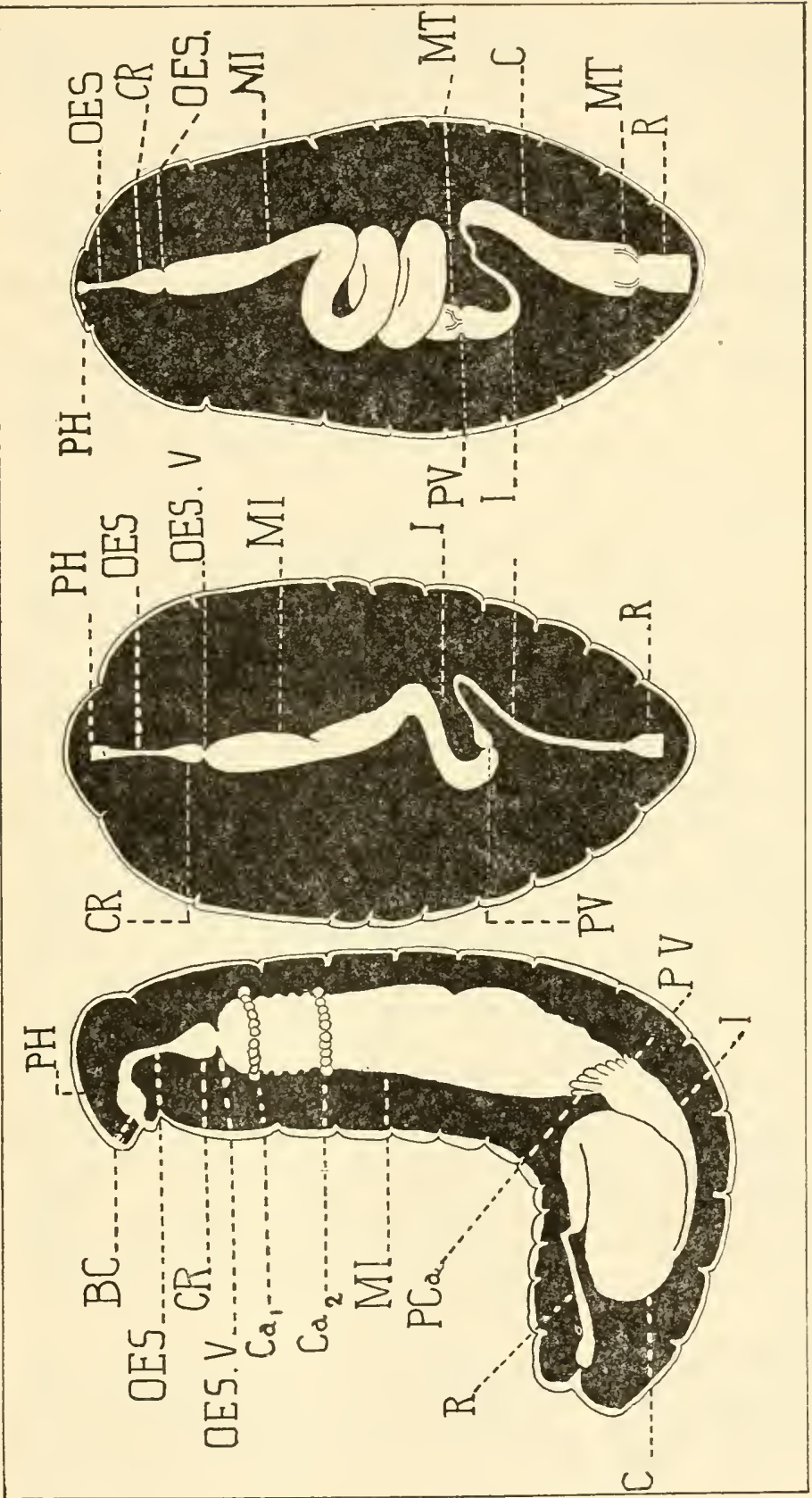

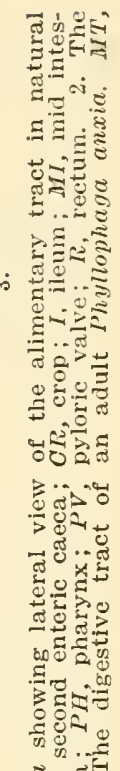

बूँ

क $2 \ldots 0$

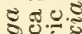

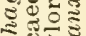

ลำ

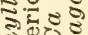

更过

पै

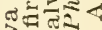

สี تีँ

$0 . .+\infty$

इ

낭ㅎㅇ읭

+0 पु

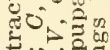

$\pm . .-5$

D.

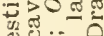

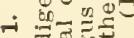

势

든

10 눙

:

N 되

เरํㅇ을

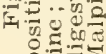


By carefully removing the membranous tergites of the abdomen the heart can be seen to consist of a thin-walled dorsal vessel with paired lateral openings into the body cavity. The blood is forced forward through the heart chambers by the pulsations of the heart walls. There are no arteries and veins, which means that the heart serves mainly as an agitator of the body fluids, helping to distribute the absorbed food to the tissues.

The tracheal system is well developed for carrying the air from the spiracles to all parts of the body.

There are many changes in the digestive system of the June bug as it passes from the larval stages to the imago. The alimentary tract of the larva consists of a straight tube, except for a bend in the colon. It is much greater in diameter than in the later stages due to the nature of the food, which consists of roots, humus, and some soil. The food passes from the mouth or buccal cavity into the esophagus and then into the crop. At this point there is a valve between the crop or gizzard and the mid-intestines. Two rows of gastric caeca are present on the anterior end of the midintestines. This is a very unique feature, as it is rarely met with in larval stages of other insects. The large saclike stomach or mid-intestine of the larva is transformed into an elongated coiled stomach in the adult, without the two rows of gastric caeca. At the posterior end of the midintestine and in front of the pyloric valve are ten pairs of pyloric caeca. The hind intestine consists of the ileum, colon, and rectum. There are four Malpighian tubules connected to the hind intestine. In the pupal stage the gastric caeca have disappeared, and the tract is becoming much elongated and coiled. In the adult the excretory organs, the Malpighian tubules, arise in the ileum just posterior to the pyloric valve. They extend into the body and then end blindly at the junction of the colon and rectum.

The nervous system consists of a ventral nerve chain, a brain, or supra-esophageal ganglion, a nerve ring which connects the brain and the foremost or infra-esophageal ganglion. There are eight ganglia in the ventral nerve chain, four in the thorax and four in the abdomen.

The life histories of the June bugs vary in length from three to four years, depending upon a number of ecological factors. The adult females dig into the ground and deposit from a hundred to two hundred eggs. The larvae are commonly known as "white 
grubs." The adults come forth in great numbers in May or June and live from one to two weeks, feeding upon the foliage of many plants.

\section{THE HONEY BEE}

The honey bee belongs to the order Hymenoptera, composed of insects with two pair of membranous wings, well-developed biting or sucking mouth parts, and the females usually with a stinging organ. Many of the Hymenoptera, such as the honey bee, live a social life, developing colonies consisting of three types of bees: a queen, drones, and workers.

The worker bee is provided with large compound eyes on the sides of the head and three small ocelli near the median part of the frons. The antennae are attached to the anterior surface of the head.

The mouth parts are adapted for both sucking up nectar and chewing. The labrum is attached to the lower edge of the clypeus. A little organ, the epipharynx, is just below the upper lip. The mandibles are attached to the ends of the labrum and lie over it. Beneath the mandibles is the proboscis made up of several separate structures: (1) the glossa or long tongue; (2) the labial palps; and (3) the maxillae, lateral to the labial palps. The maxillae and labial palps are used in sucking the nectar from the flowers.

The thorax is divided into the prothorax, mesothorax, and metathorax. Each segment bears a pair of legs. The wings are borne upon the mesothorax and metathorax. The legs are very well adapted for the work of the hive. The first pair of legs are provided with hairs adapted for various uses. On the tibia are the curved bristles, known as the pollen brush, and the large spinelike structure, the velum, which is associated with the antenna comb. The metathoracic legs have the tibia modified to form a pollen basket. There are also modified spines and structures on the last pair of legs known as the pecten, auricle, and pollen combs. The modifications found in the legs of the bee are remarkable adaptations for the specialized life of this insect.

The abdomen is composed of six external segments consisting of a dorsal tergum and a ventral sternum. At the end of the abdomen is a highly specialized organ, the sting. Associated with the sting are the poison glands, which secrete a substance composed of an acid and an alkali. 


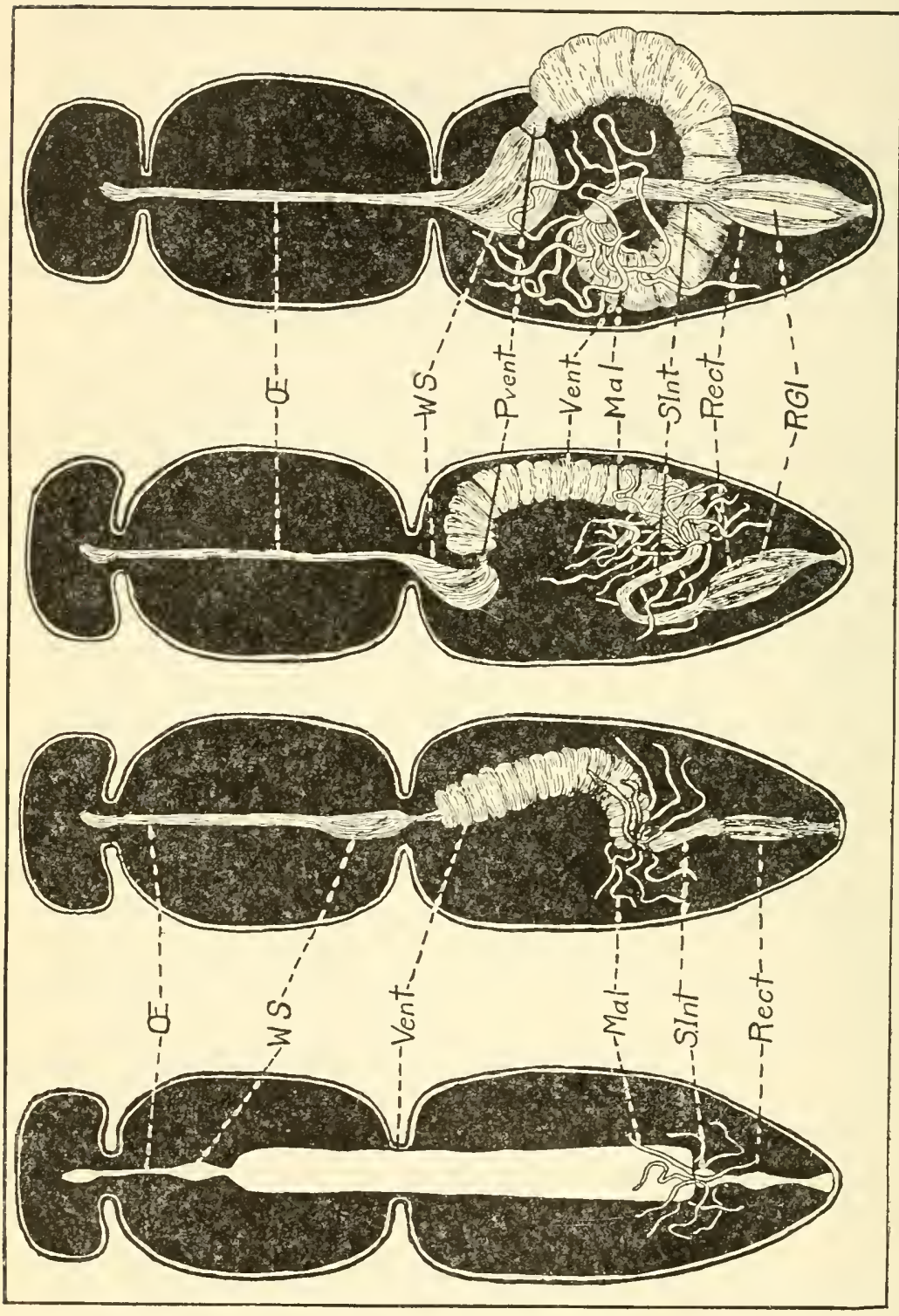

किए

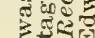

a

ฐิ

ơ

. $0 \$ \bar{E}$

A

ㄷำด

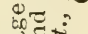

สูก สี

तथ

证。

突

Q 3 ह

당.

월 它

of 0

需.

○.

릎

$\div$ ए

เี

ㅁ․․․

0 던

$50:$

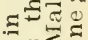

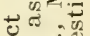

氙芯志

$\infty$

늘

ชู

تE

政

इ

ธี०

एٓ-

है

过

०.

०:

-

$-\infty$ ते चु

के

เค่

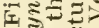

ठี० 
The digestive tract is well adapted for the specialized life of the bee, that of gathering and feeding upon the nectar of flowers. A study of the digestive system of the larva, pupa, and adult of the solitary wasp, Odynerus dorsalis, by Mr. Edwin Vest reveals that it is very similar to that of the honey bee, as well as many other Hymenoptera. In Odynerus or the honey bee the digestive tract may be divided into the fore intestines, mid-intestines, and hind intestines, as in the June bug. The divisions of fore intestines arc: the mouth or buccal cavity, esophagus; water sac or honey stomach; and the proventriculus. The mid-intestine consists of the stomach, while the hind intestine may be divided into the ileum, rectal glands, rectum, and anus. In the larval and early pupal stages the mid-intestine is a thin flat tube, but in the adult it has developed into a convoluted, looped stomach. The number of Malpighian tubules increases from the larval stage to the adult. Only four Malpighian tubules are found in the larva, while there are around one hundred in the adult. There is also a marked change in the length of the esophagus during metamorphosis. In the adult the esophagus extends from the buccal cavity through the thorax into the first abdominal segment where it enters the water sac, or in the honey bee, the honey stomach.

The body of the bee is well filled with tracheae, which are connected with two pairs of thoracic spiracles and eight pairs on the abdomen.

The nervous system is similar to that of the grasshopper. The brain is a ganglion in the head above the esophagus. It is connected by a nerve ring with the subesophageal ganglion, which is in the head but below the esophagus. The two ganglia of the head are connected with two in the thorax and four in the abdomen.

The queen bee when fully developed mates with a drone on the virgin flight. By means of the copulatory organ the male transfers a supply of sperms to the seminal receptacle of the queen. Just how the queen is able to regulate the laying of eggs that are fertilized by the sperms from the seminal receptacle or those that are not fertilized is not fully known. The fertilized eggs develop into workers and the unfertilized eggs into drones.

The life history of the bee, life in the hive, the gathering of nectar and its development into honey for table use, as well as swarming and the rearing of a queen, are fascinating subjects dealt with in the many books devoted exclusively to a study of the honey bee. 


\section{CHAPTER XXIII \\ PHYLUM CHORDATA}

Phylum Chordata (kôr dā' tá, cord) is made up of the group of animals which includes man himself and in general the more conspicuous, better known animals.

\section{Characteristics}

There is a rather wide range of variation as to form and size in the group. It includes minute sessile forms, small colonial forms, mud-burrowing forms, and on up to the largest and most complex of living animals. All individuals classified in the phylum possess three distinctive characteristics that are most conspicuous in certain primitive forms. The three features elearly distinguish the phylum from all others and bind together individuals which are widely separated in appearance but characterized by certain traits peculiar to this group alone. These three characteristics are: (1) notochord, a flexible rod extending from anterior to posterior in the longitudinal axis of the body, lying dorsal to the digestive tube and ventral to the nerve cord; (2) pharyngeal clefts or gills, a series of paired slits in the wall of the pharynx and in the body wall of some; (3) dorsally located tubular nerve cord, extending the length of the body dorsal to the notochord and other organs.

The notochord serves as a stiffening rod and is the foundation axis for the endoskeleton. It is present as such at some time during the life of every chordate animal. In the adult vertebrate it is replaced by the centra of the vertebrae. The gill clefts are present at some time in the life of all individuals placed in this phylum. Although the gills become modified to form other structures in the adult terrestrial chordates including man, they have had rather typical ones as embryos. The pharyngeal clefts or gills provide a more effective mode of respiration for aquatic animals than that used by most nonchordates because the gills are thus interposed directly in the course of the circulation, and the entire blood supply of the body passes through them. The central nervous system is derived from the ectoderm along the middorsal line of the embryo, first as a plate, then as a groove, and finally a tube which results in the spinal 
cord and brain. Iu higher forms the anterior end of the tube becomes expanded and modified to form the brain. The continuous tubular nerve cord is at the apex of the development of centralization in the nervous system, and allows for an increase in number of nerve cells, increased accessibility, and more intimate association of ganglionic masses to furnish better coordination. These are all advances in both structure and function when compared with other groups. The chordates possess segmentation (metamerism), but it is progressively obscure as one proceeds from simpler to more complex forms. There is a tendency toward fusion of metameres and shifting of superficial muscles. The internal skeleton of this group compared with the external one of others studied does not give as great a leverage for the muscles, but it greatly increases the mechanical freedom allowed and this is a distinct advantage as well as an advance in structure.

\section{Classification}

There are approximately 40,000 different species in this phylum which is divided into four established subphyla as follows:

Hemichorda (hĕm $\breve{i} k \hat{o} r^{\prime}$ dả, half cord) or sometimes known as Enteropneusta (ĕn tẽr ŏp nūs'tả) includes order Balanoglossida with its four families, ten genera and twenty-eight species, and order Cephalodiscida with its two genera Cephalodiscus and Rhabdopleura. These are all small wormlike animals.

Urochorda (ū ró kôr'dả, tail cord), or Tunicata (tū nĭ kā'tả) includes the tunicates, all of which are marine and mostly small. Adults show a high degree of degeneration so it is the larvae only that exhibit distinctive characteristics of the phylum. There are three classes: (1) Larvacea, so named because it retains the larva form throughout life. Genus Appendicularia is an example. Ascidiacea, the sea squirt, either free-swimming or sessile, simple or colonial, may reproduce sexually or by budding. Molgula, Cynthia, and Ascidia are common examples. (3) Thaliacea, free-swimming, pelagic, solitary or colonial forms, usually exhibit alternation of generation. Salpa and Doliolum are the most common examples.

Cephalochorda (sěf ả ló kôr'dà, head cord) includes approximately twenty-eight different species of marine, shore-loving, fishlike forms of which Amphioxus (Branchiostoma lanceolatus) is the most common representative. 
Vertebrata (vẽr tē brā'tá, jointed) animals with backbone-frog, man. These are the larger, more conspicuous animals and will be discussed at length in later sections of the book.

\section{Phylogenetic Advances of Chordata}

(1) Notochord and endoskeleton, (2) pectoral and pelvic girdles with limbs, (3) development of dorsally located nerve cord with anterior brain, (4) developinent of five senses, (5) pharyngeal gills and lungs for respiration, (6) voice production, (7) specialization and coordination of muscles.

\section{Protochordata (Lower Chordates)}

Until relatively recent years the two subphyla, Hemichorda and Urochorda were not classified as Chordata; the former was with Annelida and the latter was independent. With the exception of the value as biological specimens and the use of amphioxus as food by Chinese, this group is of no economic importance.

\section{SUBPHYLUM HEMICHORDATA}

One of the species of Balanoglossus or Dolichoglossus kowalevskii will serve as an example. They are wormlike animals which burrow into the mud and sand along the seashore. They range from 6 to 10 inches in length. Others of the subphylum may be as short as one inch or still other's as long as four feet. The three portions of the body are proboscis, a ringlike collar, and a segmented trunk. The proboscis, as well as the collar, is hollow and serves as a water chamber. The eavity of the proboseis is filled with water which is drawn in and expelled through a proboscis pore or vent located on its dorsal side and just anterior to the collar. Supporting the base of the proboscis is a short skeletal process which is stiff and extends anteriorly from the roof of the mouth region and assists in burrowing. This process, ealled the diverticulum, is usually referred to as the rudimentary notochord. However, it is very poorly developed and in a peculiar position. Nevertheless, it has the relationship to the digestive tube which is characteristic in the embryonic development of the notochord for certain higher chordates. The mouth opens on the ventral side just anterior to the collar and leads into the straight alimentary canal which extends to the posterior end of the body 
and ends in the anus. Like the earthworm, this animal utilizes the mud in which it lives for food, absorbing the organic matter from it as nutriment. Balanoglossus has numerous paired gill slits, located in the lateral walls of the anterior (supposedly pharyngeal) position of the digestive tube. In some of the other representatives the gills are much reduced in numbers or are lacking. Where gills are present, water is passed through them for respiratory purposes, oxygen being absorbed and carbon dioxide being discharged from the blood here. There is no differentiation of a distinct pharynx.

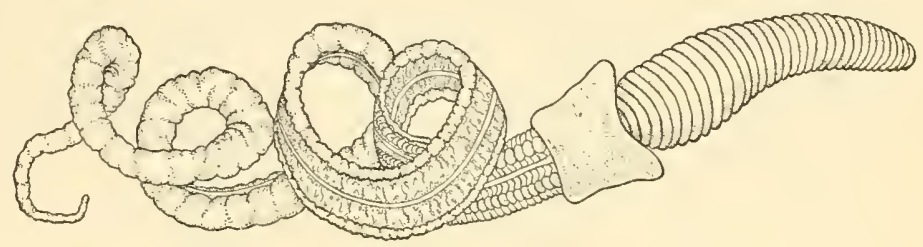
Fig. 209.-External features of Dolichoglossus kowalevskii. (Courtesy of

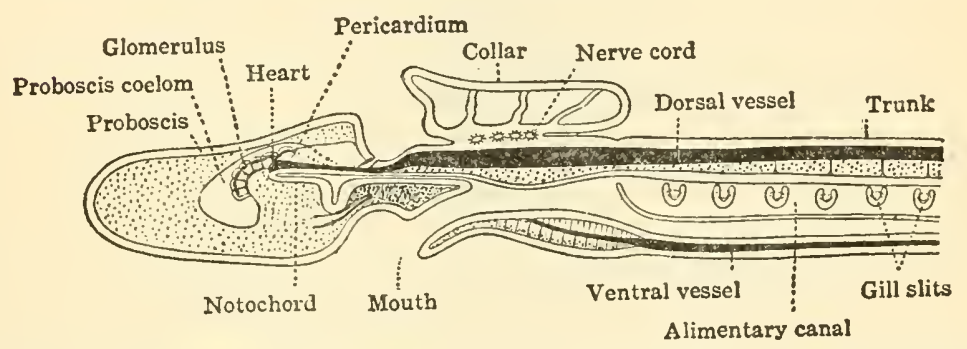

Fig. 210.-Diagram of a sagittal section through anterlor portion of Dolichoglossus. (From Hegner, College Zoology, published by The Macmillan Company. after MacBride.)

The circulatory system is rather rudimentary. It includes a sinuslike heart which is held in a pericardial sac located in the basal part of the proboscis. A dorsal vessel extends posteriorly from the heart to the posterior end of the trunk. At the collar it is joined by lateral connectives which encircle the body to connect with a ventral vessel extending posteriorly below the intestine. Sinuslike branches of these main vessels supply various parts of the body.

The nervous system is composed of a dorsal cord which is tubular in the region of the collar and extends the length of the trunk, a more or less concentrated center of nerve cells in the collar, and a 
ventral cord running longitudinally on the floor of the trunk. The ventral cord certainly is not a chordate characteristic, but the dominance and hollow structure of the anterior portion of the dorsal one, represent features which are homologous to the central nervous system of higher chordates.

Excretion seems to be accomplished by a mass of vascular tissue (glomerulus?) located in the proboscis just anterior to the heart. The excreted materials are received by the water in the proboscis cavity (coelom) and pass out the pore with the water as it is expelled. These animals are dioecious, with gonads in the form of a genital ridge extending lengthwise along each side of the anterior portion of the trunk. The mature germ cells escape through the body wall, are fertilized in the water, hatch out and become tornaria

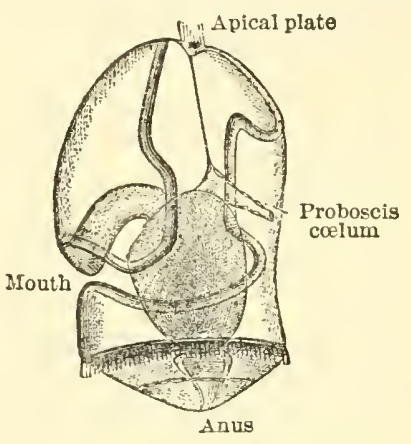

Fig. 211.-Tornaria larva of Hemichorda. (From Hegner, College Zoology, published by The Macmillan Company, after Metchnikoff.)

larvae, which are globular in shape and form a pattern of ciliated bands over the body. In this respect and in habit of life these larvae resemble the larvae of the echinoderms. On this basis a theoretical relationship has been proposed. Until relatively recent times this larva was mistaken for a form of adult nonchordate animal and went under the genus name of Tornaria.

Dolichoglossus and its subphylum, though lacking in complete conformity to ehordate characteristics, is classified here because of the diverticulum supposedly representing a rudimentary notochord, the gill clefts in the alimentary canal, and the dominance and grooved structure of the dorsal nerve cord. The group includes Cephalodiscus and Rhabdopleura which are colonial forms living in deep sea. 


\section{SUBPHYLUM UROCHORDA, IMOLGULA}

Subphylum Urochorda includes a number of common representative marine forms, such as Salpa, Cynthia, Ciona, Clavelina, Ascidia, and Molgula. The latter genus represented by $M$. manhattensis will be given particular consideration here. This animal is commonly known as sea lemon, sea peach, or sea squirt. The body of the adult is saclike and averages about one inch in diameter. In this condition it would be an outcast among chordates because as an adult it has no notochord, and no dorsally located, tubular nerve cord. However, it does present pharyngeal gill slits.

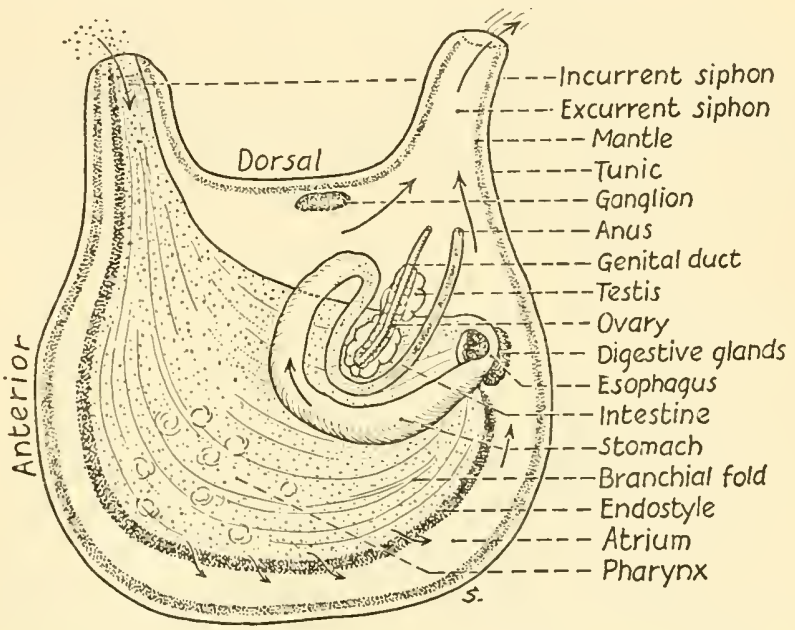

Fig. 212.-Diagram of Molgula manhattensis from the left side to show the structure with the courses of water and food through the body indicated by arrows.

It is saved to the chordates by the presence of all three of the characteristic features in the larval stage. The larva is free-swimming and shaped like a tadpole, while the adult is globular and sessile in most of the common forms. Some are brilliantly tinted with color. The adult is covered externally by a cellulose coat or tunic (test), which is secreted by the cells of the underlying mantle. Inside the mantle is the extensive atrial cavity. On the dorsal (unattached) side of the body are two funnellike siphons. The anterior one is the branchial siphon (oral funnel, incurrent siphon or mouth) and the other is the atrial siphon (atrial funnel, excurrent siphon, or atriopore). When the tunic of Molgula is removed, one may see most of 
the internal organs through the transparent mantle. Upon viewing this from the left the large saclike pharynx may be seen continuing ventrally and posteriorly from the branchial siphon, finally narrowing at its dorsoposterior extremity to become the small tubular esophagus which turns sharply downward and anteriorly to become the stomach. The esophagus is partially embedded in a dark-colored digestive gland. The stomach contimues anteriorly and upward where it becomes intestine, which turns ventrally on itself in a U-shape. It finally ends with the anus which opens into the atrial cavity shortly below the atrial siphon. A current of water carries food into the digestive system and oxygen for respiratory purposes. The water enters the branchial siphon, passes into the sievelike pharynx, and from here passes through the gill slits or stigmata in its wall into the surrounding atrial eavity, and finally leaves the body by way of the atrial siphon. Oxygen is absorbed by the blood in the walls of the stigmata. The animal's food consists of minute organisms which are entangled in mucus secreted by a glandular groove, the endostyle, which extends from the branchial siphon along the ventral midline of the pharynx to the esophagus. This food mass passes into the esophagus and out through the alimentary canal where digestion and absorption occur. The heart is a contractile tube which pulsates. It lies ventral to the stomach and forces the blood in one direction by a series of contractions and then in the opposite direction by another series. Vessels extend in one direction to the pharynx, primarily, and in the opposite direction to other organs and the body wall. These animals are hermaphroditic or monoecious. Each has two compound sets of gonads, one on the left side in the loop of the intestine and the other on the right side of the body. Some of the sessile tunicates, as Molgula, reproduce by budding. There is an oblong, closed excretory sac which may be seen from the right side. The central nervous system is reduced to a nodulelike ganglion located between the siphons in the dorsal portion. Nerves branch from this to the various parts of the body. The life history of the tunicate is one of interest. Cross-fertilization is the rule; that is, spermatozoa from one individual usually fertilize ova from another; however, there may be exceptions to this. The fertilization occurs in the water outside the body. The eggs hatch to produce larvae somewhat similar to amphibian tadpoles which are free-swimming. The larva possesses the typical notochord, gills, and nerve cord of Chordata. For some reason 
it then settles on the bottom and attaches itself by adhesive papillae located in the anteroventral position. Some authors express it by saying this larva settles on its "chin." It now undergoes regressive changes involving loss of tail, notochord, and posterior portion of nerve cord. The anterior portion of the cord becomes a simple ganglion. The paired eyes and otocysts (ear structures) also disappear. The dorsal side shortens while the rentral side lengthens. This places the mouth in a dorsoanterior position, the anus in the dorsoposterior position, and bends the alimentary canal into a U-shape. The num-
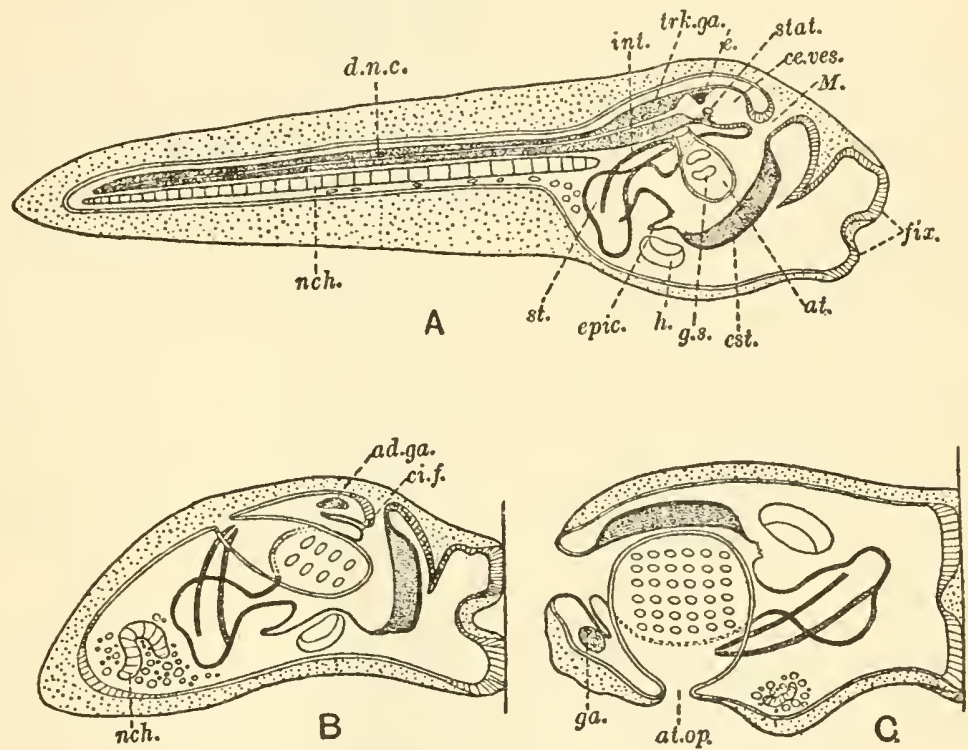

Fig. 213.-Metamorphosis of an ascidian larva. A, larva ready for fixation. $B$, an intermediate stage of metamorphosis. $C$, completion of metamorphosis. ad.ga., adult ganglion; at., rudiment of atrium; at.op., atrial opening; ce.ves., cerebral vesicle; ci.f., ciliary funnel; d.n.c., dorsal nerve cord; e., eye; epic., epicardium; est., endostyle; fix., fixation papillae; ga., ganglion; g.s., gill slits ; h., heart; int., intestine; m., mouth; nch., notochord; st., stomach ; stat., statolith trk.ga., trunk ganglion. (From Borradaile and Potts, The Invertebrata, published by The Macmillan Company.)

ber of gill slits increases greatly. The atrial cavity is formed by infoldings from the exterior on each side which surround the pharynx and meet each other. The external opening of this cavity is the atrial siphon. The outer wall of this newly formed cavity is the mantle. Later the tunic is secreted by the mantle to become a protective, cellulose covering. This process of metamorphosis has caused an active 
respectable chordate to become a lazy, stationary form which is not much more than a water-bag whose level of development has degenerated almost to that of a sponge. Certain of the sessile forms, which reproduce also by budding, develop colonies with a common tunic. This form is one of the few colonial chordate animals. In a few instances tunicates reproduce one generation sexually, and the next is produced by budding (asexually). This alternation of generation is another retrogressive feature.

\section{SUBPHYLUII CEPHALOCHORDA, AMPHIOXUS}

There are usually listed twenty-eight species in this group which are rather locally distributed over the world. There are four species on American shores: Branchiostoma virginiae, B. floridae, B. bermu$d a e$, and $B$. californiense. Amphioxus or the lancelet, Branchiostoma lanceolatus, the European form, is an admirable representative of the subphylum and has become classical in its use. However, it is likely that $B$. virginiae or $B$. floridae is more commonly studied in the United States. It is a small, fishlike, marine animal whose average adult length is about two or three inches. In its adult form it represents clearly the distinctive characteristics of the phylum in a simple condition. It is a chordate, possessing only rare essentials. It is usually referred to as a close ancestral relative of Vertebrata.

Habitat.-It is found in shore water and on the sandy beaches of the subtropical and tropical portions of the world. These animals are found along our Atlantic Coast as far north as Chesapeake Bay, at certain points in the Gulf of Mexico, and on the southern Pacific Coast. They may be found along the shores of the Mediterranean Sea, the Indian Ocean, and along the southern coasts of China.

Habits and Behavior.-It burrows rapidly, head first, in the sand by means of a vibratory action of the entire body, but comes to rest with the anterior end exposed to the water. At times, particularly at night and during breeding season, the animal leaves the burrow and swims about like a fish by means of lateral strokes of the posterior portion of the body.

External Structure.-The body of this animal is shaped like a small lance, the tail being the point. In general, it is similar to 


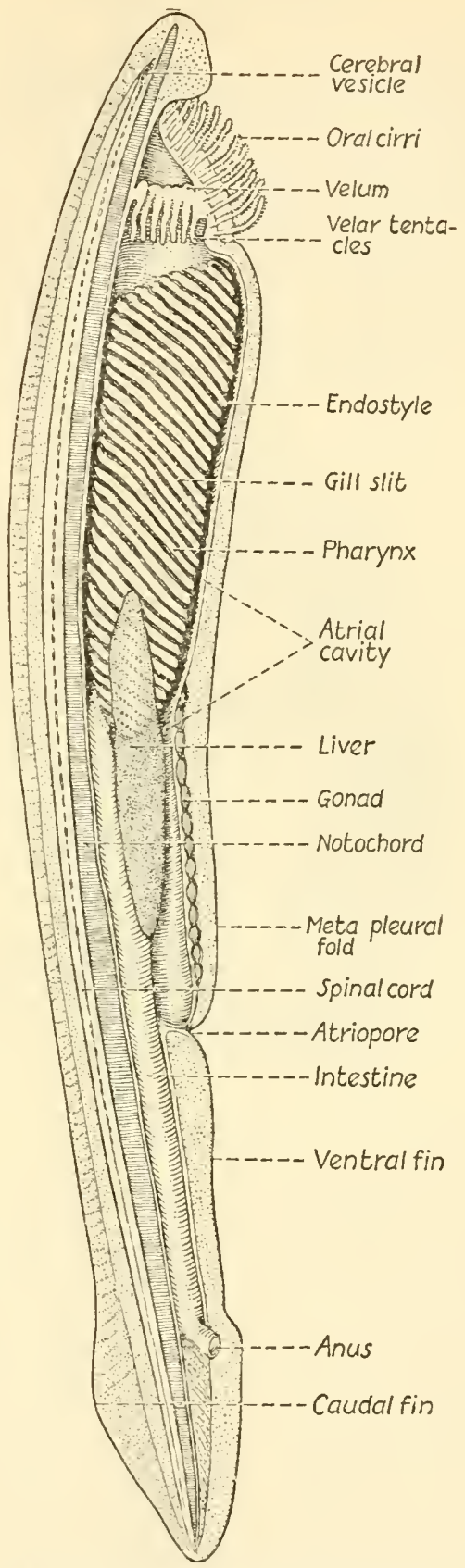

Fig. 214.-Diagram of Branchiostoma (Amphioxus) lanceolatus from the right side to show the structure. 
a small fish, but it does not have a distinct head. The mouth opens on the ventral surface of the anterior portion of the body. It is beneath a rostrumlike projection and is nestled well up in an oral hood which is shaped like an inverted funnel. This hood is fringed with sensory fingerlike oral tentacles. There is a median fin along the dorsal side, continuing around the tail as the caudal fin and anteriorly about one-third of the length of the body as the ventral median fin. There are no clearly defined lateral fins, but a pair of skin structures, the metapleural folds, extending along the anterior

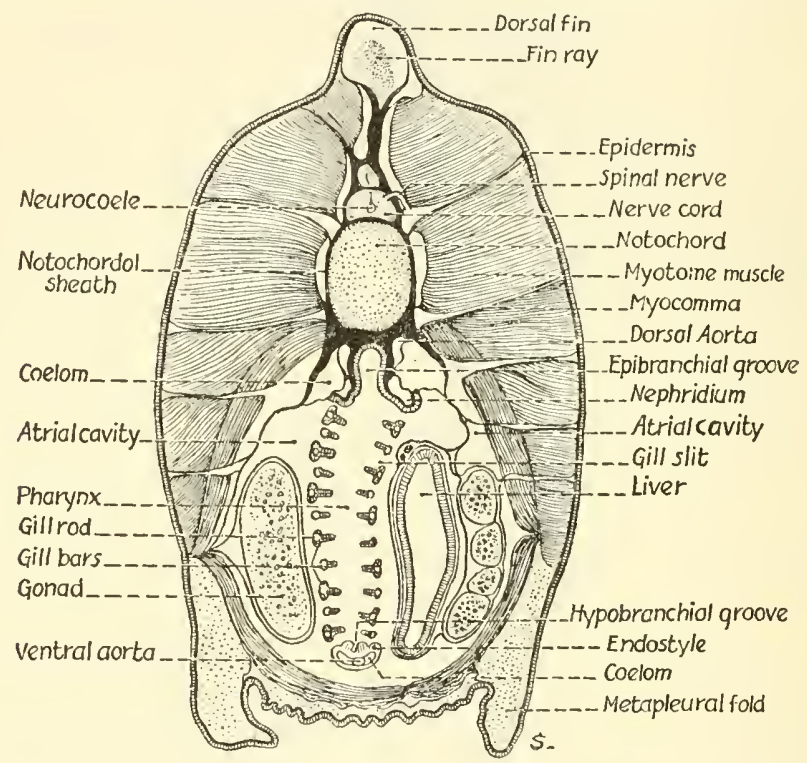

Fig. 215.-Cross section of Amphioxus through the level of the posterior portion of the pharynx.

two-thirds of the ventral surface of the body are thought to be their forerunners. The ventral and dorsal fins are supported by small vertical rodlike fin rays. On the ventral side, just posterior to the metapleural folds, is an opening, the atriopore, and beside the ventral margin of the caudal fin is the anus. The segmental divisions of the muscles are apparent on the body wall. There are from fifty-eight to sixty-four of them on each side in B. lanceolatus but sixty-nine in $B$. californiense and they are known as myotomes. The myotomes on the two sides are not paired, but alternate with each other. Adjacent ones are separated by a myocomma or myoseptum. 


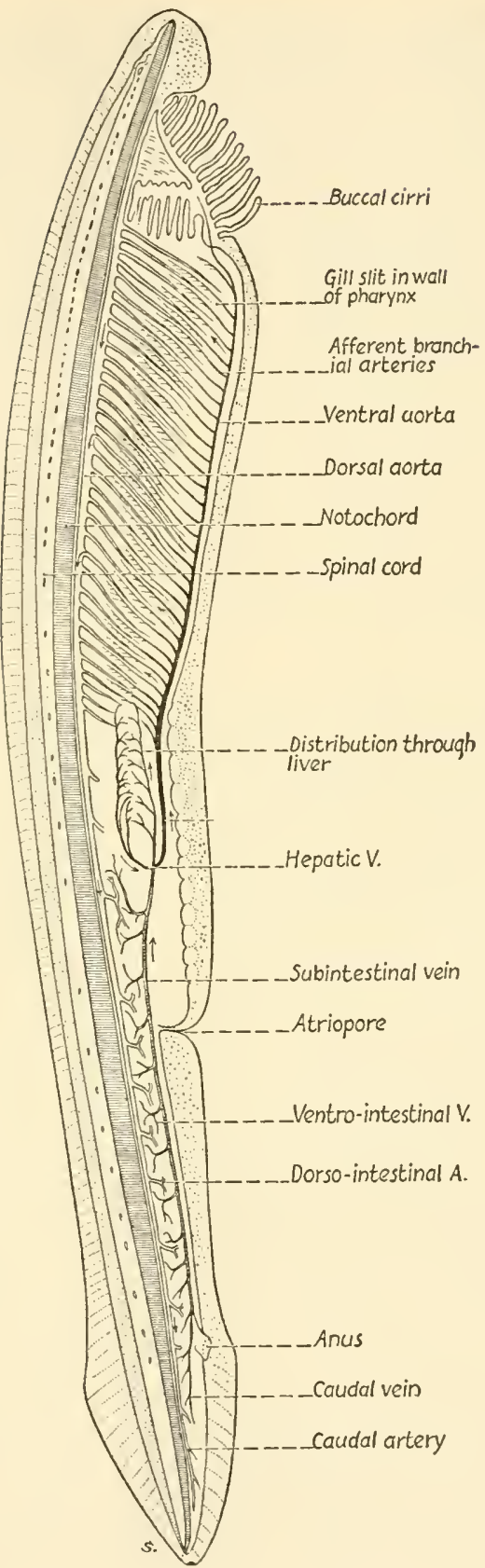

Fig. 216.-Diagram of the circulatory system of Amphioxus. 
Internal Structure and Metabolic Activities.-In small cleared specimens the internal organs are easily observed. The notochord extends the length of the body as a slender rod of vacuolated cells which are filled with fluid to give it turgor or stiffness. Immediately dorsal to this rod is the nerve cord, which also runs the length of the body. It has a small central canal or neurocoele extending lengthwise through it and is dilated at the anterior end to form the cerebral vesicle or rudimentary brain. A mass of dark pigment is located at the anterior end which is known as the eyespot. There are smaller pigment bodies distributed along the length of the cord. These are thought to be sensitive to light. The nerve cord gives off nerves to the organs of the body. The two anterior ones are paired, but those behind the cerebral vesicle alternate on the two sides. There are dorsal sensory nerves going to the skin and ventral motor nerves going to the myotomes. There are sensory cells in the skin, oral tentacles, and velar tentacles.

The circulatory system does not include a heart, but the blood is moved by the contractions of a ventral aorta, which branches to form the afferent branchial arteries to the gills. Here these vessels branch into capillaries, providing aeration for the blood. These capillaries converge to form the efferent branchial arteries which lead dorsally to join the paired dorsal aortae. The dorsal aorta extends posteriorly to the tip of the body giving off numerous branches to myotomes and internal organs along the way. The posterior direction of the flow of the blood is just opposite to that in the dorsal vessel of the earthworm. The subintestinal vein receives the blood from the intestine and continues anteriorly to the liver as the hepatic portal vein. The hepatic vein collects from the liver and leads forward as the ventral aorta. The blood in the subintestinal and hepatic portal reins is laden with dissolved nutriment. The blood in these ventral veins flows from posterior toward the anterior (Fig. 216).

Digestive System.-A current of water is carried into the mouth by the eiliated bands on the inner surface of the oral hood. These cilia form what is called a wheel organ because of their rotary motion. Surrounding the mouth is a membranous velum to which are attached twelve velar tentacles, which fold across the mouth and serve as a strainer to hold back the coarser particles, as well as being sensory. The mouth leads to the large, barrel-shaped pharynx. The gill slits are clefts in the lateral walls of the pharynx. The number of clefts 
varies, ranging between fifty and ninety pairs. These open into the atrial cavity which surrounds the pharynx and other visceral organs. In the midline of the roof of the pharynx is an inverted trough, the hyperbranchial groove, which is ciliated. In the floor of the pharynx is another ciliated groove, the hypobranchial groove. Its glandular walls, which are capable of secreting mucus, constitute the endostyle. It functions on the same plan here as in tunicates. The strings of mucus entangle the food particles and are moved anteriorly, and then by two peribranchial grooves are carried dorsally to the hyperbranchial groove. The cilia here move the mass back to the intestine. A blind, fingerlike diverticulum of the intestine, the liver or hepatic caecum, extends anteriorly from its conncction on the anterior part of the intestine to lie on one side of the pharynx. This organ is a digestive gland and empties a digestive juice containing enzymes into the intestine. The intestine extends posteriorly to the anus as a relatively straight tube. The food is digested in, and absorbed from, the intestine.

Respiratory System and Respiration.-As stated above, the water in passing through the gill slits delivers oxygen to the blood in the capillaries there and absorbs carbon dioxide from it. The water then passes back through the atrial cavity and out through the atriopore. The blood then distributes the oxygen to all tissues of the body. The gill-bars, which separate the slits, contain the blood vessels, and are supported by chitinous rods. The gills are on the faces of the gill bars and are covered with cilia which help move the water through its course.

Excretory System and Excretion.-Ciliated nephridia similar to those of the earthworm lead from the dorsal portion of the coelom to the atrial cavity. The coelomic cavity is reduced in the pharyngeal region to a narrow space surrounding the dorsal aorta above the pharynx and a narrower one around the ventral aorta below. Between the posterior end of the pharynx and the atriopore, the coelom consists of a narrow space surrounding the intestine with a thin membrane separating it from the atrial cavity. Behind the atriopore it is relatively larger.

Reproductive System and Life Cycle.-This animal is dioecious with each mature individual possessing 26 pairs of (31 to 33 pairs in $B$. californiense) nodular gonads embedded in the body wall near the base of the metapleural folds. When the germ cells mature, 
they break through the wall of the gowad into the atrial cavity and pass out through the atriopore with the water. Fertilization occurs in the water. Early summer is the breeding season, and at that time the animals are quite active during the evenings and nights. Following fertilization comes a series of cleavage divisions which are total and equal. This is followed by the infolding of one side of the spherical body to form the gastrula and this in turn becomes a freeswimming larva which reaches adult condition without metamorphosis, only to begin bashfully burying itself in the sand. 


\section{CHAPTER XXIV}

\section{THE VERTEBRATE ANIMAL: SUBPHYLUM VERTEBRATA}

In this group to which man himself belongs are found the distinctive chordate characteristics at some time in the life of the individual. In terrestrial forms there are certain modifications to produce other structures. Metamerism and bilateral symmetry are universal characteristics among vertebrates. The segmented vertebral column and other supporting structures form an endoskeleton (internal skeleton) which is the basic support of the body. Paired appendages are usually present at some time in the life of the individual. The majority have two pairs of fins or limbs in adult condition. There is a ventrally located heart which is divided into chambers. The blood contains hemoglobin bearing red corpuscles and amoeboid white corpuscles. In the vertebrate body is a well-developed coelom, which encloses advanced systems of organs for digestion, excretion, circulation, reproduction, and in terrestrial forms, respiration. Cephalization is developed in all vertebrates and along with this they possess a hollow, five-lobed brain located in the more or less distinct head. The sense organs are in an advanced state of development. The body is divided into head, trunk, and tail. The tail is a posterior prolongation of the body behind the anal opening and is found in some degree in all vertebrates. The neck which is a constricted region between trunk and head is conspicuous in terrestrial forms. The appendages are usually arranged with one pair attached to the anterior, pectoral portion of the trunk and one situated at the posterior, pelvic region. This arrangement is less consistent in the aquatic types where the weight of the body is buoyed up by the water and the limbs are used less for support and locomotion. In different types of vertebrates there are various modifications of pectoral appendages as arms, wings, pectoral fins, forelegs, and flippers. The same is generally true for the pelvic limbs.

The body wall is composed of the skin, which usually has characteristic tegumentary outgrowths, such as scales, nails, shells, feathers, and hair, as the outer layer, beneath which is the muscular 
coat and internal to this is the membranous peritoneum. In all vertebrates, except mammals, the coelom consists of only two parts: the pericardial cavity and the general abdominal cavity. In mammals it is further divided into pericardial, thoracic, and abdominal

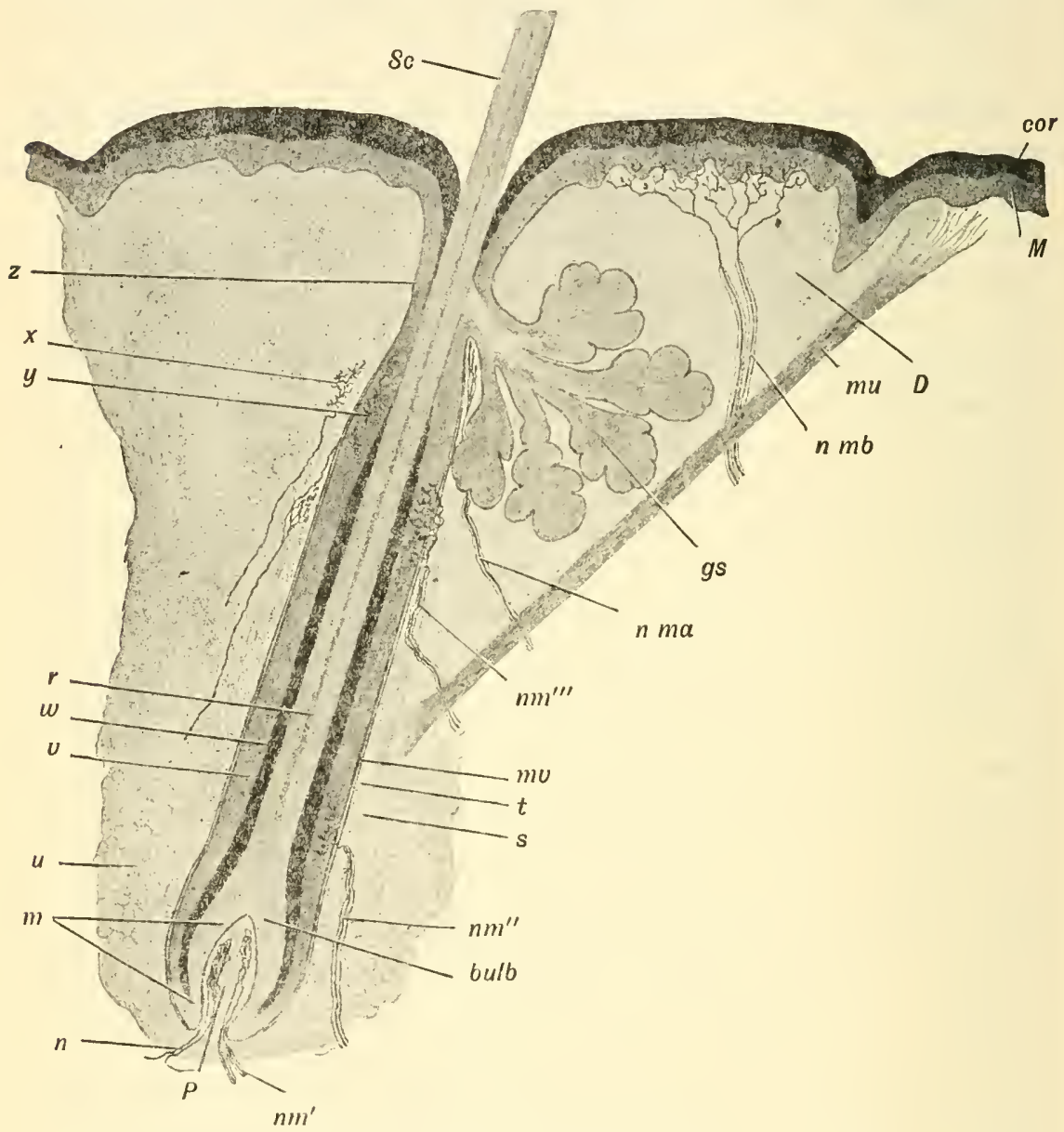

Fig. 217.-Diagrammatic section of the human skin. Cor, stratum corneum; $D$, dermis; $g s$, sebaceous gland; $M$, Malpighian layer; mu, muscle; $n$, nonmedullated nerve: $n m^{\prime}, n m^{\prime \prime}, n m^{\prime \prime \prime}, n m a$, and $n m b$, myelinated nerve flbers; $\boldsymbol{P}$, papilla of hair: Sc, hair shaft, $u$, fat tissue; $v$ and $w$, external and internal sheaths of hair root; $x$ and $y$, endings of nonmyelinated nerve fibers. (From Maximow and Bloom, Histology, published by W. B. Saunders Co.)

cavities. The first contains the heart, the second the lungs, and the third the organs of the excretory, reproductive, and digestive systems. 
The vertebrate animal is covered by an integument or skin which serves as a protective and sensory organ. It also helps in excretion through the sweat glands, mucus glands, and oil glands as well as facilitating temperature regulation in some. Such exoskeletal structures as scales, nails, hoofs, claws, feathers, hairs and enamel of teeth are produced by the skin. The integument is composed of an outer stratified epithelial epidermis which consists of several layers of cells, few nerves, and no blood vessels, and the inner fibrous dermis or corium, which consists of areolar connective tissue, nerves, nerve endings, integumental glands, blood vessels and lymph spaces. The membrane type of bone is developed in the dermis.

The maintenance of any living body requires the cooperation of several functions which will attain similar fundamental results wherever in living material they occur. The principal functions performed by the structures in the animal body are: (1) support and protection, (2) movement and locomotion, (3) digestion, (4) respiration, (5) circulation, (6) excretion, (7) reproduction, (8) reception and conduction of stimuli, and (9) internal regulation. These functions merge into one living process which involves the building up of protoplasm, transformation of energy, and reproduction. During the execution of these activities energy is constantly being changed from the potential to the kinetic form.

Metabolism.-The collective term metabolism is employed when referring to all of the interactions involved in the living process of protoplasm. It includes the processes concerned with conversion of food into protoplasm, release of energy through oxidation, production of heat, morement, elimination of wastes; or, in other words, these processes are chiefly: Ingestion, digestion, egestion, absorption, transportation, respiration, oxidation, and elimination. The processes concerned with the conversion of food material into protoplasm (building up) constitute the phase of metabolism known as anabolism. Included here are ingestion, digestion, absorption, transportation, and assimilation. The oxidation of materials of the protoplasm to liberate energy, and the elimination of wastes incidental to it, is known as catabolism or the "breaking down" phase.

Metabolism is one of the fundamental features of all protoplasm, therefore, all physiology, since it is a study of the functions of living organisms, must be concerned with metabolism. It includes all 
of the chemical changes and transformations by which energy is supplied for the activities of the protoplasm.

The skeleton is quite well developed in the vertebrates and serves them quite efficiently for support, stature, protection, and muscle attachment. It is composed of cartilage entirely in some of the simpler forms and of bone and cartilage in higher types. It is divided into an exoskeleton which is superficial and an inner endoskeleton which includes all of the deeper skeletal parts. The exoskeleton is a rather minor part in vertebrates and consists of nails, claws, seales, hair, feathers, and other outgrowths. The endoskeleton includes the axial and appendicular portions. The first is composed of the skull, vertebral column, ribs, and in some a sternum. The appendicular portion is composed of the anterior and posterior girdles and two pairs of limbs. In their development bones either replace cartilage to be called cartilage bones or they develop in the connective tissue of the dermis, to be known as membrane bones. The vertebral column is composed of segmental divisions, the vertebrae, and is divided into five regions as follows: cervical vertebrae of the neck, thoracic vertebrae of the chest, lumbar vertebrae of the small of the back, sacral, vertebrae of the hip region, and the caudal vertebrae of the tail region. Bone is a firm, hard tissue consisting of abundant matrix, composed of inorganic salts, and the bone cells which are held in pocketlike lacunae in the matrix. The outer membranous covering of bone is called periosteum. The mineral part of the bone consists chiefiy of calcium phosphate and calcium carbonate. They give it firmness and rigidity. The animal matter is composed of the bone cells and cartilage which serve to give the bone life and resilience. A weak acid, such as the acetic acid in vinegar, will dissolve the mineral matter of bone if allowed sufficient time, in which case the bone will lose its rigidity. Caustic solutions will destroy the animal matter and make the bone brittle. The following outline presents a summary of the principal parts of the terrestrial vertebrate skeleton.

Divisions of Skeleton of Terrestrial Vertebrate

I. Axial Skeleton

(a) Skull

1. Cranium

2. Sense capsules

3. Jaw apparatus

(Visceral arches) 
(b) Vertebral column

1. Cervical vertebrae (neck)

2. Thoracic vertebrae (chest)

3. Lumbar vertebrae (small of back)

4. Sacral vertebrae (hip)

5. Caudal vertebrae (tail)

(c) Thoracic basket

1. Ribs (paired)

2. Sternum (breastbone)

II. Appendicular Skeleton (girdles and limbs)

(a) Pectoral (anterior)

1. Girdle: scapula, claviele, procoracoid and coracoit

2. Limb: Humerus (upper arm), radius and ulna (forearm), carpals (wrist), metacarpals (palm), phalanges (bones of digits)

(b) Pelvic (posterior)

1. Girdle: ilium, pubis, and ischium

2. Limb: Femur (thigh), patella (knee cap), tibia and fibula (shank), tarsals (ankle), metatarsals (sole), phalanges (bones of toes)

In Protozoa there is no very elaborate adaptation toward a skeleton. The presence of a euticle in some and the secretion of a hard shell in others seem to be the particular developments related to these special functions in this group. Arcella, Difflugia, the Foraminifera, and Radiolaria exemplify this adaptation.

The skeleton and integumentary structures serve the Metazoa primarily for a support and protection. The corals of the phylum Coelenterata secrete a calcareous or horny skeleton around the external surface of the body proper. The sponges, as a rule, each have a calcareous, siliceous (glassy), or horny skeleton extending throughout the body. Such forms as snails, crayfishes, beetles and representatives of their respective phyla secrete a well-developed exoskeleton as an external cover over most of the other tissues of the body. The muscles and other tissues are attached within. There are special cells of the epidermis which function primarily in production of this skeletal material. The echinoderms, including animals like the starfish, possess calcareous skeletal plates which are essentially similar to exokeleton except that they are principally beneath the skin.

There is no well-developed endoskeletal structure known in nonchordate animals but the endophragmal structures extending into the thorax of some Crustacea are thought to be the forerunner of the endoskeleton. A number of exoskeletal modifications are used for 
protection and temperature regulation in most of the groups of vertebrates. Such structures as scales, shells, feathers, hair, nails, horns, and even enamel of teeth are of this type.

Primitively the notochord is the original endoskeletal structure of the chordate group. Around it are developed the basic structures of

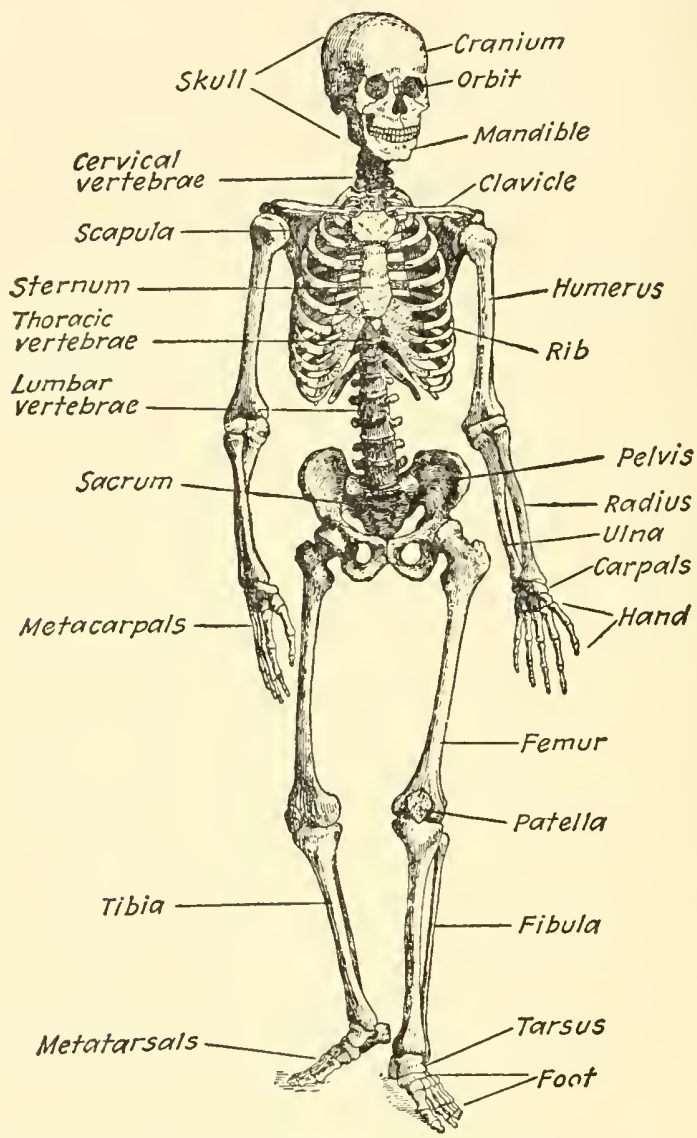

Fig. 218.-Human skeleton. (From Wolcott, Animal Biology, published by the McGraw-Hill Book Company.)

the vertebral column which functions as the principal axial support of all vertebrates. The sternum, girdles, and paired limbs have developed with the terrestrial life of vertebrates and the necessity for locomotion on land. 
The muscular system represents a system of cells highly specialized in contractility. The muscles are usually attached to the skeleton or occasionally to other muscles by fibrous cords called tendons. Voluntary muscles are usually connected with the skeleton; those of the visceral organs, e.g., intestine, are involuntary. Cardiac muscle is the highly specialized involuntary muscle which makes up the wall of the heart.

Independent power of movement is almost a characteristic of animal life. Contractility as a property of all protoplasm is the fundamental basis for all animal movement. The adult forms of certain animals, such as sponges, corals, oysters, barnacles, and others, are sessile; however, they all pass through a free, active larval stage. Most of them retain the power to move separate parts in adult condition.

Simpler forms of locomotion have already been seen in Protozoa which move from place to place by means of pseudopodia, cilia, or flagella. In ciliary movement the numerous small strands of protoplasm beat rhythmically with a stroke in one direction, so timed that the beat passes in a wavelike progression from one end of the ciliated area to the other (metachronous rhythm). The stroke of a cilium consists of a vigorous bend in one direction and a very deliberate recovery in the other. In many Protozoa the entire body is covered with eilia while in Metazoa the entire body may be covered where they are used for locomotion; but more often they cover only areas of free surface of epithelium, particularly the linings of passages. Here they serve to move materials along and keep the surface free of foreign material and excess mucus.

The development of a high degree of contractility in special cells, such as muscle cells, makes possible muscular movement which is the principal kind in higher animals. A muscular locomotor system consists of sets of opposing muscles. In muscular contraction there is a cycle of rapid chemicophysical rearrangement in the cells. Oxidation and heat production are involved in the process. Carboliydrates in the form of glucose are oxidized (burned) in the reaction. During the shortening of the muscle there is a lyydrolysis or absorption of water by the protein product, creatine-phosphoric acid. By-products of the process include carbon dioxide, lactic acid, urea, creatinine, and phosphoric acid. 
In animals without a skeleton muscle bands are arranged in both circular and longitudinal directions. The contraction of the circular group tends to lengthen the body, and the shortening of the longitudinal strands draws the body along. The pressure exerted on the coelomic fluid is thought to be a factor in bringing about an even extension of the body by this means. In echinoderms with the water vascular system the pressure is exerted on water in a system of tubes which extend to make contact with the surface over which the animal is moving.

The Digestive System.-The digestive system is typically a straight tube extending through the length of the trunk of primitive vertebrates. In the higher forms there are many outgrowths, such as digestive glands and respiratory organs. The anterior region of the digestive tube is the mouth cavity which contains teeth on the jaws, a tongue, and receives saliva from salivary glands. Following the mouth is the pharynx or throat region which receives the internal nostrils, the Eustachian tubes from the middle ears and opens into the esophagus posteriorly. The esophagus is usually tubular and propels the "swallows" of food posteriorly by consecutive waves of contraction, a process known as peristalsis. It leads to the saclike stomach, whose walls possess gastrie glands for secretion of a digestive fluid containing enzymes (ferments) and weak hydrochloric acid. The peristaltic contractions continue along the wall of the stomach to help digestion by churning and mixing the food with digestive juices. At the posterior end a pyloric valve in the form of a sphincter muscle guards the entrance to the small intestine which follows. This is a convoluted tube in most of the advanced forms of vertebrates and is divided into the anterior duodenum, middle jejunum, and posterior ileum. It is usually longer than the body and therefore it is coiled. Its walls produce digestive enzymes from glands and it receives digestive juices from two other glands: the liver and the pancreas. The small intestine serves both as a digestive organ and as the principal absorptive organ of the body. Its internal lining is provided with numerous fine fingerlike projections which increase the immer surface and enhance absorption. The digested food is taken up by the lymphatic spaces and by blood vessels which are embedded in the wall just outside of the lining epithelium. The liver is the largest organ in the body of most vertebrates. It secretes the bile which is stored in the thin-walled gall bladder, 
which is attached to one of its lobes. The liver also serves to convert carbohydrates to glycogen (animal starch) and store it for future energy production. It is also in the liver that protein wastes are converted into urea and uric acid in order that they may be excreted from the blood in the kidneys. The large intestine which is shorter than the small intestine possesses no villi or digestive glands. It receives the fecal matter and delivers it to the anus. In many forms of vertebrates the posterior portion of the large intestine is modified to become a cloaca, which receives also the products from the urinary and reproductive organs.

The chief function of this entire system is that of dissolving and converting complex food materials into a form which may be absorbed and assimilated by the protoplasm of cells throughout the body. The materials commonly used for foods have large molecules, usually called colloidal in nature. Digestion then must serve to break up these large molecules into smaller ones, thus forming solutions of substances in order that they will readily diffuse through membranes. Digestive enzymes are responsible for placing the food materials in solution. So, proteins are converted to soluble amino acids, starches and sugars to maltose and finally glucose, and fats to fatty acids and glycerin.

In general, an enzyme is an organic substance which by its presence under certain conditions will cause or hasten chemical reaction between other substances without itself being consumed. The enzymes are formed in the protoplasm of cells and their action is similar to that of a catalyst, since they accelerate chemical action. There are different types of enzymes each capable of producing specific kinds of reactions. There are oxidizing enzymes (oxidases) capable of bringing about oxidation ; reducing enzymes (reductases) which produce reduction in tissues; coagulating enzymes (coagulases) which cause clotting or coagulation; and hydrolysing enzymes (hydrolases) act by causing a reaction between a substance and water. Most of the digestive enzymes fall in this latter class. Most enzymes consist of a parent substance or precursor (zymogen) which becomes active only in the presence of a certain other substance, termed activating agent or coenzyme. As an example, the precursor of pepsin is pepsinogen which becomes activated in the presence of dilute acid. 


\section{Classes of Digestive Enzymes}

1. Diastases or diastatic enzymes-split carbohydrates

(a) Ptyalin in saliva

(b) Amylase in pancreatic juice

(c) Glycogenases-liver and muscles

Converts glycogen to glucose

2. Lipase or lipolytic enzyme-splits fats

(a) Steapsin in pancreatic juice

3. Inverting enzymes-convert disaccharids to the less complex monosaccharids (simple sugars) -intestinal juice
(a) Maltase
(b) Lactase
(c) Sucrase (invertase)

4. Proteases or proteolytic enzymes-split complex proteins

(a) Pepsin in gastric juice

(b) Trypsin in pancreatic juice-functions in small intestine

(c) Erepsin in intestinal juice

5. Clotting or coagulating enzyme

(a) Rennin in gastric juice

In higher Metazoa digestion is accomplished principally extracellularly through secretion of enzymes by certain groups of cells. Such systems consist of : (1) an alimentary canal proper; and (2) associated glands which discharge digestive juices into it. The relative length of this canal varies considerably depending on the habitual diet of the organism. In carnivores (flesh-eaters), such as cats and dogs, it is from three to five times as long as the body; while in herbivorous forms (plant-eaters), such as horses and cows, it is over twenty times as long as the body. The length of the human digestive tract is approximately ten times the length of the body. The relative proportion of the internal absorptive surface of the alimentary canal to the external surface of the body is significant. In carnivorous animals it is about one-half the area of the skin while in herbivorous animals it is about twice the area of the skin.

The process of digestion in man is quite well understood, and it is fairly typical and general because of the omnivorous food habits. The action of the several enzymes produced by different glands is a very essential part of the process. The digestion of all organic food materials is brought about by hydrolysis in the same kind of chemical change. In hydrolysis the large molecules of protein, carbohydrate, or fat first combine with water and then split into simpler products. Some foods may require more than one such 
splitting. The splitting of the disaccharide, maltose, will serve as an example of this process :

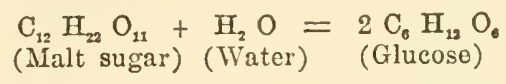

The two molecules of glucose formed are in a form for ready absorption.

Gastric Digestion.-The tubular gastric glands located in the mucous layer of the stomach secrete the acid gastric juice which is a solution of 0.2 to 0.5 per cent hydrochloric acid and two important enzymes, pepsin and rennin. The pepsin when present in the acid medium brings about the splitting of complex proteins into intermediate proteoses and peptones. Rennin causes the casein in milk to coagulate. This is the first step in its digestion. It is claimed by some that emulsified fats, such as cream, are partially digested by a gastric lipase. The digesting mass or chyme in the stomach is continually churned and mixed by muscular activity of the walls. When it becomes saturated ( 0.4 per cent) with acid and has been reduced to the consistency of soup, it is discharged through the pylorus.

Intestinal Digestion.- When the chyme is ejected through the pylorus into the dnodenum, the hydrochloric acid stimulates certain cells of the intestinal lining, causing them to secrete into the blood a substance of hormone nature, known as secretin. Upon reaching the pancreas this secretin stimulates it to secrete the digestive fluid, pancreatic juice, into the small intestine by way of the pancreatic ducts. There is some evidence that secretin also stimulates secretion in the liver.

Pancreatic juice is a clear, watery, alkaline solution containing inorganic salts (carbonates, etc.) and three enzymes; the protease, trypsin, the diastase, amylopsin, and the lipase, steapsin. These act respectively on proteins and peptones, starches and sugars, and fats. This protease is in the form of trypsinogen until it reaches the intestine and is activated by an intestinal enzyme, enterokinase. Trypsin completes the work begun by the pepsin in that it converts proteoses and peptones into amino acids, but it also digests complex proteins which have escaped the action of pepsin. It acts more rapidly and efficiently than does pepsin. There are nineteen amino acids that are regarded as building stoncs of the protcin molecule. In a complex protein like casein, as many as sixteen of these amino acids will 
be found. The tissues of the animal body must not only have available a wide range of amino acids but must also select in the proper proportion the ones needed to reconstruct their specific protein constituency.

Amylopsin (amylase) is the pancreatic diastase, and it is able to bring about hydrolysis of carbohydrates in the alkaline medium of the intestine without activation. It produces dextrin and maltose (malt sugar). The pancreatic lipase, steapsin, brings about the splitting of fats into glycerin (glycerol) and one or more fatty acids, such as stearic acid, oleic acid, butyric acid, etc. The alkaline salts which are introduced by the bile, combine with these fatty acids to form soaps which help in emulsifying the remaining fats, thus making them more readily split.

Intestinal secretions or succus entericus which are produced by glands in the mucous membrane of the small intestine include five enzymes. Enterokinase, which activates trypsinogen to form trypsin, has been mentioned already. Erepsin, the intestinal protease, supplements the activity of trypsin by converting proteoses and peptones into amino acids. Maltase converts maltose and dextrin into dextrose. Invertase changes sucrose (cane sugar) into dextrose and levulose. Lactase converts milk sugar (lactose) into galactose and dextrose, both simple sugars.

The undigested residne passes into the large intestine where probably no enzyme digestion occurs. Certain bacteria ( $B$. coli and others) attack any undigested protein and bring about putrefactive fermentation. Products of this action may be absorbed; some of them are frequently toxic and must be eliminated in either the urine or the feces. Certain other bacteria here feed upon cellulose and may produce some sugar from it. When the chyme reaches the large intestine it is about the consistency of thick cream, but it becomes more and more solid by absorption of water here until finally only concentrated fecal matter remains.

Functions of the Liver.-The secretion of the liver is bile and is discharged into the duodenum of the small intestine by way of the common bile duct. This is an alkaline solution which serves to help neutralize the acidity of the chyme as it comes from the stomach. This with the pancreatic juice brings about the emulsification of fats mentioned above. Cholesterin and two pigment materials are excreted in 
The Digestive Enzymes and Their Functions

\begin{tabular}{|c|c|c|c|c|}
\hline ENZYME & $\begin{array}{l}\text { REGION OF DI- } \\
\text { GESTIVE TRACT* }\end{array}$ & $\begin{array}{l}\text { DIGESTIVE } \\
\text { JUICES }\end{array}$ & $\begin{array}{c}\text { FOODS } \\
\text { AFFECTED } \\
\end{array}$ & $\begin{array}{l}\text { SUBSTANCES } \\
\text { PRODUCED }\end{array}$ \\
\hline Ptyalin & Mouth & Saliva & $\begin{array}{l}\text { Starch } \\
\text { (carbo- } \\
\text { hydrates) }\end{array}$ & Maltose \\
\hline Pepsin & Stomach & \multirow[t]{3}{*}{$\begin{array}{c}\text { Gastric juice } \\
\text { from gas- } \\
\text { tric glands }\end{array}$} & $\begin{array}{l}\text { Proteins in } \\
\text { acid } \\
\text { medium }\end{array}$ & $\begin{array}{l}\text { Proteoses } \\
\text { and pep- } \\
\text { tones }\end{array}$ \\
\hline $\begin{array}{l}\text { Rennin in } \\
\text { manmal }\end{array}$ & Stomach & & $\begin{array}{l}\text { Protein of } \\
\text { milk }\end{array}$ & $\begin{array}{l}\text { Coagulated } \\
\text { to form } \\
\text { paracasein }\end{array}$ \\
\hline $\begin{array}{l}\text { Gastric } \\
\text { lipase }\end{array}$ & Stomach & & $\begin{array}{l}\text { Emulsifies } \\
\text { fats }\end{array}$ & $\begin{array}{r}\text { Glycerol and } \\
\text { fatty acids }\end{array}$ \\
\hline None & Liver & Bile & $\begin{array}{l}\text { Emulsifies } \\
\text { fats }\end{array}$ & None \\
\hline $\begin{array}{l}\text { Amylase or } \\
\text { amylopsin }\end{array}$ & $\begin{array}{c}\text { Produced in } \\
\text { pancreas }\end{array}$ & $\begin{array}{l}\text { Pancreatic } \\
\text { juice pro- } \\
\text { duced in } \\
\text { pancreas } \\
\text { but acting } \\
\text { in small } \\
\text { intestine } \\
\end{array}$ & $\begin{array}{l}\text { Carbohy- } \\
\text { drates }\end{array}$ & Maltose \\
\hline Steapsin & $\begin{array}{c}\text { Produced in } \\
\text { pancreas }\end{array}$ & \multirow{6}{*}{ Intestinal } & $\begin{array}{l}\text { Lipins } \\
\text { (fats) }\end{array}$ & $\begin{array}{l}\text { Glycerol and } \\
\text { fatty acids }\end{array}$ \\
\hline Trypsin & $\begin{array}{c}\text { Produced in } \\
\text { pancreas }\end{array}$ & & $\begin{array}{l}\text { Proteoses } \\
\text { and pep- } \\
\text { tones in } \\
\text { alkaline } \\
\text { medium } \\
\text { with enter- } \\
\text { okinases } \\
\end{array}$ & Polypeptids \\
\hline Erepsin & $\begin{array}{l}\text { Snall } \\
\text { intestine }\end{array}$ & & Polypeptids & Amino acids \\
\hline Maltase & $\begin{array}{l}\text { Simall } \\
\text { intestine }\end{array}$ & & Maltose & $\begin{array}{l}\text { Glucose } \\
\text { (dextrose) }\end{array}$ \\
\hline $\begin{array}{l}\text { Lactase in } \\
\text { manmals }\end{array}$ & $\begin{array}{l}\text { Small } \\
\text { intestine }\end{array}$ & & Lactose & $\begin{array}{c}\text { Glucose and } \\
\text { galactose }\end{array}$ \\
\hline $\begin{array}{l}\text { Invertase or } \\
\text { sucrase }\end{array}$ & $\begin{array}{l}\text { Small } \\
\text { intestine }\end{array}$ & & Sucrose & $\begin{array}{c}\text { Glucose and } \\
\text { levulose }\end{array}$ \\
\hline
\end{tabular}

*The esophagus and colon do not secrete any enzymes.

the bile. Bile is secreted all of the time, but between meals it is stored in the gall bladder and supplied in quantity at meal time.

Besides these digestive and excretory functions the liver serves in another capacity. It is a storehouse for carbohydrates which it converts to glycogen (animal stareh) by enzyme action. This substance is also stored in the voluntary muscles. It is easily reconverted to dextrose for ready oxidation. Most of the protein byproduct urea (and uric acid in some forms) is formed in the liver and carried by the blood to the kidneys for exeretion. 
Absorption and Utilization of Food Materials. - The soluble products of digestion are absorbed through the semipermeable epithelial lining of the intestine into the blood of the adjacent capillaries, or in the case of fats into the lacteal lymphatics and from here into the subclavian vein. The blood supplying the intestine is collected by the hepatic portal vein and delivered to the liver.

The two functions of proteins in the body are: to rebuild debilitated protoplasm; and help supply heat and energy to the body by oxidation. They serve first and best for the purpose mentioned first. Carbohydrates and then fats are more economical and efficient as sources of fuel for production of heat and energy. Oxidation of protein requires the disposal of much more waste products. The comparative heat production values of the three are as follows:

One gram of protein $=4.100$ Calories*
One gram of carbohydrate $=4.100$ Calories
One gram of fat

Some portion of the dextrose is distributed and oxidized directly for immediate energy, but much of it is transformed into glycogen by the enzyme glycogenase in the liver. This may be stored here or in the muscles to be reconverted into dextrose for oxidation by the tissues as needed. Normally there is a constant supply of dextrose ( 0.1 to 0.15 per cent) in the blood and this level must be maintained. The final oxidation products of carbohydrates in the body are heat, kinetic energy, water, and carbon dioxide. The last two are discharged from the body as waste products. Fat is converted to dextrose and oxidized to produce heat and kinetic energy. It is usually stored as a rescrve fuel supply in adipose tissue over the body. Carbohydrates in excess may be converted to fat, and stored.

Vitamins and Their Functions.--Besides proteins, carbohydrates, fats, inorganic salts, and water there is another indispensable class of food material, the vitamins. They are natural substances found in relatively small quantities in a number of different foods. In general, their function is regulatory. They are recognized usually through the abnormal condition brought on by their defieiency. There is little danger of vitamin deficiency for adults living on a balanced and mixed diet. MIuch of our knowledge concerning the symptoms brought on by lack of different substances has been

- A Calorie equals the amount of heat necessary to raise one liter of water one degree centigrade under standard conditions. 
gained by feeding experiments on different kinds of laboratory animals and results applied to human beings. The following outline will give much of the essential information concerning vitamins.

\section{The Vitamins and Their Characteristics}

I. Vitamin $A\left(\mathrm{C}_{20} \mathrm{H}_{30} \mathrm{O}\right)$-antixerophthalmic-fat soluble.

(a) Sources: carotene $\left(\mathrm{C}_{40} \mathrm{H}_{56}\right)$ a yellow pigment in green plant leaves, carrots, and such plant tissues. Transformation of this pigment into the vitamin which is especially stored in shark, cod, halibut or other fish liver oil, egg yolk, and milk.

(b) Functions: Influences efficiency and acuity of vision, important factor in regeneration of visual purple of retina, strengthens and promotes liardiness in epithelial tissue.

(c) Effects of Deficiency: Xerophthalmia (lack of tear secretion and dry cornea), and "night blindness", in human. "Nutritional" roup in birds.

II. Vitamin $B^{*}$ "Complex."

1. $\mathrm{B}_{1}$ or Thiamin $\left(\mathrm{C}_{12} \mathrm{H}_{17} \mathrm{ON}_{4} \mathrm{~S}\right)$-Antineuritic.

(a) Sources: Germ of wheat and other cereal grains, peanuts, liver, and egg yolk.

(b) Functions: Promotes tone in alimentary tract, stimulates appetite, essential for normal growth, essential for carbolydrate metabolism.

(c) Effects of deficiency: Beri-beri (neurodigestive disturbance following diet of polished rice), loss of tonus and muscular activity of digestive tract. Cessation of growth. Polyneuritis develops in birds.

2. Riboflavin $\left(\mathrm{C}_{17} \mathrm{H}_{20} \mathrm{O}_{6} \mathrm{~N}_{4}\right)$.

(a) Sources: Eggs, liver, milk, green leaves, yeast.

(b) Functions: Necessary for growth, active relatiou to several enzymes witl intermediate metabolism of food.

(c) Effects of deficiency: Irritation and inflammation at corners of mouth in human (cheilosis). "Yellow liver" of dogs. "Curl-toe", paralysis of chickens. Dermatitis of turkeys.

3. Nicotinic Acid $\left(\mathrm{C}_{6} \mathrm{H}_{5} \mathrm{NO}_{2}\right)$-antipellagric.

(a) Sources: Meat, liver, egg yolk, green leaves, wheat germ, yeast.

(b) Functions: Produces active "coenzymes"' ( $\mathrm{I}$ and II), balances cellular function.

(c) Effects of deficiency: Pellagra in primates (man and monkeys). Black-tongue in dogs. Swine pellagra.

4. $\mathrm{B}_{6}$ or pyridoxine $\left(\mathrm{C}_{8} \mathrm{H}_{12} \mathrm{O}_{3} \mathrm{~N}\right)$.

(a) Sourees: Milk, liver, cereals, Jeast.

(b) Functions: Necessary for growth. May influence oxidation of food.

(c) Effects of deficiency: Paralysis in chickens.

- There are still other recently discovered fractions of Vitamin B. whose functions are specific. 
5. Pantothenic acid $\left(\mathrm{C}_{9} \mathrm{H}_{17} \mathrm{O}_{5} \mathrm{~N}\right)$.

(a) Sources: Liver, milk, egg yolk, yeast, molasses, peanuts.

(b) Functions: Eissential for growth.

(c) Effects of deficiency: Graying in black rats. Dermatitis in rats and ehickens.

6. Biotin $\left(\mathrm{C}_{10} \mathrm{H}_{10} \mathrm{O}_{3} \mathrm{~N}_{2} \mathrm{~S}\right)$.

(a) Sources: Egg yolk, yeast, cereal grains, molasses.

(b) Functions: Essential for growth.

(c) Effects of deficiency: Thickening of skin and dermatitis in chicks and rats.

III. Vitamin $C$ or Ascorbic Acid $\left(\mathrm{C}_{6} \mathrm{H}_{8} \mathrm{O}_{6}\right)$-antiscorbutic-water-soluble.

(a) Sources: Citrus fruits, tomatoes, turnips (most mammals except primates and guinea pig ean synthesize this vitamin).

(b) Functions: Maintains strueture of capillary walls.

(c) Effects of deficiency: Scurvy in human and guinea pig (bleeding in mucous membranes, beneath skin and into joints).

IV. Vitamin $D\left(\mathrm{C}_{28} \mathrm{H}_{44} \mathrm{O}\right)$-antirachitic.

(a) Sources: Tuna and cod-fish liver oils. Exposure of skin to ultraviolet radiation.

(b) Functions: Regulation of ealcium and phosphorus metabolism. Required for normal growtl and mineralization of bone.

(c) Effects of deficiency: Soft, deformed bones in young (rickets). Soft bones (osteomalacia) especially in women of the orient.

V. Vitamin E or Tocopherol $\left(\mathrm{C}_{29} \mathrm{H}_{50} \mathrm{O}_{2}\right)$-antisterility.

(a) Sources: Wheat germ oil, green leaves, other vegetable fats.

(b) Functions: Promotes rapid cell proliferation and differentiation.

(c) Effects of deficiency: Sterility in male fowls and rats. Failure of spermatogenesis. Death of rat embryos in uterus.

VI. Vitamin $\mathrm{K}\left(\mathrm{C}_{31} \mathrm{H}_{46} \mathrm{O}_{2}\right)$-antihemorrhagic.

(a) Sources: Green leaves, alfalfa, also certain bacteria of the "intestinal flora."

(b) Functious: Influences the production of prothrombin by the liver (prothrombin is necessary for blood clotting).

(c) Effects of deficiency: Blood fails to clot.

The Respiratory System.--The respiratory system is at least in part an outgrowth of the digestive canal. In most aquatic vertebrates respiration is accomplished by drawing water through gill slits in the wall of the pharynx. Air-breathing, terrestrial forms have developed the trachea (windpipe) and lungs as another outgrowth of the pharynx. A certain amount of respiration takes place through the skin. The respiratory process is composed of two phases: external respiration which includes the exchange of the gases, oxygen and carbon dioxide, between the external environmental medium and the blood; and internal respiration which is the 
exchange of the gases between the blood and the protoplasm of the cells over the body. Much of the carbon dioxide given up by the cells becomes carbonic acid and carbonates which may be transported by the plasma (fluid) of the blood.

Respiration has been defined as the process involving the exchange of gases between the protoplasm of an organism and its environment. All living protoplasm must be provided with a means of receiving oxygen and giving up carbon dioxide. "In protozoa and simple metazoa, such as sponges, coelenterates, flatworms, roundworms, and even some annelids, this gaseous exchange is made by almost direct diffusion through the cell membranes to the surrounding medium. This movement of gas through the cell membranes depends on the partial pressure of the particular gas on the two sides of the membrane. Gas will flow in the direction toward the least pressure.

In the larger and more complex animals where the volume of tissue is such that a more active interchange of gases is required than the general body surface will permit, special organs or modifications of the surface must be provided. Also the possibilities of oxygen absorption are greatly increased by the development of respiratory pigments like hemoglobin and hemocyanin, which are carried in a blood vascular system all over the body. These pigments readily unite with oxygen to form oxyhemoglobin in the case of the former. Thus the blood is enabled to absorb far more oxygen than an equal quantity of ordinary liquid. When the oxygen pressure of the surlounding tissue is sufficiently low, the oxyhemoglobin releases its oxygen rapidly. Carbon dioxide accumulates in excess in the tissues and diffuses from the cells to the lymph, thence to the plasma where much of it combines with sodium as sodium carbonate. Small amounts of $\mathrm{CO}_{2}$ combine with the hemoglobin.

The gills of most aquatic forms are richly supplied with a capillary supply of blood and then membranous surfaces are directly exposed to surrounding water from which the dissolved oxygen is absorbed. In many aquatic worms the gill filaments are outgrowths of the sides of the body wall. Likewise, the more or less plumelike gills of crayfish are pocketlike outpushings of the body wall. In a number of aquatic insects, worms, fishes, and turtles, the rectum serves as an accessory respiratory organ. 
Aerial respiration is accomplished in terrestrial animals through special internal surfaces which must be kept moist. In insects a system of branched tubes called tracheae, which open through spiracles along the sides of the body, distribute oxygen to and receive carbon dioxide from all of the cells of the body. In pulmonate snails the "lung" is simply an invagination of the skin, as are also the tracheae of insects. The real lung is a development found in the terrestrial vertebrate, and it is a specialized surface derived from the anterior or pharyngeal portion of the digestive tube. In higher vertebrates, such as birds and mammals, they are extensively lobed, and made spongy by the innumerable small air sacs which provide the enormous respiratoly surface necessary. It has been estimated that if all of these pitlike alveoli of the internal lining of the lungs of the average human being were spread out in an even surface, the area of it would be more than 100 square yards. The mechanism for the accomplishment of breathing in the cat and other mammals by the use of the diaphragm and thoracic wall is described in the chapter on mammals.

The muscles which control these actions are automatically stimulated through the nervous system to contract when the carbon dioxide level of the blood reaches a certain point. A respiratory center, located in the medulla oblongata, is affected by the carbon dioxide and determines the rate of respiratory movements. There are also nerves from the lungs themselves which extend to this center and contribute to the maintenance of the proper rhythm. Abundance of venous blood stimulates an increase of the respiratory action. In addition to exchanging gases the lungs also discharge moisture and give off a certain amount of heat.

The Circulatory System.-The circulatory system is a closed system of vessels supplying all parts of the body with blood and a system of spaces, sinuses, and vessels collecting lymph from the various organs to return it to the blood vessels. The blood circulatory system centers in a contractile heart from which tubular arteries lead out to various organs of the body where they branch into minute vessels or capillaries. The capillaries converge as they carry the blood away from the organs to form the veins which carry the blood back to the heart. This is a closed system of vessels. The blood is cumposed of the clear fluid, plasma, and the blood corpuscles. The red corpuscles contain the red pigment matter, hemoglobin, which 
was mentioned in eonnection with respiration. Due to this substance, the cells have oxygen-carrying power. The white corpuscles or leucocytes are of several varieties and they are amoeboid in character. These cells may make their way among cells of other tissues

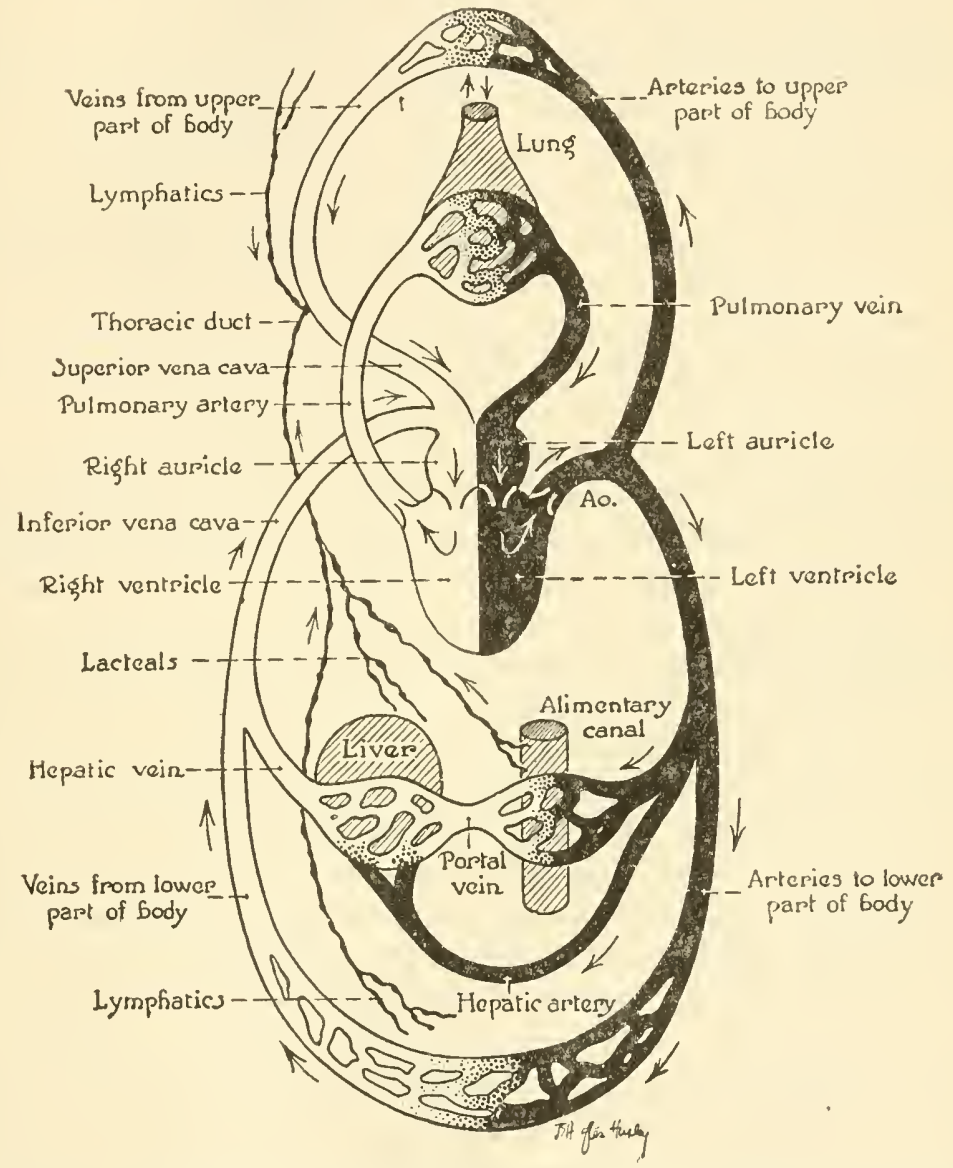

Fig. 219.-Diagram of circulation of the blood in a mammal. The oxygenated blood is shown in black; the venous blood in white. The lymphatics are the black irregular lines. (From Pettibone, Physiological Chemistry, published by The C. V. Mosby Company.)

where they engulf bacteria and foreign matter. Upon exposure to air the dissolved fibrinogen in the blood becomes fibrin and forms a clot which is semisolid and blocks flow of blood from most wounds. The remaining fluid after the blood clots is ealled serum. Lymph 
is a fluid similar to plasma which has seeped through the walls of the capillaries in the various organs, and it carries amoeboid white corpuscles. Certain of them are produced in the lymph glands. The spleen is a lymphoid organ in which debilitated red corpuscles are disintegrated and the products placed in the blood.

Circulation.-Transportation of materials through the protoplasm of a single cell or a single-celled organism and from cell to cell of the metazoan is a fundamental function among living things. In most Protozoa there is no special arrangement for this function, but the necessary exchange and movement of food materials, waste substances, and gases is accomplished by simple diffusion of materials. In a few forms, however, of which paramecium is an example, there is a definite course of movement by the endoplasm. This is known as cyclosis, and it serves to circulate the food vacuoles.

In double-walled, simple, saccular forms like hydra there is no provision necessary except an exchange of the water in the gastrovascular cavity. In flatworms, such as planaria, the necessity of increased food distribution is cared for by branching of the gastrovascular cavity into diverticula. In sponges the wandering cells assist in transporting materials. A distinct system of tubelike vessels with contractile parts is developed in the annelid worms, as was studied in the earthworm. Here a closed system of vessels forms a complete circuit to carry a circulating medium to all parts of the body. In this group the fluid is known as hemolymph because it bears no red corpuscles. The hemoglobin is borne in the fluid. The vertebrate system is closed, and the blood is circulated by the action of a single heart. The hemoglobin, an iron compound, is carried in the red blood corpuscles. In molluses and some crustaceans there is a similar respiratory pigment carried in the plasma, which is called hemocyanin. Instead of iron, copper is the principal constituent of this pigment. Vertebrate blood is largely water carrying dissolved materials and suspended corpuscles. The fluid part is known as plasma. The amount of blood in a mammal is approximately one-twentieth of the body weight, or in the average man a little more than a gallon. The plasma contains enough inorganic salts to taste slightly salty. Its salt content is about equal to that of sea water. When the body is active, the blood is very unequally distributed. One-fourth is always in the heart, large arteries, veins, and lungs. Another fourth is held in the hepatic portal system, the liver and its sinuses; the 
skeletal muscles require another fourth; and the remaining fourth is distributed through all of the other organs. Human blood contains normally about 5,000,000 red corpuscles (erythrocytes) per cubic millimeter of volume in the male and about 4,500,000 in the female. The average person, weighing 150 pounds, then, would possess approximately 20,000,000,000,000 (20 trillion) of them. Each erythrocyte is essentially a little capsule filled with hemoglobin which is a compound peculiarly fitted to unite with atmospheric oxygen. When united with oxygen it is known as oxyhemoglobin, which is readily reduced to give up the oxygen to the cells when the blood reaches the tissues. The carbon dioxide given off by the cells is collected principally in the plasma and returned to the lungs.

The leucocytes or white corpuscles are quite variable in form and number from 6,000 to 10,000 per cubic millimeter. They are amoeboid and therefore not confined to the blood vessels. One of their chief functions is the destruction of bacteria and other foreign material in the tissues. This process is known as phagocytosis. The accompanying table summarizes essential information concerning blood cells.

The plasma of the blood contains a group of substances called antibodies. These have been produced by various tissues of the body upon contact with certain foreign proteins. Since bacteria and pathogenic Protozoa react as foreign protein, they stimulate the body tissues to the production of specific protective antibodies and physicians have come to make use of these antibodies in sterile serum for prevention and treatment of several diseases. Some of these antigen substances bring about the clumping or agglutination of foreign bacteria, others dissolve the bacteria, and still others cause them to be precipitated. The chemical nature of these bodies is not yet known.

There are individuals known as hemophiliacs or bleeders whose blood will not clot, and any wound is likely to be fatal. The plasma normally contains a soluble protein, called fibrinogen and calcium in solution. Howell's theory of coagulation of blood holds that there is also an inert substance, antithrombin, which prevents the activation of the prothrombin of the plasma to become thrombin. When blood is shed and exposed to air, the blood cells and platelets produce a substance, cephalin, which, in the presence of calcium, neutralizes the antithrombin, allowing the formation of thrombin. Thrombin reacts with fibrinogen to produce fibrin, the solid fibers of the clot. The rate 
Average Characteristics of Human Blood Cells

\begin{tabular}{|c|c|c|c|}
\hline $\begin{array}{l}\text { KINDS OF CELLS AND } \\
\text { AVERAGE NUMBER } \\
\text { PER CUBIC MILLI- } \\
\text { METER OF BLOOD }\end{array}$ & $\begin{array}{c}\text { STRUCTURE } \\
\text { COLOR WITH } \\
\text { WRIGHT'S STAIN }\end{array}$ & SOURCE & FUNCTION \\
\hline $\begin{array}{l}\text { Erytlirocytes } \\
\text { (red blood cells) } \\
5,000,000 \text { (males) } \\
4,500,000 \text { (fe- } \\
\text { males) }\end{array}$ & $\begin{array}{l}\text { Nonnucleated, circu- } \\
\text { lar, biconcave; } \\
\text { orange buff ; } 7.5 \\
\text { to } 7.7 \text { microns in } \\
\text { diameter }\end{array}$ & $\begin{array}{l}\text { Endothelium of } \\
\text { capillaries of } \\
\text { bone marrow }\end{array}$ & $\begin{array}{l}\text { Transport oxygen; } \\
\text { remain in blood } \\
\text { vessels }\end{array}$ \\
\hline $\begin{array}{l}\text { a. Neutrophile } \\
60 \text { to } 70 \% \\
\text { b. Eosinophile } \\
2 \text { to } 4 \% \\
\text { c. Basophile } \\
0.5 \text { to } 1.5 \%\end{array}$ & $\begin{array}{l}\text { Colorless in life } \\
\text { Nucleus of lobes } \\
\text { joined by thread; } \\
\text { stains dark lilac, } \\
\text { cytoplasm pale; } \\
\text { blue with gran- } \\
\text { ules; } 9 \text { to } 12 \text { mi- } \\
\text { crons in diameter } \\
\text { Granules stain } \\
\text { weakly } \\
\text { Granules few, eosin } \\
\text { (red) } \\
\text { Granules deep blue }\end{array}$ & $\begin{array}{l}\text { Reticuloendothelial } \\
\text { cells outside } \\
\text { capillaries of } \\
\text { bone marrow }\end{array}$ & $\begin{array}{l}\text { Amoeboid; can } \\
\text { leave blood ves- } \\
\text { sels and enter } \\
\text { tissues } \\
\text { Defend against in- } \\
\text { fection }\end{array}$ \\
\hline $\begin{array}{l}\text { 2. Lymphocytes } \\
20 \text { to } 30 \%\end{array}$ & $\begin{array}{l}\text { Nucleus single, } \\
\text { large, round, deep } \\
\text { blue; scant cyto- } \\
\text { plasm, clear blue } \\
4 \text { to } 10\end{array}$ & $\begin{array}{l}\text { Lymphoid tissue, } \\
\text { spleen and } \\
\text { lymph glands }\end{array}$ & $\begin{array}{l}\text { Nonmotile; related } \\
\text { to immunity }\end{array}$ \\
\hline $\begin{array}{l}\text { 3. Monocytes } \\
5 \text { to } 10 \%\end{array}$ & $\begin{array}{l}\text { Nucleus single, } \\
\text { large, round, deep } \\
\text { blue; much cyto- } \\
\text { plasm, muddy } \\
\text { blue: } 12 \text { to } 20\end{array}$ & $\begin{array}{l}\text { Spleen and bone } \\
\text { marrow }\end{array}$ & $\begin{array}{l}\text { Tery motile; } \\
\text { phagocytic }\end{array}$ \\
\hline $\begin{array}{l}\text { Platelets } \\
200,000 \text { to } 400,000\end{array}$ & $\begin{array}{l}\text { Small, refractile, no } \\
\text { nucleus; dark } \\
\text { blue to lilac; } \\
2 \text { to } 3\end{array}$ & Bone marrow & $\begin{array}{l}\text { Provide substance } \\
\text { needed in elotting }\end{array}$ \\
\hline
\end{tabular}

(Reproduced by permission from Textbook of Zoolomy by Storer, copyrighted 1943, by McGraw-Hill Book Co., Inc.)

of the heartbeat for an average adult man at rest is about 72 times per minute. The contraction phase of the heartbeat is called the systole and the relaxation phase is the diastole. It has been estimated that an average circuit of the circulation of blood in man can be completed in twenty-three seconds. with about two seconds of this time being spent in capillaries.

The Excretory System.- The excretory system of vertebrates consists of kidneys, excretory ducts, and often a urinary bladder. The kidneys serve to remove from the blood, waste nitrogen products and excess salts in solution as well as to dispose of excess water. In simpler vertebrates there is a pronephros type of kidney as well 
as a mesonephros. The former is seldom functional, but the latter is the functional organ in vertebrates up to and including the Amphibia, as in frogs and salamanders. The metanephros is the higher developed kidney as found in reptiles, birds, and mammals. The ureter is the excretory duct which leads from the metanephric kidney. The life history of these animals as individuals includes successive stages as follows: the pronephros, the sole kidney for a time; followed by the mesonephros which is the dominant functional excretory organ when in its glory; and, finally, the development of the metanephros with retrogression of the others. This is an illustration of the Theory of Recapitulation which says that each individual in its development lives through abbreviated stages of the history of the development of the race.

Excretion.-A certain result of the oxidation necessary for metabolism is the production of end-products which are not only of no further use to the protoplasm but may be a distinct menace to the welfare of the organism because of their toxic effects. The substances are usually dissolved and removed as a waste liquid or occasionally as crystals by special parts of the body.

In Protozoa this function is performed by general diffusion through the plasma membrane and in many forms by the contractile vacuoles. The quantity of water which passes through the protozoan in twentyfour hours is several times the volume of the animal itself. Among sponges and coelenterates diffusion of liquid wastes through the general surfaces of the body to the surrounding water serves for excretion.

In an animal like the flatworm, planaria, excretion is accomplished by a system of canals which begins in numerous capillary-sized tubules whose blind ends are composed of individual cells called flame cells. These flame cells are irregular in shape and each bears a tuft of cilia extending into the end of the tubule. The flickering movement of the cilia in the cell gives the appearance of a flame and moves the accumulated excretion down the tubule. The waste liquid of the surrounding tissues diffuses into this cell. The main excretory ducts open to the surface of the body by excretory pores. This arrangement is sometimes called a protonephridial system.

The nephridial system is found in Annelida and has been studied in connection with the earthworm. Here a coelomic cavity is present, and a series of segmentally arranged pairs of coiled tubes or 
nephridia extend through the wall to the exterior. The excreted wastes accumulate in the coelomic cavity and are moved into the nephridia through the ciliated funnellike internal end, known as the nephrostome. This coelomic fluid is drawn into the canal of the nephridium by the beating of the cilia and is delivered to the outside of the body at the nephridiopore of the next segment.

The green glands of crayfish are much more concentrated, although they are modified nephridia. They function as a pair of unit organs, each opening by a duct on the basal segments of the antennae. In mollusks there are both nephridia, known as pericardial glands, and the special cells formed from the coelomic epithelium. The echinoderms make use of direct diffusion as well as intracellular excretion by which excreted materials are taken up from the coelomic cavity by the numerous phagocytic, amoeboid cells of the coelomic fluid. These cells wander out into the eavities of the respiratory organs where they coalesce into large masses, and finally with their enclosed granules are east out through the membranes of the respiratory papillae. Soluble materials in solution also diffuse through the membranous walls of these structures. In the insects excretion is taken care of by the Malpighian tubules, which are considered modified nephridia. They are bunched in the posterior part of the body cavity and discharge excretions into the intestine at its junction with the rectum.

Kidneys.-The chief excretory organs of vertebrates are called kidneys, and they are thonght by some authors to have developed by modification and condensation from segmentally arranged nephridial tubules. The fact that in vertebrate embryos as well as in lower cliordates, even the frog, these tubules open into the coelom as nephrostomes, makes it seem possible that in vertebrates as well as in annelids the coelom was once important in excretion. The essential structures of the kidney for taking waste substances from the blood and delivering it to the exterior of the body are the Malpighian corpuscles, each made up of a glomerulus and a Bowman's capsule, and the coiled uriniferous tubules which discharge the excretion through collecting tubules into the ureter at the pelvis of the kidney. This canal leads to the cloaca in most vertebrates below mammals (excepting some fish), or to a wrinary bladder in the mammals. 
The wall of each Bowman's capsule is very thin and readily permits diffusion of water and dissolved materials from the blood into the cavity of the uriniferous tubule on the opposite side of the membrane. The glomerulus carries arterial blood from the afferent arterial branch and discharges it into the efferent arterial branch. The latter soon spreads into a capillary network which surrounds the convoluted portions of the uriniferous tubule. Water constitutes the largest volume of materials to be excreted in most animals, except in some desert forms where water is conserved and the excretion is in crystalline form. Water is eliminated by lungs, skin, alimentary canal, and kidneys. In man the quantity of sweat discharged may amount to two or three liters a day. In the dog, which has few sweat glands, the water eliminated by the lungs, through panting, is proportionately greater than in man. The kidneys are the most important organs in the excretion of water, and the amount they eliminate is inversely proportional to the amount excreted by the skin. Most of the water to be excreted is taken from the blood in Malpighian corpuscles.

Some of the nitrogenous wastes are excreted in the form of ammonium salts and some free or combined amino acids. However, most of the ammonia which results from protein metabolism is converted into urea in the liver and is carried in that form to the kidneys where it is removed from the set of capillaries ramifying over the convoluted tubules by a process of true secretion. According to this idea, the urine which consists of urea, various salts, other soluble materials, and water is excreted by different parts of the uriniferous tubule. The substances which are excreted by the kidney are not formed there, but are merely removed from the blood by this organ.

The Nervous System.-The nervous system in this type of animal is composed of a brain and spinal cord forming the central nervous system; nerves extending to all parts of the body, ganglia which are groups of nerve cell bodies outside the central nervous system, and the sense organs which serve for receiving stimuli are usually grouped together under the name peripheral nervous system. A portion of this latter division, consisting of two longitudinal trunks with ganglia distributed along them, lies parallel to the spinal cord, and constitutes the sympathetic system. Each ganglion has a connection with the adjacent spinal nerve or cranial nerve as the case 
might be. This system controls the involuntary muscles. The peripheral system includes ten to twelve pairs of cranial nerves from the brain, and ten to thirty-one pairs of spinal nerves in different forms of vertebrates. Each spinal nerve has two roots where it joins the spinal cord. A dorsal root receives fibers from sensory endings and therefore conducts impulses toward the cord. This root has a spinal ganglion located on it. The ventral root of each of these

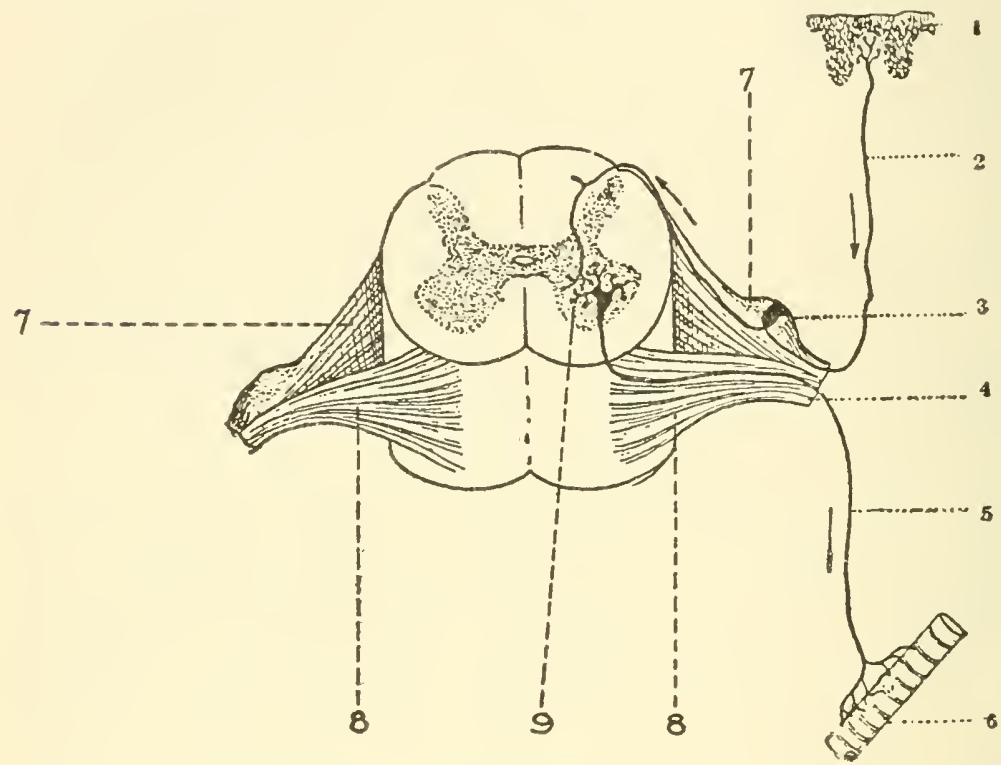

Fig. 220.-Cross section of spinal cord and roots of spinal nerves, showing a simple reflex circuit. 1 , sensory surface of skin; 2 , afferent nerve fiber with $s$, its cell of origin, located in the spinal ganglion; 4 , cut end of spinal nerve; 5 , efferent nerve fiber; 6 , voluntary muscle; 7 , dorsal root of spinal nerve; 8 , ventral root of spinal nerve; 9, dendrites of motor nerve cell body in gray matter of the cord. (From Zoethout, Textbook of Physiology, published by The C. V. Mosby Company, after Morat.)

nerves earries fibers extending from the motor cells in the cord to the motor end plates on the voluntary muscle cells. The impulses, therefore, pass from the spinal cord to the muscles over these roots. The reflex arc, which is the simplest kind of a nerve conduction cireuit, is set up by the connectives from the sense organ or receptor to the gray matter of the cord and then the return connection from the motor nerve cells over the ventral root to the muscles. In general, the relationship of parts in regard to function is similar to what has already been seen in the higher nonchordate animals. 
A high development of sense organs for the senses of sight, hearing, smell, taste, and touch is characteristic of vertebrates. The organs are receptors and they are stimulated by changes in external environmental conditions, such as light, sound waves, chemical changes, and contact. The eye, which is the organ of sight, is a highly developed organ. It is constructed on the plan of a camera with the eyeball forming the light-tight box. The wall of this is composed of an outer fibrous sclera (white of eye) which continues anteriorly as a transparent front, the cornea. Beneath the sclera is a black, pigmented and vascular layer, the chorioid, which continues anteriorly as the iris, the colored part of the eye. The iris is like a curtain

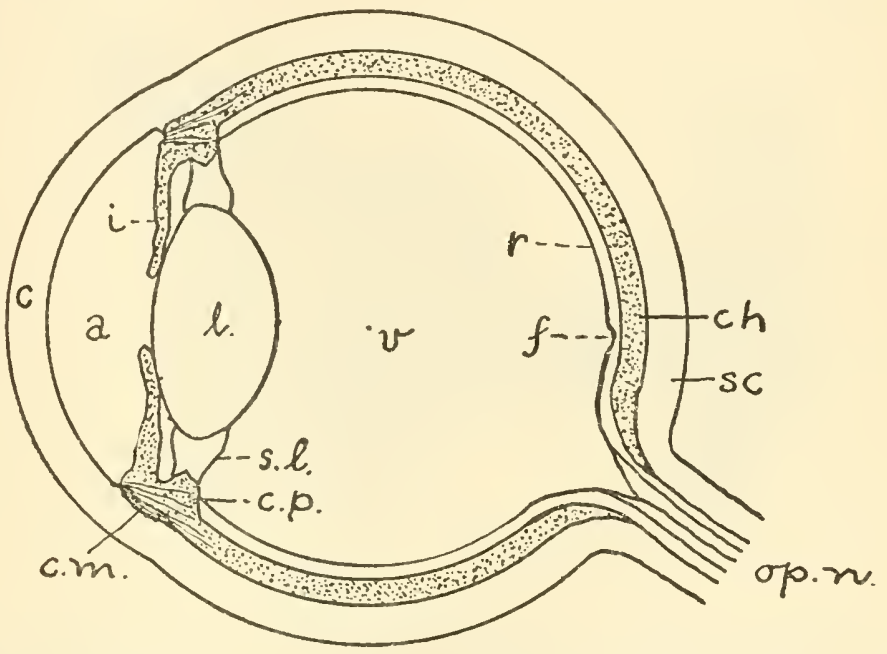

Fig. 221.-Diagram of the eycball ; $c$, cornea; $a$, aqueous humor; $l$, lens; $v$, vitreous humor: $s c$, sclerotic coat; $c h$, chorivid coat : $r$, retina : $f$, fovea centralis ; $i$, iris ; s.l., suspensory ligaments; c.p., ciliary process; c.m., ciliary muscles; op.n., optic nerve. (From Zoethout, Textbook of Physiology, published by The C. V' Mosby Company.)

surrounding a space at the anterior surface of the eye and this space between its medial margins is the pitpil. The pupil appears black because there is no light behind it. Behind the pupil is a transparent lens whose surfaces are curved to bend the rays of light in such a way as to focus them on the sheetlike retina behind. The retina is a lateral extension of the brain and is the sensory part of the eye. It lies as a lining of the inside of the posterior half of the cavity of the eye and is comnected directly with the brain by the optic nerve. The general cavity of the eyeball is divided into some chambers. The 
external or aqueous chamber between the cornea and the lens, with the iris extending into it, is filled with aqueous humor. This cavity is subdivided by the iris. Behind the lens is the large internal or vitreous chamber which is filled with a jellylike vitreous humor. The curvature of the lens can be controlled by the action of the ciliary muscle which encircles its margins. This makes possible an adjustment of the eye to near and far objects and particularly so in higher vertebrates. This power is known as accommodation. As people get older they tend to lose this accommodation because of loss of elasticity in the lens. The tension on it due to the attachment of the inside of the eyeball by the ciliary process tends to hold it out

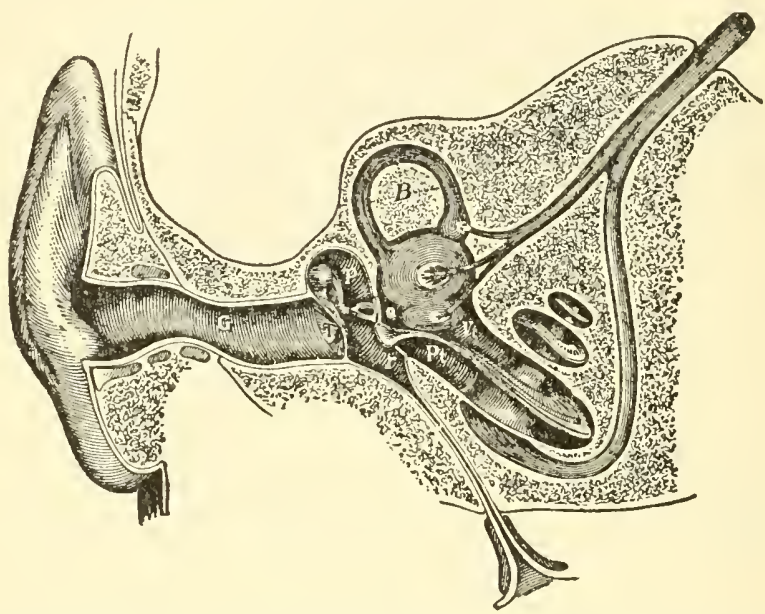

Fig. 222.-Diagram of a section through the right ear. $B$, semicircular canal; $G$, external auditory meatus; $o$, oval window (fenestra ovale); $P$, tympanic cavity containing the three auditory ossicles: $P t$., scala tympani; $r$, round window (fenestra rotunda); below $r$ is seen the Eustachian tube; $S$, cochlea; $T$, membrana tympani; $V t$, scala vestibuli. (From Zoethout, Textbook of Physiology, published by The C. V. Mosby Company, after Czermak.)

in a flattened condition. This focuses the eyes very well on distant objects but does not provide the necessary curvature of the lens to bring near objects in focus. Eyeglasses are used by older people to supply this lost phase of accommodation. A ray of light enters the eye by passing through the cormea, then aqueous humor, pupil, lens, vitreous humor and then to the retina where the sensory cells are stimulated and the impulse carried to the brain by the nerve fibers of the optic nerve. 
The ear structures provide most classes of vertebrates with facilities for two functions: hearing and equilibrium. This organ consists of an external ear, which serves in eatching and directing sound waves within, a middle ear or tympanum, containing ossicles, and the inner ear, which contains the sensory cochlea with its organ of Corti for hearing, and the semicircular canals, which are concerned with equilibrium rather than hearing. The latter are common to all vertebrates while the cochlea is limited to Amphibia and higher classes. The external ear is still further limited to reptiles, birds, and mammals. The middle ear is a space beneath a tympanic membrane which separates it from the external auditory canal. In this cavity are three bony ossicles, the malleus, incus, and stapes, which transmit the sound vibrations from the tympanic membrane to the membrane over the fenestra ovalis leading into the internal ear. The mem. branous labyrinth is the name often applied to the chambers of the inner ear. Its ventral chamber is the sacculus connected with the organs of hearing, and the dorsal portion is the utriculus which is related to equilibrium. The two semicircular canals in simpler forms and the three in higher, join the body of the utriculus in as many different planes as there are canals. In the higher forms there are two vertical canals, one anterior and one posterior, with their planes at right angles to each other, and one horizontal canal. At one end of each canal there is a bulblike swelling or ampulla which contains sensory hairs. When the position of the head is moved, the fluid in the canals changes its level and position to stimulate the sensory hairs, thus giving a sense of position. The sound waves which stimulate the sensory cells of hearing enter the external ear and set up vibrations in the tympanic membrane. These are in turn transmitted by the ossicles to the fluid endolymph within the labyrinth. The vibrations of the fluid extend through the cochlea, in which the sensory cells are supported on the organ of Corti stretched across it. These cells are connected with the brain by way of the auditory or eighth cranial nerve (Fig. 222).

The sense of smell is centralized in the epithelial lining of the nasal chamber. Special olfactory cells are stimulated by particles of material from the air dissolving on this membrane and making contact with the sensory cells. The sense of taste is similar except that it is located in sensory cells in taste buds on the tongue, epig]ottis, and 
lips (and barbels of some vertebrates). The particles come in by way of food and drink and as the material dissolves, it reaches the taste cells.

Most of the tactile and pressure sense organs are located just beneath the skin over different parts of the body. A few of the pressure sense organs are found in certain of the internal structures of the body. The lateral line system in fishes is sensory to vibrations carried in the water and is quite important to aquatic animals of this type.

Nervous Function-Reception and Conduction.-Irritability and conductivity are fundamental functions of all protoplasm, whether. it be in the body of an Amoeba or a man. The responsiveness of organisms to change of conditions both externally and internally determines their behavior. Living protoplasm is not only excitable, but it possesses the power to record or store up the effects of previous stimuli. In the final analysis, the perceptions and reactions of man are but expressions of these primitive functions in a more specialized organism.

The protozoan organism has only neuromotor apparatus and depends largely on the primitive properties of irritability and conduetivity to guide its activities. In the simpler Metazoa, such as the coelenterates, there are scattered nerve cells connected with each other by fibers to form a nerve net. The neuroepithelial or neuromuscular cells which make up this continuous net through the body are the forerunners of the typical neurone and are called protoneurones by Parker. A protoneurone transmits in every direction while a true neurone transmits in only one. In the net system there is no central exchange and no specific path of conduction. Every part of the receptor surface of such an organism is in physiological continuity with every other part of the body.

Next comes the linear type of nervous system in the form of a ladder. It is composed of an organization of neurones into a double chain of ganglia, each cord lying lateral to the digestive tract with transverse connectives and predominant ganglia at the anterior end. Such a system was studied in planaria. In Annelida and Arthropoda the nervous system is a modified ladder type in which the two longitudinal cords of ganglia have fused along most of the midventral line. Toward the anterior end, the cords separate at a paired gan- 
glionic enlargement, the subesophageal ganglion, and encircle the alimentary eanal to join on the dorsal side as the pair of supraesophageal ganglia or "brain." In Arthropoda the ganglia of the thorax have undergone considerable fusion. In Echinodermata, the starfish for example, the central group of ganglia makes up the circumoral nerve ring around the mouth, and radial branches extend into each arm. Branches from these communicate with the sensory structures of the skin and tube feet.

Concentration of the tissue of the nervous system into definite organs is carried farther in vertebrates than in the less highly organized forms. The fact that the central nervous system of vertebrates is dorsally located and hollow has been brought ont previously. Even within the group of vertebrates, the nervous system shows a progressive increase in complexity. The highly developed brain of the mammal is the climax of this tendency.

The neuroncs have been referred to before as the units of structure and function of the higher type of nervous system, from worms to man. Each neurone is a nerve cell with processes extending from it, and each of these units must conduct nerve impulses in its normal function. The exact nature of the nerve impulse is still somewhat of a question. It is thought to be transmitted as a metabolic change passing along the nerve fiber (axone or dendrite). This is at least partially a chemical change in which oxygen is necessary and a certain amount of carbon dioxide is produced, but since there is only slight inerease in temperatnre during the change, it seems not to be a typical metabolic oxidation process ; furthermore, the activity seems not to fatigue the nerve fiber. An electrical charge follows the wave of activity along the nerve fiber, but it apparently accompanies the impulse or is a result of it rather than the impulse itself. The speed of electrical transmission has been measured in a number of different animals and nervous transmission is much slower than electrical. At room temperature the sciatic nerve of a frog will transmit a nerve impulse at the rate of about 100 feet per second. Conduction over nonmedullated fibers of invertebrates is much slower than this. On the other hand, measurements of the rate of conduction in man show a velocity of about 400 feet per second.

The reflex arc and reflex actions illustrate the simple form of nervous conduction circuit. In its simplest form the reflex are is com- 
posed of one motor and one sensory neurone; however, it is usually more complex. The classical example involves the spinal cord and a spinal nerve. This is known as a reflex of the first level, because it returns the motor impulse over the motor fibers of the same nerves which brought in the sensory impulse. The motor axone carrying the impulse from the motor nerve cell in the gray matter usually ends in a muscle cell or a gland. There is no protoplasmic union between the axone of the sensory neurone and the dendrite of the motor, for these come in contact only by a synapse which brings them in close proximity. It has been found experimentally that nervous impulses may be conducted in either direction by the fiber but can cross a synapse only from axone to dendrite, thus serving like a valve in a pipeline. Reflex actions may be in the form of motion, as withdrawal from unexpected pain, or shivering or formation of goose flesh, or the contraction of the pupil of the eye with increased light intensity. Still other reflex actions include secretion by glands, breathing, movements of speech, individual actions included in walking, and others.

Functions of the Spinal Cord.-This organ serves as a system of reflex centers which control the actions of glands of the trunk, visceral organs, and skeletal muscles. The spinal cord is also a nervous pathway between the brain and numcrous organs of the body. It is said that more than half a million neurones join the cord through the doisal roots of the spinal cord.

Functions of the Divisions of the Brain.-Conscious sensations and intelligence are centered in the gray matter or cortex of the cerebrum. This section controls voluntary actions and provides memory associations. The diencephalon serves as a center for spontaneous actions. The midbrain is one of the centers of coordinated movement which has to do with posture and eye muscles. The cerebellum is another center of coordinated movement, particularly with reference to equilibrium. The impulses from the muscles, tendons, joints, and semicircular canals of the ear are coordinated so that in a movement or posture the proper muscles may be contracted to the proper extent at the proper time. Below and behind the cerebellum is the medulla oblongata which controls breathing and may be an inhibitor on heart action. It also regulates digestive secretions, movements of digestive organs, and vasomotor activity of the blood 
ressels. As a whole, the brain serves as the organ of communication between the sense organs and the body and is the coordinator of the bodily activities.

The Reproductive System.-The rertebrate reproductive system shows a fairly high degree of development. The sexes are almost universally separate, with the exception of some cyclostomes. The distinct gonads develop to produce special germ cells. The male gonads are testes, and they prodnce spermatozoa which are carried from the gonads by the vasa deferentia. The female gonads are ovaries, and they produce ova or eggs. They are carried from the body by oviducts. The males of some classes possess for use in copnlation certain accessory organs which tend to insure fertilization. The vertebrates which lay eggs are spoken of as being oviparous: in those in which the egg is retained in the body and the embryo develops there, feeding on the yolk of the egg, and is later born alive, the condition is known as ovoviviparous, and in the forms in which the fertilized ormm is retained in the uterus, the embryo being nourished by diffusion of nutriments from the blood of the parent, the condition is said to be viviparous, and here too the young are born alive. In vertebrates the possible offspring produced each season by a single individual varies from one to thousands.

Reproductive Function.-A living organism is in numerous ways similar to a machine, but reproduction of new units of living material by existing organisms is hardly comparable to any mechanical processes known in our industries. New organisms all arise from preexisting organisms of the same kind. The process of cell division is the fundamental basis for all reproduction. For centuries before the invention of the microscope it was commonly believed that living things arose spontaneously from nonliving material, or from the dead bodies of plants and animals. Certain old books carry directions for the artificial generation of mice or bees. Louis Pasteur did as much as anyone to diseredit this idea of spontaneous generation. Our present eonception is that the protoplasmic substance of the new individual is but a continuation of the specific protoplasm peculiar to an earlier individual or in sexual reproduction to two individnals. Therefore, under ordinary eircumstances the structural and physiological complexities which arise through embryonic development must be generally similar to those of the predecessors. 
In most of the single-celled organisms reproduction may occur by such equal division of the protoplasm (binary fission) that the new individuals cannot be distinguished as parent and offspring. Protozoa may reproduce also by sporulation, by which process the cell forms a protective cyst and by a series of simple divisions (fragmentation) the internal protoplasm breaks into a number of smaller units. Following this the cyst ruptures and releases these new units as independent individuals. For the most part, reproduction among protozoans is taken to be asexual, but according to a recently published work by Sonneborn, a distinct sexuality exists in paramecium. Examples of asexual reproduction by budding and fission have already been pointed out in the studies of reproduction of sponges, hydra, planaria, and even in tunicates.

Sexual Reproduction.-In certain of the colonial Protozoa, volvox for example, the colony may reproduce for several generations by asexual division of the individual cells but sooner or later the cells of the colony become specialized into conjugating individuals. In some forms this goes to the extent of certain cells becoming distinct gametes with male and female characteristics. In such forms it is possible to see foreshadowed sexual reproduction as it is known in Metazoa.

In the simplest of Metazoa, as in sponges, there are no specially organized gonads for the production of germ cells, but as a rule the germ cells are produced in such organs set apart for this purpose. The ovary produces mature or nearly mature ova and the testis produces mature spermatozoa.

Hermaphroditism is the condition in which the same individual produces both ova and spermatozoa. It occurs principally in the simpler Metazoa, a few higher ones, and rarely among normal vertebrates. Previous studies made on the reproduction of hydra have brought out that the gonads are temporary, both being formed by aggregations of formative or interstitial cells between the ectoderm and endoderm. After the seasonal production of germ cells is completed, the gonads disappear. In flatworms and annelid worms the gonads are permanent structures of the mesoderm. Both ovaries and testes are present. Even in these true hermaphrodites cross-fertilization is insured by copulation or union in such a way that the spermatozoa of one individual fertilize the ova of another. In certain 
other hermaphroditic forms (as some cyclostomes) the spermatozoa and ova of a particular individual are usually not mature at the same time.

Bisexual reproduction is the form of reproduction common to many groups of the higher invertebrates and nearly all vertebrates. Here the sexes are distinct, each with functional gonads and accessory structures capable of producing only one kind of germ cells. In some of the types of animals already studied individuals of the two sexes have simply deposited the mature germ cells in the same vicinity and at about the same time. Under the sections on reproduction in starfish and the bullhead (fish) such a procedure has been described. In animals like the toads and frogs, a special provision is made to bring the individuals of the opposite sexes together in that the male clasps the female and sheds sperm over the eggs as they are expelled from the cloaca. This act is known as amphiplexus. It will be remembered that the first and second pairs of abdominal appendages of the male crayfish are modified for transferring spermatozoa into the seminal receptacle of the female, where they remain until the eggs are laid. This represents a beginning in the development of a copulatory organ. The majority of bisexual or dioecious animals make a still greater provision to insure fertilization of the ova by copulation or coitus. At the time of breeding the mature spermatozoa are delivered to the cloaca or vagina of the female, and the ova are fertilized within the genital tract of the female.

In birds and most reptiles after the addition of nutritive and protective coats the egg passes to the outside to develop and hatch (oviparous animals) but in all mammals, except monotremes, it is retained within the uterus during the period of embryonic development, and the young are born as more or less developed individuals (viviparous). In the females of viviparous mammals the posterior portions of the two oviducts are modified into a uterus within which the young are retained and nourished until ready for birth. The internal wall of the uterus and the external embryonic membranes (serosa and allantois-chorion) cooperate to form a placenta through which food, metabolic wastes, and respiratory gases diffuse between parental and embryonic blood. The blood does not pass from parent to embryo 
or vice versa but the necessary materials are allowed to diffuse through the tissue of the placenta in which both systems are distributed.

Parthenogenesis.-In some species of invertebrates, sexual reproduction may lapse for considerable periods of time, during which period no males are developed. The female produces ova which develop into new individuals like herself without fertilization for a whole season. This is known as parthenogenesis. Usually in the fall of the year males are developed, and fertile eggs, provided with protective hard shells, arc produced by the females of this generation to live through the winter. After winter is over such fertile eggs hatch into parthenogenetic females for the next season. This process is common in many smaller Crustacea, aphids, scale insects, some ants, bees, wasps, thrips, a few moths, and rotifers. Artificial parthenogenesis may be induced in many mature eggs by change of osmotic pressure due to change of salt content in the surrounding medium. Fatty acids, saponin, solanin, bile salts, benzol, toluol, chloroform, ether, and alcohol are other substances which will induce it. Electric stimulus, mechanical pricking, and change of temperature are also used. Such methods have produced artificial parthenogenesis in eggs of sea urchins, starfish, molluses, annelids, moths, and frogs. The immediate cause of the development by an egg thus stimulated is not known.

In normal fertilization of an egg by only one spermatozoon, it has been found that the rate of oxidation then increases from 400 to 600 per cent. There are indications that this is also the case in artificial parthenogenesis. This oxidation may be the cause of the development in the ovum. Fertilization, where it occurs, has a dual function: (1) that of stimulating the egg to develop, and (2) that of introducing the properties of the male parent.

\section{Classification}

In most recent classifications this subphylum is divided into seven classes; however, the second is sometimes found as a subclass under the third. These classes are as follows:

Cyclostomata (sī klō stō'mả tả, eircle and mouth). Round-mouthed fish with only median fins, unsegmented notochord, and jawless. Lampreys and Hagfish. 
Elasmobranchii (ē lăs mō brăn'kǐ ī, metal plate and gills). Fish with jaws, cartilaginous skeleton, persistent notochord, and placoid scales. Sharks, Rays, and Chimaeras.

Pisces (Pı̌s'ēs, fishes). True fish with bony skeleton, gill respiration, with jaws and paired lateral fins. Catfish, Perch, Bass.

Amphibia (ăm fřb’í á, both lives). Cold-blooded, nonscaled aquatic and terrestrial vertebrates with five-fingered, paired appendages. Most of them breathe by gills in the larval stage and by lungs in the adult. Toads, Frogs, and Salamanders.

Reptilia (rěp tǐl'ǐ à, crawling). Cold-blooded forms which are fundamentally terrestrial, usually possessing a scaly skin and breathing by lungs. Turtles, Lizards, Snakes, and Crocodiles.

Aves (a'vēz, birds). Warm-blooded, erect forms possessing feathers. The forelimbs have become wings. All birds.

Mammalia (mă mā'lì à, mammary or breast). Warm-blooded vertebrates with hair and with mammary glands for suckling the young. Cats, Men, Monkeys, Whales, Seals, Bats, etc. 


\section{CHAPTER XXV CYCLOSTOMATA*}

Because of the absence of jaws this group is sometimes known as Agnathostomata (ăg năth ō stō' må tả). This name is in contrast to Gnathostomata (jaw mouth) which includes all other vertebrates. The mouth of the cyclostomes is round, jawless, and suctorial. There are some exoskeletal teeth located on the roof and floor of the mouth and on the tongue. The body is slender and eel-like in shape. It is covered with a slippery, smooth skin and has only dorsal and ventral median fins.

\section{Classification}

The group is divided into two subclasses (or orders according to some authors) distinguishable by presence or absence of tentacles around the mouth, number of gill slits, and the number of semicircular canals. These subclasses are Myxinoidea (Hyperotreti) including the hagfishes; and Petromyzontia (Hyperoartii) including lamprey (or improperly, lamprey eel to some).

Myxinoidea or hagfish are all included in one family Myxinidae which is divided into three genera: Myxine of the Atlantic and Pacific Oceans, Bdellostoma and Paramyxine of the Pacific. These each have specific characteristics, but they all agree in having a terminal nostril, four tentacles on each side of the mouth, ability to produce enormous quantities of mucus, and the lack of the oral funnel or sucker. They all possess twelve or more pairs of gills, only one semicircular canal in the inner ear, and a functional pronephros. The development of the hagfish does not include a metamorphosis. They usually live in the mud of the sea bottom except when they are feeding either on the dead body of a fish or attached to a live one. They frequently enter the mouth or gills of fish caught in nets or those dead from natural causes and devour all of the inter-

- If the frog or toad is to be used as the laboratory animal representing the typlcal vertebrate, and the instructor so desires, Chapters XXV, XXVI, and XXVII may be omitted until after the study of Chapter XXVIII and then assigned if time permits. 
nal organs and flesh. They frequently attack living fish which have been otherwise injured.

Subclass Petromyzontia likewise includes only one family, Petro. myzontidae, which follows the type name. There are several genera including Petromyzon, the common Atlantic form, Ichthyomyzon of the lakes and streams and Entosphenus of the Pacific coast. Entosphenus tridentatus tridentatus is the northern form and $E$. tridentatus ciliatus is the southern form. The lampreys live in both salt and fresh water, and they are quite predaceous, attacking fish of considerable size. The characteristics of the group will be brought out under the discussion of Lamprey as a typical representative.

\section{Fconomic Relations of the Class}

In a general way lampreys are both beneficial and injurious. They all serve as excellent fish food and fish bait when they are in the larval stage. Brook lampreys are classed as wholly beneficial since they feed on microscopic organisms while larvae and do not feed as adults. Sea lampreys and lake lampreys are both valuable as human food, especially just preceding the spawning season. The sea lamprey, for the two or three years it spends in the ocean, lives at the expense of marine fish. It attaches itself and rasps a hole in the side of a fish about once a month, and through the hole thus formed, it sucks the fish's blood. One will remain to a single fish for about five days, get its fill, and release itself. The fish frequently dies as a result. Since the sea lamprey does not feed after it starts up stream, it does little harm to fresh-water fish except as the newly matured ones are making their trip to sea. The lake lamprey is similar except that it spends its entire life in fresh water. They are very destructive to lake fish since they are predaceous and spend their adult lives in the lakes.

\section{THE LAMPREY}

\section{Habitat}

All live on or in the muddy bottoms of fresh-water streams during larval stages. In adult life the sea lamprey goes to the open sea and the lake lamprey goes to the deep water of the lakes. Both return to the fresh-water streams to spawn a few years later. 


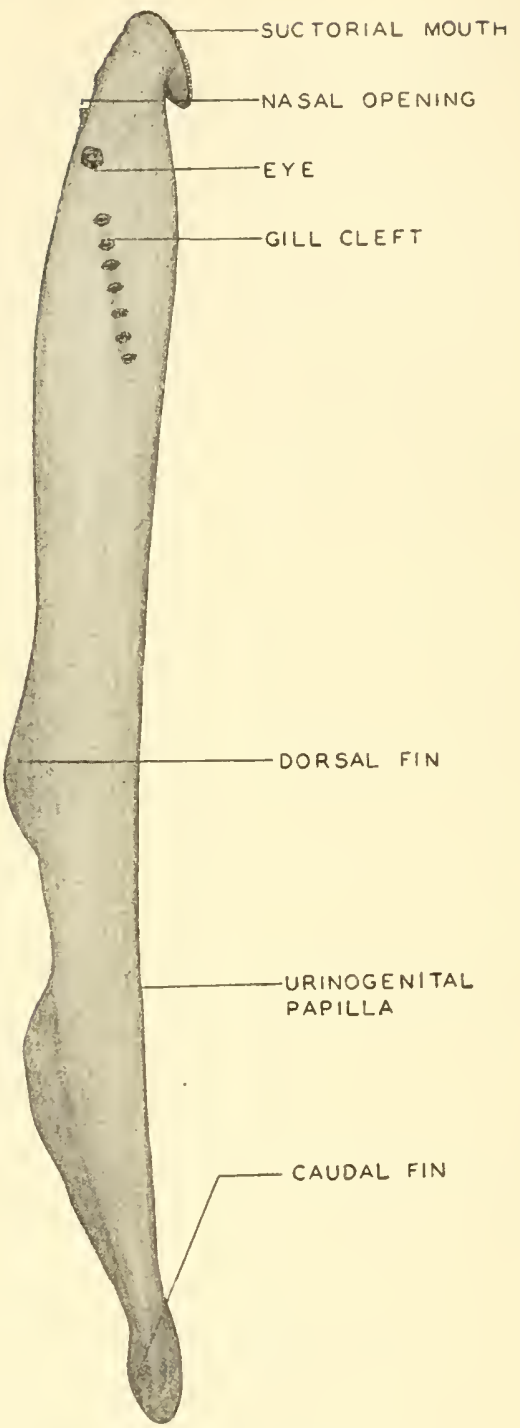

Fig. 223.-Lateral view of the Pacific lamprey, Entosphenus tridentatus. (Drawn by Titus C. Evans.) 


\section{Habits and Behavior}

The animal is a rather inebriate type of swimmer because it is long and slender and does not possess paired fins. It winds its way through the water and occasionally comes to rest by attaching itself to a rock by means of an oral funnel.

\section{External Structure}

In most respects the Atlantic lamprey, Petromyzon marinus, and the Pacific form, Entosphenus tridentatus, correspond quite closely in structure and make excellent representatives for study of the group. The following account will fit them generally. They may reach a length of three feet and three inches. The color is rather variable but might be expressed as being a variegated olive brown. Along the length of the lateral axis of the body are distributed sensory organs. There are two dorsal fins and a caudal fin, but no paired fins. At the anterior end of the animal is the mouth with the buccal funnel extending from it. This funnel is provided with chitinous teeth used in rasping through the body wall of the host fish. The annular cartilage supports the margin of the funnel and holds it open. Along the margin is a fringe of papillae. The mouth lies at the bottom of the funnel. In the floor of the mouth is a plungerlike tongue supported by a cartilage and bearing teeth. There are seven uncovered gill slits along each side of the anterior portion of the body. In front of the gills on each side of the head is a poorly developed eye. It has no lid, simply being covered with transparent skin. In a middorsal position on the head is located the single nostril which leads into an olfactory chamber, and on ventrally as a pituitary pouch or caecum. The anus is located in the midventral line a short distance anterior to the tail. Immediately behind it is the urinogenital opening at the tip of a papilla. The papilla is larger in the male specimens.

\section{Internal Structure}

The muscular system is quite primitive. It is principally a series of zig-zag myotomes along the length of the body very similar to those in Amphioxus. A large lingual muscle is differentiated for moving the tongue, and several bundles of muscular tissue radiate through the wall of the funnel to expand and contract it. 


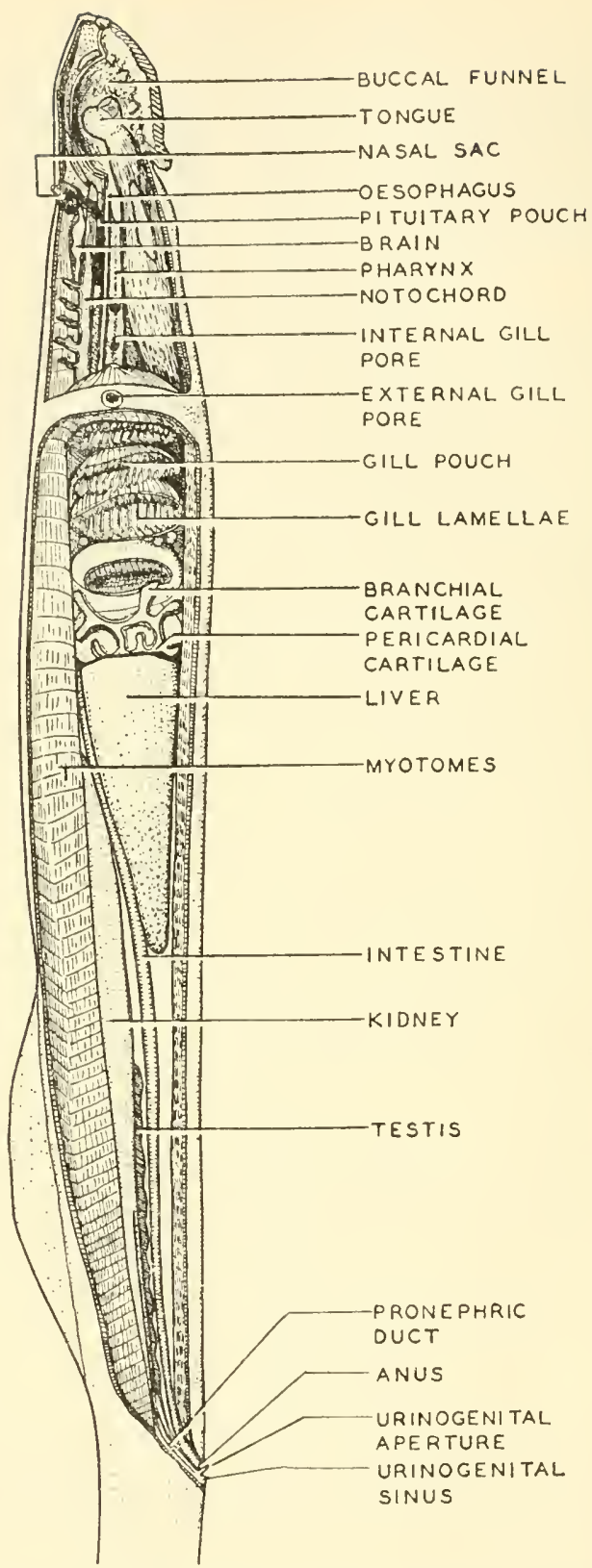

Fig. 224.-Lateral view of dissection of Entosphenus to show principal organs. 
The skeletal system is cartilaginous, developed around a nonsegmented notochord along each side of which are paired cartilages called neural arches. At the anterior end is a skull whose floor and sides are cartilaginous, while the roof is membranous, except for a transverse bar. There are two auditory capsules near the postcrior part of the skull. The buccal funnel is supported by the annular cartilage already mentioned and three sets of labial cartilages. The branchial area is supported by the cartilaginous branchial basket which is composed of a pair each of dorsal and ventral longitudinal bars, two pairs of sinuous, lateral bar's, and nine much-curved, dorsoventral bars. The anterior one of these is not in contact with a gill aperture. The cartilaginous pericardium joins the branchial basket at the posterior end.

The digestive system is not very highly developed because the adult lives entirely on blood and lymph of other fish, obtained by rasping a hole through the body wall and sucking it out. They take a meal about once in three or four weeks. The blood is passed from the mouth down the esophagus which continues into the intestine at the level of the posterior end of the branchial region. The intestine is slender and almost straight, but it has a slight internal fold which extends spirally through its length. This is called a typhlosole or spiral valve, and it tends to increase the absorptive surface. The intestine ends posteriorly at the anus. The liver is found in the anterior part of the body cavity.

The circulatory system consists of a heart with two principal chambers, arteries, capillaries, veins, and lymphatic spaces. The posterior and anterior cardinal veins located just lateral to the lower side of the notochord collect blood from the body wall and head region, and empty it into the common cardinal vein which extends ventrally to the sinus venosus. The sinus venosus receives also the single inferior jugular and the hepatic vein from the ventral region. The blood then passes through the sinuauricular valve to the single auricle, thence by the auriculoventricular aperture to the single vontricle, thence through bulbus arteriosus to the ventral aorta. Six pairs of afferent branchial arteries carry the blood to the gills where capillaries supply the gill lamellae. The efferent branchial arteries collect this blood, earry it dorsally to join the dorsal aorta which is made up by their convergence. A carotid branch of this artery supplies the brain region, and the main aorta passes posteriorly, 
giving branches to the viscera and body wall. There is no renal portal system; the caudal vein simply divides, giving one part to each posterior cardinal vein.

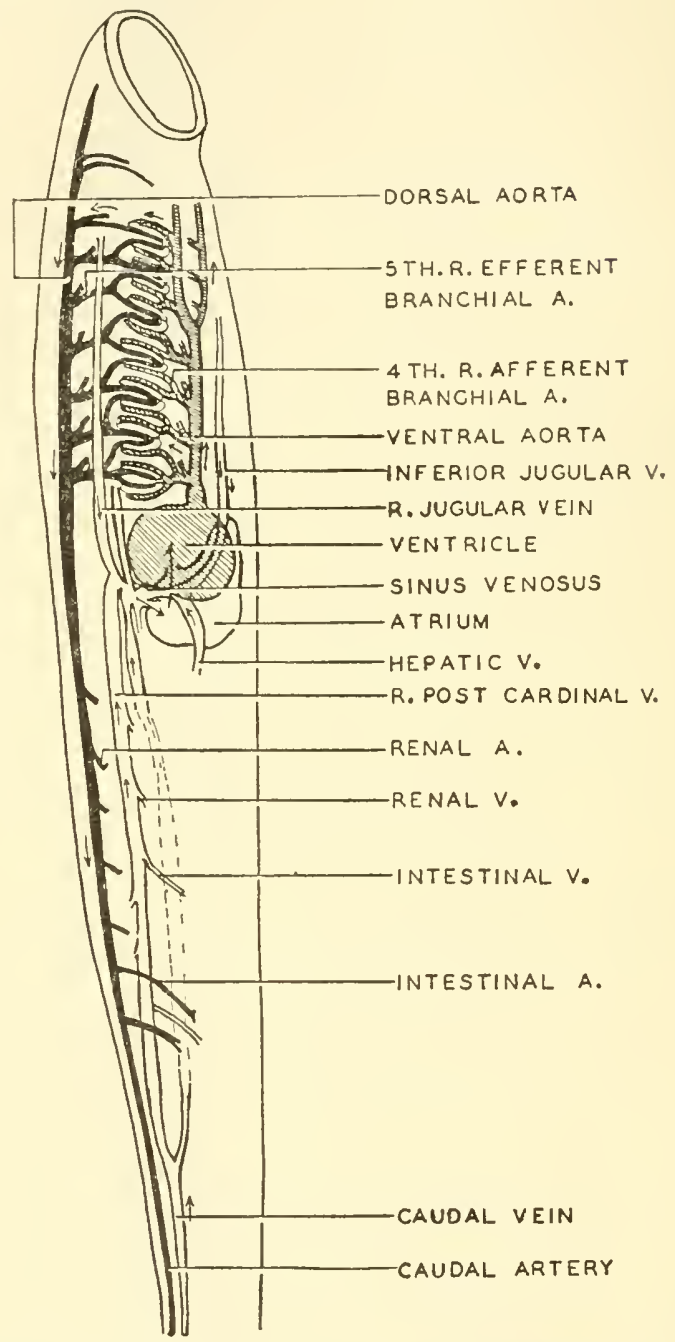

Fig. 225.-Diagram of oblique ventrolateral view of heart, arteries, and veins of lamprey. Arrows indicate direction of flow of blood. (Drawn by Titus C. Evans.)

The seven pairs of gills and respiratory tube constitute the principal features of the respiratory system of this animal. When the 
animal is not attached to a host, water may be drawn through the mouth, under the velum, through the respiratory tube, through the paired gills and to the outside through the seven pairs of external apertures. The blood in the gill capillaries is aerated from the oxygen carried in the water as it passes over the gill lamellae. While the lamprey is attached to a host fish, the water is drawn into the respiratory tube through the gill slits and then discharged through them.

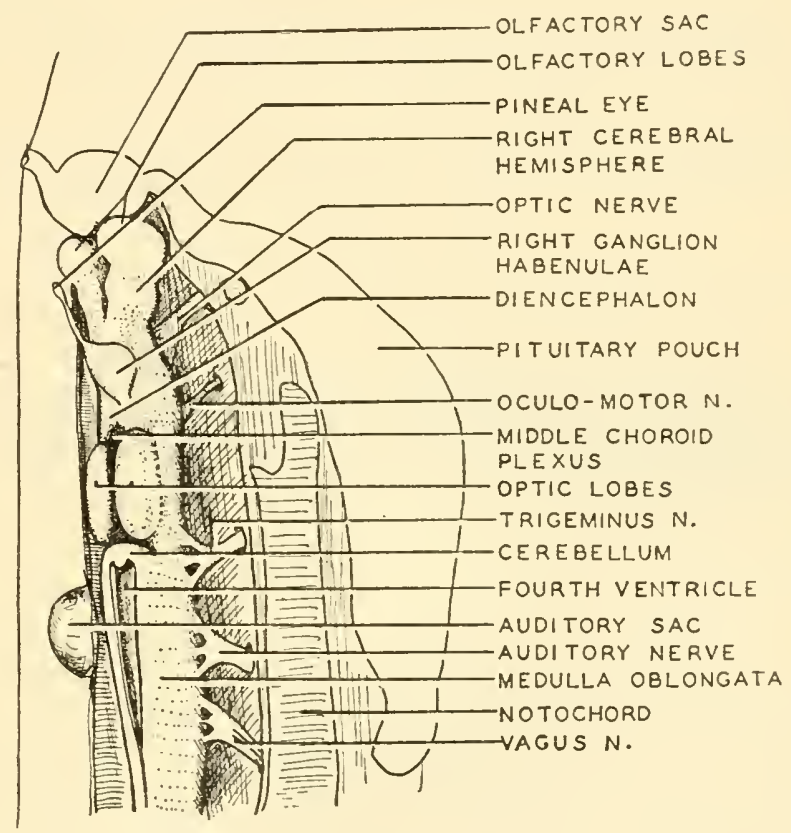

Fig. 226.-Brain of lamprey. Lateral view: dorsal view. (Drawn by Titus C. Evans.)

The nervous system shows the development of a small, primitive brain, which possesses all five principal divisions of a vertebrate brain. From anterior to posterior it is composed of olfactory lobes, cerebral hemispheres closely fused to preceding, single diencephalon with its dorsal epiphysis, midbrain with a pair of optic lobes, insignificant narrow bandlike cerebellum just behind the optic lobes, and the medulla just posterior to it. This continues directly posteriorly as the flattened spinal cord. The roof of the brain is rather membranous, as it is not entirely closed over. The sense organs include 
the single nasal chamber which is located immediately anterior to the brain. Extending ventrally from the nasal chamber and projecting beneath the brain to end blindly just above the esophagus is the pituitary pouch. As it passes beneath the diencephalon it makes contact with the infundibulum. The eyes of this animal are not highly developed, and sight is not used extensively by it. The auditory organ, which does not include an organ of hearing, is only for equilibrium; it consists simply of a vestibule and two vertical semicircular canals. The sense of taste centers in taste buds located in the respiratory tubes between the gill slits and possibly near the inner margin of the buccal funnel.

The urinogenital system shows only fair development. The ribbonlike kidneys lie, one at each side of the notochord and just dorsal

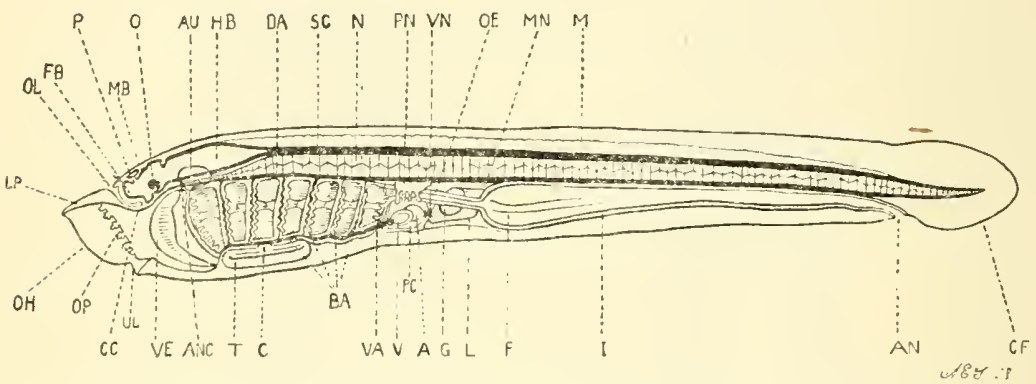

Fig. 227.-Ammocoetes larva of the lamprey, Entosphenus tridentatus. A. auricle of heart: $A N$, anus : $A N C$, anterior end of notochord: $A U$, ear: $B A$, branchial arteries (afferent); $C$, duct connecting pharynx and thyroid; $C C$, cranial cartilage, extending from tip of upper lip to a point slightly anterior to end of notochord, where it divides to form two lateral rods; $C F$, caudal fin; $D A$, dorsal aorta: $F$, folds in intestinal wall, suggesting a possible origin of the spiral valve; $F B$, forebrain; $G$, gall bladder; $H B$, hind brain; $I$, intestine; $L$, liver: $L P$, upper lip, supported by cranial cartilage; $M$, body muscles (myotomes); $M B$, midbrain : $M N$, position in which mesonephros will develop: $N$, notochord: $O$, eve; $O E$, esophagus; $O H$, oral hood; $O L$, olfactory organ; $O P$, oral papillae; $P$, pineal body : $P C$, pericardial cavity; $P N$, pronephros, showing pronephric tubules with their ciliated funnels (nephrostomes); $S C$, spinal cord; $T$, thyroid; $U L$, under lip; $\boldsymbol{V}$. ventricle of heart; $V A$, ventral' aorta; $V E$, velum: 'VN, hepatic vein. (Courtesy of Albert E. Galigher, Inc.)

to the peritoneal lining of the body eavity. A mesonephric duct extends posteriorly along the free edge of each to join the small urinogenital sinus. This is located just posterior to the rectum and opens externally by the urinogcnital papilla just behind the anus. The single gonad is rather large and is suspended by a peritoneal fold into the cnelom. The sexes are presumably separate, but hermaphroditic conditions are occasionally found. Germ cells when mature are discharged from the gonad into the body cavity and go by way of two 
genital pores into the urinogenital sinus, then out through the papilla to the environmental water where fertilization occurs.

The life history may be summarized as follows:

a. The eggs which contain considerable yolk in the vegetal portion and are about one millimeter $(1 / 25 \mathrm{inch})$ in diameter are laid in fresh-water streams, usually between March and June for all kinds of lampreys. The eggs first stick to objects, then fall in the sand. They are fertilized in the water almost immediately after laying. Cleavage follows, in about six hours when the optimum temperature of $22.5^{\circ} \mathrm{C}$. prevails. At $20^{\circ} \mathrm{C}$., this division requires nine days.

b. The adults spawn but once and then die.

c. A tadpolelike larval form, ammocoetes or mud lamprey, hatches from the egg and lives from four to five years in the mud along the streams where the eggs are laid.

d. At the end of four or five years the ammocoetes undergo metamorphosis to become adult. They remain under the mud from July or Angust to February or March while undergoing this transformation to adult condition.

e. The sea lamprey then migrates to the ocean and the lake lamprey moves down stream to a large fresh-water lake. They both become parasitic on other fish and continue this existence for from one and one-half to three and one-half years, when they return to fresh-water streams to breed again. 


\section{CHAPTER XXVI ELASMOBRANCHII*}

Unlike the cyclostomes, the Elasmobranchs are covered with scales and have two sets of paired fins on the ventrolateral surfaces of the body. In addition to these, there are unpaired or median fins. The gill apertures, except for the first, or spiracle, are slitlike instead of circular, as seen in the lamprey. The gills are supported by gill arches, and the mouth has an upper and lower jaw. The skeleton is entirely cartilaginous, there is a partially persistent notochord, and the exterior is covered and protected by placoid scales. The males have a modification of each pelvic fin known as a clasper which is used as a copulatory organ.

The mouth is not right at the anterior end of the body but is ventral or subterminal. There is present in the ileum of the small intestine a spiral valve which increases the internal surface, thus adding absorptive area. The Elasmobranchs have no operculum or air bladder.

\section{Classification}

The class is divided into two rather easily distinguished subclasses. The first group is very common to American shores and the second is rarely seen in our waters.

Subclass Selachii.-This group includes the sharks which are cylindrical in shape, possess laterally located gill slits, and are active swimmers; and the rays, which are dorsoventrally flattened, possess ventrally located gills, are less active, and dwell on the bottom of the sea. This subclass is usually divided into two orders.

Orders Euselachii and Cyclospondyli.-The sharks make up these orders. The dogfish sharks (Squalus acanthias and others), tiger shark (Galeocerdo arcticus [Faber]), cub shark (Carcharias platydon [Poey]), shovelhead or bonnethead shark (Reniceps tiburo [Linn], and man-eater shark (Carcharodon carcharias [Linn]) are forms commonly found. The majority of sharks are carnivorous and active, but they rarely attack man unless the person is already wounded.

\footnotetext{
*In collaboration with Miss Mary Fickling.
} 
The average length of most sharks commonly observed ranges between three and six or eight feet. Their natural food consists principally of crustacea, small fish, squids, and refuse. In the Gulf of Mexico and other warm seas the so-called man-eater may occasionally reach a length of thirty feet and is sometimes charged with

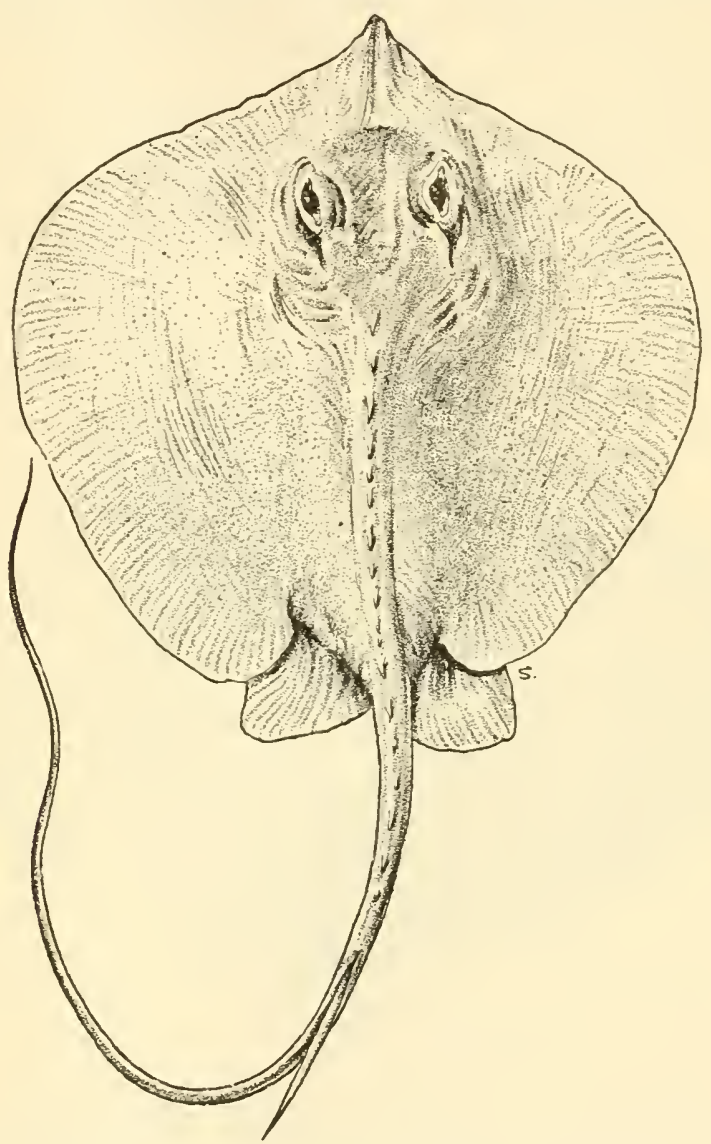

Fig. 228.- Southern sting ray, Dasyatis americana, a common form in the Gulf of Mexico.

eating human beings. The shovelnose (bonnethead) and hammerhead sharks are very interesting forms. The shape of the head of each is about the shape ascribed to it by the common name. The former has been considered sufficiently interesting to warrant further discussion of it as an example of the class. 
Order Batoidei-Skates and Rays.-This is a group of depressed or dorsoventrally flattened fishes in which the gill slits are located on the broad, flat, ventral side. 'These fish lack the anal fin and the caudal is absent or reduced. The saw-fish, Pristis pectinatus is a sharklike ray with a long tooth-bearing rostral process or snout that resembles a double-edged saw. These animals may reach a length of fifteen or twenty feet, with a saw five feet in length.

The skates are distributed along our Atlantic shores and are oviparous. The eggs are enclosed in dark brown cases or capsules, quadrate in outline and of considerable size. They have hornlike processes extending from each corner. There are about six species of skates, of which Raja erinacea, $R$. diaphanes, and $R$. ackleyi are common ones.

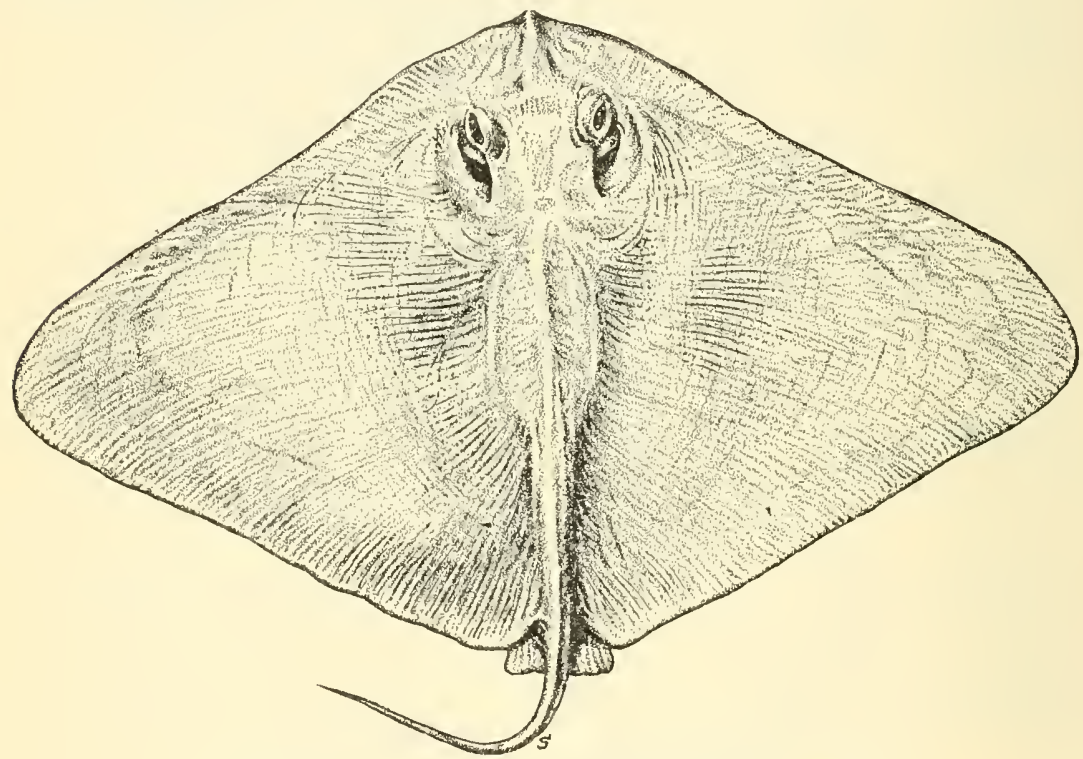

Fig. 229,-Butterfly ray, a common bottom feeder.

The rays are of similar shape, but they bear their young alive and tend to have a smoother skin. The rays are more numerous in the warmer waters. The torpedo ray of family Torpedinidae, has attracted considerable attention because of its ability to generate and store electrical energy in the muscles of the bases of the broad pectoral fins. These electric organs are capable of discharging suffcient current to paralyze other animals, ring a doorbell, or light a 
flashlight bulb. The sting ray or stingaree is very common in the Gulf of Mexico. The average width of those usually seen is from eighteen inches to two feet. They have a long, slender, whiplike tail with a strong spine or sting on the dorsal side of its proximal third. Dasyatis sabina and Dasyatis americana are two common forms. The butterfly ray, Pteroplatea micrura, is a broad-bodied form with an exceptionally short tail. They, too, are quite common in the water of the Gulf of Mcxico. The sting is usually obsolete in this form. It is called butterfly ray because of the manner of flapping the lateral expansions about as a butterfly moves its wings in flight.

Subclass Holocephali.-This group contains an order with three modern genera. Psychichthys affinis (or Chimaera affinis, as often called) is the only species taken from the waters of the coasts of North America, and then only rarely. Chimaera monstrosa is another species which is found in South American waters.

\section{Economic Relations of the Class}

Many of the smaller sharks, like Squalus acanthias and Mustelus canis are very destructive to lobsters, crabs, shrimp, squid, and valuable fish which they use for food. They also damage much fishing gear by tearing through nets. It is estimated that the damage done in this way averages $\$ 400,000$ in Massachusetts alone. Along the coasts of California and in the Gulf of Mexico both sharks and rays are a nuisance to the seining fisherman.

The sting rays or "stingarees" which are armed with the barbed stinging spine on the proximal portion of the tail are generally common in most of the warmer fishing waters. With a sudden swing of the tail one can inflict an ugly and extremely painful wound. Some people become severely ill as the result of such a sting. Bathers particularly dislike "stingaree" infested beaches as well as those infested with the less common torpedo ray.

The skins of certain sharks and skates, which have the sharply pointed, toothlike scales, are used as a polisher of wood and other materials and is called shagreen. Shark skins are now being manufactured into leather on a commercial basis. Large quantities of oil are extracted from some of the sharks, as the cub shark for example. This oil is used in currying leather in the tanning industry. 
Shark liver oil is of high vitamin content and has an important medicinal use.

In many countries, particularly of the Orient, these fish are commonly used as food. It is said that small sharks and skates are offered for sale right along with other fish in the markets of China. They are also salted and dried. In the United States there is an unfounded prejudice against eating these fish, but dogfish are now being canned and sold under the name of "grayfish." The winglike fins of skates and rays make delicious steaks. Sawfish steaks are quite desirable and the saws are preserved as ormaments. The flesh of sharks and rays is also ground up and used extensively as fertilizer. In some parts of the world the fins of sharks are used in the manufacture of gelatin. A good many dogfish and bonnethead sharks are sold for purposes of study in zoology laboratories.

\section{THE SPINY DOGFISH}

This shark is the most commonly studied representative of the Elasmobranch group. Squalus acanthias is the scientific name applied to the common form taken along the Atlantic coast and Squalus suckleyi is the name given the similar one of the Pacific coastal waters. The average length of Squalus is between two and one-half and three feet. It is a strong swimmer and is frequently seen as a scavenger in harbors as well as going out to sea for extended periods. It apparently makes a spring migration northward along the coast and a return movement in the fall. Because of the ventral location of the mouth, these fish find it necessary to turn ventral side up to eat morsels of food from the surface of the water.

\section{External Features}

The body is generally spindle-shaped (fusiform) tapering at both head and tail. There are two pairs of fins, the anterior pectoral and the posterior pelvic, or ventral fins. In addition to the paired fins, there are two unpaired, median, dorsal fins, each with a spine at its anterior margin (hence spiny). Male individuals may be distinguished from females by the fingerlike extensions, or claspers on the pelvic fins. The dorsal and ventral lobes of the caudal fin, or tail, are unequal and based on this, the tail is described as heterocercal.

There are six pairs of uncovered gill clefts in the walls of the pharynx. The anterior one, which is dorsally located and greatly 
modified, is called the spiracle. It contains a rudimentary gill structure. The mouth aperture is somewhat the shape of an inverted U, located on the ventral side of the head, and supplied with sharp teeth on the jaws. These teeth are developed by modification of the placoid scales which cover the skin over the body generally. The placoid scales are primitive exoskeletal structures with a basal plate embedded beneath the skin and a spine projecting on the surface. This spine has a pulp cavity, surrounded by dentine, which is covered on its surface by enamel. This strueture is considered to be homologous to the vertebrate tooth. The paired nostrils are openings on the ventral side of the snout, anterior to the mouth. The eyes, the lids of which are immovable, are situated on the sides of the head. The cloacal aperture is located between the bases of the pelvic fins.

\section{Muscular System}

The segmental arrangement of myotomes, separated by myocommas, is fairly complete along both sides of the body. The principal specializations of independent muscles are found in the form of myotome modification in the region of the mouth gills and paired appendages. The trapezius found above the branchial area; the superficial constrictors extending from the head to beyond the gill slits and assisting in their operation; and the adductor mandibularis, connected with the lower jaw, are all examples of special developments.

\section{Skeletal System}

The endoskeleton of the sharks is composed of cartilage. It consists of axial skeleton (skull and vertebral column) ; visceral skeleton (jaw and gill arches); and, appendicular skeleton (pectoral girdle and fins, pelvic girdle and fins). The vertebral column and skull are much more developed than in the cyclostomes. The notochord has become segmented and partially replaced by cartilage. The centrum, which has replaced a considerable portion of the notochord in each vertebra, is deeply concave at each end, and is said to be amphicoelous. Some of the remains of the notochord fills these interstices between vertebrae.

The skull is laid on a foundation of the ventral basal plate. The dorsal side is fairly well enclosed with cartilage. The anterior extension of the skull is the rostrum and the depression in its dorsal 
side is the anterior fontanelle. The nasal capsules are rounded, cartilage-encased cavities, one at each side of the base of the rostrum. These capsules house the olfactory sense organ in life. The orbits are laterally located, spherical depressions which normally hold the eyes. Each orbit is guarded anteriorly, dorsally, and posteriorly by slight extensions of the cartilage linown as preorbital process, supraorbital crest, and postorbital process respectively. The orbits are laterally located spherical depressions in which the eyes are set.

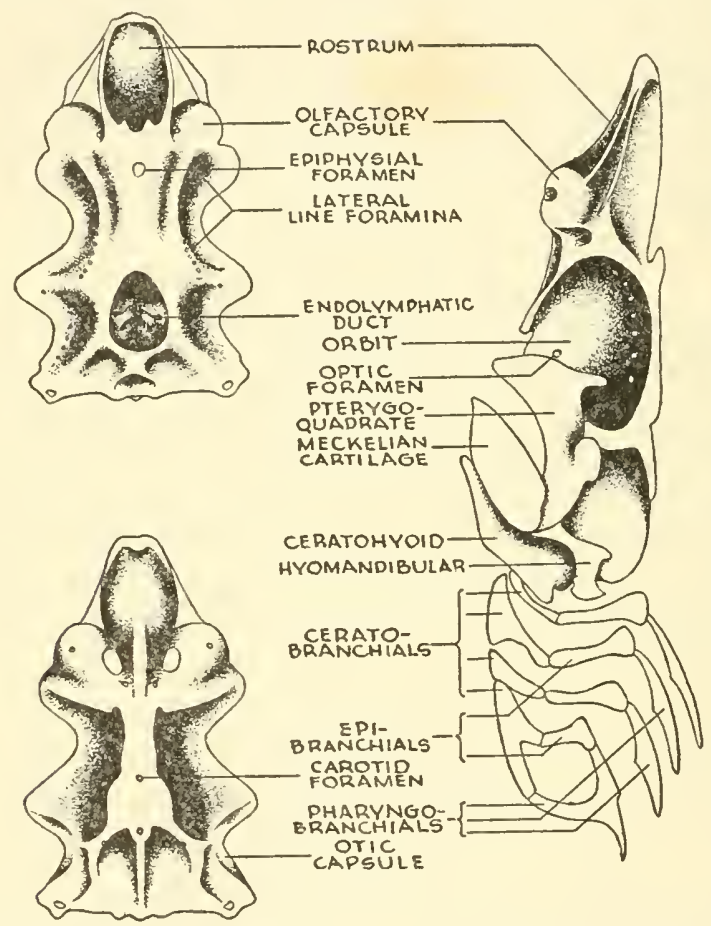

Fig. 230.-The skull and visceral arches of the dogfish shark, Squalus acanthias. Dorsal view above, ventral view below. Latiral aspect on the right. (From Atwood: Introduction to Fertebrate Zoology, published by The C. V. Mosby Company.)

The visceral skeleton consists of the upper jaw (palatopterygoid or quadratopterygoid cartilages), lower jaw (Meckel's cartilages), hyoid arch (hyomandibular, ceratohyal, basihyal), and five branchial arches (each typical one has pharyngobranchial, epibranchial, ceratobranchial, hypobranchial, and basibranchial cartilages). 


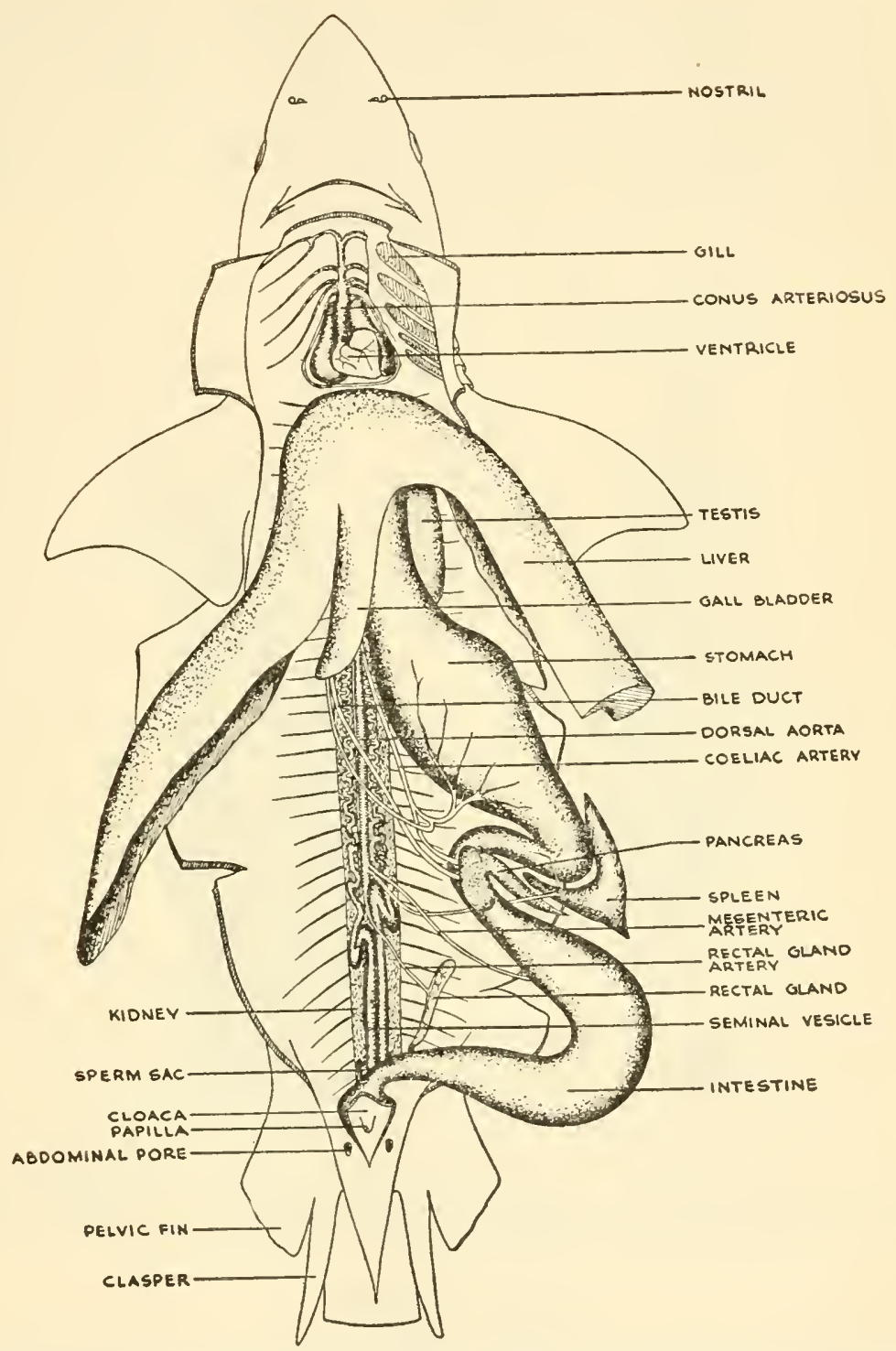

Fig. 231.-Ventral view of the visceral anatomy of the dogfish shark, Squalus acanthias, male. (From Atwood: Introduction to Vertebrate Zoology, published by The C. V. Mosby Company.) 
The pectoral girdle is composed of the ventral coracoid bar and the dorsolateral scapular process at each end of it. The fin consists of three flat basal cartilages (propterygium, mesopterygium, and metapterygium), a series of radial cartilaginous rays, and a series of exoskeletal dermal rays. The pelvic girdle is made of one cartilaginous bar (ischiopubis) with a fin joining at each end. The basals of this fin are fused into one cartilaginous plate.

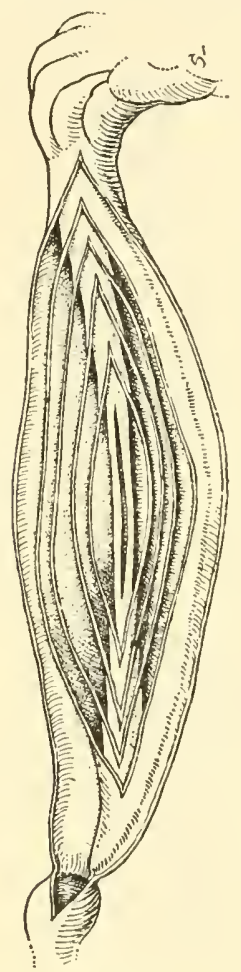

$A$.

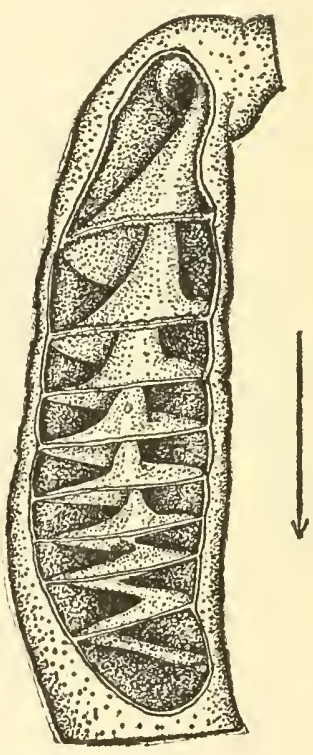

$B$.

Fig. 232, A.-Dissection of the valvular portion of the small intestine. $A$, the bonnet-head shark to show the spiral valve; $B$, spiral valve of Raia. ( $B$ reproduced by permission, from General Zoology by Wieman, copyrighted 1938 by McGraw-Hill Book Co., Inc.)

\section{Digestive System}

Most of the organs of this system and other viscera lie in the pleuroperitoneal portion of the coelomic cavity. Anterior to this the pericardial portion of the coelom contains the heart. The digestive organs are in the form of an alimentary canal with accessory glands. 
The canal begins anteriorly with the mouth, which leads directly into the pharynx, in whose lateral walls are the gill slits (and spiracle). Following this is the short tubular esophagus, which leads into the cardiac end of the stomach. This organ, which is somewhat broader than the esophagus, is rather U-shaped. The posterior, or pyloric, portion of the stomach is provided with a sphincter muscle, the pylorus, which controls the passage of food materials into the intestine. The duodenum is the short anterior portion of the intestine which follows the pyloric portion of the stomach and leads into the valvular portion of the intestine (ileum). This section of the small intestine is of considerably greater diameter than the duodenum and contains internally a spiral valve, which is a spirally arranged infolding of the mucous lining. This arrangement serves to slow the passage of food and increases the absorption surface. The principal absorption takes place through this part of the intestine. The valvular portion leads to the short, narrow, large intestine, which empties into the cloaca (Figs. 231 and 232).

The liver, pancreas, and rectal gland are accessory glands connected with this system. The liver is a large, three-lobed organ with the saclike gall bladder located just dorsal to the junction of the right and middle lobes. The gall bladder stores bile produced by the liver and delivers it to the duodenum throngh the bile duct. The pancreas is divided into two lobes, an oval ventral and a slender dorsal lobe. Ducts lead from it to the duodenum. The rectal gland is a spindle-shaped gland leading into the large intestine directly. The reddish spleen, which is a lymphoid rather than digestive organ, lies around the greater curvature of the stomach.

The digestive tract and adjacent organs in both species are suspended from the body wall by mesenteries, which are extensions from the peritoneum, or membranous lining of the coelom. The mesentery supporting the stomach is the mesogaster, the one extending between the spleen and stomach is the gastrosplenic, and the mesorectum supports the large intestine and rectal gland.

\section{Circulatory System}

This centers in the heart, which is located ventrally at about the level of the posterior pair of gills, and consists of two principal chambers and two accessory chambers. The pericardium, a membranous extension of the peritoneum, encloses the heart. The two 


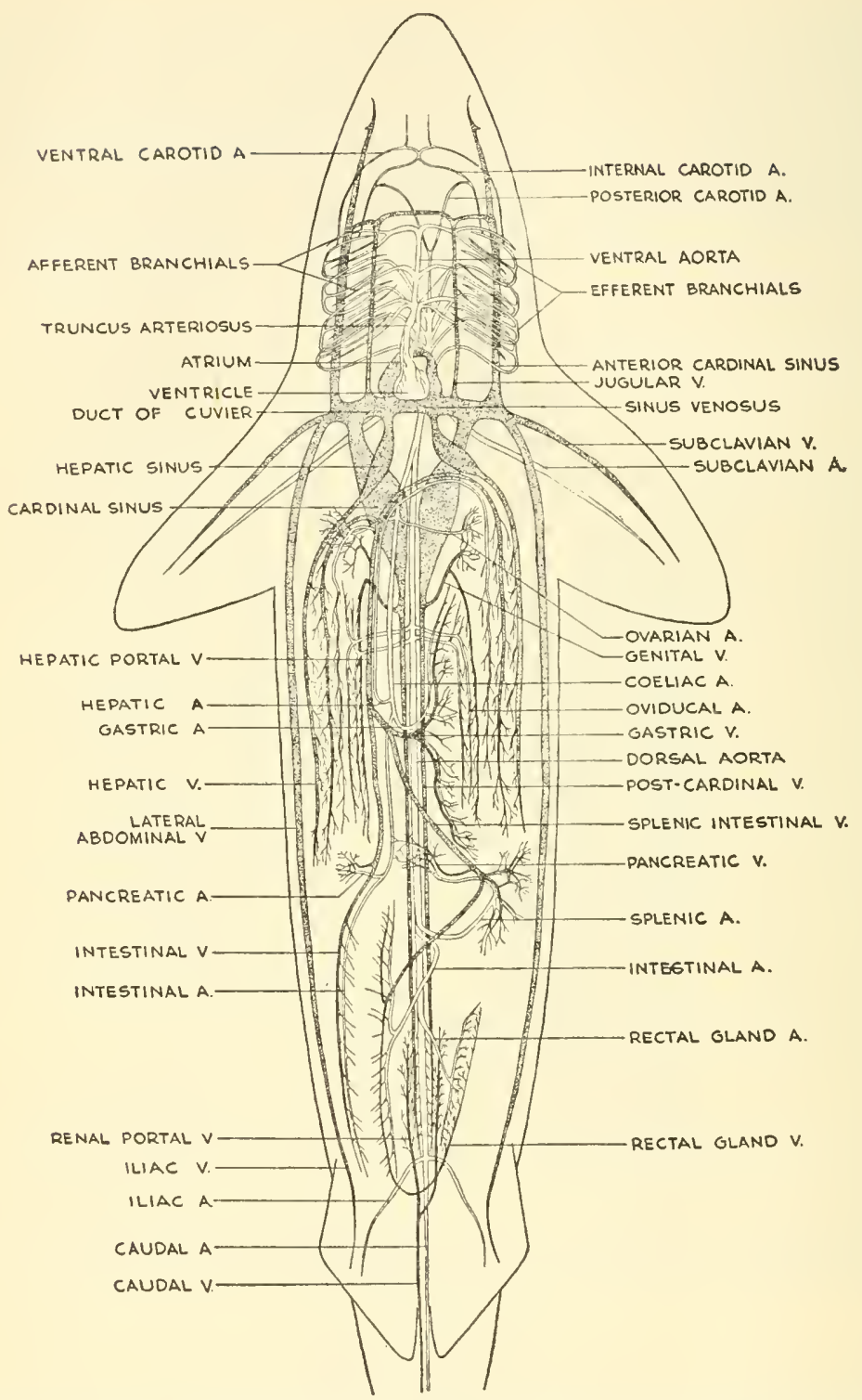

Fig. 233.-The circulatory system of the spiny dogfish, Squalus acanthias. (From Atwood: Introduction to Vertebrate Zoology, published by The C. V. Mosby Company.) 


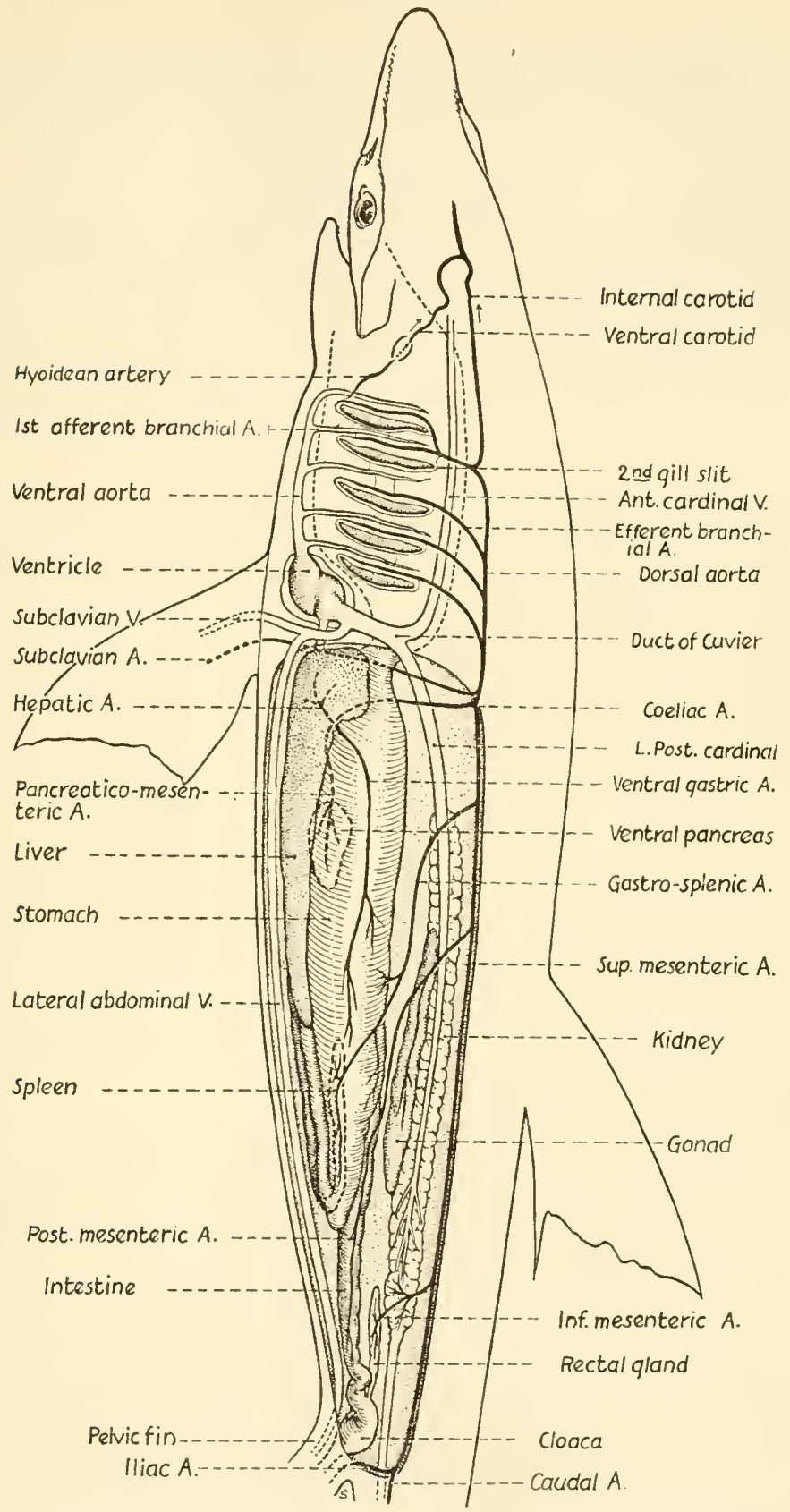

Fig. 234.-Diagram of lateral view of the circulatory system and other organs of bonnet-head shark. (From dissections by Mary Fickling.) 
main chambers are the single, more dorsal auricle, and the ventral, muscular ventricle. Leading into the auricle is the sinus venosus, which receives blood from the veins of the body. A bulblike enlargement at the base of the ventral aorta, where it leaves the ventricle, is the conus arteriosus. The ventral aorta leads anteriorly from the conus and gives off three pairs of afferent branchial arteries which branch to the five pairs of gills. The blood spreads from these by capillaries through the gill lamellae for oxygenation. The pretrematic and postrematic branches of the efferent branchial arteries which form four efferent branchials leave the gills and join the dorsal aorta, which is formed by them in the dorsal midline. The hyoidean extends from the ventral portion of the first pretrematic to the spiracle where it spreads in capillaries. The ventral carotid leads forward from the spiracle to the internal carotid. Extending anteriorly from the dorsal part of each first efferent branchial is a common carotid which supplies arterial blood to the head and brain. The dorsal aorta extends posteriorly from the junction of the efferent brachial arteries, soon giving off the subclavian arteries to the pectoral fins; coeliac to stomach, liver, and pancreas; gastrosplenic to stomach and spleen; superior mesenteric to the valvular intestine to become the posterior mesenteric artery there; renal arterics to the kidneys; the inferior mesenteric to the rectal gland and large intestine; and the iliac to the pelvic fins and cloaca. The subclavian artery leaves the aorta more anteriorly, coming off ahead of the posterior efferent branchial; the coeliac is farther back and sends a pancreaticomesenteric artery above the duodenum, through the ventral pancreas, and along the valvular intestine a gastric to the stomach and the hepatic artery to the liver; the gastrosplenic and superior mesenteric arise very near each other.

The systemic veins all return venous blood to the sinus venosus, which empties into the auricle by way of the sinu-auricular aperture. Hepatic veins lead directly from the liver to the sinus venosus. The ducts of Cuvier collect blood from the anterior cardinals of the head region and posterior cardinals of the trunk region and empty it into the sinus. A hepatic portal system collects from the stomach, intestines, pancreas, and spleen and empties into the liver. The two renal portal veins bring blood to the kidneys from the single caudal vein of the tail. This blood spreads through the capillaries of the kidneys and is collected into the postcardinals through the renal 
branches. There is also a system of lymph spaces, which supplements the blood circulatory system.

\section{Respiratory System}

The gills in the wall of the pharynx are constantly bathed in water forced through from the mouth. An exchange of carbon dioxide for oxygen is made by the blood as it passes through the capillaries of the gills. This is made possible by diffusion of these gases through the membranes of the gill lamellae. The gills are supported by cartilaginous gill arches.

\section{Nervous System}

The central nervous system consists as usual in vertebrates of the brain and spinal cord. The brain includes two large olfactory lobes at its anterior, followed by two cerebral hemispheres, a depressed diencephalon, a pair of large optic lobes, a well-developed cerebellum, and behind this the medulla oblongata. There is a very slight constriction betwcen olfactory lobes and cerebrum. The cerebellum is divided into quarters by a longitudinal groove and a transverse groove. It covers a part of the optic lobes as well as the anterior portion of the medulla oblongata. On the lateral walls of the medulla are located the acusticolateral areas, including the earlike auricles.

The cavity within the medulla, which opens to the dorsal surface beneath and behind the cerebellum, is the fourth ventricle. There are ten pairs of cranial nerves which are numbered and named from anterior to posterior: I, olfactory; II, optic; III, oculomotor; IV, trochlearis; V, trigeminus; VI, abducens; VII, facial ; VIII, auditory; IX, glossopharyngeal; $X$, vagus. The olfactory nerves extend from the olfactory lobes; optic from diencephalon and optic lobes; oculomotor from ventral side of optic lobes or midbrain; trochlear from dorsal side of optic lobes between them and cerebellum; the trigeminus, abducens, facial, and auditory all from the anterior portion of the medulla oblongata; the glossopharyngeal from a more posterior part of the sides of the medulla. These last two supply the gills, lateral line, and certain viscera.

The spinal cord is tubular and somewhat flattened. It extends the length of the vertebral column and gives off paired spinal nerves segmentally. 
The sense organs include the eyes, the olfactory organ, internal ear, and the lateral line system. The eyes are in the orbits, one on each side of the cranium. They are quite typical of the vertebrate eye described in the general chapter on phylum Chordata. The olfactory

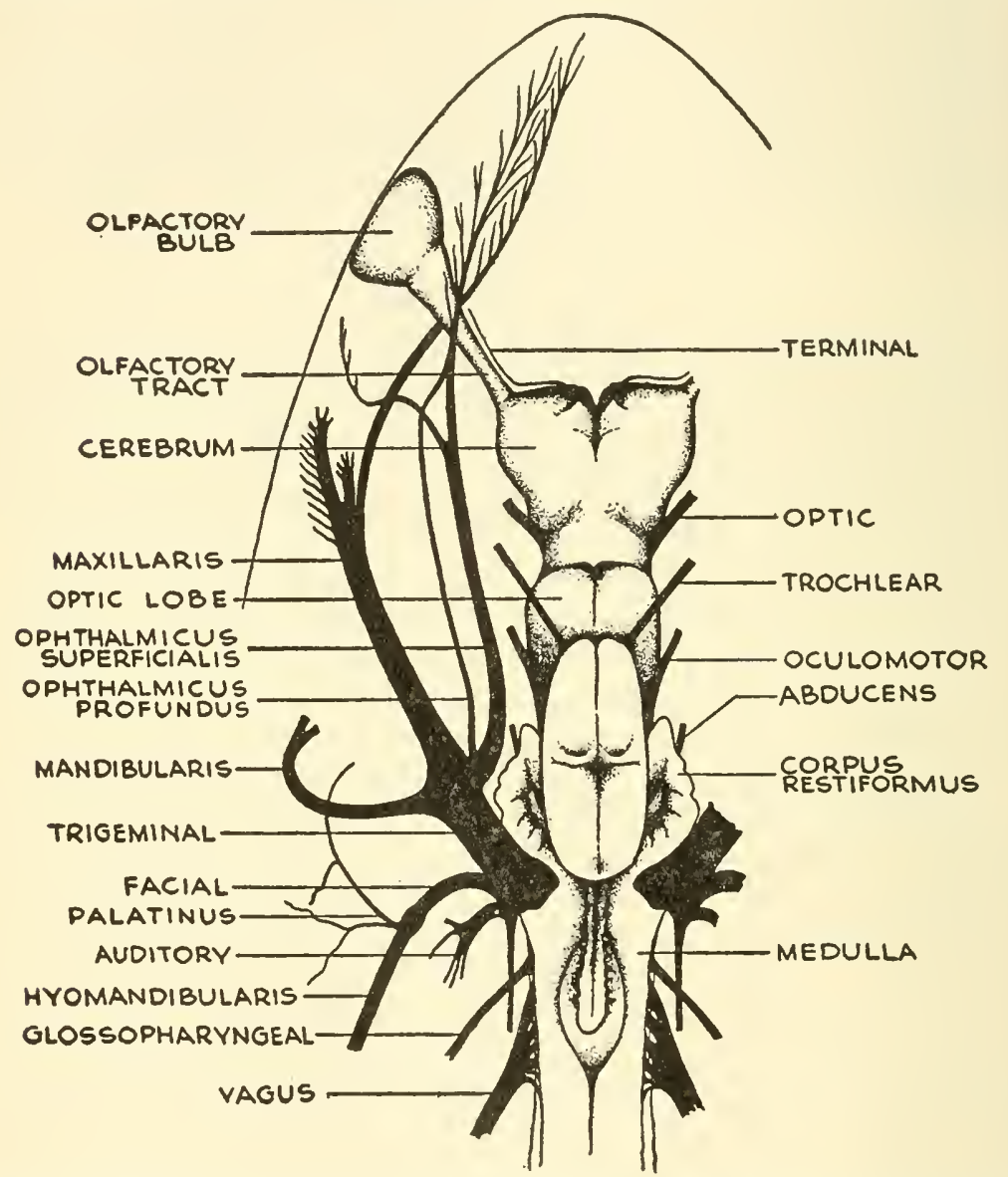

Flg. 235.-Dorsal view of the brain and cranial nerves of the dogfish shark, squalus acanthias. (From Atwood: Introduction to Vertebrate Zoology, published by The C. V. Mosby Company.)

organ consists of a pair of nasal sacs on the ventral side of the rostrum which open by nostrils. The nasal chambers are blind sacs and lined with a sensory lamellated olfactory membrane in which the olfactory nerve ends. The internal ears are composed of a vestibule 
and three semicircular canals. An endolymphatic canal leads from the dorsal exterior into the lower part of the vestibule the sacculus. A posterior pouch of the sacculus is the lagena, which is considered the forerunner of the cochlea of higher vertebrates. The ear serves the sense of equilibration in the fish.

A canal extends along the side of the body in the lateral line and forward onto the head, lying just beneath the skin. This is the lateral line system. On the head there are some pores with tubes extending beneath the skin to small bulbs called ampullae of Lorenzini. The funetion of the lateral line system and these ampullae is perception of water pressure and vibrations.

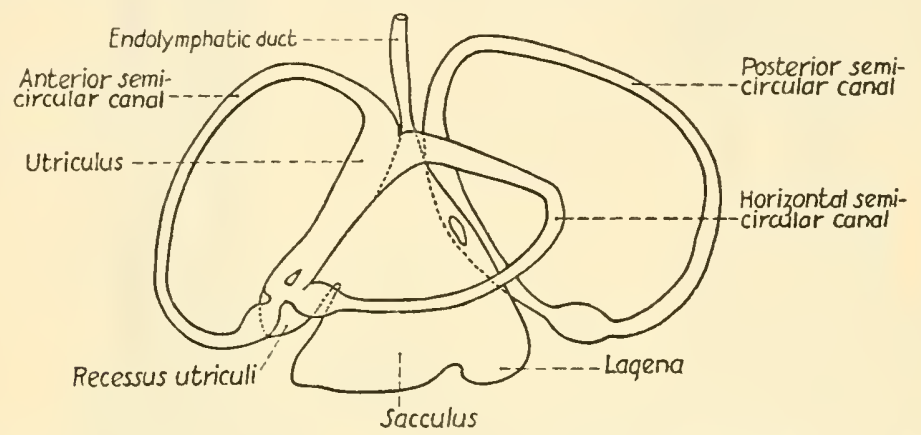

Fig. 236.-Diagram of lateral view of left internal ear of Reniceps tiburu.

\section{Urinogenital System}

The kidneys are thin, slender organs extending along the dorsal body wall, one on each side of the vertebral column. The posterior, or caudal, portion functions in excretion. There is an accessory mesonephric duct embedded in each kidney which carries urine to the urinogenital sinus of the male, and the Wolffian duct serves as the urinary duct of the female, emptying into the urinary sinus. A papilla leads from the sinus to the cloaca in both. In the male spermatozoa are produced in the testes, carried by several vasa efferentia through the mesochorium to the convoluted cranial portion of the Wolffian duct, the epididymis of each side, which continues posteriorly as the vas deferens. This tube enlarges to become the seminal resicle and continues into the inflated sperm sac which is directly connected with the urinogenital sinus. The spermatozoa then pass out through the papilla to the cloaca, thence to the outside by way of 
the anus. During copulation they are transferred to the cloaca of the female by use of the claspers. They swim by their own motility into the uteri and oviducts.

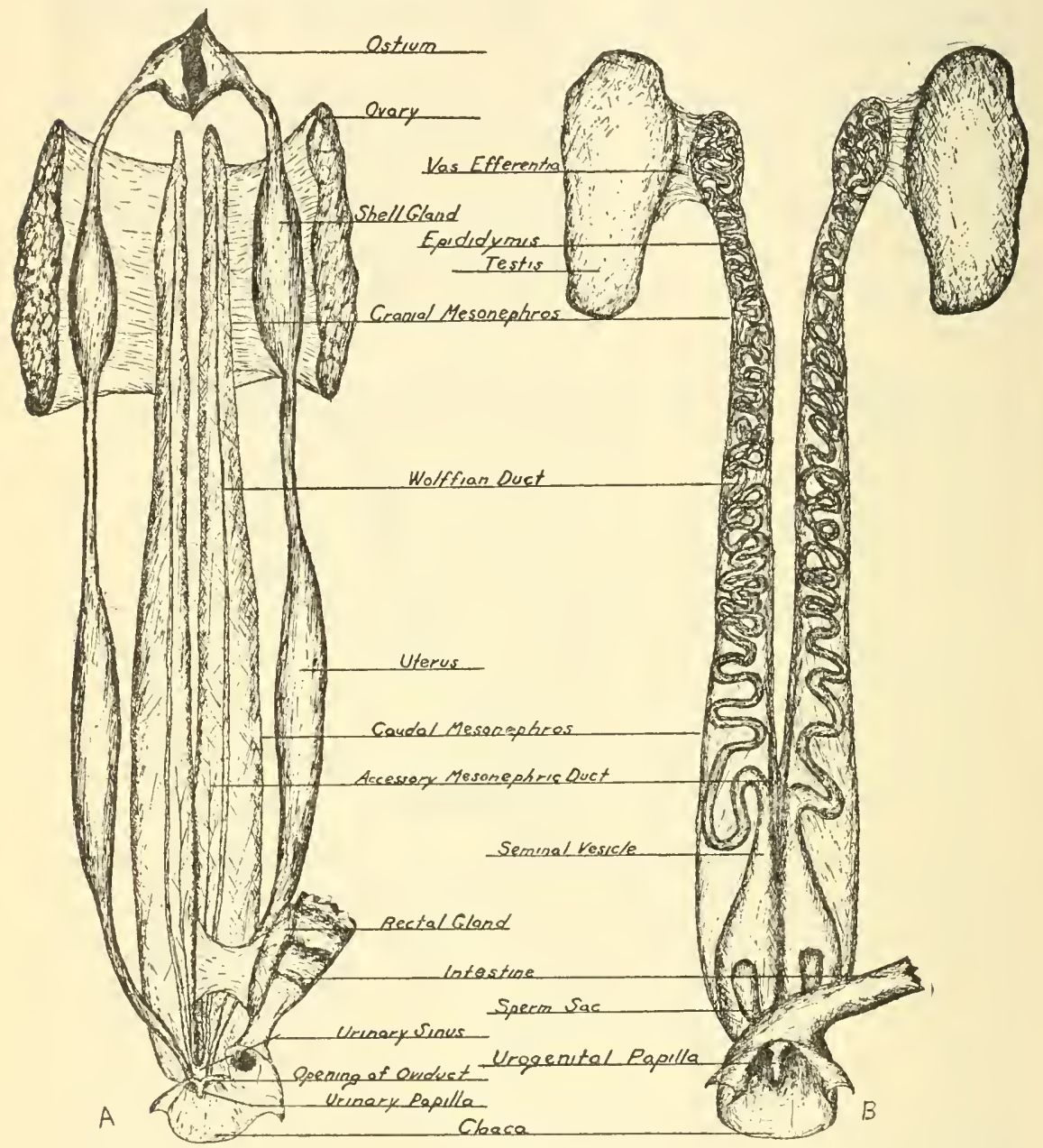

Fig. 237.-Urogenital systems of Squalus acanthias; $A$, female; $B$, male.

The ova of the female are produced in the ovaries which are located one on each side of the median dorsal line in the anterior portion of the coclom: Each gonad is suspended in a mesentery, the mesovarium. Mature ova rupture from the ovary into the body cavity and enter 
the funnellike ostium of the oviduct, which is held by the faleiforn mesentery at the anterior end of the peritoneal cavity. As the ova pass down the oviduct, they receive a covering which is secreted by the shell gland in the wall of the duct. Fertilization occurs in the oviduct and the embryo develops in the uterus which is the expanded lower portion of the oviduct. The embryo is nourished by the large yolk mass of the egg.

\section{THE BONNETHEAD SHARK, RENICEPS (SPHYRNA) TIBURO COMPARED TO SQUALUS}

The bonnethead (or shovel-nosed) shark is common in the Atlantic along the coast of the Southern States and in the Gulf of Mexico. It occurs abundantly along the Louisiana and Texas Gulf coast during May and June. It averages about the same size as Squalus. In many respects it is similar to the smooth dogfish, Mustelus canis, and the ground shark, Carcharhinns.

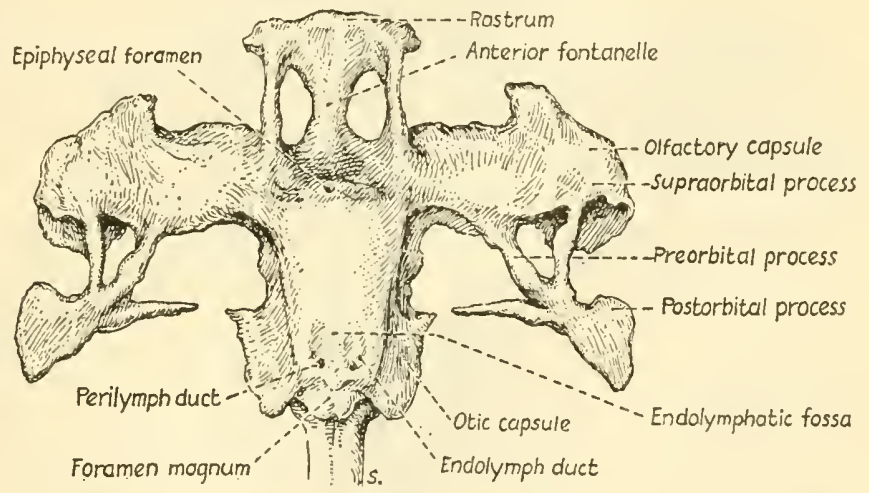
Fig. 238.-Dorsal view of the skull of bonnet-head shark. (From dissections by
Mary Fickling.)

The peculiar shovel-shaped head with the eyes out on the lateral margins is one of the striking features of Reniceps by which it differs from the others mentioned. In Reniceps there are no spines in front of the dorsal fins and a single anal fin is present on the ventral side between the anus and tail. The spiracle is absent, leaving only the five pairs of gill slits. The other external features are similar to those of Squalus.

The skeleton of the skull is shaped considerably different from that of Squalus. This is brought about by lateral extension. Each olfac- 
tory capule is extended far to the lateral of the base of the rostrum instead of lying beside it. The orbit with the modified supraorbital crest, preorbital process, and bladelike postorbital process are also

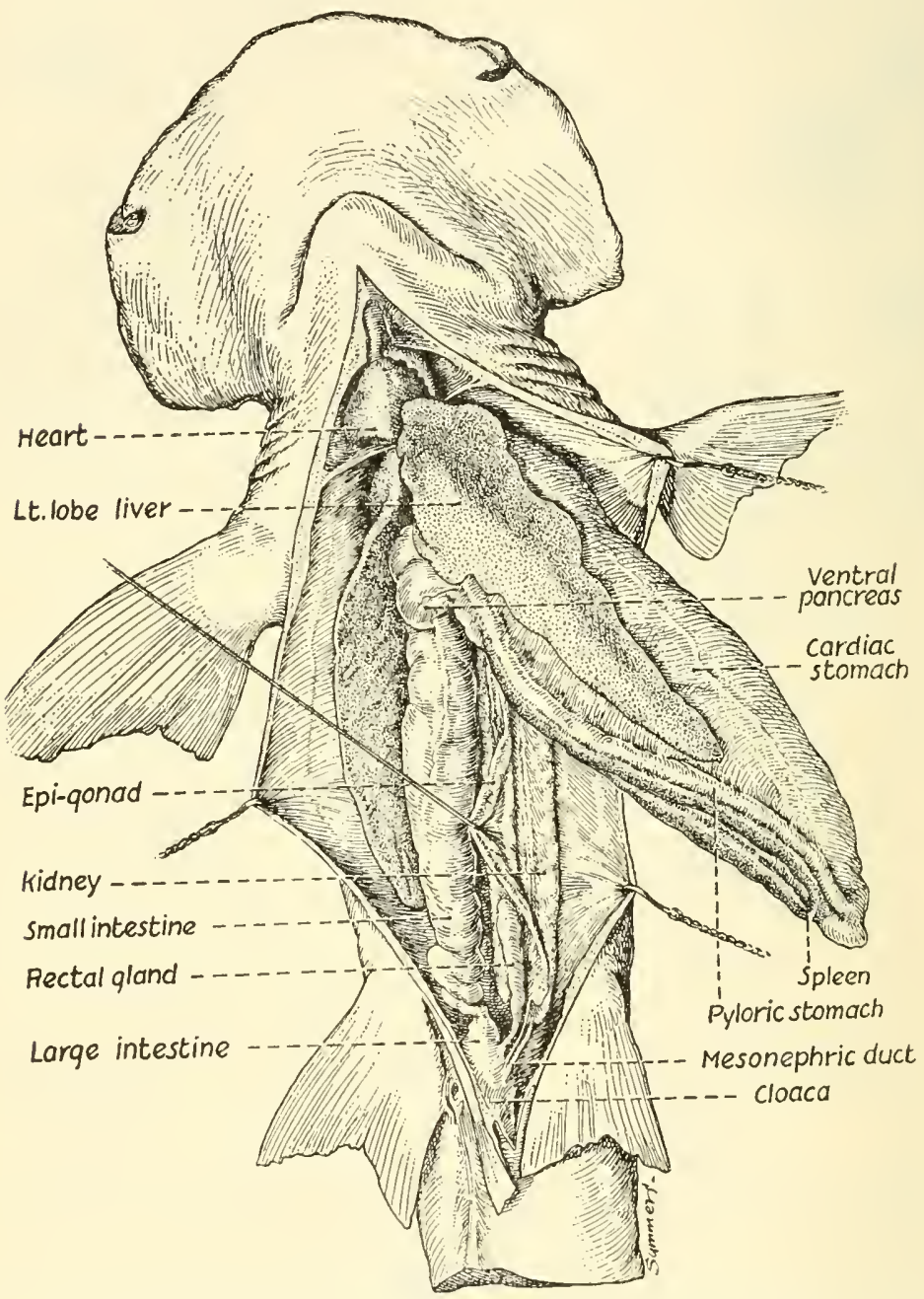

Hig. 239.-Internal anatomy of bonnet-head shark, Reniceps tiburo, from ventral view. (From dissections by Mary Fickling.)

projected at the terminal portion of this arm of cartilage. The posterior part of the skull is somewhat narrowed and flattened but otherwise similar to that of Squalus. 
There are a few differences in the digestive systems of the two. In the pharynx there are no spiracles. The stomach of Reniceps is $\mathrm{J}$-shaped instead of $\mathrm{U}$-shaped and the long slender pyloric portion is armlike. The spiral folds of the spiral valve are more telescoped into each other than in Squalus. There are only two lobes in the liver of bonnethead and the gall bladder is nearly embedded in its tissue. In the circulatory system, Reniceps has five afferent branchial arteries branching from the ventral aorta while Squalus has only three, two of which branch. The branching of the coeliac artery is somewhat different in the two animals.

The brain has the same general parts as it does in Squalus but they are quite modified. The olfactory lobes are broader and almost completely fused to each other. The cerebrum is a somewhat smaller single lobe just posterior and dorsal to the olfactory lobes. There is no line of demarcation between the hemispheres. The diencephalon is entirely hidden from dorsal view by the cerebrum and cerebellum. The latter is large, irregularly divided into three lobes, and convoluted. It covers not only the diencephalon but also most of the optic lobes (midbrain) and much of the medulla oblongata. The medulla has well-developed acousticolateral areas.

The nasal chambers of Reniceps are quite large and kidney-shaped. They eontain extensive folds or lamellae of the olfactory membrane.

The shape of the testes in male Reniceps is much longer and more slender than in Squalus. In addition to this, there is a long glandular body, the epigonad, whieh extends from the level of the gonad proper to the region of the eloaca.

Copulation in Reniceps probably occurs during May and June in the Gulf of Mexico, at the time when they are so numerous in the shore waters. Fairly mature "pups," às the developing young are called, have been found in the uteri of specimens collected off shore in Texas Gulf waters in late August and early September. 


\section{CHAPTER XXVII}

\section{PISCES, TRUE FISH}

This important class includes quite an extensive variety of different forms. They are aquatic and possess the usual adaptation of gills for respiration, and paired fins as well as median fins to assist in locomotion. Most forms within the class have scales as an exoskeletal covering of the skin. The endoskeleton is primarily bony. Pectoral and pelvic girdles are developed to support the paired fins, but the pelvic girdle is usually small. The fins are supported by fairly well-developed fin rays. The majority of families in this class possess a swim bladder. The typical shape of the fish's body is fusiform or spindle-shaped, with all of the original features of stream-lining. The shape assists in dividing the water as the fish moves through it. As the water passes over the thicker part of the body, it rushes in to push forward on the posterior slopes of the spindle form of the body. This is an adaptation for easy production of speed. The sedentary forms of fish usually tend to lose this shape and become flattened or otherwise modified. The shape of the body varies from that of the long slender eel to that of the globe-shaped box-fish and inflated puffers which can float like balloons. The sea horse is one of a group of very peculiarly shaped forms. Still another peculiar adaptation is the flying fish. The fins of fish are found singly in the form of a dorsal median fin, sometimes divided into two; a single caudal fin over the tail; a ventral median anal fin in most species; a pair of pelvic or ventral fins which are quite variable in position and in some forms rudimentary, and the paired pectoral fins. These paired fins are supported by bony girdles. The pelvic fins of the perch are located almost immediately ventral to the pectoral fins, while in the bullhead catfish they are just anterior to the anus. In this catfish there is a second dorsal, which is composed entirely of skin and is called an adipose fin. The structure of the caudal fin and posterior end of the vertebral column is distinctive and has been classified. The most primitive type of tail is the diphycercal in which both the cutaneous and osseous parts are equally divided between dorsal and ventral regions. The heterocercal tail is asymmetrical and the tip of the vertebral column ex- 
tends into the dorsal lobe as has already been seen in the dogfish. Still another type of tail is the homocercal, which is internally unbalanced but externally symmetrical. The original notochord turns

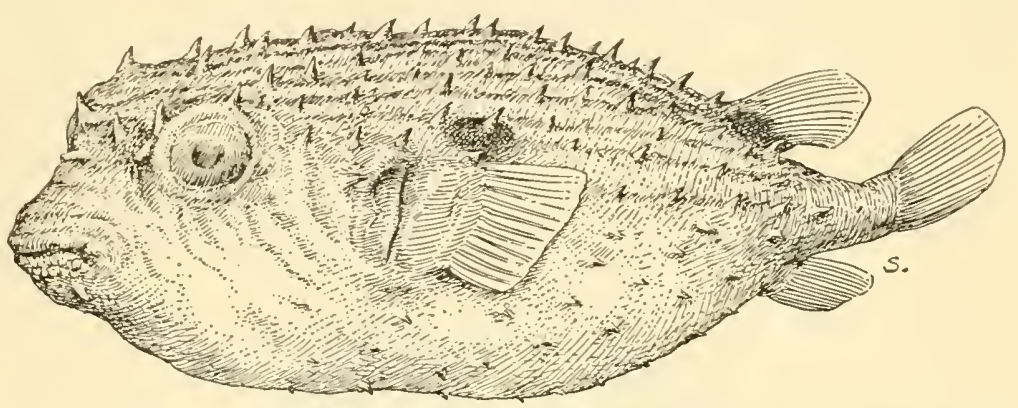

Fig. 240.-Spiny boxfish, Chilomycterus schoepfli, from Gulf of Mexico and Atlantic Ocean.

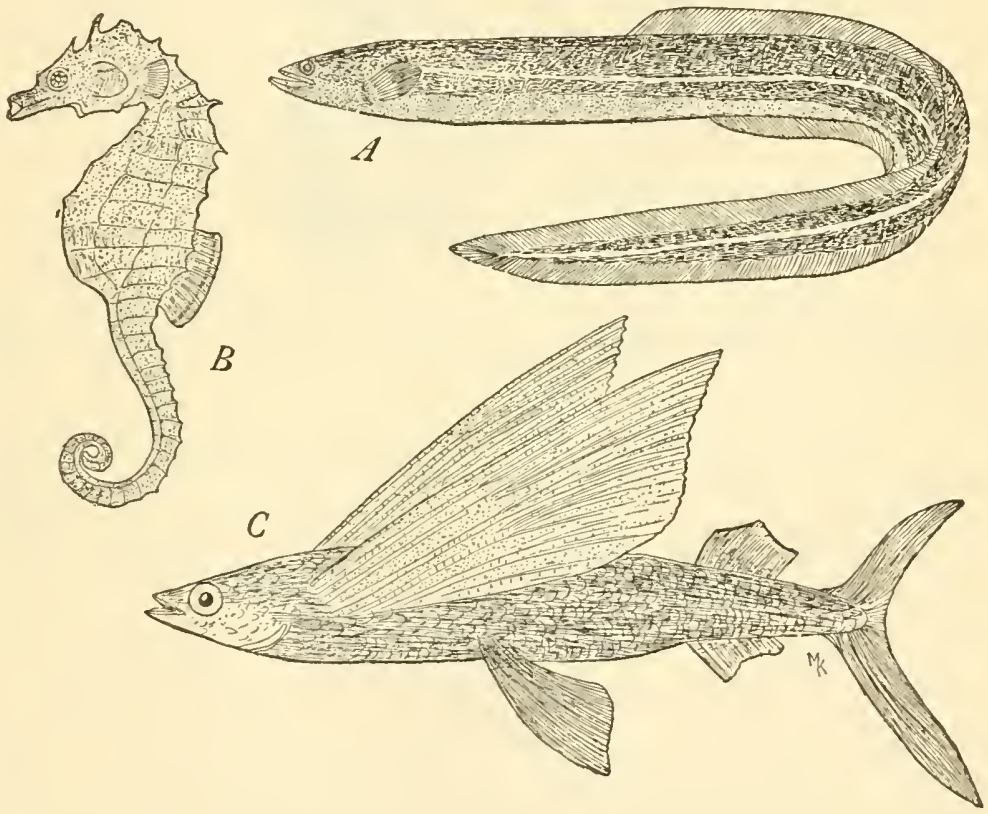

Fig. 241.-Dlagram showing some peculiar bony fish. $A$, common eel; $B$, sea horse; $C$, flying fish. (From Krecker, General Zoology, published by Henry Holt \& Company, after Jordan.)

into the dorsal lobe, but the lobes stroke the water with about equal surface and force. It forces the fish through the water in a horizontal plane and is correlated with a terminal mouth. 
There are three principal types of scales which cover and protect the body of most true fish (a notable exception is the catfish to be described later). These are: ganoid, cycloid, and ctenoid. The first are usually rhombic or oval in shape and are covered by a dentinelike substance called ganoin. Such fish as gar pikes and bowfins possess this type. Cycloid scales are rather disc-shaped with conspicuous concentric lines. They are usually imbricated on the skin, like a shingle roof. The third type is similar to the cycloid except that the free edge of the scale bears some spiny projections or cteni.
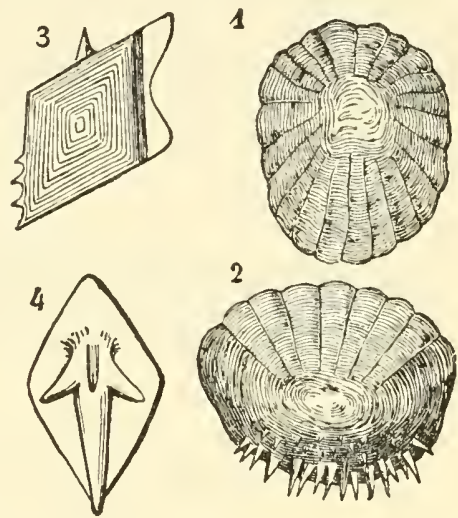

Fig. 242.-The different types of fish scales. 1, cycloid; 2 , ctenoid; $\$$, ganoid ; 4, placoid. (From Krecker, General Zoology, published by Henry Holt and Company. after Hertwig.)

Cycloid scales are found on the carp while the ctenoid are characteristic of the perch and sunfishes. The age of many fish can be determined by the distribution of the concentric lines on the scales. The lines formed during nongrowing periods fuse closely together, thus indicating seasonal periods on the scale.

The skeleton includes, besides the paired fins and girdles already mentioned, the amphicoelous (concave in both ends) vertebrae and bony cranium, which is complete and independent of the visceral skeleton. This latter portion consists of seven arches, the jaw structures, and five gill arches. The bones of the operculum arise as a part of this division.

The digestive tract is in the usual form of a canal with outgrowths. Food ranging from vegetation, insect larvae, crustacea, clams, and snails to small fish and amphibia is utilized. It passes 
through the toothed mouth, pharynx, esophagus, stomach, duodenum, ileum, and large intestine during the process of digestion. Teeth are located on the jaws, roof of the mouth, and walls of the pharynx, and are used primarily for holding prey. Gastric glands in the wall of the stomach supply some of the digestive juices. Pyloric caeca which join the anterior portion of the duodenum inerease the absorptive and digestive surface in many fish.

The respiratory system consists of the mouth, gills, and, to some extent, the swim bladder in certain fish. Water is drawn in or inspired through the mouth and forced out or expired through the gill slits. The mouth and pharynx form a water-tight pumplike arrangement with the help of the flaplike oral valves just inside the lips and the branchiostegal membrane at the margin of the operculum. The exchange of oxygen and carbon dioxide between the blood in the capillaries of the gills and the water occurs as the water passes over the gill lamellae. Oxygen is absorbed by the blood, and carbon dioxide is discharged to the water.

The circulation in most fish is, in general, similar to that described for the lamprey, except for certain specializations and phylogenetic developments. The system includes the paired anterior and posterior cardinal veins meeting in the duct of Cuvier which joins the sinus venosus, the hepatic portal vein leading to the liver, the hepatic vein from liver to sinus venosus, the two-chambered heart with its accessory sinus venosus and bulbus arteriosus, ventral aorta, branchial arteries, dorsal aorta, and the various branches.

Excretion is accomplished by a pair of dorsally located mesonephric kidneys, each of which is comnected by a mesonephric duct to a urinary sinus or bladder. This bladder opens to the exterior by an aperture located just posterior to the anus.

\section{Classification}

Few students of the fish are in complete agreement on the classification of all groups of fish, but a general scheme of the most acceptable plan will be presented. There are something like 20,000 species of fish described, of which more than 3,300 species oceur in North America. There are two subclasses. An abridged summary of their orders and families is included here, 
Subclass Teleostomi.--True Fishes. In this group are found all fresh-water fishes and many of the marine fish which frequent our waters and shores, except lamprey. In this general division of the group are four orders in the world, each including subdivisions of either families or in the larger orders, suborders and families.

Order Crossopterygii (Family Polypteridae).-Polypterus, an Afriean fish using the swim bladder as an accessory respiratory organ.

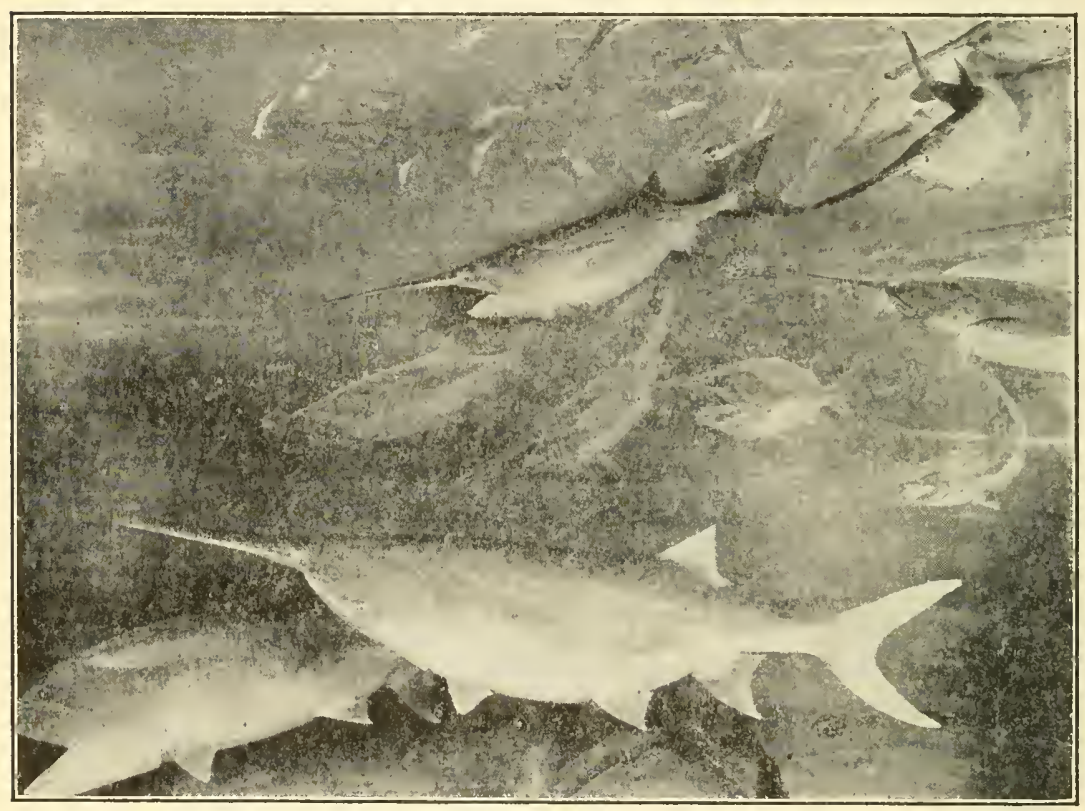

Fig. 243.-Polydon spathula, spoonbill cat or paddle fish and its associates. (Courtesy of American Museum of Natural History.)

Order Chondrostei.-Paddlefishes and Sturgeons. A ganoid type of fish with abundant proportion of cartilage in the skeleton and with bony ganoid scales.

Family Polyodontidae.-Paddlefishes or Spoonbills. Polydon spathula is found in the Mississippi Valley. It has a smooth skin and long, flat paddlelike snout, with a few ganoid scales on the tail.

Family Acipenseridae.-Sturgeons. There are only three genera usually described for this country. The body is covered with five rows of keeled, ganoid shields and the tail is distinctly heterocercal 
with the upper lobe quite extended and slender. There are no teeth in the mouth. Acipenser fulvescens is the Mississippi Valley form, and, though it was once abundant, it can now be found only occasionally. Both families of this order furnish both flesh and roe (caviar) as food for man.

Order Holostei.-Gar pikes and Bowfins. This is another ganoid type but with a more complete bony skeleton. In most of the representatives of the order, the scales are of the enamellike ganoid variety, but in a few they are cycloid and imbricated.

Family Amiidae.-Bowfin or Fresh-water Dogfish. In Amia calva, the only species in existence, we find the cycloid scales and another form capable of accessory respiration by means of swim bladder. They inhabit the fresh-water lakes and sluggish streams as far southwest as east Texas.

Family Lepisosteidae.-Gar pikes (garfishes). The long-nosed gar and alligator gar are the most common southwestern forms, while the long-nosed and short-nosed are the common species of the Middle West. These are covered with a strong armor of rhombic, enamellike, ganoid scales. The pelvic fin is abdominal and the dorsal fin is far to the posterior. The snout is conspicuously extended, and the tail is heterocercal.

Order Teleostei.-True Bony Fishes. A group which includes the majority of existing fishes. It is thought to have descended from the ganoid type. Some are soft-rayed fishes with open connection between swim bladder and alimentary canal (Physostomi), and others are spiny-rayed fishes with no air duct from swim bladder (Physoclisti). Their skeletons are highly ossified. They have cycloid, ctenoid, or no scales and their type of tail is usually homocercal.

Suborder Isospondyli.-Tarpon, Herring, Salmon, etc.

Family Elopidae.-Ten-pounders. Found in warm marine waters. Elops saurus is the typical representative.

Family Megalopidae.-Tarpons. This is a very active game type of fish with large scales and an extended filament from the dorsal fin. It is very abundant throughout the Gulf of Mexico and is a famous game fish all along the Texas-Louisiana coast.

Family Hiodontidae.-Mooneyes. Fish of this family have greatly compressed bodies which are covered with large, silvery, cycloid 
scales. There are three species in our western streams and in the Great Lakes. Hiodon tergisus is the form found in the Mississippi Valley.

Family Albulidae.-Ladyfishes. A small group of a few species found in warm marine waters.

Family Dussumuriidae.-Round Herrings. This is a scarce, small, bluish fish, with a rounded belly outline.

Family Clupeidae.-Herrings. This family includes a large number of species, and it is thought that there are more individuals in this group than in any other. They are found in most seas, and many species enter fresh water to spawn, but there are very few fresh-water species. The family includes many valuable food fishes of which Clupea harengus is the most important.

Family Dorosomidae.-Gizzard Shads. This is an abundant, widely distributed, prolific, but almost inedible, group of fish. The body is quite flat from side to side, and the edges are thin. There is a filament extending from last ray of the dorsal fin.

Family Engraulidae.-Anchovies. These fish have elongated, small, compressed bodies. They are abundant in warm seas and swim in large schools. Anchoviella (Anchovia) mitchilli is one of the most common American forms.

Family Coregonidae.-Whitefishes. There are about twenty freshwater lake species. This is an important commercial fish. Ciscos are included in this group also.

Family Salmonidae.-Salmon and Trout. This is a group of elongate, moderately compressed, large-mouthed fish with fine scales, a lateral line and, internally, a large number of pyloric caeca. They are principally northern and abound in clear waters north of latitude $40^{\circ}$. They are noted as game and food fish.

Family Thymallidae.-Graylings. These fish resemble the previous group in many ways, but they have a large dorsal fin. The Michigan Grayling, Thymallus tricolor, is a typical species. It is a northern fish.

Family Osmeridae.-Smelts. These differ from Salmonidae chiefly in the presence of a blind saclike stomach. The esophagus and pylorus join close together. 
Family Argentinidae-Argentines. This is a small group of northern or deep-sea species.

Suborder Apodes.-Eels, etc.

Family Anguillidae.-True Eels. Fishes with elongated slender body and no pelvic fins. The scales are embedded in the skin. They are among the most voracious of fishes and are quite hardy. Eels come several hundred miles inland from the Gulf of Mexico in the

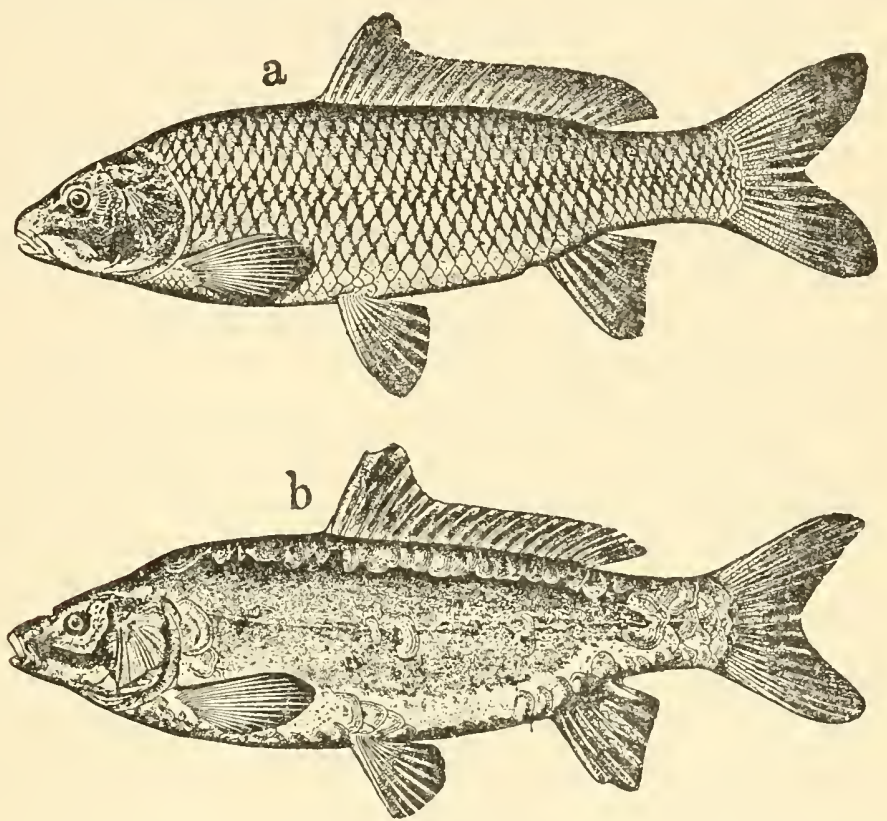

Fig. 244.-European carp, Cyprinus carpio. $a$, scale carp; $b$, leather carp. (From Metcalf, Textbook of Economic Zoology, published by Lea and Febiger, after Smith, Fishes of North Carslina.)

streams of the Southwest. They go out to sea to spawn and the young make their way back up the streams. It is thought that the female spawns once and then dies.

Family Congridae.-Conger Eels. A group of marine eels, distantly related to the Anguillidae, the common eel. They are withsut scales, and the body is often entirely black.

Family Ophichthyidae.-Snake Eels. These eels are also without ncales and have the tongue more or less adherent. 
Family Muraenidae.-Moray eels. In this type there is an absence of pectoral, as well as pelvic, fins. The skin is thick, leathery, and scaleless.

Suborder Eventognathi.-Suckers, Carp, and Minnows.

Family Catostomidae.-Suckers. This is a large and important group in fresh water. These fish have elongated bodies covered with cycloid scales, toothless jaws without barbels, and a round sucking mouth. The air bladder is large and connected with the alimentary canal. They feed on plant tissue and small animals. There are 60 species in the fresh waters of North America.

Family Cyprinidae.-Carp, Dace, and Minnows. A group of fish whose air bladders are usually large and commonly divided into anterior and posterior lobes. As in the suckers, the jaws of this group are toothless. They range from small minnows, two inches in length, to large carp of two feet in length. Some of the most abundant species of general distribution fall in this family.

Suborder Nematognathi.-Catfish.

Family Ameiuridae.-Fresh-water Catfishes. These fish are devoid of scales, have barbels on the lips, and a large, tough air bladder. As a group, they are excellent commercial and food fish, and all are tenacious of life. The bullhead catfish and channel catfish are very well known and almost universally distributed over the United States, east of the Rocky mountains.

Family Ariidae.-Sea Catfish. These, too, are scaleless and have barbels. Bagre marina, the Gaff-topsail Catfish, and Galeichthys felis, common sea cat, are very common along the shores of the southern Atlantic and the Gulf of Mexico.

Suborder Iniomi--

Family Synodontidae--Lizard Fishes. The body of this type of fish is elongate and covered with cycloid scales. The head is depressed and the mouth is quite wide with strong teeth.

Suborder Haplomi.-Mud Minnows, Pikes, and Pickerels.

Family Umbridac.-Mud Minnows. These carnivorous minnows live in the mud of sluggish streams. They have no lateral line and are covered with cycloid scales. There are at least three American species.

Family Esocidae.-The Pikes or Pickerels. These fish have a slender body, a large mouth with the lower jaw projecting, and a 
dorsal fin far to the posterior, near the base of the forked tail. Esox lucius, the common pike, and Esox masquinongy, the muskellunge, are familiar northern forms. Esox vermiculatus, the mud or grass pickerel, and Esox niger penetrate the Southwest.

Suborder Cyprinodontes.-Killifishes, Mosquito Fish, and Cave Fish.

Family Cyprinodontidae.-Killifishes. This group of minnows, well represented by Fundulus heteroclitus, the common killifish, or Zygonectes notatus, top minnow, are found commonly in shallow estuaries or fresh-water streams. $F$. heteroclitus is a much used laboratory animal along the Atlantic coast.

Family Poeciliidae.-Mosquito Fishes. This is a very common mosquito-eating fish of the South. Gambusia is a widely distributed genus.

Family Amblyopsidae.-Cave Fishes. There are a few species of these small fish living in subterranean streams of the central and southern United States.

Suborder Synentognathi.-Garfishes and Flying Fishes.

Family Belonidae.-Sea Garfishes. These are long-bodied, voracious fish of the warm seas. They range from Maine to the Texas Coast of the Gulf of Mexico.

Family Exocoetidae.-Flying Fishes. Some of these peculiar fishes are able to leave the water and glide through the air for several yards. The pectoral fins are greatly developed and serve as planing surfaces. There are about sixty different species inhabiting the tropical seas.

Suborder Thoracostei.-Pipefish, Sea Horses, and Sticklebacks.

Family Syngnathidae.-Pipefishes and Sea Horses. A group of very peculiarly-shaped fishes whose bodies are quite long and slender.

Family Gasterosteidae.-Sticklebacks. Most representatives of this family have prominent spines just anterior to the dorsal fin. They are principally northern in distribution.

Suborder Anacanthini-CCodfishes.

Family Gadidae.-Codfishes. These are large-monthed fish with numerous teeth and pyloric eaeca. They are food fish of northern seas.

Suborder Heterosomata.-Halibut Flounders, Soles, and Tongue Fish. (Flat-fishes.) 
Family Hippoglossidae.-Halibuts. The Halibut family is principally marine and is used a great deal for food.

Family Pleuronectidae.-Flounders. These fish have a laterally flattened body and both eyes on the right side of the head.

Family Achiridae.-Broad Soles. These are found quite commonly along the Gulf coast. Like the other flounders they are greatly compressed, lie on the left side, and have both eyes on the right.

Family Cynoglossidae.-Tongue Fish. In these the eyes are on the left side of the flat body and the entire body resembles a tongue in shape.

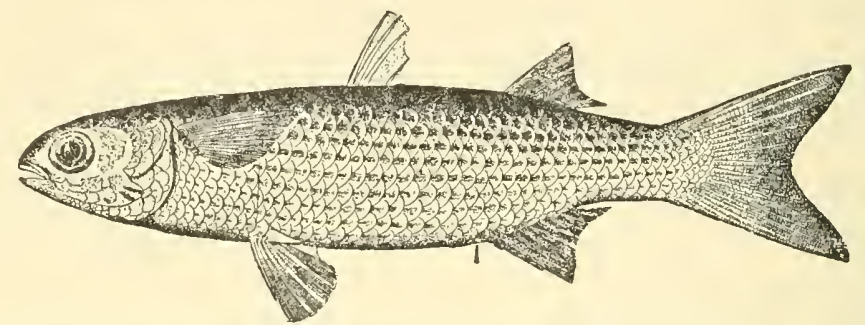

Fig. 245.-White mullet, Mugil curema. (From Metcalf, Textbook of Economic Zoology, published by Lea and Febiger, after Smith.)

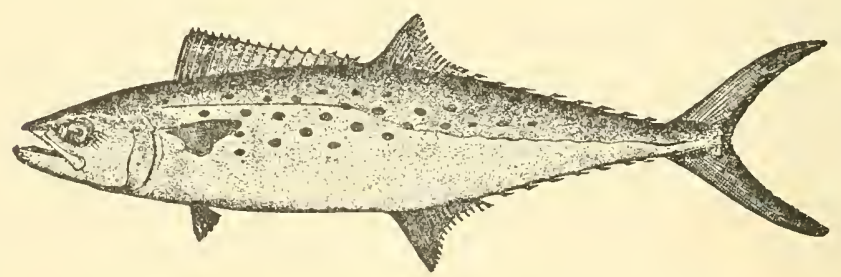

Fig. 246.-Spanish mackerel, Scomberomorus maculatus. (From Metcalf, Textbook of Economic Zoology, published by Lea and Febiger, after Smith.)

Suborder Percomorphi.-Mullets, Mackerels, Pompanos, Perches, Basses, Snappers, etc.

Family Mugilidae.-Mullets. There are mumerous species of common forms in the estuaries and river mouths along our southern shores. (Fig. 245.) This is an estecmed food fish along Atlantic coast, but it is ignored in the Gulf States.

Family Atherinidae.-Silversides. Most of these fish are small, compressed and covered with even cycloid scales. They live in schools, particularly along the coast in the warmer regions. 
Family Sphyraenidae.-Barracudas. This group includes vicious, voracious, pikelike fishes of the warmer seas. There are 15 species.

Family Polynemidae. Threadfins. This common name comes from the pectoral filaments. These are found among collections from the Gulf of Mexico.

Family Istiophoridae.-Sailfishes. Among this group are found some of the swiftest of fishes. The dorsal fin projects high above the body.

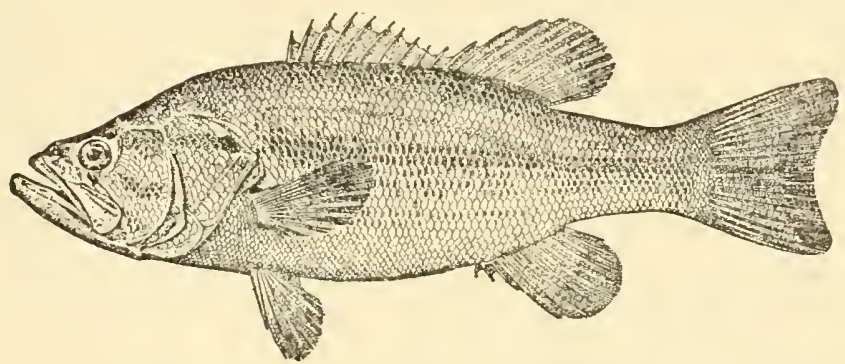

Fig. 247.-Large-mouthed black bass, Micropterus salmoides. The prize freshwater game fish. (From Metcalf, Textbook of Economic Zoology, published by Lea and Febiger, after Snith.)

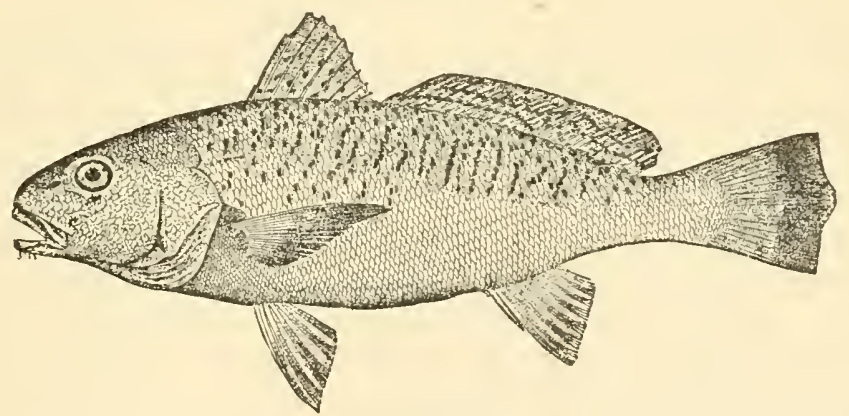

Fig. 248.-Marine croaker, Micropogon undulatus. (From Metcalf, Textbook of Economic Zoology, published by Lea and Febiger, after Smith.)

Family Scombridae.-True Mackerels. These fish are a pretty, metallic blue in color and are very important commercial fish of the Atlantic. They live in great schools.

Family Cybiidae.-Spanish Nackerel. These mackerels have a scaly body. The tail is usually quite widely forked. They are fine food fish and are very abundant in the Gulf of Mexico (Fig. 246). 
Family Thunnidae.-Tunnies and Bonitos. There is not a wide difference between these and the other mackerels. They reach large size and are quite numerous on the high seas.

Family Carangidae.-Pompanos. This family is another which is related to the mackerels. These are tropical fish and several species of them are choice food fish.

Family Centrarchidae.-Sunfishes and Fresh-water Basses. This is one of the widely distributed families with numerous species and abundant individuals. The bodies of most of the sunfishes are about the shape of a person's hand. They all build nests and are desirable game fish.

Family Etheostomidae.-Darters. A brilliantly colored group of small fish found in clear, swiftly-moving streams.

Family Percidae.-Perches. These fish have rather small fusiform bodies with ctenoid scales. Perca flavescens, the yellow perch of the northern states, is almost a classical classroom form.

Family Serranidae.-Sea Basses. There are several important food fish included in this group.

Family Lutianidae.-Snappers. These fish, particularly Lutianus campechanus, the red snapper, are abundant in the deep waters off the Gulf coast. They are prized commercial fish.

Family Sciaenidae.-Drumfishes. In this group the air bladder is usually large and constructed to enable the fish to make a grunting or drumming sound. They are carnivorous, and common on sandy shores of warm seas. Aplodinotus, the gaspergou or drum is found in fresh water.

Suborder Cataphracti.-Sea Robins.

Family Triglidae.-Gurnards or Sea Robins. The bodies of members of this family are usually covered with rough scales. They are common in all warm seas.

Suborder Discocephali.-Remoras.

Family Echeneidae.-Remoras or Shark Pilots. The spinous dorsal fin of this fish is modified to form a sucker on top of its head. The remora attaches itself to the ventral side of a shark and is carried about with it.

\section{Suborder Gobioidea.-}

Family Gobiidae.-Gobies. These small, carnivorous fish are found creeping about on sandy bottoms along shores and in mouths of rivers. There are at least 500 species, chiefly of tropical seas. 


\section{Suborder Jugulares.-}

Family Batrachoididae.-Toadfishes. This group includes a num. ber of species which have large months and somewhat the appearance of toads.

Suborder Plectognathi.-Triggerfishes, Filefishes, and Porcupine Fishes.

Family Balistidae.-Triggerfishes. The spines of the dorsal fins of these fish arc long and saw-toothed.

Family Monacanthidae.-Filefishes. The body of this type of fish is much compressed and covered with rough, velvety skin. The fins are poorly developed.

Family Diodontidae.-Porcupine Fishes. The fixed spines on the bony plates in the skin are characteristic of the group.

Suborder Pediculati.-

Family Lophiidae.-Anglers. A heary appearing, broad-bodied fish. The first spine of the dorsal fin is extended and enlarged at the tip to hang over the mouth as a bait for other fish.

Subclass Dipnoi.-Lungfishes. These fish have long, slender, paired fins and a well-developed median fin. The air bladder is connected with the pharynx and is modified to serve as a lung. They possess characteristics which apparently place them intermediately between fishes and Amphibia. There are only three living genera.

Family Ceratodontidae.-Australian Lungfish. There is only one species in this family and it is Neoceratodus fosteri, found in stagnant waters of Australia. This species is able to breathe air.

Family Lepidosirenidae.-Lungfishes of South America and Africa. Protopterus is the genus found in the marshes of Africa. They feed on insects, worms, erustaceans, and smaller vertebrates. They go into aestivation by burrowing into the mud of the marshes during the dry summer season. Here they secrete a cocoon of slime for protection. Respiration is carried on by two lungs formed from a modified air bladder. Lepidosiren paradoxa is the single form found in South America.

\section{Economic Relations of the Class}

Fish have been one of the stable sources of food for man all through history, and it is in this respect that they are most important to man in present times. Hundreds of millions of dollars 
annually are yielded by the commercial fisheries. The annual catch of salmon alone is estimated at $\$ 17,000,000$. Besides salmon, codfish, halibut, herring, shad, mackerel, mullet, red snapper, buffalo fish, carp, catfish, trout, ciscoes, and pile perch are all important food and commercial fish. In addition to the food value of the flesh, the eggs of several species, such as sturgeons and paddlefish, are in great demand as caviar. Several of the food fish are also greatly prized as game fish. Many of the game fish, such as bass, crappie, trout, and the pikes, are choice food. Some highly desirable game fish, such as the tarpon, are almost worthless as food.

Several groups of fish have a distinct negative value. The sharks destroy many other fish, lobsters, and crabs; damage perhaps onehalf million dollars' worth of fishing gear per year, as well as the larger ones, injuring or even killing a human being occasionally. Certain rays, such as Dasyatis sabina and $D$. americana, have a poisonous spine on the tail, and the pectoral fins of eatfish have a barbed poisonous spine. Either of these fish can inflict an ugly, painful wound which is dreaded by all fishermen.

The United States govermment through the U. S. Fish and Wildlife Service and most states through their Fish and Game departments are making continuous studies of the fishing industry and are conducting fish culture on a rather large scale.

The gar pike is decidedly a predator, living largely on other fish. The value of the sport of fishing is usually underestimated. Besides having recreational value, there is much money spent on the trips, tackle, clothing, etc., inspired by this sport.

There are still several other valuable relations of fish to the welfare of man. Such oils as cod-liver oil, haliver oil, shark-liver oil, especially, are much used as medicinal products for production of vitamin $\mathrm{D}$ which is a preventive for rickets. Fish scrap, which is left after the oil is extracted, is used as a fertilizer and is also put up in the form of fish meal and sold as protein feed for other animals. The hides of sharks and some other fish make fine leather when tanned. Gambusia, a top minnow of the South, called the mosquito fish, is an asset because of its appetite for mosquito larvae. It is used to control the spread of mosquito-borne diseases, such as malaria, yellow fever, and dengue fever, by destroying mosquitoes. A liquid, known as pearl essence, is extracted from certain fish scales in an ammonia 
solution. When glass beads are coated with wax and covered with this solution they become artificial pearls.

\section{TYPICAL BONY FISH-YELLOW BULLHEAD AND SOME COMPARISONS WITH YELLOW PERCH*}

The yellow bullhead catfish, Ameiurus natalis, is widely distributed through the fresh waters of the United States. Its distribution did not originally reach the Pacific coast, but during recent years it has been successfully introduced. The natural range extends throughout the Middle West, South, and well into the Southwest. These fish will be the principal subject of this description, but there will be some comparisons made with yellow perch, Perca flavescens.

The bullhead inhabits nearly all sluggish streams, ponds, and lakes. It lives along the muddy banks and around submerged rocks and $\operatorname{logs}$ in the water. It is a very hardy fish and is able to thrive in almost any aquatic condition. Perch lives in clear lakes and ponds.

\section{External Features}

The body is stout, the head is short and broad, and the mouth is wide. There is a relatively small dorsal fin located anteriorly, an adipose fin back near the tail, and a rounded caudal fin forming the tail. Just anterior to the caudal fin on the ventral side of the body is a single, broad, bladelike anal fin. Anterior to this is a pair of ventral or pelvic fins. Just posterior to the gills and in a lateral position are the pectoral fins. The skin of Ameiurus is smooth and without scales whereas the skin of perch is covered with ctenoid scales. There are two pairs of nostrils on the head, as well as eight feelerlike barbels. These are distributed, two dorsally, one attached to the maxillary process at each side of the mouth, and four palecolored ones on the skin of the lower jaw. Perch has none. Extending along each side of the body is a lateral line. The eyes are relatively small and withont lids. On each side of the head is a flaplike operculum, which covers the gills. The tail is homocercal. The colors of the upper parts of the bullhead range from yellowish green to dark brown. The sides are a lighter waxy yellow or yellowish brown, and the ventral side is yellow. The dorsal barbels are brown, while those ventral to the mouth are pinkish cream. There is a darker longitudinal band running lengthwise of the anal

- In collaboration with Rose Newman. 
fin. The general shape of the body is fusiform or spindle-shaped and hence offers little resistance to the water. In fact, the body splits the water as it passes through it, and the water closes in on the posterior slopes of the spindle shape to help force it forward.

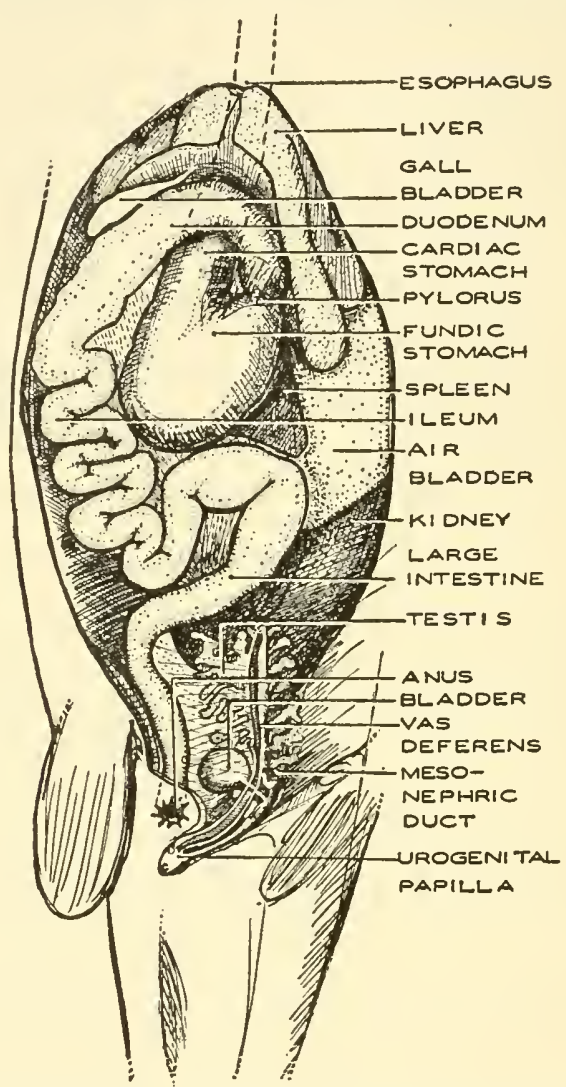

Fig. 249.-Digestive system and other visceral organs of Ameiums natalis. (Drawn by Titus Evans from dissections by Rose Newman.)

\section{Digestive System and Digestion}

Except for some habits of a scavenger, the food of the bullhead is similar to that of the yellow perch, which includes crayfishes, water snails, insect larvae, as well as insects, and other small fish. The dead bodies of almost any animal will be eaten by the bullhead. The digestive system consists of the mouth, pharynx, esophagus, stomach, intestines, and anus. The mouth is large and has teeth 
at the front supported by the maxillary jaw above and the mandibular jaw below. The teeth serve to hold the prey or food in the mouth. The tongue, which is supported by the hyoid bone, has a row of papillae running along its midline posteriorly into the pharynx. The pharynx is rather funnel-shaped and has four gill slits in each lateral wall. The bones in the roof of the pharynx bear superior tooth pads which are round or oval in shape. The esophagus is a straight muscular tube near the posterior end of which enters the ductus pneumaticus from the air bladder. As is generally the case in fish, digestion begins in the stomach which is saclike and continues directly to the pylorus in the bullhead, but is cylindrical in perch, with the pyloric portion extending from the side. Gastric glands in the walls secrete enzymes which start the digestive process. In perch there is a group of fingerlike pyloric caeca attached to the side of the pyloric region. The mass of partially digested material passes through the pyloric valve to the duodenum of the small intestine. The small intestine is shorter and less coiled in the bullhead than in the perch. It receives the bile duct from the liver and possibly some small pancreatic ducts from small masses of pancreatic tissue held in the mesenteries. There is not a distinct pancreas in either the perch or the bullhead. Digestion continues in the small intestine and most of the absorption of food by the blood occurs through the walls of the posterior portion of the ileum. Following the small intestine the short broad large intestine leads to the anus where fecal material is discharged.

\section{Circulatory System and Circulation}

The heart lies almost free in the pericardial space at the extreme anterior end of the body cavity. It is composed of two principal chambers and two accessory ones. There is a single auricle with the accessory sinus venosus leading into it, and the single ventricle which leads into the accessory conus arteriosus. The blood enters the saclike sinus venosus from the common cardinal veins and hepatic veins. It passes through a valve to the auricle, then with contraction of the auricle to the muscular ventricle through the auriculoventricular valve. The contraction of the ventricle forces the blood through the semilunar valve into the conus arteriosus, thence to the ventral aorta which branches into four pairs of afferent branchial arteries into the gill arches. Here the blood passes through finely branched capillaries 


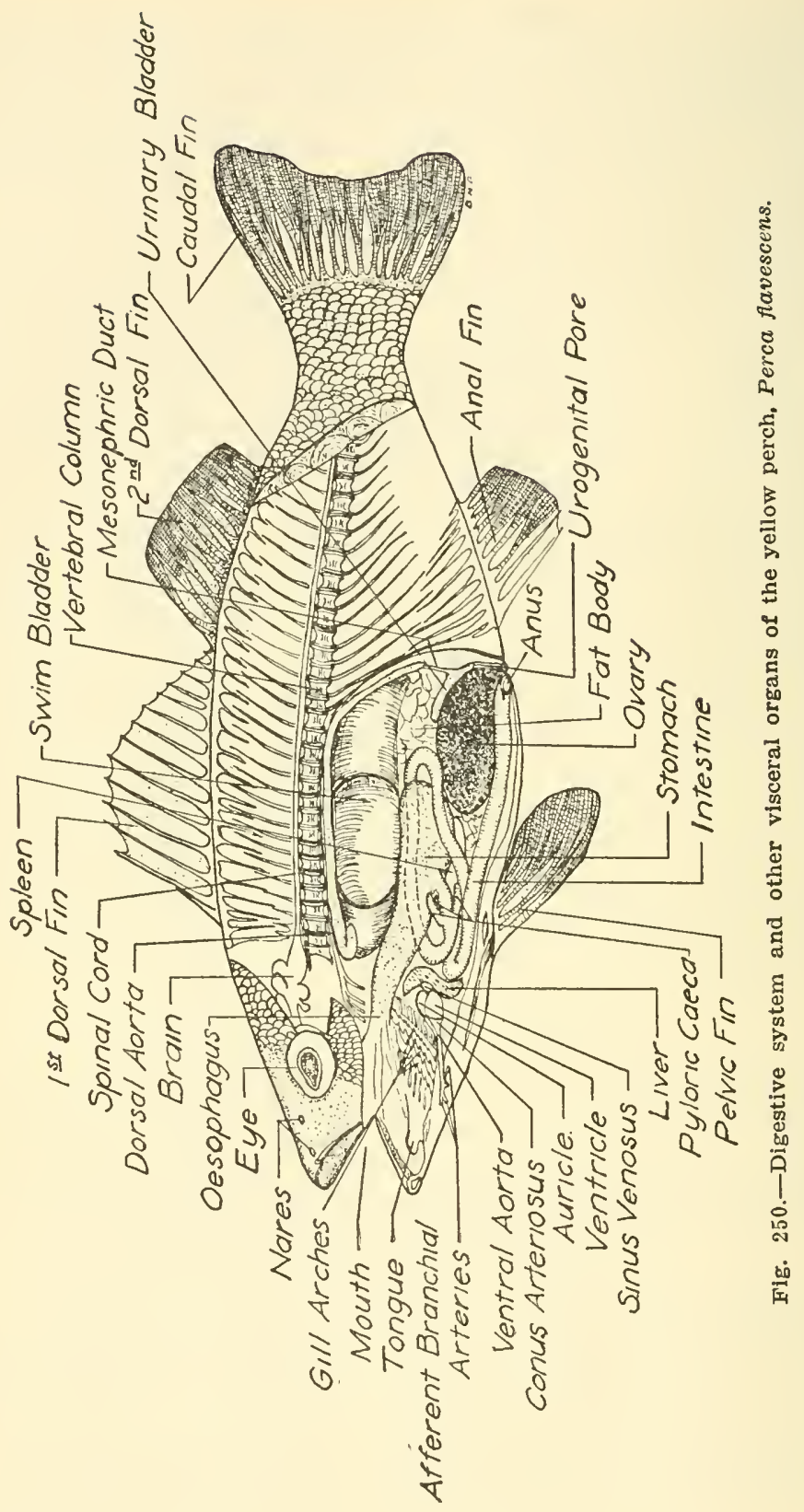




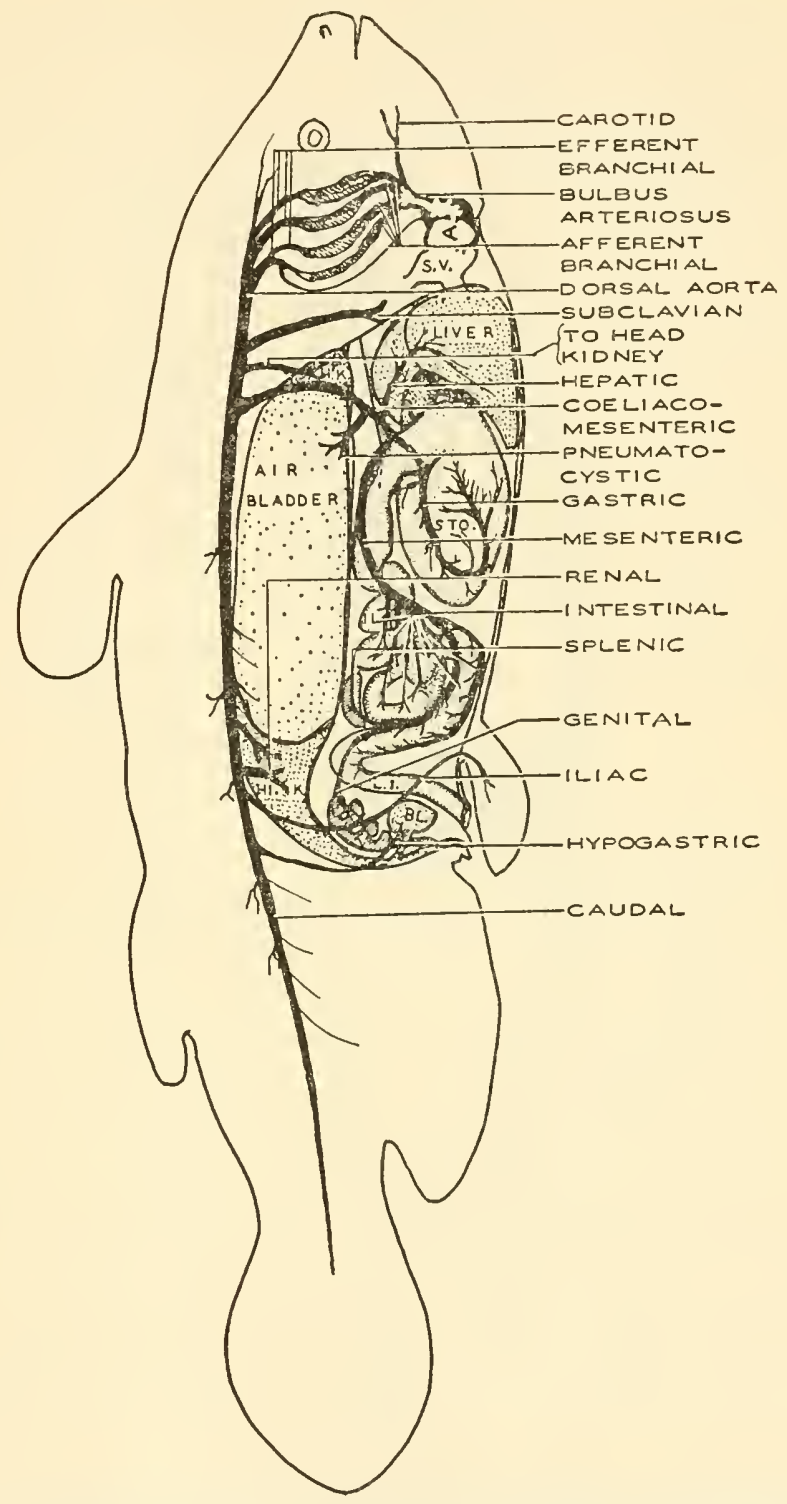

Fig. 251.-Arteries and most of the visceral organs of Ameiurus natalis Le Sueur, lateral view. $A$, auricle; $B l$., urinary bladder; H.K., head kidney; $H i . K$., kidney proper; il., ileum; L.I., large intestine; Sto., stomach; T., testis. (Drawn by Titus Evan' from dissections by Rose Newman.) 


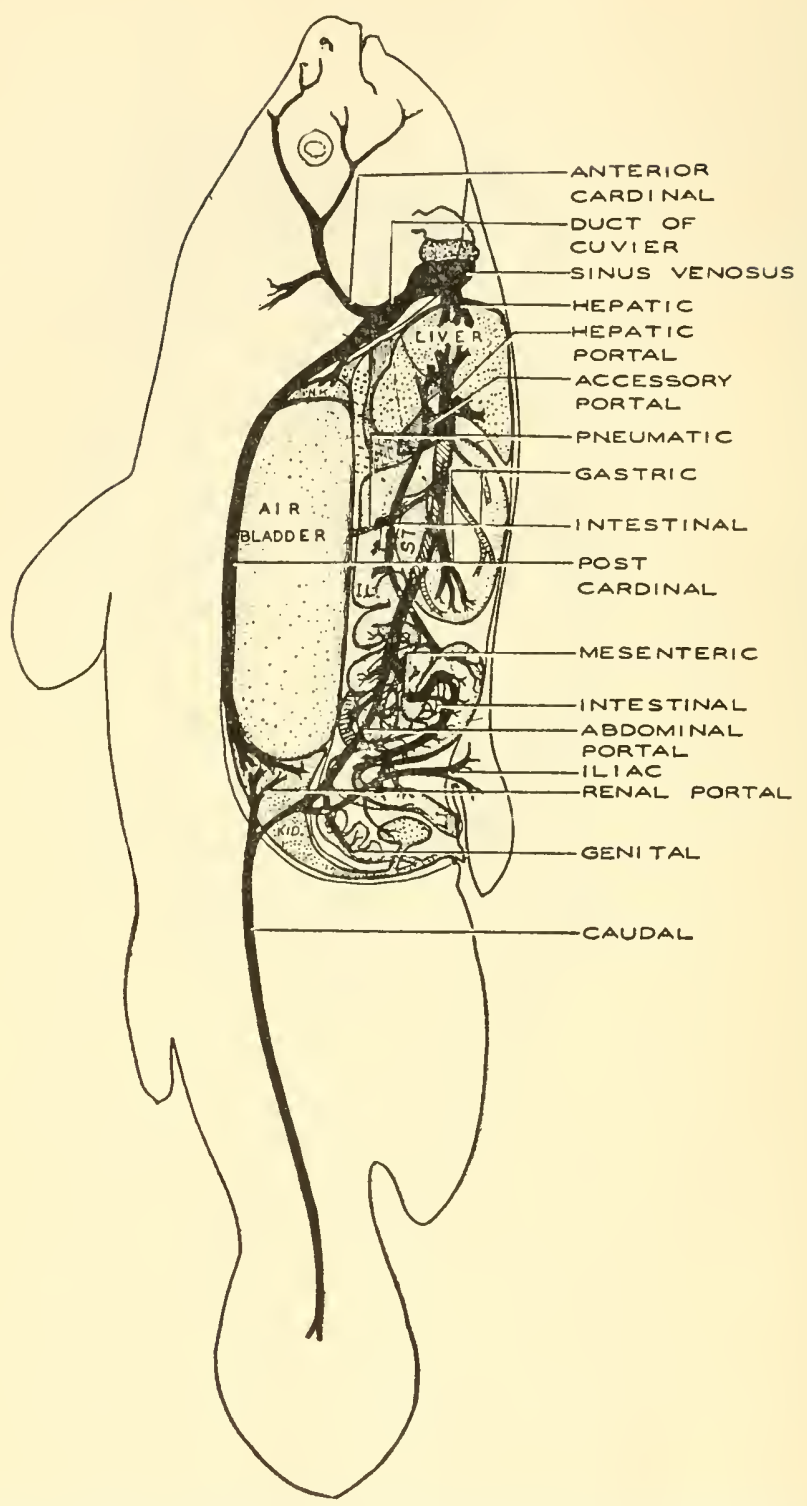

Fig. 252.-Veins and viscera of Ameiums natalis Le Sueur, lateral view. $\boldsymbol{H} . \boldsymbol{K}$., head kidney; Il., ileum; Kid., kidney; L.I., large intestine; st., stomach. (Drawn by Titus Evans from dissections by Rose Niewman.) 
where it is aerated by absorption of oxygen from the water passing over the surrounding gills. This blood continues by convergence of the capillaries into the efferent branchial arteries which lead dorsally and join in the formation of the dorsal aorta. This is the principal artery of the trunk of the body and gives subclavian branches to the pectoral fins, coeliaco-mesenteric artery to the viscera, parietal arteries to the body wall, renal arteries to the kidneys, and finally ends in the caudal artery supplying the tail. The food substances and oxygen are supplied to the tissues of the body by means of capillary branches through them. These capillaries also collect the waste products and converge to form the veins which carry the blood back to the heart. The posterior cardinals return from the posterior portion of the trunk, the hepatic portal from the visceral organs to the liver, and the hepatic from the liver to the sinus venosus, while subclavian veins return from the pectoral fin region. The blood consists of the fluid plasma, oval nucleated red corpuscles, and amoeboid white corpuscles.

\section{Respiratory System}

The mouth is used in forcing water over the four pairs of gills. Water is drawn into the mouth by lowering the floor, while the branchiostegal membranes at the margins of the opercula are closed down over the gills. The opening of the mouth is guarded by fleshy flaps or oral valves. When the mouth is filled with water, it is closed, and these valves prevent water from escaping through this aperture. The branchiostegal membranes relax and when pressure is applied to the water in the mouth, it is forced out over the gills. This process is repeated in rhythmic sequence in order that the blood passing through the capillaries of the gills will be constantly aerated.

The air bladder likely has some respiratory function. It is a large, tough sac, taking up almost one-half of the space of the abdominal cavity. The pneumatic duct extends from its midventral region to the posterior part of the esophagus. There is likely some exchange of air through this and the possibility of some degree of diffusion of gases between this and the blood in its walls. In the perch, which has no pneumatic duct, it is found that oxygen is secreted into the closed swim bladder during periods of plentiful supply in the water then drawn upon by the blood at times when the environmental supply is scant. Another function of the air bladder is to decrease 
the specific gravity of the body of the animal in water and to serve as a hydrostatic organ. In forms where the bladder is open to the exterior the volume of air in it can be regulated by direct exchange and allow the animal to take a definite level in the water without effort. In the closed type, as in perch, the volume is regulated by secretion or absorption of oxygen, as the need may be.

\section{Excretory Organs}

The kidneys of Ameiurus are similar to those in the perch. They are located in the dorsal wall of the abdominal cavity, posterior to the air bladder and just outside of the peritoneum. The head kidney

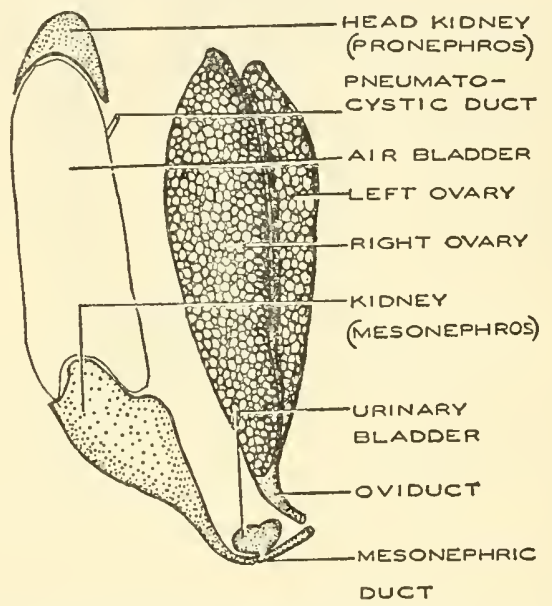

Fig. 253.-Urogenital system of bullhead, lateral view. (Drawn by Titus Evans from dissections by Rose Newman.)

(pronephros) is a paired mass of lymphoid tissue in front of the air bladder. The functional kidneys are composed of numerous uriniferous tubules supplied with blood capillaries which extract urea, creatinin, and other wastes from the blood. A slender mesonephric tube leads from each kidney to the urinary bladder. The urine is stored in the bladder and finally expelled through the urinogenital pore just posterior to the anus.

\section{Skeletal System}

Since there are no scales on the bullhead, the exoskeleton consists of the soft fin rays which support the fins. The ctenoid scales of a fish like the perch are also exoskeletal. 
The endoskeleton is principally bony and is composed of skull, vertebrae, ribs, pectoral and pelvic girdles, and bony fin supports. The main axis of the skeleton is the vertebral column and skull, known as the axial portion. The first five vertebrae of the neck or cervical region are fused together, but the remainder are separate and are called amphicoelous because each end of the centrum or body is concave. The parts of one of the simple vertebrae are the body or centrum just mentioned, and the neural arch over the spinal cord which lies in the neural canal. A neural spine extends dorsally from the neural arch, and the parapophyses extend laterally from the centrum and support the ribs. There are haemal arches supporting haemal spines on the ventral side of the posterior vertebrae. The adjacent vertebrae articulate at the centra and are held in place by ligaments. Vertebrae six to fourteen bear ribs from the transverse processes.

The skull is very largely bone with some eartilage. The bones are arranged bilaterally. The skull may be divided into cranium and visceral slicleton. The cranium encloses the brain and is composed of the frontal bones, postfrontals, parietals, supraoccipital, exoccipitals, basioccipitals, basisphenoids, alisphenoids, and parasphenoid. The ethnoids, sphenoids, epiotic, quadrates, pterygoids and nasals protect and support the auditory and olfactory organs.

The visceral skeleton supports the gills and includes the jaws. The maxillary arch supplies both upper and lower jaws. The upper jaw develops from a cartilaginous pterygoquadrate process into the pair of premaxillae and pair of maxillae bones. The lower jaw is the mandible. The premaxillae and mandible both have short spinelike teeth. Just behind the mandible is found the hyoid arch, referred to as number two. It supports the tongue, floor of the mouth, and opercle. The next five arches support the four gills and are known as gill or branchial arches. Each is composed of either four or five parts and has spiny gill rakcrs at its anterior margin. They are located in the lateral wall of the pharynx and are covered by the four opercular bones of each side which compose the framework of the operculum.

The skeleton of the paired fins is known as the appendicular skeleton. The pectoral girdle, made up of scapula, coracoid, supraclavicle, 


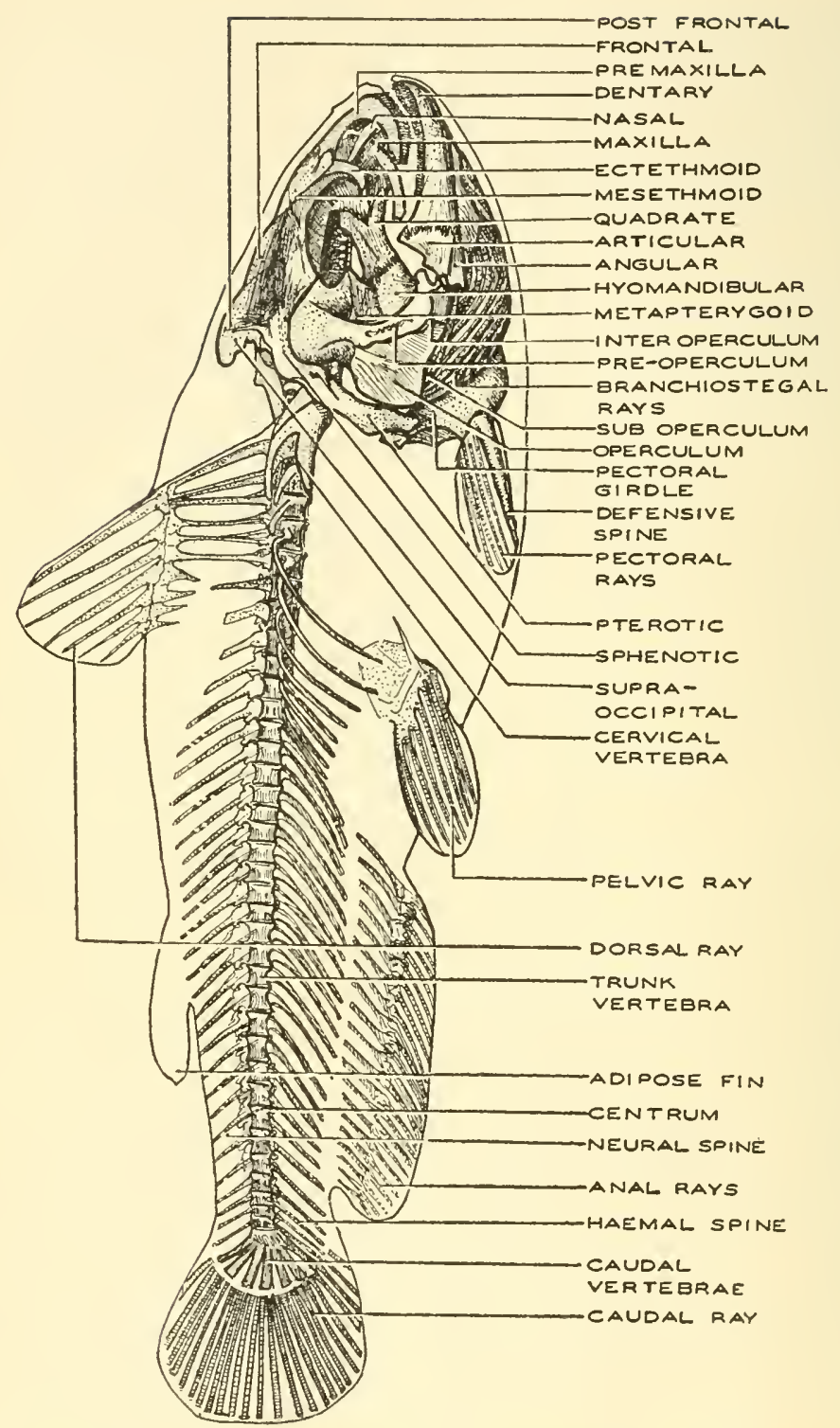

Fig. 254.- Skeleton of Ameiurus natalis Le Sueur, lateral view. (Drawn by Titus Evans from dissections by Rose Newman.) 
mesoclavicle, and infraclavicle, supports the pectoral fin which consists of a row of basal elements, and distal to this, a row of radial elements. The most anterior radial is completely ossified, terminates in a sharp spine, and has the posterior edge serrated. In addition, it has a poisonous secretion with which to inflict wounds. These are much stronger in the bullhead than in the perch. The bullhead has a rudimentary pelvic girdle, but the perch does not. It consists

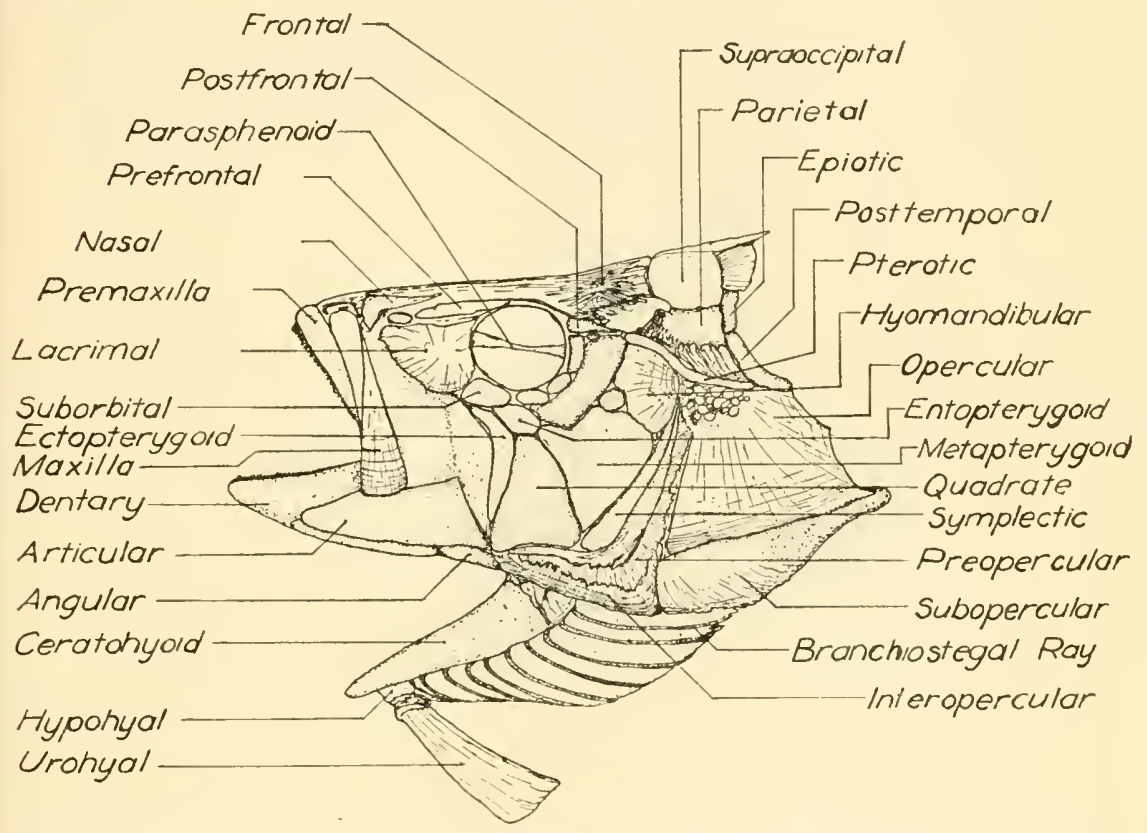

Fig. 255.-Lateral view of the skull of the yellow perch, Perca fiavescens. (Drawn by B. Galloway.)

of two similar ischiopubic plates united in the middle. Posterior to these in the midline is a platelike fusion of the basals of the fin. The radials are all fibrous. The anal fin and the dorsal fin are supported by interspinous bones. The anterior ray of the dorsal is a bony spine.

\section{Muscular System and Locomotion}

Locomotion is not the only function of the voluntary muscular system, but it is an important one. In addition to this function, 
certain muscles are specialized for feeding and others to assist in respiration. The segmental myotomes divided dorsally and ventrally by the lateral line are the chief muscles of locomotion, since most of the power is delivered by lateral strokes of the tail against the water. The myotomes are modified in the regions of the paired fins to supply certain muscles to them. The action of the fins serves

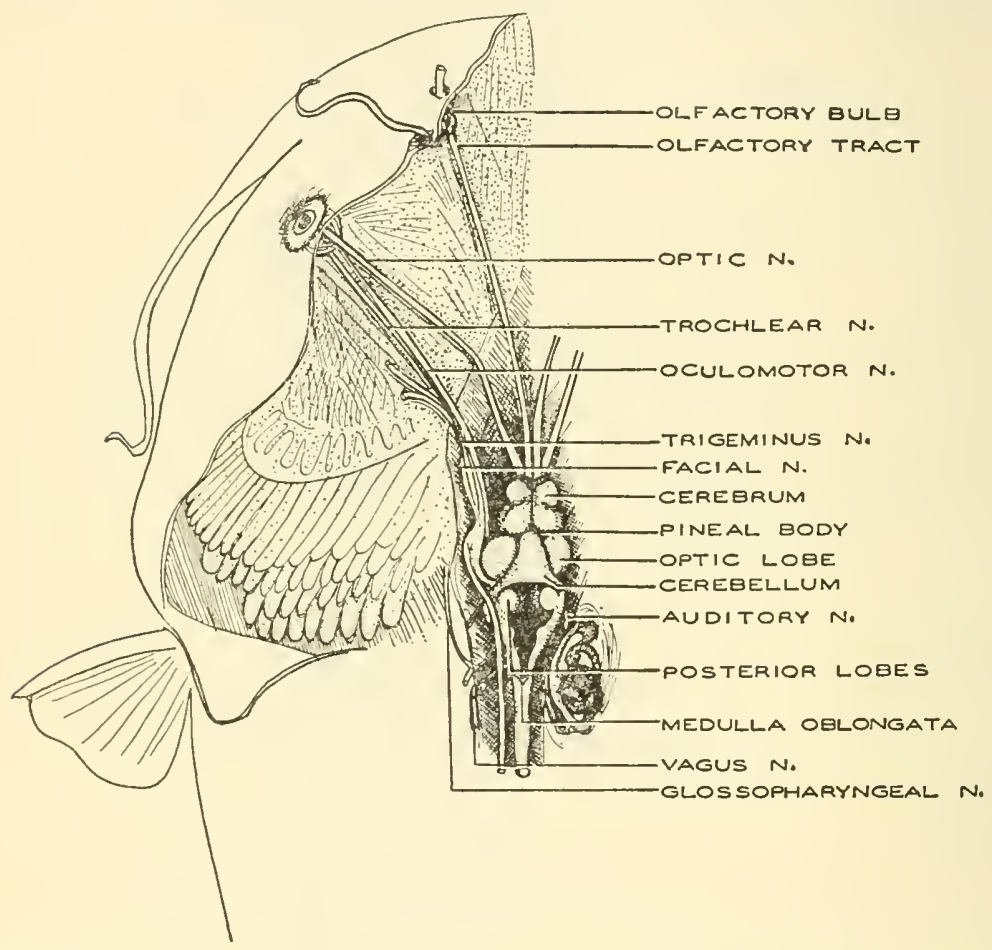

Fig. 256.-Dorsal view of brain and cranial nerves of Ameiurus natalis Le Sueur. (Drawn by Titus Evans from dissections by Rose Newman.)

to help in equilibration and in guiding the body as it is forced through the water. The fins act somewhat as a combination keel and rudder. Without them the fish is unable to hold its upright position and guide itself through the water.

In connection with the mouth and feeding process there are several distinct muscles. The adductor mandibularis elevates the lower jaw while the geniohyoid and mylohyoid raise the floor of the mouth and tongue. There are eight different sets of muscles connected with the respiratory movements of the opereula and gills. 


\section{Nervous System}

The central nervous system, composed of the brain and spinal cord, is a little more developed than it is in the lamprey or shark. The cerebral hemispheres are closely fused with the olfactory tracts which extend by long tracts anteriorly to the olfactory bulbs (Fig. 256). The diencephalon, which is immediately posterior to the cerebrum, is covered dorsally by the large dome-shaped cerebellum (much smaller in the perch). Extending dorsally from the roof of the diencephalon is a slender, fingerlike pineal body (epiphysis) which is the vestige of a third or median eye. Also partially covered by the cerebellum is the midbrain which is divided into two rounded optic lobes. The medulla oblongata lies just posterior to the cerebellum and is quite

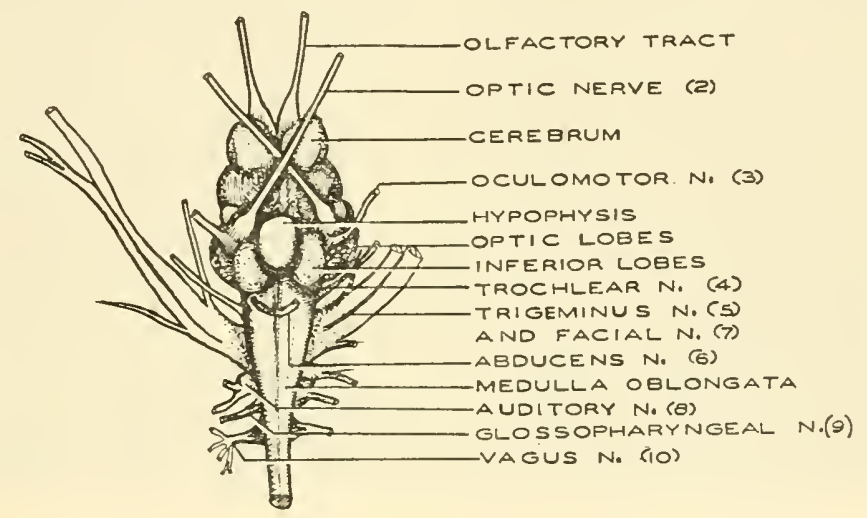

Fig. 257.-Ventral view of braln and cranial nerves of Ameiurus natalis Le Sueur. (Drawn by Titus Evans from dissections by Rose Newman.)

prominent, due to the large posterior lobes (tubercula acoustica) on each of its anterolateral positions. Dorsally, between these lobes, is a diamond-shaped slit which leads into the cavity of the brain. This is the fourth ventricle. On the rentral side of the diencephalon is the optic chiasma where the optic nerves meet, and behind this are the inferior lobes with the stalklike infundibulum joining the glandular hypophysis. Two peculiarities of the brain of Ameiurus are the large cerebellum and the large posterior lobes. There are ten pairs of cranial nerves emerging from various levels of the brain. Three of these have strictly sensory function, three are strictly motor in function, and four have both sensory and motor function. The bullhead has forty-one pairs of spinal nerves arising segmentally from 
the spinal cord. Each has a dorsal ramus, or branch, and a ventral one extending out to certain parts of the body in the region.

The sense of taste is highly developed and is centered in the numerous and well-developed taste buds which are distributed on the inside and outside of the lips, in the lining of the first three gill slits, on the barbels, and in groups over the external surface of the body, even to the tail. The eyes are small and without lids but have fair power of vision as this sense goes in fish. The focal distance is between twelve and eighteen inches, and is better for detecting motion than for recognizing objects. The fish does not have a sense of hearing;

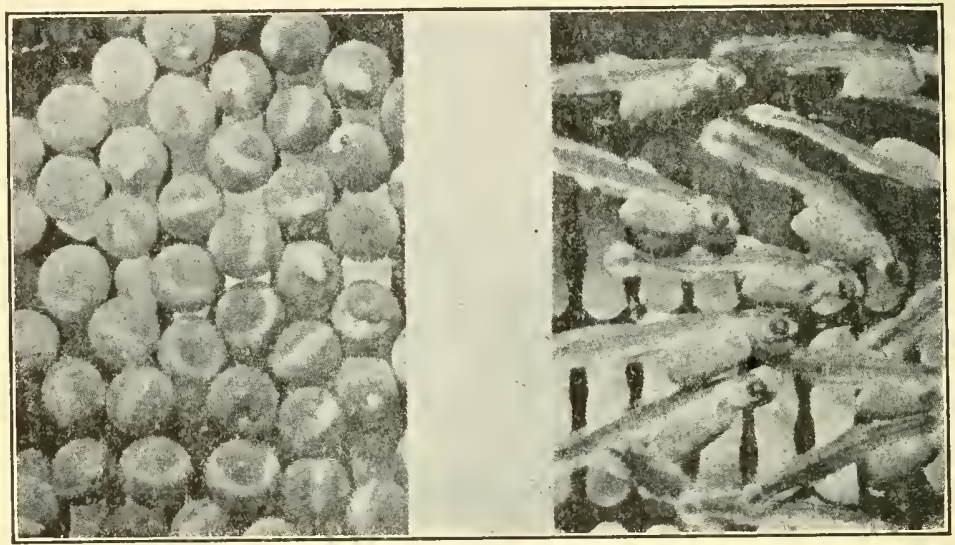

A.

$B$.

Fig. 258.-Eggs of trout with well-developed embryos, and recently hatched fry. $A$, eggs with embryos; $B$, fry. (Courtesy of General Biological Supply House.)

the ear structures serve in the sense of equilibrium. Ameiurus, perch, and other fish have a well-developed pressure and water-vibration sense centered in the lateral line system. The sense of touch is distributed over the epidermis but is particularly keen in the lips and barbels.

\section{Reproduction and the Life History}

The bullhead, perch, sunfish and many other common fish build nests of one sort or another, lay the eggs in the nest, and guard the nest until the eggs hatch. The details of the reproduction and breeding are not so well known in Ameiurus natalis as they are in 
A. nebulosus, the brown bullhead. This being the ease and since the two are very similar, a brief description will be given for the latter. The observations were made on a pair in an aquarium in Washington, D. C. They made a nest on July 3 by removing with their mouths more than a gallon of gravel from one end of the tank, leaving the slate bottom bare. On July 5 about two thousand eggs were deposited in four masses. Ninety-five per cent of them hatched in five days with the water at $77^{\circ} \mathrm{F}$. The young remained in masses until six days old; then they began to swim. By the end of the seventh day they were swimming actively and most of them collected in a school just beneath the surface, where they remained for two days, afterwards scattering. It is also reported that they ate finely ground liver on the sixth day and had enormous appetites after the eighth day. They were $4 \mathrm{~mm}$. long when hatched and had attained a length of $18 \mathrm{~mm}$. by the fourteenth day. At the age of two months their average length was $50 \mathrm{~mm}$. Both parents assume responsibility in caring for the eggs, keeping them agitated constantly by a gentle fanning motion of the ventral fins. The egg masses are also sucked into the mouth and then blown out with some force. These operations were continued until the fry (newly hatched fish) swam freely. 


\section{CHAPTER XXVIII}

\section{CLASS AMPHIBIA}

\section{(By Ottys Sanders, Southwestern Biological Supply Company)}

As there are many vertebrate animals which lead an amphibious life, it was natural for Linnaeus to group these together under the class Amphibia. This, of course, was classification based on habits rather than on structure, and as soon as such animals as the seal and crocodile were studied structurally they were removed from the class. Today the name is restricted to a group of vertebrates which we know as frogs, toads, salamanders, and caecilians. They are intermediate between fishes and reptiles. Except in caecilians, they have paired limbs, usually with fingers and toes, and never have paired fins like fishes. They have a moist, naked skin lacking the protective hair of mammals or the feathers of birds. The caecilians, none of which has been reported from the United States, are wormlike burrowing creatures of the tropics. They have small scales between their transverse body rings, although these are not usually seen unless a dissection is made. These animals and a few others such as the large South American frog ceratopharys, which has dermal bones or "scales," are the only ones of the class to have scales. The amphibians are cold-blooded vertebrates, in contrast to the warm-blooded mammals and birds.

The frogs, toads, and salamanders usually lay their eggs in water. These develop into tadpoles or larvae breathing with gills before metamorphosing to become adults which breathe with lungs. A few species of frogs and salamanders lay their eggs on land and pass their entire development in the egg. Ricord's frog, Eleutherodactylus ricordii, and the slimy salamander, Plethodon glutinosus, are examples of species that lay their eggs on land. These land eggs lack the calcareous shell of reptile and bird eggs.

There are other exceptions to the general characteristics of this diverse class. A large group of salamanders, the plethodontids, do not have lungs even as adults, and their respiration takes place in the mouth cavity and through the skin, both of which are richly supplied with blood vessels. 
Size.-While most moderm Amphibia are small creatures, paleontological species reached large proportions, as, for example, the Mastodonsaurus, which had a skull 4 feet long and a total length of probably 15 or 20 feet. Among living amphibians, the giant salamander of Japan and China, Megalobatrachus japonicus, grows to a length of 5 feet. In the Southwest, the largest salamanders are Siren lacertina, which attains a length of about 30 inches, and the "hellbender," Cryptobranchus, which commonly grows to be

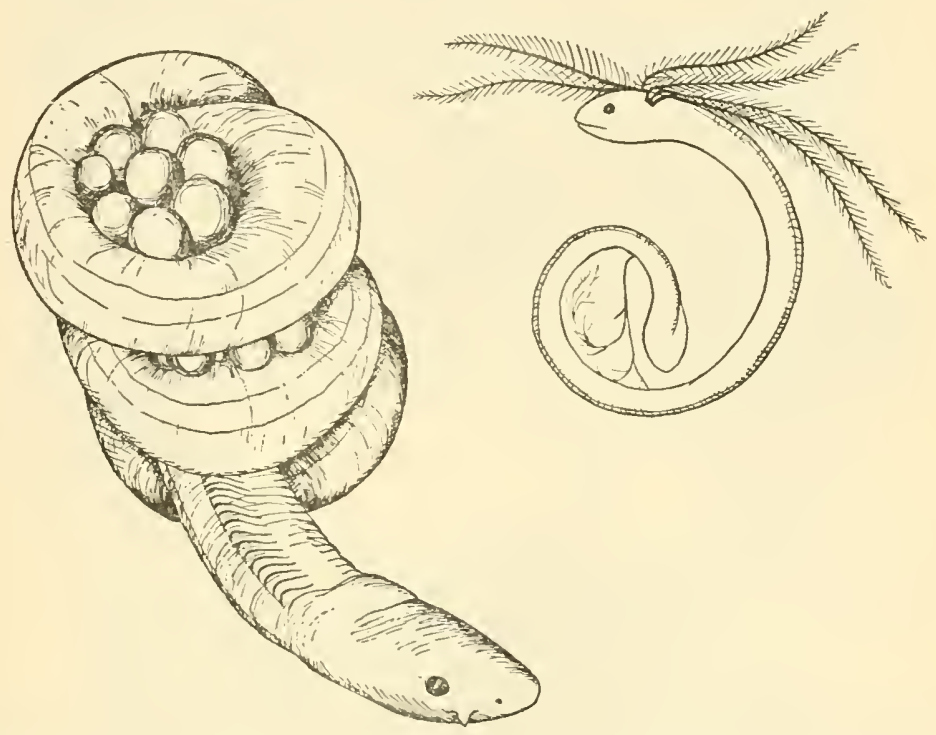

Fig. 259.-The caecilian, Ichthyophis glutinosus, adult female, guarding her eggs on the left, and a larva showing external gills on the right. Partly after Sarasins. (From Atwood: Introduction to Vertebrate Zoology, published by The C. V. Mosby Company.)

about 18 inches long and Amphiuma, the Congo eel. The goliath frog of Africa reaches a body length of nearly a foot, while southern bullfrogs, larger than their northern relatives, may grow to be over $71 / 2$ inches in body length, with a total length of 16 to 18 inches when the legs are extended. The giant toad or marine toad, Bufo marinus, is the largest of the true toads, and attains a body length of $8^{4} / 5$ inches. The smallest frog in the United States is the swamp tree frog, Pseudacris ocularis, which ranges from North Carolina to southern Florida. Adults measure only $2 / 5$ to $5 / 8$ of an inch in body length. 
As far as is known, the length of life of Amphibia ranges from ten to fifty-two years. The larger ones, in general, seem to live longer than the smaller species. Some species of toads may live about thirty years, frogs probably less.

Coloration.-Amphibians as a group al'e very colorful. The bright green tree frog, Hyla cinerea, which makes bell-like calls from the reeds and cattails in the summer months, the small grayish canyon toad, Bufo punctatus, with its red warts, the varicolored common tree frog, Hyla versicolor, with its orange groins, are but a few examples of beautiful species. Amphibians possess considerable ability to change color, and many of the tree frogs equal or surpass the chameleon in this respect.

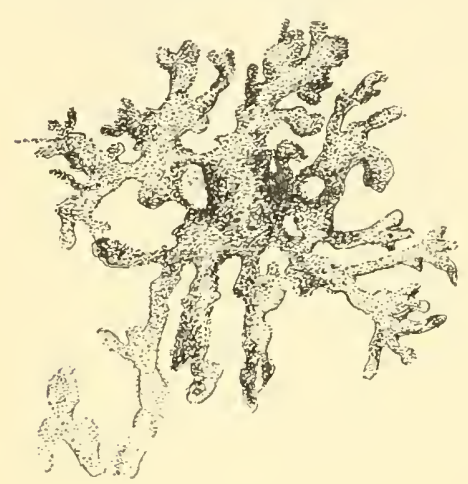

A

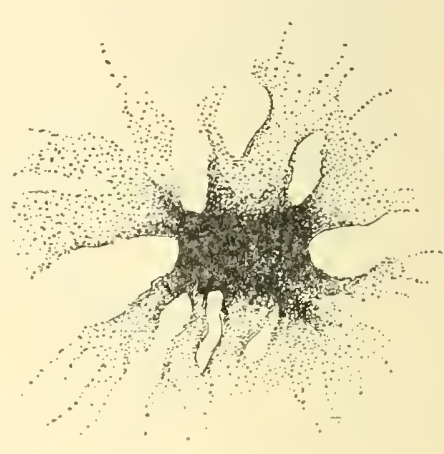

$B$

Fig. 260.-Melanophore from Rana temporia. A, pigment distributed in response to light; B, pigment contracted. (Redrawn and modified from Noble, Amphibia of North America published by McGraw-Hill Book Company.)

Their different colors are due primarily to various combinations of three kinds of pigment cells in their skiı. The black melanophores are branching pigment cells which may contract or expand, and, when these predominate, the skin appears black or brown. Yellow or red results from the action of lipophores contained in spherical cells, and white from the guanophores. Green color results from the reflection of light from guanin granules wherein all the light rays escape absorption except the green. Different arrangements of these pigment cells produce color changes which are initiated by various stimuli, such as light, temperature, moisture, and the chemical composition of the frog's habitat. These color changes are 
directly beneficial to the animal when they help it to resemble more closely its surroundings and thus avoid capture.

The Skin.-Amphibians have a soft, moist skin which is kept in that condition primarily by a rich supply of mucous glands. Aquatic

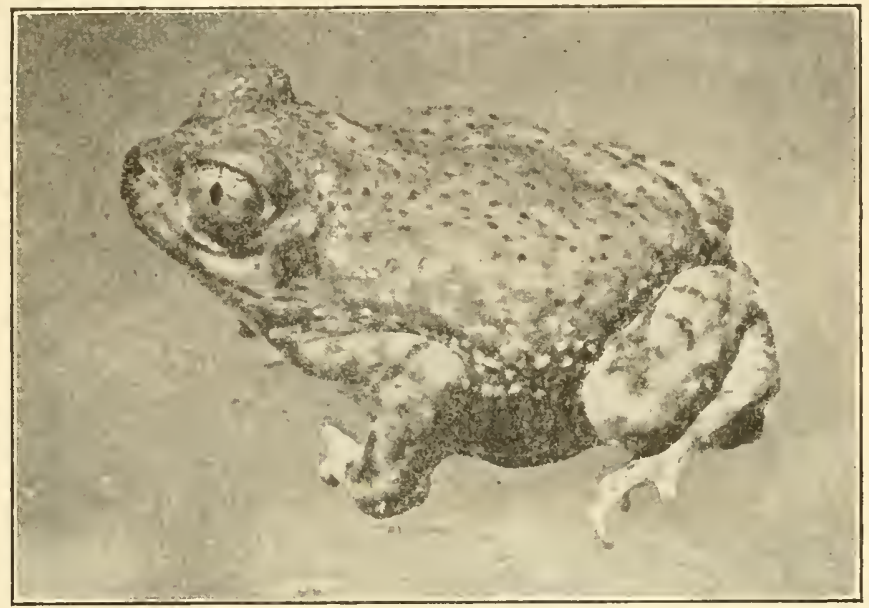

Fig. 261.-Spadefoot toad, Scaphiopus couchii, showing the shape of the pupil of the eye. (Plotograph by Thos. Mebane Jones.)

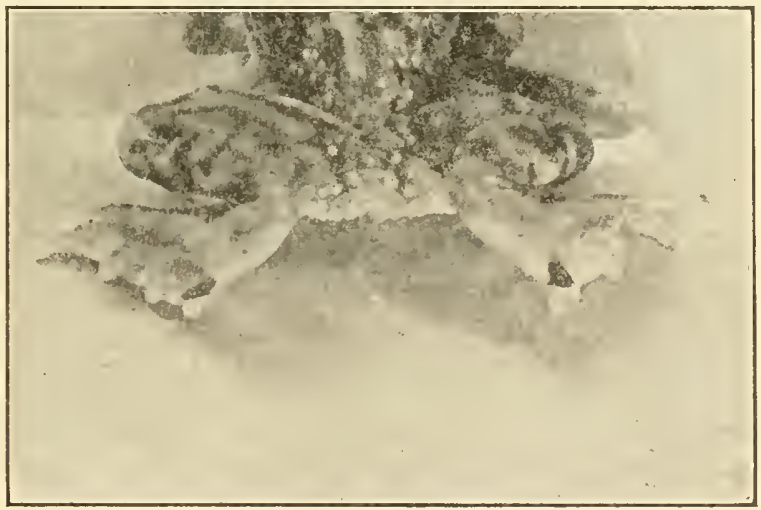

Fig. 262.-Feet of spadefoot toad, Scaphiopus couchii, showing the dark-colored, dartlike spades. (Photograph by Thos. Mebane Jones.)

and forest-inhabiting frogs and toads have a smoother skin than species which live in drier places. Burrowing frogs and toads, such as the spadefoot toad, Scaphiopus, also have thin, smooth skins, 
The skin not only protects the underlying tissues from excessive light but also has other functions. With its pigment it helps to regulate temperature by transforming light into heat. A most important function is its use as a respiratory organ. As previously mentioned, one large group of salamanders, the plethodontids, lack lungs and use the skin and buccal carity for respiration. During hibernation, practically all of the respiration of frogs is taken care of through the skin. In Africa there is a frog, with greatly reduced

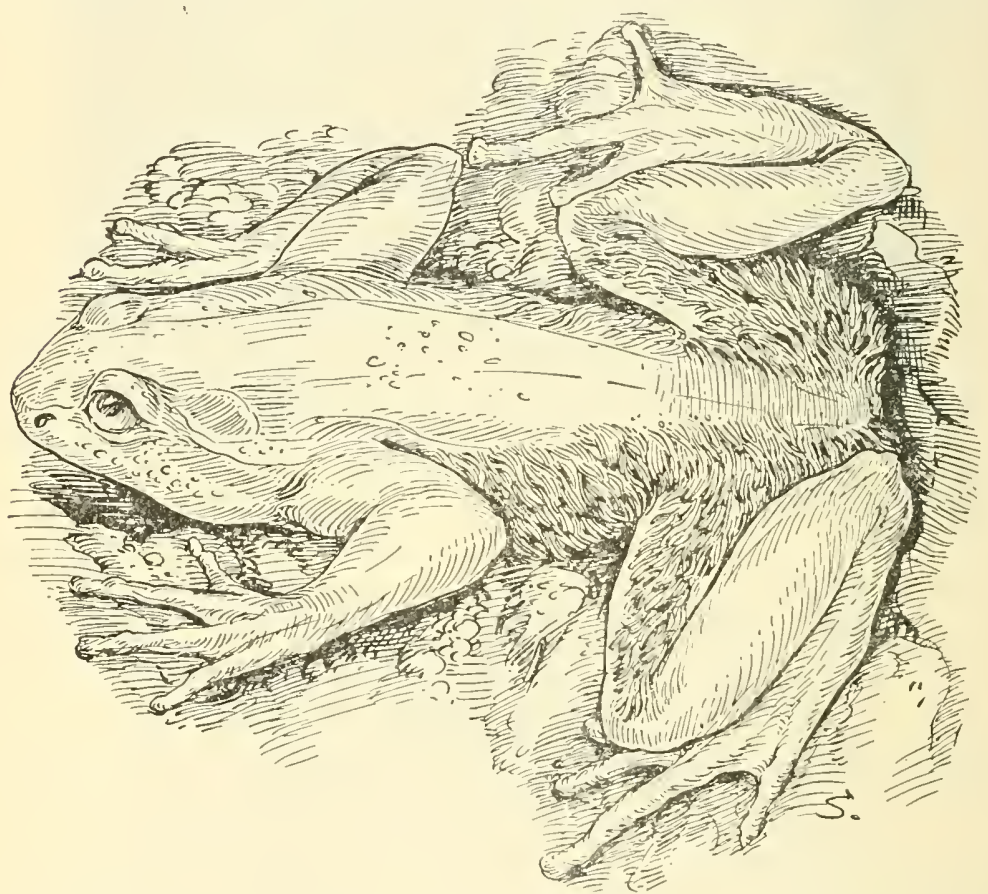

Fig. 263.- "Halry frog." (Redrawn and modified from Noble, Amphibia of North A merica, published by McGraw-Hill Book Company.)

lungs, which, in the male sex, has developed a strange aid to respiration. It has patches of vascular villosities on the thighs and sides to such an extent that it has been named the "hairy frog." These villosities help provide sufficient oxygen for its increased metabolism during the breeding season.

Since amphibians have moist skins, they are in constant danger of drying out, and therefore seek moist places where they may absorb water through their skins. Most of them are nocturnal in 
their habits and therefore can be found during the daytime under logs, in crevices or burrows in the earth, or in other situations where they can protect themselves against this constant threat of desiccation.

Food and Feeding Habits.-Adult frogs and toads eat animal food, while the tadpoles eat either animal or plant food. The food of the adults consists primarily of living insects, worms, snails, spiders and other small invertebrate animals. Many large frogs and a few smaller ones are cannibalistic. Amphibians depend to a large extent upon their sight in detecting food. While, in general, frogs and toads will seize a moving object without much examination, the toads quite often stalk their prey and inspect it. If a disagreeable insect, such as a stag beetle with strong mandibles, is swallowed, it can be disgorged because, fortunately, the toad has a wide esophagus. Most of the frogs and toads and many salamanders utilize their eyeballs in swallowing food. Their eyes can be retracted into the head and by this action they help to push food in the mouth cavity toward the esophagus.

Amphibians can go for a long period of time without food. Tadpoles may live for months, and experiments made on axolotls (larvae of the tiger salamander) have demonstrated that they may live for about a year on the food stored in their own tissues. During the hibernation season and breeding season most salamanders and frogs do not feed.

Enemies of Amphibia.- The enemies of amphibians are many. In their larval or tadpole stages they are a delicate food for giant water bugs, dragonfly nymphs, larvae of water beetles, and other aquatic insects. Small crustaceans devour the gills of salamander larvae, and fish appreciate their good flavor. Snakes, turtles, alligators, birds, and mammals feed upon the adults and young. Man enjoys the hind legs of frogs, and there is an increasing demand for these as food. Nan also destroys amphibians by polluting the streams where they breed, and his automobile kills countless toads and frogs on the highways. Nor are amphibians immune to disease and gross infestation by parasites.

Powers of Regeneration.-The power of regenerating lost parts is one way in which Nature aids the gromp. Young tadpoles may regrow limbs or tails, although adult frogs and toads are apparently unable to regenerate lost appendages. The axolotl larva of 
the tiger salamander, Ambystoma tigrinum, which is found in Texas, New Mexico, Colorado, and elsewhere, has been used extensively in experiments for studying the nature of this regeneration.

Means of Defense.-Amphibians have few ways of protecting themselves from their enemies. Their coloration often blends in with their surroundings and camouflages them, and their habit of remaining immobile frequently causes them to be overlooked. Many species practice death feints and some swell up by inflating their lungs, making themselves more difficult to swallow. The mucous glands of frogs and salamanders make them slippery, and, in the case of salamanders particularly, their writhing and twisting movements when captured make them hard to hold. A few salamanders have, in addition, the ability to break off their tails and escape.

One of the most protective weapons that amphibians have, however, is the secretion of their poison glands. This is especially effective in the case of toads, many of which have large glands on their shoulders, known as parotoid glands. An animal that has attempted to bite or swallow a toad and felt the effects of the poisonous secretion of the parotoids upon the mouth tissues will not soon forget the experience. The largest known toad of the North American continent, Bufo marinus, which ranges from Texas to Patagonia, produces one of the most virulent poisons known among amphibians. There are records of dogs which have been killed by its secretions. Glandular secretions of certain South American toads, Dendrobates, have been used by the Indians of Colombia for poisoning their arrows. The secretions of toads are ordinarily quite harmless to man, however, unless they happen to get into his mouth or eyes.

Voice.-The amphibians were probably the first vertebrates to develop a voice. The calls of modern frogs and toads are very distinctive, each species having its own particular call. Most of the croaking is done by the males, and the primary function of these calls seems to be to attract females and other males to the pond or stream. It is during the breeding season that the air resounds at night with their choruses, although certain species may croak at other times.

The croaking of frogs and toads is usually done with the mouth and nostrils closed. The air is forced back and forth between the 
lungs and mouth over the vocal cords, causing them to vibrate. Vocal saes, when present usually lying either in the floor or at each corner of the month, puff out to make resonating chambers which increase the volume of the call. Bullfrogs quite often call while under water. A few frogs, such as Ascaphus, which lives in the cold mountain streams of Washington and the northwestern United States, have given up their voice and reduced their lungs. Apparently voice would not be as useful to this species as to frogs living in quieter places, for its sound would not carry above the noise of the mountain streams. So far as is known, none of the salamander's use voice in attracting mates, and most of them are silent throughout their existence.

Breeding and Egg-Laying Habits.-Frogs, toads, and salamanders make periodic migrations to ponds and streams for the purpose of egg-laying. These periods, called the breeding season, usually occur during the spring months or, in tropical climates, during the rainy season. Salamanders often come to the pools much earlier than do the frogs and toads and may also begin their egg-laying earlier.

Most amphibians are oviparous, and their eggs are fertilized by the male after they leave the body of the female. Some salamanders and caecilians, however, have the eggs fertilized before they are laid. Among salamanders in many species, the males deposit spermatophores containing sperm which are picked up by the females and provide internal fertilization. A few species of salamanders such as the fire salamander of Europe, Salamandra salamandra, give birth to living young.

While the majority of amphibians lay their eggs in water, and the young pass through tadpole or larval stages, there are many exceptions. The eggs of the Texan cliff frog, Eleutherodactylus latrans, are laid on land, as are the eggs of its relatives in Mexico, and the tadpole stage is passed in the egg. Many salamanders lay their eggs on land. Species in the Sonthwest, such as Plethodon cinereus, usually lay their eggs in cracks and hollows in logs. The slimy salamander, Plethodon glutinosus, lays its eggs in moist places, often in the walls of caves. Some species of Oriental frogs are reported to lay their eggs in trees high out of the water. There is also reported a South African frog, Arthroleptella lightfooti, which undergoes its entire development on land and cannot swim when placed in water. 
The marsupial frogs of South America, Gastrotheca, carry their eggs in a dorsal sac or brood pouch which is found in the female. The Amazonian frogs Pipa and Protopipa carry their eggs and tadpoles in individual dermal chambers on the back of the female. In the case of a small frog (Rhinoderma) in Chile, eggs are carried in the vocal pouch of the male where they metamorphose and hatch as fully formed young. Phyllobates and Dendrobates, two frogs from the northern part of South America and Central America, transport their tadpoles on the back of the male to the stream where they pass the rest of their tadpole stage and metamorphose. In the case of the obstetrical toad of Europe (Alytes obstetricans), the male carries the eggs wrapped around his legs until they hatch.

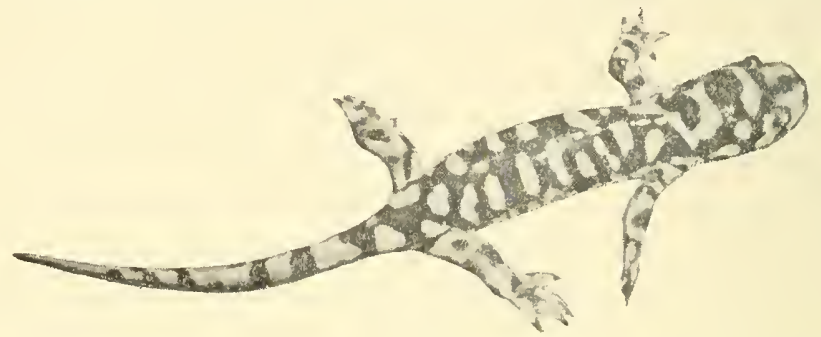

Fig. 264.-Adult Ambystoma tigrinum, tiger salamander. (Photograph by Sanders.)

Secondary Sexual Characters.-Secondary sexual characters compose those differences, exclusive of the reproductive organs, between males and females of a species. These differences may be both structural and physiological. Familiar secondary sexual characters are the nuptial pads of male frogs, their swollen thumbs during the breeding season, and, in the male bullfrog, the size of the tympanum, which is larger than that of the female. These sexual characters may be various. In some salamanders the teeth of the male may elongate; in others, glandular masses at the base of the tail or elsewhere may be present in the males and absent in the females. One of the most bizarre secondary sexual characters is found in an African frog (Petropedetes newtoni). In this frog the male has the columella of the ear pushed through the drum to form a noticeable projection.

Hibernation.-Amphibia are more or less adapted to their environment; and, when winter comes, bringing low temperatures and 
a scarcity of food, most of them hibernate. Frogs crawl into the mud in the bottom of ponds or other damp spots, dig into the ground under logs, or crawl into cracks and crevices. Toads burrow into the ground, the depth to which they go depending on the type of soil. They may go as far as 18 inches underground in sandy soil. Salamanders may bury themselves in the mud, under rocks in running streams, in rotting tree stumps or in burrows in the ground.

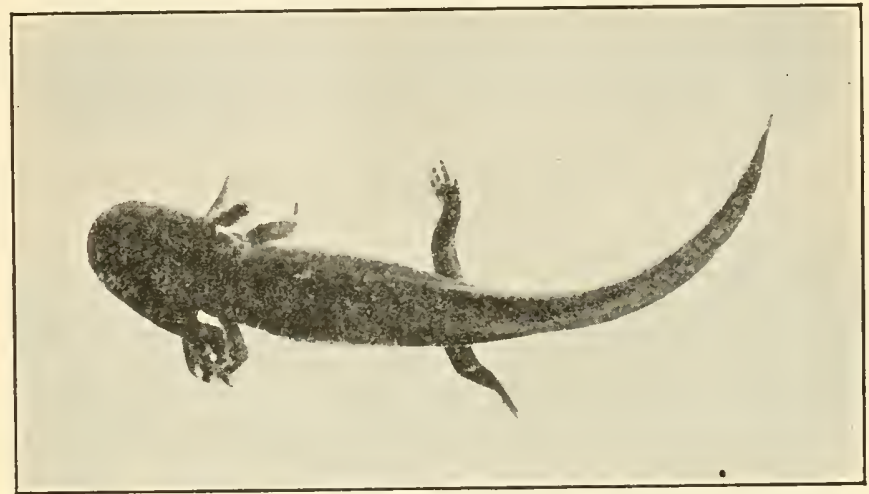

Fig. 265.-Axolotl larva of the tiger salamander, Ambystoma tigrinum. (Photograph by Sanders.

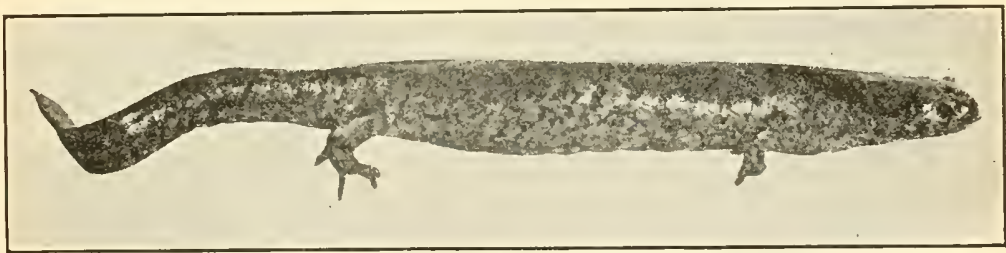

Fig. 266.-Ambystoma texanum, one of the most common salamanders in Texas. (Photograph by Sanders.)

After establishing itself in hibernation quarters the amphibian reduces all vital activities to a minimum. Respiration is carried on entirely through the skin, and the body in its dormant state secures the slight amount of nutriment needed from the food stored in its tissues. In some hot countries during the dry, torrid season amphibians aestivate in a protected moist place, reducing their activities until the severest weather is over. 


\section{Classification}

There are estimated to be about 1,900 known species of living frogs, toads, and salamanders in the world, and about 60 species

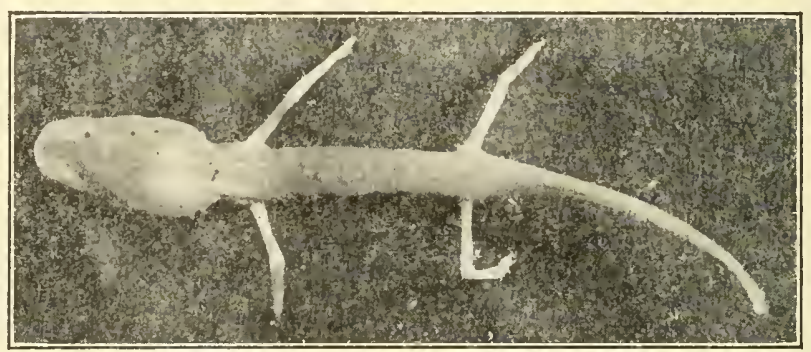

Fig. 267.-Typhlomolge rathbuni, the blind cave salamander of Texas. (Photograph by Sanders.)

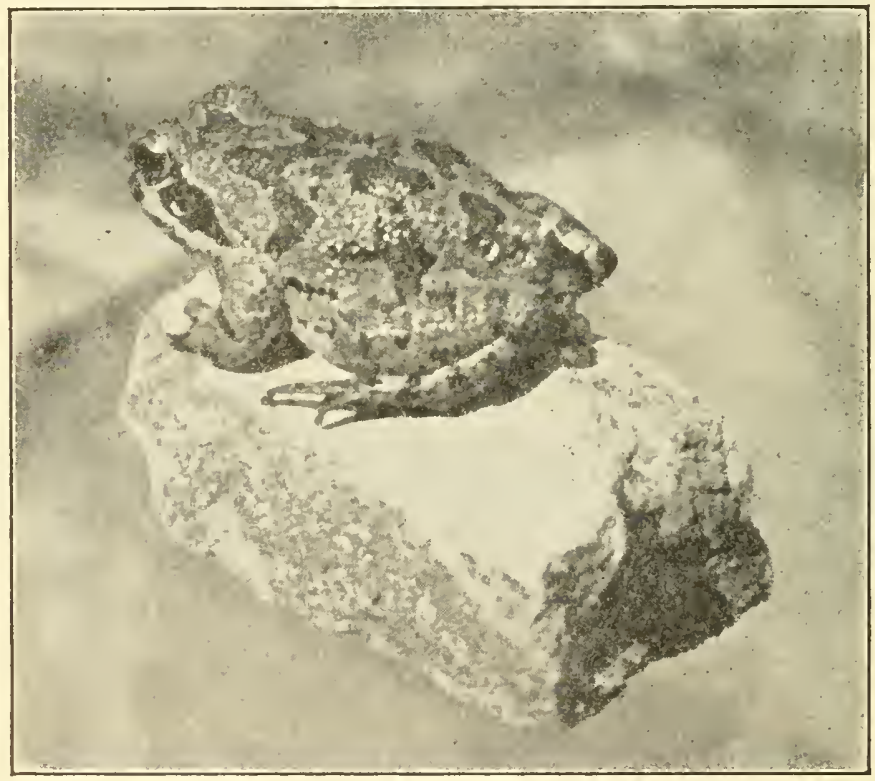
Fig. 268.-Pseudacris streckeri, Strecker's ornate chorus frog. (Photograph by
Thos. Mebane Jones.)

of caecilians. None of the caecilians have been reported from the United States. In the United States there occur about 79 species of salamanders and about 70 species of frogs and toads.* Many of

According to the Check List of North America Amphibia and Reptiles by Stejneger and Barbour, 4th edition. 
these species are subdivided into several subspecies. The Southwest contains a large proportion of all of these.

Some characters used in classifying salamanders are: the presence or absence of gills, either external or internal; color markings; shape and appearance of body; length; number of costal grooves; number of digits; position of teeth; presence or absence of a nasolabial groove; plantar tubercles; shape of vertebrae; form of cranial bones and cartilages; presence or absence of lungs; presence or absence of ypsiloid cartilage.

Some characters used in classifying adult frogs and toads are: color markings; length of body and of lind limb; shape of head;

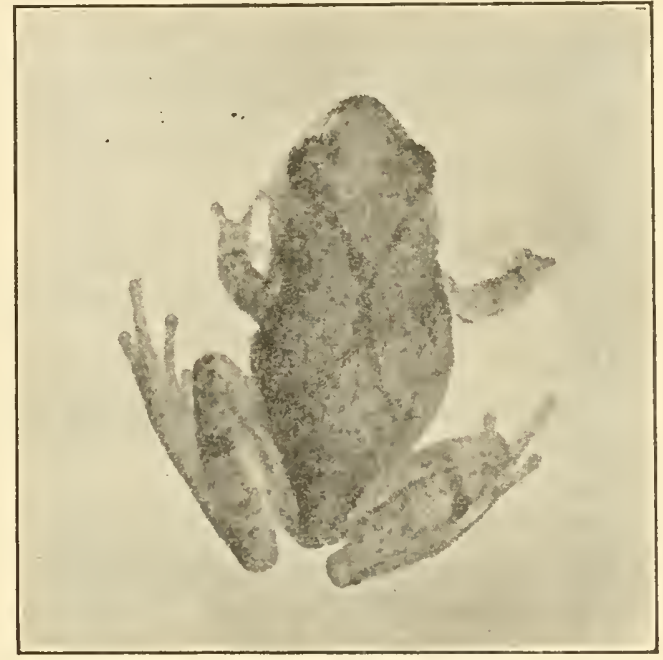

Fig. 269.-Tree frog, Hyla crucifer. (Photograph by Thos. Mebane Jones.)

nature of skin; presence or absence of parotoid glands and their shape; presence or absence of tympanum; presence or absence of cranial crests and their shape; presence or absence of teeth and their situation; the shape of the vertebrae; shape of the sacrum and pectoral girdle; shape of pupil of the eye; presence or absence of adhesive dises at the ends of digits.

The student interested in classification and identification of species should consult appropriate keys for the various groups of Amphibia. There is appended at the end of the book a list of references dealing with this class of animals. 


\section{A List of Families of the Amphibia in the United States}

The ranges cited below are not exact but give an idea of the distribution of the genera.

\section{Order Caudata (Urodela) (Tailed Amphibians)}

\section{Suborder Cryptobranchoidea}

Family Cryptobranchidae

Cryptobranchus alleganiensis (1 species). This so-called "hellbender" ranges from the eastern states west to Iowa, south to Louisiana.

Suborder Ambystomoidea

Family Ambystomidae

Ambystoma (13 species). Common species in the Southwest are: the Tiger salamander (A. tigrinum); the Texan salamander (A.texanum); and the Marbled salamander (A. opacum).

Dicamptodon ensatus (1 species). Region of San Francisco, Calif.

Rhyacotriton olympicus (1 species). Olympic Mountains, Wash.

\section{Suborder Salamandroidea}

Family Salamandridae

Triturus (5 species in the United States). The common newt of the Southwest is Triturus viridescens louisianensis. The other species represented is T. meridionalis.

Family Amphiumidae

Amphiuma (2 species). A. tridactylum, the three-toed congo eel, ranges from northern Florida to eastern Texas.

Family Plethodontidae

Gyrinophilus porphyriticus (1 species). Eastern states west to Kentucky, south to Georgia.

Pseudotriton (2 species). Pennsylvania to Louisiana.

Eurycea (6 species). Range from New England to Texas.

Manculus quadridigitatus (1 species). North Carolina to Texas. This dwarf salamander has only four toes.

Stereochilus marginatus (1 species). Dismal Swamp, Virginia to Georgia.

Typhlotriton spelaeus (1 species). The blind salamander of the cares of Missouri and Arkansas.

Typhlomolge rathbuni (1 species). The blind eave salamander of Texas.

Leurognathus marmorata (1 species). North Carolina mountains.

Desmognathus (5 species). Southern Canada to the Gulf of Mexico, eastern states westward to Illinois. Most common species in Southwest is $D$. brimleyorum, Brimley's triton.

Plethodon (15 species). Distributed over almost the entire United States. Common in the Southwest is $P$. glutinosus, the slimy salamander.

Hemidactylium scutatum (1 species). Canada to Louisiana. Another fourtoed salamander. 
Plethopsis wrighti (1 species). Oregon.

Batrachoseps (2 species). The worm salamander. Both species on the Pacific.

Ensatina (3 species). All on the Pacific Coast.

Aneides (4 species). On Pacific Coast and in southeastern states.

Hydromantes platycephalus (1 speeies). Yosemite salamander.

\section{Suborder Proteida}

Family Proteidae (with external gills and 2 pairs of limbs)

Necturus. According to a recent revision of the genus by Mr. Percy Viosca, of New Orleans, describing two new species from Alabama and two new species from Louisiana, the number of species in the U. S. is increased from three to seven. The common large Necturus from the Great Lakes region is $N$. maculosus; the species which seems to be the most common in southern states is $N$. beyeri Viosea, which extends into Texas.

\section{Suborder Meantes}

Family Sirenidae (with external gills, without hind limbs)

Siren (2 species). Eastern Virginia to Texas. Both S. lacertina and $S$. intermedia are found in the Southwest.

Pseudobranchus striatus (1 species). South Carolina to Florida.

\section{Order Salientia (Anura) (Tailless Amphibians)}

Suborder Amphicoela

Family Liopelmidae

Ascaphus truei (1 species). Washington and a few other points on the Pacific Coast.

\section{Suborder Anomocoela}

Family Pelobatidae (Spadefoots)

Scaphiopus (5 species). One species in the East, one each in Florida and California; four species in the Southwest. These are the spadefoot toads, the pupils of whose eyes are vertical when in daylight.

Suborder Procoela

Family Bufonidae (Toads)

Bufo (17 species). Species of Bufo are distributed over the entire United States. Among common species in the Southwest are B. cognatus, B. compactilis, B. debilis, B. insidior, B. marinus, B. fowleri, B. punctatus, $B$. valliceps, and $B$. woodhousii.

Family Leptodactylidae (Robber Frogs)

Leptodactylus labialis (1 species). Found only in Texas.

Eleutherodactylus (3 species). One species in Texas (Texas cliff frog), one species in Arizona, one species in Florida.

Syrrhophus (2 species). Both species limited to Texas.

Family Hylidae (Tree Frogs)

Acris gryllus ( 1 species). The cricket frog, widespread throughout eastern and central United States, including the Southwest. 
Pseudacris (6 species). Throughout the same regions as Acris. Various subspecies of the swamp cricket frog ( $P$. nigrita) are common in the Southwest. The recently described $P$. streckeri Wright, ranging throughout Texas, is a very colorful species, and its high-pitched staccato chirp is one of the earliest to be heard at breeding pools in Texas.

Hyla (12 species). Various species in all of the United States. They are the most colorful of all the frogs. Common species in the Southwest include: II. arenicolor, H. cinerea, H. crucifer, H. squirella, H. versicolor.

Suborder Diplasiocoela

Family Ranidae (True Frogs)

Rana (18 species). Various species occur in all parts of the United States. Common species in the Southwest are: $R$. sphenocephala, $R$. pipiens, $\boldsymbol{R}$. catesbeiana, R. clamitans.

Family Brevicipitidae (Narrow-mouthed Toads)

Hypopachus cuneus (1 species). In southern Texas.

Microhyla (3 species). Ranges from Virginia to Texas, northward to Missouri and Indiana.

\section{Economic Importance}

The entire group of Amphibia are of considerable economic value because they feed to such a large extent on insects, thus becoming valuable aids to the farmer in controlling noxious insects. In the flooded rice fields of Louisiana, bullfrogs grow fat eating insects, crayfish, and other small animals.

Frogs are used throughout the world as an article of food by man as well as by other animals. In the eastern United States, large quantities of the leopard frog and wood frog are consumed. In the southern states, bullfrog legs have been a favorite food for years. Within recent years businesses have developed which are devoted to supplying bullfrog legs, and the demands for these from all parts of the country have been so great that it may become necessary to afford some protection to prevent the rapid depletion of these animals. Attempts have been made to operate frog farms and raise a supply. Most of these attempts have been failures because of the high overhead cost. The axolotl is used in Mexico as food; and water dogs, such as our Necturus, are reputed to have a good flavor.

Dried frogs and toads have been used in China both as a source of food and for medicinal purposes. It is reported that toad skins have been used in Japan and elsewhere for making a fine type of 
leather. Dried salamanders have been used as a vermifnge. Adult frogs and salamanders, as well as larval stages, are widely used as laboratory animals.

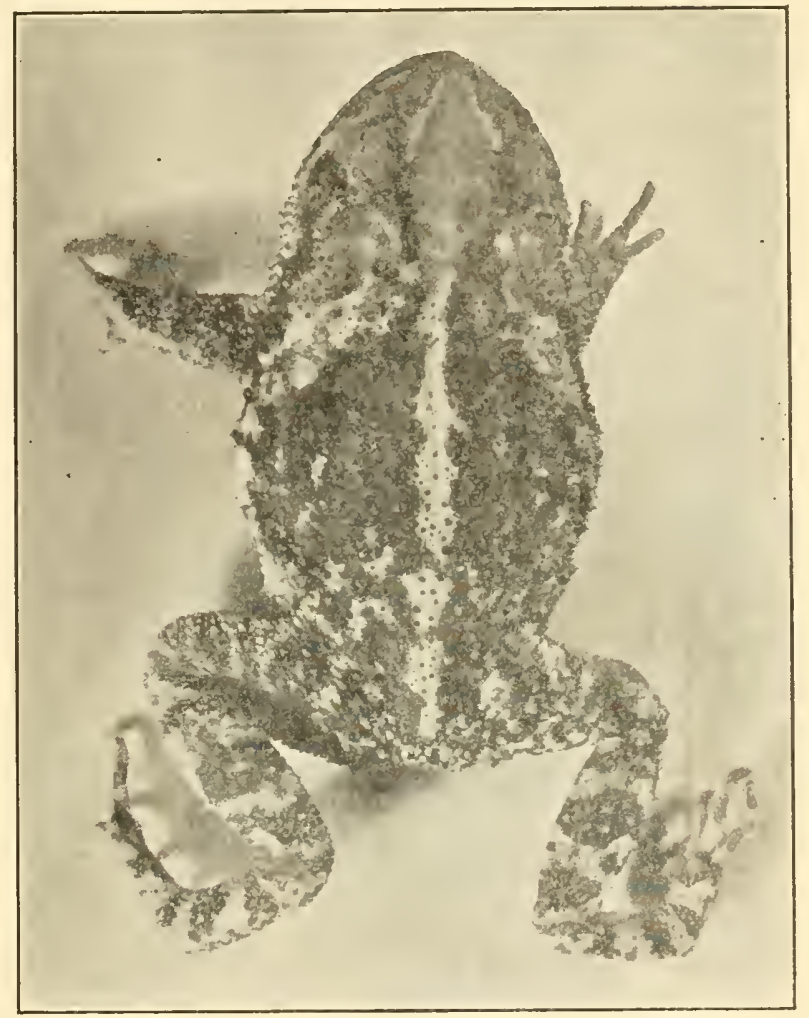

Fig. 270.-Bufo valliceps is a common toad. (Photograph by Thos. Mebane Jones.)

\section{NECTURUS MACULOSUS, THE MUD PUPPY}

Necturus maculosus, the mud puppy or water dog, is a very common example of the salamander division of Amphibia found from the Mississippi basin eastward, and is the one most commonly used for laboratory study. It lives in ponds and streams, spending most of the time in the mud at the bottom, but swimming and crawling about at night. It comes ashore only occasionally. Insect larvae, crayfish, worms, frogs, and occasionally fish comprise much of its food. 


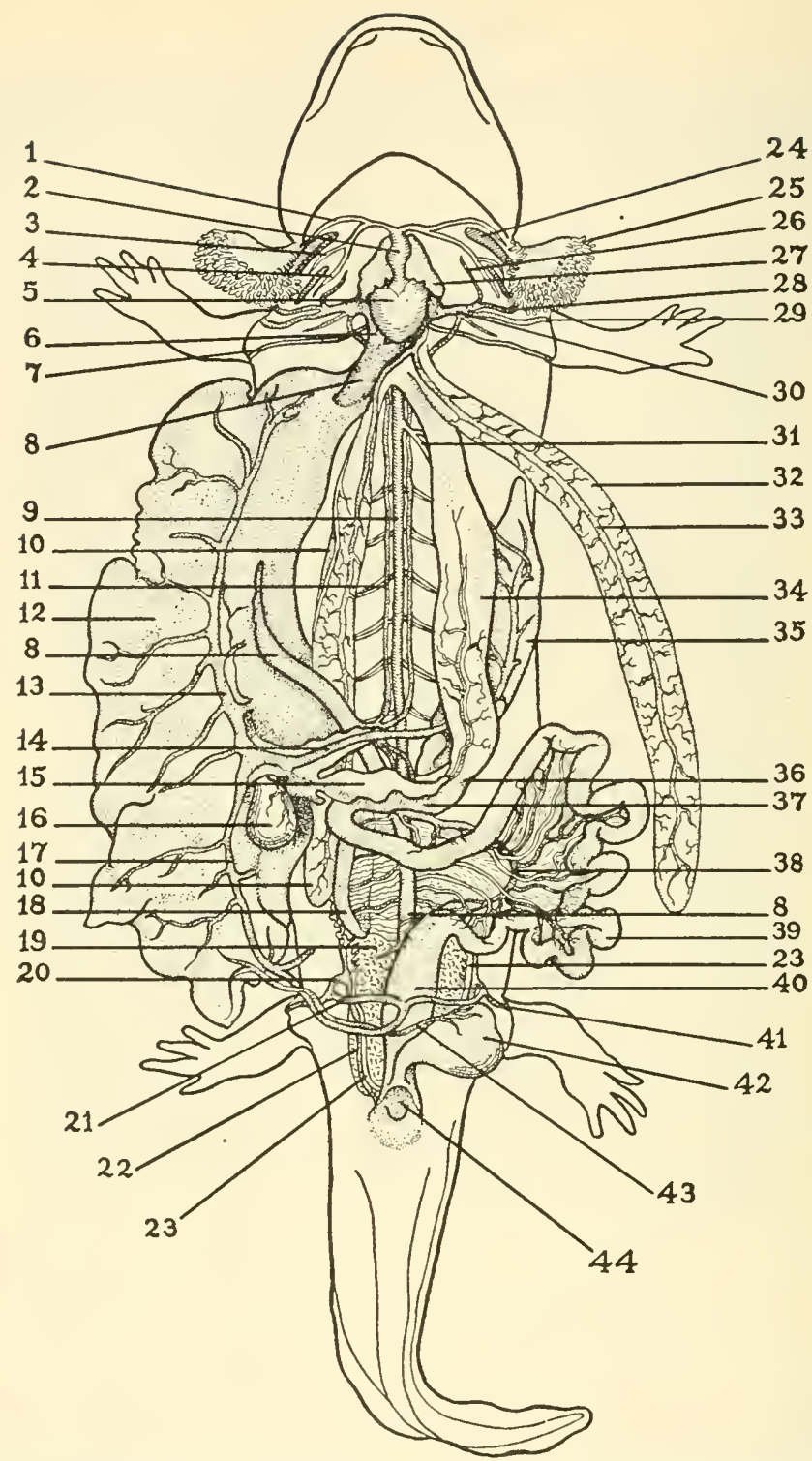

Fig. 271, A.-Diagram of dissection to show principal organs of Necturus. 1, afferent branchial artery, I; 2 , bulbus arteriosus; 3 , afferent branchial artery, II; 4 , afferent branchial ártery, III; 5, ventricle; 6 , hepatic sinus; 7 , subclavian artery; 8 , postcaval vein ; 9 , dorsal aorta; 10, right lung; 11 , postcardinal vein; 12, liver; 1s, hepatic portal vein; 14, gastrosplenic vein; 15 , pancreas; 16, gall bládder; '17, ventral abdominal vein; 18 , testis; 19 , kidney; 20 , renal portal vein; 21, pelvic vein: 22 , caudal vein: 23 , mesonephric duct; 24, gill slit; 25, external gills; 26 , internal jugular vein; 27 , left auricle: 28 , external jugular vein; 29 , subclavian vein; 30 , common cardinal vein or duct of Cuvier: $\$ 1$, gastric artery ; $\$ 2$, left lunng ; $s 3$, pulmonary vein ; 34 , stomach ; 35 , spleen ; $s 6$, pylorus ; $s 7$, duodenum ; 38 , mesenteric vein: 39 , ileum; 40, large intestine; 41 , femoral vein; 42 , urinary bladder: 43, vesical vein; 44, cloaca. (Courtesy' of General Biological Supply House.) 
The group of vertebrates which Necturus represents is of particular interest because of its transitional position between aquatic and terrestrial forms. Necturus is aquatic and fishlike in its possession and use of external gills, although it has only three arches. The body is used like that of a fish in swimming; that is, by lateral strokes of the tail against the water. It is terrestrial in the development of pectoral and pelvic girdles and limbs for crawling. Also lungs are developed for aerial respiration, although not highly functional. The sixth or last pair of aortic arches of the primitive series gives off a pulmonary artery and still retains the connection to the dorsal aorta. This portion of this arch is known as the duct of Botallus. The heart has become three-chambered; there are now two auricles instead of only one as in fish. The posterior cardinal veins are still present, but their function is partially taken over by the newly developed post cava.

Necturus remains in a larval condition throughout its life, becomes sexually mature, and reproduces without metamorphosis. Such a condition is referred to as neoteny. The retention of external gills is a very marked larval feature.

\section{Food and Digestive System}

This animal is quite inactive and requires relatively little food. It does make use of several aquatic inhabitants including erayfish, other small crustaceans, snails, insect larvae, leeches, some minnows, and occasionally fish eggs for food.

The mouth is located in the anterior, terminal position and with a fairly wide gape. Teeth are located on the premaxillae, vomer, and palato-pterygoid bones of the upper jaw, and the dentary and splenial bones of the lower jaw. The tongue is broad and only slightly movable. The internal nares enter the mouth cavity as a slit on each side between the two dorsal rows of teeth near their posterior terminations. More posteriorly, in the lateral walls of the pharynx are the two pairs of gill slits or pharyngeal clefts. Still more posteriorly there is a very small inconspicuous pharyngeal prominence with a tiny slit, the glottis. The esophagus leads posteriorly from the pharynx and joins the anterior or cardiac portion of the prominent stomach. The stomach has the typical shape and appearance of this organ in the lower vertebrate groups, possessing only the cardiac portion anteriorly and the posterior narrowed pyloric portion. This 
leads into the anterior section of the small intestine or duodenum, which includes the first S-shaped turn of the tube. The more coiled part of the small intestine following this is the ileum. This empties into the short but somewhat broadened large intestine, which opens into the cloaca, the common receptor of faccal matter from the intestine and urinogenital products from the wolffian ducts, urinary bladder and müllerian ducts. The urinary bladder is a thin-walled sac hanging at the ventral side of the cloaca, whose lumen it joins.

The liver is an elongated, dark-colored and somewhat serrated organ lying in the ventral portion of the body cavity. The gall bladder is a membranous sac attached to the margin of the liver (usually at the right side). The bladder is connected with the duodenum by a bile duct which is obscured by a mass of pancreatic tissue. The pancreas is divided into slender lobes and lies in the vicinity of the junction of stomach and duodenum. One lobe extends to the tip of the spleen which lies dorsolateral to the stomach. Another slender lobe extends posteriorly in the mesentery that supports the anterior part of ileum and the mesenteric vein. Both of these lobes join the mass of pancreatic tissue around the bile duct and the pancreatic ducts enter the duodenum at this level.

\section{Circulatory System}

There is a partial conversion from the straight branchial type of circulation of the fish to the pulmonary type of the terrestrial vertebrates, in that the number of functional aortic arches is reduced, pulmonary vessels are added to supply the lungs, and the atrium of the heart is divided into two parts to keep the systematic and aerated, pulmonary blood partially separated. With certain modification of the situation in fish, the system consists of heart, aortic arches, systemic and pulmonary arteries, as well as systemic, renal portal, hepatic portal, and pulmonary veins. The heart consists of right and left atria (auricles) and one ventricle with the two usual accessory chambers, the sinus venosus which joins the right atrium and the conus arteriosus which leads from the ventricle to the ventral aorta. The right atrium receives the systemic blood and the left atrium, the pulmonary blood by way of pulmonary veins. Blood in passing through the heart may be traced by entering the sinus venosus from systemic veins, pass by way of sinu-atrial (sinuauricular) valve to right atrium, thence through the atrioventricular valve to the ventricle which it enters simultaneously with aerated blood from left atrium. 
The blood is expelled from here through the conus arteriosus into the ventral aorta. As will likely be remembered, the theoretical, primitive, and embryonic typical number of aortic arches in vertebrates is six. This number is modified in most adult rertebrates, usually by reduction. Even teleost fish have only four branchial arches. In salamanders this number may be referred to as four but considerable modification has occurred. The first (anterior), second, and third original arches have been rearranged and combined to form the common external, and internal carotid arteries. The fourth and fifth supply the external gills with the fourth becoming the systemic arches which meet dorsally to form the dorsal aorta. The sixth arch is modified to supply a large pulmonary artery from each side to the respective lung. The portion of the sixth aortic arch which continues on dorsally to join the aorta, from the point where the pulmonary branches off, is known as the duct of Botallus.

The special modification of the veins is centered around the development of the post cava which is formed posteriorly by the junction of urinogenital veins. The pair of posterior cardinals, which are characteristic of fish, are retained also but are greatly reduced. They usually join the post cava some distance anterior to the kidneys and parallel the aorta to the heart where they enter the ducts of Cuvier, one on each side. Another modification is the pelvic-ventral abdominal complex which connects the renal portal arrangement with the hepatic portal. A pelvic vein branches from the femoral on each side before it joins the renal portal. The two pelvic veins pass ventrally to meet each other at the midrentral point of the pelvis and this union forms the ventral abdominal vein which either enters the liver or a branch of the hepatic portal before it enters the liver, thus providing a cut-off in the course of the venous circulation in going anteriorly from the posterior limbs. The lateral veins of sharks form a similar cut-off but enter the duct of Cuvier instead of the hepatic portal system.*

\section{Respiratory System and Breathing}

The respiration may be divided into cutaneous, performed through the wet skin; branchial through the gills; and pulmonary through the lungs. There are several parts to the latter arrangement. The small external nares lead by way of passages to the slitlike intemal nares which open into the mouth between the posterior ends of the two

* Helpful illustrations of the circulatory system of Necturus may be found in Stuart: Anatomy of Necturus maculosus, Denoyer-Geppert Co., Chicago. 
dorsal rows of teeth. The mouth is made airtight by the shape and fitting of the lips. The general portion of this cavity posterior to the angle of the jaws is the pharynx. Well back in the floor of it, is the tiny slitlike glottis in the midst of a slightly thickened laryngeal prominence, the opening of which would receive only an object the size of the liead of a pin. The glottis leads into a recess called the $\operatorname{larynx}$ and the two smooth-walled, saclike lings extend posteriorly from this. These saclike lungs have a fairly abundant vascularization (blood supply). The air is pumped into and from the lungs by the movements of the floor of the airtight mouth and change of position of visceral organs within the body cavity. Branchial respiration is accomplished largely by waving the highly vascularized external gills back and forth in the water. The capillary branching of aortic arches 4 and 5 provides most of this blood supply to the gills. The pulmonary artery supplying the lungs is formed by a large branch from aortic arch number 6 .

\section{Urinogenital System}

The following organs constitute this composite system: a pair of mesonephric kidneys, a pair of gonads (testes in male, ovaries in female) numerous vasa efferentia from testes, one pair of Wolffian or mesonephric ducts (ducts of Leydig in male), one pair of Müllerian ducts or oviducts (in female, only vestigial in male) single cloaca, the urinary bladder and the mesenteries (mesovarium, mesotubarium, and mesorchium).

The kidneys are somewhat elongated and flat but thicker toward the posterior, suspended in the dorsal peritoneum and lying dorsal to the large intestine. The kidney of the female is smaller than that of the male. The Wolffian duct leads from the lateral margin of the kidney in either sex and proceeds directly from the posterior portion of the kidney to make a dorsolateral entrance into the cloaca. Inside the kidney the Malpighian corpuscles, including glomeruli, are connected with the uriniferous tubules, which in turn join the collecting tubules and they lead to the Wolffian duct. After the urine enters the cloaca it collects in the urinary bladder which hangs ventrally and serves as a storage reservoir. Upon becoming filled with urine the bladder contracts and forces the urine back into the cloaca and from here it passes to the exterior by way of the anus. 
In the male specimen the yellow or brown-colored cylindrical testes are located one in either side of the dorsal part of the body cavity and each suspended by a fold of the dorsal peritoneum, the mesorchium. The vasa efferentia, which are tiny sperm tubules about the size of very fine threads, and the spermatic blood vessels are suspended in this mesentery. The vasa efferentia enter the medial side of the kidney (except at its anterior) and deliver spermatozoa to a longitudinal Bidder's canal just within. This canal is connected with the medial ends of collecting tubules and through them the spermatozoa reach the Wolffian duct, as does the urine. The Wolffian duct carries them to the cloaca. When the spermatozoa reach the cloaca and bladder they clump into bundles called spermatophores, and are stored until breeding time. A IVolffian duct (mesonephric duct) which serves both for conveying urine as well as spermatozoa is called a duct of Leydig.

In the female the pair of ovaries can usually be recognized by the presence of eggs of some stage of development in them. Then fully mature each ovary seems to be a large sac full of large yellow mature eggs about the size of small peas. In specimens with immature ovaries the eggs may be about the size of pinheads. Each ovary is suspended from the dorsal peritoneum by a mesentery, the mesovarium. There is a prominent, coiled, white oviduct or Müllerian tube in the body cavity at each side of the other organs whose anterior end is suspended in the anterior portion of the body cavity and spreads into a wide membranous funnel called the ostium. The mesentery which supports the oviduct is the mesotubarium. When the ova reach maturity inside the ovary they escape by a rupture in its wall which frees them in the coelomic cavity. Due to the shape of the body cavity and position of visceral organs these eggs move to the anterior part of the cavity and the ciliated mouths of the two ostia receive them one at a time in each. As these ova pass down the Müllerian tube (duct) they are met by spermatozoa, fertilization occurs, a mucous substance is added as a cover by the glands in the oviduct. These fertilized cells in a pouchlike posterior part of each oviduct which is called the uterus and after a few accumulate they are deposited by passing from the body by way of the cloaca and anus. These zygotes (fertilized eggs) are deposited by attachment to the under sides of rocks, logs, etc. in the water in small clutches of from 25 to 90 individuals. The embryonic stages are passed here and the larvae hatch out as tiny fishlike organisms. 
At about a year of age they are one and one-half or two inches in length with a stripe down the side which gives them a peculiar appearance.

The actual breeding and copulation activities (if any) do not seem to be very well understood. There is a prevailing idea that the spermatophores are passed from the male to the female in the autumn and held in the genital tract of the female until the succeeding spring when the eggs mature and pass down the oviducts. The act of transferring spermatophores is described as occurring in shallow water or on the muddy margin of the pond or stream by the male, depositing them here while the female follows and collects them into the cloaca by use of its swollen lips, the papillae there, and the mucus which is secreted by the cloacal glands that lie at the sides of the cloacal aperture.*

\section{Skeletal System}

The skeleton of these animals is classified as a bony skeleton but is not completely ossified and a considerable part of it is cartilage. The axial portion consisting of skull, vertebral column and ribs; and the appendicular portion, consisting of the two girdles with limbs constitute the essential parts of this system. The skull is platybasic (flat and broad) with a marked fusion and loss of primitive bones when compared with the teleost fish. The anterior, dorsal surface of it is covered by a single, fused frontal bone, posterior to which, and extending beneath and somewhat lateral to this, is the large pair of parietals. At the anterior tip of the frontal are the premaxillae which bears teeth. Just posterior to this and somewhat covered by the frontal is the vomer, which also bears teeth. Both the nasals and maxillae are absent. The braces, at the side of the skull, are the palatopterygoid bones, each of which bears a few teeth; the quadrate cartilage; quadrate bone, which articulates with the lower jaw; and the squamosal, which appears more dorsally. The otic group is represented only by the prootic, a small, irregular one which lies between the anterior part of the squamosal and the parietal, and another small one, the opisthotic, which is at the posterolateral corner of the skull. The foramen magnum (large opening) is located at the mid-posterior position and an occipital condyle is located at each side of it for articulation with atlas, the first vertebra. The principal part of the floor of the skull consists of the large flat

* Helpful illustrations of the urinogenital systems of Necturus may be found in Stuart: Anatomy of Necturus maculosus, Denoyer-Geppert Co., Chicago. 
parasphenoid. The lower jaw is composed of a pair of each, dentary bones, which bear teeth; splenial bones, which bear the last few teeth; and the angular bones, devoid of teeth and articulating with the quadrate of the skull. Necturus usually has forty-six amphicoelous vertebrae. They articulate with each other by anterior and posterior zygapophyses as well as the ends of the centra. There is one cervical vertebra, atlas, with which the skull articulates. Posterior to this one are about eighteen thoracolumbar vertebrae each of which bears a pair of short Y-shaped ribs. Each rib has a double head (bicipital), the dorsal head or tuberculum articulating with the transverse process of the vertebra and the ventral head or capitulum articulating with the side of the centrum. Following the thoracolumbar group is a single sacral vertebra to which the ilium of each side is attached by way of the saeral rib. The remainder of the series, posterior to this point, consists of caudal vertebrae.

The pectoral girdle is principally cartilage in structure. The velltral portion is formed by a posterior coracoid cartilage in the muscles of the body wall, and an anterior procoracoid. Projecting dorsally and laterally is the third unit of each side, the scapula. The most dorsal, free margin of this is frequently referred to as suprascapula. The recess formed at the junction of scapula with the rentral parts into which the arm articulates is called the glenoid fossa. The skeleton of the anterior appendage includes the proximal humerus (in the brachium), the radius and ulna in the forearm (antebrachinm), six carpals in the wrist, four metacarpals in the palm, and four digits each composed of joints or phalanges.

The pelvic girdle is likewise largely cartilage, but it is fused in the midventral line. The anterior, ventral part is the pubic plate consisting of eartilage, posterior to this is the pair of ischia which are partly ossified. Extending dorsally on each side is a slender ilium which joins the sacral rib and this in turn the saerum. In the lateral position where the ilium meets the two ventral parts of the girdle is a concave recess into which the head of the femur of the thigh articulates. This recess is called the acetabulum. Distal to the thigh is the shank with two bones, the tibia and the fibula lying parallel to each other. There are six somewhat fused tarsals in the ankle. Distal to this are the four elongated metatarsals and beyond each is the digit, composed of phalanges.*

- Illustrations of the skeleton of Necturus may be found in Stuart: Anatomy of Necturus maculosus, Denoyer-Geppert Co., Chicago. 


\section{Muscular System}

The muscles of the body are divided into segmental myotomes with intervening connective tissue sheets or myosepta. A horizontal septum along the side of the body divides the muscles into a dorsal, epaxial portion and a ventral hypaxial portion. The principal sets of super-

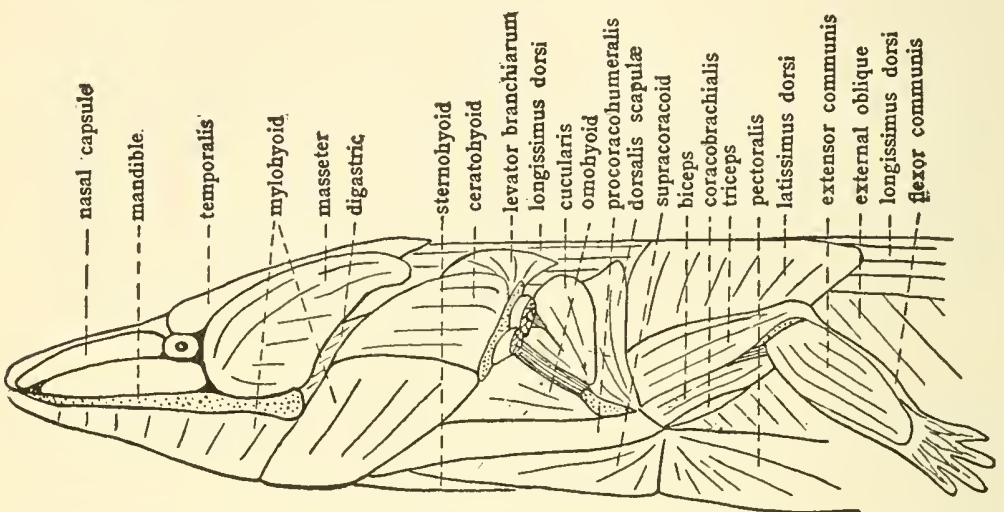

Fig. 271, B.-Left lateral vlew of the muscles of the head and shoulder reglon of the salamander, Necturus maculosis. (From Atwood, Comparative Vertebrate Dissection, The Blakiston Company.)

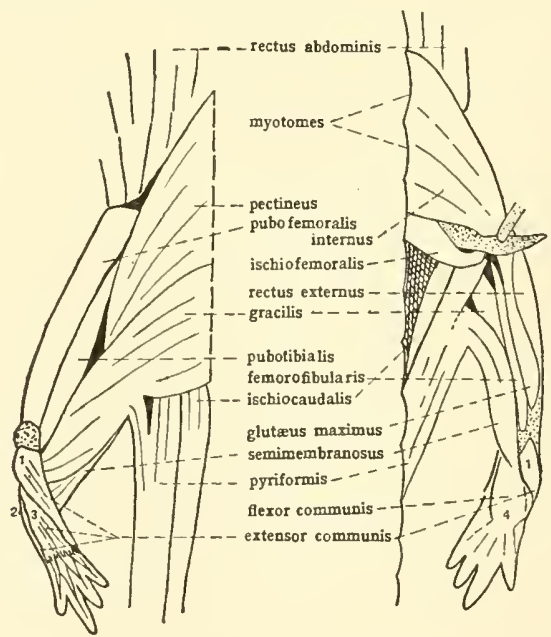

Fig. 271, C.-The muscles of the hind legs of Necturus maculosus; ventral view on the left, dorsal view on the right. In the dorsal view the ilium has been cut from the pelvic girdle and deflected downward. 1,2 , and $\&$ are extensors of the poot, and 4 is a flexor. (From Atwood, Comparative Vertebrate Dissection, The Blakiston Company.) 
ficial muscles or those of the head and gills, body wall, and the appendages. Because of the development of the terrestrial limbs, the latter group is much more complicated in this amphibian than it was in the fishes. For the detailed information concerning the specific muscles the student will depend on the accompanying illustrations and the laboratory study.

\section{The Nervous System and Sense Organs}

Since this system resembles that of the fish which has been studied already, and is so closely similar to that of the frog, which is described in the next section of the book, it seems unnecessary to describe it here.*

\section{THE BULLFROG}

\section{Habitat}

The bullfrog is a solitary animal except during the breeding season. It is strictly aquatic and does not leave the pools as does the leopard frog. It prefers bodies of quiet water where there are both shallows and deeper water, such as lagoons, small lakes, and the cypress ponds of swampy regions. In such a situation, the shore is protected by low willows or other trees, and the shore waters are filled with aquatic plants, pickerel weeds, and floating lily pads. These furnish not only a good hiding place but a good hunting ground for the crayfish, insect larvae, water beetles, snails, and other aquatic organisms which make up the bullfrog's diet. This diet is quite varied and may even include younger frogs.

Bullfrogs are found in North America east of the Rockies from Canada to Mexico. They have also been introduced into the western portion of the United States and into various foreign countries.

\section{External Structure}

Bullfrogs obtained in the South and Southwest are usually of two species, Rana catesbeiana Shaw, the common bullfrog, or Rana grylio Stejneger, the southern bullfrog. Individuals of the former species attain larger sizes, and the giant bullfrogs of the southern swamps usually are Rana catesbeiana. The two species differ not only in size but also in external appearance, particularly when alive. However, they are essentially the same anatomically, and this chapter is based on a study of Rana catesbeiana.

- Iliustrations of this system may be found in Stuart: Anatomy of Necturus maculosus, Denoyer-Geppert Co., Chicago. 
The common bullfrog is ordinarily greenish or olive brown. Underparts are mottled with dark spots on a white background, and the upper surfaces may be plain or marked with large dark splotches. The legs are marked with crossbars and other splotches of dark color. Preserved specimens appear brownish gray with the dark mottling lighter in color than on the living specimen.

The body of the bullfrog includes the head and trunk. Attached to the trunk on either side anteriorly are the forelegs and posteriorly the hindlegs.

The head has two prominent eyes which protrude above its surface. These call be drawn back into their orbits and forced some-

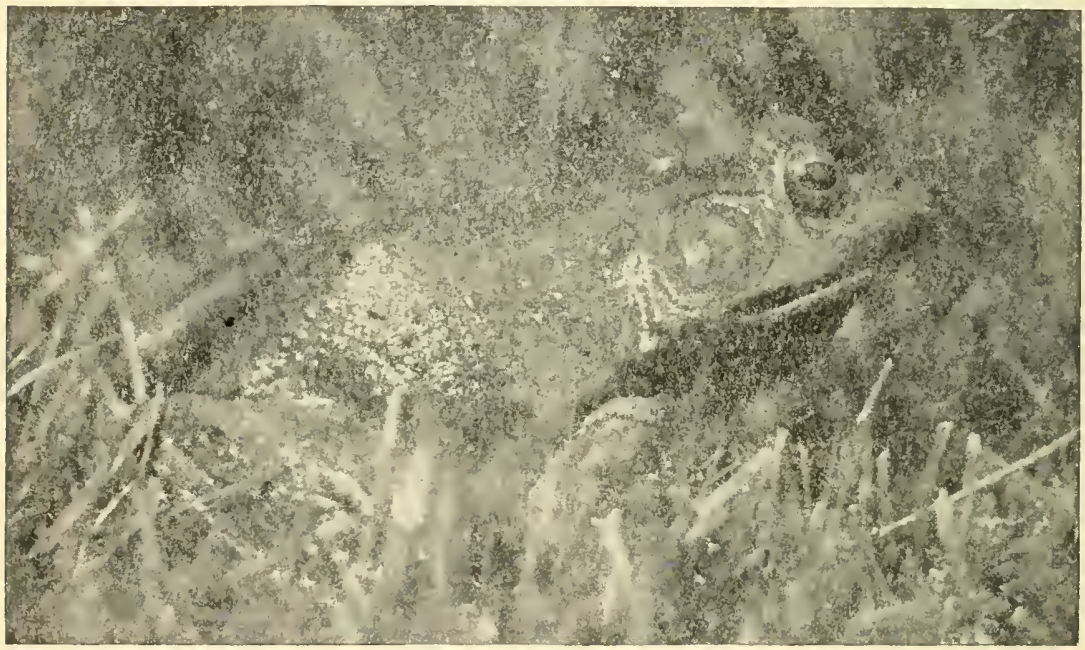

Fig. 272.-External features of the common bullfrog, Rana catesbeiana. (Courtesy of Southern Biological Supply Company.)

what into the mouth cavity. The lower lid of the frog's eye with its attached nictitating membrane is drawn up over the eye, not by independent movement of the eyelid, but as a result of the retraction of the eye into the orbit. The upper eyelid is immovable. Back of each eye is a circular oval area, the tympanum or eardrum. In the females this is about the size of the eye, while in the males it is larger than the eye. A small fold of skin, the tympanic fold, runs from the eye around the posterior margin of the tympanum. The two nostrils or nares are near the anterior part of the head, and each is guarded by a valve. The mouth reaches from one side of 
the head to the other and has an upper and lower jaw. The anus or vent is at the extreme posterior end of the trunk.

The forelimbs are composed of the upper arm, which joins the trunk, the forearm, wrist or carpus, and the hand with its four digits. In the male, particularly during the breeding season, the innermost digit, or thumb, is enlarged, whereas the thumbs of females remain apparently the same size. The digits may have tubereles on them, and their positions in relation to various bones of the hand give rise to specifie names for these tubercles. The forelimbs are used not only to help support the body but also as an aid in pushing food into the mouth.

The hindlimbs are long and have powerful museles. Bullfrogs ordinarily leap about three feet but ean easily cover a distance of five or six feet. The hindlegs are composed of the thigh, which joins the trunk; the shank; and the ankle, or tarsus. Following the tarsus is the foot with five digits (toes), which are conneeted by a web, produeing a very efficient swimming organ.

The smooth damp skin, which is soft and loosely attached to the body except in the head region, is composed of two layers, an outer epidermis and an inner dermis. The skin is pigmented and very rich in mueous glands, which aid in keeping it moist. Bullfrogs moult or shed the superfieial layer of epidermal eells of their skin at varying intervals.

\section{Digestive System and Digestion}

The mouth cavity, or buccal cavity continues direetly into the phar$y n x$ with no sharp line of boundary between them. The latter narrows toward the esophagus, which is a short gullet leading direetly from the pharynx to the stomaeh. The lining of the esophagus has a number of longitudinal folds and is eiliated. The stomach normally lies on the left side of the body. It is eurved, with the convex side toward the bullfrog's left. Its anterior or cardiac end is wide, and the pyloric or posterior end is narrowed and constricted where it joins the small intestine. The duodenum, or anterior part of the small intestine, runs forward almost parallel with the stomaeh. At the point where the intestine turns baek posteriorly the duodenum becomes the ileum, which composes the remainder of the small intestine and is considerably coiled. The large intestine or rectum is sharply 
marked off from the small intestine and is wide and short. It passes directly into a muscular part, the cloaca, which terminates in the anus or vent.

The buccal cavity has in its roof near the end of the snout two patches of small conical teeth, called vomerine teeth. In addition, the upper jaw has a single series of small conical teeth on its edge known as maxillary teeth. These teeth serve primarily to help hold the crayfish, insect, or other animal captured for food, and they may help at times in crushing it. The tongue is somewhat leaflike in shape and is deeply notched behind, making it bicornute. Its anterior half is attached to the floor of the mouth just back of the tip of the lower jaw,

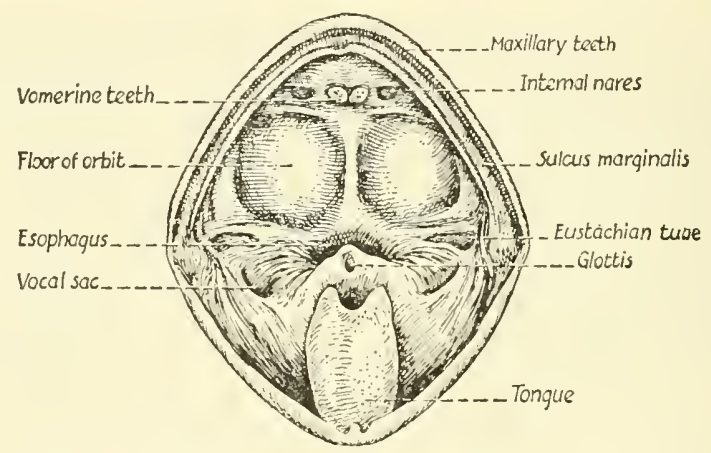

Fig. 273.-Mouth or buccal cavity of the bullfrog.

and its posterior end is free. In order to get the tongue out of the mouth the posterior part has to somersault over the attached anterior part. The tongue of the bullfrog is somewhat smaller proportionally than that of the grass frog, as might be expected, for the latter is more dependent on this organ when it hunts insects inland. Taste buds are present on the tongue and palate.

Esophagus, stomach, and intestine have an outer longitudinal and an inner circular layer of smooth muscle. The peristaltic contractions of these muscles pass the food through the digestive tract and aid in mixing it with the gastric juice in the stomach. They may also be used to regurgitate a disagreeable substance swallowed by the frog, in which case the stomach turns inside out and protrudes into the mouth cavity. The stomach can be greatly expanded and acts as a reservoir for food which may be available only at irregular intervals and the frog has to take advantage of a food supply when 
it is present. The mucosa of the intestines has a number of longitudinal and transverse folds which produce a great absorptive surface through which the digested food can be taken up by the blood stream and transported to different parts of the body.

The liver lies on each side of and behind the heart. It is threelobed, two lobes being on the left and one on the right, connected by narrow bridges of liver tissue. Between the right and left lobes is the gall bladder, which receives an alkaline secretion known as bile from the liver and stores it until needed in the process of digestion. Bile is carried from the gall bladder to the duodenum by the bile duct, which passes through the pancreas on its way. The liver is not primarily a digestive gland, for, while the bile it secretes permits the fats to be more easily digested by a lipase from the pancreas, the bile itself contains no digestive enzymes. Although its function in altering fatty substances is important, of prime importance is its ability to store glycogen and the fat upon which a hibernating frog lives. It is also concerned in the formation of urea and in the destruction of red blood corpuscles.

The pancreas lies in the loop between the stomach and duodenum. It is a long, whitish, irregularly-lobed gland whose alkaline secretion is of considerable importance in digestion, for it contains three digestive enzymes. This secretion is taken from the pancreas by pancreatic ducts which empty into the bile duct that passes through the pancreas before entering the duodenum near its beginning.

Intestines, liver, and pancreas are covered with peritoneum. The mesenteries which hold the body organs in position and the internal surface of the body wall likewise are made up of this peritoneal membrane.

Digestion.-Since frogs live primarily on insects, crayfish, and other small invertebrate animals, their food is very rich in proteins. Their vomerine and maxillary teeth are too feeble to do more than slightly crush their prey, so digestion begins in the stomach. Here the gastric glands secrete hydrochloric acid and an enzyme, pepsin, which converts the proteins to peptones. Peristaltic contractions of the stomach cause a thorough mixing of the gastric juice with the food and then this partly digested food (chyme) is passed posteriorly into the small intestine. Here, activated by the acid nature of the food, the intestinal glands release into the blood stream a substance, secretin, which on reaching the pancreas causes it to pour forth into the duo- 
denum its highly alkaline secretion. In addition, this pancreatic juice contains three digestive enzymes: trypsin, which continues the digestion begun by pepsin in the stomach, converting proteins to amino acids; an amylase, amylopsin, which changes starches into sugars; and a lipase, steapsin, which, aided by the bile, causes a splitting of the fats into glycerol and fatty acidis. Bile also contributes to the alkaline condition here.

The process of digestion is completed in the intestine and the food products are taken up by absorption in its mucosa layer. These foods in solution are taken by the blood stream and lymph vessels to various parts of the body where they are utilized for building tissue or for supplying energy, leaving as by-products urea and carbon dioxide. Sugars that are not used are stored as glycogen in the liver and in voluntary muscles. The liver also serves to store fats and to secrete urea and sugar dircetly into the blood stream.

Food that is not digested passes to the large intestine where it is retained for a time and then passed to the outside through the anus as feces.

Other Glands.-Attached by a mesentery to the wall of the intestine near the anterior end of the rectum is the spleen. It is a small, reddish, spherical, lymphoid organ, the functions of which are but incompletely known. The destroying of red blood corpuseles is an important duty, as possibly also is the formation in its tissues of lymphocytes, one type of white blood corpuscle. In mammals the spleen is also believed to accumulate iron freed by the metabolism of other tissues. This iron is subsequently used in the formation of hemoglobin.

The two thyroid glands are small and lie in front of the glottis under the floor of the mouth. There is one on each side of the hyoid apparatus. The secretion and functions are discussed in the chapter on Internal Regulation.

A thymus gland lies under the skin behind the tympanic membrane on each side. It is partly covered with muscle and is small. Further discussion of it will be taken up in the chapter on Internal Regulation.

\section{Circulatory System}

The circulatory system comprises the blood vascular system and the lymphatic systcm. The two systems are closely interrelated in that they both carry to the tissues of the body nutritive material neces- 
sary for metabolism and remove from them to the excretory organs, waste products of body activity. They differ in several respects; the lymph neither contains red blood corpuscles for transporting oxygen nor moves in a continuous closed vascular circuit as does the blood. Other differences will be noted in the discussion.

The Blood Vascular System.-The blood moves through a closed system of tubelike vessels of various sizes which distribute it to all parts of the body. The pump is the heart, which, by its contractions, forces the blood to flow to the tissues. Since the system is a closed one, the blood eventually returns to the heart.

The blood ressels leading away from the heart are the arteries. When these reach the tissues, they break up into very small vessels, the capillaries. The ressels leading back to the heart are the veins. The arteries and reins are connected by the capillaries.

Blood is comprised of a clear liquid called the plasma, suspended in which are blood corpuscles of three kinds, the red blood corpuscles or erythrocytes, the white blood corpuscles or leucocytes, and the spindle cells or thrombocytes. In addition, the blood may contain dissolved nutritive substances from the digestive system, waste products from tissue repair and destruction, hormones being transported from organs of one part of the body to another, or foreign substances accidentally introduced.

The capillaries are very small ressels, the walls of which are made up of endothelium continued from the linings of arteries and veins. They connect the distal ends of the arteries with the proximal ends of the veins, but in so doing they branch extensively and anastomose to form fine networks in the tissues invaded. Through their thin walls, acting as semipermeable membranes, food products brought by the arterial blood pass into the tissues, oxygen is unloaded from the red blood corpuscles, and carbon dioxide and waste products are taken up to be conducted into the veins. Leucocytes are able to get out of the capillaries, squeezing their way between the cells of the capillary walls, and thus become free in the surrounding tissue to engulf bacteria or other harmful objects.

The abundance of the capillaries varies with the activity of the organ; the greater the rate of metabolism the greater their abundance. Examples of such are the various glands and the mucous membrane of the digestive tract. In contrast, a tendon has few capillaries. 
The arteries are large vessels with elastic walls and carry blood from the heart to the capillary networks in the various organs and tissues of the body. The arteries arise from the conus arteriosus

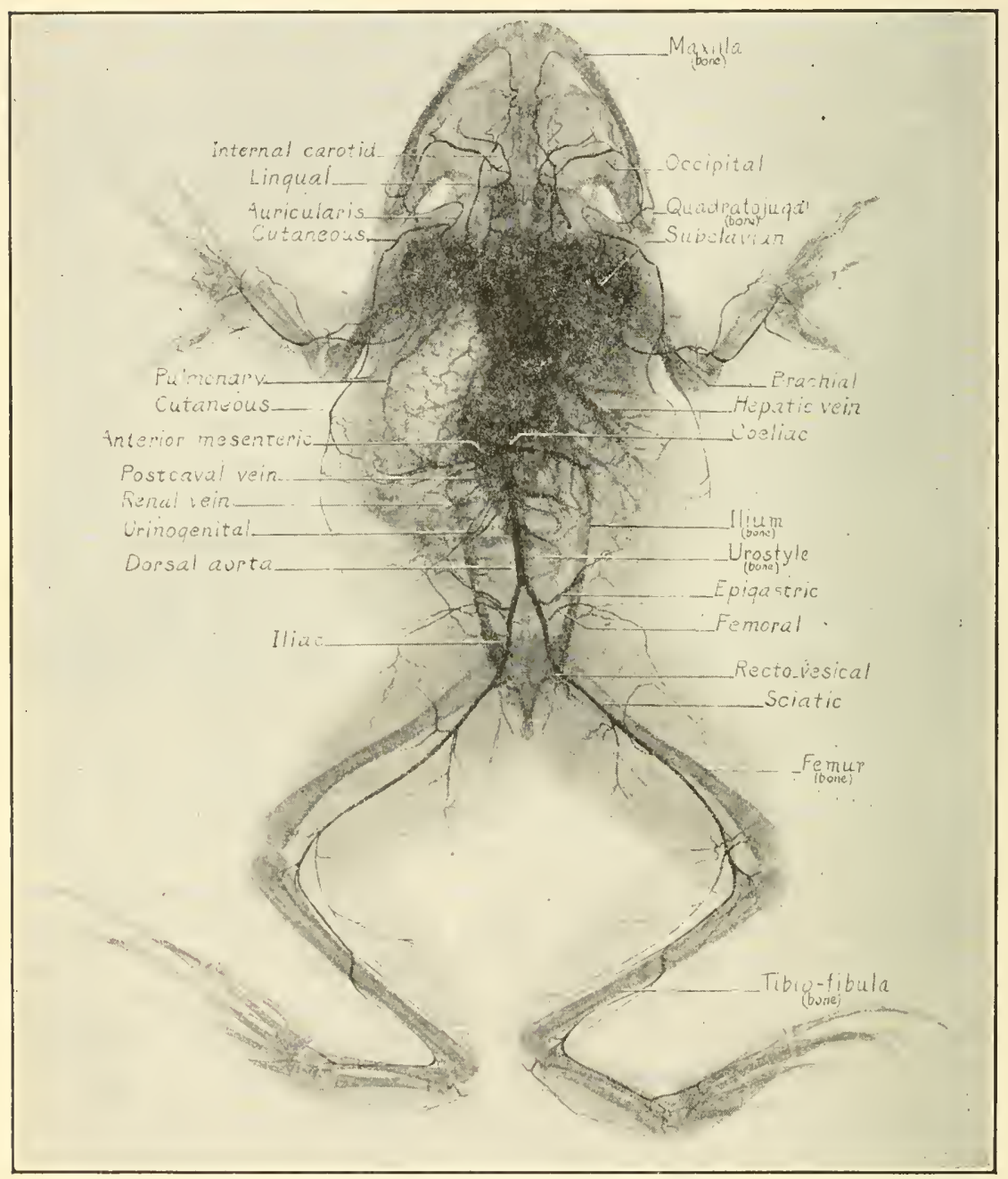

Fig. 274.-X-ray picture of bullfrog with arterial system and a portion of venous system injected. All labels indicate arteries except where otherwise noted. (X-ray courtesy of Dr. Malcolm B. Bowers.)

which divides just above the auricles into a right and left truncus arteriosus. Each of these trunks splits into three arches going to 
each side of the body, the anterior carotid arch, the middle, systemic arch, and the posterior, pulmocutaneous arch.

The Carotid Arch.-Each carotid arch divides into two branches. The more ventral, lingual artery, or external carotid, passes forward, giving branches to the thyroid, pseudothyroid, muscles of the hyoid

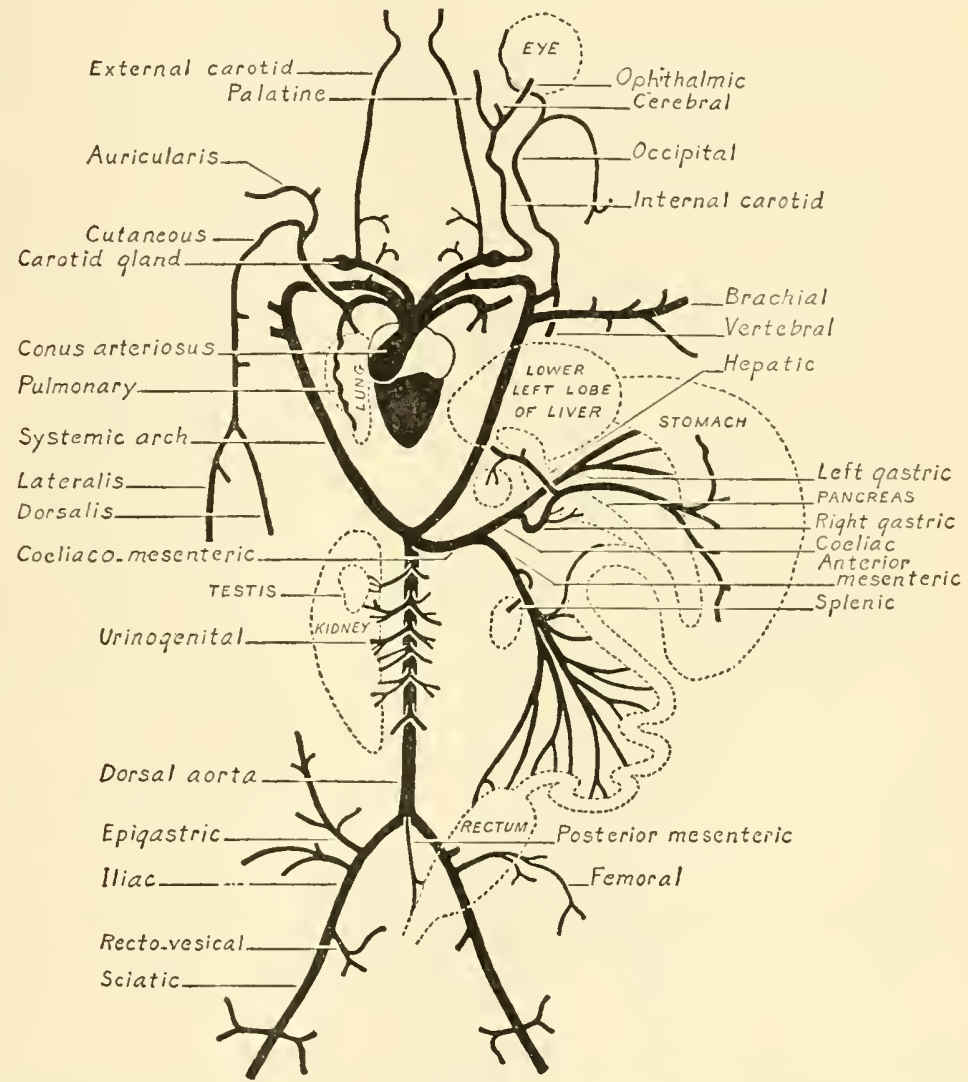

Fig. 275.-Arteries of the bullfrog from ventral view. (Drawn by Ruth $M$.

and tongue, and then extends along the edges of the lower jaw. The internal branch is larger and is called the internal carotid. It has at its base a spongy enlargement known as the carotid gland which by its structure serves to steady the pressure of blood passing into the artery. This artery follows the side of the neck to the base 
of the skull, giving off the palatine artery to the roof of the mouth, the cerebral carotid which enters the skull and supplies the brain, and the ophthalmic artery to the eye.

The Systemic Arch.-The systemic arch soon after it leaves the truncus supplies a small laryngeal artery to the larynx and muscles of the hyoid. It then curves downward and around the esophagus on each side. It gives off an occipitovertebral artery which sends a small artery to the dorsal side of the esophagus, then branches at the spinal cord into the occipital artery, rumning anteriorly on the dorsal side of the skull to the orbit and tympanum, and the vertebral artery, turning posteriorly along the spinal column. Immediately posterior to the occipitovertebral artery the large subclavian artery arises from the systemic arch. It branches to the shoulder and adjacent body wall and enters the arm as the brachial artery.

The systemic arches from each side, after curving under the alimentary canal, meet near the anterior end of the kidneys and fuse into a single large artery, the dorsal aorta, which extends posteriorly. At or just posterior to this meeting point, there arises from the aorta the large coeliacomesenteric artery which divides into an anterior branch, the coeliac artery, and a posterior branch, the anterior mesenteric artery. The coeliac artery divides into right and left gastric arteries. The latter runs directly to the dorsal or left side of the stomach, while the former sends off small pancreatic arteries to the pancreas; a larger hepatic artery to the pancreas, gall bladder, and liver; and continues to the ventral side of the stomach, where it is distributed. The anterior mesenteric artery gives off the splenic (lienal) artery to the spleen and then divides into two parallel vessels which send numerous smaller arteries to the small and large intestines.

The urinogenital arteries consist of about four to six small muchdivided arteries which are given off from the ventral side of the dorsal aorta to right and left, supplying the kidneys, reproductive organs, and fat bodies. A few small lumbar arteries arise either as branches of these or directly from the aorta and go to the body wall on each side. The small posterior mesenteric artery is given off near the posterior end of the aorta, passing to a portion of the rectum and, in the female, to the ovisac. It often anastomoses on the rectum with descending branches of the anterior mesenteric. 
Near the posterior end of the body cavity the dorsal aorta divides into two iliac arteries going to the hind legs. Each of these gives off (1) an epigastric artery supplying the bladder and dorsal and ventral body walls of the region, and (2) just below it, a femoral artery passing to the body wall, skin, and proximal muscles of the thigh. As the iliac artery enters the leg, a rectovesicular artery is sent

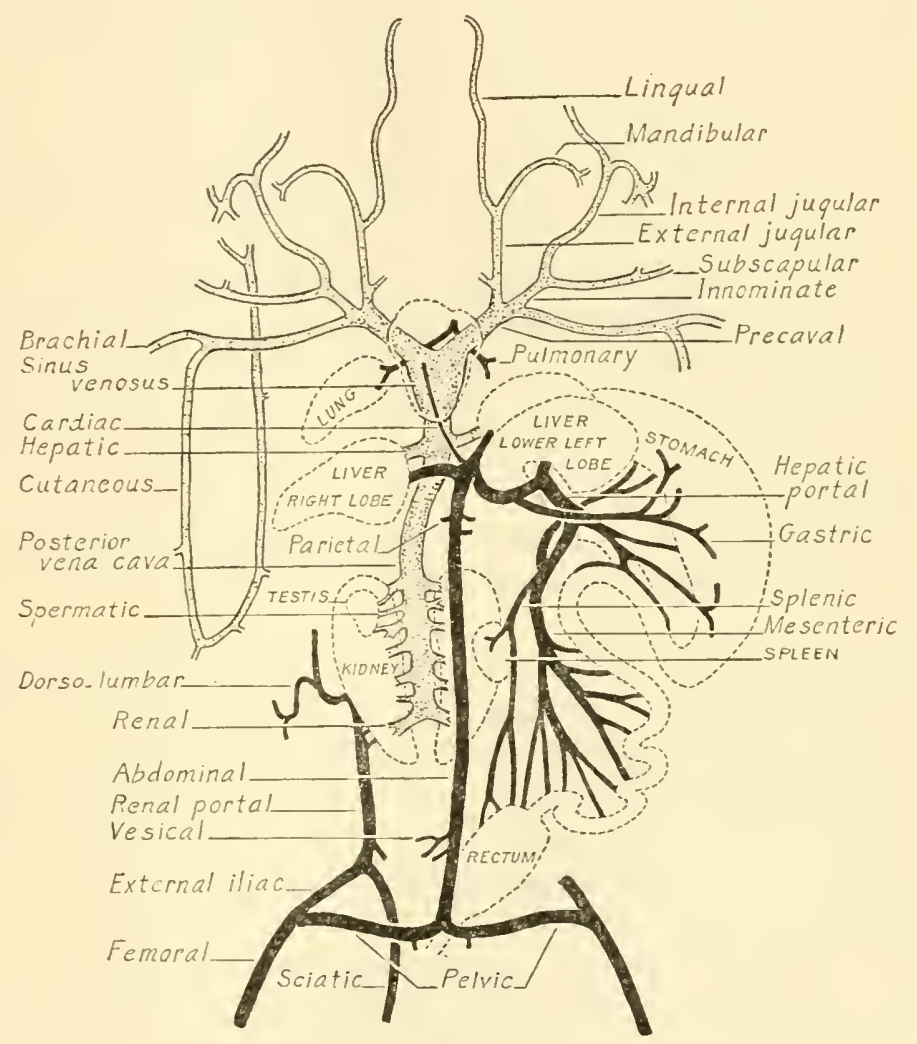

Fig. 276.- Veins of bullfrog from ventral view. (Drawn by Ruth M. Sanders.)

off to the rectum, bladder, and skin on the dorsal surface of the thigh. In the upper leg the continuation of the iliac, now called the sciatic, gives off a branch to the right and to the left, supplying the muscles, and then continues down the leg, sending off several branches at the knee.

The pulmocutaneous arch takes blood to the respiratory organs: the lungs, skin, and buccopharyngeal cavity. The pulmocutaneous 
arch on each side divides into a pulmonary artery to the lungs and a large cutaneous artery, which passes outward to the skin. Important branches of the cutaneous are: the auricularis, supplying the tympanum and adjacent head region; the dorsalis, supplying the skin of the back; and the lateralis, which is distributed to the skin of the side.

The Veins.- These vessels usually parallel the arteries that brought blood to the tissues from which the veins are returning it. The walls of the veins are thinner and not as elastic as those of the arteries. Many veins, particularly those of the limbs, have semilunar valves on the internal surface of the wall which open in the direction of flow and prevent the backflow of blood.

In returning blood to the heart, the venous system carries some of the blood through the kidneys or through the liver, providing renal or hepatic filters to eliminate urea and other waste products from the blood or to alter it chemically. Pulmonary veins from the lungs carry oxygenated blood, which differs from the type of blood found in the other veins.

The venous circulation, therefore, may be divided into four main systems: the systemic, hepatic portal, renal portal, and pulmonary systems.

The systemic veins carry the greatest load of blood to the heart. The larger collecting veins of the system consist of two precavals receiving blood from the anterior parts of the body, except the lungs, and a single postcaval or posterior vena cava receiving blood from the posterior parts of the body. The two precavals empty into the anterior end of the sinus venosus of the heart, and the posterior vena cava empties into its posterior end.

Each of the two anterior precavals receives blood from three branches: (1) the external jugular bringing blood from the tongue, hyoid, thyroid, pseudothyroid, and floor of the mouth; (2) the innominate vein, made up of a fusion of the internal jugular returning blood from the brain and other parts of the head, and the subscapular vein bringing blood from the back of the arm and shoulder; and (3) the subclavian vein, a fusion of the brachial vein, returning blood from the forelimb, and the large musculocutaneous vein, which forms an ellipse down the side of the body and extends up into the head region, returning blood from the skin and outer muscles in these regions. 
The large posterior vena cava originates between the kidneys and receires blood from each kidney by five or six renal veins, from the gonads by small spermatic or ovarian veins, and from the fat bodies by other small branches. Near the heart the vena cava receives two large hepatic veins from each side of the liver.

The Hepatic Portal System.-This system is comprised of two chief veins, the hepatic portal vein and the ventral abdominal vein. These veins, instead of carrying blood directly to the heart, bring it to the liver to pass through a network of sinusoids (modified capillaries). It is returned to the systemic system through hepatic veins that join the posteaval.

Veins from the large and small intestines unite to form the mesenteric vein which is joined as it progresses forward by the splenic vein from the spleen, pancreatic veins from the pancreas, and gastric veins from both sides of the stomach. The ressel resulting from these unions is the hepatic portal vein. It passes through the anterior portion of the pancreas and sends a large branch into the lower left lobe of the liver. At about this point it often receives a final gastric branch which has passed on top of the pancreas to join it. It then continues a short distance to join the abdominal vein just below the heart.

The abdominal vein arises as follows: Two large veins, the sciatic and femoral, bring blood from the hindlimbs. The femoral, as it enters the body cavity, gives off the pelvic vein. The pelvic veins from each side of the body join in the middle to form the large ventral abdominal vein. As the abdominal vein runs toward the heart along the median portion of the ventral body wall, it receives vesicular veins from the bladder, parietal veins from the body wall and, at its anterior end, a cardiac vein from the heart. In the region of the liver it leaves the body wall, is joined by the hepatic portal vein, and enters the right and upper left lobes of the liver by short branches, discharging its blood into sinusoids.

The Renal Portal System.-This system, like the hepatic portal system, diverts blood to a purifying organ instead of carrying it directly to the heart. In this case, the blood is taken to the kidneys.

The outer femoral vein and the medial sciatic vein collect blood from the hindlegs. The femoral vein, after giving off the pelvic vein, runs anteriorly and joins the sciatic, to make the renal portal vein. Near the kidney this vein receives the dorsolumbar vein from the body 
wall and, in the female, several vessels from the ovisacs (uteri). The renal portal vein follows the dorsolateral margin of the kidney, sending numerous transverse branches into the organ, where they break up into capillaries. Blood which passes through these capillaries is purified of some of its waste products and then leaves the kidney through the renal veins which empty into and originate the posterior vena cava of the systemic system.

Pulmonary Veins.-These veins run along the inner walls of each lung, returning the oxygenated blood to the heart. The right and left pulmonary veins unite to form a single vessel which empties into the left auricle on its dorsal side. Other veins which take on oxygen are those coming from the skin and buccopharyngeal cavity.

The Heart.-The heart is enclosed in the pericardial cavity, which is lined by a transparent tissue, the pericardium, and is separated from the remainder of the body by the transverse septum. It is the rhythmically contracting organ that circulates the blood. It is conical in shape and in the frog consists of a right and left thin-walled auricle above a single thick-walled ventricle. On the ventral side is a muscular tube, the conus arteriosus, described with the arteries. It conducts blood away from the heart. On the dorsal side of the heart is a thin-walled sac, triangular in shape, the sinus venosus, which receives venous blood from the systemic veins.

The sinus venosus empties into the right auricle through the sinuauricular aperture. This aperture has liplike valves on each side to prevent the blood from flowing back into the sinus when the auricle contracts. The smaller left auricle receives oxygenated blood from the pulmonary vein. Valves are not necessary at this opening, for pressure on the auricular walls tends to close the small oblique aperture when the auricle contracts.

Both auricles pass blood into the ventricle through a common opening, the auriculoventricular aperture, which is divided by the interauricular septum separating the two auricles. This aperture has two large valves on each side and two small valves at each end which regulate the discharge of blood into the ventricle and prevent its backflow.

Blood leaves the ventricle and enters the arterial system through the conus arteriosus. The opening into the conus is protected by three pocketlike semilunar valves which open inwardly into the conus when blood is passing out but are tightly closed at other times. The 
proximal portion of the conus is known as the pylangium, and the distal portion as the synangium. Rumning through the length of the pylangium is a longitudinal spiral valve, one edge attached to the dorsal wall of the pylangium and the other edge lying free in the vessel. Upon contraction of the conus this structure is brought into contact with the ventral wall and helps direct the flow of blood into the arches.

Near the anterior free end of the spiral valve where it is the widest, there is a pair of small synangial valves which, together

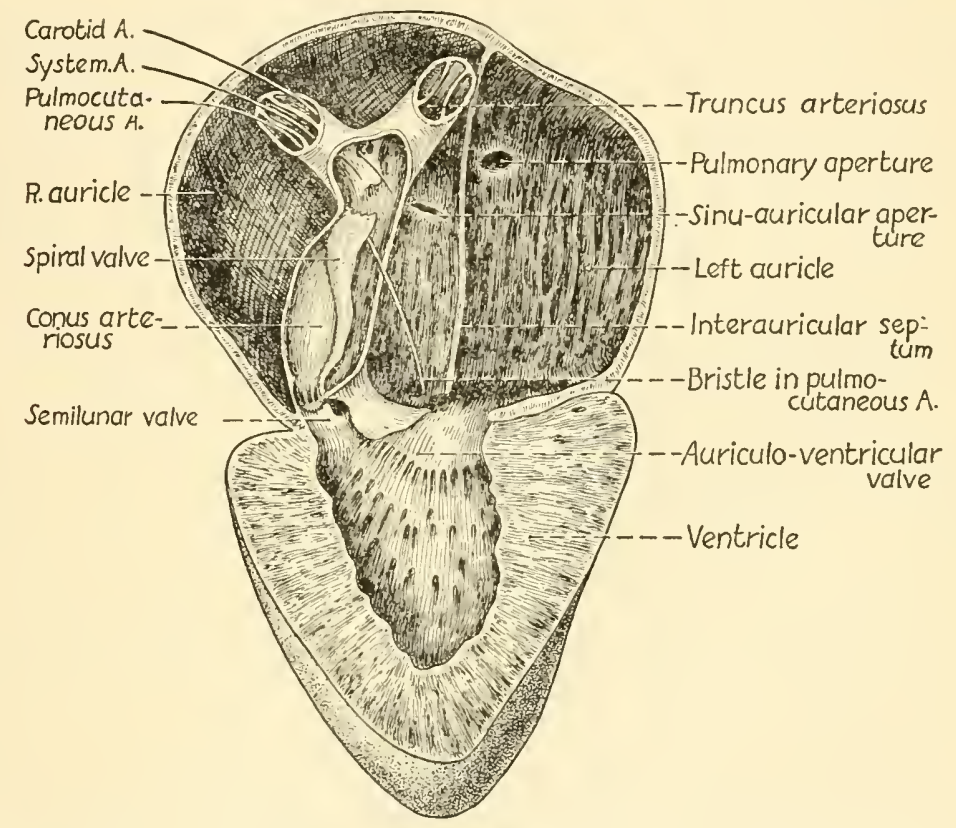

Fig. 277.-Heart of frog with the ventral wall removed and bristles shown through the arteries of the truncus arteriosus.

with the end of the spiral valve, separate the pylangium from the synangium. Just below these valves is an aperture which leads into the trunk formed by the union of the two pulmocutaneous arteries.

The synangial chamber is very short and gives off almost immediately two large branches, one to the right and the other to the left. In each of these branches originate the three main trunks or arches of the arterial system. They are formed by two longitudinal septa dividing the vessel into three compartments. All three trunks 
are therefore enclosed in one large vessel for a short distance before breaking up into three separate vessels. The carotid arch originates from the anterior compartment, the systemic arch from the middle compartment, and the pulmocutaneous arch from the posterior compartment. Blood enters the anterior and middle compartments from the synangium, but enters the posterior compartment, or pulmocutaneous arch, from the pylangium.

The heart beats in a wavelike peristaltic manner. The sinus venosus contracts first, then the auricles (the right auricle preceding the left by a moment), then the ventricle, and finally the conus.

Venous blood from the right auricle enters the right side of the ventricle, and oxygenated blood from the left auricle enters the left side. Muscular ridges of the ventricular wall tend to hold the blood and reduce mixing. Since the heart's contractions are wavelike, the ventricle immediately forces the blood into the conus through the semilunar valve. Venous blood from the right auricle is closest to the conus, and it passes out first, flowing into the closest opening offering the least resistance. This is the opening in the pylangium to the pulmonary arch, leading to the lungs. As the contraction of the ventricle comes to an end, forcing out the remaining oxygenated blood, the pylangial part of the conus contracts, bringing the spiral valve against its ventral wall. This action, together with that of the synangial valves which are anterior to the common opening of the pulmonary arches, completely shuts off the flow of blood into these arches. The blood therefore passes into the synangium and enters the chambers leading to the systemic arteries or the carotid arteries. Since the carotid arteries offer some resistance to blood flow, the blood tends to enter the larger systemic arteries first. As the systemic arteries fill, they offer more resistance to the blood, while resistance in the carotid arteries decreases due to their emptying into capillaries; so the last oxygenated blood from the ventricle passes into the carotids and is conveyed to the head region.

The heart must beat sufficiently fast and pump a sufficient volume of blood at each stroke to insure an adequate supply of oxygen and food to the body tissues, as well as to remove waste products as they form. The rate of pulsation is influenced greatly by temperature up to a certain maximum rate, for the activity and metabolism of the bullfrog are considerably affected by temperature. Blood 
pressure is increased by a constriction of the smaller arteries or arterioles. Their muscular walls may contract from stimuli received from the nervous system or from hormones.

Blood corpuscles, which are of three kinds, float in the plasma. The erythrocytes are flattened and elliptical, with an oval nucleus in the center. They contain a pigment, hemoglobin, which has the property of absorbing oxygen. The colorless thrombocytes or spindle cells are not as large as the erythrocytes but resemble them except for their tapering ends. When these cells contact certain foreign bodies, they break up, releasing a substance that causes, upon contact with air, the coagulation of certain proteins in the blood plasma in which blood corpuscles become entangled, forming a clot. The insoluble protein strands thus formed are called fibrin (see chapter on The Vertebrate Animal). After the frog has been injured, the formation of a clot prevents indefinite bleeding and makes it possible for the tissues to begin repair.

The white blood corpuscles or leucocytes are of three kinds: lymphocytes, monocytes, and granulocytes. Their outline is irregular, due to their amoeboid movement, and the shape of their nuclei varies greatly. They are much less numerous in the blood stream than are the red blood corpuscles and spindle cells. Leucocytes may escape from blood capillaries and engulf bacteria and other harmful substances in the tissues. They are finally returned to the venous system by lymphatic vessels. Worn out corpuscles are removed from the blood stream by the liver and spleen. The spleen seems to be the primary organ concerned in supplying new blood corpuscles except for a period in the spring when the bone marrow may produce some. Leucocytes may also increase by fission.

Lymphatic System.-The lymphatic system of the bullfrog is an open system comprised of a series of large irregular sinuses in various parts of the body. It collects lymph from the tissues and eventually returns it to the veins. The lymph is a colorless fluid containing leucocytes but no erythrocytes. It is derived from seepage of plasma from the capillaries. It bathes all of the cells, collects wastes, and distributes food products. In the region of the intestinal tract, Iymphatics absorb a considerable amount of fat and are ealled lacteals. Lymph removes cellular debris and transports leucocytes which engulf harmful material and cleanse the tissues of the body. 
Between the skin and muscle are a series of subcutaneous lymph sacs; other sinuses are in the mesenteries, around the vertebral column, and elsewhere. The peritoneal and pericardial cavities are connected with the lymphatic system. Nephrostomes on the ventral surface of the kidney convey lymph from the peritoneal cavity into the renal veins.

\section{Respiratory Organs and Respiration}

Air enters through the nostrils, passes into a small olfactory chamber and then into the mouth cavity through the internal nares, which open in the roof of the mouth. The mouth is kept tightly closed in breathing. Air is sucked in by lowering the floor of the mouth and is then forced into the lungs by raising the floor, the external nares being closed by valves. This pushes the air through the slitlike glottis immediately behind the tongue in the floor of the mouth, thence into a short larynx which connects with the lungs.

The walls of the larynx are reinforeed by a framework of cartilage, and the laryngeal chamber supports two horizontal fleshy folds, the vocal cords, which extend across the passageway. When a frog croaks, its mouth and nostrils are kept tightly elosed, and the air is forced back and forth between lungs and mouth cavity, causing the vocal cords to vibrate. The sound is amplified in male frogs by the vocal sacs which act as resonating chambers. In the bullfrog the two internal vocal sacs have openings into the floor of the month at each corner, and, when inflated, they swell out under the throat and sides of the body in the region of the lungs. Bullfrogs frequently call under water.

The two lungs lie dorsal to the heart on each side and dorsal to the liver. They are very elastic sacs with their inner walls raised into a number of ridges, forming chambers which are called alveoli These chambers are richly supplied with a network of blood vessels for facilitating the oxygenation of the blood. In the bullfrog the lungs are also important as a hydrostatic organ.

While the lungs play the major role in respiration, other factors are of considerable importance. The lining of the mouth of the bullfrog contains a large number of blood vessels and serves for a type of respiration known as buccopharyngeal respiration. With the glottis closed, air is drawn into the month eavity and forced out by rhythmi- 
cal morements of the throat. Oxygen is taken up by blood vessels in the lining of the mouth by diffusion.

The skin of the bullfrog plays a large part in its respiration, and frogs that are not protected from drying out soon die. Gaseous exchange of carbon dioxide and oxygen can take place through the moist vascular skin, and, since its area is large, it serves effectively as a respiratory organ. This type of respiration is known as cutaneous respiration. During hibernation, practically all respiration of the bullfrog is of this nature. Even at other times, the skin releases more carbon dioxide than do the lungs. The functions of respiration are discussed in the chapter on The Vertebrate Animal.

\section{Excretory System and Excretion}

The two kidneys lie between the parietal peritoneum and dorsal body wall in the posterior region of the body cavity. They are dark red in color, flattened and elongated. They are made up of a very great number of uriniferous tubules. A mesonephric duct runs from the posterior lateral border of each kidney and empties into the dorsal side of the cloaca. The urinary bladder also opens into the cloaca but does so on its ventral surface, and the ducts do not join the bladder. The bladder is a two-lobed sac with very thin walls which stores the urine collected from the cloaca. When filled, the bladder contracts and forces the urine back through the cloaca and outside through the anus. Embedded in the ventral surface of each kidney is a yellowish red patch, the adrenal gland, which will be discussed in the chapter on Internal Regulators.

The waste products resulting from the vital processes of destruction, repair, and growth in the body must be removed if the organism lives. These are taken from the tissues by the blood and more especially by the lymph. We have already mentioned the expulsion of carbon dioxide and water through the skin and lungs. Another product of protein metabolism is urea. This soluble crystalline substance, formed to a large extent in the liver from the nitrogen of protein metabolism, entcrs the blood stream and is removed by the kidneys. The kidneys also remove foreign substances from the blood and pass these to the outside through their mesonephric ducts and the cloaca.

Frogs and toads excrete considerably more urine per day proportionally than does man, although this may vary considerably, for in some forms the bladder may act as a filter for water which is used 
over and over. It has been estimated that, while man excretes about one-fiftieth of his weight per day, the frog excretes about one-third of its weight. During hibernation and aestivation, however, in common with the slowing down of its other body functions, the kidney function of the frog is practically stopped.

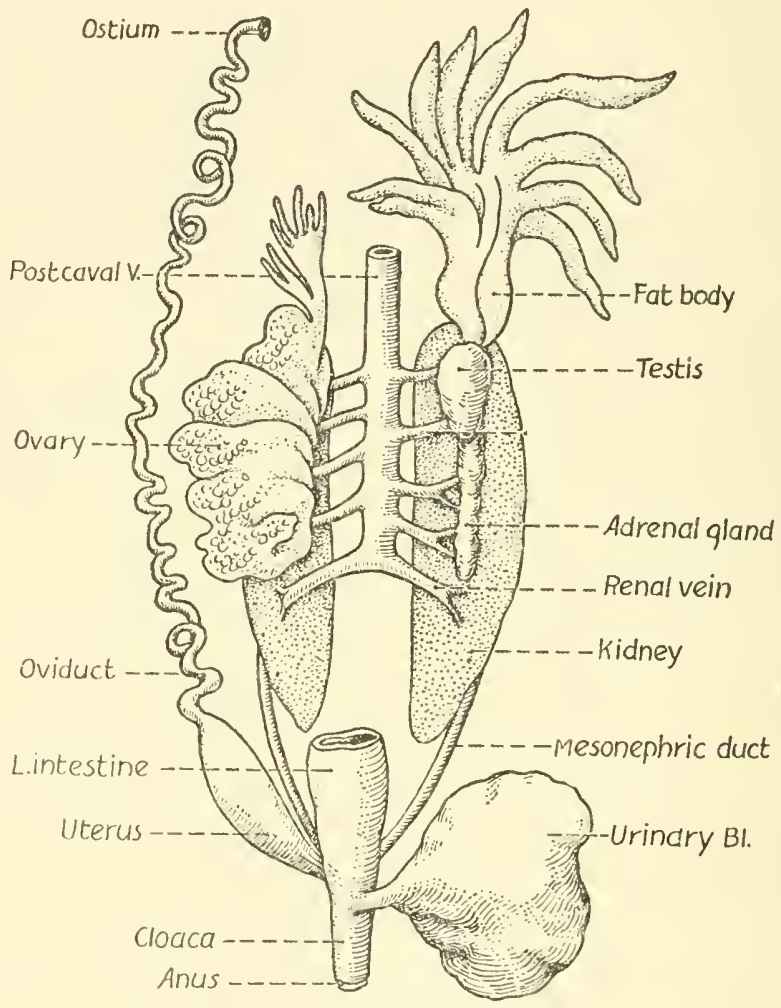

Fig. 278.-Urogenital system of the frog from ventral view. Male organs shown on one side, female on the other.

The kidney is not only concerned with the elimination of waste products but also has other functions. One of these is the reabsorption by its tubules of useful substances, such as some of the salts and glucose which have filtered out, and their reintroduction to the blood stream. In their food frogs obtain less sodium cliloride than do mammals, and this is compensated for in part by a retention of salts from the water taken in, while in mammals water is retained and the salts are eliminated. 
Another function is in maintaining the concentration of body fluids. Frogs absorb water through their skin at a rather constant rate, varying with the temperature. The kidney in turn expels water at the same rate and thus maintains the proper balance. In addition to its usual function the urinary bladder may be used as a storage reservoir for water during temporary drought. The water may be absorbed from it by other tissues until the proper osmotic equilibrium of the tissues with the blood is produced. The excretory function is further developed in the chapter on The Vertebrate Animal.

\section{Skeletal System}

The bullfrog has no exoskeleton, its body being covered by smooth skin. The endoskeleton may be considered in two main divisions, the axial and appendicular portions. The axial part includes the skull and vertebral column; the appendicular portion consists of the bones of the limbs and their supports, the pectoral and pelvic girdles.

Bones are joined to one another by structures made up of connective tissue which allow varying degrees of movement between them. These structures are called joints or articulations. In some cases, as in the skull, the joints are immovable and the bones are separated only by a thin sutural ligament of connective tissue. In other cases, the joints are slightly movable, as in the vertebral column where a plate of dense tissue and cartilage connect the vertebrae. In still other cases the bones are freely movable, as in the limbs, and here the bones are entirely scparated, but are held in place by ligaments.

The Axial Skeleton.-The skull, which is composed of cartilage, cartilage bones, and membrane bones, forms a case for the brain and capsules for the sense organs. The frog's cranium has considerably more cartilage than do the skulls of higher vertebrates and less than those of lower vertebrates. The cartilage bones are so called because of their origin in cartilage which has subsequently been partly replaced by ossified tissue, forming bones separated by sutures. These cartilage bones are found at various points on the cartilage box that composes the foundation of the cranium. Cartilage bones are the sphenethmoids, pro-otics, exoccipitals, pterygoids, palatines, and cartilaginous quadrates. The membrane bones develop from ossifications of membranes which cover the cartilage and cartilage bones. They are thin and may be separated from the others. The membrane bones 
are the premaxillaries, maxillaries, nasals, frontoparietals, quadratojugals, squamosals, parasphenoids, and vomers. The bones enclosing the brain constitute the cranium.

On the dorsal surface of the cranium, the two frontoparietals form most of the roof, the pro-otics form the roof of the auditory

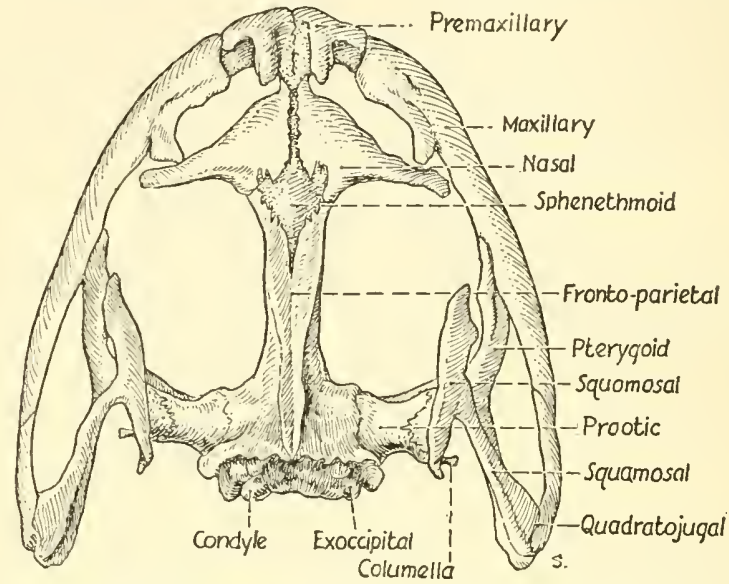

Fig. 279.-Dorsal view of the skull and upper jaw of the bullfrog.

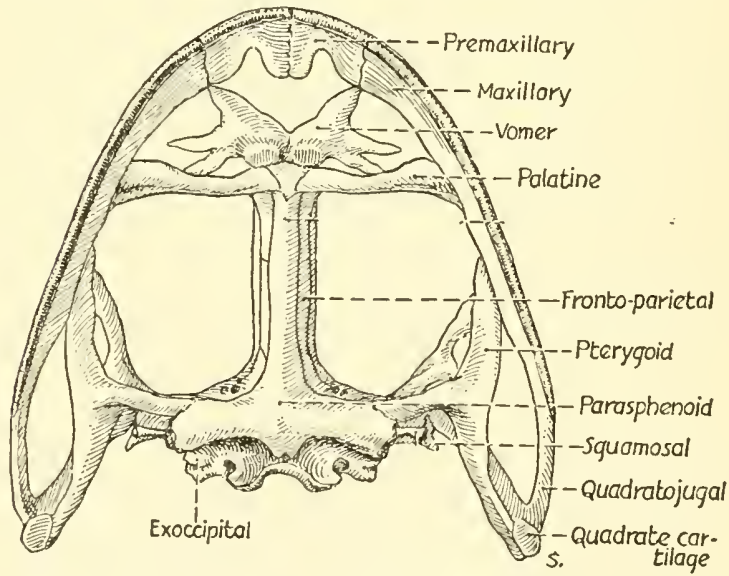

Fig. 280.-Ventral view of the skull and upper jaw of the bullfrog.

capsule (inner ear capsule), the sphenethmoids form the posterior wall of the olfactory capsule (nasal chamber), and the two triangular nasal bones lie above. On the ventral surface of the cra- 
nium are the slender palatines extending laterally on each side from the anterior end of the sphenethmoid to the upper jaw. The vomers form the floor of the olfactory capsules, and their ventral surfaces bear the vomerine teeth. The parasphenoid forms the floor of the brain case.

At the posterior end of the cranium is a large opening, the foramen magnum, through which the spinal cord passes. On each side of this opening are the exoccipital bones. Each bone has a rounded projection at its base, an occipital condyle, which articulates with the vertebral column.

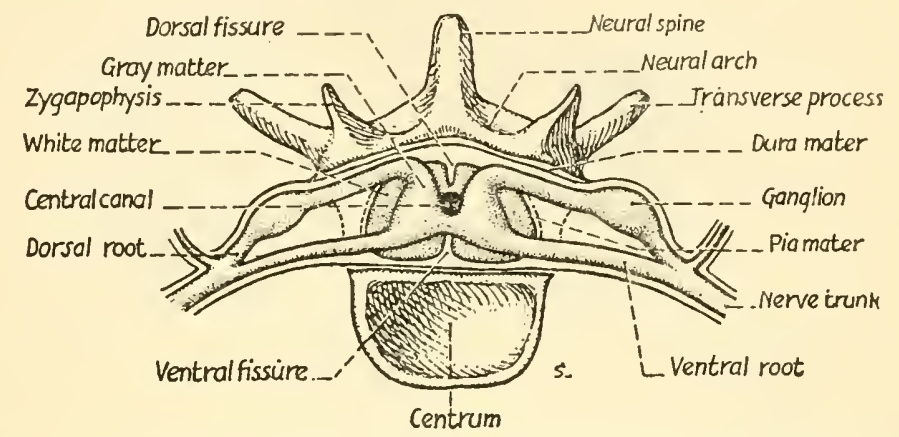

Fig. 281.-Structure of a single vertebra and cross section of the spinal cord. (Redrawn and modified from Holmes, Biology of the Frog, by permission of The Macmillan Company, after Howe, Atlas of Zootomy.)

Visceral Skeleton.-The visceral skeleton is that part of the axial skeleton which consists of the jaws and hyoid apparatus in the adult. The gill arches of the tadpoles are included in this portion. These parts originate in cartilage which is later partially replaced and reinforced by ossifications. The hyoid apparatus is primarily cartilaginous and serves as a support for the base of the tongue and the larynx. According to some authors, the jaws and the hyoid were originally the branched arches supporting the gills, and evidence of this is seen when the frog tadpole breathing with gills transforms to the frog breathing without gills.

The upper jaw consists of a pair of short premaxillary bones in front, a pair of long maxillae forming the sides, and a pair of short quadratojugals as the posterior portions. The premaxillae and maxillae each bear a row of small conical tecth. 
The lower jaw is formed primarily of a cartilaginous rod known as Meckel's cartilage. At the extreme anterior tip of the jaw the rod is ossified to form two small bones, the mentomeckelian bones. It is subsequently covered anteriorly by a dentary bone and posteriorly by an angulosplenial bone. The jaws are attached to the cranium by a combination of three bones on each side, the squamosal, pterygoid, and palatine, to form a suspensory mechanism.

The vertebral column is made up of a series of nine typical vertebrae and a long bone, the urostyle, which includes a fusion of the vertebrae of the tadpole tail.

In the neck region, there is one cervical vertebra, the atlas, which articulates with the skull. This is followed by seven trunk vertebrae, then one sacral vertebra whose processes support the pelvic girdle, and finally the urostyle, which contains all of the caudal vertebrae fused into one piece.

The basal portion of the typical vertebra is known as the centrum. The centrum is concave in front and convex posteriorly, and therefore is procoelous except one vertebra which is amphicoelous in Rana. Attached to the centrum is a bony arch, the neural arch, which extends dorsally from the centrum around the spinal cord. The neural arch has extending from its sides, at the point of union with the centrum, a pair of riblike transverse processes to which muscles are attached. A dorsal projection of the neural areh is the neural spine. In addition, the neural arch has at each its anterior border and posterior border a pair of processes known as zygapophyses by which the vertebrae are coupled together, the posterior zygapophyses of one vertebra overlapping the anterior zygapophyses of the succeeding one (Fig. 281). This arrangement furnishes a protected canal for the spinal cord and a firm axial support which also allows bending of the body. The spinal nerves emerge between vertebrae through intervertebral foramina protected by the cartilaginous pads between the vertebrae.

Appendicular Skeleton.-The anterior portion of the appendicular skeleton is composed of the pectoral girdle, sternum, and bones of the forelimbs. The posterior portion has the pelvic girdle and bones of the hindlimbs.

The pectoral girdle and sternum furnish a support and place of attachment for the forelimbs and their muscles. They also provide a case to protect the heart, lungs, and other organs in the anterior part of the body. This girdle is not connected to the vertebral column. 
Each side of dorsal part of the girdle is composed of a large flat bone, the suprascapula, which curves ventrally and joins the scapula, narrowing as it does so. From the ventro-anterior end of the scapula two bones extend to the midventral line of the body and would meet their fellows from the opposite side except that the narrow epicoracoid cartilage intervenes. The anterior of these two bars is the clavicle and the posterior one the coracoid. At the junction of coracoid and scapula a depression is formed, known as the glenoid fossa, into which the forelimb articulates. The ventral sternum is separated

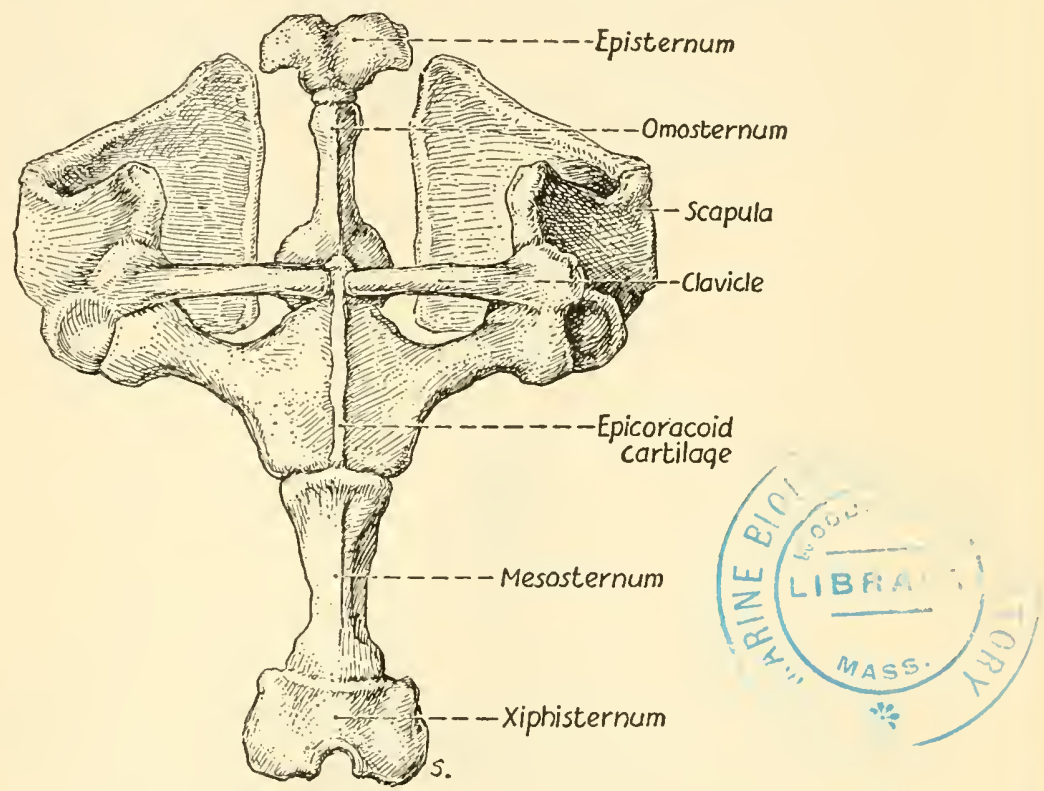

Fig. 282.-Diagram of the ventral view of the pectoral girdle of Rana catesberana, natural position.

into two portions by the pectoral girdle. The anterior portion is composed of a bone, the omosternum, to which is attached anteriorly a rounded plate of cartilage, the episternum. The posterior portion is composed of a bone, the sternum proper (mesosternum), and a rounded cartilage, the xiphisternum, which has a notch at its posterior margin through which the abdominal vein runs as it leaves the body wall.

The pelvic girdle furnishes a place of attachment and support for the hindlimbs. Each half of the pelvic girdle is composed of three bones, the ilium, ischium, and pubis. The more slender ilium is at- 
tached anteriorly to the transverse process of the ninth vertebra, and posteriorly it fuses with the pubis and ischium, forming a dishlike concavity, the acetabulum, which receives the hindlimb. The pubis forms the ventral part of the acetabulum, the ischium the posterior, and the ilium the anterodorsal.

The forelimbs join the body by a ball and socket joint at the glenoid cavity in the pectoral girdle. The large bone which makes this articulation is the humerus. The succeeding bone of the forcarm is the radio-ulna, a fusion of two originally distinct bones. The wrist, which follows, contains six carpal bones arranged in two rows. Each hand, or manus, contains four metacarpals following the carpals, and distal to these are four complete digits and an exceedingly small rudimentary fifth near the thumb, the prepollex, consisting of only

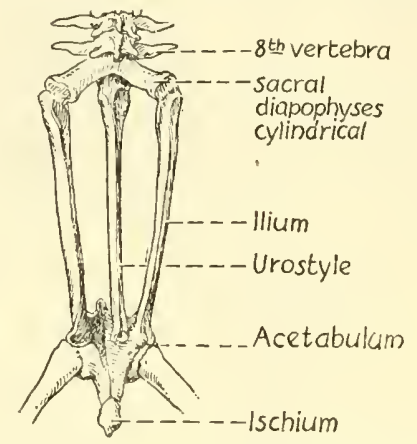

Fig. 283.-Pelvic girdle of the bullfrog, dorsal view.

a single bone. Each of the four digits, or fingers, extends from a metacarpal bone. This is followed in digits II and III by two phalanges and in digits IV and $\mathrm{V}$ by three phalanges.

The hindlimbs have essentially the same structure as the forelimbs. The large bone which joins the girdle at the socketlike acetabulum is known as the fenur. This bone articulates with the tibiofibula, which, like the bone of the forearm, is a fusion of two bones. The tarsus or ankle differs from the wrist, being composed of two long bones, the tibiale and fibulare, and two small tarsals. There are also two extremely small bones forming the prehallux, or rudimentary sixth toe. Distal to the tarsals are five long metatarsals. Each foot contains five complete digits, each following a metatarsal bone. In digits I and II are two phalanges, in digits III and $\mathrm{V}$ three phalanges, and in digit JV four phalanges. 


\section{Muscular System}

Muscular tissue controls the movements and positions of various parts of the body of the bullfrog. This it does by contracting, that is, by shortening and thickening its elements.

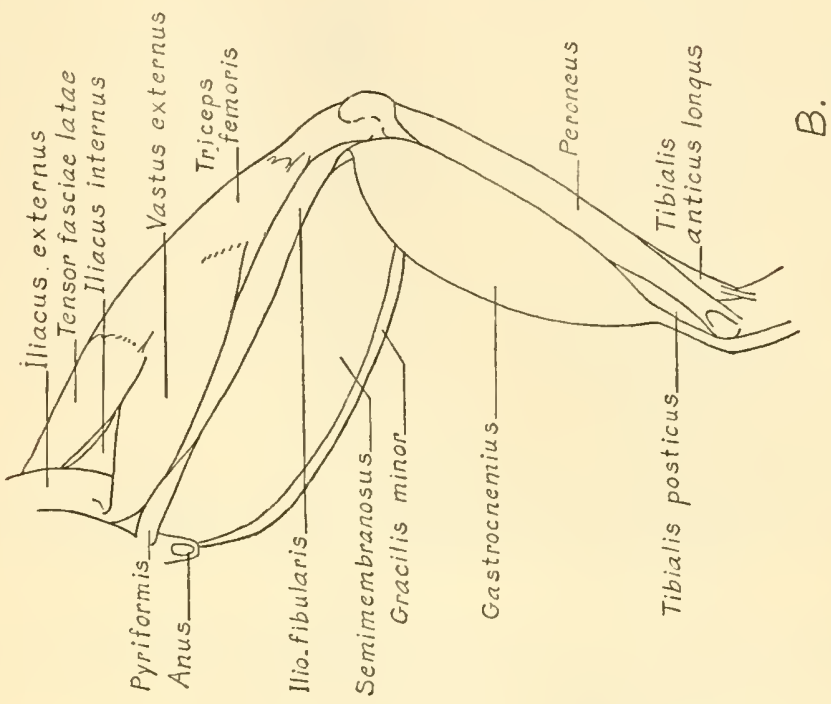

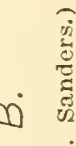

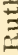

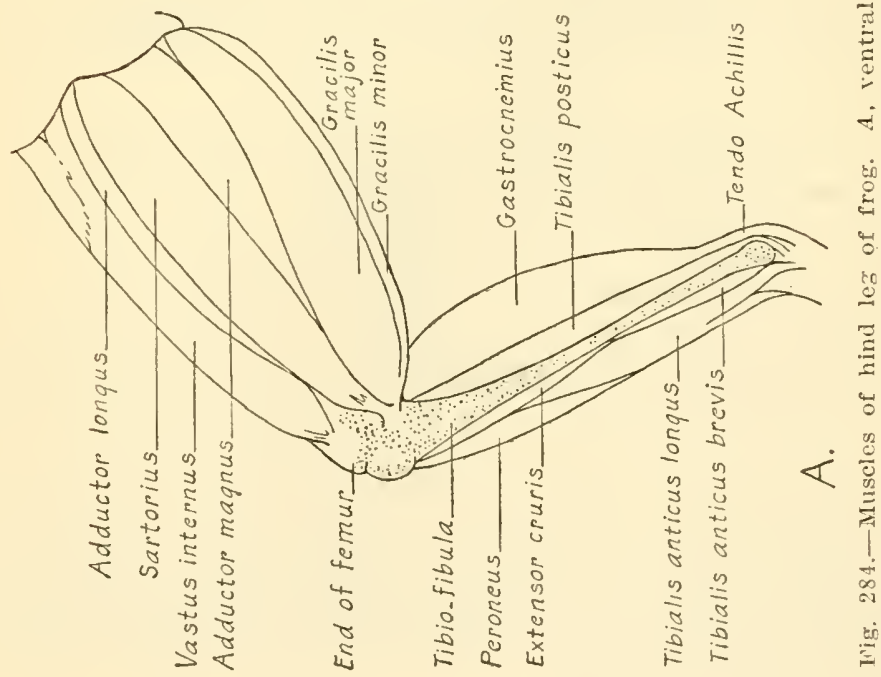


Movements may be under voluntary control, as the skeletal muscles, involved in moving the limbs, in which case the muscle fibers are striated and are known as voluntary muscle. Other movements, such as the heartbeat and the peristaltic movements in the intestines, are not under control of the will. Muscles concerned in these actions are known as involuntary and are usually made up of smooth muscle fibers except in the heart, which contains striated cardiac muscle.

Most voluntary muscles are attached to bones at one end or at both by specialized connective tissue bands known as tendons. The end of the muscle which is attached to a relatively fixed and immovable part is called the origin; the end which is attached to the part which moves when the muscle contracts is known as the insertion. A typical voluntary muscle is made up of three parts: the tendons attached at its ends; the membrane surrounding the muscle, known as the fascia; and the belly, or fieshy part, of the muscle.

The different actions performed by the various skcletal muscles give rise to descriptive names applied to them. Some of these are as follows :

Extensor-one that straightens a part, such as extending the foot.

Flexor-one that bends a part, such as a joint.

Adductor-one that draws the limb toward the median ventral line.

Abductor-one that draws the limb away from the median ventral line.

Levator-one that raises a part, such as the lower jaw.

Depressor-one that lowers a part, such as the lower jaw.

Rotator-one that rotates one part on another.

The pectoral muscles cover the chest and ventral portion of the upper body region; the rectus abdominis extends along the median ventral region; the paired obliquus externus and internus cover most of the sides of the trunk. The muscles of the limbs are numerous. There are some eighteen separate muscles which control various movements of the legs. A detailed description of these and other muscles of the frog would be confusing to the elementary student and therefore is not included. The major muscles of the hind leg are illustrated in Fig. 284 and can be clearly understood after a careful dissection in the laboratory. 


\section{Nervous System}

The three divisions in which the nervous system of the bullfrog may be considered are: (1) central nervous system, (2) peripheral nervous system, and (3) sympathetic nervous system.

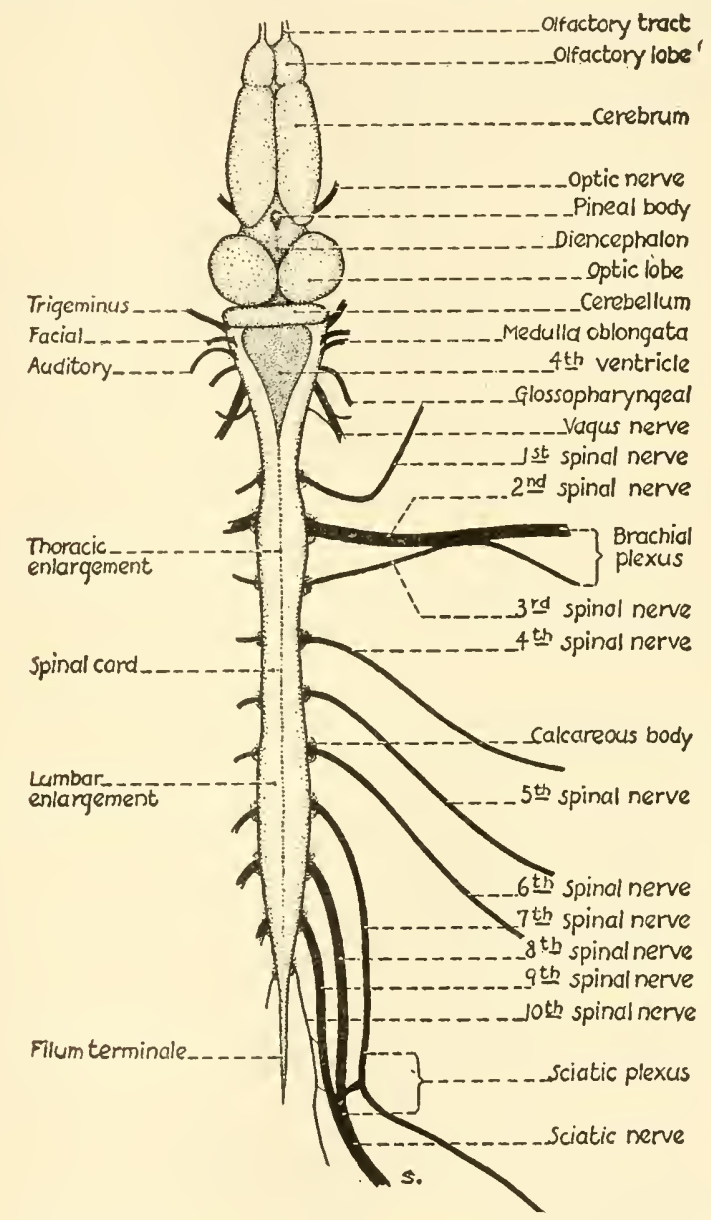

Fig. 285.-Dorsal vlew of the nervous system of a frog.

The central nervous system, so called because it comprises the larger number of nerve centers, consists of the brain and spinal cord. The peripheral nervous system consists of (1) the paired cranial and spinal nerves which connect the brain and spinal cord with other 
organs of the body and (2) a large number of small nerve centers, ganglia, distributed throughout the body. The sympathetic nervous system is a part of the peripheral nervous system. It is made up of a large number of small ganglia, two rows of which form the sympathetic trunks on each side of the vertebral column and connect with the spinal nerves. The branches of these sympathetic trunks connect with numerous small ganglia throughout the tissues of the body. This system controls and regulates primarily the involuntary movements of such organs as the heart, digestive tract, glands, organs of respiration, and walls of blood vessels.

Central Nervous System.-The brain is covered with a pigmented membrane known as the pia mater. The brain has three main divisions, the forebrain, midbrain, and hindbrain. The forebrain consists of a pair of elongated cerebral hemispheres, separated from each other by a fissure, and two enlargements at the anterior end of the hemispheres known as the olfactory lobes. These lobes are fused on the dorsal side but separated by a groove on the ventral side. Immediately behind the forebrain is the diencephalon. On its dorsal surface is a vestige of the pineal organ which was more developed in the tadpole. On its ventral surface is the optic chiasma, a crossing of the optic nerves formed by fibers from the right and left sides, each crossing to supply the eye of the opposite side. Just behind the optic chiasma is the infundibulum, and somewhat behind this is the pituitary body, or hypophysis. The pituitary is of dual origin, developing in part from the diencephalon and in part from the roof of the mouth cavity. The midbrain contains two large rounded optic lobes. The ventral part of the brain below these lobes is the crura cerebri.

The hindbrain consists of the cerebellum and the medulla oblongata. The cerebellum in the frog is almost rudimentary and consists of a transverse fold of tissue immediately posterior to the optic lobes. The cerebellum is in close connection with the large triangular medulla oblongata which constitutes the most posterior part of the brain and is continuous with the spinal cord.

Internal Organization.-The central nervous system is hollow. In embryological development the central cavity is large; but, as maturity is approached, the walls thicken, and the cavity, particularly in the spinal cord, is much reduced. In the brain these cavities, known as ventricles, form a continuous channel for the flow of cerebrospinal fluid. The ventricles are comnected one with another 
by openings known as foramina. The cavities are large in four regions: (1) the paired lateral ventricles in the cerebral hemispheres, (2) the single third ventricle in the diencephalon, (3) the paired optic ventricles in the optic lobes, (4) the single large triangular fourth ventricle in the medulla oblongata. Vascular nets of blood vessels in the much-folded pia mater constitute choroid plexuses that form the roofs of the third and fourth ventricles and extend into the other ventricles somewhat. Most of the cerebrospinal fluid is derived from the blood vessels of these plexuses.

The spinal cord is continuous with the medulla oblongata anteriorly, runs posteriorly through the canal formed by the vertebrae, and finally tapers to a narrow filament which ends in the urostyle. It is covered by two membranes, an outer dura mater and an inner pia mater. It is somewhat flattened, and a median fissure occurs on both its dorsal and ventral sides. The central part of the cord comprising its bulk is made up of gray matter consisting primarily of nerve cells. In the center of this gray matter is a small hollow canal, the neurocoele, which communicates with the ventricles of the brain. Surrounding the gray matter is white matter consisting chiefly of nerve fibers.

Peripheral Nervous System.-The peripheral nervous system is composed of the cranial, spinal, and sympathetic nerves, the last of which will be considered separately.

The cranial nerves arise from the brain, and there are ten pairs of them in the bullfrog. Counting from the olfactory lobes backward, they are as follows: olfactory, optic, oculomotor, trochlearis, trigeminus, abducens, facial, auditory, glossopharyngeal, and the vagus. All of these, with the exception of the tenth or vagus nerve, run to parts of the head. The vagus nerves branch to the heart, lungs, and digestive system.

The bullfrog has ten pairs of spinal nerves. Each spinal nerve originates from the gray matter in the spinal cord by a dorsal and a ventral root. These roots pass out of the vertebral column between vertebrae throngh an opening or intervertebral foramen and unite into a nerve trunk, branches of which extend to the muscles and skin of the body and limbs. The dorsal root is known as the sensory or afferent root and has a ganglion; the ventral root is known as the efferent or motor root and has no ganglion. Where these roots meet 
after leaving the spinal cord, they are covered on the ventral side by a large calcareous body, the periganglionic gland, or "gland of Swammerdam."

The first spinal nerve arises between the first and second vertebrae, the second between the second and third vertebrae, and so on until the tenth, which is small and emerges from the urostyle near its anterior end. These nerves frequently send branches to preceding or succeeding nerves to form plexuses. Two large plexuses in particular are present. Branches from the first and third nerves join with the large second nerve to form the brachial plexus, which supplies nerves to the muscles of the forelimbs and shoulder. Nerves number seven, eight, and nine fuse to form the large sciatic plexus which supplies the sciatic nerve to the hind leg.

Sympathetic Nervous System.-From the first sympathetic ganglion, nerves are given off which form a cardiac plexus on the heart. Another plexus, formed primarily from nerves of the third, fourth, and fifth sympathetic ganglia, is the solar plexus on the dorsal surface of the stomach. In addition, numerous ganglia are scattered throughout the tissues of the body, all being connected by sympathetic nerve fibers and finally communicating with the sympathetic trunks. The cooperation of certain cranial and spinal nerves with the sympathetic in relation to the involuntary actions of a number of the vital internal organs is referred to as the autonomic function.

\section{The Sense Organs}

The olfactory sacs, or nasal chambers, are located internal to the external nares. The median portion of the nasal chamber is lined with olfactory epithelium which contains sense cells possessing protoplasmic processes known as olfactory hairs on their free ends. These olfactory hairs are stimulated by chemical substances present in the air and pass the stimuli received through the olfactory cells to the olfactory nerves.

The degree to which the sense of smell is used by amphibians is not known. It is likely, however, that it may cause the frog at times to approach objects and may serve to test the food substances it takes into its mouth.

The eyes lie in cavities, or orbits, on the dorsolateral sides of the head. The exposed portion of the eyeball is covered by a transparent membrane, the cornea, which is continuous with the opaque connec- 
tive tissue sheath covering the remainder of the eyeball and known as the sclera. Attached to the sclera are sereral muscles which move the eye in various directions. The iris of the bullfrog is colorful, being either golden or reddish bronze, and is clearly visible through the transparent cornea. In its center is an oval opening, the pupil, which can be contracted or expanded by the action of muscle fibers in the iris and, like the shutter of a camera, regulates the amount of light which enters the inner chambers of the eye. The lens lies behind the iris and is flattened on its outer surface. It is enclosed in a membrane and held in place by delicate fibers to the ciliary body. The space between the cornea and lens is filled with a watery transparent substance, the aqueous humor.

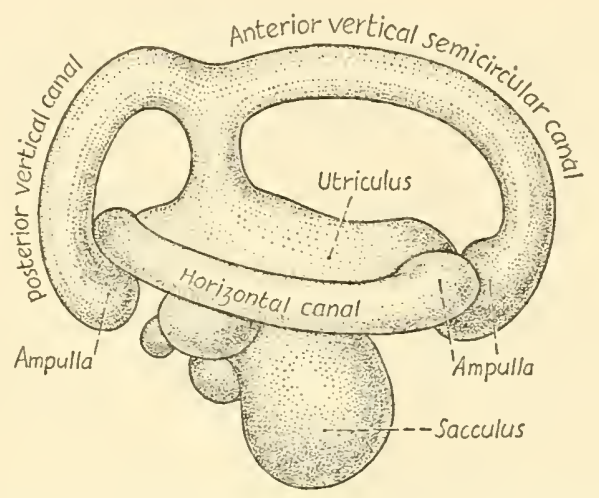

Fig. 286. - The right internal ear of the frog, lateral view.

The main cavity of the eye back of the lens is filled with a gelatinous tissue, the vitreous humor. The walls of this carity are made up of three layers, the outer sclerotic coat, previously mentioned, then a vascular pigmented chorioid and the innermost layer, the retina. The anterior portion of the chorioid forms the ciliary body, which subsequently is continuous with the iris.

The retina contains the photosensitive cells of the eye which pass the stimuli received on to the optic nerve. These sensitive cells, known as the rods and cones, lie embedded in the tissue so that light has to pass through several layers of nerve fibers, as well as much supporting tissue, before reaching them. The rods and cones communicate with fine branches of the optic nerve, which enters the eye posteriorly. 
Sharpness of vision is dependent on both the proper focusing of the lens and the proper amount of light reaching the retina. When the light is too strong, the pupil of the iris contracts and euts down the volume. The eye of the frog has little if any accommodation or focusing of the lens. It therefore has very imperfect vision.

The ear of the bullfrog is covered externally by a membrane, the tympanum. A Eustachian tube runs between the middle ear and mouth cavity. The tympanum has attached to it a bony rod, the columella, the other end of which is joined to a portion of the inner ear. This rod transmits sound vibrations from the tympanum to the inner ear.

The inner ear lies in a cavity of the skull known as the auditory capsule. The structures of the inner ear compose a membranous labyrinth which is surrounded by a lymphlike fluid, the perilymph. The labyrinth is formed of a dorsal utriculus concerned with equilibrium and a ventral sacculus functioning as an auditory organ. The utriculus is connected with three semicircular eanals which are placed in planes almost at right angles to one another. Two are vertical canals, and the third, on the outer side of the utriculus, is horizontal. The sacculus is irregular, pouchlike, and filled with a fluid, the endolymph. It also contains the nerve endings which receive the stimuli and convey them to the auditory nerve.

Sound progresses in the following fashion. The tympanic membrane vibrates to sound waves, and these are transported by the columella to the inner ear. These vibrations are taken up by the endolymph of the sacculus and are received by the nerve endings which lead to the auditory nerves. These nerves convey the impulse to the brain, subsequently giving rise to auditory sensations.

In a similar manner, movements of the endolymph in the utriculus affect sensory cells and cause a reaction associated with a sense of position or equilibration.

Sound and hearing play an important role in the life of frogs, the calls of the males serving to attract the females and others to the ponds during the breeding season. They are of prime importance in the daily life of the terrestrial toad, who is on the alert when an insect has announced its location by a sound. 


\section{Reproductive Organs}

The ovoid testes (Fig. 278) of the male bullfrog are attached to each kidney by a fold of peritoneum. In this fold of peritoneum, running between the testes and kidneys, are several small ducts, the vasa efferentia. These ducts connect with the mesonephric duct through the collecting tubules of the kidney. Spermatic fluid containing the spermatozoa passes from the testes through the rasa efferentia into the kidney, then into the mesonephric duct, which opens into the cloaca, and thence to the outside through the anus. In some species, this duct is slightly expanded prior to its opening into the cloaca to form the seminal vesicle, a reservoir for spermatozoa. This is poorly developed in the bullfrog.

The two ovaries of the female bullfrog, when filled with eggs, occupy a large part of the body cavity and consist of folded sacs covered with peritoneum. They originate in about the same position as do the testes and lie in a fold of the peritoneum ventral to the kidneys. The eggs lie in the outer surface of the ovary and during their growth are surrounded by a network of blood ressels and follicle cells.

The two oviducts are greatly convoluted white tubes, one on each side of the body eavity, ruming from near the base of the lungs to the dorsal wall of the cloaca. Their anterior ends are funnel-shaped and open into the body eavity. Their posterior ends are dilated to form thin-walled ovisacs or uteri which open into the cloaca near the entrance of the mesonephric duct. They are not connected at any point with the ovaries.

When the eggs are mature at the breeding season, they break through the walls of the ovary and its peritoneal covering and are free in the body cavity. They make their way to the funnel-shaped opening, or ostium, of the oviduct and, probably by ciliary action or movements of the female, are squeezed into it. The oviducts contain a large number of glands which secrete a clear, jellylike material. As the eggs are forced down the oviduct by ciliary action, they become coated with the gelatinous material, which swells enormously when it contacts water.

Fertilization in the bullfrog is external, and the spermatozoa of the male enter the eggs after they have been laid in the water. 
Attached to the anterior end of the testes of the male frog and to the ovaries of the female are fingerlike projections known as fat bodies. These serve to store a reserve fat supply which the bullfrog may draw on during hibernation or at other times. They are largest before hibernation and smallest after egg laying. Recent experiments have also shown that these fat bodies are essential for allowing the normal development of the sex organs and for maintaining their health. When they are removed, there is a deterioration of eggs and sperm.

\section{Embryology}

The bullfrog lays its eggs in a large floating mass, forming a surface film on the water, usually among brush or plants near the pool's edge. This mass may be from 1 to $2 \frac{1}{2}$ feet in diameter and may contain ten to twenty thousand eggs. In Texas, bullfrogs may lay their eggs as early as February, though it is more common for them to be laid later in the season.

The eggs of the bullfrog are smaller than those of the leopard frog. They hatch in about four or five days, depending on the temperature. After hatching, the tadpole normally spends about two years in the water before transforming as a young bullfrog. The tadpole may grow to be four to six inches long, but the average body length of the young bullfrog as it metamorphoses is about $13 / 4$ to 2 inches. It usually takes about three to four years for this young frog to attain maturity and begin egg laying.

The embryology of the bullfrog does not difter materially from that of the leopard frog, and the following account is based, except where otherwise noted, upon the development of the latter.

The egg when laid is a single cell. The upper portion of the egg has considerable pigmentation, making it black. This part of the egg is known as the animal hemisphere, and it is thought that the pigmentation serves to absorb and retain heat necessary for development. The lower portion is white and is known as the vegetal hemisphere. The bullfrog egg is surrounded by a layer of transparent jelly, but does not have an inner envelope of jelly, as does the leopard frog egg. This jelly protects the egg and helps it to retain heat. The nucleus of the egg, or germinal vesicle, lies near the animal pole. The boundary of the egg is known as the vitelline membrane. 
The eggs are fertilized externally by the male, who is clasping the female as the eggs are laid and discharges spermatozoa into the water. The first spcrmatozoon to swim to the egg and enter it by piercing the vitelline membrane initiates fertilization. After the sperm has entered, a fertilization membrane is formed which prevents the entrance of additional spermatozoa. Only the head of the spermatozoon enters, the remainder being discarded. This head, which is composed primarily of the male spermatozoon nucleus, fuses with the nucleus of the egg to complete fertilization and start development.
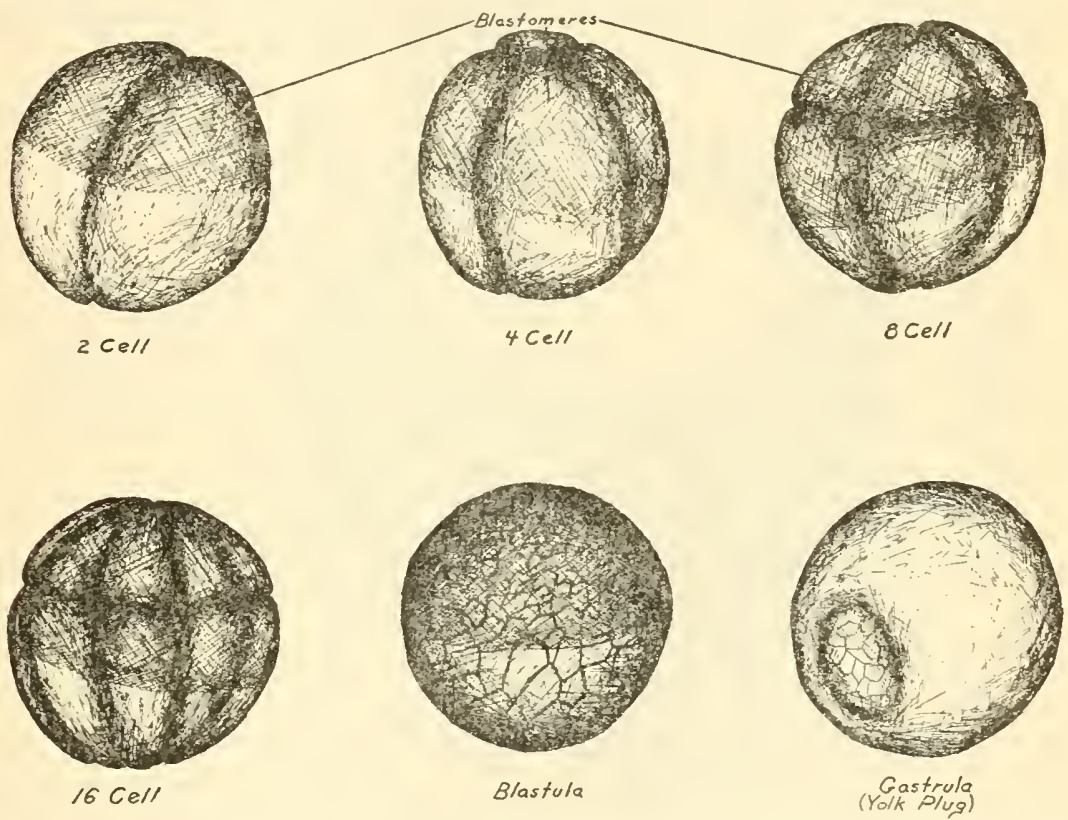

Flg. 287.-Diagrams of early cleavage stages, blastula and gastrula of the frog. Thls is holoblastic, unequal type of cleavage. The upper, shaded portion of each of the frrst five diagrams represents the animal hemisphere, and the lower portion of each, the vegetal hemisphere. The circular plug seen in the gastrula stage is the yolk plug.

Development begins with cleavage which is a series of mitotic divisions. Cleavage results in the rearrangement of nuclear material in relation to the cytoplasm. The furrow made by cleavage cuts through the entire egg, and such cleavage is known as total, or holoblastic.

Cleavage and Blastula Formation.-The first and second divisions run from pole to pole at right angles to each other and divide the egg into four equal blastomeres. The third cleavage is parallel to 
the equator of the egg and somewhat above it. This produces four cells at the animal pole that are smaller than the four cells at the vegetal pole. Subsequently the cells at the animal pole (micromeres) divide more rapidly than the cells at the vegetal pole, for they contain less yolk. Such eleavage is known as unequal, and as divisions proceed the cells at the animal pole become smaller and more numerous than those at the other pole. The final result of the following divisions is to form a hollow sphere, the blastula, whose cavity is known as the blastocoele. The blastula is essentially one cell layer thick, althougl in reality some cells have been crowded from the surface, giving the appearance of additional layers.

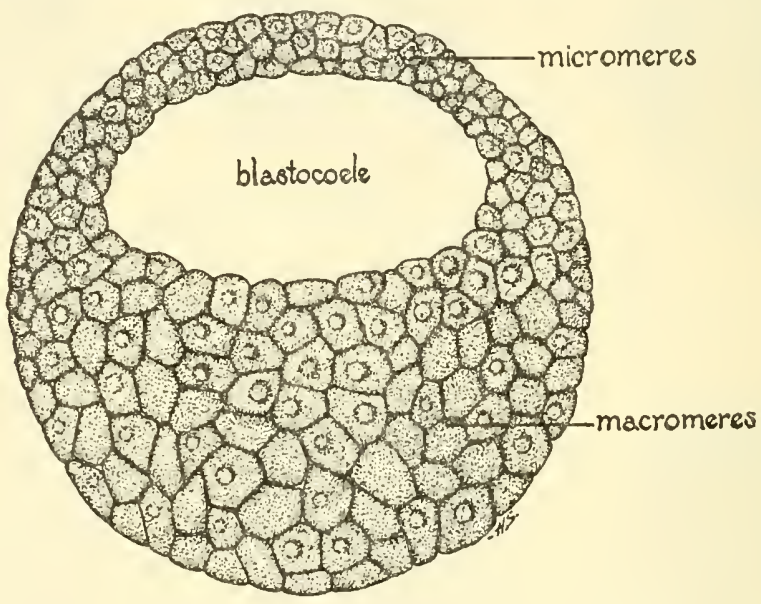

TROG EGG, CROSS SECTION OF BLASTULA

Fig. 288.- Section through blastula stage of developing frog. (Courtesy of General Biological Supply House.)

Gastrulation.-Gastrulation begins with the appearance of a small groove slightly below the equator of the egg. The upper edge of this groove is known as the dorsal lip of the blastopore. Coincident with the appearance of this groove, the pigmented animal cells begin to grow down over the white vegetal, or yolk, cells; and the dorsal lip moves downward as the line advances. It also extends its edges in a crescent shape laterally around the egg until they finally meet to form a circle. The area enclosed by this circle shrinks as the cells grow down from all sides and its rim moves downward. Cells on the side where the groove began advance more rapidly than 
the others; and the rim on this side, or the dorsal lip, is forced down to the vegetal pole and a little beyond on the other side before gastrulation is complete. The area enclosed by the circle or blastopore is finally very small, and the white vegetal cells which fill it are called the yolk plug.

While the animal cells liave been advancing and covering the vegetal cells, changes have been going on internally in the egg. The appearance of the groove or dorsal lip of the blastopore was caused

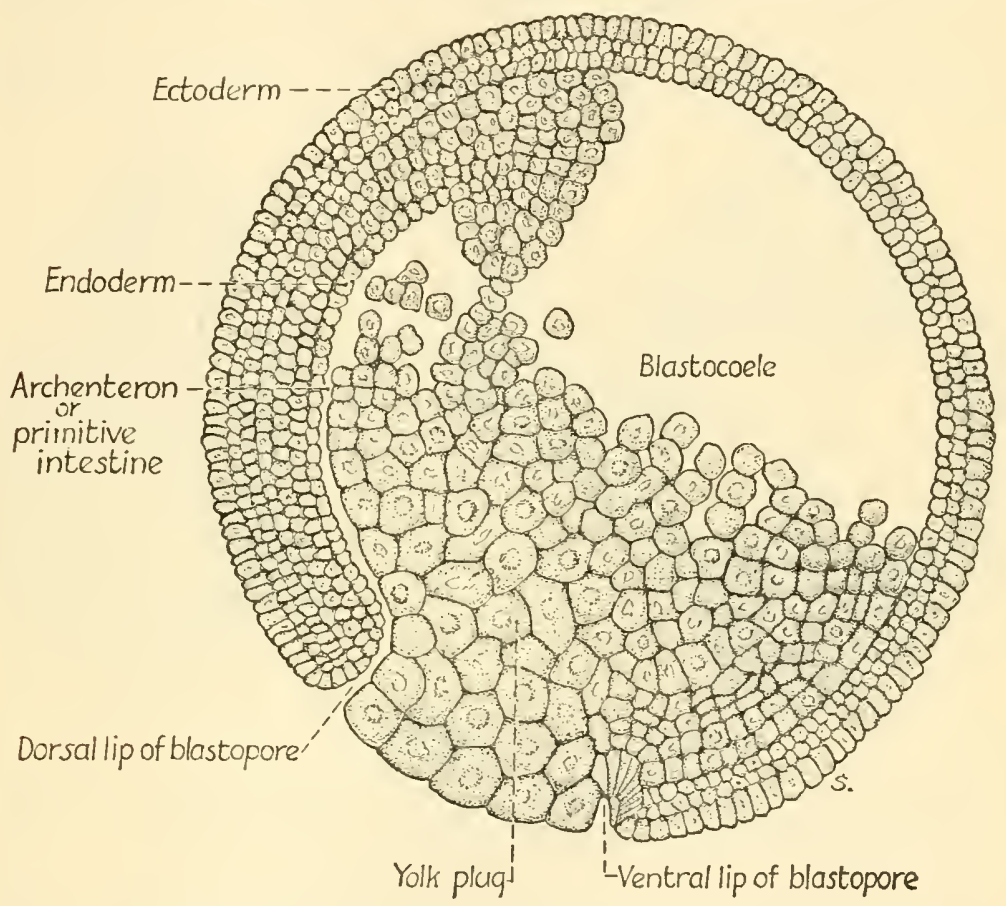

Fig. 289.-Gastrula stage of developing frog in section.

by an infolding of outside cells. This ingrowth of cells progresses around the egg as the crescent groove extends itself. The cells which consequently come to lie inside are known as endodermal cells, those on the outside as ectodermal cells.

By this invagination a cavity known as the archenteron is formed, the walls of which are made up of the invaginated endoderm cells. At first it is quite flat, but it expands as invagination and the other 
processes of gastrulation proceed, and soon it takes up the space formerly occupied by the cleavage cavity or blastocoele. Its posterior end is the blastopore, and this, as previously mentioned, is plugged with yolk or vegetal cells known as the yolk plug. As a final result, the gastrula forms a two-layered embryo of ectoderm and endoderm cells, each layer of which may be several cells thick.

Mesoderm Formation.-Before the process of gastrulation is completed, a sheet of cells forms between the ectoderm and endoderm cells. This sheet of cells is known as mesoderm. As the mesoderm grows it splits into two sheets, an outer, or somatic layer, which lies next to the ectoderm of the embryo wall, and an inner, or splanchnic

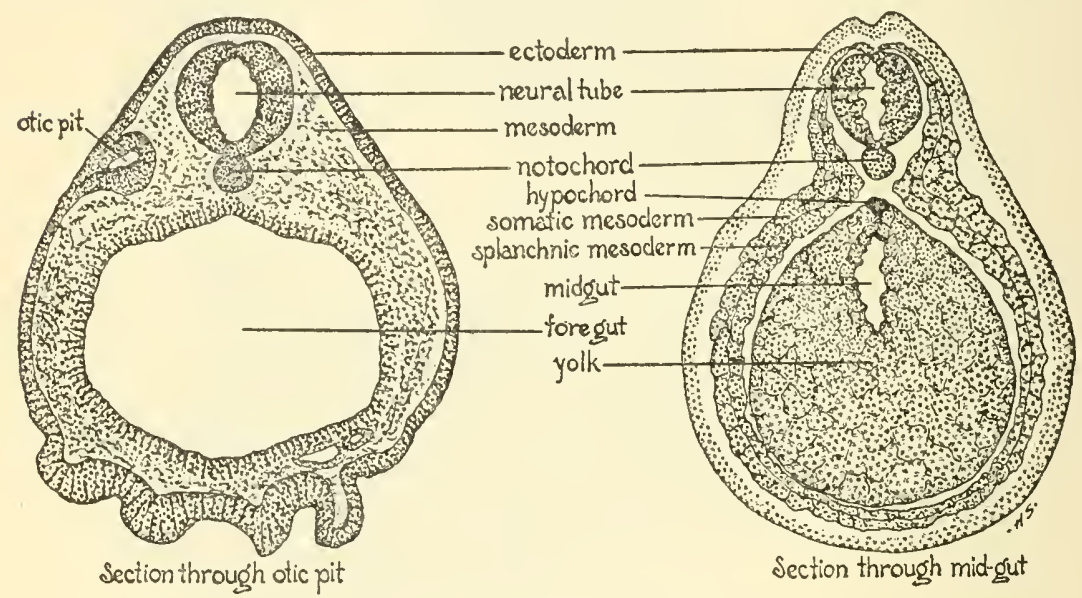

FROG EMBRYO, SHOWING MESODERM FOLDS

Fig. 290.-Two levels through the body of the neural tube stage of the developing frog. (Courtesy of General Biological Supply House.)

layer, which lies next to the endoderm cells of the archenteron. The cavity formed between these two layers is the beginning of the coelom, or body cavity. From these three layers, ectoderm, endoderm, and mesoderm, all the body structures are formed.

Formation of Nervous System.- With the reduction of the blastopore to a very small area, there appears on the dorsal side of the embryo a thickened plate of ectoderm known as the neural plate which is wide in front but narrows posteriorly. This plate soon is flanked on each side and in front by ridges known as neural folds. As these folds arise, the remainder of the plate becomes a longitudinal 
groove known as the neural groove. The neural folds or lateral edges of the plate grow rapidly and curve in to meet in the dorsal midline, where they fuse to form the neural tube. Anteriorly the neural tube soon closes to end blindly while posteriorly it remains open to the exterior at the blastopore for a time. Subsequently this communication with the blastopore closes. The neural tube soon separates from the ectoderm above, its walls thicken and through other changes it develops into the central nervous system. The anterior part becomes the brain and the posterior part the spinal cord.

During these changes, the egg, which previously was spherical, has elongated in the axis of the nenral tube. The blastopore has been covered by the folding of edges of the neural groove extending to its borders. Subsequently the embryo takes on form so that definite body regions can be identified.

Later Development.-In the head region appears an elevated side plate, the gill plate, where later the gills develop. Anterior to this, a swelling on each side of the head denotes the beginning of certain sense organs. A depression on the anterior ventral surface is a forerunner of the mouth, and posterior to this a crescent-shaped area indicates the beginning of a ventral sucker by which the newly hatched larva may attach itself to objects. At the posterior end of the body a tail bud appears, and the region of the blastopore becomes the anus. The embryo soon hatches, branched external gills which serve as respiratory organs make their appearance, and definite sense organs can be found on the head. This is the external gill stage. The intestine becomes coiled; the tail elongates; and muscle segments, or myotomes, can be seen along the sides of the body. Shortly after hatching, the external gills are absorbed and internal gills take their place. A fold of skin, the operculum, develops over this region. There remains on the left side, however, a small opening, the spiracle, through which water, taken in at the mouth, may pass out after going over the internal gills. The skeleton is cartilaginous; lateral line sense organs are present on the sides; the pineal organ is evident; and the animal is similar to a fish.

The tadpoles feed primarily on plant substances. In the mouth of the bullfrog tadpole is one row of teeth above and three rows below, plus a border of projections, known as papillae, for testing food substances. Between the lips is a horny beak somewhat like that of a 
bird's with which the tadpole can scrape thin pieces from leaves of aquatic plants, or algae and other plant material from sticks and stones.

Near the end of the larval period (about two years in the bullfrog), which varies considerably with species and environment, the tadpole prepares to metamorphose into a frog. First the hind legs push through the skin at the base of the tail; then the forelegs appear, forcing their way through the operculum on the right and the spiracle on the left side. As the lungs develop, the tadpole has to come to the surface of the water frequently to give out a bubble of impure air and take in a purer one. The tail is gradually absorbed, the intestines shorten, the homy beak disappears, the mouth widens, the gills are resorbed, the legs develop, and the tadpole becomes a frog.

In general, it may be stated that the ectoderm gives rise to the nervous structures, the epidermis and its outgrowths. The endoderm forms the epithelial lining of the intestine, and outgrowths of the intestine, such as the epithelial lining of gills, lungs, liver, pancreas, gall bladder, urinary bladder, etc. From the mesoderm are formed the muscular, vascular, and skeletal systems. Most of the organs are formed not from a single germ layer but from a combination of these tissues. The elementary tissues have been discussed in the chapter on Metazoan Organization.

\section{THE TOAD}

One of the common toads is the American toad, Bufo americanus. It resembles elosely its relatives, the Woodhouse's toad (Bufo woodhousii Girard) and Fowler's toad (Bufo fowleri), and only by close scrutiny can they be distinguished. Bufo woodhousii ranges, in general, from Texas to Kansas and Nebraska and westward to Arizona and southeastern Califormia.

\section{Habitat}

In contrast to the bullfrog, the toad is entirely terrestrial except when it goes to the water during the breeding season to lay its eggs. Although the toad's skin is tougher and better protected from drying out, water evaporates through it rapidly, and it cannot endure dry heat. Most of the toad's supply of water is obtained 
by absorption through the skin. Toads usually hide away in some erevice, burrow, or moist place during the daytime, coming out in the late evening to get their meal of insects.

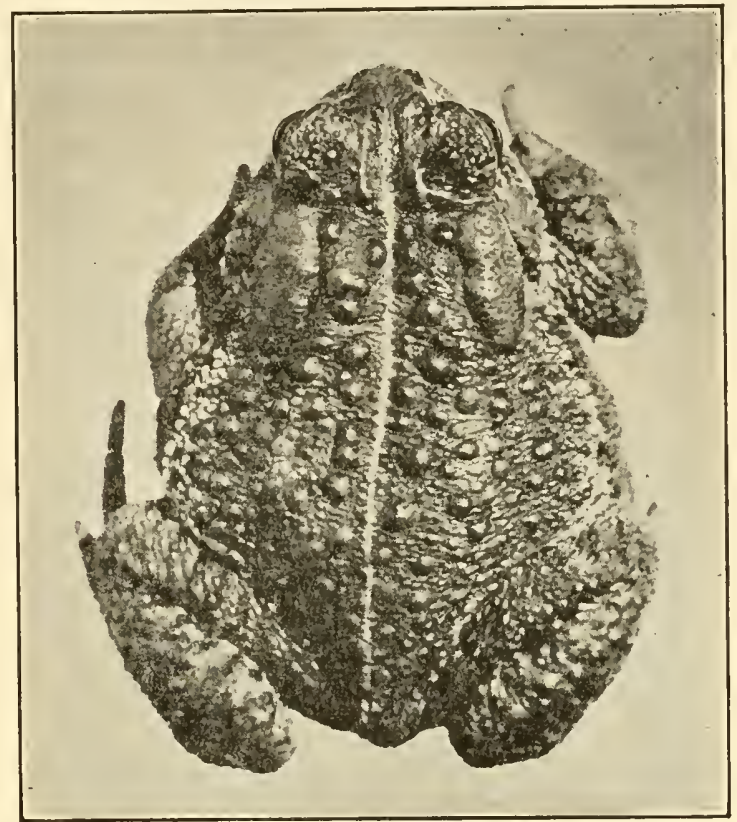

Fig. 291.-Woodhouse's toad, Bufo woodhousii. (From photograph taken by A. H. Wright from specimen furnished by L. M. Klauber.)

\section{External Features}

The toad differs markedly from the bullfrog in external appearance. Its body is broad and thick, and its hind limbs are short. It reaches a body length of $2 \frac{1}{2}$ to $43 / 4$ inches. Its skin is rough and covered with warts. Its color is not as drab as a casual glance would cause one to believe. If its skin is not covered with soil from its burrow, the upper parts are grayish or a dull yellowish brown often blotched with darker irregular areas. A light vertebral stripe runs down the middle of the back from between the eyes to the vent. The limbs are faintly barred, and the underparts are a light yellow with or without black markings on the breast. The largest warts are encircled at their base with a narrow black border. There is usually only one large wart in a spot of this nature, although this 
may vary. Individual toads of a species may vary widely in color and other characteristics.

The single vocal sac is a rounded throat pouch which balloons out when the toad calls. The toad does not have internal sacs as does the bullfrog or sacs between the ear and shoulder as does the leopard frog.

On each shoulder is a large oblong gland known as the parotoid gland, which is not found in frogs. Anterior to it and on the side of the head is the vertically oval tympanum. According to Kellogg, the secretion of the parotoid gland "is an acid irritant causing pain in cuts and producing a bitter astringent sensation in the mouth."

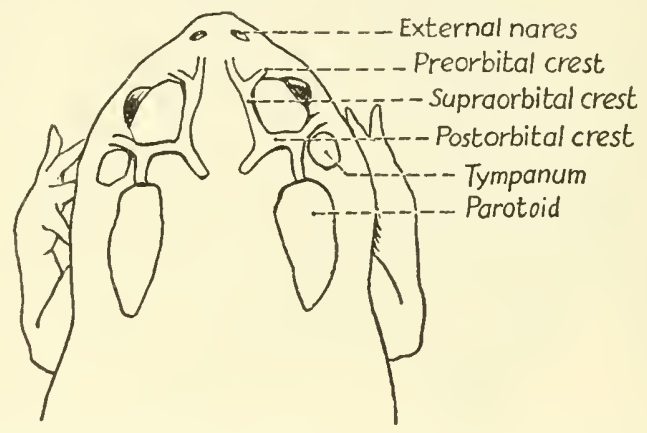

Fig. 292.-Dorsal view of head region of a toad to show parotoid glands and

It may act as a poison or, at any rate, as a powerful repellent when the toad is seized by other animals. A dog that picks up a toad lets it go at once and does not soon forget the experience.

The ridges between the eyes and back of them are known as cranial crests. They are made by the bones of the head and are variously named according to their position. Those between the eyes are known as superciliary or supraorbital; the transverse crests back of the eyes and at right angles to the superciliary crests are the postorbital crests. A crest that is absent in Woodhouse's toad but often occurs in other toads is one running from the postorbital crest to the parotoid gland and known as the preparotoid crest. The parotoid glands are usually in contact with the postorbital crests in Woodhouse's toad.

The toes are about one-third webbed, the webs being fleshy. There are two tubercles on the sole of the foot known as metatarsal tu- 
bercles. The inner of these is large and has a hormy black edge which is used in digging when the toad burrows into the ground. The other metatarsal tubercle is small.

The toad, like the frog, has four fingers on each hand and five toes on each foot. The first finger is slightly longer than the second.

\section{Internal Structure}

The internal organs of the toad are similar to those of the frog, and the previous description of these is referred to. Only striking differences will be pointed out. The word "frog" used subsequently refers to bullfrogs or leopard frogs.

\section{Respiratory and Digestive Organs}

Since the toad is terrestrial and has a thicker epidermis than the frog, it needs to depend to a larger extent on its lungs; these are large and well vascularized, being more spongy than those of the bullfrog or leopard frog.

The mouth is large and toothless, lacking the maxillary and vomerine teeth of the frog. Two openings, one on each side of the tongue, are apertures to the single vocal sac. The tongue of the toad is not notched behind like that of the frog. It is thicker and rounded with more of its posterior end free. The surface of the tongue is sticky and holds the captured insect as it is pulled back into the mouth. The liver is two lobed, in contrast to the threelobed liver of the frog.

\section{Urinogenital Organs}

The mesonephric ducts from the kidneys, which in the frog opened separately into the cloaca, unite in the toad and open into it in a single duct. The urinary bladder in the toad is large and may function also as a reservoir for water to prevent the animal's drying out. It is held in place by sheets of peritoneum and has a sphincter muscle at its mouth which permits its contents to be emptied rapidly. This discharge may lighten the toad and make it easier to escape from its enemies.

The testes of the toad are elongated and extend along a good portion of the length of the kidney. At their anterior ends, between them and the fat bodies, occurs an irregularly shaped granular 
body, Bidder's organ, which is thought to represent a rudimentary ovary. Some experiments on the toad in which the testes were removed have indicated that this body may develop into a functional ovary. In male toads there also may be found alongside the mesonephric duct a coiled tube which is the remains of a rudimentary oviduct that is nonfunctional. This rudimentary structure is not found in the male bullfrog, although it is encountered in the male leopard frog.

\section{Blood Vascular System}

For studying the blood vascular system, as well as some other systems, the toad is quite superior to the leopard frog. The vessels are of larger caliber, and the broad interior of the body makes the dissection more easily examined. The bullfrog, of course, is superior to either of these.

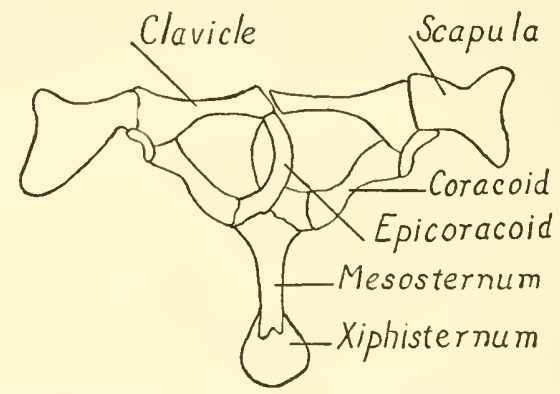

Fig. 293.-Diagram of ventral view of the arciferal, pectoral girdle of toad. (Modified from Kellog, Mex. Tailless Amphibia, U. S. National Museum Bulletin No. 160.)

Arterial System.-The subclavian artery is larger in proportion to the size of the animal than in the bullfrog. It sends large branches to muscles of the pectoral girdle, forelimb, and to the upper portion of the bulky side muscles. Two or three conspicuous branches of the vertebral artery run diagonally across the dorsal wall of the body cavity to the lateral muscles of the body which they enter. One large femoral branch, instead of two small ones, as in the bullfrog, is given off from the sciatic artery in the upper leg and runs ventrally, branching into the surrounding muscles.

Venous System.-The parietal branches of the ventral abdominal vein are relatively larger in the toad and may extend horizontally across the ventral body wall to the large side muscles. The dorso- 
lumbar vein is often quite large, with branches running the entire length of the body cavity and others coming from the lateral muscles.

\section{Skeleton and Muscles}

The pectoral girdle of the toad is quite different from that of the bullfrog and leopard frog. In the latter specimens, the two halves of the girdle grow together in the midventral line, separated by a cartilage, and the chest is not expansible. This type of pectoral girdle is known as firmisternal. In the toad, however, the epicoracoid cartilages separating the two halves of the girdle overlap in front, and the chest is expansible. This type of pectoral girdle is known as arciferal (Fig. 293).

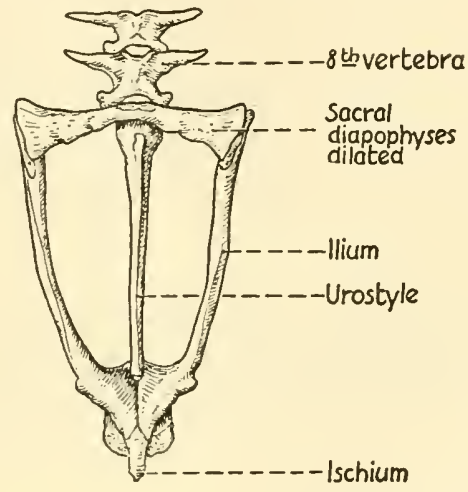

Fig. 294.-Pelvic girdle and urostyle of the common toad, Bufo americanus.

As in the bullfrog, the toad has nine typical vertebrae and a tenth which is included in the urostyle. Like the frog's, the vertebrae are procoelous. Unlike that of the frog, however, the single sacral vertebra which precedes the urostyle has its transverse processes (sacral diapophyses) dilated or expanded. In the bullfrog they were more circular.

The muscles of the legs, pectoral girdle, and body wall are large and thick. In preserved specimens their origins are clearly outlined, and they are more easily separated one from another in dissection than those of the leopard frog.

\section{Nervous System and Sense Organs}

The large calcareous bodies around the spinal ganglia, seen in the bullfrog, are not evident in the toad. While both bullfrogs and 
toads have poor vision, due to the lack of accommodation in the eye, the toad appears to see better. Toads also retain sound impulses longer than do frogs. Sound is of greater importance to the toad, for in his terrestrial hunting ground he is dependent to a large extent on sound in locating insect food.

\section{Embryology}

The eggs pass continuously through the oviduct with the result that they are laid in long strings or tubes of albuminous jelly. The egg mass is laid underwater, and the toad moves around during the egg laying process with the result that the strings may be considerably tangled around submerged vegetation. Shallow creeks provide a favorite breeding ground.

The length of time taken for the eggs to develop and hatch depends upon the temperature. They usually hatch in from two to four days. The tadpoles may transform into young toads in from 30 to 60 days after hatching. They measure $2 / 5$ to $3 / 5$ of an inch at this time.

Toad tadpoles are black. Usually toad tadpoles can be distinguished from frog tadpoles by the position of the anus. The anus in toad tadpoles is median, while in frog tadpoles it is somewhat on the right side. The spiracle of toad tadpoles is very small and is on the left side. 


\section{CHAPTER XXIX}

\section{REPTILIA}

(By Leo T. Murray and James E. Blaylock)

Class Reptitia includes among living forms, turtles, snakes, lizards, alligators, and similar animals. These are the only living vertebrates which are cold blooded, breathe by lungs, and have a single median occipital condyle. A more complete list of distinguishing characteristies has been given by Gadow as follows:

" 1 . The vertebrae are gastrocentrous.

"2. The skull articulates with the atlas by one condyle, which is formed mainly by the basioccipital.

" 3 . The mandible consists of many pieces and articulates with the cranium through the quadrate bones.

"4. There is an auditory columellar apparatus fitting into the fenestra ovalis.

“" 5 . The limbs are of the tetrapodous, pentadactyl type.

"6. There is an intracranial hypoglossal nerve.

"7. The ribs form a true sternum.

"8. The iliosacral connection is postacetabular.

" 9 . The skin is covered (a) with scales, but (b) neither with feathers nor with hairs; and there is a great paucity of glands.

"10. Reptiles are poikilothermos (cold blooded).

"11. The red blood corpuscles are nucleated, biconvex, and oval.

"12. The heart is divided into two atria and an imperfectly divided ventricle. It has no conus, but semilunar valves exist at the base of the tripartite aortic trunk.

"13. The right and left aortic arches are complete and remain functional.

"14. Respiration is effected by lungs; functional gills are entirely absent, even during embryonic life.

"15. Lateral sense organs are absent.

"16. The metanephric kidneys have no nephrostomes. Each kidney has one separate ureter.

"17. There is always a typical eloaca. 
"18. The eggs are meroblastic.

"19. Fertilization is internal, and is effected, with the single exception of Sphonodon, by means of malc copulatory organs.

"20. An amnion and an allantois are formed during development.

"Numbers 1, 2, 6, 7, 8, 14, 16, 18, and 20 separate the Reptiles from the Amphibia. Numbers 9 (b), 10, 12, and 13 separate them from the Birds and Mammals. Numbers 3, 8, and 11 separate them from the Mammals."

The majority of living forms are covered by scales. The turtles have epidermal scutes as an external covering; and the crocodiles have a tough skin with osseous plates in it. Many living reptiles are capable of very rapid movement; and the Pterosauria, an extinct order, had wings. Only one lizard and a relatively small number of snakes are poisonous. It is thought that the poison gland is a recent development among reptiles.

At present herpetologists place all known reptiles into nineteen groups or orders. Only four of these orders have living representatives. This indicates that Reptilia is an old class of vertebrates which is now decadent.

\section{FOSSIL REPTILES}

A study of the fossils of reptiles reveals that during the Triassic, Jurassic, and Early Cretaceous geological periods reptiles were the dominant animals on earth. They occupied and dominated all types of habitats then much as mammals do now. Many of these ancient reptiles were no larger than the common lizards of today, but others were the largest vertebrates that have ever lived. Brontosaurus, "the thunder lizard," was nearly seventy feet long and weighed approximately forty tons. This enormous reptile lived in the shallow waters of ponds and bays and fed on the plants that grew in the mud along the margins of the water. Tyrannosaurus, "the king lizard," reached a length of forty-seven feet and a weight greater than that of any elcphant. Since it was cntirely carnivorous in its feeding habits, it is easily the most terrible animal that has ever lived on earth. Many variations in form and size have bridged the gap between the largest and the smallest. The five or six known families of flying reptiles varied in expanse of wings from a few feet to as much as twenty-five feet. As a group, they dominated the air for several million years. A few small wormlike burrowing 
lizards less than two feet in length are known from the strata deposited in the Oligocene time in North America. Though reptiles had become completely adapted to living on land, many of them returned to the water. Most living turtles spend the greater part of their lives in water but must return to land to deposit their eggs. In the heyday of reptiles there were many other aquatic and marine forms showing numerous and diverse adaptations to living in a liquid medium. The ichthyosaurs (fish lizards) were the most perfectly adapted to aquatic life, being very fishlike in form.

\section{CLASSIFICATION OF LIVING REPTILES}

Many different plans have been proposed for grouping the reptiles. All of these plans have attempted to express the relationships of the various groups to each other; and the relations of representatives of a group to other members of the same group. The following arrangement is adapted from Williston, and includes only those groups having living representatives.

\section{Class-Reptilia}

Subclass-Anapsida. Temporal region of skull not perforated.

Order-Testudinata. A single coracoid; ten dorsal vertebrae, their ribs expanded to meet on the dorsum or a dermal layer of bony plates. Turtles.

Suborder-Pleurodira. Neck retracted laterally; pelvis united with the plastron.

No representatives of this suborder are found in North America.

Suborder-Cryptodira. Neck retracted rertically; pelvis not united witl the plastron; carapace with marginal plates.

The majority of the living turtles of North America belong to this suborder.

Suborder-Trionychoidea. Neck retracted vertically; carapace with no marginal plates.

The "soft-shelled" turtles make up this suborder.

Subclass-Parapsida. Temporal region of skull with one opening.

Order-Squamata. Quadrate freely articulated proximally (streptostylic) or secondarily fixed. Lizards and Snakes.

Suborder-Lacertilia (Sauria). Parietals never united to basisphenoid by descending plates; the brain case more or less membranous anteriorly.

The one hundred and seventy-four kinds of lizards known from the United States and Lower California belong to this suborder. 
Suborder-Ophidia (Serpentes). The braincase enclosed by descending plates from parietals and frontals; no external limbs present. This suborder includes all snakes.

Subclass-Diapsida. Temporal region of the skull with two openings.

Order-Rhynchocephalia. Amphicoelous vertebrae, premaxillae with a decurved beak; pineal eye present.

Suborder-Sphenodontia. Has the characters of the order. Sphenodon, the single living genus of this order is famous as being one of the primitive living reptiles. It is found in New Zealand and on some smaller islands of that region.

Order-Crocodilia. Procoelous vertebrae; premaxillae never decurved; no pineal eye present. Crocodiles and Alligators.

Suborder-Eusuchia. Has the characteristics of the order.

The living crocodiles and alligators belong to this suborder.

\section{Order Testudinata (Chelonia)}

Suborder Pleurodira.-Turtles of this group are found in South America, Africa, and Australia. They are known as "side neck" turtles because they do not retract the head and neck under the carapace but lay it along the periphery of the shell. Some members of the group have a pair of bones, the mesoplastra, in the plastron that is not present in other living turtles, though it was common among forms now known only as fossils. The bones of the pelvic girdle are sutured to the plastron as well as to the sacral vertebrae. This is also a characteristic unique among living forms but more common in extinct species. Hence, it is thought that members of this suborder are more primitive in structure than the members of either of the other two suborders having living representatives.

Suborder Cryptodira.-There are sixty-one species of turtles in North America north of Mexico or in the oceans that bound the shores of this region. Fifty-seven of this number belong to this suborder. Six families are represented.

Family Kinosternidae.-This family includes the turtles commonly known as the "mud turtles," "stinkpots," or "musk turtles." They are all small, brown or black turtles, sometimes with white or yellow lines on the head and neck. Many turtles are frequently mistaken for members of this family that belong to some other family. In California, where no kinosternid turtle is found, a member of the family Emydidae is called the "mud turtle." All kinosternids possess musk glands that open through pores on 
the margins of the carapace just anterior and just posterior to the bridge. If a dry musk turtle be disturbed, a drop of yellow liquia ean often be seen to appear at each one of the openings of the ducts from the four musk glands. This liquid gives off a disagreeable odlor. If it touches hands or elothing, it is very difficult to remove.

Family Chelydridae.-The common suapping turtles and the alligator snappers are the living members of this family. Both turtles have rows of raised prominences along each side of the carapace; and a row of large bony ossicles along the middorsal region of the tail. The head is large and formidable. The alligator snapper has a pair of wormlike appendages in the mouth, which it is said are used to entice fish within reach of its powerful jaws. Both turtles are a plain brown color dorsally and dirty white to black ventrally. The alligator snapper may attain a weight of one hundred and fifty pounds, while the common snapper will seldom exceed forty pounds. Large specimens of either turtle can easily amputate a finger or possibly a hand. Both kinds of snapping turtles possess scent glands very similar to those found in the kinosternids. This family is an excellent example of discontinuous distribution. It was long thought that it was confined to the Western Hemisphere but a genus is also found in New Guinea.

Family Emydidae.-Many turtles, diverse in habits and appearance, belong in this large family. All of the "hard-shelled" pond and river turtles commonly called "sliders," the painted turtles, the red-bellied turtles, the box turtles and many others fall into this group. While there is great variation among members of the family, they all exhibit certain tendencies as well as fundamental structural similarities. Many species are brightly colored. The painted turtles have bright red colors patterned with dark green and black. Different speeies of the genus Pseudemys possess various types of pretty colorations. The wood turtle has a somber carapace, a bright plastron with pleasing black spots, and a rich reddish-orange skin on the legs and neck. The males of many species of this family have long straight claws on the toes of the fore feet. Members of this family are found on all the continents except Australia. Emys blandingii, found in the Great Lakes region, is very similar to Emys orbicularis of Europe. 
Family Testudinidae.-Only three species of turtles found in the United States belong to this family; namely, the gopher tortoise, Berlandier's tortoise, and Agassiz's tortoise. Members of this family are numerous in Africa, on the Galapagos Islands, and in other widely separated localities. How these three species of turtles come to be in North America is a most interesting problem in animal distribution. All three species found in this country are dark brown or black on the carapace, often lighter ventrally. Young individuals usually show a light area in the center of each dorsal scute. Since they are all dry-land turtles, they lack the streamlined form of water dwellers. The most distinct characteristic of our species is a narrow extension of the plastron into a gular process.

Family Cheloniidae.-This family includes the green turtles, the shell turtles, and the loggerhead turtles. All are marine animals, and show modifications for an aquatic existence in the form of the body and in the modification of feet into flippers. Many members of this family may weigh five hundred pounds, but most specimens seen in the markets weigh much less.

Family Dermochelidae.-This family contains but one genus with two species. They are commonly known as the leatherback turtles, the trunk turtles, or the harp turtles. Unlike most turtles, they lack the covering of horny scutes, being covered instead with a leathery integument. These are the largest of living turtles. Large individuals may weigh as much as one thousand pounds.

Suborder Trionychoidea.-Members of this suborder are found in North America, Africa, Asia, and New Guinea. They are among the most aquatic of all land and fresh-water forms. The only occasion on which they leave the water is to deposit eggs.

Family Trionychidae.-This is the only family of the suborder having representatives in North America. It is represented by five species and one subspecies, all in the genus Amyda. All are "softshelled" turtles covered with a soft rubberlike skin instead of the horny scutes present on most turtles. The color on the dorsal side is olive brown, while the ventral side is white. Any of these species will bite viciously when angered and can inflict painful wounds. It was probably one of these turtles that gave rise to the belief that a turtle would not loose its hold until it heard thunder. They have a habit of retaining their grip on a victim very tenaciously. 


\section{Order Squamata}

Suborder Lacertilia (Sauria) (The Lizards).-This group contains more different kinds of living animals than any other suborder among the reptiles. There are more than 2,500 living species known on earth. Of this number, about 175 species are found in North America, north of Mexico. Representatives of nine families are found among them.

Family Gelikonidae.-There are about fifty genera, containing some 300 species, in this family. They are found around the world in the tropical and semitropical regions. Seven species are known from the United States and neighboring regions.

All of our species are small, seldom attaining a length of six inches; but some tropical forms may be over a foot in length. The colors vary considerably but are often bright, as is usual in nocturnal animals. The scales of the skin are very minute. This gives the geckos a soft, smooth, appearance unlike that of any of the other lizards. The eyes usually have vertical pupils and are without lids, though they are covered by a transparent, cutaneous membrane. As in some other lizards, there is nothing obstructing the auditory passage through the head. It is possible to see through this passage. Members of this family are among the few lizards that can make a sound other than hissing. Their characteristic call sounds like the word "gecko."

In a great many species the toes are flattened on the end to form adhesive discs. These enable a gecko to walk across the ceiling of a room with ease. In most species the tail is short and thick.

Geckos sleep during the day but come forth at nightfall in search of their insect prey, which they capture by means of their short sticky tongues.

Family Iguanidae.-This family of lizards has more representatives in the United States than any other single family. Of approximately 175 species of lizards known from this country, 90 species and 19 subspecies belong to this family. Representatives are found in all parts of the United States except the most northern portion, as well as throughout Central and South America, and the West Indies. Two genera are found in Madagascar and one in the Fiji Islands. As might be expected in so large a group, great varia- 
tion in size, form, and coloration occurs. The chameleons (Anolis) change from various shades of brown to light green in response to changes in the intensity of the light. Members of the Central American genus Basiliscus are remarkable for erectile middorsal crests. The horned lizards (Phrynosoma) bear conspicuous osseous spines or "horns" on the postelior and lateral borders of the head. All the members of the family, however, have certain structural characteristics in common; such as, fleshy tongue, and eyes with round pupils and well-developed lids. Femoral pores are usually present on the males. Most of the species found in the United States lay eggs, though some species are known to be ovoviviparous. Various types of habitats have been adopted by different iguanid lizards. The chameleons and many species of the genus Sceloporus are essentially arboreal, while the horned lizards (Phrynosoma) are strictly terrestrial.

Crotaphytus collaris, the collared lizard, is a rather large lizard with a long tail and a heavy body. It is brightly colored and has a yellow collar bordered with black. Its distribution is in the Southwest, from the Plains westward.

Certain tropical species are semiaquatic and one species is semimarine. The majority of species are insectivorous, though three genera are herbivorous.

Family Anguidae (Alligator lizards, "glass snake," joint snake, etc.).- This interesting group of lizards is represented in the United States by 10 species. Approximately 40 other species are found in other regions of the world. Most of these other species are native to the tropical regions of the Western Hemisphere, though some are found in Europe, Asia, and Africa. A reduction in the size and strength of the limbs is common in this family. Many species, such as our "glass snake," are entirely legless. Other common characteristics are a fold in the skin where the ventral plates join the body wall; a long, brittle tail ; eye with a lid; emarginate, protractile tongue; and solid teeth. Many of our species have large auditory openings connected by an unobstructed passage. Our largest species reaches a maximum length of approximately one foot, while a form found on the Balkan peninsula may be three feet long. Our alligator lizards (Gerrhonotus) are ovoviviparous while the "glass snake" (Ophisaurus) lays eggs. All members of this family feed on animal food, such as insects, snails, and small mammals. 
Family Anniellidae (blindworm, "worm snake," worm lizard).This family consists of one genus and two species found in southern California. These small, legless, wormlike lizards are burrowing in habit. The ears are concealed and the eyes are covered by translu-

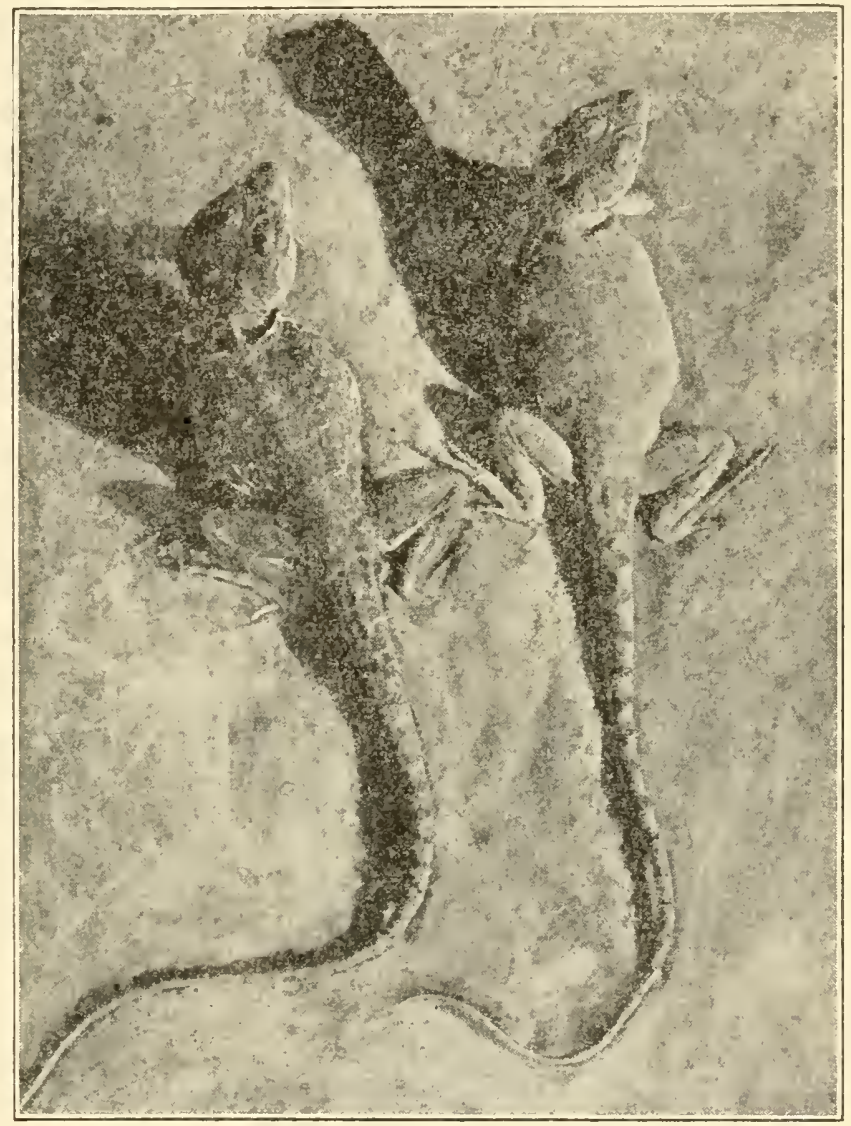

Fig. 295.-Collared llzards, Crotaphytus collaris, female and male. This is a beautiful lizard of the Southwest. (Courtesy of Ottys Sanders, Southwestern Biological Supply Company.)

cent skin and poorly functional. The tongue is protractile as in the members of the Anguidae.

Family Helodermatidae (Gila monsters, beaded lizards).-This family contains one genus and two species. One species, Heloderma suspectum, is found in Arizona, New Mexico, and northern Mexico. 
The other species, H. horridum, ranges through central and western Mexico to northern Central America. These lizards may reach a length of 2 feet though smaller ones are more commonly seen. The surface of the body is totally unlike that of any other lizard, being covered by beadlike ossicles or tubercles. The most interesting and distinctive structural characteristic of these lizards is their grooved teeth with ducts from poison glands opening at the base of the grooves. No other family of lizards in America is venomous. The color of $H$. suspectum is black marbled with pale pink, salmon, or flesh. The Mexican species is black with yellow or lemon spots or bars. The short thick tail becomes more slender when the animals fast. The natural food of the animals is not known. They take eggs readily in captivity and thrive on them. Reproduction is oviparous, the eggs being laid in warm, moist sand where they hatch in twenty-eight to thirty days.

Family Xantusiidae (night lizards).-This small family contains only three genera with a total of seven species. Five species, all of the genus Xantusiidae, are found in southern California, Lower California, and Arizona. One other genus is found in Central America; and a third in Cuba.

These lizards are seldom over six inches in length. The color changes from dark brown in subdued light to lighter hues in stronger light. The pupils are vertical, and the eyes are without lids. The tongue is only slightly extensible.

These little reptiles are strictly nocturnal, hiding by day under fallen Yucea plants or in crevices between boulders. So far as is known they are insectivorous in feeding habits. At least one species is known to be ovoviviparous.

Family Teiidae (striped lizards, race rumers, sand lizards).--This family contains 40 genera with more than a hundred species. Twentytwo species and subspecies, all belonging to the genus Cnemidophorus, are found in the warmer parts of the United States. All other members of the family are found in South America and the West Indies.

The species of Cnemidophorus are long slender, active lizards capable of surprising speed in running. The ground color is usually some shade of brown. Lines, bands, or spots of lighter color form various patterns on different species. The tongue is black, forked, and protractile. 
Our species are found in open, sunny, sandy places. If disturbed they skim over the ground with great rapidity, but if hard pressed they take refuge in burrows. Insects make up the bulk of their food. All the species lay thin-shelled eggs which are deposited in shallow excavations in the sand to be hatched by the heat of the sun.

Family Scincidae (skinks, or smooth lizards).-This is one of the largest families of lizards, being composed of over four hundred species arranged in thirty genera. Three genera containing sixteen species are found in the United States. They are more abundant both in number of kinds and in number of individuals in tropical regions, especially in tropical parts of the old world and in the Australian regions. South America has fewer skinks than any other region in the world.

All the skinks are relatively small lizards, the largest in this country seldom attaining a length of ten inches. The scales are smooth and usually shining. The color varies with age. The young are darker than the adults and color patterns of lines present on the young often disappear on adults. There is great variation in the development of the limbs. Most of our forms have one or both pairs of legs.

The skinks are diurnal, feeding by day and seeking a hiding place at night. Many old world kinds are burrowers in sand, but of all American forms only one Florida species is a burrower. The majority of species are to be found under bark, logs, stones and in other dark, cool places. Some kinds of skinks have been observed to guard the eggs by curling about them. Some old world species are ovoviviparous.

Family Amphisbaenidae (worm lizards).-This highly modified family is represented in the United States by one genus with one species in Florida and another genus with one species in southern California. Forty species are known from the American tropics and others from northern Africa and the Mediterranean region.

These remarkable lizards are all limbless except Bipes biporus, the two-footed lizard of Lower California, which has the anterior pair of limbs well developed. The skin is without scales and forms numerous rings about the body, suggesting an annelid worm in appearance. The eyes are absent or reduced. There is usually no external ear opening. 
Both of our species lead a subterranean existence, boring tunnels in which they move backward and forward with equal ease.

Suborder Ophidia (Serpentes) (Snakes).--No group of reptiles is of greater natural interest to man than the snakes. Superstitions and stories relating to snakes are as old as written language. Many religions and cults have used the serpent as a symbol of good or of evil. In many regions of the world today many of the most poisonous of snakes are venerated and protected by the natives. The snake dance of the Hopi Indians of our own country is a wellknown example of the symbolic use of snakes.

The ophidians are highly modified vertebrates. Their anatomical structure indicates that they have been derived rather recently, geologically speaking, from lizardlike ancestors. Some lizards are totally limbless, while some snakes of the family Boidae have vestiges of the posterior pair of limbs. In the structure of the jaws there is close similarity between some snakes and some lizards.

Upon the basis of structure and arrangement of teeth snakes have been arranged into the following four groups:

The Aglypha, or those with solid, ungrooved teeth. Our harmless snakes all have this type of dentition.

The Opisthoglypha, or those having the posterior maxillary teeth grooved. These snakes are venomous but seldom dangerous to man. The position of the venom conducting teeth makes it difficult for the snake to inflict a wound on man. The lyre snakes (Trimorphodon) of the Southwest, the black-headed snakes (Tantilla) of the Southern States, and a few other rare snakes belong to this group.

The Proteroglypha are those that have the anterior maxillary teeth grooved and often enlarged and elongated. Many of the most dangerous snakes in the world belong to this group. The coral snakes, cobras, and sea serpents have this type of dentition.

The Solenoglypha, or those having hollow, hinged fangs in the anterior part of the mouth. The rattlesnakes, copperheads, water moccasins and their relatives make up this group. They are all venomous and dangerous to man.

There are approximately 2,300 known species of snakes. Of this number, some 225 species are venomous; but 75 of these poisonous species are so small or rare that there are only 150 to 175 species that man need fear. 
In the United States there are 234 recognized species and subspecies of snakes. Of this number, 51 species and subspecies are venomous but 13 of these are too small or rare to be considered dangerous to man. Hence, there are 38 kinds in our country that we must avoid.

Family Leptotyphlopidae (worm snakes).-Three species belonging to this family are found in southwestern United States. In Mexico, Central America, Asia, and Africa, there are about thirty species. One of these, a Syrian species, is the smallest of all adult snakes. Those found in our own country are small, seldom attaining a length of more than a few inches. They are all plain flesh, or various shades of pale pinkish lavender in color. An iridescent, silvery sheen extends over all. The head is blunt and of the same diameter as the neck and body. The small eyes are covered by translucent scales. It is probable that they have very poor powers of vision. The tail is likewise blunt and very short. There is a vestige of a pelvis present in some members of the family.

All of the North American species are burrowers, making long tunnels in which they find insect larvae and worms. They seldom come to the surface except when forced out of their burrows by heavy rains.

Family Boidae (boas and pythons).-There are sixty to seventy species in this family, some of which are found in all tropical parts of the world. In the United States there are three species, all found in southern California or neighboring desert regions. Our species are all small, but the largest of living snakes are members of the family. There are authentic records of specimens 30 feet long and weighing approximately 300 pounds. There is usually some external evidence of vestigial limbs present. Though none are venomous, many species have elliptical pupils. All members of the family are constrictors in feeding habits, preferring warm-blooded animals as a rule. The females lay eggs and some species are known to coil about them until the young are hatched.

Family Colubridae.-This is the largest of all the families of snakes, containing 90 per cent of the living species. In the United States more than 100 species of snakes belong to this family. Members of the family range farther north and south of the equator than those of any other family of snakes. Being so numerous and widespread, it is not surprising that some species should have adopted every available habitat. Hence, the variety in size, form, 
and color is great. The arboreal species are slender and green. The terrestrial species are heavier in body and varied in color. The subterramean, the semiaquatic, and the aquatic forms all show adaptations to their environments. Most of our Coluberine snakes are nonvenomous, but some are mildly poisonous opisthoglyphs. A majority of the suakes in this family lay eggs but some bring forth the young alive.

Family Elapidae (corals, harlequins).-Twenty-nine genera with about 140 species make up this family. All except two genera are found in the Old World only. Africa, Asia, Malay Archipelago, and Australia have representatives of this family. In Australia there are only a few representatives of other families. In the United States there are two genera containing one species each. Together they cover most of the southern half of our country.

All the suakes in this family are deadly poisonous proteroglyph serpents. The cobras of Asia and Africa kill thousands of persons every year. This is due partly to superstitions and religious beliefs that protect these snakes in those regions. The venom of this family of snakes is largely neurotoxic in action; i.e., it acts on the nervous centers. Hence, it usually acts much more quickly than the slower hemolytic and hemotoxic venoms of the pit vipers. Some cobras may attain a length of ten to twelve feet. Men have been known to die in less than an hour after being bitten by such a snake. However, the venom of the coral suakes, the American representatives of this family, is more deadly per unit volume. The coral snakes are seldom more than two feet long and are not capable of injecting such large quantities of venom.

The coral snakes are beantiful little snakes marked with briliiant cross bands of red, yellow, and black. There are three harmless snakes found in the same parts of our country that have the same colors in their patterns. None of them, however, duplicates the sequence of the bands on the coral snakes. In these poisonous snakes the order of the colored bands is red, yellow, and black. In the harmless species the order is red, black, and yellow. The following jingle is a good device for remembering these color schemes:

Red and yellow

Kill a fellow.

Red and black

Venom lack. 
The small, conical head and slender, cylindrical body of the coral snakes fit them for their subterranean life. They seldom come to the surface during the day, but may be found at night crawling about in search of food. They eat other snakes and small lizards. These snakes lay eggs.

Family Hydrophidae.-Members of this family are marine relatives of the Elapidae. Only one species is found in the New World. It occurs off the west coast of Mexico and has been reported as very common in some localities at certain seasons. The females come into shallow coastal waters to give birth to their young. Here the young have some protection from their enemies and access to small fish suitable for food. Adults of old World species have been sighted one thousand miles from land. All species have the tail flattened for swimming.

Family Crotalidae (pit vipers).-This family is composed of six genera which contain about eighty species. Members of the family are found over all the temperate and tropical parts of the Western Hemisphere. In the Old World they are found in India, China, and neighboring regions.

Three of the six genera in the family have representatives in the United States. In fact, all the dangerously poisonous snakes in this country, except the coral snakes, belong to this family. They are all solenoglyph snakes. There is a prominent pit on each side of the head between the eye and the nostril. The rattlesnakes (Crotalus and Sistrurus) bear rattles on the end of the tail. All members of the family liave elliptical pupils.

The poison glands and highly developed fangs enable these snakes to capture their food with a minimum of effort on their part. 'The venom is injected so quickly and so unexpectedly that the prey has little chance to avoid it. Most small animals die very soon after being bitten. The reptile then swallows the carcass at its own pleasure. Rattlesnakes prefer mammals. In regions where these snakes abound wild rats and mice are rare. Water moccasins take frogs and other cold-blooded aquatic animals for food. The copperhead appears to enjoy both warm-blooded and cold-blooded animals.

All members of this family give birth to living young or lay thinshelled eggs which hatch in a very short time, usually less than an hour. 


\section{ORDER RHINCOCEPHALIA}

Suborder Sphenodontia (Sphenodon, Tuatara).--The only living representative of this order is Sphenodon punctatum, a lizardlike animal found on a few small islands off the coast of New Zealand.

It is often called a "living fossil" because many of its anatomical characters are found in no other living reptile. Some of these characters are old, even in relation to many extinct reptiles. The entire brain is said to be smaller than one of its eyes. Unlike all other living reptiles, it has no external copulatory organ. There are ten separate carpal bones present. This is a primitive number. Many other skeletal features indicate a close relation to reptiles of other geological periods.

The adults usually attain a length of about twenty inches. They are dull yellowish or olive brown in color. A middorsal row of spinelike scales extends from the occipital region to the end of the tail. There are other rows of smaller excrescences along the sides. One of the most interesting features of the animal is its pineal eye. This "third eye" is located in the center of the head between the eyes. It is surrounded by a rosette of small scales and covered by a translucent plate. The nerve from this eye is well developed and passes to the brain through a foramen in the cranium. There are a retina and a comea in the structure of this organ, but the extent of its function as an eye is unknown.

The habits of the animal are as unique and interesting as its structure. It lives along the shore in burrows with a small petrel, a shore bird of that region. It is said that the reptile and the bird have special sides in the enlarged chamber at the end of the burrow and neither trespasses on the other's space. The food of these animals consists of insects, spiders, and crustaceans. In captivity they have been known to thrive on a diet of earthworms. They are nocturnal, hiding by day and hunting along the beaches at night. Reproduction is oviparous, about ten eggs being a usual complement.

\section{Order Crocodilia}

Suborder Eusuchia (crocodiles and alligators).--This order is represented by about twenty living species. They are found along the coastal plains in our Southern States, southward through Mexico, Central America, and in the warmer regions in South America. In 
the Old World they occur in tropical Africa, Southern Asia, Java, Sumatra, and Northern Australia.

In the United States two genera with one species each are found. One, Alligator mississippiensis, is found in all our coastal states from the Rio Grande to the Carolinas. The other, Crocodilus acutus, is found in the southern tip of Florida, Mexico, Central and South America.

The general appearance of alligators is well known to everyone. The animals are not corered by scales but are protected by rows of dermal ossicles in the skin. Formerly a crocodile or an alligator might be found that was as long as thirty feet, but it is doubtful whether such an animal can be found today.

The females lay eggs in a nest built of sticks and decaying vegetation. The heat generated by decomposing vegetable matter aids in incubating the eggs. It was formerly thought that the growth of alligators was very slow but specimens in captivity have been brought to a length of five and one-half feet in five years.

\section{THE HORNED LIZARD}

The horned lizard is a convenient reptile to study since one or more species is found locally throughout southwestern United States. It is usually found in abundance and is relatively easy to capture. The "horns" on the head are unique among living reptiles. The general plan of structure is sufficiently generalized to illustrate most of the characteristics typical of reptiles.

\section{Habits and Behavior}

These lizards are diurnal animals, feeding by day and at night burrowing into the ground until only their head spines can be seen. Their food consists of small beetles, flies, smooth caterpillars, moths, ants, and other small insects. The animals are solitary, not being found in groups in deus. Each one burrows underground to hibernate during the cooler season of the year. After mating, a female of an oviparous species digs a cup-shaped hole and deposits twenty to twenty-five eggs in it, covering the eggs with several layers of earth. Females of the ovoviviparous species bring forth the young alive. 


\section{External Structure}

The horned lizards are typical iguanid lizards, with broad, flat bodies covered with horny, strongly keeled scales and spines. There is at least one row of spines in a marginal fringe at the lateral edges of the belly. Erect, scattered spines of various shapes and sizes are apparent on the animal's back. The body is devoid of a dorsal crest but is covered with small granular scales and with four rows of enlarged, sharply pointed spines. The rentral parts are covered with small, smooth, light-colored, rectangular scales.

The body of the animal is divided into three well-defined regions: head, neck, and trunk. The trunk bears two pair of well-developed limbs and tapers posteriorly to a broad, short tail.

The head is short and the muzzle descends steeply in profile, but is not separated from the front by a conspicuous angle. It is covered with small scales, and is bordered posteriorly by a row of osseous spines. The most anterior structures are paired, small nostrils. These are small rounded apertures situated one on each side of the snout, a short distance from its anterior end. Just posterior to the nostrils are the eyes. They are situated one on each side of the head about midway between the nostrils and the tympanic area. Each eye is guarded by a short, thick upper eyelid and a thimner lower lid. The lower lid covers most of the eye when that organ is closed. Within the eyelids and attached to the anterior comer of the eye is a thin, transparent nictitating membrane which closes backward over the eye. Above each orbital socket is a bony structure which forms a posterior superciliary angle by being produced into a short postorbital horn. Behind the eye and a little posterior to the mouth is the auditory aperture. A thin tympanic membrane is stretehed over it. Above the auditory aperture and between it and the eye are three temporal horns. They form the postlateral border of the head. The occipital horns mark the dorsoposterior boundary of the head. The gape of the large mouth begins at the snout and extends postcriorly to within a short distance of the auditory aperture. The lower labial scales vary from the small rounded anterior ones to the prominent, acute posterior spinelike scales.

The cervical region is thick and stout but well differentiated. A transverse gular fold is present on the ventral surface of the neck. 
A row of enlarged gular scales parallel the gular fold and a small, single spine may be found on each side at the posterior gular border. Two longitudinal folds are present on each side of the cervical region. These folds descend forward and obliquely downward to the plane of the gular fold.

The trunk is depressed and is broadly fusiform in shape. It is flattened ventrally and tapers posteriorly toward the pelvis.

The anterior limbs spring one from each side of the body near the anterior end of the trunk. They are divided into three divisions: proximal or brachium, middle or antebrachium, and distal or manus. The distal division is terminated by five clawed digits. The first digit is the shortest. It is designated as the thumb or pollex.

The posterior limbs arise one from each side of the body near the posterior end of the trunk. They are also divided into three regions: proximal or thigh, middle or shank, and distal or foot. The foot, like the hand, ends with five clawed digits. The first digit, or hallux, is the shortest. The ventral surface of the thigh bears twelve to fifteen femoral pores, whose function is obscure. They are present in adult males only.

Between the thighs on the ventral aspect of the body is a slitlike, transverse vent, or anus. It is the common outlet for the digestive and urinogenital systems. The short tail is broad at the base but tapers quickly toward the distal end. In males two genital swellings lie one on each side of the ventral surface of the broadened proximal region of the tail just posterior to the vent.

Like most reptiles, these lizards shed their thin epidermal covering periodically. The variable color pattern is brighter for a time after this skin is cast.

\section{Digestive System}

The alimentary canal is composed of the following organs in order: mouth, esophagus, stomach, small intestine, large intestine, rectum, cloaca, anus. Several accessory organs and structures are teeth, tongue, pancreas, and liver.

The upper and lower jaws form the anterior boundary of the aperture of the mouth, and are each provided with a single row of small, faintly tricuspid teeth. These teeth are not adapted for mastication but for seizing and holding prey. The posterior nares are apertures situated a little behind the end of the snout and separated from one 
another by a vertical partition supported by the vomerine bones of the skull. The Eustachian pits are paired diverticula from the dorsal wall of the pharynx continuous with the tympanic cavities, which are closed externally by the tympanic membranes. The mouth cavity ends posteriorly as the pharyngeal cavity which is produced posteriorly into the horizontal slit which leads to the esophagus. The glottis, or aperture of the trachea, is located centrally on the summit of the laryngeal chamber. The tongue is a fleshy organ lying on the floor of the mouth. It is furnished with many tactile and gustatory cells, and is usually covered with a sticky secretion which assists it in catching and holding prey. It can be extended from the mouth and is used in capturing insects.

The tubular esophagus opens into the mouth cavity by way of a horizontal slit posterior to the pharyngeal cavity. It is deeply pigmented anteriorly and lies dorsal to the trachea. The walls are thick and muscular. It opens posteriorly into the cardiac portion of the stomach.

The stomach is a cylindrical, muscular organ with relatively thick walls. When empty it is but little greater in diameter than the esophagus or the intestine. Since it lies chiefly on the left side of the body, it is largely concealed by the left lobe of the liver. It is larger at the anterior or cardiac end and constricted at the posterior or pyloric end. The pyloric valve is an annular ridge of muscular tissue which narrows or closes the aperture between the stomach and the small intestine. The inner surface of the walls of the stomach is thrown into longitudinal folds or rugae. Distributed over the entire inner surface are the openings of minute ducts from the numerous gastric glands which secrete the gastric juice. This digestive fluid normally begins the digestion of proteins in the food.

The intestine forms several coils in the posterior third of the coelom, and is held in place by the mesenteries. As is commonly the case in insectivorous or carnivorous animals the intestine is relatively short. The anterior part of the small intestine is the duodenum. The com. mon bile duct from the liver and the pancreatic duct from the pancreas join this section of the intestine. There is no definite division between the duodenum and the ileum, the succeeding portion of the intestine. After several turns the ileum passes into the large intestine on the right side of the body. An annular ridge of tissue, the ileo- 
caecal valve, narrows the aperture between the ileum and the large intestine. The large intestine can be traced from the right side of the body downward and medially to a point where it passes out of sight beneath the pelvis to enter the cloaca. There is a very small

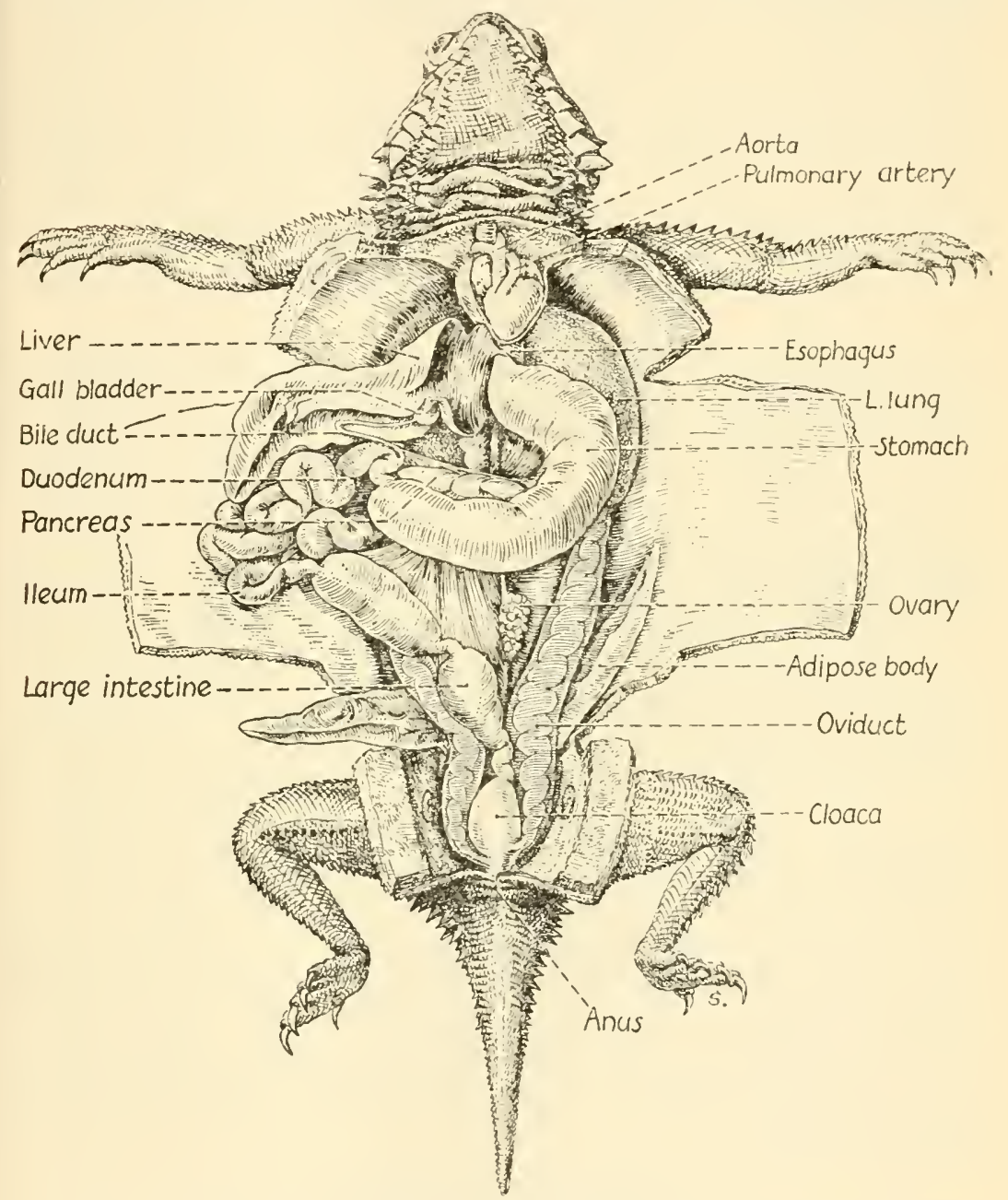

Fig. 296.-Dissection of a horned lizard to show internal organs, ventral view.

blind pouch or cecum, if any, at the position of the ileocaecal valve, where the ileum joins the large intestine. Posteriorly the large intestine becomes the rectum hefore entering the cloaca which is di- 
vided into an anterior and posterior chamber. The anus is the posterior end of the alimentary canal.

The pancreas is an elongated branched body of glandular material located within the first loop of the intestine between the stomach and the duodenum. It secretes an alkaline digestive fluid which is emptied into the duodenum.

The liver is a large, bilobed organ divided imperfectly into right and left lobes. It secretes bile, an alkaline digestive fluid. This fluid is stored in the gall bladder until food enters the duodenum. It then passes into the intestine through the common bile duct.

\section{Respiratory System}

A basic feature that distinguishes reptiles and other higher vertebrates from amphibians and fish is that they do not breathe by gills during development. 'The respiratory system of all lizards is typical of air-breathing vertebrates. A tubular trachea conducts air from the mouth to the highly vascularized lungs. This tube is prevented from collapsing by rings of cartilage. A gaseous exchange is effected between inhaled air and the blood. The oxygen is absorbed and carbon dioxide exhaled. Breathing is effected by the motion of the ribs. Air passes through the nostrils, or external nares, into the olfactory chamber. From there it passes through the internal or posterior nares into the mouth cavity to be forced from there through slitlike glottis into the chamber of the larynx. The larynx continues posteriorly as the trachea, which lies in the midventral line of the throat and extends to the lungs. The posterior end of the trachea bifurcates to form two smaller tubes, the right and left bronchi. Each bronchus connects with a corresponding lung a short distance from the apex of the lung. Within the lung each bronchus divides into secondary bronchi and these in turn give off tertiary branches. The smallest tubules, bronchioles, carry the air into air spaces or alveoli. It is in the alveoli that an exchange of gases takes place.

\section{The Circulatory System}

The circulatory system of reptiles shows distinct advances over the circulatory system of amphibians. Since respiration is carried on exclusively by the lungs, changes which are in keeping with this 
modification are apparent in the pulmonary circulation. The system consists of a heart, arteries, capillaries, and veins, forming a continuous or closed set of vessels throughout the body.

The heart lies at the extreme anterior end of the body cavity and is enclosed in a thin-walled sac, the pericardium. The heart consists of a dorsal sinus venosus, a right and a left auricle, located anteriorly, and a posterior ventricle. The auricles are thin-walled chambers which communicate with the ventricle by means of the auriculoventricular aperture. This aperture is guarded by an auriculoventricular valve which consists of two flaps. The thin vertical interauricular septum separates the auricles. The ventricle is a thick, cone-shaped, muscular chamber. The base is directed anteriorly and is connected to the auricles at the auriculoventricular aperture. The thick, spongy walls of the ventricle leave only a small cavity within its chamber just below the auriculoventricular aperture. It is completely divided into two chambers by the perforated interventricular septum only when in a state of contraction.

Three arterial trunks arise from the rentral surface of the ventricle. The pulmonary artery arises from the right portion of the ventricle. Its aperture is separated from that of the right aorta by means of a muscular partition.

The pulmonary artery is visible for only a short distance. It originates from the right sicle of the ventricle, passes anteriorly, then turns dorsal to the heart. It soon divides into right and left trunks supplying the respective lungs.

The right aortic arch arises from the left half of the ventricle and at first is dorsal to the other two arterial trunks. Soon the pulmonary artery assumes the dorsal position and the right aortic arch becomes ventral and passes to the right and anteriorly between the auricles. At the anterior end of the heart it forks to form a Y. The branch passing to the left is the left common carotid artery. The right branch forks again. The anterior branch is the right common carotid artery, and the other is the continuation of the right aortic arch, which gives off the two subclavian arteries.

The left aortic arch is the most ventral of the three arterial trunks and it also arises from the left side of the ventricle. The right aortic arch is in close relation to the left arch. The left aortic arch then passes laterally and diagonally across the pulmonary artery and the 
right aortic arch, and continues eephalad on the left side of the interauricular space.

The aortic arches may be traced laterally and are seen to bend around the esophagus and unite with one another on the dorsal side of the esophagus, ventral to the vertebral column, to form the median dorsal aorta. An esophageal artery may branch from the left aorta.

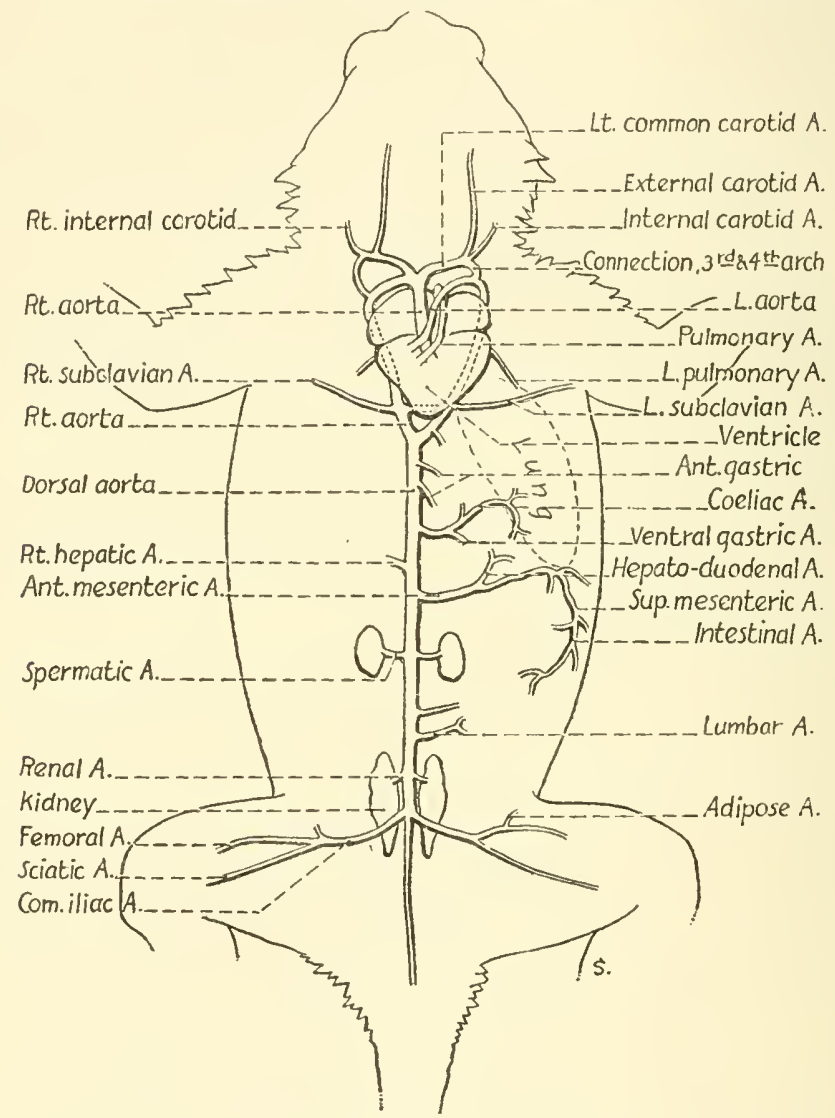

Fig. 297.-Heart and arterial system of horned lizard, ventral view.

The right and left common carotid arteries arise from the right aortic arch at the point where it leaves the left aortic arch. The first branch of each is the external carotid which supplies the lower jaw. Each of the common carotids next gives off an internal carotid artery which supplies the right and left sides of the head. Now the common 
carotids pass laterally and parallel with the corresponding aortic arches. At the distal end of the common carotids each anastomoses with the corresponding aortic arch.

The right and left subclavian arteries arise from the right aortic arch just anterior to its junction with the left aortic arch to form the dorsal aorta. Each passes laterally; one to each of the forelimbs.

The dorsal aorta is a median, unpaired, longitudinal artery lying in the mid-dorsal line ventral to the vertebral column. A little further caudal small gastric arteries pass to the stomach. Near the posterior end of the stomach the large coeliac artery is given off. This soon divides into two trunks. The anterior coeliac artery supplies the digestive glands, stomach, and duodenum. The posterior branch, the ventral gastric supplies the ventral surface of the stomach. The anterior mesenteric is the next unpaired branch of the aorta and it soon divides into the hepatoduodenal to the left side of the liver and the duodenum, and the superior mesenteric to the ileum. The right hepatic artery springs from the dorsal aorta and passes to the right lobe of the liver. The inferior mesenteric arteries include three small vessels arising from the dorsal aorta at the level of the pelvis, which supply the large intestine and rectum. Two pairs of small branches, the genital and renal arteries, supply the gonads and kidneys respectively. The right and left itiac arteries branch from the aorta and pass into the respective hindlimbs and bifurcate to become femoral and sciatic artery of each limb. Further posteriorly the dorsal aorta continues into the tail as the caudal artery.

The veins of the body, with the exception of the pulmonary veins, enter the sinus venosus. This is a thin-walled chamber located dorsally to the right auricle. It communicates with the right auricle by means of the sinuauricular aperture. This aperture is guarded by the two-lipped sinuauricular valve.

The pulmonary veins bring blood from the lungs. They unite into a short common trunk which enters the left auricle on its dorsal wall.

The postcaval vein, which receives the wide hepatic vein from the liver, extends from the anterior border of the liver to the sinus venosus. Posteriorly it lies between the gonads, and is formed by the union of a pair of efferent renal veins which lie parallel to the epididymis or oviducts. The postcava also receives a spermatic or ovarian tributary from each gonad. 
The hepatic portal vein is formed by the union of the gastrosplenic, mesenteric, and the ventral abdominal veins. It passes anteriorly and enters the tissue of the liver. The principal branch of the hepatic portal is continued posteriorly as the abdominal vein. The gastrosplenic is a large vessel and lies within the duodenohepatic mesentery,

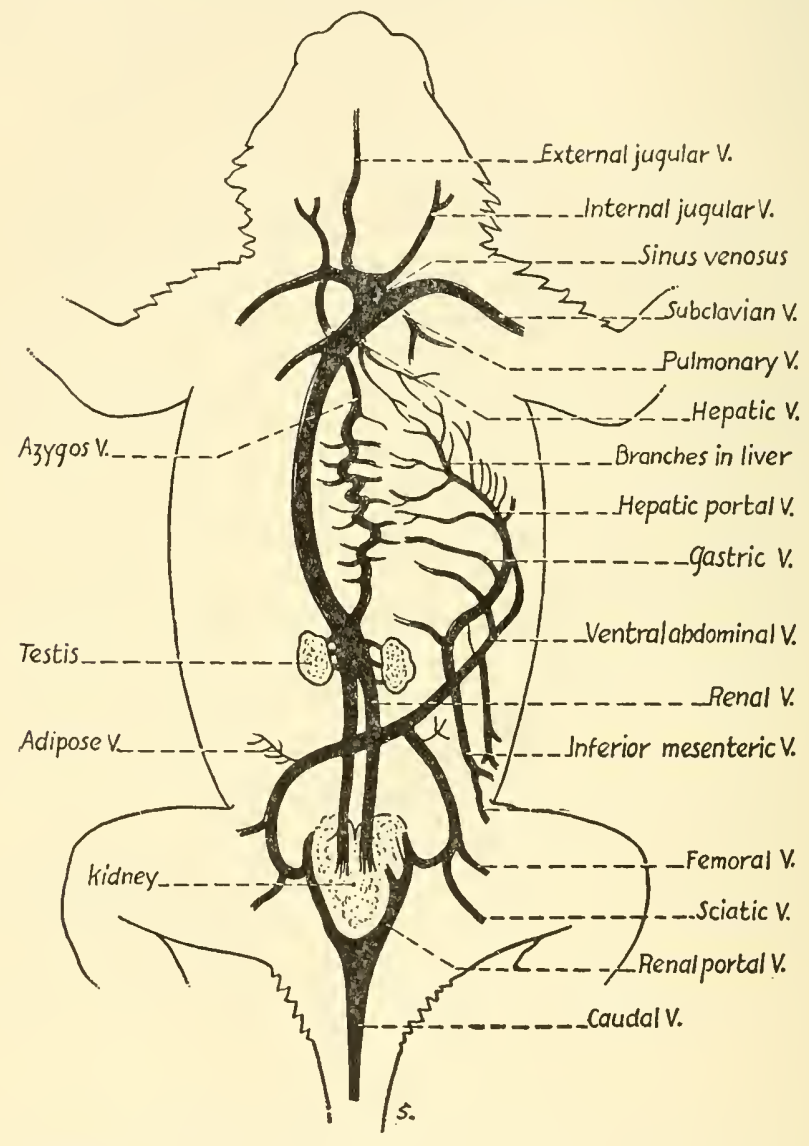

Fig. 298.-Venous system of horned lizard, ventral view.

parallel to the pancreas. It receives gastric veins from the stomach and small pancreatic veins. It is formed at its distal end by the union of the splenic and mesenteric veins from the spleen and intestines. Several separate gastric portal veins take blood directly from the stomach to the liver. 
The renal portal system drains a considerable portion of the body. The venous blood from the tail is carried by means of a caudal vein. It lies ventrally to the caudal vertebrae, and bifurcates at the base of the tail to form the two lateral pelvic veins. Each of these passes across the ventral face of the corresponding kidney to contact the posterior edge of the corpora adiposa. Each receives efferent veins from these bodies, at the anterior border of which the pelvic veins empty into the epigastric or anterior abdominal vein. This vein extends anteriorly along the inner face of the body wall to enter the left lobe of the liver on the posterior face. The renal portal veins receive the pelvic veins near the kidneys. The pelvic veins are formed by the union of the femoral and the sciatic veins from each hindlimb.

The right precava is located in the lateral neck region and passes posteriorly to the sinus venosus. It is formed by the union of the internal and external jugulars. For a short distance it is the common jugular. Then it receives the right subclavian vein which returns the blood from the right forelimb. From that point on it is the right precava. The left precava is formed by the union of the left internal jugular and the left subclavian veins. The left external jugular is not present. The right and left precavae converge with the postcava and enter the siuus venosus.

\section{The Urinogenital System}

The reptilian urinogenital system shows decided advances over the types of urinogenital systems found in lower vertebrates. The mesonephric type of kidney found in lower groups is replaced by a metanephric type of kidney in the reptiles. The products of the gonads are conducted through special ducts from these glands and not through the kidney, as in lower animals. Instead of producing hundreds of small eggs in a season, as in certain amphibians and fishes, the horned lizard lays twenty to twenty-five eggs of moderate size. The eggs of all vertebrate classes below reptiles are without shells. This makes external fertilization possible and necessitates an aquatic nesting site. The eggs of reptiles are covered by a tough, thick shell. Fertilization must occur internally before the shell is put on; hence, the males have intromittent copulatory organs for introducing spermatic fluid into the females. Such eggs will develop on land and often in rather dry places. The oviducts 
are much shorter in animals producing only a few ova than in animals producing many.

Certain similarities, however, are evident in the urinogenital system of reptiles and of lower forms. The urinogenital products are voided into the common cloacal chamber as in lower animals.

The kidneys of the horned lizard are reddish brown, paired bodies lying on each side of the middorsal line, between the large intestine and the body wall. Technically they are outside of the body cavity since they are dorsal to the peritoneum. Each kidney has a delicate excretory duct, the ureter. In the males this duct opens into the

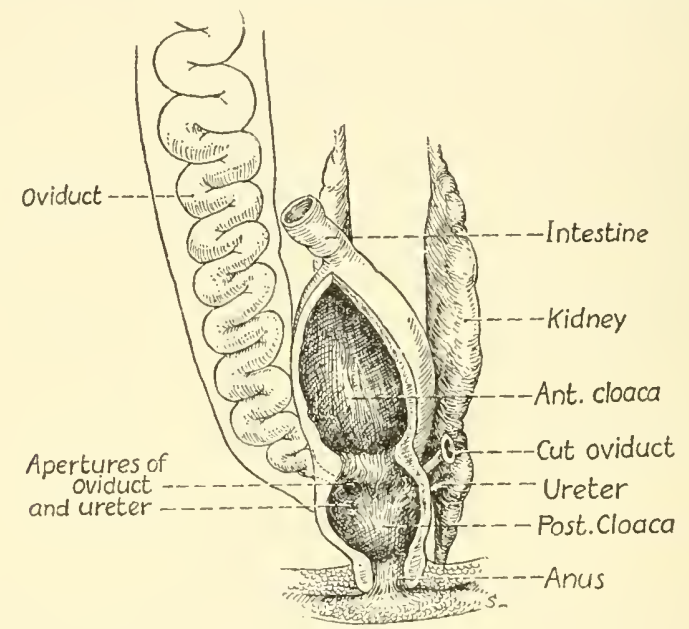

Fig. 299.-Urinogenital system of female horned lizard from ventral view.

vas deferens and a common duct leads to the cloaca. In the females the ureter connects the posterior end of the kidney with the dorsal wall of the cloaca.

The male reproductive organs consist of a pair of testes, a pair of eversible hemipenes, and various connecting ducts. The testes are white oval bodies lying in the posterior part of the body cavity, one on each side of the mesorectum. Each testis is attached to the dorsal body wall by a sheet of peritoneum, the mesorchium. The series of ducts connecting each testis with the cloaca includes the rete testis, the epididymis, and the vas deferens in order. The vas deferens passes posteriorly, joins the ureter, and enters the cloacal wall. The paired, eversible hemipenes, or copulatory organs, are vascular 
sacs embedded in the musculature of the ventral side of the base of the tail. They are elongated, smooth-walled structures which open into the posterior chamber of the cloaca. During copulation these organs protrude through the vent of the cloaca by everting themselves; i.e., turning themselves wrong side out. They are pulled back into place by the contraction of a retractor muscle attached to the distal end of each organ. Often only one hemipenis functions during copulation, but this is not an invariable rule.

The female reproductive organs consist of a pair of ovaries and a pair of oviducts. Each ovary is attached to the dorsal body wall in the posterior end of the body cavity by means of a double sheet of peritoneum, the mesovarium. The folded oviducts lie dorsolateral to the ovaries. The anterior end of each oviduct is expanded into a thin-walled ostium; posteriorly, each duct opens into the dorsal wall of the posterior cloacal chamber. The oviducts are held in position by a broad fold of the peritoneum, the broad ligament.

The common cloacal chamber of both sexes receives the products discharged by the alimentary canal, the kidneys, and gonads. The cloaca is divided into two regions, an anterior and a posterior, by an incomplete ridge of mucous membrane.

\section{The Nervous System}

The reptilian nervous system shows certain advances over the nervous system of amphibians. Twelve pairs of cranial nerves are present, as in birds and mammals. The various regions of the brain are better developed than in lower vertebrates; however, the reptilian brain is still a comparatively simple structure.

The nervous system of horned lizards, as in other vertebrates, consists of the central nervous system, the peripheral nervous system, and the sympathetic nervous system.

The central nervous system consists of a brain located within the skull, and a spinal cord located within the neural canal. The brain has all the divisions of the typical vertebrate brain. The peripheral nervous system is composed of the twelve pairs of cranial nerves from the brain and sixteen pairs of spinal nerves from the spinal cord. These nerves are arranged scgmentally. Typically, there is one pair to each segment. The sympathetic system includes two conspicuous white trunks that extend along each side of the vertebral column and the lateral fibers from these trunks. Each of the sympathetic 
trunks is connected with all of the spinal nerves on the same side of the body by a number of ganglionic enlargements. The usual sensory and motor functions are carried on by these systems. The autonomic system helps to regulate involuntary reactions.

The special sense organs of the head are the eyes and the ears. Each eyeball reposes in an orbital cavity, and is attached to the walls of the cavity by voluntary muscles which serve to move it in all directions. The innervation is from the optic lobes and other parts of the brain by way of the optic nerves. The structure of the eye itself is similar to that of other vertebrates.

The ear consists of two principal parts: the middle ear, or tympanum, and the internal ear, or membranous labyrinth. The cavity is closed outwardly by the tympanic membrane. It communicates with the mouth eavity by means of the Eustachian passage. A small rod of bone and cartilage, the columella, stretches across the cavity from the tympanic membrane and is fixed internally into the membrane covering the fenestra ovalis. The internal ear is enclosed by the bones of the auditory region.

\section{The Skeletal System}

The reptilian skeleton retains many features of the amphibian skeleton and forecasts many features found in birds and mammals.

The divisions of the skeleton of the horned lizard are the skull, the vertebral column, ribs, sternum, the limb girdles, and limbs.

The skull proper is triangular and somewhat pyramidal in form. The base of the pyramid is represented by the posterior aspect of the skull, and the apex is represented by the snout. It is more completely ossified than in amphibians and is composed of a greater number of bones. There are sharp protuberances and excrescences that form the skeletal support of the "horns" of horned lizards. Were these magnified to the size of dinosaurs, they would be equally imposing. The skull articulates posteriorly with the atlas, or first cervical vertebra.

The vertebral column consists of several distinct regions and a number of vertebrae. There is a cervical region composed of seven vertebrae which form the skeleton of the neck. A thoracicolumbar region consists of fourteen vertebrae and with the ribs and sternum 
make up the skeleton of the trunk. A sacral region of two vertebrae gives attachment to the pelvis. A caudal region of fourteen vertebrae supports the tail.

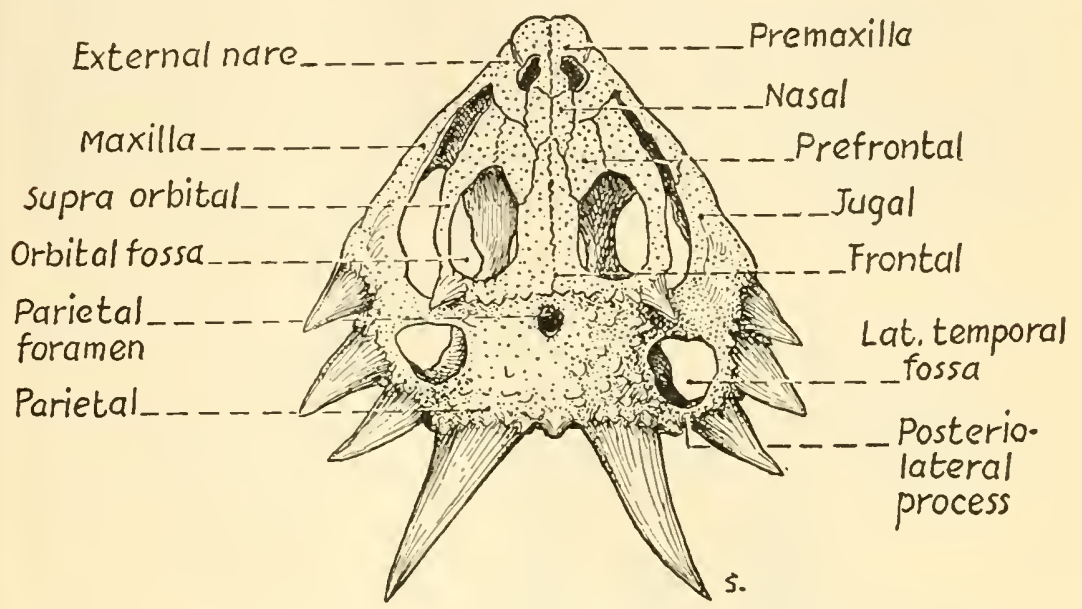

Fig. 300.-Dorsal view of the skull of horned lizard.

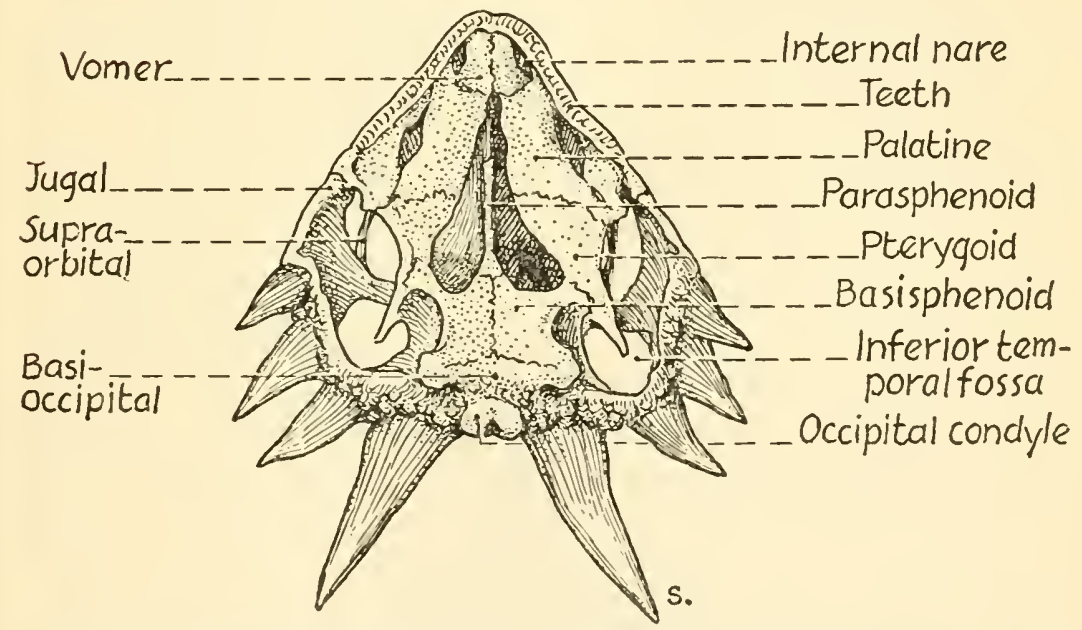

Fig. 301.-Ventral view of the skull of horned lizard.

The pectoral girdle is composed of bone and cartilage. The two anterior limbs articulate with it. The limb is a rather generalized example of a typical pentadactylous vertebrate limb. The pelvic girdlo 
is composed of a pair of triradiate bones, or innominate bones. Each innominate bone consists of three separate bones: the ilium, ischium, and pubis. These three bones radiate from a triradiate suture at the acetabulum or hip socket. The pelvic girdle articulates with the sacral vertebrae by means of the ilia. The ischia are the most posterior bones of the three. The pubic bones extend anteroventrally and unite at the pubic symphysis. As in the forelimb, the structure of the hindlimb is of the characteristic vertebrate type.

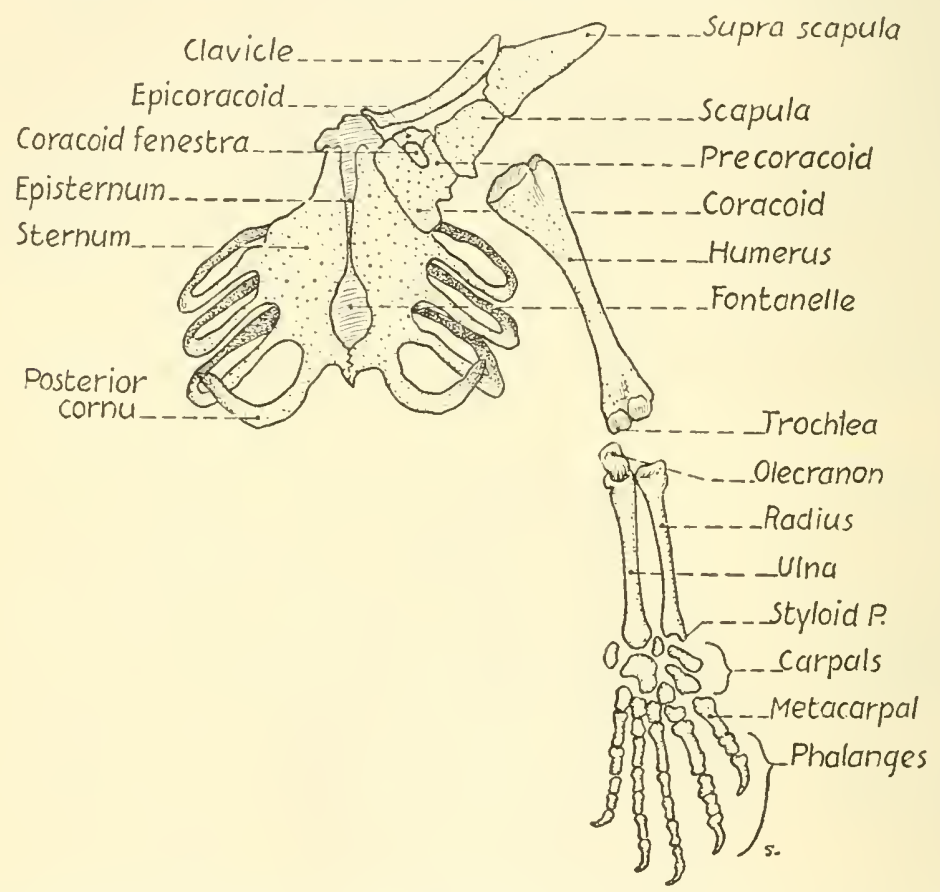
Fig. 302.-Sternum, pectoral girdle, and disarticulated forelimb of horned lizard,
ventral view.

\section{Muscular System}

The reptilian musculature shows few unusual characteristics. In a general way it may be said to be intermediate between that of amphibians, on the one hand, and birds and mammals, on the other.

Since the horned lizard is a generalized terrestrial animal, the musculature follows the typical vertebrate plan rather closely. 


\section{THE TURTLE}

The pond turtles of various genera belonging to Family Emydidae have long been used as convenient and useful examples for studies of reptilian characteristies. While there are many variations in details, the main features of Troost's turtle, Psendemys troostii elegans (Wied), are found, very little modified, in other members of the family. This is one of the most abundant species of the Mississippi Valley region and is very frequently the turtle sold by supply houses for laboratory use.

One of the most interesting characteristics of this species ( $P$. troostii elegans) has only recently been discovered. The adult males are frequently melanistic; i.e., have the normal color pattern concealed by a superimposed layer of black pigment. The young males have the same coloration as the females and only gradually take on the melanistic coloring. Hence, all stages from normal coloration to jet black can be found among them.

\section{Habits and Behavior}

These turtles are commonly seen sumning themselves on logs along the margins of ponds and rivers. When disturbed, they fall into the water with a splash and swim hastily for the deeper parts of the body of water. This activity in daylight hours might lead one to suspect that they are entirely diurnal, but turtles are known to feed at night. Hence, it seems that they are not as strictly regulated by hours of light and darkness as are most vertebrates. Since all turtles are cold-blooded animals, they hibernate in winter in temperate regions. Turtles are often seen in considerable numbers in a restricted locality, but there is no congregating or communal instinct among them. Each one is totally oblivious of the presence of all the others, except during the mating season, when the males seek out the females. Turtles have been observed to mate at all times from spring to autumn but the chief mating period occurs soon after the animal comes out of hibernation in the spring. The eggs are laid soon afterwards in a hole or pit in the earth excavated by the female for that purpose. When the full complement has been deposited, she covers them and returns to the water. Turtles taken in the autumn months are often found to contain a full complement of completely shelled eggs. This indicates that the mating that commonly occurs immediately prior to the laying of these eggs 
does not fertilize the eggs of the year but those of succeeding years. In experimenting upon this phase of the life history of turtles, the United States Fish and Wildlife Service has discovered the surprising fact that a female turtle may lay fertile egg's for as long as four successive years after a single mating.

\section{External Structure}

No vertebrate is so unique in structure as the turtle. No turtle could be mistaken for any other animal. The arched or domeshaped dorsal covering, the carapace, and the plane floor of this armor, the plastron, set the turtles apart from all living vertebrates. The head and neck can be completely withdrawn into the shelter of this covering; and legs, feet, and tail can be partially protected in this manner.

The body of a turtle may be divided into the following regions: head, neck, trunk, and appendages.

The head is covered with thin, smooth skin. Various colored lines and patterns may be present on the skin of the head and neck. Troost's turtle has an oblong red patch on each side of the head and neck unless it has been completely obliterated by black pigment deposited over it. The neck is relatively long and slender, and retractile within the shell by vertical sigmoid flexures. The skeletal elements of the trunk region are completely co-ossified into an immobile boxlike structure. This carapace and plastron are covered by scutes of epidermal tissue. These scutes are definite in number and arrangement. Tarious color patterus are found on or under them. The legs are typical pentadactyl limbs. The toes are united by various degrees of webbing in aquatic forms. The tail is relatively small and short.

\section{Digestive System}

The organs of the digestive tract are mouth, pharynx, esophagus, stomach, small intestine, large intestine, rectum, cloaca, and anus. Various accessory structures and glands are the cutting edges of the beaklike jaws, the liver and the pancreas. The size and relative length of the various parts of the alimentary canal are correlated with the food habits of the animal. Turtles eating largely vegetable food have intestines many times the length of the body. Those taking 
chiefly animal matter possess intestines only a little longer than the body. The accessory glands, liver and pancreas, function as in other vertebrates.

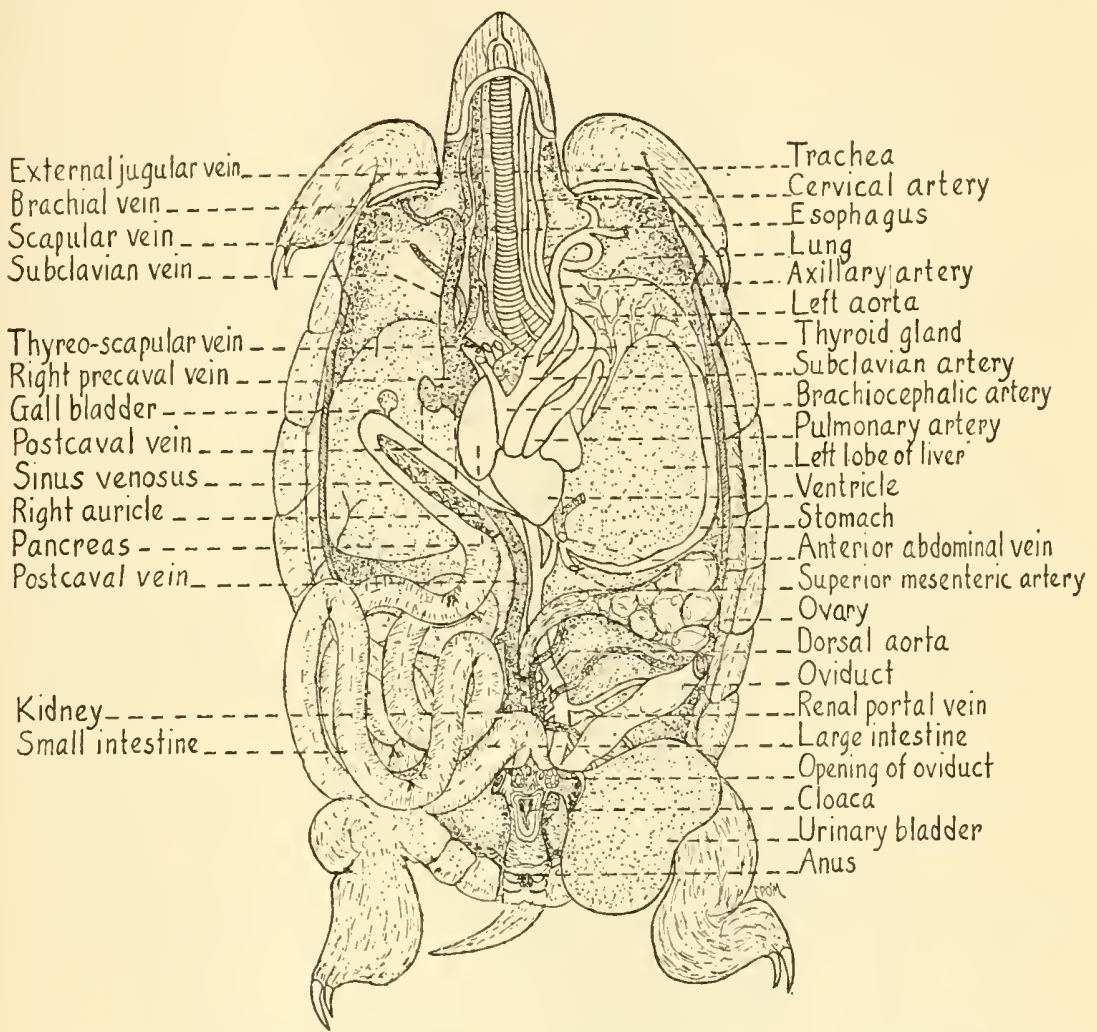

Fig. 303.-Dissection to show internal organs of the turtle, Pseudemys troostit elegans, ventral view.

\section{Respiratory System}

The respiratory system is adapted for breathing air only, regardless of the fact that turtles spend the greater part of their lives in water. The organs composing the system are the nostrils, pharynx, trachea, bronchi, and lungs. An indispensable accessory structure is the hyoid apparatus. Since the ribs are made fast in the carapace, breathing cannot be accomplished in the usual vertebrate manner; hence, the hyoid apparatus, located in the gular region, functions as a pump and the turtle seems to swallow air. The lung 
space is relatively great in some turtles. This enables them to remain submerged for considerable periods. Accessory urinary bladders also serve in aquatic respiration.

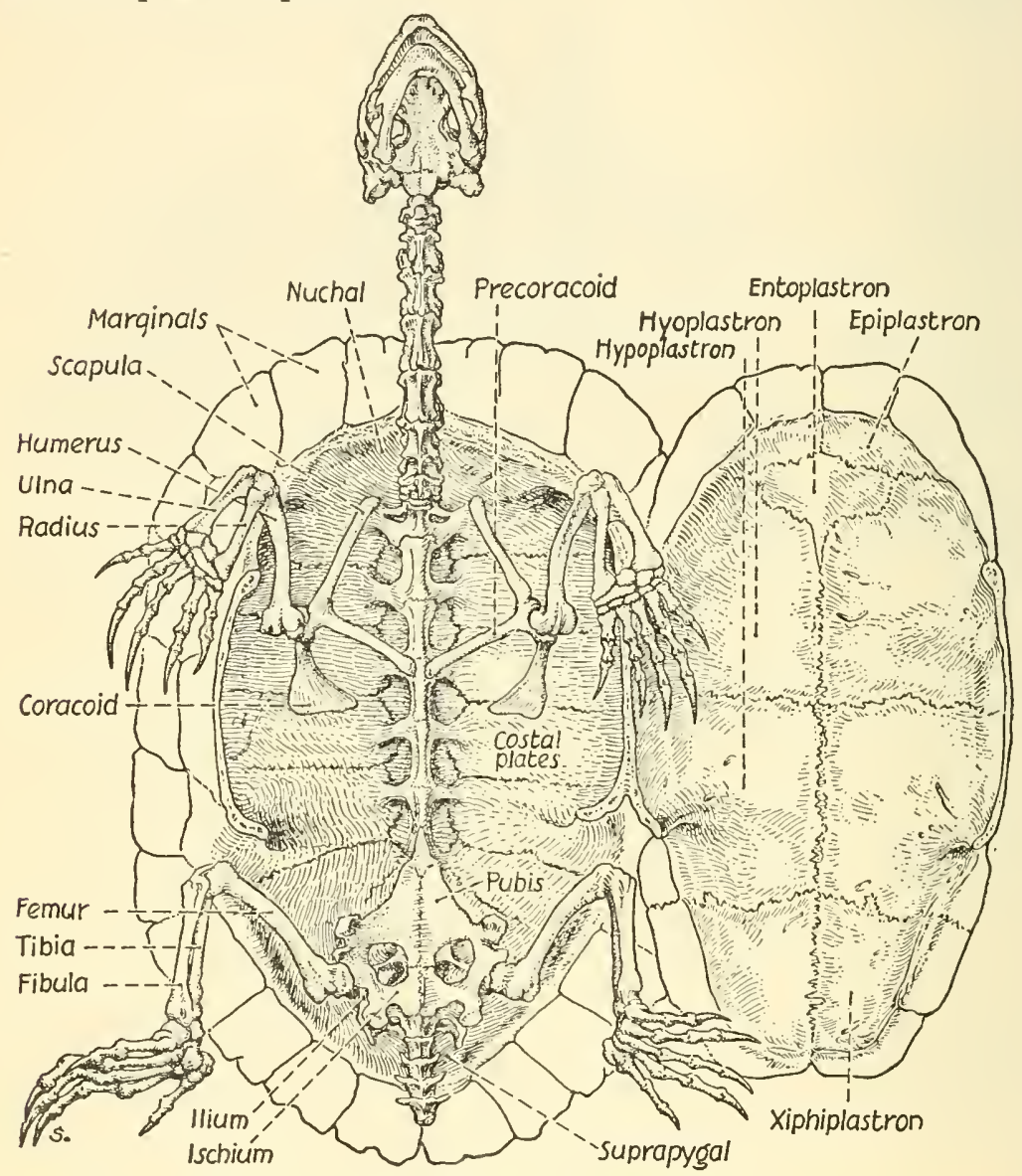

Fig. 304.- Skeleton of the turtle, Pseudemys troostii elegans, showing ventral view of carapace and dorsal view of plastron.

\section{Circulatory System}

The circulatory system of the turtle consists of the heart, arteries, connecting capillaries, and veins.

The heart is typically reptilian in structure, having two auricles and one ventricle with a perforated partition dividing it. The pulmonary circulation returns blood to the left auricle, but this aerated 
blood is partially mixed with venous blood in the ventricle before being sent into the aortic arches.

\section{Urinogenital System}

The excretory system consists of two kidneys, their ureters, the cloaca, and a bilobed urinary bladder. The urine is voided into the cloaca and stored in the urinary bladder until expelled through the anus.

The sexes are separate. The male reproductive system is made up of a pair of testes, a pair of vasa deferentia, and an evertible penis located on the anterior wall of the cloaca. The female genital organs are ovaries, oviducts, and cloaca.

\section{The Nervous System}

This system is typically reptilian (see page 573 ). In some turtles adaptations in structure of nervous tracts to the eye are known to have occurred, probably in response to feeding habits. That part of the peripheral nervous system which normally innervates the musculature of the costal region is absent.

\section{The Skeleton}

The skeleton of turtles is the most characteristic of their systems Embryological studies have shown that in the early stages the skeleton follows the typical vertebrate plan. Soon, however, the ribs rise above the limb girdles; those above the pectoral girdle bend anteriorly and those over the pelvic girdle bend posteriorly. The process of co-ossification goes on over a period of years and is not altogether completed until maturity is reached. The result is a vertebrate animal with the limb girdles enclosed by the ribs. No other vertebrate follows such a plan.

\section{The Muscular System}

This system shows modifications corresponding to those in the skeleton. The intercostal muscles of the body wall are not present as such. The muscles of the neck are highly developed and adapted to moving the head with great rapidity. The muscles of the legs are little modified, being similar to those of other vertebrates. 


\section{CHAPTER XXX}

\section{AVES}

Class Aves ( $\overline{\mathrm{A}}^{\prime}$ vēz), which includes all birds, has several distinctive characteristics. The fundamental structural features of this class are quite closely related to those of reptiles. The outstanding characteristics of birds are as summarized in the following statements. (1) The skin is covered with feathers which are exoskeletal outgrowths of it. Birds are the only animals with feathers. (2) The jaws are toothless in the adult and are covered with a hard horny beak. (3) All modern birds are bipedal, the forelimbs being modified into wings, or undeveloped. (4) The pelvic girdle is securely anchored to the vertebral column to support the bird adequately on two legs. These limbs serve the animal in locomotion on land and in water as well as for perching and climbing. (5) The caudal vertebrae are greatly reduced in number and all except the free anterior ones are fused into a single bone, the pygostyle. (6) In most birds the digestive system is modified to provide a crop for storage of food, and a muscular portion of the stomach for chewing food. They are warm-blooded vertebrates.

Most people associate birds with flight, but there are numerous birds which do not have this power. In fact, several have practically no wings. The wings of the New Zealand kiwi are so rudimentary that they are completely concealed by the body feathers.

Many birds are migratory, moving north for the summer season and south for the winter. This is a very aristocratic habit around which the living activities are centered. The migration routes are precisely laid out; however, birds occupying the same general breeding range do not necessarily spend the winter in the same region or vice versa, and they do not necessarily travel the same route in the fall as was traveled in the spring. The birds return to the same breeding range and winter home, season after season, which seems to demonstrate a definite homing sense. An example of extreme migration is the Arctic tern which nests within the Arctic circle and winters within the Antaretic circle, a distance of 10,000 or 11,000 miles. 
This is traversed fall and spring and largely over water. The average migrating bird travels only about 23 miles a day on migration, but this tern makes at least 150 miles a day. Recently the U. S. Army Signal Corps reported that one of its messenger pigeons had flown 600

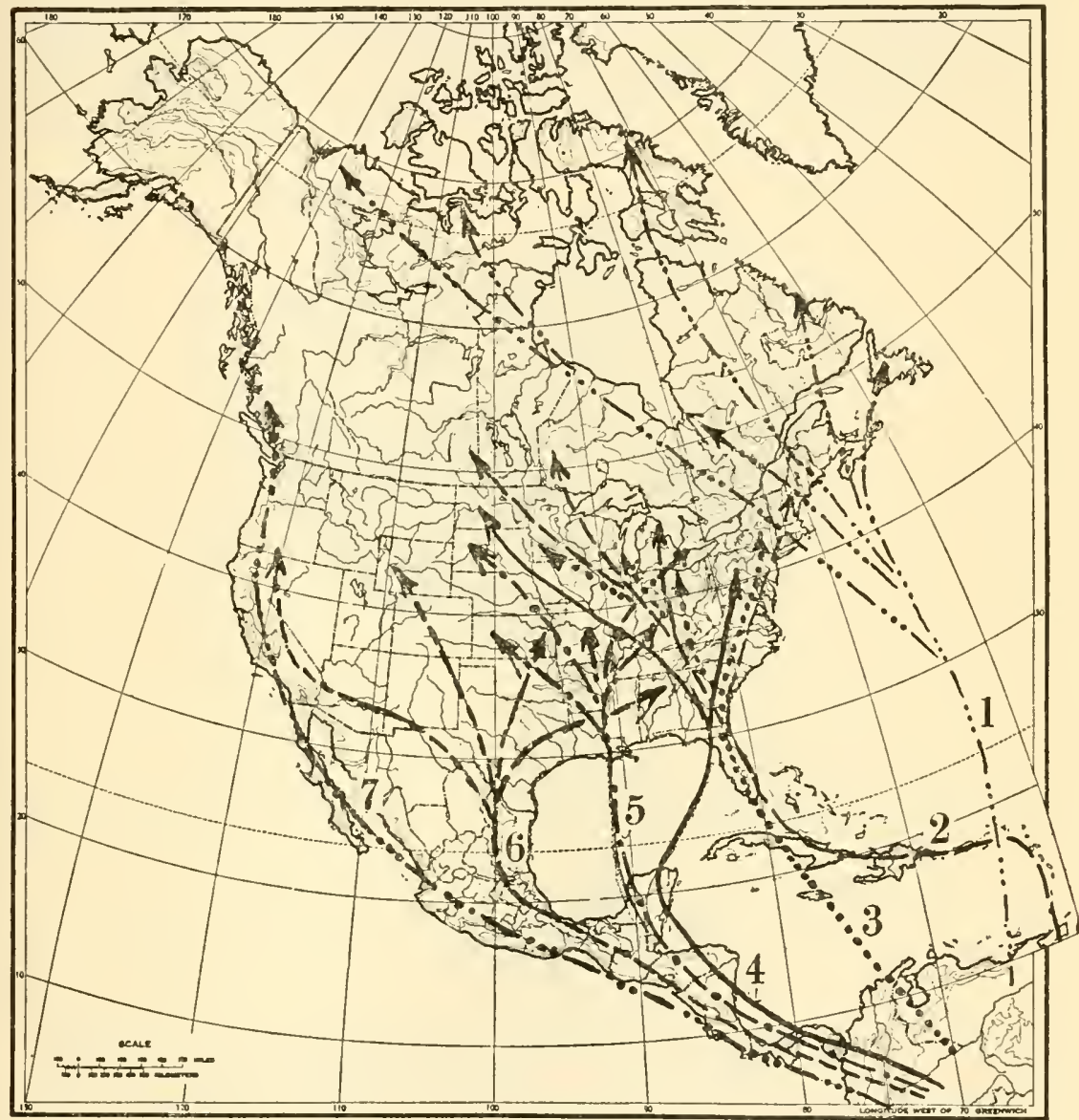

Fig. 305.-Migration airways of birds in North America. (From Metcalf, Textbook of Economic Zoology, published by Lea and Febiger.)

miles in less than 14 hours (42 miles per hour) without the aid of a tail wind. Several theories attempting to explain bird migration involve such factors as food conditions, temperature, changing length of days, and hormone control. The explanation is still an open question. 


\section{Classification}

Birds constitute a relatively uniform group of animals. They are much more similar throughout the class than any other group to be studied. The differences between the most dissimilar birds are no greater than the differences seen among frogs in the order Salientia, in class Amphibia. Such characteristics as size, color, wing development, type of beak and feet, are the bases for distinguishing the

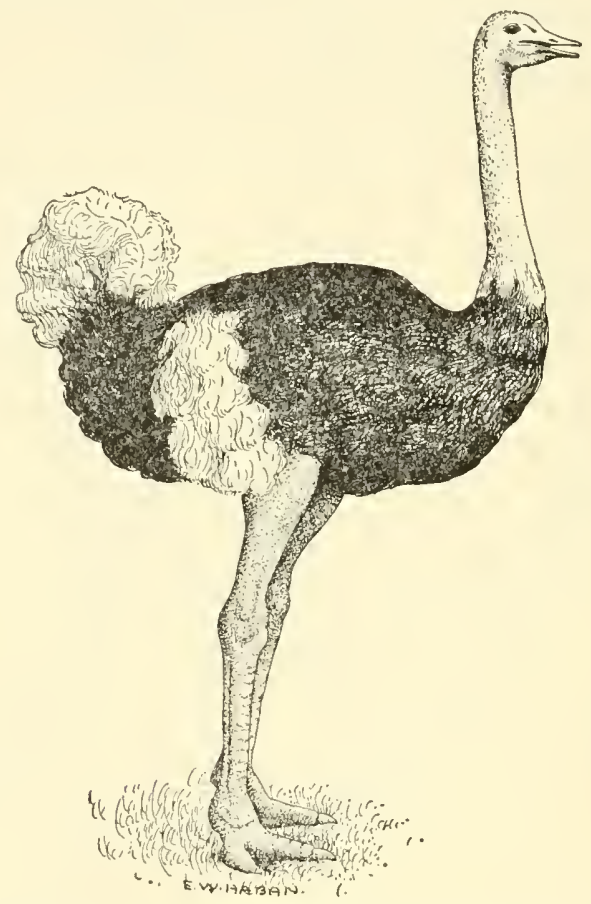

Fig. 306.-African ostrich, Struthio camelus. (From Metcalf, Textbook of Economic Zoology, published by Lea and Febiger.)

orders of birds. There are four orders of flightless birds which are sometimes placed in a group designated as subclass Ratitae. This group includes such birds as ostriches, rheas, cassowaries, and kiwis. The sternum has no keel in any of these, the feathers are without barbules, and the wings are either absent or reduced. All other birds would be grouped in subclass Carinatae according to this plan. More than 14,000 species of birds are classified into twenty-five orders. 
A brief summary of the orders of birds will be given in the following pages. Some of the orders are divided into numerous families.

Struthioniformes (Ostriches).- These are the largest known birds and they are native to Africa, but they are growing in domestication in numerous parts of the United States. The large eggrs weighing three or four pounds are laid in communal nests in the sand; they are then incubated by the sun. The wings are very rudimentary, and there are only two toes.

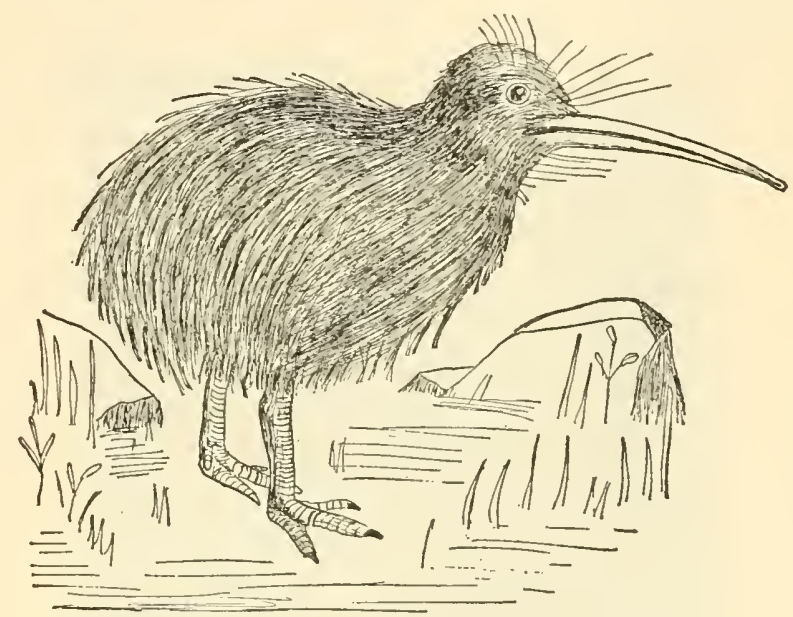

Fig. 307.-Kiwi, or apteryx, a wingless bird from New Zealand which is about the size of the domestic fowl and looks like an overgrown chick. (From Krecker, General Zoology, published by Henry Holt and Company, Inc., after Evans.)

Rheiformes (Rheas).-This is another form of ruming bird. They are partially feathered on the neck, have three toes, and inhabit South America.

Casuariiformes (Emus and Cassowaries).-These are flightless, rumning birds with very small wings. The former inhabits Australia and the latter New Guinea. The cassowaries, which are smaller than ostriches and have shorter necks, have a headgear with bright colors on the head and neck.

Apterygiformes (Kiwis).-This is the fourth order of running, flightless birds. The feathers of this form are hairlike. The kiwi is about the size of a hen, but its wings are much reduced. It is nocturnal, and it makes a nest in a hole in the ground. 
Crypturiformes (Tinamous).-This is a group of little known quaillike birds of northern South America, Central America and southern Mexico.

Sphenisciformes (Penguins).--These are flightless, diving birds. The feathers are almost scalelike, and the wings are modified as "flippers" to be used under water. They dive for fish which can be swallowed under water. They live on barren rocky shores of the Antarctic. Here they nest in colonies, each female laying one or two eggs in depressions among the rocks.

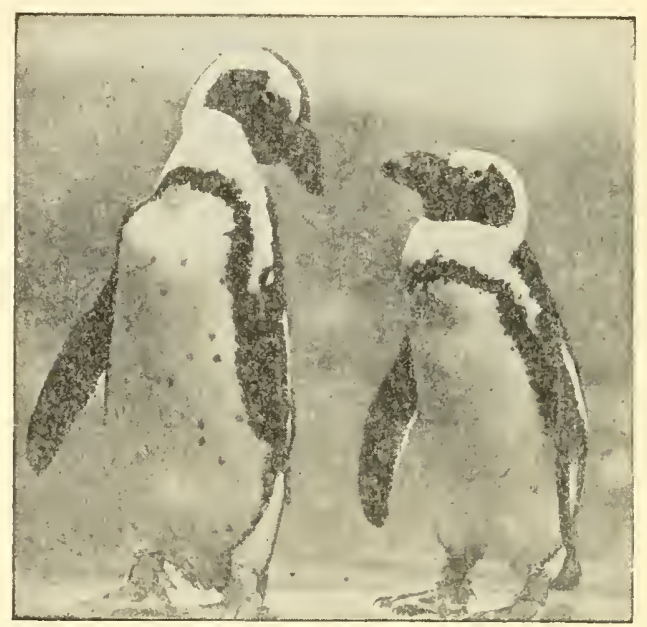

Fig. 308.-Penquins. The wings and feet are highly adapted to swimming. (From Hegner, College Zoology, published by The Macmillan Company.)

Gaviiformes (Loons).--Our common loon is checked with black and white over the back. These birds are also expert divers and swimmers under water. They have a very peculiar call that sounds like a weird or crazy langh. Their nesting range is between northern United States and the Arctic circle. The wintering ground is principally the Gulf Coastal States.

Colymbiformes (Grebes).-This is a group of small or mediumsized diving birds with lobed feet. The most common one is the piedbilled grebe or hell-diver. The legs are far posterior on the body. There are about twenty-five species generally distributed over the world. 
Procellariiformes (Albatrosses and Petrels).-The former are birds of the open sea with a wing spread of ten or twelve feet. They will follow ships for days without landing by gliding on nearly motionless wings. They come to land usually on islands in colonies, to lay their eggs. Laysan Island, far out in the Pacific, is noted for them. The petrels are small and may be found in midocean. They nest in crevices on rocky shores and islands. The members of this order have tubular external nostrils, fully webbed toes, and long narrow wings.

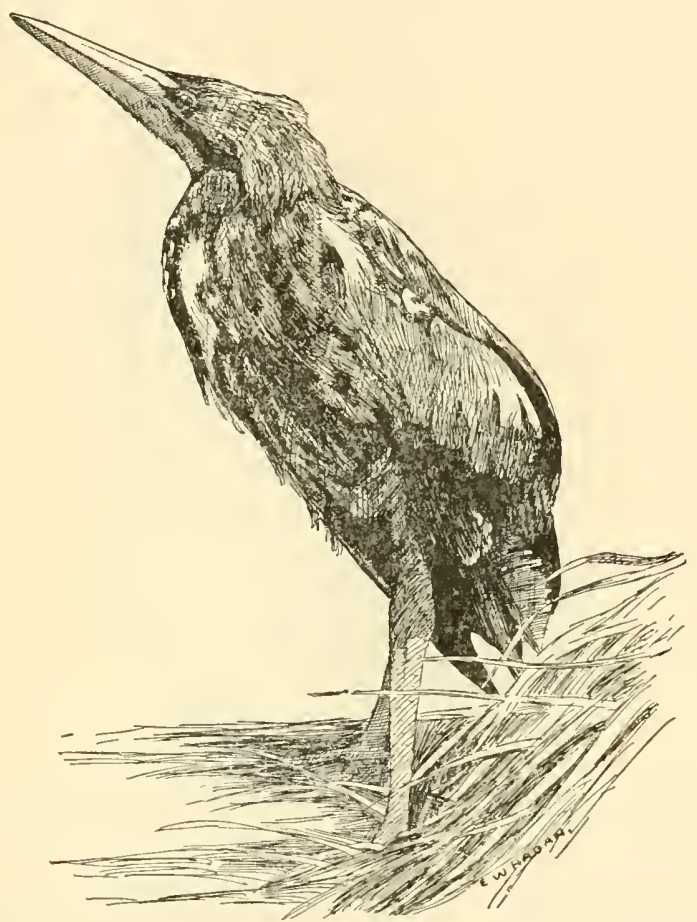

Fig. 309.-American bittern, Botaurus lentiginosus, stake driver. Sometimes called shikepoke in common parlance. (From Metcalf, Textbook of Economic Zoology, published by Lea and Febiger.)

Pelecaniformes (Totipalmate swimmers).-The cormorants are the widely distributed, well-known representatives of the order. They have long necks, long, hooked bills, and webbed feet. Their habits are gregarious, and they dive for fish which can be eaten under water. The oriental peoples take advantage of this ability to catch fish, and train them to retrieve for the master. 
Ciconiiformes (Long-legged Waders).-This order includes two prominent families: Ardeidae, including bitterns, herons, egrets, and storks, and Phoenicopteridae, including flamingos; of the latter, one species, Phoenicopterus ruber, inhabits the Gulf States. It is a tall bird with long, rosy colored legs and a long, curved neck. The bill is large and peculiarly shaped, with a curved matching of upper and lower parts. It is used for seining food in the form of small animals out of the mud. The tall, conical nests are built of mud on mud flats. Two eggs are laid in the hollowed top. The birds of the first

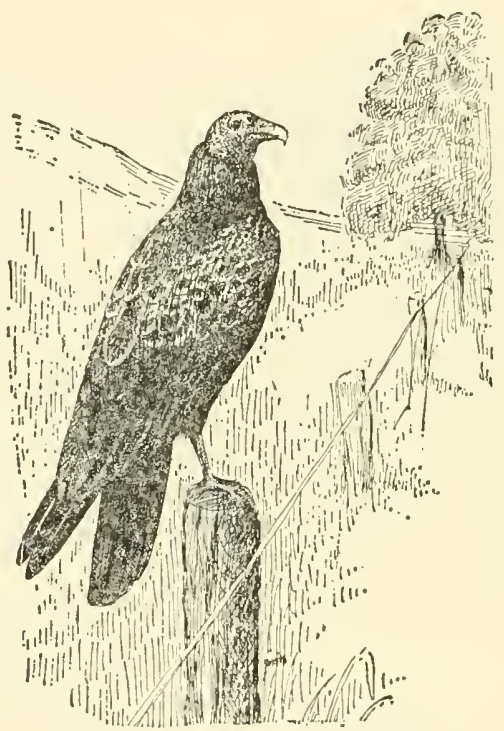

Fig. 310.-Turkey vulture (buzzard), Cathartes aura septentrionales. (From Metcalf, Textbook of Economic Zoology, published by Lea and Febiger, after Snyder.)

family, particularly bitterns, herons, and egrets, are structurally similar but their habits are quite different. They are medium-sized to large birds with long bills, necks, and legs. The bitterns live in marshes and grasses along shores while herons live in open shore waters. The great blue and lesser blue herons with some color variations are quite common all through the middle United States, and in the South and Southwest. The green heron is another medium-sized bird. The American bittern, or shikepoke is a long-billed fishing bird as are also the herons. The Southern States have two species of egrets, Herodias cgretta and Egretta candidissma, with long plumes 
or aigrettes on the head which were formerly hunted for millinery trade until the birds were almost extinet. Fortunately this has been prohibited and the birds are reported as on the increase.

Anseriformes (Ducks, Geese, Swans, and Mergansers).-These are swimming birds with short legs and completely webbed toes. The bill is rather broad and flattened. Nearly all of them are strong, swift fliers, and are excellent game and food birds. There are more than two hundred species of these ducklike birds, and many of them frequent the waters of the United States. Geese and swans are somewhat larger than ducks. They migrate to the Sonthern States for the winter, flying in V-shaped formation.

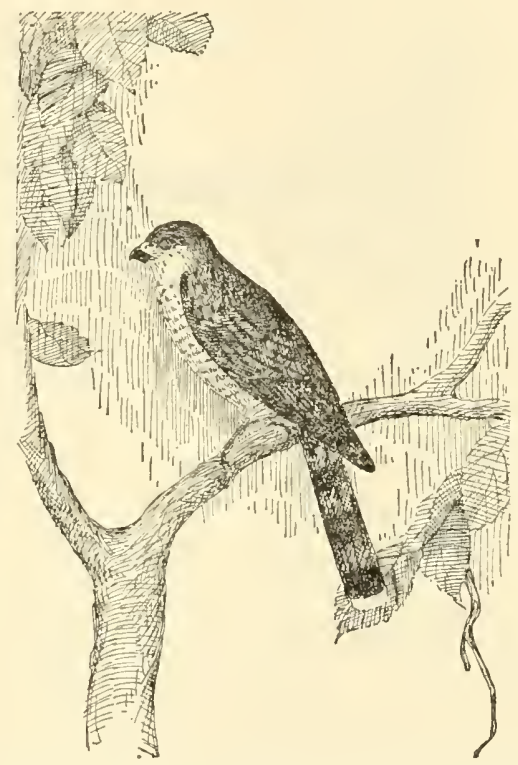

Fig. 311.-One of the "blue darters," Cooper's hawk, Accipiter cooperi. (From Metcalf, Textbook of Economic Zoology, published by Lea and Febiger, after Snyder.)

Falconiformes (Birds of Prey).--In this order there are three families of these strong birds with powerful wings, stout hooked bills, and strong elaws. It includes vultures, eagles, hawks, falcons, kites, and ospreys. The turkey vulture (buzzard) and black vulture are common birds of the Southwest. The California vulture, Pseudogryphus californianus, and the condor of the Andes Mountains of South America are very large birds. Most of our species of hawks, 
including the large red-tailed and red-shouldered, are extremely beneficial because of the rodents and insects they devour. Only the sharp-shinned hawk, Accipiter velox, and Cooper's hawk, Accipiter cooperi, are particularly destructive to other birds and poultry. They are bluish gray, swift fliers, frequently called "blue darters." There are two American eagles quite generally distributed over the continent. They are: the national bird, Aquila chrysaëtos, or golden eagle, and the bald eagle, Haliaetus leucocephalus.

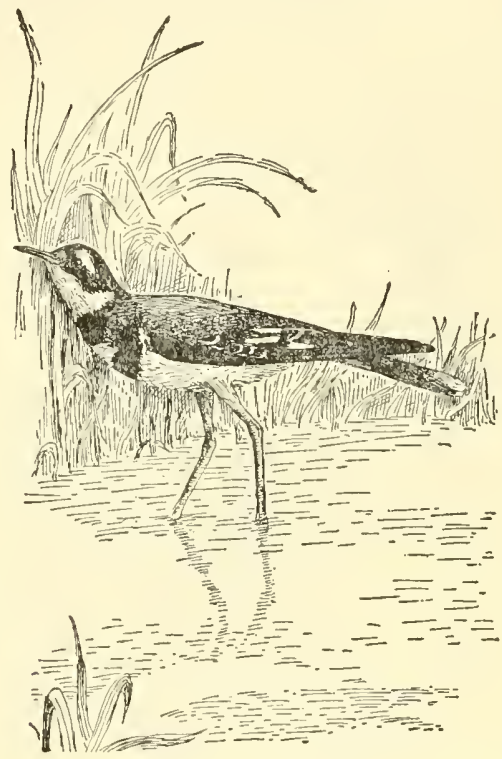

Fig. 312.-Killdeer, Oxyechus vociferus. A common shore bird. (From Metcalf, Textbook of Economic Zoology, published by Lea and Febiger, after Snyder.)

Galliformes (Fowllike Scratchers).-Turkeys, quails, pheasants, prairie chickens, and domestic chickens are the most notable representatives of this group. Here are some of the most famous game as well as commercial birds. The wild turkeys and prairie chickens are becoming scarce, and considerable effort is being made to save and rebuild the remnant in the Southwest. Pheasants have been very successfully introduced to some parts of our country from Asia. They make excellent game birds. The group are all scratchers, living largely on the ground and feeding on seeds and insects. Some of them roost in trees. They have stout bodies, hard bills, and short wings. 
Gruiformes (Cranes and Rails).-The rails, gallinules, and coots are rather small birds, the first two live in fresh- or salt-water marshes. The common coot or mud hen, Fulica americana, lives on the water like a duck. It swims about in small flocks, dives, and can perch in trees. Its feet are not webbed, but the toes are fringed. The cranes are large heronlike birds with long necks and legs. The large whooping crane has a wingspread of about eight feet. These birds like many of our larger ones need more protection to keep them extant.

Charadriiformes (Plovers or Shore Birds).-There are representatives of nine families of these birds in North America. The plovers, suipes, sandpipers, killdeers, curlews, gulls, terns, woodcocks, avocets, phalaropes, and auks are all fairly well known except the last three. The jacana bird, Jacana spinosa gymnostoma, a tropical form, ranges into Texas.

Columbiformes (Pigeons and Doves).-These birds have a distinctive appearance and are seldom confused with anything else. They are of medium size and lave general distribution. They feed on seeds and fruits. They produce "pigeon mills" which is regurgitated as food for the young. The passenger pigeon, Ectopistes migratorius (Fig. 411), which is now extinct, was at one time our most abundant bird. The flocks were supposed to contain billions of individuals. They were killed out by the market-hunters and by the encroachments of civilization. The mourning dove, Zenaidura macroura, is now a much sought after game bird. It is known for its very plaintive call. They breed in pairs, then flock and feed in pastures and grain fields.

Psittaciformes (Parrots, Parakeets, Lovebirds, and Macaws).The one native species of southeastern United States is now thought to be extinct or nearly so. The larger parrots are sought after because of their ability to talk, and all of the others are brilliantly colored. The lovebirds are small parrots with affectionate dispositions from Africa; they are commonly used as cage birds. Macaws are the largest of parrots and range from Mexico to Argentina. They are exceptionally colored.

Cuculiformes (Cuckoos, Chaparral bird, and Ani).-The "rain crows," as the cuckoos are called because of their peculiar call which is said to forecast rain, are long, slender birds with long wings, a long tail, distinctive bill, feet with two toes in front and two behind. They build their nests in the form of loose platforms of small twigs 
in bushes and trees. The road runner or chaparral bird is quite common along the roads from central Texas on southwest. It is a slender bird of fair size with a tail as long as the body. It feeds on grasshoppers, mice, lizards, and small snakes. Many strange stories are

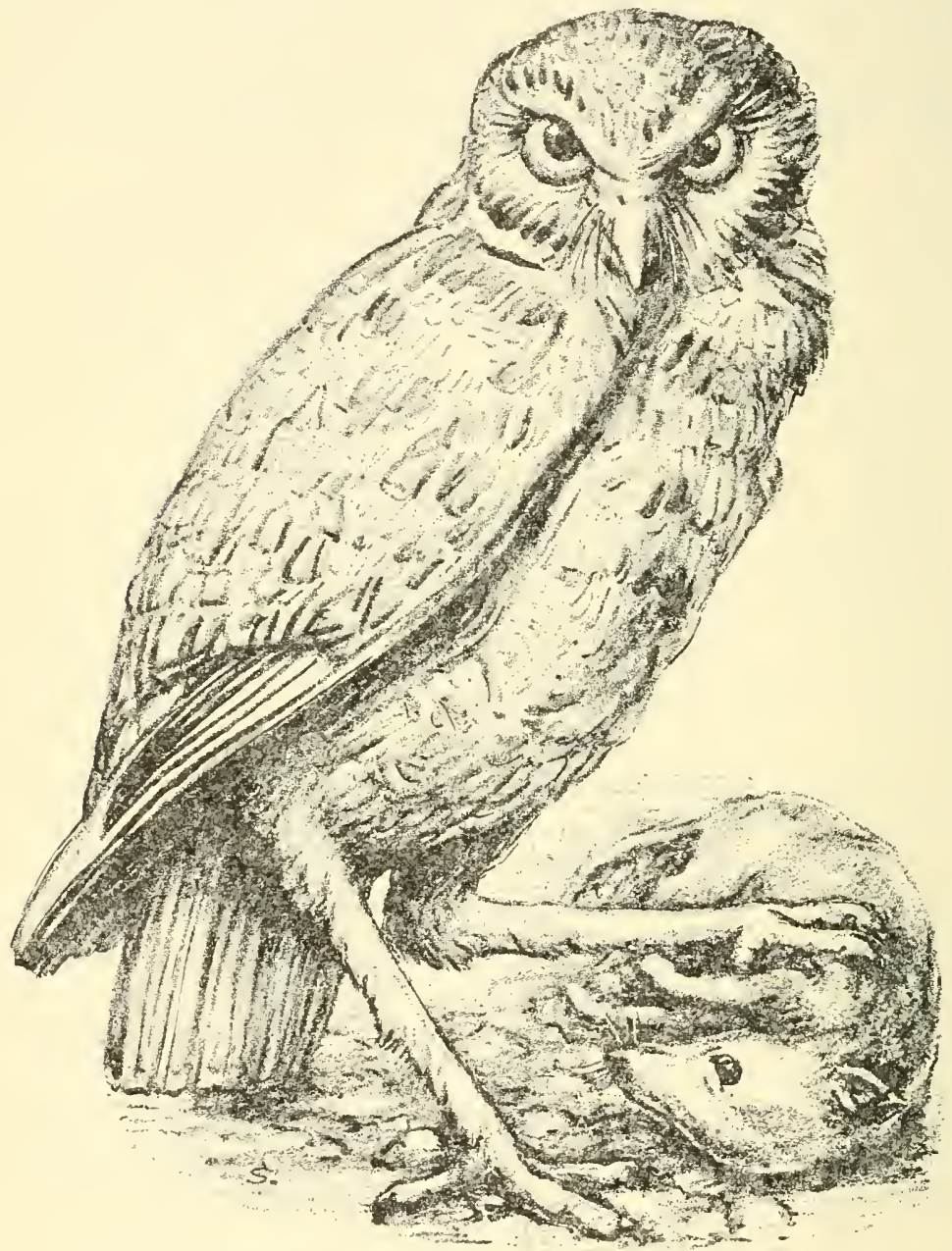

Fig. 313.-Burrowing or prairie dog owl, Speotyto cunicularia. Common on the plains around prairie dog towns.

told about its battles with rattlesnakes. The ani is common in Mexico and gets over into the United States occasionally. They are thought to lay their eggs in community nests somewhat as the ostrich does. 
Strigiformes (Owls).-These are large birds of prey with large eyes, soft feathers, strong hooked beaks, and vicious claws. Their food consists of mice, rats, insects, small reptiles, occasionally small birds, and snails. The group, as a whole, is beneficial to man. The great horned owl, Bubo virginianus, the screech owl, Otus asio, the burrowing owl, Speotyto cunicularia hypogaea, the barred or hoot owl, Strix varia, and the barn owl (monkey-faced), Strix pratincola, are all well known.

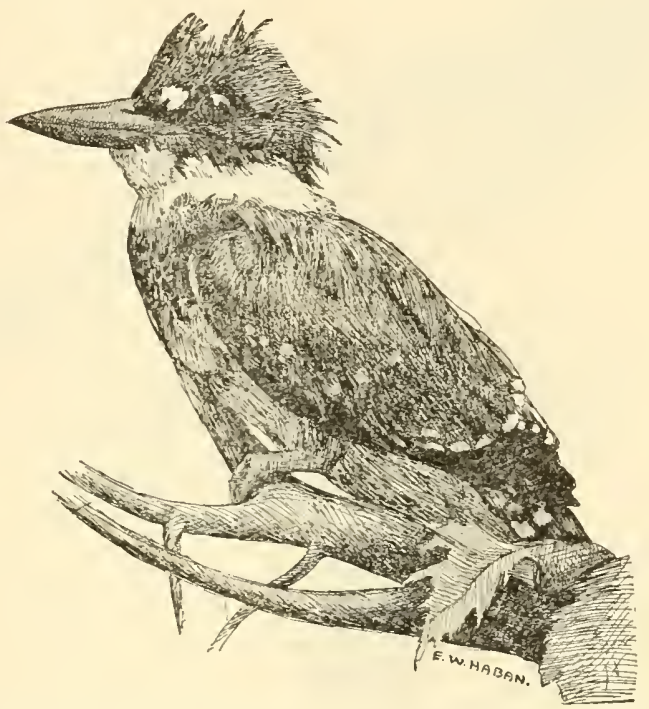

Fig. 314.-Kingfisher, Ceryle alcyon. (From Metcalf, Textbook of Economic Zoology, published by Lea and Febiger.)

Caprimulgiformes (Goatsuckers).-The nighthawk and the whippoorwill are the best known representatives. They are chiefly noctumal, flying during the erening and morning hours and catehing insects as they fly. They are medium-sized bircts with a large gape and weak bill. They excel in their ability to dodge while flying. No nest is built; the eggs are laid in almost any convenient place.

Micropodiformes (Hummingbirds and Swifts).- The former are small, swiftly flying, long-billed birds which are confined to the Americas and are more abundant in the tropies. The feathers of the male are often brightly colored, and the tail in both sexes is long, slender, and usually forked. Their wings beat the most rapidly of 
any birds. They feed on nectar from flowers. The chimney swifts are universally distributed. They have a short bill, broad mouth, short tail with terminal stiff quills, and long, pointed wings. They nest in chimneys, caves, hollow trees and on cliffs, in nests made of twigs and leaves glued together with adhesive saliva. Some forms make the nest entirely from saliva and in China these nests are eaten by man.

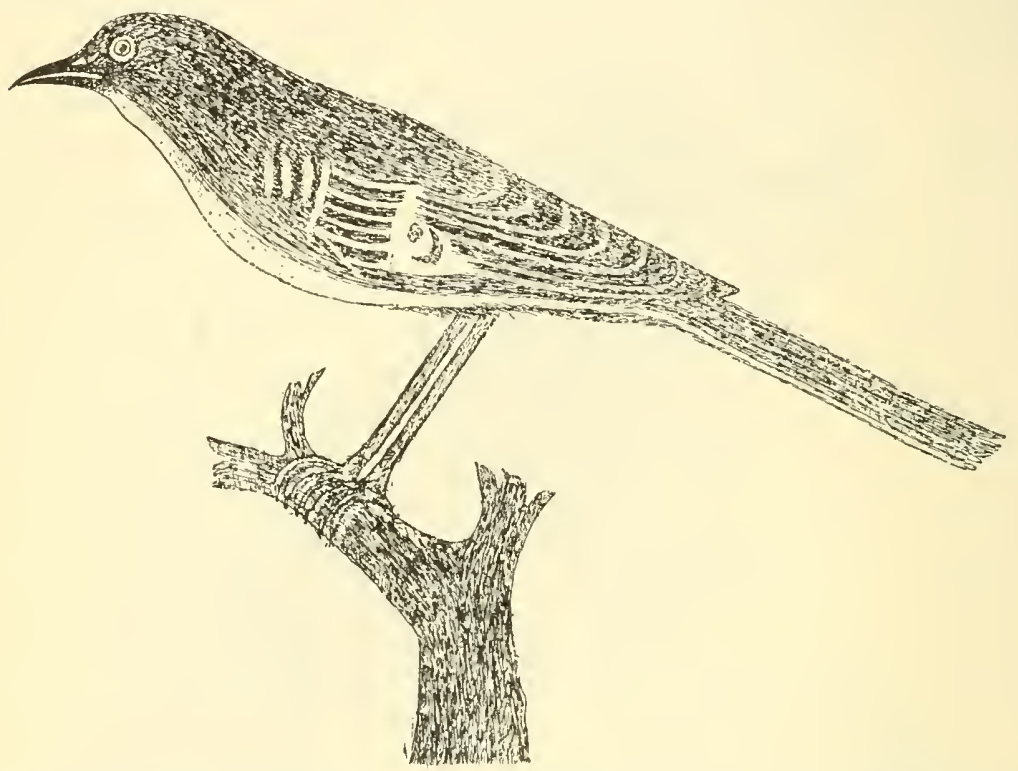

Fig. 315.-Mocking-bird, Mimus polyglottos leucopterus. One of the most common birds of the South and Southwest. (Drawn by Edward O'Malley.)

Coraciiformes (Kingfishers).-This group is represented by the belted kingfisher, Ceryle alcyon, which breeds from northern Canada to the Gulf States. It lives along streams and near ponds to dart down and eatch a fish at every opportunity. They nest in holes in a bank, usually four to six feet deep.

Piciformes (Woodpeckers).-These are arboreal (tree living) birds with stout beaks for boring holes in tree trunks. They feed largely on wood-boring insects, their larvae, and ants. The tongue can be extended some distance beyond the bill in capturing prey. The tail feathers are stiff and are used to prop the animal against the tree while it is working. The flicker (yellowhammer), Colaptes auratus, 
the red-headed woodpecker, Melanerpes erythrocephalus, the hairy, and the downy woodpecker, Dryobates pubescens, are the most common species. These birds are quite destructive to some orchard trees, telephone poles, and occasionally roofs of buildings.

Passeriformes (Song or Sparrowlike Birds).-More than half of the known birds belong to this order. They all have four toes, three in front and one behind on the same level as the other three. There are about sixty families described. They are usually small and most of them have characteristic songs. The arrangement of the toes is an

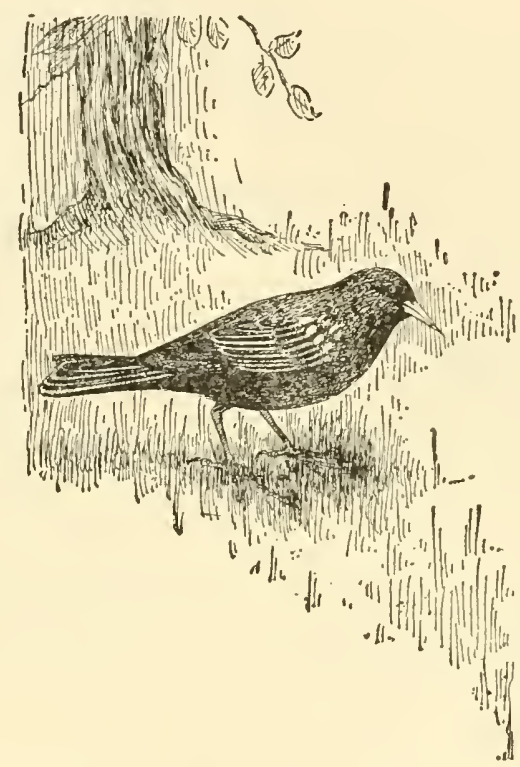

Fig. 316.-Starling, Sturnus vulgaris. An introduced European bird. (From Metcalf, Textbook of Economic Zoology, published by Lea and Febiger, after Snyder.)

adaptation for grasping and perching. There are nineteen families of common birds which make up this large group, including such birds as sparrows of several kinds, cardinals, buntings, tanagers, swallows, kingbirds, larks, phoebes, scissor-tailed flycatchers, crows, jays, starlings, magpies, blackbirds, grackles, cowbirds, bobolinks, swallows, martins, waxwings, shrikes, vireos, warblers, wrens, thrashers, mockingbirds, nuthatches, chickadees, robins, and bluebirds. The starling, Sturnus vulgaris, was introduced from Europe in about 1850. Since that time it has spread restward from the Atlantic seaboard until 
at the present time it reaches the Rocky Mountains and has gone far into Texas on the southwest. It became noticeable in central Texas in the winter of 1935-1936 and plentiful during 1936-1937. It has increased rapidly and it promises to be a nuisance. It may be recognized by its gregarious habits, its rapid darting flight in flocks, its short, thick body, and the fact that it walks on the ground instead of hopping. It is about the size of a cowbird and its color is greenish or purplish black with lustrous brown flecks or streaks.

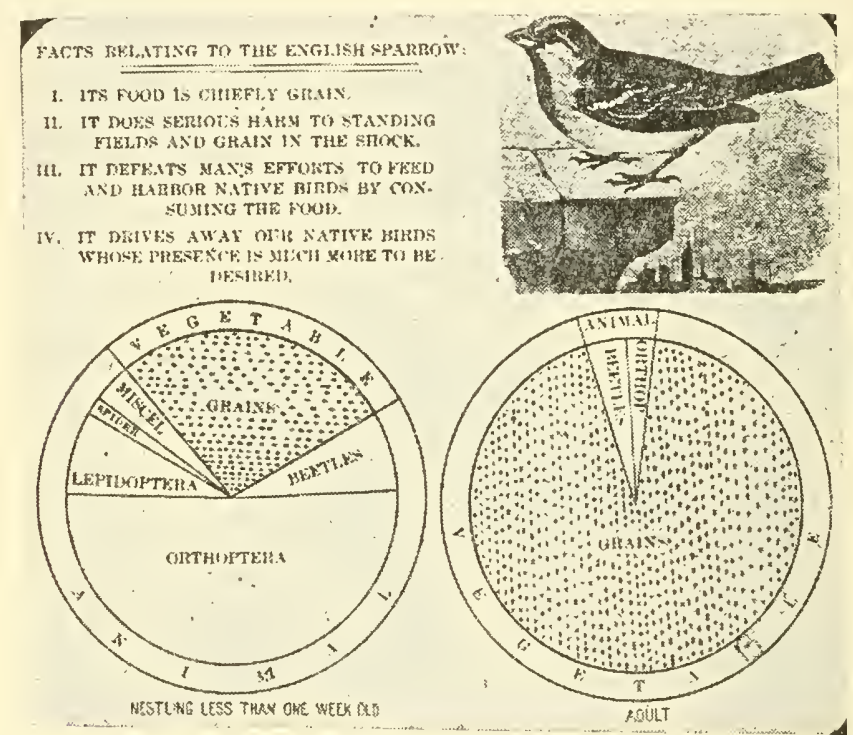

Fíg. 317.-Facts concerning English sparrow. (Courtesy Conrad Slide and Projection Company.)

\section{Economic Relations}

Birds constitute one of our very valuable groups of animals because of their several services to man. At the same time there are some forms which have only a negative importance. The meat of several birds has long been used as food for man. The chief groups among the wild birds that are used as food are ducks, geese, quail, doves, grouse, pheasants, and turkeys. Many other birds are abundant enough and palatable enough, but they are too small. The sparrow is such an example. There are others that are abundant and easy to secure, but are not particularly palatable; this is 
true of the flesh-eating, fish-eating, and particularly carrion-eating forms, such as vultures, eagles, and hawks. During the last few years the common crow has been coming into use as an article of food. Since it lives on an omnivorous diet largely of plant origin, it has a palatable flesh. It certainly is abundant, and it is great sport hunting it. Some restaurants serve it occasionally as a regular part of the menu. The wild ducks and geese have been hunted so extensively that they are no longer a very important source of food; too, they are necessarily strictly protected at present.

The chief domestic birds that are used for food are chickens (domestic fowls), ducks, geese, guinea fowl, pigeons, and peacocks. The domestic chicken is the chief one of these. It likely descended originally from the jungle fowl of India and the East Indian Islands. The original birds were used all through that part of the world as gamecocks. The present game chickens are nearest the jungle fowl of any existing breeds. From this beginning have arisen dozens of valuable breeds of domestic chickens. In thickly populated areas poultry raising is on the increase, not only because the hen is an efficient apparatus for converting grain and garbage into meat and eggs, but also because a large number of chickens may be kept in a relatively small area. Most of the commercial egg production comes from the chicken, which produces more than $2,600,000,000$ dozen eggs a year in the United States alone.

The turkey is a domesticated form of the native wild turkey of North America. It is a very popular and profitable meat-producing bird. Millions are produced for the markets in the southwestern part of the United States each year. The guinea fowl is the descendent of a small turkeylike bird of Africa. It has dark flesh which is prized by some people. The pigeon is used as food principally while it is young. The young pigeon is known as a "squab," and it can be raised in close quarters, such as back yards, in cities.

Besides the production of meat and eggs there are some other important products and services which come from birds. Feathers are used both for ornaments and for pillows. Guano, which is a mixture of the excreta, soil, feathers, unhatched eggs, and dead birds, is rich in nitrogen and is one of the best sources for nitrogen fertilizer. Hundreds of thousands of tons of it are mined each year and sold. It is estimated that in the areas of the bird roosts the 
guano accumulates at the rate of about four inches a year or 750 tons per acre per year. Large numbers of different kinds of birds render service of almost inestimable value in destroying insects, rodent pests, and weed seed. The hawks and owls are very important as rat and mice killers. Finches, sparrows, and quails are destroyers of weed seed. Woodpeckers, chickadees, creepers, wrens, bluebirds, robins, thrushes, flycatchers, kingbirds, meadow larks, swallows, warblers, tanagers, vireos, and many others are very important insect feeders. This asset, if computed financially, would run into millions of dollars a year for the country. At the same time the value of the birds as game for sportsmen to hunt cannot be measured wholly in dollars and cents.

\section{DOMESTIC CHICKEN}

Gallus domestica (bankiva), the domestic fowl, is a convenient animal for study because of its size, its availability, and its universal distribution with man. As previously stated, it is thought to have arisen from the Indian jungle fowl. It is of great interest, not only because of its immense economic value, but because it shows many of the adaptations of this form of animal to its type of life. Although the chicken has partially lost its power to fly, it still retains the features which adapt birds to a life in the air, sucl as feathers, wings, air sacs, hollow bones, and a rigid skeleton. The principal modification of the chicken from its wild ancestors is the relative increase in weight to give the body a stocky build, failure to develop and exercise the short wings, and a great increase in egg production in most breeds.

\section{Habits and Behavior}

This is a diurnal perching bird which spends the time between dusk and daylight sleeping in a squatting position on a perch. During the daylight hours it is an extremely busy animal at hunting food, dusting feathers, running, walking, scratching, and for the females, egg-laying. The flocking habit is fairly well developed, and usually onc cock establishes himself as master of a certain flock and all other cocks are more or less subservient to him. They are entirely polygamous and the mating is promiscuous. 


\section{External Structure}

The body of the gamecock, the Leghorn, and the Bantam is quite spindle-shaped and graceful, while that of the Plymouth Rock or Rhode Island Red is stocky and almost awkward. The body is divided into three general divisions: head, neck, and trunk. The

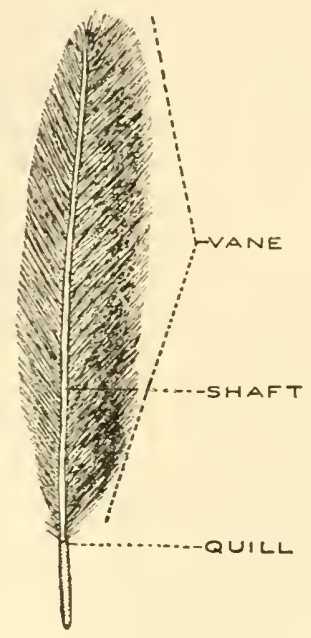

A

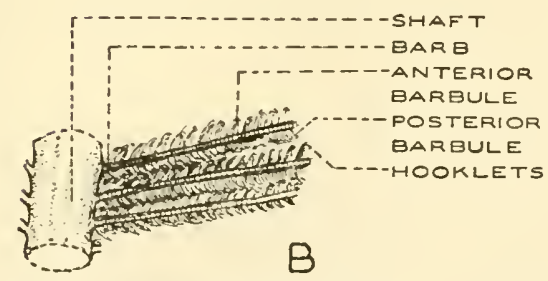

Fig. 318.- $A$, flight feather showing general features; $B$, details of a portion of a feather.

head is prolonged at the mouth region by the pointed beak, whose horny covering is derived from the skin and is true horny material. At the base of the beak there is a small fleshy structure which has a naked, waxy appearance. This structure is known as the cere. In the birds of flight, this is larger and serves as a shield to keep the air from blowing directly into the slitlike nostrils and interfering with breathing. One relatively large eye is located on either side 
of the head. From the location, the vision is necessarily monofocal. The eyeball is slightly movable and is provided with an upper lid, lower lid, and a semitransparent nictitating membrane which extends across the eye from the inner corner. The ear is lacking in external conchal cartilage, but appears below and behind the eye on each side as the opening to the external auditory meatus. This is usually guarded by a few stiff short feathers. On top of the head is the comb and below the angles of the jaws are the wattles. These are highly vascularized, fleshy modifications of the skin in these regions, and are frequently different in the two sexes.

The mobile neck is quite long and joins the dorsoanterior point of the trunk. The trunk is more or less basket-shaped, with the pair of wings folded at the sides. The skin stretching from the body to the wing is the alar membrane. The body stands almost erect on the strong legs. The distal part of the hindlimbs are devoid of feathers but are covered with scales. The toes are provided with nails or claws which originate in the epidermis. The dorsal surface consists of a horny plate which is set in a matrix. The forelimbs and girdle are modified for flight, while the hindlimbs and girdle are modified for bipedal locomotion. The skin which covers the chicken is very thin and does not contain sweat glands, neither does it have a general distribution of oil glands. The oil is supplied by a tail gland or uropygial gland. The bird squeezes a quantity of oil from this gland into its beak and passes the beak over all feathers one at a time until they are all preened. This treatment renders the feathers practically impervious to water.

The feathers are skin developments arising in dermal papillae which are well set in the skin. There are three types of feathers: contour (fight or quill) feathers with a stiff shaft and firm texture; down (plume) feathers with a soft shaft and a free fluffy arrangement of the barbs; and filoplume feathers with liairlike structure consisting of a slender shaft and a few or more branches. A typical feather is composed of a quill which sets in the feather follicle of the papilla of the skin and continues up into the feather as the shaft. The shaft bears slender barbs which extend obliquely from it to make up the principal surface of the feather. The barbs in turn bear still smaller and more slender projections known as barbules, which hook the barbs together. The barbs in normal position make up the vane, and give the feather a smooth surface. At the end of the quill there is a small 
opening called the inferior umbiticus and at the point where the quill emerges from the skin there is another small opening, the superior unbilicus. In many feathers the hyporachis or after-feather arises from this point. The shaft is often called the rachis. The feathers in many parts of the body are developed in rows with long intervals between the rows in which there are no papillae, but these areas are simply covered over by feathers growing in the adjacent areas. The definite feather areas over the body are called pterylae while the intervening featherless areas are apteria (apterylae).

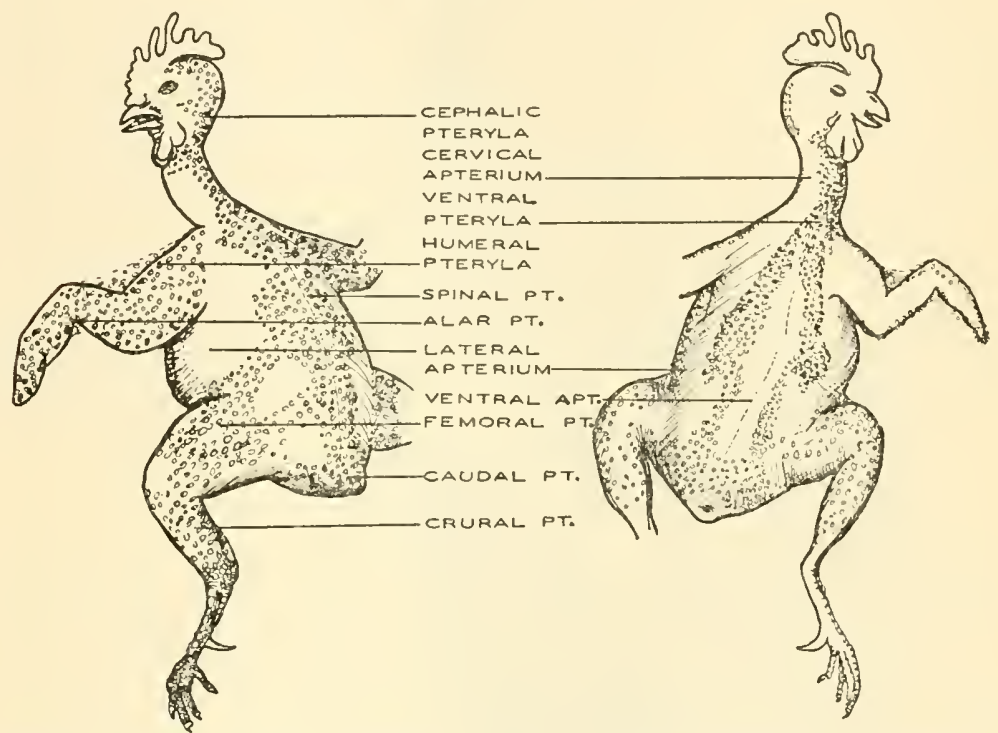

Fig. 319.-Diagram showing feather tracts, or pterylae, and featherless areas, or apterylae (apteria) of a rooster. (Drawn by Titus Evans.)

There is a complete but gradual shedding of the feathers or molt in the fall and a partial one in the spring. Of course, new feathers replace the old as fast as they are shed. There may be some color change of the plumage aecompanying the molt.

\section{Digestive System}

The alimentary eanal consists of the principal organs in order: mouth, pharynx, anterior esophagus, crop, posterior esophagus, proventriculus, gizzard (ventriculus), intestine, large intestine, cloaca, anus. The several accessory organs are beak, tongue, salivary glands, liver, and panereas. The proventriculus and gizzard constitute the 
stomach. The chicken feeds largely on vegetal food, particularly grain and plant seeds. This food is ground in the gizzard by the muscular activity of the wall crushing it against the sand and gravel which have been swallowed and lodged there. The walls of the

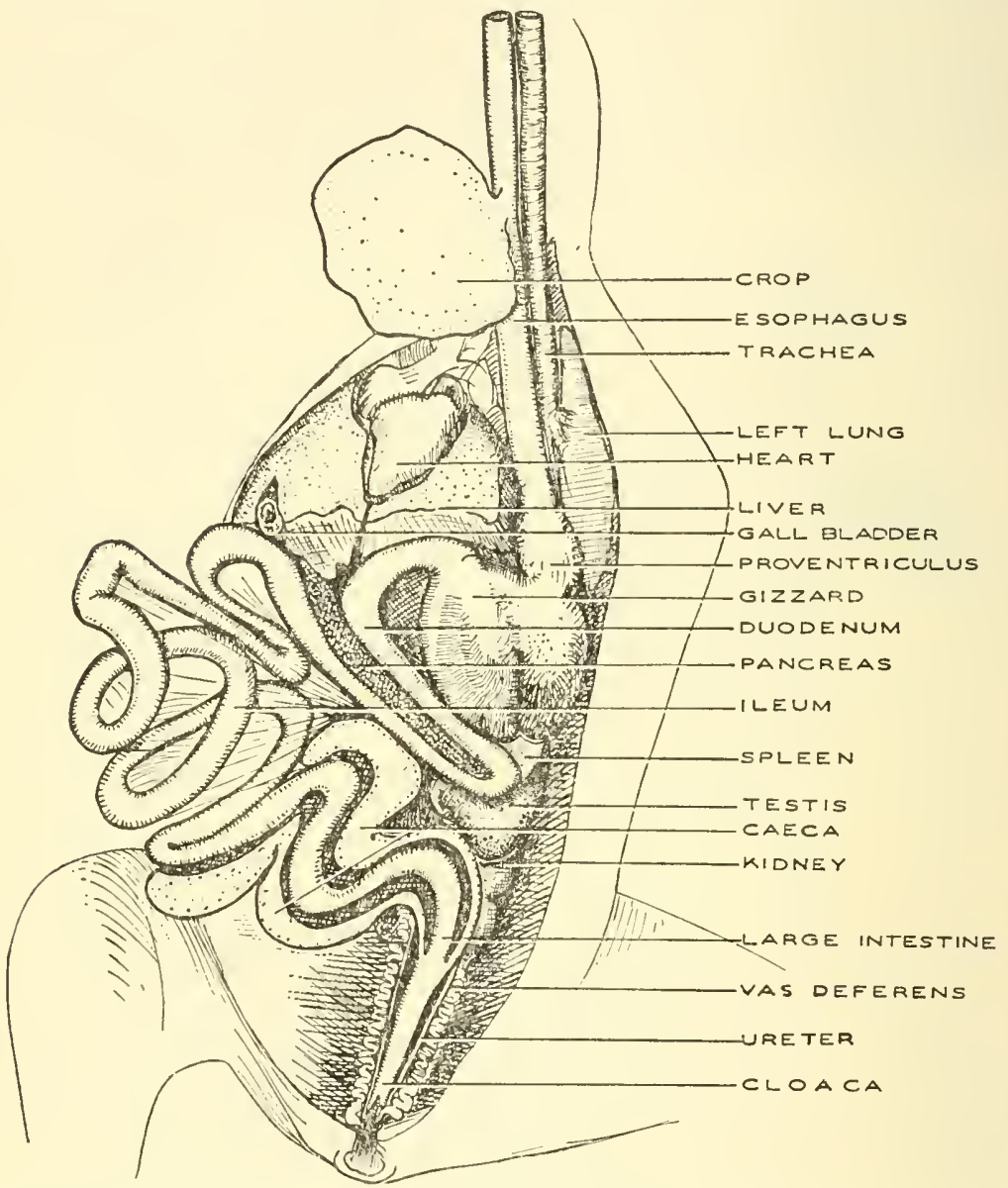

Fig. 320.-Visceral organs of male chicken, ventrolateral view. (Drawn by Titus Evans.)

proventriculus are glandular and secrete gastric juice which softens the food and begins digestion of the protein. The crop, which is a modification of the esophagus, is used as storage space for food as it is ingested. In the pigeon the crop is glandular and secretes "pigeon 
milk" on which the young are nourished. The small intestine is about 60 inches long and is made up of duodenum first, beyond the stomach, and then at the end of the first turn, the ileum begins. It is coiled considerably and leads to the large intestine. The bile ducts coming from the gall bladder and the liver enter the small intestine about fourteen inches below the stomach. The pancreas, which lies beside the duorlenum, also pours its contents into the small intestine. Two blind saes, each about seven and one-half inches in length, and called caeca, extend forward from their point of origin at the juncture of the small and large intestine. They are usually partly filled with a soft, pasty material. The rectum of the large intestine opens into the saccular cloaca. One portion of this space receives the fecal material from the intestine and another portion receives products from the urinogenital organs. The cloaca opens to the outside by the anus. The ileum and caeca serve as the principal organs in which absorption of digested food by the blood occurs. The digestive functions of the bird are very potent and rapid. This seems to be a necessary compensation for the waste caused by their extensive and energetic motions, and their high state of irritability.

\section{Respiratory System}

The chicken and other birds breathe through the nostrils, nasal chambers, pharynx, superior larynx, trachea, inferior larynx (syrinx), bronchi, bronchial tubes, lungs, and air sacs.

Air is brought into the nasal chambers through the slitlike nostrils in the upper jaw. Within the nasal chamber the air is warmed by passing over three scroll-like folds which are the turbinated lamina supported by the turbinated bones. Next it passes to the pharynx through a narrow slit in the hard palate. There is a row of filiform papillae or fingerlike projections marking the junction of mouth and pharynx, and another transverse row of horny ones at the juncture of the roof of the larynx and esophageal margin. There is no epiglottis but simply the slitlike glottis through the anterior wall of the larynx. This is provided with two lips which can be brought tightly together so that nothing can fall through into the larynx when food is being swallowed. From the boxlike superior larynx the air passes through the tubular trachea to the inferior larynx or syrinx where it bifurcates. The walls of the trachea are supported by cartilaginous 
rings which in different kinds of birds will vary from 90 to 120 , depending on the length of the neek. This inferior larynx is the true voice box in the bird because it is here that the vocal apparatus is located. The two semi-lumar internal tympanic membranes, one as a fold on each side of the cavity, are caused to vibrate by the air in sound production. In song birds there is a membrane with a second glottis across the lower end of the trachea. The bony bar supporting it is called the pessulus.

The air goes next into two bronchi which enter the tissue of the lungs. Within the lung each primary bronchus divides into secondary bronchi and beyond this each of these gives off smaller tertiary bronchial branches. The further branches are considered a part of the lung. The lungs occupy only about one-seventh of the thoracic space. Bronchioles which are small branches of the tubules carry the air into the air spaces or alveoli of the lungs where most of the respiratory exchange of gases is made with the blood. Birds lave some bladderlike extensions of the bronchial tubes in the form of air sacs which increase the respiratory surface as well as making the body more buoyant in the air or on water. These sacs are arranged in the neck, thorax, and abdomen, and extend into the cavities of bones. The principal ones are: a single anterior thoracic, a pair of cervical sacs along the neck, a pair of posterior diaphragmatic sacs behind the diaphragm, a pair of anterior diaphragmatic sacs, and a pair of abdominal saes.

\section{Circulatory System}

This system, as a whole, includes the blood vascular system and the lymphatic system. The first consists of heart, arteries, capillaries, and veins, making a closed system through the body, while the latter is composed of spaces, vessels, and capillaries which empty into the blood vascular veins near the heart. The lymphatic system is somewhat of an open system.

The heart of the chicken and most other birds is relatively large and is located near the median line of the thoracic cavity. The double-walled membranous sac, the pericardium, surrounds and holds the heart in place. There are two distinct, thin-walled auricles, and two distinct muscular ventricles. Blood is drawn into the right auricle from the systemic veins; right and left precava, and the single postcava. This blood needs aeration and passes through the 
right auriculoventricular valve to the right ventricle. Then it is pumped, with the contraction of the heart, through a semilunar valve into the pulmonary artery which divides to supply each lung. The aerated blood from the lungs returns to the left auricle by way of the two pulmonary veins. It passes from the left auricle to the left ventricle through the bicuspid or left auriculoventricular valve. Then

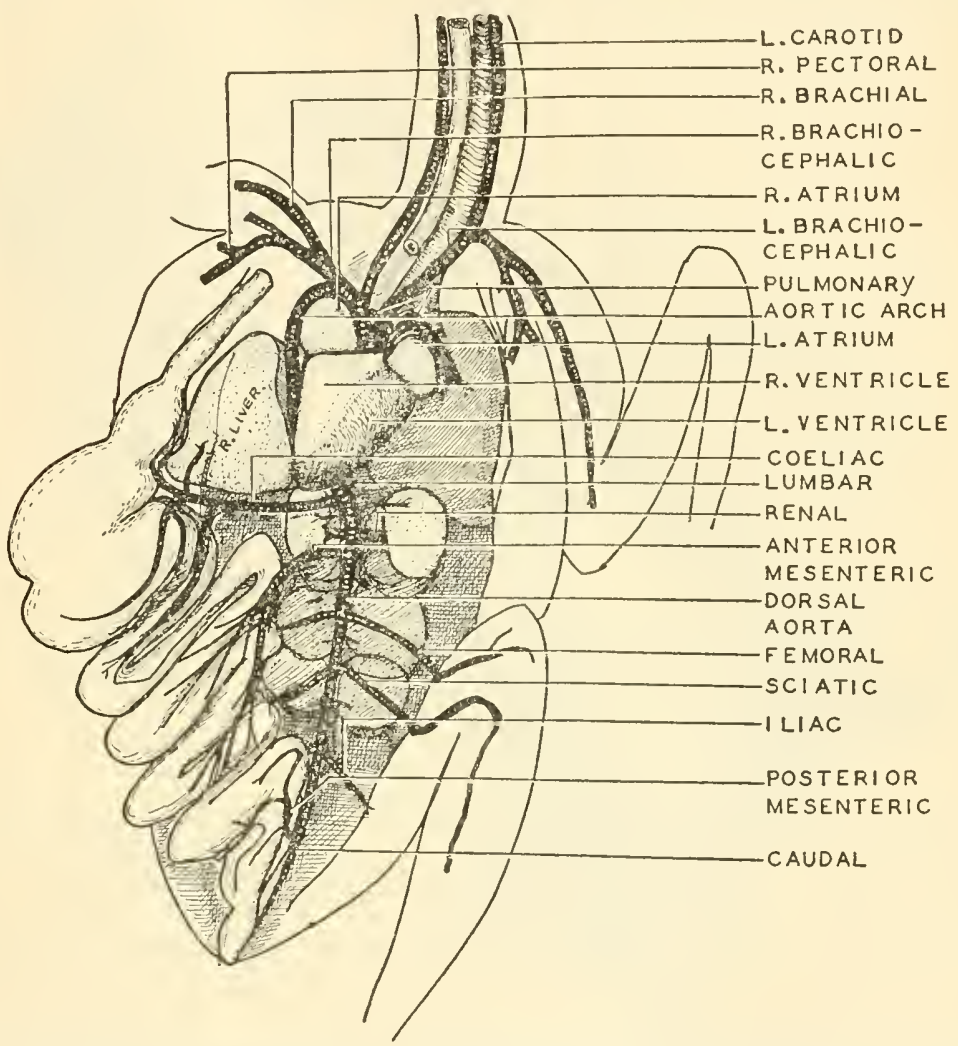

Fig. 321.-Heart and arteries of domestic chicken from ventral view. (Drawn by Titus Evans.)

it is pumped through the semilunar valve in the base of the aorta and out the right aortic arch. Just at the anterior level of the heart this arch passes through the pericardium and gives off the right and left brachiocephalic arteries to the neck, head, and arms; then it curves to the right and extends posteriorly around the heart as the dorsal aorta. The dorsal aorta supplies csophageal branches to the 
esophagus, intercostal arteries to the ribs, lumbar branches to the small of the back, ovarian or spermatic arteries to the gonads, the coeliac axis to the crop, gizzard, duodenum, spleen, and liver; the anterior mesenteric to the small intestine; posterior mesenteric to the large intestine; external iliac or crural arteries branching to the pelvis and thigh; and, the ischiadic continues one to each posterior limb where it branches to all parts.

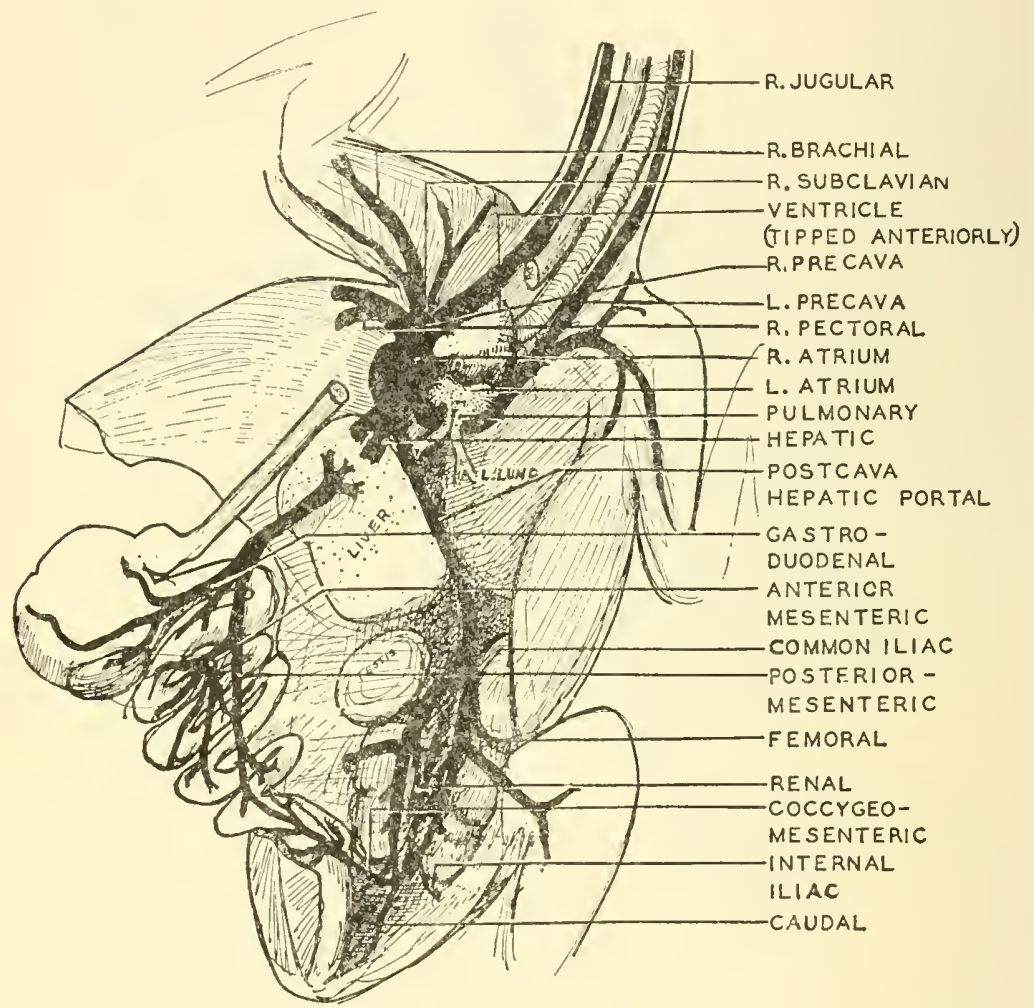

Fig. 322.-Veins of chicken from ventral view. (Drawn by Titus Evans.)

\section{Excretory System}

The urinary apparatus of the bird consists of two metanephric kidneys, from each of which a ureter extends and cmpties into the cloaca. The kidneys are about $2 \frac{1}{2}$ inches long, made up of three irregular lobes, and located above the peritoneum in the lumbosacral region. Each is made up of Malpighian bodies, uriniferous tubules, 
and the surrounding tissue. Urine passes from the collecting tubules in the kidneys to the pelvis of each of the ureters, and through the ureter's into the cloaca. From here it passes to the exterior through the anus with the feces. There is no urinary bladder.

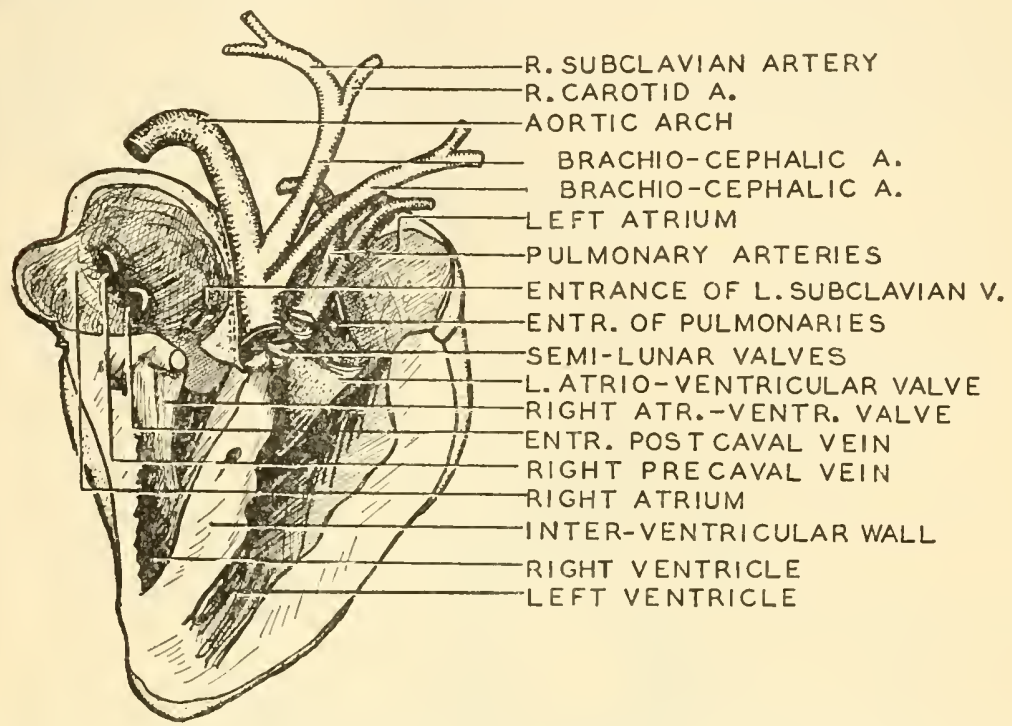

Fig. 323.-Horizontal section of heart of chicken to show chambers and valves from ventral view. (Drawn by Titus Evans.)

\section{Nervous System}

The bird has a relatively well-developed brain and nervous system. In an average chicken the brain weighs about 10 grams. The cerebrum, the anterior division of the brain, shows fair development but no convolutions, while the olfactory lobes which are accessory to it are very small. The optic lobes of the midbrain are well developed and the cerebellum is exceptionally developed. The cerebellum consists principally of a median lobe called the vcrmis or worm. The ventral part of this division of the brain forms the roof of the fourth ventricle of the medulla oblongata.

The sense organs of sight and hearing show improvement when compared with the reptiles. The eye is large, rounded laterally, and rather flattened anteroposteriorly. It has the usual layers of outer sclerotic coat continnous with the cornea in front, the pigmental 
chorioid just beneath, and the membranous retina lining the inside of the posterior portion. The crystalline lens is of a soft texture, is nearly round, and is enclosed in a capsule. The cornea is of a horny consistency and is transparent. Light thus passes rapidly through it to the posterior part of the eyc. There is a comb-shaped membrane stretching from the entrance of the optic nerve at the posterior wall of the eye to the posterior surface of the lens. This structure is called the pecten, and it is supposed that it has something to do either with the nutrition of the eye or with the keen accommodation possessed by the bird. The chicken has a lacrimal duct and sheds tears.

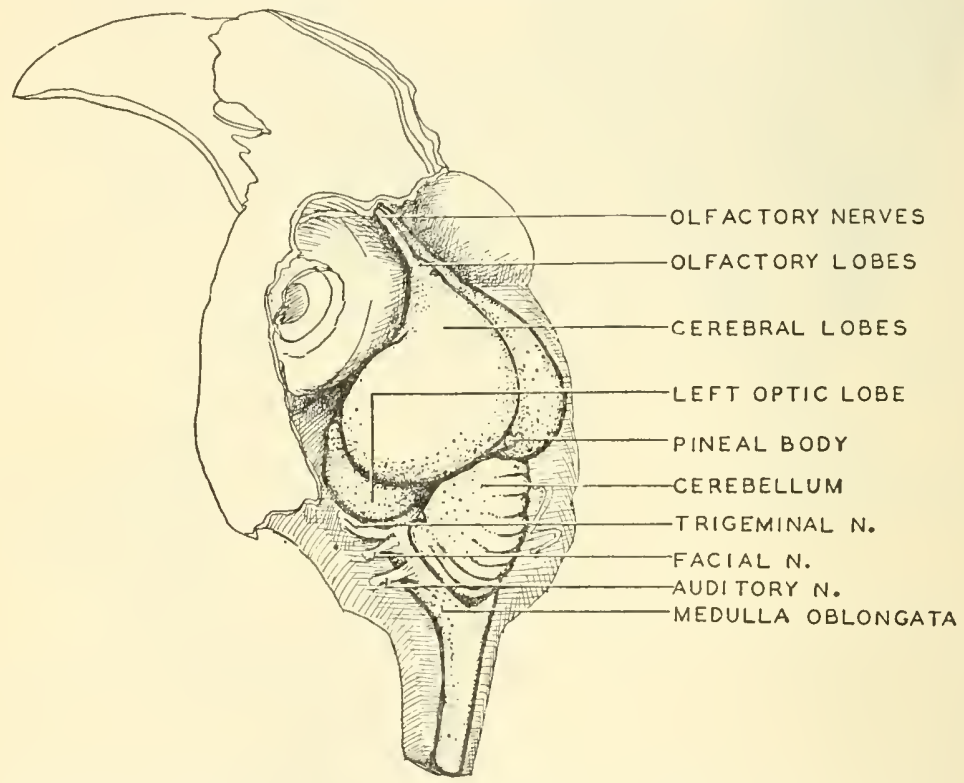

Fig. 324.-Dorsal view of brain of chicken. (Drawn by Titus Evans.)

The organ of hearing is embedded in the skull and consists of the external meatus, tympanic membrane, one ossicle membrane, and the columella which is attached to the tympanic membrane by a cartilage. The inner ear is enclosed in the bony labyrinth and contains the vestibule, three semicircular canals, and the membranous cochlea. The cochlea has arisen from a pocketlike lagena on the vestibule, and is much better perfected in birds than in simpler vertebrates. The Eustachian tube provides a connection between the tympanic cavity 
and the pharynx. This provision allows for equalization of air pressure on the two sides of the tympanic membrane.

The sense of smell and the olfactory apparatus are poorly developed in the chicken. The sense of taste is centered in the taste buds located on the surface of the tongue and on the dorsal palate. This sense is only fairly developed. Touch is distributed over the skin and in certain feathers. The edge and point of the beak are sensitive to touch. Herbst's touch corpuscles are found in all parts of the skin.

\section{Skeletal System}

The skeleton of the bird is remarkable for its rigidity with light weight. Even in the heavy, nonflying chicken, this is noticeable. The strongly keeled sternum serves as the attachment for the strong pectoral muscles which are so important in the flight of most birds. There are short bracing bones between the ribs, called uncinate processes. Many of the bones, even in a chicken, have air cavities in them. These are better dereloped in the better fliers. Teeth are entirely lacking in the adult.

The skull is quite well developed and has a relatively large eranial cavity. The orbit is also large. The cranium consists of three single bones, the occipital, the ethmoid, and the sphenoid. There are also three pairs including the frontals, the parietal, and the temporal. The free quadrate connects the lower jaw with the cranium.

The long flexible neck is composed of sixteen cervical vertebrae, the anterior two of which are named the atlas and the epistropheus (axis). The articulation of the vertebrae is such that the head is well adapted for use in feeding, fighting, and nest building in some birds. The centra of the vertebrae are heterococlous or saddle-shaped. Following the cervical vertebrae there are five thoracic, thirteen lumbosacral, and seven coccygeal in order. The total number is forty-one. The cervical and coccygeal alone have free movement. The pygostyle at the caudal end supports large tail feathers, which are movable. There are seven pairs of ribs, two of them articulating with cervical vertebrae and five pairs related to thoracic vertebrae. The thoracic ribs join the sternum by sternal ribs. The cervical ribs are floating. The sternum joins the pectoral girdle by a union in front with the coracoid. The coracoid articulates dorsally with the scapula, another girdle bone, and with the humerus of the wing. Besides the scapula or shoulder blade the two clavicles join the upper 


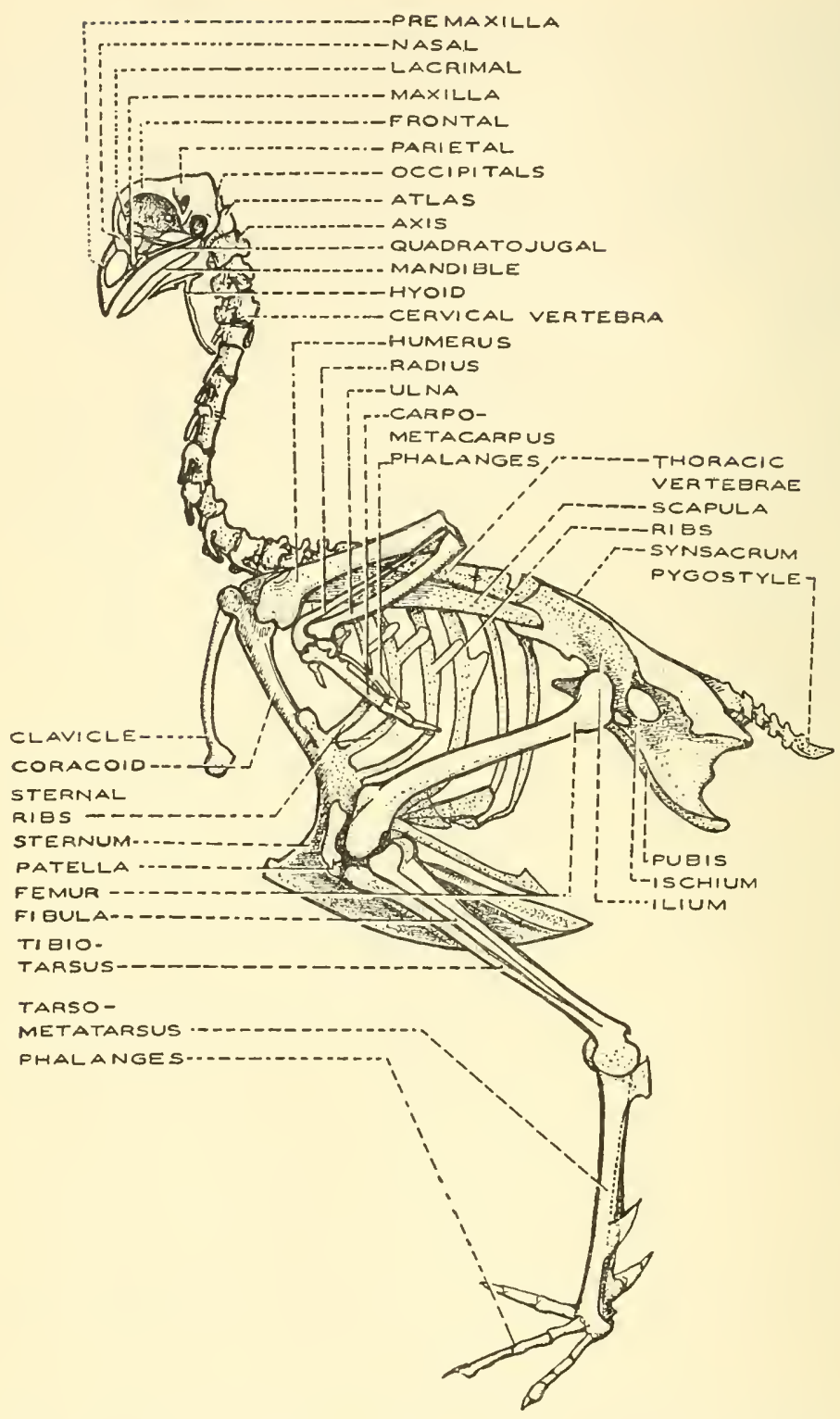

Fig. 325.-Lateral view of skeleton of chicken. (Drawn by Titus Evans.) 
ends of the coracoids and fuse with each other ventrally to form the "wishbone" or "collar bone." The wing is a greatly modified front limb, but strictly homologous to the arm. There is a humerus in the upper arm whose head fits in the glenoid cavity. The radius and ulna support the forearm. The carpus or wrist is composed of two bones, scapholunar and cuneiform, followed by two metacarpals. The first digit or pollex has only one phalanx or joint, the second has two phalanges, and the third is located at the end of the metacarpal bone.

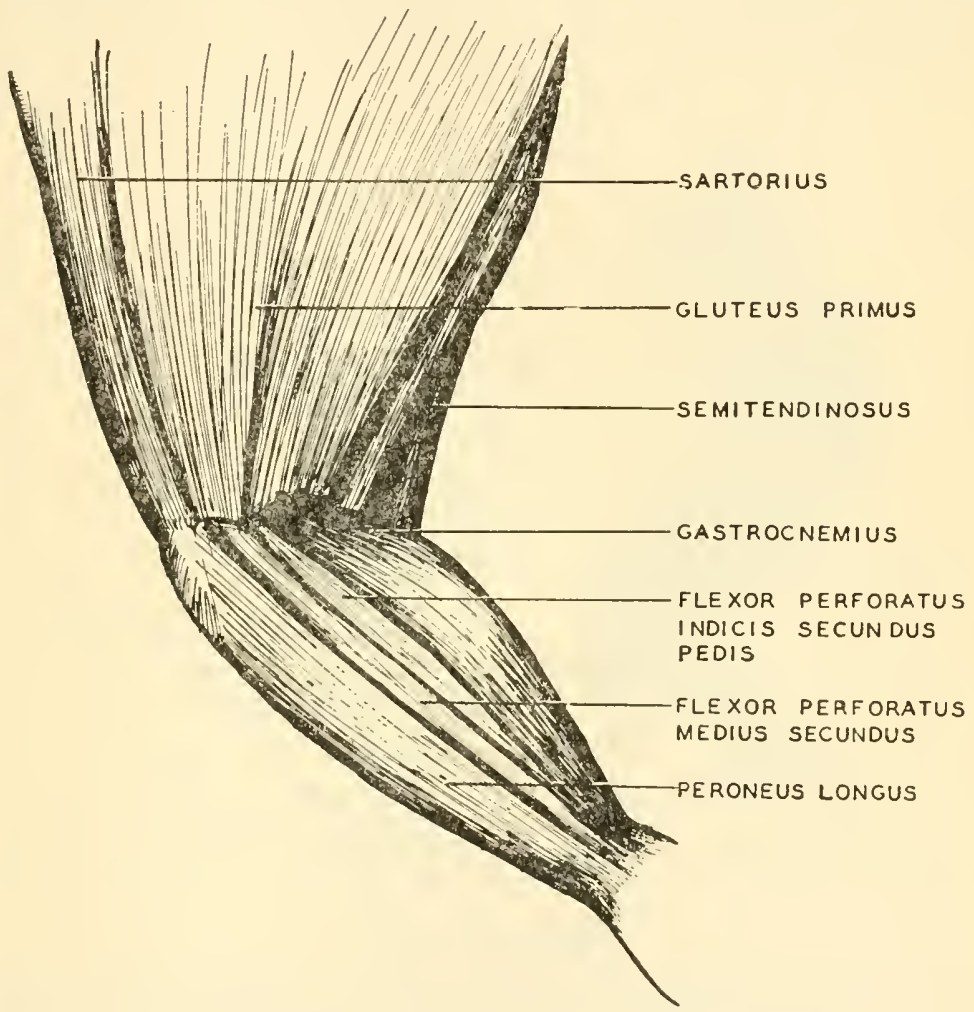

Fig. 326.-Lateral view of the muscles of the hind leg of the chicken. (Drawn by Titus Evans.)

The pelvic girdle is fused quite rigidly to the sacrum. The ilium, ischium, and pubis of cach side are closely fused with each other. The pelvic limb fits into the rounded concavity on each side of the girdle. This socket is called the acetabulum. The hindlimb supports the body in nearly an upright position when the bird is walking. The 
femur is the bone of the thigh which fits close to the body and is covered by the feathers. The section below the knee (shank) is composed of the long, larger tibiotarsus and the slender fibula which is more or less free at the proximal end but is bound to the tibiotarsus along its side. The next portion is a single bone, the tarsometatarsus, which has resulted from a fusion of several. Distal to it are attached the four digits, each composed of phalanges. The first toe is directed backward and the other three forward, as an adaptation for perching. In the male chicken there is a horny spur projecting backward from the tarsometatarsus.

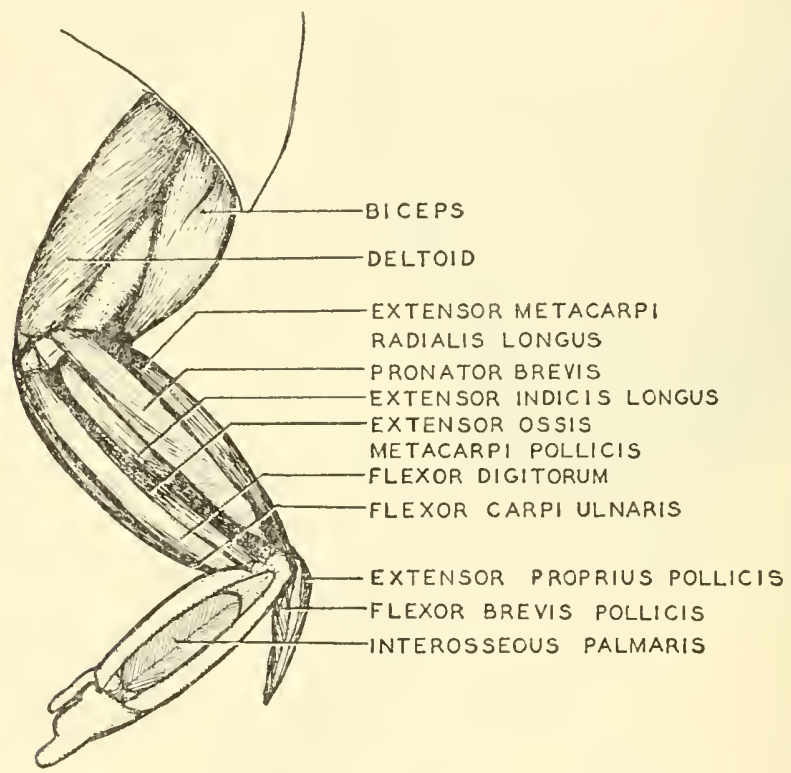

Fig. 327.-Ventral view of the muscles of the wing of a chicken. (Drawn by

\section{Muscular System}

In the chicken there are 162 voluntary, striated muscles, single or in pairs. These muscles are named in some cases from their location, others from their attachments, some for their shape and form, others from their use or function, and still other's from their direction in the body. They are covered and bound together by the white fibrous comnective tissue sheath, called fascia.

The muscles of the chest and forelimb are quite well developed as an adaptation to flight. The muscles of the thigh and shank are 
well developed for bipedal locomotion and perching. The muscles of the long flexible neck are quite intricate. Since this animal does no chewing, the jaw muscles are not strongly developed. The large pectoral muscles which extend from the sternum to the upper arm (Fig. 327 ) constitute from 15 to 20 per cent of the weight of the body and make up the breast meat of the bird. The muscles of the thigh and shank or "drumstick" are quite well developed, and the chicken is a strong rumner (Fig. 326). Certain muscles and liganents of the legs are arranged in such a way that the bird is able to eling to the pereh due to the down pull of the weight of the body. This makes it possible for the chicken to sleep on the perch without clanger of falling off.

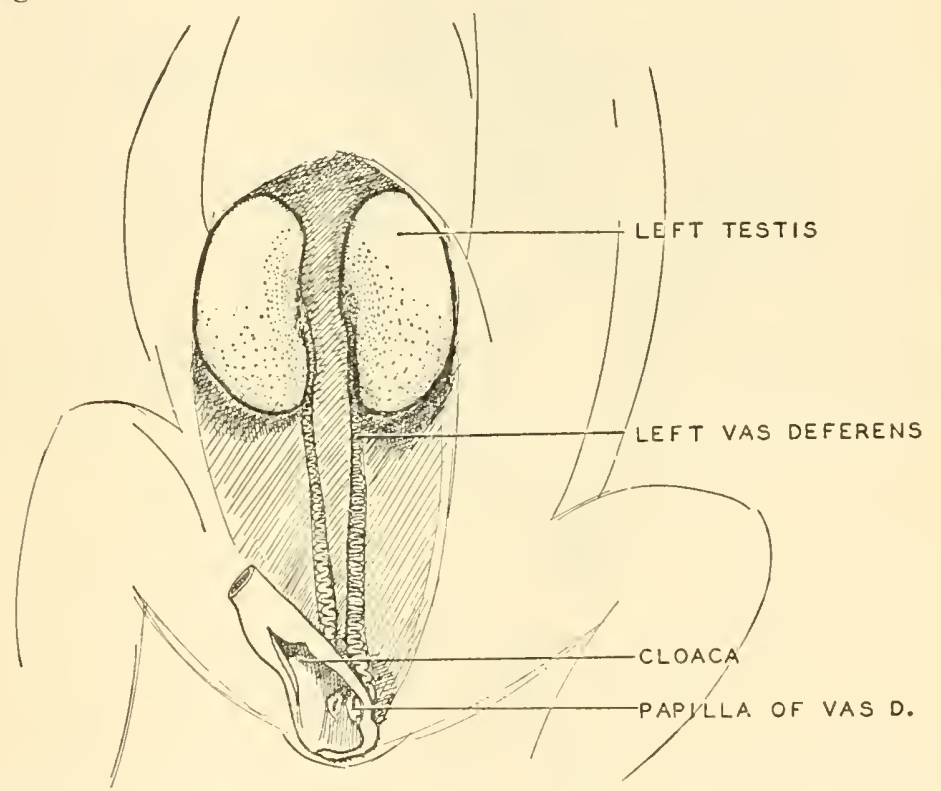

Fig. 328.-Reproductive organs of male chicken. (Drawn by Titus Evans.)

\section{Reproduction and Life History}

The sexes are distinct but there is no definite pairing for repro. duction; one cock may mate with several hens. The eggs are usually laid in a rounded nest constructed of hay or other soft material. Some birds build no nest at all, while others build rery elaborate ones, such as the hanging model of the Baltimore oriole. Copulation is necessary for the transfer of mature spermatozoa from 
the cloaca of the male to the cloaca of the female. This is accomplished by the cock covering the back of the hen and bringing the cloacal apertures of the two together. The motile spermatozoa migrate up the uterus and oviduct to meet the mature eggs and unite with them in fertilization. In the cock there is a pair of oval, light-colored testes located near the dorsal body wall anterior to the

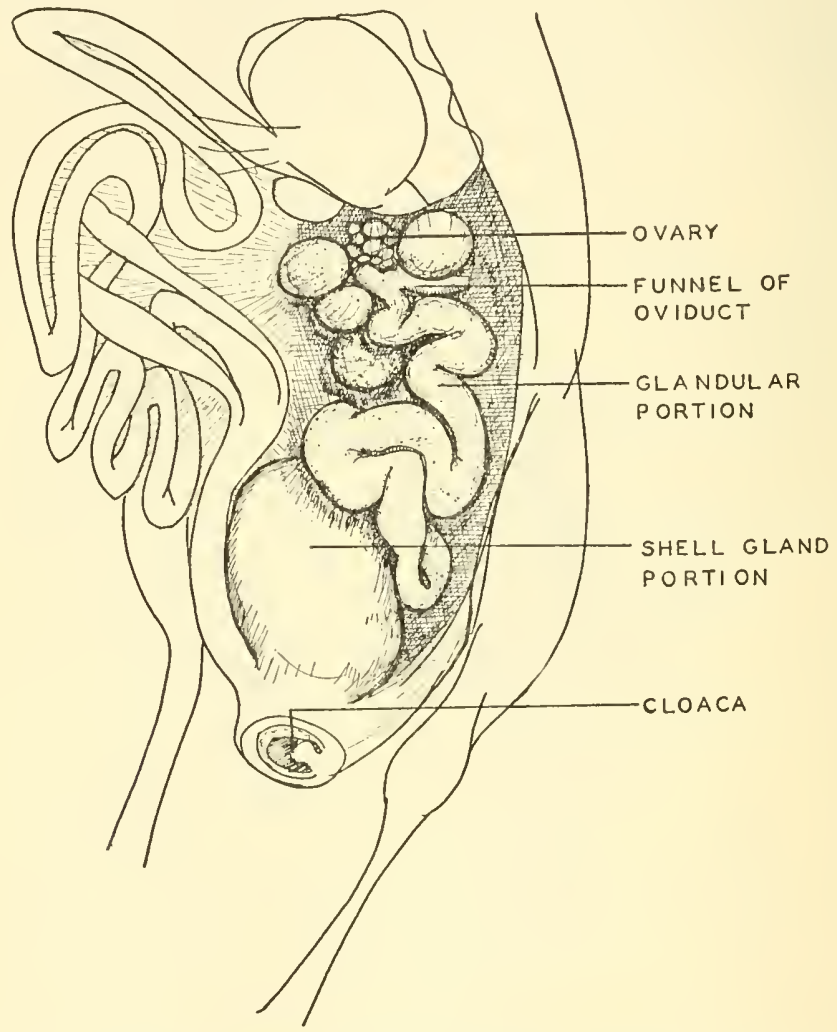

Fig. 329.-Reproductive organs of female chicken. Notice the single left ovary and oviduct. (Drawn by Titus Evans.)

kidneys. From each testis the vas deferens, a duct, leads posteriorly to open in the cloaca. Just before joining the cloaca this duct is dilated to form a storage sac, the seminal vesicle.

The adult hen has only the left ovary and oviduct, the right having atrophied during embryonic development. The mature ova rupture the wall of the ovary and pass into the oviduct. The 
glandular wall of the oviduct secretes much of the albumen or "white" around the egg. In the lower part of the oviduct, the shell membranes are added, and the calcareous shell is deposited in part here and completed in the uterus and vagina. The time required for the egg to pass from the ovary to the exterior at laying averages about twenty-two hours. The average-sized hen's egg weighs about two ounces of which 11 per cent is shell, 32 per cent yolk, and 57 per cent albumen. A dozen or fifteen eggs are usually laid in the same nest by the hen and then incubated with her body temperature by sitting on them almost constantly for twenty-one days. They are kept at a temperature between $98^{\circ} \mathrm{F}$. and $100^{\circ} \mathrm{F}$., and are turned over each day to avoid internal adhesion of the embryo to shell membranes. At the end of this period they hatch by breaking through the shell with the temporary "egg tooth" on the beak. The chicks are covered with down, have their eyes open, and can run as soon as they are dry. For this reason they are said to be precocial, and since they leave the nest immediately, they belong to a general group called the nidifugae. Another type of bird which requires parental care, such as feeding, is said to be altricial, and since it remains in the nest, it belongs to the group nidicolae. The individual reaches maturity in from six to ten months, depending on the breed. 


\section{CHAPTER XXXI}

\section{MAMMALIA}

The class Mammalia (măm'mā'lĭ á, milk-forming) includes many of the animals most familiar to man. Nearly one-third of the total number of them occur in America. Besides our common domestic animals, such familiar forms as rats, mice, ground hogs, bats, foxes, bears, deer's, seals, whales, man and many other's belong in this group. The skin of them all is provided with sweat glands and sebaceous (oil) glands and is more or less covered with hair. These animals are typically quadrupeds with five digits on each limb. The females have well-developed mammary glands which secrete milk for the nourishment of the young. There is usually a distinct division of the body into head, neck, trunk, and tail regions. All mammals have a definite temperature regulation and are said to be homoiothermal, or warm-blooded. The body temperatures of different species vary from $77^{\circ} \mathrm{F}$. to $104^{\circ} \mathrm{F}$. In mammals, as in birds, the heart is completely divided into four principal chambers. The single systemic arch of the aorta is the left one. Respiration is carried on by lungs and the anterior end of the trachea is modified to form a larynx capable of sound production. In general, Mammalian blood contains nonnucleated, circular red corpuscles; however, in the camel they are oval in shape. The thoracic cavity of the mammal is separated from the abdominal cavity by a complete diaphragm.

\section{Classification}

The entire class includes approximately 4,000 species of living mammals and 3,500 fossil forms. The class is divided into two subclasses, several divisions and a number of orders.

Subclass Prototheria.-The primitive, egg-laying mammals. This group includes only a single order.

Order Monotremata.-The most primitive mammals and the only ones that lay eggs are placed here. They are limited in their distribution to Australia, Tasmania, and New Guinea. The eggs are similar to turtle eggs and are laid either in a ponch on the female's abdomen, as in the spiny anteater, or in a tunnel in the ground near 
water, as in the duckbill. The oviducts in these animals do not unite to form a vagina, but empty directly into the cloaca, which is present in this subclass of mammals only. After hatching, the young are nourished for a time on milk from the mammary glands of the parent. These glands secrete their milk on to the hair of the abdomen and the young either suck or lick it up from here. There are two quite representative animals of this group. One is the duckbill or duck mole, Ornithorhynchus anatinus, which is about a foot and a half long; it is covered with hair, has webbed feet, and a peculiar duck bill snout. It feeds on worms which it digs from the mud with its bill. During the daytime it sleeps in its grass-lined tumnel, the entrance of which is under water. The eggs

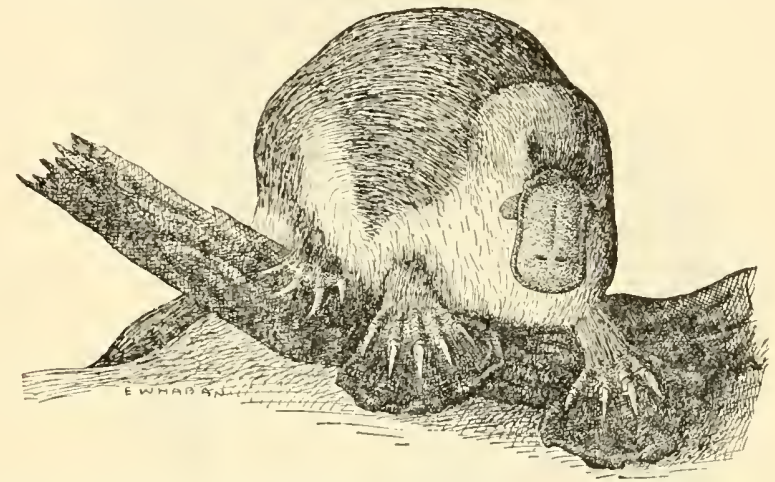

Fig. 330.-The duckbill, Ornithorhynchus anatinus. The "duck mole" of Australia. (From Metcalf, Textbook of Economic Zoology, published by Lea and Febiger.)

are laid and the young reared in this underground chamber. On the heels of the hind feet of the mole are some spurs which are supplied with poison from a gland located in each thigh.

A second representative of the order is the spiny anteater, Tachyglossus aculeatus, which is about one foot long and covered with stiff spines mixed with coarse hair. It has a head and mouth drawn out into a long proboscis with a long, slender tongue which is protruded for picking up ants and other insects. This animal lives in a burrow.

Subclass Eutheria.-The group includes the true viviparous mammals and is divided into two divisions, the marsupials or pouched mammals (Didelphia) and the placental mammals, Monodelphia. 


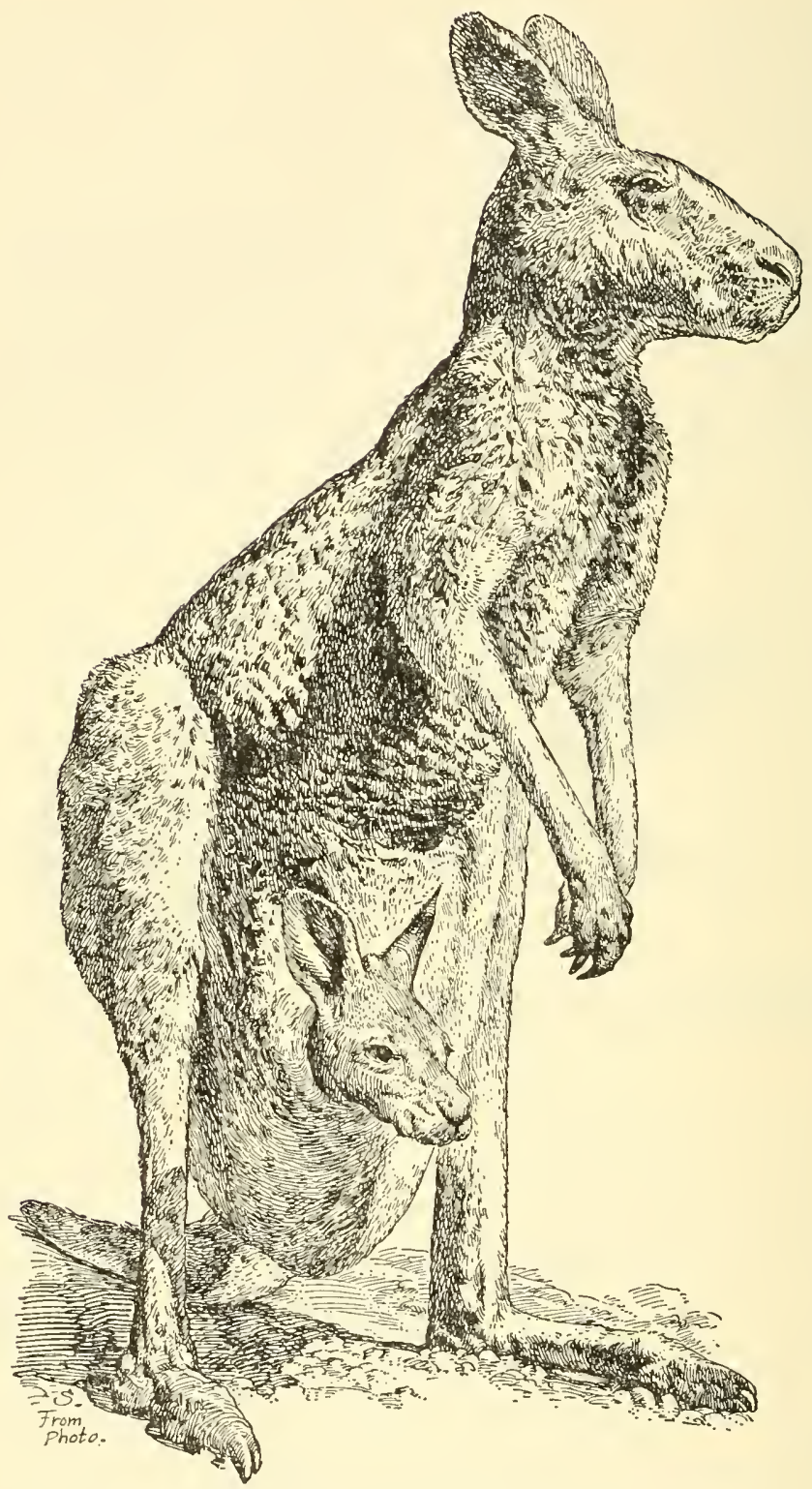

Fig. 331.- Kangaroo carrying young in the marsupial pouch. 
Order Marsupialia.-This group of mammals lias no well-developed placenta and is sometimes designated as a separate subclass, called Metatheria. Their shell-less eggs absorb food from the wall of the uterus. The young are born in a very immature state and make their way to the marsupial pouch where they cling to the teats and are nourished on milk until they ean shift for themselves. The group is at its best in Australia and nearby islands. Here is found the true kangaroo with its short, poorly developed forelimbs, powerful hindlimbs and tail, and peculiar upright posture and leaping locomotion. The seven families of the order include not only kangaroos, but also opossums, phalangers, wombats, bandicoots, dasyures, and Caenolestes. There are several species in South America. Some of these are no larger than mice or rats and are frequently brought into this country on bunches of bananas. In the United States, the opossum, Didelphis virginiana, is the only representative of the group. It is about the size of the common cat, with a long scaly tail and fur of dirty yellowish white color. Ten or twelve young are born at a time and are carried in the pouch of the mother. The young remain with the mother about two months, often riding as a group on the mother's back during the latter part of this period. The opposum is quite active at night, but it usually sleeps through the day.

Order Insectivora.-This group includes the common mole, Scalopus aquaticus, the hairy-tailed mole, Parascalops breweri, the star-nosed mole, Condylura cristata, the shrews and short-tailed shrews. They are quite well distributed throngh North America and Europe, but are absent from Australia and most of South America. The group is largely burrowing and noeturnal in habit. They feed chiefly on insects which they seize with their sharp, projecting incisor teeth. The moles are well adapted to the burrowing habit of life. They have rudimentary eyes, no external ears, short stout forelimbs with strong sharp claws for digging. Their tumnels are just under the surface of the sandy loam in which they live. They occasionally throw up molehills along the tunnel.

The shrews are small, mouselike animals with conical, pointed heads, ratlike feet, small eyes, and external ears. They may live in burrows or on the surface of the ground under logs, rocks, or heavy vegetation. The long-tailed shrew, Sorex personatus, of the North and the East, and the short-tailed shrew, Cryptotis parva, ex- 
tending into the South and Southwest, are the common forms. Because of their small size and retiring habits, these animals are seldom observed.

Order Chiroptera.-Bats are the mammals which have developed. the power of flight and are not always distinguished from birds by the layman. The forcarm and fingers are extended, and the skin stretches between them as well as to the hind limbs. Most of them are small and nocturnal. They usually have the toes of the hind feet developed for grasping and are able to hang by them, head downward, when at rest. The brown bat, Eptesicus fuscus, is the most common form in the United States. The Mexican free-tailed

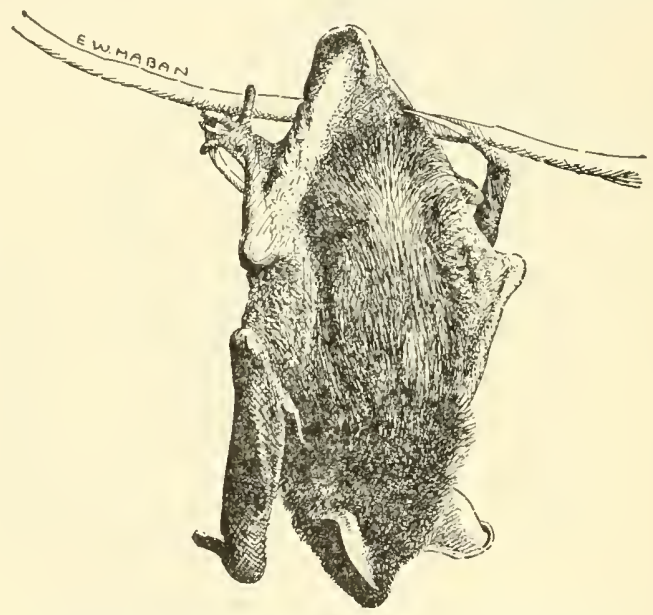

Fig. 332.-Little brown bat, Myotis lucifugus, in resting position. (From Metcalf, Textbook of Economic Zoology, published by Lea \& Febiger.)

bat, Tadarida mexicana, extends as far north as central Texas. Carlsbad Caverns near the eastern boundary of New Mexico is famous for its evening bat flight. The bats come out like a cloud of smoke, make a definite flight of about sixty miles along two streams and back to the caverns before daybreak. Here they remain until the next evening.

Becanse of their ability to fly the bats are widely distributed mammals, being found on all of the continents and even on isolated islands. In the East Indies, Australia, Africa, and Asia, there are several different fruit-eating bats. In tropical America there are true and false vampire bats. The true vampires live on the blood of horses, sheep, cattle, and occasionally sleeping human beings. Their 
teeth are well adapted for bringing the blood on the victim. It is then lapped up from the wound.

Order Edentata.-This is a group including the giant anteaters, sloths, and armadillos. The giant anteater is the only one in which the teeth are entirely absent; they are modified in other forms by lack of enamel. The giant anteater, Myrmecophaga jubata, reaches a length of six or seven feet. Its long claws are used to open the anthill, and the long prehensile tongue is used for taking up the ants. The sloths live in trees, clinging to the underside of the limbs by the long, clawed feet. The animal is ventral side up. They can even sleep in this position. Their food is principally leaves and buts. They are very slow moving animals and inhabit Sonth and Central America.

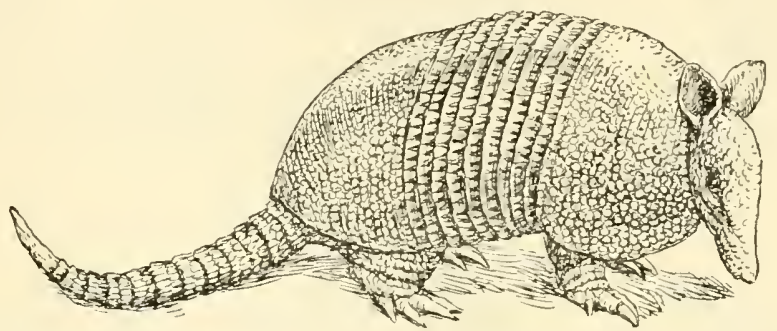

Fig. 333.-Nine-banded armadillo, Dasypus novemeinetum.

The nine-banded armadillo, Dasypus novemcinctum, is the only one of this group inhabiting the United States. It ranges from Argentina to southern New Mexico and northeast to central and east Texas. The head is small, tail elongate, and the body is covered dorsally by bony plates. The ventral parts of the head and body are covered with bristly hair. The nine bands are formed around the trunk by the arrangement of the scutes in that region. These animals dig very rapidly in the ground, and when they are frightened they can roll themselves into a ball as does a pill bug. The normal litter of young is quadruplets produced from a single fertilized ovum (Fig. 334).

Order Pholidota.-This is a group of scaly anteaters found in Africa and eastern Asia. They are well protected by epidermal scales and can roll up like armadillos. They are from one to five feet long.

Order Rodentia.-These animals are the gnawers and compose one of the largest orders of mammals. Such forms as pocket gophers, 
rats, mice, ground squirrels, squirrels, chipmunks, prairie dogs, kangaroo rats, cotton rats, wood rats, muskrats, woodchucks, porcupines, guinea pigs and beavers are representative. They all have long chisellike incisors but no canine teeth.

The prairie dogs (Cynomys ludovicianus) are heavy-bodied, burrowing, rodents that live on the plains west of the Mississippi River.

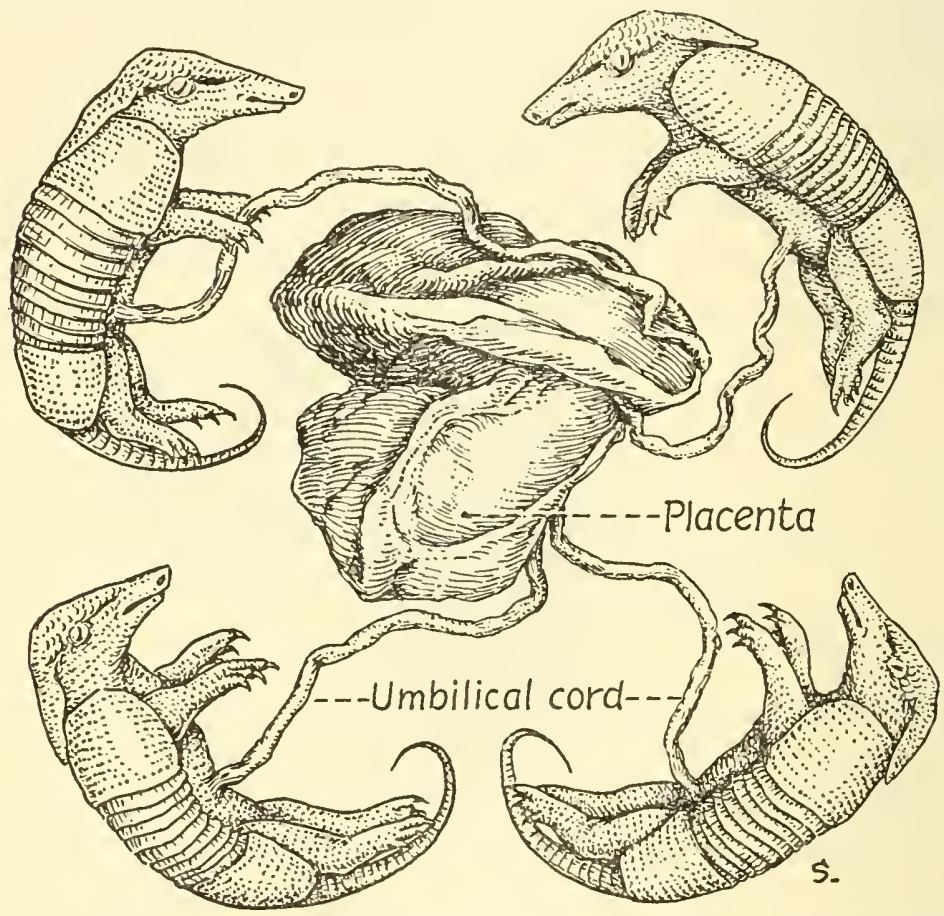

Fig. 334.-Identical armadillo quadruplet embryos attached to the placenta.

These animals are gregarious, living in "towns" or colonies. The burrows are provided with a mound around the entrance, and they are usually quite deep and fifteen to thirty feet apart. Forty or fifty acres of land may be covered by one town.

The ground hog or woodchuck, Marmota monax, is a larger solitary, burrowing animal which hibernates during the winter. It bears about six young in the burrow early in the spring. The ground squirrels are also burrowing animals somewhat similar to the ground hog, but much smaller. They are found from the Mississippi basin 
to the West Coast in shallow burrows in the open fields or low brush. The striped spermophile is a common midwestern form. The chipmunk, Tamias striatus, a small striped squirrel, with a slender nonbushy tail, usually lives on the border of the woods. It feeds

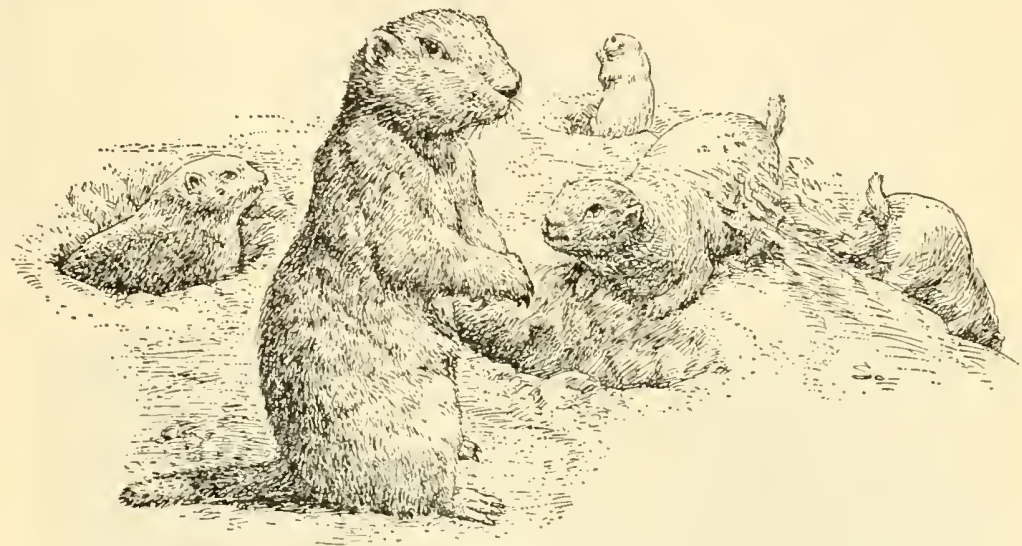

Fig. 335.-Prairie dog, Cynomys hudovicianus. Lived in large colonies or "towns" in great abundance over the prairie country at one time.

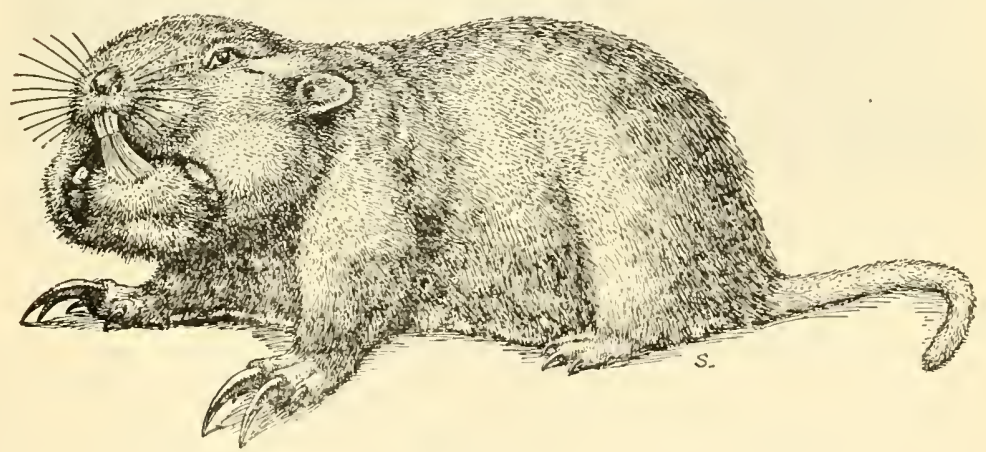

Fig. 336.-Pocket gopher, Geomys bursarius. A burrowing rodent with destructive habits.

on seeds and nuts which it usually stores for times of need. The squirrels are excellent tree climbers and in fact live in trees most of the time. The red squirrel, Douglass squirrel, gray squirrel, Abert squirrel, fox squirrel, and flying squirrel are the common species. The gray squirrel is more common in the South while the Abert is found in the Rocky Mountain region and the Douglass is the Pacific 
form of the red squirrel. Most of these squirrels live on nuts, acorns, and seeds primarily.

The pocket gopher, of the family Geomyidae, is another burrowing animal which ranges through the South, Southwest, and Midwest. They are provided with strong incisors, large cheek pouches, and large digging claws. They are very able digger's and construct extensive burrows about 12 to 18 inches below the surface. Surplus earth is pushed to the surface in mounds. The cheek pouches are used for gathering tubers, such as potatoes, roots, and seeds, for food. The pocket mice and kangaroo rats are other related forms found in

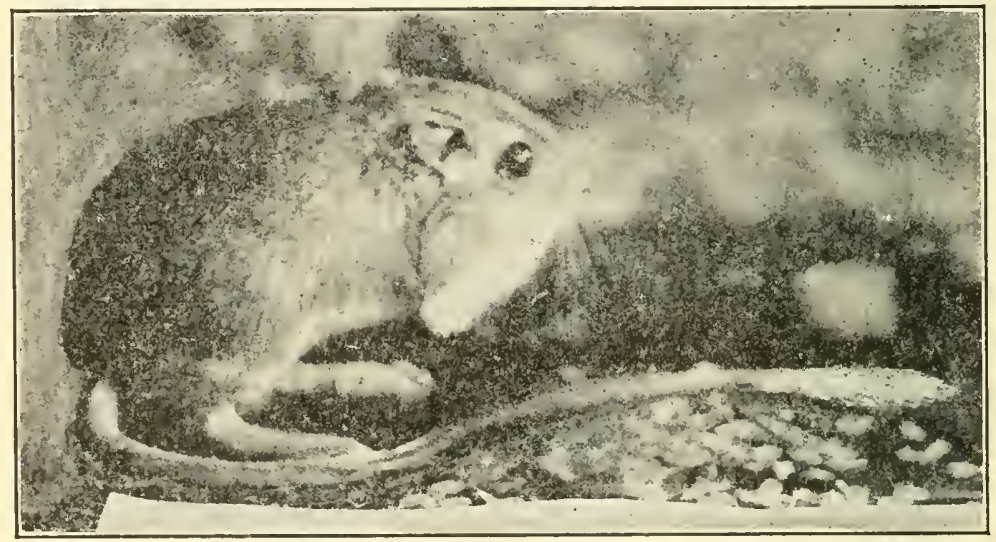

Fig. 337.-Banner-tail kangaroo rat, a common resident of the plains and desert. (Courtesy of Nature Magazine.)

the Great Plains region and Southwest. These mice have cheek pouches and long, bush-tipped tails. The kangaroo rats usually live in colonies in sandy land. They build extensive tunnels. These animals are built like small kangaroos with long hind legs and strong tails.

The beaver, Castor canadensis, is the largest animal of this order, reaching a length of from three to three and one-half feet. At one time beavers were distributed over most of North America, but now they are reduced to a few in out of the way mountain streams. The beaver has a stout body, strong eutting teeth, webbed hind feet, and a broad, flat naked tail. It is well adapted to aquatic life and builds 
dams for its home. The dams are built of trees which are cut with its teeth, floated into position, and chinked with mud.

The muskrat, Fiber zibethicus, belongs in a large family with lemmings, rats, meadow mice, and white-footed mice. It lives in slow streams, ponds, and swamps, and feeds on roots of water plants, fresh-water mussels, dock, corn, and other grain when it is available. It builds houses of stalks, leaves, cattail leaves and mud out in the water of swamps. In ponds it uses a burrow in the bank. The home is lined with cattail down or grass; here it rears the young and spends the winter.

The porcupine, Erethizon dorsatus, is principally a northerm animal but is found in the mountains as far south as Virginia and also

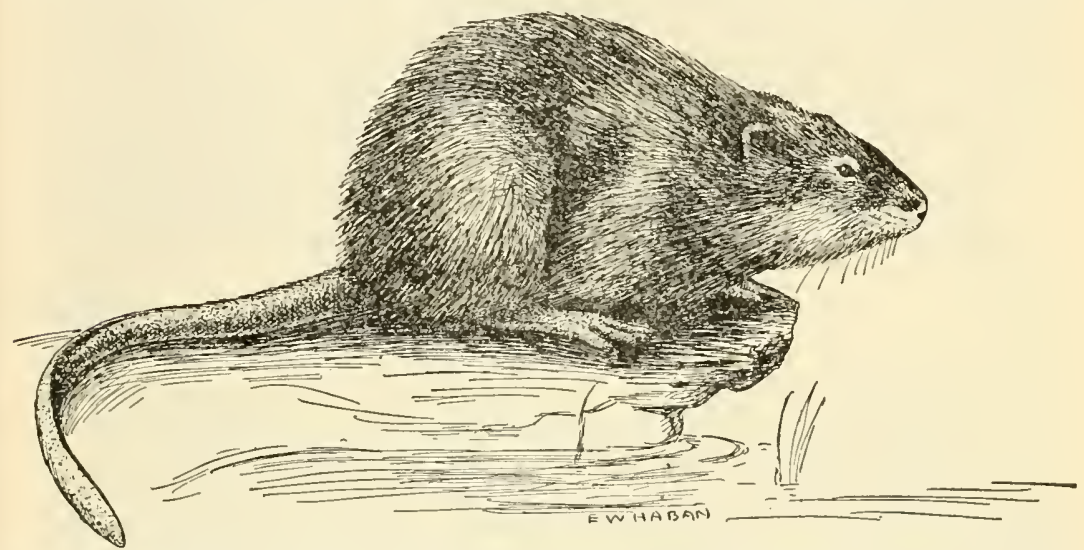

Fig. 338.-Muskrat, Fiber zibethicus, an important fur bearer. (From Metcalf, Textbook of Economic Zoology, published by Lea and Febiger.)

in the mountains of the Southwest in Texas and Arizona. These animals have the hairs of the back modified into spines which normally lie flat, but which can be elevated by muscles when the animal is frightened or angered. The black rat, Rattus rattus, the Norway rat, Rattus norvegicus, and the common mouse, Mus musculus, have all been introduced into this country from Europe. The chinchillas, viscachas, and the cavies (guinea pigs) have all been introduced from South America.

Order Lagomorpha.-The rabbits and hares constitute a very interesting and important group. The jack rabbit is the most common hare of the western plains, mountain region, and Southwest. It is 


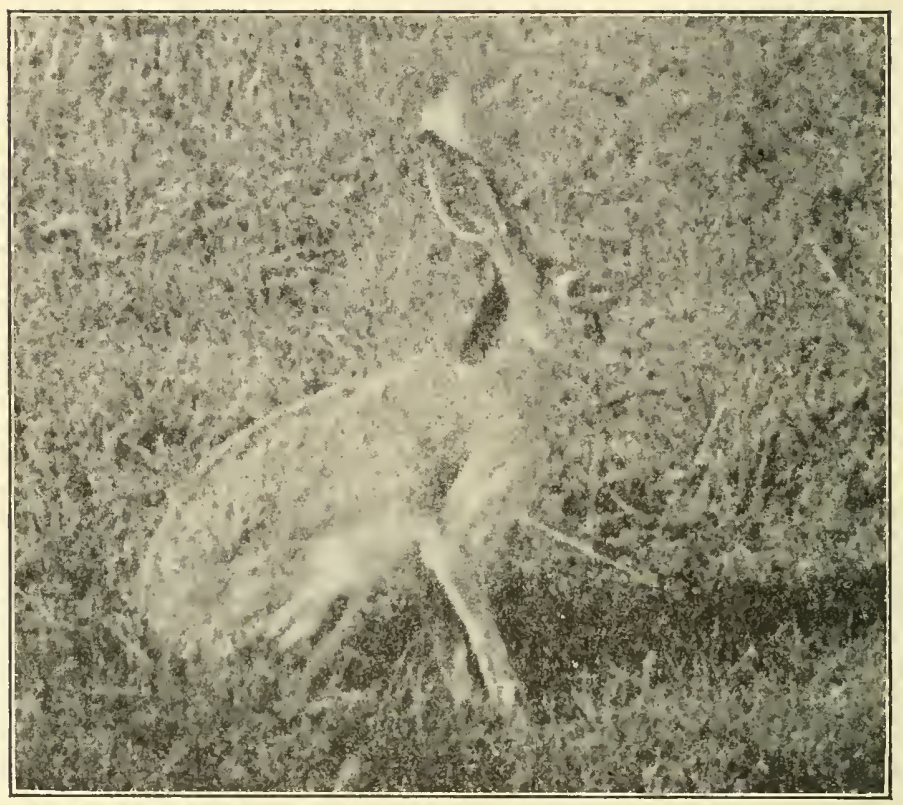

Fig. 339, A.-Jack rabbit, Lepus californicus. A common prairie sight. (Courtesy of Nature Magazine.)

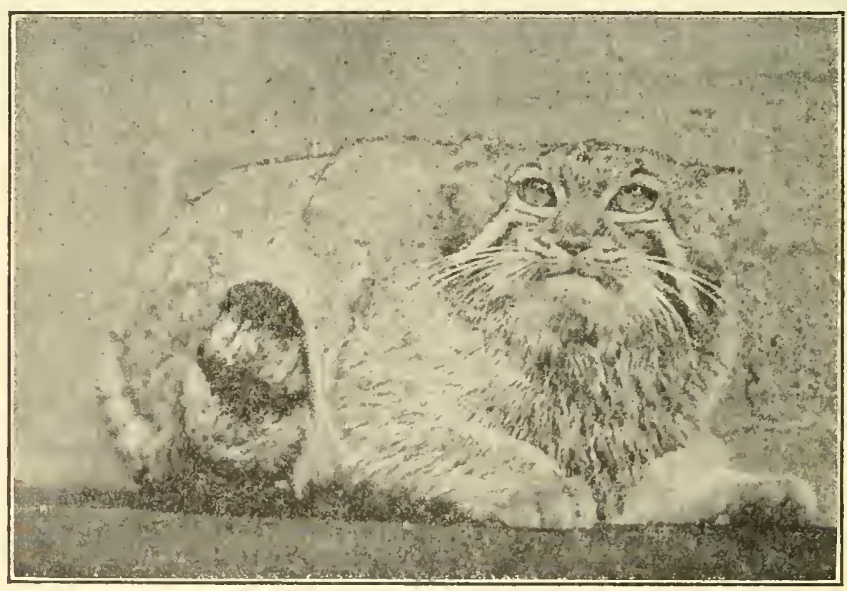

Fig. 339, B.-Mexican ring-tail cat. (Courtesy of Nature Magazine.) 
famous for its long legs, long ears, and speed. All of the hares build nests in heavy grass and bear the young here. The cotton tail rabbits, Sylvilagus of several species, are generally distributed over the country. They are grizzly gray above and lighter below, with the underside of the tail cottony white. They dig their own burrows, or borrow burrows from prairie dogs, ground hogs, or badgers. Several litters of blind, helpless young are born in the burrow or in a nest above the ground during the breeding season. In the Gulf States, extending

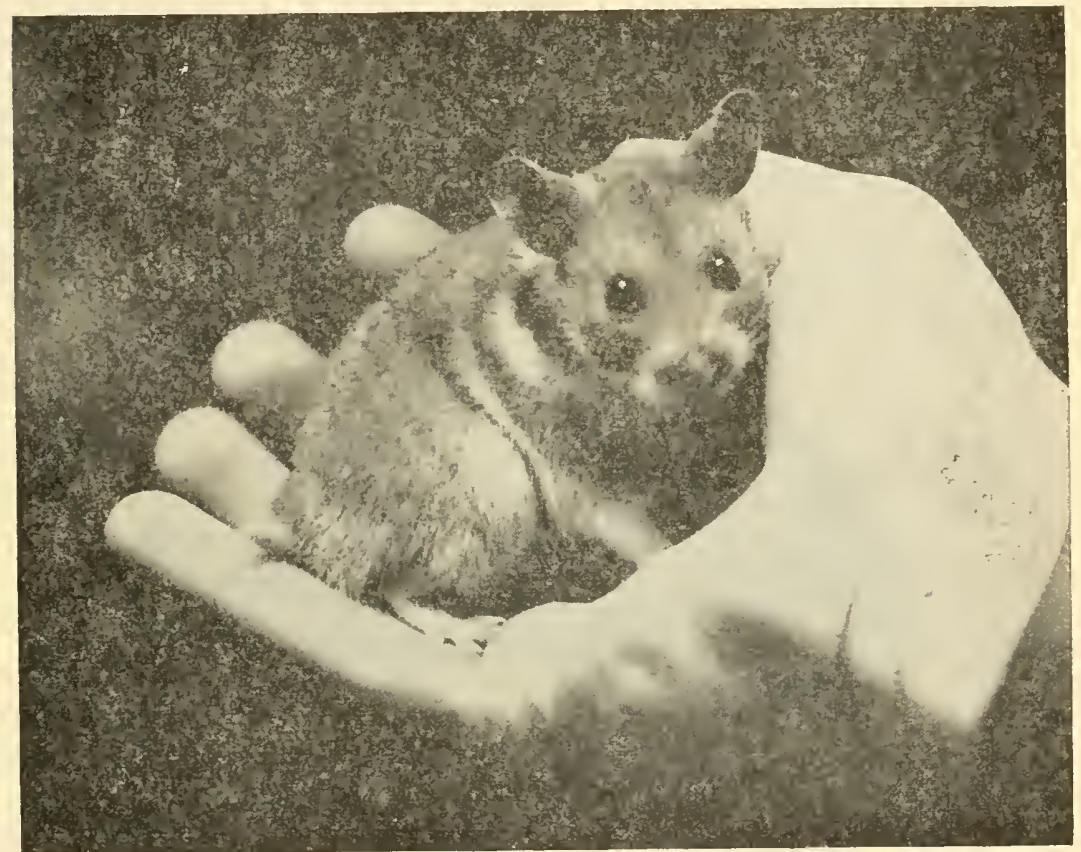

Fig. 340.-Adult golden harnster. (Courtesy Biological Survey House, Chicago.)

as far west as central Texas, is a larger semiaquatic relative of the cottontail, the swamp rabbit. It is brownish gray above and is said to have the habit of concealing itself in the water with only the tip of the nose exposed. In the region above the timber line are found the tailless rock rabbits or pikas. They live among the rocks and look a little like guinea pigs.

Order Carnivora.-Most of the mammals of this order are flesheating, although a few are omnivorous and one or two are vegetarians. They have the canine teeth well developed and conical premolars as 
an adaptation to their feeding habits. The suborder Fissipedia includes the terrestrial carmivores with walking feet, while a second suborder Pinnipedia includes the aquatic carnivores with swimming feet.

There is a large group of common mammals that belong to the first suborder. The fishers, martens, minks, weasels, ferrets, otters, skunks, and badgers constitute a group of very blood-thirsty killers. The skunks of genus Mephitis are quite common and are well known because of their conspicuous white stripes on black fur background and powerful scent glands. Besides the striped forms there are the smaller, spotted forms. The badger, Taxidea taxus, extends down the plains, and there meets the Mexican variety coming up from the south. It is heavy-set and has short legs with long, sharp, strong claws. It can dig almost as fast as a man with a spade and usually comes out winner in a fight with any dog. It is strictly nocturnal and lives in a burrow.

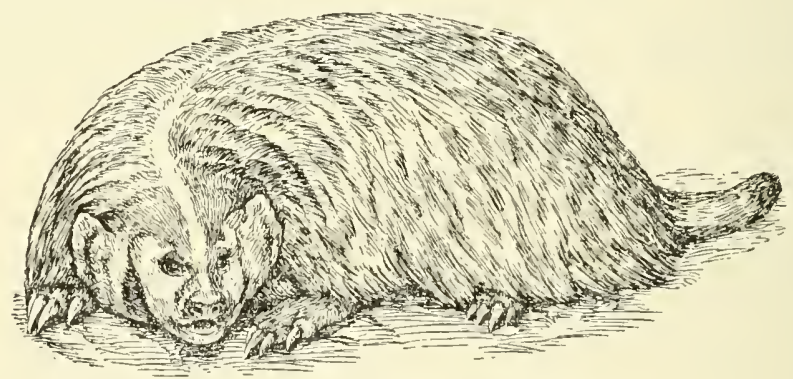

Fig. 341.-The badger, Taxidea taxus.

In the family Canidae there are besides the domestic dog, the fox, the coyote, and the wolves. The red fox, Vulpes fulva, is a common form through the North and East, and has been introduced into the Southwest by sportsmen who enjoy fox-hunting. It is a cunning animal and quite difficult to catch or trap. It digs burrows or builds dens in rocky hillsides where the young are raised. The chosen food of the fox includes mice, insects, wild birds, and occasional poultry. Besides the red fox there are the gray fox, the kite fox, and the Arctic fox. The coyote, Canis latrans, is somewhat larger than the foxes and is quite crafty. It has managed to keep up its numbers in spite of persistent control measures, such as poisoning, trapping, and hunting. It lives in dens and among the rocks and feeds largely on 
rabbits, mice, poultry, game, and small livestock. The wolves are larger than coyotes and frequently hunt in packs. Canis gigas is the timber wolf.

In the family Frelidae are classified the domestic cat, bobcat (lynx), tiger, leopard, mountain lion, jaguar, and ocelot. Most of the wild representatives of this group have been pushed far back into the more remote parts of the Southwest and the Rocky Mountain area. Felis couguar, the mountain lion (puma, panther, or cougar); Felis hernandesii, the jaguar; Felis pardalis, the ocelot; and Lynx rufus, the bobcat or catamount, can all be found in southwestern Texas and New Mexico. The common North American coon or raccoon (Fig. 412 ) is a plantigrade (walks on entire foot with heel tonching the ground) animal about two and one-half feet long and generally distributed east of the Rockies. Procyon lotor is its name, and it is much hunted throughout the South for its fur and its flesh. The bears include the black or brown bear, Euarctos americanus, which is the most widely distributed and most common; the large, grizzly, Ursus horribilis, of the Rocky Mountains, and the white polar bear, Thalarctos maritimus, of the Arctic region. Brown bears and grizzlies are rather omnivorous, feeding on fruit, insects, flesh, honey, and even tourists' lunches. The grizzly bear is more partial to meat than is the brown bear. The polar bear feeds quite largely on fish.

There are several carnivores which are considerably modified as an adaptation to a life in the ocean. The body has become fishlike in its shape and specializations. The appendages in particular have become swimming organs. Callorhinus alascanus, the fur seal, occurs along the Pacific coast and goes to the Pribilof Islands of the Bering Sea at breeding season. At this time one male or "bull," depending on his ability to fight other males, will set himself up in charge of from four or five to twenty-five or thirty females. Under present laws, only the unmated males ("bachelors") are allowed to be killed for the furs.

Besides the fur seal, there are the Califormia sea lion, Zalophus californianus; the Pacific walrus, Odobenus divergens or O. obesus; and the Atlantic walrus, Odobenus rosmarus. The walruses have very long canine teeth in the upper jaw and use these for digging mollusks which are used as food.

Order Artiodactyla.-This is one of the four orders of hoofed animals and includes those with the even toes. The group includes 
cattle, camels, llamas, goats, sheep, pigs, alpacas, reindeer, deer, elks, moose, giraffes, bisons, hippopotamuses and gazelles. The deer family, Cervidae, peceary family, Tayassuidae, cattle family, Bovidae, and the pronghorn antelope family, Antilocapridae all have native representatives in North America. The family Cervidae is the largest in the order. Nearly all males in the deer group have horns which are solid outgrowths of the skull. These are shed cach year and a new but larger set grown the next season. The moose, Alces americana is the largest of the group, and it may reach a weight of one thousand pounds. It is found in the mountainous parts of the Northwest. It has large, broadly flattened antlers. The elk, Cervus canadensis, is another large representative which is found only in isolated parts of our western states. In recent years there has been much effort made to conserve the remaining ones, particularly in Wyoming, Montana, and Utah. The antlers of the elk are long, with numerous slender points. The most commonly distributed deer is the whitetailed or Virginia deer, Odocoileus virginianus. It thrives in semidomestication as well as in the wild. The black-tailed deer, Odocoileus crooki and the mule deer, Odocoileus hemionus are both a little larger than the white-tailed. The mule deer is common through the Rocky Mountain states. The black-tailed deer does not range so far north. Both have doubly branching antlers while the white-tailed does not.

The caribous, which are native, and the imported European reindeer are important meat animals of Canada and Alaska. The caribou, Rangifer caribou, is heavy-bodied with stout legs and heavy, irregular antlers in both sexes. The pronghorn antelope, Antilocapra americana (Fig. 410) which once covered the Great Plains and ranged over most of the westem states is now limited to a few scattered, isolated herds. Much attention is now being given to its restoration and fortunately so. It is nearly as large as a small whitetailed deer, has peculiar short, single-branched horns in both sexes, long pointed ears, and a striking white rimp patch. It sheds the horns each year.

The fanily Bovidae, of course, includes the domestic cow which is not a native of this continent. The horns of this group are hollow, occur in both sexes, and are permanent. Rocky Mountain sheep, Ovis canadensis, is found in the higher western mountains. The horns of the male are greatly dereloped and curved. They are the basis for 
the common name, Bighorn. It is very sure-footed and can live on mountain ledges. The Rocky Mountain goat, Oreumnos montanus, lives in a similar habitat, but farther north. It has short, dark horns, shaggy wool, and a beard. The musk ox, Ovibos moschatus, is a heavy bodied, hump-shouldered animal with stout, down-curved horns. It lives above the timberline, principally in Canada and

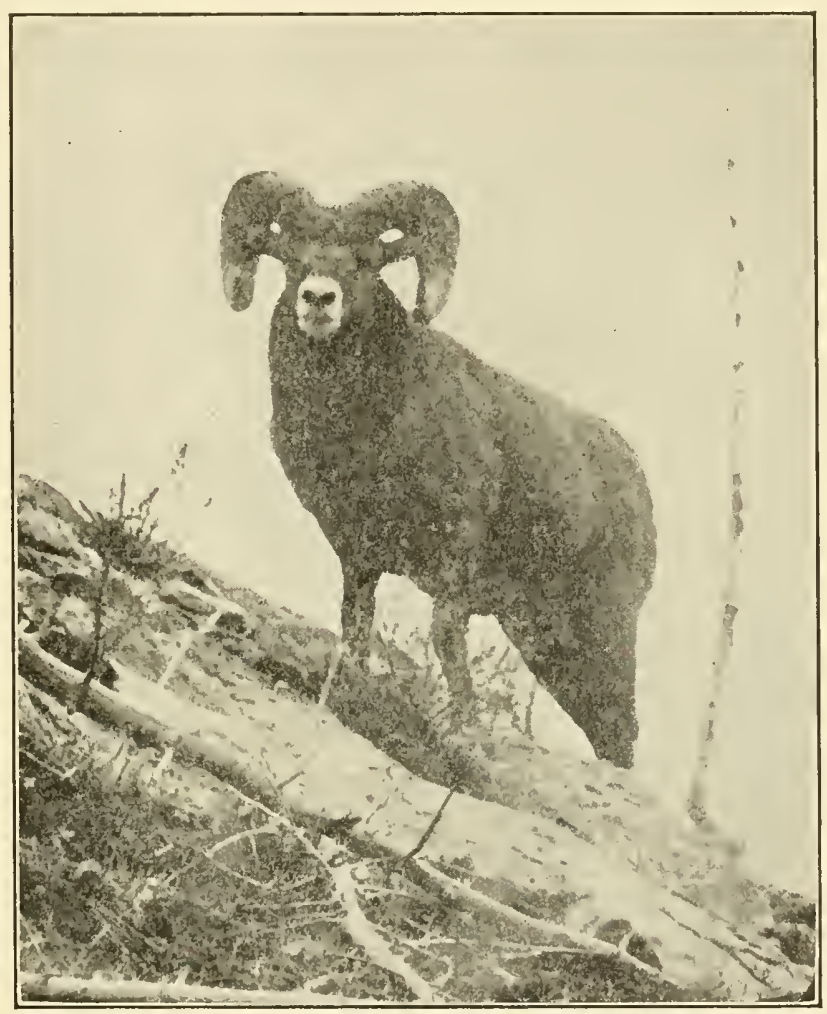

Fig. 342.-Bighorn mountain sheep are still to be found in the Rockies. (Courtesy of Nature Magazine.)

Alaska. The buffalo, Bison americanus, was extremely abundant all over the Great Plains until the last quarter of the nineteenth century when they were killed out by hunters and crowded out by civilization. They arc powerful, heavy-headed, hump-shouldered animals. There are still a few herds, such as the one at Yellowstone National Park, that are kept under semidomesticated conditions. Our domesticated 
hogs belong in the family Suidae and have come down to us from the European wild boar. In southern Texas and on south to South America is a piglike animal, known as the peccaries, or javelinas, Pecari angulatus. They are gregarious, nocturnal, and feed on roots and nuts primarily. Their heads are large, bodies slender, and tails

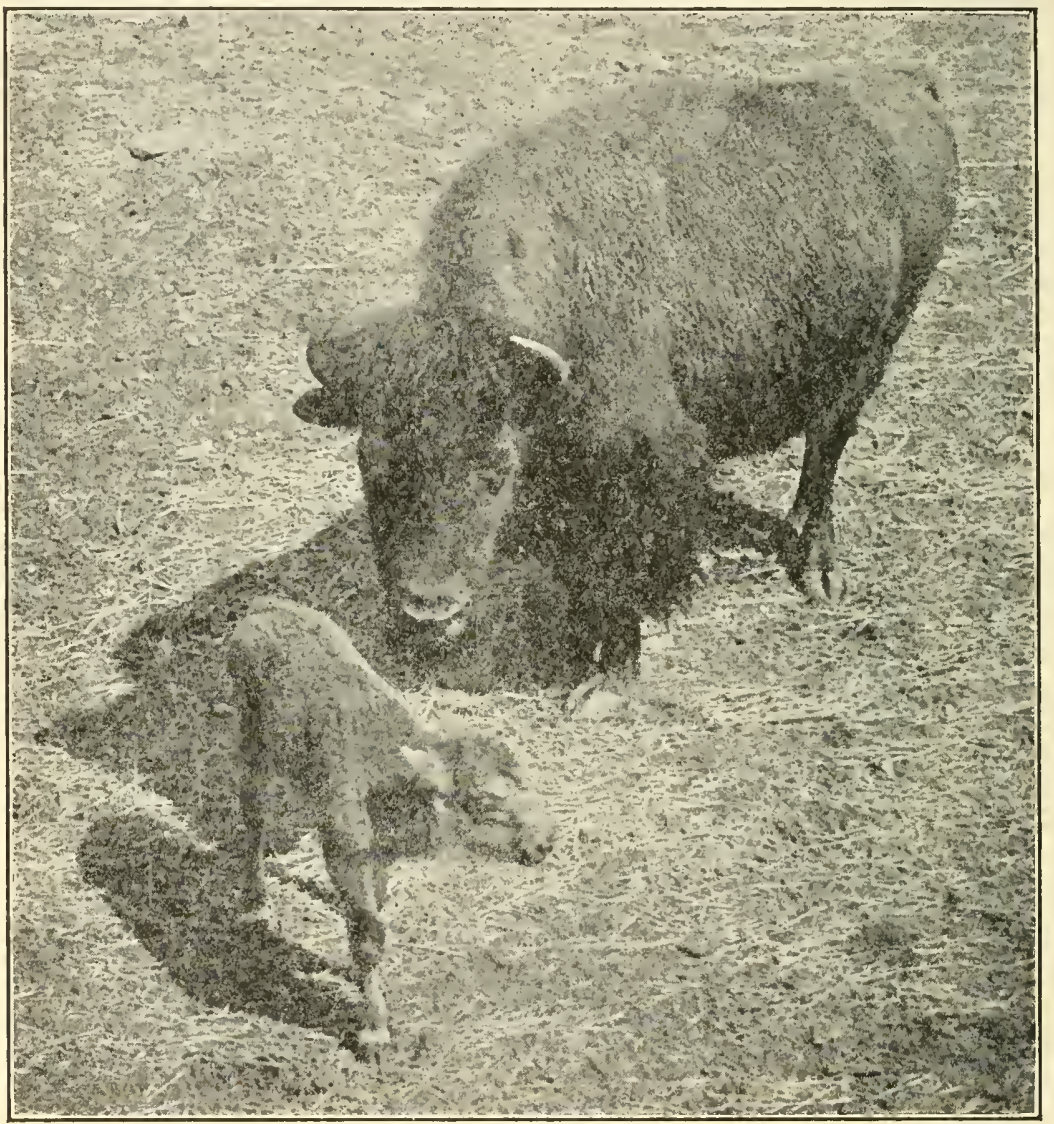

Fig. 343.-Buffalo cow and calf. A sight which is no longer seen except in special preserves. (Courtesy of Nature Magazine.)

short. Hippopotamuses, camels, and giraffes are numerous and important African members of this order. The llama and alpaca are useful South American forms.

Animals, such as camels, cattle, sheep, deer, giraffes, and pronghorn antelopes, chew their cud and are therefore said to be ruminants. 
They swallow their food partially chewed and, because of the structure of the stomach, they are able to regurgitate it later for further chewing. For this reason such animals can consume large quantities of bulky food in a short time, then retire to the shade and chew while reclining. The stomach of such an animal is greatly modified by having four divisions. Following the esophagus is the pouchlike rumen at the left, then the small middle reticulum, at the right, is another pouchlike part, the omasum or psalterium, which continues on to the right and posteriorly into the more elongated abomasum, which in turn joins the duodenum. On the first trip to the stomach, the food passes into the rumen, is stored and moistened. It then goes in small quantities at a time into the reticulum, and this "cud"

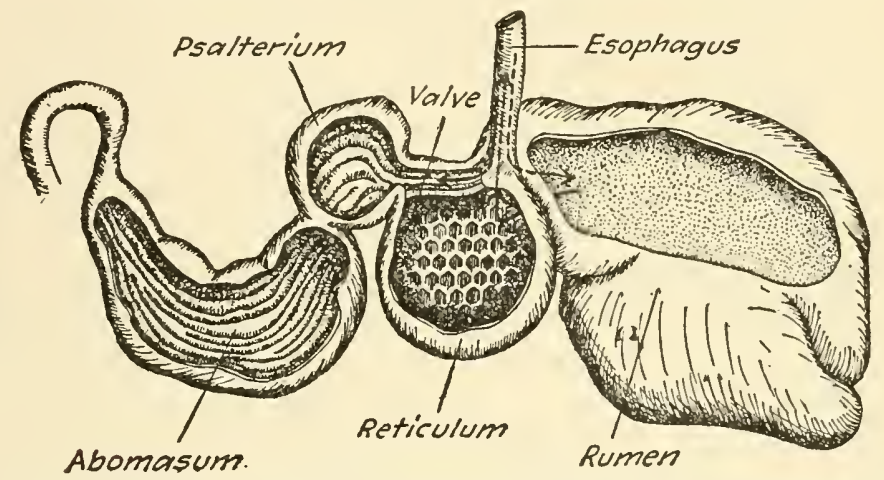

Fig. 344.-A ruminant stomach. The arrows indicate the direction of the movement of the food in the formation and regurgitation of a cud. (From Wolcott, Animal Biology, published by McGraw-Hill Book Company.)

may pass back to the mouth from here. After it is chewed and reswallowed it passes through a valve at the entrance to the stomach into the omasum, and on to the abomasum.

Order Perissodactyla.- This is the group of odd toed mammals in which the axis of the foot is through the third toe. There are no modern forms which are natives of this country with the possible exception of the horse, and this is very indirect. In the horse, ass, and zebra, the foot is reduced to one hoofed toe. The ass and zebra are African and Asiatic forms. Tapirs are piglike with four toes in front and three behind. They are found in southern Asia, and in Central and South America. The rhinoceros is a large Asiatic and African form. 
Order Proboscidea.-This small order includes only two genera of elephants with one species each of the largest terrestrial animals. One species, Elephas indicus, is Asiatic, and the other, Loxodonta africana, lives in the tropical forests of Africa. Both have the nose extended several feet as a muscular trunk or proboscis which is a very handy and useful appendage. The skull is very thick, with air spaces, and the molar teeth are very large, with prominent ridges.

Order Sirenia.-This is a very limited order of sea-cows. They are aquatic mammals with a pair of flexible anterior flippers and a strong, rounded tail. The dugongs of the Indian Ocean and Australia, and the Manatees of the Atlantic Ocean represent the group. The Florida manatee, Trichechus latirostris is only rarely found.

Order Odontoccti (The Toothed Whales).-All of the whales are mammals which have become adapted to a strictly aquatic life. The body is modified for swimming by the reduction of appendages, the horizontal flatteuing of the tail and its division into two lobes or "flukes." The head of these animals is large with long jaw bones. The nostrils open by a single aperture from which the breath is spouted when the animal comes to the surface. A thick layer of fat or "blubber" is deposited beneath the skin and this serves to conserve heat in the body. The porpoise, Phocaena phocaena, is very common in the Gulf of Mexico and elsewhere. It is about six feet long and rolls around in the water. It catches such fish as mackerel and squeteague for food. The sperm whale, Physeter catodon, is a large whale of about seventy-five feet in length. Such other animals as the beaked whale, dolphin, and narwhal belong in this order. The killer whale, Orcinus orca, is generally distributed. It is about twenty feet long, ferocious and predatory on fish, seals, and even other whales.

Order Mystacoceti.-This is the whalebone whale group. Their teeth do not develop beyond the embryonic stage, but they are replaced by cordlike plates of baleen or whalebone. Whalebone was once an important item of commerce, being used in making whips, stays, and other flexible articles. The largest species in the order and, in fact, the largest of all animals is the sulphur-bottom whale, Sibbaldus musculus. It reaches a length of one hundred feet and lives in the Atlantic and in the Pacific off the coasts of Central America, Mexico, and Califormia. The gray whale, Rhachianectes glaucus, is another Pacific form. The Greenland right whale, 
Balaena mysticetus, is a polar inhabitant, each of which yields about 3,000 pounds of whalebone and 300 barrels of oil. The finback and humpback whales also belong to this order. In feeding, all of these whales take large quantities of water into the mouth, pass it out through the whalebone which serves as a sieve, and retain all of the small organisms and particles of organic matter as food.

Order Prinates. - This is the order which includes the lemurs, monkeys, apes, and man. Because of the large number of primitive characteristics of representatives of this order, some authors place it near the first or middle of the list of orders of mammals instead of at the end. Mrost of them are tropical, arboreal, and live on nuts, seeds, fruits, insects, and birds. They have a particularly welldeveloped brain. The thumb and also the great toe in most forms are placed in opposition to the other digits as an adaptation for grasping. Usually only one young is born in a fairly advanced stage, but rather helpless at first and requiring considerable care. The order is usually divided into two suborder's, the Lemuroidea and Anthropoidea. The first includes the lemuroids which have the front teeth separated. These are typically small or medium-sized quadrupeds of Madagasear, with long, bushy tails. The aye-aye is a nocturnal arboreal animal with long ears, large ratlike teeth, bushy tail, and long digits with sharp claws. Its body is about one foot long. The tarsiers are about the size of rats with suckerlike dises on the ends of their fingers and toes. They are also arborcal and nocturnal. The true lemurs have an elongate face and a small cranium. Their hindlimbs are longer than the forelimbs. Most of them live on Madagascar and nearby islands as well as in Africa and Isia.

The second suborder, Anthropoidea, includes several families of monkeys and apes.

The marmosets make up another family found in South and Central America. They have a flat nail on the big toe, while the thumb is not opposable to the other digits. The brain case is large, and the space between the nostrils is broad. The Cebidae, or New World monkeys, have long prehensile tails, broad flat noses, and all digits have nails instead of claws. This group includes the common monkey of hand-organ fame, the spider monkeys, squirrel monkeys, and howlers, all of which are natives of Central America and the northern part of South America. 
The Old World monkeys, family Cercopithecidae, have narrow high-ridg'ed noses. Some have 'long tails, others have short tails. Certain of them are almost bipedal. Most of them have heavy callouses on the hips which are used as cushions while sitting. Some of

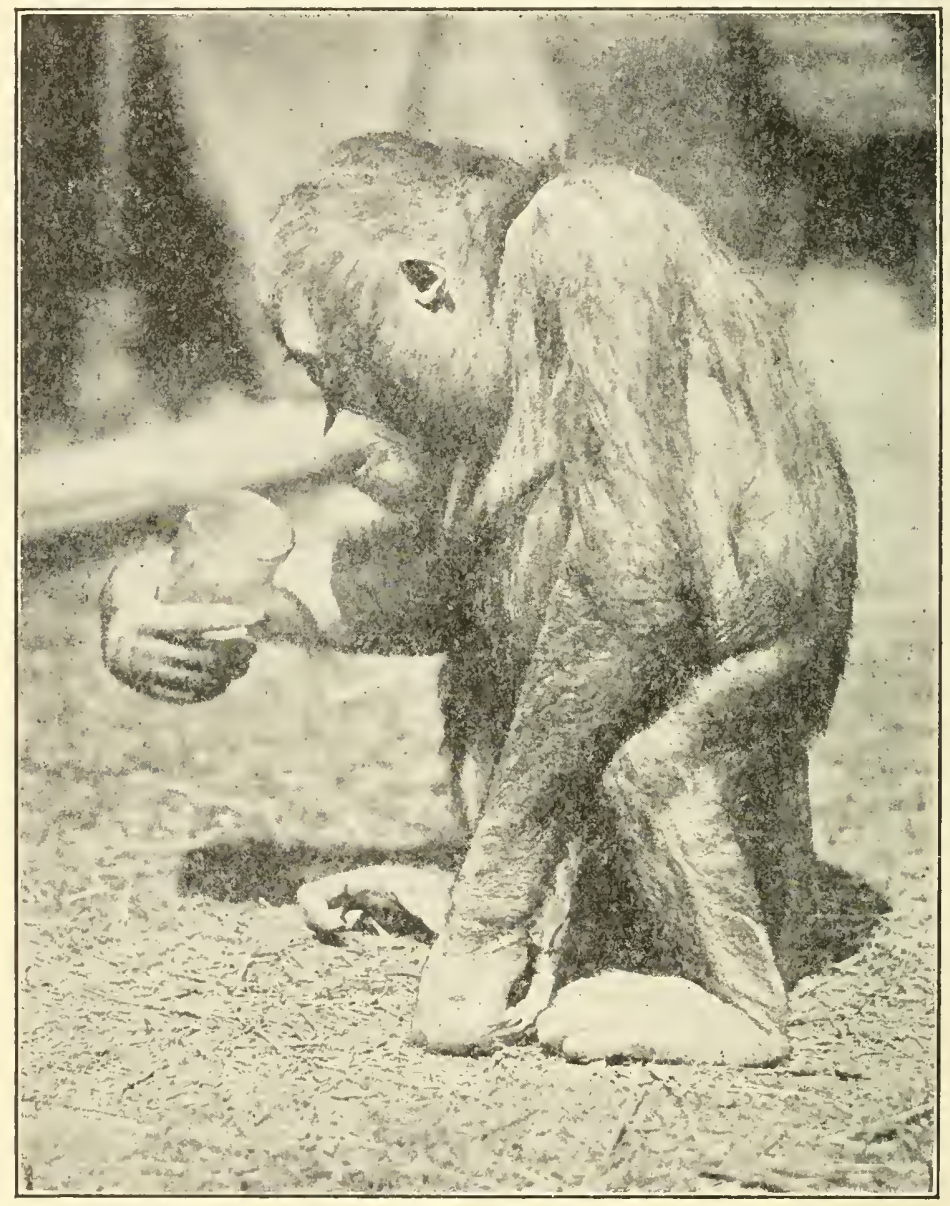

Fig. 345.-Orangutan holding a glass. (Courtesy of Nature Magazine.)

them have cheek pouches. With the exception of the Barbary ape, Macaca sylvana, of the Rock of Gibraltar, this entire family is confined to Africa and India. The baboon, of genus Cynocephalus, a doglike monkey with a short tail, lives in central Africa. The mandrill, drill, and macaque monkeys are others in this group. 
The manlike, or anthropoid, apes are grouped in the family Simiidae which includes the gibbons, Hylobates; the orangutan, Simia satyrus; the chimpanzee, Anthropopithecus troglodytes; and the gorilla, Gorilla gorilla. The gibbons are tailless apes with long arms. They are arboreal and omnivorous. They can accomplish bipedal locomotion. They are only three feet tall and strictly arboreal; they live on the Malay Peninsula and in the East Indies. The orangutan builds nests in the trees and feeds principally on fruits. It is between four and five feet tall and has an arm spread of seven feet. The chimpanzee is perhaps the most intelligent ape. It is easily tamed and in many respects is more manlike than most of the others. It lives in central and western Africa. The arms are somewhat shorter and the skull rounder and smoother than are those of the gorilla. The gorilla, which is the largest of the group and somewhat more ferocious, is about five and one-half feet tall. It walks on the soles of the feet and on the knuckles of the hands. It has prominent canine teeth and feeds mainly on plants and foliage.

\section{Economic Relations}

Nearly all of the important beasts of burden, such as horses, asses, mules, elephants, camels, llamas, reindeer, oxen, and dogs, are mammals. The history of the origin of the domestication of most of these has been lost to antiquity. Cattle, sheep, hogs, goats, reindeer, alpaca, and rabbits are the chief meat-producing mammals. A few years ago it was estimated that more than 20,000,000 cattle, $15,000,000$ sheep, and $80,000,000$ swine are required to supply the meat demand in the United States each year. Cattle and goats are the most important commercial milk-producing animals. The milk of camels, reindeer, and llama is also used in some parts of the world. The leather produced by tamning the hides of meat-producing domestic animals particularly is worth millions of dollars. It is used for making shoes, saddles, harness, belts, and for ornamentation. Wool is a very important animal fiber used in the manufacture of fabrics which are resistant to dampness and cold. It is produced principally by sheep and goats in this country. In some parts of the world the alpaca and camel are important wool-producing animals.

The skins and furs of many wild mammals, the fur-bearers, are exceedingly important commercially. They were used as clothing and ornamentation even by primitive people. The modern people are 
demanding more and more furs. The most eommonly used and abundantly sold furs are in the following order: mole, rabbit, skunk, muskrat, opossum, squirrel, fox, ermine, wallaby, mink, wolf, civet eat, and raccoon. There are several very valuable fur-bearing animals coming very near the point of extinetion, and there should be an earnest effort made to restore them. In this group would be ineluded beaver, fur seal, otter, Russian sable, and ehinchilla. A single, choiee, silver fox fur may bring a thousand dollars or more, a fisher is valued at about three hundred dollars, the beaver and otter, each at about one hundred; a wolf is worth about forty as also is the blaek bear; the skunk is valued at about six dollars, and the muskrat at about three dollars. Fur farming is being praeticed with some species, such as silver fox, mink, muskrat, and rabbits. Rabbit and muskrat farming have the added advantage of producing salable meat.

Many of the undomesticated mammals become serious pests at times when the usual balanee in nature is disturbed. Rats and mice are very destructive of stored provisions, such as fabries, clothing, grains, and varions foodstuffs. These rodents, along with squirrels, gophers, prairie dogs, and groundhogs which normally live on wild plant tubers, seeds, acorns or nuts, are frequently destruetive to grain crops. Field miee, rats, and rabbits sometimes damage young fruit trees by gnawing the tender bark just at the surface of the ground until the tree is girdled, thus eausing subsequent death. The burrowing forms may be killed by fumigating the burrow with carbon disulphide, calcium cyanide, or carbon monoxide from the exhaust of an automobile.

Many of the larger earnivorous mammals are quite destructive of young domesticated animals. The wolf, coyote, fox, and, in some parts of the Southwest, the puma, are quite predatory, killing calves, lambs, kids, and poultry. Certain wild mammals are earriers of disease. In the Rocky Mountain region, ground squirrels and other rodents carry Rocky Mountain spotted fever and transmit it to man through the bite of the spotted fever tick. Bubonie plague is earried by rats and other rodents and is transferred to man by the rat flea.

The deer and bear are about the only large mammals left that are classified as game animals and hunted for sport. Several others, sueh as the pronghorn antelope, buffalo, and elk, have been almost 
completely destroyed. With proper protection, however, these species might still be restored in many parts of the country. Squirrels and rabbits are the most abundant game mammals of present times.

\section{THE CAT, A REPRESTNTATIVE IMAMIMAL}

The common house cat, Felis domestica, is a carnivore which is familiar to everyone. It is so common that it is always available for study. The cat is a quadruped which is well adapted for walking and climbing, at which activities it is very adept. The eyes of the cat are well adapted to sight at night when it is quite active. As a result of domestication the carnivorous diet of the cat has been somewhat modified. The cat is clean in its sanitary habits.

\section{External Structure}

The entire body is covered with a ligh quality hair or fur which may be one of several colors. On the npper lip and around the eyes are some especially long stout hairs, whiskers or vibrissae. The npper lip is somewhat cleft in the center. In the hare this cleft is extremely prominent, and it is from this that the abnormally cleft lip in man came to be known as "harelip." The tall, flexible external ear or pinna partially surrounds an ear opening, the external auditory meatus, which leads to the interior of the skull and the tympanic membrane. The large, oblong nostrils are located in the fleshy nose, the end of which is naked. Their large eyes have both upper and lower lids, and each has a sheetlike nictitating membrane which may be drawn over the eye from the medial corner.

The trunk is conveniently divided into a thorax or chest, supported by the ribs, and posterior to this the abdomen. Extending posteriorly is the long slender tail. Along the ventral side are four or five pairs of nipples or teats which are prominent in the female but rudimentary in the male. They contain the openings of the milk or mammary glands. Ventral to the tail is the perineal region in which is located the anus and external genital organs. The two pairs of limbs extend ventrally from the sides of the trunk. The forelimb is divided into upper arm, forearm, wrist, palm, and five digits with retractile claws at their tips. The hindlimb extends from the hip and is divided into thigh, shank, ankle, arch, and four digits with similar claws. 


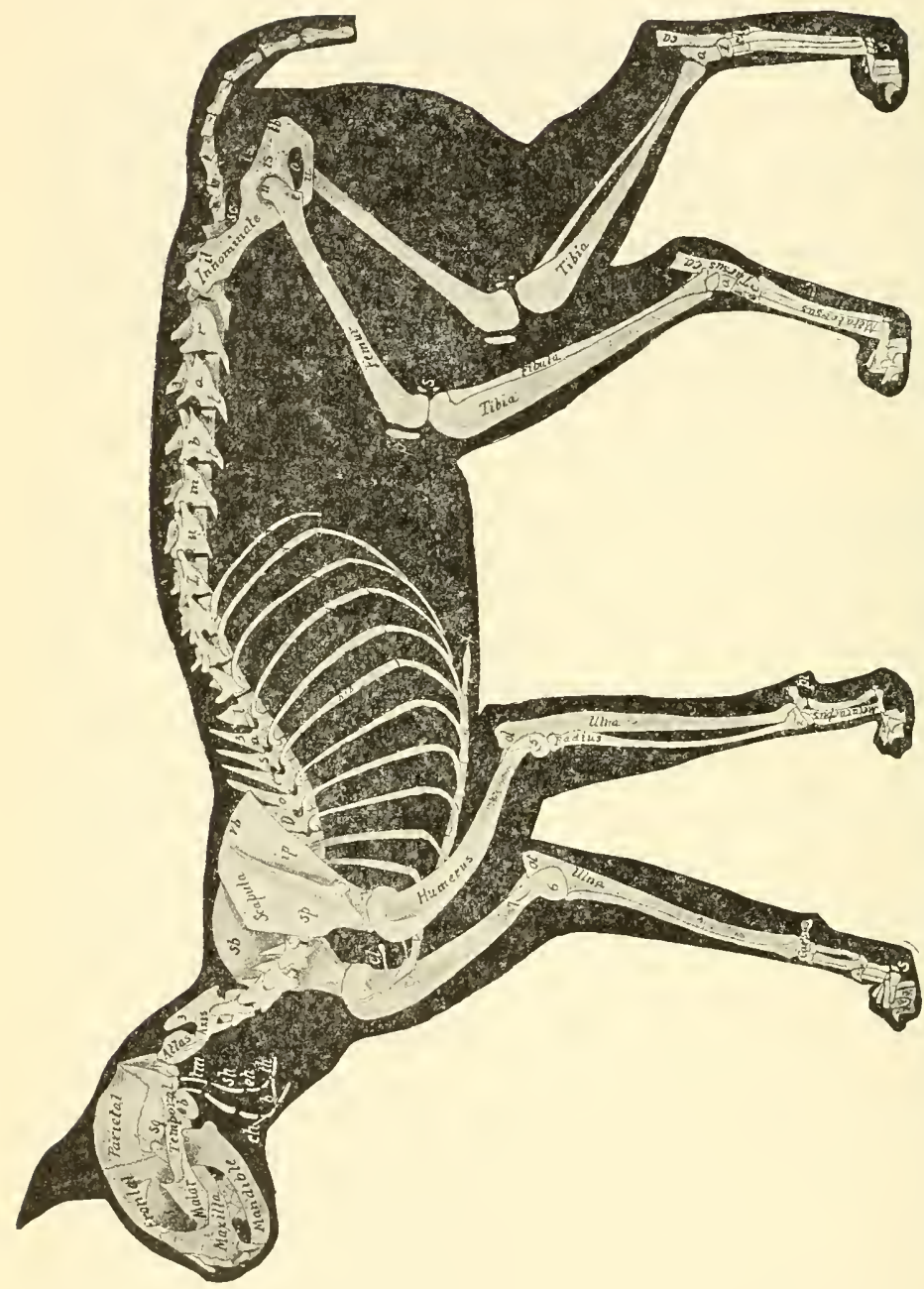

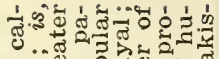

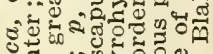
년. व्टें.. है ํํ워 उ. कि..... है

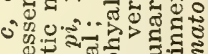
-... - 00 फ … जु हैं ०ैक ……

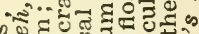
..००

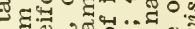

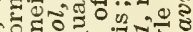

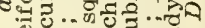
늠 ษึ

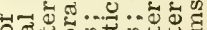

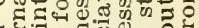
5 政... 40 $00 \%$ 구응 웅 정 พั

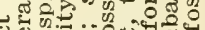
ठण कू ส ธง จ…ํ.

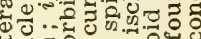
का † जिंतु० ต ह ह

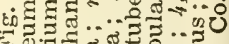
द दु० 
The eat walks on the digits with the remainder of the hand or foot failing to reach the ground. This is known as the digitigrade gait. Man and bears use the plantigrade or primitive gait in which the entire sole and leel make contact with the ground.

\section{Skeleton}

Exclusive of the teeth, ear bones, chevron bones, and sesamoid bones a young fully developed cat has 233 bones. In addition to the bones there is some cartilage. This skeleton is made up of cartilage-bones, which are preformed in cartilage and replaced by bone, and membrane-bones, a bone formation in the dermis of certain portions of the skin. The sesamoid bones are pieces formed in tendons. The kneecap is the largest of these. Chevron bones are paired and extend ventrally from the anterior ends of several of the caudal or tail vertebrae.

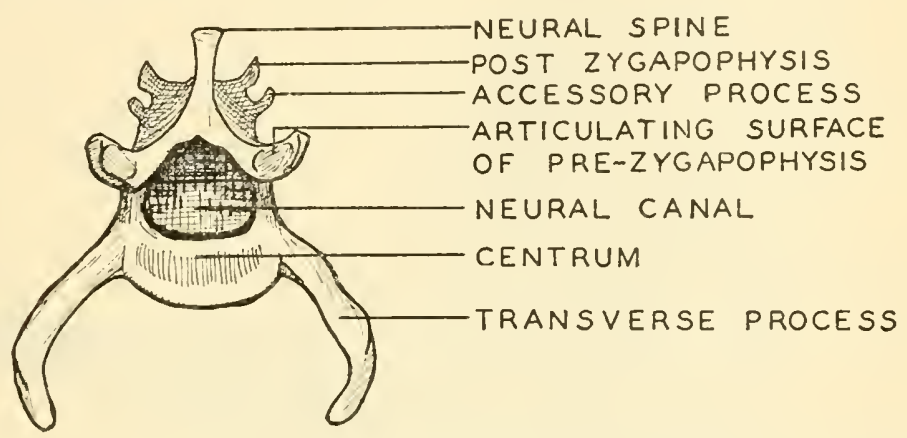

Fig. 347.-Anterodorsal view of lumbar vertebrae of cat. (Drawn by Titus Evans.)

The axial skeleton, as is usual in vertebrates, is the portion extending in the main axis of the body and is composed of skull, vertebral column, ribs, and sternum. There are twenty-six bones that are usually named and described in the cat's skull. These bones are for the most part immovably fused together at sutures. In the most anterior or frontal segment of the skull proper are the two frontal bones, dorsally, the presphenoid and two orbitosphenoids. Behind this is a middle or parietal segment composed of the basisphenoid, two alisphenoids, and the two dorsal parietals. The most posterior or occipital segment represents a fusion of four bones. It consists of basioccipital, two exoccipitals, and the supraoccipital. In addition to these rings or segments there are three sense capsules, the olfactory 


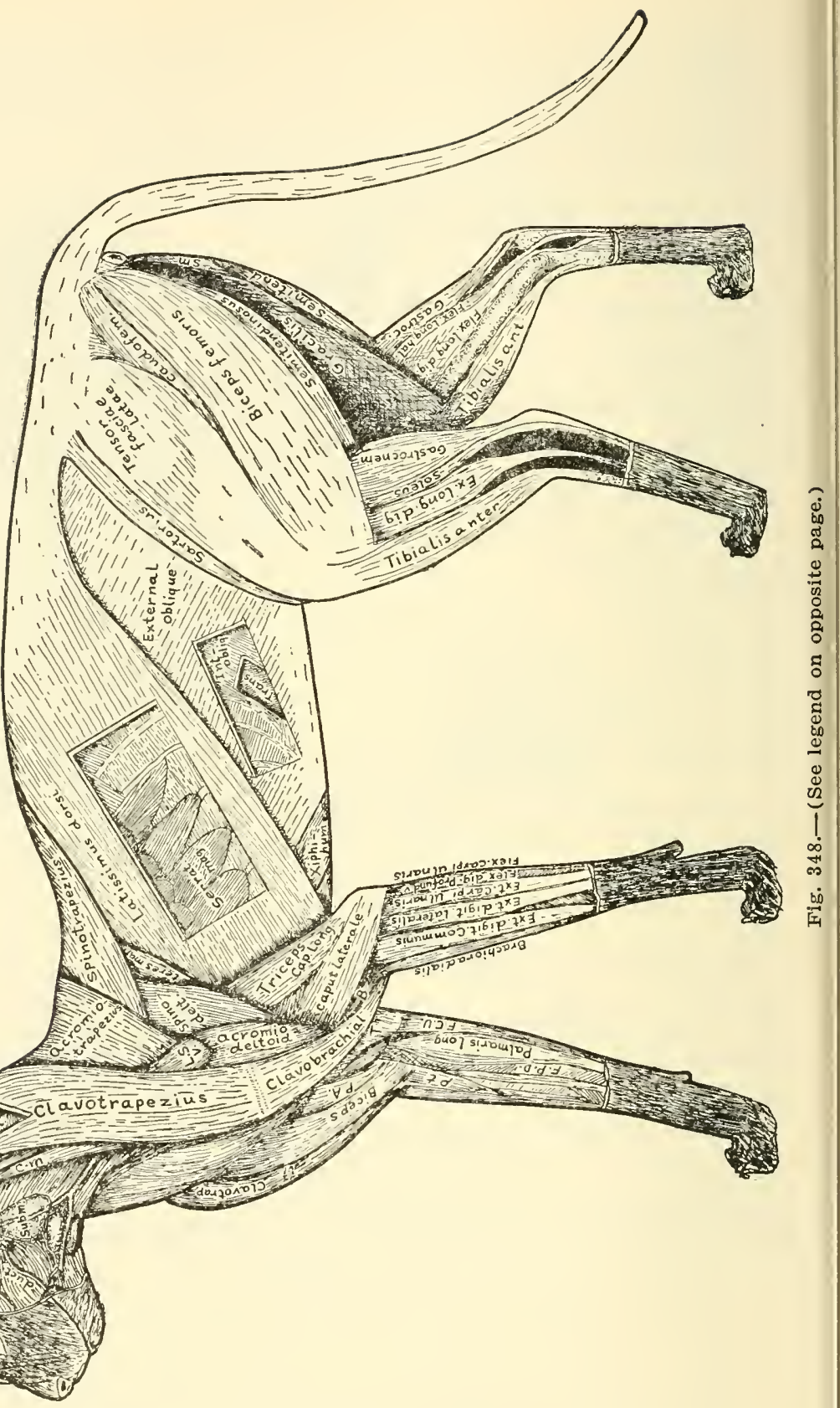


capsule, auditory capsule and optic capsule, as well as the jaws. The walls of the olfactory capsule consist of the vomer, mesethmoid, pterygoid, palatine, maxilla, premaxilla, lacrimal, nasal, and nasal processes of frontal bones. The auditory capsule is enclosed in a portion of the temporal bone. This bone is formed by fusion of the squamous, petrous, and tympanic bones.

The cat and other mammals have two sets of teeth, the milk or deciduous set, and the permanent set. In the first set, the dental formula for one-half of the mouth is incisors $3 / 3$, canines $1 / 1$, and premolars $3 / 2$. These teeth begin to appear when the kitten is two weeks old and are complete at eight weeks. At the end of the fourth month the milk incisors are being replaced by permanent teeth. The formula for one-half of the mouth with permanent teeth is: incisors $3 / 3$, eanines $1 / 1$, premolars $3 / 2$, molars $1 / 1$ or a total of thirty teeth. Because of the different forms these teeth are said to be heterodont. They develop like placoid scales from the epithelial lining of the mouth, and each tooth consists of an outer, hard covering, the enamel. under which is the dentine and then the pulp. The permanent set in man includes 32 teeth and the formula for one-half of the set is incisors $2 / 2$, canines $1 / 1$, premolars $2 / 2$, molars $3 / 3$.

The vertebral column or backbone is composed of five groups of vertebrae; seven cervical in the neck, thirteen thoracic in the chest, seven lumbar in the small of the back, three sacral in the hip region, and from four to twenty-six caudal in the tail. These vertebrae articulate on each other. They are separated by intervertebral discs of cartilage, except the fused sacral group. Intervertebral ligaments serve to connect the vertebrae. The principal parts of the typical vertebrae are: the body or centrum, neural arch over the central eanal, a spinous process or neural spine projecting dorsally from the arch, two transverse processes projecting laterally one on each side, zygapophyses or articular processes, a pair projecting anteriorly and a pair posteriorly to articulate with adjacent vertebrae. Articulating laterally with the thoracic vertebrae are thirteen pairs of ribs which

Fig. 348.-Nuscles of the cat. a.s., occipitoscapularis (rhomboidens capitis); c.m., cleidomastoid; ex. long. dig., extensor longus digitorum; $F$. $C$. $U$., flexor carpi ulnáris: fex, long. dig., flexor longus digitorum; flex. long. hal., flexor longus hallucis: $F, P . D$. flexor profundus digitorum; gastroc, gastrocnemius ; int. obliq., internal oblique; $L$. S. V., levator scapulae ventralis ; P.A., pectoralis : parot., parotid gland; $\dot{P} t$, pronator teres; spl., splenius; subm., submaxillary gland; $T$, triceps brachii; trans., transverse abdominis. (From Stromsten, Davison's Mammalian Anatomy, published by The Blakiston Co.) 
extend in an arch, ventrally. The nine anterior ones are called true ribs because they articulate dorsally with the thoracic vertebrae and ventrally with the sternum, or breastbone. The other four are termed false ribs, three pairs of which articulate with other ribs, while the fourth has no ventral articulation and is called a floating rib. The sternum is divided into eight pieces, called sternebrae, which lie in a row in the median line of the ventral side of the thorax. The anterior sternebra is called the manubrium and the posterior one is called the xiphoid or ensiform process.

The two girdles and pairs of limbs are quite well developed. The forelimb and pectoral girdle are composed of a scapula, poorly developed clavicle, humerus, ulna, radius, seven carpals, five metacarpals, and fourteen phalanges. The hindlimb and pelvic girdle are composed of the innominate bone (ilium, ischium, pubis), femur, patella, tibia, fibula, seven tarsals, five metatarsals, and twelve phalanges.

\section{Muscular System}

The study of the muscular system is known as myology. All muscles moving the bones are voluntary; i.e., they are under control of the will. There are over five hundred voluntary muscles in the cat, each of which is attached by one or both ends to the periosteum of the bone, usually by tendons. The point of attachment on the fixed bone, which is not moved by the contraction, is the origin. The end of the muscle attached to the bone which is moved is known as the insertion. According to function the skeletal muscles are grouped as flexors, extensors, adductors, abductors, rotators, elevators, depressors, and sphincters. A flexor muscle is one which bends the part on itself. An extensor is one which by its action tends to extend or straighten the part. An adductor muscle is one attached to a long bone in such a way as to move it toward the ventral midline of the body. An abductor opposes the action of the adductor. The caudofemoralis is an abductor of the thigh and flexor of the shank. The gracilis on the medial surface of the thigh is an adductor of the thigh. A rotator muscle is one which causes a part to rotate on its own axis. An elevator raises or elevates a part, as the splenius of the neck may elevate the head. A depressor is the antagonist of an elevator. A sphincter muscle is one encircling an aperture which it closes by contraction. The orbicularis oris in the lips around the mouth is an example. A 
dilator is antagonistic to a sphincter muscle. The relations of most of the superficial muscles may be observed by reference to Fig. 347 . Over four hundred of the muscles of the eat are found in man, and they have approximately the same relative location, function, and nerve supply.

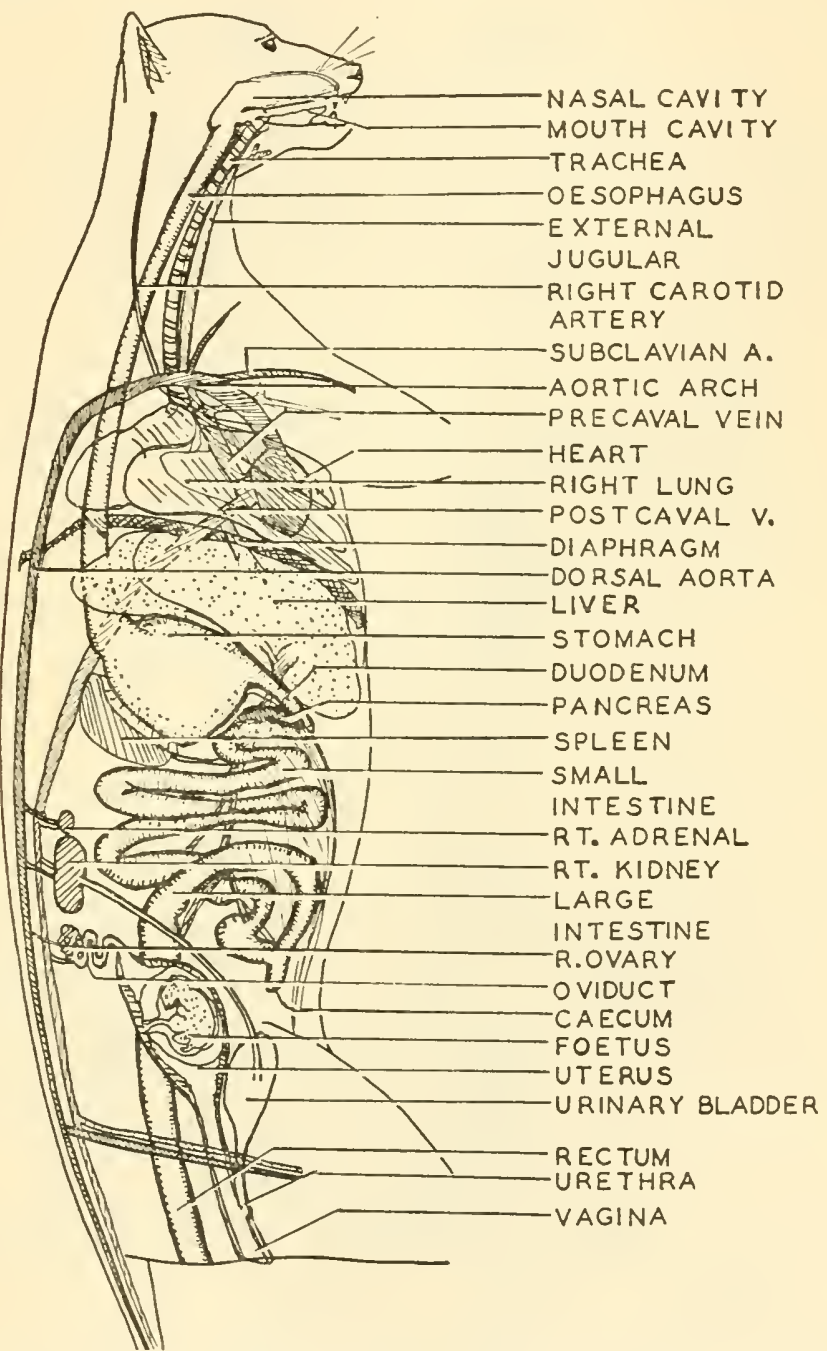

Fig. 349.-Lateral view of visceral organs of a female cat. (Drawn by Titus Evans.) 


\section{The Digestive System}

The most anterior portion of this system is the buccal cavity or mouth. It contains the teeth, which are set in alveoli or sockets in the jaws, and the tongue on which are located taste buds. The roof

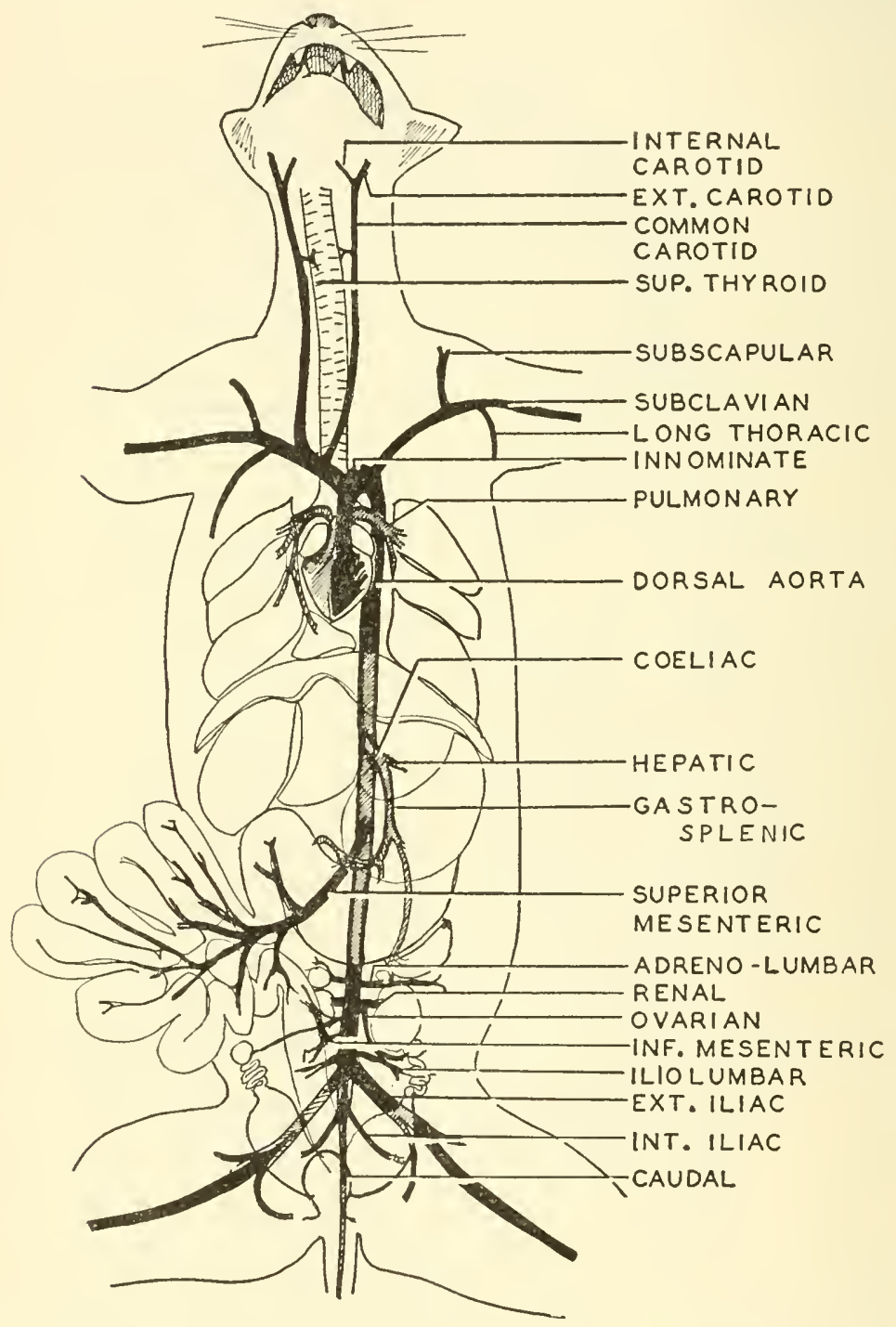

Fig. 350.-Heart and arteries of cat (ventral view). (Drawn by Titus Evans.) 
of the mouth is composed of the hard palate at the posterior margin of which is a transverse muscular flap, the soft palate, which hangs down to separate the mouth from the pharynx just behind. At the lateral extremities are found the tonsils, which are masses of lymphoid tissue each held in a pit or fossa. The space between the base

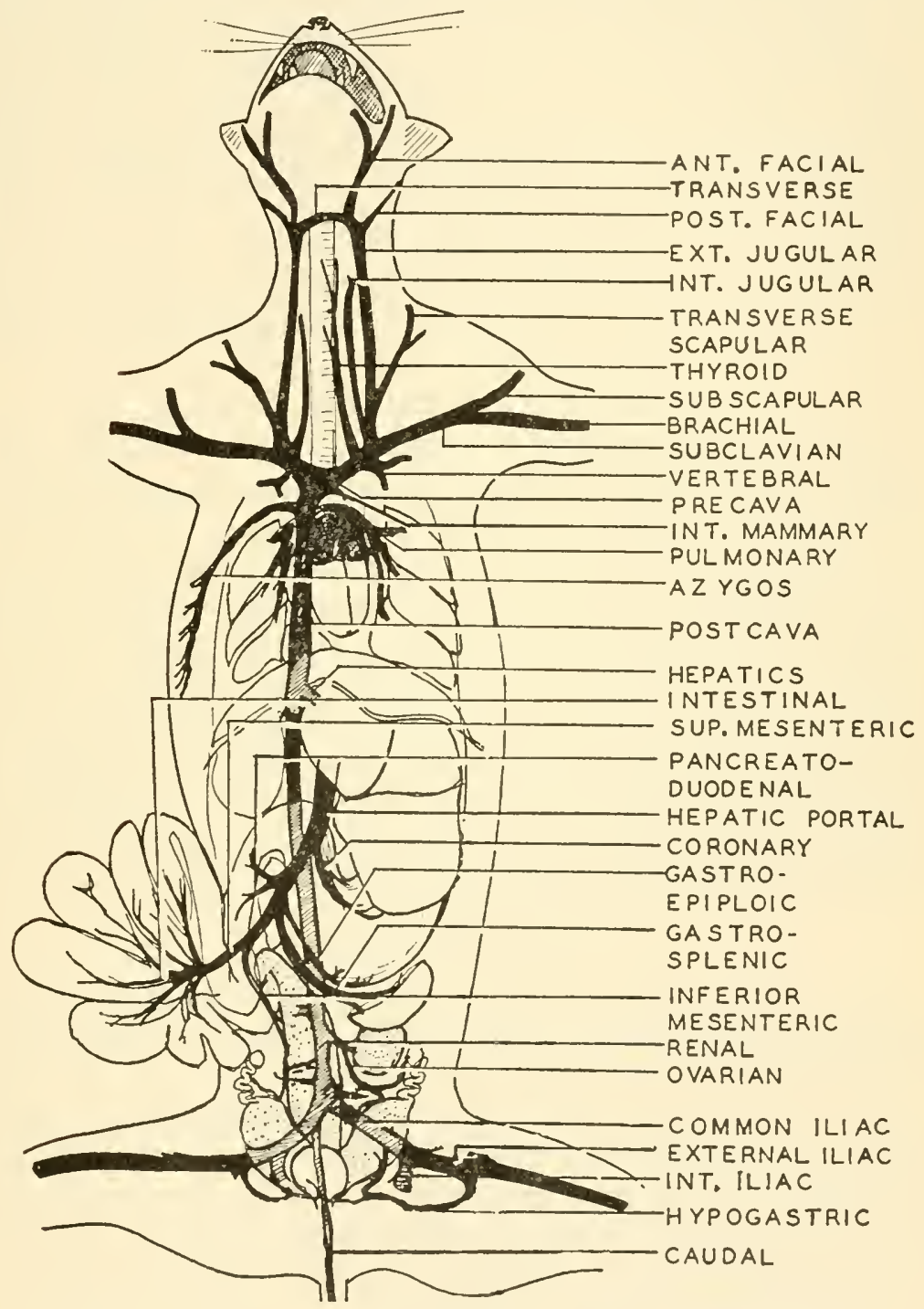

Fig. 351.-Veins of cat (ventral view). (Drawn by Titus Evans.) 
of the tongue and palate, which provides the opening from mouth to pharynx, is known as the fauces. Ducts from the salivary glands open into the mouth (Fig. 349).

The pharynx receives the two openings of the internal nares from above the hard palate and the two Eustachian tubes from the middle ears. In the floor of the pharynx is the glottis which opens to the respiratory tract and is guarded by the cartilaginous, flaplike epiglottis. Posteriorly the pharynx leads to the aperture of the esophagus which is a narrow, muscular tube leading posteriorly to the stomach, which lies caudal to the diaphragm on the left side. The esophageal end of the stomach is known as the cardiac portion, and the intestinal end is the pyloric portion. Inside, the gastric glands are embedded in the fine foldings of the internal epithelium. At the posterior end is a circular fold of the epithelium, embraced by a sphincter muscle which serves as a valve to open and close the pylorus or gateway to the intestine. Following the stomach is the small intestine which is seven or eight feet long and is divided into the anterior curved duodenum, the jejunum and the much-coiled ileum. The duodenum receives the pancreatic duct from the pancreas and the bile duct from the liver. The small intestine leads into the ascending portion of the colon, which turns transversely across the abdomen and follows posteriorly as the descending colon to the rectum. At the anterior end of the colon is a short, blind sac extending beyond the point of entrance of the ileum; this is the cecum. In human beings a narrowed extension of this is the vermiform appendix. In the rabbit the cecum is about twenty inches in length. Carnivorous animals usually have a reduced cecum.

\section{Circulatory System}

This system includes both the blood vascular portion and the lymphatic system. The blood system is responsible for the transportation of oxygen, food, and excretions. The lymph of the lymphatic system can be compared to blood without red corpuscles. It makes intimate contact with the tissues. The plasma of the blood seeps through the capillaries and is collected as lymph in the lymph spaces of the tissues over the body. These spaces are joined by vessels and many of them empty into the thoracic lymph duct which leads into the large subclavian vein of the thorax. 
The heart has four prineipal chambers, as it does in the chicken. It is fairly large and compact. The pericardial sae holds it in position in the body. There is only one systemic arch leading from the heart, and it turns to the left. The anterior part of the body is supplied largely by the branches of the innominate artery which represents the right aortie areh (Fig. 350). The two precaval veins with their branches eollect blood from the anterior, and the single posteaval vein and its branehes from the posterior, parts of the body. They bring it to the right auricle of the heart (Fig. 351). The hepatic portal system is present, serving to eollect blood from the visceral organs and deliver it to the liver. There is no renal portal system. The blood is composed of the fluid plasma, white eorpuscles, and red corpuscles. The latter are small, dise-shaped, bieoneave, and without nuelei, as is eommon among mammals.

\section{Respiratory System}

In mammals, the special organs of respiration are the lungs, wherein the earbon dioxide is taken from the blood and the oxygen from the air is supplied to it. The nasal passages lead through the nasopharynx to the pharynx and from here through the glottis to the larynx, thence by trachea to the lungs. The air is warmed as it passes in through the nasal chambers and pharynx. The air passes over the true and false vocal cords of the larynx in the produetion of sound. The walls of the trachea are prevented from eollapsing by about forty-five C-shaped rings. The trachea divides into right and left bronchi just before reaching the lungs. Each lung is completely invested with a sae of delieate, transparent serous membrane, ealled pleura, and is divided into lobes by deep elefts. The left one is eomposed of two large unequal lobes and one small lobe while the right consists of four unequal lobes.

Breathing is effected by inereasing and deereasing the size of the thoraeic eavity. To do this the ribs are moved forward and spread while the diaphragm which usually arches anteriorly is contracted to a flat position. As the ehest eavity is thus enlarged, a raeuum is produced, and to balanee the pressure, air naturally rushes into the lungs from the outside. When relaxed the wall of the thorax and the diaphragm both return to their original position and force the air out. The intaking of the air is called inspiration and the diseharge is expiration. 


\section{Nervous System}

The nervous elements of the cat, as of most vertebrates, form systems, known as the contral, peripheral, and autonomic (sympathetic). The first includes the brain and spinal cord and is protected by three membranes called neninges. The outer one is the dura mater, under it the arachnoid, and on the surface of the nervous tissue, the pia mater. The peripheral system includes twelve pairs of cranial nerves emanating from the brain, and about forty pairs from the spinal cord. The autonomic system is composed of two trunks which bear ganglia and extend one on each side of the vertebral column through its length of the trunk. Branches from these trunks reach all visceral organs.

The brain presents a much enlarged ccrebrum with fairly welldeveloped convolutions. It has spread until the diencephalon and midbrain have been covered by it. The cerebellum, which is also quite well developed, is divided into two lateral hemispheres and a convoluted, central vermis (Fig. 352).

The sense organs are relatively well developed. The olfactory organ is located in the deep posterior portion of the nasal cavity, which is quite large. The sense of taste is located in the vallate papillae and in some fungiform papillae on the dorsal surface of the tong'ue, as well as sparsely scattered in the mucous membrane of the mouth and pharynx. The ear, an organ of hearing, is composed of the pinna, or external ear, which directs sound waves to the tympanic membrane, from which they are transferred by three bony ossicles to the vestibule of the spiral-shaped cochlea. The latter contains the sensory organ of Corti of the inner ear. The eye is of typical vertebrate form and is developed for keen vision.

\section{Excretory System}

The kidneys, two urcters leading from the kidneys, the bladder, and the urethra constitute the principal organs of this system. Each kidney is composed of an outer cortical layer about one-half centimeter thick and an inner medullary substance. In the cortical substance are hundreds of renal corpuscles, each composed of a ball of capillaries, the glomerulus, and an epithelial wall, Bowman's capsule. Urine is taken from the blood here and carried through the parts of the uriniferous tubules to the pelvis of the ureter embedded in the 


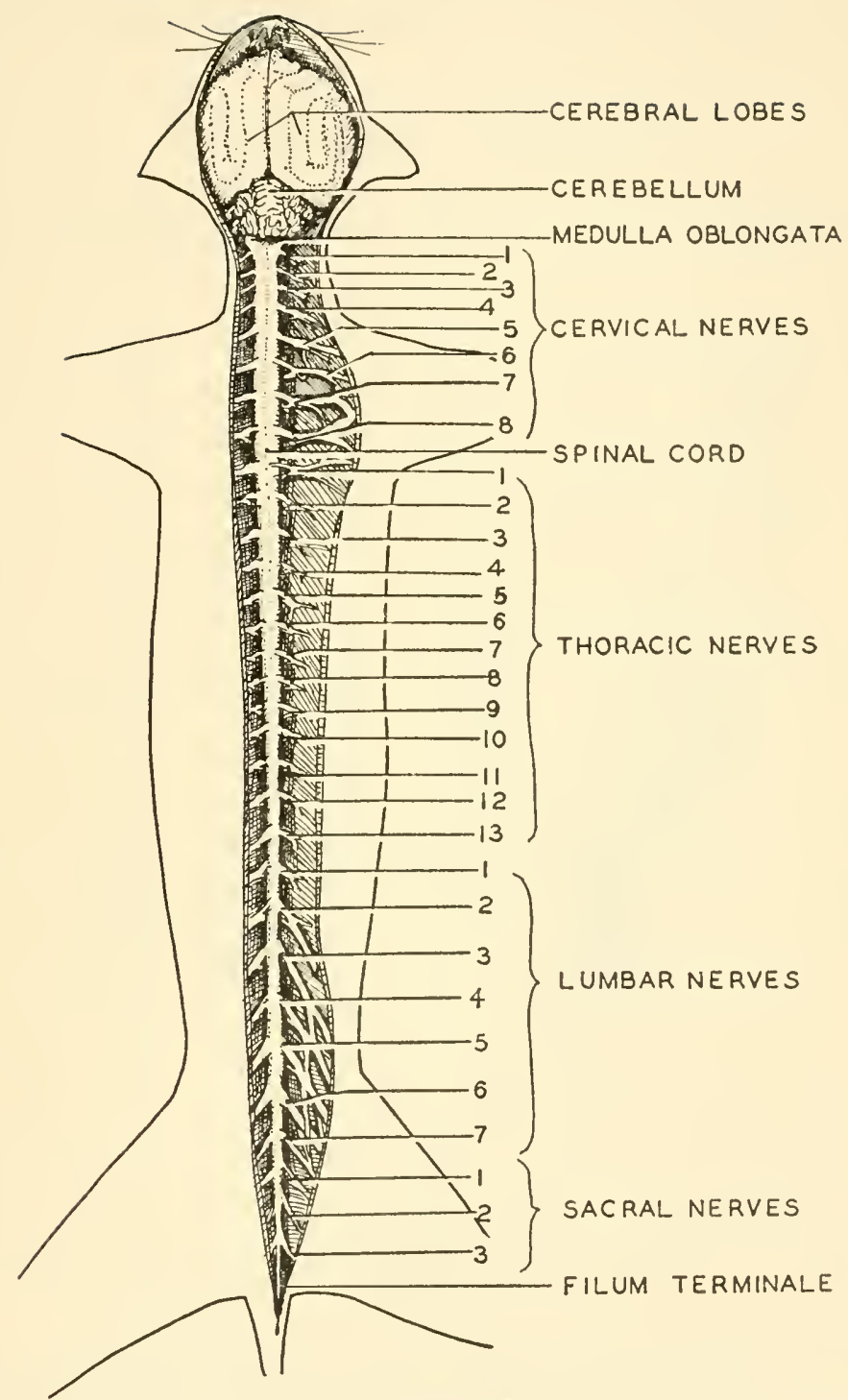

Fig. 352.-Dorsal view of nervous system of cat. (Drawn by Titus Evans.) 
hollowed hilus of the kidney. The slender ureter carries the urine from each kidney to the urinary bladder. From here it is carried to the exterior by the urethra.

\section{Reproduction and Life History}

The ovaries of the female cat are small, yellowish, oval bodies each about the size of a large navy bean and located against the dorsal

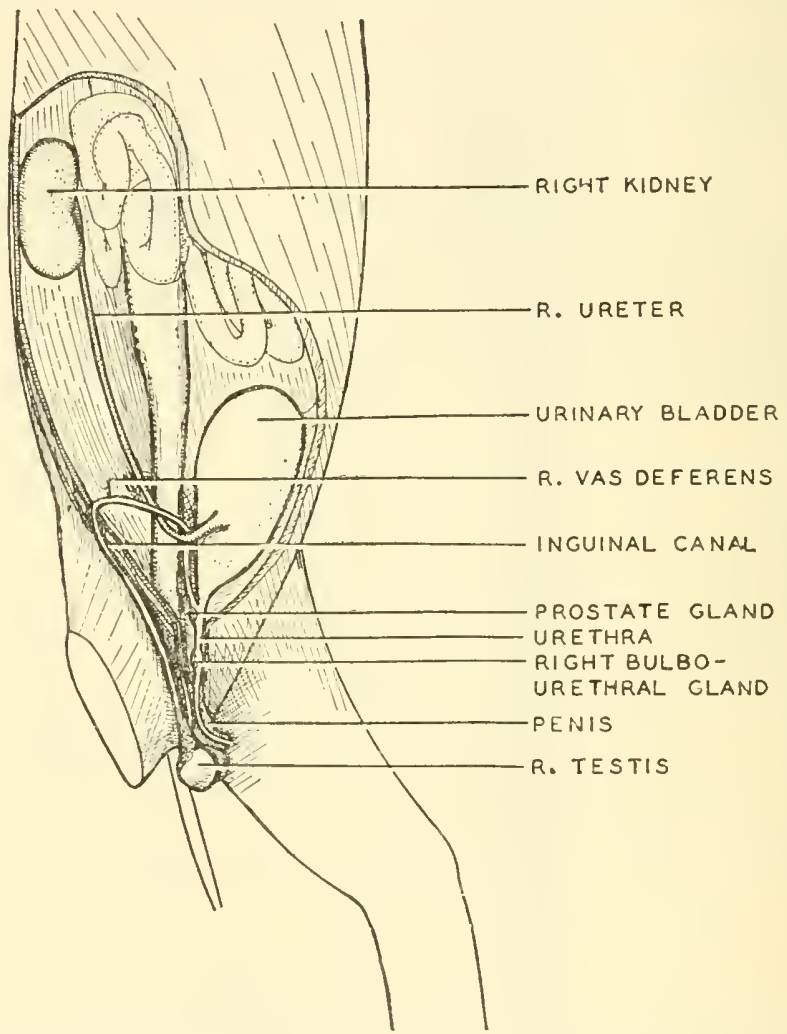

Fig. 353.- Urinogenital system of male cat. (Drawn by Titus Evans.)

body wall just posterior to the lidiney. On the surfaces of the ovaries are a number of projections, the graafian follicles, in each of which is a devcloping egg or ovum. Each of the two oviducts or Fallopian tubes has a fumnel-shaped end or ostium which fits loosely around the ovary. The oviduct leads into the horn of the uterus. The two uterine horns join in the body of the uterus which continues pos- 
teriorly as the vagina. In the ventral wall of the vagina, near the aperture, is a small body of erectile tissue, the clitoris, which corresponds to the penis of the male. The pair of testes of the male are contained in a pouch of integument called the scrotum, which liangs beneath the anus. Each testis is about one and a half centimeters long by one centimeter thick. The epididymis is a mass of coiled tubules coming from the testis and lying at its dorsal side as a part of the testicle. The vas deferens is the canal leading from this cranially through the abdominal ring and inguinal canal into the abdominal cavity, where it enters the urethra on its dorsal side near the prostate gland. During copulation the mature spermatozoa pass into the urethra and are discharged into the ragina of the female by the erected penis. The secretions of the prostate glands and of Cowper's glands just posterior to them, provide a considerable part of the seminal fluid.

The cat will first breed at the age of one year. The female will receive the male at certain periods only. MIature ova rupture through the wall of the follicle of the ovary and are normally received by the ostium of the oviduct which covers it. Occasionally an ovum falls into the body cavity. In the oviduct the ova are moved posteriorly where, if copulation has occurred, they will meet spermatozoa and be fertilized. Cleavage, or divisions of each zygote follows, and each resulting multicelled embryonic mass moves down to the uterus where it becomes implanted in the wall. Here a placenta is formed from a union of certain embryonic membranes with the internal lining of the uterus. Parental blood carries nourishment and oxygen to this membrane where it diffuses through to the embryonic blood in other vessels in the membrane. The time between copulation and birth of young (gestation period) in the cat is from fifty-five to sixty-five days. From three to six young are nsually born in each litter. They are fed from the four nipples of the mammary gland along each side of the ventral surface of the trunk. 


\section{CHAPTER XXXII}

\section{ANIMAL ANOMALIES}

It is rather generally understood among students of biology that no two animals, even of the same species, are exactly counterparts of each other. There is a certain normal range of variation in size and structure as well as in functional efficiency. Any variations beyond these rather fixed limits are classified as malformations or anomalies. A study of such abnormalities is known as teratology. Abnormalities may occur at almost any stage in the life of the individual, but a large majority result from abnormalities in the process of development. Many are due to failure of development and some to overdevelopment. In turn, much of this is due to malregulation resulting from failure of balance in the functioning of the endocrine system. The occurrence of striking malformations in newborn human babies is in approximately the ratio of one to 165 . Fortunately, many of the most grotesque anomalies do not reach full development and are born dead.

Anomalies have been classified into several groups on the basis of the following conditions:

a. Failure of Development.--The embryonic structure fails to appear or at least fails to develop to a significant degree. Examples of this account for single kidneys or legs where they are usually paired.

b. Arrested Development in which the development stops before adult condition is reached, as in the cleft palate, harelip, or diaphragmatic hernia.

c. Overdevelopment.--In such cases growth is exaggerated or the number of parts increased as in gigantism (macrosomia) or increased number of digits or accessory mammary nipples.

d. Fusion.-The kidneys are sometimes fused together to form a horseshoe kidney.

e. Splitting, thus forming extra structures, as in the case of accessory spleens or the splitting of the ureter. 
f. Displacement of Organs.-Cases are found where organs occupy abnormal positions, as both kidneys on the same side, a finger at the wrist, or the appendix on the left side.

Causes of Anomalies.-There are both internal and external agencies which bring about malformations in the individual. Both embryology and pathology contribute to the explanation of the causes of these abnormal conditions. The development may be perfectly normal and a subsequent disease may be the cause of striking abnormality. On the other hand, certain discases of the parent will influence the normal development of the fetus. Even twinning by itself is an abnormal process in most animals. It, however, is not usually thought of under the title of anomaly, except when they are
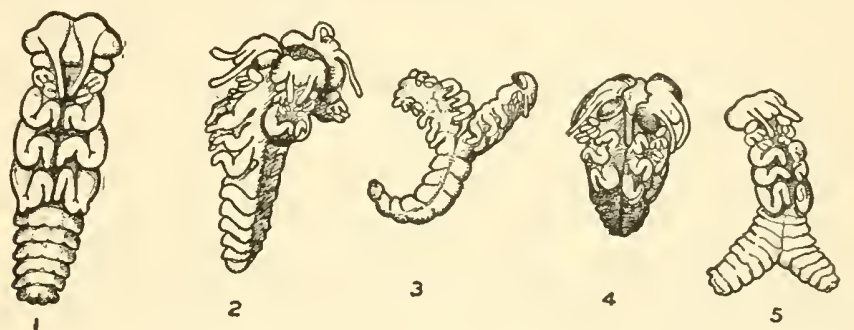

Fig. 354.-Grasshoppers at time of diapause, showing some of the abnormalities which very infrequently occur in their natural development. 1, Normal embryo: 2 , embryo with two extra heads and mouth parts; 3 . embryo with a lateral twin joined at the abdomen: 4, almost complete twins back to back: 5 , embryo with a double abdomen. (From Evans, "Effects of Roentgen Radiation on Embryonic Organization and Regulation in Melanoplus differentialis [Orthoptera]," Physiological Zoology, Vol. X.)

physically connected or the individuals are otherwise malformed. The causes of anomalies may be summarized as follows:

\section{Internal Causes.}

a. The germ plasm carries liereditary causes for some, and these characteristics are transmitted as are normal traits. Color blindness and hemophilia (bleeder condition) are examples.

b. Diseases which cause abnormal growths and conditions, as elephantiasis. (Fig. 389.)

c. Unbalancing the chemical regulators (hormones) which are produced by the endocrine glands. (Overactivity of the hypophysis causes gigantism; cretinism, a dwarf condition, results from deficiency physically connceted or the individuals are otherwise malformed. in thyroid activity.) 
d. Fortuitous abnormalities whose causes are not apparent or are influenced by certain variations in other organs. Such anomalies show up most frequently in the circulatory and nervous systems. Venous or nervous supplies to organs are often modified. Another example is the rearrangement brought about by diaphragmatic hernia.

2. External Causes.

a. Environmental agencies may affect almost any individual whose development occurs outside the body of its parent. Exposure to radium or $x$-ray radiation, sharp variations in temperature, excessive salt content, or contact with toxic substances may all be responsible for various degrees of abnormality. The same factors are also effective, if present, in the uterine environment of the placental type of animal.

b. Mechanical factors, such as abnormal pressure, blows, and falls, may cause some abnormalities.

c. Abnormal implantation in the wall of the uterus resulting in deficiencies in nourishment and support of the fetus.

d. Such diseases as syphilis, which may be transmitted from mother to offspring, are responsible for some types of defects, as impaired vision.

e. Developmental inhibition or arrest brought about by deficiencies in metabolism at a time when the rate or efficiency should be high. The work of several embryologists seems to indicate that the production of twins either as normal individuals or otherwise is related to this condition.

\section{Harelip and Cleft Palate}

These two defects are related and are sometimes found together. The lateral palatine processes may fail to complete growth and unite properly, thus leaving a gap in the roof of the mouth which opens directly into the nasopharyux above.

Haretip (cheiloschisis) is a very common defect and is due to the failure of union of the nasal and maxillary processes. There may be double harelip or single harelip. In comnection with double harelip it sometimes happens that the premaxilla projects beyond the outline of the face to form what is called wolf-snout. Harelips are frequently remedied by a surgical operation early in life. 


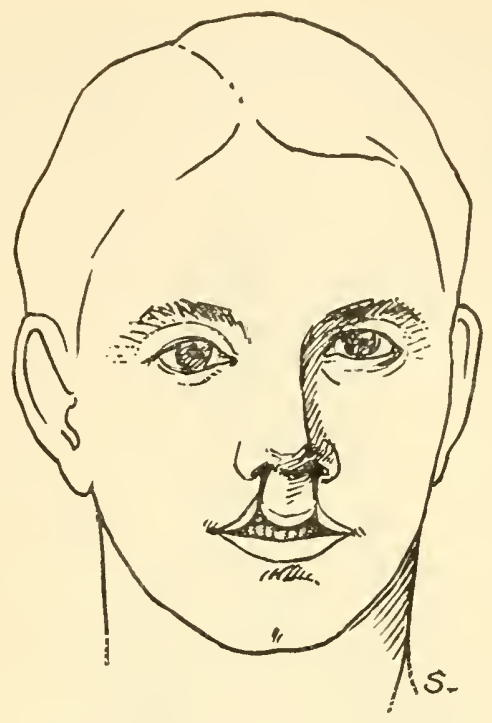

Fig. 355.-A case of harelip due to arrested development.

\section{Diaphragmatic Hernia (Open Diaphragm)}

An extreme case of this was found in a cat which was being used for dissection purposes. The animal had lived an apparently normal life and had been killed for laboratory study without showing evidence of its abnormality until dissected. From all appearances the diaphragm had not completed its development, but had formed a fringelike projection which reached inward about half an inch from the thoracic wall and extended the entire circumference of the inside of the thorax. The aperture in its center measured one and three-fourths inches in diameter.

Due to this condition the arrangement of several visceral organs was greatly affected. The thorax was somewhat elongated, and the right side of the cavity comprised about two-thirds of the space of the chest. The mediastinum (supporting median mesentery of heart and lungs) had its attachment more than half an inch to the left of the midline.

Almost the entire liver was turned forward to occupy the right two-thirds of the chest eavity, and this placed the gall bladder at the level of the junction of the auricle and ventricle of the heart. 
Approximately half of the spleen, the pyloric portion of the stomach, and a large portion of the omentum had been drawn through the aperture in the diaphragm. The right lung was extremely crowded and small.

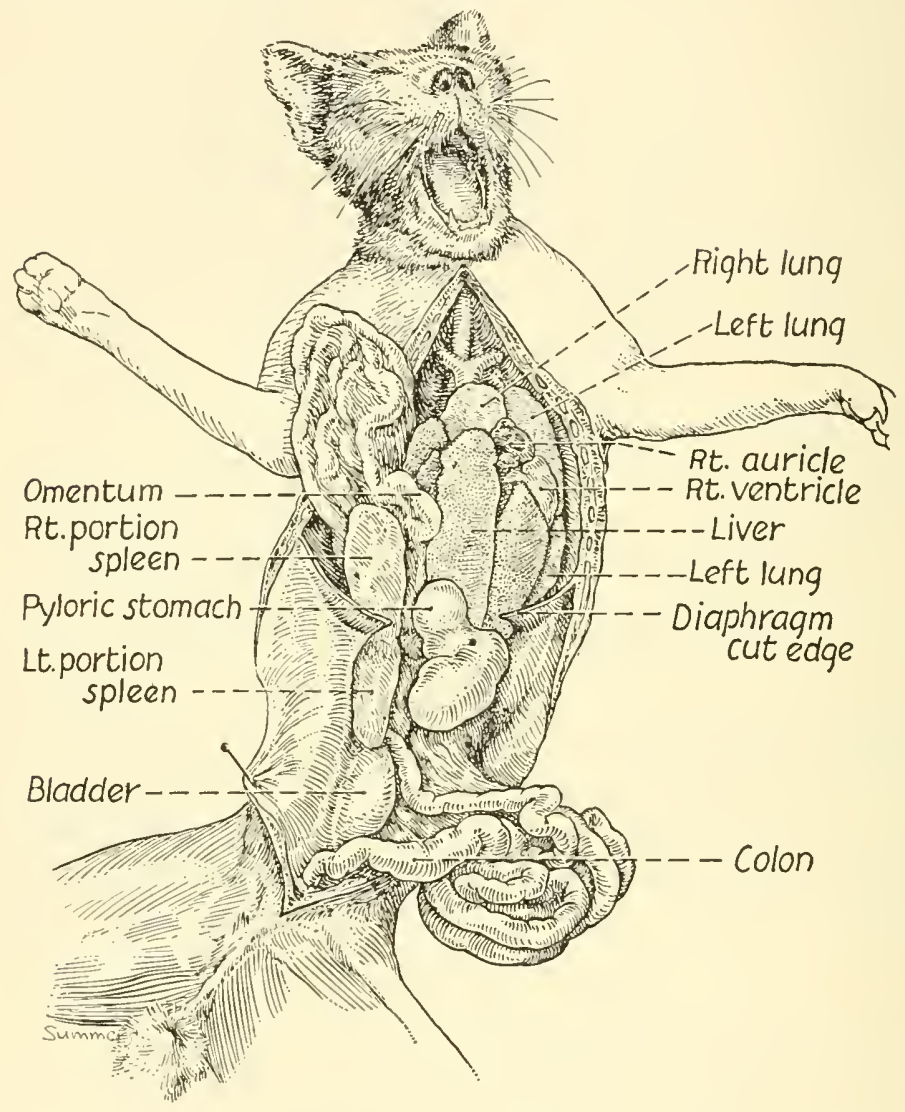

Fig. 356.-Dissection of a fully-grown cat with a congenitally open diaphragm (diaphragmatic hernia), showing many of the abdominal organs everted into the thorax.

This anomaly occurs occasionally in human beings, but usually in much less degree. It is not likely that a man would be able to reach maturity and carry on normal activities with such an extensive hernia. 


\section{Polydactylism (Extra Digits)}

There are numerous abnormal variations in the number and arrangement of digits, ranging from a stumplike structure of no digits through the "lobster claw" condition of two or three, to as many as two more than normal. The polydactylous condition is rather frequently found in cats. In fact, Wilder once reported that possibly 25 per cent of the cats of the vicinity of Ithaca, N. Y., possessed an extra digit on each foot. The forefeet shown in Fig. 357 are from a living cat which came from a litter of four, two of which showed these complete characteristics on all feet, one of the litter had only an extra toe on one foot, and the fourth was normal. Both hind feet of this animal had five well-developed digits instead of the usual four. This condition is a hereditary one and is brought about by partial duplication of elementary structures.
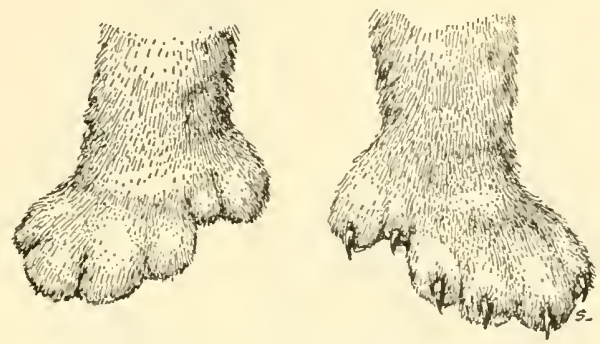

Fig. 357.-Front feet of a half-grown kitten with six toes. The claws are protracted on the left foot and retracted on the right. There were five toes on the hind feet of this cat.

\section{Conjoined Twins}

Instances occur in which individuals of about equal size or of unequal size are fused together. If this occurs at the hips with dorsal sides together, the condition is usually known as Siamese twins. There are a number of different varieties of fusion, including the head region, chest region, or complete fusion of trunk into a single body with two heads and vice versa. A chicken with two pairs of legs and wings, two backs, and a single head has been dissected and described by Dr. F. L. Fitzpatrick. The feather tracts were double on the neck and trunk. Internal dissection showed the single digestive system to extend between the two necks, follow through the combined body cavity with some modifications, and empty into the cloaca of the right back region. No cloaca was pres- 
ent in connection with the left back. There was a single heart, two very unequal lungs, two pulmonary arteries and veins to the larger lung, while there was only one common carotid artery, that being the right. Two trachea were present, the right being rather normally developed while the left was rudimentary. The brain was normal, but joining the medulla were two separate and complete

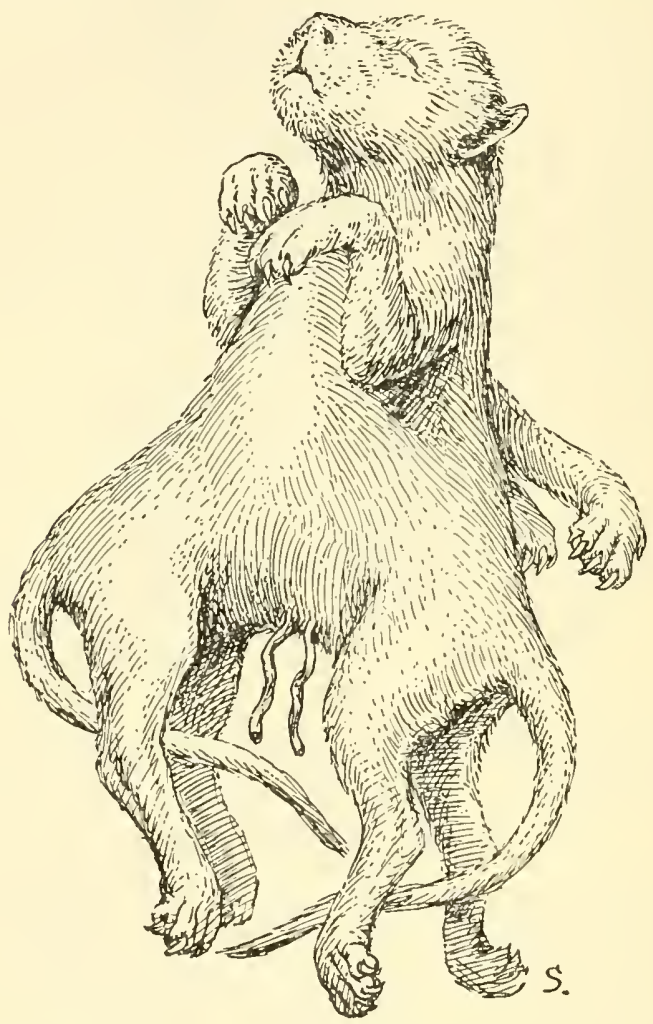

Fig. 358.-Conjoined twins of cat showing single head, but double trunk and appendages.

spinal cords, one passing to each back region. Most of the internal organs "favored" the right side, except the lungs, of which the left was much more developed.

Dr. F. L. Fitzpatrick reports upon the anatomy of a double pig also. This freak had two tracheae leading to two sets of lungs. The brain consisted of a three-lobed cerebrum, two cerebellums, 
and two medullas. There was a spinal cord in each of the two backs. A third or median eye was present on a ventral stalk beneath the third or transverse lobe of the cerebrum. Two pairs of kidneys, two bladders, four ovaries, double sets of oviducts and uteri, as well as

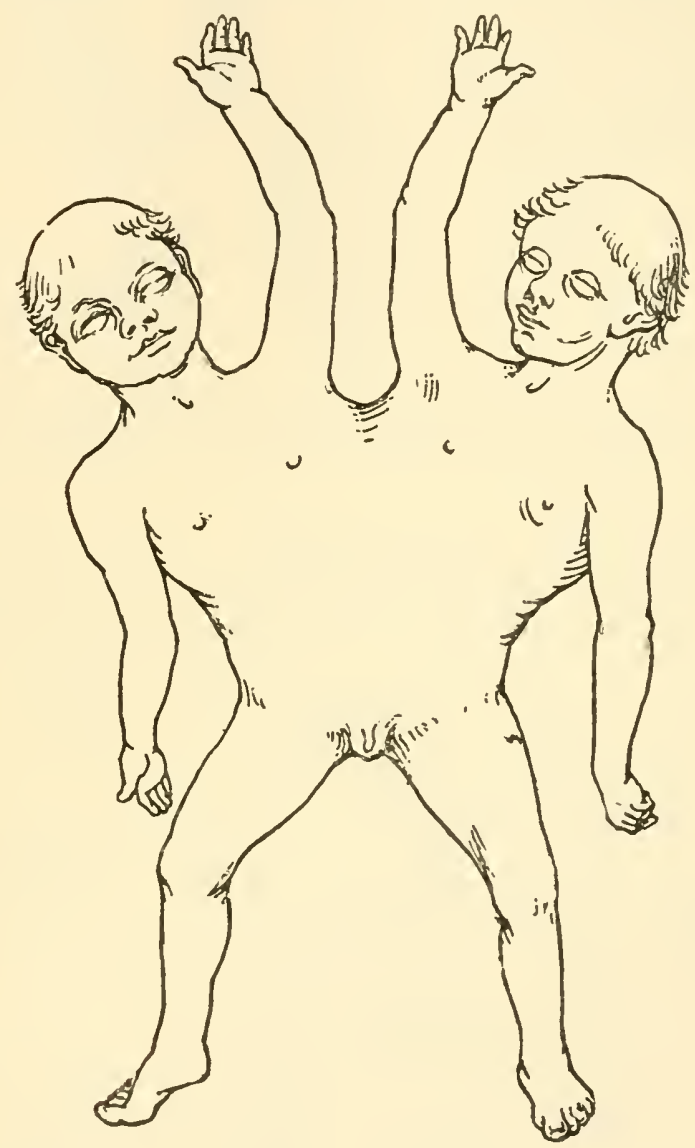

Fig. 359.-Conjoined human twins showing single hips but double trunk and head. (Redrawn and modified from Arey, Developmental Anatomy, published by W. B. Saunders Co.)

a double cloaca, were present. There was a single but modified heart and two aortas leaving it. Externally the animal had two sets of limbs, two tails, two sets of mammae, two anal apertures, and two urinogenital openings. 


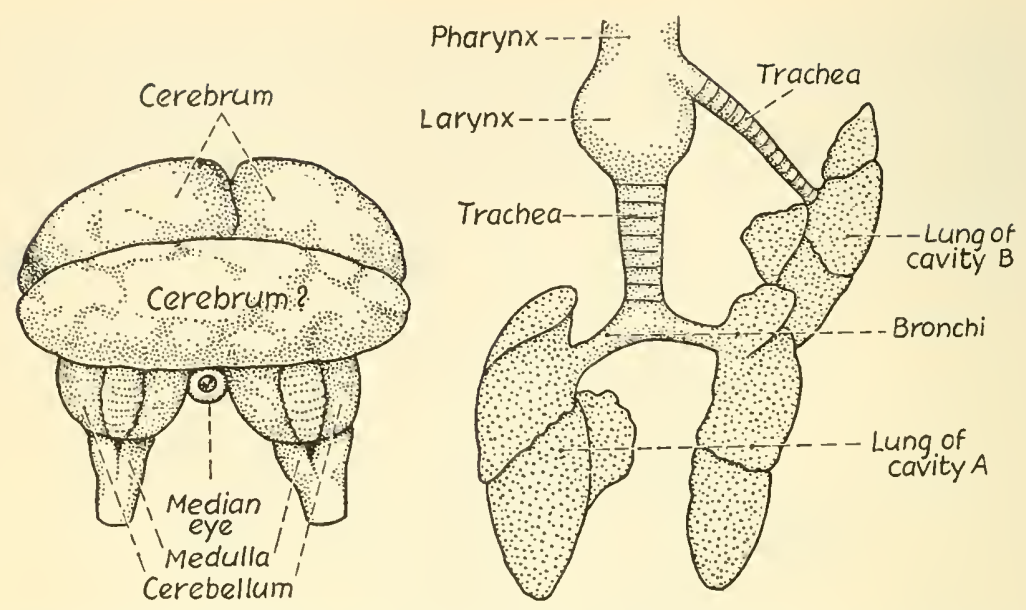

Fig. 360.-Left, dorsoposterior view of brain from pig embryo with double body and single head. Right, larynx, tracheae, and lungs of pig embryo with double trunk. (After Fitzpatrick.)

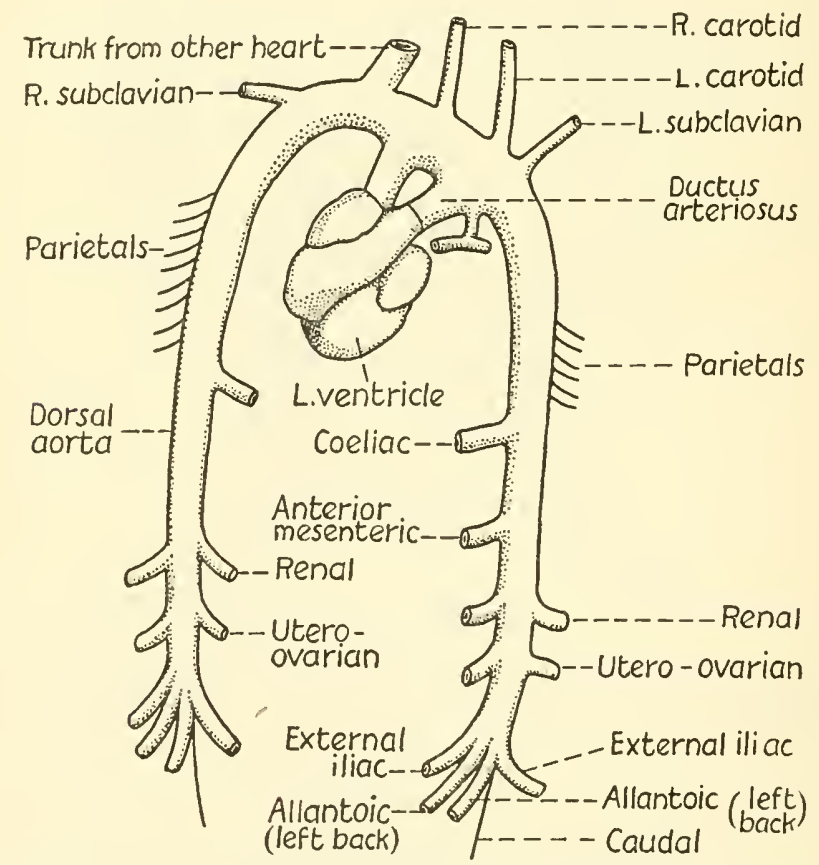

Fig. 361.-Dorsal aortae and branches in embryo pig which had a single head and two bodies. (After Fitzpatrick.) 
It is suggested that such individuals have developed from a single zygote which underwent an abnormal cleavage. Examples of these conditions are shown as they occur in cats. Similar fusions occur in human beings as is illustrated in the accompanying diagrams.

There are also cases of unequal conjoined twins in which a much smaller and less developed individual is fused to the abdomen, head, palate, or sacrum of an otherwise normal individual. Such an individual is referred to as a parasite on its larger mate.

\section{Hermaphroditism}

There are abnormal cases of sexual development in vertebrates including man, in which the organs of both sexes are combined in one individual. In true cases there are present both ovaries and testes in the one individual, but no cases are known in which both have functional capacity. The external organs are partly male and partly female, and the secondary sexual characteristics, such as voice, mammae, stature, and beard, may be of a mixed nature. Hermaphroditism is the normal condition in the coelenterates, flatworms, annelid worms, and molluses. Here both sets of organs are capable of function. Birds and mammals are rarely subject to this condition.

A condition spoken of as false hermaphroditism involves the presence of gonads of one sex but the secondary sexual characteristics and external genitalia of the other. In masculine hermaphroditism testes are present but not usually descended into the scrotum, while the external genitalia and secondary characteristics are those of the female. In feminine hermaphroditism ovaries are present, even descended into a scrotum in rare cases, but the clitoris is enlarged and labiae are fused to resemble the penis of the male. In some instances the lips of the slitlike urinogenital aperture on the under side of the penis fail to fuse.

There is a very close homology in the organs of the two sexes. The external genitalia are indifferent or sexless until the end of the seventh week of embryonic development in human beings. Then the determination comes, causing normally the modifications of development to form the organs of one sex or the other. The development of the external organs of the two sexes is strictly parallel. 
It is likely that this development is controlled by hormone relationships, and it is in cases in which this balance is disturbed that hermaphrodites occur. There is still much to be learned concerning the causes of this condition.

\section{Cardiac Anomalies}

Transposition of the heart to the right side of the midline of the body is known as dextrocardia and occurs rarely. It is usually associated with displacement of other visceral organs.

There has been a case reported recently in which the autopsy of a 73-year-old farmer disclosed that he had died because of a ruptured left ventricle of an extra heart. One of the hearts of this man was in the normal position, the other (the one that failed) was located just above the spleen and below the left lung in the lower part of the chest. The hearts were both of about normal proportions and both functioned actively. They both joined the aorta.

Due to faulty development, an incomplete interventricular septum is occasionally found in the four-chambered hearts. The failure of complete development of the septum between the auricles to close the foramen ovale is a more common anomaly. It has been reported that this occurs in some degree in one case in four for the human being. Actual mixing of auricular blood sufficient to interfere with normal function is much less common, however, because of the overlapping of the membranous walls which are pressed together by the pressure of contraction. In a small number of human cases the aerated blood and unaerated blood from the two auricles do mix and produce a purplish colored blood and in turn affects the color of the skin. Such a case is known as a "blue baby" and often leads to early death.

\section{Abnormalities of Brain and Sense Organs}

Encephalocoele is due to the protrusion of a sac of the meninges and part of the brain through a defect in the roof of the cranium. An abnormally large brain, which is usually associated with the distention of the cranium by superabundance of cerebral fluid, is known as hydrocephalus, or the size may be macrocephalus. The opposite extreme in which the head and brain are abnormally small due to failure of development is known as microcephalus. 
There are cases of cleft nose in which the nostrils are in independent projections. This condition is usually associated with harelip and cleft palate. With regard to the eyes there are several possible abnormalities. Cyclopia is a condition in which there is a single median eyc like that of the Cyclops instead of the usual paired arrangement. In such cases the nose is usually at the base of the forehead, above the eye, and cylindrical in shape. Failure of complete development of the iris or chorioid, thus leaving a gap or open sector in the margin of the pupil, is known as coloboma. 


\section{CHAPTER XXXIII \\ THE ENDOCRINE GLANDS AND THEIR FUNCTIONS}

The great complexity of the structure of organisms, particularly of those animals in the higher ranks of the animal kingdom, makes necessary a means of regulation and coordination of the functions of the organ systems individually and a means of intercommunication between them. This work is cared for in part by the nervous system; but another agency of regulation is present in all higher organisms and many of the lower forms of life, which is of first importance in this respect, one so complex and interwoven with other organs in carrying out its functions that it is not thoronghly understood at the present time. The group of organs doing this work we designate as the endocrine glands. They manufacture and furnish the body with chemical compounds called hormones, a term which refers to compounds formed in the body and capable of encouraging or exciting activity in another part of the body; they are chemical messengers. The endocrine glands pour their hormones directly into the blood stream without the assistance of ducts, and the blood carries them to other parts of the body; but they are never carried to the outside of the body directly, as in the case of secretions from the glands of external secretion, and for this reason the endocrines are often referred to as organs of internal secretion, or ductless glands.

The functions of the hormones are numerous, and current research and investigation in the new field of endocrinology are constantly presenting new activities and interrelationships of these secretions. The field of endocrinology is comparatively new, and a complete understanding of the complexities involved is impossible until further research is completed. The mysterious manner in which the endocrine organs produce their secretions, the hormones, the manner in which they choose the raw materials, combine them, and give forth products which are vital to the welfare and happiness and often to the very existence of an organism, is of most intense interest and of the greatest importance. 
The hormones may be classified, arbitrarily, according to function in three main groups. The first of these groups includes those hormones which arouse specific responses in particular organs or in localized parts of the body. A second group is composed of those hormones which affect the general metabolism of the body. The third group is that of the hormones which affect, in particular, the growth and development of the organism. Another general method of classifying substances of this nature terms those substances which serve as excitors or accelerators "hormones" and those which inhibit or depress activities as "chalones." Illustrations of each of these will be found in the discussion of the individual organs and their functions.

Hormones are found in plants as growth hormones, in the invertebrate animals, and are best known in vertebrate animals. The following examples will serve to demonstrate the naturc of endocrine function in invertebrates: Earthworms whose testes are completely destroyed, do not develop the clitellum, the band-like organ which funetions during and following copulation. In Bonellia, another annelid worm, there seems to be a relation between sex determination and certain hormones, in that the week-old larvae are indifferent sexually, but those that attach to the proboseis of the female parent become males due to some agent received there. All others develop to become normal females. Among the crabs there is a parasite which attacks and destroys the gonads, and it is found that the parasitized male crab will take on distinctly female characters due to the lack of some humoral agent lost with the destruction of these glands. Too, using radium to destroy the gonads of Asellus, another crustacean, seems to indicate that the development of the brood pouch is controlled by a substance produced in the normal ovaries. Molting and metamorphosis both are regulated by hormones in insects. The supraesophageal ganglion seems to produce a substance which initiates pupation activities in moths. The endocrine glands of vertebrates which are best known and most clearly understood at the present time are the thyroid gland, the parathyroid glands, the suprarenal bodies, the pituitary body or hypophysis, the thymus, the gonads, and the pancreas.

\section{The Thyroid Gland}

The thyroid gland is the most familiar of the endocrine organs to the layman. It is a body of two lobes of about the size of walnuts, slightly flattened, placed one on each side of the upper part 
of the trachea just below the larynx, or voice box, the two lobes being connected by a saddle-shaped isthmus. The thyroid is well supplied with blood, receiving in proportion to weight, three and one-half times as much blood as the brain; this permits an easy access of the hormone to all parts of the body. The thyroid is normally not visible externally, but the pathogenic condition of the organ caused by overgrowth, and known as goiter, is familiar to everyone.

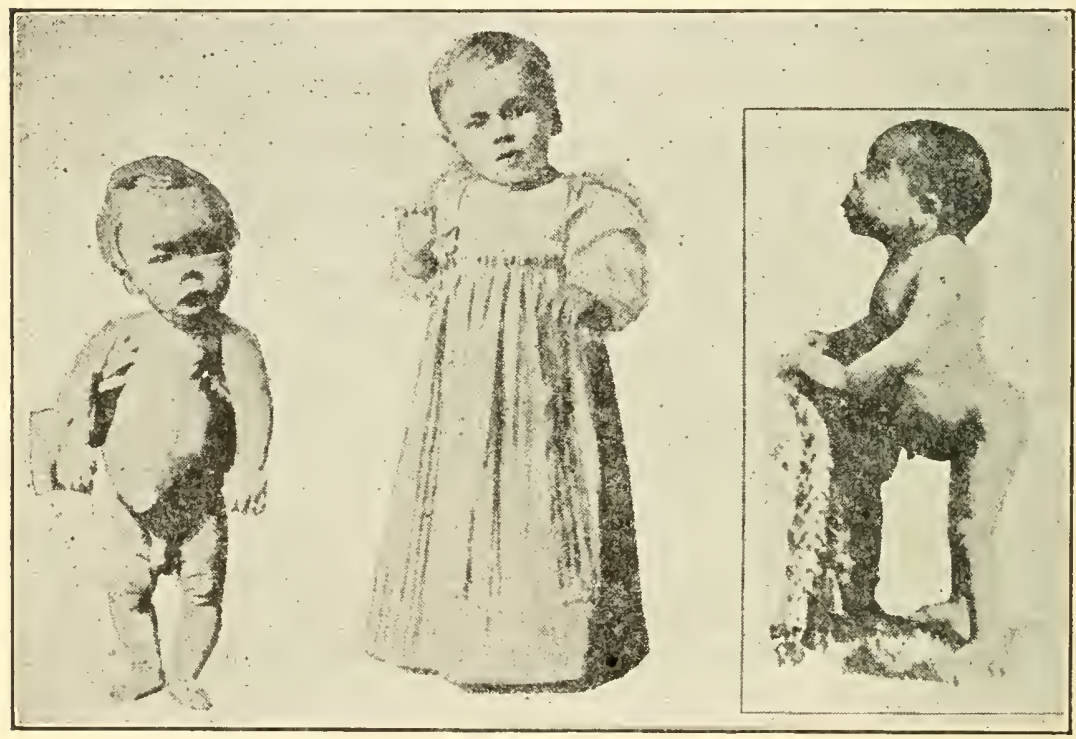

A.

$B$.

C.

Fig. 362.--Illustrating cretinism. $A$, a cretin, 23 months old and $B$, the same child after having received thyroid treatment for eleven months. $C$, an untreated cretin 15 years old. (From Zoethout, Textbook of Physiology, published by The C. V. Mosby Company, after Osler.)

The functions of the thyroid gland, as recognized today, are two: that of control, in conjunction with other endocrine organs, of the growth and development of the body; and, second, a most important role in the regulation of metabolism. Any upset of the normal functioning of the gland or the removal of the gland results in serious physical and mental disorders, if not in death itself. The hormone produced by this organ, designated ihyroxine, has been prepared from the fresh thyroid glands removed from various animals and has also been manufactured synthetically. Chemical diag- 
nosis of the hormone reveals the presence of iodine in its composition, and the amount of iodine available in the body seems to be the determining factor in the degree of control which this hormone exerts on bodily functions. In regions of the world in which iodine is scarce in water and food, goiter is a prevalent disorder, and only in recent years has the understanding of the eause been complete enough to suggest as a remedy and a preventive the introduction of additional iodine into the diet by use of iodized salt. Since the amount of iodine required for normal purposes is almost infinitesimal, a sufficient quantity is supplied by this means. The correction of disorders due to a deficient functioning of the thyroid gland by use of natural thyroxine or by use of the synthetic product is quite common today among human beings.

The removal of the thyroid gland in animals which have not obtained their complete growth results in delayed or arrested development. An interesting example of this is found among certain amphibians. The proper functioning of the thyroid gland is essential for the accomplishment of metamorphosis of the frog tadpole into the adult frog. The tadpole lacking sufficient thyroid extract may grow to an unusually large size, but metamorphosis never occurs without the encouragement of this hormone. In the development of human beings, a deficient supply of thyroxine in early years results in a condition known as cretinism. The growth of the bones does not take place, the entire body is stunted and deformed, mental development ceases, the facial features are misshapen, growth of hair is scant, and the development of the sexual organs is incomplete. Before work on the thyroid gland was undertaken, cretins were often seen in certain parts of Europe and occasionally in America; but the present understanding of the hormone and its use has made possible the prevention and cure of most eases of this nature.

Improper functioning of the gland in adult years results often in a condition known as myxedema, in which there is a thickening and drying of the skin, a puffiness of the eyelids and lips, loss of hair due to the condition of the skin, a slowing down of metabolism and heartbeat, a depression of body temperature, the deposition of large quantities of fat, and a final result, in many cases, of imbecility. The administration of thyroxine, cspecially in the early stages, accomplishes a complete, or at least a temporary, remedy.

An overfunctional thyroid gland, in which the condition is known as hyperthyroidism, results in an increased metabolic rate, a loss of 
body fat, and a condition of hyperirritability of the nervous system. It seems, therefore, that the difference between an overly energetic and a sluggish person, and a lean and an obese person, may often be traced directly to the degree of functioning of the thyroid gland. Hyperthyroidism is accompanied by increased excretion of ealcium. It differs from the calcium upset due to parathyroid disturbance in that in hyperthyroidism its concentration in the blood remains normal.

Work on the lower vertebrate groups suggests the probability of an important function of the thyroid gland in determining the hibernation periods of certain animals. The thyroid performs additional functions in conjunction with other of the endocrine glands; e.g., the control of sexual activity; but these interrelationships will not be discussed here. Peculiarly, thyroxin, although an accelcrator of oxidation in vertebrates, has a depressor effect on cell division and differentiation in such invertebrates as Paramecium, sea urchin, and the hydroid Pennaria. This has not been completely explained.

\section{The Parathyroid Glands}

Connected with the thyroid body are four little glands about the size of small peas, so insignificant in appearance that they were overlooked for many years. The removal of these small bodies along with the thyroid gland in certain operations provoked such startling results, however, as to attract attention to their presence and to evoke considerable interest in their investigation. A complete removal of the parathyroids results in unbalaneing the blood calcium and in a type of convulsion known as tetany; death is the usual result. A deficient supply of parathormone or parathyrin, the hormone of the parathyroid glands, may be responsible for defective growth of the bones and for deficient formation of enamel and dentine of the teeth. Calcium is necded for both the teeth and the bones and the introduction of either calcium or parathormone into the body is made to supplement a deficient supply of calcium due to malfunctioning of the parathyroids. Removal of the parathyroids also brings about a fall in the renal excretion of phosphorus, and the injection of parathormone canses an immediate rise in the level of renal phosphorus. Parathyroid activity is particularly useful to laying hens where so much calcium is needed in shell formation. There is evidence of a close functional relationship between the pituitary gland and the parathyroid. In dogs the removal of the pitnitary causes atrophy of the parathyroids, particularly if the pancreas has been removed also. 
Conversely it has been noticed that injections of extract from anterior pituitary raises blood calcium in some species because of increased activity of the parathyroids.

Too great an activity on the part of the parathyroid glands results in a decalcification of the bones, and an inereased content of calcium in the blood and in the excretion of the kidneys. The final result of this softening of the bones may cause serious disfiguration and stunting of the body. Accompanying these results are flabbiness of the muscles, decreased irritability of the nervous system, and other unfavorable conditions. These may be remedied by the removal of a portion of the parathyroids.

\section{The Suprarenal Bodies}

Lying close to and slightly anterior to the kidneys are two small yellow or reddish masses of tissue, which play a prominent role in the regulation of the body and one of such complexity that much is yet to be learned concerning its method of functioning. These adrenal glands, or suprarenal bodies, are made up of an inner and an outer portion, the medulla and cortex respectively. The former secretes a substance designated as adrenalin (cpinephrine or adrenin), which acts upon various organs and raises the level of their functioning. Adrenalin, at times of excitement or emergeney, may cause constriction of blood vessels, increased rate of heartbeat, a greater discharge of glucose from the liver to provide additional energy, erection of hairs, stimulation or inhibition of the various visceral muscles, etc., to provide greater efficiency. The range of control of the medullary portion of the adrenals is thus wide and complex, definitely interrelated with the functions of the sympathetic nervous system, with other endocrine glands, and numerous processes of the body, so that its true importance is difficult to estimate. Adrenalin has been obtained from various animals for use in the treatment of certain disorders, as an anesthesia in minor operations, and to stop small hemorrhages. It has also been successfully employed in the relief of asthma and similar troubles.

A hormone known as cortin has been isolated from the suprarenal cortex, and, while the removal of this portion of the adrenals results in death, the exact functions of the hormone produced therein are not entirely understood. It does relieve the condition known as Addison's disease. There is, without doubt, a close relationship between the eortex and sexual development; and some workers 
believe that the cortex regulates the normal flow of blood, which would account for the fatal results of its removal. An extract has been secured from the adrenal cortex of cattle in particular and is used for the treatment of conditions resulting from malfunctioning of the cortical portion of the adrenals in other organisms. Some have shown cortin to have capacity for delaying the onset of scurvy in vitamin $\mathrm{C}$ deficiency.

After complete bilateral removal of the adrenal cortex, the following were the average survival periods for these several animals: opossums, six days; guinea pigs, seven days; dogs, ten days; cats, twelve days; while rabbits and rats may live on quite normally, because of the presence of accessory inter-renal tissue. Symptoms of insufficient cortin are loss of appetite with particular distaste for fats, vomiting, diarrhea, muscular twitching, tetanic convulsions, lowering of blood pressure and body temperature, and decline in urine secretion and heart rate. Lack of cortin is said to disturb the salt relation in the blood (particularly sodium chloride and potassium), the water metabolism and redistribution in the body, the metabolism (especially absorption) of intermediate carbohydrates and fats, and milk production in females (at least in cats).

\section{The Pituitary Gland}

The pituitary gland is the human being is a body weighing about $0.5 \mathrm{Gm}$., lodged in a depression at the base of the brain. It consists of two principal parts: the anterior lobe and the posterior lobe. These two portions have distinet functions. The vital importance of this body was not realized for many years, but a series of observations has placed it in a position of such importance that it has been referred to as the regulator of the glandular system. The pituitary gland is now known to be the source of a number of hormones, and their functions are so closely connected with those of other endocrine secretions that they apparently have a part in all hormonal processes of the body.

The secretions of the anterior lobe of the pituitary affect the growth and development of the organism in general, the general metabolism of the body, the development of the sex organs, and work with other hormones in controlling additional processes. Inefficiency in the anterior lobe, furnishing the body with either too much or too little of the growth-promoting hormone, phyrone, re- 
sults in the production of giants or dwarfs. A decreased supply of the hormone in an immature individual, if the condition is not remedied by administration of the hormonal extract, retards the growth of the body and may cause a complete cessation of growth. The dwarfs of the circus furnish examples of this unfortunate condition, although not all dwarfism must be thought due to this cause. For example, the cretin described previously is the result of thyroid disorder and is usually a mental dwarf as well as a dwarf in body, while dwarfism resulting from deficient phyrone is accompanied in most eases by a normal mental development.

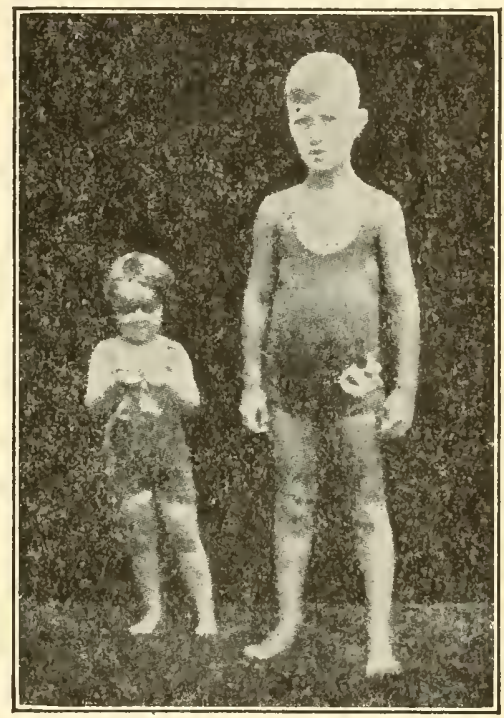

Fig. 363.-A pituitary dware at the age of nine and one-half years, compared with a normal child of the same age. (From Zoethout, Textbook of Physiology, after Engelbach.)

An overfunctional anterior pituitary results is a marked increase in the growth of bones, although the general development of the individual thus affected may be symmetrical and the physiological processes may be normal in every respect. Cases of this type are commonly seen. One such instance may be cited in which a nineyear-old boy measured six feet and one inch and weighed 178 pounds. His mental condition was normal.

Another condition, known as acromegaly, may result from an overfunctional anterior lobe of the pituitary during adult years. The 
facial bones enlarge, particularly in the ridges above the eyes, the nose, and the lower jaw, and the soft tissues of the face undergo an overgrowth resulting in a coarsening of the features. The hands and feet may enlarge, also. The disease may prove fatal if it contimues sufficiently long.

A second hormone secreted by the anterior lobe has a direct influence on the sex organs. This hormone, prolan, stimulates growth and activity of the gonads, the testes and ovaries, and therefore controls the production of the gonadal hormones, which will be diseussed later. The absence of prolan results in an atrophy of the testes and ovaries, and the cessation of the production of the spermatozoa and the ova; its injection increases the activity of the sex organs.

The posterior lobe of the pituitary also produces more than one hormone, although pituitrin is the one concerning which we have the most definite knowledge and which is commonly associated with this portion of the organ. Pituitrin is known to stimulate the muscles of the arterial system, increasing or decreasing the blood pressure according to the amount of the hormone released in the blood. It is also a stimulant for the musculature of the uterus and the intestinal muscles.

It is concerned also with the regulation and disposal of carbohydrates in the body. The body is able to use an increased quantity of sugar when the secretion of pituitrin is reduced; and, on the other hand, when the quantity of the hormone is more than normal, the body needs less sugar; earbohydrates not actually needed are stored as fat, resulting often in abnormally fat people, extreme cases of which are seen in the circus. The posterior lobe of the pituitary probably does not affect the development of the bones, but the function just discussed is quite definite.

The posterior lobe of the pituitary is concerned also with the regulation of the secretion by the kidneys. A diseased condition, known as diabetes insipidus, in which the patient voids large quantities of urine, is treated by injection of the postpituitary hormone. It appears that the hormone probably enables tissues to utilize and store larger amounts of water than is possible in its absence.

Still another effect of the secretion is found in the case of certain amphibians and reptiles; that is, its effect on the pigmentation of the skin of these animals. The removal of the pituitary gland of a 
frog results, among other things, in the bleaching of the animal and the inability of the frog to alter the color scheme of its skin to agree with the surroundings. When in a strong light or on a light background the retimas of the frog's eyes are stimulated by light rays, and some of the impulses reach the pituitary's posterior lobe, resulting in a suppression of its secretion, and consequently a lightening of the frog's skin. When the light is decreased the pituitary increases its secretion and the frog has a darker pigmentation. These reactions probably do not occur so directly in the higher groups of vertebrates. Removal of the pituitary gland tends to cause atrophy of the other endocrine glands.

\section{The Thymus Gland}

The thymus, a small glandular structure located in the chest between the upper part of the sternum and the pericardium, is a temporary organ, which normally atrophies in human beings by the time of the onset of puberty. When the gland is too active, a condition is found in children in which an enlargement of the organ results, and breathing is rendered difficult. No distinct hormone has been obtained from this gland.

It has been claimed recently that accruing acceleration in the rate of growth and development occurs when successive generations of rats are given daily injections of thymus extract. In third and fourth generations, the rats at twelve days of age compared farorably with controls of twenty days. Introduction of thymus extract in young tadpoles causes them to grow rapidly to the size of the adult frog but still retain their tadpole form and appearance. The disappearance of the thymus at the time of puberty permits the differentiation of mature animals and particularly the onset of activity of the sex glands. Some workers claim that the thymus contributes to the orderly and proper development of the bones of the skeleton.

\section{The Gonads and Sex Hormones}

In addition to the usual function of producing germ cells for reproduction, the gonads produce hormones which influence the development of secondary sexual characters and which have a regulatory effect on the reproductive processes and activities. Sex differences are caused in part by various hormones which have a selective action on the male or on the female secondary and accessory sex 
characteristics. The earlier concept that the male sex produces "male" hormones exclusively and the female produces only "female" hormones is no longer held. For example, extracts have been prepared from the urine of women as well as from men which on injection into capons caused growth of the comb which ordinarily fails to develop as it would in the cock. The sex hormone substances affecting the male are spoken of as androgenic and those affecting the female as estrogenic. The important sex hormones are androsterone, testosterone, theelin, and progesterone (progestin). The first two are male hormones, and the others are female.

Androsterone is found in male urine and can be crystallized from it. It has a stimulating effect on development of secondary sex characteristics and a definite regenerative effect on accessory organs (seminal vesicles, prostate glands, and penis) of castrated male animals. Testosterone is produced in the interstitial tissue of the testis but is absent from the urine. This hormone is several times as effective as androsterone in bringing about regeneration of accessory sex organs in castrated males. Recently androgenic hormones, which are potent enough to affect the growth of comb in the capon, have been found in the urine and ovaries of female animals.

Theelin (oestrin, estrogen, folliculin, menoform, progynon) has been isolated from liquor folliculi, pregnancy urine, the placenta, and amniotic fluid. This substance causes (1) increased growth of the accessory female organs (uterus, oviducts, etc.), including changes in the glands of their linings and in vascularization; (2) contraction of the smooth muscle of the uterus; (3) initial growth of mammary glands and nipples; and (4) sudden lowering of theelin concentration in blood (suggested as cause for bleeding during menses). The secretion of theelin is influenced by the gonadotropic principle of the anterior pituitary.

Progesterone (lutein hormone, corporin, luteosterone progestin) is a female hormone produced by the corpus luteum, the yellow body of material which forms in the ruptured Graafian follicle after the escape of the ovum. It produces the following effects: (1) sensitization of the lining of the uterus so that implantation or attachment of the zygote may take place in case of fertilization; (2) development of placenta; (3) arrest of rhythmic contractions of the smooth muscle of the uterus; (4) inhibition of ovum production and uterine bleeding. 
The influence of other hormones of the body is noted in the reproductive and sexual processes. The effect of the pituitary has already been mentioned. The thyroid secretion probably plays a role, not clearly understood, in the female reproductive processes, since the thyroid always enlarges at puberty and during pregnancy. Another hormone is thought by some investigators to be formed in the placenta during the development of an embryo. The interrelationships of these hormones are involved, and doubt exists in some cases as to their exact functions.

\section{The Pancreas}

This is one of the organs serving a dual purpose in the body; it secretes from a group of its cells, called the islands of Langerhans, a hormone designated as insulin. Experiments have shown the action of insulin to be concerned with the metabolism of carbohydrates and fats. Its presence facilitates the combustion of carbohydrates, regulates the rate of sugar production by the liver, and promotes the storage of sugar as glycogen in the muscles. It therefore decreases the amount of sugar in the blood.

Extracts of insulin are obtained from the pancreas of animals and are used commercially for the treatment of the condition known as diabetes. This disorder is due to a disturbance of the metabolism of sugars, provoked by a deficiency of insulin. The blood contains too great a percentage of sugar, but this is not turned into needed energy, and much water is excreted by the kidneys in order to eliminate the excess sugar. The patient suffers, therefore, from fatigue, excessive hunger, and thirst. The injection of insulin subcutaneously assists in regulating the condition by restoring the power to transform glucose into glycogen in the muscles. The patient regains strength and weight as a result, but the treatment does not perfect a complete cure and additional insulin must be injected at intervals to maintain normal health. Overdoses of insulin result in very serious disturbances, which may be relieved by ingestion of glucose.

Among the functions of the hormone, adrenalin, is the acceleration of the production of glucose in the blood; it is apparent, therefore, that the hormones, insulin and adrenalin, are antagonistic in their effect on sugar metabolism, and an upset of the normal production of these secretions results in metabolic disturbances. 


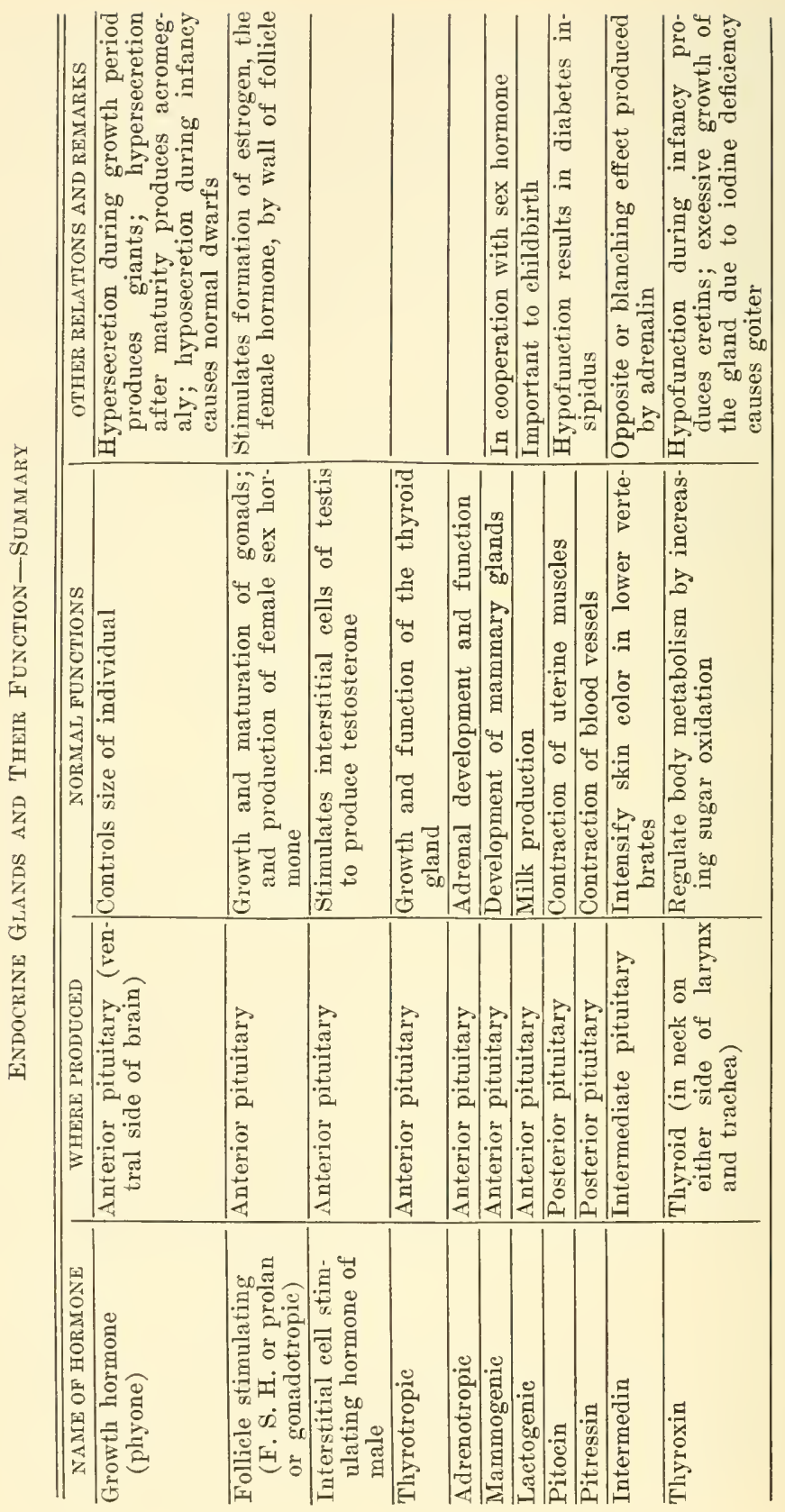




\begin{tabular}{|c|c|c|c|c|c|c|c|}
\hline 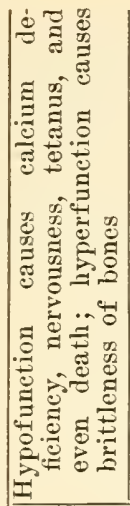 & 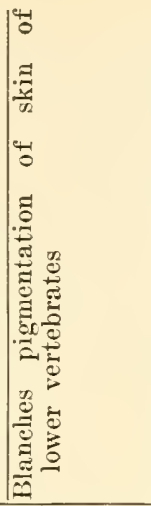 & 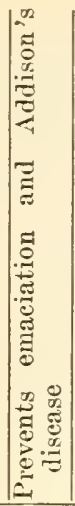 & 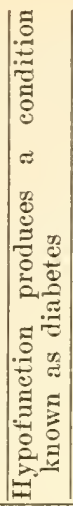 & 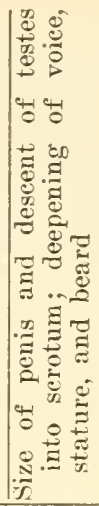 & 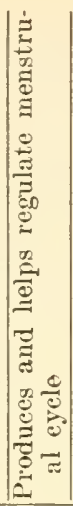 & 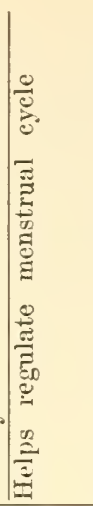 & \\
\hline శ్ & 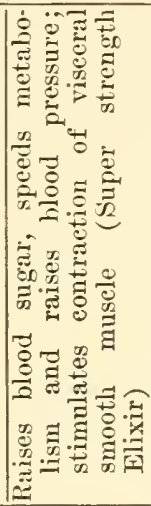 & 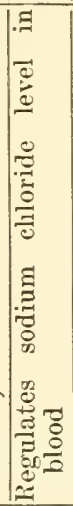 & 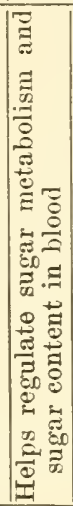 & 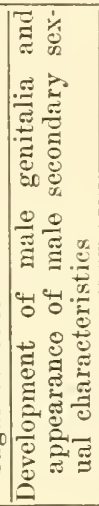 & 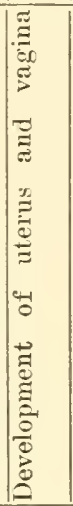 & 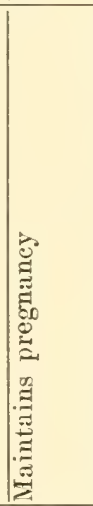 & 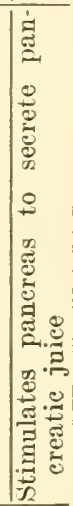 \\
\hline 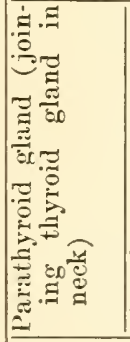 & 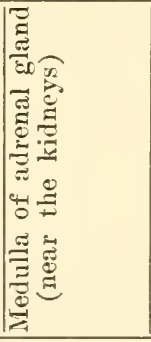 & 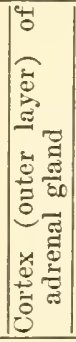 & 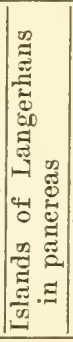 & 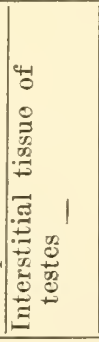 & 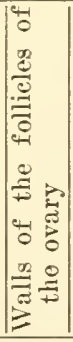 & 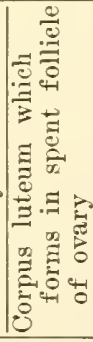 & 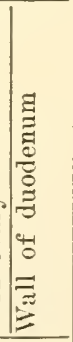 \\
\hline 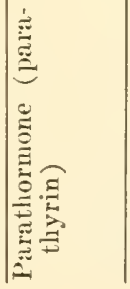 & 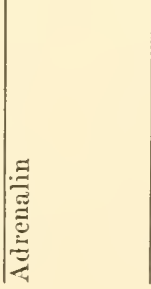 & & 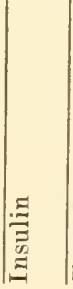 & 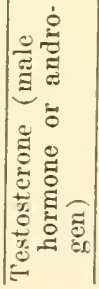 & 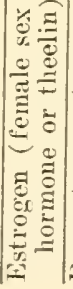 & 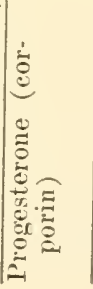 & 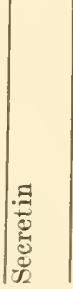 \\
\hline
\end{tabular}


Thus we see the complexity of the performance of these secretions in the body. When they are present in correct proportions, the organism enjoys a smooth functioning of its physiological processes, regulated by the hormones. The malfunctioning of any one endocrine organ results, however, in a disturbance of those processes which it specifically regulates and usually of others which it influences in conjunction with its sister glands of internal secretions or with other regulatory organs of the body. Each gland is called upon, therefore, to function in full cooperation with the other glands as well as to perform duties peculiar to itself. 


\title{
CHAPTER XXXIV
}

\section{REGENERATION}

\author{
(By T. C. Byerly, Poultry Research Division, United States \\ Department of Agriculture)
}

\section{INTRODUCTION}

Regeneration may be defined as the formation of new tissue to replace that which has been lost. The fact of regeneration was familiar to Aristotle and Pliny but Spallanzani, who published the results of his researches in 1768 , was the first to apply the experimental method extensively to the study of the problem. Examples of regenerative ability have been given in previous descriptions of the various animal phyla. In general, the ability to regenerate lost parts is more extensive in the simpler animal organisms and more restricted in animals of great complexity. In every case, regeneration involves the loss of tissue followed by reorganization of the remaining tissues. Reorganization is usually followed by growth of the regenerating portion until it reaches approximately normal size in proportion to the total size of the body. Discussion of regeneration will include consideration of the control by the organism of its parts and of the integration of the parts.

\section{REGENERATIVE CAPACITY}

\section{Protozoa}

Regenerative capacity varies inversely with complexity of the organism but also varies a great deal among animals belonging to the same phylum. Among the protozoa, nucleated fragments are generally capable of regeneration while nonnucleated fragments are not. Thus, if Stentor is cut into three transverse pieces, each containing a portion of the chainlike nucleus, each fragment regenerates by rearrangement of material, forming a small Stentor which subsequently grows to normal size. In forms such as Amoeba, only one fragment produced by cutting is likely to be nucleated. This fragment will regenerate while nonnucleated fragments will round up but fail to regenerate further. 

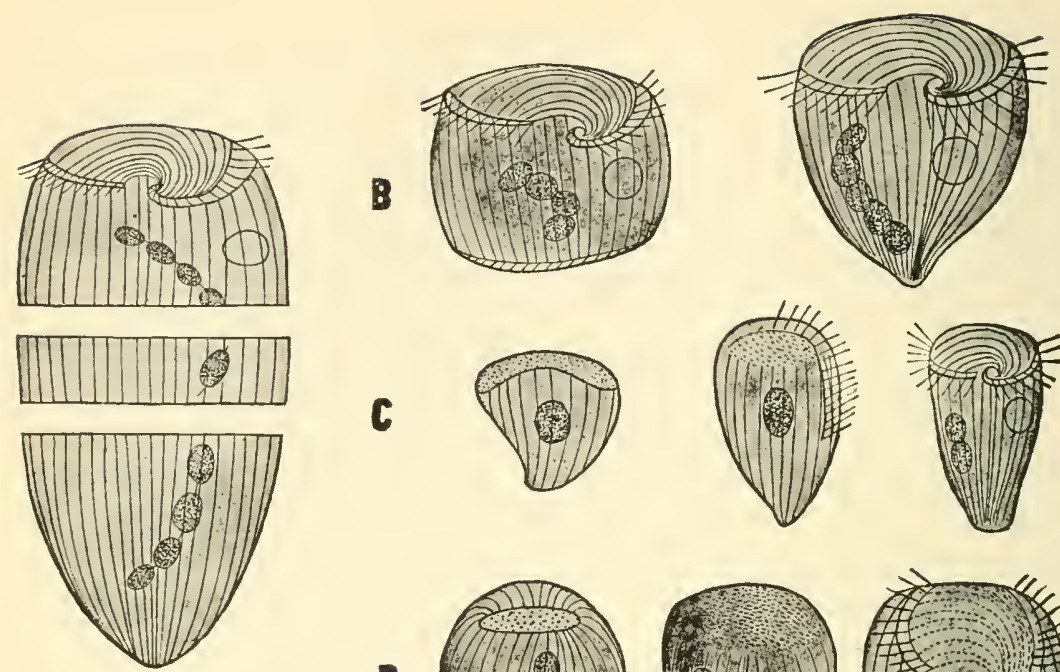

A
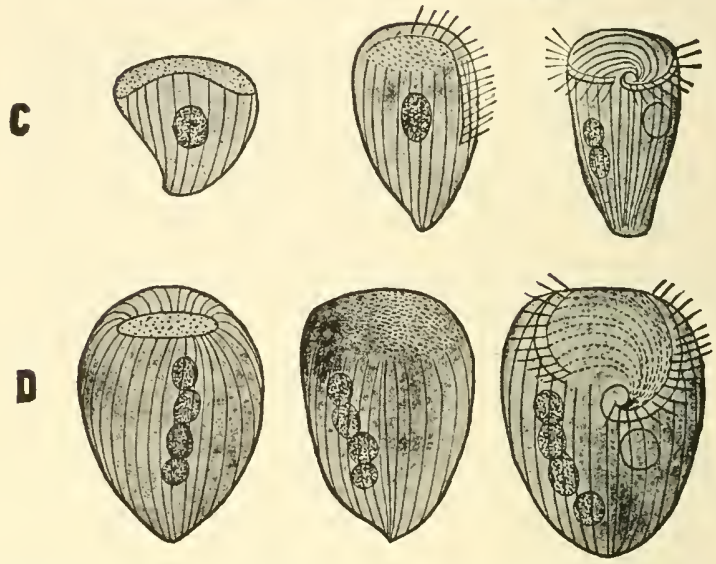

Fig. 364.-Stentor coeruleus, showing regeneration. $A$, cut into three pieces; $B$, shows progress of development of anterior portion; $C$, progress of regeneration of middle piece; $D$, progress of regeneration of posterior piece.z (From Morgan. Regeneration, by permission of The Macmillan Company.)

\section{Porifera}

Some of the sponges have very extensive capacity for regeneration. A series of classic experiments by Prof. H. V. Wilson of North Carolina demonstrated that regeneration is possible after the dissociation of the cells comprising the bodies of monaxonid sponges by pressing them through gauze. The individual cells thus separated come together in groups. These cell groups organize themselves into spheres, then grow and develop into new sponges. Differentiated cells, as well as the undifferentiated amoebocytes, take part in, regeneration.

\section{Coelenterata}

The coelenterates have been the subject of very extensive experiments on regeneration. In general, nembers of this group may be cut into fragments and each fragment will regenerate a complete individual. Some forms, such as Eudendrium, are capable of regeneration after dissociation in the same manner as the Porifera. 
Transverse sections of hydroid stems must be of minimum length, characteristic for the individual and level from which they were taken. Pieces shorter than this minimum length may regenerate tentacles and other structures characteristic of the oral region at each end of the piece or may fail to regenerate such structures entirely.

It is possible to split hydroids partially and get individuals with $2,4,8$, or more, complete oral regions with a single foot.

\section{Platyhelminthes}

The free-living flatworms have served as experimental material for a great many researches on regeneration. Planaria, like the hydroids, may be eut into several transverse segments each of which
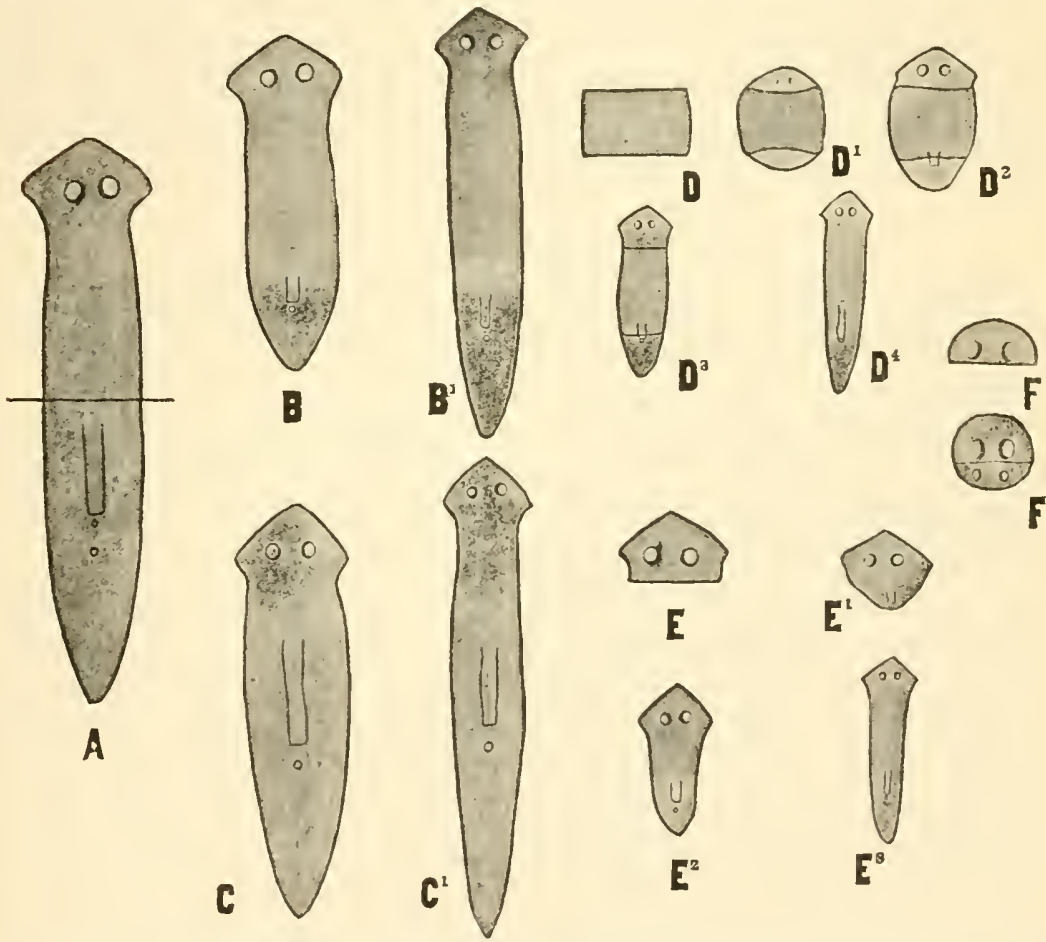

$F^{1}$

Fig. 365.-Regeneration in Planaria. A, normal animal with transverse line to show level of cut to divide the animal; $B, B^{1}$, regeneration of anterior half: $C, C^{1}$. regeneration of posterior half: $D$. piece cut from middle of body; $D^{1}, D^{2}$, $D^{3}, D^{4}$, regeneration of $D ; E$, anterior piece: $E^{1}, E^{2}, E^{3}$, regeneration of $B ; F^{3}, F^{1}$. regeneration in head of another species. (From Morgan, Regeneration, published by The Macmillan Company.) 
undergoes reorganization and growth to regenerate a new individual. Like the coelenterates, very short pieces may regenerate a head at each end or fail to regenerate a head at all.

Experiments with $x$-rays have shown that it is possible to prevent regeneration by this means. X-rays prevent cells in a physiological condition preliminary to cell division from undergoing division, though they do not prevent completion of division in cells undergoing mitosis at the time of application.

Some of the flatworms exhibit a type of agamic reproduction indistinguishable from regeneration. This is especially true of the tapeworms. The tapeworm forms new segments just distal to the scolex, growing to very considerable length if undisturbed. The more distal segments break off as they become exhausted.

Vermifuges cause the death and shedding of many segments but are often ineffective in destroying or dislodging the scolex and the growing region next to it, especially if the scolex is barbed. The growing region subsequently produces new segments and the worm may be completely "regenerated."

\section{Annelida}

Regeneration is a more complicated process in the annelids than in the simpler animals and regenerative capacity is somewhat more restricted.

If a Lumbriculus be cut into a dozen transverse pieces, a dozen worms may be formed. The regenerated head consists of but a few segments but the tail continues to grow by intercalation of new segments just anterior to the anal segment.

When the anterior end is cut off, the cut surface is closed very quickly by contraction of the body wall. The cut end of the digestive tract is contracted and closed. No further extensive changes are visible for a day or two but new ectoderm is soon formed over the cut surface. Old ectoderm gives rise to the new. As the new ectoderm increases in amount, the old is pushed back, leaving a single layer of cells over the cut surface. The new ectoderm proliferates, producing a bulge in the new head. New cells arise from the ventral side of the bulge to form the brain, commissures, and ventral nerve cord. Other ectodermal cells form the muscles.

The blind end of the gut proliferates and grows forward toward the ectoderm. Invagination of the ectoderm takes place so that 
gut and ectoderm are flattened against each other. The cells draw away from the region of contact and a new mouth is formed. Tail end regeneration is similar to that at the head end, but simpler since neither brain nor commissures are formed.

Regeneration in the earthworm is similar to that in Lumbriculus in many respects but is somewhat more complicated. The anterior end, when cut off, regenerates a new tail. When a head is cut off, another is formed.

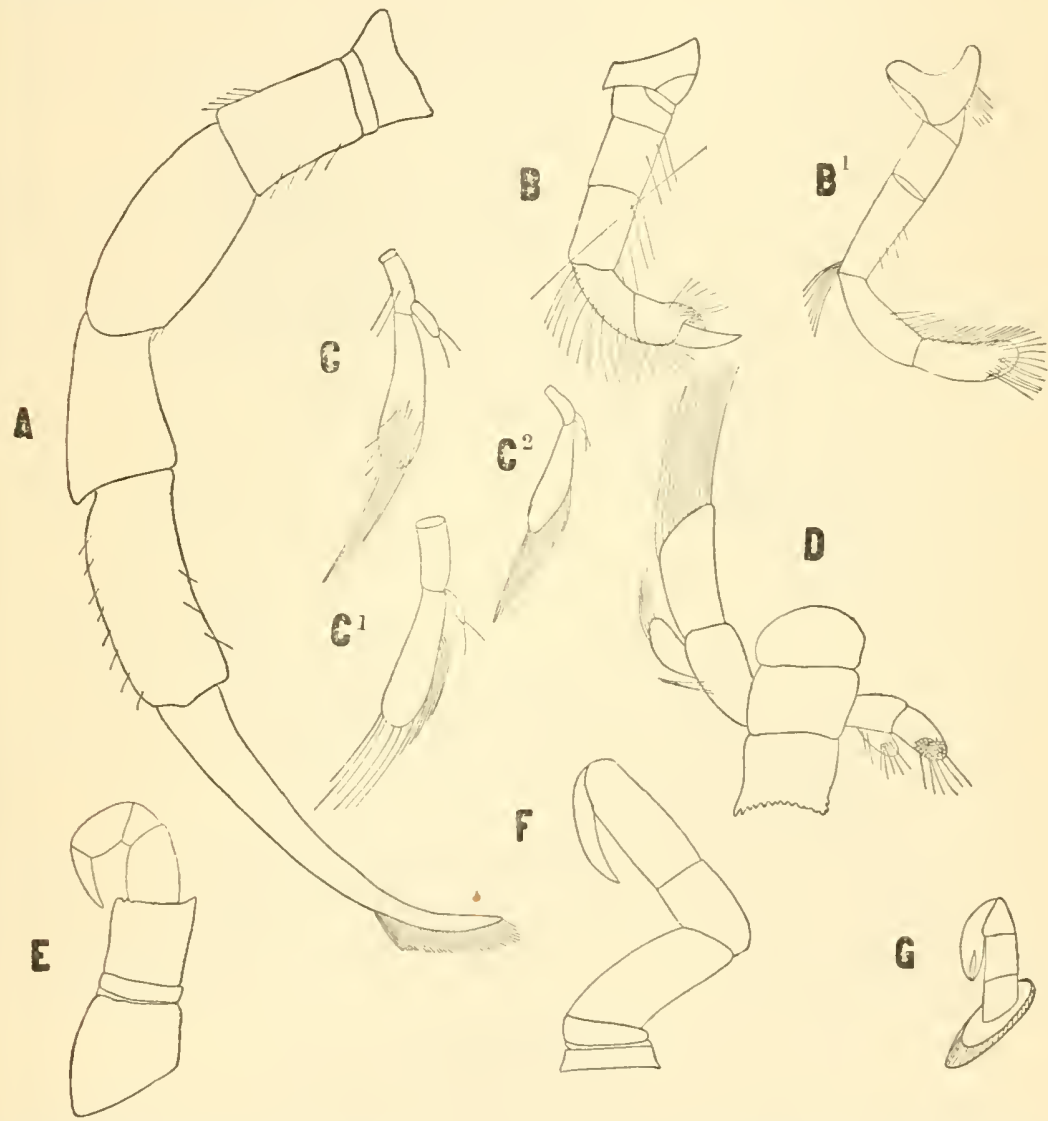

Fig. 366.-Loss and regeneration of appendages in the hermit crab, Eupagurus longicarpus. $A$, third walking leg; $B$, next to posterior thoracic leg: $B^{1}$, posterior thoracic leg; $C, C^{1}, C^{2}$ abdominal appendages of male ; $D$, telson and sixth segment with last pair of abdominal appendages; $E$, regeneration of new leg from cut-end distal to "breaking joint": $F$, new leg from cut made proximal to the "breaking joint": $G$, leg regenerating from cut made very near the body. (From Iorgan, Regeneration, published by The Macmillan Company.) 


\section{Mollusca}

Capacity for regeneration is extensive in this phylum. Foot or head, mantle or shell, may be replaced under favorable environmental conditions.

\section{Arthropoda}

Regenerative capacity of organisms in the phylum Arthropoda is more limited than in the preceding groups. Regeneration takes place in the walking legs of decapod Crustacea, such as shrimps, crabs, lobsters, and crayfish. Spiders, which are members of the order Arachnida, are also able to regenerate appendages, but the insects generally lack this capacity.

The hermit crab protrudes three pairs of thoracic legs from the snail shell in which it lives. These three pairs of legs have breaking joints, clearly demarked levels at which these legs break when caught. The last two pairs of thoracic legs are not protruded from the snail shell and have no breaking joint. When a leg of the first three pairs is injured, it is thrown off at the breaking joint and the leg regenerates from the breaking joint outward. Such a regenerated leg is small and of little use until the crab molts. The regenerated leg grows to its proper proportions during the molt. If a leg of the first three thoracic pairs is cut off between the body and the breaking joint, a new leg forms with a new breaking joint. Thus, the breaking joint is not essential to regeneration (Fig. 366).

The last pairs of legs, without breaking joints, are not readily subject to injury. If, however, they are cut off at any level, the missing parts are regenerated.

The eyes of Crustacea are also capable of regeneration. When the eyestalk of Palaemon (shrimp) is cut off near the tip, a new eye regenerates, but when the eyestalk is cut off near the base, an antenna is formed.

\section{Echinodermata}

Regenerative capacity is very great among members of this phylum. If an arm be torn from a starfish, not only will the major fragment of the starfish regenerate a new arm but the detached arm will also regenerate a new starfish provided even a small portion of the disc remains with it. 
Some Holothurians (sea cucumbers) eviscerate themselves when roughly handled or kept in an unfavorable environment. Such individuals may regenerate a complete new viscera.

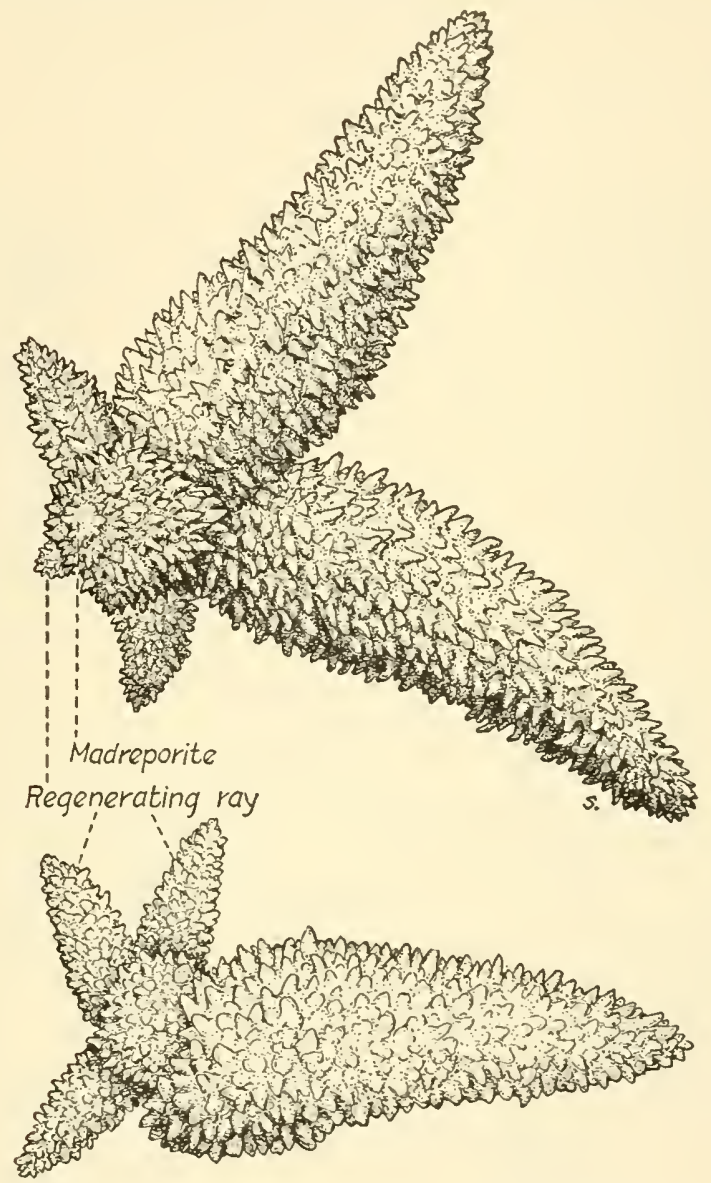

Fig. 367.--Regeneration in the starfish. Above, starfish regenerating part of the central disc and three arms; below, arm of starfish regenerating the remainder of the body.

\section{Chordata}

Regenerative capacity varies widely in this phylum. Increased complexity is generally accompanied by reduced regenerative capacity within the phylum, but the Pisces form a notable exception. This order is relatively primitive in many respects, but many fishes have a relatively limited eapacity to regenerate lost parts. 


\section{Amphibia}

Among the Amphibia, the Caudata are able to regenerate appendages. Adult Salientia are unable to do so, though they are more primitive in many skeletal characters than the Caudata. A great deal of research pertinent to an understanding of regeneration has been done with amphibian material.

Salamander larvae frequently snap off each other's legs or gills. Some salamanders will snap off their tails if they are seized. Gills, legs, and tails are regenerated in both larvae and adults, though regeneration is slower in the adult than in the larva.

Regeneration may be accomplished through the activity of previously highly differentiated cells. When the lens of either a frog or a salamander eye is destroyed, the cells of the iris undergo a loss of pigment, differentiate, and form a different type of structure which develops into a new lens. This process is quite unlike lens formation in the embryo in which the lens is formed from superficial ectoderm cells under the influence of the optic cup.

New legs are formed by the assembly of undifferentiated connective tissue cells beneath the surface of the wound and by dedifferentiation of muscle and other cells. Such a group of cells is termed a blastema. The new leg is formed by the proliferation and differentiation of the cells of the blastema in a manner similar to the development of the leg in the embryo.

After the leg of a salamander is cut off, new leg bones, muscle, and other tissues are formed from the blastema over the old stump, not from the bones and muscle of the old stump directly. The cells that form the blastema are assembled from their resting place in nearby tissues. They are not brought together from distant points in the body. Under local influences they are induced to form a new leg. The strictly local nature of this induction has been demonstrated by transplantation experiments. A boneless leg transplanted to the back of a salamander regenerated its proper complement of bones. Split limbs develop partial limbs complete as to structures normally distal to the point of cleavage, producing duplicated limbs.

Nerves are not essential to regeneration of a new leg, but if they are prevented from growing into it, the regenerated leg is much smaller than is normal. 
Regenerative eapacity has been limited in the salamanders so that while the whole will regenerate certain lost parts, the lost part even under favorable eireumstances is incapable of regenerating the whole body. A tail eut off and transplanted to the back, and proper cireulation established to insure good nutrition, will regenerate another tail if a proper wound is made, but it will not regenerate a new hody.
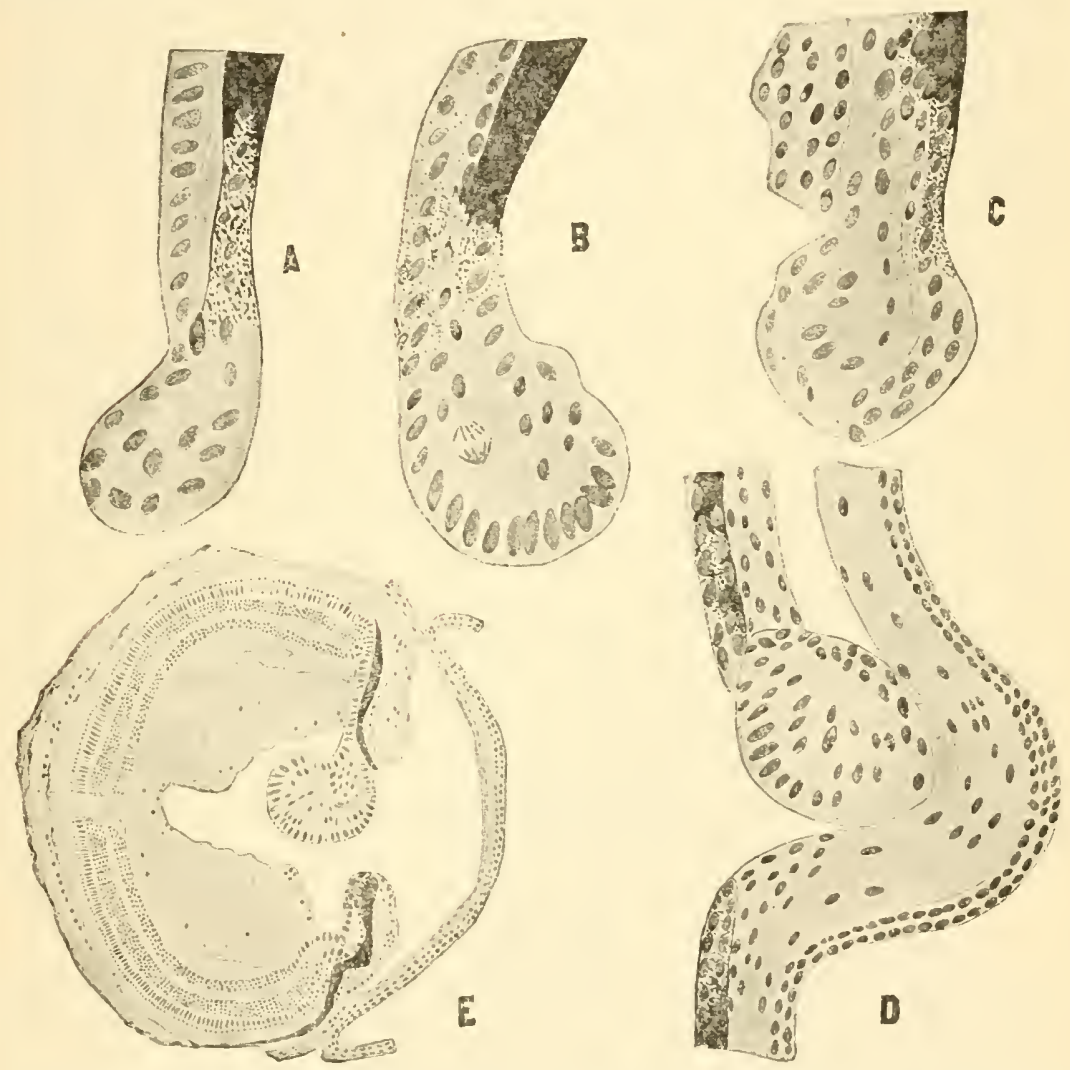

Fig. 368.--Regeneration of the lens of the eye from the iris in Triton. (After Wolff.) $A$, edge of iris with beginning of lens; $B, C$, later stages; $D$, still later stage, showing rounded formation of lens structure; $E$, entire eye with regenerating lens. (From Morgan, Regeneration, published by The Macmillan Company.)

Regeneration of the lens of the eye from the edge of the iris in Triton is a different feature in regeneration. Here the new structure eomes from an entirely different germ layer. 
Regeneration may be prevented by x-rays, or sometimes by simply sewing the edges of the wound together. After application of $\mathbf{x}$-rays, epithelium migrates over the wound surface, but no blastema is formed.

\section{Reptilia}

Some of the lizards are capable of regenerating new tails. Indeed, some of them have special areas developed where breaking occurs when the tail is seized just as do the legs of the hermit crab. Sphenodon, the most primitive of the reptiles, is capable of regeneration of a new tail. The regenerated tail contains a central cartilage cylinder instead of vertebrae and the regenerated skin and other tissues are not quite normal. None of the reptiles is capable of regenerating new legs.

\section{Aves}

The most striking regenerative capacity among birds is the ability to grow new feathers. Each feather is subtended by a feather germ, a sort of permanent blastema, from which a new feather is formed if the old one is lost throngh molt or accident.

Regeneration of the ovary is quite extensive if operative injury is inflicted in the young chick. If the left ovary of a young female chick is removed, the right, normally rudimentary, grows to form an ovary, an ovotestis, or a testis. Experiments indicate that the medullary portion of the primary gonad gives rise to a testis and that some medullary substance remains in the rudimentary right ovary. When the inhibiting influence of the normally functioning left ovary is removed, this medullary substance may proliferate to form a testis. Such sex-reversed birds are usually sterile.

\section{Mammalia}

No mammal is capable of growing a new appendage if an old one is lost nor of completely regenerating any other major organ. Regeneration of tissues, however, is quite general. Hair, hoof, horn, and epithelium regularly regenerate from germinal layers or areas. If these germinal areas are destroyed, regeneration fails. Baldness is, of course, a familiar example of failure of regeneration.

Regeneration in the group is largely limited to repair rather than replacement. In this, as in the case of agamic reproduction, it is difficult to differentiate between processes which may be classified 
as normal growth and those which are regenerative in nature. The ability of various tissnes to regenerate will be described briefly.

Epithelial Tissue.-Cuts and other wounds of the body surface are healed by the migration of cells from the old epithelium over a substrate formed by the underlying vascular and connective tissues. Proliferation of the epithelial cells by mitosis follows.

Glands, such as the mammary and salivary glands, are capable of considerable regeneration by proliferation of the small cells of their secretory ducts. The liver is capable of regeneration by proliferation of cells of the small bile ducts. The relation of the regenerated tissue to the old tissue is usually atypical. If a lobe of the liver is removed, the other lobes increase in mass, but the lost lobe is not replaced by a new lobe. This process is called compensatory hypertrophy. The kidney has a very limited capacity for regeneration, but if one kidney is removed, the remaining one does undergo considerable compensatory hypertrophy.

Connective Tissues.-Blood cells of all sorts are formed continually in the mammalian body by special groups of cells. White blood cells are formed largely in patches of lymphoid and myeloid tissue scattered throughout the body. Red cells are formed largely in the red bone marrow. When considerable amounts of blood are lost, regeneration takes place through increased proliferation of these special groups of cells.

Small blood vessels are regenerated by sprouting from pre-existing capillaries. These sprouts may unite distally. The sprouts thicken and become channeled to complete the vaseularization of the injured area.

Cartilage is regenerated largely from cells of the perichondrium. Bone is not regenerated primarily from pre-existing bone cells. The cells of the periosteum or marrow form a cellular connective tissue or the perichondrium forms hyaline cartilage to form a mass called a callus. Osteoblasts then invade this tissue to form new bone and complete the repair.

The fibrous connective tissues regenerate by the division of undifferentiated cells. A homogeneous ground substance is formed between the cells. Fibers appear in the ground substance and complete the regeneration. 
Muscle Tissue.-Smooth muscle has slight regenerative capacity. Healing is effected largely through the formation of fibrous tissue. In the case of striated muscle, sarcolemma cells sometimes divide after injury and a limited amount of regeneration takes place.

Nervous Tissue.-Nerve fibers do not reunite after they are divided. The distal portion of the severed fiber degenerates completely. The neurilemma cells undergo proliferation and unite with the neurilemma of the proximal portion. The axome may then grow into the united sheath. The process takes months. Nerve cells of adult mammals do not proliferate.

\section{Basis for Regeneration}

Certain facts must be reviewed to furnish a basis for an explanation of regenerative capacity. Differentiation of body cells during embryonic development gives rise to an integrated organism. Genetic experiments indicate that every cell in the body, with sporadic exceptions, contains the same genes as every other cell. Differentiation does not consist of segregation of genes for the various organs. Different expressions of the same genes at different times in development or at different places in the organism or the expression of the effects of particular genes only at a particular time and place in the organism must be responsible for differentiation of organs and tissues.

The zygote is capable of giving rise to a whole organism. When the zygote of some organisms divides, each of the two cells produced is capable of producing a whole organism, if separated from the other, regardless of the complexity of the adult organism; e.g., in the sea urchin and in man. Such a separation is indicated in the case of identical twins. In other animals, due to differentiation of the cytoplasm, the first cleavage reduces the potency of the resulting cells so that they will not produce a whole organism if they are separated. Obviously, if the first two cells remain in normal position, each produces but a half organism in any case, demonstrating the importance of the relationship between cells in limiting the potency of each. The first two cells formed by division of the zygote are thus physiologically different though genetically identical so long as they remain in their normal relationship to each other. As development and differentiation proceed, potencies of the cells tend to be limited more and more, the final limitation of potencies in the adult being slight in simple organisms, great in complex organisms. 
Reduction of potency of individual cells of the adult organism is really only another way of stating reduction of regenerative capacity. As illustration of this parallel, it has been shown that a few isolated cells from some of the sponges, kept in a proper environment, will regenerate the whole organism. In contrast to the formation of an entire organism by each of the first two blastomeres, if a few cells from an aclult mammal are isolated in a favorable environment, as in tissue culture, the cells will only reproduce cells of their own kind, not a new organism. Of course, some cells, such as nerve cells, will not even reproduce their own kind and thus loss of reproductive or regenerative potency is complete.

Why does the position of a particular cell, in relation to other cells, determine its subsequent history?

Every animal organism exhibits polarity. The frog's egg is a familiar example with its animal and vegetal pole; the nerve cell with its afferent axone and its efferent dendrites is another. Polarity may be determined in many ways; in the frog's egg by the relation of the egg to its nutritive supply, in the nerve cell by the orientation of the cell with respect to nervous center and periphery or to other parts of the central nervous system. Polarity implies the presence of a gradient of some sort between the poles-whether a gradient of materials as in the frog's egg, or of permeability, of rate of metabolism, or of some other sort. Usually the region at the more rapidly functioning end of such a gradient dominates at least a portion of the gradient.

Dominance of one region over another may be exerted in various ways. Thus the central nerrous system dominates a major portion of man's body by assorting stimuli from the varions portions of the body and sending impulses to the proper effectors. The endocrines respond to other stimuli and send substances throngh the blood stream. In other cases, substances are produced locally and produce their characteristic effects after diffusion to nearby cells. Bio-electric currents may also serve as an agency in the exercise of dominance. Polarity, with its resulting gradients, dominance of the more active region of the gradient over the less active, integrate the developing organism. When a region grows sufficiently large, a formerly subordinate level may become so far removed that it escapes from the dominance of the more active region and itself becomes locally dominant, resulting in agamic reproduction in the simpler forms, or in 
the formation of a characteristic organ for that time and place in the development of one of the more complex organisms.

Dominant areas sometimes produce substances which diffuse to surrounding cells and determine their subsequent proliferation and differentiation. Such dominant areas are called organizers. The dorsal lip of the blastopore of the amphibian egg, which determines the formation of the central nervous system, is a classic example. Cells from the vegetal pole of the amphibian egg will form nervous tissue under the influence of the organizer if such cells are placed over it instead of the cells normally occurring there. Organizers are formed in the region of every regenerating structure, if not previously present. Thus, the stump of the leg of a salamander induces the cells of the blastema to form those structures which are lacking. In some cases, as in hydroids, if the dominant area itself is injured organs characteristic of that region are regenerated rather than the portion removed.

In the complex organisms, there finally results a maze of interdependent and integrated, locally controlled regions under the general dominance of the body as a whole. Each organ has a characteristic size and rate of activity in proportion to the other parts. These relations may be maintained from a food-supply in the blood stream kept at a level which is relatively uniform for all portions of the body, through different rates of absorption and utilization of food by the different kinds of cells.

As development proceeds, an ever greater proportion of cells becomes functionally differentiated, and the rate of proliferation decreases correspondingly. Some cells, such as the germ cells and the cells which replenish the supply of blood cells, maintain their physiological youthfulness and rate of proliferation, while others, such as nerve cells, become irreversibly differentiated and cease proliferation altogether. Animals cease to grow when a balance is reached between normal wearing out of tissue cells, on the one hand, and proliferative activity on the other.

Experiments with tissue cultures have clearly demonstrated that animal cells are potentially immortal. Removed from the dominance of the organ and body of which they form a part and given adequate, sanitary, nutrient conditions, they multiply indefinitely, forming other cells like themselves. Tissue cells from rertebrate animals do not organize new animals in tissue culture. Apparently, vertebrate 
cells older than cells of the earliest cleavage stages in development have either undergone differentiation which is not completely reversible or else tissue culture media have not yet been developed which permit the formation of proper organizers and fields of dominance.

When a group of cells is isolated from its dominating region, those cells may regenerate a new body, as in the sponges, or only other cells like themselves, as in tissue culture. Usually they lack a favorable environment, in the case of the more complex animals, and die. The removal of the cell group reduces asymmetrically the remaining portion of the body.

When an integrated animal body is disturbed by a wound or by removal of a part, certain conditions are set up under which healing and sometimes more extensive regeneration take place. These are: interference with or destruction of nervous control; stagnation in the transport system so that food and metabolic products accumulate; change in diffusion gradients and the formation of new bio-electric currents. The normal partition of foods is disturbed. Migration of cells occurs to close the wound. Cells capable of growth assemble and proliferate to reproduce the lost part under the influence of remaining local tissues. These cells are physiologically younger than the differentiated cells of surrounding parts, utilize food faster, and grow faster, so that regeneration takes place even when the organism as a whole is starving.

Dedifferentiation, when it occurs, is a sort of rejuvenation process resulting in physiologically young cells with potencies comparable to embryonic cells.

\section{ADAPTABILITY AND REGENERATION}

The capacity for minor repairs is exhibited universally by animal organisms and is essential to their success. There is certainly no close correlation, however, between regenerative capacity and success of species. In general, regenerative capacity is a limiting factor to the individual, not to the species. In many eases, it seems to be more economical from the viewpoint of the species to grow new individuals by means of sexual reproduction than to repair damaged ones by regeneration. Fishes and insects are highly successful 
because of profligate rates of increase. Man suceeeds because he avoids physical injury. These three dominant groups all have limited regenerative capacity. On the other hand, it would be foolish to maintain that extensive regenerative capacity is of no value to those species possessing it.

\section{Summary}

All animals have some regenerative capacity. Some of the simpler ones may regenerate a new organism from a small portion of an older one, while in the most complex animals only relatively small parts may be regenerated after loss.

Regeneration consists of the assembly of physiologically young cells at the site of injury, usually through migration of such cells, but sometimes by dedifferentiation, a kind of physiological rejuvenation, of local, differentiated cells. These physiologically young cells proliferate and differentiate under the inductive influence of surrounding parts to reproduce the lost part in so far as regenerative capacity is characteristic of the species. 


\title{
CHAPTER XXXV
}

\section{BIOLOGICAL EFFECTS OF RADIATIONS}

\author{
(By Titus C. Evans, Colleges of Physicians, New York)
}

The study of biological effects of radiations is making such rapid progress that it is difficult to cover all of the important phases. It is hoped that this chapter will serve as an outline of the subject, and that the reader will study the references listed in the back of the book for detailed information. A few points in physics and chemistry must be reviewed to understand this subject.

\section{The Structure of the Atom}

The atom is thought to consist of (1) a central nucleus bearing a positive charge and possessing nearly all of the mass of the atom, and (2) one or more electrons (negatively charged particles of small mass) revolving around the nucleus in definite orbits. The nucleus contains protons (positively charged particles) and electrons. The protons are in excess and this gives the nucleus its positive charge. The number of electrons in the orbits is determined by the number of protons in the nucleus in excess of the ones neutralized by the nuclear electrons. Hydrogen, for example, consists of one proton in the nucleus (no nuclear electrons) and one orbital electron.

Atomic Weight.-Atomic weight is the total weight of the nucleus. Heavy hydrogen has a nucleus twice the weight of hydrogen. It contains, besides the proton, another particle of approximate equal mass which is electrically neutral. This particle is termed a neutron and is thought to be composed of a proton and an electron ir close association.

The atomic number is rclated to the charge on the nucleus. Helium, for example, contains two neutrons and two protons in its nucleus. This gives it an atomic weight of four and an atomic number of two. The atom is electrically neutral, so two orbital electrons are present to balance the charge on the nucleus. The chemical properties of an element depend upon the number of orbital electrons (atomic number). Those in the outermost shell are valence electrons by which one atom may be joined to another. 
Radioactive Atoms.-The nucleus of radium is unstable and in changing to a more stable condition it emits radiation of two kinds. These are particle and wave radiation. Particle radiations have mass and vary in velocity. Alpha particles are positively charged (2 plus) and have a mass of four. They are produced by nuclear disintegrations. Beta particles are high speed electrons that have been removed from atomic orbits. They carry a negative charge and have very little mass.

Neutrons have a mass equal to that of the nucleus of heavy hydrogen but carry no charge. These are produced by nuclear disintegrations.

The cyclotron is a machine which swings particles, such as the nuclein of heavy hydrogen, through an electric field increasing the velocity of the particle at each cycle until it finally emerges with a charge of several million volts. Such particles are able to remove neutrons from metals such as berillium and are able to produce artificial radioactivity in atoms such as phosphorus, sodium, carbon, ete. Particles may also be accelerated to high velocities by static generators such as the Van de Graaf type.

Wave radiation or electromagnetic radiation is different from particle radiation in several respects. The units, photons, have no mass, no charge, and travel at a constant velocity (equal to that of light). The wave lengths, and some other characteristics, of the various radiations are shown in Fig. 369. The frequency of the vibration is inversely proportional to the wave length. The amount of energy associated with a photon is termed its quantum. The quanta of short wave length radiations are large whereas those of long wave length are relatively small.

Roentgen radiation is produced by bombarding a metal target with high speed electrons in a vacuum. If the voltage of the electrons is high enough, it will remove electrons from an orbit near the nucleus of the target atom (tungsten, for example). As an outer electron drops into the vacated orbit near the nucleus it gives up its excess energy in the form of a photon of roentgen radiation. High voltage roentgen radiation will penetrate iron and copper to a depth of several millimeters but low voltage $\mathrm{x}$-rays will be stopped by even thin sheets of aluminum.

Enough energy can be given to atoms by heat or electricity to cause them to emit radiation resulting from rearrangement of outer 
electrons. Such radiations are heat, infrared, visible, and ultraviolet. Heat and infrared radiation penetrate slowly and the quanta are eomparatively small. The quanta of visible light are larger and the wave lengths are shorter. Such radiations are easily absorbed by even light elements except that some light is transmitted by

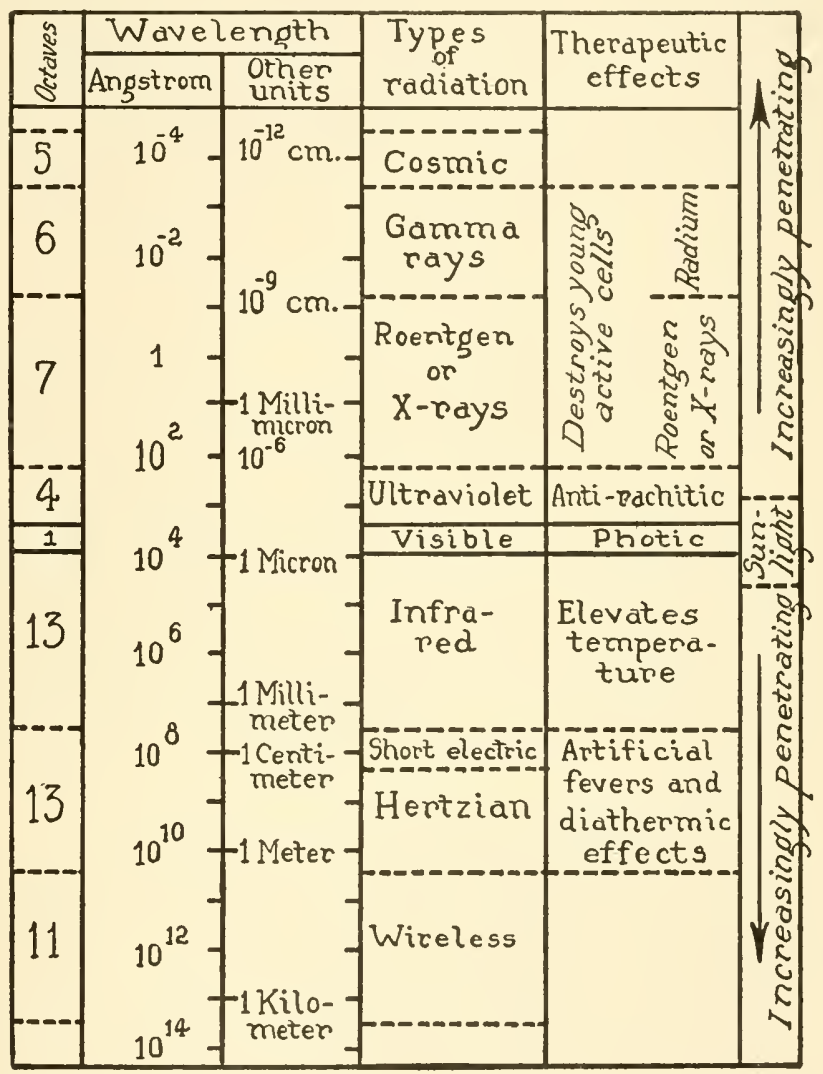

Fig. 369.-Chart of wave radiations. Visible light contains different radiations which appear as colors to the human eye. The long wave length radiation is the red, and in order of decreasing wave length the others are orange, yellow, green, blue, and violet. Visible light covers a range of one octave, as the wave length of the red radiation is twice that of the violet. The range of wave length for the infrared is much greater as it can be seen that the wave length doubles itself thirteen times. (From Sheard, Lifegiving Light, published by Williams \& Wilkins Company.)

glass. Ultraviolet radiations have wave lengths shorter than that of visible light and penetrate opaque substances to a greater degree. Ultraviolet is also more chemically active since the contained quanta 
are large. The sun is continually transforming mass into radiation consisting of cosmic rays visible, ultraviolet, and infrared radiations.

\section{Biological Effects of Sunlight}

Organisms living on the surface of the earth are dependent on solar radiation either directly or indirectly. It would be difficult to imagine human life, for example, without the benefits of food, heat, fresh water, and light. Most biochemical processes are exothermic (give off heat) and lose energy. Photosynthesis constitutes the chief means of counteracting the "running down" in the energy of our planet. Green plants contain chlorophyll which absorbs energy from sunlight, decomposes the carbon dioxide of the air and combines its carbon with the oxygen and hydrogen of water to make carbohydrates (simple sugars). This process of photosynthesis is a very complex one and some essential factors are chlorophyll, light, carbon dioxide, oxygen, water, temperature, certain minerals, and enzymes (organic catalysts which accelerate chemical reactions). The energy absorbed from the sunlight is employed to activate atoms which results in the synthesis of new compounds capable of oxidation and release of energy. Plants also show other reactions to light. The intensity and wave length of light affects size, time of fruiting, amount of fruit produced, and transpiration (evaporation of water). Animals, by eating the plants, liberate the energy and ntilize it in doing work, in producing heat, and in building more living tissue.

The eye is man's most important receptor of radiation. The light coming into the eye is focused by the lens and the image (inverted) is thrown upon the retina. Retinal cells contain photosensitive substances which are affected by the quantity and quality of the radiation. The photochemical reactions incite nerve impulses which travel to the visual centers of the brain. Moderate amounts of sunlight are not injurious to the eye, but extreme intensities are harmful and may result in temporary or permanent blindness, eye fatigue, and cataracts. The glare of carbon ares and the reflection of bright sunlight should be avoided.

\section{Infrared Radiation}

Both sunlight and artificial light sources have much of the radiant energy output in the infrared. The wave lengths of the radiation are too long for visibility and the energy is absorbed in tissues 
by inereasing molecular motion. This results in a local rise in temperature. At low temperatures the chemical activities of a living organism are slowed to such an extent that extremes will produce death. A physical factor in eausing death by freezing is the formation of ice crystals which disrupt the cellular structure of the tissues. Biological processes are accelerated by increases in temperature and, within limits, the activity may be doubled by each 10 degrees (centigrade) increase in temperature. If, however, the temperature becomes too high biological processes are retarded and even death may result. Physical factors associated with death resulting from ligh temperature are loss of water, inactivation of enzymes, oxidation, and coagulation of the protoplasm.

\section{High Frequency Oscillations}

Hertzian (radio) waves are longer in wave length than the infrared radiation. They apparently are not biologically effective under usual conditions of radio transmission, but high frequency oscillations of shorter wave lengths applied across two terminals at a high intensity will subject the intervening material to an immediate rise in temperature. This "internal heat" is being used in the treatment of certain diseases in which fever aids the body in fighting the infection. The physiological action is the result of electrieal stress. It has been found to destroy certain bacteria (in milk, for example), to increase germination of seeds, and to produce abnormal development of certain embryos.

\section{Effects of Ultraviolet Radiation}

The radiation contained in sunlight whose wave length is too short to be visible is the ultraviolet. It may be produced by mercury are or mercury vapor lamps. Small amounts of ultraviolet radiation retard growth of bacteria, protozoa, and fungi. Continued irradiation may cause the death of the organisms. There is evidence that ultraviolet radiation increases the permeability of cells. Proteins are altered chemically by long exposures to the radiation.

The importance of certain fish oils in the treatment of rickets has long been recognized. Likewise, the importance of sunlight is not a new discovery. A great step, however, was taken recently when it was discovered that certain inert substances could be activated by ultraviolet radiation so that they would function, as cod-liver 
oil in the treatment of rickets. It appears that in the body the sunlight acts on the sterol-bearing fats of the skin to produce the necessary vitamin $\mathrm{D}$.

\section{Roentgen Radiation}

In 1895, W. C. Roentgen, a professor of physics in the University of Würzburg (Bavaria), discovered that the cathode ray tube with which he was working threw some sort of invisible radiation on a

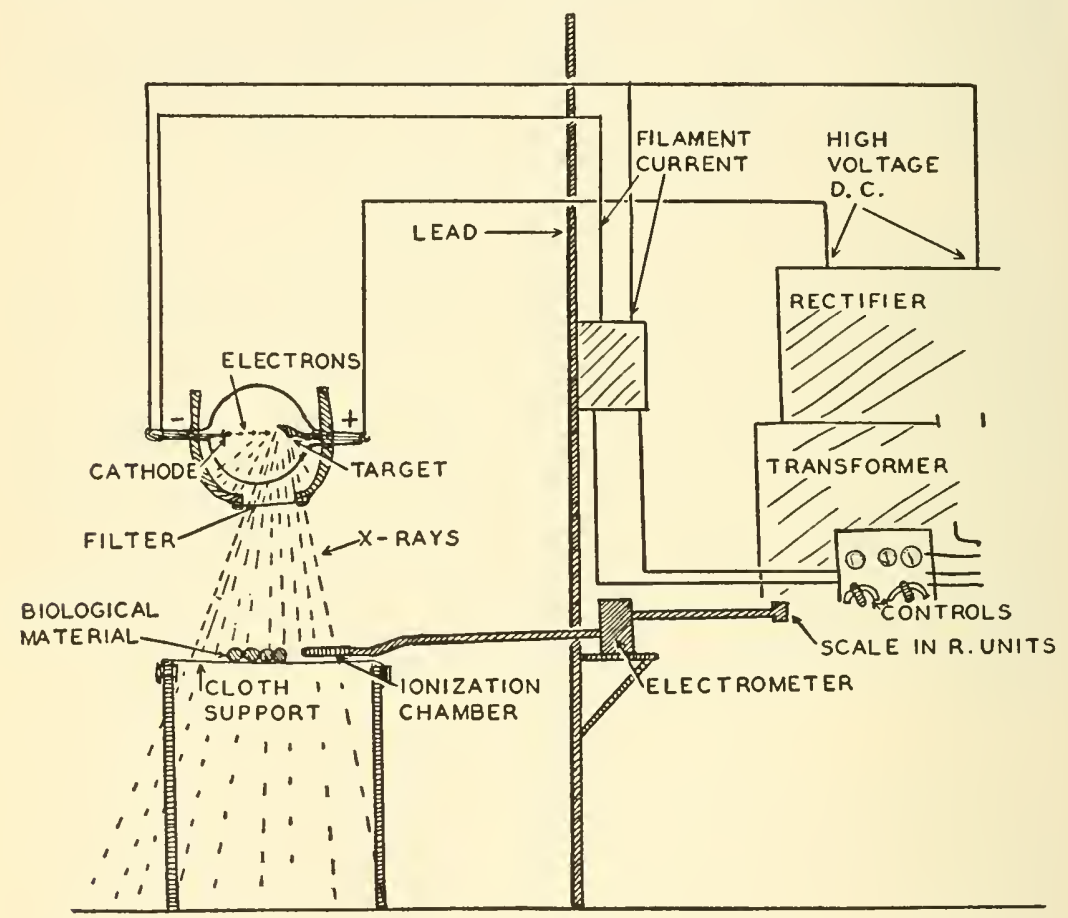

Fig. 370.-Diagram of a roentgen ray machine arranged to irradiate some biological material. The line current is comparatively low and has to be stepped up by the transformer. The input is usually alternating current and must be changed by the rectifier to direct current. The roentgen ray tube is of glass, is evacuated, and contains a flament at the cathode end and a target at the anode end. The filament is heated to incandescence by means of a low voltage current and gives off electrons. A high negative potential is applied to the flament and this drives the electrons across the tube where they are attracted to the positively charged target. The energy of the electrons is given up to the target (usually tungsten) as they are stopped and part of this energy is emitted as roentgen radiation.

barium platinum cyanide sereen, causing it to glow. He found that when his hand was placed between the tube and the fluorescent screen that bones would absorb the radiation while the softer tissues 
allowed the passage of the rays. It was also found that the radiation would darken a photographic plate entirely protected from light. These new rays which he called "x-rays" are usually spoken of as "roentgen rays" in his honor. These x-rays were found to penetrate paper, wood, aluminum, etc., but were completely absorbed by thick sheets of heavier metals, such as iron and lead.

It was not long after the physicists had perfected the roentgenray machine until physicians began to apply it in diagnosis of bone injuries and in locating bullets and other foreign objects embedded in the tissues of the body. By having the patient ingest a barium compound it is now possible to obtain a detailed radiograph of the digestive tract. Use of the fluorescent sereen has allowed immediate diagnosis of gastrointestinal disorders and has been of value in the setting of broken bones. The roentgen ray machine has also proved of great value to the dentist in locating cavities and pus pockets in teeth, and in finding teeth that have never broken through the gums.

The biological effectiveness of roentgen radiation was discovered in a tragic manner. Many technicians and physicians, who had been exposing their hands and other parts of their bodies to the radiation while making radiographs or using the fluoroscope, began to develop severe burns on the exposed areas. Before the severity of the affliction could be appreciated and before the knowledge of the danger could become widespread many pioneers in the field of $\mathrm{x}$-ray diagnosis had unwittingly become martyrs to the cause of medical science. It was thus realized that the radiation was biologically effective, and that even low dosages delivered at frequent intervals might result in incurable burns. Short exposures, such as are employed in diagnosis, are not sufficient to be harmful to the patient, but the technician who is around an x-ray machine day after day should be protected by lead shields. White blood cells and germinal tissues are especially susceptible to roentgen radiation, whereas other tissues such as those of the nervous and skeletal systems are more resistant. This differential susceptibility of cells has allowed the application of roentgen radiation to the treatment of cancer. Malignant growths arising from radiosensitive tissue are susceptible to roentgen radiation. If a cancer is composed of undifferentiated. rapidly growing cells, it is usually more sensitive to the injurious action of the roentgen radiation. There are a few notable exceptions to the above generalizations. 
One of the first problems to confront the radiologist was whether nonkilling dosages would have any subsequent effects on the normal cells. This was of interest especially in cases where the gonads had been irradiated. Cases of temporary sterility have been reported following roentgen ray treatment of the gonads and it was wondered whether the offspring of such treated individuals would develop abnormally. Evidence has come in abundance from the geneticists to the effect that the mutation rate of Drosophila is increased to an amazing degree by irradiating the germinal cells. If the gonads of cither sex are irradiated, it is found that inbreeding will bring out many recessive and harmful mutations in the third generation (F-2). It should be pointed out that the danger of harmful mutations is not of the same consequence in the human because brother-sister matings are not permitted. As the mutant genes are usually recessive, they would probably never be expressed unless the practice were widespread enough to allow the mating of two people both of whose parents had treated gonads.

A contribution of greater theoretical importance than this warning to the x-ray therapist was the new method of increasing the mutation rate. This addition of so many new characters in Drosophila which would breed true allowed the geneticist to make rapid advances in supporting and clarifying the chromosome theory of inheritance. This has made possible the chromosome maps which indicate the position of genes on the chromosome. Cytologists have worked hand-in-hand with the geneticists, so that the approximate location of the genes can be demonstrated visibly under the microscope.

The question naturally arose as to how the x-rays bring about such changes in the hereditary material of the germ cells. It has been found that most of the changes in the chromatin were so minute as to defy attempts to demonstrate them morphologically. Occasionally, however, the eytologist has found that the x-radiation has caused parts of a chromosome to be moved from their normal position. Moreover, in some irradiated cells a part of one chromosome has become attached to another. Other experiments which have proved of great value to genetics have been concerned with the action of $\mathrm{x}$-rays in increasing the rate of crossing over and in producing somatic mutations. 
Organisms vary widely in their susceptibility to roentgen radiations. Protozoa and bacteria in vitro are unusually resistant.

There is no doubt but that x-radiation produces changes in the physiological activity of cells, but these changes are not as evident as the morphological disturbanees occurring in the nuclens and cytoplasm. Such structural changes as lagging of chromosomes in mitosis, failure of ehromosomes to separate in meiosis, disintegration of chromatin, and coagulation of the protoplasm are produced. Some physiological disturbances that have been noted are increased permeability, increase in acidity, and changes in rate of respiration.

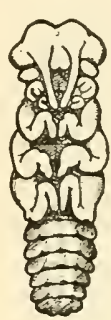

1

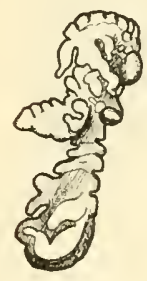

4

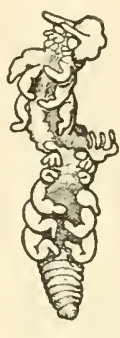

2

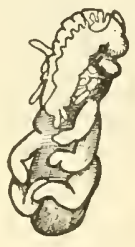

5
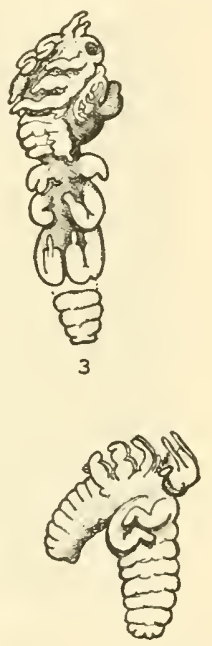

6

Fig. 371.-Drawings of grasshopper embryos. The first figure in the upper left is a normal embryo. The others are abnormal due to the $\mathrm{x}$-radiation treatments. Note that affected empryos have attempted to produce a secondary embryo.

Mitotically active cells are usually more susceptible than resting cells, and irradiated cells in the quiescent condition may appear normal until the onset of mitosis and then disintegrate. Cells irradiated while in the process of dividing may complete the process but be unable to negotiate a succeeding division. Destruction of cells following irradiation may be delayed by holding them inactive by low temperature or by some natural inhibitor. Some recovery has been noted in a few instances in which quiescent cells were kept inactive for a long period following irradiation. 
In all biological experiments the amount of radiation applied is very important. It has been found that a very slight dosage may be temporarily accelerative, a moderate dosage may have no visible effect, a heavier dose may have a temporary inhibiting effect, and a very heavy dose will result in permanent injury. There is usually a lapse of some time following irradiation before the effects appear; and it has been found that, as the dosage is increased, this latent period becomes shorter. There are several physical factors which affect the amount of radiation produced; these are the roltage, the current, the type of target, the distance from the target to the irra-

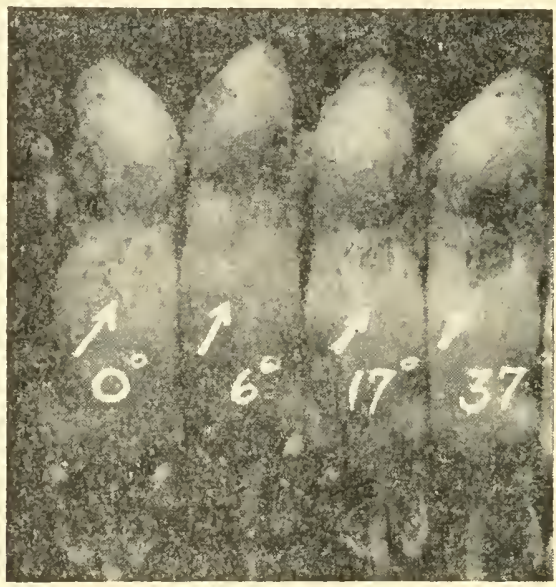

Fig. 372.-Effect of temperature during the irradiation upon the amount of injury produced. These rats are now two weeks of age. They were irradiated when one day of age. Each rat was given a dosage of 2,010 roentgens to a small region of the back, indicated by the arrow. The radiation inhibits hair formation and it can be seen that this injury is much greater in the animals irradiated at the higher temperatures.

diated object, the filtration, the back scatter, and the exposure time. Radiological experiments have become much more aceurate and dependable with the advent of the dosimeter. Such a device measures the amount of ionization in air and records this change on a scale calibrated to read in roentgens. This mechanical measure allows the operator to determine the intensity and the total dose delivered.

Roentgen radiation has proved to be a very valuable tool in the study of experimental embryology. It has been found that the first cleavage is retarded by appropriate exposures. It has been demonstrated that development can be stopped by heavy dosages. It has 
also been found that embryos become more resistant to radiation as they develop. Exceptions to this conclusion that the embryos become more resistant as they develop are stages of extreme susceptibility, such as gastrulation which involves many eritical processes not experienced later, and stages of unusually high resistance, such as occur in some insects during early clearage when the undifferentiated embryo has remarkable regulative powers. Roentgen radiation has been employed in upsetting the normal differentiation of embryos, and the results have shed some light on inter-relationships of cells at different stages in derelopment. It has been found that injury to young embryos as a result of irradiation tends to be widespread, whereas radiation injury in older ones is more localized. Evidence indicates that differential susceptibility of tissues of the adult is not the same as in embryos. For example, the arlult nerve and bone tissues are apparently resistant, whereas in growing embryos these tissues are susceptible.

One of the interesting and more practical types of experiment with roentgen radiation has been that of determining the effects on regeneration. It has been found that the effects are localized to regions of a body which have been exposed, and regeneration of amputated limbs can be inhibited by proper exposures even though the wound may heal over. This may be considered as evidence, which is in accord with other embryological experiments, that cellular differentiation can be effected without necessarily destroying the ability of cells to increase in number.

The radiologist (x-ray physician) and the x-ray manufacturers, as well as the biologist, have long recognized the need of more information concerning the fundamental action of roentgen radiation upon cells. Radiological organizations, some x-ray manufacurers, and such scientific groups as the Committec on Radiation of the National Research Council and the American Association for the Adrancement of Science have aided such investigations.

\section{The Fundamental Action of Roentgen Radiation}

The photon of roentgen radiation is capable of removing an extranuclear electron from an atom leaving it temporarily charged as an ion. The electron removed by the photon is called a photoclectron and is eapable of removing other electrons from lower energy levels. Thus the path of the photon (until all of the encrgy is absorbed) is marked, momentarily, by a trail of ions. In such a manner mole- 
cules become dissociated and form new molecules on recombination. This production of ionization is the basic action of roentgen radiation on protoplasm. It is thought that the production of an ion-pair in a chromosome produces the alteration or destruction of the gene at that point. Chromatin, especially in stages of synthesis, is apparently the most radiosensitive of cell structures. Some investigators have theorized a "sensitive volume" for a cell or an organism. This conclusion may be derived from certain analyses of dose-effect curves. Such interpretation of quantitative data also yields ideas that in some instances single "hits" kill a cell whereas others require many "hits" on the sensitive area before being killed. This interpretation of destruction indicates that the amount of radiation absorbed is the only factor involved in the killing action. The "target" theories of radiation action appear to apply best to the killing action on bacteria and protozoa, and to the production of mutations and chromosomal abnormalities.

Two important objections to the "target" theories, from a biological point of view, are (1) the shape of the dose-effect curves may be explained on the basis of individual variation in sensitivity, and (2) the metabolic condition of the cell at the time of the radiation affects the amount of injury produced by the radiation. It therefore appears that ionization is the fundamental action, but that the full expression of the radiation injury depends upon the number of vital processes in a susceptible condition (one of nolecular interchanges of energy) at the time the ionization takes place. The importance of the environmetal condition at the time of irradiation is shown, for example, in Fig. 372 where the amount of injury produced was different even though the dosage applied was the same for all of the animals. The lower temperature decreases the physiological activity of cells and this apparently increases the resistance to the radiation.

\section{BIOLOGICAL ACTION OF RADIUM}

Unfiltered radium emits three types of radiation: (1) alpha particles, (2) beta particles, and (3) gamma rays. The alpha particles produce dense ionization paths and are very active biologically. However, they do not penetrate deeply into tissue, and may be entirely absorbed by a sheet of paper. The beta particles may also be filtered out relatively easily by enclosing the radium in a small tube of platinum or gold. The biological action of beta particles 
is intense but most of them do not penetrate deeply into tissue. The gamma rays are similar to high voltage roentgen radiation and their biological action is very much the same. There is a difference in their application in that the radium is usually placed close to the biological material and then effects are more localized than that of roentgen radiation.

\section{Effects of Other Radiations}

The ionization produced by neutrons in tissue is initiated in a manner different from that of the roentgen and gamma radiations already mentioned. The neutron ionization is much more localized and intense. The neutron releases its energy by knocking the hydrogen nucleus, the proton, free from its outer electron. This recoil proton is so large that it comes in contact with many atoms (thus producing ions) within a short range before it dissipates its energy, picks up an electron and becomes an ordinary hydrogen atom again. Neutrons are more readily absorbed in light substances rich in hydrogen (as biological tissues and water), than in denser substances such as iron or lead. In terms of ionization in air, the nentrons appear to be from five to fifteen times more effective than roentgen radiation in killing cells. Qualitatively, the therapentic effects of the fast neutrons have so far been similar to those of roentgen radiation.

Radioactive substances have proved to be valnable as tracers in following atoms through metabolic processes in the body. Radioactive iodine, for example, can be fed and within a short time the region of the thyroid will be giving off radiation that can be detected by the Geiger counter (sensitive ionization chamber). Radioactive phosphorus shows promise of becoming of value in the treatment of diseases related to excess bone marrow development. It appears that phosphorus is concentrated in malignant bone marrow cells and thus the concentration of radioactivity becomes great enough to destroy them.

Cosmic rays abound in the atmosphere and penetrate to depths of. even the heavier materials. Apparently not enough of the radiation is absorbed by biological material to be effective. However, from time to time one hears of the theory that natural mutations may be the result of cosmic rays affecting the chromosomes of the germ cells. 


\section{Summary}

Biological effects are related to the type of radiation being absorbed. High frequency oscillations penetrate body tissues and produce molecular agitation ("artificial fever"). Infrared also increases body temperature (although more peripheral in location). More resistance is offered to radiations of the visible spectrum, although the energy changes involved are not extensive enough to bring about striking systemic effects. Ultraviolet radiation contains more energy and its absorption causes changes in atomic associations which may have profound effects on chemical processes.

Roentgen and gamma radiations are very penetrating and when absorbed they displace electrons and produce ionization. These changes are fundamental in character and may result in extensive injury to cells.

The physiological condition of cells appears to affect the reaction to radiation. Embryonic and highly active cells are apparently the most susceptible.

The quantity of radiation absorbed is an important factor in producing biological effects.

The knowledge of biological effects of radiation at present offers possibilities of theorizing about the origin and development of life itself. The most dependable contributions, however, have been the production of artificial fever by high frequency oscillations; infrared therapy; the understanding of the importance of sumlight in the production of food by plants; the role of ultraviolet radiation in the conversion of ergosterol to vitamin $\mathrm{D}$; the use of roentgen radiation and radium emanations in increasing mutation rates which has been of great value to genetical studies; the use of these radiations in the study of embryological development; and their use in the treatment of cancer, etc. 


\title{
CHAPTER XXXVI
}

\author{
ANIMAL DISTRIBUTION \\ (By Willis Hewatt, Texas Christian University)
}

\section{Life Regions and Zones of the Earth}

Every area of the earth has its animal and plant life. The apparently barren sun-baked desert, the ice-capped polar regions, the highest mountain tops, and the tropical rain forests are all inhabited by their famas and floras. Life in the seas extends from the shore line to the greatest depths. After extensive taxonomic surreys have been made in practically all areas of the earth and these data are studied, it is found that the earth can be divided into fairly definite horizontal regions and vertical zones. These two phases of the distribution of organisms on the earth are very closely related to each other but are usually studied separately as geographic or horizontal distribution and bathymetric or vertical distribution, respectively.

Geographic Distribution.-Many attempts have been made to divide the earth into horizontal life regions based upon the distribution of various groups of animals and plants. The exact boundaries of the regions are not fully agreed upon since several groups of animals have been used as criteria for the division into life regions. P. L. Sclater (1829-1917), the earliest zoogeographer, worked with the perching birds. These forms were well adapted to such a study since they have little power of flight, they are widely distributed, and they had been very closely studied. The early (1876) classic work of the Englishman, Alfred Russell Wallace, in which the earth was divided into six primary regions, is the one most commonly followed by modern biologists. Wallace based his divisions upon the distribution of the mammals. Other significant studies in geographic distribution have been based upon the geographic ranges of mollusks, earthworms, moths, butterflies, spiders, fresh-water fishes, reptiles, and many others. From works dealing with such varied groups of animals it is readily understood that any classification of the regions of the earth will depend greatly upon the group of animals used as a criterion. On the other hand, there are many correlations found in the studies of the geographical ranges of all of these groups of animals. The zoogeographical regions of Wallace and the 
general boundaries of these areas are given below. The boundaries of the regions do not necessarily follow the outlines of continents as will be seen in Fig. 373 .

1. The Palaearctic Region includes all of Europe, Africa north of the Sahara, and all of Asia north of the Himalaya Mountains.

2. The Nearctic Region embraces the North American continent as far south as Mexico.

3. The Ethiopian Region consists of Africa south of the Sahara, southern Arabia, and Madagascar.

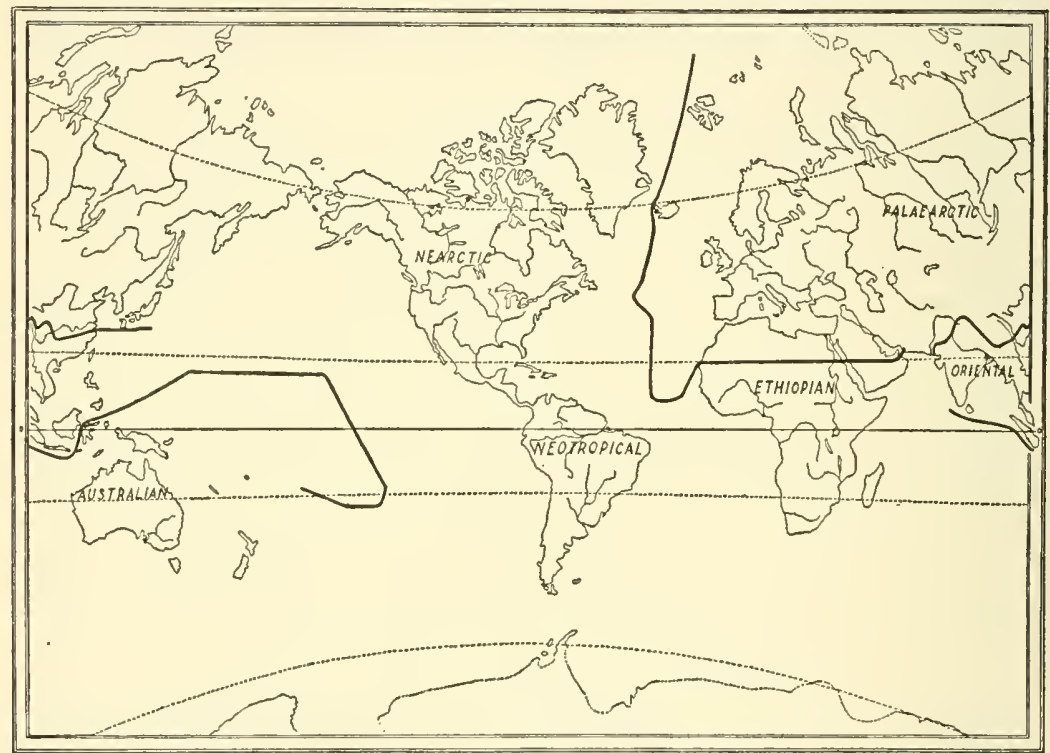

Fig. 373.-Map showing the life regions of the earth. (Base by permission of A. J. Nystrom Company.)

4. The Oriental Region includes Asia south of the Himalayas, and that portion of the Malayan Archipelago which lies northwest of Wallace's line. This famous line dividing the Oriental and Australian Regions passes east of the Philippines between the islands of Bali and Lombok, and between Borneo and Celebes. Bali and Lombok are separated by a very narrow but deep strait, and there is a very remarkable contrast between the faunas of the two islands.

5. The Australian Region is made up of Australia and the neighboring islands as far west as Wallace's line. It includes the great number of small islands east of Australia. 
6. The Neotropical Region consists of Mexico, Central America, and South America.

The present distribution of animals on the earth gives us many evidences concerning the past history of the earth. The North American fauna is notably different from the South American fauna. This fact apparently indicates that North and South America were completely separated from each other by an extensive water barrier until a relatively recent period. The great contrast between the faunas of the islands of Bali and Lombok indicate that the Australian continent and its neighboring islands have been separated from the Asiatic mainland for a relatively long period of time. The study of distribution also gives many clues to the ancient changes of climate on the earth.

Bathymetric Distribution.-In discussing the vertical or altitudinal distribution of animals two fundamental types of habitats must be considered; namely, the water habitat or Hydrobios and the land habitat or Geobios. The inhabitants of these two realms differ greatly, but some few forms oceupy both regions.

The hydrobios includes both fresh- and salt-water realms. The variation in vertical distribution of fresh-water animals is relatively insignificant when compared to that of marine animals. The seas have been variously classified into vertical zones. The divisions most commonly recognized are:

1. The Littoral which is the area between tide marks. It is characterized on rocky shores by growths of barnacles, mussels, and snails. Life in sandy littoral regions, such as that along the Texas Gulf Coast, is relatively scarce and consists chiefly of annelid worms and other boring forms.

2. The Sublittoral includes the subtidal region to a depth of approximately 100 meters. This is about the lowest depth at which plants can grow abundantly. It is the most productive region on the earth as far as variety and numbers of animals are concerned.

3. The Abyssal zone is marked by the absence of liglit and extends to the greatest depths of the oceans. Life is not very abundant in this zone. Plants cammot live at such depths, therefore, the animals which live in the abyssal region are dependent upon other animals as a source of food, or they subsist upon the floating plant life which is continuously dying and sinking to lower levels. 
The vertical or altitudinal distribution of terrestrial animals depends primarily upon the vertical distribution of the vegetation. The variation in the kinds of plants found at different altitudes depends to a large extent upon the elimatic belts which extend around the earth between the equator and the poles. The vertical belts of plants encountered in traveling from a lowland to a high mountain top correspond very closely to the climatic zones surrounding the poles of the earth. The communities of animals and plants found in each vertical zone are similar throughout the world.

The seven vertical life zones and a few of the characteristic animals and plants found in each zone, as it is represented in the Southwest, are here given.

1. The Alpine zone (usually above 10,500 feet) is the area above the timber line. This highest zone is represented in the Southwest

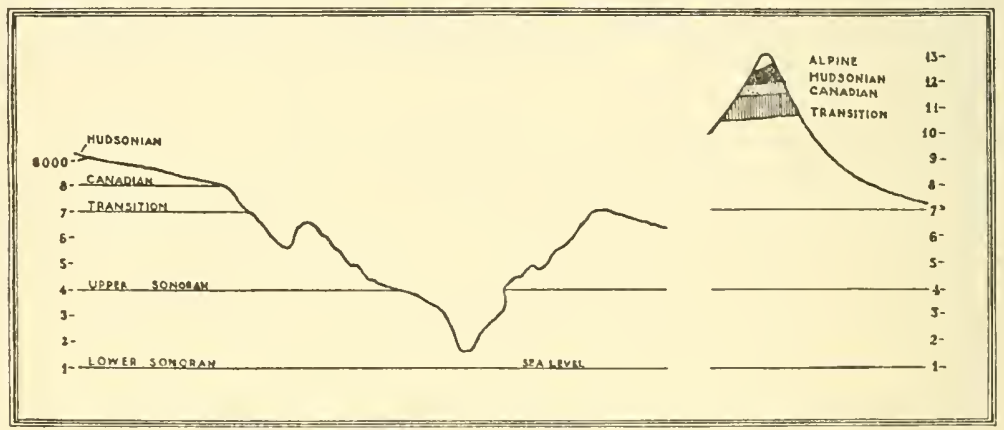

Fig. 374.-Diagrammatic sections of the Grand Canyon and San Francisco peak showing the vertieal life zones. (After N. N. Dodge and Merriam.)

on the top of San Francisco Mountain in Northern Arizona and in the Sangre de Cristo range of the Rockies in Northern New Mexico. This zone is characterized by the presence of few plants, such as saxifrages and dwarf willows. Among the fauna are found the golden eagle, some weasels, and mountain sheep.

2. The Hudsonian zone (9,000-10,500 feet) consists of a forest of spruces and some firs, and harbors the dusky lorned owl, bears, shrews, and red squirrels. The Hudsonian zone is found in the same region as the Alpine zone.

3. The Canadian zone (8,000-9,000 feet) is distinguished by the presence of the Douglas fir and the aspens. Common animals of this zone are the three-toed woodpecker, one species of shrew, and two 
species of field mice. This zone is found in the Rockies as far south as southern New Mexico. There is some evidence of Canadian fauna in the Chisos Monntains of Texas.

4. The Transition zone (7,000-8,000 feet) is found in the same regions as the above zones. It is also found in the Davis and Chisos and Guadalupe Mountains of Texas. It is covered with pine trees

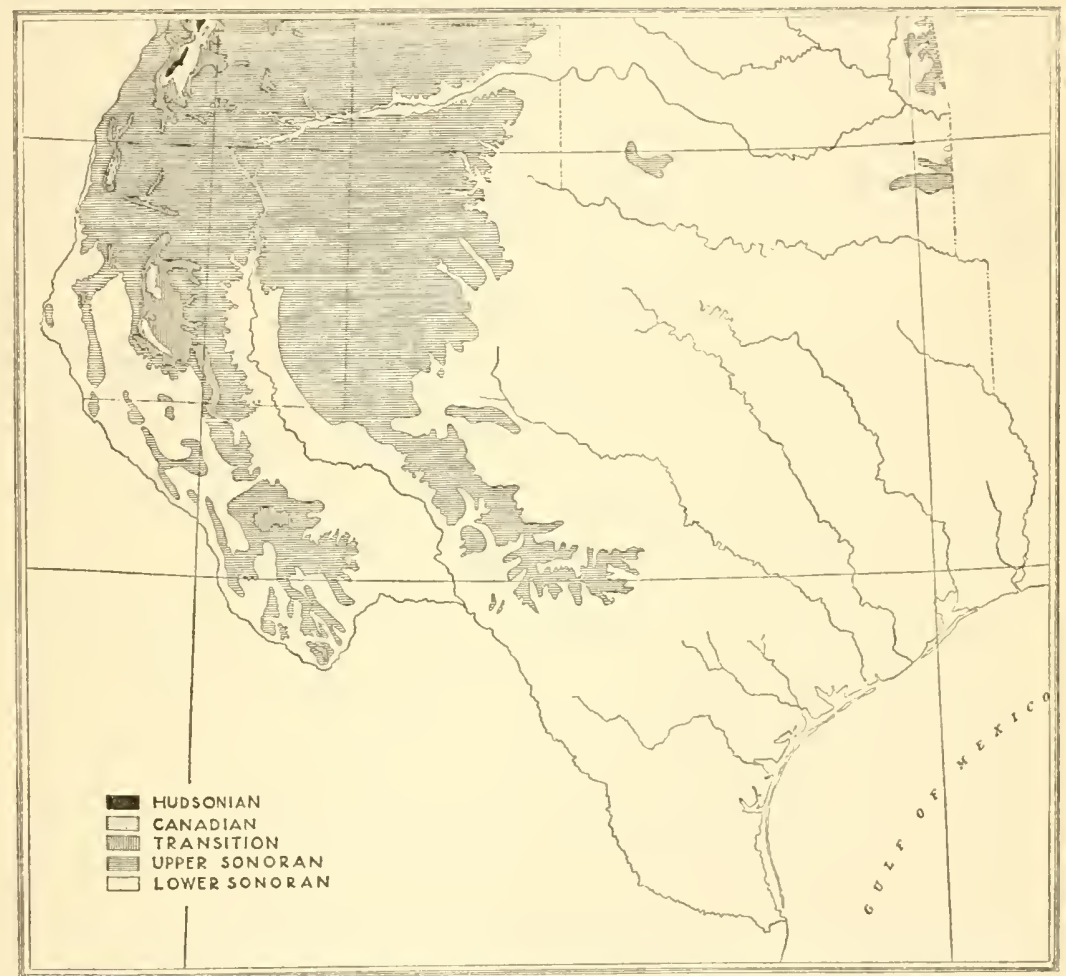

Fig. 375.-Map of the vertical life zones of Texas and adjacent areas. (After Bailey.)

(mainly Pinus ponderosa). Abert's squirrel is found in the Transition zone as is one species of horned lizard.

5. The Upper Sonoran zone (6,000-7,000 feet) is the piñon belt distinguished by the nut or piñon pine and cedars. The zone extends from western Texas through New Mexieo, Colorado, Arizona, and into southern California. Characteristie animals are the piñon jay bird, and the large rock squirrel. 
6. The Lower Sonoran zone is less homogeneous in its flora and fauna than are the other zones. In the Southwest it includes most of Texas, Oklahoma, southern New Mexico, southern Arizona, and southern California. The eastern third of Texas is typical of the lower Sonoran of the southeastern states which are characterized mainly by long-leafed pines, magnolia, and live oaks. Some animals of the eastern portion are mockingbirds, painted buntings, and wood rats. The western lower Sonoran is inhabited by typical desert and semidesert flora and fauna.

7. The Tropical zone is of very little significance in the southwestern United States, but many tropical plants, such as the Texas palms and bananas grow in a narrow strip of the lower Rio Grande Valley.

\section{Migration of Animals}

The migrations of many animals are not well understood but in most cases they involve breeding habits, food, or shelter. Most of these migrations are seasonal, but many permanent changes of location have been made by groups of animals as a result of permanent changes of climate on the earth.

The seasonal migrations of the North American caribou and the bison are among the most noted examples of migrations of animals which move about in search of food. The caribou migrate southward in Canada during the winter and follow the melting snow northward in the summer.

The remarkable migrations of the eels of the Mediterranean area and of the easter'n coast of the United States are among the best known examples of breeding migrations. The eels from the rivers of these two regions migrate into the Caribbean Sea and spawn. Although the two spawning territories overlap, the young eels of each species journey hundreds of miles back to the rivers inhabited by their ancestors. A great many species of birds migrate from the tropics and semitropics and breed in northern regions. The scarlet tanager spends the winter in northwestern South America and migrates across the Gulf of Mexico into northeastern United States and southern Canada and breeds during the summer months. The upland plover is familiar in the Southwest, since it migrates through this region twice each year on its journeys between Argentina and British Columbia. 


\section{Means of Dispersal and Barriers}

The wide distribution of species of animals on the earth depends largely upon their means of dispersal or means of being carried from one place to another and upon the barriers which they encounter. Among the members of a species as well as among the related and nonrelated species of animals there is a continuous struggle for existence. Those forms which, by some means, are able to enter new environments where competition is less severe will have the better chance at survival.

In practically all marine organisms there exists a means of locomotion during some stage of the life history. In a great number of the forms, especially those which are sessile in the adult stage, there is a free-swimming larval stage: planula larva of the coelenterates; trochophore larva of annelids; and the various free-swimming larvae of mollusks, echinoderms, and crustaceans. The planktonic larvae and adults, i.e., those which swim or float free of the bottom, depend greatly upon oceanic currents for their wide dispersal.

The dispersal of oceanic animals is also greatly enlanced by the large numbers of eggs and larvae produced by these forms. For example, one investigator found that a "sea hare," a marine gastropod, deposited 478 million eggs during one four-month spawning period. The eggs were laid at the rate of 41,000 per minute.

Marine animals are limited in their distribution by such barriers as temperature, land masses, and salinity of the water. The marine animals on each side of the narrow Isthmus of Panama are entirely different. The extreme changes in temperature prevent most of these species going around the southem tip of South America. Littoral animals are often limited in their distribution by large river mouths which empty great quantities of fresl water into the oceans. Only specially adapted forms can live in these brackish waters.

Fresh-water faunas are restricted in their distribution by land barriers which usually separate the bodies of water in which they live. These forms depend mainly upon other animals, such as birds and insects, for the dispersal of their eggs and dormant stages. Many fresh-water animals, such as snails and clams, attach themselves to the bodies of birds or insects and are carried into new habitats. 
Among land animals the birds and flying insects appear to be least restricted in their ranges. Even these, however, are often limited by mountains, deserts, and large rivers which act as the most effective barriers to the wide distribution of land animals. Many of the birds and mammals found on the south rim of the Grand Canyon of the Colorado River are not able to reach the north rim of the Canyon. For example, the Abert squirrel is found in New Mexico and Arizona, south of the Canyon, while the Kaibab squirrel oceurs only north of the Canyon. Small islands, which are located great distances from the mainland, are often inhabited by small mammals and reptiles similar to those found on the mainland. Such forms have probably reached the islands by way of floating rafts of vegetation, and hollow trees which are known to be carried several thousands of miles by oceanic currents.

Wind currents may carry birds across the Atlantic Ocean. It is not uncommon for American birds to land on the coast of England after severe storms. These same currents also carry spores of Protozoa, small insects, and the eggs of many invertebrates.

\section{Effects of Man Upon Distribution}

The advent of man upon the earth ard the development of his more efficient means of travel have greatly enhanced the wide distribution of many species of animals. Marine invertebrates attach themselves to the bottoms of ships (ship fouling) and are carried to practically all parts of the earth. Rodents and insects are accidentally distributed from one country to another by ships. During the past man has purposely transported animals from one country to another. In some instances the animals multiply more rapidly in the new environment than they did in the original habitat. English sparrows were introduced into North America in 1859, and since that time they have become so numerous as to be a great pest in this country. The starling, which was introduced at about the same time is rapidly increasing in numbers and in recent years has been reported as far south and west as Central Texas. By cultivating extensive prairie lands, man has created insurmountable barriers to grazing animals which once roamed these areas. Deforestation of wooded regions has destroyed the shelter necessary for such animals as deer, foxes, wolves, bears, and many others. 


\section{CHAP'TER XXXVH}

\section{THE ANIMAL AND I'S ENVIRONMEN'T}

\section{(By A. O. Weese, University of Orlahoma)}

Ecology is that division of biology which has to do with the relations between organisms and their environment. The environment of an organism, for convenience, may be divided into two parts, the nonliving, including physical, chemical, and climatic factors, and the living, including other organisms of the same and of different kinds. The science of ecology, in a sense, is as old as man, because from the very begimning of his conscious existence it was necessary that man take cognizance of the fact that his environment was made up in part of plants and animals and that these organisms in turn harl relations to their environment. It was not until comparatively recently, however, that ecology eame to be recognized as a separate department of biology. Modern ecology may be said to have begun with the recognition of the community. Plants and animals are distributed as they are over the surface of the earth, not because of any chance coincidence, but because of a combination of circumstances, one of which is the fitness of the physical environment for the proper completion of their life histories and another of which is the presence of such other organisms as are necessary to furnish food and to provide other requisite conditions. We can think of a community of organisms in much the same way as we think of a community of human beings. The analogy eannot be followed too closely, for, after all, human beings are much alike while the organisms in an ecological community are of many different kinds, having different requirements in detail as to food and environmental conditions. We shall arrive at a better concept if we think of a human community as made up, not only of butehers and bakers and candlestick makers, each with their particular functions as producers and as consumers, but also of the domestic animals which furnish materials for food or clothing or which perform labor, the household pets and pests, the eultivated plants which are utilized in the manufacture of food, clothing or shelter, and the host of wild animals and plants which enter into some relation with those previously 
mentioned. The characteristics of such communities vary from time to time and from place to place. A difference in climate may be sufficient to change almost every component of the community. The domestic animals and plants associated with man in the tropics are quite different from those in arctic regions. Perhaps one species, the dog, almost as adaptable as man himself, might be considered as a member of both communities. The removal of a single species or the addition of a new one may alter profoundly the aspect of the community. Consider, for example, a human society from which all cows were removed, or the changes made in the life of certain sections of the United States with the introduction of the cotton boll weevil.

Some idea of the complexity of the relationships involved in a community of organisms may be gained by citing Charles Darwin's example of the dependence of clover on cats, or Thomas Huxley's extension of the chain of cause and effect to the responsibility of the old maids of England for the supremacy of that nation on the seas. Obviously, since old maids are fond of cats, the number of the latter is greater when old maids are numerous. Cats eat field mice, which in turn prey upon the nests of bumblebees. Thus, a large cat population is favorable for the development of clover which is fertilized by the bumblebee. Clover is fed to cattle and it is well known that Britain's sea power is due to "The roast beef of old England."

Most of the relationships suggested in this series have to do with food. It is often possible to gain a better idea of relations within a community by the use of a diagram indicating the more obvious influences. The accompanying figure, modified from Shelford (Fig. 376 ), represents food relationships only in a hypothetical prairie community and may be called a "food chain diagram." Many very obvious food chains are omitted in order to avoid a complexity too great for easy reading, and, of course, any real community would include many more kinds of animals. The chart is very incomplete, also, in that no relations other than those directly con. cerned with food are indicated.

Life exists on the earth only in a relatively very limited space. We might represent the relationship of the various parts of the planet on which we live by a series of concentric circles, the area within the inner circle corresponding to the "solid" portion of the 
earth, the lithosphere. If the lithosphere were uniform in diameter, it would be covered by a layer of water, the hydrosphere, which is in turn surromnded by a gaseous layer, the atmosphere. It is only

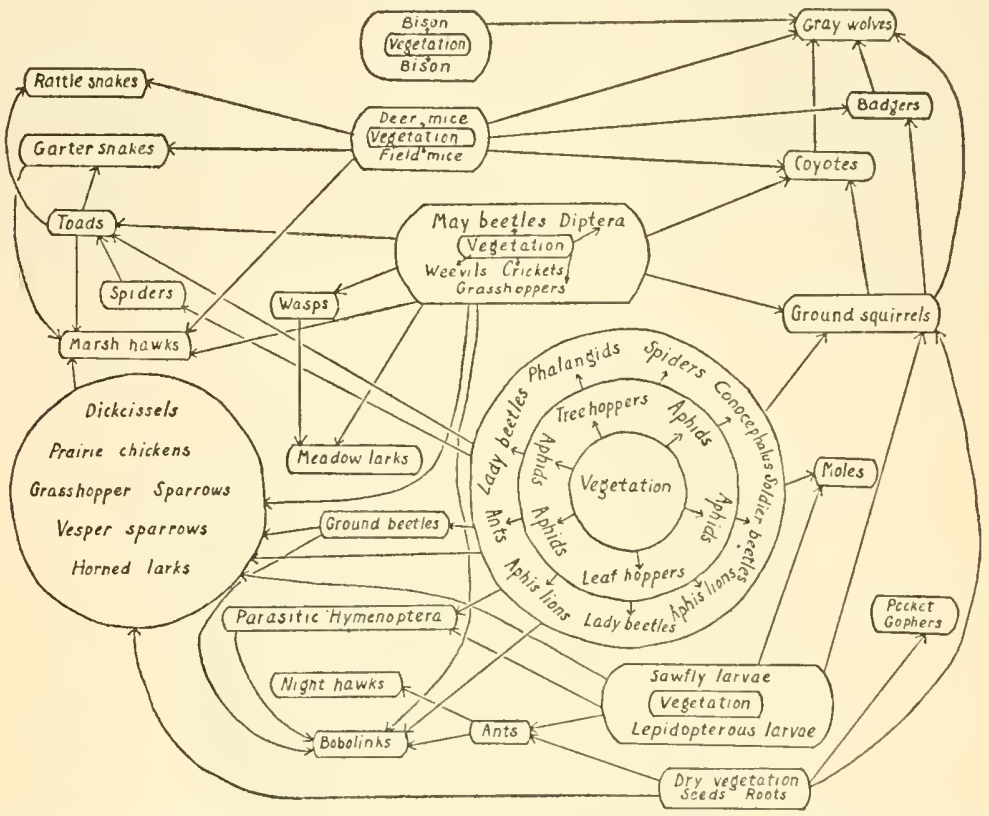

Fig. 376.-Diagram to show food relationships in a hypothetical prairie community. (Redrawn and modified from shelford. Animal Communities in. Temperate America, published by University of Chicago Press.)

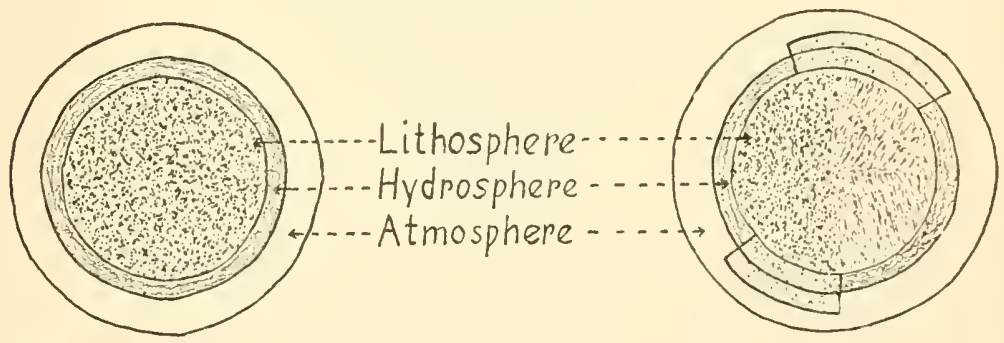

Fig. 377.-Diagram to show the relationship of the general areas of our planet.

where the components of these three layers interact that life is possible. Living organisms cannot exist in the lithosphere (Fig. 377) without the presence also of water, and of oxygen from the atmosphere. Similarly life in water is possible only where it contains in solution 
both solids and oxygen. Air is habitable only temporarily and animals spending time there must return frequently to water or soil. Free interaction between the three components of the earth is made possible by the fact that the lithosphere is not of exactly uniform diameter so that certain portions project above the hydrosphere, forming continents (and islands). These elevated areas constitute a rather small proportion of the whole surface of the earth, but it is with these areas that we shall be concerned, not because there is no life in the submerged areas, but because the space available in this chapter is too small for us to consider the great subject of marine ecology.

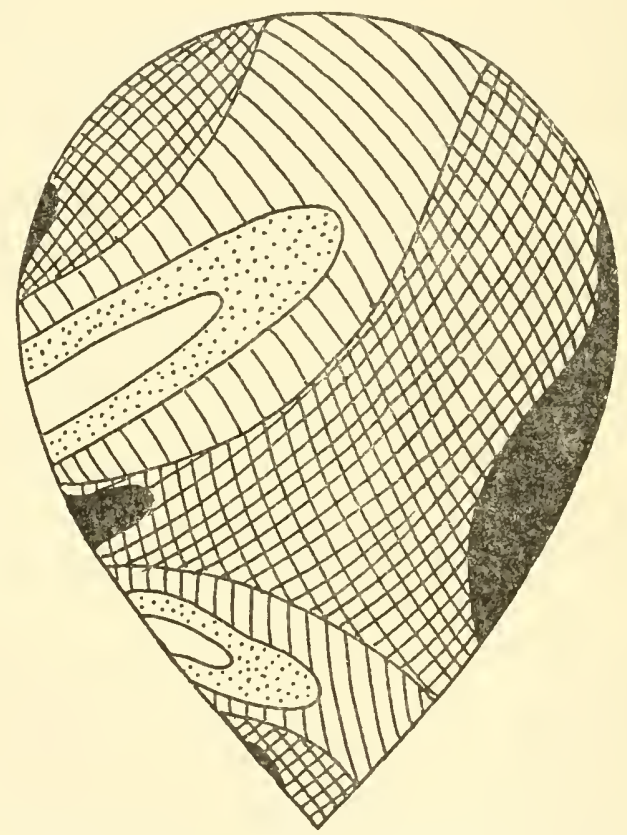

Fig. 378.-Distribution of "precipitation effectivity" on an ideal continent. (Modified after Thornthwaite. Drawn by Edward O'AIalley.)

The inclination of the earth's axis of rotation to the plane of the earth's orbit about the sun and the relative positions of the continents are factors which influence the distribution of climates. The two principal climatic factors are, of course, temperature and moisture. If the effect of altitude is not considered, the former is distributed rather uniformly, so that the familiar torrid, tem- 
perate, and frigid zones express rather well the comparative temperature conditions on a continent. NIany factors, however, operate in the control of the amount of precipitation and its distribution. In general, the distribution of available rainfall on an "ideal" topshaped continent withont mountain ranges might be expected to follow the plan of Fig. 378 in which the darker areas indicate maximum rainfall and the unshaded areas represent very arid regions. Each climatic type makes possible the occupancy of the region concerned by a definite type of biotic community which can

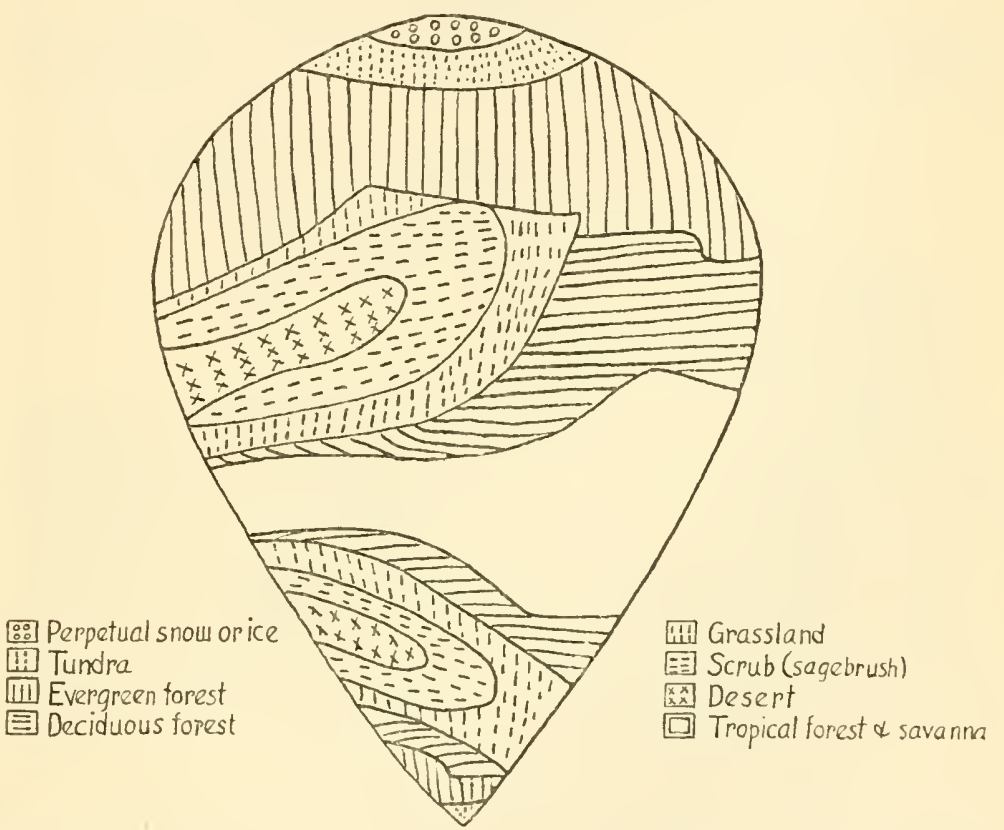

Fig. 379.-Hypothetical distribution of biotic communities on an "ideal continent."

be most easily designated by the character of the conspicuous or dominant vegetation. Taking into consideration the seasonal distribution of arailable moisture and the annual variations in temperature, the "ideal continent" might be expected to present an aspect something like that represented in Fig. 379. It will be seen at once that in the regions of deficient temperature heat is the determining factor, and that in warmer regions the amount of moisture is the major influence. How elosely this expectation is realized in 
the case of North America may be seen by a comparison with Fig. 380. As will be seen later, the animals of a climatic region are as distinctive as the plants. The soil, which is the result of interaction between the climate, the plants and animals, and the rock substratum, also is characteristic of each climate. Thus, in the area influenced by each climatic type, there is a definite biotic community or formation, characterized by plants and animals whose relations to their environment are similar or equivalent.

\section{The Principal Biotic Formations}

A rough classification of the climatic formations of North America includes the following:

1. The Tundra Formation

2. The Coniferous (Evergreen) Forest Formation

3. The Deciduous Forest Formation

4. The Grassland Formation

5. The Sagebrush Formation

6. The Desert Formation

In addition there are transitional communities difficult of representation on a map because of their discontinuous distribution or small area. Some of these may be referred to, provisionally, as Woodland, Desert Scrub, Chaparral, and Swamp communities.

1. The Tundra Formation (Sedge-Musk Ox Biome).-This is the community of the aretic "barren grounds," beyond the northern limit of trees and between it and the polar region of perpetual snow and ice. Included, also, is the region above timber line on high mountains, where climatic conditions are similar to those of the far north. The vegetation of the tundra is composed mainly of grasses, sedges, and lichens (e.g., reindeer moss), although there are also patches of dwarf willows and other woody plants from a fraction of an inch to a few feet in height. Typical animals of the Arctic tundra are the musk ox and the arctic caribou which feed on the low vegetation. Here, also, are found great hosts of rodents, including lemmings, whose enormous increases in numbers and periodic migrations in Europe have been known for many hundreds of years. Arctic hares and aretic ptarmigan, with those animals previously mentioned, furnish food for the aretic fox and the arctic 
wolf. Many of these animals, including the arctic fox, the aretic hare, the ptarmigan, and the collared lemming, are adapted to the climatic rhythm of their environment by changing in color from white in winter, when the entire landscape is snow-covered, to a darker color during the summer. On the tundra nest vast numbers of migratory birds, some of which, like the golden plover, fly to winter homes in South America.

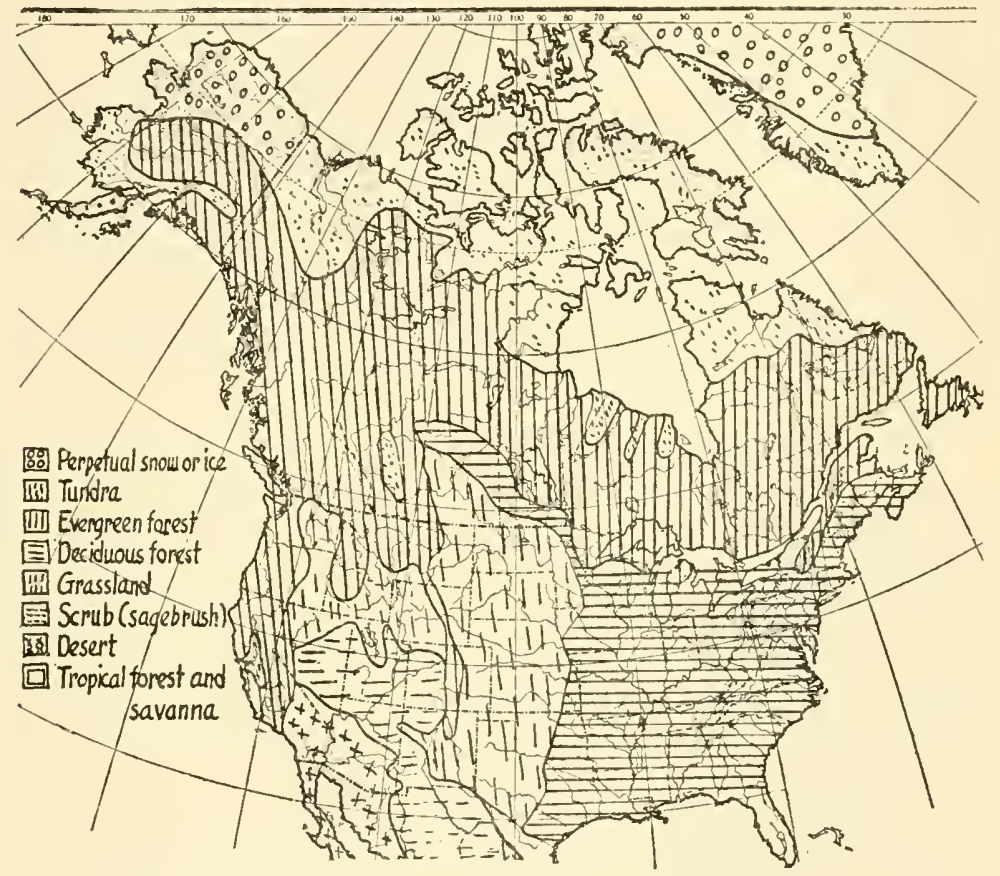

Fig. 380.-Distribution of major biotic regions in North America.

\section{The Coniferous Forest Formation (Spruce-Moose Biome).-} South of the Arctic tundra and below the high mountain tundra along the Rockics and the Sierras is the great coniferous forest whose dominant regetation is evergreen, composed chiefly of spruces, firs, and pines of many species. The moose and the woodland caribou are characteristic animals over the greater part of the area. They feed upon grass and shrubs, chiefly in open areas near streams or lakes. The principal carnirore is the timber wolf. The Canada 
lynx is also a creature of the evergreen forest. Black bears are numerous but are widespread, also, beyond the borders of this formation. The wolverine and the red fox are lesser carnivores. The varying hare is similar to the aretic hare but lives in more protected situations.

3. The Deciduous Forest Formation (Oak-Deer Biome).-Most of the United States lying east of the Mississippi River is characterized by deciduous forest. In this area the dominant vegetation consists of trees whose leaves are shed during the unfavorable winter season, such as oaks, beech, maple, hickory, and elm. The most widespread large herbivorous animal is the Virginia deer, although the wapiti (elk) was formerly of frequent occurrence. The wolf and the cougar were once widely distributed but are almost extinct now. The bay lynx, a close relative of the Canada lynx, preys upon the numerous rodents and birds. Other characteristic animals are the opossum, the raccoon, the flying squirrel, and the woodchuck. Many of these inhabit open areas in the woods rather than the dense forest.

4. The Grassland Formation (Grass-Bison Biome).-No single grass is included in the "common" name of this community because it is probable that the name of no single genus of grass is sufficiently familiar. Important grasses in the great central grassland area are the bluestems (or beard grasses), June grass, porcupine grass, grama grass, and buffalo grass. The grassland region was the habitat of the bison which once ranged over the prairies from Texas to Saskatchewan in enormous numbers. It is estimated that at the coming of the white man there were over 75 million of these animals, upon which the plains Indians depended for food, clothing, and shelter. The American pronghorn or antelope was also abundant. Both were held in check by the gray wolf. The black-tailed jack rabbit is an animal of the open grassland while the cottontail occupies wooded or at least bushy areas. In the drier western portion of the grassland the prairie dog and the badger (Fig. 341) are common. There are many small rodents, including the pocket gopher, the ground squirrels, and prairie deer mice.

5. The Sagebrush Formation (Sagebrush-Jack Rabbit Biome).This community is centered in the Great Basin of Nevada, Utah, and neighboring states. It is an area of deficient rainfall, and sagebrush is a prominent constituent of the vegetation. Most of 
the larger animals belong to groups which are also found in neighboring formations, although the smaller mammals are mostly of different subspecies. Rabbits and rodents are especially abundant.

6. The Desert Formation (Creosote Bush-Kangaroo Rat Biome). -It is difficult to characterize this community by the name of a dominant plant and an important mammal, as there are many types of descrt associations. They have, however, one characteristic in common-a great deficiency in available water. Plants must guard against water loss and conserve water between the infrequent moist periods. Some of the adaptations which meet such conditions are: reduction of leaves (thorns), fleshy stems, thick euticle, extensive underground organs, etc. Animals conserve water by remaining underground or in sheltered places during the hotter parts of the day and coming out only at night or at dusk. Reptiles and birds are able to reduce water loss by the absence of sweat glands and by giving off their nitrogenous excretion in the form of uric acid which does not need to be dissolved in water.

\section{Adaptation}

From what has been said above, it appears that each particular set of climatic and other environmental conditions accommodates a particular group of plants and animals. The activities and structure of these animals are such as enable them to survive best under the conditions in which they are found. The barren ground caribou is not fitted for life on the central grassland area nor is the bison able to survive on the tundra. Each is said to be adapted to the particular conditions under which it exists. Many so-called adaptations, however, seem to be worthless. One might think, for example, that the shovellike brow tines of the caribou would be a remarkable adaptation for shovelling snow from the vegetation on which the animal must feed during the winter-but the antlers are shed in the late autumn and do not grow out again until the following spring. One must be careful not to assign adaptive functions to animal structures without careful consideration of the habits and life histories of the animals concerned.

\section{Succession}

It must not be thought that, within the areas of the great climatic formations, there is entire uniformity in the communities of plants 
and animals. This is far from true. There exist minor differences of climate, soil, and physiographic conditions and of biological history which result in differences in plant and animal population. There are always areas which, for example, have been denuded by physiographic or other processes. Fire, flood, and human utilization are among the more common influences which may cause partial or total destruction of the biotic communities of a region. When denudation occurs, a legion does not long remain unpopulated, but the slow process of redevelopment of a community characteristic of the existing climate begins as soon as the destructive force has ceased its action. This process of development is called succession because it is characterized by the appearance, first, of pioneers, which give way to other groups of organisms which, in turn, alter conditions in such a way that still others find conditions favorable for their existence. The final result, after a long time, is the development of a community which is in equilibrium with its environment and will not change unless the environment changes. Such a community is called a climax community. An analogy might be sought, again, in human experience. The human pioneers who first penetrated into the broad plains of the "old west" conquered the wilderness to such an extent that some who required more of the comforts of civilization were able to find suitable homes. Many of the original pioneers, however, moved on to new frontiers when the land became too thickly settled. The "climax human community" has not yet been attained, as man is forever seeking more perfect adjustment to his environment.

It is often possible to study the stages in the development of a community without waiting for the entire process to take place in one locality. For example, the broad flood plains of many of our rivers are subject to frequent inundations. Such inundations may remove all living things from their paths, but since the flood plains are so broad and the course of the river is never the same from year to year, the same tracts are not covered each spring, and areas may be found which have been undisturbed for a few weeks, a few months, a year, two years, five years, fifty years, etc. In the flood plain of the Canadian River (in Oklahoma) mud flats from which the water has receded recently are soon occupied by an assemblage of organisms including blue-green algae, two small beetles, and a 
Succession on the Canadian River Flood Plain. (Data From Hefley.)

\begin{tabular}{|c|c|c|c|c|c|c|}
\hline STAGE IN DEVELOPMENT & 1 & 2 & 3 & 4 & 5 & 6 \\
\hline $\begin{array}{l}\text { Paralimna appendiculatum } \\
\text { Fly } \\
\text { Heterocerus pallidus } \\
\text { Beetle (Algae and detritus) }\end{array}$ & $x^{*}$ & & & & & \\
\hline $\begin{array}{l}\text { Bembidion laevigatum } \\
\text { Beetle (Algae and detritus) }\end{array}$ & $x$ & $x$ & & & & \\
\hline $\begin{array}{l}\text { Cicindela hirticollis } \\
\text { Tiger Beetle (small insects) }\end{array}$ & $x$ & $\mathrm{x}$ & $\mathrm{x}$ & & & \\
\hline $\begin{array}{l}\text { Cicindela cuprascens } \\
\text { Tiger Beetle (small insects) }\end{array}$ & $\mathrm{x}$ & $x$ & $\mathrm{x}$ & & & \\
\hline $\begin{array}{l}\text { Cicindela punctulata } \\
\text { Tiger Beetle (small insects) }\end{array}$ & & $\mathbf{x}$ & & & & \\
\hline $\begin{array}{l}\text { Mutillidae } \\
\text { Velvet ants (insects and spiders) }\end{array}$ & & & $x$ & & & \\
\hline $\begin{array}{l}\text { Apion pennsylvanicum } \\
\text { Weevil (cocklebur) }\end{array}$ & & & $x$ & & & \\
\hline $\begin{array}{l}\text { Haltica bimarginata } \\
\text { Beetle (willow) }\end{array}$ & & & $\mathrm{x}$ & $\mathrm{x}$ & $\mathrm{x}$ & \\
\hline $\begin{array}{l}\text { Phalacrus politus } \\
\text { Beetle (willow) }\end{array}$ & & & & $\mathrm{x}$ & & \\
\hline $\begin{array}{l}\text { Stictocephala lutea } \\
\text { Tree hopper (willow, cottonwood) }\end{array}$ & & & & $x$ & & \\
\hline $\begin{array}{l}\text { Cicadella gothica } \\
\text { Leafhopper (willow) }\end{array}$ & & & & & $\mathrm{x}$ & \\
\hline $\begin{array}{l}\text { Dorytomus squamosus } \\
\text { Weevil (willow) }\end{array}$ & & & & & $x$ & \\
\hline $\begin{array}{l}\text { Strongylocornis stygicus } \\
\text { Bug (coral berry) }\end{array}$ & & & & & & $x$ \\
\hline Epitrix brevis & & & & & & $x$ \\
\hline
\end{tabular}

Flea Beetle (Miscellaneous plants)

Brief descriptions of stages:

1. Mud flat with blue-green algae, later liverworts.

2. Sedges, willow and cottonwood seedlings in addition to 1 .

3. Second lerel. Sand, sedges, willows, cottonwoods, cockleburs.

4. Third level. Grasses, willows, cottonwoods.

5. Third level. Cottonwoods, fewer willows.

6. Fourth level. Elm-oak forest with shrub undergrowth.

* $x$ indicates the presence of the species as an important member of the community.

fly. Tiger beetle larvae come a little later, and then seedlings of willow and cottonwood. During the course of a year or so blowing sand is deposited so that the level of the ground is raised several feet, willows and cottonwoods increase in size, and other plants 
are added. Soil and sand continue to be deposited, the trees increase in size, and the ground becomes more thickly covered by vegetation. Finally, after a hundred years or so a still higher level is attained and the willows and cottonwoods give way to oaks and elms. The accompanying table indicates some of the insects to be found in each stage of this developmental series. After the name of each insect is given, in parentheses, its principal food.

\section{Animal Populations}

A great deal can be learned about the relations of organisms by quantitative methods making possible an estimate of the numbers of various species present in a given community at any one time. Comparisons may then be made between the populations of communities differing in some observable respect, or between the population of the same community at different times of the year. It is comparatively easy, although somewhat tedious, to determine the relative numbers of various plants in a given region by blocking off sample areas and counting the plants. Most animals, however, will not remain statiouary while a census is being taken, and various less exact methods of obtaining population data must be employed. Some of these methods may be suggested. The number of birds may be estimated by the number seen within a given area or during a certain time, or a census of nesting pairs may be taken by counting nests. Larger mammals may be counted by experienced observers, burrows of rodents may be counted (but it is also necessary to determine the average number occupying a burrow) or the relative frequency of tracks, fecal pellets, or other evidences of the presence of the animals may give an idea of their numbers. The relative number of rodents is often estimated by the frequency with which they are caught in traps. The most frequently used method of estimating the number of insects is the use of the insect net. A net is swept through vegetation a definite number of times and all insects caught are counted. While all such methods are necessarily inaccurate, a great deal of valuable information may be obtained.

A list of the ten most abundant groups of insects found in two adjacent areas, one heavily overgrazed and the other lightly grazed, is given in the accompanying table. The figures indicate their relative 

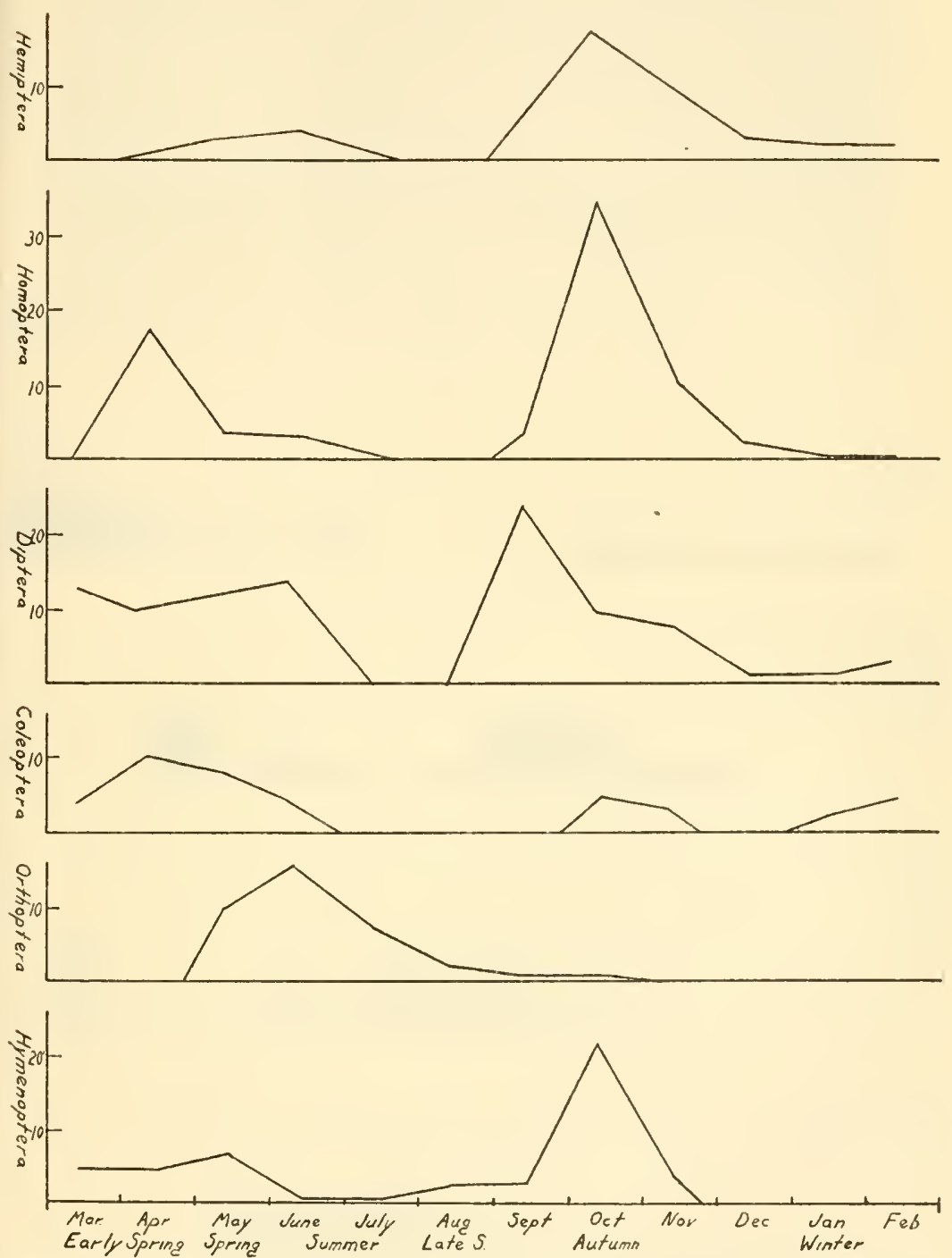

Fig. 381.-Numbers of insects collected in average catch with 100 sweeps of an insect sweep net in a prairie ravine (Oklahoma) arranged according to orders through the seasons of the year. (Data from Carpenter.) 


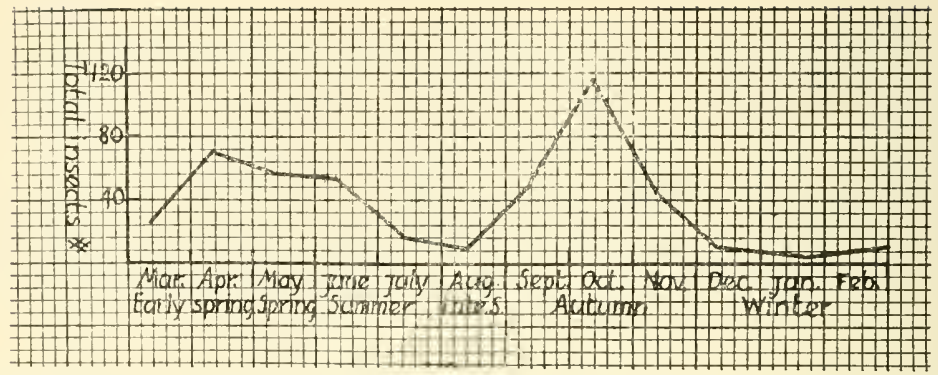

Fig. 382.- Total numbers of insects collected in average catch with 100 sweeps of insect net in prairie ravine (Oklahoma). (Data from Carpenter.) The scale for this graph is one-fourth of that in the preceding figure.

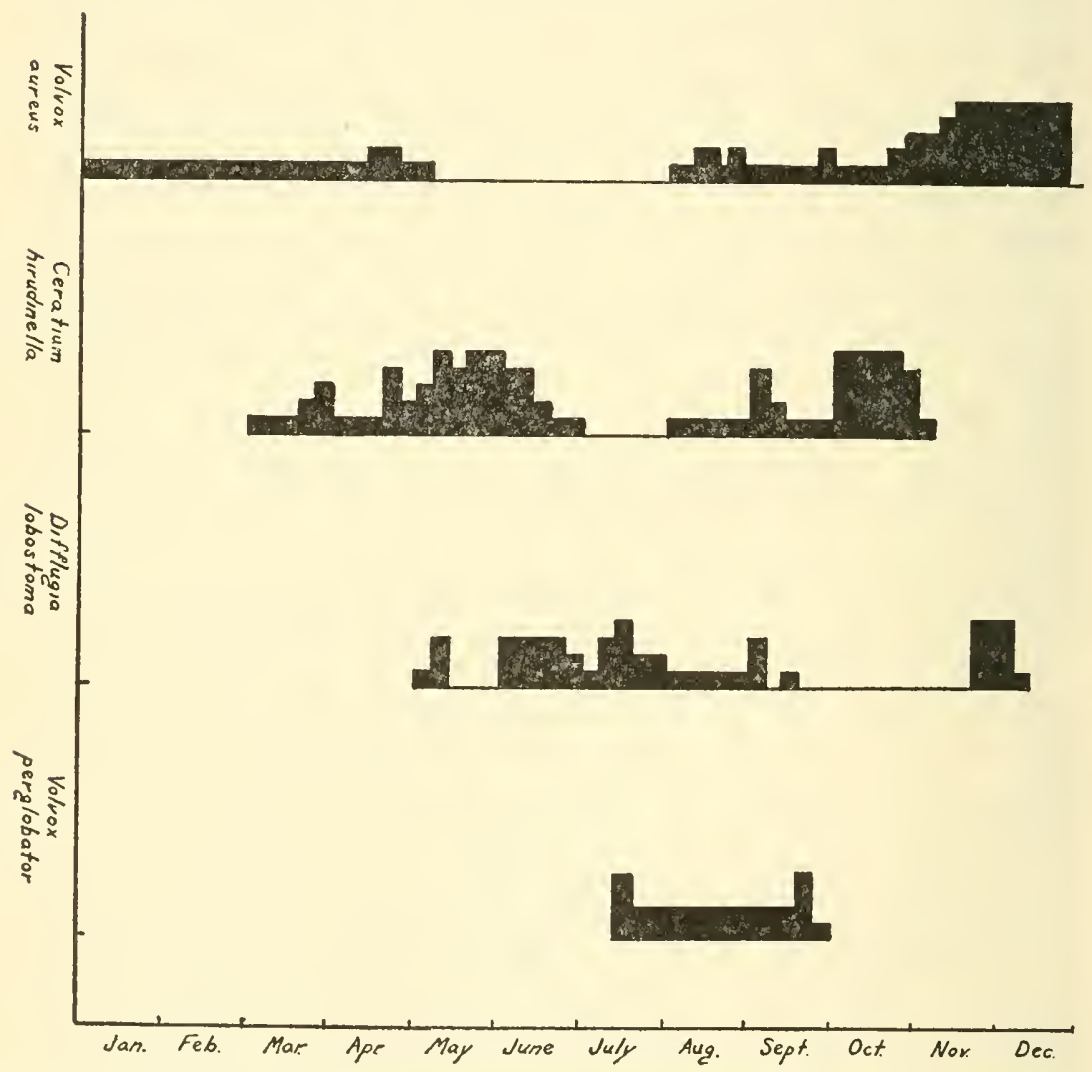

Fig. 383.-Fluctuations in populations of four Protozoa in an artificial lake (Oklahoma). (Data from Bragg.) 
Relative Numbers of Ten Most Abundant Insects From Overgrazed and Normal Grassland. (Wichita Mountains Wildlife Refuge.)

\begin{tabular}{llcc}
\hline \hline \multicolumn{1}{c}{ GENUS } & COMMON NAME & NORMAL & OVERGRAZED \\
\hline Melanoplus & (grasshopper) & 11.0 & 220.0 \\
Campylenchia & (tree hopper) & 0.5 & 144.0 \\
Mermiria & (grasshopper) & 14.0 & 138.0 \\
Scolops & (plant hopper) & 10.0 & 120.0 \\
Elleschus & (weevil) & 0.9 & 80.0 \\
Poeciloscytus & (leaf bug) & 1.2 & 54.0 \\
Agallia & (leaf hopper) & 8.0 & 38.0 \\
Harmostes & (plant bug) & 8.0 & 17.0 \\
Deltocephalus & (leaf hopper) & 0.5 & 3.4 \\
Bruchomorpha & (plant hopper) & 11.0 & 0.4 \\
\hline
\end{tabular}

abundance in ten collections of fifty sweeps of the insect net, each. These collections were taken over a period of a month and indicate a very real difference in the insect populations of the two areas.

\section{Seasonal Changes}

It is a matter of common observation that the animals observed in any one place vary greatly from season to season during the year. This is not only true of those animals which, like many birds, migrate southward on the approach of winter and return to northern climates for nesting. Some animals hibcruate or aestivate, and others spend a part of the year in an inactive stage, such as the egg, or the pupa. The life cycle of an organism must be adjusted to the annual climatic cycle of the climate in which it lives. Some idea of the variations in the number of insects during the year may be gained from a study of the accompanying charts (Figs. 381 and 382) giving the average catch with one hundred sweeps of an insect net at different seasons.

A study of the abundance of Protozoa in an artificial lake shows a similar difference in the time of abundance of the various species.

\section{Summary}

This chapter has considered very briefly the distribution of the biotic communities of North America in relation to climate. The phenomena of succession and seasonal fluctuation of populations have been discussed, with examples. Attention has been directed toward the community rather than toward the individual organism 
or the species. Similarly the sum total of physical environment as expressed in climate has been stressed rather than single factors, such as moisture, temperature, light, etc. The animal in nature is subject always to the action of a complex environment and its distribution and reactions are the result of its response to the whole. The student is referred to the chapter on Animal Behavior for a discussion of the response of the individual organism to modifications in single environmental factors. 


\title{
CHAPTER XXXVIII
}

\author{
ANIMAL PARASITISM \\ (By Sewell H. Hopeins, Texas A. and M. College)
}

\section{SOCIAL RELATIONS OF ANIMALS}

It has been explained in the previous chapter that no animal is ever entirely independent of others, since all plants and animals are influenced, directly or indirectly, by all the other organisms in the community. Most animals, however, can and do catch and eat their own food, and such animals are said to be "free-living." But there are thousands of species which depend either completely or partially on others to provide them with a livelihood. The varying degrees of dependence are called commensalism, mutualism, and parasitism. The term symbiosis is applied to all cases of two different kinds of animals living together, and thus includes commensalism, mutualism and parasitism.

In commensalism, one animal receives all of the benefit from the association while the other is neither benefited nor harmed. The jackal which follows the tiger and cleans up the carcass of the prey when the tiger has eaten his fill, the small fishes which accompany sharks and feed on the scraps wasted by the shark in feeding, and the oyster crab which lives inside the oyster's shell and feeds on the organisms brought in by the oyster's feeding movements, are examples of commensalism.

Mutualism is the kind of symbiosis in which both animals receive benefit from their association. One species of hydra (Hydra viridis) is green in color because a certain species of green alga lives within its cells; the alga receives protection and some nourishment from its host, while the hydra benefits from the food manufactured by the green plant. A case of mutualism so far developed that the two animals cannot live separately is the relationship between wood-eating termites and their intestinal protozoa. The termite cannot digest the wood which it eats; the protozoa in the termite's intestine break down the wood into a form in which it can be used 
by the host; on the other hand, the protozoa are absolutely dependent on the termite for food and the proper environment; neither termite nor protozoan can live without the other partner.

The word parasitism in its broad sense applies to all cases in which one animal depends on another to furnish it with food; for instance, ornithologists call cowbirds parasites because they lay their eggs in the nests of other birds and leave the foster-parents to feed and eare for the young cowbirds. Most zoologists, however, use the word parasitism only for cases in which the parasite lives in or on the body of its host; for example lice live on the bodies of many animals, and tapeworms live in them.

\section{Origin of Parasitism}

How did it happen that some animals became dependent on others to furnish their food, that is, how did parasitism arise? There is a considerable amount of evidence for the belief that all parasites are descendants of free-living ancestors, and that these descendants, in the course of generations, gradually became more and more dependent on certain hosts, until in some cases they are now absolutely unable to make their own living. For example, certain species of nematodes which are free-living inhabitants of the mud at the bottom of ponds and streams are able to live in the large intestine of a frog if they happen to be swallowed by a frog. Other species, very similar to the mud-dwelling nematodes, have found the intestines of frogs such a good habitat that they live nowhere else; in other words, they have become parasites. Some intestinal parasites, in the course of many generations, have lost their locomotor structures or even their digestive organs and yet continue to thrive because there is little or no need for locomotion or digestion when all food is brought to the parasite already digested by the host's intestine. Since such degenerate parasites are unable to secure food elsewhere, they are condemned by their peculiar structure to live as parasites in the intestine of their host.

\section{Degrees of Parasitism}

Free-living animals which sometimes become parasites when they get into another animal (by swallowing, for instance) are called accidental or occasional parasites, as in the ease of the mud-dwelling 
nematodes mentioned above. "Vinegar eels," nematodes in vinegar, sometimes establish themselves as harmless parasites in the human urinary bladder. Facultative parasites are able to live almost equally well as free-living animals or as parasites; many leeches are facultative parasites. Obligate parasites, on the other hand, cannot live without the host. Parasites which are free-living during part of the life cycle, as in the case of the horsehair worms and some ticks and mites, are called temporary parasites, while animals like Acanthocephala and tapeworms which are parasitic during the entire life cycle are called permanent parasites.

\section{The Successful Parasite}

Like all other ways of living, successful existence as a parasite requires certain modifications or adaptations in structure and function. Parasites which live on the outside of the host's body are called ectoparasites; they must have special organs for attachment in order to maintain their hold on the host; for example, lice have hooklike feet with which they hold on to the skin, hair, or feathers of the host, and ectoparasitic trematodes have either muscular suckers or chitinous hooks for attachment to the outside skin or to the gills of the fishes on which they live. On the other hand, ectoparasitic insects have no need for wings, so fleas and bedbugs continue to thrive without them. Nany ectoparasites, such as fleas, lice, bedbugs, mites, and ticks, also have specially constructed mouth parts for piercing their host's skin and sucking blood. Endoparasites, which live inside their hosts, also require special adaptations. For maintaining their positions in the intestine or other organs they must have some sort of attachment organ, such as the muscular suckers of trematodes and tapeworms and the hooks of thorny-headed worms. On the other hand, they live in the dark so eyes may be entirely lacking without inconveniencing the endoparasite; usually all sense organs are either absent or very poorly developed. There is little or no need for rapid locomotion, so most endoparasites have locomotor structures much reduced or even entirely lacking. Many endoparasites also have less of a digestive system than their free-living relatives; parasites in the liver, lungs, blood vessels, etc., usually have some sort of digestive apparatus, but many intestinal parasites, such as tapeworms and thorny-headed worms, have no sign of digestive organs whatever, but depend on 
the host to furnish them with food already digested and ready for absorption. On the other hand, most endoparasites have their reproductive organs enormously developed, sometimes so much so that 90 per cent of the body is taken up by the reproductive system. This is in keeping with the general rule that animals whose offspring

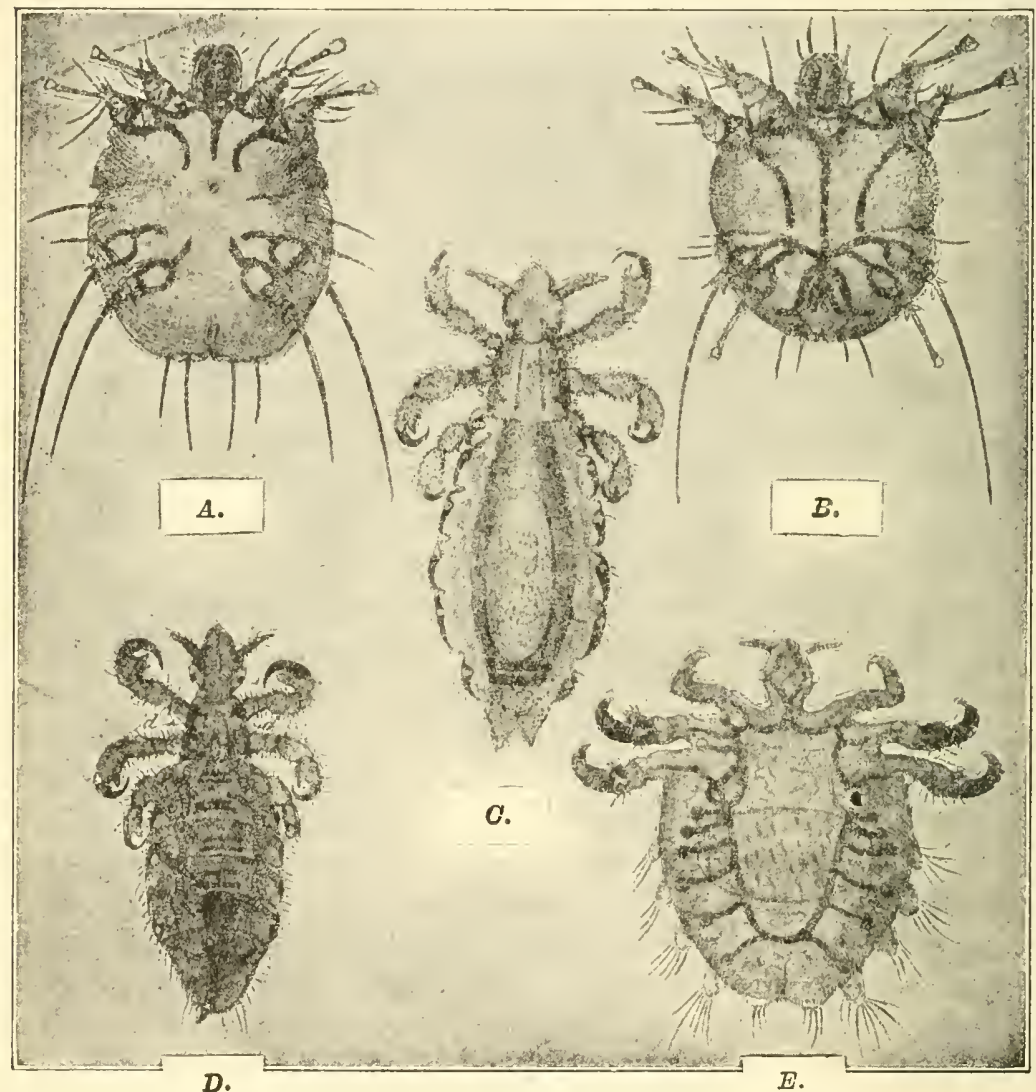

Fig. 384.-Arthropod parasites. A, human itch mite, female, Sarcoptes scabiei, ventral surface; $B$, ventral surface of male itch mite; $C$, body louse (cootie). Pediculus humanus corporis; $D$, head louse, $P$, humanus capitis; $E$, crab louse. Phthirius pubis. (From Sutton, Diseases of the Skin, published by The C. V. Mosby Conpany.)

have the least chance to survive usually produce the largest number of offspring. In the case of a tapeworm, for instance, the chance of any one egg being eaten by the right kind of host, so that it ean develop into another tapeworm, is only one in a million, and tape- 
worms would long ago have become extinct except for the fact that each tapeworm produces many millions of eggs. The peculiar habitat and mode of life of endoparasites also makes necessary peculiar adaptations in the functions or physiology of the parasite. A parasite in the intestine, for instance, must be able to carry on respiration in almost complete absence of oxygen, must secrete substances to counteract the digestive juices of the host in order to prevent its being digested, must be adapted to a high concentration of salts, acids, and other substances in solution in the fluid around it, and if in a warm-blooded animal must be able to live at a relatively high constant temperature. The fact that no host is immortal makes it necessary for a parasite to have some special provision for its offspring to escape to another host, in order to maintain the existence of the species; this necessity is met by various peculiar adaptations in the life cycle or development, such as the complex succession of larval stages in the endoparasitic trematodes and cestodes.

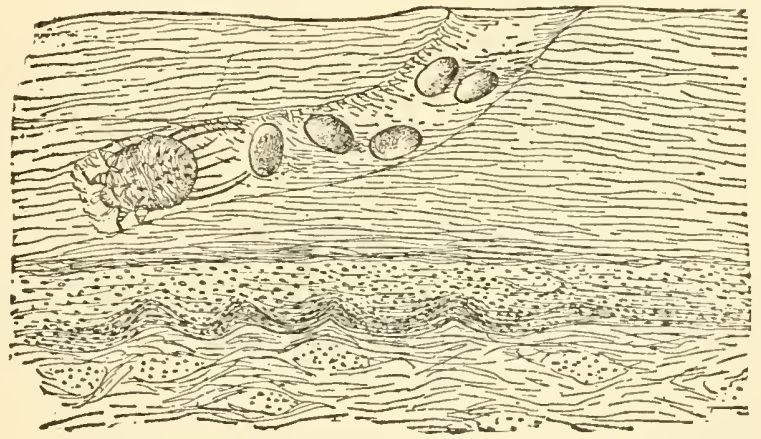

Fig. 385.-Diagram of the tunnel of an itch mite in human skin. The female animal is depositing eggs. (Reprinted by permission from Introduction to Human Parasitologu by Chandler, published by John Wiley and Sons, Inc. Adapted from Riley and Johannsen.)

Some parasites are able to carry on parasitic activities without injuring their hosts, while others may weaken or destroy the host. Parasites which injure their hosts are said to be pathogenic (diseaseproducing), while those which cause no appreciable injury are said to be nonpathogenic or commensal. Since most parasites cannot live without their hosts, a parasite which shortens the life of its host destroys its own home and means of livelihood; nonpathogenic parasites are more likely to be successful in the long run, and are there- 
fore more abundant. Some parasitologists consider pathogenic parasites to be imperfect parasites because they are not quite perfectly adapted for successful parasitic life, while nonpathogenic species are considered perfect parasites. However, no hard and fast line can be drawn between the two. Many parasites which are so perfectly adapted to their customary host that they produce no illeffects have been found to be strongly pathogenic to other hosts where the adaptation is less perfect; for example, certain trypanosomes which are harmless to the antelopes of Africa, their natural hosts, produce the highly fatal African sleeping sickness when injected into men.

\section{Means of Infection and Transmission}

Many different means of transfer from host to host have been developed by the various kinds of parasites. These may be classified as below:

A. Passive transmission.

1. In food or in water.

2. By bite of insects.

3. By sexual intercourse.

4. By direct contact.

B. Active invasion under own power.

By "passive transmission" is meant the transfer of eggs or larvae from one host to another without any aetion of their own. For example, the eggs of cestodes and of some nematodes, such as Ascaris, pass out of the host's intestine in the feces; if food of other animals is contaminated by these feces, animals which eat this food will swallow the eggs, which hatch into larval worms within the digestive system of the second host and thus establish a new infection. Sheep may become infected with liver flukes by eating the encysted larvae on grass or swallowing encysted larvae while drinking water. Trichina larvae encysted in hog meat develop into adult worms in the human intestines if infected pork is eaten raw or improperly cooked.

The second means of passive transmission is also widely used. Nalaria parasites, the trypanosomes which cause African sleeping sickness, and many parasites of domestic and wild animals are earried 
from infected individuals to new hosts by biting insects which suck up the parasites with the blood and inject it into the new host with their salivary secretions.

The third means is used by only a few parasites, most of them Protozoa. The spirochete which causes syphilis, a flagellate protozoan called Trichomonas vaginalis, and a trypanosome parasitic in the reproductive organs of horses are examples. Some nematode parasites of insects are also transmitted in this way.

The fourth means includes a few cases, such as the acquiring of dog tapeworm by people who kiss dogs and the transmission of pinworm from the hands of infected people who do not have clean habits.

Hookworms and the human blood flukes, called schistosomes, are examples of parasites that invade new hosts under their own power. Their larvae are able to penetrate the skin.

\section{Parasitism and Host Specificity}

Since the beginning of the scientific study of parasitism, it has been recognized that different animals have different parasites; for instance, the parasites found in and on goats are nearly all different from those of man. Some of the early parasitologists leaped to the conclusion that each species had its own peculiar species of parasites found nowhere else, and carried this idea of species specificity so far that they considered presence in different hosts to be sufficient evidence of specific difference of the parasites.

Modern knowledge reveals that while some parasites are actually speeies specific others have a wide range of hosts. Thus the beef tapeworm, Taenia saginata, is found in the adult stage in man only, but the fish tapeworm, Diphyllobothrium latum, seems to be able to live in nearly all mammals which eat fish.

Three main factors determine whether a parasite will infect any given host: (1) opportunity for infection of host, determined by habits or mode of life of parasite and host (malaria parasites may' be injected into any land animal by bite of mosquito, but strictly aquatic animals, such as fish, would not be bitten); (2) the environmental condition of the habitat furnished by the body of the host, involving such factors as body temperature, nature of outside sur- 
face, size, chemical content of internal organs, etc. (intestinal parasites of birds are seldom found in mammals, which have lower body temperatures, and parasites adapted to the oxygen-rich interior of a frog's lung can find no suitable habitat in a lungless fish); and (3) ability of the parasite to adapt itself to the wide range of environmental conditions found in different hosts; thus $D$. latum, though it finds its optimum conditions in the human intestine, is adaptable enough to survive under the very different ehemical conditions found in the dog, while Taenia saginata is usually unable to survive under these conditions.

\section{PARASITES AND THE GROUPS IN THE ANIMAL KINGDOM}

What kinds of animals are infested by parasites? Not only all phyla and classes, but all known species and probably all individuals of higher forms serve as lrosts for some kind of parasite. Even in

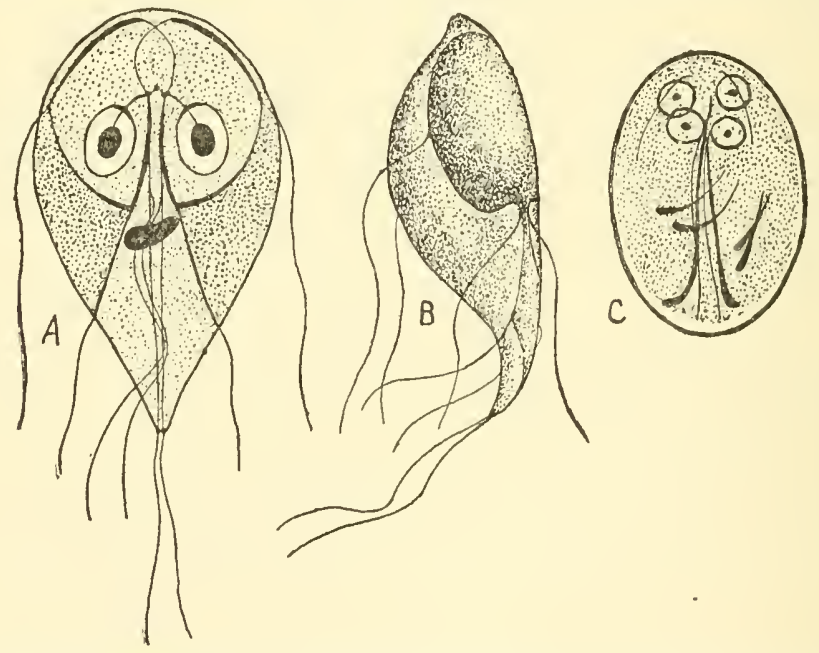

Fig. 386.-Giardia lamblia, an intestinal flagellate. $A$, face view; $B$, semiprofle view ; $C$, cyst. (Reprinted by permission from Introduction to Human Parasitology by Chandler, published by John Wiley and Sons, Inc.)

the microscopic Protozoa many individuals harbor still smaller protozoans. For example, several species of parasitic Protozoa are found in Amoeba proteus.

To which of the main groups of animals do parasites belong? All animal phyla, except Echinodermata, include some species which 
live as parasites, but the great majority of parasites belong to one of these four phyla: Protozoa, Platyhelminthes, Nemathelminthes, and Arthropoda.

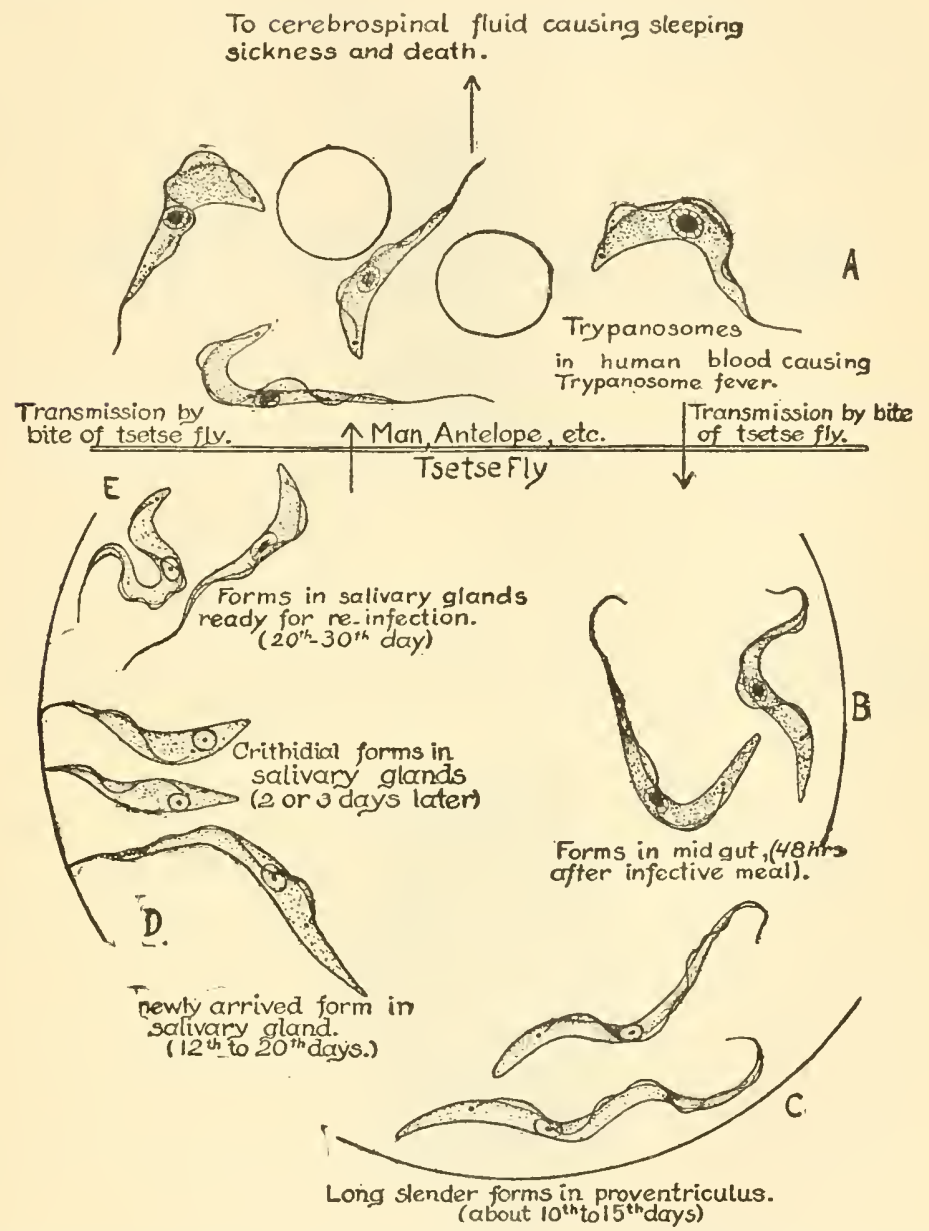

Fig. 387.-Life history of Trypanosoma gambiense. (Reprinted by permission from Introduction to Human Parasitology by Chandler, published by John WViley and Sons, Inc.)

Protozoa.-Of the four classes in this phylum, one, Sporozoa, is entirely parasitic; the other three (Sarcodina, Mastigophora, Infusoria) also contain a number of parasitic forms. Examples of parasitic Sarcodina are the three common human amoebae, Enda- 
moeba histolytica (Fig. 391), which invades and destroys the intestinal lining, thus causing amoebic dysentery; Endamoeba coli, a harmless

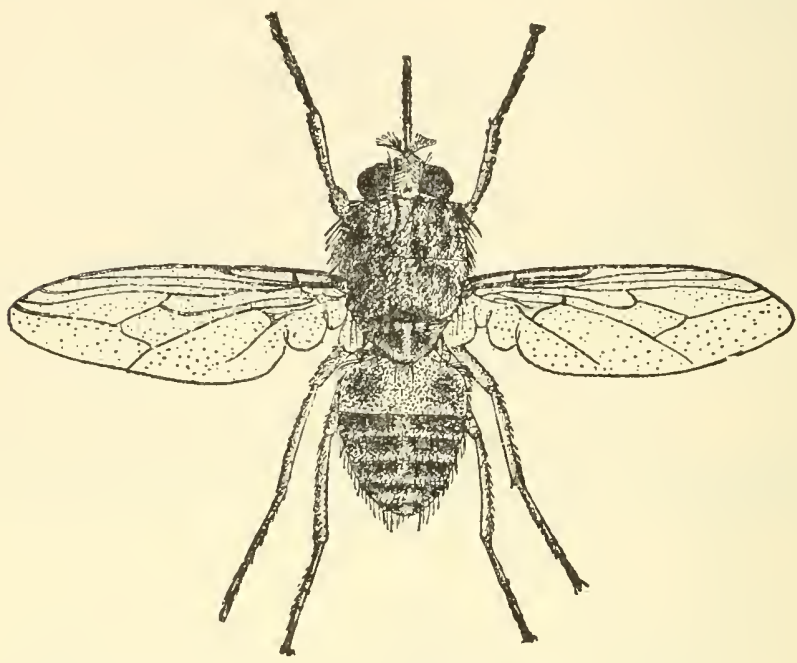

Fig. 388.-Tsetse fly, Glossina, the transmitting agent for trypanosoma, which causes African sleeping sickness. (Reprinted by permission from Introduction to Human Parasitology by Chandler, published by John Wiley and Sons, Inc.)

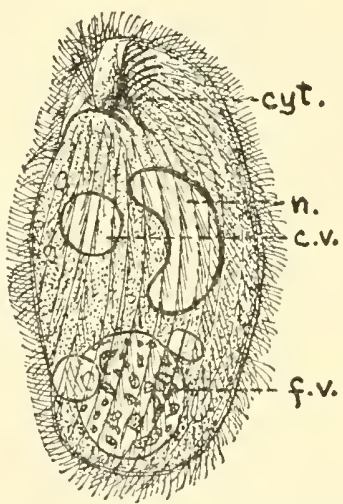

Fig. 389.-Brantidium coli, an infusorian parasite of the intestine. Active form from intestine. c.v., anterior contractile vacuole; cyt., cytostome; f.v. food vacuole; $n$, nucleus. (Reprinted by permission from Introduction to Human Parasitology by Chandler, published by John Wiley and Sons, Inc.)

commensal in the intestine; and Endamoeba gingivalis, a very common parasite in the human mouth, usually harmless but sometimes apparently injurious to the gums. Examples of parasitic Mastigophora 
are the human intestinal flagellate, Giardia lamblia (Fig. 386), and the blood-inhabiting trypanosome, Trypanosoma rhodesiense, eausative agent of African sleeping sickness which is carried by the Tsetse fly, Glossina (Fig. 3S8). Examples of parasitic Infusoria are the human intestinal ciliate, Bulantidium coli (Fig. 389), the various species of Opalina, and related genera found in the excretory bladder or cloaca of frogs and toads. Of the thousands of species of Sporozoa, all of which are parasitic, probably the best known are the three species of the genus Plasmodium (Fig. 393), which eause human malaria, and Babesia bigemina, which produces Texas tick ferer of eattle.

Platyhelminthes.-This phylum also eontains four elasses, two of which, Trematoda (flukes) and Cestoda (tapeworms), are all parasitic, while the other two, Turbellaria and Nemertinea, are mainly freeliving but eontain some speeies whieh are parasitic on aquatie invertebrates. Among the best known examples of Trematodes are Fasciola hepatica (Figs. 398 and 399), the sheep liver fluke; Clonorchis sinensis (Fig. 397), the Chinese human liver fluke; and Schistosoma haematobium, one of the three speeies of human blood flukes. Probably the best known tapeworms are Taenia saginata (Fig. 402), the beef tapeworm, Taenia solium, the pork tapeworm and Diphyllobothrium latum, the broad fish tapeworm, all three common parasites of the human intestine, and Echinococcus granulosus, a dog and wolf tapeworm whose larval stage is the cause of a horrible human disease.

Nemathelminthes.--The single elass Nematoda includes at least 95 per eent of the speeies in this phylum; most of them are freeliving, but there are also thousands of parasitie species. Examples of parasitic speeies are the human hookworms, Necator anericamus, the American hookworm, and Ancylostoma duodenale (Figs. 394 and 395), the Old World hookworm; Ascaris lumbricoides (Fig. 90), the large intestinal roundworm of hog and man; Dracunculus medinensis, the Guinea worm, often over a yard long, whieh crawls around under the human skin (believed by some to be the "fiery serpent" mentioned in Exodus); Trichinella spiralis, which eauses the often fatal human disease, trichinosis, when its larvae, eneysted in pork, are eaten by man; and Wuchereria bancrofti, the filaria which is injeeted into the human blood by eertain tropical mosquitos and eauses elephantiasis, a disease in whieh the infeeted limbs may become larger than the body of the vietim. 
Onchocerca volvulus, transmitted by certain biting flies, is a common cause of blindness in some parts of Mexico. Besides the human nematodes there are thousands of others parasitizing lower animals, both vertebrates and invertebrates. The other two classes of the phylum Nemathelminthes are entirely parasitic; the Acanthocephala, or thorny-headed worms, are common intestinal parasites of many vertebrates, including the hog and occasionally man;
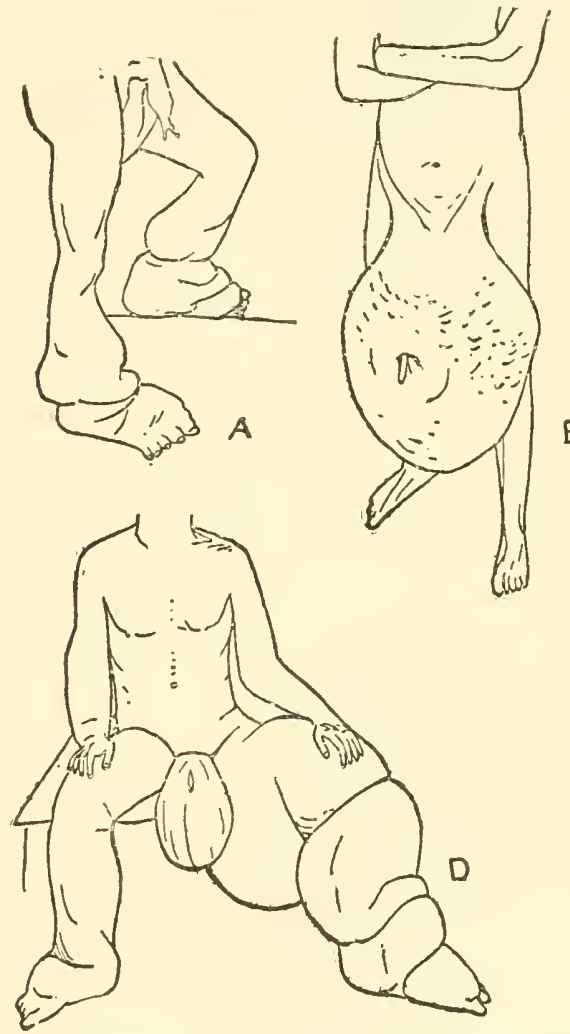

B
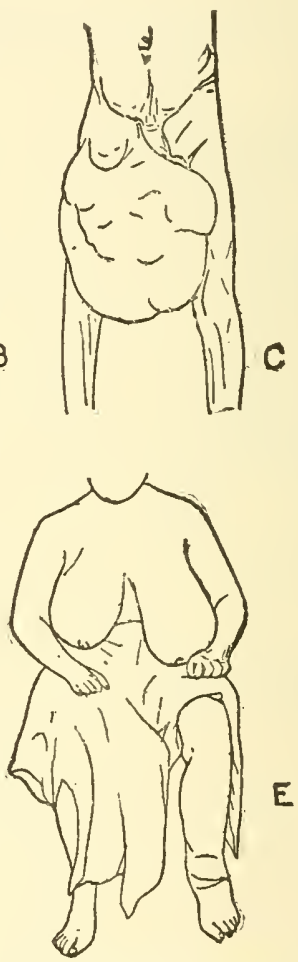

Fig. 390.-Elephantiasis, some extreme cases. $A$, of legs and feet; $B$, of scrotum; $C$, varicose groin gland: $D$, of scrotum and legs ; $E$, of mammary glands. (Reprinted by permission from Introduction to Human Parasitology by Chandler. published by John Wiley and Sons, Inc. $A$ and $B$ sketched from photograps from Castellani and Chalmers; $C, D$, and $E$ from Manson.)

the Gordiacea or horsehair worms (Fig. SS) are parasites of insects until nearly mature; they erawl out of their insect hosts when the latter fall into water, become sexually mature, and lay their eggs.

Arthropoda.-All of the classes in this phylum are predominantly free-living, but several classes also include parasitic species. The 
class Hexapoda or Insecta contains, besides several hundreds of thousands of free-living insects, the parasitic fleas, lice, and bedbugs; the class Arachnida, characteristically free-living, contains the parasitic ticks and mites, and the class Crustacea, though mostly free-living, includes a number of species parasitic on fishes and other aquatic animals. While most of the parasitic arthropods are ectoparasites, there are also a few endoparasitic species. For example the horse bot, Gastrophilus, which is the larva of a fly, is parasitic in the stomach of horses; long wormlike arachnids known as Linguatulids or tongue worms are found in the intestines of some reptiles and mammals; and Sacculina (Fig. 404), a crustacean, parasitic on crabs and lobsters, sends rootlike outgrowths all through the body of its host, although the saclike body remains on the outside.

\section{Some Representative Parasites}

Protozoa.-The very small amoebalike protozoans of the genus Endamoeba are examples of parasites only slightly modified for parasitic life. There are two distinct stages in the life cycle, the

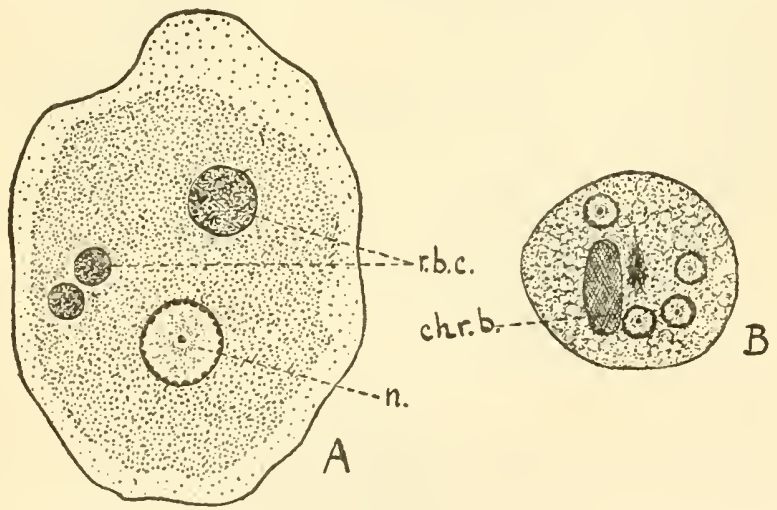

Fig. 391.-Amoeba histolytica, one of the important protozoan parasites. It is the causal agent of amoebic dysentery. $A$, Stained vegetative amoeba; $B$, cyst with four nuclei; $n$, nucleus, showing peripheral chromatin granules and central karyosome; r.b.c., ingested red blood corpuscles; chr.b., chromatoid body. (Reprinted by permission from Introduction to Human Parasitology by Chandler, published by John Wiley and Sons, Inc., after Dobell.)

active form being much like a small amoeba except that the pseudopodia are shorter and more more slowly; these active forms finally round up and become surrounded by a semirigid, resistant cyst wall. In this encysted condition Endamoeba is passed from the host with the feces or other body excrements. While in the encysted con- 
dition the parasite divides by binary fission into first two, then four, and finally (in $E$. coli) eight little amoebae. If the cyst is swallowed by another host, the eyst wall is dissolved and the four or eight young amoebae come out to begin the active stage again. The common Endamoebae of man are $E$. gingivalis which lives in the mouth and is usually transmitted by kissing, and the two intestinal species $E$. coli (nompathogenic) and E. histolytica. The latter species breaks down the cells of the intestinal lining by means of enzymes which it secretes, and then ingests the broken cells in the same way that the common free-living amoebae take in their food. The disease caused by $E$. histolytica is known as "amoebic dysentery." Infection occurs as the result of eating food or drinking water which has been contaminated by the feces of infected people, as in the case of the

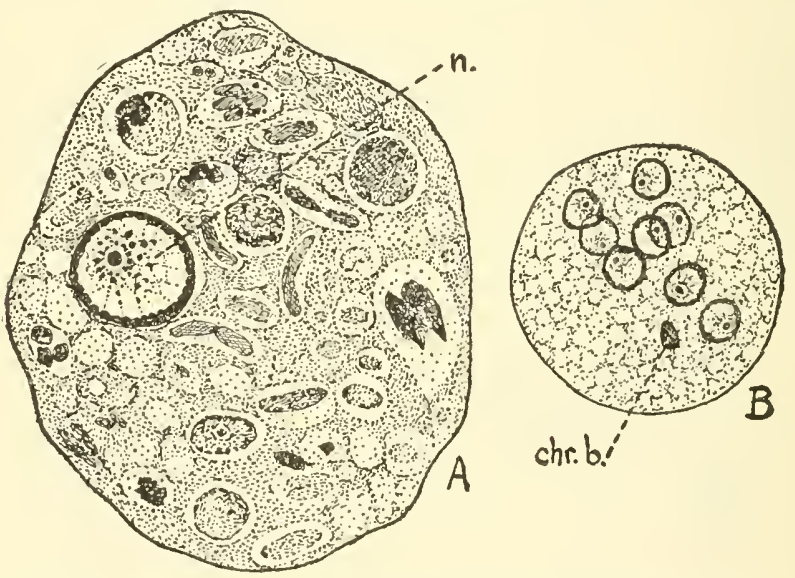

Fig. 392.-Endamoeba coli. A, stained vegetative amoeba: $B$, cyst with eight nuclei, n., nucleus, showing coarse peripheral chromatin granules, chromatin granules in "clear zone" between periphery and karyosome which is eccentric in position; chr.b., remnant of chromatoid body. Numerous food vacuoles in vegetative form. (Reprinted by permission from Introduction to Human Parasitology by Chandler, published by Joln Wiley and Sons, Inc., after Dobell.)

Chicago hotels where contamination of drinking water by water siphoned up from the toilet drains into the water pipes caused a serious outbreak in 1933.

The malaria parasites, of which there are three species infecting man (Plasmodium vivax, $P$. falciparum, and $P$. malariae, each causing a different form of malaria), are Protozoa belonging to the class Sporozoa, and are very highly modified for parasitic life. The adaptations for parasitism and for transmission from host to host involve a very complex life cycle. The two main phases of the life 


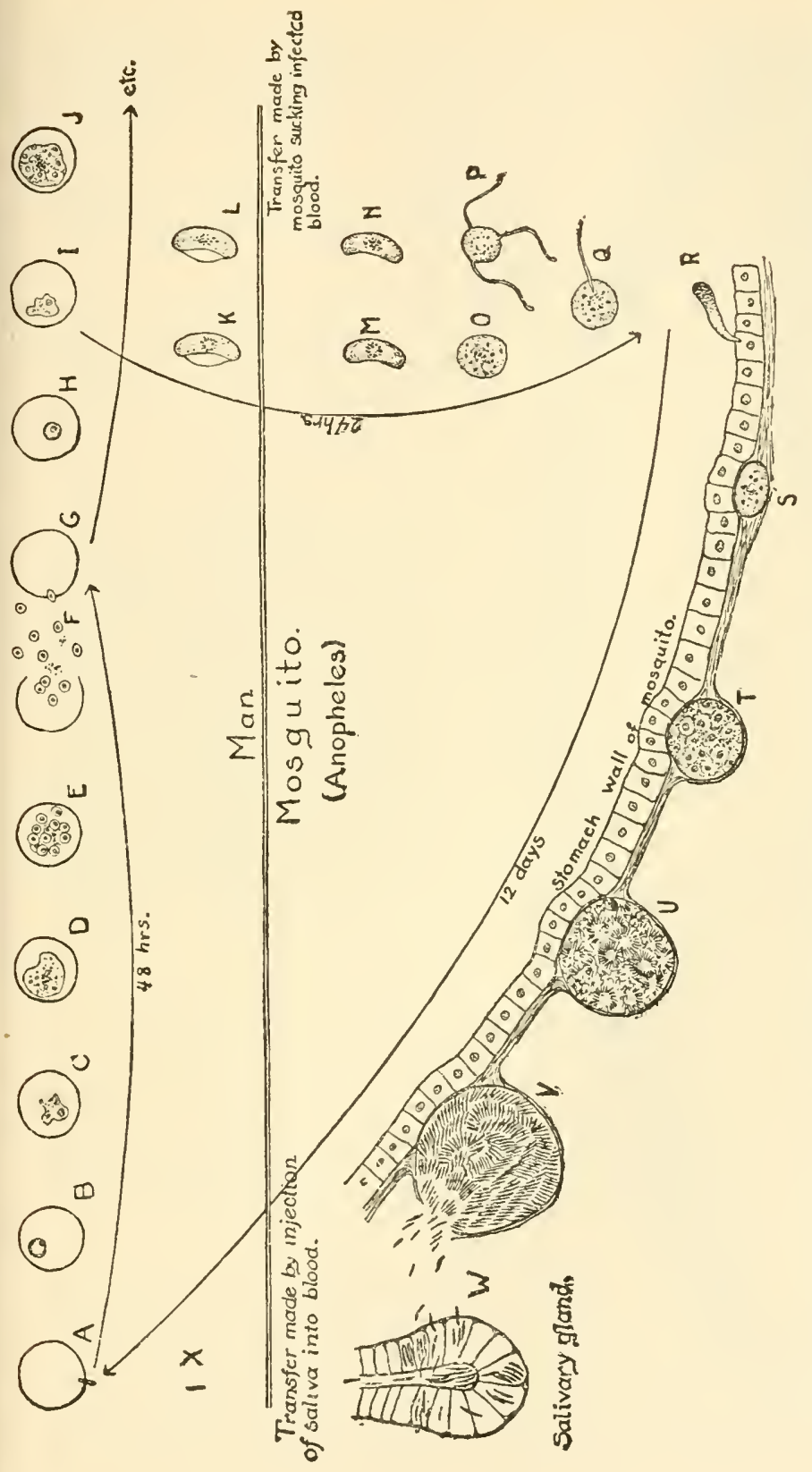

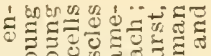
잉.

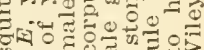
$0 . \cdots$ 的 $\S$ \$ 4 군 万o

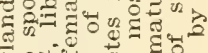

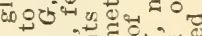

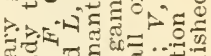
ॠ..

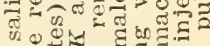

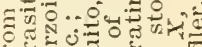
ज政政 证 U.

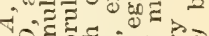

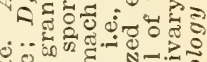

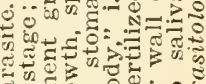
$\approx-0.00 \%$ \%

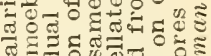

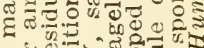

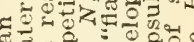
of N. 政

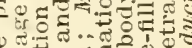
w F

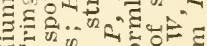

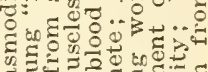

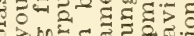

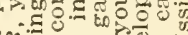
की >0 कु

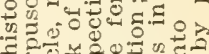

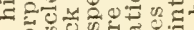
0 ज证考 100 6. - $20 .$.

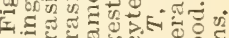

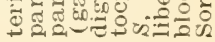


cycle are the vegetative or schizont stage (merozoites) and the sexually reproductive or sporont stage. The biology of this parasite has been discussed in the earlier chapter on Protozoa under class Sporozoa and Economic Relations of Protozoa.

Nematodes.-Of the thousands of species of parasitic nematodes, space permits mention of only a few which are particularly important because of their danger to man.

Hookworms.-In the United States the most important human nematode parasite, from the public health viewpoint, is the American hookworm, Necator americanus. Although called the "American hookworm" this species probably came originally from Africa and was introduced into America by the negro slaves. The pioneer work on hookworm in the United States was done by Dr. Charles W. Stiles in 1901. Hookworms are slender threadlike nematodes about onehalf inch long; the females are tapered to a point at each end, while the slightly smaller males have on the posterior end a fanlike expansion, the copulatory bursa, with curved riblike supports. Both sexes have a large mouth containing hooklike chitinous teeth by means of which they tear holes in the walls of the intestine and start blood flowing from the wounds. A muscular esophagus leading back from the mouth cavity gradnally broadens into a large muscular bulb; by means of rhythmic contractions and expansions of the bulblike esophagus blood is drawn into the mouth and forced down into the straiglit intestine where some of it is digested and the rest passes on through and out of the anus near the posterior end. Because of the large number of worms present there is a serious loss of blood resulting in anemia and lack of energy; in children the growth is stunted or retarded by hookworms, and often there is also a lack of proper mental development. Individuals, very heavily infected during childhood and early youth, may fail to develop sexually. Treatment is fairly easy, hookworms being easily killed by doses of anthelmintics, such as carbon tetrachloride and hexylresorcinol (which are poisonous and should be taken only under doctor's supervision).

Each female hookworm produces 9,000 eggs per day; these eggs pass out with the feces of the host; if the infected person defecates on the ground, the eggs hatch and the larvae crawl around in the soil; there they develop into infective larvae which live for several months on the surface of the ground. If bare human skin comes in contact with these microseopic worms they bore through it to the 
Fig. 394.-Ancylostoma duodenale, female and male, with head of Necator americanus drawn to same scale. an., anus; $b$, bursa; b.c., buccal capsule; cem.gl., cement gland; cerv.gl., cervical gland; cerv.p., cervical papilla; cl., cloaca; c.sp., caudal spine: ex,d. so-called excretory duct: int. intestine: n.ceph.gl. nucleus of cephalic gland; $n . r$, nerve ring; oes., esophagus ; ov., ovary ; ovej, ovejector: $s p$, spicules: $t$ testes; ut., uterus; vag., vagina; v.s., vesicula seminalis. (After Looss from Chandler, Hookworm Disease. Reprinted by permission from Introduction to Human Parasitology by Chandler, published by John IViley and Sons, Inc.)

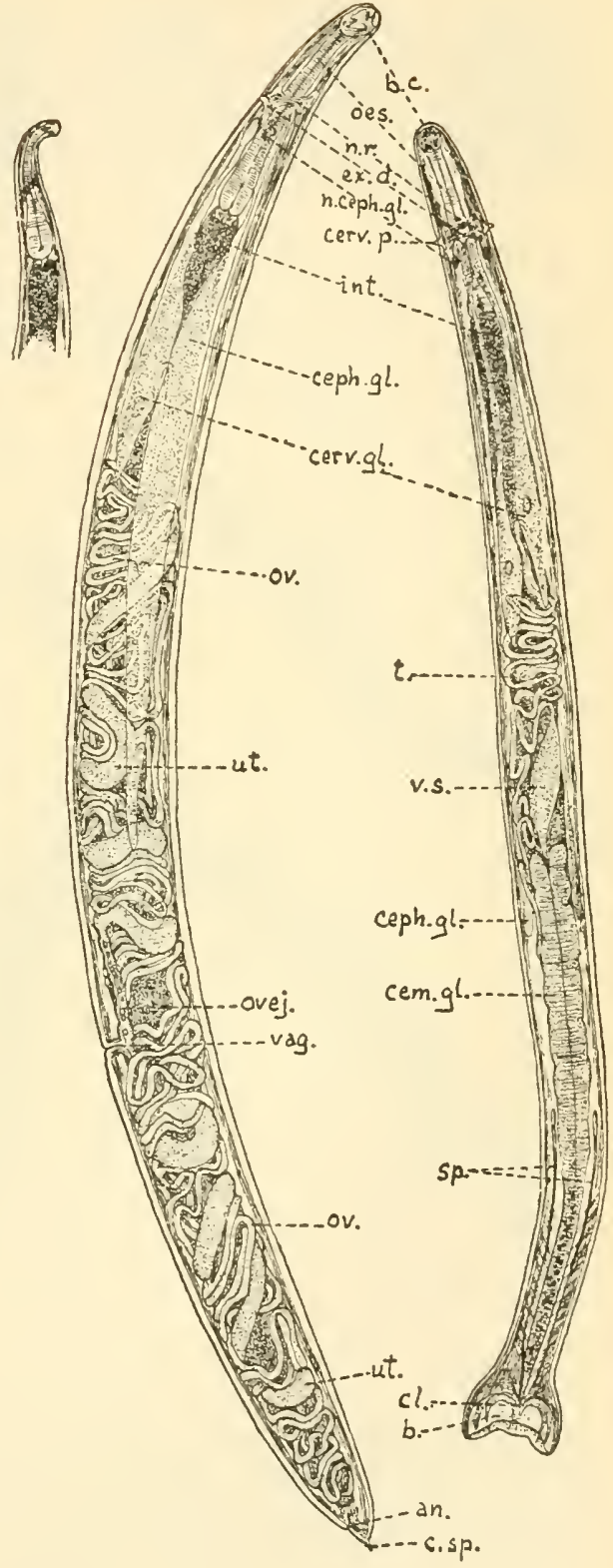



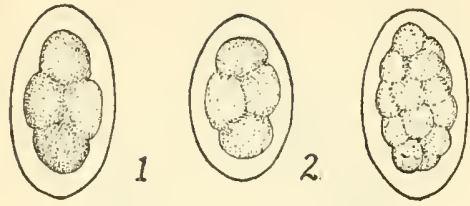

3
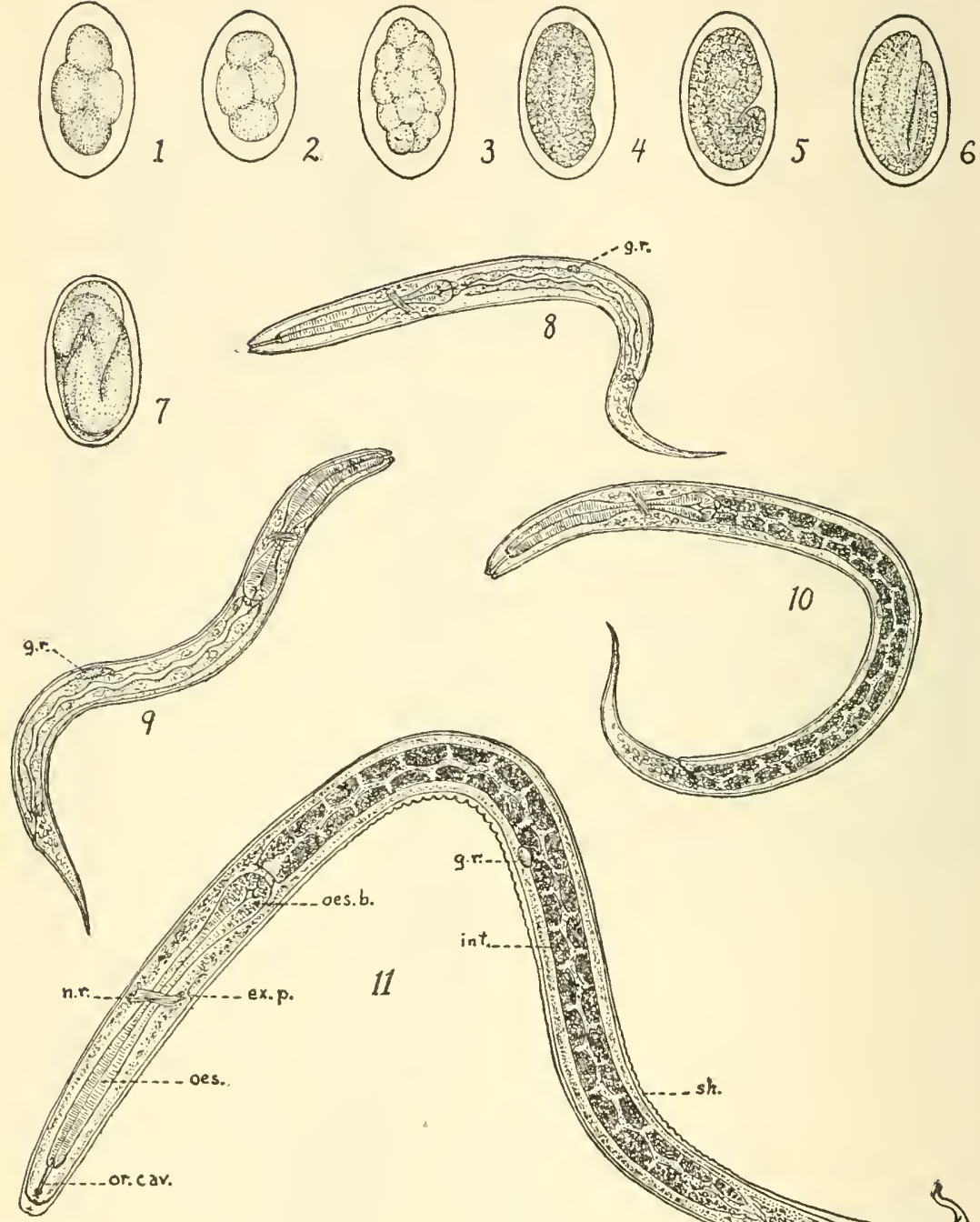

11

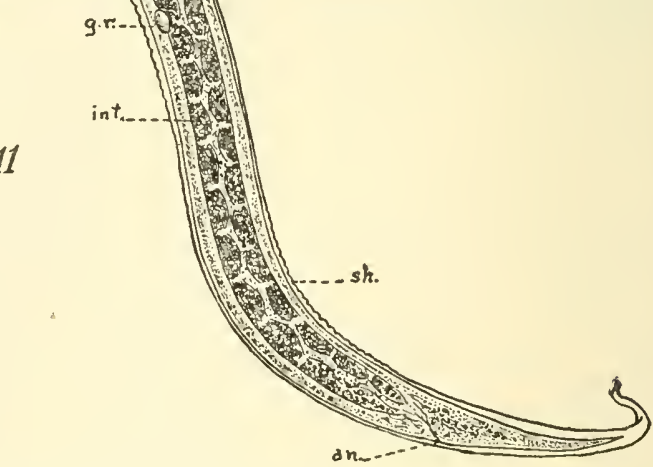

Fig. 395.-Life history of hookworms from egg to infective larva. 1, egg of Necator americanus at time of leaving host; 2, same of Ancylostoma duodenale; 3 to 7 , segmentation and development of embryo in egg; 8 , newly hatched embryo; 9 , same of Strongyloides for comparison (note length of oral cavity and size of genital rudiment, g.r.) ; 10 , second stage larva; 11 , fully developed larva ; an., anus; ex.p., excretory pore; g.r., genital rudiment; int., intestine; $n . r$. , nerve ring; oes., esophagus; ocs.b., esophageal bulb; or. cav., oral cavity; sh., sheath. (After Looss from Chandler, Hookworm Disease. Reprinted by permission from Introduction to Human Parasitology by Chandler, published by John Wiley and Sons, Inc.) 
blood vessels, are carried by the blood to the lungs, then migrate up to the trachea and pharynx, into the esophagus, then down through the esophagus and stomach to the small intestine; meanwhile they increase in size, so that on arrival in the small intestine they are ready to attach themselves to the wall of the intestine, feed on the blood of the host, and become adults.

Since the larval hookworms must go through part of their development in the soil, and a person can become infected only by direct contact with contaminated soil, the distribution of hookworm in the United States is determined by the following factors: (1) freezing of soil in winter (kills the larvae); (2) texture of soil (hookworm larvae live best in light, sandy loams); (3) moisture (hookworm larvae can live only in damp soil) ; (4) customs of the people in disposal of feces; the bad hookworm districts are sections in which sanitation is very primitive and sanitary toilets are not in universal use; deposit of feces on the ground is particularly conducive to spread of these animals.

From the public health standpoint hookworm disease is a social problem rather than a medical problem. Few if any people are killed by hookworm, and infected individuals are easily eured if they go to a physician for treatment. On the other hand, snch a large proportion of the population, in hookworm territory, are kept in bad health and a listless condition that the social welfare of the whole community is injured. Prevention of hookworm disease is theoretically easy; hookworms could be killed out of a community in a few months if everyone would defecate only in sanitary toilets, if everyone would take treatments for hookworms at the same time, or if everyone would wear good shoes; but so far, it has been impossible to get the cooperation of all the people in hookworm districts. The work of the medical profession, with the help of certain state agencies, has reduced hooliworm disease in the United States, but there are still considerable districts in which over 20 per cent of the populations are infected. In parts of East Texas 33 per cent of the people examined have hookworm, even now.

Trichina.-Trichinella spiralis, commonly known as Trichina, is an example of a nematode with an alternation of hosts and a passive means of transmission. The microscopic larvae are encysted in the muscles of various meat-eating animals, being particularly common in hogs and rats; within the cyst, the larva is coiled in a tight spiral, 
which gives the species its name. If pork containing trichina cysts is eaten by a man, the cysts are digested off in the stomach, the larvae become active and penetrate the mucosa of the small intestine to moult. They soon become full grown, sexually mature adults, sometimes beginning copulation only forty hours after being swallowed. The adult worms are short-lived and are not harmful to the host, but each fertile female, about a week or ten days after the infected meat was swallowed, begins to produce thousands of microscopic larvae which she deposits in the walls of the intestine, usually directly into

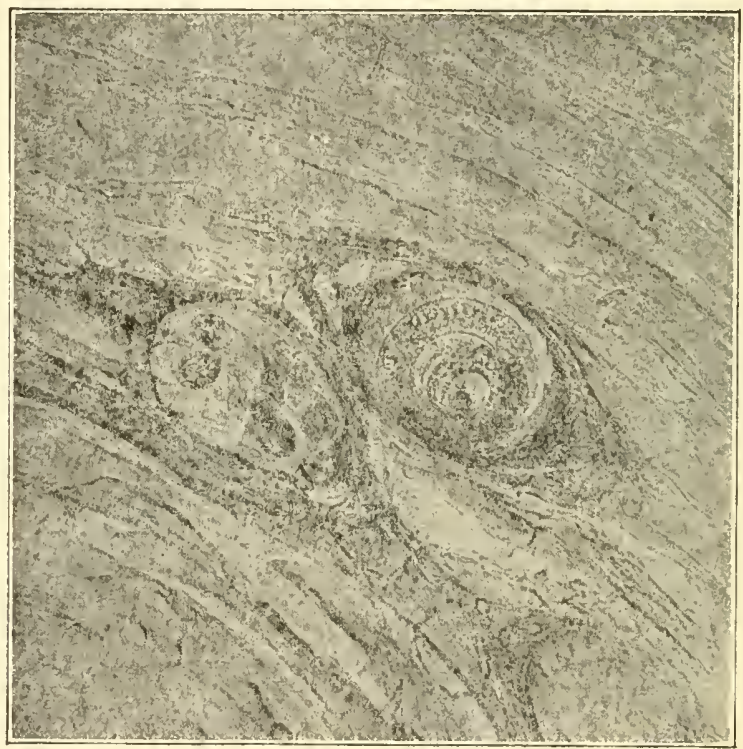

(c)

Fig. 396.-Larvae of Trichinella spiralis, encysted in voluntary muscle. The adults are parasites of the intestine. (Photomicrograph by Albert E. Galigher, Inc.)

a lymph vessel or blood vessel. The larvae are carried by the blood or lymph to the heart, and from there they are carried by the blood to all parts of the body. The larvae which reach voluntary muscle enter the muscle fibers and coil up into spirals, grow rapidly to a length of about $1 \mathrm{~mm}$., and in a few weeks become enclosed in a cyst of connective tissue which grows around them. Trichina larvae are likely to be most abundant in active muscles, such as the diaphragm, the intercostals, and the muscles of the larynx, tongue, and eye. The 
symptoms of trichinosis (the disease caused by trichina worms) vary according to the stage of the infection. In the first week, when the adult females are burrowing into the walls of the intestine to deposit their larvae, the symptoms are so much like those of typhoid fever that many cases are diagnosed as typhoid by good physicians. Nine or ten days after the beginning of infection, when the larvae are migrating to the muscles, there are severe muscular pains and aches (sometimes diagnosed as rheumatism), and the inflammation of the muscles used in breathing, chewing, etc., may interfere with these functions. As the parasites become encysted, about six weeks after infection, pains become worse and swelling of the infected parts occurs, accompanied by anemia and skin eruptions. If the victim survives this period he usually recovers, as the parasites are now walled off by cysts of connective tissue formed by the host; later calcium carbonate is deposited in these cysts, walling off the parasites so completely that they die, but the calcified cysts remain as hard grains in the muscles and may cause some rheumatic pains for years. Until the worms are completely walled off fever is caused by poisonous substances produced by the larval worms. Recent studies of the cadavers used in medical schools have revealed that about 20 per cent of the American population probably have cases of trichinosis in some degree at some time during life, since about this proportion of the cadavers had trichina cysts in the muscles.

All danger of trichinosis can be avoided by cooking pork thoroughly before eating it, as the larvae are killed by a temperature of $55^{\circ}$ C. $\left(131^{\circ}\right.$ F.). Investigations of the United States Bureau of Animal Industry, in which the author assisted, indicate that prepared sausages seldom contain living worms, most of them being killed by the salts and seasoning or by long-continued cold storage; the greatest danger is from fresh pork. Contrary to popular impression, Federal inspection does not guard against trichina, as there is no effective way to inspect meat for trichina on the large scale that would be necessary.

Trematodes._Schistosoma haematobium, a Human Blood Fluke.The blood flukes (Family Schistosomatidae) are distinguished from all other trematodes by having separate sexes. The male has a thick body with the lateral edges bent ventrally, thus forming a long groove on the ventral surface, the gynecophoric canal. The female is long and slender, almost threadlike in some species; when an adult male and 
female happen to come in contact, the male folds his body around the female so that she is held fast in the gynecophoric canal, and the pair begin copulation. After once becoming paired they remain in copula during the rest of their lives. Three species of the genus Schistosoma are human parasites: Schistosoma mansoni, found in Africa and the tropical parts of the New World, S. japonicum of Japan and China, and S. haematobium, the Egyptian blood fluke found in north Africa and southwestern Asia. Schistosoma haematobium is a parasite in the large blood ressels of the rectum and urinary bladder. The female lays an enormous number of eggs, which collect in the capillaries of the bladder and intestinal walls and block the flow of blood, causing the infected parts to become swollen and ulcerlike. Eventually the eggs are released into the Iumen of the rectum or bladder and pass out of the host's body in the feces and urine, along with considerable quantities of blood from the torn tissues. Aside from the pain caused by the egg-filled swellings, the loss of blood is the most serious effect of blood-fluke infection. Infected individuals are kept in a run-down anemic condition. The parasite is a serious public health problem in Egypt, where 80 per cent of the population of the Delta region is infected.

The egg must fall into water for the embryo to develop into a ciliated larva, the miracidium. When the miracidium is fully developed, the cap or operculum on the end of the egg shell is pushed open and the liberated miracidium begins to swim around in the water. Certain species of snails seem to emit a chemical which attracts schistosome miracidia; if a snail of the right species is in the vieinity, the miracidium swims to it and enters its body. If no suitable snail is available, the miracidium dies after swimming a few hours. After penetration into a snail the miracidium loses its cilia and develops into a long sausage-shaped sac, the sporocyst, without any recognizable organs. Germ cells within the sporocyst develop into a number of young sporocysts, which escape from the mother sporocyst into the tissues of the snail, grow to full size, and then in turn give birth to a new generation of sporocysts, or, under some conditions, these sporocysts may give lise to a different kind of larva, the cercaria, which has a long, forked, muscular tail, a pair of eyespots, two suckers (one anterior and one midventral), and a rudimentary digestive system. When fully developed, the cercaria forces its way out of the snail and begins to swim through the water. If unsuccess- 
ful in finding a host, the cercaria dies in a few hours, but if it comes in contact with the skin of a man, or any other mammal, the cercaria enters the skin, with the aid of glands in the head region which seem to be used in digesting or destroying the skin tissues. After penetrating the skin the cercaria soon finds its way into a blood vessel and begins a voyage through the circulatory systen, carried along by the current of the blood stream, meantime growing into an adult fluke. The mating of males and females usually occurs in the larger veins, and the pair mores to the reins in the walls of the rectum and bladder, where egg-laying begins.

Irrigated districts, such as the Nile Delta, are especially favorable for the development of blood flukes because the eggs have more chance of getting into water and because the field workers often get into the water while working around the irrigation ditches. The chances of infection are increased by the customs of defecating and

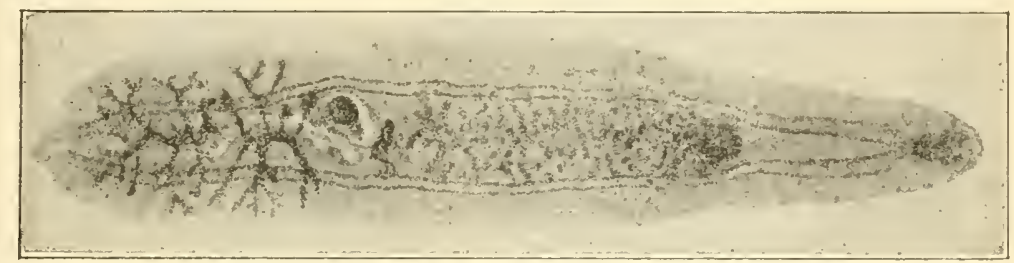

Fig. 397.-Clonorchis sinensis. Oriental human liver fluke, showing male and female reproductive organs. (Photomicrograph by Albert E. Galigher, Inc.)

urinating into the water, and using water from irrigation ditches for drinking and washing. In Japan the number of human blood fluke cases has been greatly decreased by improred sanitation and by killing the host snails. In Egypt, public clinies (by injecting fuadin into the blood) treat thousands of cases of this disease, but it will probably remain a public health problem for years because of the refusal of the Egyptian peasants to change their old customs.

Clonorchis sinensis, the Chinese Liver Flule.-This is an important human parasite in parts of the Orient. Clonorchis sinensis also occurs in other fish-eating mammals, including dogs, cats, and pigs. The adult worm lives in the bile passages of the liver. In man it often causes enlargement of the liver, diarrhea, jaundice, anemia, and extreme weakness, sometimes resulting in death. Hundreds of worms may be found in a badly infected man. The eggrs laid by the 


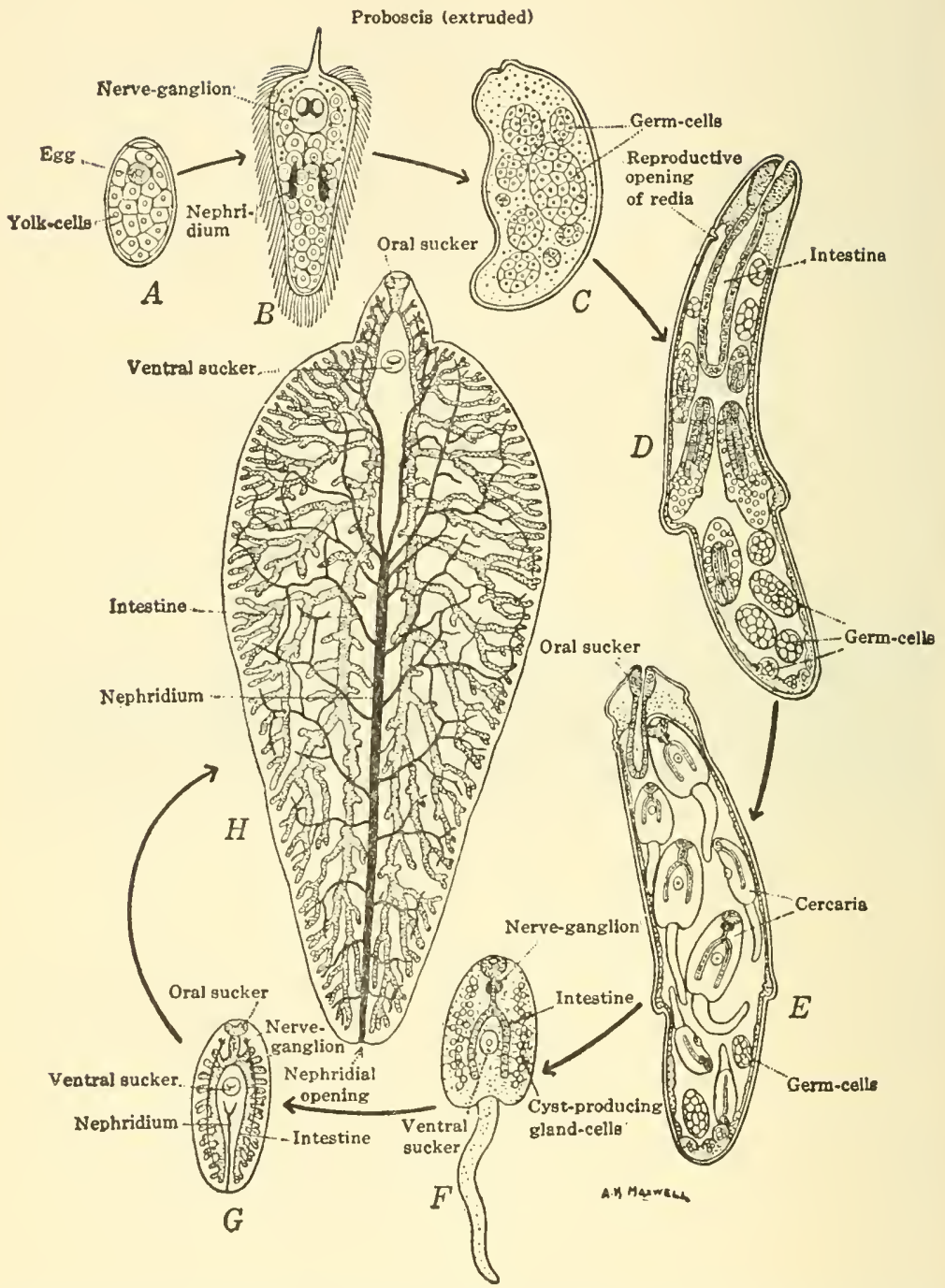

Fig. 398.-The liver fluke, Fasciola hepatica. A, egg; $B$, miracidium; $C$, sporocyst: $D$ and $E$. rediae: $F$, cercaria; $G$. tncysted stage; $H$, adult (showing diges tive and excretory systems). (From Hegner, College 'Zoology, published by The Macmillan Company.) 
adults pass from the liver to the intestine of the host by way of the bile duct, then pass from the body in the feces. Snails probably become infected by swallowing the eggs, while feeding on fecal matter in the water. After hatching, the miracidia migrate into the lymph spaces of the snail and develop into elongated sporocysts, each of which gives birth to a number of redia (differing from sporocysts by possessing a pharynx and a rudimentary gut). Each redia gives birth to six or eight cercariae, which emerge from the snail and swim around in the water by means of a very large, undivided tail. When a cercaria comes in contact with a fish, it enters the skin and encysts either in the skin or in the muscles just below the skin. It is now called a metacercaria or agamodistomum. Man becomes infected by eating these metacercariae in poorly cooked fish. When swallowed, the cysts are dissolved by digestive juices of the host, the larva escapes into the duodenum, migrates up the bile duct to the liver, and there develops into an adult. There is evidence that the adult Clonorchis may live as long as twenty ycars in the liver of man.

Treatment of clonorchiasis is not very satisfactory. Prevention is simple: avoid eating fish which are not thoroughly cooked.

It will be noted that the Clonorchis life cycle involves three hosts: a mammal as the final host, a snail as the first intermediate host, and a fish as the second intermediate host. Infection of the fish is by active invasion of the cercariae, and infection of the final host is passive.

Other Trematodes.-One of the best known parasites of domestic animals is the sheep liver fluke, Fasciola hepatica, which occurs in all sheep-raising countries in which wet pastures are common. It is also a common parasite of goats and cattle. Like Clonorchis, Fasciola lives in the bile passages, and its eggs pass out with the feces of the host, but unlike Clonorchis, the eggs hatch in water and the free-swimming miracidium actively seeks and penetrates the snail host. Sporocysts in the snail give rise to rediae which produce cercariae, but the cercariae encyst on any surface, including grass blades and even the surface film of the water. Sheep become infected by eating grass bearing encysted larvae or by swallowing floating cysts while drinking water. Fascioloides magna, the large liver fluke of cattle and sheep in Louisiana, Arkansas, and Texas, is very similar in structure and life history. 
Other important flukes are the human intestinal fluke, Frasciolopsis buskii, which is common among the Chinese, who become infected by eating the cysts on various aquatic food plants; the human lung fluke, Paragonimus westermanii of eastern Asia, where the natives become infected by eating the encysted larvae in fresh-water crabs

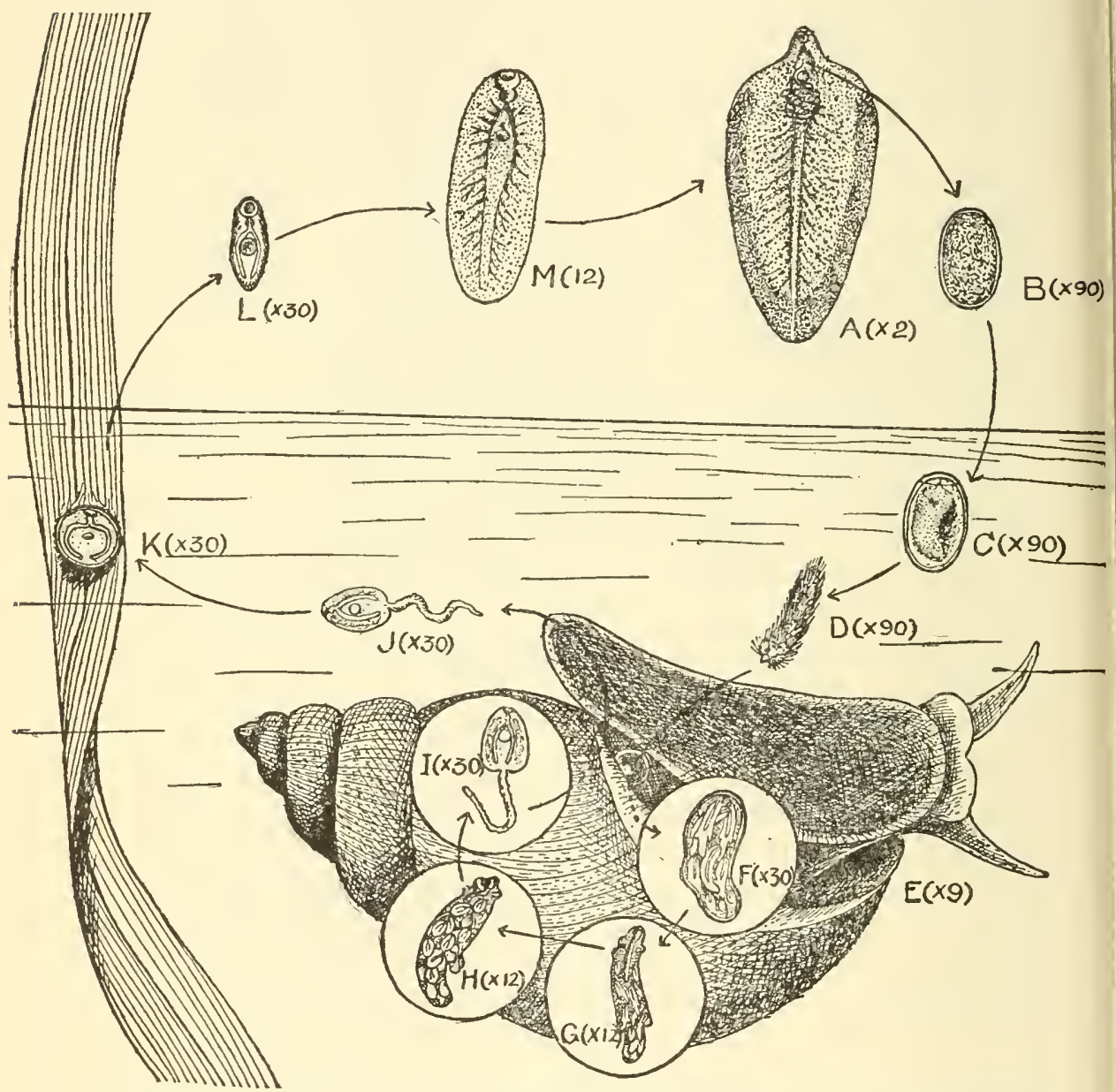

Fig. 399.-Life history of the liver fluke. Fasciola hepatica. A. adult in liver of sheep: $B$, freshly passed egg as it leaves the body of the host; $C$, developing embryo ready to hatch in the water: $D$, ciliated miracidiun emblyo in the water and about to enter the pulmonary chamber of snail; $E, F$, sporocyst containing rediae; $G$, redia containing daughter rediae; $H$, redia of the second generation containing cercaria; $J$, same having emerged from snail into water; $K$, cercaria encysted on blade of grass; $L$, cercaria liberated from cyst after ingestion by sheep ; $M$, young fluke developing in liver of sheep. (Reprinted by permission from Chandler, Introduction to Human Parasitology, published by John WViley \& Sons Inc.) 


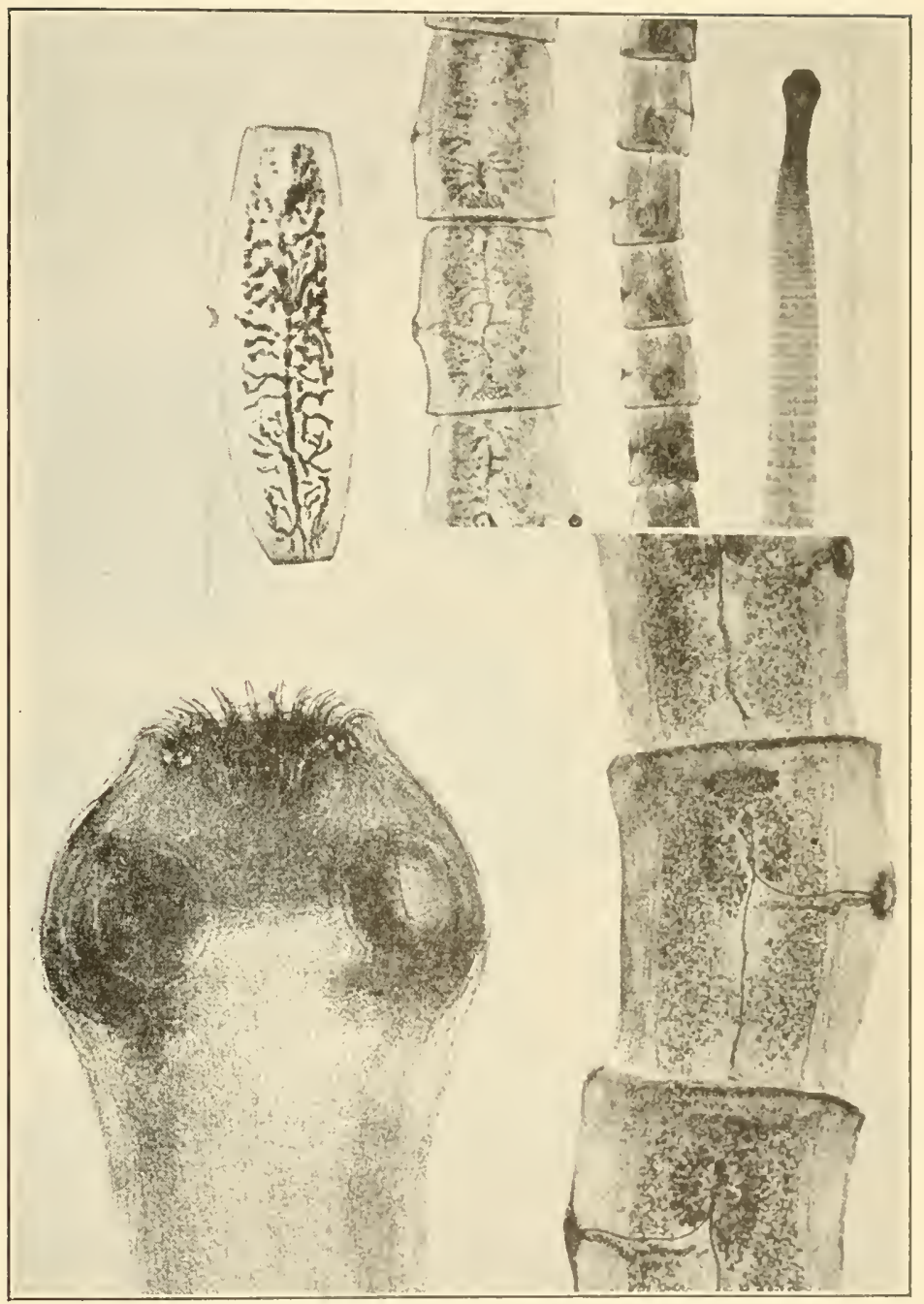

Fig. 400.-Structure of tapeworn to show different stages of maturity. At lower left, Taenia pisiformis, dog tapeworm, scolex with hooks and sucker dises. It lover right, mature proglottids. Above, scolex and proglottids of three ages. The reproduetive organs and pore are shown in most proglotticls. (Courtesy of' $\|^{\prime i l}$ 'd's Natural Science Establishment, Inc.) 
and crayfishes; Cotylophoron cotylophorum, a stomach parasite of cattle in Louisiana, and Dicrocoelium lanceatum, a common liver fluke of herbivorous mammals in Europe and Asia.

Numerous ectoparasitic trematodes, of the Subclass Monogenea, occur on the skin and gills of fish and are often of economic importance because they kill goldfish and other aquarium fishes, and also young fishes in state fish hatcheries.

A

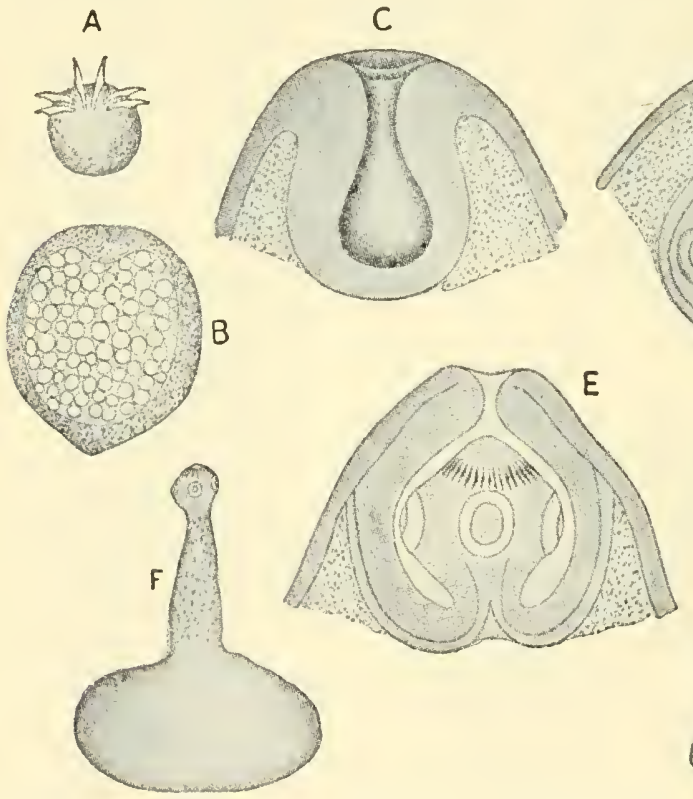

D

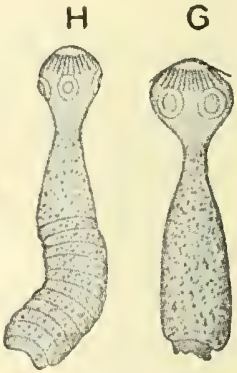

Fig. 401.-Development of tapeworm. $A$, six-hooked embryo ready to become embedded in muscle: $B$, cysticercus, or bladder worm as encysted; $C$, section through developin scolex in cysticercus; $D$, later stage: $E$, scolex everting as it protrudes from bladder: $F$, extension of scolex from bladder; $G$, later stage; $H$, formation of proglottids. (From Parker and Haswell, Zoology, published by 'The Macmillan Company, after Jijima and Hatschek.)

The Tapeworms (Class Cestoda).-Cestoda differ from Trematoda in the complete lack of a digestive system. In fact, Cestodes never have any sign of a digestive organ at any time during life; they receive their nourishment by absorbing through the surface of their bodies the food already digested for them by the host. Most cestodes also differ from trematodes by having the body divided into a series of segments, one behind the other, each segment having a complete set of reproductive organs. This structure characteristic of 
tapeworms is usually referred to as segmentation of the body, but it is probably more correct to consider a tapeworm as a linear colony, in which the segments are really individuals in various stages of maturity.

Taenia solium, the pork tapeworm of man, may be taken as an example to illustrate the structure and life history of a cestode. The adult tapeworm consists of a scolex or head provided with four muscular suckers and a snoutlike rostellum surrounded by a row of

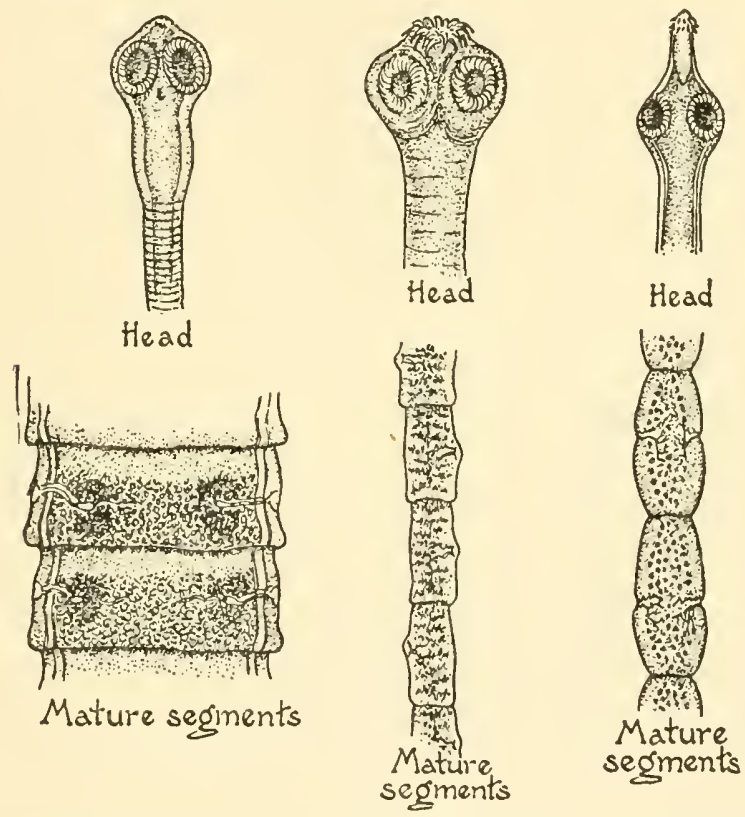

Fig. 402,-Common tapeworms, showing different regions of the body. At the left above, scolex of Taenia saginata, beef tapeworm : left below, proglottids of Moniezia, sheep tapeworm; middle, scolex and proglottids of Taenia solium, pork tapeworm; right, scolex and proglottids of Dipylidium caninum, a dog tapeworm. (Courtesy of General Biological Supply House.)

chitinous hooks, which serve as means of attachment to the wall of the human intestine; a narrow unsegmenfed neck behind the scolex, and then a series of several hundred proglottids (the segments) becoming progressively larger as they get farther from the scolex. The whole chain of proglottids is called the strobilus. New proglottids are constantly budded off from the neck; consequently, the youngest proglottid is the first one back of the neck and the oldest one is 
the one at the end of the strobilus farthest from the scolex. The youngest proglottids contain no recognizable structures, except the paired longitudinal nerve cords and longitudinal excretory vessels which run the full length of the strobilus, and a transverse excretory vessel in each proglottid. As the proglottids become older and are pushed farther away from the scolex, the reproductive organs begin to develop; each proglottid develops a complete set of both male and female reproductive organs; when these become mature and ready to function, the proglottid is a mature proglottid. Each mature proglottid is capable of copulating with itself, by bending the cirrus down into its own vagina, or it may copulate with another mature proglottid of another tapeworm if one is present close by. After copulation the male organs begin to degenerate, the uterus becomes filled with fertilized eggs and takes up more and more space, then the ovaries, vitellaria, and other female organs degenerate and leave the uterus to occupy nearly the whole proglottid; in this condition the proglottid, now hardly more than a sack of eggs, is called a gravid proglottid. The gravid proglottid at the extreme end of the strobilus breaks off and passes out of the host's intestine with the feces. It continues to live and erawl slowly through the feces like an independent animal for a few hours, then usually dies, but the embryos within the proglottid remain alive much longer. If gravid proglottids or separate eggs are eaten by a hog, the six-hooked hexacanth larva hatches in the hog's intestine, bores through the intestinal wall, and migrates to other parts of the body where it changes into a bladder-worm or cysticercus, which is a saclike larva with an inverted scolex. The cysticerci remain in the flesh of the hog until the pork is eaten by man; under the influence of human digestive juices the cysticerci become everted so that the scolex is on the outside of the saclike part, then the scolex attaches itsclf to the wall of the human intestine, proglottids begin to bud off from the neck, and an adult tapeworm is formed within a few weeks.

Taenia saginata, the beef tapeworm, has a similar life history, but uses cattle instead of logs as intermediate hosts. Taenia serrata, a common dog tapeworm often used as a laboratory specimen, is very similar to the two human species in structure and life cycle. 
Other important cestodes are Hymenolepis nana of man and mice; the broad fish tapeworm, Diphyllobothrium latum, which man gets by eating raw or poorly cooked fish; the peculiar Echinococcus granulosus, adult in dogs, whose cysticercus stages are dangerous parasites of man (man being the intermediate host in this case); and many tapeworms of domestic animals, such as Dipylidium caninum of dogs, Thysanosoma and Moniezia of sheep, goats, and cattle, and many others. 


\section{CHAPTER XXXIX}

\section{MARINE ZOOLOGY}

It is true that a good many people never have an opportunity to study the conditions present in the ocean or to observe the animals found in its waters and on its beaches. However, with the improved transportation and awakened interest in the subject, there are more and more students of this fascinating subject. Many of the animal forms seem peculiar and spectacular to those of us who reside inland. With the modern facilities for travel four hundred miles is not remote from the seashore, and every student majoring in biology in colleges or universities within that distance should be given marine experience, first hand.

The life of the ocean is known as halobios. Marine animals are affected and limited by many factors which in turn influence their distribution. They are affected by the temperature of the water, the height of the tides, the velocity of currents, salinity of the water, its turbidity, light, pressure, oxygen content, and the nature of the bottom and the shore. The occurrence of the proper food greatly affects the range and abundance of any particular species. Rocky shores harbor the choice food of certain species and muddy lagoons supply other groups. The adaptation and adjustment of the marine animal to the salinity of the water is one of the first considerations. The salt content is effective both quantitatively and qualitatively. If an oyster is taken from the ocean and placed in a fresh-water pond or stream, it absorbs water and swells up excessively. On the other hand, a clam taken from fresh water and placed in the ocean loses water and shrinks. In either case the effects will finally become lethal. A few animals, such as lamprey, eels, shad, salmon, and even gar pike and mullet are able to make the transfer from marine to fresh water and vice versa.

The salinity of the water of the marine habitat is an important and interesting feature to be studied. Along the Texas coast the water is either that of the Gulf of Mexico proper or of the various bays. The analyses* which have been made on the Gulf water out

\footnotetext{
*Reported by Mr. J. G. Burr, Texas Game, Fish, and Oyster Commission.
} 
from Matagorda and out from Port Aransas show a high degree of salinity. The readings range from 36.6 to 37.1 parts of salt per one thousand. The average given for the Atlantic Ocean is between 35 and 36 parts per one thousand. These readings on the Gulf are being checked by further data, but the cause of this difference has not becn explained.

The salinity of the bays is quite variable, and Galtsoff in 1926 made comparative studies of a number of them. It was found that in bays with a large fresh-water stream entering, as Nueces Bay,

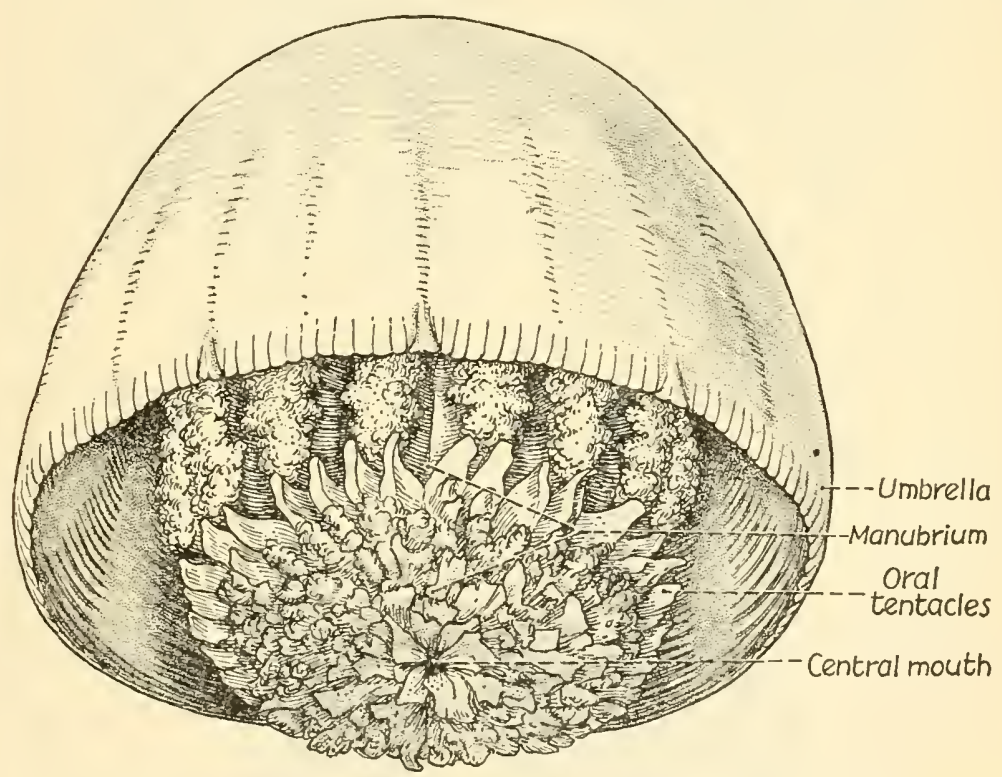

Fig. 403.-Cabbage-head jellyfish, Stomolophus meleagris, a very common form in the Gulf of Mexico.

the range is from 6.06 parts per thousand in June to 33.06 parts per thousand in September. In Mesquite Bay at Belden Dugout Beacon the range is from 5.03 parts per thousand in June to 18.44 parts per thousand in October. In Aransas Bay, where there is relatively small fresh-water intake, the salinity ranges on the average between 14.79 parts of salt per thousand in June and 25.47 parts per thousand in September.

These wide ranges of salinity in the bays create problems for the organisms attempting to live there. It requires a high degree of 
adaptation to salinity changes to be able to do it. Not only do floods coming in from streams bring serious disturbances, but the opposite effect may be brought about by storms rolling the waves
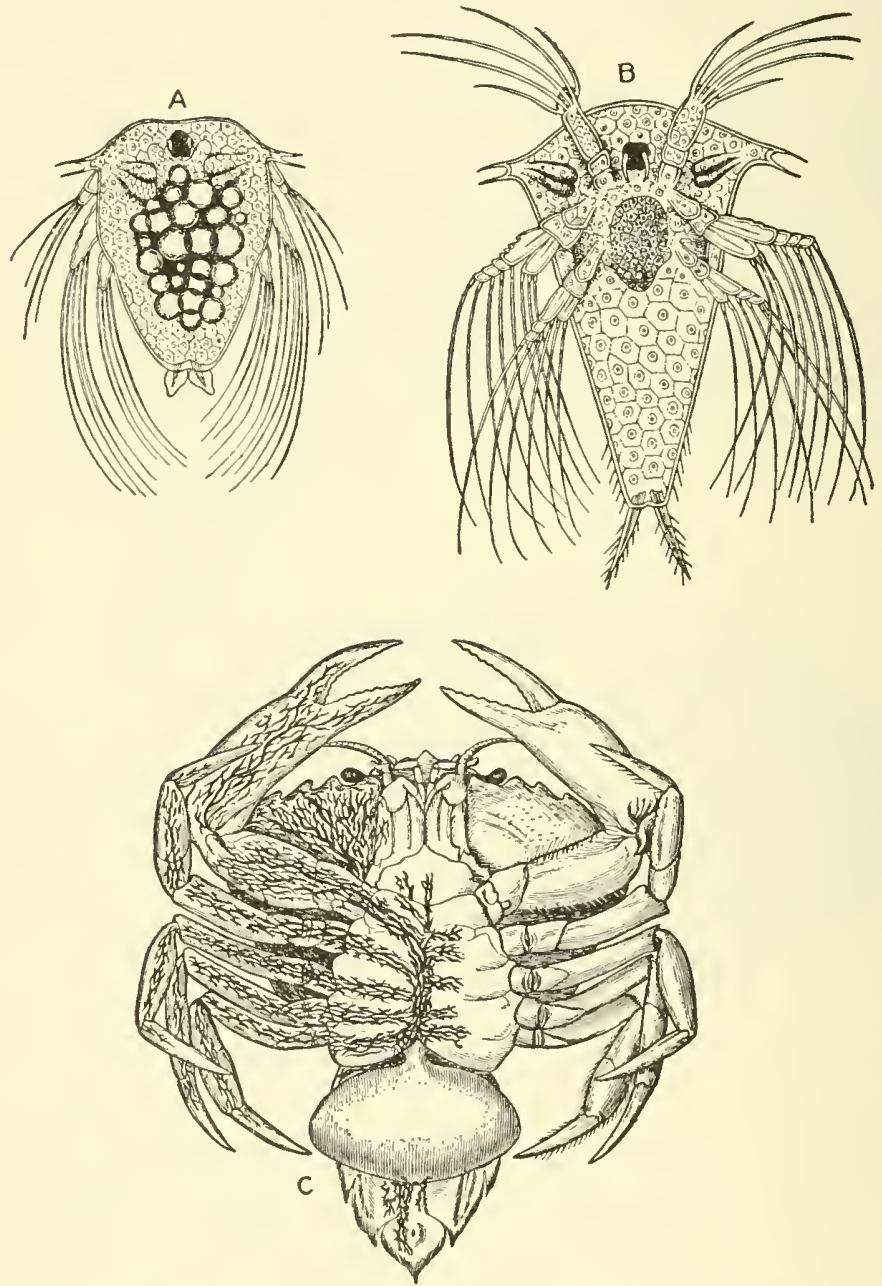

Fig. 404. - Sacculina. A common parasite on the crab. It is recognized as an arthropod only by its larval stages because the adult looks like a sponge or fungus. $A$ and $B$, larval stages with jointed appendages: $C$, crab infested with adult Sacculina. The figure shows it on one side only. (From Lull, Organic Evolution, published by The Macmillan Company.)

in from the sea. There are opportunity and need for much more study of the effects of these phenomena. 
In general, marine animals are adapted to one of three habits of life: dwelling on the bottom, swimming at various levels, or simply floating at the surface. There are three or four forms of marine animals according to the position they take in the water. Benthos include all nonswimming bottom-dwelling forms, and they may be vagrant or sessile (stationary). Hydroid and erinoid types exemplify the sessile form and urchin or starfish the vagrant. The benthos may extend from the shore line to the deep sea. (2) Nekton is the name of the entire group of swimming animals that are able to maintain themselves in the water and do not rest on the bottom. The fishes, whales, and porpoises are typical examples. (3) Plankton is the collective name for plants and animals that float in the water. Many of the plankton animals are nearly transparent, and the smaller ones are surprisingly abundant. Because of their transparency and size, the casual observer seldom sees this group of animals. The nature of the tissue of the bodies of these animals is largely of gelatinous material and the shape is either that of an umbrella or of a mass with projecting processes. The composition of the tissue is such that it is bulky without great weight. In fact, it is largely water and has a specific gravity slightly greater than water. These adaptations make it possible to float with an easy distribution of weight and very little if any effort on the part of the animal. A large number of Protozoa, jellyfishes, and Entomostraca (microscopic Crustacea) are typical plankton. (4) Pelagic animals are all of the surface-living forms away from the shore in the open sea.

Another expression of the distribution of marine animals is by the following zones. (1) Littoral zone, which is the shallow water along the shore. Here the fluctuations of wave action, temperature, and depth (because of tides) are extreme, and the light intensity is at its maximum. Such animals as certain coelenterates, worms, Crustacea, echinoderms, and molluses are common inhabitants of this zone. It requires a rather sturdy type of body and lardy protoplasm to withstand the hardships of the shore and near-shore. Some clams and oysters, also corals, sea urchins, and starfishes can live out of the water for several hours. The majority of corals live where they can depend on wave action to carry floating objects to 
them for food. The zone extends from shoreline to a depth of from 50 to 75 feet. (2) Intermediate (sublittoral) zone includes relatively shallow water of from 50 to 500 feet and supports hydroids, sponges, some corals, sea eneumbers, sea urehins, and starfish. There is a gradual inerease in stability of eonditions through this zone. (3) Bathyal (abyssal) zone is in the depths of the ocean ranging from 5,000 to

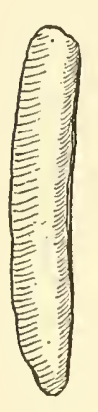

1.

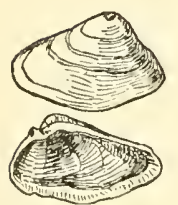

5.

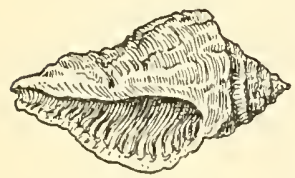

9.

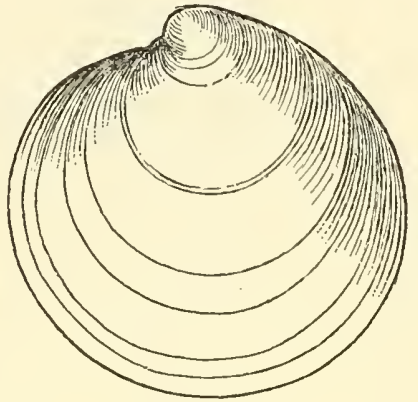

2.

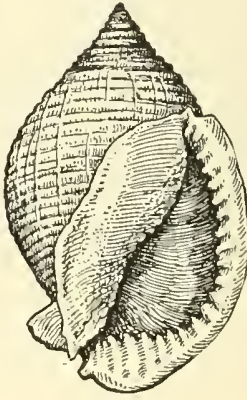

3.

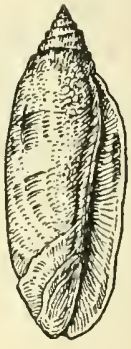

4.

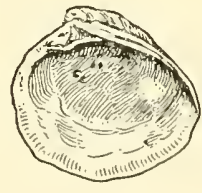

6.

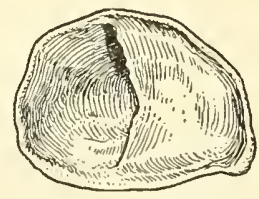

7.

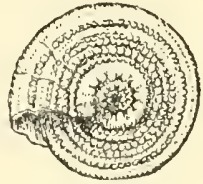

10.

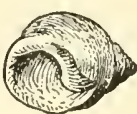

11.

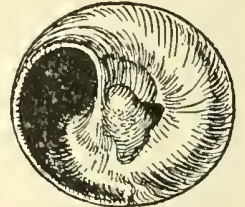

8.

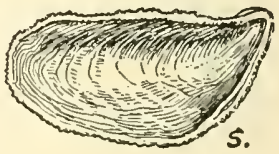

12.

Fig. 405.-A group of typical mollusks occurring along the Texas coast of the Gulf of Mexico. (From specimens belonging to Dr. Elmer P. Cheatum). 1, Solen viridis. 2, Dosina discus. 3, Semicassis gibba. 4, Oliva sayana. 5, Donax roemeri. 6. Venus crmpechiensis texana. 7, Crepidula fomicata. 8, Polinices duplicatr. 9, Thais haemostoma floridana. 10, Architectonica granulata. 11, Littorina irrorata. 12, Modiolus tulipus.

25,000 feet. The pressure inereases fiftecn pounds per square inch with each thirty feet of inereased depth. The conditions become even more constant in this zone. Below 800 feet there is no light and enormous pressure. In the great depths the temperature is nearly constant and is about $2^{\circ}$ C., or just above freezing. Most of the animals found in this zone are relatively small and somewhat modified 
structurally as well as physiologically in order to adjust to the conditions. Approximately 150 species are known to exist at 15,000 feet or deeper. There is a tendency toward the development of phosphorescent organs and telescopic eyes among animals inhabiting the bathyal regions.

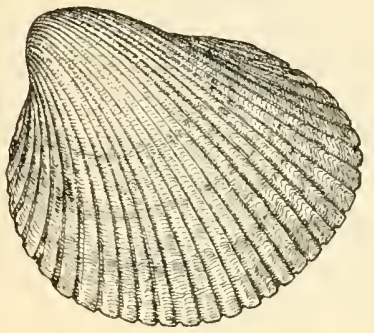

1.

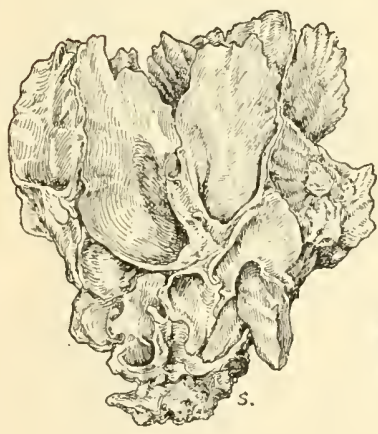

4.

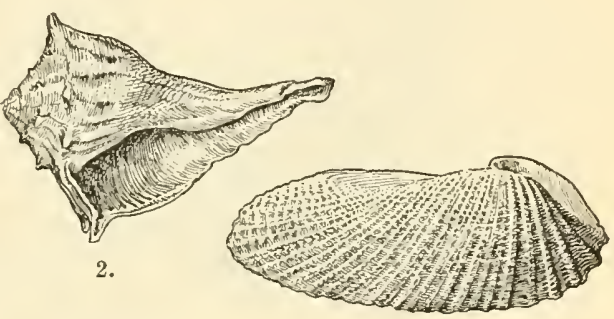

3.

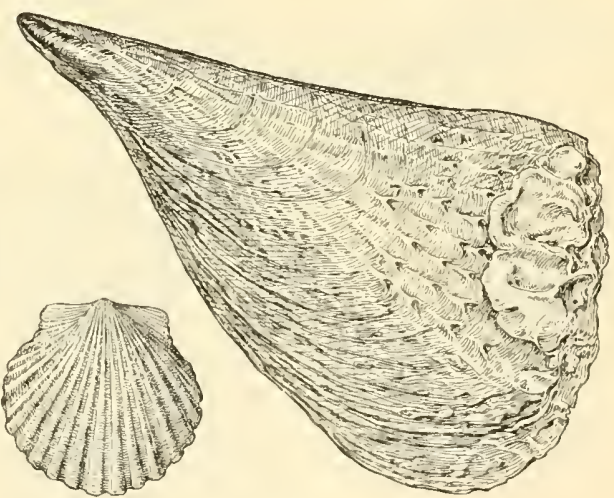

6.

Fig. 406.-Comnon bivalve and univalve mollusks that occur along the Texas coast of the Gulf of Mexico. (From specimens belonging to Dr. Elmer P. Cheatum.) 1, Dinocardium robustm. 2, Buswcon periersus. 3, Barnea costata. 4, Ostraea virginica. 5, Plagioctenium irradiens, 6, Atrina seminuda.

The methods of studying the conditions of the ocean are largely by survey methods, use of light disks, pressure gauges, electric thermocouple temperature recorders, hydrogen ion analysis apparatus, oxygen tension analyses, and chemical analysis for salinity. Samples of sea water can be obtained from various depths by use of a sampling tube or cylinder which is lowered while open so that water passes right through it. When the sample is desired, a lead messenger is sent down the cable to trip the trigger and allow the cylinder to snap shut at both ends. The cylinder with the sample 


$$
\frac{d}{2}
$$


can then be drawn up. Many of the smaller animals will be included in such samples. Other methods of collecting the animals is by use of metal dredges, dredge nets, shrimp nets, cord mops or tangles, dragnets along shore, townets, and fishing tackle generally. It is necessary to use a spade and seine in the beach sands and some sharp instrument for scraping pilings and rocks. The shore and the drift line offer many opportunities of collecting and observing a wide range of animals with no special apparatus. Studies of distribution and migration of marine animals have been made by tagging large numbers of individuals and compiling records of the return of these tags.

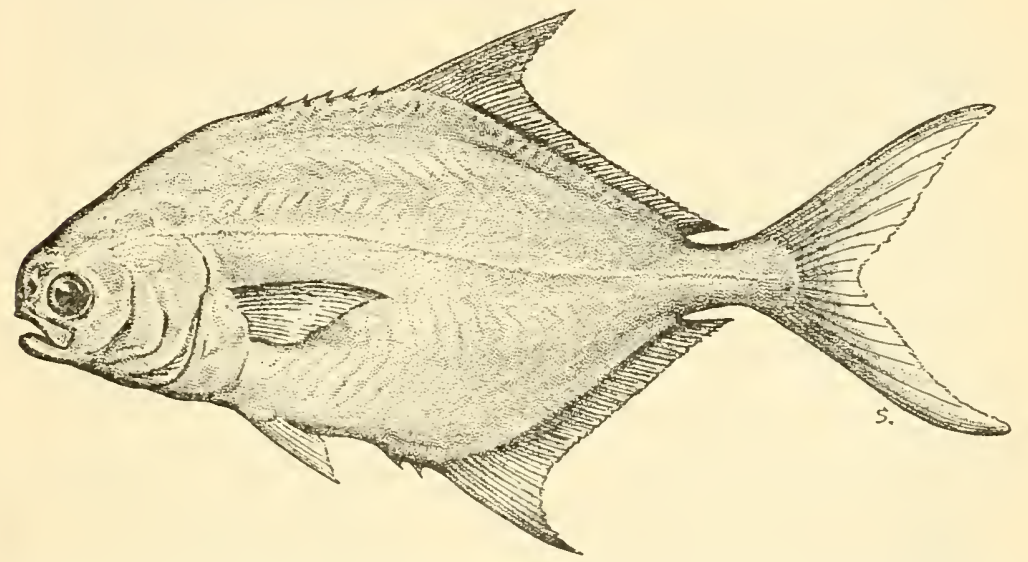

Fig. 409.-The common pompano, Trachinotus carolinus, a valuable food fish which is taken abundantly along the southern part of our Atlantic Coast and in the Gulf of Mexico.

In an effort to give an idea of the typical animal life of the seashore and shore waters, two tables of representative animals are included. The first includes many of the marine and shore animals, excluding vertebrates, of the Pacific coast, while the second provides a similar representative group including fish from the shores and waters of the Texas portion of the Gulf of Mexico. The rocky shores of our western coast furnish an abundance of life. To the casual observer standing almost anywhere on the Texas beach, it seems almost like a desert at the seashore. The shore fauna is rela. tively sparse because the bare sand and shell fragments are almost sterile of food. Closer observation, however, will reveal much more life than at first thought, as will be indicated by the representative lists which follow. 


\section{Typical Pacific Shore Animals}

\section{NAME}

HABITAT ZONE

\section{Porifera}

Reneira cineria, purple sponge

Plocamia karyloinos, red sponge

Leucosolenia eleanor, cream colored sponge

Leuconia heathi, sharp-spined sponge

\section{Coelenterata}

Garveia annulata, hydroid

Eudendrium californioum, hydroid

Tubularia marina, solitary hydroid

Aglaophenia struthionides, ostrich plume hydroid

Gonionemus vertens, hydroid medusa

Velella lata, siphonophore

Stylatula elongata, sea pen

Renilla amethystina, sea pansy

Cribrina xanthogrammica, large green anemone

Cribrina elegantissima, verrucose anemone

Epiactis prolifera, brown anemone

Metridium dianthus, anemone

Balanophyllia elegans, orange-red coral

\section{Ctenophora}

Pleurobrachia bachei, "cat's eye" comb jelly

Bolinopsis microptera, comb jelly

\section{Platyhelminthes}

Planocera californica, large flatworm

Leptoplana acticola, polyclad worm

Polychoerus caudatus, red flatworm

Nemertina

Amphiporus bimaculatus, orange nemertine worm

Emplectonema gracile, yellowish green nemertine worm

\section{Bryozoa}

Bulgula californica, purple Bryozoa

Bulgula pacifica, bryozoan

\section{Annelida}

Audounia luxuriosus, hairy gilled worm

Nereis vexillosa, mussel worm

Nereis brandti, large mussel worm

Lumbrinereis sp., nereid worm

\section{Mollusca}

\section{Pelecypoda}

Siliqua patula, razor clam

Tivela stultorum, pismo clam

Mytitis edulis, edible clam

Mytilis californianus, mussel

Ostraea lurida, western oyster
Middle tide pool rocks

Middle tide pool rocks

Middle tide pool rocks

Middle tide pool rocks

Lower tide pools

Lower tide pools

Lower tide pools

Lower tide pools

Eel grass at water edge

Driven asliore by storm

Mud flat below tide

Sand flats

Rocks, lower tide pools

Rocks, middle tide

Rocks, middle tide pools

Low tide pilings

Rocks, middle tide pools

\section{Cast ashore (Planktonic)}

Cast ashore (Planktonic)

Rocky shore, high tide levels Rocky shore, upper tide pools Middle tide pools

Middle tide pools

Middle shore

Lower tide pools

Middle tide pools

Between rocks and in mud

Cosmopolitan

Cosmopolitan

Mud and sand flats

Sandy beaches

Sandy beaches

Sandy beaches

Sub-tide and half-tide

Low tide, rocky bay 
Typical Pacifio Shore ANIMals-CONT'D

\begin{tabular}{|c|c|}
\hline NAME & HABITAT ZONE \\
\hline Pecten hindsii, scallop & Low tide, rocky bay \\
\hline Pecten circularis, thick scallop & Low tide, rocky bay \\
\hline Cardita subquadrata, bivalve & Low tide pools \\
\hline Schizothaerus nuttallii, gaper elam & Substratum burrow \\
\hline \multicolumn{2}{|l|}{ Gastropoda } \\
\hline Anisodoris nobilis, sea lemon (nudibranch) & Middle tide pools \\
\hline Dialula sandiegensis, nudibranch & Rocks, middle tide pools \\
\hline Pleurophyllidea californica, nudibranch & Low tide level, open bay \\
\hline Tethys californica, sea hare & Rocky middle tide pools \\
\hline Littorina planaxis, gray littorine & Rocky shore, high tide level \\
\hline Littorina scutulata, checkered littorine & Rocky shore, upper tide pools \\
\hline Littorina sitchana, Sitka periwinkle & High tide bay \\
\hline \multicolumn{2}{|l|}{$\begin{array}{l}\text { Littorina rudis, North Pacific white peri- } \\
\text { winkle }\end{array}$} \\
\hline Purpura foliata, a conch & Rocky shore, lower tide pools \\
\hline Cypraea spadicea, the corrry & Rocky shore, lower tide pools \\
\hline Conus californicus, cone shell & Rocky shore, lower tide pools \\
\hline Tegula funebralis, black turban snail & Upper tide pools \\
\hline Tegula brunnea, brown turban & Rocks, middle tide pools \\
\hline Norrisia norrisii, smooth turban & Rocky shore, lower tide pools \\
\hline Tegula ligulata, banded turban & Rocky shore, lower tide pools \\
\hline Thais lamellosa, wrinkled purple, snail & Rocky shore, bay \\
\hline Polinices lewisii, moon snail & Low tide level, open bay \\
\hline Olivella biplicata, purple olive snail & Low tide lecel, open bay \\
\hline Urosalpinx cinereus, oyster drill & Mud flats, intertidal \\
\hline Crepidula adunca, hooked slipper & Middle tide pools \\
\hline \multirow[t]{2}{*}{ Lottea gigantea, owl limpet } & Rocky shore, high intertidal \\
\hline & $\begin{array}{l}\text { zone } \\
\text { Rocky shore, upper tide pools }\end{array}$ \\
\hline Acmaea limatula, file limpet & Rocky shore, upper tide pools \\
\hline Acmaea digitalis, limpet & Rocky shore, high tide level \\
\hline Acmaea scutum patina, plate limpet & Rocks, middle tide pools \\
\hline Acmaea cassis pelta, shield limpet & Rocks, middle tide pools \\
\hline Acmaea mitra, white cap limpet & Rocks, middle tide pools \\
\hline Acmaea depicta, painted limpet & Rocks, lower tide pools \\
\hline Haliotis rufescens, red abalone & Lower tide pools - \\
\hline \multicolumn{2}{|l|}{ Amphineura } \\
\hline Mropalia muscosa, moss chiton & Intertidal rocks \\
\hline Katharina tunicata, black chiton & Intertidal rocks \\
\hline Cryptochiton stelleri, giant chiton & Rocks at low tide \\
\hline Lepidochitona lineata, lined chiton & Low tide level \\
\hline \multicolumn{2}{|l|}{ Cephalopoda. } \\
\hline Polypus bimaculatus, octopus & Lower tide pools \\
\hline Loligo opalescens, opalescent squid & Washed ashore occasionally \\
\hline \multicolumn{2}{|l|}{ Echinodermata } \\
\hline \multicolumn{2}{|l|}{ Asteroidea } \\
\hline $\begin{array}{l}\text { Pisaster ochraceous, fire-rayed starfish } \\
\text { Pisaster giganteus capitatus, fire-rayed }\end{array}$ & Lower tide pools under rocks \\
\hline starfish & Lower tide pools under rocks \\
\hline Pisaster brevispinus, short-spined starfish & Below tide line \\
\hline Evasterias troschelii, small starfish & Below tide line \\
\hline Pycnopodia helianthoides, large starfish & Below tide line \\
\hline Leptasterias aequalis, six-rayed starfish & Middle tide pool rocks \\
\hline Patiria miniata, webbed starfish & Middle tide on rocks \\
\hline
\end{tabular}


Trpical Pacific Shore Animals-Cont'd

\begin{tabular}{|c|c|}
\hline NAME & HABITAT ZONE \\
\hline \multicolumn{2}{|l|}{ Ophiuroidea } \\
\hline Ophioderma panamensis, brittle starfish & Lower tide pools under rocks \\
\hline $\begin{array}{l}\text { Ophioplocus esmarki, sand-colored brittle } \\
\text { star }\end{array}$ & Middle tide, under rocks \\
\hline Amphipholis pugetana, small brittle star- & 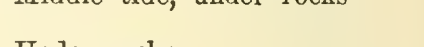 \\
\hline $\begin{array}{l}\text { fish } \\
\text { Amphiodia occidentalis, brittle star }\end{array}$ & $\begin{array}{l}\text { Under rocks } \\
\text { Low tide }\end{array}$ \\
\hline Ophiopholis aculeata, brittle starfish & Low tide \\
\hline $\begin{array}{l}\text { Echinoidea } \\
\text { Stronaulocentrotus franciscanus }\end{array}$ & \\
\hline $\begin{array}{l}\text { strongylocentrotus franciscamus, large sea } \\
\text { urchin }\end{array}$ & Lower tide pools \\
\hline $\begin{array}{l}\text { Strongylocentrotus purpuratus, purple sea } \\
\text { urchin }\end{array}$ & Lower tide pools \\
\hline Lovenia cordiformis, red heart urchin & Sand flats \\
\hline Dendraster excentricus, sand dollar & Sand flats \\
\hline $\begin{array}{l}\text { Echinarachnius parma, sand dollar } \\
\text { Holothuroidea }\end{array}$ & Sand flats \\
\hline Cucumaria miniata, sea cucumber & Lowest intertidal \\
\hline $\begin{array}{l}\text { Leptosynapta inhaerens, dirty white sea } \\
\text { cucumber }\end{array}$ & Middle tide pools \\
\hline Stıchopus californicus, brown sea cucumber & Lower tide pools \\
\hline \multicolumn{2}{|l|}{ Crustacea } \\
\hline Copepoda & \\
\hline $\begin{array}{l}\text { Tigriopus fulvus, copepod } \\
\text { Cirripedia }\end{array}$ & Upper tide pools \\
\hline Balanus cariosus, large barnacle & Rocky bay shore \\
\hline Balanus glandula, acorn barnacle & High tide rocks \\
\hline Chthamalus fissus, small brown barnacle & High tide rocks \\
\hline Balanus mubilis, large barnacle & Pilings and boats \\
\hline Mitella polymerus, goose barnacle & Intertidal rocks \\
\hline $\begin{array}{l}\text { Balanus tintinnabulum, barnacle } \\
\text { Amphipoda }\end{array}$ & Pilings and boats \\
\hline Amphithoe sp., amphipod & Middle tide pool rocks \\
\hline Melita palmata, amphipod & Upper tide pools \\
\hline Caprella scaura, amphipod & Kelp, sand, or mud \\
\hline $\begin{array}{l}\text { Orchestia traskiana, beach lopper } \\
\text { Orchestoidea californiana, large beach hop. }\end{array}$ & Sand above tide \\
\hline per & Sandy beach among rocks \\
\hline $\begin{array}{l}\text { 1sopoda } \\
\text { Cirolana harfordi, dral }\end{array}$ & \\
\hline Ligyda occidentalis, larg & Under rocks, all levels \\
\hline $\begin{array}{l}\text { Ligyaa occidentalss, large isopod } \\
\text { Stomatopoda }\end{array}$ & shore, above tide level \\
\hline Pseudosquilla bigelowi, mantis shrimp & Lower tide pools \\
\hline Decapoda & \\
\hline Crangon dentipes, snapping shrimp & Lower tide pools under rocks \\
\hline Betaeus longidactylus, long-fingered shrimp & Middle tide pools under rocks \\
\hline Pamulirus interruptus, spiny lobster & Lower tide pools \\
\hline Pagurus samuelis, hermit crab & Upper tide pools \\
\hline Pagums hirsutinisculus, common hermit & Middle tide pools of bays \\
\hline Pagurus granosimanus, small hermit & Upper tide pools of bays \\
\hline Pagurıs beringanus, large hermit & Lower tide pools of bays \\
\hline Pagurus hemphilli, hermit crab & Middle tide pools of bays \\
\hline Petrolisthes cinctipes, porcelain crab & Middle tide pools of bays \\
\hline
\end{tabular}


Typical Pacific Shore ANimals-Cont'D

\begin{tabular}{|c|c|}
\hline NAMIE & HABITAT ZONE \\
\hline Pugettia gracilis, erab & Lower tide pools \\
\hline Pugettia producta, kelp erab & Lower tide pools of bays \\
\hline Cancer oregonensis, round Oregon crab & Lower tide pools of bays \\
\hline Randallia ornata, round crab & Lower tide pools of bays \\
\hline Portumus zantusii, sand crab & Kelp, sand or mud \\
\hline Pachygrapsus crassipes, striped shore crab & Upper tide pools \\
\hline Hemigraspus nudus, shore crab & Middle tide pools \\
\hline Cancer antennarius, edible rock crab & Lower tide pools under rocks \\
\hline Cancer productus, crab & Lower tide pools under rocks \\
\hline Lophopanopeus leucomanus, crab & Lower tide pools under rocks \\
\hline $\begin{array}{l}\text { Speocarcimus californiensis, burrowing } \\
\text { crab }\end{array}$ & Mud flats, burrow \\
\hline Uca crenulata, common fiddler & Mud flats, burrow \\
\hline Arachnoidea & \\
\hline $\begin{array}{l}\text { Pycnogonum stearnsi, pycnogonid (sea } \\
\text { spider) }\end{array}$ & Lower tide pools \\
\hline \multicolumn{2}{|l|}{ Chordata } \\
\hline \multicolumn{2}{|l|}{ Hemichordata } \\
\hline Dolichoglossus pusillus, acorn tongue worm & Below tide \\
\hline $\begin{array}{l}\text { Urochordata } \\
\text { Styela stimpsoni, red tunicate }\end{array}$ & Rocky shores, low tide \\
\hline Halocynthia nanstor, large tunicate & Rocky shores, low tide \\
\hline Perophora annectens, greenish tunicate & Lower tide pools \\
\hline $\begin{array}{l}\text { Glossophorum planum, lobed tunicate } \\
\text { Amaroucium californicum, colonial tuni- }\end{array}$ & Lower tide pools \\
\hline $\begin{array}{l}\text { cate } \\
\text { Cephalochordata }\end{array}$ & Lower tide pools \\
\hline Branchiostoma californiense, Amphioxus & Below tide \\
\hline
\end{tabular}


Txpical animals From the Gulf of Mexico-Texas Coast*

\begin{tabular}{c}
\hline NAME \\
\hline
\end{tabular}

Porifera

Clione celata, yellow-boring sponge

Common on oysters

\section{Coelenterata}

Obelia hyalina, branched hydroid

Plumularia inermis, branched hydroid

Clytia naliformis, simple hydroid

Tubularia crocea, matted hydroid

Velella mutica, siphonophore (sp. not definitely determined)

Physalia pelagica, Portuguese-man-of-war

Porpita linnaeana, (sp. identification not yet confirmed)

Dactylometra quinquecirrha, jellyfish

Stomolophus meleagris, cabbage-head jelly. fish

Renilla reniformis, sea pansy (sp. not definitely determined)

Ptilosarous quadrangularis, sea pen (sp. not definitely determined)

Stylatula elongata, sea pen (sp. not defnitely determined)

Leptogorgia carolinensis, yellow gorgonid coral

Astrangia danae, common coral (sp. not definitely determined)

Aurellia aurita, clear jellyfish

Sea anemone (Classification not determined)

Chiropsalamus sp.

\section{Ctenophora}

Beroe ovata, sea walnut or comb jelly

Bolinopsis vitrea (not definitely determined)

IInemiopsis sp., small sea walnut

\section{Molluscoidea}

Bugula turrita

Zobotryon pellucidum, clear bryozoan

(Another bryozoan of family

Valkeriidae encrusting sargassum; classification undetermined)

Platyhelminthes

Eustylochus meridianalis, flatworm

Attached to floating sargassum Common on Sargassum

Attached to floating sargassum

Washed ashore, and taken in shrimp nets

Floats, but may be cast up

Floats generally, cast up

Floats, may be cast ashore

Floating in bays and coves

Floating in gulf, bays, and coves

Sand bottom, shallow water, gulf

Bottom, deep water

Mud bottom, shallow water

Encrusting sleells

Low tide sands, encrusting small shells

Floating in gulf and bays

Attached

Floating in bays and bayous

Floating in bays and bayous

In gulf and bays on the bottom

Among oysters and barnacles

\section{Annelida}

Glycera abranchiata

Nereis limbata, sandworm

Nerine minuta

Oyster beds and beaches

* Appreciation is expressed to Mr. Gordon Gunter, Marine Institute of Science, University of Texas, and to Professor Elmer P. Cheatum, Southern Methodist University, who have assisted in the revision of this section. 
Typical Animals From the Gulf of Mexico-Texas Coast-Cont'd

NAME HABITAT ZONE

Euphole grubei

Lumbrinereis sp. (possibly young floridana), red sandworm

Capitellides teres

Paraxiothea (Clymenella) torguata

Polydora ciliata, burrowing worm

\section{Mollusca}

\section{Gastropoda}

Tethys protea, sea hare

Pleurophyllidia, nudibranch

Crepidula plana, flat slipper shell

Crepidula fornicata, arched slipper shell

Littorina irrorata, periwinkle

Acmaea antillarum, limpet

Sinum perspectivum, ear shell

Eupleura candata, oyster drill

Thais haemostoma floridana, purple

Polinices duplicata, duplicid moon shell

Buscycon pyrum, pear conch

Busycon canalioulatus, channeled whelk

Busycon (Fulgur) perversa, left-h a n d e d whelk

Oliva sayana, lettered olive shell

Irurex spinicostata, spine-ribbed murex

Murex pomum, apple murex

Semicassis gibba, helmet shell

Fasciolaria tulipa, tulip band shell

Fasciolaria gigantea, giant band shell

Architectonica granulata (Solarium), granulated sundial shell

Triton tigrinis, tiger triton

\section{Pelecypoda}

Arca pexata, ark shell

Arca incongrua

Arca ponderosa, ponderous ark

Arca transversa, rhomboid ark

Plagiocteniun irradians, calico shell

Pecten nodosus, knobbed scallop

Ostraea virginica, oyster

Pinna seminuda (atrinia serrata), halfnaked pen sliell

Tellina tenera, small sunset shell

Dinocardium robustum, large cockle

Cardium muricatum

Dosinia discus, disk Dosinia

Modiolus dismissus, horse mussel

Barnea costata, angel's wings

Chione cancellata, cross-barrerl venus

Venus mercenaria, hard-shelled clam or round clam

Tenus campechiensis texana

Domax roemeri, wedge shell

Donax tumida, wedge shell

Between tide levels of sandy beach

Common in oyster shells

Attached to shells and rocks Attached to shells and rocks

Jetties

Jetties

Common in gulf

Jetties, oyster beds

Jetties, shallow water

Feeds on clams, etc., just under the surface of the sand

Sandy ocean floor

Sandy ocean floor

Under surface on sandy beach In gulf

Creep and swim on sandy floor

Scarengers in both shallow and relatively deep water

Rocky crevices

Rocky crevices

Beach

Beach

Sandy beach

Beach

Beach 
Typical Animals Fron the Gulf of Mexico-Tezas Coasi-Cont'd

\begin{tabular}{l}
\hline NAME \\
Donax variabitis, variable wedge shell \\
Solen viridis, green razor clam \\
Brachiodontes hamatus (Mytilus), hooked \\
mussel \\
Brachiodontes recurvus (Mytilus) \\
Spisula similis, dish shell \\
Macrocallista nimbosa \\
Mulina sp. \\
Echinochama sp. \\
Martesia cuneimeris, boring clam \\
Anomia simplex, false oyster or jingle \\
shell navalis, shipworm \\
Teredo navalo \\
Cephalopoda \\
Loligo brevipennis, squid \\
Loligo opalescens \\
Octopus (not identified), devil fish \\
Arthropoda, Crustacea \\
Stomatopoda \\
Chloridella empusa, mantis shrimp \\
Decapoda \\
Clibanarius vittatus, hermit crab \\
Pagurus longicarpus, hermit crab \\
Pagums floridamus, hermit crab \\
Peneus setiferus, common shrimp \\
Penacus aztecus \\
Palaemonetes vulgaris, common prawn \\
MIcrobrachinm acanthurus, tropical shrimp \\
Crangon armillatus, snapping shrimp or \\
pistol crab \\
Callinectes sapidus, blue crab \\
\end{tabular}

Callinectes sapidus, blue crab

Portunus gibbesii, swimming crab Ovalipes ocellatus, lady crab (probable)

Sesarma cinereum

Ocypode albicans, sand crab

Panopeus herbstii, mud erab

Eurypanopeus depressus, mud crab

Metoporhaphis calcarata, spider crab

Libinia emarginata, spider crab

Arenaeus cribrarius, calico crab

Menippe morcenaria, stone crab

Persephona crinita, purse crab

Persephona punctata aquilonaris

Calappa flammea

Hepatus ephelitious

Pinnixa chaetopterana

Uca minax, fiddler crab

Uca pugnax, fiddler crab

Uca prigitator, fiddler erab

\section{Amphipoda}

Orchestia platensis, beach hopper

Orchestia grillus, beach hopper

Caprella acutifrons
HABITAT ZONE

Beach

Beach

Brackish water

Beach

In oysters

On oyster reefs

Wooden pilings

Muddy and gravelled shore

Muddy and gravelled shore

Muddy and gravelled shore

Shallow water

On muddy bottom

Brackish to fresh water

Near jetties; shallow water and bayou

Sandy beaches

Back of high-water mark, sandy beaches

In oyster reefs

Muddy bottom and oyster beds

GuIf waters along shore

Gulf waters along shore

Gulf, shallow water

Oyster reefs

Gulf, along shores

Around worm tubes

Salt marshes, burrows

Salt marshes, burrows

Burrows in sandy or muddy beaches

Under beach debris

Under beach debris

In algae growing on jetties 
Typical aninals from the Gulf of Mexico-Texas Coast-Cont'D

NAME

Grubia compta

Hyale brevipes

Allorchestes sp. beach fiea

Amphithoe sp. beach flea

Jassa sp.

Cirripedia

Lepas anatifera, goose barnacle

Balanus eburneus, acorn barnacle

\section{Isopoda}

Aega psora, parasitic isopod

Ligyda exotica, common isopod

\section{Echinodermata}

Astropecten articularis, yellow starfish

Echinaster sp. violet starfish

Ophioderma sp. brittle star

Mellita quinquiesperforata, keyhole urchin Moira atropos, heart urchin (probable identity)

Sea cucumber, sp., has not been identified

\section{Chordata}

\section{Urochordata}

Tunicate sp. (Has not been identified)

\section{Elasmobranchii}

Galeocerdo arctious, tiger shark

Carcharias platyodon, cub shark

Reniceps (Sphyrna) tiburo, bonnethead or shovelhead shark

Sphyrna tudes, liammerhead shark

Alopias vulpes, thresher shark

Carcharodon carcharias, man-eater shark

Dasyatis sabina, stingaree

Dasyatis americana, southern sting ray

Pteroplatea miomura, butterfly ray

Manta birostris, great manta

Stoasodon narinari, whip or eagle ray

Narcine brasiliensis, electric ray

Rhinoptera bonasus, cow-nosed ray

Pristis pectinatus, sawfish

Pristis sp.

Scoliodon terrae-novae, sharp-nosed shark Raja ackleyi, ray

\section{Pisces}

Atractosteus spatula, alligator gar

Tarpon atlanticus, tarpon

Elops saurus, ten pounder

Dorosoma cepedianum, gizzard shad

Signalosa alchafalayae

Galeichthys felis, sea catfish

Bagre marina, gaff-topsail catfish
Under beach debris (wrack)

Under beach debris (wrack)

On floating wrack, such as logs and boards

Jetties and piles

On sharks

Jetties and beach debris

Off shore

Tide pools in jetties and off shore

Sandy beach, low tide

Sandy beach, low tide

Specimen came from ship channel, Galveston

Off shore

Along shore and harbors

Along shore and harbors in spring

Along shore and harbors in spring

Off shore

In bays and off shore

Along shore and bays

Along shore and bays

In gulf

In oyster beds

Oyster and clam beds

Bays and open gulf

Bays and open gulf

Along shore

Bays

Open gulf

Bottom open water

Along shore

Bays and gulf

Along shore and shallow bays

Along shore and shallow bays 
Typical Animals From the Gulf of Mexico-Texas Coast-Cont'd

\begin{tabular}{|c|c|}
\hline NAME & HABITAT ZONE \\
\hline $\begin{array}{l}\text { Ogcocephalus radiatus, short-nosed batfish } \\
\text { Chilomycterus schoepfi, spiny boxfish }\end{array}$ & Shallow bays, sandy bottoms \\
\hline Trichiurus lepturus, cutlass fish & \\
\hline Synodus foetens, lizard fish & \\
\hline Ancylopsetta quadrocellata, ocellated fluke & \\
\hline Symphurus plagiusa, tongue fish & Bottom, bays and off shore \\
\hline Chaetodipterus faber, spadefish & Bays \\
\hline Spheroides marmoratus, southern swellfish & Along shore \\
\hline Lagocephalus loevigatus, smooth swellfish & Shore waters \\
\hline Sphyraena guachanco, barracuda & Open gulf \\
\hline Trachinotus carolinus, common pompano & Aliong shore \\
\hline $\begin{array}{l}\text { Menticirrhus littoralis, kingfish } \\
\text { Brevoortia patronus, menhaden }\end{array}$ & Open gulf \\
\hline Lactophrys tricornis, cowfish & Shrimp trawls \\
\hline Pogonias cromis, black drum & Deep water gulf \\
\hline Stellifer lanceolatus, star drum & Deep water \\
\hline Cynoscion nebulosus, speckled trout & Along shore and bays \\
\hline Cynoscion arenarius, sand trout & Along shore \\
\hline Cynoscion nothus, silver squeteague & $\begin{array}{l}\text { Open gulf } \\
\text { Off shore }\end{array}$ \\
\hline $\begin{array}{l}\text { Nautopaedium porosissimus, midshipman } \\
\text { Opsanus beta, toadfish }\end{array}$ & $\begin{array}{l}\text { Oft shore } \\
\text { Shallow water, among weeds }\end{array}$ \\
\hline Astroscopus y-graeoum, southern stargazer & Sandy bottom \\
\hline Prionotus punctatus, spotted sea robin & Bottom, along shore \\
\hline Prionotus tribulus, southern sea robin & Bottom, along shore \\
\hline Anchoviella epsetus, striped anchovy & Usually open gulf \\
\hline Hippocampus stylifer, sea horse & Bottom, deep water \\
\hline Syngnathus louisianae, pipefish & \\
\hline Gobius hostatus, sharp-tailed goby & Along shore \\
\hline $\begin{array}{l}\text { Gobius lyricus, lyre goby } \\
\text { Micropogon undulatus, croaker }\end{array}$ & Along shore \\
\hline Conodon nobilis, barred grunt & About docks, shallow water \\
\hline $\begin{array}{l}\text { Lepophidinm brevibarbe, short-bearded } \\
\text { cusk eel }\end{array}$ & Off shore, deep water \\
\hline Remora remora, pilot fish & \\
\hline Sciaenops ocellata, redfish & Along shore, bay, and gulf \\
\hline $\begin{array}{l}\text { Leiostomus xanthurus, spot } \\
\text { Lutianus campechanus, red snapper }\end{array}$ & Aloug shore, bay, and gulf \\
\hline Lutianus griseus, gray snapper & Along shores \\
\hline $\begin{array}{l}\text { Scomberomorus maculatus, spanish mack- } \\
\text { eral }\end{array}$ & Off shore \\
\hline Mugil cephalus, common mullet & Along shore \\
\hline Vomer setapinnis, moonfish & Gulf \\
\hline Selene vomer, look-down & Gulf in shrimp trawls \\
\hline Bairdiella chrysura, silver perch & \\
\hline Poronotus triacanthus, butterfish & Taken in shrimp trawls \\
\hline Chloroscombrus chrysurus, bumper & \\
\hline $\begin{array}{l}\text { Citharichthys spilopterus, spot-finned whiff } \\
\text { Lobotes surinamensis, triple-tail }\end{array}$ & \\
\hline $\begin{array}{l}\text { Centropomus undecimalis, pike, snook, or } \\
\text { roballo }\end{array}$ & \\
\hline Lagodon rhomboides, pinfish & \\
\hline Orthopristes chrysopterus, pigfish & In shrimp trawls \\
\hline Hypsoblennius ianthas, freckled blenny & Oyster reefs \\
\hline Chasmodes bosquianus, striped blenny & Oyster reefs \\
\hline
\end{tabular}


Typical animals From the Gulf of Mexico-Texas Coast-Cont'd

\begin{tabular}{|c|c|}
\hline NAME & HABITAT ZONE \\
\hline $\begin{array}{l}\text { Trinectes maculatus (Achirus fasciatus), } \\
\text { log choker } \\
\text { Achirus lincatus, striped sole } \\
\text { Paralichthys lethostigmus, , southern fluke } \\
\text { or "commercial flounder", } \\
\text { Etropus crossotus, fringed flounder } \\
\text { Polynemus octonemus, threadfin } \\
\text { Caranx hippos, common jack or Jiguagua } \\
\text { Caranx ruber, shipjack } \\
\text { Fundulus simitis, killifish } \\
\text { Fundulus heteroclitus grandis, killifish } \\
\text { Cyprinodon variegatus, broad killifish }\end{array}$ & $\begin{array}{l}\text { Oyster reefs and pilings } \\
\text { Silversides, along shore and in } \\
\text { bays } \\
\text { Muddy or sandy bottom, shal- } \\
\text { low } \\
\text { Bottom in shallow water } \\
\text { Bottom in shallow water } \\
\text { Taken in shrimp trawls } \\
\text { Sandy shores } \\
\text { Off shore } \\
\text { Along shore } \\
\text { Shore waters and bays } \\
\text { Shore waters and bays } \\
\text { Shore waters and bays }\end{array}$ \\
\hline $\begin{array}{l}\text { Reptilia } \\
\text { Chelonia mydas, green sea turtle } \\
\text { Caretta kempil, bastard loggerhead turtle }\end{array}$ & $\begin{array}{l}\text { Along shore, Gulf water } \\
\text { Open sea }\end{array}$ \\
\hline $\begin{array}{l}\text { ammalia } \\
\text { Tursiops truncatus, porpoise, bottle-nosed } \\
\text { dolphin } \\
\text { Trichechus manatus latirostris, manatee, } \\
\text { very rare }\end{array}$ & $\begin{array}{l}\text { Bays and along shore } \\
\text { Back bays and estuaries }\end{array}$ \\
\hline
\end{tabular}




\title{
CHAPTER XL
}

\section{WILDLIFE CONSERVATION}

\author{
(By Walter P. Taylor,* Senior Biologist, United States \\ Fish and Wildlife Service)
}

\section{The Abundance of Wild Animals}

"The manor of living nature is so ample that all may be allowed to sport on it freely. The most jealous proprietor cannot entertain any apprehension that the game will be diminished or even perceptibly thinned." So wrote Dr. Riehard Harlan, in his book, Fauna Americana, published in Philadelphia in 1825, just 122 years ago.

What a panorama of abundant wildlife must have greeted the explorers and pioneers, east, west, north, and south, to inspire so universally the blind faith in the inexhaustibility of our natural resourees. This was a false faith that even yet has not been wholly eradieated, although the evidenee of pitifully small remnants of some of our valuable wildlife speeies, and some of these still diminishing, should teach us better.

It is true that in some of the unbroken forests, deer may not have been so abundant as they are today in the same areas; for deer and similar wildlife like borders and developmental stages of vegetation better than they do pure forest stands. Similarly, bobwhite quail may have been less generously represented in the broom-sedge of the southeastern United States than in the more varied environment of the present. On the whole, however, taking aecount of variations in numbers dependent on habitat, the generous abundanee of the wildlife resourees eneountered in those early days is diffieult for us to conceive. Let us examine some of the records.

Buffalo were widely distributed on the North American continent, almost from the Atlantic to the Pacifie, and almost from the Aretie barrens to the Gulf of Mexico. Their numbers were estimated at not less than sixty or seventy million individuals. The American bison, as they grazed the plains country or migrated with the seasons, must have been one of the outstanding wildlife phenomena of all time.

*In charge, Texas Cooperative WVildife Research Unit. (The Agricultural and Mechanical College of Texas; the Texas Game. Fish, and Oyster Commission; the American Wildlife Institute; and the Fish and Wildife Service, U. S. Department of the Interior, cooperating.) 
It is well known that the pronghorned antelope is found on the continent of North Ameriea and nowhere else in the world. This ereature, combining as it does some of the charaeters of the cow and the deer, is unique. It is the only member of its speeies, genus, and family. While North America shares such animals as the bear, deer, moose,

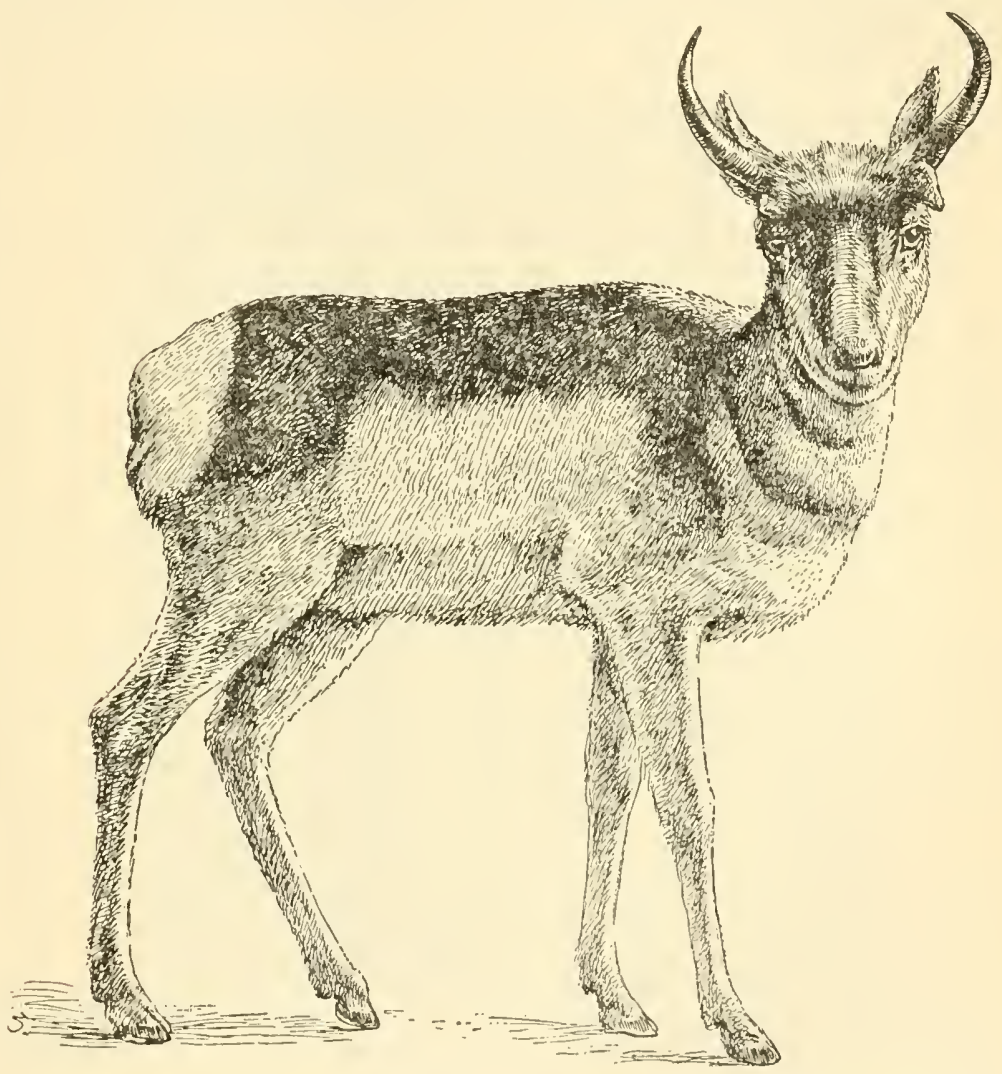

Fig. 410.-Pronghorn antelope, Antilocapra americana. A strictly American animal of the open plains and foothills.

elk, and beaver with the Old World, the pronghorn is peculiarly our own. Like the buffalo, the antelope was found very widely over the plains, although its range was not so extensive as that of the buffalo, and by some authorities it is estimated to have been equally abundant.

In order to obtain a hint of the number of passenger pigeons in the early part of the nineteenth century let us examine a calculation of 
pigeon numbers made by John James Audubon, the eelebrated early Ameriean artist and ornithologist. He estimated a total of nearly five billion birds in the three states of Kentueky, Ohio, and Indiana along the Ohio River in one year's flight. Old-timers still living in eastern Texas report passenger pigeons as still numerous in the 1870 's in parts of that State. So abundant were these birds over their original range that when, after a long period of exploitation,

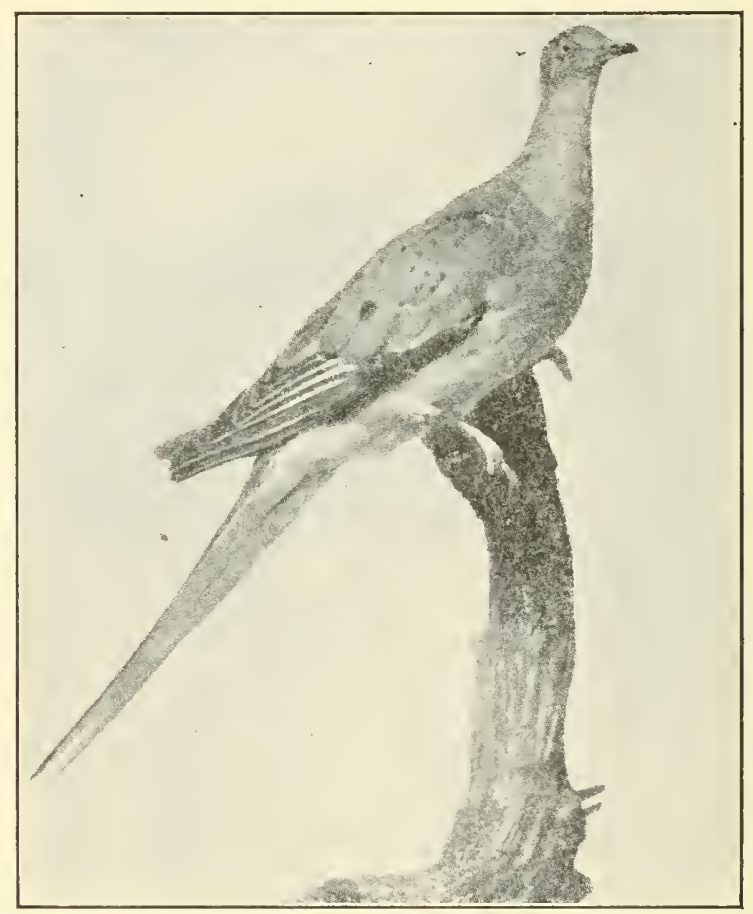

Fig. 411.-Passenger pigeon, Ectopistes migratorius. This bird was once extremeiy abundant but is now extinct. (Courtesy of General Biological Supply House.)

the inevitable happened, and the speeies disappeared, many and varied were the explanations offered. But there is really no mystery at all. Carloads of birds were shipped direet to the big eity markets for weeks during the nesting season. If there is any mystery, it is that the birds persisted as long as they did.

The deer family, ineluding some of the most magnifieent animals in the world-earibou, moose, elk, mule deer, black-tailed deer, and the Virginia deer-has fortunately, with few exceptions, proved to be 
tough and tenacious. Caribou may still be observed in large numbers in the Mount McKinley region and in other suitable parts of Alaska. Moose, although they have been extirpated from New York, Pennsylvania, and some other states, still persist over much of their former range, a remarkable fact when the size and character of the animal are considered. Elk, or wapiti, now reduced to a few favorable localities in the West, were originally of general distribution nearly throughout the country. True deer of the several varieties mentioned above have persisted over much of their former ranges, at least wherever habitat conditions coupled with an interest in deer conservation on the part of intelligent landowners have made such persistence possible. Although in a few places, as in parts of Pennsylvania, deer may be more abundant now than formerly, as a result primarily of manmade openings in the forests, in most instances deer have decreased. Strecker points out that between 1845 and 1853 no fewer than 75,000 deer hides were marketed by a single firm dealing in furs and hides and operating out of Trading House Creek near Waco, Texas. At present deer are scarce in this general region. This is a striking example of what has happened throughout the country.

The collared peccary is the only North American wild pig. Formerly found widely throughout southwestern Texas, and still occurring over a considerable area in that state as well as in southern Arizona, New Mexico, and old Mexico, the peccary has been forced back into a relatively restricted range. This has been given legal protection recently as a game animal in Texas.

The former range of the beaver, one of our most valuable rodents, was almost coextensive with the outlines of North America, excepting only in the extreme North, the ultra-arid region, and parts of the lowlying South and Southeast. Its numbers must have been correspondingly great. It is said that some of Canada's great modern cities are founded on the sites of former beaver colonies. The experience of W'isconsin, Michigan, New York, and Pennsylvania, however, indicates the entire practicability of the restoration of this highly useful animal over vast stretehes of its former domain. The desirability of such restoration is increasingly appreciated as man recognizes the value of the beaver as a soil conservationist; for this most interesting rodent is the original check-dam engineer. The beaver was in the business of soil-building, soil conservation, and flood prevention a hundred thousand years ago. 
Along with the beaver, certain other fur animals, among them the mink, raccoon, marten, fisher, otter, wolverine, skunk, opossum, lynx, muskrat, badger, and fox, have for long been sought for their furs. Some, as the otter and fisher, were formerly present in far greater numbers than they are now. All, save possibly the opossum, muskrat, and fox in certain areas, are much reduced. From a total of $\$ 500,000,000$ in 1929 , the retail turnover in the fur business of the United States declined to $\$ 200,000,000$ in 1935 , owing partly to curtailment of business and deflation of values and partly to actual reduction in the numbers of wild fur animals. In Texas, where a fur-

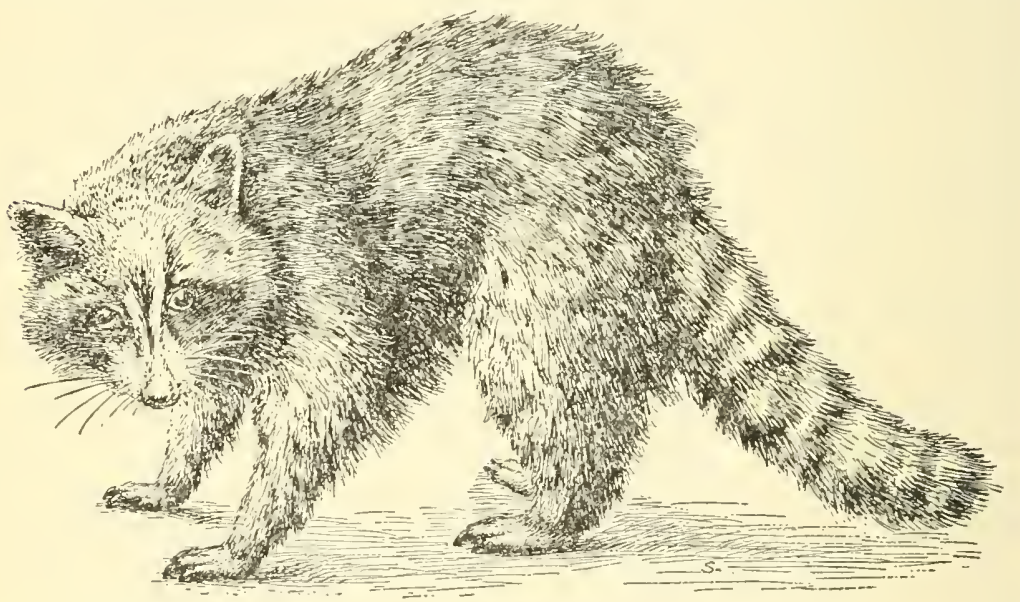

Fig. 412.-Raccoon, Procyon lotor, a valuable fur-bearing animal.

taigging law permitted an accurate check to be made, there was an actual decline in the number of furs taken from 1,407,884 in 1932-1933 to 912,276 in $1934-1935$. This means a 35 per cent decrease- $-500,000$ pelts, worth $\$ 325,000$-in three years. This same decline has been noticed over the entire country.

Ducks, geese, and swans have been favorite objects of sport from the earliest days of our Republic, and formerly were present in such numbers that little concern was felt, although spring shooting was the rule in many localities and the birds were freely sold in the markets. During the past few years, however, difficulties in securing good hunting have been so obvious that extraordinary efforts have had to be exerted to save these popular game birds. During 1938 and since, the ruddy, wood duck, canvasback, redhead, and bufflehead are 
given complete protection under the Federal Migratory Bird Act. The season, bag limit, and manner of hunting of all the other speeies are rigorously regulated.

We can only guess at the numbers attained by some of the smaller animals, but it is certain that, in many instances, they were very large indeed. In some parts of the country the squirrels are important objects of sport. They function also as tree planters and as possible balance wheels on the increase of certain other forms of wildlife. Basing his figures on the known abundance of the gray squirrel in some localities and on the extent of its geographic range, Seton estimated that the gray squirrel population may easily have numbered several billions in eastern North America at the beginning of the nineteenth century.

Bailey, of the United States Biological Survey, in 1905 made a study of the prairie dog (Fig. 335) in western Texas. Usually, as he pointed out, the animals were found in scattered colonies, ". . . but over an extensive area lying just east of the 'Staked Plains' they cover the whole country in an almost continuous and thickly inhabited dog town, cxtending from San Angelo north to Clarendon in a strip approximately 100 miles wide by 250 miles long," a 25,000 square mile colony containing 400,000,000 prairie dogs. Their numbers have been extremely depleted by poisoning.

The whales of the sea were formerly numerous enough to support an extensive industry maintained by nationals representing various maritime countries. The American share in this business centered in the village of New Bedford, Massachusetts, and reached large proportions. Like most other wildlife resources, the whales were subjected to unreasonable slaughter; indeed, the history of the whaling industry as a whole has been a rather sad story of declining possibilities and steadily more restricted opportunities, owing to the remorseless pursuit and crassly unbusinesslike exploitation to which these greatest of living creatures have been subjected by man.

\section{The Natural Range of Wild Animals}

As indicated in the foregoing paragraphs, the natural range of many game animals of great potential value was formerly much more extensive than it is at present. The buffalo which ranged so widely over the continent is now virtually extinct in a wild state. The prong- 
horned antelope, considered by Seton to have occupied a primitive range of approximately 2,000,000 square miles, or 1,280,000,000 acres, is now represented by seattered remnants only.

The wild turkey, a strictly North American product, once occurred generally throughout the wooded sections of the eastern, southern, and southwestern United States, as well as in Mexico. Now it is gone from many of its former homes and its future is decidedly uncertain unless there is marked change in its treatment by man.

Conservationists are becoming much concemed about the prairie chickens. Formerly represented in the Northeast by the now extinct heath hen, and at present in the northern Great Plains area and the Middle West by the greater prairie chicken, on the southern plains by the lesser species, and on the Gulf Coast by the Attwater prairie chicken, these birds are worthy of real attention. Handsome and grouselike birds, weighing up to one and a half pounds and exhibiting some of the most interesting courting habits known among American game birds, the loss of any of them is a real calamity. The heath hen has become extinct in recent years. The greater and lesser prairie chickens are much reduced. They have been so crowded out by field-erop agriculture and eut down by drought, overgrazing, and too much shooting, that their status is not encouraging in any part of their range. The Attwater prairie chicken, which formerly occurred on the Gulf coastal prairie from Louisiana westward into Texas and south to a point beyond Corpus Christi, has been all but eliminated. It is gone from more than 90 per cent of its former range, and is probably in worse condition than any other of the still existing forms.

The jaguar, el tigre of the Mexicans, really an American leopard and justly entitled to protection as an object of sport wherever it would not interfere too much with livestock, formerly ranged north in Arizona occasionally to the Grand Canyon of the Colorado, and was found widely throughout Texas. Of recent years there are few authentic records of its occurrence in the United States, though the species is seemingly quite abundant still in parts of Mexico. This is likewise true of the ocelot or leopard eat. Now within the United States it is restricted to a limited locality in the brush country of southern Texas. Likewise, the puma is becoming greatly reduced and restricted. 
One last instance, the grizzly bear, noblest of American flesh-eaters, was formerly present widely over the western United States, as well as in Alaska and the extreme northern parts of North America, and in northern Mexico. Merriam's studies show what a wealth of forms were represented. Unlike the black bear, which is among the shyest of animals, the grizzly was courageous and much more inclined than the black to dispute man's encroachment on his hereditary range. In this way poor old Ursus horribilis (the name itself has a majestic sound) proved to be not so well adapted to modern conditions as the black bear. Now all the grizzlies are gone from the United States, except in a few scattered localities in the West, where they maintain a precarious existence under special protection or other unusual conditions. Fortunately, there are a good many of these splendid animals still remaining in Canada and Alaska.

Detailed consideration of our valuable wildlife shows a discouraging decrease in almost every instance during the three hundred years or more of our occupation of North America. However, there are a few cases in which the natural range of wild animals has been increased as a result of man's activities. Everyone is familiar with the English sparrow and the starling in America, the muskrat in England and on the continent of Europe, the rabbit in Australia, the house mouse and the Norway rat generally wherever civilized man is found. In most cases, unfortunately, these increases result from man's unintelligent meddling with natural conditions, and result in much damage to his interests. The Norway rat is undoubtedly the most dangerous and costly manmalian enemy of mankind in the whole world.

In a few places, favorable ecological conditions combined with protection by man or a reduction in the number of natural enemies have resulted in an abnormal increase in numbers of game species ordinarily considered valuable, and sometimes dereloped detrimental surpluses. Protection of deer on certain royal forests in England and on the continent of Europe has sometimes seriously interfered with the normal growth of the trees and other vegetation. On the Kaibab Plateau north of the Grand Canyon of the Colorado River, in the state of Arizona, the deer, numbering normally perhaps 3,000 or 4,000 individuals, increased to a number estimated at 30,000 to 100,000 animals. The future of the deer's own food supply, and indeed of the Kaibab Forest, was threatened, There is no more reason for op- 
posing an open season on does or "antlerless" deer in sueh cases than for opposing the marketing of breeding cows on an overstocked livestoek range.

\section{The Coming of Civilization and a Declaration of Indefensibles}

The story of the development of Ameriea is full of meaning as it bears on our natural resourees. Man's inventive genius, coupled with his occupation of the land, has "modified the earth," to use Marsh's expressive statement, and has helped, in all too many instanees, to make his surroundings less favorable for his own future.

Below are reviewed some of the things for which eivilized man is responsible. Here is a sort of Declaration of Indefensibles:

Man has eut and burned the trees of the forest, in many instances leaving the affected area so poor that it eannot reeover without costly plantings.

He has preeipitately drained the marshes for his agrieulture or for the alleged protection of his health, sometimes wisely, often wastefully. Not seldom have drainage enterprises turned out to be of little value for agriculture or for mosquito eontrol, but they nearly always eliminate much valuable wildlife (waterfowl, fishes, fur animals, and a host of other creatures).

With his roads man has penetrated nearly every remaining fastness. The wild game has few remaining refuges. Except for a few preserves, the man with the gun can ride nearly anywhere and enjoy a "suceessful" hunt with a minimum of physieal exertion or mental stimulus.

Man has polluted the streams and lakes of America, and even the waters of the sea itself. Salt water from oil wells, chemical wastes from industry, and raw sewage from towns and great eities are poured into our great drainage ways. Formerly elear and sparkling, with an abundanee of fish, what are they now? All too often merely nauseating reminders of what once was a splendid past.

Man has plowed much land that should have been kept for livestock and wild game. He has grazed a great deal more land that should have been reserved for wildlife. He has planted his field crops in such a manner that erosion of the soil, mother resource of all the rest, is aecelerated often to the point of destruetion. The results of all this land mismanagement are becoming annually more obvious, through the inerease in number and volume of floods, the augmented turbidity 
and even opacity of streamways that should be clear as crystal, the destruction of valuable fish resources, the silting and choking up of reservoirs and of irrigation works, the lowering of water tables, the drying up of springs, the increase in black blizzards of dust, and the abandonment by settlers of homes and lands.

Man has unnecessarily and unintelligently reduced the food and cover for wildlife. He seems not to have realized that every time he cuts a needed tree or a bush, every time he cleans a fence row or grazes the underbrush out of a woodlot, he thereby removes the lifegiving food and essential cover of many living creatures-insects, mammals, birds, and game. It is of little use to liberate fish in streams if no fish food is there.

Man has perfected his mechanical devices so that all creatures are threatened: guns, stronger, better, more of them; more efficient ammunition; automobiles, comfortable, speedy, easily capable of taking the sportsman five hundred miles in a single day to the place where good shooting or fishing is reported; airplanes that still further accelerate the process; traps and scents that lessen the chances for unwary fur animals to escape; huge juggernauts, called tree-dozers, to remove the jungles which in some cases afford the only remaining protection to some of our most interesting forms of wildlife!

Man has thoughtlessly removed the dead trees and brush which are so necessary to such forms as woodpeckers, owls, and raccoons, as well as to game; he has "cleaned up" the native plants on which the bobwhites principally feed at certain times of the year; he has campaigned against the marsh hawks and other hawks and owls that must be relied on to help keep the snakes, rodents, and insects in a proper balance; he has at times eliminated rodents that are the necessary food of fur animals and valuable raptores; he has fought the predators, sometimes quite unnecessarily, seeming not to realize that in themselves the predators hold possibilities, on certain areas, of developing into as high class objects of sport as can be found anywhere, as well as being one element in a balanced wildlife program.

Man has devleoped an agricultural system so efficient that a seeming overproduction is one of its acute problems. He has built railroads, bridges, cities, and mechanical devices of all sorts that enable the well-to-do to live on a scale of luxury scarcely imaginable in other days. In short, he has developed the mightiest mechanical civilization of all time. 
But what does this civilization rest on? The answer is clear: First, on the renewable natural resources - the soil, the waters, the forage growing on the open range, the trees of the forest, and the wildlife; and second, on the nonrenewable natural resources-oil, sulphur, stone, and minerals. Wildlife conservation is but one aspect of the larger world problem of conservation, on the solution of which depends the entire future of the human race.

\section{The Problem of Restoration}

A rather discouraging outlook, it seems, and so it is, but restoration is by no means hopeless. Some farmers are encountered who have decided to get their share of game while the getting is good, and scientists, who have almost turned defeatist in the fight for conservation, have concluded that we are living at the close of the "Age of Nammals," and that our interesting mammals and birls - and in all probability we ourselves-will shortly pass out of the picture. Perhaps so, but, in my opinion, there is no justification for such a view.

It is the considered opinion of the writer that if the American people want fish in their streams, bobwhite quail in their coverts, deer and wild turkey in their forests, song birds and insectivorous species about their farms and homes, they can have them in generous abundance. This opinion is based on some concrete instances in which actual results constitute unmistakable demonstrations of the possibilities. Three of these demonstrations will now be considered.

1. Bobwhite Quail.-The painstaking scientifie research work of H. L. Stoddard and C. O. Handley in southern Georgia and northern Florida has resulted in an encouraging increase in the bobwhite population on the great plantations of the region. Here is a positive demonstration on a grand scale of the possibility of substantially increased production of bobwhites throughout the vast sonthern pine forest area, extending over parts of at least fifteen southern states.

2. Big Game in the National Forests.-During the past several years, under the enlightened management policies of the Federal Forest Service, big game is reported to have increased 140 per cent in the national forests. Nor has this increase entailed a rigorous program of absolute protection, except in unusual cases, but rather, true conservation through wise use. Here, then, we have a second demonstration, on a series of forests and ranges that embrace more than 
$180,000,000$ acres of well-handled public land, of the possibility of saving our big game, whenever we wish to do it.

3. Deer and Wild Turkey.-It is well known that deer and wild turkey are more abundant in the Edwards Plateau country in Texas than in many similar places, and that the receipts of many ranch owners from hunting leases are important ranch assets in the region. It will be remembered that the county agents of six Edwards Plateau counties in Texas estimated the income derived from outside hunters entering these counties to hunt at not less than a million dollars in a single season (1935-1936).

The Edwards Plateau situation, like the others, convincingly indicates the entire practicability of building up the deer and turkey population over a vast section of the Southwest which at present is characterized by a shortage or even absence of these excellent game animals.

There are some rather simple measures, however, that will have to be adopted if American wildlife is to be properly maintained and increased. The willingness and ability of the public to do these things will be a measure of their real desire for game. Here are some of the essentials :

1. Support a strong educational program, including research, teaching, and extension, so that the present and future generations will have the facts, and will be inspired to act in the light of them.

2. Take politics out of the state game departments in all instances, so that merit and good behavior will be the sole criteria for service in conservation administration.

3. Give the responsible state agency full powers and responsibilities to set seasons and bag limits, and to make other regulations regarding the taking of game and the conservation of wildlife, subject to legislative approval.

4. Give the game department money enough to work with. The easiest way to do this, at present, is to adopt the so-called universal hunting license law, which, while it means a slightly inereased cost of hunting, will afford the sportsman a chance to get something when he goes afield.

5. Provide food and cover for wildlife. For the most part this can best be done, not by an expensive planting program, but by protecting the native vegetation from overgrazing, and letting Nature her- 
self do the job. Fencing off the heads of gulleys so that valuable plants will have a chance. Eliminate overgrazing. If the vegetation is removed to get rid of the boll weevil or other insects, numerous song and insectivorous birds will be eliminated at the same time, and the game will go, too. Earlier ideals of ultra clean farming might well be replaced by a better balanced program according to the best information available at present.

6. Know your wildlife. Count your quail, turkeys, and deer, and so regulate the take that the seed stock will not be drawn upon. If you are a landowner, join with your neighbors to form a game preserve demonstration association in cooperation with the Extension Service at your nearest agricultural and mechanical college.

7. Work out a cooperative agreement between farmers and sportsmen, such that the interests of both will be more adequately safeguarded. The farmer will have to be equitably reimbursed for his game, the sportsman assured of better hunting.

8. Keep in touch with your game department, with your agricultural and mechanical college, with the United States Fish and Wildlife Service, and practice the best modern methods of game management. The game wardens, county agents, and teachers of vocational agriculture will help you if you call on them.

9. Do not forget the fur animals. As rapidly as practicable, work out a plan whereby trapping can be permitted on a basis that will avoid depletion of the stock, but will pay an appreciable return to the landowners as well as the trapper himself.

10. Wildlife restoration, in the opinion of the most enlightened and advanced wildlife managers, is not so much a matter of expensive plantings as of letting Nature grow her own vegetation; it is less a matter of propagation in captivity, and more a matter of encouraging maximum natural production of game; it is less a matter of introducing exotics or stocks of game from somewhere else, and more a matter of making conditions favorable for wildlife already on the ground. Restoration is not only possible, but is highly desirable. It should supplement the existing income of the landowner, and promote the utilization of lands not now used to best advantage. It should result in the substantial increase of a resource already estimated to be worth more than a billion dollars to the United States each year. It should add to the store of those natural treasures that, 
all money considerations aside, make life more interesting, more worth living.

11. Make full use of the natural crops on wild land-forest land, marshland, bottom land, range land. Perhaps such areas will produce more actual income through the natural crops: fur animals, fishes, lumber (harvested on a sustained yield basis), stovewood, wildlife, reereation, water supply, a moderate amount of grazing, and natural woods' products (nuts, berries, etc.), than they would if made over into farms. Much land is not well suited to field crops or grains. 


\section{CHAPTER XLI}

\section{COMPARATIVE EMBRYOLOGY}

\section{(By A. Richards, University of Oklahoma)}

Embryological knowledge has been a common property of mankind since before the period of recorded history, and Aristotle embodied with surprising accuracy in his extensive treatises on animal life a great many facts in regard to the development of animals. Because of the nature of embryos, however, it is obvious that there was a very definite limit to any except the most superficial knowledge until the development of the microscope carly in the seventeenth century opened the way for scientific embryology. The first extensive study of the embryo was made by William Harvey and was pub. lished in his work, Gencration of Animals, in 1651. He was followed by Malpighi, who published $D e$ formatione pulle in ova, in 1672. These investigators studied especially the chick embryo, but Malpighi's account includes also various invertebrates. In the middle of the eighteenth contury came the great question of preformation versus epigenesis, with which the names of Wolff, Haller, and Bonnet are connected. Workers later came to see that neither the egg nor the sperm contains a perfectly formed small embryo, and that development is a gradual growth from small beginnings. They realized also that Wolff's elaims that the germs develop from homogeneous material do not fully represent facts, although his concepts were better than those of his opponents. To the student of presentday embryology these difficulties are seen to be concerned with the nature of organization of the embryo. The earlier investigators were trying to understand the mamer in which the mechanism of life begins to operate, as well as the steps by which the structure of the animal is produced. The next step came in 1828 when von Baer published his germ-layer doctrinc, according to which all the parts of an embryo develop from three sheets of tissue, and the organisms are formed by outpocketings, foldings, thickenings, and other mechanical devices applied to thesc three sheets of tissue. This theory is said to have established embryology as a science. It was a very stimulating generalization and contains much of truth; yet it 
would probably not have had quite the place in the history of embryology that it does if the cell doctrine had not developed before it. The eell doctrine, coming in 1838-1839, made it possible to carry knowledge of the organization of the embryo back beyond the stages of germ-layer formation and it gave the basis from which an understanding of the formation of tissues and organs could proceed. The cell theory, the improvements on the microscope, and the development of new methods of fixing, scetioning, and staining material made possible the accumulation of a great deal of information about the origin and the details of development of the embryos of all kinds of animals, and this was a special contribution of the last third of the nineteenth century. The twentieth century has extended this body of knowledge and interpreted it upon the basis of extensive experimentation.

For convenience, the developmental history of an animal may be divided into stages, but the student must remember that this is an arbitrary action on our part, and that the development from the animal's standpoint is a continuous process. It must be borne in mind that ontogeny, which means the entire life history of an organism from the earliest beginnings to old age and death, is a continuous and ever-changing set of processes, and the sum of the characteristics of all the stages of development makes up the characteristies of the animal. The hen's egg is as much a representative of the genus and species, Gallus domesticus, as is the feathered animal to which most of us attach that name.

Embryology really begins with the formation of the germ cells in the bodies of the parents, and it is known in some forms that the prospective germ cells are set aside very early in the development of the organism, even in the cleavage stages, and that they may remain latent until the organism has become independent in its existence. The development of the germ cells, or gametes, through the active stages of gametogenesis (Fig. 47) involves the multiplication period during which they are known as "gonia" (oogonic and spermatogonia), the growth period, the maturation period proper, that is, the period of the "cytes" (oocytes and spermatocytes), ending with the fully formed or mature oogonia and spermatogonia. These gametes are now ready for the process of fertilization, which takes the form of two steps, the first the initiation of development on the part of the egg following the entrance of the sperm; and, second, the union of 
the egg and sperm pronuclei, by means of which the chromosomal complex of the egg is restored to the diploid condition and the hereditary mechanism is enabled to function. The last period is that of cleavage, during which the egg segments into many cells and the egg substances
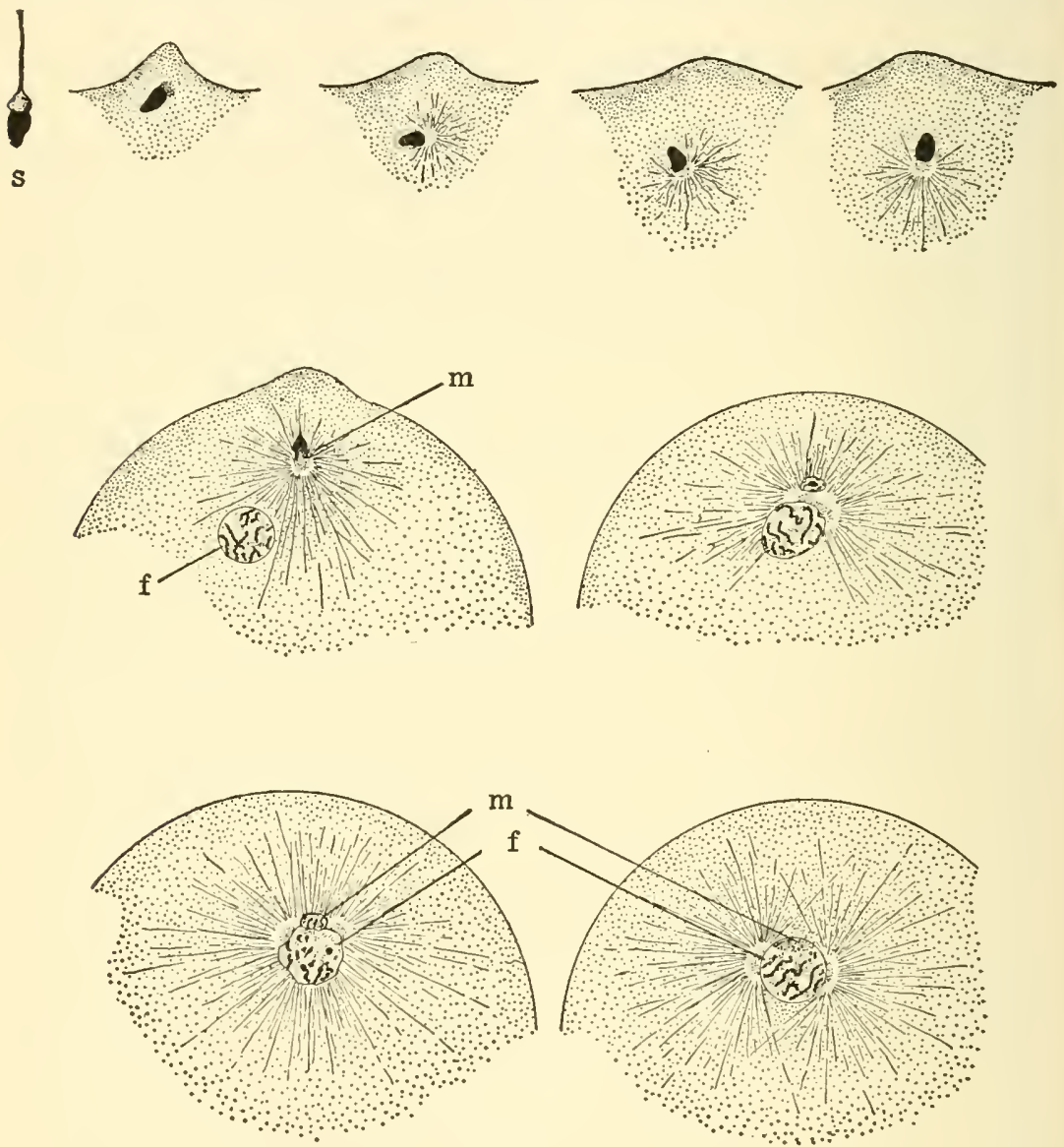

Fig. 413.-Fertilization in the sea urchin, Toxopneustes. S, mature spermatozoon; $m$, transformation of spermatozoon into male pronucleus : $f$, female pronucleus. (Reprinted by permission from Outline of Comparative Embryology by Richards, published by John Wiley and Sons, Inc., after Wilson.)

are distributed into the appropriate cells, so that differentiation and subsequent division of labor can take place. The formation of germ layers follows the organization of the blastomeres into the three sheets of tissue whose further development leads to the formation of the 
organs. The period of organ formation is succeded by a period of histological differentiation, the result of which is a fully formed but small organism. Its subsequent growth is accompanied by changes which are spolien of as metamorphosis, leading to the adult animal. Summarizing the stages in the embryological history of an organism, we find: gametogenesis, fertilization, eleavage, germ layer formation, organ formation or organogenesis (Fig. 110), histological differentiation or histogenesis. The ontogenetic cycle may be completed if we carry the history of derelopment on through adolescence, adulthood, and old age.

The first problem to which the developing embryo must be adapted is that of a store of food to enable it to grow until it can obtain food for itself. At the same time it must be protected from the adversities of the environment; these are of chemical and physical nature and also include other organisms which would prey upon it for food. The first of these problems is solved by the storage of a sufficient amount of yolk (Figs. 287 and 288) in the maturing egg to carry the embryo through its period of helplessness. The duration of this period depends upon the habitats in which the embryos develop, and hence there are various kinds of yolk with respect to form and deposition in the egg.

To solve the second problem, that of protection, many devices are to be seen throughout the animal kingdom. Conspicuous among them are the types of membrane, including shells and horny structures which protect embryos from physical harm and regulate the chemical exchanges with the surrounding medium. There are also many delicate adjustments, as for example, the oil droplets which cause some eggs to have a lighter specific gravity and thus to float at the surface of the water where certain forms of animals cannot so readily destroy them. Egg membranes are usually classified into three groups according to their origin. Those formed from the egg itself are spoken of as "primary membranes," and of them the vitelline is an example. Those formed from the follicular cells are "secondary membranes," and of these the true chorion is an example, and finally those formed from the wall of the oviduct or uterus are "tertiary membranes," of which the albumen and shell of the bird egg are examples.

The amount and kind of distribution of yolk are to no small extent responsible for the types of eleavage found in the animal kingdom. Without explaining this relation in detail, we may say that there are 
six types of cleavage, as given below. In general, according to the amount of yolk present, eggs are spoken of as of three kinds. Isolecithal or homolecithal eggs are those in which only a small amount of yolk is present and that uniformly distributed throughout the cytoplasm. Telolecithal eggs are those in which the vegetative half of the egg contains most of the yolk (the regetative pole is opposite the animal pole, where the polar bodies are given off). In some telolecithal eggs the amount of yolk is not very large, and it is in the form of distinct globules; in others, it is concentrated into a dense mass in the form of a large yolk spherule, which is not penetrated by the cytoplasm and therefore cannot be divided by it when the cell undergoes cleavage. The third characteristic mode of division is that in centrolecithal eggs in which the entire egg is well filled with yolk at the beginning of cleavage, with a nucleus surrounded by cytoplasm forming an island in the center of the egg. Successive divisions carry the plasma islands to the surface and leave the yolk as a centrally located mass.

Cleavage is therefore divided first of all into two kinds, total and partial, depending on whether or not the cytoplasm is able to divide the yolk completely. Eggs having total cleavage are spoken of as holoblastic; those having partial cleavage, as meroblastic. In each of these subdivisions the cleavage patterns take different forms, and therefore allow us to recognize six distinct modes by which the separation of egg substances into definite areas is accomplished; thus the different plans of embryonic organization are established. These six types are radial, disymmetrical, bilateral, and spiral, which occur in holoblastic eggs; and superficial and discoidal, in meroblastic eggs.

Radial cleavage (Fig. 415) occurs in eggs which are not heavily yolk-laden, and hence is marked by a high degree of regularity and symmetry. Eggs of the echinoderms are commonly chosen to illustrate this type, which also occurs in the Porifera and Coelenterata. The first cleavage plane cuts the egg into half from the animal to the regetal pole, and may pass through any diameter of the egg. The second cleavage plane again cuts through the poles at right angles to the first. The third cuts the egg somewhere near the equatorial region; it is therefore best to speak of it as latitudinal. The sixteen-cell stage is reached by a meridional eleavage, and meridional and latitudinal cleavages alternate until there is developed a well-formed blastula, the coeloblastula (see below). 

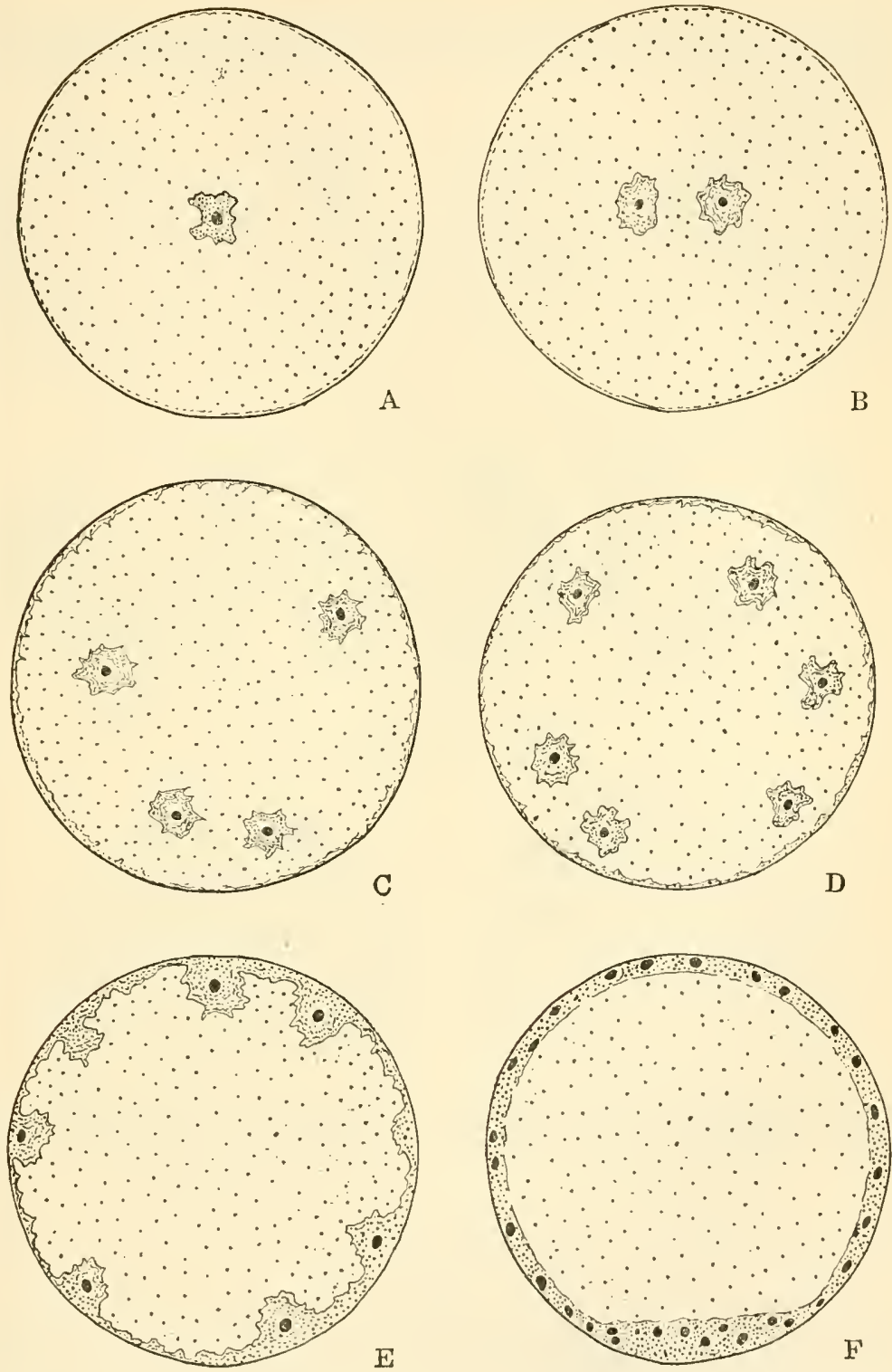

Fig. 414.-Superficial cleavage in the centrolecithal egg of the bristle-tail Insect, Campo in $A$ the fertilized egg appears as a nucleus surrounded by cytoplasm forming an island in the center of a yolk mass. $B, C, D, E$, stages cleavage. In $F$ the thickening on the ventral side will give rise to the blastoderm. (Reprinted by permission from outline of Comparative Embryology by Richards, published by John Wiley and Sons, Ine., after Uzel.) 
Disymmetrical cleavage is found in only one group of animals, the Ctenophora, and is so specialized that it may be omitted from the discussion here.

In eggs having bilateral cleavager a degree of organization of the egg substances is present only in the single-celled condition, a fact which is discoverable in various ways in different kinds of eggs belonging here. The restriction of yolk to certain portions of the egg; pigmentation in the outer layers, or granular cytoplasm as distinguished from clear areas may be the indications of bilaterality in
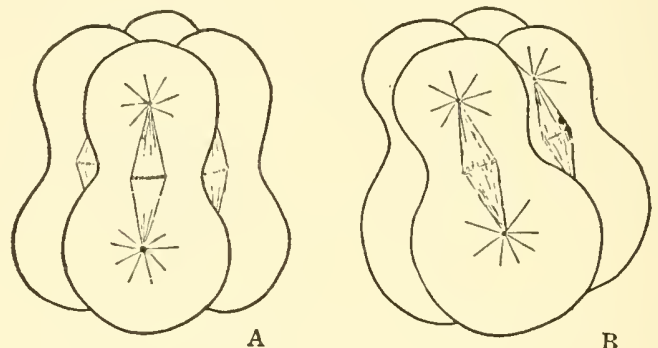

A

B
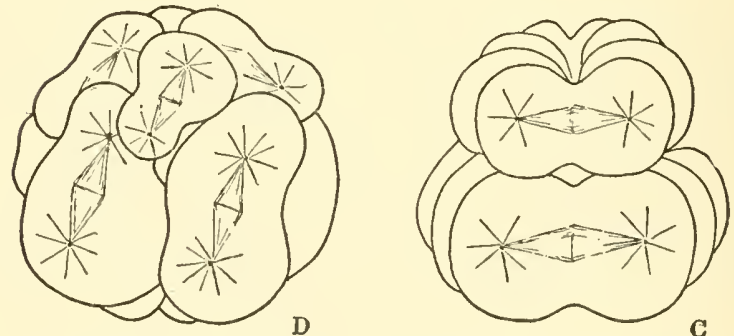

Fig. 415.-Comparison of radial and spiral cleavage. $A$, radial type; $B$, spiral type in third cleavage: $C$ and $D$, radial and spiral types respectively in fourth cleavage. (Reprinted by permission from Outline of Comparative Embryology by Richards, published by John Wiley and Sons, Inc., after Korschelt and Heider.)

the egg even before the first cleavage plan appears. There can only be one plane which will divide the egg into two equal halves in examples of this kind and, as a result, the two blastomeres contain approximately equal amounts of all the egg substances. Again the second plane is at right angles to the first and the egg is divided into quarters. In some animals the order of sequence of these two planes is interchanged, but in the four-cell stage of all bilateral eggs two of the cells must belong to the right and two to the left half of the 
future animal. Bilateral cleavage is fairly widespread throughout the animal kingdom, occurring in the Nematoda, Tunicata, amphioxus, and in all the Vertebrata which have holoblastic cleavage; that is, all
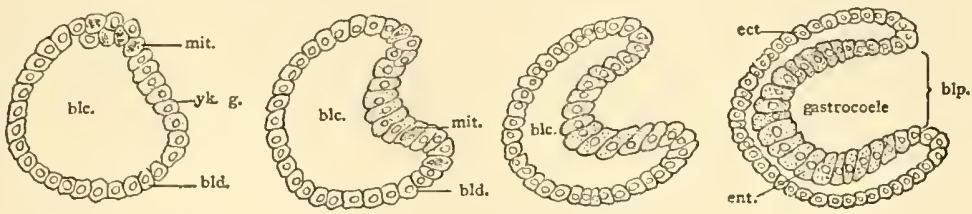

GASTRULATION IN FORM WITH ISOLECITHAL EGG HAVINGALMOST NO YOLK-AMPHIOXUS.
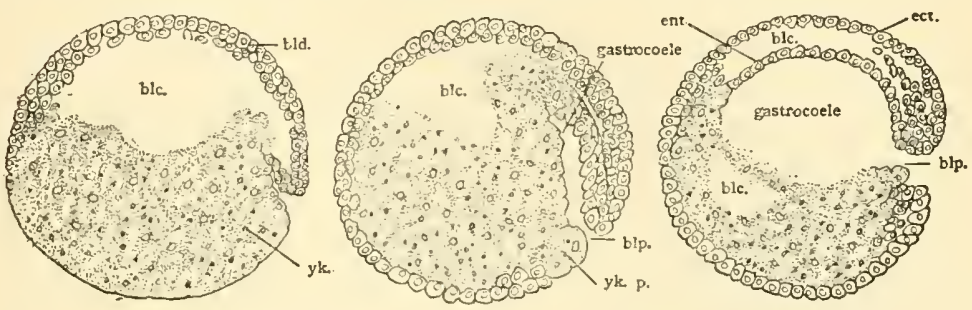

GASTRULATION IN FORM WITH TELOLECITHAL EGG CONTAINING MODERATE AMOUNT OF YOLK-AMPHIBIA.
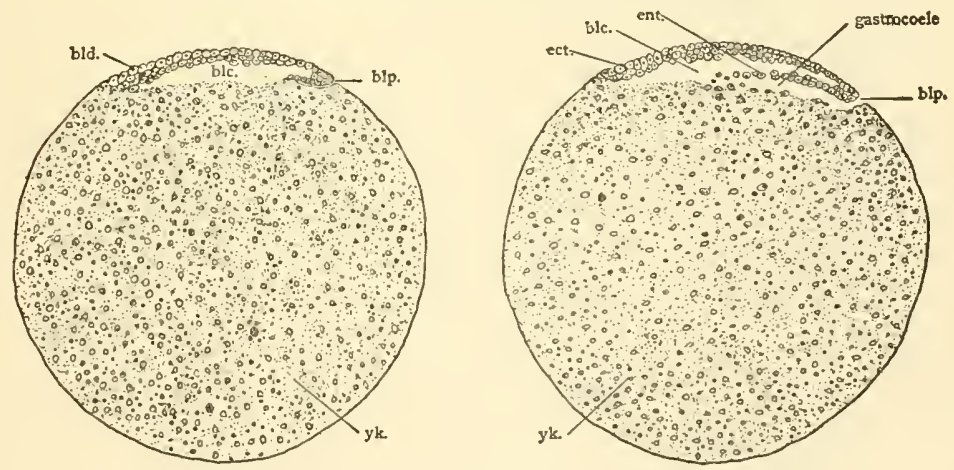

GASTRULATION IN FORM WITH TELOLECITHAL EGG CONTAINING LARGE. AMOUNT OF YOLK-BIRDS.

Fig. 416.-Gastrulation in three types of embryos. Typical discoidal blastula formation (left) and gastrulation (right) in the telolecithal egg of the bird, below. blc., blastocoele ; bld., blastoderm; blp., blastopore; ect., ectoderm; ent., endoderm: mut., cells undergoing mitosis. (From Patten, Embryology of the Chick, published by permission of The Blakiston Co.)

except the selachians, teleosts, reptiles, and birds, in which the cleavage is meroblastic, and the mammals, which are so specialized as not 
to permit a clear-cut designation of the types of cleavage. Bilateral cleavage is greatly influenced by the amount of yolk, and irregularities in the cleavage pattern develop much earlier than in the radial type. The degree of organization present in the egg at the beginning indicates earlier differentiation, both physiological and morphological, and embryos of this type are correspondingly reduced in their power of regulatory adjustment.

In spiral (Fig. 415) cleavage the tendency toward earlier organization of the egg is carried to a further extent, and consequently these eggs are among the most extreme of the types spoken of as determinative; that is, they have a very much reduced power of regulation and a much more precise sequence of stages leading to the formation of the embryonic body. This type of cleavage occurs in the annelids and mollusks, and in some smaller groups, for example, the subclass, Polycladida, of the flatworms.

Eggs of the meroblastic type contain so much yolk that their cleavage is quite modified from the more simple ones, previously mentioned, of the holoblastic type. There are two general kinds, discoidal and superficial. In the discoidal eggs (Fig. 416) the yolk is in the form of a single, fairly large and fairly dense body, surrounded by a very thin cytoplasmic film which is totally unable to divide the entire mass. It is possible to derive this type of cleavage from the extremely telolecithal eggs of the bilateral sort by considering the amount of yolk to increase and concentrate and the amount of cytoplasm to decrease in proportion to the egg. Upon fertilization the cytoplasm accumulates on one side of the egg to form a small disc-shaped or lensshaped mass. It is only this discoidal portion of the cytoplasm that undergoes cleavage; hence the term applied to it. It divides successively into two, four, eight cells, all lying in one plane, but at the fourth or fifth cleavage some cells are eut off interiorly and there arises a disc-shaped mass, several cells in thickness. With continued cell division the discoblastula is produced. After gastrulation and the formation of the germ layers, this cap of cells grows down, so that it entirely encloses the yolk mass; but at no time does the yolk undergo any sort of division. Discoidal cleavage oceurs chiefly in the vertebrate classes, Elasmobranchii, Teleostei, Reptilia, and Aves. There is also a type of discoidal cleavage to be found in the Cephalopoda, which is an extreme case of the spiral type, and a further 
illustration is seen in the scorpions, which might be considered as an extreme case of superficial cleavage.

Superficial cleavage (Fig. 414) predominates in the arthropods, although not all arthropods have this type of cleavage. It occurs also in some coelenterates, and it has been described for one sea cucumber. In superficial cleavage the yolk is in the form of a great many spheroids which are separated from each other by very thin eytoplasmic films. In the center of the uncleaved egg the nucleus lics in an island of cytoplasm. Cleavage is accomplished by the repeated divisions of the nucleus and the surrounding cytoplasm. These small nuclear cytoplasmic islands gradually move about, and as their division continues, they are arranged into the form of a layer of cells which finally reaches the surface of the egg and forms the blastoderm there. This is a superficial layer with the yulk mass left in the center of the egg and constitutes the so-called superficial blastula. Some cells remain behind to help digest the yolk and are known as vitellophages.

The cleavage period, regardless of the type, terminates with the formation of the blastula, a one-celled embryo. Of the blastulae there are seven kinds. Most students of embryology think of cleavage in terms of the holoblastic eggs, and of blastulae as hollow spheres of cells of the type of the cocloblastula. Actually this is only one of the varied kinds of structures, all of which answer the definition of a blastula, "a one-layered embryo."

Eggs having radial cleavage usually develop into coeloblastulae, as do those other holoblastic types in which the amount of yolk is small. Coeloblastulae, that is, blastulae containing cleavage cavities, may be either equal or unequal, depending upon the amount of yolk present. If the size of the cavity is greatly reduced, we approach the second type, the stereoblastula, which is a solid embryo, but here again the dimensions of the cells may be uniform or quite variable, and we likewise have equal and unequal stereoblastulae. In the stereoblastula, however, all of the cells reach the surface of the entire embryo, none of them being cut off at the interior as in the case of the type of blastula known as the morula. Formerly, it was common to speak of the morula as a stage in development of any embryo, but this usage has been abandoned and the term is now given to a particular type of solid blastula in which certain cells are entirely cut off from the surface of the embryo. The placula is a type of blastula in which the cavity is reduced by a shortening of the egg axis which runs from 
the animal to the vegetal pole, so that instead of being spherical it is a mere flattened slit. The circumference of the placula corresponds to the equator of the coeloblastula much as if two saucers were placed with their cavities together. The amphiblastula of a sponge (Fig. 56) is a free-swimming larval stage, the cells of the animal half being sharply distinguished from those of the vegetal half by their smaller size and ciliated covering. The superficial blastula, as already described, is formed when the dividing plasma islands reach the surface of the egg and form a blastoderm covering it. The discoblastula results from the repeated divisions of the dise on the surface of these eggs, and is in the form of a dise several cells in thickness.

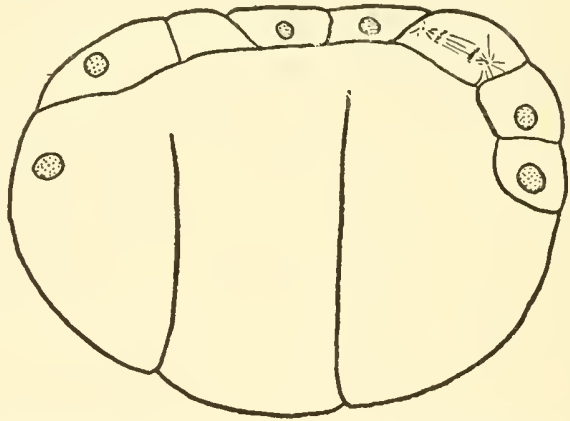

Fig. 417.-Unequal stereoblastula of the moilusk Crepidula (boat shell). (Reprinted by permission from Outline of Comparative Embryology by Richards, pubIIshed by John Wiley and Sons, Inc., after Conklin.)

Examples of these various types of blastulae occur as follows: equal coeloblastulae are found among the echinoderms and in amphioxus; unequal coeloblastulae (Fig. 288), in the frog, annelids (Fig. 110) and molluses; stereoblastulae, in those forms having spiral cleavage in which there is a large amount of yolk; morulae, chiefly in the coelenterates; placulae, in ascidians and in some nematodes; amphiblastulae, limited to the sponges; superficial blastulae, chiefly among the arthropods, and discoidal blastulae, of the same disposition as discoidal cleavage.

The formatiun of the blastula marks the end of the first great period of embryological development. It is followed by germ-layer formation which takes plase in two steps; the first, endoderm formation or gastrulation (Fig. 410), and the second, the formation of primary germ layers (primary ectoderm and primary endoderm), from which are derived the definitive germ layers, ectoderm, mesoderm, and endo- 
derm. The processes by which the formation of these definitive germ layers is brought about we speak of as mesoderm formation. There are really four methods by which gastrulation is accomplished in the animal kingdom: embolic or invaginate gastrulation, epibolic gastrulation, gastrulation by polar ingression, and gastrulation by delamination. As in the case of the coeloblastula (Fig. 48), the invaginate gastrulation is the kind people usually think of in following the story of development, but it again is only one of the several types. It is the most simple type, however, and occurs in those embryos which have a cleavage cavity of fair size. The cells at the vegetal pole flatten and gradually push in, so that they come to form a lining layer enclosed by cells of the other half of the egg. The new cavity formed

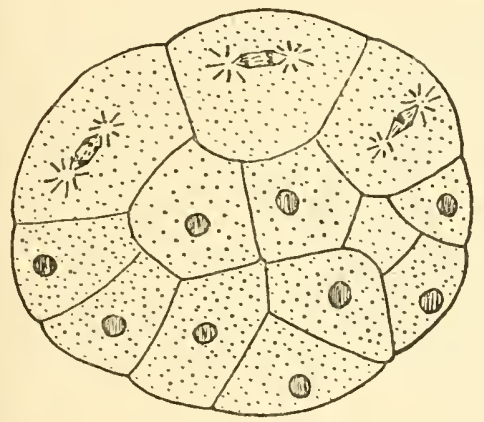

Fig. 418.

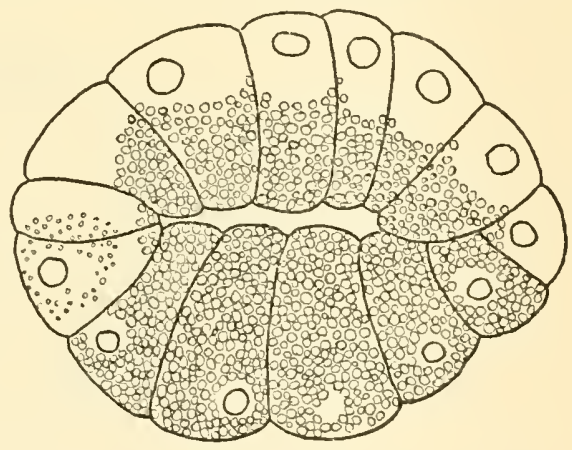

Fig. 419.

Fig. 418.-Morula of the coelenterate, Clava squamata. Notice the "mulberry" appearance. (Reprinted by permission from Outline of Comparative Embryology by Richards, published by John Wiley and Sons, Inc., after Harm.)

Fig. 419.-Placula of the tunicate, Cynthia partita. (Reprinted by permission from Outline of Comparative Embryology by Richards, published by John Wiley and Sons, Inc., after Conklin.)

by their inward movement becomes the gastrocoele or primitive gut cavity, and will develop into the archenteron of the cmbryo. The best illustration of this type of gastrulation is found in echinoderms, but many others are scattered throughout the animal kingdom.

Epibolic gastrulation (Fig. 289), on the other hand, is a process of overgrowth occurring in those embryos with very large yolk-containing cells, small ectodermal cells with no cavity between, and spiral cleavage. The ectodermal cells begin a gradual process of overgrowth, which when completed converts the stereogastrula into an embryo containing a relatively few primary endodermal cells surrounded by a layer of numerous small primary ectodermal cells. 
Polar ingression resembles invagination except in one particular. In invagination the cells, whether few or many, move inward as a definite layer. In ingression the cells move inwards, but not as a definite layer. They do not retain their mass formation, but move as individual cells or as very small groups of cells and rearrange themselves into the form of a layer with an interior cavity.

The fourth means of gastrulation is delamination, which means the formation of a second layer, divided off from the primary ectoderm because the spindles of the cells are directed at right angles to the surface of the embryo. The second layer is split off, so to speak, from the first. The net result of gastrulation is a two-layered embryo of primary ectoderm and primary endoderm.

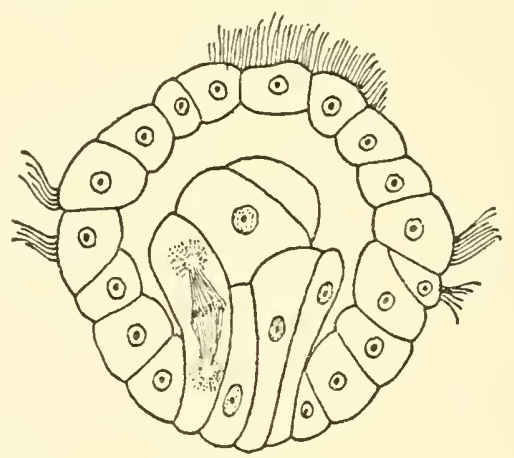

Fig. 420.-Formation of the endoderm in the gastropod, Patella, by unipolar ingression. (Reprinted by permission from Outline of Comparative Embryology by Richards, published by John Wiley and Sons, Inc., after Patten.)

The formation of mesoderm changes the two-layered embryo into a three-layered or triploblastic one. Obviously there are only two sources from which the mesoderm can arise, primary ectoderm or primary endoderm, and when it has been produced, definite endoderm, ectoderm, and mesoderm result. If the mesoderm is derived from cetodem, it is known as ecto-mesoderm. In general, ecto-mesoderm may be looked upon as of temporary importance, often being lost when larval metamorphosis is accomplished. Important embryological questions are connected with the formation of this kind of mesoderm, and it has a bearing also upon the phylogeny of different animal groups. If the mesoderm is formed from the endoderm, it is known as endo-mesoderm, and is the type with which we are most familiar in the animals usually studied in courses in embryology. 
There are several processes leading to the formation of endo-mesoderm, but it always arises from a group of cells lying at the sides of the gut and gradually extending themselves to make a mass which later hollows out to form the mesoblastic somites.

Enterocoele formation is the evagination of a series of pockets from the cavity of the primitive gut and occurs, for example, in amphioxus. The third type of coelome formation is by a solid outgrowth of cells arising from the enteron, a common method among the vertebrates. Lastly, is mesenchymous coelome formation, the formation of mesoblastic somites by the fusion of scattered mesenchymous cells. This is rather rare and of little importance in general embryology.

Mesoderm formation results in a larva consisting of three germ layers and completes the second great period of embryological development. From the three germ layers the different organs of the body are developed by invagination, evagination, foldings, and the retention of embryonic thinness in certain regions while neighboring areas, because of differential growth, thicken and become elaborated into the organs as we know them; this latter process is especially well illustrated in the development of the brain.

In many animals development is direct, the embryo passing directly from a stage of organ formation into a small, but adultlike creature. Others, especially those which develop in the sea and the terrestrial insects, undergo a process of metamorphosis, their earlier adaptations to one type of habitat being insufficient to fit them to live in the different habitats of the adult. A small organism essentially like the adult is the result, and only the processes of histological differentiation and of growth are necessary to produce the mature organism. Organ formation and histological differentiation complete the processes which we commonly speak of under the heading of embryology. 


\section{CHAPTER XLII}

\section{MAMMALIAN DEVELOPMENT}

In mammals the reproduction is entirely sexual and in all of the higher forms the development is intrauterine (within the uterus). With the exception of those of the lowest group, Prototheria, the eggs of mammals bear only a meager amount of yolk.

The ovaries are small, ovoid, paired organs which are attached one on either side of the wall of the coelom by the supporting mesenteries, mesovaria. The histological structure of the ovary includes the outer (germinal) epithelium, the stroma (connnective tissue and few smooth muscle cells), ovarian follicles with ova (eggs) in different stages of development. Fig. 421 shows the origin of a follicle from the germinal epithelium in the formation of an ovigcrous tube. Certain cells of the epithelium are the primordial germ cells, which become ova and the adjacent cells form the lining of the primary follicle. The wall of the follicle cells thickens by multiplication of these cells. Soon a cavity forms in this wall of cells as the beginning of antrum or space of the follicle. The space does not entirely surround the developing ovum. The layer of the cells immediately surrrounding the ovum (corona radiata) are joined at one side to the wall of the follicle. The band of cells extending to the wall is known as the cumulus. The outermost layer of a mature follicle consists of two thin bands of connective tissue, the internal theca and external theca. The human ovum measures only 0.22 millimeter in diameter and that of a dog is still smaller, measuring only 0.15 millimeter. The follicle becomes distended with fluid (follicular liquor) and at maturity projects beyond the general surface of the ovary.

The ovum develops in an ovarian (Graafian) follicle. It passes through the stages of oögenesis to the secondary oocyte here (Fig. 421). At this point of development the follicle reaches its maturity and its outer wall ruptures to free the maturing ovum. The ruptured follicle from which the ovum has been discharged, immediately fills with a blood clot in which are many of the follicle cells. It later takes on a yellowish color and appears as a mass of epithelial cells. If pregnancy ensues, this body becomes a functional corpus luteum and produces essential hormones, if not, it degenerates to become a 


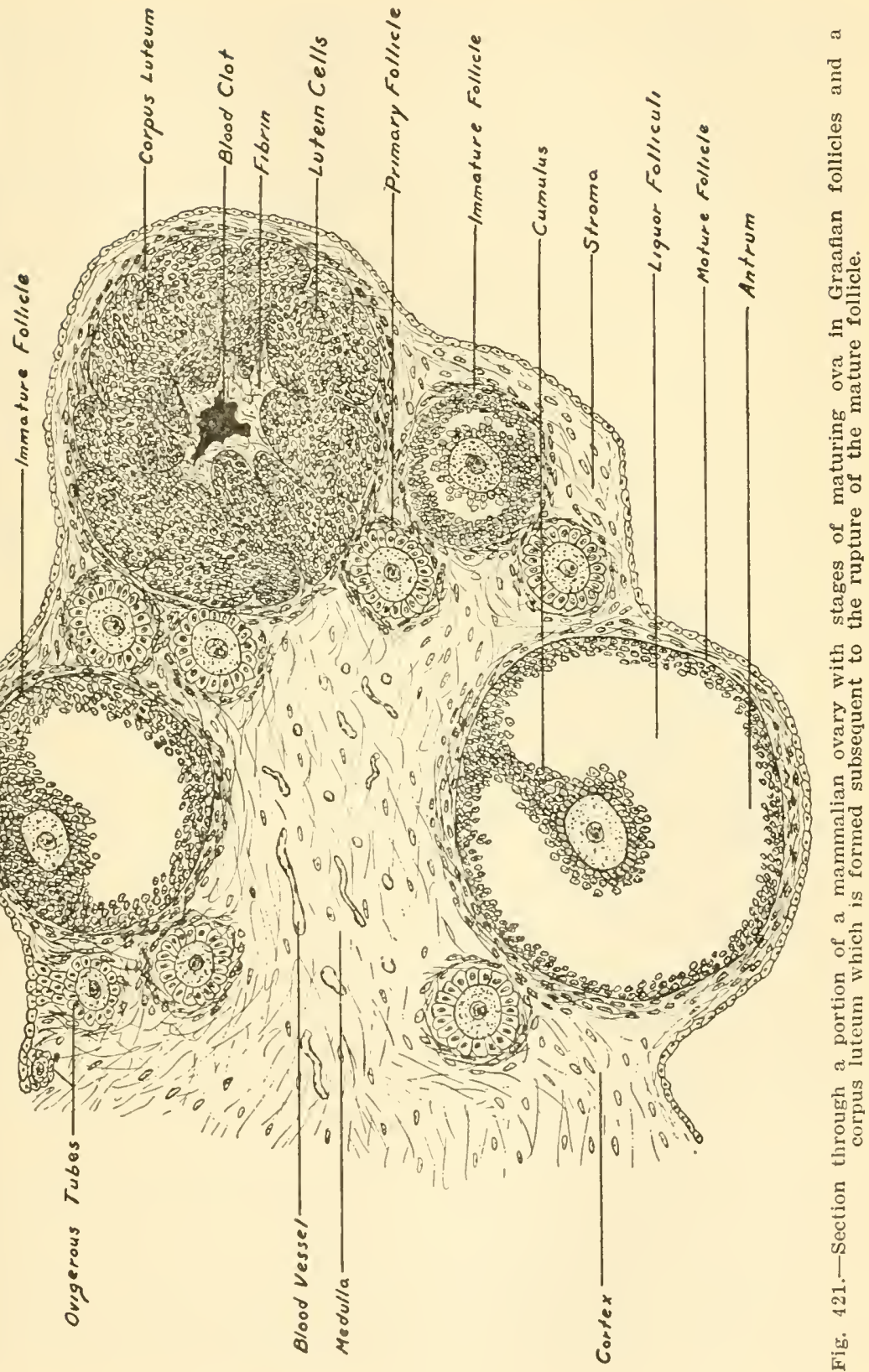


scarlike corpus albicans. In human beings the usual procedure is for only one ovum to mature in one of the ovaries each four weeks (approxinately). At the end of the succeeding period an ovum matures in the other ovary. This alternation proceds from month to month in the female from puberty to menopause, except during pregnancy. When the ovum leaves the ruptured follicle of the ovary it is technically in the body cavity, but in practice the funnellike ostium of the oviduct receives it immediately and starts it down the oviduct. It is here in the upper part of the oviduct that maturation of the ovum is completed and fertilization occurs. Spermatozoa, carried in semen, are introduced into the vagina of the female genital tract in the act of copulation or coitus. These motile spermatozoa swim up the oviduet and meet the ovum shortly after it enters. Fertilization of the ovum by union with a spermatozoön occurs and the zygote continues to move slowly down the oviduct. Cleavage, the next step in development, takes place while the embryo proceeds along the oviduct. The later stages are completed normally in the uterus. This process of successive cell divisoin is modified somewhat when compared with that described for starfish on page 116 and frog on page 533, but the same ultimate purpose of rapidly increasing the cells is accomplished. Cleavage in mammals is complete and nearly equal. The stages of the earlier divisions have been obtained and observed in such mammals as Macacus monkey, rabbit, guinea pig, rat, pig, sheep, and horse. It is thought that cleavage in the human being is similar to these. One of the four cells resulting from the second cleavage division is different from the others. It is the forerunner of a differentiated group of cells which soon becomes surrounded by the other cells (Fig. 422). This enclosed group is then known as the inner cells. The outer layer which surrounds these inner cells is known as the trophoblast. Shortly, small pouches or vesicles filled with watery fluid which is secreted by the newly formed trophoblast, appear beneath it. These join to form a common cavity between the trophoblast and the inner cells, except at one pole, where the two groups of cclls remain in contact. This cavity goes under the name of blastocyst cavity and the whole structure, which is comparable to a specialized blastula, is called a blastocyst or blastodermic vesicle. The embryo's body will develop from the inner cell mass. The trophoblast becomes closely associated with the inner lining of the uterus of the mother and soon plays a part in nutrition, respiration, and excretion of the embryo. It sinks into the uterine lining carrying 
the inner cell mass with it. This is known as implantation. The entire blastocyst makes a rapid growth and the cavity becomes distended with the lymphlike fluid.

Next, a simplified process of gastrulation takes place, in that two successive layers of cells are shed into the blastocyst cavity from the free margin of the inner cell mass. These are layers of endoderm cells. The ends of the layers join to form an elongated enclosure. The inner cells which are joined to the trophoblast become the ectoderm of the body and the trophoblast remains as extraembryonic ectoderm. The cavity enclosed by the endoderm is the archenteron or primitive gut. In coelenterates and sponges development stops with this two-layered or gastrula condition.

In higher forms, the mesoderm or third germ layer is formed immediately following gastrulation. At this stage in mammals and most other vertebrates a primitive streak appears along the dorsal midline of the posterior portion of the blastoderm (embryo). When sectioned, the primitive streak appears as a thickened band which is continuous with the ectoderm. The ventral side of this streak produces many cells which organize as a sheet of mesoderm at each side and spread both laterally and caudally between the other two germ layers. The mesoderm continues to spread until it finally eneircles the endoderm. The mass of cells thus formed, is soon divided into two layers by the development of a small cavity in it at each side of the archenteron. These cavities grow ventrally in the mesoderm until they meet each other at the ventral side of the archenteron. This cavity is the coelom or body cavity. The layer of mesoderm forming the outer or lateral wall (next to the ectoderm) of the coelom is known as somatic or parietal mesoderm and that on the side next to the endoderm is the splanchnic or visceral mesoderm. Later in development, when the somatic layer unites with the ectoderm, it forms the somatopleure (body wall). Similarly the splanchnic layer unites with the endoderm to form the splanchnopleure or wall of the alimentary canal.

Almost coincidental with the early development of the mesoderm, but independent of it, is the formation of the neural plate as a thickening and depression in the ectoderm along its dorsal midline. This begins just above the primitive streak mentioned previously and grows anteriorly. This is the portion from which the nervous system develops. This plate sinks, neural folds develop along its sides form- 

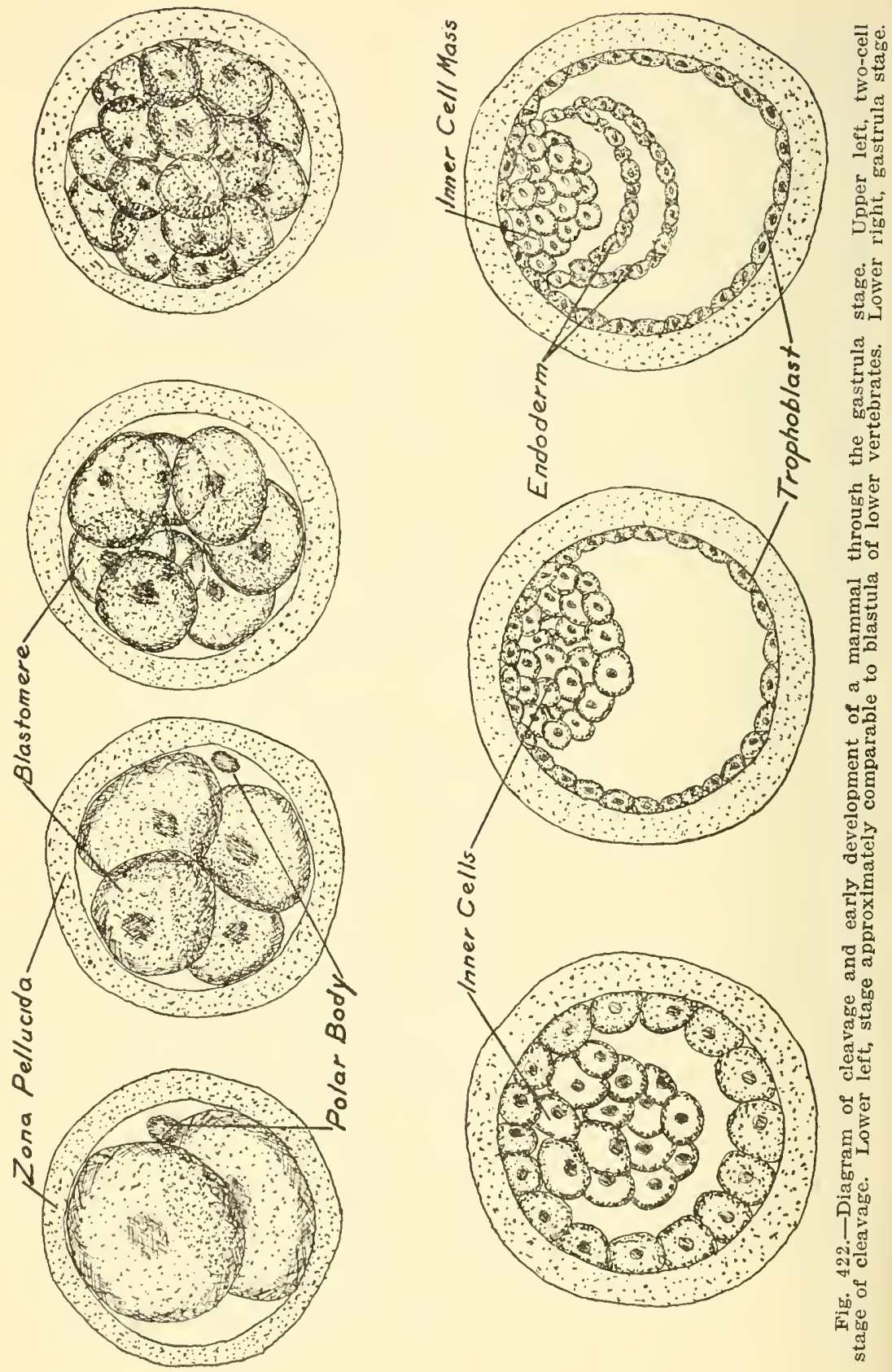
ing a neural groove. These folds meet each other over the groove to form the neural tube (Fig. 423).

The mesoderm along each side of the neural plate becomes organized into blocklike thiekenings. These are somites, and they are paired opposite each other, marking segmentation in the body. In a ehick embryo of thirty-six hours of incubation there are fourteen pairs of somites and in a pig embryo 6 millimeters long there are thirty-two pairs.

The younger embryos of different groups of vertebrates are so similar that it is impossible to distinguish them from each other. This is illustrated in Fig. 444 in a later chapter. At a sufficiently early stage, the embryos of the human being, the pig, the rat, the alligator, the salamander, and the fish all appear very similar. The gill slits and segments are conspieuous in all of them. Mueh later the limbs develop from lateral pairs of limb buds in the mesoderm. The hind limbs develop first and the front ones follow. The human body ean barely be discerned in embryos of one month, and the embryo must be nearly two months of age before it ean be identified definitely as human on the basis of morphologieal features.

\section{Organs and Systems}

After the three germ layers (ectoderm, endoderm, and mesoderm) have been established in the embryo, the next step is differentiation of these layers each in various ways for the formation of partieular organs and systems of organs. The fate of the germ layers has been coneisely summarized in the last paragraph in the chapter on metazoan organization.

Embryonic Membranes.-All three of the terrestrial groups of vertebrates (reptiles, birds, and mammals; i.e., amniota) produce extensive embryonic membranes as a feature of their development which is not found in the aquatie forms. These membranes serve to give the developing embryo added protection, as well as inereased faeilities for the functions of nutrition, respiration, and excretion. In birds and reptiles the early embryo is flat and the somatoplenre extends over the surface of the yolk far beyond the limits of the embryo proper. A fold of this sheet of somatopleure (ectoderm and parietal mesoderm) grows dorsally along each side of the embryo (Fig. 424). These folds finally meet each other above the embryo and fuse together, thus forming an enelosed eavity between the dorsal sur- 
face of the body and this new membrane. The name of this membrane is amnion and the eavity formed is the amniotic cavity. The superficial limb or layer of the original fold of somatopleure over the body is the serosa. In mammals the serosa unites with another embryonic membrane, the allantois, to form the real chorion which is highly vascularized and serves as the embryonic portion of the placenta. The allantois is a ventral evagination (outgrowth) from the ventral side

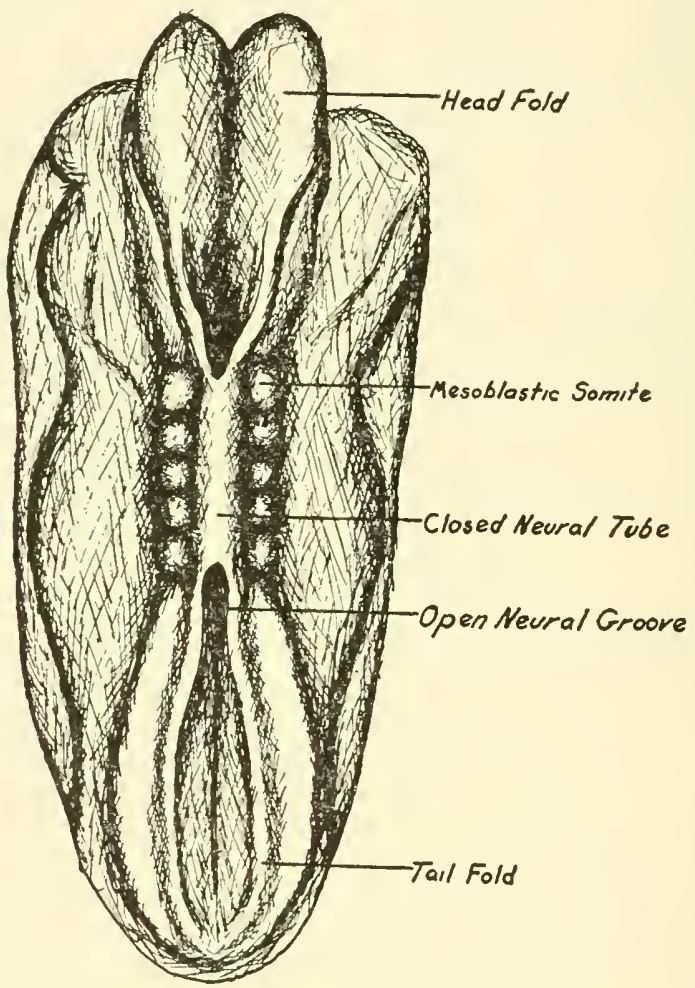

Fig. 423-Dorsal view of an embryo in the neural tube stage of development. Both ends of the tube are still open. The brain forms at the anterior end later.

of the cloaca of the embryo. It extends out through the open, ventral side of the body and spreads between the amnion and serosa until it covers most of the body like a double-walled sac. After development is complete, its stalk within the body cavity is retained as the urinary bladder. In embryonic birds and reptiles the allantois thus lies quite close to the shell membrane and since it is richly vaseularized, serves as the respiratory organ. 
In mammals, the allantois spreads between the amnion and serosa in about the same way, and becomes fused with the inner side of the serosa to form the chorion. Many branched processes, chorionic villi, extend from the outer or serosa surface of this membrane and come to fit into corresponding pits in the internal uterine wall of the mother.

The yolk sac is another membrane which extends ventrally from the mid-gut and out through the ventral side of the body wall just anterior to the allantois. This structure is present in the fish and amphibia as well as in the amniotes. It is large in the shark for

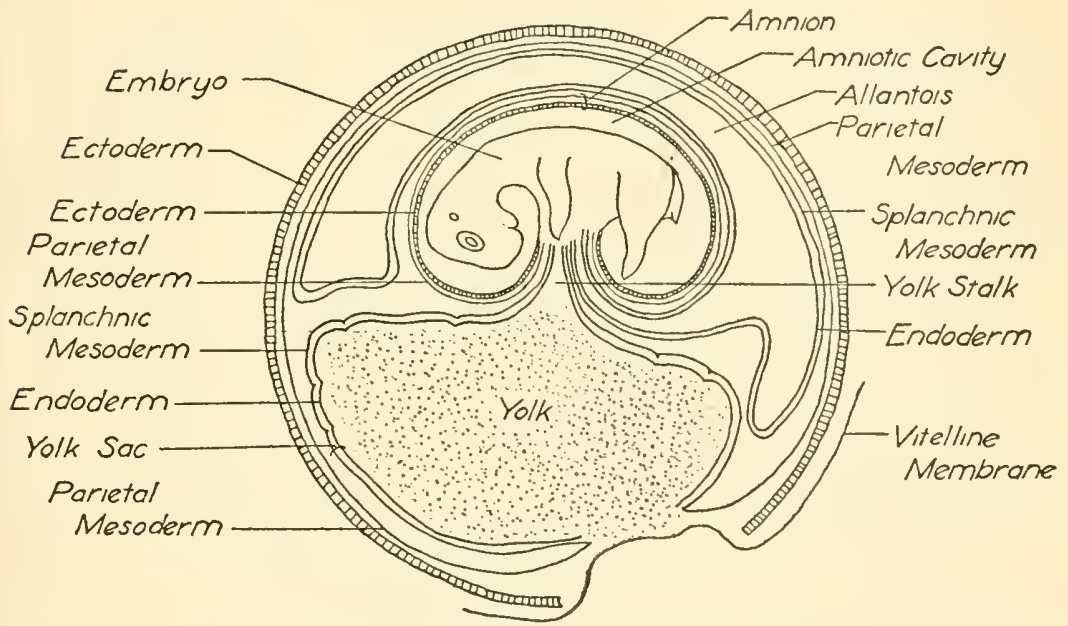

Fig. 424.-Diagram showing development of the embryonic membranes of a vertebrate. The amnion and serosa (chorion) each consists of a fusion of ectoderm and somatic mesoderm: while the yolk sac and allantois each consist of a fusion of endoderm and splanchnic mesoderm. The serosa is composed of the two outer layers of the diagram. Notice that the allantois is a double-walled sac from the ventral stalk, and it extends well around the embryo.

example. In reptiles and birds it carries the large mass of yolk used for nutriment by the embryo. In mammals it is much reduced beeause the embryo soon develops a means of nourishment through the placenta.

Placenta.-This membranous sae in which the embryo and later the fetus (designation after distinct body form is apparent) is formed from two sources, one embryonic and the other maternal. As suggested in the paragraph above, the chorion of the embryo and the mucous membrane (internal lining) of the uterus unite (actually 
fuse in some forms) to form this saclike organ, the placenta. The chorion is richly supplied by blood from the fetus while the uterine layer is similarly supplied by maternal blood. Although the embryonic and maternal parts of the placenta may be almost interwoven in mammals like the human, there is no actual circulation of maternal blood through the vessels of the fetus. The exchange of materials (nutrition, respiration, exeretion) is by diffusion through the tissues here in this intimate relation. As in the ease of circulatory connec-

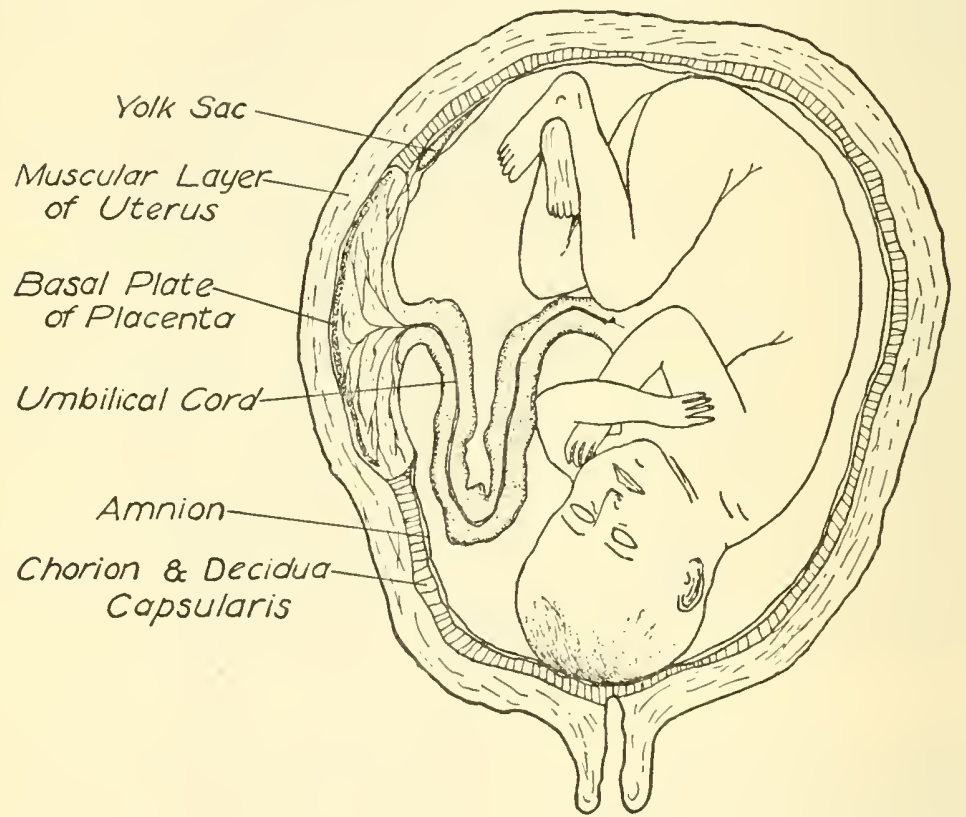

Fig. 425.- - Sectional view of a fetus in normal position in the uterus, showing also the intimate relations of embryonic membranes and uterine wall. The chorion is the outer embryonic membrane. (Modified after Ahlfeld.)

tions, there are no nerves passing from parent to fetus. The stalklike extension from the abdomen of the fetus to the placenta is the umbilical cord. It carries the umbilical arteries and vein, extending from the chorion to the fetus; the allantoic stalk; and, a vestigial yolk stalk from the intestine. The navel of the adult is the scar where the body wall has elosed in about the umbilical cord at the point of its severance at birth. The fetus is suspended within the amnistic eavity which is filled with the watery, lymphlike amniotic fluid. 


\section{CHAPTER XLIII}

\section{GENETICS AND EUGENICS}

(By Frank G. Brooks, Cornell College, Iowa)

\section{The History of a Great Discovery}

"Like father like son" is an ancient adage. Since man has been able to think, he has pondered the problem of heredity. Although very early he had observed evidence of the inheriting of parental characters by offspring of the various forms of life with which he was familiar and was convinced by these observations that heredity did take place, he has not known the "how" or "why" of it until recently. The fundamental law on which heredity is based was announced by Gregor Johann Mendel in 1866. However, Mendel, an Austrian monk, published his discovery in an obseure journal and it did not receive general recognition until its rediscovery in 1900. Therefore, genetics is really a twentieth century science.

Mendel's success in finding the underlying principle of hercdity was due, in part, to his choice of an experimental unit. Instead of following the usual trend by considering how a parent conveys his various traits to his offspring, thus making the individual the unit of observation, he chose a definite inheritable character and considered how it was transferred from many parents to all their offspring. In addition to this wise choice of an investigational unit, the patience, mathematical ability, skill as a gardener, and analytical insight of the investigator contributed also to the success of the research.

Mendel chose the garden pca as the material for his work. This was a fortunate choice for, although the law that he was to discover underlies practically all inheritance, it is not always as free from complications as it is in the case of those traits of the pea which he investigated.

\section{Mendel's Law}

For one of his projects he planted seeds from stock that had been known to produce nothing but tall plants for many generations. $\mathrm{He}$ planted also seeds from stock that had produced nothing but dwarf plants. When the two types of plants were in blossom, he transferred the pollen from the stamens of the one to the stigmas of the other. 
The seeds that resulted from the cross were collected and planted. To the amazement of the investigator they produced nothing but tall peas; peas as tall as the original tall parents. Dwarfness seemed to be lost and tallness was certainly dominant. A less thorough investigator might have called his experiment finished and have proclaimed as a rule, the fact that a trait can disappear when it is crossed with its opposite. But not so with the patient monk. He pollinated these plants with their own pollen and planted another generation. This

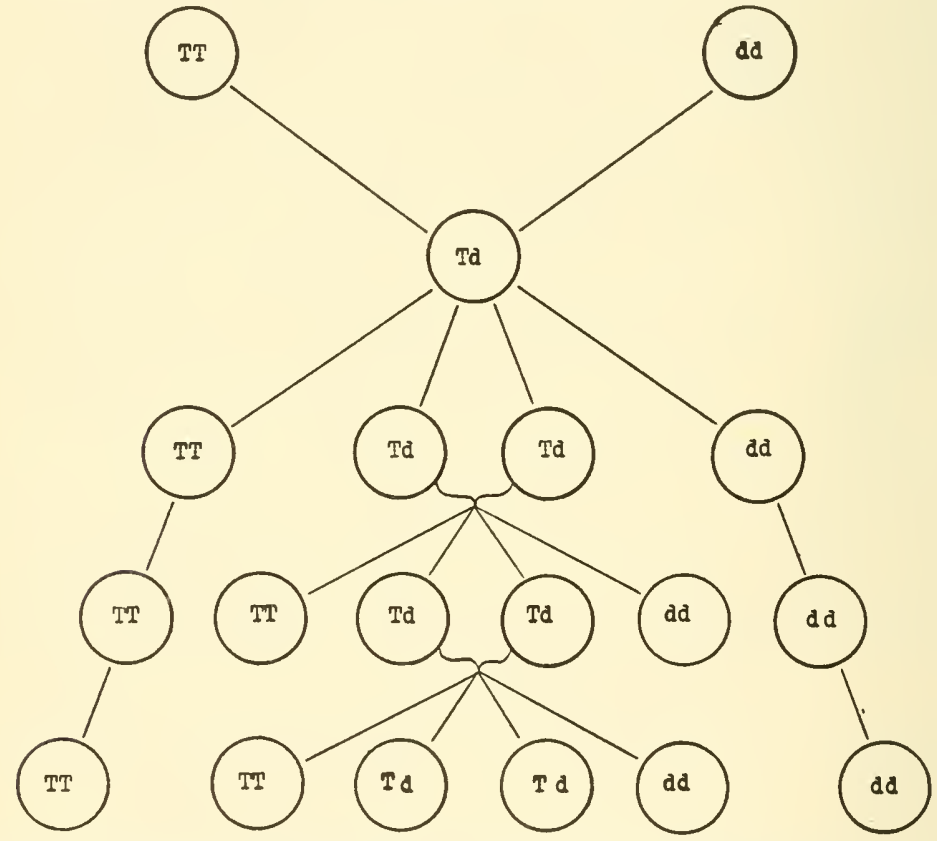

Fig. 426.-Diagram to show the result of crossing tall and dwarf peas. The 1:2:1 ratio appears in the $\mathbf{F}_{2}$ generation.

time three-fourths of the plants were tall and one-fourth of them were as short as the original dwarf parents. Not being ready even yet to formulate a law, he self-pollinated his plants for several more generations and got results that required his best mathematical skill to interpret. The dwarf peas produced only dwarf peas. Of the tall peas, one-third of them (constituting one-fourth of the whole number) produced tall peas without deviation, but the other two-thirds (half of the whole number) produced stock that repeated the produc- 
tion of tall and dwarf peas in the same proportion as did the preceding generation. Here, then, was a definite ratio of $1: 2: 1$. Mendel tested his findings with six other traits of the pea and with more than a dozen other kinds of plants, and after verifying his results he was able to announce the following law: When members of a species having contrasting characters are crossed, all the immediate offspring will show the trait that is dominant, but if the members of this generation are bred to themselves, one-fourth of the offspring will show the dominant trait and breed true for it; one-fourth will show the recessive trait and breed true for it; the remaining two-fourths of the offspring will show the dommant trait, but will reproduce the contrasting characters in the same proportion as did their immediate parents.

\section{Derivatives of Mendel's Law}

From the fundamental law which Mendel discovered, several corollaries can be drawn, based on factors responsible for the distributional behavior of inheritable characters. These corollaries are:

Principle of Dominance.-The determiner for one member of a pair of contrasting characters (pairs of contrasting characters are called allelomorphs) may take precedence over the other member when the two have been brought together in a cross between unlike parents. Which trait is dominant and which is recessive can be determined only after a cross has been made, and the determination holds only for the species observed. Thus, tallness may be dominant over dwarfness in one species of plant and recessive in another. In many cases, dominance is not complete, and in some instances it is lacking altogether. These cases will be discussed later in this chapter.

Independence of Unit Characters.-The determiner for any given character acts as an independent hereditary unit as it is passed along from generation to generation. In each generation it may have a different set of associates, but its associations in one generation do not affect the company it may keep in the next. Thus tallness in peas may be associated with yellow pod and with wrinkled seed coat in one generation, but it may form a combination with green pod and smooth seed coat in the next. This important principle is responsible for the great variation we find in plants and animals and makes it possible for a breeder to bring about any desired combinations of the traits 
possessed by the species of plant or animal with which he is working. Thus the modern Shasta daisy has a combination of the three important characters: size of flower, gloss of petal, and prolific growth, each of which was procured from an original variety having one, but not the other two, of these traits.

Principle of Segregation.- Since unit characters are independent, it follows that they can go into various combinations and are free to segregate out again. This was illustrated by dwarfness in Mendel's peas which went into a cross with tallness, but segregated out again to form peas that were as consistent for dwarfness as was the original dwarf stock. To illustrate both the principles of unit characters and the related phenomenon of segregation we might use an analogy. If a gallon of white marbles were mixed with a gallon of black marbles, they would make a mass of marbles that would look speckled-gray. But the mixture is not irrevocably gray as would be the case if a gallon each of black and white paint had been mixed. Inheritable traits, like marbles, can be placed in various combinations. These may last for one generation only, after which they can make new combinations with other traits.

\section{The Physical Basis}

With the simple facts in mind of how heredity works, it is desirable to consider the germinal background of the laws governing it.

The tiny sperm cells of both plants and animals and the egg cells which, deprived of their food-containing yolk, are about equally small, contain within them something that determines all the traits which the individual resulting from their combination will develop. In fact, the body of the sperm cell and the nucleus of the egg contain little else than a material that carries the combined inheritable traits of one generation over to the next. This important material is called chromatin and during mitosis it becomes arranged in series of hereditary units called genes. Each gene has a definite causal relationship to an inheritable trait. Both the sperm cell and the egg cell contain a complete gene complex, and each, under favorable conditions, could produce a complete individual.

When the two totipotential gametes are brought together at fertilization, the resulting zygote contains two genes for each allelomorph. When the two genes are for the same trait, the resulting individual 
is said to be homozygous for that trait and, of course, shows it. When each of the two genes is for a different member of the allelomorph, in which case we use the term heterozygous, one gene takes precedence over the other, but the recessive gene is in no way destroyed; it simply lies dormant and bides its time. How these genes are arranged into a limited number of "packages" called chromosomes and how genes and chromosomes are distributed in the process of sperm and egg formation was explained in Chapter VIII.

\section{Plotting Crosses}

The Monohybrid Cross.-When the genetic constitution of parental stocks is known, it is possible to plot ont the results of various kinds of crosses. The checkerboard is the simplest device for such

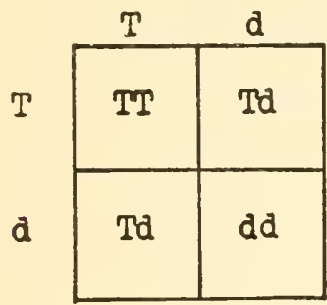

Fig. 427.

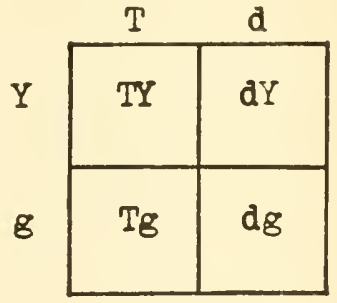

Fig. 428.

Fig. 427. - The outcome of a monohybrid cross between two heterozygous individuals is according to the ratio $1: 2: 1$, i.e., (TT), (Td), (Td), (dd).

Fig. 428. - The checkerboard may be used to determine the possible gene combinations in the ova and sperm of a dihybrid cross.

computations. The genetic constitution of the various kinds of male gametes are set down along the ordinate and that of the various kinds of female gametes along the abscissa. These values are then copied into the squares horizontally and vertically and their sums give the values of the various kinds of gametes that will result from the cross. In the $\mathrm{F}_{2}$ cross of Mendel's experiment with tall and dwarf peas, half of the male gametes and half of the female gametes contained a gene for tallness $(\mathrm{T})$ and half in cach case contained a gene for dwarfness (d). The outcome, therefore, is shown in Fig. 427.

The Polyhybrid Cross.-It is often desirable to know the outcome of a cross in which two or more allelomorphs are considered together as in the case of crossing a tall, green-podded pea $(\mathrm{Tg}) *$ with a dwarf,

- It is customary to Indicate the dominant trait by using a capital letter and the recessive trait by using a lower-case letter. 
yellow-podded pea $(d Y)$. The determination of the possible kinds of gametes may be simplified by first making a small checkerboard for them (Fig. 428). Thus we find that in a dihybrid cross there are four kinds of male gametes and four kinds of female gametes which may be listed as TY, Tg, dY, dg. Now we make a checkerboard of sixteen squares and proceed as we did before. (Fig. 429.)

The computation of the dihybrid cross indicates that ninc-sixteenths of the progeny will show the two dominant traits because (Fig. 429) we find at least one $\mathrm{T}$ and one $\mathrm{Y}$ together in that many squares. Threesixteenths will show the dominant trait of the first allelomorph and the recessive trait of the other. Another three-sixteenths will show the re-

\begin{tabular}{|c|c|c|c|}
\hline$T Y$ & $\mathrm{Tg}$ & $d Y$ & $d g$ \\
\hline $\begin{array}{l}T Y \\
T Y\end{array}$ & $\begin{array}{l}T g \\
T Y\end{array}$ & $\begin{array}{l}d Y \\
T Y\end{array}$ & $\begin{array}{l}d 8 \\
\text { TY }\end{array}$ \\
\hline $\begin{array}{l}T_{Y} \\
T_{B}\end{array}$ & $\begin{array}{l}T_{B} \\
T_{B}\end{array}$ & $\begin{array}{l}d Y \\
T_{B}\end{array}$ & $\begin{array}{l}d g \\
T_{g}\end{array}$ \\
\hline $\begin{array}{l}T Y \\
d Y\end{array}$ & $\begin{array}{l}T_{B} \\
d Y\end{array}$ & $\begin{array}{l}d Y \\
d Y\end{array}$ & $\begin{array}{l}\mathrm{dg} \\
\mathrm{dY}\end{array}$ \\
\hline $\begin{array}{l}T Y \\
d_{g}\end{array}$ & $\begin{array}{l}\mathrm{T}_{8} \\
\mathrm{~d}_{8}\end{array}$ & $\begin{array}{l}d Y \\
d g\end{array}$ & $\begin{array}{l}\mathrm{dg} \\
\mathrm{dg}\end{array}$ \\
\hline
\end{tabular}

Fig. 429.-The outcome of a dihybrid cross between two heterozygous individuals is according to the ratio $9: 3: 3: 1$.

cessive trait of the first allelomorph and the dominant trait of the second. One-sixteenth of the offspring will show both recessive traits. It will be noticed, however, that the nine squares showing individuals that will be tall and yellow vary with each other in regard to their entire content. Further examination of the squares will indicate that there are nine different combinations of letters and that in only one case as many as four are exactly similar. Individuals whose genes cause them to look alike are called phenotypes; those whose genes are exactly alike are called genotypes.

A trihybrid cross, such as one between a tall yellow-podded pea with a wrinkled seed-coat (TYw) and a dwarf green-podded pea with a 
smooth seed-coat ( $\mathrm{dgS}$ ) can be plotted by using a checkerboard of sixty-four squares. The ratio of phenotypes of a trihibrid cross is $27: 9: 9: 9: 3: 3: 3: 1$.

The Back Cross.-The checkerboard is also useful in computing the results of a cross between a heterozygous and a homozygous individual as would be the case in which the offspring of a cross between a homozygous dominant and a homozygous recessive individual is bred back to either of the parental stocks; a procedure often followed in practical animals and plant breeding. When such a cross is made to the homozygous dominant stock the results, in terms of pea traits, are TT, TT, Td, Td. (Fig. 430.) All four will look alike, i.e., they are phenotypes, but there are two pairs of genotypes.

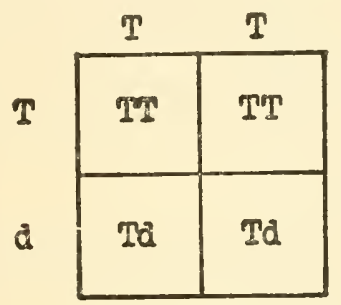

Fig. 430.

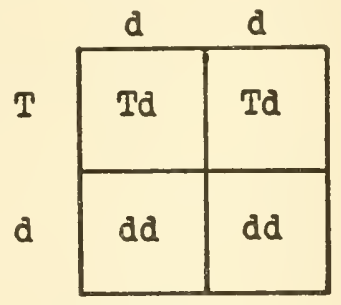

Fig. 431.

Fig. 430.-A cross between heterozygous and homozygous tall peas produces a $2: 2$ ratio.

Fig. 431. A cross between heterozygous tall and homozygous dwarf peas also produces a $2: 2$ ratio.

When the heterozygous stock is bred back to the homozygous recessive parental stock, a $2: 2$ ratio is also produced. This time the two kinds are not only genetically unlike, but they also appear unlike (Fig. 431).

\section{Complications of Mendelian Inheritance}

If all inheritable characters followed Mendel's law in as simple a fashion as do the pea traits that Mendel first investigated, the science of genetics would be much more easily mastered. Unfortunately this is not the case. Although Mendel's law is found so consistently as the underlying principle of heredity that it can be said to be practically universal in its application, it is often modified and complicated in various ways.

Multiple Allelomorphs.-Instead of the allelomorph always consisting of the usual two factors, a larger number of alternatives sometimes 
appears. Dominance in these cases occurs in a graded series, each member, between the extremes, being dominant to the lower members and recessive to the higher members of the series. A simple case of multiple allelomorphs is found in the inheritance of color in rats where there are three factors; namely, ordinary pigmentation, ruby-eyed dilution, and albinism. In a cross between heterozygous parents any two of the three factors may be brought together in the offspring. If ordinary pigmentation is present with either of the other two, it will determine the color, since it is first in the series of dominance. If the other two are the ones present, the color will be ruby-eyed dilution, the second member of the series.

A larger series is presented by eyc color in the common fruit fly, Drosophila melanogaster, in which ease there are eleven members which are, in the order of their dominance: red, apricot, coral, ivory, ecru, buff, tinged, blood, cherry, eosin, and white. Each parent may have any two of these in its germ plasm and transmit either of them to the offspring.

Multiple Genes.-Several cases formerly interpreted to be simple blending inheritance not conforming to Mendel's law have been explained by the discovery that there ean be more than one allelomorphic pair conccrned with the inheritance of the trait. Thus, instead of there being one gene located at some definite place on a chromosome, there are two or more genes variously located. Cases of multiple genes fall into two categories. In the one, each gene inherited as a dominant, produces part of the result, and the effects are cumulative. In the other type of case, the inheritance of one dominant gene produces the entire effect.

A common example of the cumulative type is the inheritance of skin color in man. It has long been known that a cross between a white person and a pure-blooded negro produces offspring of a medium shade called mulatto, and that a cross between two mulattocs will produce offspring with a range of color varying from intense black to a shade that may allow the person to pass for white. This is explained by the fact that there are two allelomorphic pairs concerned with the inheritance of color, the dominant of either of which will produce a certain amount of pigment per square millimeter of skin surface. The negro of pure skin inheritance is homozygous, having the dominant genes of each pair. Using $\mathrm{P}$ and $\mathrm{P}^{\prime}$ to represent the dominant factors of these two allelomorphs and $p$ and $p^{\prime}$ the 
recessives, the homozygous negro would have $\mathrm{PPP}^{\prime} \mathrm{P}^{\prime}$ while the homozygous white person would have $\mathrm{ppp}^{\prime} \mathrm{p}^{\prime}$ and the mulatto resulting from the first cross would have $\mathrm{PpP}^{\prime} \mathrm{p}^{\prime}$. The cross between two mulattoes would be a dihybrid eross which could be plotted by a checkerboard essentially similar to that of Fig. 432. The accompanying chart (Fig. 432) shows that there can be four, three, two, one, or no dominant factors present, thus accounting for the series known under the following technical terms: "negro," "chocolate," "mulatto," "quadroon" and "pass for white."

\begin{tabular}{|c|c|c|c|c|}
\hline & & $-p$ & $g_{1}$ & \\
\hline & $\begin{array}{c}\text { PP' } \\
\text { Pp: } \\
\text { negro }\end{array}$ & $\begin{array}{c}\text { PP' } \\
P P^{\prime} \\
\text { choc. }\end{array}$ & $\begin{array}{c}P^{\prime} \\
P P^{\prime} \\
\text { choc. } \\
\end{array}$ & $\begin{array}{c}\text { pp' } \\
\text { Pp' } \\
\text { mulat }\end{array}$ \\
\hline & $\begin{array}{l}\text { PP' } \\
\text { Pp' } \\
\text { choc. }\end{array}$ & $\begin{array}{c}\text { Pp' } \\
\text { Pp' } \\
\text { mulat }\end{array}$ & $\begin{array}{c}\mathrm{pP} \\
\mathrm{Pp} \\
\text { mulat }\end{array}$ & $\begin{array}{c}p p^{\prime} \\
\text { Pp: } \\
\text { quadr }\end{array}$ \\
\hline & $\begin{array}{c}\mathrm{PP} \\
\mathrm{pP} \\
\text { choc. }\end{array}$ & $\begin{array}{c}P p^{\prime} \\
p P^{\prime} \\
\text { mulat }\end{array}$ & $\begin{array}{c}\mathrm{pP} \\
\mathrm{p} \mathrm{P}^{\prime} \\
\text { mulat }\end{array}$ & $\begin{array}{c}\mathrm{pp}^{\prime} \\
\mathrm{pp} \\
\text { quadr }\end{array}$ \\
\hline & $\begin{array}{l}\text { PP' } \\
\text { pp } \\
\text { mulat }\end{array}$ & $\begin{array}{l}\mathrm{Pp} \\
\mathrm{pp}\end{array}$ & $\begin{array}{c}\mathrm{pP} \\
\mathrm{pp} \\
\text { quadr }\end{array}$ & $\mathrm{pp}_{\text {wh }}^{\prime}$ \\
\hline
\end{tabular}

Fig. 432.-The inheritance of color by children of mulatto parents can be shown by the checkerboard for a dihybrid cross.

The other type of case in which multiple genes oceur is exemplified by a certain brown-seeded variety of oats in which there are two pairs of genes concerned with the determination of color. Here the presence of the dominant gene of either pair produces the entire effect. This case, too, can be plotted on the dihybrid checkerboard, but the result is a $15: 1$ ratio, for fifteen of the sixteen squares would show at least one dominant gene. Crosses between a red and a white variety of wheat in which there are three pairs of genes for color, produce a $63: 1$ ratio. This is a trihybrid cross in which each dominant gene can produce the entire effect.

Complementary Genes.-In a number of cases color is produced by two allelomorphic pairs of genes, the dominants of which must react on each other to produce the color effect. In the sweet pea, if the dominant factor of the red allelomorph $(\mathrm{Rr})$ is acted on by the 
dominant faetor of the color allelomorph (Cc), then red is produced. If either of these dominant genes is present without the other, white flowers are produeed, and if two white-flowered plants of the genetie constitution Rree and rrCe are erossed that part of the progeny will be red in which both $\mathrm{R}$ and $\mathrm{C}$ are inherited. A student familiar with elementary chemistry may understand this better if he thinks of the red factor as being represented by a colorless solution of phenolphthalein and the color faetor as a eolorless alkaline solution. When both solutions eome together, red is produced, but neither solution ean produce the color without the other.

Supplementary Genes.-But sometimes purple sweet peas appear when two white varieties are crossed or when a red variety is crossed with a white variety. Purple is produced by a third gene which intensifies red if it is already present, but has no effect unless the $\mathrm{RC}$ genes are likewise inherited. Thus we find that its effeet is to supplement that of the two genes $\mathrm{R}$ and $\mathrm{C}$ which are complements to each other in the production of red. The aceompanying list shows some of the surprising erosses that may be made with sweet peas. Of course the eombination of genes shown in the last column is not the only one that will come about in each of the various cases, but it is the one that emphasizes the point desired. Bb represents the intensifying allelomorph.

$$
\begin{aligned}
& \text { White + White = Purple } \\
& \text { Red + White = Purple } \\
& \text { Purple + Red = Red } \\
& \text { Purple + White = Purple } \\
& \text { Purple + White = White } \\
& \text { Purple + White = Red }
\end{aligned}
$$$$
\begin{aligned}
& \mathrm{ReB}+\mathrm{r} C b=\mathrm{RCB} \\
& \mathrm{RCb}+\mathrm{ReB}=\mathrm{RCB} \\
& \mathrm{RCB}+\mathrm{RCb}=\mathrm{RCb} \\
& \mathrm{RCB}+\mathrm{reb}=\mathrm{RCB} \\
& \mathrm{RCB}+\mathrm{reb}=\mathrm{reB} \\
& \mathrm{RCB}+\mathrm{reb}=\mathrm{RCb}
\end{aligned}
$$

Lack of Dominance.-In several classic eases, some of which are among plants and others among animals, neither factor of an allelomorph is dominant over the other. In the $\mathrm{F}_{1}$ generation, these eases seem to produce perfect examples of blending inheritance, but the $\mathbf{F}_{2}$ generation exemplifies the $1: 2: 1$ ratio so beautifully that these exceptional eases are often used to explain Mendel's law to beginning students.

The Andalusian breed of ehickens includes both black and white individuals. When black fowl are crossed with white fowl, all the offspring are of a slate color technically known as "blue." When a blue 
ehicken is crossed with another blue chicken, one-fourth the progeny are black, one-fourth are white and the remaining two-fourths are blue. The blacks and the whites are homozygous for their respective colors while the blues are heterozygous. The inheritance of color in short-horn cattle, and of the color in the four-o'clock flower are also examples of this. In still other cases in which there is not neutrality of dominance, the dominant effect may not be complete. Very often the heterozygous individuals can be picked out from the homozygous dominant ones by casual inspection.

\section{Inheritance of Sex}

In certain lower phyla of animals and in the early embryos of higher forms, including mammals, both male and female reproductive systems are present in each individual. Typically in

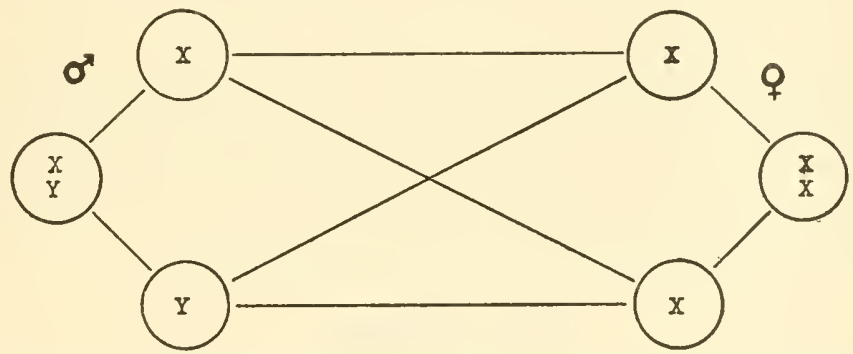

Fig. 433.- Half of all possible fertilization combinations will be (XY) and half will be (XX). The XY combination will be males and the $\mathrm{XX}$ will be females.

higher forms, one of these systems is repressed, and we speak of the individual as being either male or female. The determination of whether the male or the female system will develop is an inherited trait. The chromosome that carries the gene of sex determination has come to be designated as the $\mathrm{X}$-chromosome. In typical cases, the female has in each of her germ cells, two of these X-chromosomes and the male has only one. Therefore, when, in the process of spermatogenesis, the chromosomes match with their homologous chromosomes and separate again to give each of the resultant cells one complete set of ehromosomes, the X-chromosome cannot pair with another like itself, and when the chromosomes are distributed in sets, half the sets will lack an X-chromosome. The sperm cells that do not have an X-chromosome will produce males while those that have such a chromosome will produce females. 
In other cases that include man and Drosophila, the X-chromosome will pair with a Y-chromosome which has been described as "an empty case" or a "ghost chromosome." In cases in which a Y-chromosome is present, we cannot say that the male has one less chromosome than the female, but it practically amounts to that.

\section{Linkage}

Since the number of inherited traits of an animal or plant is very great while the number of chromosomes is comparatively small, it is apparent that a single chromosome must contain many genes. This being so, there is not absolutely free assortment of traits as they are passed from parent to offspring, but rather the offspring must inherit his traits in groups, that are not, ordinarily, broken up. Therefore, when two genes, $\mathrm{A}$ and $\mathrm{B}$, are on the same chromosome of one of the parents, if the offspring inherits gene $A$, he must inherit gene $B$. In Drosophila we know that the gene which determines whether or not the wing will be fringed occurs on the same chromosome as the one that is responsible for the body being or not being black. Now if one of the parents is fringed-winged and black-bodied, the offspring will have to inherit both these traits from that parent if he receives either one of them. This phenomenon is called linkage.

\section{Sex Linkage}

Since there are other genes than those concerned with sex on the $\mathrm{X}$-chromosome, it is to be expected that their inheritance will differ somewhat in the two sexes. This is strikingly illustrated by certain abnormalities of man in cases where the abnormal condition is inherited as a recessive; for example, color blindness, blindness resulting from atrophy of the optic nerves (Leber's atrophy), and hemophilia or bleeder's disease, in which great loss of blood occurs even from slight wounds because clotting will not take place. Since the abnormality is a recessive trait produced by a gene on the $\mathrm{X}$-chromosome, its effect can be offset by a dominant for normal in the female, but not in the male, because in the latter case there is not another $\mathrm{X}$-chromosome present. Therefore, the daughter of a color-blind father will not show the defective trait but may pass it to her son.

Fig. 434 shows a cross between a color-blind father and a normal mother. Two kinds of male gametes are produced, half of which carry the father's gene for color blindness on the X-chromosome, and half of 
which have no X-ehromosome and, therefore, have no such gene. All female gametes have one $\mathrm{X}$-elromosome bearing a gene for normality. The various possible combinations of the two kinds of sperm with the single type of egg will produce two types of offspring; namely, sons who will be strietly normal in regard to the trait in question and daughters who will inherit the gene, but who will not show the trait because its effeet will be overeome by the presence of a dominant gene

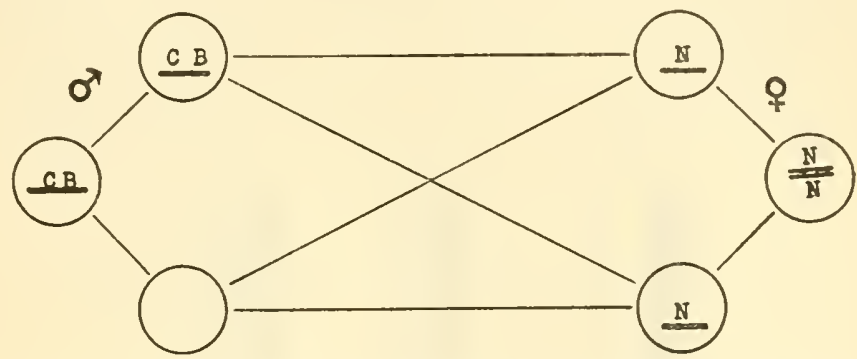

Fig. 434.-In the cross between a color-blind male and a normal female, the results $(\mathrm{N}, \mathrm{cb}),(\mathrm{N}, \mathrm{cb}),(\mathrm{N}),(\mathrm{N})$ indicate that all daughters, being heterozygous, will not show the defect though they might transmit it to their offspring, while the sons are entirely free from it.

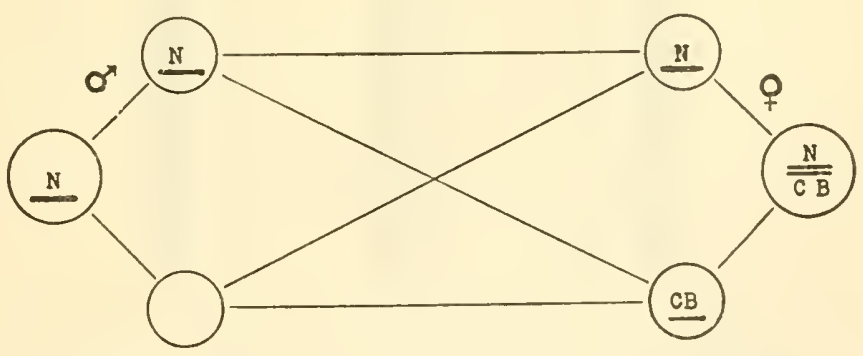

Fig. 435.-Results ( $N, N),(N, c b),(N),(c b)$. In a cross between a normal male and a female who is heterozygous for color blindness, half of the sons will be normal and half will be color blind. Half the daughters will be homozygous for normal and half will be heterozygous.

for normal. Therefore, the daughters will be heterozygous for the trait and might be spoken of as carriers. Fig. 435 shows a eross between sueh a daughter and a normal male.

Inspection of this figure will show that sueh a eross will produee four types of offspring; namely, normal daughters, "earrier" daughters, normal sons, and eolor-blind sons. Thus, we see that half the grandsons of a color-blind man will receive the trait through their mothers, and that all daughters and half the granddaughters of the 
color-blind male will be earriers of the trait, but will have normal color vision. Of course, from the mating of such a person with a colorblind man, all the sons and half the daughters will be color blind, but a young woman who is used to having her father buy pink neckties thinking they are blue, is not likely to marry a man similarly defective.

\section{Crossing Over}

Genetics might be defined as an exact science which has exceptions to many of its rules. A previous paragraph describes the principle of linkage which provided for the inheriting as groups of those traits whose genes occur together on a chromosome. Certain gametogenetic

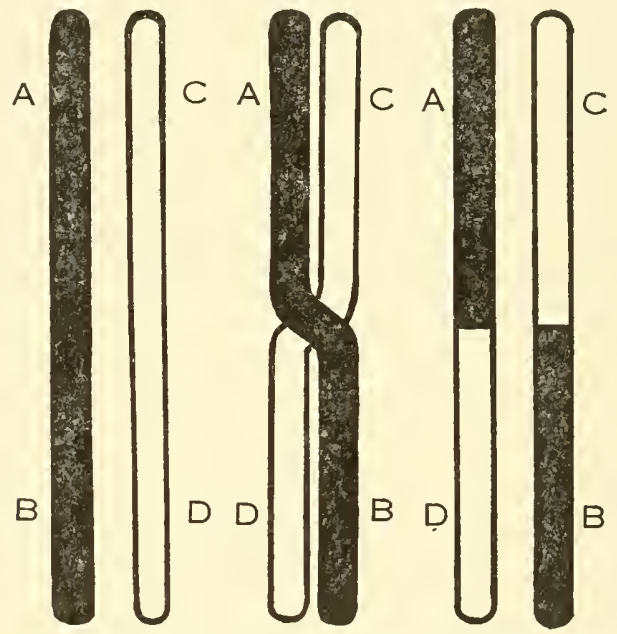

Fig. 436.-Crossing over occurs when chromosomes break apart after synapsis.

accidents bring about occasional exceptions to this principle. It will be remembered (page 114) that at synapsis, the chromosomes pair and the homologous chromosomes become loosely attached to each other. At times, the pairing chromosomes will even twist around each other. When they come to separate in the maturation division, instcad of making a complete separation, they sometimes break off at some point so that a part of each will be joined to a reciprocal part of the other, as is shown in Fig. 436. Thus, as a result of crossing over, instead of traits $\mathrm{A}$ and $\mathrm{B}$, and $\mathrm{C}$ and $\mathrm{D}$ being associated in the offspring as they were in the respective parents, $\mathrm{A}$ will be associated with $\mathrm{D}$, and $\mathrm{C}$ with $\mathrm{B}$. 


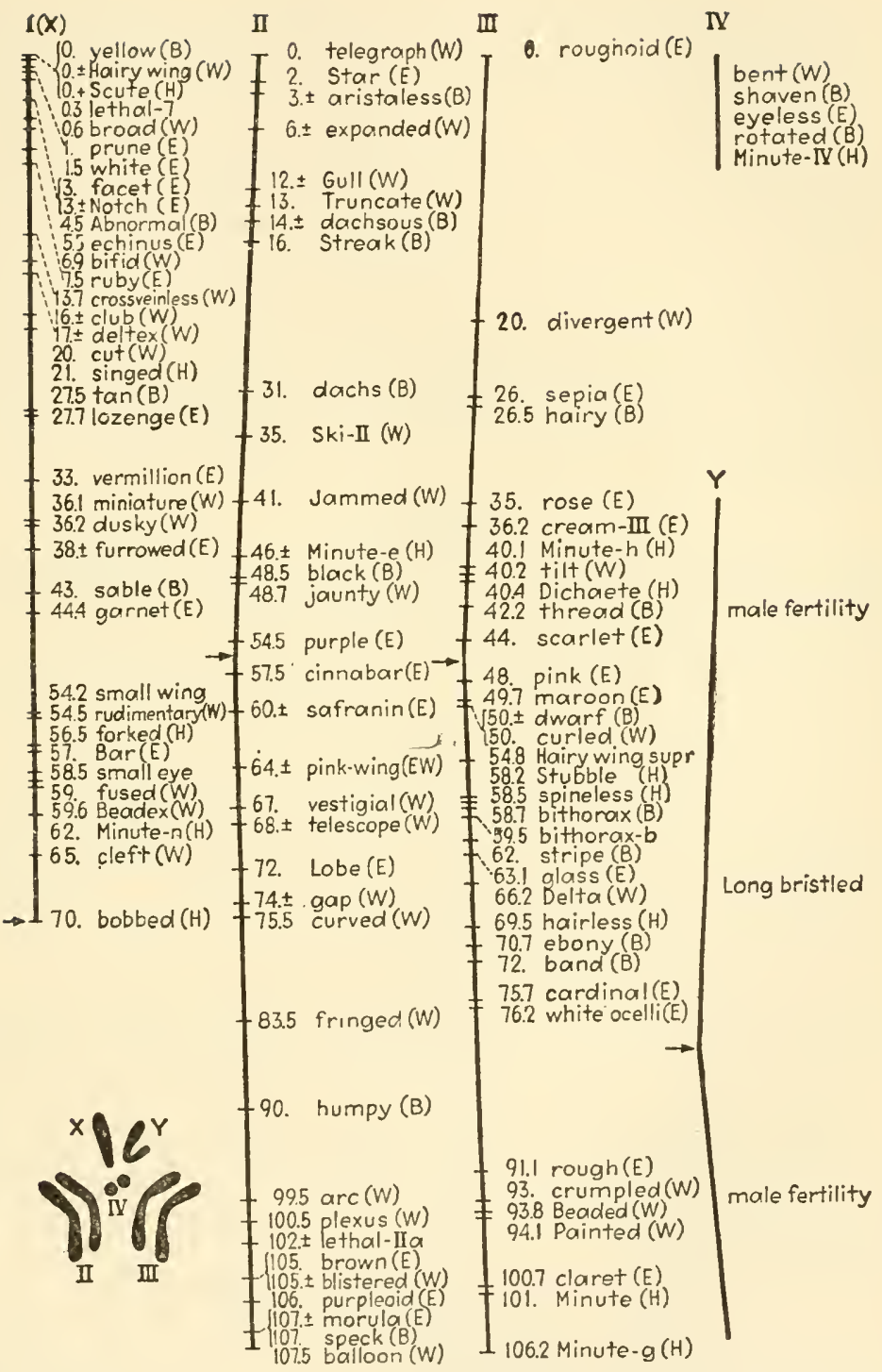

Fig. 437.-Chromosome map of drosophila. (From Sharp, Introduction to Cytology, published by McGraw-Hill Book Company.) 
Crossing Over, a Useful Tool.-The process of crossing over has proved to be a valuable means of determining something of the nature of genes and chromosomes and, especially in Drosophila, it has enabled us to locate the positions of many of the genes on their respective chromosomes. If two linked traits are separated every time erossing over oecurs, it is obvious that their genes lie at opposite ends of the ehromosome, but if they are separated only half the times, it is apparent that half the length of the chromosome lies between them. Therefore, by computing the percentages of such separations and noting which traits are affected, it has been possible to make chromosome maps showing the positions of the various genes and the distances separating them. The chromosome maps for Drosophila are quite complete (Fig. 437).

\section{Mutations}

When chromosomes separate from each other at synapsis, aside from breaking so that a piece of one will adhere to the correlative piece of the other, various additional "ehromosomal aceidents" will oceur which change the organization of the chromosome. A piece of chromosome, for example, might break off from the end of one ehromosome and adhere to the end of its homologue. Any disarrangement of the genes of a ehromosome results in structural or physiological ehanges in the organism into which it goes. Such changes are inheritable and are ealled mutations. Since there are places on the chromosomes where these accidents oceur more frequently than at other places, there is a tendency for certain mutations to oceur with fairly determinable regularity, say once in every hundred thousand births. Some mutations are useful and are preserved to the species to such an extent that De Vries believed they were the principal factor in bringing about evolution. Other mutations are a disadrantage to the speeies, and the organisms possessing them are eliminated in Nature's fierce struggle for existence unless they are sared from that cruel fate by man's interference.

\section{Human Heredity}

Man, very naturally, is interested most in the heredity of man. In spite of this supreme interest, his knowledge of his own heredity is much more limited than his knowledge of. inheritance in any of a number of other organisms. There are two reasons for this: namely, he is not free to experiment with his own kind; and, as would be expected, 
the application of Mendel's law to inheritable traits in this, the most complex of all living forms, is correspondingly complicated. Although there are cases in which the 1:2:1 ratio occurs in as simple a form as in Mendel's peas, there are many more cases in which the Mendelian principle is manifested as multiple allelomorphs, multiple genes, modifying factors, complementary factors, incomplete dominance, changeable dominance, irregular dominance, etc. There is a tendency among some to depreciate our knowledge of human heredity on the ground that there is so much that we do not know. It is right to admit the extent of our lack of knowledge, but it is wise to give proper credit to our present store of information and to take cognizance of the rapidity with which the gaps in our knowledge are being eliminated. One by one the complicated problems of human heredity are being solved by patient investigators, and by putting what we already know to use, we stimulate the accession of more data.

Some Cases of Human Heredity.-To give a complete summary of our knowledge of human heredity would be beyond the scope of this cliapter. The accompanying table gives some of the data that have been accumulated:

Data on HuMaN HeREdity

\begin{tabular}{|c|c|c|}
\hline TRAIT & HOW INHERITED & DOMINANCE \\
\hline $\begin{array}{l}\text { General Physique and Skeleton } \\
\text { Stature }\end{array}$ & $\begin{array}{l}\text { A composite } \\
\text { character }\end{array}$ & Many genes for shortness \\
\hline Body build & $\begin{array}{l}\text { Multiple genes, } \\
\text { two or more } \\
\text { pairs }\end{array}$ & $\begin{array}{l}\text { Factors for stoutness are } \\
\text { dominant }\end{array}$ \\
\hline Polydactyly (extra digits) & Simple & Abnormality dominant \\
\hline $\begin{array}{l}\text { Brachydactyly (short digits and } \\
\text { limbs) }\end{array}$ & Simple & Abnormality dominant \\
\hline $\begin{array}{l}\text { Symphalangism (fused fingers and } \\
\text { toes) }\end{array}$ & Simplo & Abnormality dominant \\
\hline $\begin{array}{l}\text { Zygodactyly (webbed fingers and } \\
\text { toes) }\end{array}$ & Simple & Abnormality dominant \\
\hline Club foot & Simple & Abnormality recessive \\
\hline Lobster claw (split hands and feet) & Simple & Abnormality dominant \\
\hline Exostoses (outgrowths of long bones) & Simple & Abnormality dominant \\
\hline Abnormal fragility of bones & Simple & Abnormality dominant \\
\hline $\begin{array}{l}\text { Amputation (entire absence of liands } \\
\text { and feet) }\end{array}$ & Simplo & Abnormality dominant \\
\hline Shortened arms & $\begin{array}{l}\text { Irregular dom- } \\
\text { inance }\end{array}$ & Abnormality dominant \\
\hline $\begin{array}{l}\text { Skin, Hair and Countenance } \\
\text { Ichthyosis (scaly skin) }\end{array}$ & $\begin{array}{l}\text { Simple. Lethal } \\
\text { when homozy- } \\
\text { gous, }\end{array}$ & Abnormality dominant \\
\hline
\end{tabular}


Data on Human Heredity-Cont'd

\begin{tabular}{|c|c|c|}
\hline TRAIT & HOW INHERITED & DOMINANCE \\
\hline $\begin{array}{l}\text { Keratosis (thickened skin on palms } \\
\text { and soles, suggestive of hoofs) }\end{array}$ & Sex-linked & Abnormality dominant \\
\hline Cutis laxa (rubberlike skin) & Simple & Abnormality dominant \\
\hline Thick lower lip ("Habsburg") & Simple & Abnormality dominant \\
\hline$t$ chin & Simple & Abnormality dominant \\
\hline ostrils (negroid) & Simple & $\begin{array}{l}\text { Dominant to Caucasian } \\
\text { type }\end{array}$ \\
\hline High nasal bridge & $\begin{array}{l}\text { Multiple genes, } \\
\text { four pairs }\end{array}$ & $\begin{array}{l}\text { Dominant to low or } \\
\text { "snub", }\end{array}$ \\
\hline Normal pigmentation & Plural genes & Cumulative \\
\hline Piebald (spotted skin) & Simple & Piebald dominant \\
\hline ture grayness & Simple & Abnormality dominant \\
\hline (a white forelock) & Simple & Blaze dominant \\
\hline $\begin{array}{l}\text { Epidermolysis (excessive formation of } \\
\text { blisters) }\end{array}$ & Simple & Abnormality dominant \\
\hline $\begin{array}{l}\text { Albinism (lack of pigment in skin, } \\
\text { eyes, and hair) }\end{array}$ & Simple & $\begin{array}{l}\text { Recessive to all types of } \\
\text { pigmentation }\end{array}$ \\
\hline Beaded hair & Simple & Abnormality dominant \\
\hline hair & Probably simple & Partial dominance \\
\hline hair (negroid) & Simple & Dominant \\
\hline Alopecia (baldness) & Sex-linked & Dominant in men \\
\hline Hair color (brunette or blonde) & Multiple genes & Dark shades dominant \\
\hline Red hair & Probably simple & Red pigment dominant \\
\hline \multicolumn{3}{|l|}{ Eyes } \\
\hline Eye color & $\begin{array}{l}\text { Modifying fac- } \\
\text { tors and varia- } \\
\text { tion in domi- } \\
\text { nance }\end{array}$ & $\begin{array}{l}\text { Dark shades tend to be } \\
\text { dominant over blue }\end{array}$ \\
\hline Hereditary cataract & Simple & Abnormality dominant \\
\hline Color blindness & Sex-linked & $\begin{array}{l}\text { Abnormality usually re- } \\
\text { cessive }\end{array}$ \\
\hline Night blindness & Sex-linked & Abnormality usually re- \\
\hline Atrophy of optic nerve & Sex-linked & Abnormality recessive \\
\hline Large, irregu & Simple & $\begin{array}{l}\text { Abnormality, an irregu- } \\
\text { lar dominant }\end{array}$ \\
\hline Almond eyes (Mongolian) & Simple & Recessive \\
\hline Ear Structure and Hearing & & \\
\hline Complete absence of external ears & Simple & Abnormality dominant \\
\hline $\begin{array}{l}\text { Cup ears (small, deformed, inverted } \\
\text { pinnae) }\end{array}$ & Simple & Abnormality dominant \\
\hline $\begin{array}{l}\text { Otosclerosis (progressive hardening of } \\
\text { ear drums) }\end{array}$ & Simple & Abnormality dominant \\
\hline Deaf mutism & Simple & Abnormality recessive \\
\hline Nervous System & & \\
\hline Feeblemindedness & Can be simple & Abnormality recessive \\
\hline Amaurntic family idiocy & Simple & Abnormality recessive \\
\hline $\begin{array}{l}\text { Huntington's chorea (St. Vitus' } \\
\text { dance) }\end{array}$ & Simple & Abnormality dominant \\
\hline Manic depressive insanity & Plural genes & $\begin{array}{l}\text { Abnormality chiefly dom- } \\
\text { inant }\end{array}$ \\
\hline
\end{tabular}


Data on Human Heredity-Cont'd

\begin{tabular}{|c|c|c|}
\hline TRAIT & HOW INHERITED & DOMINANCE \\
\hline Dementia praecox (schizophrenia) & $\begin{array}{l}\text { Plural genes, } 32 \\
\text { pair }\end{array}$ & $\begin{array}{l}\text { Abnormality seems to oc- } \\
\text { cur as an incomplete } \\
\text { recessive }\end{array}$ \\
\hline $\begin{array}{l}\text { Dipsomania } \\
\text { Average intelligence }\end{array}$ & Probably simple & $\begin{array}{l}\text { Abnormality recessive } \\
\text { Dominant to very high or } \\
\text { very low }\end{array}$ \\
\hline $\begin{array}{l}\text { Diseases and Diatheses } \\
\text { Hemophilia }\end{array}$ & Sex-linked & Abnormality recessive \\
\hline $\begin{array}{l}\text { Allergy (predisposition to hay fever, } \\
\text { asthma, eczema, migraine, etc.) }\end{array}$ & Simple & Abnormality dominant \\
\hline $\begin{array}{l}\text { Gower's muscular atrophy } \\
\text { Diabetes insipidus (excessive produc- } \\
\text { tion of urine) }\end{array}$ & $\begin{array}{l}\text { Simple } \\
\text { Simple }\end{array}$ & $\begin{array}{l}\text { Abnormality recessive } \\
\text { Abnormality dominant }\end{array}$ \\
\hline
\end{tabular}

\section{Matings Among Defectives}

It is obvious that persons who have inherited scaly skin, lobster claws, amputated hands and feet, exostotic bones, or who might have any of many other inheritable defects that incite pity or repulsion will find difficulty in securing mates. When these abnormalities oceur as mutations, the aftlicted persons are not likely to marry on their own social level, but will probably mate with others who are of lower grade mentally or who have other abnormalities that make them objectionable to normal people. Thus there is a tendency for defeetiveness to be precipitated to a social group that can clearly be called dysgenic. By her process of eliminating the unfit who could not survive the fieree struggle for existence, Nature formerly kept this group at minimum size. Today its numbers are being added to, not only by recruits from higher groups who have a poor heredity either by unfortunate segregation of undesirable genes or by the occurrence of such mutations as have been mentioned, but also by increased reproduction by the members of the dysgenic group itself.

\section{The Differential Birth Rate}

Nature keeps her creatures fit by giving reproductive advantage to the best members of each species. Various dioecious animal forms produce from dozens to millions of young per pair from which, on an average, two individuals are selected to replace the parents. As a rule, the two selected are the ones that are strongest and the most free of defects-these are usually the ones that are best adapted to their environment. Man has, in the ease of his own kind, preserved the weak and defective individuals that Nature would have eliminated. This has 
been done through the applieation of medieal seienee, together with public health and other measures that have come with the development of a humanitarian consciousness. Nothing but praise should be given to an altruism that saves lives and relieves suffering, but the effect on our race of man's present practice of preserving individuals that Nature would have destroyed, without safeguarding the reproductive advantage of the fitter group, is worthy of eonsideration.

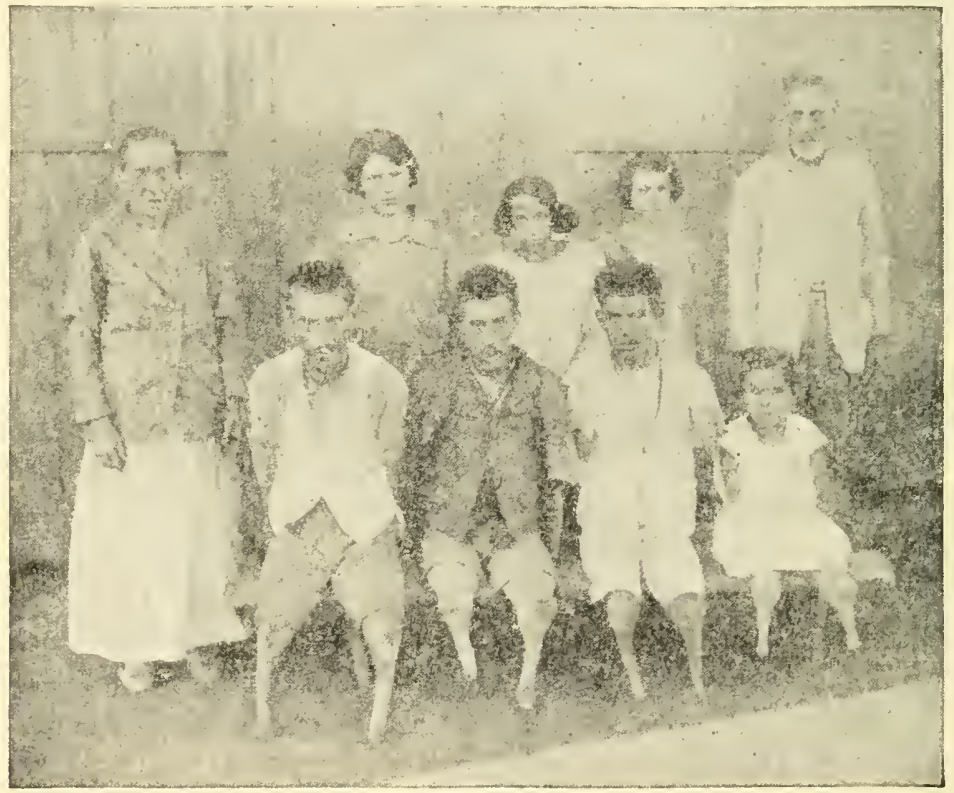

Fig. 438.- Family from Brazil showing hereditary absence of hands and feet. The man in the picture is the uncle of the children shown. The father, who is dead, had the same deformity. Of the twelve children six were normal and six were deformed. (From Holmes, Human Genetics and Its Social Import, published by McGraw-Hill Book Company.)

It has been shown by Lorimer and Osborn* that certain large groups are inereasing so rapidly while others are so diminishing, that the surviving children of a million women of reproductive age of the first eategory will be twiee as numerous as the surviving children of a similar group of women of the second classification. Carried on at the same rate for three generations (which is only a long lifetime) the descendants of the first groups will be sixteen times as numerous as those of the second groups.

*Lorimer, F., and Osborn, F.: Dynamics of Population, Macmillan. 
Casual observation will make evident that such grouping is likely to be on a basis of engenicity and dysgenicity. A number of studies have been made of the reproductive rate of groups classified by vocation. These studies reveal that passing from the professional and successful business classes through the various occupations to that of the unskilled, transient, agricultural laborer, the number of children per family rises steadily.

\section{Family Size in Eugenic Groups}

The vocational group made up of college teachers might be taken as an example of a profession whose members have a low reproductive rate. A recent study made by Kunkel* shows that 4,567 college teachers have 5,932 living children, an average of 1.3 children per teacher. Dividing the teachers surveyed into three groups according to age, he found that in the oldest group there is an average of 1.6 children, the middle group averages 1.42, and in the youngest group, which consists of those of less than forty-three years of age, there are 0.86 children per teacher. Since the families of this last group are not complete, the average of the other two groups, or about 1.5 children per family, might be taken to indicate the reproductive rate of faculty families.

A correspondingly low birthrate is found among other groups whose members would be expected to possess traits that should be preserved for our race. Cattell reports that the average number of children in the families of the persons listed in American Men of Science is 1.88. But small families are not limited to college professors and scientists, for those distinguished persons whose names are recorded in Who's Who in America have families averaging only slightly more than two.

Since the families from which college students come can reasonably be taken as a eugenic group, several studies have been made of the sizes of the families represented on the campuses of various American colleges and universities. The writer kept a record for a ten-year period of the sizes of the families represented by the students of a city university of the Southwest. The average number of children in those families was found to vary from year to year from slightly under three to slightly over three. Since there were no childless families represented, these figures are high for the social stratum concerned.

*Kunkel, B. W.: A Survey of College Faculties, Bulletin of the Association of American Colleges 23: No. 4, Dec., 1937. 
What effect college education may have on family size may be inferred from other studies. Harvard graduates whose year of graduation would give us reason to suppose that their families are complete, have produced 1.9 children per married alumnus; allowing for the members of the group who did not marry, the average falls to 1.6 children. Corresponding averages for Yale are 1.9 and 1.5, for Swarthmore 2.15 and 1.9, and for Vassar 2.15 and 1.25.

A false sense of eugenic security might be prompted by the belief that the figures exceeding two in the foregoing citations indicate that the parents are being replaced and that any residual value represents a gain. But in the cases of two-child families, what assurance have we that those children will live to reproductive age, that they will marry, and if they marry that they in turn will have children? Considering these possibilities, it is evident that fertile families must provide for more than replacement if the group to which they belong is to be perpetuated. Various computations have been made of the average number of children per fertile family necessary to maintain the numerical strength of a group. These estimates range from 3.1 to 4 . Considering that the current incidence of childless families in America is 20 per cent, probably the higher number is more nearly correct, and it is not safe to place the figure at less than 3.5, or stated another way, it is only when $\frac{\text { Grandmothers }}{\text { Mothers of daughters }}=1$ that a given group is maintaining itself.

\section{Family Size in Dysgenic Groups}

Various studies have shown that larger families occur among people who have but a poor store of those qualities of intelligence, stability, and physical traits that go to make up racial excellence. Lorimer and Osborn found in their study of the school children of selected eastern cities that children with the lowest intelligence scores (I.Q. below 60) came from families that averaged nearly six children; that those with medium scores (I.Q.'s 90 to 110) came from families averaging less than four; and that the children of superior intelligence (I.Q.'s 140 and over) came from families that averaged less than two and a half children.

The writer's study of nearly a thousand improvident families of a type well known to the social workers of southwestern cities revealed 
that in this group the number of births occurring in completed families averaged 7.9 and the average number of children born to mothers of all ages was 5.7. The average number of surviving children of the two groups was 6.1 and 4.6 respectively. The significance of these data in comparison with those concerned with the size of the families from

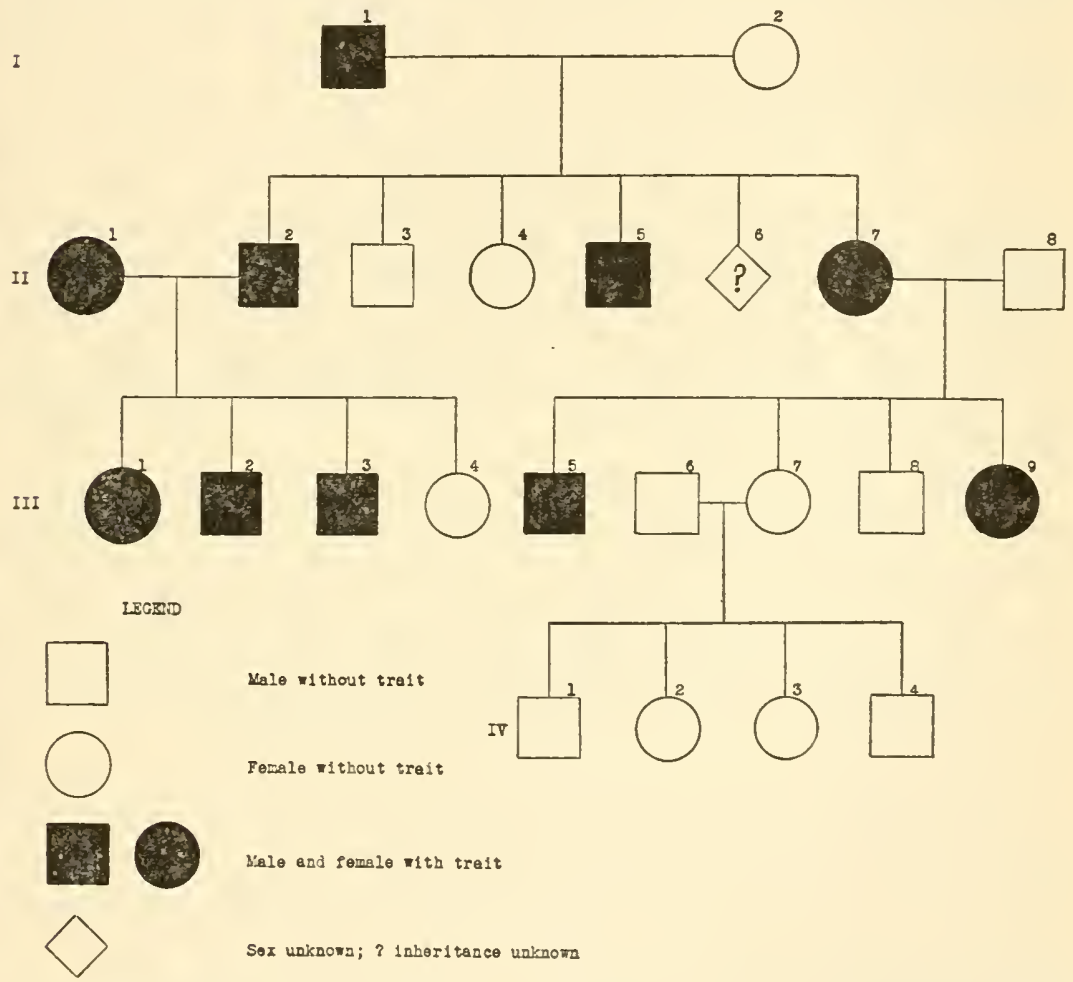

Fig. 439.- Standard pedigree chart. Charts such as this can be used in tracing a trait through several generations of a family. This particular chart shows the inheritance of a dominant trait starting with a cross between a male who was heterozygous for the trait and a female in whom it was homozygously recessive.

which college students come can be realized from the fact that if the reproductive rates in these two groups continue for ten generations, the descendants of one hundred families of the dysgenic group will number more than twenty-eight thousand, while in the same generation, one hundred families of the present-day college-student group will be represented by eleven persons. 


\section{What Can Be Done?}

It must be admitted that the present racial situation has been brought about through the advance of humanitarianism and seience. The cure for the situation must eome through the application of greater humanitarian and scientific measures. The many corrective panaceas that have been suggested must be tested carefully and applied cautiously. Some reforms ean be applied at once and are being applied today to some degree; others must wait until the time for their application is more opportune.

\section{Some Eugenic Measures}

The step that would be most fruitful of racial betterment is general education along eugenic lines. If a sufficient number of thoughtful eitizens were informed about racial trends, a eugenic eonseiousness would be developed that would cause every proposed social or economie change to be considered from the standpoint of its genetic significance.

The marriage and divoree laws of the various states should be unified and rewritten along eugenic lines. They should provide for advance notification of applications for marriage licences as is provided for by the California laws, and should contain provision for health examinations as already enforeed in a number of states. The latter practice should be extended to include the examination of family histories.

Many positive measures have been proposed for granting aid of various kinds to large families of good eugenic stock. At the present time most of these proposals are impractical, but we might look forward to wage adjustment to family size starting with gorermment employecs, and rental rates of gorernment-owned houses based on a fixed pereentage of the family income regardless of the size of the house required.

It has been proposed that those who are elearly unworthy of parenthood should be segregated in colonies of their own sex. The expense of this as well as the probability of many social and other problems that would arise in such a situation ehallenges the wisdom of such a measure beyond the degree it is now being practiced in our eleemosynary and punitive institutions.

Twenty-nine of our states have adopted laws providing for the eugenic sterilization of such persons as those who have been eommitted 
to sanitaria for mental eases because of an inheritable type of insanity and who are to be returned to their families. A few states provide also for such sterilization of habitual criminals and those who are clearly feebleminded. Eugenie sterilization consists of vasectomy and salpingectomy-operations that bring about sterility without interfering with endocrine function or normal sexual reactions. Over twenty-five thousand such sterilizations have been performed under the present laws.

It is evident from the data previously diseussed that family limitation is being practiced by the eugenic group. It is suggested by many who are facing squarely the problems of racial welfare that those who are mentally and in other ways far bolow the majority of our citizens should have made available to them the means of similarly limiting the sizes of their families. The number of clinies where such measures are made available to persons who will make proper use of them already number in the hundreds.

Some of these eugenic measures are questioned on the grounds that they violate human rights. We should also be considerate of the rights of the unborn. It is reasonable to say that every child that is to be born into this world has:

The right to be born with a sound mind.

The right to be born with a strong and normal body. The right to be born into an environment in which his inherited potentialities will have a fair chance to develop. 


\title{
CHAPTER XLIV
}

\section{ANIMAL BEHAVIOR}

\author{
(By Ina Cox Gardner)*
}

\section{Introduction}

To most people the study of either human behavior or the behavior of other animals means a study of the mind. This conception came from the early definition of psychology, which meant the science of soul or mind. The term mind is an abstract one, and whether or not any animals including human beings have what we call mind, is a matter of inference. In the past we have sought to explain behavior by the use of such terms as instinct, consciousness, mind, intelligence, memory, and many others. These abstract terms became supposedly concrete entities and much valuable time was spent in a search for them. Psychologists in the modern period reject these nouns and make use of adjectives such as conscious or mental, which have more precise meaning in the description of behavior.

The movement which led to the development of animal psychology into a distinct scientific discipline began with the work of Darwin. This does not mean that the field had been altogether neglected up to that time. Even prehistoric man must have been interested in the behavior of animals that supplied him his daily food. The ancient Greeks observed animal activities intensively with the definite aim of attempting to understand and explain them. From this time onward the problem of the nature of infrahuman behavior has attracted the serious attention of scientists. In fact, this problem has led to almost endless controversy from age to age.

Two extreme views have grown out of this controversy. They are antipodal-at one end of the pole animal behavior is interpreted in the best possible light. The higher, nonhuman animals are often regarded as almost human in intelligence. This view arises from the use, mure or less uncritically, of anthropomorphic analogy. The basic inference in such analogy seems to be that if an animal acts like a man it must also feel and reason as a man does. While at the other

*Acknowledgment is made to Warden, Jenkins, and Warner, and the Ronald Press of New York for permission to use certain of the materials included in this chapter. 
end of the pole, other animals are rated very low in intelligence. Simple organisms are usually regarded as mere living machines without mental life of any sort. The bchavior of even the higher, nonhuman animals is explained as due, in the main, to the operation of blind instincts, although a certain degree of intelligence may be admitted. Both of these views have been rejected by present-day comparative psychologists.

The history of the study of animal behavior may be divided into two periods. The first, known as the anecdotal period, had its origin about the time of Darwin, and the collection of Romanes aneedotes may be taken as a representation of the better classes of anecdotes. Scores of anecdotal collections appeared in which the tendency to humanize the mental powers of the other higher animals was carried to the ridiculous. The anecdotes were often taken from unreliable sources and accepted without critical comment. The anecdotal collections were widely read and the popular imagination was deeply stirred. This movement which lasted from about 1859 to 1890 was not altogether without value, in spite of its absurdities and vagaries, since the interest aroused was later turned into more scientific channels.

A reaction against the humanizing tendency began about 1890 and led into the experimental period. This period was characterized by a more critical use of anthropomorphic analogy which led in time to a rejection of it altogether in favor of a strictly objective position, and the use of more precise observation and the gradual development of carefully controlled experimental methods.

The new movement arose from the work of Lubbock, Loeb, Newman, and Lloyd Morgan. Lubbock, the English naturalist, became interested in the behavior of insects through the personal influence of Darwin. Loeb and Newman, both physiologists of the German school, sought to analyze the behavior of lower organisms along rigidly scientific lines. The work of Morgan, an English biologist and philosopher, was restricted mainly to the higher vertebrates. Lubbock was the first of the group to make use of experimental methods, his studies on insect behavior appearing in collection form in 1S82. Each of these men, however, was an original and independent worker : Lubbock and Morgan were most directly influential in the spread of the animal laboratory among psychologists after the turn of the century.

Morgan was the first to extend experimental methods to the higher vertebrates. As early as 1891 , he spole of the "trial and practice" 
factor in learning, and in 1893 announced the famous Canon of Morgan. This runs as follows: "In no case may we interpret an action as the outcome of the exercise of a higher psychical faculty if it can be interpreted as the outcome of the exercise of one which stands lower in the psychological scale." This is merely the law of parsimony applied to animal psychology. While it does not rule out the use of anthropomorphic analogy entirely, it did serve to introduce a measure of restraint into such speculation. This eanon was very influential among those, who, while objecting to the extreme view of Loeb, were ready to reject the absurd anthropomorphism of the anecdotalists.

Two observation methods are used in the study of animal behavior: (1) The genetic method, wherein observations are made upon an animal's behavior as it naturally occurs from the beginning to the end of the life of the animal; (2) The training method, wherein the observations are made upon an animal's behavior during training and after it has been trained in certain habits of action or habits of discrimination among stimuli of different sorts. Every act in an animal's behavior is a response to one or more stimuli, produced by conditions internal or external to the organism. In the whole phylogenetic series, the internal stimulus is frequently, if not usually, due to the metabolic processes going on constantly within the protoplasm. The restlessness of all organisms is to be ascribed to impulses generated by the metabolic processes going on within that organism. An external stimulus is any change in the environment which affects the organism generally, or some special organ or part of it in particular, so that the animal's behavior is modified to some extent at least. No organisms, as a rule, live under conditions that are always the same, i.e., all organisms are subject to the effects of stimuli, and their responses are termed reactions. One-celled organisms react to all classes of stimuli that call forth reactions in the many celled organisms. The nervous system and sense organs constitute merely a more delicate, complex, and effective mechanism for the reception of the stimuli to which even the undifferentiated protoplasm of Amoeba responds.

Such stimuli may be classed as chemical, clectrical, thermal (heat), mechanical, or photic (light). The reactions of animals to any and all stimuli are classed as tropisms, reflexes, instincts, and habits. The first three of these are used to connote the many types of native behavior, whereas acquired activities are spoken of as habits. In so far as these terms imply a sharp distinction between heredity and en- 
vironment, they are objeetionable. They are seldom used in the same sense by different writers in the classification of types of behavior.

\section{Tropistic Behavior}

The concept of tropism has its origin in plant physiology where it was applied to the positive or negative orientation of plant to light, gravity, or other external stimuli. The term was later used by Loeb to eover similar growth responses in lower organisms and simple responses of the reflex type as well. In time, Loeb extended the eoncept to inelude practically all reflex and instinctive actions. Loeb used the tropistie theory as a definite explanatory principle. He assumed that symmetrieal parts of the body surface of an or'ganism possess identical irritability value. Orientation behavior is due to a forced turning of the organism so that the corresponding sides are equally stimulated. This simple explanation ignores the factor of internal regulation. This has been pointed out by Jennings, Mast, Buddenbrock, and others. The tropistic theory is useful, however, in describing the behavior of Protozoa. Since these forms are eomposed of but a single eell, any response is likely to involve the whole body. But even in Protozoa it has been asserted that the metabolie or internal activities influence the tropisms. Thus the chemotropic response might be entirely different toward a substance that was food to Protozoa, if the animal was hungry rather than satiated. Also it has been found that the reactions of an animal to a strong stimulus may be the opposite of its reaction to a weak stimulus. Some insects are negatively phototropic to daylight while they are positively phototropic to weak artificial light. And again it has been demonstrated that tropistic reactions may be modified through experience. Parameeium is a freeswimming organism, and when it finds its progress in one direetion hindered by some obstacle, such as a strand of thread, it reverses its course and heads in a new direction. If in the next trial, the obstaele is not avoided, the little animal continues its trials until it does succeed in avoiding the obstacle. The analysis of the behavior of many organisms shows the trial and error method to be a very important factor in their behavior. Many revisions have been made in our concepts of the tropisms during the past few years, but it has not altered the significance of the theory as a means of deseribing the behavior of Protozoa and lower vertebrates. Various tropisms are described by the response animals make to these stimuli. Thus we 
have phototropism, response to light; thermotropism, response to heat; chemotropism, response to ehemicals; thigmotropism, response to contact; geotropism, response to gravity.

\section{Reflex Behavior}

Tropistic behavior is the dominant type of behavior in the organism without a well-differentiated nervous system. The protozoa are without a nervous system, and though a few of them have threadlike struetures running through their bodies in such a way as to suggest a correlating mechanism of some sort, it seems that, in general, this sensitivity and conductivity are properties of the protoplasm itself. In such animals as Hydra and the jellyfish, as well as in all higher animals, we have to do with activities promoted by nervous tissue. In such animals, we no longer eall their behavior tropistic but speak of their simplest reactions as reflexes. In principle there is little difference between a tropism and a reflex exeept in the relative complexity of the mechanism involved. The reflex is the dominant type of behavior in the segmented animals which have a simple ganglionie type of nervous system. Also according to Coghill reflexes represent secondary patternings of behavior in the developing vertebrates. The sensitivity and conductivity involved in the production of a reflex are the same qualities of protoplasm as are concerned in tropisms, but they are enhanced by the special differentiation of nervous tissue in these particular directions.

The human being, as well as other animals, exhibits reflexes. They are sometimes called physiological refiexes and are reactions, such as the wink of the eyelids which follows the entrance of a grain of sand into the eye or which follows the sudden approach of an object to the eye. Other reflexes are the knee jerk, contraction of the pupil of the eye (pupillary reflex) when a strong light is flashed into the eye, and the general start of the whole body when a sudden and unexpected noise oceurs.

These reflexes have two characteristics which are important. First, they are brief in duration, consisting, in general, of a single contraction or relaxation pattern of a group of muscles. Second, they are predictable; a partieular stimulus always, in the normal subject, produces the reaction. If the reaction (such as the knee jerk or the pupillary reflex) does not follow the appropriate stimulus (the blow on the 
tendon or the flash of light) this failure is evidence of an abnormal condition of the reaction.

Reflexes terminating in action patterns of smooth muscles and glands also occur. The saliva starts to flow when the odor of food assails the nostrils. It has been conventional to describe reflexes as unconscious reactions, but as a matter of fact there are no such reflexes in the normal human being, and apparently none in other vertebrate animals. Striking a blow on the patellar tendon, for example, does not merely produce a reaction terminating in contraction of the quadriceps muscle which propels the lower leg forward. Effects are demonstrable in other parts of the body and included in the total response is the perceptional awareness of the blow. Expectant attention of the blow and attention to other stimuli affect the leg movement. The predictability and brevity of the reflex are only relative. The important difference between a reflex and a perceptional reaction is that the reflex would occur and be still more predictable, even if the animal were so mutilated that the neural patterns, aside from the parts most directly affected, were eliminated. For example, if by accident the spinal cord of a person is broken above the lumbar region, and several months are allowed for the effects of shock to disappear, the knee jerk may be elicited and is more pronounced and more uniform for a given force of blow than before. The person in such a condition does not perceive the blow unless he is allowed to see it or hear it, and he cannot perceive the jerk of the leg except by sight. The discrimination of the lower part of the spinal eord, through which the essential part of the reflex transit oceurs from the upper part of the cord and brain, prevents the stimulus from affecting more than the leg muscle. Similar results can be obtaincd from dogs and eats by experimental severing of the cord at proper levels. In these cases the reflex becomes a real uneonscious reaction, which it is not in the normal animal, and it is unconscious because it is restricted to a part of the organism.

\section{Chain Reflex Behavior}

Chain reflex behavior in animals is also often called instinctive behavior. Watson defines instinct as a series of concutenated reflexes which unfold serially upon proper stimulation. In insects and other animals, we find that reflexes often occur in series; one directly called out by some appropriate stimulus, itself becomes the cause of another which follows it, and so on through a longer or shorter series. These 
are often called chain reflexes or instincts. Psychologists are inclined to drop the word instinct, because of the disagreement in definition. The term chain reflex seems to describe better the reaction of animals. A chain reflex, like a simple reflex, is an inherited type of reaction and may appear so complicated and purposeful in character that the inexperienced observer is often led to describe it as intelligent experience or reason. It differs from a simple reflex, furthermore, in that it involves generally the reaction of the animal as a whole rather than that of single parts of organs. In its most complex forms it often defies analysis, as in the case of migration of birds, or can be only incompletely analyzed, as in nest-building, eare of young, and many others.

\section{Habitual Behavior}

The terms instinct and habit are usually applied to relatively complex types of behavior--the one native and the other acquired. Bernard has shown that psychologists find it difficult to separate complex behavior into the two categories. This is true because most instinctive acts involve a considerable amount of practice in becoming established. Practice is the carmark of habit. The capacity to form habits is Dunlap's definition of instinct. Habits are also defined as the modification of response tendencies which show probabilities of certain high degree, that particular responses will occur in particular circumstances.

As we have seen, the animal has a set of native reactions to external stimuli which we call reflexes. A habit is an acquired response that somehow came to be associated with a stimulus to which it is not ordinarily attached. Formerly the animal, learning this new association, was said to possess associative memory and this was thought to be indicative of consciousness in such animals. Associative memory was aceredited only to higher animals. Today in many of the lower animals experimentalists find a reaction at first directly called forth by a certain stimulus may later be provoked by another, even an unrelated stimulus. Almost every modern student of psychology or physiology knows of Pavlov's experiment with the dog whose mouth watered when a bell was rung as a signal of feeding time. This Pavlov called a conditioned reflex. It has been produced experimentally in a great many kinds of animals. Conditioned reflexes years ago were ealled associations and were taken to indicate the presence of associative memory. The term associative memory implies a certain subjective 
interpretation, and psyehologists today are secking to leave out all such terms in the study of animal behavior.

Most of the behavior of animals can be explained on the basis of tropisms, reflexes, ehain reflexes, and conditioned reflexes used in the formation of habits. In most eases the other animals' eapacity to form conditioned reflexes and handle themselves in a complex environment is much more limited than in the average man. Whether the higher animals, other than man, can reason is a question that has been much debated. Reasoning is a term upon which there is not unanimous agreement as to meaning. If it merely means problem solving, one would say the individual is able to reason because most higher animals ean solve a problem if the problem is suited to the animal. If reasoning involves the use of symbolic processes or abstract ideas, then we are much more in doubt.

The cases of so-called intelligent animals such as "Clever Hans" and "The Horses of Elberfield," when examined by comparative psyehologists were found to be making use of motor eues, and these had been conditioned by trainers. These so-called intelligent horses could add, subtract, multiply, divide, and extract square root and eube root. They could solve difficult problems, as well as easy onesbut they could not give correct responses when answers were unknown to experimenters and audiences. Reasoning could not be aceredited to them.

In Hunter's experiments on delayed reaction, the rats and dogs sueceeded in going immediately to the lighted box after a period of delay only when the body or a part of the body was liept oriented toward the lighted box. The monkeys and ehildren were able to suceed when the body was not oriented, thus giving evidence of the use of some other process than bodily orientation.

If we hold to the definition that reasoning involves the use of symbolic processes, there is little evidence of reasoning in animals, other than human. 


\section{CHAPTER XLV}

\section{PALEONTOLOGY}

(By W. M. Winton, Texas Christian University)

Paleontology deals with fossils. One of the most difficult biological concepts to define satisfactorily is the term fossil. Broadly speaking, the word should refer to records left by organisms and not by nonvital forces. Expressions such as "fossil ripple marks," "fossil sand dunes" and the like should never be used.

On the other hand, any recognizable trace left by an ancient organism is a fossil. Bones and shells, of course; but also scales, plates, impressions, burrows, leaf prints, intestinal droppings or coprolites, all are fossils.

Among the most interesting fossils are tracks. The poet's "footprints on the sands of time" in Triassic valleys of New England, the "thunderbird dance floors" of the Navajo reservation, and the huge tracks around Glenrose, Hamilton, and Hondo, Texas, are all dinosaur tracks. Abundant as are skeletons of Mesozoic dinosaurs, many are known only by their footprints hardened in the shores of ancient seas.

Other indirect records may be of considerable biological importance. In the deposits of the Lower Cretaceous division of Texas a few examples of the extinct oyster, Exogyra, have been found which exhibit pearls. These pearls (the best specimen is in the Museum of the Bureau of Economic Geology) are of the kind known as body pearls. A quarter of a century of research by the Marquesan commissions of the French Government has shown us that pearls of this kind are caused only by parasitic flatworms. With the Gallic romantic touch evident even in a scientific report, one writer puts it, "our most beautiful pearl, then, is but the sarcophagus of a miserable worm."

Pearls in Lower Cretaceous oysters tell us that parasitic flatworms appeared on the earth at least as early as mid-Mesozoic 
times. With this great span of time $(150,000,000 \text { years })^{*}$ one can partly understand how each species of mammal today has one or more species of tapeworms adapted to life in the body of its host only.

Some pathogenic bacteria when attacking an animal body leave unmistakable lesions in various parts of the skeleton. The late Dr. Roy L. Moodie, working first at the Baylor Medical School and later at the University of Illinois, was able to date the appearance on the earth of many important disease bacteria. Dr. Moodie studied large collections of skeletons of ancient reptiles and mammals and found lesions which the modern pathologist associates with the various bacteria in question.

Before leaving the matter of indirect records, one more instance will be mentioned. Some predatory carnivorous dinosaurs left on the vertebrae and other bones of their victims, deep tooth prints and scoriations as the only record which keeps them from oblivion. Are these tooth scratches fossils?

The last fifteen years have seen an unprecedented rise in the interest in paleontology and in the number of full-time workers in this field. Shortly after World War I, the pressing search for more petroleum brought out strongly the value of fossils in establishing "horizons" or levels which the oil geologist could use in determining favorable domes and other structures into which to drill. Immediately, workers in what had, hitherto, been one of the most academic subjects were lured from classrooms and museum benches into one of the most competitive and commercial industries.

At first attention was given only to the usual larger fossils found at the surface, but as the science of petroleum geology advanced, it became more and more important to know the levels or horizons

* How we have come to change such expressions of the earlier geologlsts as "ancient," "very ancient" and "extremely ancient" to round figures is a long and fascinating story which cannot be taken up here. The students of geochronology or age of the earth after many unsuccessful attempts based on rate of increase of salinity of the sea. change of the ellipticity of the earth's orbit, addition of the elements of the known geological column and others which produced figures which made us human beings, earth's most recent inhabitants, gasp, have now found a dependable check on absolute time.

The earlier estimates, and that is all they were, are characterized by only one feature common to all and that is a large figure for the age of the earth. As large as these figures were, they have been replaced by others which are truly staggering, and the new figures are believed accurate. At least different workers get closely parallel results.

The unvarying time clock of a disintegrating radio-active mineral and its use in determining the absolute length of time since the beginning of the various geologic perfods, are discussed fully in any new book on historical geology or perhaps more entertainingly in Wells' Science of Life or Jeans' Through Space and Time. 
which were successively penetrated by the drill. Only microscopic specimens persisted, as the drill crushed the others to unrecognizable bits. Then came the remarkable rise of the young science of micropaleontology, a field in which women have distinguished themselves and proved themselves equal to or better than men.

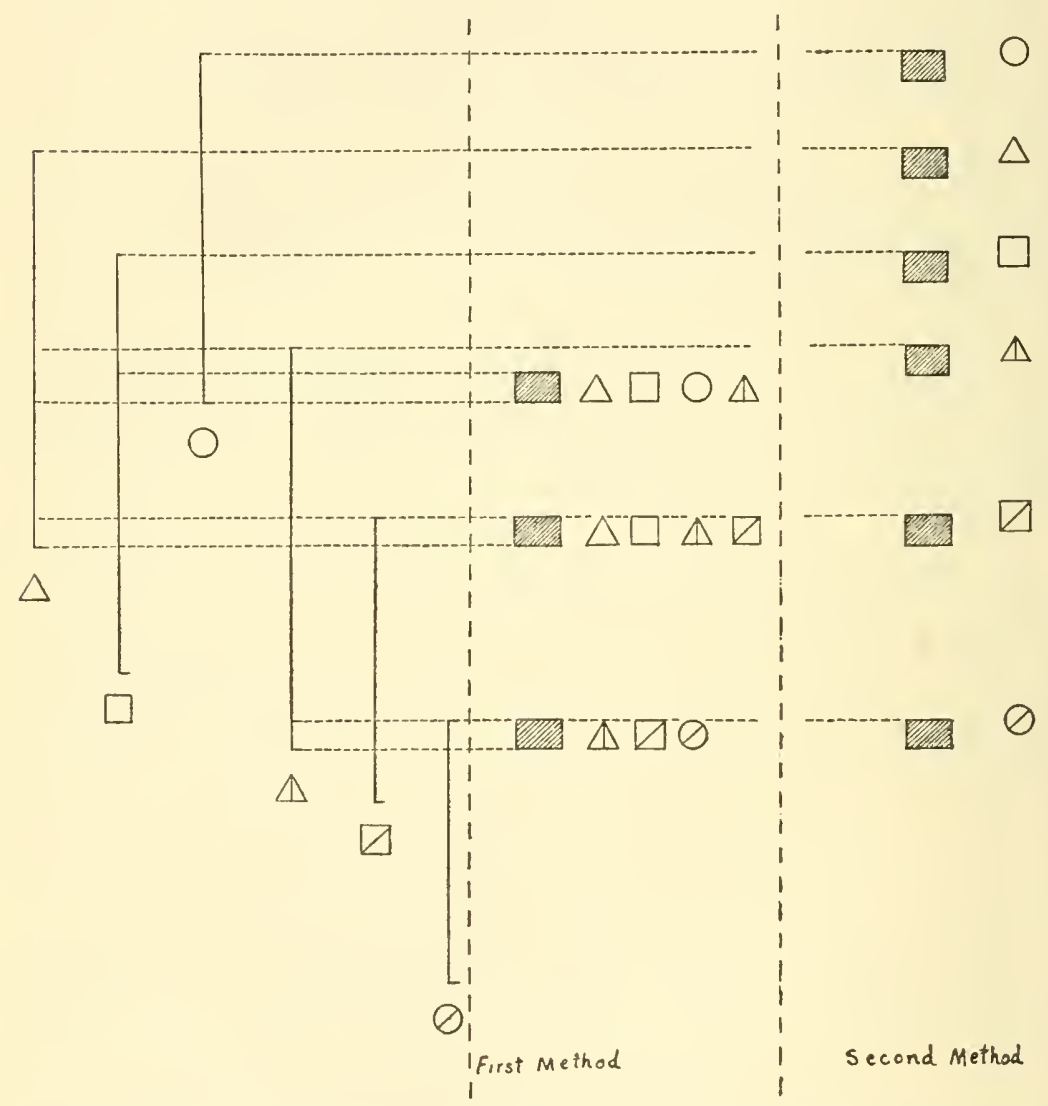

Fig. 440.-Diagram to show the vertical distribution of fossils. $\triangle, \square, 0, \triangle, \square, 0$ are characters, each indicating a species which has a thousand feet or more definite vertical range. The middle of the foure illustrates the frst method described in the text, and the right portion represents the second method.

Some changes have been made in the classic methods of the science of paleontology, although in the main the newer methods are identifiable as merely refinements of the older.

Men have long known that some species lived on the earth much longer than others. In the case of the ordinary marine species, the 
relative length of the life of the race in question is shown by its vertical extent in the sediments. A short-lived species left its fossils in a very few feet of sediments; others are recorded in hundreds of vertical feet; others in literally thousands; and a few species (of Globigerina) have lived uninterruptedly from the Cretaceous period to the present, representing many miles of vertical extent in the sediments.

Whether dealing with larger fossils or with microscopic ones, certain features are desirable when doing geologic work. An ideal fossil species: (1) had a short species life; (2) should have left abundant fossils, since a species which sharply marks off a narrow part of the column is valueless if it is very difficult to find; (3) should have had wide distribution, leaving its record over a large area; (4) should be readily recognizable, requiring a minimum of time-consuming measures, such as the counting of plates and spines or measurements of different features.

The only fossils which completely satisfy the above-mentioned requirements are the ammonites (Fig. 148). The various species were short-lived; in some cases the fossil specimens are actually thicker than the sediments which were laid down during the life span of the species. Most ammonite species occurred in large numbers, literally dominating the seas. Most ammonites had a wide distribution, sometimes world-wide. Often the chambered shells, comparable to those of nautili, acted as airtight bulkheads after the death of the animals, and the shells floated to great distances. Thus they dated sediments of the same time, whether the animals lived in the waters above any particular locality or not. Finally within a region, most of the ammonite species are characteristic and easily identified.

But ammonites are valuable only when working with rocks exposed at the surface and only in a limited part of the geologie column.

Efforts have been made, naturally, to use less perfect fossils, and methods have been developed to overcome difficulties when the species concerned lack one or more aspects of the ideal set forth above.

Since too great a vertical range is the most serious difficulty encountered in establishing precise levels in the strata, a brief sum. 
mary will be given of the particular methods which apply to this problem. Two of the methods are here described diagrammatically.

First, is the device of bracketing or use of associations, the term being used here with much less exactness than in ecology. Several long-lived species are represented by the symbols (triangles, squares, circles). Each is a species which has a thousand feet or more of vertical range, and the ranges are known. In a well sample the appearance of no single one of these species would be of much use to the paleontologist. Certain associations might indicate limited horizons upon which the petroleum geologist could carry his structures from well to well.

Using this method of bracketing on the larger fossils in the Lower Cretaceous period of North and West Texas during the past twenty years the writer has been able to locate for engineers, where highways should run to have the greatest volume of the cuts in soft marls. He has also, on many occasions, been able to foretell for builders the exact deptl to which foundations would have to be carried for large buildings, dams, and other masonry structures.

The greatest development of this method has been in the work with microfossils. As long ago as 1922, three young women,* working in the laboratories of oil companies in Houston, astounded a large audience of petroleum geologists by demonstrating beyond question that they could locate horizons as narrow as twenty feet. This was done in the soft Tertiary sediments of Southeast Texas, where geologists, up to that time, had been satisfied with divisions running from five hundred to a thousand feet.

The second method $\dagger$ is based on the fact that many marine organisms suddenly disappear from the seas which they have long occupied. This extinction is caused by a change in food supply, a change in temperature or other factor, or by the appearance of new enemies. A rapid extinction of a species, in terms of the record in the sediments, may be said to be almost instantaneous. In other words, the end of a species is more likely to be startlingly abrupt than is its begimning. This is true, notwithstanding the fact that with favorable conditions a species can populate an area of land or a portion of the sea in a very short time. An everyday example

- Misses Hedwig Knicker and Alva Ellisor and Mrs. P. L. Applin.

tFirst published by Mr. N. L. Thomas, paleontologist for the Pure Oil Company. although not claiming the method as original. 
of the rapid disappearance of a species from the face of the earth is the tragic case of the passenger pigeon.

Working on this basis, the paleontologist often will use the "top" of an extended horizon as a fairly precise marker. This method is especially favorable for the micropaleontologist working with well samples, as the drill encounters the top first.

Biologists working exclusively with living organisms or at least living species sometimes are puzzled by the conventions which paleontologists have developed. Show a new flower to a botanist and his first effort is to place the specimen in the family to which it belongs. Show an insect to a zoologist and his first thought is to refer it to its proper order. Just as the botanist recognizes families and the zoologist recognizes orders the paleontologist recognizes (or attempts to do so) both of these divisions in his fossils. But the working unit in paleontology is the genus. Undoubtedly, many of the so-called genera of fossils, if we could see the animals restored to life, might actually be families or in some cases might be species.

The old question of the biologist of "what is a species?" is even more difficult to answer in the ease of fossils. A convention which is fairly well adopted is to consider the different examples of a number of varying fossils as the same species if the varying characters exhibit gradations between the extremes. Thus, sometimes, quite variable fossils are put in the same species. On the other hand, examples which do not appear superficially to differ very greatly may be placed in separate species because certain differing characters do not intergrade.

Some paleontologists consider, also, that vertical range should be taken into consideration. The time factor is an important one in all branches of geology. No one has ever seen a part of any living species of plant or animal change into a new species. Such changes are common in fossils. No observant person denies the customary assumption that the Kaibab squirrel of the north rim of the Grand Canyon descended from an isolated colony of the more familiar Abert squirrels. This change required many thousands of ycars, yet in a vertical cliff face of forty or fifty feet, exposing ancient sea bottoms, we may see a record of a quarter of a million years of the history of the sea and its life.

The paleontologic picture, however, is a badly distorted one, and the imagination must be exercised vigorously to reconstruct the 
ancient seas and their life. If we examine the life in a modern tropical sea as shown in a Williamson motion picture, it is difficult to realize how few of the objects we are viewing are apt to leave a permanent record.

The corals, of course, will survive eternally. The sea anemones will leave no trace unless some individual may die and leave the impression of its body in the mud before the tissues are disintegrated by bacteria. Most of the gorgeous fishes will leave no record. As one grows ill and weak it is attacked by its fellows and the leftover scraps if they reach bottom will be snatehed up by Crustacea. Only an oceasional tooth or vertebra will survive.

Sometimes in geologic history a great underwater volcanic detonation would kill all of the fish in a small area of the sea, laying them down to be buried in the mud. Thus we get an occasional glimpse of masses of tangled fish skeletons furnishing us a clue to the great numbers of fish which have lived and of which we have no record. Volcanic detonations of this sort were more common in Devonian time than in any period before or since. For a long time geologists incorrectly called the Devonian "The Age of Fishes," although we know now that since this time, there have been many more kinds of fishes and more of a kind.

Returning to our under water movie, we may note that the holothurioids will leave only a few microseopic denticles and dermal plates. The marine worms, if they are tube builders, will leave their tubes. As for the clams and pectens, some of them will leave their shells, althongh most of the shells will be crushed by octopuses and other scavengers and the small bits will be passed repeatedly through the bodies of marine worms in an effort to squeeze the last milligram of nutrient material from them. Some of the bivalves will settle in the mud, and even if the shells disintegrate, neat mud casts will be left. In the Southwest mud casts are the most common fossils.

The foraminifera, ostracods, diatoms and other minute organisms, which the picture does not show us but which we know are there, will likely leave a record, provided they have hard tests. Many thick deposits of shales exhibit no fossils which may be seen by the unaided eye, but are teeming with microfossils, principally foraminifera. 
The chances of the ordinary marine animal persisting as a fossil are decidedly slim but are infinitely better than those of a land animal. Land animals are most likely to leave a fossil record in some natural trap in which large numbers of individuals became concentrated. Well-known examples of this phenomenon are the La Brea asphalt pits of California and the sandstones of the Dinosaur National Monument in Utah. In one, asphalt underlay shallow pools of water where Pleistocene mammals came to drink. The larger herbivores often became mired and their cries wonld attract their fellows and also the predatory carnirores. Many would become trapped. The concentration of some kinds of skeletons in these pits is astonishing. The hard Mesozoic sandstone from which so many reptilian remains have been chiseled in the Dinosaur Monument was originally a quicksand which served as a trap.

Since most finds of vertebrate fossils are fragmentary, the art of reconstruction has been highly developed. An exaggerated idea has become widespread that a paleontologist needs only a single bone or tooth to reconstruct the entire animal. It is true that some fossil species have been named, based on a single tooth or bone. In some cases, more of the skeleton has been found or remains of other individuals of the species have been turned up.*

When the famous fossil Archaeopteryx was found in the lithographic limestone of Solenhoffen, comparative anatomists and embryologists already were agreed that the birds sprang from a branch of the reptiles. The finding of this specimen, cxhibiting a true halfway stage and showing both reptilian and avian structures, started another incorrect idea which has persisted to this day. This is the notion commonly held by unscientific persons that paleontologists are constantly searching for "missing links."

Another incorrect idea, spreading unfortunately to scientific circles, concerns the gaps in the geological record. Admittedly, gaps still exist, but they are far fewer and far less significant than they were in the days when Darwin and Wallace commented so vigorously upon this matter. In fact, the major developments in paleontology have been made since the days of Darwin and Wallace, and the findings have been incorporated in the general disciplines of bio-

*The methods used in reconstructing vertebrate fossils are described in Scott's History of Land DIammals of the Western Hemisphere. 
logical science. Courageous, indeed, is the modern biologist who will utter generalizations which contradict the ever-growing story told by fossils.

Some points, of course, may never be settled. Paleontologists, for example, without exception are neo-Darwinists and believe implicitly in natural selection as the major cause of the new species constantly appearing on the earth. In this respect, they are considered by some biologists as being old fashioned. Certainly paleontologists could profitably inform themselves on some of the newer aspects of geneties, and it is equally certain that biologists should know more about fossils. 


\section{CHAPTER XLVI \\ PHYLOGENETIC RELATIONS OF ANIMAL GROUPS AND THE THEORY OF EVOLUTION}

It is likely that no student of modern biology has gone far before he has realized something of a progressive relationship between the various groups of animals in the animal kingdom. Phylogeny refers to the background of what has gone before in producing a race or phylum and incorporates the thought that different species have arisen from common ancestors. The general idea of it, as now accepted by most biologists, is that all living organisms have been derived through normal reproduction and variation by adaptation from simpler, more generalized ancestors. Phylogenetic relationships of organisms and the origin of species have long been topics of exceptional interest to biologists. Two questions which have intrigued the thought of many are: first, whence came living material? and second, what has been its course of events since the time of origin?

The observations and thought of Charles Darwin gave the first substantial support to the idea of all modern species originating from preexisting organjsms. Results of his careful studies were presented in $1859 \mathrm{in}$ his book The Origin of Species, which is now a classic in the field. There is still lack of agreement among scientists concerning the details of origin of life and species, but there seems to be little doubt that species, genera, and even larger groups have progressively developed since life originated on earth. The ideas of continuity, development, and differentiation of living organisms are quite generally accepted by those who have studied these problems. The lines of kinship of animal groups are traced only through common ancestry, and it is not ordinarily assumed that direct relationship exists between representatives of modern groups.

Sources of evidences of relationships are quite widespread, coming from such studies as zoogeography, comparative anatomy, embryology, physiology, paleontology, and even others. By combination of information gained from such sources much has been learned of the past history of many groups of organisms. Even these available sources give incomplete and sometimes inconclusive evidence of the history of any particular organism. The individuals are recognized 
as being only points on a long line of modifications. The two ends of this progressive line are seldom recognized with any finality. Darwin, and many others since his time, recognized three fundamental facts that bring about a progressive continuity among living things: heredity, with the tendency for organisms to resemble their parents; variation, in that no two organisms are exactly alike, hence the resemblance between parent and offspring is not absolute; and constant production of more offspring than can survive. The phylogenetic relations of animals show all indications of having been brought about by the operation of the above-mentioned factors in an orderly, evolutionary progress.

Variation is one of the most obvious facts and consistent phenomena in the animal kingdom. As long as differences among individuals of a group are minor or irreguarly distributed the group is apt to be considered a species. However, the species is not rigidly bounded and it must be remembered that continuous variation is in progress within this group as one generation follows another. It is becoming apparent to man that new groups have arisen and are arising within old groups of animals, also that old groups have broken up, some to become new ones and others to become extinct. These new groups were separated by small differences at first but gradually they attained greater and greater divergence from the common form. This is particularly likely where the different groups have become widely separated geographically or exist under distinctly different conditions of life. Frequently, it is only the widely separated extremes of the group which earry on the posterity, the intermediate forms having died out. In this way rather widely separated species may have arisen from common stock, and the lack of intermediate forms may accentuate the wide divergence between them. During the millions of years which life has existed on earth it seems evident that this process of divergence between groups has been in operation until there now exist many definable groups with distinct lines of relationship. Our natural system of classification is based on the relations and differences established for the different animal groups. Comparative studies of numerous animal groups will help to show some of these relationships.

\section{Colony Formation in Certain Protozoa}

Due to incomplete separation of cells following division in Protozoa, colonies are begun. In some instances groups of cells in some 
such colonies, e.g., volvox, become specialized as gametes for reproductive purposes and the other cells remain as somatic cells. Because of the similarity between various mastigophoran colonies and blastula stages in metazoan development, this group has frequently been cited as the predecessors from which Metazoa at some time originated.

\section{Development of the Gastrula}

The primitive gastrula is thought to have had its origin from a spherical colony of Protozoa by a more rapid growth of the cells at one pole which brought about an infolding or invagination to produce a new cavity from the exterior. This development estab. lishes a body composed of two general layer's. Modern adult coelenterates, like hydra, and others demonstrate precisely this condition today, with the outer ectoderm and the inner endoderm, each composed of cells serving vital general functions. This allows all cells surface exposure either directly on the exterior or to the cavity. By this means larger and more complex organisms were developed and have lived.

\section{Trochophore Larva}

In Platyhelminthes, Annelida, Molluscoidea, Trochelminthes and Mollusca there occur larval forms of the trochophore type. Although the surface ciliation and some other superficial features differ somewhat, a direct relationship among these forms is traceable through the larva. The fundamental morphology of the ctenophore is similar to that of the trochophore larva, and the Platy. helminthes are generally thought to have been derived from Ctenophores. The regular arrangement of gonads and the even, orderly distribution of the diverticula from the intestines of the flatworms are interpreted by many to indicate preparation for segmentation as it appears in annelids.

\section{Peripatus and the Wormlike Ancestry of Arthropoda}

Peripatus, the only representative genus of class Onychophora, was considered a segmented worm for a long time because of its shape, its even, conspicuous segmentation, and possession of nephridia for excretion. But later it was discovered that the respiration is carried on by tracheae, and the body cavity serves as a blood space, both of which are typical arthropod features. In addition, the paired 
legs are jointed, although similar in appearance to paropodia of Annelida. There are two jointed antennae on the head and some jawlike plates in the mouth. Situated as it is, midway between annelids and arthropods, this form seems to show an immediate transition from the one group to the other. Upon this basis it is usually held that the arthropods have a wormlike ancestry.

Interestingly enough, there exists another idea of arthropod ancestry from the fact that all of the lower forms of Crustacea pass through a characteristic stage known as the Nauplius. This larva does not correspond very closely to any strictly annelid stage, but with its short body and three pairs of appendages it resembles a modified trochophore larva. The nauplius larva has some features in common with the rotifers which authorities feel may have arthropod tendencies.

\section{Echinoderms and Their Larval Relations}

Although the adult echinoderms possess radial symmetry, that seems not to have been in the immediate phylogenetical background. The larvae of all echinoderms possess bilateral symmetry. These larvae all have definitely arranged bands of cilia over the body. Embryologists have pointed out the fact that these larvae are not directly related to the trochophore. They show more resemblance to the Tornaria larva of Hemichordates than to any of the nonchordate forms.

\section{Ancestry of the Vertebrates}

The establishment of relationships between the chordate and nonchordate animals has been one of the perplexing problems in the study of phylogeny. Different students of this problem have investigated the possible relationships of such nonchordate groups as the flatworms, nemertine worms, annelids, arachnids, insects, and echinoderms. Their investigations have resulted in the formulation of a number of theories putting forth the various ones of the abovementioned groups as the progenitors of, or claiming common ancestry with, chordates.

Some authors insist that the chordates have arisen from some segmented form; others conclude from their evidence that this is not necessarily true or essential. All of the theories establish their relationships to the vertebrates through the protochordates, which are represented by Amphioxus, the tunicates, and hemichordates. 
Each of these has been considered as ancestral stock, which has contributed to the origin of vertebrates. It is usually conceded by authorities in this field of study that Amphioxus is a modified ancestor of the vertebrates, due to the clear-cut and well-defined condition of the distinctive characteristies of the chordates and the presence of a midventral endostyle. Next in the line of thought would come the possibilities of ancestors of Amphioxus. The tunicates have been given this distinction by some. The adult has lost most of its typical primitive characteristics, but the larva possesses the distinctive characteristies of chordates and also the ventrally located endostyle, very similar to these structures in adult Amphioxus. It has been suggested that probably the adult tunicate once existed as an animal similar to its larva of today, and that its forebear was not only the ancestor of modern tunicates but also the form from which the Amphioxus group has descended.

Hemichordata, which is usually considered the most primitive of chordates, is regarded as a possible ancestor of, or as possessing common ancestral stock with, tunicates and Amphioxus. As will be remembered from the previous study of Hemichordata, its representatives possess gill slits, a support in the base of the proboscis which may be homologous to the notochord, and four longitudinal nerve cords of which the dorsal is the most highly developed. The above protochordate relations are rather generally conceded, but there is much less agreement concerning their origin, and several theories have arisen of which the following are important.

Annelid Theory.--The segmental condition of this group, the relationship of the digestive system to other circulatory and nervous systems, and presence of the coelom with related nephridia, present a close comparison to what is found in the embryonic development of the vertebrates. It has been suggested by some scholars that by inverting the body of the nonchordate annelid, the fundamental systems are bronght to resemble their relative locations in vertebrates. A fibrous cord has been found in some groups of the annelids, and this structure is held to take the place of a notochord in function and position. These fibers are found just dorsal to the chain of ganglia in the annelids.

Arachnid Theory.-Such forms as scorpions, Limulus, and other arachnids have been favorably compared to vertebrates. By comparison of these arachnids with the extinct, fishlike ostracoderms, 
an elaborate theory of the possible origin of the vertebrates from this ancestry is derived.

Echinoderm Theory.-This theory of vertebrate descent goes again to Hemichordata. The developing egg of this animal becomes a larva known as Tornaria, which floats in marine waters, has bilateral symmetry, is almost transparent, and possesses bands of cilia used in locomotion. This larva is almost exactly like that of the starfish and other echinoderms which live in the same habitat. The close correspondence of features of these two groups of larvae has suggested the conclusion that these two types of animals have descended from a common ancestor which was similar to these larvae. The line of descent of one branch of this stock has presumably passed through Hemichordata, Tunicata, Amphioxus, and Vertebrata. The nonchorclate ancestors are not yet conclusively determined, but the foregoing theories snggest the thinking and evidence along that line.

Within the subphylum Vertebrata the relations are somewhat more evident, but the phylogenetic sequence is rather obseure at some points. Cyclostomes, the simplest vertebrates, are most closely related to Amphioxus, which has been suggested as the protochordate most similar to vertebrates. These very primitive fish have an eel-like body without paired fins and without jaws. They have from seven to fourteen branchial (gill) apertures in different species, and all of them possess skeletons composed of cartilage. The cartilaginous skull is not entirely closed dorsally but resembles a trough with bars over the roof. The anterior end of the nerve cord has expanded to become a brain (Chapter XXVI). Next in order of complexity are the Elasmobranchii, which possess well-developed, paired appendages (fins) and jaws (Chapter XXVI). They also have a cartilaginous skeleton, but the skull is much more complete dorsally. The number of gill arches is reduced to five, but the apertures are uncovered as in cyclostomes. The number of aortic arches has been reduced from the sixty to ninety pairs of Amphioxus or seven pairs of lamprey to five pairs. The group of ganoid fishes, which was the dominant Devonian animal, is generally conceded to have Elasmobranch ancestry. Nost ganoids have more or less cartilage along with the bony structure of the skeleton. Their gills are covered with an operculum, and there are only four aortic arches. The bony ganoids are usually thought to be the ancestors of true bony fish. 
There is an extinct form of Amphibia, Stegocephalia, which shows relations to the ganoid fishes and for this reason the ganoids are usually named as ancestors of Amphibia. Some authors hold that the lungfishes, which represent an independent branch of the Elasmobranch group, are the ancestors of Amphibia because of their ability to breathe air and live out of water. However, the former view of the phylogenetic relation between fish and amphibia is most commonly held. Amphibia have well-developed bony skeleton with paired appendages for locomotion on land. Lungs have appeared as a means of aerial respiration, and the aortic arches have been reduced to three.

Reptiles are supposed to have descended from Stegocephatia also, with most modern reptiles coming by way of Rhynchocephalia which is represented by one living species, Sphenodon punctatum. The snakes, lizards, crocodiles, and the extinct dinosaurs have probably branched from this group, while the turtles are thought to have descended through Theromorpha, another extinct branch of Stegocephatia. The dinosaurs are credited with the ancestry of birds by way of a toothed, feathered, extinet form known as Archaeopteryx. It was essentially a flying reptile. The mammals probably descended from the reptilian group Theromorpha by way of our modern monotremes which lay eggs, hatch them out, and then suckle the young with milk from mammary glands. The marsupials, such as kangaroos and opossums, are next in order, and from these it is thought the Placentalia have arisen. Within this group some authorities hold the view that the Primates, the order including man, have arisen from Insectivora. The apes and monkeys belong to the Primate group, and there has been some misunderstanding among laymen generally in regard to the possible relationship of man and the apes. Most people have the misconception that this is a linear descent in which the most advanced member of the lower group represents the immediate ancestor of the next higher group. As a matter of fact, the theory is not that the higher monkeys are in the process of becoming apes and the higher apes becoming men, but that all three of these groups have had origin as different lines from a common primitive form.

Recapitulation Theory.-In the early part of the nineteenth century von Baer observed that the early stages of vertebrate embryos of different classes had a very close resemblance to each other. $\mathrm{He}$ 
did not subscribe, however, to the recapitulation theory when it was formulated later. Haeckel, coming a little later, became convinced that the developing embryo lives over again the stages through which its whole race has passed, and he formulated the recapitulation theory or biogenetic law from this idea. In other words, the organism in its individual life tends to recapitulate the different stages through which its ancestors have passed in their racial history. Briefly this same statement is, ontogeny recapitulates phylogeny. The rehearsal of the phylogeny is in rather slurred form in some details, but the basis for the idea is readily seen. In brief, the theory is applied by comparison. Nearly all metazoan organisms begin life by the union of the two germ cells to form a singlecelled zygote which is the new organism. At this time it is comparable to the Protozoa. During the ensuing cleavage divisions a colonial form is represented. Following this, when one side infolds to form a gastrula with two germ layers, the embryo is almost identical to the diploblastic coelenterates as represented by Hydra. Following this, the third germ layer forms between the others and results in the triploblastic metazoan.

\section{Basis for the Theory of Evolution}

One who has thoughtfully studied the field of zoology soon realizes many relationships or homologies in structural make-up of certain different groups of animals; much similarity of embryonic developmental processes, and fundamental coincidences in physiological activities in all living material. Too, it is recognized that fauna and flora are not the same in all parts of the earth at the present, and have not been the same in the past as now. These realizations and otlier similar ones have been based on scattered sources of evidence, much of which is indirect. The evidences which have been discovered, however, have led to the formulation of the theory of evolution and its rather general acceptance as a working hypothesis among biologists. There has been confusion regarding this subject through failure to distinguish between the existence of progressive evolutionary changes, the course of these changes, and the cause of such a progressive series of events. The latter point has been the basis of most of the questions concerning the whole idea, and it is the most speculative of the three. These three are separate though related factors. 
In general, scientists are convinced, according to the evidence they have examined, that organic evolution exists in the form of a progressive change, which has generally proceeded from simpler to more complex organization through a long period of time. Although some sections of the course of this series of developmental events cannot be charted as completely as a ship's course at sea, numerous landmarks serve to indicate what has taken place. Biologists are free to admit that the causes of this process are not understood, and treat it strictly as a scientific problem.

Most estimates of the time when living organisms first came into existence range between $60,000,000$ and 1,200,000,000 years ago, coincident with Pre-Cambrian deposits. The manner of origin of protoplasm is purely hypothetical. It is suggested that when conditions became suitable, as to chemical elements present, temperature, pressure, etc., a relatively simple colloidal, protoplasmic mixture arose, having properties of life though perhaps in a very elementary way. It is assumed that all organisms which have lived are descendants of this simple origin. Cellular organization, with division of labor between nucleus and cytoplasm, is taken to be a first step in the development of protoplasm. It is the simplest vital unit now recognized. If this speculation is followed further, it may be supposed that the unicellular organisms of modern times have descended from such early cells without changing from the simplecelled state, but have developed many specializations as individual types, while metazoans have adranced from certain of these simple forms to a more complexly organized cellular condition.

From the nature of protoplasm and from the evidence in the geological records, it is usually assumed that organisms first appeared in the water near the shores of the primitive oceans. Presumably the first oceans were boiling hot, and the land at that time was a molten volcanic mass. If this is true, there must have been a long period after the formation of the atmosphere, seas, and land before protoplasm, as we know it, could have existed. After these cooled and became favorable for life the seas are thought to have supported an abundant life before the land became suitable for its existence. Along with these several speculative aspects of the subject there have been offered several forms of evidence to support the existence of an evolutionary progress of development in organisms. 


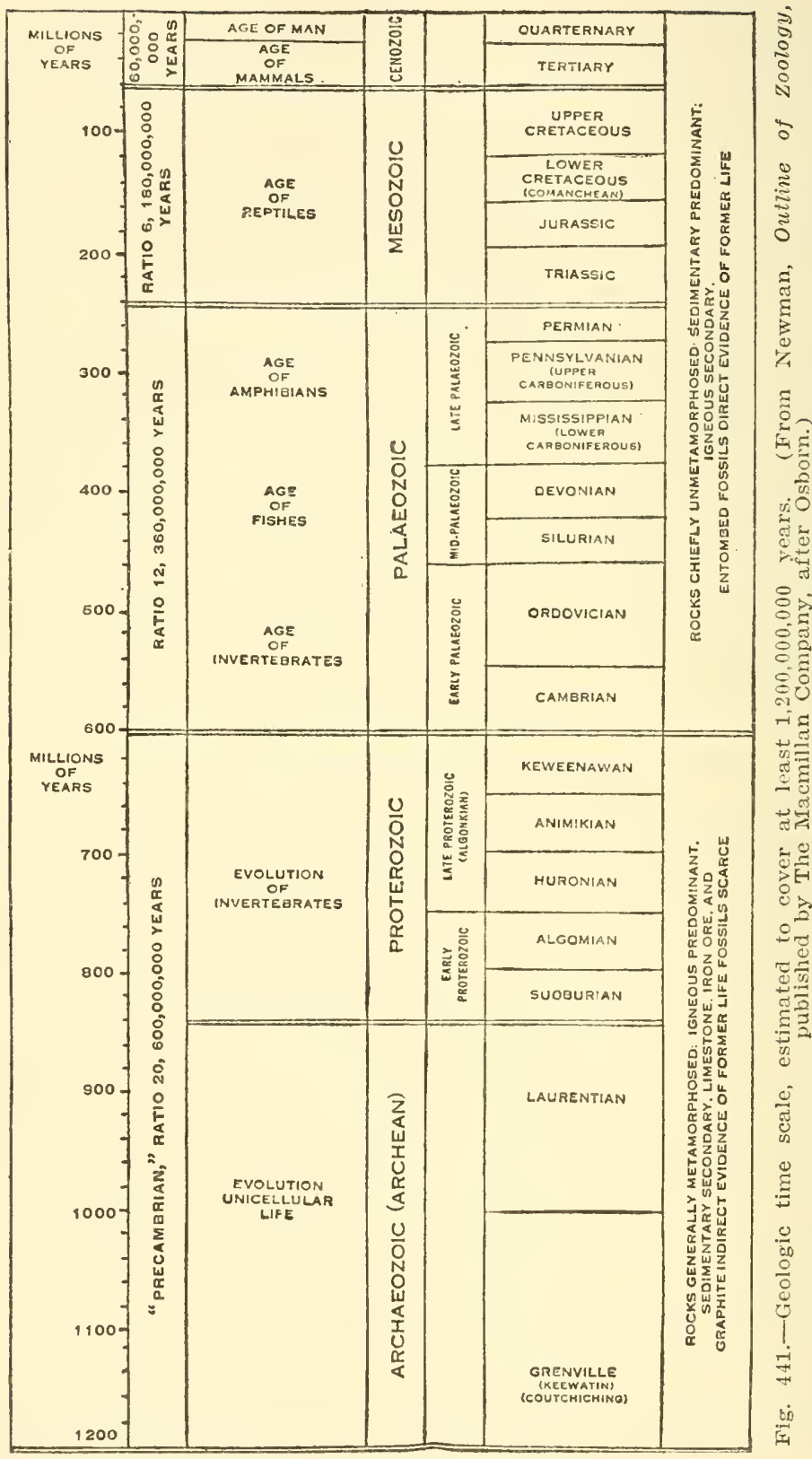


Geological Evidence.-Paleontology is the study of fossil remains of organisms deposited in the strata of the earth's crust. Shells and other hard parts are mineralized or petrified; in other cases mud impressions, or tracks, or pitch-preserved individuals, such as insects, and a few frozen forms constitute the majority of fossils. It must be kept clearly in mind that the geologist is able to determine quite accurately the sequence of time or chronological succession of the layer's of the earth's crust. The geological time scale shows a long period before any life existed, then the appearance of unicellular plants, then unicellular animals, then colonial forms, simple many-celled forms, and then the more complex ones. Such a timetable estimates the relative period of time during each era and shows some fusion and overlapping of certain types of life. Certain types of unicellular forms are continuous through the entire scale.

The principal facts shown by the fossil record may be summarized: (a) The fossil forms are not strictly identical with any living species, and the remains of plants and animals of each geologic stratum are at least specifically different from the forms in any other stratum, but they may belong to the same genus; (b) the oldest strata containing fossils have represented in them most of the simpler forms of nonchordate animals, while the upper strata contain fossils of all groups mole nearly like modern forms, including chordates; (c) in studying these in sequence, there may be observed a gradual progression from simpler and generalized types toward more specialized and complex forms as one proceeds from the older toward the upper or newer strata; (d) only the more generalized varieties have persisted within the groups that, as a whole, have become specialized; many of the others have long since reached their climax of specialization and have become extinct; (e) many of the dominant groups of organisms have arisen near the close of a period during which great climatic changes were taking place and have enjoyed dominance during the following period because such a group probably arose in response to the conditions; (f) although many nonchordate phyla had reached an advanced stage of development in the early Cambrian period, where early fossil records occur, many ancestral sequences have been observed, and these have supplied information making possible the detailed description of the course of events that has led to the surriving animals of modern 
times; $(\mathrm{g})$ the developmental changes of the chordate groups are more completely read in the fossil record, with the history of the mammals in fullest outline, partly because they are relatively recent and partly because the mammalian skeleton is readily fossilized. The most complete pedigree in fossils has probably been worked out for the horse, and a great deal of its material has been located in the southwestern part of the United States.

The Rise of the Horse--Primitive horselike animals are thought probably to have arisen from an extinct group called Condylarthra, which had five toes on each foot and a large part of the sole resting
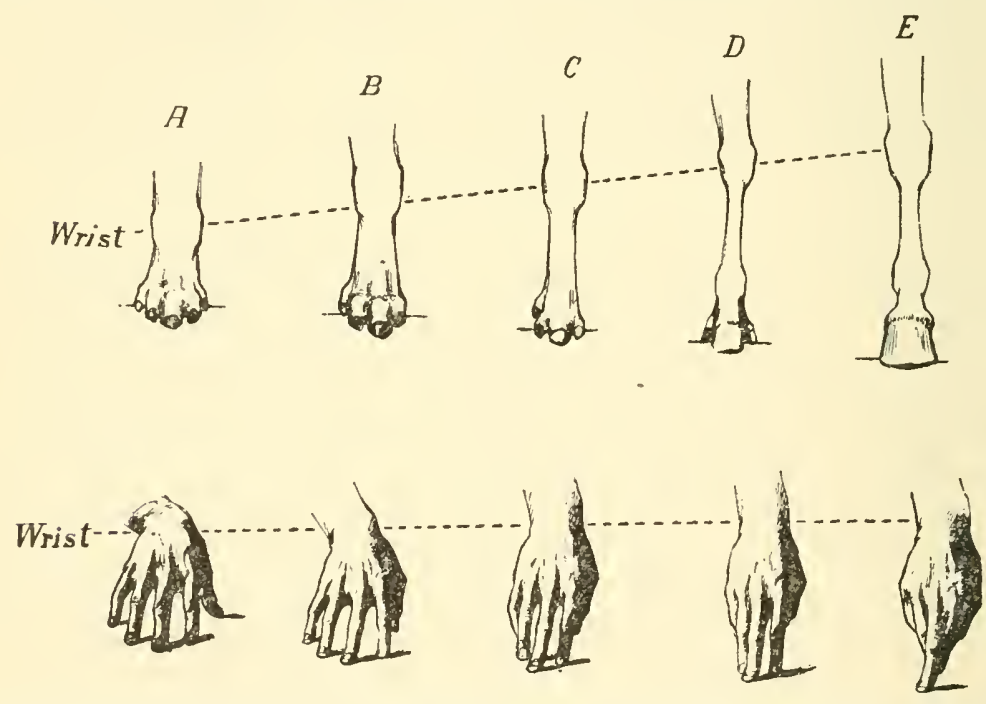

Fig. 442.- Positions of the human hand to show the comparative stages of elevation of the horse's foot to the tip of the middle toe. (Courtesy of American Museum of Natural History.)

on the ground. The first unquestionable horselike form found in America is the small Eohippus which was about one foot tall and the fossils of which came from the rocks of Eocene times. It had the outer four digits complete on the forefoot, but no trace of the thumb, while the hind foot had three complete digits with vestiges or splints of the first and fifth. Following the foxlike Eohippus, later in the Eocene period came the Orohippus with an enlarged central digit in the forefoot and the loss of the splints in the hind foot. Mesohippus, about the size of a large dog with a three-toed 


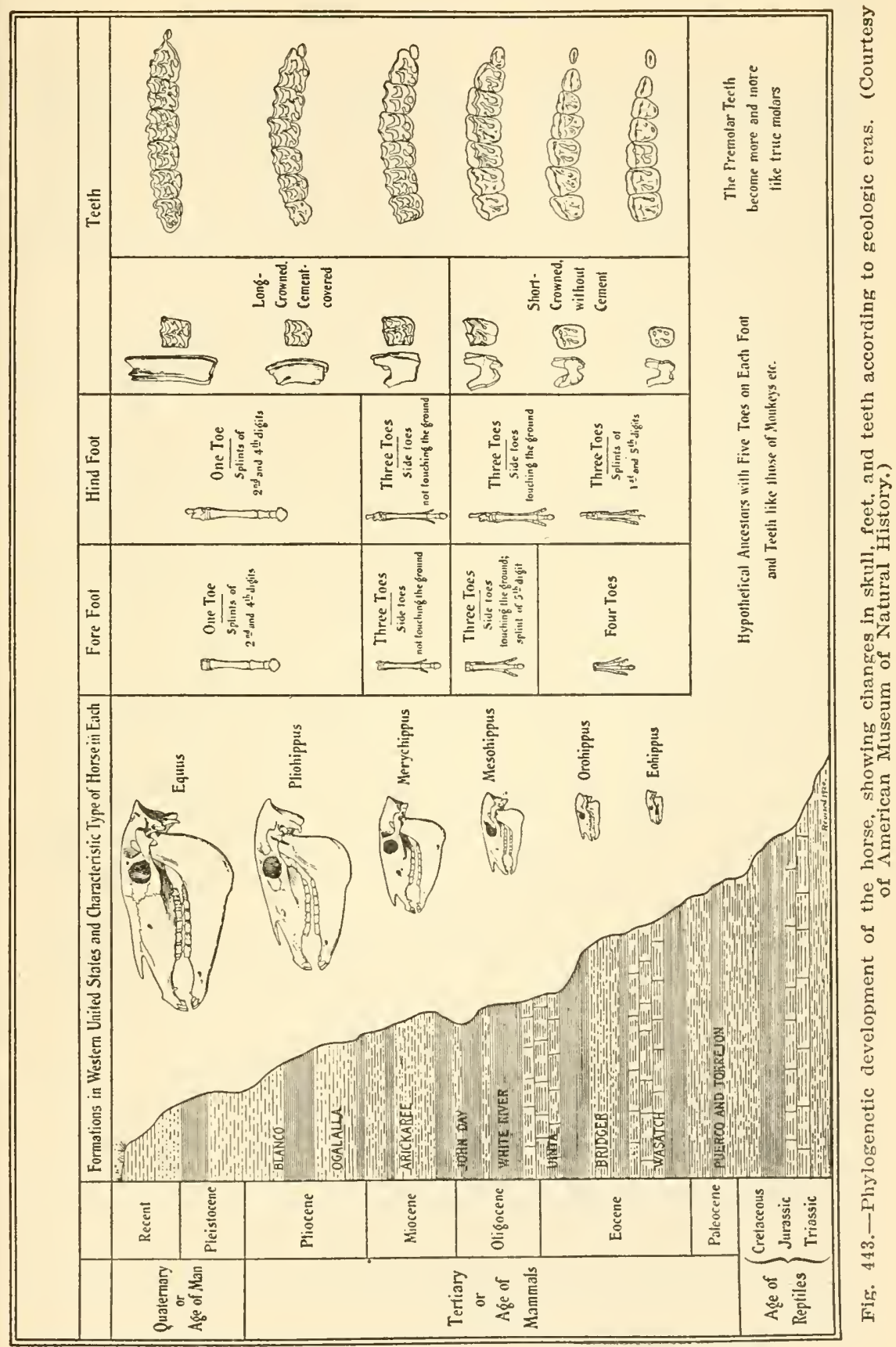


foot both in front and behind but with the side toes much reduced and a trace of the fifth digit in the forefoot, appcared during the Oligocene epoch. Merychippus of Hiocene times and Pliohippus of the Pliocene epoch show a continuation of this reduction to a onetoed type which leads to Equus caballus of modern times. The modern horse walks on the tip of the middle toe of each foot with the vestiges of digits 2 and 4 persisting as splints.

Arthur Dendy, in 1911, wrote that the horse is an example of the adaptation of a lowland type to become a plains type, as the extensive, dry, grass-covered, plains developed. The adaptation has proceeded along two lines. The limbs have become elongated by the elevation of the heel, thus putting the animal on tiptoe and fitting it for rapid flight from its enemies on the grass-covered open prairie. At the same time the neck and head became elongated to enable the animal to graze the ground without bending the legs. Along with this the teeth changed from a carnivorous form to a complex, broad grinding type for feeding on grass. In addition to these changes the brain advanced.

These changes took place gradually through millions of years and the intermediate forms give the paleontologist a graphic picture of the history of this modern species. The sequence of these stages seems to fit in exactly with the theory that each has been derived from the preceding by a continued adaptation to the changing conditions of life. The horse's pedigree is essentially similar to that of numerous other forms, such as the elephant, camel, and certain birds, which have been worked out.

The molluscan, echinoderm, and arthropod fossils give similar stories among invertebrates. The numerous fossils of fish, Amphibia, Reptilia, particularly dinosaurs, and birds with the famous Archaeopteryx, all have their testimony to contribute.

Distributional Evidence.-The paleontological distribution of animals is considered vertically while geographic distribution is in horizontal plane. It is impossible to separate these two completely. The study of geographical distribution gives essentially a cross section of the vertical distribution, thus giving a sort of "still picture" of the complex developmental relations of animal groups at one moment in geologic time. In studying this subject, it is necessary to have in mind two fundamental conceptions. The first is that the ancestors of related genera first appeared or originated in a 
locality which is designated as the common conter of origin. A second conception is that as the ancestral form became established and multiplied, migration in search of food and more suitable conditions occurred. Barriers, many of which were geographic, determined the direction and extent of this migration. Large bodies of water blocked the passage of terrestrial animals, as of course land was a barrier to aquatic animals. High mountains or deserts were barriers to all animals unable to withstand low temperatures and altitudes on the one hand, or high temperature and dryness on the other. These forces, and others, are believed to account for the natural distribution of animals. The cases of the camel group, originating in North America, migrating to South America and Asia by the land connections of the Eocene to Pliocene epoch, and the tapirs, which are represented by distinct species in two widely separated regions, South Asia territory, and the Central America-South American territory are examples. Here again paleontological data show that in the Pliocene epoch tapirs were distributed over nearly all of North America, Northern Asia, and Emrope. Following that time they were gradually decreased due to discontinuous distribution until the one-time world-wide distribution is now isolated in two widely separated regions. Long isolation of genera in different environments will bring about definite specific differences. As an example, a litter of foreign rabbits was introdnced to the island of Porto Santo during the fifteenth century and by the middle of the nineteenth century the descendants had become so distinct from the original ancestors that they were described as a new species. There are numerous instances of this effect, demonstrated by isolation on ocean islands. The islands are either continental, with fauna similar to those of the nearby continent from which the animals have come, as the British Isles, or they are oceanic, with a very bizarre assemblage of animals which have either drifted in or have been carried there, as the Hawaiian Islands. Many of the animals on these oceanic islands are peculiar and are found nowhere else on eartl. Australia has a group of animals, examples of which are very different from those of Asia because the two have been so long separated. Europe, Asia, Africa, and North America have been comnected with each other by land bridges in recent enough times that the mammals show similarity. The distribution of the species of a genus often radiates from the more generalized specics which occupy the 
center of the range of the genus, and the more specialized species are found in the scattered outskirts of the range.

From the preceding statements concerning distribution it seems that any given species originates in a definite locality, that it multiplies there and migrates in all possible directions. It modifies as it goes in response to the various new conditions prevailing and becomes divided into local varieties which in the course of time become species. Thus the working method of animal distribution, as it has been presented, is the principle of descent from preceding generations with modification.

Morphological Evidence.-Classification of the animals shows in fact something of the morphological evidence, since current classification is based chiefly on anatomical features and comparative anatomy. The groups of the classification are established largely on anatomical similarities. The differences existing among the representatives of all the classes of vertebrates are relatively slight when set over against the fundamental similarities. Closely related groups show numerous similarities in the form of homologies. The flipper of the whale, the wing of the bat or the bird, the foreleg of the cat and the arm of man all show the same general type of structure in spite of certain specific differences. There is seldom any question of their phylogenetic relationship.

The presence of series of similar parts on different segments of the same animal and the various specializations of these parts show a progressive development. The highly specialized walking legs, uropod, claws, and antemnae of crayfish, for example, have all developed from the simple swimmeret type of appendage. They form a serial homology and are also homologous to the appendages of all other Crustacea as well.

In higher forms of animals, such as man, there are numerous structures which seem to be useless and are even harmful in some instances. These are spoken of as vestigial structures. Such parts correspond in structure and plan to functional parts in other related forms, but are reduced morphologically and without the original function. In man, one probably thinks first of the vermiform appendix of the colon as such a structure. This is apparently funetionless in human beings and can be removed with no loss, but this same organ in rabbits, some birds, and other animals is an extensive and functional digestive organ. Man has a $\operatorname{coccy} x$ or vestigial tail, 
and the frog has the urostyle. Pythons and porpoises, neither of which has the least use for them, have vestigial hind limbs similar to the functional ones of their relatives. The salivary glands of certain snakes have become adapted as poison glands, certain sweat glands have become milk glands, and gill arches have become sup. porting structures of the tongue, larynx, and throat of adult terrestrial animals. The blood supply and nerves both follow the phylogenetic changes of these organs.

Most animals seem not to have originated in these present forms, and they do seem to have changed through the long periods of geologic history. The explanation offered by modern biologists for these anatomical relations and resemblances between animals is that the individuals in any group have inherited a similar plan of structure from the ancestors which was common to all members of the group. In a group, such as the vertebrates, there have been numerous modifications of various fundamental structures in different subdivisions in relation to the particular habits of life; still they remain fundamentally alike because they have developed from the underlying plan of organization found in the ancestors. The seal and the bird, although quite different, show similarities in habits and otherwise because of common ancestry long ago. The conclusion of biologists of today is that all of the animals in a group, such as the vertebrates, have arisen by descent with change from a primitive organism which possessed the fundamental organization as shown from cyclostomes to man.

Embryological Evidence.-Evidences from this field really continue directly from the previous discussion. The animal is to be thought of as an individual from the single-celled zygote stage to the mature stage of old age, no matter what its complexity. Intimately related types of animals parallel through a large portion of their development to diverge somewhat in adult condition, more remotely related forms take separate developmental courses rather early in life, and unrelated forms may be different almost from the beginning. In numerous instances the developing stages of more advanced forms resemble very closely the mature stages of the less advanced types in a serial fashion. The history of the individual animal often corresponds in a general way to the history of the advances of the animal kingdom, up to its state of development. This apparent repetition of the ancestral development in individuals 


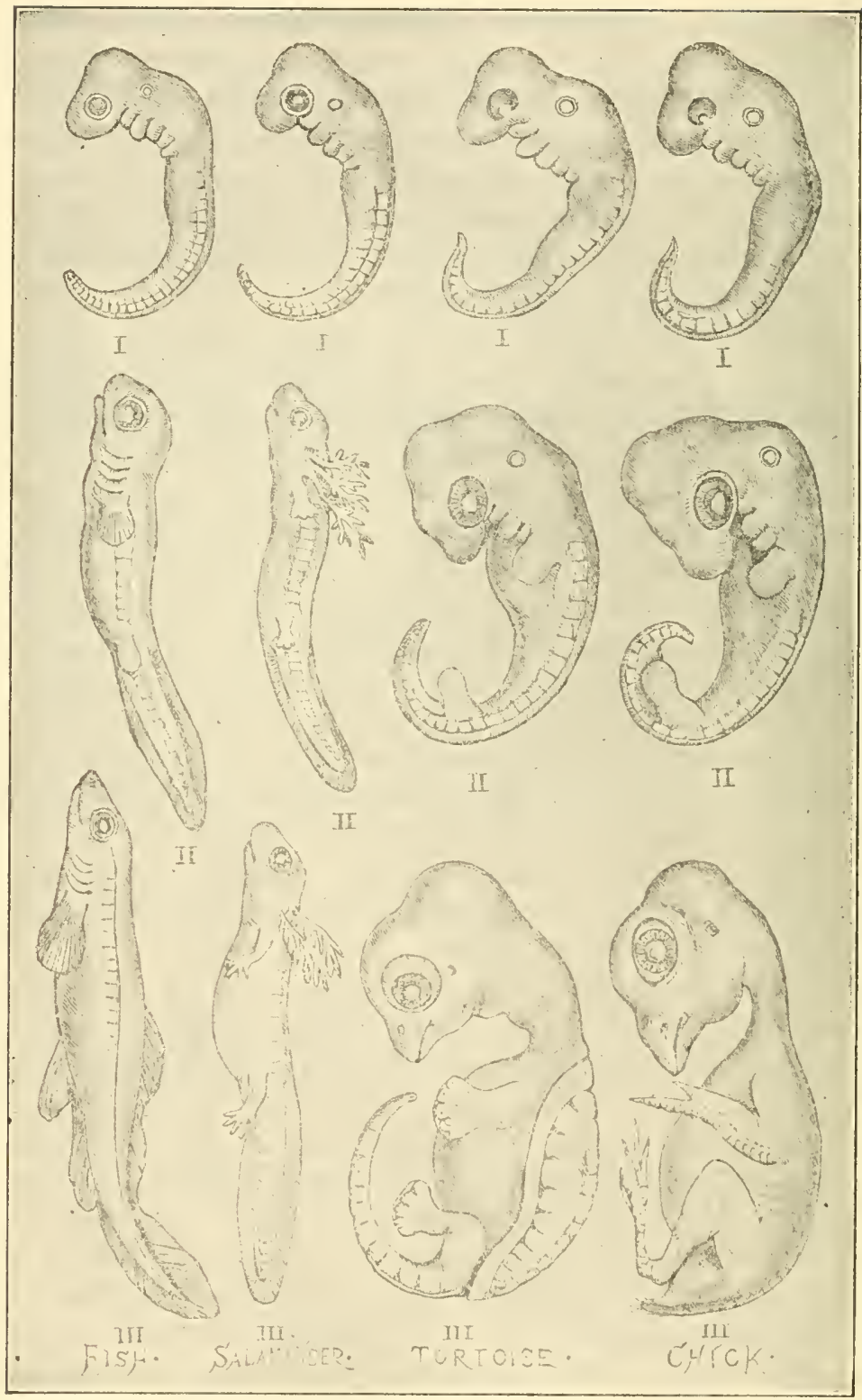

Fig. 444.-Diagram to show three parallel stages of development of several (From Romanes, Darwin and After Darwin, published by the Open Court Publish- 


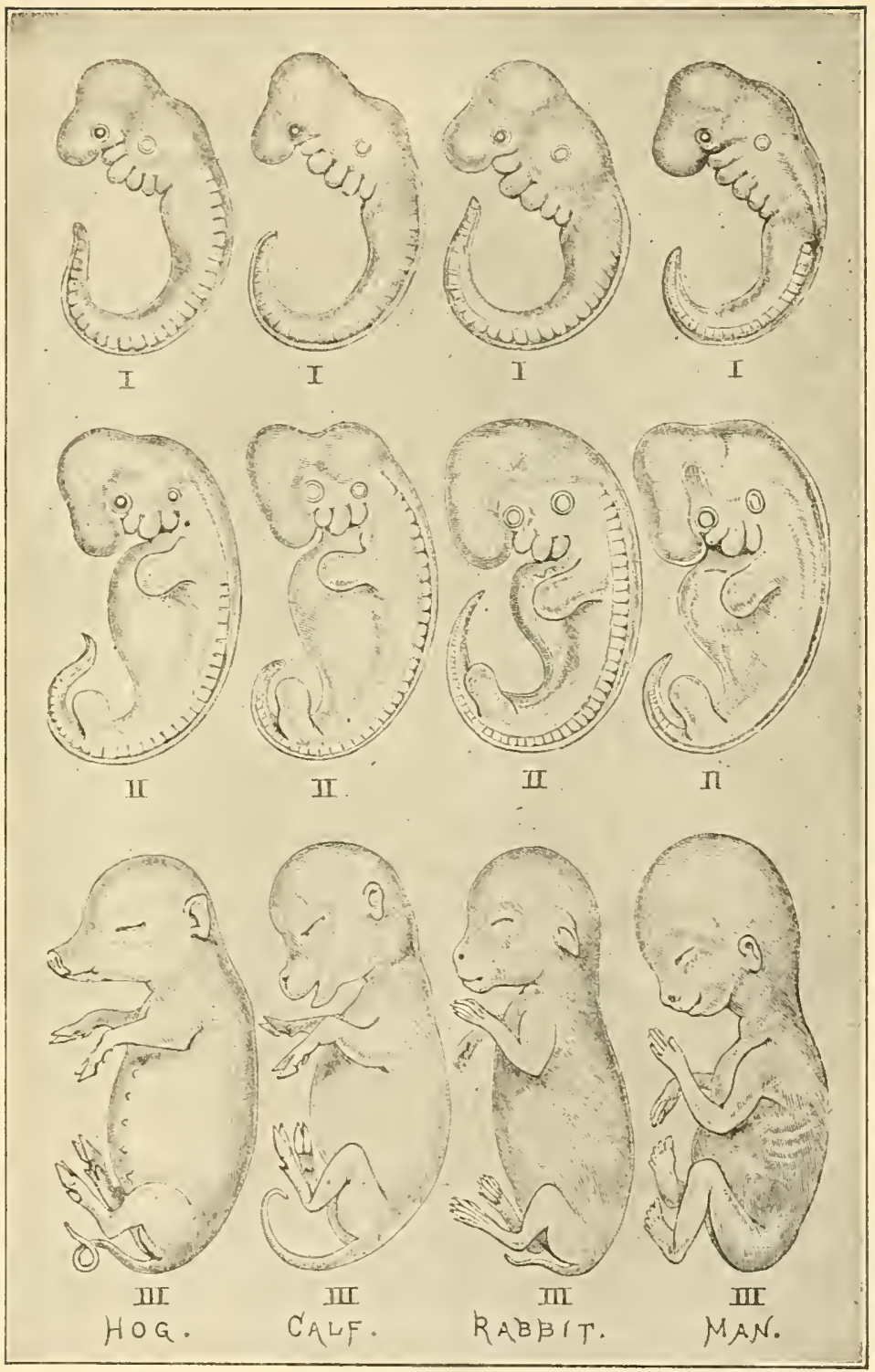

vertebrate animals. Notice the close similarity of the first two stages in all. ing Company.) 
was what led Haeckel to formulate the recapitulation theory, expressed briefly: ontogeny repeats or recapitulates phylogeny, as has been discussed earlier in the chapter.

In the vertebrate group these apparent relations are much shown. Most of the embryos of this group are so similar that it is nearly impossible to distinguish them. They pass through the identical
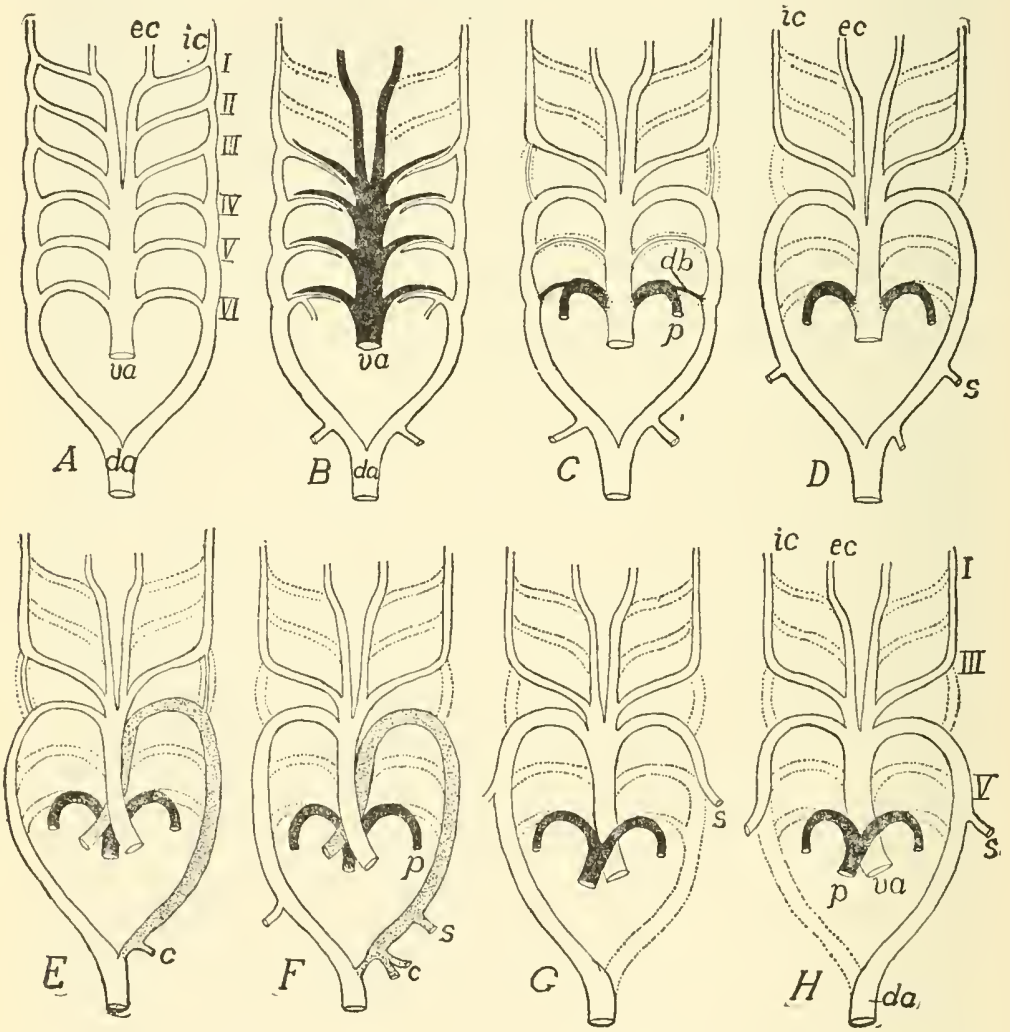

Fig. 445.-Diagram to show the modifications of aortic or branchial arches in different vertebrates types. $A$, primitive scheme; $B$, lungfish: $C$, primitive amphiblan (urodele); $D$, frog; $\dot{E}$, snake; $F$, lizard; $G$, bird; $H$, mammal; $e c$, ic, external and internal carotids: va, ventral aorta: da, dorsal aorta; db, ductus Botalli ; $p$, pulmonary artery; $s$, subclavian; $c$, coeliac. Vessels carrying venous blood are black; those with mixed blood are shaded; those which disappear are dotted outlines. (After Boas, Reprinted by permission from Kingsiy, Comparative Anatomy of Vertebrates, published by The Blakiston Co.)

stages of development. Systems, such as the circulatory, nervous, digestive, and respiratory, follow the same course of development in all of the vertebrates, no matter how simple or complex. In earlier stages the similarity is strikingly close. 
The course of development and the modifications shown in the aortic arches or main arteries leaving the heart and passing through the gill regions is a specific example of the manner in which a specific set of structures follows out a repetition of ancestral stages in the development of the individual. Fig. 445 shows a comparison of the arrangement of the branchial (aortic) arches from the primitive set of six through the fishes, Amphibia, reptiles, to the birds and mammals, with a modified condition of three arches.

Along with the aortic arch situation are other examples of similar stages of development in all forms of the group. Gills are present in all chordates at some time. In the primitive ones the gills are functional throughout life; in the more adranced types they are only transitory gill structures. In frogs and toads, the tadpole stage is essentially fishlike, and they become amphibian at metamorphosis. The notochord is a definite structure throughout the life of the primitive chordates, but is present as such in only the embryo stage of all others. The development of the heart from the tubular condition through the two-chambered, three-chambered, and finally fourchambered condition, illustrates the same progressive development.

Physiological Evidence.-The fact that all protoplasm possesses the same set of fundamental properties or capacities as contractility, irritability, metabolism, etc., is in itself a definite indication of relationship of all organisms, since they are all composed of protoplasm. Too, all protoplasm acts under similar laws and conditions.

Such natural substances of animal bodies as the hormones, or antibodies or even enzymes are almost universal in their reactions among chordates and even among nonchordates. MIost of them are interchangeable from one animal group to anotler. A deficiency of pepsin or adrenalin in man may be supplied from a cow, a hog, a cat, a rat, or a dog. The blood of all vertebrates has certain physiological similarities and some specific differences. The blood of large groups of human beings will mix without precipitation and is said to "match." Other individuals' blood may not "match" in this type but will mix with blood from another group. The agglutination (clumping of red corpuscles) which occurs when samples of blood from two such individuals are put together, is due to the reaction of two substances produced by the corpuscles in one or both of the blood samples. The designation of the blood group depends upon the presence or absence of one or both of these substances. One 
blood group contains neither, another contains one substanee, a third the second substance only, while a fourth group eontains both substanees. Serum of the first group will agghntinate corpuseles when mixed with any of the other three. The groups with the single substance will agglutinate corpuscles in blood having only the other substanee or blood with both substanees. The serum from blood with both substances will not eanse agglutination in any of the others.

In lower mammals some similar blood groups have been found, but it is only in the apes that the groups correspond to those in the human. This is an indication of the rather elose relationship of these animal groups. Whether in human or other animals this blood characteristie is permanent in the individual and it is hereditary.

Serum (blood minus eorpuscles and fibrinogen) studies also establish certain relationships among animals. If small quantities of human blood serum are introduced at intervals into the blood stream of a rabbit, in time there will be developed in the blood of the rabbit a substance (antibodies) which, when mixed with normal human blood will eause preeipitation of the proteins here. Serum from such a rabbit is ealled antituman serum. When this serum is mixed with serum in a certain dilution taken from human, chimpanzee, gorilla, or monkeys, it will cause precipitation. If the dilution is increased there is no precipitation when mixed with monkey serum; at still higher dilution there is no preeipitation when mixed with gorilla blood; and higher none for chimpanzee blood, until finally none for human blood. On the basis of these sera preeipitation tests the chimpanzee is elosest in its relation to man, then gorilla, and then the monkeys.

In making similar tests on other vertebrate groups it is found that crocodiles are more closely related to birds than are the other reptiles; also among reptiles, that snakes and lizards are more closely related to each other than to turtles. Too, croeodiles show a closer relation to turtles than to the other groups of reptiles. Blood studies of the various gromps of vertebrates indicate that there is more similarity in blood of closely related forms than of others. At the same time, it is seen that a chemical relationship persists in the blood throughout the chordate phylum.

The breeding of plants and animals through long series of generations of domestieation and laboratory experiments has yielded much 
information concerning the ways of adaptation and phylogenetic development. A significant result is the demonstration of changes oecurring in animals and plants. From such studies it seems quite obvious that organisms now living have come to be what they are by gradual ehange from generation to generation through a course of descent from preexisting and varied ancestors, rather than by a sudden and eompletely new development. Most of the various breeds of cattle, chickens, dogs, horses, sheep, erops, ete., have been developed in each case from a preexisting common ancestor.

\section{Darwin and Studies of Evolution}

Most discussions of organic evolution usually begin with mention of Darwin's monumental work on this subject and diffieult it is to get away from his fundamental basic thinking on the subject. $\mathrm{He}$ was the first to thoronghly survey the fields of distribution, morphology, embryology, and paleontology, and logically relate the data found there to the theory of evolution. From his studies, many of which were done along the east and west shores of South America while he was naturalist of a British Naval expedition on the ship "Beagle," Darwin formulated a clear-eut and definite argument for evolution on the basis of Natural Selection. Beginning with Malthus' Law of Population, published in 1838, which stated that since man reprodices in a geometric ratio, the earth would be overpopulated in a few gencrations except for such checks as the arithmetric ratio of inerease in food production, disease, war, flood, earthquake, fire and other natural eatastrophes reducing population, Darwin formulated the Natural Selection Theory. This theory includes among other things the application of Malthus' law to all living organisms. The fonr basie points on which this theory is developed, may be named in order as follows: (1) overproduction, (2) struggle for existence, (3) variation and heredity, and (4) survival of the fittest (natural selection).

Overproduction is in operation in all thriving normal species. A single codfish will produce several million eggs in one season. If every codfish egg were to be fertilized, reach maturity, and reproduce with no loss from one generation to the next, it would not be more than a dozen years until the entire face of the earth would be eovered with codfish and all other animals would be crowded out of existence completely. Even a form like the elephant, which lives to 
be ninety or a hundred years of age and averages only six progeny, could soon oceupy all of the standing room on the face of the earth. Beginning with one pair of elephants and providing every individual lived and reproduced even at the average slow rate mentioned, $19,000,000$ individuals would be produced in 750 years. If every elephant alive today were to enter into a program like that, both food and space would become quite scarce before many generations. However, this doesn't happen on a large scale. All plants and animals tend to produce more offspring than can ever reach maturity and reproduce.

The Struggle for Existence is ever present because there are more individuals produced than the habitat will support. The two most fundamental needs for which organisms struggle are: (1) food and (2) opportunity to reproduce and rear young. Of these two, the struggle for food is very immediate and the food supply is an important limiting factor on population from season to season. Since the food supply, on the average, remains quite constant, it is evident that only a limited number of the increase in individuals can be supported in a particular habitat. A struggle ensues with each individual attempting to secure the necessities of life. Not only is there a struggle for food but also with many factors in the environment like climate, geographic changes, ete.

Survival of the Fittest was the outcome which Darwin saw resulting from such a struggle. Those individuals which were best adapted to the environment into which they were born have been the ones to win out in the struggle and leave offspring for a future generation. The inheritance of favorable or unfavorable characters influences very strongly the success of the individual in maintaining itself. The survivors in any generation are those which inherit the most favorable combination of variations. Many variations, both favorable and unfavorable to the success of the individual, are hereditary.

When changes in environment come and bring about new living conditions the animals in the particular habitat must meet these changes, be able to migrate, or perish. The standard for fitness has changed under such circumstances and animals with somewhat different characters and adaptations may now be the "fittest." The individuals whose variations have brought them to most nearly fit the requirements for life in their particular habitat will be the ones most likely to obtain sufficient food supply and adequate provision for reproduction to rapidly increase their population. As one group 
is able to do this, it invariably reduces or perhaps entirely eliminates other species in the locality. Evolutionary changes result from survivial of the fittest which has come about by accumulation of favorable variations in each of successive generations. Those individuals or races which have not been as well adapted to conditions of the habitat have become inconsequential or extinct. The appearance of characters in an animal is a matter of chance as far as the individual is concerned. Natural selection may act as an eliminating agent and determine whether the character or trait shall survive after it appears.

Mutation Theory.-Hugo de Vries is the name most prominently connected with the origin of this theory. He was a Dutch botanist and in 1886 found some evening primrose plants (Oenothera lamarkiana) which exhibited discontinuous variation or sudden appearance of new characters. These sudden sharp variations came to be known as mutations. There are two types of variations recognized: (1) contimuous or fluctuating variation, such as height of individuals of a species where they are expected to fall within a normal range thus allowing a degree of variation among individuals of the species, and; (2) discontinuous or sport variation (mutation) where the variation falls outside the normal range of variation and not connected with it by intermediate changes.

In the course of several generations of this primrose and involving approximately 50,000 individuals, six different mutations were found. The new characters which appeared, were quite different from those of the typical species as well as being inherited. Since this was the case, de Vries concluded that he was observing the origin of new species. He was sufficiently convinced of this to discount Darwin's conception of the development of new species by the gradual accumulation of continuous variations through natural selection. He pointed out that mutations are due to changes occurring in the germ plasm while the continuous variations, individually, are due to changes in somatic cells.

Following de Vries' work there has been much study of mutations and numerous ones have been found in nature. Too, it has been found that spontaneous mutations could be produced in Drosophila (fruit fly) by x-ray radiation. It is thought that mutations come as the result of physiological changes in the chromosomes or genes. 
While this mutation theory of origin of new speeies has prompted much study and thought on evolution it seems not to have displaeed Darwin's general coneeption of the origin of new speeics. So far as Darwin's theory is concerned, the occurrenee of mutations only hastens the process of evolution since they produce quick abrupt variations instead of the slower smaller continuous variations. Natural selection will operate with either. Biologists now consider both small and large variations as mutations, and have turned back to Darwin's idea of natural selection as the most likely explanation of the development of new kinds of animals. 


\section{REFERENCES}

A limited number of selected works which are suitable for reference and collateral reading are presented in the following pages.

Potter, G. E.: Laboratory Outlines for General Zoology, St. Louis, 1938, The C. V. Mosby Co. A very useful guide to the laboratory portion of the study.

\section{Biology in General}

American Men of Science. Biographical sketches of outstanding scientists, ed. 4, 1927, Science Press.

Biological Abstracts. Review of the current biological literature. 1926-1947, Philadelphia.

Carpenter, K. E.: Life in Inland Waters, New York, 1928, The Macmillan Co.

Child, C. M.: Individuality in Organisms, Chicago, 1915, The University of Chicago Press.

-: Senescence and Rejurenescence, Chicago, 1915, The University of Chicago Press.

Conklin, F. G.: Heredity and Environment in the Derelopment of Men, Princeton, 1922, Princeton University Press.

Garrison, F. H.: History of Medicine, Philadelphia, 1923, IV. B. Saunders Co.

Geiser, S. W.: Naturalists of the Frontier, Dallas, 1937, Southern Methodist University Press.

Hegner, R. W.: The Parade of the Animal Kingdom, New York, 1935, The Macmillan Co.

Locy, W. A.: Biology and Its Makers, New York, 1915, Henry Holt and Co. -: The Growth of Biology, New York, 1925, Henry Holt and Co.

Loeb, Jacques: The Organism as a Whole, New Iork, 1918.

Morgan, Ann: Fieldbook of Ponds and Streams, New York, 1930, G. P. Putnam's Sons.

Needham, J. G., and Needham, Paul R.: Guide to Freshwater Biology, American Viewpoint Society, 1927.

-, and Lloyd, J. T.: The Life of Inland Waters, Ithaca, N. I., 1930, Comstock Publishing Co.

Nordenshiod, E.: History of Biology, New York, 1928, Alfred A. Knopf.

Ward and Whipple: Fresl-TVater Biology, New York, 1918, John Wiley \& Sons.

White, E. Grace: General Biology, St. Louis, 1937, The C. V. Mosby Co.

Williams, S. H.: The Living World, New York, 1937, The Macmillan Co.

Woodruff, L. L.: The Development of the Sciences, New Haren, 1923, Yale University Press.

Young, R. T.: Biology in America, Boston, 1922, Gorlam Press.

\section{General Zoology}

Atwood, Henry W.: Introduction to Vertebrate Zoology, St. Louis, 1940, The C. V. Mosby Co.

Borradaille, L. A., and Potts, F. A.: The Invertebrata, New York, 1932, The Macmillan Co.

Buchsbaum, Palph: Animals Without Backbones, Chicago, 1938, The University of Chicago Press.

Curtis, W. C., and Guthrie, Mary J.: 'Textbook of General Zoology, New York, 1947 , John Wiley and Sons.

Guyer, M. F.: Animal Biology, New York, 1941, Harper and Brothers.

Haldane, J. B. S., and Huxley, Julian: Animal Biology, 1927, Oxford at Clarendon Press. 
Hegner, R. W.: College Zoology, New York, 1942, The Macmillan Co. -: Invertebrate Zoology, New York, 1933, The Macmillan Co.

Jordan, D. S.: A Manual of the Vertebrates, New York, 1929, World Book Co. Kingsley, J. R.: Comparative Anatomy of Vertebrates, Philadelphia, 1926, P. Blakiston's Son and Co.

Krecker, Frederick H.: General Zoology, New York, 1934, Henry Holt and Co. Lane, Henry H.: Animal Biology, Philadelphia, 1929, P. Blakiston's Son and Co.

Metcalf, Z. P.: Economic Zoology, Philadelphia, 1930, Lea and Febiger.

Newman, H. H.: Vertebrate Zoology, New York, 1920, The Macmillan Co.

-: Outlines of General Zoology, New York, 1936, The Macmillan Co.

Parker, T. J., and Haswell, William A.: Textbook of Zoology (two volumes), New York, 1930, The Macmillan Co.

Petrunkevitch, Alexander: Morphology of Invertebrate Types, New York, 1916, The Macmillan Co.

Pratt, H. S.: A Manual of the Common Land and Fresh-Water Vertebrates of the United States, Philadelphia, 1935, The Blakiston Co.

-: A Manual of Common Invertebrates, Philadelphia, 1935, The Blakiston Co.

Reese, Albert M.: Economic Zoology, Philadelphia, 1924, P. Blakiston's Son and Co.

Shull, A. F.: Principles of Animal Biology, New York, 1934, McGraw-Hill Book Co.

Storer, Tracy I.: General Zoology, New York, 1943, McGraw-Hill Book Co.

Van Ćleave, H. J.: Invertebrate Zoology, New York, 1931, MeGraw-Hill Book Co.

Walter, H. E.: Biology of the Vertebrates, New York, 1928, The Macmillan Co. Wolcott, Robert H.: Animal Biology, New York, 1946, McGraw-Hill Book Co. Woodruff, L. L.: Foundations of Biology, New York, 1932, The Macmillan Co.

\section{Protoplasm and the Cell}

Cowdry, E. V. (editor): General Cytology, Chicago, 1924, University of Chicago Press.

Doncaster, L.: An Introduction to the Study of Cytology, London, 1920, Cambridge University Press.

Heilbrunn, L. V.: Colloidal Chemistry of Protoplasm, Berlin, 1928, Borntraeger.

Huxley, T. H.: On the Physical Basis of Life, 1868, Collected Essays, Vol. Method and Results, D. Appleton \& Co.

Loeb, Jacques: The Dynamics of Living Matter, New York, 1906, Columbia University Press.

Painter, Theophilus S., and Griffen, Allen B.: The Structure and the Development of the Salivary Gland Chromosomes of Simulium. Genetics 22: 612633, November, 1937.

Sharp, L. W.: Introduction to Cytology, ed. 2, New York, 1926, McGraw-Hill Book Co.

Wilson, E. B.: The Cell in Development and Inheritance, ed. 3, New York, 1925, The Macmillan Co.

-: The Physical Basis of Life, New Haven, 1923, Yale University Press.

\section{Protozoology}

Calkins, Gary N.: Biology of Protozoa, Philadelphia, 1926, Lea and Febiger. Hegner, R. W., and Taliaferro, W. H.: Human Protozoology, New York, 1924, The Macmillan Co.

Kudo, Richard R.: Handbook of Protozoology, Springfield, Ill., 1931, Charles C Thomas, Publisher. 
MTarshall, C. E.: Microbiology, ed. 3, Philadelphia, 1926, P. Blakiston's Son and Co.

Woodruff, L. L.: The Origin and Sequence of the Protozoan Fauna of Hay Infusions, J. Exper. Zoöl. 12: 205-264, 1912.

\section{Porifora, Coelenterata, Platyhelminthes, and Nemathelminthes}

Geiser, S. W.: The Distribution of Pectinella magnifica Leidy in the United States, Field and Laboratory 2: 1934.

Mayer, A. G.: The Medusae of the World, Washington, 1910, Carnegie Institute.

Moore, H. F.: The Commercial Sponges and Sponge Fisheries, Bulletin of U. S. Bureau of Fisheries 28: 1910.

Nutting, C. C.: American Hydroids, I, II, III, Bulletin of U. S. Nat. Museum, $1900,1904,1905$.

Old, M. C.: Taxonomy and Distribution of Freshwater Sponges of Michigan, Michigan Academy of Sciences, Arts, and Letters, No. 15, 1932.

-: A Contribution to the Biology of the Ereshwater Sponges, Michigan Academy of Sciences, Arts, and Letters, No. 17, 1933.

Potts, E.: Freshwater Sponges, Proc. Acad. Nat. Sci., Philadelphia, 1887.

Smith, F.: Distribution of the Fresh-Water Sponges of North America, 111. State Lab. Nat. Hist. Surv. No. 14, 1921.

\section{Annelida}

Beddard, F. E.: Earthworms and Their Allies, London, 1901, Cambridge University Press.

Harmer, S. F., and Shipley, A. E.: Worms, Cambridge Natural History Series, Vol. II, New York, 1909, The Macmillan Co.

\section{Mollusca}

Rodgers, Julia: The Shell Book, Garden City, N. Y., 1909, Doubleday Page. Tryon, G. W., and Pilsbry, H. A.: A Manual of Conchology, Phil. Acad. Nat. Sci., 1878.

\section{Crustacea}

Creaser, E. P.: Decapod Crustaceans of Wisconsin, Trans. Wisconsin Academy Sciences, Arts, and Letters, No. 27, 1932.

Harris, J. A.: An Ecological Catalogue of the Crayfishes Belonging to the Genus Cambarus, Kansas Univ. Sci. Bull. 2: 51-1\$7, 1913.

Hay, W. P.: Crustacea of Iudiana, Proc. Indiana Acad. Sci., 1896.

-: Life History of the Blue Crab, U. S. Bur. Fish. Rept., 1905.

Herrick, F. H.: Natural History of the American Lobster, Bull. Bureau Fish. 29: 1909.

Herriek, C. L., and Turner, C. H.: Synopsis of the Entomostraca of Minnesota, Minnesota Geol. and Nat. Hist. Surv., 2d. Rept. State Zoologist, 1895.

Rathbun, Mary J.: The Spider Crabs of America, Smithsonian Inst. N. S. Nat. Mus. Bull. 129.

-: The Grapsoid Crabs of America, Bull. 97 U. S. Nat. Mus., 1917.

\section{Myriapoda}

Bollman, C. H.: The Myriapoda of North America, U. S. Nat. Mus. Bull. 46, 1893.

Chamberlin, R. V.: The Chilopoda of California, Pomona Jour. of Ent. 4: 1912.

-: The North American Chilopoda and Diplopoda, Ann. Ent. Soe. Am. 5: 1912. 


\section{Arachinida}

Baerg, W. J.: The Effects of the Bite of Latrodectus mactans, J. Parasitol., Urbana, 1923, pp. 161-169.

Banks, N. A.: A Treatise on the Mites, Proc. U. S. Nat. Mus., 1904.

-: The Acarina or Mites, Bur. Ent. Report 108, U. S. Dept. Agric., 1915.

Chamberlin, R. V.: Revision of the North American Spiders of the Family Lycosidae, Proc. Acad. Nat. Sci., Philadelphia, 1908, pp. 157-318.

Comstock, J. H.: The Spider Book, Garden City, N. Y., 1912, Doubleday Page. Fabre, J. H.: Life of the Spider, New York, 1913, Dodd, Mead \& Co.

Petrunkevitch, A.: A Synoptic Index-Catalogue of Spiders of North, Central, and South America, New York Bull. Amer. Mus. Nat. Hist., 1911, pp. 1-791.

Ran, P.: Some Life Notes on the Black Widow Spider, Latrodectus mactans, Psyche 31: 162-166, 1924.

Reese, A. M.: Tenomous Spiders, Science 51: 382-385, 1921.

Watson, J. R.: Bite of Latrodectus mactans, Science 55: 539, 1922.

\section{Insecta}

Blatchley, W. S.: Coleoptera of Indiana, Indianapolis, 1910, Nat. Pub. Co.

Boyce, R. W.: Mosquitoes or Man\& The Conquest of the Tropical World, London, 1910, Jolnn Murray.

Carpenter, G. H.: Insects, Their Structure and Life, Dent, 1928.

-: The Biology of Insects, New York, 1928, The Macmillan Co.

Comstock, J. H.: Introduction to Entomology, Ithaca, N. X., 1933, Comstock Pub. Co.

-, and Comstock, A. B.: How to Know Butterflies, Ithaca, N. Y., 1920, Comstock Pub. Co.

-, and -: A Manual for the Study of Insects, Ithaca, N. Y., 1930, Comstock Pub. Co.

Dyar, H. G.: The Mrosquitoes of the United States, Proc. U. S. Nat. Mus. 62: 1922.

Essig, E. O.: The Insects of Western North America, New York, 1926, The Macmillan Co.

Fernald, H. G.: Applied Entomology, New York, 1926, McGraw-Hill Book Co.

Folsom, J. W., and Wardle, R. A.: Entomology With Reference to Its Ecological Aspects, Pliiladelphia, 1934, P. Bialiston's Son \& Co.

French, G. H.: Butterflies of Eastern North America, Philadelphia, 1886, J. B. Lippincott Co.

Kerms, W. B.: Medical and Veterinary Entomology, New York, 1923, The Macnillan Co.

Herrick, Glenn W.: Manual of Injurious Insects, New York, 1925, Henry Holt $\&$ Co.

Holland, W. J.: The Moth Book, Garden City, N. Y., 1922, Doubleday Page. -: The Butterfly Book, Garden City, N. Y., 1931, Doubleday, Doran \& Co.

Howard, L. O.: The Insect Book, Garden City, N. Y., 1922, Doubleday Page \& Co.

Imms, A. D.: A General Textbook of Entomology, New York, 1925, E. P. Dutton \& Co.

Kellogg, V. L., and Doane, R. W.: Economic Zoology and Entomology, New York, 1915, Henry Holt \& Co.

Kofoid, C. A.: Termites, Berkeley, Univ. California Press.

Lefroy, H. M.: Manual of Entomology, London, 1923, Edward Arnold and Co. Lubbock, Sir John: Ants, Bees, and Wasps, New York, 1929, E. P. Dutton \& Co. Lutz, F. E.: A Fieldbook of Insects, New York, 1921, G. P. Putnam's Sons. 
Metealf, C. L., and Flint, W. P.: Destructive and Useful Insects, New York, 1925, MeGraw-Hill Book Co.

-, and -: A Textbook of Practical Eutomology, New York, 1932, McGraw. Hill Book Co.

Packard, A. S.: Textbook of Entomology, New York, 1898, The Macmillan Co. Plath, O. E.: Bumblebees and Their Tays, New Fork, 193t, The Macmillan Co.

Reamur, R. A. F.: A Natural History of Ants (translated by W. M. Wheeler), New York, 1926, Alfred A. Knopf.

Snodgrass, R. E.: The Anatomy and Physiology of the Honeybee, New York, 1925, McGraw-Hill Book Co.

-: The Principles of Insect MIorphology, New York, 1935, McGraw-Hill Book Co.

Wardle, R. A.: The Problems of Applied Entomology, New York, 1929, McGrawHill Book Co.

Weed, C. M.: Butterflies (Little Nature Library), Garden City, 1922, Doubleday Page \& Co.

Wheeler, W. M.: Ants, Their Structure, Derelopment, and Beliavior, New York, 1910, Columbia Univ. Press.

Entomological News, published in Philadelphia, the Journal of Economic Entomology, and the Aunals of the Entomological Society of America contain current articles of importance. Biological Abstracts summarizes all of the current literature of the world and is an excellent reference for scattered publications. The Zoological Record is also an invaluable source for bibliographical studies.

Fishes and Amphibia

Adams, L. A.: Necturus-A Laboratory Study, New York, 1926, The Macmillan Co.

Campbell, Nelle: Organograplyy of $10 \mathrm{~mm}$. Ameiurus, Thesis, 1934, Baylor University Library.

Creaser, C. W.: The Structure and Growth of the Scales of Fishes, etc., Unir. of Michigan Zool. Pub. 17, 1920.

Daniel, J. F.: The Elasmobranch Fishes, Berkeley, 1928, Univ. California Press.

Dickerson, Mary C.: The Frog Book, Garden City, 1906, Doubleday Page \& Co. Forbes, S. A., and Richardson, R. E.: The Fishes of Illinois, Bull. Ill. State Lab. Nat. Hist. III, 1908.

Gage, Simon Henry: The Lampreys of New York State, Life History and Economics, A Biological Survey of the Oswego River System, Supple. mentary to 17 th annual report, 1927, State of New Iork Conservation Department, 1928.

Goode, A. M.: American Fishes, Boston, 1926, C. Page and Co.

Holmes, S. J.: The Biology of the Frog, New York, 1927, The Macmillan Co. Hubbs, C. I.: A Check List of the Fishes of the Great Lakes and Tributary Waters, With Keys, Univ. Michigan Mus. Zool. Misc. Pub. 15, 1926.

Jordan, D. S.: A Guide to the Study of Fishes, 2 vols, New York, 1905, Henry Holt \& Co.

-, and Ererman, W. E.: The Fishes of Midale and North America, U. S. Nat. Mus. Bulls., 1896.

Kellogg, Remington: Mexican Tailless Amphibians in the United States, National Museum Bull. 160, U. S. Nat'l. Museum.

-: The Toad, U. S. Dept. Agr. Bur. Biol. Survey, M. S., 1922.

Kyle, H. M.: The Biology of Fishes, New York, 1926, The Macmillan Co.

Marshall, A. M.: The Frog, New York, 1923, The Macmillan Co. 
Noble, G. K.: The Biology of the Amphibia, New York, 1931, MeGraw-Hill Book Co.

Potter, G. E.: Respiratory Function of the Swim Bladder in Lepidosteus, J. Exper. Zool. 49: No. 1, 1927.

-, and Medlen, A. B.: Organography of Gambusia patruelis, J. Morphol. 57: No. 1, 1935.

- and Self, J. Teague: Derelopment of Posterior Cardinal Veins in Relation to the Swim Bladder in Lepidosteus, J. Morphol. 56: No. 1, 1934.

Ruthven, A. G., Thompson, C., and Gaige, H. T.: The Herpetology of Michigan, Michigan Handb. Ser. 3, 1928.

Stejneger, L., and Barbour, T.: A Check List of North American Amphibians and Reptiles, ed. 3, Cambridge, Mass., 1933, Harvard University Press.

Strecker, J. K.: Reptiles and Amphibians of Texas, Baylor Bull. 17: No. 4, 1915.

Stuart, Ricliard R.: Anatomy of the Bullfrog, Chicago, 1940, Denoyer-Geppert Co.

-: Anatomy of Necturus, Chicago, 1940, Denoyer-Geppert Co.

Wright, A. A., and Wright, A. H.: Handbook of Frogs and Toads, Ithaca, N. Y., 1933, Comstock Pub. Co.

Wright, A. H.: Life-Histories of the Frogs of Okefinokee Swamp, Georgia, North American Salientia, No. 2, New York, 1932.

\section{Reptiles, Birds, and Mammals}

Adams, Charles C.: The Conservation of Predatory Mammals, J. Mammalogy 6: No. 2, May, 1925.

Agassiz, Louis: Turtles, Contrib. to Nat. Hist. of U. S., No. 1, 1857.

Anthony, H. E.: Field Book of North American Mammals, New York, 1928, G. P. Putnam's Sons.

Apgar, A. C.: Birds of the United States East of the Rocky Mountains, New York, 1898, American Book Co.

Bailey, Vernon: Beaver Habits, Beaver Control, and Possibilities in Beaver Farming, Bull. 1078, U. S. Dept. Agric., 1922.

-: Mammals of New Mexico, U. S. Dept. Agric. Bur. Biol. Survey, North Amer. Fauna 53 (1931).

Barrows, W. B.: Michigan Bird Life, Bull. Michigan Agriculture, 1912.

Beddard, F. E.: The Mammalia, Cambridge Nat. Hist. Series, New York, 1923, The Macmillan Co.

Beebe, C. W.: The Bird, Its Form and Function, New York, 1906, Henry Holt \& Co.

Bent, A. C.: North American Diving Birds, U. S. Nat. Mus., Bull. 107 (1919).

Blanchard, F. N.: A Key to the Snakes of the United States, Canada, and Lower California, Michigan Acad. Sci. Arts and Letters 4: Part 2, 1925.

Bradley, O. Charnoch: Structure of the Fowl, 1915, published by A. \& C. Black, Ltd., 4 Solıo Sq., London.

Bready, M. B.: The European Starling on His Westward Way (Sturnus vulgaris vulgaris), 1929, Knickerbocker Press.

Burroughs, John: Squirrels and Other Fur Bearers, Boston, 1909, Houghton Mifflin Co.

Butler, A. W.: Birds of Indiana, Indiana Geol. and Nat. Res. Rep. 20: 1897.

Camp, C. L.: Classification of the Lizards, Bull. Amer. Mus. Nat. Hist. 48: 1923.

Chapman, F. M.: Color Key to North American Birds, Garden City, 1903, Doubleday Page \& Co.

-: Handbook of Birds of Eastern North America, New York, 1932, D. Appleton \& Co. 
Cope, E. D.: A Critical Review of the Characters and Variations of the Snakes of North America, Proc. U. S. Nat. Mus. 14: 1892.

-: The Crocodilians, Lizards, and Snakes of North America, Ann. Rep. U. S. Nat. Mus., 1898.

Ditmars, R. L.: Snakes of the World, New York, 1931, The Macmillan Co.

-: The Reptile Book, Garden City, 1908, Doubleday, Doran \& Co. Well illustrated and interesting accounts of the turtles, tortoises, crocodilians, lizards, and snakes whicli inhabit the United States and northern Mexico.

Gadow, Hans: Amphibia and Reptiles, Cambridge Nat. Hist., VIII, London, 1901.

Greene, Eunice C.: Anatomy of the Rat, Philadelphia, 1935, Tr. Am. Philo. Soc., New Series, Vol. XXVII.

Headley, F. W.: The Flight of Birds, London, 1912, Witherby and Co. (Charles Scribner's Sons, New York, U. S. agents.)

Heaps, W.: Emigration, Migration, and Nomadism, Cambridge, England, 1931, W. Heffer and Sons.

Herrick, C. L.: The Mammals of Minnesota, Minnesota Geol. and Nat. Hist. Surv. Bull. 7, 1592.

Hollister, N.: A Systematic Account of the Prairie Dogs, Bur. Biol. Surrey, North Amer. Fauna 40 (1916).

Hornaday, T. W.: The Destruetion of Our Birds and Mammals, 2d Ann. Rep. of New York Zool. Soc., 1901.

Howell, A. B.: Aquatic Mammals, Springfield, Ill., 1930, C. C. Thomas.

Hurter, Julius, Sr.: Herpetology of Missouri, Trans. Missouri Acad. Sc. Vol. 20, St. Louis, 1911.

Jackson, H. H. T.: A Review of the American Moles, U. S. Biol. Survey, North Amer. Fauna 38 (1916).

Kalmbach, E. R.: The Crow in Its Relation to Agriculture, U. S. Dept. Agric., Farmers' Bull. 1102 (1920).

Kaupp, B. F.: Anatomy of the Domestic Fowl, Philadelphia, 1918, W. B. Saunders Co.

Lantz, D. E.: An Economic Study of Field Mice, U. S. Dept. Agric. Biol. Survey Bull. 31, 1907.

Lewis, John B.: Sight and Scent in the Turkey Vulture, The Auk 45: pp. 467$470,1928$.

Louisiana Conservation Dept. Birds of Louisiana, 1931.

McAtee, W. L.: How to Attract Birds, U. S. Dept. Agric. Farmers' Bulls. 621 (1914), 760 (1918), S44 (1917), 912 (1918).

Merriam, F. A.: Handbook of Birds of Western United States, Boston, 1927, Houghton Mifflin Co.

National Geographic Society, The Book of Birds, 1927.

Neff, J. A.: A Study of the Economic Status of the Common Woodpeckers in Relation to Oregon Horticulture, Free Press Print, 1928.

Nelson, E. W.: Wild Animals of North America, Nat. Geog. Soc., 1918.

Pearson, T. G.: The Bird Study Books, Garden City, 1923, Doubleday Page $\& \mathrm{Co}$.

Phillips, J. C.: American Waterforwl, Boston, 1930, Houghton Mifflin Co.

Reese, Albert M.: 'The Alligator and Its Allies, New York, 1915, G. P. Putnam's Sons.

Reighard, Jacob and Jennings, H. S., Anatomy of the Cat, New York, 1901, Henry Holt \& Co., Ine.

Ridgway, Robert: The Birds of North and Middle Ameriea, Parts I to VIII, U. S. Nat. Mus., 1901 to 1919.

Ruthven, A. G., Thompson, C., and Gaige, H. T.: The Herpetology of Michigan, Michigan Handb., Ser. 3, 1928.

Seton, E. T.: Life Histories of North American Animals, New York, 1909, Charles Scribner's Sons. 
-: Lives of Game Animals, Vols. 1, 4, Garden City, 1925 to 1928, Doubleday Page \& Co.

Stejneger, Leonard: The Poisonous Snakes of Nortl America, Rep. U. S. Nat. Mus. for 1893.

Stoddard, H. L.: The Bobwhite Quail: Its Habits, Preservation, and Increase, New York, 1931, Charles Scribner's Sons.

Stoner, D.: The Rodents of Iowa, Iowa Geol. Surv., 1918.

Stromsten, F. A.: Davison's Mammalian Anatomy, Philadelplia, 1937, P. Blakiston's Son \& Co.

Thomson, J. A.: The Biology of Birds, London, 1923, Sedgwick and Jackson.

Viosea, Percy, Jr.: A Contribution to Our Knowledge of the Water Snakes, Contr. No. 2, Research Dept., Southern Biol. Sup. Co., Inc., 1924.

Vorhies, C. T., and Taylor, W. P.: Life History of the Kangaroo Rat, U. S. Dept. Agric. Bull. No. 1091 (1922).

Warren, E. R.: The Mammals of Colorado, New York, 1910, G. P. Putnam's Sons.

-: The Beaver: Its Work and Its Ways, Baltimore, 1926, Williams and Wilkins Co.

Wetmore, Alexander: The Migration of Birds, Cambridge, Mass., 1926, Harvard Univ. Press.

\section{Anomalies}

Arey, L. B.: Developmental Anatomy, Pliladelphia, 1947, W. B. Saunders Co.

Evans, Titus C.: Effects of Roentgen Radiation on Embryonic Organization and Regulation in Melanoplus Differentialis (Orthoptera), Physiol. Zool. 10: No. 3, July, 1937.

Fitzpatrick, F. L.: Abnormal Foetal Pig (Double Body), Proc. Iowa Acad. Sci. 35: 1928.

-: A Case of Abnormal Development in Gallus domestica, Proc. Iowa Acad. Sci. 32: 1925.

\section{Internal Regulation}

Allen, Danforth, Daisy, et al.: Sex and Internal Secretions, Baltimore, 1939, Williams and Wilkins Co.

Banting, F. G., Best, C. H., and Macleod, J. J. R.: The Internal Secretion of the Pancreas, Am. Jour. Physiol. 59: 1922.

Guyer, M. F.: Internal Secretions and Human Well-Being, Science 74: 159, 1931.

Schafer, E. A.: The Endocrine Organs, ed. 2, London, 1916, Longmans, Green $\&$ Co.

\section{Regeneration}

Child, C. M.: Physiological Foundations of Behavior, New York, 1924, Henry Holt \& Co.

Morgan, T. H.: Regeneration, New York, 1901, The Macmillan Co.

\section{Radiation}

Baitsell, G. A.: Science in Progress, New Haven, 1939, Yale University Press, Ch. I and II.

Butler, E. F.: The Effect of X-rays on Tissue Regeneration, Scientific Monthly, December, 1934, pp. 511-518.

Clark, George C.: A General Survey of Radiobiology, Cold Spring Harbor Symposia on Quantitative Biology 2: 249-263, 1934.

Clark, G. L.: Applied X-rays, New York, 1940, McGraw-Hill Co.

Duggar, B. M.: Biological Effects of Radiation, New York, 1936, MeGraw-Hill Book Co.

Evans, T. C.: Effects of Roentgen Radiation on Embryonic Organization and Regulation in Melanoplus Differentialis (Orthoptera), Physiol. Zool. 10: 258-268, 1937. 
Fricke, Hugo: The Chemical-Physical Foundation for the Biological Effects of $\mathrm{X}$-rays, Cold Spring Harbor Symposia on Quantitative Biology 2: 241-248, 1934.

Glasser, Otto: The Science of Radiology, Springfield, Ill., 1933, C. C. Thomas.

Henshaw, P. S.: I. Studies of the Effect of Roentgen Rays on the Time of First Cleavage in Some Marine Invertebrate Eggs. II. Recovery From Roentgen Ray Effects in Arbacia Eggs, Am. J. Roentgenol. 27: 890-898, 1932.

Henshaw, P. S.: Radiation and the Cell, J. National Cancer Inst. Vol. 1, pp. 277-290.

Laurens, H. K.: Physiological Effects of Radiant Energy, Chemical Catalogue Company, New York, 1933.

Patterson, J. T.: X-rays and Somatic Mutations, J. Hered. 20: 261-267, 1929.

Pohle, E. A.: Theoretical Principles of Roentgen Therapy, Philadelphia, 1938, Lea and Febiger.

Quimby, E. H.: The Physical Basis of Radiation Therapy, Ann Arbor, Mich., 1939, Edwards Bros., Inc.

Sheard, Charles: Life-Giving Light, Baltimore, 1933, Williams and Wilkins Co.

\section{Animal Distribution}

Dice, L. R.: Life Zones and Mammalian Distribution, J. Mammalogy 4: No. 1, 1923.

Merriam, C. Hart: The Geographic Distribution of Animals and Plants in North America, U. S. Dept. of Agric. Yearbook, 1894-1895, pp. 203-214.

Newbingin, Marion: Animal Geograplyy, London, 1913, Oxford Univ. Press.

Parker, T. J., and Haswell, Wm. A.: Textbook of Zoology, New York, 1928, The Macmillan Co. (Introduction).

Scharf, R. F.: Origin and Distribution of Life in America, London, 1911, Constable \& Co.

Wallace, A. R.: The Geographical Distribution of Animals, 2 vols, New York, 1876, The Macmillan Co.

\section{Ecology}

Adams, C. C.: Guide to the Study of Animal Ecology, New York, 1913, The Macmillan Co.

Birge, E. A., and Juday, C. A.: A Limnological Study of the Finger Lakes of New York, Bull. 32, U. S. Bureau of Fisheries.

-, and - : The Inland Lakes of Wisconsin, Wisconsin Geol. and Nat. Hist. Survey Bull. 27, 1914.

Chapman, R. N.: Animal Ecology, New York, 1931, MeGraw-Hill Book Co.

Elton, Charles S.: Animal Ecology, London, 1927, Sedgwick and Jackson.

Henderson, L. J.: The Fitness of the Environment, New York, 1913, The Macmillan Co.

Hesse, Richard, et al.: Ecological Animal Geography, New York, 1937, John Wiley \& Sons.

Mast, S. O.: Light and the Beharior of Organisms, New York, 1911, John Wiley and Sons.

Pearse, A. S.: Animal Ecology, New York, 1926, McGraw-Hill Book Co.

Shelford, V. E.: Animal Communities in Temperate America, Chicago, revised 1927, Univ. Chicago Press.

—: Laboratory and Field Ecology, Baltimore, 1929, Williams and Wilkins Co.

-: Naturalist's Guide to the Americas, Baltimore, 1926, Williams and Wilkins Co.

Stromsten, F. A.: Lake Okoboji as a Type of Aquatic Environment, Univ. of Iowa Studies in Nat. Hist., No. 12, 1927. 
Viosca, Percy, Jr.: An Ecological Study of the Cold-Blooded Vertebrates of Southeastern Louisiana, Copeia, No. 115, 1923.

-: Distributional Problems of the Cold-Blooded Vertebrates of the Gulf Coastal Plain, Ecology 7: No. 3, 1926.

Welch, P. S.: Limnology, New York, 1935, McGraw-Hill Book Co.

Williams, S. H.: A Systematic Guide to Field Zoology, Author's mimeographed edition. Univ. of Pittsburgh, 1927.

\section{Parasitology}

Chandler, A. C.: Introduction to Parasitology, New York, 1940, John Wiley and Sons.

Doane, R. W.: Insects and Disease, New York, 1910, Henry Holt \& Co.

Ewing, H. E.: Manual of External Parasites, Springfield, Ill., 1929, C. C. Thomas.

Faust, E. C.: Human Helminthology, Philadelphia, 1929, Lea and Febiger.

Johansen, O. A., and Riley, W. A.: Handbook of Medical Entomology, Ithaca, N. Y., 1915, Comstock Pub. Co.

Linton, E., and Stiles, C. W.: Information Concerning Parasitic Worms in Fish, U. S. Bur. Fisheries, Econ. Circular No. 21, 1916.

Matheson, Robert: Medical Entomology, Springfield, Ill., 1932, C. C. Thomas.

Riley, W. A., and Christensen, R. B.: Guide to the Study of Animal Parasites, New York, 1930, McGraw-Hill Book Co.

Shipley, A. E.: Nemathelminthes, Vol. II. Cambridge Nat. Hist., London, 1922.

Stunkard, H. W.: Parasitism: A Biological Phenomenon, Scientific Monthly, 1929, pp. 349-362.

\section{Marine Zoology}

Arnold, A. F.: The Sea Beach at Ebb Tide, New York, 1901, Century Co.

Bassler, R. S.: The Bryozoa, or Moss Animals, Smithsonian Rept. for 1920, pp. 339-380. (Publication 2633.)

Berry, S. Stillman: A Review of the Cephalopods of Western North America, Bull. Bur. Fish. 30: 267-336, 1910.

-: Notes on Some Cephalopods in the Collection of the University of California, Univ. California Pub. Zool. 8: 301-310, 1911.

-: Notes on West American Chitons I. Proc. California Acad. Sci., 4th Series, Zool. 7: 229-248, 1917.

-: A Distributional Note on Haliotis, California Fish and Game 7: 254-255, 1921.

Breder, Charles M.: Field Book of Marine Fishes of the Atlantic Coast, New York, 1921, G. P. Putnam's Sons.

Bush, Mildred: Revised Key to the Echinoderms of Friday Harbor, Pub. Puget Sd. Biol. Sta. 3: 65-77, 1921.

Clark, A. H.: Animals of Land and Sea, New York, 1927, D. Van Nostrand Co.

-: The Holothurians of the Pacific Coast of North America, Zool. Anz. 24: $162-171,1901$.

-: North Pacific Ophiurans in the Collection of the United States National Museum, Bull. U. S. Nat. Mus., No. 75, 1911.

-: Hawaiian and Other Pacific Echini, Mem. Mus. Comp. Zool. Harvard 34: Nos. 2 and $3,1909$.

Coe, Wesley R.: The Echinoderms of Connecticut, Connecticut Geol. and Nat. Hist. Surv., 1912.

-: Nemerteans, Harriman Alaska Expedition 11: 1-220, 1904.

-: Nemerteans of the West and Northwest Coasts of America, Bull. Mus. Comp. Zool. Harvard 47: 1905.

Crowder, William: Dwellers of the Sea and Shore, New York, 1923, The Macmillan Co. 
-: Naturalist at the Seashore, New York, 1928, Century Co.

- : Between the Tides, New York, 1930, Dodd, Mead \& Co.

Daris, B. M.: The Early Life-History of Dolichoglossus pusillus Ritter, Univ. California Pub. Zool. 4: 1\$7-226, 1908 .

Flattely, F. W.: The Biology of the Seashore, London, 1922, Sedgwick and Jackson.

Fraser, C. McLean: The Hydroids of the West Coast of North America, Bull. from Laboratories of Natural History of State University of Iowa 6: No. 1, 3-91, 1911.

-: Some Hydroids of the Taneouver Island Region, Trans. Roy. Soc. Canada, Series 3 8: 99-216, 1914.

-: On the Development of Aequorea forstilea, Trans. Roy. Soe. Canada, Series 3 10: 97-104, 1916.

George, W. C., and Wilson, H. V.: Sponges of Beaufort and Vicinity, Bull. U. S. Bur. Fisheries 37: 1917-1918.

Gunter, Gordon: A List of Fishes of the Mainland of North and Middle America. Recorded From Both Fresh Water and Sea Water, American Midland Naturalist 28: 305-326, 1942.

Harmer, S. F., and Shysley, A. E.: Flatworms, Metazoa, ete. Cambridge Nat. Hist. Series, Vol. 2, New York, 1909, The Macmillan Co.

Heath, Harold: California Clams, California Fish and Game 2: 175-178, 1916. --: Devilfish and Squid, California Fish and Game 3: 103-108, 1917.

Heilprin, Angelo: Animals of Our Seashore, Philadelphia, 1888, J. B. Lippincott Co.

Johnson, M. E., and Snook, H. J.: Seashore Animals of the Pacifie Coast, New York, 1935, The Macmillan Co.

Mayer, A. G.: Seashore Life, New York Zool. Soc., 1911.

Miner, Roy Tr.: Fieldbook of Seashore Life, New York, 1933, G. P. Putnam's Sons.

-: Animals of the Wharf Piles, Am. Mus. Nat. Hist. Jour. 13: Feb., 1913.

-: Outposts of the Sea: Animals of the Tidal Zone, Am. Mus. Nat. Hist. Guide Leaflet, Ser. 74, 1929.

Nutting, C. C.: American Hydroids. I. Plumularidae, U. S. Nat. Mus. Bull., 1900 .

-: The Hydroids, Papers from Harriman Alaska Expedition, Proc. Wash. Acad. Sei. 3: 157-216, 1901.

-: American Hydroids. II. Sertularidae, U. S. Nat. Mus. Bull., 1904.

-: American Hydroids. 1II. Campanularidae and Bonneviellidae, U. S. Nat. Mus. Bull., 1915.

Rankin, E. P.: The Mussels of the Pacific Coast, California Fish and Game 4: $113-117,1918$.

Ritter, W. E.: The Movements of the Enteropneusta and the Mechanism by Which They Are Accomplished, Biol. Bull. 3: 255-261, 1902.

-: The Pelagic Tunicata of the San Diego liegion, Excepting the Larvacea, Univ. California Pub. Zool. 2: 51-112, 1905.

-, and Forsyth, Ruth A.: Ascidians of the Littoral Zone of Southern California, Univ. California Publ. Zool. 16: 439-512, 1915.

Robertson, Alice: Studies in Pacific Coast Entoprocta, Proc. California Acad. Sci., Zoology 2: 323-348, 1900.

Schmitt, Waldo L.: The Marine Decapod Crustacea of California, Univ. California Publ. Zool. 1: 1-104, 1902.

Torrey, H. B.: The Hydroida of the Pacific Coast of North America, Univ. California Publ. Zool. 23: 1-470, 1921.

-: Anemones, With Discussion of Variation in Metridium, Proc. Washington Acad. Sci. 4: 373-410, 190:2.

-: Biological Studies on Corymorpha. 1. C. palma and Environment, J. Exper. Zool. 1: $395-422,190 t$. 
-: On the Habits and Reactions of Sagartia davisi, Biol. Bull. 6: 203-216, 1904.

-: The Hydroids of the San Diego Region, Univ. California Publ. Zool. 2: 1-43, 1904.

-: Ctenophores of the San Diego Region, Univ. California Publ. Zool. 2: 45-50, 1904.

-: The Behavior of Corymorpha, Univ. California Publ. Zool. 2: 333-340, 1905.

-: The California Shore Anemone, Bunodactis xanthogrammica, Univ. California Publ. Zool. 3: 41-45, 1906.

-: Biological Studies on Corymorpha. II. The Development of C. palma from the Egg, Univ. California Publ. Zool. 3: 253-298, 1907.

-: The Lpeomedusae of the San Diego Region, Univ. California Publ. Zool. 6: $11-31,1909$.

-: Biological Studies on Corymorpha. III. Regeneration of Hydranth and Holdfast, Univ. California Publ. Zool. 6: 205-221, 1910.

-: Biological Studies on Corymorpha. IV. Biol. Bull. 19: 280-301, 1910.

Treadwell, A. L.: Polychaetous Annelids of the Pacific Coast in the Collections of the Zoological Museum of the University of California. New Syllidae from San Francisco Bay, Univ. California Publ. Zool. 13: 175-238, 1914.

Ulrey, A. B.: The Starfishes of Southern California, Bull. So. California Acad. Sci., July, 1918, pp. 39-52.

Verrill, A. E.: Monograph of the Shallow-Water Starfishes of the North Pacific Coast From the Aretic Ocean to California, Harriman Alaska Series, Smithsonian Institution 14: Parts 1 and 2 (Part 2 consists of plates), 1914.

Weese, A. O., and Townsend, M. T.: Some Reactions of the Jellyfish, Aequorea, Publ. Puget Sd. Biol. Sta. 3: 117-128, 1921.

Weymouth, Frank W.: The Edible Clams, Mussels and Seallops of California, Fish Bulletin, No. 4, California Fish and Game Commission, 1920.

-: The Life-History and Growth of the Pismo Clam, Fish Bulletin, No. 7, California Fish and Game Commission. 1923.

Wilson, C. B.: North American Parasitic Copepods: A List of Those Found Upon the Fishes of the Pacific Coast, Proe. U. S. Nat. Mus. 35: 431-481, 1909.

Wilson, H. V.: The Sponges, Mem. Mus. Comp. Zool. Harvard 30: No. 1, 1904. (Report of an exploration off the west coasts of Mexico and South America.)

\section{Wildlife Conservation}

Gabrielson, Ira N.: Wildlife Couservation, New York, 1941, The Macmillan

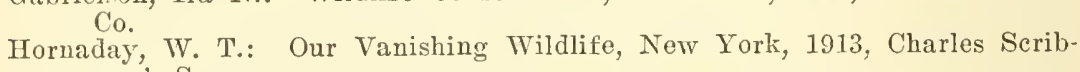
ner's Sons.

Lydekker, R.: Wildlife of the World, New York, 1915, Fred Warne \& Co.

\section{Embryology}

Arey, Leslie B.: Developmental Anatomy, ed. 3, Philadelphia, 1947, W. B. Saunders Co.

Hartman, Carl G.: Studies in the Development of the Opossum, J. Morphol. 27: 1-85, 1916.

-: Breeding, Habits, Development and Birth of the Opossum, Smithsonian Institution Pub. 2689, 1923.

Lillie, Frank: Development of the Chick, 2nd revised edition, New York, 1927, Henry Holt \& Co. 
McEwen, Robert S.: Textbook of Vertebrate Embryology, New York, 1931, Henry Holt \& Co.

Patten, Bradley M.: Embryology of the Chick, ed. 3, Philadelphia, 1929, P. Blakiston's Son and Co.

-: Embryology of the Pig, ed. 2, Philadelphia, 1931, P. Blakiston's Son and Co.

Richards, A.: Comparative Embryology, New York, 1931, John Wiley \& Sons.

Wieman, H. L.: An Introduction to Vertebrate Embryology, New York, 1930, MeGraw-Hill Book Co.

\section{Heredity}

Altenberg, E.: How We Inherit, New York, 1928, Henry Holt \& Co.

Baber, R. A., and Ross, E. A.: Changes in the Size of the American Family in One Generation, Univ. Wisconsin Studies, 1924, $99 \mathrm{pp}$.

Baker, J. R.: Sex in Man and Animals, London, 1926, George Routledge \& Sons.

Barnes, I.: The Inheritance of Pigmentation in the American Negro, Human Biol. 1: 321-381, 1929.

Bauer, E., Fiseher, E., and Lenz, F.: Human Heredity, New York, 1931, The Maemillan Co.

Blacker, C. P.: The Chances of Morbid Inheritance, London, 1934, H. K. Lewis \& Co.

Bonar, J.: Malthus and His Work, New Iork, 1924, The Macmillan Co.

Bowen, E.: An Hypothesis of Population Growth, New York, 1931, Columbia Univ. Press.

Carr-Saunders, A. M.: The Population Problem, Oxford, 1932, Clarendon Press.

-: Eugenies, New York, 1926, Henry Holt \& Co.

Castle, WV. E.: Geneties and Eugenies, ed. 4, Cambridge, Mass., 1930, Harrard Unir. Press.

Cattell, J. MeK., Families of American Men of Science, Popular Science Montlily 86: 504-515, 1915; Scient. Monthly 4: 248-262; 5: 368-377, 1917 .

Conklin, E. G.: The Direction of Human Erolution, New York, 1921, Charles Scribner's Sons.

Cowdry, E. V., and Embree, E. R.: Human Biology and Racial Welfare, Vol. 2, New York, 1930, Paul B. Hoeber.

Darwin, L.: The Need for Eugenic Reform, New York, 1926, D. Appleton \& Co.

Davenport, C. B.: Heredity of Slin Color in Negro-White Crosses, Pub. Carnegie Inst., No. 18s, $1913 b$.

-, and Muncey, E. B.: Huntington's Chorea in Relation to Ileredity and Eugenies, Am. J. Insanity 73: 195-222, 1916. Also in Bull. Eug. Ree. Off. 17: 1916.

East, E. M.: Mankind at the Crossroads, New York, 1923, Charles Seribner's Sons.

Fasten, N.: Principles of Genetics and Eugenies, Boston, 1935, Ginn \& Co.

Gates, R. R.: Heredity in Man, New York, 1929, The Macmillan Co.

Gosney, E. S., and Popenoe, P.: Sterilization for Human Betterment, New York, 1929, The Macmillan Co.

Gun, W. T. J.: Studies in Hereditary Ability, London, 1928.

Guyer, M. F.: Being Well-Born, Indinapolis, 1927, Bobbs-Merrill Co.

Herskorits, M. J.: The American Negro, A Study in Race Crossing, New York, 1929, Alfred A. Knopf.

Hodson, C. B. S.: Human Sterilization Today, London, 1934, Watts.

Hogben, I.: Genetic Principles in Medieine and Social Science, London, 1931, Williams and Norgate.

-: Nature and Nurture, London, 1933, Williams and Norgate.

Holmes, \&. J.: The Trend of the Race: A Study of Present Tendencies in the Biological Development of Civilized Mankind, New York, 1921, Hareourt, Brace \& Co. 
-: Studies in Evolution and Eugenies, New York, 1923, Hareourt, Brace \& Co.

-: The Size of College Families, J. Hered. 15: 407-415, 1924.

-: Human Geneties and Its Social Import, New York, 1933, Harcourt, Brace \& Co.

Hunt, H. R.: Some Biological Aspects of War, New York, 1930, Galton Publ. Co. Huntington, E.: Tomorrow's Children, New York, 1935, John Wiley \& Sons. Jennings, H. S.: The Biological Basis of Human Nature, New York, 1930, IV. W. Norton \& Co.

-: Geneties, New York, 1935, W. W. Norton \& Co.

Jordan, D. S.: The Human Harvest, Boston, 1907, Beacon Press.

Kuczynski, R. R.: The Balance of Births and Deaths. I. Western and Northern Europe, New York, 1928, The Macmillan Co.

Landman, J. H.: Human Sterilization, New York, 1932, The Macmillan Co.

Laughlin, H. H.: Eugenical Sterilization, Am. Eugenics Soc., 1926.

Lidbetter, E. J.: Heredity and the Social Problem Group, Vol. I, London, 1934, Edward Arnold \& Co.

Lindsey, A. W.: A Textbook of Geneties, New York, 1932, The Macmillan Co.

Lorimer, F., and Osborn, F.: Dynamies of Population, New York, 1934, The Macmillan Co.

Ludoviei, A. M.: The Choice of a Mate, London, 193 4 , John Lane.

Morgan, T. H.: The Physical Basis of Heredity, Philadelphia, 1919, J. B. Lippincott Co.

Myreson, A.: The Inheritance of Mental Diseases, Baltimore, 1925, Williams and Wilkins Co.

Newman, H. H.: Evolution, Genetics, and Eugenies, ed. 3, Chicago, 1932, Univ. Chicago Press.

Pearl, R.: Studies in Human Biology, Baltimore, 1924, Williams and Wilkins Co.

-, and Pearl, R. DelT.: The Ancestry of the Long-Lived, Baltimore, 1934, Johns Hopkins Press.

Popenoe, P.: The Child's Heredity, Baltimore, 1929, Williams and Wilkins Co.

-, and Johnson, R. S.: Applied Eugenics, ed. 2, New York, 1933, The Macmillan Co.

Rollins, TV. A.: Fertility of College Graduates, J. Hered. 20: 425-427, 1929.

Shull, A. F.: Heredity, New York, 1931, McGraw-Hill Book Co.

Sinnott, E. W., and Dunu, L. C.: Principles of Geneties, New York, 1932, MeGraw-Hill Book Co.

Stockard, C. R.: The Physical Basis of Personality, New York, 1931, W. W. Norton Co.

Terman, L. M.: Genetic Studies of Genius. Vol. I, Mental and Physical Traits of a Thousand Gifted Children, 1925, Stanford Univ. Press.

Thompson, W. S.: Population Problems, ed. 2, New York, 1935, MeGraw-Hill Book Co.

Wralter, H. E.: Genetics, New Iork, 1930, The Macmillan Co.

Whitney, L. F.: The Case for Sterilization, New York, 1934, Frederick A. Stokes Co.

Wiggam, A. E.: The Fruit of the Family Tree, Indianapolis, 1924, Bobbs-Merrill Co.

\section{Animal Behavior}

Beruard, L. L.: Instinct, a Study in Social Psychology, New York, 1924, Henry Holt \& Co.

Buddenbrock, W. T.: A Criticism of the Tropism Theory of Jacque Loeb, J. Animal Behavior 6: 341-366, 1916.

Coghill, G. E.: Anatomy and the Problems of Behavior, New York, 1929, The Macmillan Co. 
Dunlap, Knight: Elements of Psychology, St. Louis, 1936, The C. V. Mosby Co. Holmes, S. J.: Studies in Animal Behavior, Boston, 1916, R. G. Badger.

Howes, Paul G.: Insect Belavior, Boston, 1919, R. G. Badger.

Hunter, W. S.: The Delayed Reaction in Animals and Children, Behavior Monographs 2: No. 1, 1913.

Jennings, H. S.: Behavior of Lower Organisms, Part III, New York, 1906, Columbia University Press.

Loeb, J.: Forced Movements, Tropisms, and Animal Conduct, Philadelphia, 1918, J. B. Lippineott Co.

Mast, S. C.: Light and the Behavior of Organisms, Part I, New York, 1910, John Wiley \& Sons.

Morgan, C. L.: Introduction to Comparative Psychology, el. 2, London, 1906, W. Scott.

Romanes, G. J.: Animal Intelligence, London, 1851, Keagan Paul.

Washburn, M. F.: The Animal Mind, New York, 1926, The Macmillan Co.

Watson, J. B.: Introduction to Animal Psychology, New York, Henry Holt \& Co.

\section{Physiology}

Bayliss, Wm.: Principles of General Physiology, New York, 192t, Longmans, Green \& Co.

Burton-Opitz, R.: An Elementary Manual of Physiology, Philadelphia, 1936, WV. B. Saunders Co.

Heilbrunn, L. V.: An Outline of General Plysiology, Philadelphia, 1937, W. B. Saunders Co.

Howell, WV. H.: Textbook of Physiology, Philarlelphia, 193., TV. B. Saunders Co.

Macleod, J. J. R.: Physiology in Molern Medicine, St. Lonis, 1938, The C. V. Mlosby Co.

Mitchell, P. H.: General Plyysiology, New Tork, 1922, McGraw-Hill Book Co.

Rogers, C. G.: Textbook of Comparative Physiology, New York, 1927, MleGraw Hill Book Co.

Stiles, P. G.: Human Physiology, Philadelphia, 1939, T. B. Saunders Co.

Zoethont, Wm. D.: 'I'extbook of 'hysiology, St. Lonis, 1935, The C. V. Mosby Co.

\section{Paleontology}

Bassler, R. S.: Shelled Invertebrates of the Past and Present, Smithsonian Scientific Series 10: 19:3.

Berry, E. W.: Paleontology, New York, 1929, MeGraw-Hill Book Co.

Crabtree, J. H.: Rocks and Fossils and How to Identify Them, London, 1921, Epworth Press.

Davies, Arthur M.: Introduetion to Paleontology, London, 1920, Thomas Murby Co.

Hawkins, Herbert L.: Invertebrate Paleontology; An Introduction to the Study of Fossils, London, 1920, Methuen \& Co.

Lucas, H. A.: Animals of the Past, Am. Mus. Nat. Hist. Handbook No. 4, 1929.

Lull, Richard S.: Organje Evolution, New York, 1929, The Macmillan Co.

Lydekker, L.: The Horse and Its Relatives, New York, 1912, The Marmillan Co.

Noddie, Roy L.: A Popular Guide to the Nature and Environment of the Fossil Vertebrates of New York, New York State Mus. Handhook No. 12, 1933.

Osborn, H. F.: The Age of Mammals, New York, 1910, The Macmillan Co.

Scott, W. B.: History of the Land Mammals of the Western Hemisphere, New York, 1929, The Macmillan Co.

Scudder, Samuel H.: Some Inseets of Special Interest From Florissant Colorado and Other Points in the Tertiaries of Colorado and Utah, U. S. Geol. Surv. Bull. No. 93, 1892.

Shimer, Hervey W.: Introduction to a Study of Fossils, New York, 1933, The Macmillan Co. 


\section{Phylogeny}

Darwin, Charles: Origin of Species, ed. 6, London, 1880.

-: Variation in Animals and Plants Under Domestication, London, 1868. -: The Descent of Man, London, 1871.

Dendy, Arthur: Outlines of Evolutionary Biology, New York, 1911, D. Appleton \& $\mathrm{Co}$.

Gaskell, W. H.: The Origin of the Vertebrates, London, 1908, Longmans, Green \& Co.

Jordan, D. S., and Kellogg, V. L.: Evolution and Animal Life, New York, 1908, D. Appleton \& Co.

Kellogg, V. L.: Darwinism Today, New York, 1908, Henry Holt \& Co.

Lindsey, A. W.: Problems of Evolution, New York, 1931, The Macmillan Co. Lull, R. S.: Organic Erolution, New York, 1940, The Macmillan Co.

Newman, H. H., et al.: The Nature of the World and of Men, Chicago, 1926, Univ. Chicago Press.

Newman, H. H.: Evolution Yesterday and Today, Baltimore, 1932, Williams and Wilkins Co.

Osborn, H. F.: From the Greeks to Darwin, New York, 1894, The Macmillan Co.

-: The Origin and Evolution of Life, New York, 1917, Charles Scribner's Sons.

Patten, W.: The Erolution of the Vertebrates and Their Kin, Philadelphia, 1912, P. Blakiston's Son \& Co.

Romer, A. S.: Man and the Vertebrates, Chicago, 1933, Univ. Chicago Press.

Thomson, J. A.: Darwinism and Human Life, New York, 1908, Henry Holt \& Co.

Wilder, H. H.: History of the Human Body, New York, 1909, Henry Holt \& Co.

-: The Pedigree of the Human Race, New York, 1926, Henry Holt \& Co. 


\section{GLOSSARY*}

Abdomen (ăb dō'mĕn), the portion of the trunk posterior to the thorax of an animal.

Aboral (ăb ónăl), opposite tlie mouth.

Absorption (ăb sôrp'shŭn), the process of taking in soluble foods by the circulatory medium or by the protoplasm directly.

Accommodation (ăkŏm ódä'shŭn), the power of adjustment of the eye to near and far objects.

Acetabulum (ăs êtăb't̂̀ lŭm), the socket in each side of the innominate bone of the pelvic girdle into which the femur fits.

Achromatic figure (ăk rò măt'ǐk), the mitotic figure without chromosomes.

Aestivation (ěs tĭ vā'shŭn), state of torpidity induced by heat and dryness.

Allelomorphs (ă lëlồ môrfs), a pair of corresponding genes in homologous cliromosomes, but each produces a different character.

Allergy (ăl'ẽr jĩ), aeute seusitiveness to a foreigu sulstance, as foreign protein in the body.

Altermation of generation (ăl tẽr nā'shŭn of jĕn ẽr ä'shŭn). (See Metagenesis.)

Altricial (ăl trish'ăl), in reference to lirids which are hatched without feathers and in a helpless condition.

Alveolus (ăl véto lüs), a small cavity or pit, such as the air sacs in the lung of a higher vertebrate.

Ambulacral (ăm bù lā'krăl), area of echinoderm related to tube feet.

Amino acids (ăm'ک $110 \overline{)}$, organic acills with a $\left(\mathrm{NH}_{2}\right)$ radical, and derived from complex proteins.

Amitosis (ăm ìtō'sĭs), direet cell division, occurring without chromosomal activity.

Amnion (ămiň ŏn), iuner embryonic membrane of terrestrial vertebrates.

Amoeboid movement (áméboid), the streaning of protoplasm in a cell to extend the cell in some direction with the formation of pseudopodia.

Amphiaster (ăm'füăstẽr), the complete mitotic figure of a dividing cell.

Amphiblastula (ăm fĭblăs'tù lá), the free-smimming larval form in sponges.

Amphimixus (ăm fĭmǐk'šss), union of nuclear material from two different cells, as in fertilization.

Ampulla (ăm pŭl'á), a bulblike dilatation.

Amylopsin (ăm ĭlŏn'sĭn), a pancreatic enzỵe capable of converting starch into sugar.

Anabolism (ăn ăb'ò liz'm), the building up of living protoplasm.

Analogous (ă năl'ô gǔs), differing in structure and origin, but similar in function.

Anaphylaxis (ăn ȧfŭlăk'šs), acute reaction of the body to foreign protein materials which have a toxic effect; this may he an increased sensitivity to the material because of previous contact with it.

Anatomy ( $\dot{a}$ năt'ô mǐ), the science that treats of the structurc of organic bodies. Anus (a'nuis), the posterior opening of the alimentary canal.

Appendicular skeleton (ăp ěn d̆̉k'ū làr), skeleton of the paired fins of Pisces.

Archenteron (är kén'tẽro ón), the cavity of the gastrula which is the primitive digestive cavity.

Artery (är'tẽr İ), the larger blood vessels leading away from the heart.

Asexual reproduction (à sěk'shû ăl), reproduction without sex cells.

Assimilation (ăs sim $\check{i}$ là'shŭn), the transforming of digested food into protoplasm.

*Phonetics according to Webster's New International Dictionary. 
Asymmetry (ả sim'ět rĭ), a condition in which the two sides of an animal are dissimilar.

Autonomic nervous system (ô tô nóm'îk), that portion of the nervous system controlling the involuntary muscles.

Autotomy (ô tǒt'ồ mì), self-mutilation.

Axial gradient (ăk'sĭ ăl grā' dı̆ ěnt), the graduation of the rate of metabolism along the principal axis of an axiate animal.

Axone (ăk'sŏn), a nerve fiber serving to conduct impulses away from a nervધ cell body.

Barrier (băr'ĩe ër), any physical, chemical, or biological obstruction that prevents migration of animals.

Benthos (běn'thos), life of the deep sea bottom.

Binary fission (bī'nả rì fǐsh'ŭn), division of a cell into two daughter cells.

Biramous (bī rā'mŭs), a two-branched condition.

Bisexual (bī sĕk'shōăl), a condition in which both male and female organs are present in one individnal.

Bivium (bĭv'í üm), one side of an echinoderm including a pair of ambulacra.

Blastocoele (blăs'tồ sēl), the cavity present in the blastula stage of development; also cleavage of segmentation cavity.

Blastomere (blăs'tơ mēr), one of the segments first formed by the division of the ovium.

Blastula (blăs'tülá), a sphere of cells with a hollow cavity resulting from cleavage of the zygote.

Blepharoplast (blěf'á rŏ plăst), the body in a cell from which a flagellum arises.

Brachium (brā'kĭ ı̆m), arm.

Branchial (brăng' kĭ ăl), pertaining to gills or branchiae.

Buccal (bǔk'ăl), pertaining to the mouth eavity.

Budding (bǔd'ing), reproduction involving the branching of new individuals from the external surface of the old one.

Byssus (bis'ťs), a tuft of fiberlike threads which attach certain mussels to the substratum.

Caecum (sē'kŭm), a blind pouchlike pocket of the intestine; usually at the junction of the small and large intestines.

Calcareous (kăl kâr'ề̆s), composed of lime or calcium salts.

Calciferous (kăl siff'ēr ŭs), glands which are thought to secrete an alkaline secretion into the esophagus of the earthworm.

Canaliculus (kăn'å lǔk' ù lŭs), one of the tiny canals extending from lacuna to lacuna to distribute nutriment in bone.

Capillary (kăp' $\mathrm{i}$ lẽr $\mathrm{i}$ ), a microscopic branch of an artery which extends into a tissue and finally joins a small vein.

Carapace (kăr'à pās), shell-like external covering.

Carbohydrate (kär bō hī'drāt), organic compound of carbon, hydrogen, and oxygen, such as starch or sugar.

Cardiac (kï̈r'dĭ ăk), pertaining to the heart.

Carnivorous (kär nìv'ò rŭs), flesh eating.

Caste (kảst), any group of distinet fornis within a species, as found in some insects.

Catabolism (kả tăb' o lizm), process of oxidation or break-down of protoplasm; destructive phase of metabolism; dissimilation; the oxidation of organic substances of the body to release kinetic energy and heat.

Catalysis (kả tăl'ř sǐs), the initiation or acceleration of a chemical reaction by the presence of a substance which itself does not enter into the reaction, as an enzyme.

Caudal (kô'dŏl), pertaining to the tail. 
Cell theory (sĕl thē'or ră), the theory that all living things are composed of cells.

Centimeter (sěn'tĭ mëttẽr), one-hundredth of a meter and the equivalent of 0.393 inches; or 1 inch equals 2.54 centimeters.

Central nervous system (sěn'trăl), that portion of the nervous system composed of the brain and the spinal cord.

Centriole (sěn'trīol), a small granule within the central part of the aster in the mitotic figure; also known as centrosome.

Centrolecithal (sěn'trō lěs ǐ thăl), refers to the type of egg with the yolk mass in the center, as the egg in insects.

Centrosome (sčn'trō sôm), usually considered synonymous to centriole.

Cephalic (sẽ făl' îk), pertaining to the head.

Cephalothorax (sěf'ả lo thō'răks), a fusion of the head and thorax or chest, as in crayfish.

Cerebellum (sĕr ê bĕl'lŭm), the large lobe of the hind brain, in front of and above the medulla.

Cerebrum (sěr'ê brŭm), the anterior division of the brain.

Cercaria (sûr kā'rĭ à), a tailed larval stage of the liver fluke.

Ctenoid scale (ténoid), a type of fish scale with spines at the free margin.

Cervical (sûr'vĭ kăl), has reference to the neck region.

Chaeta (kétả), one of the bristlelike structures in the body wall of many annelids, used as organs of locomotion.

Chelicera (kê lis'ér á), an anterior pair of appendages in arachnids.

Cheliped (kéli pěd), most anterior thoracic leg of crayfish; large pincher.

Chemotropism (kē mǒt'rō pĭz'm), response of an organism to chemical changes.

Chlorophyll (klō'rố fíl), the green coloring matter in plants and a few animals which is active in plotosynthesis.

Chromosomes (krö'mō sōmz), bodies formed in the nucleus during mitosis which constitute the physical basis of inheritance.

Chitin (kítin), the hard material composing the exoskeleton or shell of Crustacea, insects, and others.

Chloragogue cells (kló'rà gŏg), compose the outer layer of the intestine of the earthworm.

Chondrin (kŏn'drĭn), the material of which cartilage is composed.

Chorion (kóprĭon), the outer embryonic membrane of mammals.

Chorioid (kó'rí oid), middle or vascular coat of vertebrate eyeball.

Chromatin (krónátĭu), dark-staining substance of the nueleus of the cell.

Chromatophore (krō'mà tō fōr), a colored pigment cell.

Chromidia (krō míd'ía), scattered chromatin graules through the cytoplasm of some cells.

Chromonemata (krō mồ nĕm'á tả), threads of chromatin distinguishable within chromosomes during mitosis; seen in the resting phase of some.

Chyme (kim), partially digested food material which is in semiliquid condition.

Cilia (sill'ĭà), hairlike cytoplasmic processes, used by certain protozoaus for locomotion.

Cirrus (sǐr'ǔs), a bristlelike appendage.

Cleavage (klêv'ij), the cell divisions changing the zygote into an organism of many cells.

Clitellum (klĭtěl'ǔm), a broadened area in the earthworm about one-third of the length of the body back from the head. It is glandular and serves in producing the cocoon.

Cloaca (klō à'kă), the common chamber into which the intestine, and urinary and genital canals discharge in some forms.

Cnidoblast (nī'do blăst), the type of cell of the coelenterate in which the sting cell or nematocyst develops. 
Cochlea (kŏk'lēa), a coiled structure of the inner ear in which is located the sensory ending of the auditory nerve.

Cocoon (kŏ kōon'), a covering which protects a larva, pupa, or even the adult stage of certain animals.

Coeloblastula (sē lō blăs'tū lả), blastula having a hollow center.

Coelom (sé'lŏm) or coelome (sélōm), the space between the walls of the body and the inclosed viscera.

Commensalism (kŏmĕn'săl $\breve{z m}$ ), an association of different species of animals in which at least one benefits without injury to the other.

Commissure (kŏm'i shoor), a strand of nerve fibers or nerves joining two centers or ganglia.

Conditioned reflex (kŏn dĭsh'ŭnd), a reflex action which is modified or established by previous experience.

Conductivity (kŏn'dǔk tǐv ǐtı̌), the power of conducting or of receiving and transmitting.

Congenital (kŏn jĕn'ĭ tăl), conditions existing at birth.

Conjugation (kǒn jō gā'shŭn), a temporary union of two individuals with exchange of nuclear material.

Copulation (kōp ū lä'shŭn), union of genital regions of two individuals during which spermatozoa are transferred from one to the other.

Corium (kō'rĭ ŭm), the deeper layer of the skin or dermis.

Cornea (kôr'nèa), transparent coat of modified epithelial tissue over the front of the eye.

Cortex (kôr'těks), superficial portion or outer layer, as of the brain or kidney.

Cranial (crā'ni ăl), pertaining to the portion of the skull enclosing the brain.

Cretin (krëtinll), a defective individual due to abnormality of the thyroid gland.

Ctenidia (těn ǐd’̌̃a), gill structure.

Cutaneous (kù tă nề ùs), pertaining to the skin.

Cuticle ( $\left.k \bar{u}^{\prime} t \hat{i} k l\right)$, the outer surface of the skin of many animals.

Cycloid scale (si'kloid), a scale which is thin and shows concentric lines of growth without serrated margin.

Cyclosis (si klō'sǐs), the rotation of the endoplasm of protozoan forms.

Cyst (sist), an organism enclosed by a resistant wall.

Cysticercus (š̌stĩ sûr'kŭs), the bladder worm or encysted stage in the life history of the tapeworm.

Cytology (sī tŏl'o jŭ

Cytopharynx (si tốfăr'inks), channel from surface to endoplasm in Euglena.

Cytoplasm (si'to plăz'm), the protoplasm of the cell not including the nucleus.

Dactyl (dăk'tǐl), refers to finger.

Daughter cells (dô'tẽr sělz), the two cells resulting from a division of one cell.

Delamination (đề lăm ì nā'shǔn), the formation of a new layer of cells parallel to the old by the division and migration of cells of the primary germ layers.

Dendrite (dĕn'drīt), a nerve fiber which carries impulses toward the nerve cell body.

Dermis (đûr'mîs), same as corium.

Dialysis (di ăl'̌ siss), separation of dissolved materials in crystalloids and colloids by means of semipermeable membrame.

Diaphragm (d’’’a frăm), a muscular partition between the abdominal and thoracic cavities in mammals.

Diastase (dì'à stās), the class of enzymes capable of bringing about conversion of starches to sugars.

Diencephalon (di ĕn sĕf'à lŏn), a region of the brain just posterior to the cerebrum. 
Differentiation (dı̆f ẽr ĕn shĭ $\bar{a}^{\prime}$ shŭn), the formation of special parts, tissues, or cclls from the primitive unspecialized layers.

Diffuse (dífūs'), to mix with or to spread through completely and thoroughly another substance.

Digestion (dĭ jĕs'chŭn), the conversion of complex unabsorbable food materials into a form capable of bodily absorption.

Dihybrid (dī hībrìd), progeny or offspring of parents differing in two characters.

Dominance (dŏm'innăns), a condition in which one of two characters present in the individual appears to the exclusion of the other.

Dimorphism (dì môr'fı̆m), difference of form between members of the same species.

Dioecious (dī è'shŭs), the male and female germ cells being produced by different individuals.

Diploblastic (dı̌p lồ blăs'tǐk), composed of two germ layers.

Diploid (dip'loid), having the base number or double number of chromosomes, as in soma ic cells.

Dissimilation (đ’̉ š̀m ı̆ lā'shŭn). (See Catabolism.)

Diurnal (dī ûr'năl), active by day.

Diverticulum (dī vẽr tĭk'tu lŭm), a blind tube branching out of a larger one.

Duodenum (dūô dènŭm), the part of the small intestine between the stomach and the jejunum.

Ecdysis (čk'dĭ siss). (See Molt.)

Ecology (êkol'o jí), the science of the relation of an organism to its environment.

Ectoderm (ĕk'tô dûrm), the outer cell layer of the wall of a gastrula and its later derivatives.

Ectoplasm (čk'tô plăz'm), substance of the outer layer of cytoplasm or ectosare of a protozoan animal.

Ectosarc (ĕk'tósärk), the superficial layer of cytoplasm of a single-celled aninal.

Egestion (ê jĕs'chŭn), the casting ont by the body of indigestible food material.

Electrolyte (ē lěk'trồ lìt), a substance whose molecules dissociate into ions.

Electrotropism (è lěk'trŏt'rồ pìz'm), response of an organism to electric currents.

Embryology (ĕm bry̆ŏl'o jŭ), the science of the origin and development of the individual.

Endacrine system (ĕn'dō krinn), a system including those ductless glands which secrete hormones.

Endoderm (ĕn'dō dûrm), the inwer cell layer of the wall of the gastrula and its later derivatives.

Endomixis (ĕn dô m̌̌k'šss), nuclear reorganization within a protozoan which does not involve conjugation.

Endeplasm (ĕn'dô plăz'nu). (See Endosare.)

Endopodite (ĕn dōp'o dit), the internal or principal branch of a biramous appendage of Crustacea.

Endosarc (ěn'dò särk), the area of cytoplasm within a cell which is surrounded by ectoplasm; substance of this is endoplasm.

Endoskeleton (ĕn dôskĕl'êtŭn), the bony, cartilaginous, or other internal frame work of an animal.

Endothelium (ĕn dôthēll̆ ŭm), the mesodermic lining layer of such closed spaces as blood ressels and lymph spaces.

Enteric (ĕn'tēr ı̌k), adjective form of enteron.

Enterocoele (ĕn'tẽr ố sēl), a portion of the coelomic eavity that arises by outgrowth from the enteric eavity.

Enteron (ěn'tēr ŏn), a digestive cavity or tube. 
Entomology (ĕn'tó mŏl ôj ju), the branch of zoology that deals with the study of insects.

Entozoic (ĕn'tó zō'ilk), forms which live within the bodies of other animals. Enzymes (ĕı'zimz), substances that bring about chemical transformation.

Ephyra (ěf'i rå), the free-swimming larval form of the Scyphozoa.

Epiboly (ê píb't lì), posterior growth of a fold of the blastoderm over the surface of an embryo in the formation of the enteron during gastrulation.

Epigenesis (ĕp ı̆ jĕn'ê siss), the conception that the parts of the organism arise from an undifferentiated germ cell.

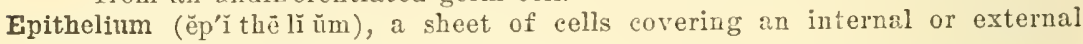
surface of the body.

Equatorial (ēkwá tō'rĭăl) plate, the platelike arrangement of chromosomes lying in the plane of the equator of the mitotic spindle during cell division.

Erepsin (è rĕp'sin), an intestinal enzyme which splits peptones into amino acids.

Estivation (ĕs tĭ vā'shŭn), a dormant condition adopted by certain animals during summer.

Eugenics (tu jĕn'îks), the science of genetics applied to human kind, usually for the purpose of improvement.

Euglenoid (ù glènoid), resembling a Euglena, as euglenoid movement.

Eustachian (ù stā'kĭăn) tube, the tube extending from the middle ear to the pharynx.

Evagination (ē văj'ı̌ nā'shŭn), the unequal growth outward of a surface layer, one of the processes by which diferentiation of organs is produced.

Eviscerate (è vıs'êr āt), to remove or cast out the internal organs.

Exopodite (ěks ŏp'ò dit), the external branch of the appendages.

Exoskeleton (ĕk sơ skěl'ề tŭn), the hardened parts of the external integument of an animal.

Expire (ĕk spir'), to expel water or air in the process of respiration.

Factor (făk'tẽr), one of several interacting elements in a complex process. Agency influencing the development of an individual, as those carried in the genes of chromosomes.

Fauna (fô'nà), a term referring to animal life.

Feces (fé'sēz), the indigestible portion of the food which passes through the alimentary canal and is discharged by way of the anus.

Fertilization (fûrtĭlì zâ'shŭn), the union of a mature ovum and a mature spermatozoon to produce a zygote.

Fetus (féturs), an advanced stage of the embryo of a mammal before birth.

Fibrin (fílbrin), the fibrous material in a blood clot; formed when fibrinogen of the blood is exposed to air.

Filtrable virus (fil'trả b'l vī'rŭs), an organism too small to be seen with the mieroscope and usually within cells of other organisms.

First filial (fûrst fřl'ı̌ ăl), generation, the individuals arising from a particular mating.

Fission (físh'ŭn), division of an organism into approximately equal parts. Flagellum (flà jêl'ŭm), a whiplike locomotor strueture of a cell or singlecelled animal.

Follicle (fŏl'̌́ k'l), a cellular sac or pocket.

Fragmentation (frăg mĕn tā'shŭı), a process by whieh individuals of certain Protozoa and simple Metazoa may divide internally to form several new individuals.

Freemartin (frē'märtinn), a modified female member of a pair of cattle twins which shows certain male features. 
Gametes (găm'ēts). (See Germ cells.)

Gametogenesis (găm êtô jĕn'ê sĭs), the series of cell divisions in the develop. ment of germ cells.

Ganglion (găng'li ŏn), a group of nerve cell bodies outside the central nervous system.

Ganoid scale (găn'oid), rhombic in shape, composed of an inner layer of bone and outer layer of enamel.

Gastrula (găs'troo lä), the two-layered stage in the development of an embryo.

Genes (jênz), the units of material which function in the transmission of characters in heredity.

Genetics (jê nĕt'iks), the science that treats of variation, resemblances, and their inheritance from parent to offspring.

Genotype (jĕn'ôtip), the genetic constitution of genetically identical organisms.

Genus (jē'nŭs), pl. Genera (jen'ẽr å), a division of the classification, a sul)division of a family, and is divided into species.

Geotropism (jề ŏt'rô pĭz'm), response of an organism to gravity.

Germ cells (jërm) (gametes), cells specialized for reproduction.

Germ layer (jërm), one of the primary cell layers in an embryo.

Germ plasm (jẽrm plăzm), the liereditary material of an organism, the ehromatin.

Gills (gils), (pharyngeal clefts), a series of paired slits in the wall of the pharynx and body.

Gonads (gŏn'ăds), reproductive organs.

Gonophore (gŏn'ófōr), a reproductive individual which bears gonads, as in Hydroids.

Glochidium (glồ kĭd’ŭ ŭm), the larva of a fresh-water clam.

Glomerulus (glồ mĕr'ù lŭs), a body of capillaries enclosed at the end of each kidney tubule of the mesonepliric and metanephric types of kidneys.

Glycerol (glis'ër ōl), one of the alcohols which enters into the composition of fats; glycerin.

Glycogen (gli'kô jěn), a form of carbohydrate food material as formed and stored by the liver.

Habitat (hăb'ítăt), the place or area in which an animal or species lives.

Haploid (hăp'loid), the reduced or half number of chromosomes of the mature germ cells.

Heliotropism (hē lǐ ŏt'rồ p̌̃z'm), a response of an organism to light.

Hemoglobin (hē mô glōbìn), a protein pigment substance of the blood which is capable of absorbing oxygen and is red when comlined with it.

Hemolysis (hê mŏl'ǐ šss), disintegration of red blood corpuseles.

Hepatic (hê păt'ǐk), pertaining to the liver.

Herbivorous (hûr bĭv'ô rŭs), herb or plant-eating animals.

Hermaphoditic (hûr măf rồ dĭt'îk) (monoecious), having both male and female germ cells produced in one individual.

Heterozygote (hĕtër ơ $\mathbf{z i}^{\prime}$ gōt), an organisnı which is carrying sets of mlike characters in its genetical constitution.

Hibernation (hỉ bẽr nā'shŭn), the cessation of activity or dormancy of an animal during the winter season.

Histogenesis (hîs tô jĕn'ê š̌s), the development and differentiation of tissue cells.

Histology (his tŏl'o jĭ), the science that treats of the microscopic structure of the various parts of the animal body.

Holoblastic (hŏl ó blăs'tǐk), having the type of egg structure in which cleavage divides the entire egg.

Holozoic (hŏl ố zóñ organic material.

Homoiothermal (hố moi'ô thûr măl), laving a temperature regulation. 
Homolecithal (hō mơ lěs'ĩ thăl) (isolecithal), eggs having a uniform distribution of the yolk.

Homologous (hơ mŏl'ō gŭs), similar in structure and origin, but different in function.

Homonomous (hơ mŏn'ô mŭs), slight or no differentiation of body segments.

Homozygote (hō mō zĩ'gōt), a zygote or resulting organism in which the corresponding genes are alike.

Hormone (hôr'mōn), the essential substance of an internal secretion which serves to help in metabolism regulation. Produced by endocrine glands and carried by the blood.

Hyaline (hī'ålĭn), semitransparent or glassy.

Hybrid (hī’brīd), a cross, or offspring of parents differing in genetical constitution.

Hydrolysis (hỉ drŏl'ǐs sis), chemical rearrangement of a substance by combining with water.

Hydrostatic (hì drồ stăt'îk), a type of organ which regulates the specific gravity of an aquatic animal.

Hypertonic (hīpẽr tŏn'îk), possessing greater osmotic pressure than some related substance.

Hypostome (hīpố stōm), a conical projection around and below the mouth in coelenterates.

Hypothesis (hì pŏth'ê sǐs), an idea as it first develops as the result of preliminary observation and experiment.

Ileum ( Il'te ŭm), the posterior and longest part of the small intestine.

Iiium (Ǐl'̌ ŭm), dorsal bone of pelvic girdle of terrestrial vertebrates.

Immunity ( $\breve{1}$ mū'nı̆ tr̆ , freedom of susceptibility to disease.

Ingestion (in jěs'chŭu), the taking in of food material by an organism.

Insectivorous (Ĭ sĕk tǐv'ô rŭs), insect-eating animals.

Inspire (in spir ${ }^{\prime}$ ), the drawing in of water or air in the respiration.

Instar (in'stär), the period between molts in insect development.

Insulin (in'sutlin), a hormone produced by the panereas and essential to the proper metabolism of earbohydrates.

Integration (ĭn tề grā'shŭn), development and correlation to give unity in an organism.

Integument (Ĭn tĕg'ù mĕnt), the outer covering of the body.

Intracellular (in trả sĕl'ù lër), within the cell.

Intracellular differentiation (Ĭn trả sěl'ut lẽr dîf êr ěn sh̆̌ ă'shŭn), the presence of a variety of cells within one body.

Invagination (in văjĭna'shŭn), the unfolding process by which the primary endoderm is withdrawn into the blastular cavity and becomes enclosed by the primary ectoderm.

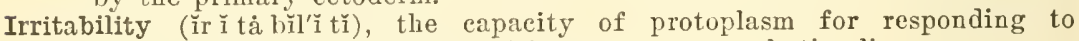
changes in environmental conditions or to external stimuli.

Isolecithal (i sồ lěs'ı̌ thăl). (See Homolecithal.)

Isotonic (î sótón'ík), possessing the same osmotic pressure in related substances.

Jejunum (jềjō'nŭm), the middle division of the small intestine, between the duodenum and the ileum.

Karyokinesis (kăr ì ô kĭ nē'sǐs), mitotic cell division.

Karyolymph (kăr'ì olimf), the more fluid material of the nucleus.

Karyoplasm (kăr'ío plăz'm), the protoplasm which constitutes the nucleus.

Karyosome (kăr'í ồ sōm), a "net knot" or a part of the chromatin which forms a distinet body in the nucleus.

Katabolism (kả tăb'ôlǐz'm). (See Catabolism.) 
Keratin (kĕr'à tĭn), a nitrogenous substance forming the chemical foundation of hair, horn, feathers, nails, claws, etc.

Kinetic energy (kỉ nět’ǐk), energy inherent in motion of a body.

Labium (lā'bĭ ŭm), posterior boundary or lower lip of an insect's mouth.

Labrum (lā'brŭm), the exoskeletal anterior boundary or upper lip of the insect's mouth.

Lacrimal (lăk'rĭ măl), pertaining to tears.

Lacteal (lăk'têăl), pertains to milk; refers to lymphatics of the intestinal region because of their light color following absorption of fat.

Lacuna (la kü'nà), a cavity or space, particularly that of bone, which contains the bone cells.

Lamella (là mèl'á), a thin layer or plate.

Larva (lär'vả), the young stage of an animal, which changes form during life.

Larynx (lăr'inks), the expanded upper end of the windpipe or trachea; voice box.

Lethal (lē'thăl), eapable of producing death.

Leucocyte (lü'kot sit), a white blood corpuscle.

Ligament (ligg'à měnt), a band of white fibrous connective tissue connecting structures other than muscles; particularly joining bones at the joints.

Limpet (lïm'pět), a small type of gastropod (Mollusca) with a simple uncoiled shell.

Linin (li'nin), the delicate threadlike structure which supports the chromatin granules in the nucleus.

Linkage (lingk'ĩj), the constant association of particular genes in certain chromosomes.

Lipase (li'pās), a fat-splitting enzyme.

Lipin (li'pin), fatty substance.

Lipoid (lip'oid), fatlike substance.

Lophophore (lö'fóför), a dise which surrounds the mouth and bears the tentacles of the Bryozoa.

Lumbar (lŭm'bēr), pertaining to the region usually known as the small of the back.

Lumen (lü'měn), internal cavity of a tubular duct, gland, vessel, etc.

Luminescence (lū mĭ něs'ĕns), the emission of light from the body.

Lymph (IImf), the blood plasma and white corpuscles in the lymph spaces about the tissues.

Lymphatic (lìm făt’ík), a vessel which earries lymph. In general, pertaining to lymph.

Macronucleus (măk rô nū'klê ŭs), the large nucleus of certain protozoans supposed to control vegetative functions.

Madreporite (măd'rè pô rît), the strainerlike external aperture of the watervascular system of echinoderms.

Màlpighian (măl prg'ĭăn) body, a structure in the cortex of the kidney, composed of a glomerulus and Bowman's capsule which serves to take urine from the blood.

Mantle (măn't'l), a fold of the body wall which partially envelopes the body; present in most mollusks and here secretes a shell.

Marsupial (mär sī'pĭăl), liaving a pouch for carrying the young.

Marsupium (mär sü'pĩ úm), an external pouch used in carrying the young, as in the kangaroo or opossum.

Matrix (mä'trìks), the mother substance, such as that which encloses anything; the intercellular material of cartilage or other sustentative tissue.

Maturation (măt t̀ rā'shŭn), the series of changes occurring in the development of germ cells before fertilization, including a reduction in the number of chromosomes in the cells. 
Maxilla (măk sil'’a), the major bone of the upper jaw of vertebrates or the accessory mouth part just back of the mandibles in many invertebrates.

Medulla (mê dŭl'à), posterior portion of the vertebrate brain; also the median area of many organs.

Medullary (měd'ŭ lẽr $\mathrm{i}$ ), pertaining to the medulla.

Medullated (mĕd'ŭ lāt ĕd), term used in reference to a nerve fiber which possesses a fatty or myelin sheath.

Medusa (mê dū'sả), a free-swimming individual coelenterate, such as a jellyfish.

Meiosis ( $m i \bar{~} \bar{o}^{\prime} \mathrm{sĭs}$ ), the reduction division in nuaturation of germ cells.

Menopause (měn'ô pôz), natural cessation of menstruation in women between. forty-five and fifty years of age.

Meridional (mě rĭd'ío năl), a condition in which planes extend from pole to pole of a spherical body.

Meroblastic (měr ó blăs'třk), having the type of egg structure in which cleavage is only partial, owing to the accumulation of yolk in the egg.

Mesencephalon (měs ěn sẹf'à lŏn), the third region of the vertebrate brain, commonly called midbrain.

Mesenchyme (měs'ěng kim) (Parenchyma), undifferentiated mesoderm composed of large cells.

Mesoderm (měs'ô dûrm), the middle germ layer and its later derivatives.

Mesoglea (měs ô glē'à), a jellylike substance found in Coelenterata between the ectoderm and endoderm.

Mesonephros (niěs ô nĕf'rŏs), the vertebrate kidney of forms from lamprey to amphibians inclusive.

Mesorchium (mě sôr'kĭ ŭm), the mesentery or membrane supporting a testis.

Mesothelium (měs ôthélĭ ưm), the mesodermic, membranous lining of the peritoneal cavity.

Mesovarium (měs ô vâ'rĭ ŭm), the mesentery in which the ovary is suspended.

Metabolism (mě tăb'olľz'm), the building up of living protoplasm and its concurrent oxidation.

Metagenesis (mĕt à jěn'ếsis), an alternation of sexual and asexual generation in the life cycle of an organism.

Metameres (mět'à mērs), one of a series of similar parts that follow one another in a vertebrate or articulate animal.

Metamerism (mêt tăm'êr $\mathrm{iz}$ 'm), serial symmetry or succession of segments.

Metamorphosis (mět à môr'fó š̌s), the transformation of one developmental stage into another without intermediate steps.

Metaphase (mět'à fāz), the phase of mitosis involving the longitudinal splitting of the chromosomes on the equatorial plate.

Metazoa (mět'á zō á), animals whose bodies consist of few or many cells functioning as a unit.

Micronucleus (mĩ krốn nū'klè ús), the small nucleus of certain protozoans supposed to control reproduction.

Micropyle (míkropil); the small opening in the egg where sperm enter in certain forms of animals.

Milt (milt), the light-colored spermatic fluid of male fish.

Miracidium (mi rá sìd’ì ŭm), the early larval stages in the flukes.

Mitochondria (mit o kŏn'drìa), small structures in the cytoplasm of animal cells; their significance is not entirely understood.

Mitosis (mîtós'sis), indirect cell division, invalving the formation and splitting of chromosomes and their equal distribution to daughter cells.

Molt (mōlt), a complete or gradual shedding of the outer covering.

Monodelphia (mŏn ô děl'fr à), having a placenta.

Monoecious (mồ nē'shǔs). (See Hermaphroditic.)

Monohybrid (mŏn ò híbrid), an offspring of parents which differ by only one character. 
Morphology (môr forl'ô jü), the science that treats of the form and structure of the bodies of animals.

Morula (mǒr'úlá), a type of blastula characterized by the absence of a seg. mentation cavity.

Mucosa (mù kō'sả), a cellular membrane lining such cavities as those of the digestive tract.

Mucus (mū'kŭs), a viscous secretion which cantains mucin (mū'sĭn). Mucous is the adjective form.

Mutation (mùtā'shŭn), a heritable change in an organism due to changes in one or more genes of germ cells.

Mutualism (mū'tú ăl ìz'm), animals of different species assaciating together for the mutual advantage of each.

Myelencephalon (mì ĕlĕn sĕf'à lŏn), the fifth or most posterior division of the vertebrate brain; the medulla oblongata of the adult.

Myelin (mï'è linn), fatty substance surrounding the axone in medullated nerve.

Myoneme (mì'ò nēm), contractile fiber or strand in the cytoplasm of certain protozoans.

Myotomes (mi'ôtōms), segmental divisions of the muscles.

Myxnoidea (mīk sĩ nō̄' dèá), subclass of Cyclostomes including hags. The name means slime and form.

Nares (nā'rēz), the openings into the nasal chambers in vertebrate animals.

Nauplius (nô'plǐ ǔs), a larval stage of certain Crustacea.

Nekton (nĕk'tŏn), the pelagic aquatic animals which are independent of the effect of wind and waves.

Nematocysts (nĕm'a tò šsts), stinging bodies found in the tentacles of certain coelenterates.

Nematode (něm'à tōd), a roundworm belonging to class Nematoda of phylum Nemathelminthes.

Neoteny (nē ŏt'è nì), the indefinite persistence of the immature condition of an animal.

Nephridium (nē frĭd'ĭ ŭm), a form of excretory organ, as found in the earthworm.

Nephrostome (něf'rô stōm), the funnel-shaped aperture at the medial end of a nephridium.

Neural (nū'răl), pertaining to the nervous system or to a nerve.

Neurilemma (nū rĭ lěm'á), the membranous outer coat of a nerve fiber.

Neuroid transmission ( $\bar{u}^{\prime}$ roid), primitive transmission of impulses from cell to cell.

Neuron (nū'rŏn), a nerve cell together with its processes.

Nidamental gland (nid'á měnt ăl), one of the reproductive organs of the female squid.

Notochord (nō'tôkôrd), a flexible rod extending anterior to posterior in the longitudinal axis of the body dorsal to the digestive tube and ventral to the nerve cord in chordates.

Nocturnal (nŏk tûr'năl), reference to night. Contrasted to diurnal which pertains to daytime.

Nodes of Ranvier (ränvyä'), constrictions in medullated nerve where the myelin sheath is interrupted.

Nomenclature (nō'mĕn klātûr), a system of naming objects or ideas.

Nondisjunction (nŏn dis jŭngk'shŭn), the failure of homologous chromosomes to separate after synapsis and both go to one daughter cell with none to the other.

Nucleolus (nū klē'ôlŭs) (Plasmosome), a body within the nucleus containing material that is not chromatin. 
Nucleus (nûtklè ŭs), a typically spherical body within the cell that contains the chromatin.

Nymph (nimf), the larval stage of an insect. which undergoes incomplete metamorphosis; also the larval stage of a few vertebrates.

Ocellus (ò sĕl'ŭs), a simple type of eye, as in some insects.

Ommatidium (ŏmàtı̆'ı̆ ŭm), one of the numerous rodlike units of the compound eye.

Ontogeny (ŏn tŏj'êñ̌), the entire development and life history of an individual organism.

Oocyte ( $\bar{o}^{\prime} \hat{0}$ sit), the female germ cell before maturation is completed.

Oogenesis (ōo jĕn'ě sis), the maturation of the female germ cell.

Oogonium (óo gō'n ŭm), the female germ cell during the multiplication and growth stages of maturation.

Operculum (ô pûr'kû lŭm), a fold of skin, bone, and scales, which covers the gills of fishes and certain Amphibia; also the bony structure closing the aperture of certain snail shells.

Organ (ôr'găn), an arrangement of two or more tissues as a part of the body which performs some specific function or functions.

Organism (ô $\mathbf{r}^{\prime}$ găn $\mathrm{z}^{\prime} \mathrm{m}$ ), any independent living being.

Orthogenesis (ôr thồ jĕn'ê sìs), the theory which holds that animals tend to develop along lines leading constantly in the same direction because they are determined by internal factors.

Osmosis (ŏs mō'sǐs), diffusion of substances dissolved in fluid, through a semipermeable membrane.

Ossicle ( $\left.\mathrm{os}^{\prime} \mathrm{i} \mathrm{k}^{\prime} \mathrm{l}\right)$, a small bony structure.

Ostium (ŏs'tr̆ ŭm), a mouthlike opening or entrance.

Otocyst (o'tồ sist), the primitive organ of hearing.

Ova (óñă), mature female germ cells. Sing., ovum ( $\bar{o}^{\prime}$ vum).

Ovary ( $\bar{o}^{\prime}$ vả rĭ), the female gonad.

Oviduct ( $\bar{o}^{\prime} v i$ dŭkt), the duet for the passage of ova from the ovary to the exterior of the animal.

Oviparous (ô vịp'á rŭs), pertaining to those animals which lay eggs that hatch after exclusion from the body.

Ovipositor (ō vĩ pǒz'îtẽr), an organ of female insects and others which serves in helping to deposit the egg.

Ovoviviparous (ō vồ vĩ vịp'á rŭs), a condition of retention of the egg in the nother's body where it is nourished by the yolk of the egg.

Ovulation (ō vot la'shŭn), the process of discharging mature eggs from the ovary.

Oxidation (ŏk sĭ dā'shŭn), a chemical combination of oxygen with another element.

Paleozoology (pā lè $\delta$ zó̀ ól'ồ jü), the science that treats of the animals of the past as represented by fossil remains.

Parasite (păr'á sīt), an organism that lives on or within and at the expense of another organism.

Parenchyma (pả rĕng'kĭ mä). (See Mesenchyme.)

Parietal (pà rí'étăl), pertaining to the walls of the coelom.

Parthenogenesis (pär thè nō jĕn'è siss), the development of an egg without fertilization.

Pathology (pá thŏl'ojī), the study of abnormal structures and abnormal functioning of life processes.

Pedal (pĕd'ăl), pertaining to the feet.

Pedicellaria (pěd ı̌ sě lā'rĭ à), pincherlike struetures found over the surfaces of sea urchins and starfishes.

Peduncle (pè dŭng'kl), the stemlike attachment of certain shells and barnacles to other objects. 
Pelagic (pè lăj'ìk), floating near the surface of water.

Pericardial (pēr i kär'di ăl), situated around the heart.

Periosteum (pěr ı̆ ŏs'tè ŭm), the membranous covering of bone.

Peripheral nervous system (pě rĭf'êr ăl), that part of the nervous system exclusive of the brain and spinal cord.

Peristaltic (pěr ǐstăl'tǐk), forcing the food along the intestine by rhythmical contractions of the intestinal wall.

Peritoneum (pěr i tō né'ŭm), the menbrane that lines the coelom of vertebrates.

Petromyzontia (pět rō mī zón'tĭ à), subclass of Cyclostomes including the lampreys. The name means stone and suek.

Phagocyte (făg'ô sit), a white corpuscle which engulf's and destroys bacteria and other foreign material.

Pharynx (făr'ingks), the region between the mouth and the esophagus.

Pharyngeal (fă rin'jê ăl), pertaining to the pharynx.

Phenotype (fënòtip), a tỵpe of organism possessing a complex of characters in its exterual features.

Phenotypic (fē nơ tǐp'ík), pertaining to phenotype.

Photosynthesis (fõ tô sin'thè sis), the process by which green plants manufacture starch from raw materials.

Phototropism (fồ tŏt'rơ pĩz'm), response of an organism to light.

Phylogeny (fì lŏj'ê nì), the study of the origin and relationships of the different groups and races of organisms.

Physiology (fiz í ŏl'ó jī), the study of the function of the parts of an organism as well as its living processes as a whole.

Pia mater (pi'a mā'tër), the membrane which is the immediate covering of the brain and spinal cord.

Pilidium (pi līd'í ŭm), helmet-shaped larva of certain forms.

Pineal (pin'ểa al) body, a dorsal projection from the diencephalon and thought to be the vestige of a third or median eye in vertebrates.

Pituitary (pi tü'îtër i) body, a glandular structure attached to the neutral side of the diencephalon of the vertebrate brain. It is an endocrine organ.

Placenta (plä sĕn'tả), the vascular membrane which connects the embryo with the varent.

Placula (plăk'ù là), a type of blastula in which the animal and vegetative halves are somewhat compressed toward each other.

Plankton (plăngk'tön), the small pelagic organisms which are at the mercy of the waves.

Plasma (plăz'mà), the fluid portion of the blood.

Plasmagel (plăz'mả jēl), the viscous or semisolid portion of protoplasm.

Plasmasol (plăz'má sŏl), the more fluid phase of protoplasm.

Plasmosome (plăz'mó sōm). (See Nucleolus.)

Pleural (ploor'ál), pertaining to the cavity which contains the lungs.

Plexus (plĕk'sŭs), a network.

Polar (pó'lēr) body, a small nonfunctional cell or oocyte produced during the maturation divisions of the female germ cell.

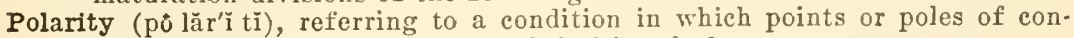
centration or dominance are established in a body.

Polocyte (pó'lö sit), a technical name for a polar body.

Polyandry (pöl'ìn drì ), the practice of one female mating with several males.

Polygamy (pó lĭg'á mî), having more than one mate at the same time.

Polygyny ( $p \delta$ lij'ín $\mathbf{i})$, the practice of one male mating with several females.

Polymorphism (pöl ì môr'fiz'm), the occurrence of two or more forms of individuals within a species.

Polyp (porl'ip), the attached phase of the life history of a coelenterate animal.

Precocial (prê kó'shăl), type of bird which leaves the nest and has downy covering at time of hatching. 
Predaceous (prè dā'shŭs) animal, one which preys on others.

Predatism (prěd'à tǐz'm), the practice of one animal preying on another.

Primordial (prī môr'dĭ ăl), the first or primitive form.

Proboscis (prổ ǒs'is), an extension of the head or mouth parts. May be nose, as in elephant; mouth parts, as of moth; or pharynx, as of planaria.

Proctodeum (prŏk tơ dé'ŭm), the pocket in the ectoderm ventral to the posterior part of the enteron of the embryo; primordium of the anus.

Proglottid (prót glót'id), one of the sections or individuals of the chain making up the body of a cestode, sueh as the tapeworm.

Pronephros (prò nĕf'rŏs), the first kidney structure to form in the developing vertebrate.

Pronucleus (prồ nıtúklè ŭs), one of the two nuclei within a fertilized egg before cleavage occurs.

Propagation (mrǒp $\dot{\mathbf{a}}$ gáa'shŭn), the production of new individuals.

Prophase (prō'fāz), the preparatory stages of mitosis during which the formation oceurs of spindle, spireme, and chromosomes.

Proprioceptor (prōprĭosěp'tẽr), the receptor or end organ of the nervous system located within a certain tissue receiving stimulations in reference to bodily position or orientation.

Prostate (prŏs'tát) gland, one of the male reproductive organs producing part of the semen.

Prostomium (prồ stō'mĭ ŭm), portion of the anterior segment of annelids which overhangs the mouth.

Protein (prōtềin), one of the organic compounds found in protoplasm. It contains the elements carbon, oxygen, hydrogen, and nitrogen.

Protoplasm (prō'tó plăz'm), the living matter of which all organisms are composed.

Protopodite (prô tŏp'ô dit), the proximal section of the crustacean appendage. It includes coxopodite and basipodite.

Prototroch (prōtotrŏk), the band of cilia extending around the equatorial region of trochophore larva.

Protrusible (prót trō'síb'l), the ability to be put out or extended from the body.

Proventriculus (prō věn trĭk'tù lŭs), the anterior, secretory portion of the stomach in certain animals, as the bird.

Pseudopodia (sū dò pō'dì a), protoplasmic processes (false feet) formed by certain protozoans and used for locomotion.

Ptyalin ( $\mathrm{i}^{\prime} \mathbf{a}$ lĭn), the stareh-digesting enzyne of saliva; a diastase.

Pupa ( $p \bar{u}$ 'pa), the encased, inactive stage between the larva and adult condition in many insects and other animals.

Pyloric ( $p \bar{i}$ lŏr $\left.r^{\prime} \mathrm{l} l s\right)$, pertaining to the pylorus.

Pylorus (pi lo'rŭs), the junction of the posterior portion of the stomach with the small intestine.

Radial symmetry (rā'dĭăl sim'ětrù), applied to a body that can be equally divided by several radial planes.

Radiant energy ( $r \overline{a ̆}^{\prime}$ dĭ ănt ěn ẽr jŭ), inherent power or energy transmitted through space, as that from the sun, radium, or $x$-ray.

Radula (răd't̀ là), the sheetlike rasping structure of the mouth of gastropods; used in mastication of food.

Recessive (rè sĕs'iv), in reference to a gene which is carried in the cell without expressing its character unless there is absence of its dominant mate.

Recapitulation (rē kà pit aै lä'shŭn), repetition in development of an individual organism of its phylogenetic history.

Redia (rế'día), second phase of the life history of the fluke.

Reflex ( $\left.r \bar{c}^{\prime} f l c ̌ k s\right)$ action, automatic reaction to a stimulus from a receptor neuron and passed on to an adjustor neuron; performs an involuntary, appropriate act. 
Regeneration (rē jĕn ẽr à'shŭn), the replacement of nutilated parts or an entire animal from a portion of one.

Renal (rè'năl), pertaining to the kidney.

Rennin (rěn'ı́n), an enzyme constituent of gastric juice of mammals and capable of coagulating the protein portion of milk.

Reproduction (rē pró dŭk'shŭn), the production by an organism of others of its kind.

Respiration (rěs pr rā'shŭn), the exchange within an organism of oxygen entering the protoplasm and carbon dioxide leaving it.

Response (rè spŏns'), the reaction of an organism to a stimulus.

Rete (rètê), a limited meshlike arrangement or network.

Reticulum (rê tǐk'ù lŭm), a fibrous or tubular network.

Retractile (rê trăk'tīl), that which can be withdrawn.

Retrogression (rět rò grěsh'ŭn), going behind or moving baekward.

Rhabdites (răb'dits), speeial structures found interspersed among the epidermal cells of flatworms.

Rheotropism (rēŏt'rồ pĩz'm), response of an organism to meehanical currents.

Roe (rō), ovary and eggs of fish.

Rudiment (roo'dĭ mĕnt), partially developed or embryonic strueture; usually without function.

Rugose (rōo'gōs), possessing many ridges and folds.

Ruminants (rō'mínănts), animals which ehew the cud.

Saprophyte (săp'rō fït), an organism which absorbs nonliving organic matter in solution directly through the surface of the body.

Sarcolemma (sïir kôlěm'à), the delicate membrane immediately enclosing the striated voluntary muscle cell.

Sarcoplasm (sär'kô plăz'm), the eytoplasm of muscle cells exclusive of the sarcostyles or fibrils.

Sarcostyles (sär'kôstils), cytoplasm filırils in the strueture of cytoplasm of voluntary muscle cells.

Schizogony (skī zŏg'ò ny̆), asexual reproduetion.

Sclerotic (sklè rŏt'ík), the dense fibrous outer eoat of the vertebrate eye.

Scolex (skō'lěks), knolllike "head" on anterior proglottid of cestode.

Scute (skint), a plate on the ventral side of the body of the snake.

Sebaceous glands (sể bā'shŭs), small subeutaneous glands, usually eonnected with hair follieles.

Sedentary (sěd'ěn těr ǐ), unattached forms wlich remain in one place.

Semen (sē'mĕn), fluid which carries the spermatozoa in the males of most animals.

Seminal (sĕm'ĭ năl), pertains to spermatozoa.

Semipermeable membrane (sěm ì pûr'mè à bl), one which permits the passage of solvents through it but not solutes, unless they dissolve in the menibrane.

Senescence (sè něs'ěns), period of old age and its effects.

Septum (sěp'tŭm), a wall dividing two cavitics.

Serial homology (sêr'ĩ ăl hố mŏl'ô jĭ), presence of struetures of similar origin and form in different segments of the same animal.

Serosa (sề rō'sả), membrane covering peritoneal surface of internal organs; one which secretes a watery fluid.

Sertoli cells (sěr tō'lè sěls), modified, supporting or nurse cells for forming spermatozoa in the testes.

Serum (sē'rŭm), the fluid part of the blood which remains after elotting.

Sessile (sěs'ìl), attached directly and incapable of loeomotion.

Seta (sè'tả), hairlike spine or bristle, found in animals.

Sinus (si'nŭs), a eavity in a bone or other part, or a dilated vessel or canal. 
Siphon (sīforn), a canal or passageway, as the waterways in clams or tunicates.

Somatic cells (sô măt'ik), the cells of the body exclusive of the germ cells; body cells.

Somites (sō'mits), segments of the body of a segmented animal.

Spermatogonium (spûr má tơ gō'nĭ ŭm), a male germ cell during the period of multiplication and growth in maturation process.

Spermatocyte (spûr'mà tó sit), the male germ cell before its maturation is com. pleted.

Spermatogenesis (spûr má tô jĕn'ề šss), the maturation of the male germ cells. Spermatozoa (spûr má tơ zō'à), mature male germ cells.

Sphincter (sfingk'tẽr), a muscular band surrounding a tube or aperture which, by its contraction, closes the lumen.

Spicule (spik'suli), one of numerous needlelike, solid structures found supporting the tissues in the body wall of sponges.

Spiracle (spir'ả k'l), openings of air tubes in insects, or modified opening of first gill slit of certain fish.

Spireme (spi'rēm) thread, the coiled bead-like string of chromatin material that appears during the prophase of mitosis.

Splanchnic (splăngk'ňlk), has reference to tlie visceral organs.

Spongin (spŭn'jin), the skeletal material of a sponge.

Sporulation (sporr ù lā'shŭn), production of spores by division of a protozoan while encysted.

Statoblast (stăt'ó blăst), an encased, ascxual winter bud of a bryozoan.

Statocysts (stăt'ós šsts), sense structures assisting in maintaining equilibrium in certain forms.

Steapsin (stè ăp'sŭu), one of the pancreatic enzymes which is capable of changing fats to fatty acids and glycerin.

Stereoblastula (stĕr è ó blăs'tù lả), a blastula in which all the cells are in close contact and no blastocoele is formed.

Stigma (stig'má), a sensitive pigment spot of Protozoa or the opening of a spiracle of insects.

Stomatodeum (stō mà to dé'ŭm), the opening of the developing alimentary tract in an embryo.

Stratum (strā'tŭm), a layer of a series.

Striated (strīât ěd), type of muscle with more dense areas across the fibers.

Strobila (strô bì'là), a series of individuals produced by linear budding, as certain Scyphozoa and tapeworms.

Succession (sŭk sèsh'ŭn), the successive occupatiou of a given area by several species, either hourly, daily, or seasonally.

Sustentative tissue (sŭs'tĕn tā tĭv), binding together or supporting the various parts of the body.

Suture (sū'turr), to sew together; a line of junction.

Symbiosis (

Synapsis (sí năp'siss), the pairing of the chromosomes in the germ cells at one stage of maturation.

Syncytium (š̉n sǐsh'r ŭm), a mass or layer of protoplasm with numerous nuclei but without distinct cell boundaries.

Syngamy (š̃n'gả mǐ), union of mature gametes to form a zygote.

System (sis'těm), an aggregation of organs to perform some general function of life.

Taxis (tăk'š̌s), a tropismal response involving movement of an organism as a whole.

Taxonomy (tăks ŏn'ô mŭ) (systematic zoology), the classification or orderly arrangement of organisms according to their natural surroundings.

Tegumentary (tĕg ù měn'tả rì), referring to the skin. 
Telencephalon (tĕl ĕn sĕf'å lŏn), the anterior division of the vertebrate brain. Telolecithal (těl ô lěs'îthăl), tỵpe of egg with abundant yolk unequally distributed.

Telophase (těl'ò fāz), the final stage in mitotic divisions.

Tentacle (těn'tảk'l), flexible, armlike extension of the body of many nonchordates.

Terrestrial (těr rĕs'trı̆ ăl), a land form; living on or in the ground.

Testis (tĕs'tǐs), male gonad.

Thermotropism (thẽr mŏt'rơ păz'm), response of an organism to temperature.

Thigmotropism (thĭg mŏt'rồ pĭz'm), response of an organism to contact.

Thoracic (thò răs'ǐk), of or pertaining to the thorax or chest.

Thorax (tho'răks), the middle region of the body.

Threshold (thrĕsh'old), the minimum strength of stimulus necessary to get a response.

Thrombin (thrŏm'linn), the substance of the blood which plays an important part in clotting.

Thyroxin (thī rǒk'sēn or -sin), the hormone which is produced by the thyroid body.

Tissue (tish'ū), an organization of similar cells into a layer or group for the performance of a specific function.

Toxin (tŏk'sin), any poisonous substance.

Trachea (trā'kề à), the windpipe or a tube for conveying air to the lungs; air tubes in insects.

Trichocyst (trǐk'ósist), saclike structure in the ectosare of Paramecium.

Triploblastic (trīp lô blăs'tǐk), composed of three germ layers.

Trivium (trǐv'ĩum), the three anterior ambulacra of Echinodermata, collectively.

Trochophore (trŏk'ô fōr), a semispherical tỵpe of larva with cilia; found among flatworms, annelids, mollusks, etc.

Tropism (trō'piz'm), the movements of an organism in response to a stimulus.

Trypanosome (trīp'á nơ sōm), genus of parasitic Protozoa (Mastigophora) including the causal agent of African sleeping sickness.

Trypsin (trip'sin), a pancreatic enzyme which converts proteins to amino acids.

Tsetse (tsĕt'sê) fly, a species of fly which serves to transmit the causal agent of African sleeping sickness.

Tundra (toon'drả), level plains region of the aretic region.

Tympanum (tim'pá núm), cavity of the middle ear or more generally any organ serving to receive sound waves.

Umbilical (ŭm bĭl'ĭkăl) cord, the cordlike connection between the fetus and the placenta.

Umbilicus (ŭm bul'i kŭs), the navel or the point of attachment of the umbilical cord to the abdomen.

Uncinate (ŭn'sì năt), in the shape of a hook.

Ungulate (ŭng'gù lât), honfed.

Unguiculate (üng gwìk'ù làt), having claws.

Urea ( $\bar{u}$ ré’áa), a nitrogenous compound which is produced as a protein byproduct in metabolism.

Ureter (ù rē'tēr), the duct which conveys urine from the metanephric kidney to the cloaca or bladder.

Uretbra (ú réthrá), the duct which leads from the urinary bladder to the exterior of the body.

Uropods (ū'rō pŏdz), the sixth pair of abdominal appendages of a crustacean.

Vacuoles (văk'ù ōlz), small carities in a cell filled with water, gasses, or oils.

Vagina (váji'ná), the cavity between the uterus and the external genital aperture of the female in many animals. 
Vascular system (văs'kû lẽr), the circulatory system.

Vascular tissue (văs'kù lẽr), fluid tissue consisting of cells known as corpuscles in a fluid medium, plasma.

Vein (vān), the larger blood vessels leading to the heart.

Ventral (věn'trăl), side away from the back; literally belly; opposite to dorsal.

Villus (vil'ǔs), a fingerlike, vascular process of the internal lining of the small intestine.

Vitamins (vī'tá mins), substances which occur in small amounts in numerous foods and are essential regulatory substances for the animal body.

Vitelline (vĭ těl'linn), the outer membrane of an egg.

Vitreous (vit'rèu ŭs), glassy in appearance.

Viviparous ( $v \bar{i}$ vip'á rǔs), the retention and development of the egg in the mother's body and nourishment of the embryo from the blood of the mother.

Volant (vō'lănt), able to fly.

Zoogeography (zōo jè ŭg'rả fü), the study of the geographical distribution of animals.

Zoology (zōólŏ jì) (animal biology), the study of the science which treats of animals.

Zygote (zì'gōt), a fertilized egg, or embryo, after fertilization.

Zymogen (zìmó jën), a pre-enzyme; a substance which is produced in a gland cell and becomes an enzyme when it is discharged and activated by some other substance, perhaps another enzyme. 


\section{INDEX}

A

Abdomen, 348, 639

Abducens nerve, 527

Abductor muscle, 524

Abomasum, 633

Abyssal zone, 713,770

Acanthocephala, class, 175, 178, 179

Acarina, order, $266,295,297$

Accipiter, 590

cooperi, 589

Accommodation, 402

Acetabulum, 495, 522, 611

Achiridae, family, 452

Aciculum, 196

Acidian larva, 367

Acipenser fulvescens, 447

Acipenseridae, family, 446

Acnidosporidia, 73

Acoela, 160

Acontia, 141

Acris gryllus, 485

Acroniegaly, 673

Actinaria, order, 140

Actinophrys, 67,69

Acusticolateral areas, 435

Adambulacral plates, 229

Adaptability and regeneration, 695

Adaptation, 727

Addison's disease, 671

Adductor mandibulare, 468

muscles, 250,254

Adhesive papillae, 367

Adipose fin, 442,457

Adrenal glands, $6 \pi 1$

Adrenalin, 671

Adrenin, 671

Aedes, 80

Aegeria exitiosa, 328

Aegeriidae, family, 328

deolis, 241

Aeolothrips fasciatus, 320

Agamodistomum, 759

Agassiz, 43

Agassiz' tortoise, 550

Aglypha, 556

Agnathostomata, 412

Agriculture, 34

Albatrosses, 587

Albulidae, family, 448

Albumin gland, 246

of hen's egg, 615

Alces americana, moose, 630

Alcyonacea, order, 143

Alcyonaria, subclass, 143

Alcyonium, 143

Alfalfa beetle, 324

Alimentary canal, 103

Allantois, 818

Allelomorph, 823, 824

Allelomorphs, multiple, 827

Allergy, 839

Alligator mississippiensis, 561

Alligators, 560

Allocosa parva, 295

Alopecia, 838

Alpacas, 630

dlpine zone, 714

Alteration of generation, 112

Altricial birds, 615

Alveoli, 566

of lungs, 514
Alytes obstetricans, 480

Amblema costata, 248

Amblyopsidae, family, 451

Ambulacral groove, 218, 224, 227, 228 ossicle, 229,230 plate, 228

Ambystoma, 484 axolotl larva, 481

texanum, 480

tigrinum, 478, 480

Ambystomoidea, suborder, 484

Ameiuridae, family, 450

Ameiurus, arteries, 461 cranial nerves, 469 internal structure, 458

life history, 470

natalis, 457

skeleton, 466

veins, 462

Ametabolous, 308

Amidae, family, 447

Amino acids, 57

Amitosis, 61

Ammocoetes, 420

Ammonite, 259

Amnicola, 245

comalensis, 247

Amnion, 818

Amoeba, 85

assimilation, 88

behavior, 91

digestion, 87

excretion, 88

histolytica, 744

locomotion, 91, 92

metabolism, 86

proteus, 67

reproduction, 89

tmoebic dysentery, 78

Amoebina, 67

Amoebocytes, 233

moeboid acivity, 152

imphiaster, 63

Amplibia, breeding habits, 479

class, 411,472

classification, 482

coloration, 474

economic importance, 486

enemies of, 477

families of, 484

hibernation, 480

means of defense, 478

regeneration, 477

secontlary sexual characters, 480

Amphibian voice, 478

Imphibians, tailless, 485

Amphiblastula, 128,808

Anmphicoela, suborder, 485

imphicoelous centrum, 444

Amphids, 175

Amphineura, 256

imphioxus, 361,368

circulatory, 371,372

respiratory system, 373

Imphiplexus, 409

Imphipoda, order, 265

implipods, 283

Amphisbaenidae, family, 555

Amphitrite, 195

Ainphiuma, 473

Amphiumidae, family, 484

Ampulla, 222, 229, 230, 231 
Ampullae of Lorenzini, 437

Amyda, 550

Amylopsin, 385, 386, 502

Anabolism, 86, 377

Anabrus simplex, 315

Anacanthini, suborder, 451

Analogy, 271

Anaphase, 62, 63

Anapsida, subclass, 547

Anasa tristis, 318

Anastomosis, 107

Anatomy, gross, 20

Ancestry of Arthropoda, 865

Anchoviella mitchilli, 448

Anchovies, 448

Ancylis comptana, 326

Ancylostoma, hookworm, 745, 751

Andalusian chickens, 830

Androgenic hormone, 676

Androsterone, 676

Anecdotal period, 847

Anglers, 455

Anguidae, family. 552

Anguillidae, family, 449

Angulosplenial bone, 520

Ani. 591

Animal and its environment, 719

anomalies, 654

behavior, 846

cell, 58

distribution, 711

kingdom, 25

parasitism, 735

Animals of Gulf of Mexico, 778

Anisoptera, suborder, 321

Annelida, 26, 194

phylogenetic advances, 216

theory of evolution, 867

Anniellidae, family, 553

Annuli, 212

Annulus, 268

Anodonta, 249

stewartiana, 248

Anolls, 552

Anomocoela, suborder, 485

Anopheles mosquito, 74, 79

Anoplura, order, 266, 333

Anseriformes, order, 589

Ant lions, 266, 322

Anteater, giant, 621

scaly, 621

spiny, 617

Antebrachium, 495

Antedon, 225

Antelope, 630,785

Antennae, 269, 271

Antennata, division, 265

Antennules, 269,271

Anterior cardinal veins, 434

fontanelle, 428

Anthelmentics, 750

Anthonomus grandls, 324

Anthozoa, class, 140

Anthropoid apes, 637

Anthropoidea, suborder, 635

Anthropopithecus troglodytes, 637

Antibodies, 395,884

Antigen 395

Antilocarpa americana, antelope, 630 . 785

Antipathes, 143

Antipathidea, order, 143

Antithrombin, 395

Antrum, 812

Ants, 266,331

Anura, order, 485

Aortic arches of vertebrates, 882

Apes, 635, 637
Aphelodactyla, 224

Aphid, beet, 317

black cherry. 318

rosy apple, 316

Aphididae, family, 318

Aphids, 318, 340

Aphis lions, 266 maidi-radicis, 340

roseus, 318

Aphrodita, 195

Apical organ, 237

Ap on proclive, 324

Aplodinotus, 454

Apodes, suborder, 449

Apopyles, 124

Alpendicular skeleton of frog, 520

Appendicularia, 361

Apvendix, vermiform, 878

Apteria, 601

Apterygiformes, 585

Ap،erygo،a, subclass, 309

Apteryx 585

Aqueous humor, 529

Aquila chrysaëtos, 590

Arachnid theory of evolution, 867

Arachnida, class, 266, 292 classification, 295

Arachnoid, 650

Arachnoidea, division, 266

Aranea gemma, 296

Arane,da, order, 266, 295

Arbacia, 220, 222

Arcelia, 67

Archaeopteryx, 861, 869

Archenteron, 117, 210,535

Archiannelida, 194,212

Architeuchis princeps, 260

Arciferal pecioral girdle, toad, 542

Ardeidae, family, 588

Arenicola, 195

Argen ines, 449

Argen.inidae, family, 449

Argiopldae, family, 296

Argulus, 264, 265, 284

Aridae, family, 450

Aristotle, 36

Aristotle's lantern, 221

Armadillidium, 282

Armadillo, nine-banded, 621 enubryos, 622

Arrowworms, 193

Artemia, 283

Arteries, efferent branchial, 463

of frog. 503

of horned lizard, 568

of toad, 542

Arthrobranchiae, 271

Arthrolepcella lightfooti, 479

Arthropuda, phylogenetic advances, 286 phylum, 27, 263, 746 wormlike ancestry, 865

Arthropodial membrane, 268

Arthrorhabuinus, 291

Articular bone, 467

Artiodactyla, order, 629

Ascaphus, 479 truei, 485

Ascaridia lineata, 176

Ascaris, 175, 176, 179

iniernal anatomy, 181

intestinal worm, 745

life cycle, 182,183

relations to man, 183

Ascaroidea, order, 176

Ascidiacca, 361

Ascon, 124, 125

A scorbic acid, 390

Asellus, 281, 282, 283 
Asllidae, famlly, 330

Asuhalt pits of La Brea, 861

Aspidiotus pernlciosus, 316, 318

Ass, 633

Assimilation, 278

in Euglena, 81

in Hydra, 152

Astacus, 266

Aster, 62, 63

Asterias, 228

cleavage, 116

Asteroidea, class, 217, 226

Asteromeyenia, 121

Asthma, 839

Astrangia, 142,143

Astropecten, 218

Atherinidae, family, 452

Atlas, 494

Atom, 697

Atomic weight, 697

Atrial cavity, 365,370

Atrina seminuda, pinna shell, 771

Atriopore, 370

Attidae, family, 296

Auditory meatus, 639 nerve, 527

Audubon, John J., 786

Auks, 591

Aurellia, 138

life cycle, 139

Australian region, 712

Autonomic function, 528

Autotomy, 219, 234, 281

Aves, class, 411, 582

classification, 584

economic relations, 596

Avicularıa, 184,185

Aviculariidae, family, 296

Avocets, 591

Avoiding reaction, 101

Axial gradient, 173

skeleion, 378

Axoloil larva of Ambystoma tigrinum, 481

Axone, 107

Aye-aye, 635

\section{B}

Babesia bigemina, 80

Baboon, 636

Back cross, 827 swimners, 318

Badgers, 627, 628

Bagre marina, 450

Bailer, 270

Baker's mealy bug, 318

Balaena mysticetus, right whale, 635

Balance in life, 32 in nature, 31

Balanoglossida, order, 361

Balantidium coli, 70, 71, 744

Balanus, 265, 285

Baleen, 634

Balistidae family, 455

Barb, 599, 600

Barbary ape, 636

Barbels, 457

Barbule, 599, 600

Barnacle, 264

acorn, 264

goose, 264

Barnacles, 284

Barnea costata, 771

Barracudas, 453

Barriers to distribution, 717

Basal disc, 140,146,147

Basiliscus, 552
Basipodite, 270

Basisphenoid bone, 465

Basket star, 219

Basommatophora, 258

Bass, freshwater, 454

Basses, sea, 454

Bat, brown, 620

Mexican iree-talled, 620

Baihyal zone, 770

Bathymetric distribution, 713

Batoidei, order, 424

Batrachoididae, family, 455

Batrisodes, $3+0$

Bats, vampire, 620

Bdellostoma, 412

Bears, 629, 791

Beaver, 624,787

Beavers, 622

Bêche-de-mer, 235

Bedbugs, 318

Bee thies, 330

Bees, 266, 331

Beele, Colorado potato, 323

Beetles, 265, 322

ambrosia, 335

passalus, 335

Behavior, chain reflex, 851

habitual, 852

reflex, 850

tropistic, 849

Belfrage, G. W., 48

Belonidae, family, 451

Belostomatidae, family, 318

Benthos, 769

Beroë ovata, 157, 159

Bidder's organ, 542

Bighorn, 631

Billbugs, 32

Binary fission, 90

Biogenetic law, 870

Biological effects of radiations, 697 point of view, 17

Biolozy, 17,20

Biomes, 724

Bios, 17

Biotic communities, 723 formations, 724

Biotin, 390

Bipes biporus, 555

Bipininaria larva, 232, 234

Biramous appendages, 270

Bird migrations, 582

B.rds, 411, 582 economic relations, 596

Birth rate, 839

Bison americanus, buffalo, 631

Bisons, 630

Bittern, 587

Bivium, 227, 228

Black bass, 453 widow spider, 293

Blackbirds, 595

"Blasendarm," 190

Blastema, 688

Blastocoel, 210

Blastocoele, 117, 534, 535, 805

Blastocyst, 814

Blastoderm, 805

Blastodermic vesicle, 814

Blastomeres, $171,533,800$

Blastonore, 534.805

Blastostylés, 132

Blastula, 11 $7,134,155$

earthworm 210 of frog, 533

Blatella germanica, 315

Blattidae, family, 315

Blind cave salamander, 482 
Blindworm, 553

Blister beetle, 322

Blood corpuscles, 107

matching, 883

white cells, 513

Blubber, 634

Blue baby, 664

crab, 282

darter 589

Bluebirds, 595

Boar, wild, 632

Bobcat, 629

Bobolinks, 595

Body louse, 738

Boidae, 556, 557

Boll, Jacob, 48

Bombylidae, family, 330

Bonellia, 214, 215

Bonitos, 454

Bonnethead shark, $42 \%, 439$ circulatory system, 433

Book lice, 333

scorpion, 266

Bot fly, 747

Botallus, duct of, 491

Botaurus lentiginosus, 587

Botflies, 330

Bovidae, family of cattle, 630

Bowfin, 447

Bowman's capsule, 398, 650

Box-elder bug, 318

Brachial artery, 506

Brachiolaria larva, 232, 234

Brachiopoda, 27,187

Brachium, 495, 563

Brachypepluus magnus, 302

Brain of frog, 526

ventricles, 526

Branclial árches, 465

Branchiata, 264

Branchiopoda, order, 264, 283

Branchiostegal membrane, 463

Branchiostegites, 268

Branchiostoma, 361,368

Branclipus, 264

Breeding habits of amphibia, 479

Bremidae, fanily, 338

Breviciptidae, family, 486

"Bristle jaws," 193

Brittle star, 218

Bronchi, 566, 604, 649

Bronchioles, 566, 604

Brontosaurus, 546

Brown, Robert, 49

Brownian movement, 55

Brucophagus funebris, 334

Bryobia plactiosa, 2ys

Bryozoa, 27, 184, 185

Bubo virginianus, 593

Buccal cavity of frog, 500

Buccopharyngeal respiration. 514

Buffalo, 631,632

Buffon, 44

Bufo americanus, 538

marinus, marine toad, 473,478

punctatus, 474

toad, 485

valliceps, 485,487

woodhousii, 485,538

Bufonidae, family, 485

Bugs, 266

Fulgula, 184, 185

Bulimulus dealbatus liquabilis, 239

Bullfrog, 473, 497

Bulliead, internal structure, 458 life history and reproduction, 470 nervous system, 469

skeleton, 466

yellow, 457
Bumblebees, 338

Bunchgill, 321

Buprestidae, family, 324

Bursa, copulatory, 179

Bursaria, 71

Busycon, 248

egg capsules, 247

shell, 771

Butterflies, 266, 325

Butterfly ray, 424, 425

"Buzzard," 588

Byssus, 253

C

Cabbage-head jellyfish, $13 \mathrm{~s}$

Cadtlis flies, 266, 321

Caeca, 603

pyloric, 459

Caecilianella, $2: 37$

Caecilians, 472

Calciferous gland, 204

Calcispongie, class, 120

Calendra mormon, 324

Callinectes, blue crab, 2S2, 772

Callorhinus alascanus, fur seal, (iz)

Callus, 691

Calorie, 3 s's

Calyx, 224

Cambarus, $266^{\circ}$

clarkii, 267

Camels, 630

Campanella, 136

Campanularia, 131

Campeloma decisum, 2t7

Campodeidae, family, 311

Canadian zone, 714

Canidae, family, 628

Canis gigas, timber wolf (ion

latrans, coyote, 628

Canon of Morgall, 848

Cantliaridin, 341

Canthon simplex, 335

Capillaries, 392

Capitophorus potentillae, 326

Capitulum, 195

Caprella, 264

Caprimulgiformes, order, 59:

Carabidae, 324

Carangidae, family, 45t

Carapace, 265, 578

Carbolyydrates, 56

Carbon cycle, 28

Carcharhinus, 439

Carcharias, platydon, 422

Carcharodon carcharias, 422

Carchesium. 72, 75

Cardiac chamber, 272

muscle, 381

plexus, 528

Cartinals, 595

Carlo, 305, 345

Calibous, $6: 30$

Carinatae, 584

Carnivora, order, ti27

Carnivores, aquatic, (62)

Carotid arch. 50. gland, 505

Carp, 450

leather, 419

lice, 284

scale, 449

Carriers, $8: 3$

Carrion bcetles. 324

Carunculina texasensis, 248

Case flies, 321

Cassowaries, 5 s.

Castor canadensis, beaver. 624

Casuiriformes, order, 585 
Cat, 411, 639

circulatory system, 648

digestive system, 646

excretory system, 650

Jexican ringtail, 626

muscles, 642

nervous system. 650

reproduction, 652

respiratory system, 649

Catabolism, 66. SS, $153,278,377$

Catamount, 629

Cataphracti, suborder, 454

Catfish, 411, 450

freshwater. 450

sea, 450

Cathartes aura septentrionales, 5ss

Catostomidae. family, 450

Cats, 629

Cattle, 630

Caudata, order, 484

Caudina, 20t

Cave fishes, 451

Caviar, 447,456

Cavies, 625

Cebidae, family of monkeys, 635

Cecum, 645

Cell division, 61

principle, 49

theory, its influence. 52

Cellular differentiation, 104 organization, 105

Centipedes, 265

Central disc, 217

Centrarchidae, family, fist

Centrolecithal egg. SOs

Centrosome, 59,60

Centrum, amphicoelous, $4(1.5)$

procoelous, 520

Cephalic gauglion, 165

Cephalin, 395

Cephalization, 375

Cephalochoria, subphylum, 361, 26s

Ceplualodiscida, order, 361

Cephalodiscus, 301,364

Cephalopoda, 2.s

Cephaloporls, evolution of. 259

Cerambycidae, 324

Ceratite, 259

Ceratium, 75

Ceratobranchial cartilage. $\$ 24$

Ceratodontidae, family. 4.5

Ceratohyoid bone, 467

Ceratopharys, $4 \pi 2$

Ceratosa, order, 122

Cercaria, $756,758,759$

Cerci, 348

Cercomonas, 66

Cercopithecidat, family of monkeys, 636

Cere, 599

Cerebellum, 406, 469, 607 frog, 526

Cerebral commissure, 252

ganglia, 245

ganglion of Helix, 248

hemispheres, 469

vesicle, 372

Cerebratulus, 163

Cerebropleural ganglion, 252

Cerebrum, 406, 607

Cerianthus, 140

Cervical groove, 268

vertebrae, frog, 520

Cervidae, family of deers. 630

Cervus canadensis, elk. 630

Ceryle alcyon, 593

Cestoda, class, 161, i62

Cestodes, 160, 161. 17t

Cestus veneris, 157

Chaetae, 197
Chaetognatha, class, 27, 184, 193

Chaetopoda, 194

Chagas disease. $7 ?$

Chain reflex, 8.51

Chalcis-fly, 334

Chalones, 667

Chambers, Dr. Robert, (i1

Chaos diffluens. S5

Chaparral bird, 591

Characters, unit, $\$ 23$

Charadriformes, order, 591

Charybulea, 137

Cheek pouches, 624

Cheiloschisis, 65

Chela, 270

Chelicerae, 292

Chelonia, order, 548

Cheloniidae, family, 550

Chelydridae, family, 549

Chemotropism. if

Chenopodium, 1S3

Chernidae, family, $31 \mathrm{~s}$

Chevron bones. 641

Chicken, 590, 597, 59S

circulatory system, 604

digestive system, 601

muscles of hind limb, 61!

muscular system, 612

reproduction and life history, il:?

respiratory system, 603

skeletal system, 609, 610

Chilodon, To

Chilomastix, 67

Chilomonas, $\mathbf{6 6}$

Chilomycterus schoepfli, 443

Chilopoda, order, 265, 285, 290

Chimaera affuis, 425

monstrosa, 425

Chimaeras, 411

Chimpanzee, 637

Chinchillas, 625

Chipmunk, 622

Chiroptera, order, 620

Chitin, 106, 343

Chitinous exoskeleton. 256

Chloragogen cells, 204

Chlorella vulgaris, 114

Chlorochroa sayi, 315

Chlorohydra. 144

Chloropisca glabra, 331

Choanocytes, $119,120,120$

Chondrostei, order, 446

Chorlata, 27. 360

classification, 361

phylogenetic advances, 31:'

Chorioid, 401

layer of exe, 529

plexus, 527

Chorion. $81 \mathrm{~S}$

Chorionic villi. 819

Chromatin. 59, 824

Chromatophore. S:

Chromomeres, 60,63

Chromonema. 63

Chromonemata, 60. (i1

Chromosome map. 835

Chromosomes, 61, 114

Chrysaora. 139

Chrysomelidae, 324

Chrysopa californica, 322

Chrysopirlae, family. 322

Chyme 355, 501

Cicadas, 266,318

Cicadellidae, family, $32 n$

Cicinlelidae, family, 32

Ciconiiformes, order, $55 s$

Cilia, 94. 102

Ciliata. 70 
Cimex lectularius. 318

Cimicidae, family, 318

Cinclides, 142

Circulatory or vascular system, 110

Circulatory system, cat. 648

chicken, 604

open type, 263. 273

phrynosoma. 566

pisces. 459

toad, 542

turtle. 580

Circumesophageal commissures, 274

Circumoral canal, 221, 231

Circumpharyngeal connectives, 208 ring, 176

Cirri, 197, 224

Cirripathes. 143

Cirripedia. 284

order, 265

Cirrus, 179

Clam, circulation, 252

digestive system, 250

excretion. 253

fresh water, 248

nervous system and sense organs, 252

reproduction, 253

respiration. 251

Class Infusoria, 93

Sarcodina. 85

Classification of $\operatorname{man}, 22$

Clavicics, 609

Cleavage, 280, 374, 502

in asterias, 116

in cat, 653

disymmetrical. S04

divisions, 374

earthworm. 211

in frog, 533

holoblastic 802

in mammal. 816.814

neroblastic, 802

spiral. S04

superficial, 803

cleft palate, 656

Click beetles, 324

Cliona. 121

Climax community, 728

clitellum. 199, 2000,209, 214

Clitoris of cat. 653

Cloaca, 613

Clonorchis, 161, 759

sinensis, Chinese liver fluke, 745, 757

Cluwea harcngus, 448

Clupeidae, family, 448

Clypeaster, 222

Clypeus, 345,357

Cnemidophorus, 554

Cnisloblast, 148, 149, 150

Cnirocil, 149, 149

Cnidosporidia, 73

Cobras. $55 \mathrm{~s}$

Coccilia 7 .

Coccinellidae family, 324

Coccyceal vertebrae. 609

Coccyx. 878

Cochineal. 341

Cochiopa texana, 247

Cochlea, 403, 608, 650

Cockroaches, 313

Cocoon, 171, 214 carthworm. 209

Corlfishes, 451

Corthliver oil, 456

Codonosiga, 66,75

Coelenterata, classification. 131 economic relations of 150

phylum, 26, 130

Coelenterates, phylogenetic advances of. 156
Coeliac axis. 606

Coeliacomesenteric artery. 506

Coeloblastula, 807

Coelom. 194, 211

development, 536

Coitus, 409, 814

Colaptes auratus, 594

Coleoptera, order, 265, 322

Coleps, 70

Collar cells, 119

Collared lizard, 55

Collembola, order, 265. 311

Colloid. 53

Colloidal state, 53

Collum, 2ss

Coloboma, 665

Colony formation. $\mathrm{S} 64$

Color blind inheritance. 833

Coloration in amphibia, 474

Colubridae, 557

Columbifornes, order, 591

Columella, 574, 608

Colymbiformes, order, $5 \$$

Comb jellies, 157

Commensalism, 735

Commissure, 199

Conditioned reflex, 852

Conductivity, 54

Condvlarthra, 874

Condylura cristata, star-nosed mole, 619

Conger eels. 449, 473

Congridae, family, 449

Coniferous forest formation, 725

Conjugation, 96, 98

Conocephalus vicinus, 310

Consciousmess, 54

Conservation of wildlife, 784

Continuity of germ plasm, 45

Contractile vacuole, S8, 94

Contractility, 54, 381

Conus arteriosus, 490. 510

Coon, 629

Cooties, 266

Coots. 591

Cope. E. D.. 48

Copeport. 284

Copepoda, order. 265

Copperheads, 556,550

Copulation, 613, 653, 814

earthworm. 203

Copulatory bursa. 179

Coracifformes, order. 594

Coracoid bar. 430

bone, 465, 195, 521, 609

Coral, 140. 143

snake, 55 s

Corallium rubrum, 143

Coregonidae, family, 448

Coreidae, family, 318

Corizidae, family, 318

Colmorants, 557

Corn-ear worm. 326

Cornea, 276, 301, 401, 528, 607

Corneagen cells, 276, 277

Corona radiata, 812

Corporin, 676

Corpus albicans, 813

luteum. 812

Corrodentia, order, 266, 333

Corti, organ of. 650

Cortin, 671

Corythucha distincta. 318

Cosmic rays, 700

Cotton boll weevil. 324

Cotylophoron. 762

Cougar. 629

Cow. 630

Cowper's glands, 653 
Coxa, 293, 306, 347

Coxopodite, 270

Corote, 628

Crabs, 264, 265, 772

Cranial crests, toad. 540 nerves, 527

of bullhead, 469

(ranium, 380

of fish. 46.5

of frog. 518

Craspedacusta. 131

Crawfishes. 267

Crayfish. 264, 265. 266

appendages, 269. 270

development, 279,280

rigestive system, 272

excletory system, 274

habitat and behavior, 267

metabolism. 27.5

regeneration. 281

reproduction, $27 \mathrm{~S}$

respiratory system. 271

vascular system 273

Creatinin, 381, 464

Cremastocheilus angularis. 340

creosote buch-kangaroo rat biome, 727

Crepidula. 808

Cretinism, 655, 668

Cricket frog acris, 485

MIormon. 312

Crickets, 265

Crinoid, 225

Crinoiriea, class, 224

Cristatella, 186

Croaker. 453

Crocoriles, 411, 548, 560

Crocodilia, order, 548,560

Crocodilus acutus, 561

Crop, 601, 602

('rossing over, S34

Crosscpterygii, order. 446

Crotaliclae, family, $5 \grave{5} 9$

Crotalus. 559

Crotaplivtus collaris, 552

Crows, 595

rrura cerebri, 526

Cuustacea, class, 264, 266

Cryptobranchidae, family. $4 S t$

rryptobranchoiclea. suborder, 484

Cryptobranchus, $473,48 t$

Cryptodira, suborder, 547, 54s

Criptotis parva, short-tailed shrew, 619

r..nturifores, order, 5\$6

Crystalline cone, 276 style. 251

Cteni, 444

Ctemoid scale 444,464

Ctenonhora, nhylum, 26, 15\%

Cub shark, 422

Cubomedusae. 137

Cuckoos, 591

Cuculiformes order, 591

Cucumaria. 224

Culicidae, family, 330

Cumulis, 81 ?

Cuneiform, 611

Curculionidae. 324

Curlews, 591

Cutaneous artery of frog, ans

Cuticle, 81, 201

nouchitinous, 194

Cuticula, 308

Cybilitae, family, 453

Cicloid scale, 444

Cyclophylliriea, order. 161

Cyclopia, 665

Cyclops, 2fit, 265. 281, 29:

Ciclosis, 95

Cyclospondyll, order, 422
Cyclostomata, class, 410,412

economic relations, 413

Cynocephalus, a baboon, 636

Cynoglossirlae, family, 452

Cynomys ludovicianus, prairie dog, 622. 623

Cynthia, 361

Cyprinidae, family, 450

Cyprinodontes, 451

Cyprinus carpio, 449

Cypris, 264

Cyrtoceracone, 259

Cysticerous, 762 . 764

Cytology, 21

Cytopharynx, 81

Cytoplasm, 60

Cytosome, s6

Dace, 450

Dactylozooids, 135, 137

Daddy longlegs, 266. 299

Damsel flies, 26.5, 321

Danaidae, family, $32 \%$

Danaüs menippe, 327

Daphnia, 264, 2S1, 253

Dart sac, 246

Darters, 454

Darwin and Evolution, s5.

Charles, 44

Dasyatis, 456

americanus, 423

Dasypus novemcinctum, a rmadillo, $6: 21$

Daughter cells, 62,64

Decapoda, order, 265,282

Deciduous forest formation. 726

Declaration of indefensibles, 792

Dedifferentiation. 69.

Deduction, 18

Deer, 630,786

Delamination, 810

Dementia praecox, 839

Demospongiae, class, 121

Dendrobates, 480

Dendrocoelum lacteum. 165

Dengue fever, 80

Dental formula, 643

Dentalium, 257. 258

Dentary bone, 495, 520

Dentine $6+3$

Dermal branchiae, 226, 220, 233

Dermaptera, orler, 26.5. 220

Dermochelidac, family, 550

Desert formation. 727

Desmognathus, 4@4

Desor's larva, 10is

Development, arrosterl. (6.)1

of nervous system. 530

of sexual reproduction, 111

of starfish. 232, 234

Developmental staces of sereral (1mbryos. SSO, SS1

ie Vries, Hugo, 49, SST

nextral, 240

Dextrocalritia, 664

Diabetes. 67t

insipiclus, 674

Diaphragm, 109

Diaphragmatic hernia, 0.5

Diaponhrsis, $5+3$

Diapsirla, subrlass, $54 \mathrm{~s}$

Diantomus. 283

Diastase, ist

Diastole 396

Dibranchiata, 260

Ticamptorion encatus, 494

Dicrocoellum. 762

Didelnhta, fi1 7

Dirielphis virginiana, 619 
Didinium, 70

Diencephalon, 419,469 of frog, 526

Differential birth rate, $\$ 39$

Differentiation, 801

intercellular, $10 t$

Difflugia, 67

Digenea, 161

Digestion in frog, 501

intracellular. 130

Digestive enzymes and their functions, 357

system, 109

cat. 646

chicken, 601

toad, 541

tract, solitary wasp, 358

Digitigrade gait. 641

Dihybrid cross, 826

Dinocardium, shell, 771

Dinosaurs, 869

Dioctophyme, 176

Dioctophymoidea, order, 176

Diodontidae, 455

Dioecious, 112

Diphyllobothrium, 161

latum, fish tape, 741,742 tape, 765

Diplasiocoela, suborder, 486

Diploblastic condition, 130 form, 120

Diploid condition, $\$ 00$ number, 114

Diplopoda, 265, 2S8, 290

Dipnoi, subclass, 455

Dipsomania, $\$ 39$

Diptera, order, 266,329

Dipyliclium caninum, dog tape, 763 . 76.5

Discoblastula, 806

Discocephali. suborder. 4.54

Discomedusae, 137

Dispersal in distribution. 717

Dissosteira spurcata, $34 t$

Distribution of animals, 711

Diverticula, 167

Diverticulae. 213

Dobson flies, 322

Dog, (i2S

Dosfish, freshwater, 447

shark. 422

Dolichoglosils, nervous system, 363

kowalevskii, 362

Doliolum, 361

Dolphin, fist

Dominance, principle of, $\$ 23$

Dominant. 45

Doodlebugs, 322

Dorosomidae, family, 448

Dorsal a orta. 605

Dosimeter, 706

Dourine. $\mathrm{SO}$

Doves, $\bar{\jmath} 91$ mourning, 591

Dracunculus, Guinea worm. T4.

Dragonflies, 265, 319. 321

Drassidae, family, 29.5

Drassids, 29.5

Drassus neglectus, 296

Drill, 630

Drosophila melanogaster, 82S

Drumfiches, 454

Dryobates pubescens, 59.

Duck mole, 617

Duckbill, 617

Ducks, 589, 788

Duct of Botallus, 491

of Cuvier. $4 \frac{1}{2} 5$

Dujarlin, 49

Duorlenum, 382
Dura mater 527,650

Dussumuridiae, family, $44 S$

Dwarfism, 673

Dysgenic. $\$ 39$

Eagles, 5S9

bald, 590

golden. 590

Ear structure, $40^{2}$

Eardrum, 498

Earthworm, 199

circulatory system, 205

development, 210

internal anatomy, 201

Earwigs, 265

Echrsis, 263

Echeneidae, family, 454

Echinarachnius, 222

Echinaster, 218

Echinococcus, 756 log tape, 745

Echinoderm theory of evolution, 868

Echinodermata, 26

Echinoderms, larval relations, 866

Echinoidea, class, 219

Echiuroidea, order, 194, 214

Ecology, 24, 719

Economic importance of entomology, 340 relations of birds, 596 of mammals, 637

Ectethmoid, 466

Ectoderm, 117, 130

of frog. 535

Ectoparasite, 737

Ectopistes, 786

migratorius, 591

Ectoprocta, 181

Fetopterygoid, 467

Ectosarc, 81. 86

Edentata, order, 621

Eel. 443

Eels, 419

conger, 449

moley, 450

Eelwworm, 175, 180

Ero, centrolecithal, s03 guides. 349 of hen. 615

"Egg tooth," 615

Egret, 5ss

Egretta candidissma. 58

Elapirlae, family, 55

Elasmobranchii, class, 111,422

conomic relations. 425

Elateridae, family, 324

Flectrons, 697

Electrotropism, 77

Flephantiasis. 745,746

Flephants, 634

Elephas indicus, elephant, 634

Fientherodactylus latrans. 479 ricordii, 472

Elks, 630

Elops saurus, $44 \pi$

Elytra, 320, 3ist

Embilina, order, 26i5, 333

Embids. 265

Embryology, 23

comparative, 79 of frog. 539

later stages, 537 of toad, 544

Fmbryonic membrancs, S17

Fmposea filamenta, 317, 320

Fmulsoil. 53

Fmus, 5.5.

Enyrlidae, family of turtles, 549, 5it

Emis blandingii, 549 
Enamel, 643

Encephalocoele, 661

Endamoeba, 747

coli. $7 \mathrm{~s}$

gingivalis, is

histolytica, $67,7 \mathrm{~S}$

Endocrine glands, 666 summary, $6 \bar{\top} \bar{S}$ system. 110

Endoderm, 117, 130, 155 of frog, $\mathbf{5 3 5}$

Endolimax nana, $7 \mathrm{~s}$

Endolymph, 403, 50:

Endomixis, 97

Endoparasite, 737

Encioplasm, 81

Endopodite, 269, 270

Endoprocta, 184

Endosarc, \&1, 86

Endoskeleton. 3S0, 517

Endostyle, 366

Engranlidae, family, 448

Ensiform process, 641

Enterobius vernicularis, 176

Enterocoele formation, 811

Enterokinase, 385

Enteronomas, 67

Entomobryidae, family, 312

Entomostraca, subclass, 32, 264, 293

Entosphenus, 413

tridentatus, 415

Entozoic animals, 66

Enzymes, 56, 383

Eohippus, ST

Ephemerella grandis, 321

Ephemerida, order, 265, 321

Ephyclatia, 121

Ephyra, 139, 140

Epibolic gastrulation, 809

Epibranchial cartilage, 428

Epicauta puncticollis, 323

Epicoracoin cartilage, 521

Epicranium, 343

Epirtermis, 499

Fpididymis, 43S, 572, 653

Fpigrnum, 293

Epinephrine, 671

Epiotic, $46 . \overline{5}$

Epipharynx, 357

Epiphragma, $23 \mathrm{~S}$

Epiphysis, 419,469

Epipoclite. 269, 270

Episternum of frog, 521

Epistropheus, 609

Fpistylis, 72, 75

Epitheliomuscular cell, 148. 150

Eptesicus fuscus, brown bat, 620

Equatorial plate, 62,63

Equus caballus, horse, s7t

Fromobates, 298

Erethizon dorsatus, porcupine, 625

Eriophyes pyri. 298

Erythucytes, $10 \mathrm{~s}, 395$ of flos. 503

Erythroneura comes, 320 ziczac. 317

Escocidae, 450

Esophagus, 203

Esox, 451

Estrogenic hormone, 670

Ftheostomiclae, family, 454

Ethiopian region, 712

Ethmoil bone, 465

Euarctos americanus, brown bear, 629

Fubranchipus, 283

Euconulus chersinus trochulus, 240

Fucosmidae family, 328

Eudorina. 7.5

Eugenic grouns S.11

measures, 844
Eugenics, $\$ 21$

Euglandina singleyana, 240

Euglena, 67, S1

behavior. $S$

reproduction, $\$ 3$

respiration, $\$ 3$

Euglenoid movement. 84

Eulamellibranchiata, 256

Euophrys monadnock, 296

Eupagurus, regeneration, 695

Euplectella, 120

Euplotes, 71

Eupomatus larva, 237

Eurypelma steindachneri, 297

Euselachii, order, 422

Euspongia, 122

Eusuchia, suborder, 519, 560

Eustachian tube, 608 frog, 530

Eutheria, subclass of mamnalia, 617

Eutherodactylus, 4S5

Eventognathi, suborder, 450

Fiolution, annelicl theory, S67

arachnid theory, $\mathrm{S} 67$

distributional evidence. Sif

echinoderm theory, $86 \varsigma$

embryological evidence. 879

geological evidence, 873

morphological evidence, $S T$

phrsiological evidence, 893

theory, S63

Excitor hormones. 667

Fxcretion, starfish, 233

Excretory organs, cat, 650

chicken, 606

fish, 464

frog 515

or urinary system, 110

Exoccipital bones, $46.5,641$

Exocoetidae, family, 451

Fxogyra. 854

Fxoporlite, 269, 270

Exumbrella. 182

Eye of chicken, 007

of fros. $52 \mathrm{~s}$

worm, 176

Eyeball, 401

Eyespot, 233

Facets, 345

Facial nerve, 527

Fairy shrimps, 2S:

Falconiformes, order, 5s9

Falcons, 589

Fallopian tubes, 652

Fanily elopidae, 447

size, $\$ 41$

in dysgenic groups, S42

Fances, 648

Fascia, 612

Fasciola. 161

liepation liver fluke, $74.7,759,759,760$

Fascioloicles, 759

Fasciolopsis, 760

Fat bolies of frog, 532

Feathers, 5\$2, 600

Feeblemindedness, s3s

Feliriac, family, 629

Felis cougre, mountain lion, 609

demestica, house eat, 639

hernandesii, jaguar, 629

partalis, ocelot, 629

Femoril vein. frog, 509

Fenuur, $806,: 347$

of from, $52 \mathrm{mo}$

Fenestra ovalis, 574

Ferrisia, 240

excentrica, 238 
Fertilization, 614 in bullfrog. 531

membrane. 533

Fiber zibethicus, muskrat. 625

Fibrogen, 393, 395

Filaria, 175, 176, 745

Filaroidea, order, 176

Filefishes, 455

Filibranchiata, 256

Filiform papiliae, 603

Filoplume. 600

Filum terminale, 651

Firmisternal girdle, 543

Fish, bony, 442

excretory organs, 464

flying, 442

lice, 284

moth, 310

respiratory system, 463

skeletal system. $46 . t$

typical bony, 457

Fisher, 638

Fisheries and zoology, 34

Fission 111

Fissipedia, suborder, $62 \mathrm{~s}$

Flagellum, S1, 82, 126

Flame celis, 160,167, 189, 190, 397

Flat-fishes, 451

Flatworms, 26

Fleas, 266, 329

Fleming, 62

Flicker. 59

Flies, 266. 329

Flounders, 451

Fluke, human blood. 755

Flukes, 160

Fly, big-headed. 332

Flying fishes 451

Folliculin. 676

Folsomides decemoculatus, 312

Foot-gland, 190

Foramen magnum, 519

ovale, 664

Foramina, 527

Foraminifera, 65, 78

Formicidae, family. 339

Fossil. 854

reptiles, 546

Fosslls, distribution. 856

Fourth ventricle, 435. 469

Fox. 628

Frog, appendicular skeleton, 520

blastula and gastrula, 533, 534

blood. 503

brain. 526

circulatory sistem, 502

cleavage, 533

common tree $\mathbf{4 7}$

digestive system, 499

embryology, 532

excretorv system. 515

feeding habits 477

fertilization. 531

hairy. 476

reart. 510

heart beat. 512

internal ear. 529

later development, 537

muscular system, 523

nervous svitem. 525

pelvic girdle. 521

reproductive organs, 531

respiratorv syctem, 514

sence organs, $52 \mathrm{~s}$

skeletal system. 517

skin. 475

skuli. 517

spinal cord. 527

Strecker's, 482

awamp tree. 473

Texan cliff. 470

\section{Frog-Cont'd}

ts ce. tou

vascular system, 803

veins. 508

verteblal column, 520

visceral skeleton, 519

Frogs, 411, 472

Frons. 343

Frontal bones. 465

Frontonia, 70, 71

Frontoparietal bone, 517

Fruit flies, 330

Fulica americana. 591

Fungiform papillae, $6 \overline{50}$

Funiculus, 185, 186

Furs, 637

G

Gadidae, family, 451

Galea, 305, 315

Galeichthys felis, 450

Galen, 21, 37

Galeocerdo arcticus. 422

Galliformes, order, 590

Gallinules, 591

Gallus domestica. 598

Galvanotropism, 77

Ciambusia, 451

Game, big, 794

Gametes, 111

of hydra. 154

Gametocytes. 7t. 749

Gametogenesis, 113, 799

Gammarus, 32, 264, 283

Ganglia, 399

Ganoid scale, 444

Ganoin 144

Gapes in chickens, $\mathbf{1 7} 6$

Garfishes. 451

Garpike, 447

Gasterosteidae, family, 451

Gasterostomata. order, 161

Gastric rigestion, 385

mill, 272

Gastrocnemius. 523

Gastrocoele. 805

Gastrocopta armifera, 240

Gastrophilus, holse bot. 747

Gastropora. 257

Fastrotheca, 480

Gastrotricha 188

Gastrovascular cavity, 130, 148, 150, 158. 167

Gastrozoids, 135. 137

Gastrula, 117. 15.5

revelopment. 80is

eirthworm, 210

of froc. 533.534

starfich. 234

Gastrulation. S05. SOS

earthworm, 211

in mammal. $\$ 15$

ravifformes, order, 586

Сrizelles. 630

Cohia stellata, 239

"Gerko." 5.51

reese. 599

Fricer. S. W.. 4S

Gekknnirlae family, 551

Fel state. 53

Crommules, 121

Genze, 343

Genes, 61. \$24

comn'smentary, $\$ 29$

multinle. sec

sinnlemeritary. $\$ 30$

Genetics. 23

and eugenles, 821

feniohvold muonle, 469

Cronital ninte, 221

rachls, 221 
Genotype, 826

Geobios, 713

Geographical distribution, 711

Geologic t.me scale, 872

Geomyidae, famlly of gophers, 621

Geomys bursarius, pocket gopher, 623

Geotropism. 77

Gephyrea, 194. 214

Germ cells, 104

layers, 117

fate of. 118

plasm. 101

continuity of. 48

Gerrhonotus, 552

Gerridae, family, 318

Gestation, 653

Giant water bugs, 318

Giardia, 67

lamblia, $\mathbf{7 4 2}$

Gibbons, 637

Gigantism, 655

Gill plate, 197

rakers, 465

Giraffes, 630

Gizzard, 203, 204, 601 shads, 448

Glands, ductless, 666 of internal secretion, 666

"Glass snake." 552

Glenoid fossa. 495. 521

Globigerina, 69,78

Glochidium, 253, 254

Glomerulus, 398, 650

Glossa, 357

Glossary, 905

Glossina, 74

palpalis, 79

Glossiphonia, 214

Glossopharyngeal nerve, 527

Glottis, 492, 564, 603

Glucose, 56

Glycogen, 5f, 393, 387

Glycogenase, $3 \mathrm{sS}$

Gnawers, 621

Goats, 630

Rocky Mountain, 631

Goatsuckers, 593

Gobies, 454

Gobiidae, family, 454

Grobioidea, suborder, 454

Goiter. $66 \mathrm{~s}$

Golden eagle, 590

fonadotropic hormone, 676

Gonads, 104

Gonangium. 134

Goniatite, 259

Goniobasis, 245, $25 \mathrm{~s}$

comalensis, 247

fonionemus. 131, 132

Gonotheca, 133

Gonozooids, 137

fooseberry fruitworm, 327

fophers, pocket, 621, 623

Gorrliacea, rlass, 175, 177

Gordius, 17\%, 178

Gorgonacea. order, 143

Gorgonia, 143

Gorronocenhalus, 219

Gorilla. 637

gorrilia, $63 \pi$

Graaftan follicles, 652,812

Gracilis. 644

muscle of frog, 523

Grackles, 595

Grantig. 121. 122

Granulocytes, 513

Grass-Bison Biome 726

Grasshopper, circulatory system, 349

Grasshoppers, 265, 343

Grassland formation, 726

Grayllngs, 448
Grebes, 586

Green glands, 110, 2S6

Gregaloid colony, 76

Gregarina, $\div 2$

Gregarinida, 73

Grew, 50

Ground beetles, 324

hog, 622

squirrels, 622

Growth, 5 t

Gruiformes, order, 591

Grynophilus porphyriticus, 454

Guanin, 474

Guano, 597

Guanophores, 471

Guinea pigs, 620, 625

worm, 175,176

Gular fold, 5 ti2

Gullet, 140

Gulls, 591

Gurnards, $45 \frac{4}{4}$

Grnecophoric canal, 755

Gyroceracone, 259

$\mathrm{H}$

Habit. $\$ 52$

Habitual behavior, 8.52

Hadurus hirsutus, $29 \mathrm{~S}$

Haeckel, 43, 2S

Haemal arches. $46 \mathrm{~s}$

Haemoglobin, 196

Haemosporidia, 73

Hagfishes, 412

Hags, 410

Haliaeetus leucocephalus, 590

Halibut, 451

Haliclona, 121

Haliclystus, 137

Haliver oil. 456

Halobios, 766

Hamster, golien, 6:5

Haploid number, 115

Haplomai, suborder, 450

Haplosporidia. 73

Harelip, $639,6.56$

Hares, 625

Harvestmen, 266, -99

Harrey, WVilliam, 40, 799

Hawk, Cooper's, 5⿺9

Hawlks, $5 \$ 9,590$

Heart anomalies, 664

Heart beat. branchial, 261

of chicken, 607

of frog. $5 \mathrm{io}, 512$

systemic. 261

Hectocotylizer arm, 261

Helicina Orbiculata, 25 tropica, 240

Heliothis obsoleta, $321 ;$

Heliozoa, 6?

Helisoma, 244

trivolvis lentum, $23 \mathrm{~s}$

Helix, 237

anatomy of. 243

circulation, $24 \cdot t$

excretory olrans, 24.

genitalia, 246

nervous system, 245

reproduction and life history, 24.

respiration, 244

Hellbender, ist

Hell-river, 5 S6

Heloderma suspectum, 5.53

Helorlermaticlae, family, 5.53

Hemichordata, subphylum. 361, 362

Hemiclactrlium scutatum. th4

Hemimetabolous, 308

Hemipenes,, 12

Hemiptera, order, 266, 318

Hemocyanin, 24t, 394 
Hemoglobin, 57, 205, 375, 513

Hemolymph, 394

Hemophilia, 839

Hemophiliacs, 395

Hepatic caeca, 229

portal vein, 434, 509

Heredity, S21

physical basis, $82 t$

Hermaphrodite, 154

Hermaphroditic eondition. 112

Hermaphroditism, 40s, 663

Hermit crab, regeneration, 655

Herodias egretta, כSS

Heron, 5ss

Herring, 447, 448

Herrings, round, 448

Hertzion waves, T01

Hetaerina americana, 321 .

Hetaerius tristriatus, 310

Heterocera, suborder, 325

Heterocercal tail. $426^{\circ}$

Heterocoela, order, 121

Heterocoelous vertebrae, fio9

Heterodont condition, 64 :

Heteronomous condition, 26.3

Heterophyllices, order, 161

Heterosonata, suborler, 4.j

Heterotrichida. 71

Heterozygous individual, 825

Hexylresorcinol, 183

Hibernation, 476

in amphibia, 450

Hiodon tergisus, 448

Hiodontidae. 4.17

Hippiscus, 315

corallipes, 310

Hippoboscidae, 330

Hippoclates, 36

Hipporlossidae, family, $4: 2$

Hippopotamuses, 630

Hirudinea, 191, 212

Hirudo, 195. 212

Histogenesis, 801

Histology, 21

History of zoology, 36

Hogs, 632

Holoblastic cleavage, S02

Holocephali, subclass, 425

Holometabolous, $30 \mathrm{~S}$

Holostei, order 417

Holothuria, 224

Holothuroidea, class, 222

Holotrichicla, 70

Holozoic animals, 66

Homo sapiens, 22

Homocercal tail. 413

Homocoela, order, 120

Homoiothermal conution. (j16

Homology, 271, 663 serial, 271

Homonomous condition, 263

Homoptera, order, 266, 316, $31 \mathrm{~s}$

Homozygous inclividual 895

Honey bee. 357

Honeydew, 340

Hoofed animals, 629

Hooke, Robt. 49

Hormones, 666

of non-chordates, 607

plant growth, 667

sex, 675

Hornel lizard. 561

arteries, 568

internal structure, $560 \mathrm{i}$

reproduction, 571

urinogenital system, 5it

veins, 570

Horned "toad," 561

Horntails, pigeon, 331
Horse, 633

Equus caballus, 876

its rise, sit

phylogenetic development, sij

Horsefly, green-headed. 331

Horsehair snake, 175, 17

Horseshoe crab, 266

Host specificity, 741

Housefly, life history, 330

Hudsonian zone, 714

Human blood cells. 396 heredity, $\$ 36$

Humboldtiana, 242

chisosensis, 239

Hummingbirds, 593

Huxley, Thomas H., 18, 47

Hyaline cartilage, 107

Hyaloplasm, 60

Hyalospongiae, class, 121

Hydatina, life cycle, 191

Hydla, 1:1, 144

life cycle, 153

locomotion, 145

metabolism, 151

regeneration, 156

viridissima, 144,146

Hydranths, 132

Hydrobios, 713

Hydrocaulus, 132

Hydrocephalus, 604

Hydrocorallina, 136

Hydrolysis, 381

Hydromedusae. 135

Hydrophidae, family, 559

Hydropsyche, partita, 322

Hydrorhiza, 132

Hyrrostatic organ, 464

Hydrotheca, 133

Hydrozoa, class, 131

Hyla, $486^{\circ}$

cinerea, $4 \overline{7} 4$

crucifer, tree frog, $4 \mathrm{~S} 3$

Hylobates, gibbons, 637

Hyman, Libbie H., 14

Hymenolepis, 161

nana, 765

Hymenoptera, order, 266, 331, 357

Hyoid arch, 46j

Hypaxial portion of borly. 496

Hyperbranchial groove. 373

Hyperoartii, 412

Hyperotreti, 412

Hyperthyroiclism, 669

Hypertrophy, 691

Hrpnotoxin, 149

Hypobranchial groove, 372

Hypodermis, 308

Hrpopharynx, 305

Hypophysis, 469

frog, 526

Hrpolachis, 601

Hrpostome, 134, 147

Hypothesis, is

Hypotrichida, 71

Irlothyomyzon, 413

Ichthyophis glutinosus, the caecilian, 473

Icius similis, ?ari

Icieal continent. 722

Idotea, 2:?

Iguanidae, family, 551

Ileocaecal valve, 565

Ileum, 499, 564

Iliac arteries of frog. 507

Ilium, 611

of frog. 521 
Imago, 309

Implantation, 815

Incus, 403

Indefensibles, 792

Infrared, 699

Infundibulum. 15S

Infusoria. 71

class, $T 0$

Ingestion in amoeba, $\$ 7$

in hydra, 151

Ingression, polar, $\$ 10$

Inguinal canal, 652, 653

Iniomi, suborler 450

Inner cell mass. $\$ 16$

Innominate vein of frog, .00s

Inseet characteristics, 301

Inseeta, class, 265, 300

Insectivora, order, 619

Insects, economic relations, 340 henimetabolous, 320

holometabolus, 321

nerve winged, 322

representative, 343

shellac scale, 341

social life, 334

Inspiration, 648

Instinct, 846,852

Insulin, 677

Integument, $37 \pi$

Integumentary system, 10s

Interambulacral plates, 219,220

Interauricular septum. 5]0

Intercalary disks, 107

Intercellular digestion, $1 \overline{5} 1$

Intercostal muscles, 5SI

Intermedin, 67s

Internal carotid artery, 43:3

Interradial pouches, 222

Interstitial cell, 149 , I.50

Intervertebral discs, 64:

Interzonal fibers, 62

Intracellular digestion, 119

Invertase, 386

Iris, 401

of frog, 529

Irritability, 54

Ischiopubis, 430

Ischium of frog 521

Ischnochiton, 257 conspieuus, 256

Islands of Langerhans, 110, (iit

Isopoda, order, 26.

Isoptera, order, 265, 320

Isospondyli, suborder, 447

Istiophoridae, family, $45 \%$

Itch mite, 739

Ixodilae, family, 298

\section{$\mathbf{J}$}

Jacalla spinosa gymnostoma, 591 Jaguar, 629

Japanese beetle. 31

Japyx lubbirrili, 311

Javelina, 632

Jays, 59.5

Jellyfishes, 20i

Joint snnkis.

Jugular veins of frog, $50 \mathrm{~S}$

Jugulares, suborder, 45.)

Julidae, fomily, 24!

Julus, 289

Jumping spirlers, 260

June beetle. 324

bug. 2.54

life history, 3inf

Jungle fowl, 597
Kangaroo, 6Is

rat, 621

Faryolymph, 61

Karyoplasm, 6I

Ḱaryosomes, 61

Katydids, 315

Felatosis, $\$ 33$

Kerona. 7 I

Kidneys, $39 \mathrm{~S}$

of cat, 6.50

Killdeers, 590, 591

Killifishes, 441

ling crab, 260

hingbirds, 595

kingfisher, 593, 594

Kinosternidae, family, 515

Kissing bug, 79

Iiwi. 582, 585

I.

Labia minor, 320

Labial palps, 35

Labium. 315

La Brea asphalt pits, $\$ 61$

Labrum 345, 3.5

Lacertilia, suborder, 547, 551

Lacewings, 322

Laeinia, 345,305

Lacrimal bone, 467, 643

Lacrymaria, 71

Lactase, 386

Licteals, 513

Lactogenic hormone, 6is

Ladybird bectles, 324

Lady fishes, 44 s

Lagena, 437, 608

Lagomorpha, orler, (;2:-

Lamalek, 43

Lampres, 413

larva, 420

life history, 421

Lampreys, 410

Lampsilis, 248

anodontoides, 249, 254

Langerhans, islanis of, (7T)

Lillia, hexacanth. Tit

Larvacea, 361

Larrngeal chamber,. (6-

Lasius, 340

Lateral line system, 470

Latrolectus, 293

mactans, 295

Laveran and Manson, 7!)

Leaf hoppers, 320

roller, strawbejry, 328

Leber's atrophy, \$32

l. rouwenhock. 49

I.emuroidea, suborder. 63.5

Ienvurs, (iB:

Lens, 401

l.eopard, 629

Iepas, $20 \%$

Jepidoptera, order, 266,325

Lepiclosiphes becki, 320

Lepidosiren pararloxa. 4.

Lepidosireniriae, family, ..,.

Lepidurus, 264

Lepisma saccharina, 310

I,pismitlac, family 310

Iepisosteidae fanily, 117

Leplorlactylicie, fanily, 44.

Leptinotarsa deeimlinenta, ?23

Leptocoris trivittatus, $31 \mathrm{~s}$

Leptodea fragilis, $24 \mathrm{~s}$

Leptolora, 264

Lepton squamosum, 239

Leptosrnanta. 224

Leptotymlopirlae, family, 557 
Lepus, 626

Lestes uncatus, 321

Leucocytes, 393

of frog, 503, 513

Leucosolenia, 120

Levatol muscle, 524

Leydig, duct of, 493

Libinia, spider crab, 772

Lice, 266, 333

Life, attributes of, 30

history, ameiurus, 470

housefly, 330

lamprey, 421

monarch butterfly, 328

mosquito, 329

zones, 713,714

Ligula, 305

Ligulae, 345

Limacina australis, 241

Limulus polyphemus, 299

Iineus socialis, 162

Linin, 59 net, 61

Linkage, 832

Linneas, 42

Lion, 629

Lionotus, 71

Liopelmidae, family, 485

Lipase, 384

Lipophores. 474

Liriope, 136

Litanseutria obscura, 318

Littoral zone. 713,769

Liver, function, 386

Lizard, alligator, 552 collared. 552 fishes, 450

horned, 552

Lizards, 411, 551

nicht, 554

sand, 554

worm, 555

Tlamas, 630

"Lobster claw," 659, 837

Lobsters, 265

Locust, 343

Locustidae, family, 315

Loligo, 258

brevipennis, 257,260

Long-horned wood beetles. 324

Loons, $5 \mathrm{~S} 6$

Lophiclae, family, 455

Lophophore, 185, 186, 188

Lovebirds, 591

Lower jaw of frog, 520

Loxodonta africana, elephant, 634

Lubber grasshopper, western, 302

Lucernaria 137

Lucunae, 107

Lumbricus, 195

terrestris, 199

Lungfishes, 455

Iutein, 676

Tutianidae, family, $\mathbf{4 5 4}$

Lutianus campechanus, 454

Lycosa, 295

Lycosidae, family, 295

Lygaeidae, family, 318

Lygus pratensis. 315

Lymnaea, 242

bulimoirles, 256 techella, 238

palustris, 237

stagnalis, 238

Iymph, 108

Lymphatic system, 502, 513

Jymphocytes, 513

Lynx, 629

Lyre gṇake, 556
Macaca sylvana, 630

Macaque monkey, 630

Macaws, 591

Machilidae, family, 310

Machilis orbitalis, 310

Mackerels, 453

Spanish, 452

Macrocephalus, 664

Macrogametes, 74, 112

Macromeres, 534

Macroperipatis, 2ss

Madrepora, 142

Madreporaria, order, 142

Madreporic plate, 218

Madreporite, 217, 220, 221, 223, 226, 22, 230,231

Magellania, $18 \mathrm{~S}$

Maggots, 330

Malacostraca, subclass, 205

Malaria, $74,79,748$

Malformations, 6.54

Malleus, 403

Mallophaga, order, 266, 333

Malpighi, 40,49

Malpighian bodies, 606

colpuscles, 39S, 492

tubules, $110,286,352$

Malthus' law, $8 \$ 5$

Mammalia, 411

class, 616

classification, 616

economic relations, 637

Mammalian development, S12

Mammary gland, 653

glands, 639

Mammogenic hormone, $67 \mathrm{~s}$

Man, $6: 35$

Manatee, 634

Mandrill, 636

Man-eater shark, 422

Manikin, human, 109

Mantidae, family, $31 \mathrm{~s}$

Mantispids, 322

Mantle, 365

cavity, 250

Manubrium, 131, 138

Manus, 522

Marine Biological Laboratory, 43 zoology, 766

Marmosets, 635

Marmota monax, woochchuck, fi:?

Marsupia, $2 \pi 3$

Marsupial frogs, 480

pouch, 61S, 619

Marsupialia, order, 619

Marsupials, 617

Martins, 505

Mastax, 159

Mastigamneba, 66

Mastigophora, 66 class, $\mathrm{S1}$

Miastodonsaurus, 473

Mating among defectives, $\$ 39$

Iaturation of germ cells, 113

Naxillae, 270 . 357

Maxillipers, 269, 270

May beetles, 354

Mayflies, 265, 319,321

Meantes, suborder, 4S5

Meckel's cartilage, 520

Mecoptera, order, 266,333

Mediastinum 6.5

Medulla oblongata, 435, 697 of fish, 469 of frog 526

Medusae, $113,132,133,134$

Megalopidae, family, 447 
Megalops, 285

Melanerpes erythrocephalus, 595

Melanophores, 474

Melanoplus atlanis, 343 femur-rubrum, 310

Melophagus ovinus, 330

Membranous labyrinth, 403, 530, 574

Itemory, associative, 852

ilendel, Gregor J., 45̃, \$21

Mendel's law, $\$ 21$

Menetus dilatatus, 238

I $\in$ ninges, 650

Menoform 676

Nentomeckelian bone, 520

Mentum, 305, 345

Mergansers, $5 \$ 9$

Mermiria bivittata, 310

Ieroblastic eggs, 546

Nerozoites, $7 \pm$

Mervchippus, $\delta \bar{t} t$

Merzoites, 749

Mesenchyme, 165

Mesethmioid, 466, 643

Miesoblast ceIl, 210, 211

Mesoderm, 117, 809

of frog. 536

parietal, $\$ 19$

splanchinic. 815

Mesoglea, 132. 150,151

Mesohippus, 874

Mesonephric tube, 464

Mesopterygium, 430

Mesorchium, 492, 572

Mesothelium, 201

Mesothorax, 305, 347, 357

Mesotubarium, 492

Nesovaria, $\$ 12$

Mesovarium, 438, 573

Mesozoa, 120

Tetabolism, 2S, 54, 377

Metacarpals, 522

Metacercalia, 759

Metacrinus, 205

Metagenesis, 113, 135

Netamere, 211

Metameles, 194, 361

Metamerisn, 375

Metamorphosis, 308, $\$ 01$ in aciclia, 367

starfish, 230

Metanephros, 397, 545

Metaphase, 62,63

Metapleural folds. 370

Netapterygium, 430

Metargiope trifasciata, 296

Metatarsal bones of frog. 522 tubercles, toad, 540

Netatheria, 619

Metathorax, 305, 347, 357

Metazoa characteristics, 103

Metazoan organization, 10

Metridium, 1.40, 141

iicrocephalus, 664

Microgametes, $7 t$

Microhyla, 486

Micromeres, 534

Micronuclei, 9S

Micropocliformes, order, 593

Mocropogon unduIatus, 453

Micropterus salmoides, 453

Microsporidia, 73

Midbrain of fish, 469

Hiescher's tube, 73

Migration of a nimals. 716 airways, birds, 583

Millepedes, 265

Millepora, 136

Millers, 327

Millipede, 289
Mimus polyglottos, $59 \pm$

Mind, 846

Mink, 638

Minnows, 450

mud, 450

Miracidium, 756, 758, 759

Miridae, family, 318

Mirids, 318

Misumena vatia, 295

Nite, human itch, 738

Mites, 266

Mitochonuria, 60

Mitosis, 62

Mnemiopsis, 157

Mockingbırd, 594, 595

Mole, 619

Molgula, 361

nanhattensis, 365

reproduction, 366

Mollusca, 27

classification. 256

economic relations, 255

internal morphology, 243

ploylum, 236

Molluscoida, 27, 184

Mollusk shells, 771

Iolt, 280, 601

Monacanthidae, family, 455

Monarch butterfly, $326^{\circ}$ life history, 32

Monaxon, 124

Monaxonida, order, 121

Moniezia, sheep tape, 763,765

Monkeys, 635

Monocytes, 513

Monodelphia, 617

Monoecious condition, 112

Monogenea, 161

subclass, 762

Monohybrid cross, 825

Munos,ga, 66

Ionotiemata, suborder, 616

Mooneyes, 447

Moose, 630

Moray eels. 450

Mormon cricket, 312

Morphology, 20

Morula, S07, 809

Mosaic vision, 277, 278

Mosquito fish, 80,451

life history, 329

Mosquitoes, 266

Mother-of-Pearl. 255

Moths, 266i, 325

Votor cells, 400 end plates, 400 nerve root, 527

Mouse, 625

Mouth parts of insects, 301

Mud hen, 591

puppy, 487

Mugil curema, 452

Mugilictae, family, 452

Mulatta, Sas

Müiller, Johannes, 43

Iüllerian tube, 493

Iullets, $45^{2}$

Muraenidae, family, 150

Murex tenuispina, 211

Murgantia histrionica, $31 \mathrm{~s}$

Mus, 625

Musca domestica, 330

Muscidae, family, 330

Iuscle, actions of, 524

attachments, 524

involuntary, 524

Muscles of crit, 642,644 of fish, 467

of hind limb, chicken, 611 
Muscular or contractile tissue, 106 system, 169

chicken, 012

frog, 523

phrynosoma, 576

Musculium ferrissi, 218

Musculocutancous vein, jos

Musk ox, 631

turtle, 549

Muskrats, 622,625

Mustelus canis, 425

Mutation theory, 887

Mutations, S36

Mutualism, 735

Mycetozoa, 69

Mylohyoid muscle, 46S

Myocomma, 370

Myocytes, 125

Myofibrils, 106

Myology, 64t

Myosepta, 490

Myoseptum, 370

Myotomes, $370,372,427,496,537$

Myriapoda, class, 265, 287. 289

Myrmecophaga jubata, giant anteater, 621

Myrmecophiles, 339

Myrneleonidae, iamily, 322

Mysis, 285

Mystacoceti, olcler of whales, (i34

Myxedema, 600

Myxidium, 73

Mryine 412

Myxinirlae, family, $\$ 12$

Myxinoidea, subclass, 412

Myxobolus, 73

Mrxosporidia, 73

Myzus cerasi. 318

\section{$\mathrm{N}$}

Nabidae, family, 318

Nabis ferus, 318

Nacreous layer, 250

Nagana, 80

Narwhal, 631

Nasal bones, 465

Natural resources, restoration, 794 selection. $8 \$ 5$

Natulalists of the frontier. 48

Nauplius larva, 285

Nautilus, 258

Navel, 820

Nearctic region, 712

Necator, 750

hookworm, 745

Nectocalyces, 137

Necturus, 485

circulatory system, 490

digestive system, 489

internal structure, 458

maculosus, 487

muscular system, 496

respiratory system, 491

skeleton, 494

urinogenital system, 492

Neelidae, family, 312

Nekton, 769

Nemathelminthes, 26,745

phylum, 175

Nematocyst, $13 t$

Nematoeysts, $136,138,142,147,148$

Nematorla, class, 175

Nematode, 750

Nematognathi, suborder, 450

Nemertina, class, 161

Nemertinea, 27, 192

Neoceratodus fosteri, 455
Neocomatella, 225

Neoechinorhynchus emydis. 17s

Neoscona benjamina, 296

Neotropical region, 713

Nephridia, 110, 18S, 195, 206, 207, 214, 39 :

Nephridial ducts, 189

Nephridiopore, 195, 206, 207

Nephridiopores, 201

Nephridium, 203

Nephrostome, 195, 199, 206, 207

Nephrostomes, on frog kidney, 514

Nereis virens, 195,196

Nerves, cranial, of frog, 529

Nervous function, 404

system, 110 of bullhead (ameiurus), 4 (i9

of cat, 650

chicken, 607

tevelopment, 530

of frog. 525

phrynosoma, 573

starfish, 233

sympathetic, 528

toad, 543

vertebrates, 399

tissue, 107

Nettling cells, $13 \%$

Neural arch, 165

folds, 536

plate, 536; 815

tube, $537,817,81 \mathrm{~s}$

Neurocoele, 372,527

Neuroepithelial, 40t cells, 153

Neuron, 107

Neurones, 405

Neuropodium, 196, 197

Neuroptera, order, 266, 322

Neutrons, 698

Newt, common (triturus), 484

Nicotinic acid, 389

Nictitating memblane, 562, 600, 639

Nidamental gland, 26:

Nidicolae, 615

Nidifugae, 615

Niglit lizards, 554

Nipples, 653

Nitrogen cycle, 29

Noctiluea, $(i 7$

Noctuidae, family, 327

Nodosaria hispida, 69

Nomenclature, binomial system, 22, 43

Notochord, 360, 372

Notonecticlae, family, $31 \mathrm{~s}$

Notopodium, 197

Nuclear membrane, 59, (60)

Nucla, 157

Nutritive-muscular cell, 151

Nymph. 319

Nysius ericae, 318

\section{0}

Oak-deer biome, 726

Obelia, 113, 131, 132

Occipital condyle, 494

Ocean, collditions, 771

Ocelli, 301, 345

Ocelot, 629

Ocular plate, 221

Oculina, 142

Oculomotor nerve, 527

Odobenus, walrus, 629

Odocoileus, deer, 630

Odonata, orter, 265, 320

Odontoceti, order of whale 4.63

Odynerus dorsalis, 335, 359

Oestridae, family, 330 
Oestrin, 676

Oligochaeta, 194, 196, 199

Omasum, 633

Ommatidia, 277, 302

Ommatidium, 276

Omosternum, 521

Onchocerca, volvulus, 745. 746

Oniscus, 282

Ontogeny, 113, 255

recapitulates phylogeny. 87()

Onychophora, class, 265, 287

Oöcyst, T4

Oöcyte, secondary, 115

Ooecium, 185, 187

Oögenesis, 113, 114

Oügonia, 114, 799

Ö̈kinete, 74

Opalina, 70,745

Operculum, 537

Ophichthyidae, family, 449

Ophidia, suborder 548, 50

Ophioclerma, 219

Ophiothrix, 219

Ophisaurus, 552

Oplivira, 219

Ophiuroidea, class, 219

Opisthobranchiata, 258

Opisthoglypha, 556

Opisthotic bone, 494

Opossum. 619

Optic chiasma, 526

ganglion, 262

lobes, 469

Oral groove, 93

hood. 370

lobes. 138

ossicles, 229

Orangutan, 636, 637

Orcinus orca, killer whale. 634

Oreamnos montanus, 631

Oreaster, $21 \mathrm{~S}$

Organ, 108

of corti, 403, 650

Organ pipe coral. 143

Organic catalysts. 57

Organizers, 694

Organogenesis, 801

Organs and system, 817

()iental region, 712

Origin of species, 43,863

Ornithorhynchus anatinus, 617

Orohippus, 8Tt

Orthoceracone, 259

Orthoptera, order, 265. 313

Orytricha, 71

Usculum, 122

Osmeridae, family, $44 \mathrm{~S}$

Osphradium, 253

Ospreys, 589

Ossicles, 227, 228

Osteoblasts, 691

Ostia, 122, 124, 273

Ostium, 531, 573. 652

Ostracoda, order, 264, 284

Ostraea virginica, oyster, it

Ostrich, African, 584, 5S5

Otocyst, 237, 367

Otter, 633

Otus asio, 593

Ova, 104

Ovarian follicle, $\$ 12, \$ 13$

Ovaries of cat, 652

Ovary of hen, 614

Ovibas moschatus, musk ox, 631

Oviducts, 407

of frog. 531

Oviparous, 407

Ovipositor, 318
Ovis canaderisis, mountain sheep, (;30

Ovotestis, 243

Oroviviparous condition, 248, 457

Ovum. 652

Owls, 593

bariled or hoot, 593

great horned, 593

prairie dog, 592

screech, 593

Oxyechus vociferus, 590

Oyster drill, 241

extinct, sơ 4

shell. 77

Oyster's, 255

\section{P}

Pacific shore animals, $7 \cdot$

Paddlefishes, $\$ 40$

Pagurus, 252

hernit crab. 772

Palacarctic region, 712

Palatine bones, 517

Paleontology, 554

Paleozoology, 24

Pallial line, 249, 250

Palpigradi, or ler, 266

Palps, 197

labial. 250

Palpus, 305

Pincleas, 490, tiт

Pidnullat. To

Panther, 629

Pantothenic acid, 390

Papillae, filiform, 603

fungifolm, 650

vallate, $6 \% 0$

Papula, 220

Parabacillus coloradus, 31s

Paragastric canals, $15 \mathrm{~S}$

Paragonimus, 161, 760

Paragordius, $17 \mathrm{~s}$

Parakeets, 591

Paramecinm, 70,93, 100

locomotion, 102

metabolism, 9.

reaction to temperature. 100

reproduction, 96,98

structure, 93

Paramylum, $\$ 3$

Paramyxine, 412

Parapodia, 196

Parapophyses, $4(i)$

Parapsirta, subclass, 517

Parascalops breweri, hairy-tailed mole, 619

Parasite, acciffental, Tisi occasional, 736

Parasites, facultative, 737

their transmission, 740

Parasitism, 735

Parasplienoid bone $465,495,518$

Parathormone, 670

Parathyrin, 670

Parathyroict glands, 670

Parenchyma, 165

Parietal bone, 46.5, 494

Parotoid glane, toak, 510

Parrots, 591

Parthenogenesis, 112, 191, 410

Pitrthenogonirtium, Fi:

Passalidae, family, 335

Passalus cornutus, 33.5

Passenger pigeon, 5!11, 786

Passeriformes, order, 595

Pasteur, 47

Patella, 240. 293, (\$44

larva, 237 
Pathology, 23

Paurometabolous, 308

Pavlov, 852

Pealis, altifical, 255

Peas, dwarf. 822

Pecari angulatus, javelina, 632

Peccaly, 630, 632, 787

Pecten, 35T, 608

Pectinatella, 186. 187

Pectine, 298

Pectoral girdle, toad, 542 muscles, 524

Perlal gauglion, 245

Pedicellariae, 218, 226, 229

Pediculati, suborder. 455

Pediculus, louse, 738

Pedigree chart, 843

Peripalpi. 292 orcler, 266

Peduncle, 187

Pelagic animals, 769

Pelecaniformes, order, 587

Pelecypoda, 256

Pellicle, 94

Pelmatohydra oligact1s, 144

Pelobatidae, family, 4S5

Pelvic girdle, frog, 521

Pemphigus betae, 318

Penguins, 586

Penial setae. 181

Penis, 246. 261

Penuatula, 144

Pennatulacea, order, 143

Pentacrinus, 225

Pentastomoicls, 292

Pentatomiclae, family, 318

Pepsinogen, 393

Peranema, 60

Perca flavescens, 454, 457

Perch, 411, 454

internal structure, 460

skull, 467

rellow, 457

Percidae, family, 454

Percomorphi, suborder, 452

Pereiopods, 270

Pericardial cavity, 376 sinus, 272,274

Pericardium, 567, 604, 619 of frog, 510

Perichondrium, 691

Pericolpa, 137

Periganglionic gland, 528

Perihemal space, 229

Perilymph, 530

Perioral nembrane, 227

Periosteum, 378, 644

Periostracum. 249

Peripatus, 265. 2S7, S65

Periphylla, 137

Periplaneta americana, 315

Periproct, 221

Perisare 132

Perissodactyla, order, 633

Peristalais, 382

Peristaltic contractions, 151, 196

Peristome, 227

Peristomium. 197

Peritoneum, 376

Peritrichicla, 71

Perivisceral coelom, 229 space. $27 t$

Perla modesta, 321

Peromerlusae, order, 137

Perophora, a tunicate. 772

Pessulus, 604

Petrels, 587

Petromyzon, 413

marinus, 415
Petromyzontla, subclass, $\mathbf{t 1 2}$

Petropedetes newtonl, 4S0

Phacus, 66

Phagocytosis, 395

Phalanges, 611

Phalangida, order, 266, 295, 299

Phalanx, 611

Phalaropes, 591

Pharyngeal clefts, 360

Pharyngoblanchial, 428

Phasmidae, family, 318

Pheasants, 590

Phenotype, 826

Phidippus workmanii, 296

Philodina, 190

Philomycus carolinensis, 240

Phocaena phocaen , porpoise, 634

Phoenicopteridae, family, 5ss

Phoenicopturus ruber, 588

Pholcidae, tanily, 2.7

Pholcids, 297

Pholcus phalangoides, 297

Pholiclota, order, 621

Phoronidea, 27

Phosphorescent organs, $\mathbf{7 7 1}$

Photoelectron, 707

Photons, 69s

Photosynthesis, 28, 29, 700

Phototropism, 77

Phrynosoma, 552

circulatory system, 566

digestive system, $\mathbf{5 6 3}$

ear, 574

muscular system, 576

nervous system, 573

respiratory system, 566

skeleton, 574

skull, 575

Phthirius pubius, crab louse, 738

Phyllophaga, 354 anxia, 355

Phylogenetic advances, arthropoda, 286 relations of animals, 863

Phylogeny, 23, 285

Physa anatina, 238

eggs, 247

gyrina, 237

humerosa, 238

Physalia, 136, 137

Physeter catodon, sperm whale, 634

Physiology, 22

Fhysoclisti, 447

Physocyclus, 297

Physostomi, 447

Phytonomus posticus, 324

Pia mater, 526, 650

Piciformes, order, 594

Pickerels, 450

"Pigeon milk," 591, 602

Pigeons, 591

Pigment cells, 474

Pigmentation of skin, 674

Pigs, 630

Pikas, 627

Pikes, 450

Pilidium larva, 162,163

Pill bug, 264, 265

Pine scale, 320

Pineal body of fish, 469

Pinna, 639

Pinnipedia, 628

Pinnules, 224

Pinworm, 176

Pipa. Amazonian frog, 480

Pipefish, 451

Pipunculus subvirescens, 332

Pisaster, 218

ochraceus, 227 
Pisces, 442

circulatory system, 459

class, 411,445

economic relations, 455

nuuscles, 467

Pitocin, 678

Pitressin, 678

Pituitary body of frog, 526

gland, 672

Pituitrin, 674

Placenta, 653, 819 of a rmadillo, 622

Placold scales, 422,427

Placula, 807,809

Plagioctenium irradiens, pecten, 771

Planaria, 160, 163

metabolism, 170

rezeneration, 173

reproductive system, 168

Plankton, 769

Plant lice, 318

Plantigrade gait, 641

Planula, 128, 134

larva, 139,140

Plasma, 108, 205, 392

of blood, 503

membrane, 59,60

Plasmagel, 86

Plasmalemma, 86

Plasmasol, 86

Plasmodiun, 79, 745

falciparum, 74

life cycle, 749

malarla parasite, 748

malariae, 74

vivax, 74

Plasmosome, 61

Plastron, 578

Platyhelminthes, 26,745

economic relations, 174

phylogenetic advances, 174 phylum, 160

Platyphylax designata, 322

Platypodidae, family, 335

Plecoptera, order, 265,321

Plectognathi, suborder, 455

Pleopods, 270

Plethodón cinereus, 479 glutinosus, 472

Plethodontidae, family, 484

Plethodontids, 472

Plethopsis wrighti, 485

Pleura, 649

Pleural ganglion, 245

Pleurobrachia bachei, 157

Pleurobranchiae, 271

Pleurocera, 245, 258

Pleurodira, suborder, 547

Pleuron, 268, 347

Pleuronectidae, family, 452

Pleuroperitoneal cavity, 430

Pleurum, 306

Pliny, 36

Pliohippus, 876

Plovers, 591

Plumatella, 186, 187

Pneumatic duct, 463

Pneumatophore, 135,136

Podlcal plates, 348

Podobranchiae, 271

Podophrya, 72

Poduridae, family, 312

Poecilidae family, 451

Poikilothermous condition, 545

Polar body, 115

ingression, 810

"Polian vesicles," 222, 223

Polistes, 338
Pollen basket, 357

brush, 357

combs, 357

Pollex, 563, 611

Polyaxon spicule, 125

Polybia, 338

Polychaeta, 194, 196

Polycladida, 160

Polydactylism, 659

Polydactyly, 837

Polydesmidae, family, 290

Polydon spathula, 446

Polygordius, 212, 213

Polygyra dorfeuilliana, 240

roenieri, 239

texasiana, 240

Polyhybrid cross, 825

Polynemidae, tamily, 453

Polyp, 130, 131

Polypide, 184

Polypteridae, family, 446

Polypterus, 446

Polypus, 258

bimaculatus, 257

Polystomella crispa, 68

Polyzoa, 184

Pompano, 454, 773

Populations, 730

Porcellio, 282

Porcupine, 622, 625 fishes, 455

Porifera, 26 phylum, 119

Porocyte, 126

Porospora gigantea, 59

Porpoise, 634

Portuguese man-of-war, 136

Postcaval vein of frog, 508

Postorbital crest, toad, 540

Potamobius, 266

Potato psylidd, 318

Prairie chickens, 590

dog, 623

Precocial bird, 615

Prehallux, 522

Prepartoid crest, toad, 540

Prepollex, 522

Prezygapophyses, 641

Prickleback, 321

Primates, order, 635

Primordial germ cell, 113

Pristis pectinatus, 424

Proboscidea order, 634

Proboscis, 161, 164, 179, 197, 362

Procellariiformes, order, 587

Procyon lotor, coon, 629, 788

Progesterone, 676

Progestin, 676

Proglottids, 161, 761, 763

Progynon, 676

Pronotum, 345

Pronuclei, 800

Pronucleus, 116

Pro-otic bone, 494, 517

Prophase, 62, 63

Proptera purpurata, 248

Propterygium, 430

Prorodon, 71

Prosobranchiata, 258

Prosopyles, 124

Prosostomata, order, 161

Prostate gland, 653

Prostoma rubrum, 162

Prostoma rubrim, 198, 200, 212, 215

Protandrous condition, 245

Protease, 384

Proteida, suborder, 485

Proteins, 56 
Proteoses, 385

Proteroglypha, 556

Proterospongia, 119

Prothorax, 305, 345, 357

Prothrombin, 395

Protobranchiata, 256

Protogynous condition, 245

Protons, 697

Protopipa, Amazonian frog, 480

Protoplasm, 49

and the cell, 49

characteristics, 53

chemical nature, 56

fundamental activities, 54

physical nature, 55

structure, 53

Protopodite, 270

Protopterus, 455

Prototheria, subclass, 616

Prototracheata, division, 265

Protozoa, 25

classification, 65

colonial, 75

economic relations, 77

phylum of, 65

Protractor muscle. 249

Proventriculus, 601

Prozoea, 285

Psalterium, 633

Pseudacris, 486 ocularis, 473

streckeri, 482

Pseudemys, 549

troostii elegans, 577

Pseudobranchus striatus, 485

Pseudococcus maritimus, 316,318

Pseudocoel, 181

Pseudogryphus califormianus, 589

Pseudolamellibranchia ta, 257

Pseudopliyllidea, order, 161

Pseudopodia, 67, 70, 86

Pseudopodiospore, 90

Pseudoscorpionida, order, 266

Psittacifornes, order, 591

Psocids, 333

Psychichthys affinis, 425

Psyllid, 316

Pteronarcella bodia, 321

Pteroplatea micrura, 425

Pteropods, 258

Pterosauria, 546

Pterotic bones, 466

Pterygoids, 465, 517, 613

Pterygoquadrate, 165

Pterygota, subclass, 302,313

Pterylae, 601

Pubis, 521

Pulmocutaneous arch, 507

Pulmonary veins of frog, 510

Pulmonata, 258

Pulmonate snails, 238

Pulvillus, 347

Pulvinaria vitis, 318

Puma, 629

Pupa, 330

Pupil of frog eye, 529

Pupipara, 331

Pupoides marginatus, 240

Purkinje, 49

Pycnogonids, 292

Pygidium, 316

Pygostyle, 582, 609

Pylangium, 511

Pyloric caéca, 228, 229, 459 chanber, 272

Pyralididae, family, 327

Pyrenoid bodies, 81

Pyridoxine, 389

\section{Q}

Quadrate bone, 465,467

Quadratojugal bone, 517

Quadratopterygoid, 428

Quadroon, 829

Quadrula forsheyi, 248

Quadrupeds, 616

Quadruplets, 621, 622

Quail, 590, 794

Quantum, 698

Rabbits, 625

$\mathrm{R}$

cotton tail, 62 ?

jack, 625,626

swamp, 627

Raccoon, 629, 788

Race runners, 554

Racemose bodies, 230

Rachis, 601

Radial canals, 124

cleavage, 802

nerve, 229

Radiata, 217

Radiations, 697

Radio waves, 701

Radioactive atom, 698

Radiolaria, 69,78

Radium radiation, 708

Ractula of snail, 243

Rails, 591

"Rain crow," 591

Raja erinacea, 424

Rana catesbeiana, 497

grylio, 497

pipiens, 486

Range of wild animals, 789

Rangifer caribou, 630

Ranidae, family, 486

Raphididae, family, 322

Rat, Banner-tail kangaroo, 624

Ratitae, subclass, 584

Rats, 622,625

Rattlesnakes, 556, 559

Rattus, 625

Ray, butterfly, 421

Rays, 421

Reason, 853

Recapitulation theory, 284, 869

Recessive, 45

Rectal caeca, 229, 233

Rectus abdominis, 521

Red corpuscles, 395

Rediae, 758,759

Red-legged locust, 310

Reduction division, 114

References, bibliographic, 889

Reflex arc, 405

behavior, 850

circuit, 400

Reflexes, 852

Regeneration, 681

earthworm, 211

in amphibia, 477

in chordata, 688

in hydra, 156

in planaria, 173

in platyhelminthes, 683

of starfish, 231

Regenerative capacity, 681

Reichenbachia, 340

Reindeer, 630

Pelations of animals and plants, 27

Remoras, 454

Renal corpuscles, 650

portal system, 509

vein, 434,491 
Reniceps, internal anatomy, 440 tiburo, 422,439

Renilla, 144

Rennin, 385

Reproduction, 54 and life cycle, starfish, 234 and life history, chicken, 613 asexual, 111, 153

in cat, 652

Reproductive function, 407 system, frog, 531

Reptiles, classification, 547 flying, 546

fossil, 546

Reptilia, 411 class, 545

Resources, nonrenewable, 794 renewable, 794

Respiration, aerial, 392

Respiratory sy-stem, 110

clicken, 603

frog, 514

phrynosoma, 566

vertebrates, 390

tree, 223

Resting cell, 62

Restoration of natural resources, 79

Rete testis, 572

Reticulitermes hesperus, 320

Reticulum, 633

Retina 401, 529

Retinella indentata paucilirata, 240

Retinula, 276

Retortamonas, 67

Retractor muscles, 224, 230

Rhabdamina, 69

Rhabdocoelida, 160

Rhabdom, 276, 277

Rhabdopleura, 361, 364

Rhaianectes glaucus, gray whale, 634

Rhagon, 124, 125

Rheas, 585

Rheiformes, order, 585

Rheotropism, 77

Rhincocephalia, order, 560

Rhinoderma, frog of Chile, 480

Rhizocrinus, 225

Rhizopoda, 67

Rholia microptera, digestive systen, 350

reproductive system, 353

Rhopalocera, suborder, 325

Rhyacotriton olympicus, 484

Rhynchites bicolor, 324

Rhynchocephalia, 869 order, 548

Riboflavin, 389

Ricord's frog, 472

Ring canal, 223, 230

Roaches, 313

Robber flies, 330

Robins, 595

Robler frogs, 40

Rock rollers, 321

Rocky Mountain spotted fever, 80

Rodentia, order, 621

Roe of starfish, 235

Roentgen, 698

radiation, 702

Roman scholars, 36

Ross, Major Ronald, 79

Rostellum, 763

Rostrum, 268, 427

Rotifers, 27, 188

Roundworms, 26, 175

Rugae, 564

Rumina decollata, 238

Ruminants, 632
Sable, Russian, 638

Sacculina, 265, 284, 747, 768

Sacculus, 403,530

Sacrum, 380

Sagartia, 140

Sagebrush formation, 726

jack rabbit biome, 726

Sagitta hexaptera, 192

Sailfishes, 453 blind cave (Typhlomolge), 482

Salamanders, 411, 472

Salamandra salamandra, 479

Salamandroidea, suborder, 484

Salientia, order, 485

Salinity of ocean, 767

Salmon, 447, 448

Salmonidae, family, 448

Salpa, 361

Salticus senicus, 296

Salts, inorganic, 58

Sand cricket, 315

dollar, 222

fleas, 265

Sandpipers, 591

Santonin, 183

Saprophytic, 66

nutrition, 82

Sarcode, 49

Sarcodina, 67

Sarcolemma, 106

Sarcoptes scabiei, itch nite, 738

Sarcosporidia, 73

Sartorius, 611

Sauria, 551

Saw-fisll, 424

Scale, ctenoid, 444, 464

cycloid, 444

ganoid, 444

insects, 266

San Jose, 316

Scalopus aquaticus, mole, 619

Scaphiopus, 485

couchi, 475

Scaphognathite, 270

Scaphopocla, 258

Scapula, 465,495

Scarabaeidae, family, 335

Sceloporus, 552

Sceroblasts, 125

Schistocerca, shoshone, 310

Schistosoma, blood fluke, 745,755

Schizogony, 73

Schizont, 750

Schizopod, 285

Schleiden, 43,50

Schneider, 62

Schwann, 43, 50, 51

Sciaenidae, family, 454

Sciatic vein of frog. 509

Scientific method, 18

Scincidae, family, 555

Sclera, 401 of frog eye, 52 ?

Sclerites, 301,305, 345

Scolex, $161,761,762,763$

Scolopendra, 291

Scoly-tidae, family, 335

Scomberomorus maculatus, 452

Scorpion flies, 266,333

Scorpionida, order. 266, 295

Scorpions, 266,298

Screech owl, 593

Scrombridae, family, 453

Scrotum of cat, 653

Scudderia, 315 
Scutigera, 290

Scypha, 121, 122

anatomy of, 123

Scyphomedusa, 137

Scyphozoa, class, 137

Sea butterflies, 258

cows, 634

cucumber, 223

eggs, 235

horse, 443,451

lion, 629

robins, 454

slug, 241

walnuts, 26,157

Seal, 629

Seasonal changes, 733

Sebaceous glands, 616

Secondary sexual characters, amphibia, 480

Secretin, 385, 501

Sedge-musk ox biome, 724

Segregation, 824

Selachii, subclass, 422

Self, J. Teague, 194

Semicircular canals, 437, 529, 608

Semilunar valves, 459,510

Seminal groove, 200, 209

receptacles, 202

vesicle, 437,614

frog, 531

vesicles, 202

Sense organs of frog, 528

Septae, 201

Serial homology, 271

Serosa, 818

Serous membrane, 649

Serpentes, 556

suborder, 548

Serranidae, family, 454

Serum, 393

antihuman, 884

Sesamoid bones, 641

Setae, 195, 196, 200, 201

Sex hormones, 675 inheritance of, 831

Sexual reproduction, 408 development of, 111

Shagreen, 425

Shark, nervous system, 436

pilots, 454

skeleton, 427

urinogenital system, 437

visceral skeleton, 428

Shark-liver oil, 456

Sharks, 411,422

Sheep, 630

Rocky Mountain, 630

Shikepoke, 587, 588

Ship worm, 241

Shore animals of Pacific, 774

Shovelnose shark, 422

Shrews, 619

Shrimps, 265

Siamese twins, 659

Sibbaldus musculus, whale, 634

Silphidae, 324

Silver moth, 265, 310

Silverfish, 310

Silversides, 452

Simia satyrus, orangutan, 637

Simiidae, family, 637

Simulium vittatum, 332

Sinistral, 240

Sinu-atrial valve, 490

Sinus venosus, 459

Siphlurus occidentalis, 321

Siphonaptera, order, 266, 329
Siphonoglyphe, 141, 142, 143

Siph onophora, order, 136

Siphonophore colony, 135

Siphons, 222, 365

excurrent, 250

exhalant, 252

Siphuncle, 259

Sipunculoidea, 27, 194, 215

Siren lacertina, 473,485

Sirenia, order, 634

Sirenidae, family, 485

Sistrurus, $\mathbf{5 5 9}$

Skates, 424

Skeletal system, 108

chicken, 609

fish, 464

frog, 517

phrynosoma, $\mathbf{5 7 4}$

Skeleton, appendicular, of fish, 465 of Ameiurus, 466

of cat, 640

of chicken, 610

of shark, 427

of toad, 543

of turile, 580,581

Skin, frog, 475

human, 376

Skınks, 555

Skull, frog, 517

perch, 467

phrynosoma, 575

Skunk, 638

Sleeping sickness, African, 78

"Sliders," 549

Slimy salamander, 479

Sloths, 621

Slug, 323

Smelts, 448

Sminthuridae, family, 312

Snail, 237 egg masses, 247

Snails, 257

pulmonate, 238

terrestrial, 240

Snake eels, 449

Snake, harlequin, 558 pit vipers, 559

Snakes, 411, 548, 556

coral color bands, 558

lyre, 556

worm, 557

Snappers, 454

Snipes, 591

Social life, insects, 334

relations of animals, 735

Sol state, 54

Solaster, 218

Solenoglypha, 556

Soles, 451

Solitary wasp, digestive tract, 358

Solpugida, order, 298

Somatic cells, 104

mesoderm, 536

Somatoplasm, 105

Somatopleure, 815,817

Sonoran zone, lower, 716

Sorex personatus, long-tailed shrew, 619

Sound production in mammals, 616

Sow bugs, 265

Spadefoot toad, 475

"Spanish fly." 341

Sparrows, 595

English, 596

Speoty to cunicularia, 592

hypogaea, 593

Spermatid, 114, 115

Spermatogenesis, 113

Spermatogonla, 799 
Spermatophores, 171, 493

Spermatophoric sac, 262

Spermatotheca, 246, 353

Spermatozoa, 104, 115, 407

Sphaerella, 66

Sphaeriidae, 256

Sphenethmoid bone, 518

Sphenisciformes, order, 586

Sphenodon, 546, 54S, 869

punctatum, 560

Sphenodontia, suborder, 548

Sphincter, 644

Sphyraeniaae, family, 453

Spicules, 121, 125

types of, 125

Spider, digestive system, 293

Spiders, 266, 292

crab, 295

comb-footed, 295

orb-web, 296

Spinal cord, frog, 527

Spiny dogfish, circulatory system, 432

Spiracles, $349,422,427$

Spiral valve, 422

Splanchnic mesoderm, 536

Splanchnopleure, 815

Splenial bones, 495

Splints, 874

Sponge, histology of, 126

netabolism, 127

reproduction of, 127

Sponges, 26, 119

diagrams of different types, 124

economic relations, 129

fresh water, 121

phylogenetic advances, 129

Spongilla, 121

Spongin, 125

Spongioblasts, 125

Spongioplasin, 60

Spongovostox apicedentatus, 320

Spoonbills, 446

Sporublasts, 74

Sporocyst, $756,758,759$

Sporogony, 73

Sporont stage, 750

Sporosac, 135

Sporozoa, 111 class, 72

Sporozoites, 74

Sporulation, $74,90,749$

Springtails, 265,312

Spruce-moose biome, 725

Squalus acanthias, 422 internal anatomy, 429

Squamata, order, 547,551

Squamosal bone, 494, 518

Squamous epithelium, 107

Squash bug, 318

Squid, dissection of, 261 eye of, 262

Squirrels, 623

Staves, 403

Staphy-linidae, family, 340

Starfish, 226

development, 232,234

metamorphosis, 232

regeneration, 234

Starling, 595

Statoblasts, 187

Statocysts, 121, 133, 159, 253, 262, 276

Statolith, 159, 253

Stauromedusae, order, 137

Steapsin, 385,502

Stegocephalia, 869

Stegomyia, 80

Stem-mother, 112
Stenopelmatus fasciatus, 312

Stenostomum, 160

Stentor, 70,71

regeneration, 681

Stereoblastula, 807

Sternal artery, 272

Sternebrae, 644

Sternum, 268, 306, 345

Sticklebacks, 451

Stigma, 81

Stigmata, 366

Stimuli, classes, 848

Sting, 357

ray, 423,456

"Stingaree," 425

Stipes, 345

Stomach, ruminant, 633

Stomodeum, 140,158

Stomolophus, 138

Stone canal, 221, 230, 231

flies, 265,321

Strawberry leafroller, 326

Strecker's frog, 482

Strepsiptera, order, 265, 333

Streptostylic condition, 54 ?

Strigiformes, order, 593

Strix varia, 593

Strobilization, 140

Strobilops labyrinthica texasiana, 240

Strobilus, 763

Strobula, 139, 140

Strongylocentrotus, 222

Strongyloidea, order, 176

S rongyloides stercoralis, 176

Struggle for existence, 885, 886

Struthio camelus, 584

Struhioniformes, order, 585

Sturgeons, 446

Sturnus vulgaris, 595

Stylommatophora, 258

Stylonychia, 71

Siylopids, 265

Subclavian veins of frog, 508

Subesophageal ganglia, 275

Subzenital plates, 348

Sublittoral zone, 713, 770

Submentum, 345

Subpharyngeal ganglion, 199, 208

Subumbrella, 132

Succession, 727

Succinea avara, 240

Suckers, 450

Suctoria, 70,72

Suidae, family, 632

Sunfishes, 454

Sunlight, biological effects, 700

Superciliary crest, toad, 540

Supraclavicle, 465

Supracondyloid fossa, 640

Supraoccipital bone, 465

Suprapharyngeal ganglia, 199 ganglion, 208

Suprarenal glands, 671

Suprascapula of frog, 521

Surra, 80

Survival of the fittest, 885,886

Sustentative tissue, 106

Suture, 343, 641

Swans, 589

Sweat glands, 616

Swifts, 593

Swimmerets, 270

Swimming clam, 284

Sycon, 124, 125

Sylvilagus, cotton tail rabbit, 627

Symbiont, 340

Symbiosis, 144,735 
Sympathetic nerves of frog, 526 nervous system, 528 system, 399

Sympetrum rubicundulum, 321

Symphysis, pubic, 576

Symplectic bone, 467

Synangium, 511

Synapse, 406

Synapsis, 107, 114

Syndronlidae, family, 450

Synentognathi, suborder, 451

Syngamus trachea, 176

Syrinx, 603

Syrphidae, family, 330

Syrphids, 330

Syrrhophus, 485

System, 108

Systemic arch, frog, 506

veins, 508

Systole, 396

\section{$\mathrm{T}$}

Tabanus phaenops, 331

Tachardia lacca, 341

Tachinidae, Iamily, 330

Tachinids, 330

Tachyglossus aculeatus, 617

Tadarida mexicana, 620

Taenia, 161

pisiformis, dog tapeworm, 761 saginata, beef tapeworm, $7+1,712$ serrata, dog tape, 764

solium, pork tape, 745, 763

Taeniolae, 137

Tailed amphibia, 484

Tantilla, 556

Tapeworn development, 762, 764

Tavewolms, 762

Tapirs, 633

Tarantula, 266, 296

Tarpon, 447

Tarsals, 495

Tarsiers, 635

Tarsometatarsus, 612

Tarsus, 293, 347

Taste buds of fish, 470

Taxidea taxus, badger, 628

Taxis, 77

Taxonomy, 21

Tayassuidae, family, 630

Tegmina, 347

Teiidae, family, 554

Teleostei, order, 447

Teleostomi, subclass, 446

Telophase, 62,64

Telosporidia, 73

Telson, 268

Tendons, 106, 524, 644

Ten-pounders, 447

Tentacles, $131,133,137,147,197$

Tentaculata, 157

Tentaculifera, 72

Tentaculocysts, 137

Teratology, 65

Teredo navalis, 241,255

Tergum, 268, 306, 347

Termites, 265,320

Ternitophiles, 339

Termopsis nevadensis, 320

Terns, 591

Test, 219

Testes, 572

of cat, 653

Testicle, 653

Testosterone, 676

Testudinata, order, 547, 548

Testudinidae, family, 550
Tetany, 670

Tetrabranchiata, 258

Tetragnatha laboriosa, 296

Tetranychus telarius, 298

Tetraphyllidae, order, 161

Tetrastemma, 163

Tetraxonida, order, 121

Tettigonidae, family, 315

Texas fever, 80

Thalaretos maritimus, polar bear, 629

Thaliacea, 361

Theca, 812

Theelin, 676

Thenea, 121

Theory, 18

of evolution, 863

of recapitulation, 397

Therididae, family, 295

Thermotropism, 77

Theromorpha, 869

Thiamin, 389

Thigmotropisn, 77

Thomisidae, family, 295

Thoracostei, suborder, 451

Threadfins, 453

Threadworms, 26, 175

Threshold stimulus, 77

Thrips, 265, 320

tabaci, 320

Thrombocytes of frog, 503

Thunnidae, family, 454

Thymallidae, family, 448

Thymallus tricolor 448

Thymus gland, 675

Thyone, 22:, 224

Thyroid gland, 110, 667

Thyrotropic hormone, 678

Thyroxine, 668

Thysanoptera, order, 265, 320

Thysanosoma, 765

Thysanura, order, 265, 309

Tibia, 293, 306,347

Tibiofibula of frog, 522

Tibiotarsus, 612

Tick, sheep, 330

Ticks, 266, 298

Tiedemann's bodies, $230,231,233$

Tiger, 629

beetles, 324

salamander, 484

ambystoma tigrinum, 480 shark, 422

Time scale, geologic, 872

Tinamous, 586

Tingitidae, family, 318

Tissue 105

epithelial, 105

muscular, 106

nervous, 107

sustentative, 106

vascular, 108

Toad, alteries, 542

common, 487

digestive systern, 541

embryology, 544

marine, 473

muscles, 543

narrow-mouthed, 486

nervous system, 543

obstetrical, 480

spadefoot 475

skeleton, 543

urinogenital system, $5+1$

vascular system, 542

veins, 542

Toadfishes, 455

Toads, 411, 472, 538

Tocopherol, 390 
Tomato fruitworm, 326

Tongue fish, 451

"Tornaria" larva, 364

Totipalmate swimmers, 587

Toxocara canis, 176

Toxopneustes, 800

Tracheae, 110, 603

Tracheata, section, 265

Traclielmonas, 66

Trachinotus carolinus, pompano, 773

Trachylina, order, 136

Tramatodes, $160,161,174,755$

Transitional zone, 71 J

Transmission of parasites. 740

Trapezius muscle, $42 \pi$

Trematoda, 161

Trepang, 235

Triatoma, 79

Triaxon, 125

Trichechus latirostris, manatee, 631

Trichina, $176,745,753$

Trichinella, $175,176,745,753$

Trichinelloidea, order, 176

Trichocysts, 94, 102

Trichomonas, 67

Trichoptera, order, 266, 321

Tricladida, 160

Trigeminus nerve, 527

Triggerfishes, 455

Triglidae, family, 454

Trihybrid cross, $\$ 26$

Trimorphodon, 556

Trionychidae, family, 550

Trionychoidea, suborder, 547, 550

Triploblastic embryo, 810

Tripneustes, 222

Triradiate spicule, 125

'Triturus, common newt, 484

Trivium, 227, 228

Trochanter, $293,306,317$

Trochelminthes, 27,184

Trochlea, 576

Trocllearis nerve, 527

Trochophore larva, 188, 192, 199, 213 , 237,865

Trochospongilla, 121

Trophoblast, 814

Tropical zone, 716

Tropicorbis liebmanni, 238

Tropism, 77, 849

Tropisnis in hydra, 146

in planaria, 16.

Tropistic behavior, 849

Trout, 448

eggs and embrjos, 470

Truncus arteriosus, 504

Trypanorhyncha, order, 161

Trypanosoma, 79,743

Trypanosonjes, 80

Trypetidae, family, 330

Trypsin, 502

Trypsinogen, 385

Tsetse fly, 741

Tuatara, 560

Tube foot, 221

Tuberculum, 495

Tubularia, 131

Tundra formation, 721

Tunic, 365

Tunicata, 361

Tunnies, 454

Turbellária, class, 160

Turbinated bones, 603

Turkey vulture, 588

Turkeys, 590

wild, 795
Turtle, circulatory system, ¡80

digestive system, 578

leatherback, 550

loggerliead, 550

mud, 548

painted, 549

pond, 577

resiuratory system, 579

skeleton, 580,581

snapping, 549

Troost's, 577

urinozenital system, 581

Turtes, $111,545,548$

Twins, conjoined, 659

Tympanic membrane, 403, 562, 604

Tympanum, 498, 530, 574

Typhlomolge rathbuni, 482

Typhlosole, 204

Tyrannosaurus, 546

U

Uca, 252

fiddler crab, $7: 2$

vitraviolet, 699

Unbilical cord, $\$ 20$

Unibilicus, interior, 601

Lmbo, 249

Umbridae, family, 450

Uncinate processes, 609

Cndulating membrane, 94

Unionidae, 256

Unit characters, 823

Upper Sonoran zone, 715

Ureter, 398, 572, 606

Ureters of cat, 650

Urethra, 653

of cat 650

Urinaly bladder, 490, 650

Urine, 650

Uriniferous tubules, $398,464,515$

Urinogenital system, 541

horned lizard, 571

turtle, 581

Urochorda, subphylum, 361, 365

Urodela, order, 484

Uroglena, 67

Uropods, 270

Uropygial gland, 600

Urosalpinx, 241

cinerea, 255

Urostyle, 520

Ursus horribilus, grizzly bear, 629

Uterus of cat, 652

Utriculus, $403,43 i$

frog ear, 530

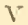

Vagina of cat, 653

Viaris uelve 527

Vallate papillie, 650

Valve semilunar 511

van Lecuwenhoek, 41

Vane, 599

Variation, 864

Vas defelens, $572,613,653$

Vasa efferentia, 531

Vascular sistem, functions, 110 tissue, 108

Veins of frog, 508 of horner lizard, 570 of toad, 5 1'

Yejovidae, family, 298

Vejovis mexicamus, 298

Ventral abdominal vein, 491, 509

Ventricles of brain, 526

Ventriculus, 601 
Venus's comb, 241 flower basket, 120,121 girdle, 157

Vermetus spiratus, 240, 241

Vermiform appendix, 878

Vermis, 650

Vertebra of frog, 519,520

Vertebral column, 378 cat, 643 frog, 520

Vertebrata, classification, 410 digestive system, 382 subphylum, 362,375

Vertebrate circulatory system, 392 excretory system, 396 skeleton, 378

Vesalius, 21,37

Vespidae, family, 338

Vespula, 338 pennsylvanica, 333

Vestibule, 608

Vestigial structures, 878

Vibracula, 185

Vibracularia, 185

Vibrissae, 639

Villosities, 476

Vinegarroon, 266

Virchow, 64

Viscachas, 625

Viscera, 109

Visceral ganglia, 245 skeleton, fish, 465 frog, 519

Vitamin D, 390 K. 390

Vitamins and their functions, 388

Vitellarium, 190, 191

Vitelline cells, 171 membrane, $115,532,819$

Vitellophages, 807

Vitrella, 276

Vitrina glacialis, 237

Vocal cords, 649

Voice of amphibia, 478

box of frog, 514

Voluntary muscle, 524

Volvox, a colony, 76, 77

Vomerine teeth, 500

von Baer, K. E., 23, 43, 284

von Mohl, 49

Vorticella, 70, 71

Vulpes fulva, red fox, 628

Vulıure, 589

California, 589

Walking legs, 270

Wallace, Alfred R., 711

Walrus, 629

Wasps, 266, 331 solitary, 335

Water, 58

dog, 487

flea, 264

moccasins, 556, 559

striders, 318

Water-vascular system, 230, 231

Wattles, 600

Wave radiations chart, 699

Waxwings, 595
Weevils, 265, 324, 325

Weismann, 47

Whale, killer, 634

sperm, 634

sulphur-bottom, 634

toothed, 634

Whalebone, 634

Wheel animalcules, 188

Whipworm, 176

White corpuscles, 513

Whitefishes, 448

IVild turkey, 795

Wildlife conservation, 784

Wolff, 798

Wolf-snout, 656

Wolves, 629

Woodchuck, 622

Woodcocks, 591

Woodpeckers, 594

downy, 595

red-headed, 595

Worm lizard, 553

shell, 241

Wrens, 595

Wuchereria bancrofti, filaria, 745,746

\section{$\mathrm{X}$}

Xantusidae, family, 554

$\mathrm{X}$-chromosome, 831

Xenodusa, 340

Xerophthalmia, 389

Xiphisternum of frog, 521

Xiphoid process, 644

Xiphosura, order, $266,295,299$

X-rays, 703

Xysticus nervosus, 295

\section{$\mathrm{Y}$}

Yellow-jacket, 333

Yolk glands, 169,191

plug, 535

sac, 819

Z

Zalophus californianus, sea lion, 629

Zebra, 633

Zenaidura macroura, 591

Zoantharia, subclass, 140

Zoea, 285

Zona pellucida, 816

Zonitoides arboreus, 240

Zooecium, 184, 187

Zoogeographical regions of Wallace, 712

Zoogeography, 20,24

Zoodd, 135

Zooids, 184

Zoology, 19

history of, 36

subdivisions of, 19

Zophodia grossulariae, 327

Zoraptera, order, 333

Zorotypus, 333

Zygapophyses, 495, 520,643

Zygodactyly, 837

Zygonectes notatus, 451

Zygoptera, suborder, 321

Zygote, 111, 115, 134, 139, 493, 824

Zymogen, 383 



